New Horizons for Lattice Computations RIKEN/RBRC Workshop with Chiral Fermions May 14-16, 2012

Proceedings of RIKEN BNL Research Center Workshop

Volume 109
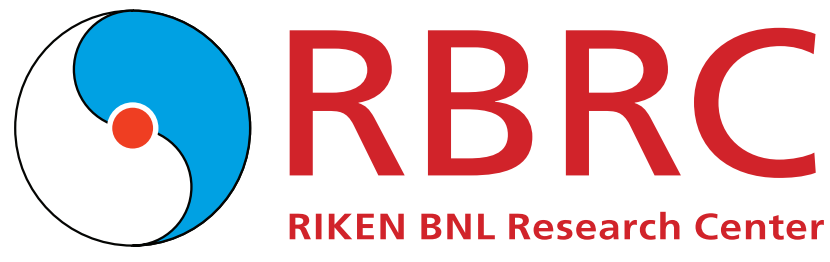


\section{DISCLAIMER}

This work was prepared as an account of work sponsored by an agency of the United States Government. Neither the United States Government nor any agency thereof, nor any of their employees, nor any of their contractors, subcontractors or their employees, makes any warranty, express or implied, or assumes any legal liability or responsibility for the accuracy, completeness, or any third party's use or the results of such use of any information, apparatus, product, or process disclosed, or represents that its use would not infringe privately owned rights. Reference herein to any specific commercial product, process, or service by trade name, trademark, manufacturer, or otherwise, does not necessarily constitute or imply its endorsement, recommendation, or favoring by the United States Government or any agency thereof or its contractors or subcontractors. The views and opinions of authors expressed herein do not necessarily state or reflect those of the United States Government or any agency thereof.

Notice: This manuscript has been authored by employees of Brookhaven Science Associates, LLC under Contract No. DE-AC02-98CH10886 with the U.S. Department of Energy. The publisher by accepting the manuscript for publication acknowledges that the United States Government retains a non-exclusive, paid-up, irrevocable, world-wide license to publish or reproduce the published form of this manuscript, or allow others to do so, for United States Government purposes. 


\section{Preface to the Series}

The RIKEN BNL Research Center (RBRC) was established in April 1997 at Brookhaven National Laboratory. It is funded by the "Rikagaku Kenkyusho" (RIKEN, The Institute of Physical and Chemical Research) of Japan. The Memorandum of Understanding between RIKEN and BNL, initiated in 1997, has been renewed in 2002 and again in 2007. The Center is dedicated to the study of strong interactions, including spin physics, lattice QCD, and RHIC physics through the nurturing of a new generation of young physicists.

The RBRC has both a theory and experimental component. The RBRC Theory Group and the RBRC Experimental Group consists of a total of 25-30 researchers. Positions include the following: full time RBRC Fellow, half-time RHIC Physics Fellow, and full-time postdoctoral Research Associate. The RHIC Physics Fellows hold joint appointments with RBRC and other institutions and have tenure track positions at their respective universities or BNL. To date, RBRC has $\sim 100$ graduates of which 29 theorists and 15 experimenters have attained tenure positions at major institutions worldwide.

Beginning in 2001 a new RIKEN Spin Program (RSP) category was implemented at RBRC. These appointments are joint positions of RBRC and RIKEN and include the following positions in theory and experiment: RSP Researchers, RSP Research Associates, and Young Researchers, who are mentored by senior RBRC Scientists. A number of RIKEN Jr. Research Associates and Visiting Scientists also contribute to the physics program at the Center.

RBRC has an active workshop program on strong interaction physics with each workshop focused on a specific physics problem. In most cases all the talks are made available on the RBRC website. In addition, highlights to each speaker's presentation are collected to form proceedings which can therefore be made available within a short time after the workshop. To date there are over one hundred proceeding volumes available.

A 10 teraflops RBRC QCDOC computer funded by RIKEN, Japan, was unveiled at a dedication ceremony at BNL on May 26, 2005. This supercomputer was designed and built by individuals from Columbia University, IBM, BNL, RBRC, and the University of Edinburgh, with the U.S. D.O.E. Office of Science providing infrastructure support at BNL. Physics results were reported at the RBRC QCDOC Symposium following the dedication. QCDSP, a 0.6 teraflops parallel processor, dedicated to lattice QCD, was begun at the Center on February 19, 1998, was completed on August 28, 1998, and was decommissioned in 2006. It was awarded the Gordon Bell Prize for price performance in 1998. The next generation computer in this sequence, QCDCQ (600 Teraflops), is expected to be fully operational in June 2012.

N. P. Samios, Director

May 2012 


\section{Contents}

\section{Presentations}

AOKI, Sinya.............................. Chiral Symmetry Restoration \& Eigenvalue Density of Dirac Operator at Finite Temperature

AOKI, Yasumichi..................... Exploring for Technicolor from QCD

AUBIN, Christopher ................An Approach to Non-leptonic B-decays on the Lattice

BERNARD, Claude ................... Status and Prospects for Some MILC and Fermilab/MILC Projects

BORICI, Artan ...........................Chiral Fermions via Energy Level Splitting

BROWER, Richard................... Prospects of Multi-grid and Domain Decomposition Dirac Solvers on GPU

CHIU, Ting-Wai .......................... Optimal Domain-Wall Fermions

COHEN, Saul ............................ Multigrid Acceleration for Domain-Wall Fermion Inversion

COSSU, Guido ......................... The Axial Symmetry at Finite Temperature in Lattice QCD with Overlap Fermions

CREUTZ, Michael ...................... On Quark Masses

DOI, Takumi.............................. Exploring Three-Nucleon Forces in Lattice QCD

FENG, Xu................................The Two-Photon Decay of Neutral Pion from Lattice QCD

FODOR, Zoltan ........................ High-precision Scale Setting in Lattice QCD

GIUSTI, Leonardo.....................Thermodynamic Potentials from Shifted Boundary Conditions

GOLTERMAN, Maarten ......... On the Hadronic Vacuum Polarization Contribution to g-2

HASENFRATZ, Anna.............. A Novel Phase with Many Flavors of Staggered Fermions

JANSEN, Karl ........................... Status of Four Flavor Simulations using Maximally Twisted Mass Fermions

JIN, Xiao-Yong........................... Exploring the Phases of QCD with Many Flavors

KANEKO, Takashi .................. Kaon Semileptonic Decays in QCD with Exact Chiral Symmetry

KELLY, Christopher................Progress Towards Delta I=1/2 K to pi pi Decays with G-parity Boundary Conditions

KIM, Hyung-Jin......................... Recent Progress in Multi GPU Conjugate Gradient Convertor with Staggered Fermion

KURAMASHI, Yoshinobu .....1+1+1 Flavor QCD+QED Simulation at the Physical Point

KUTI, Julius .............................. The Composite Higgs Mechanism and the Conformal Window

LEE, Weonjong.......................... Recent Progress in Calculating B_K using Staggered Fermions

LIN, C.-J. David .........................Lattice Study of the Phase Structure of the Strong-Yukawa Model

LIN, Huey-Wen......................... Medium-Modification Effects on Pion Structure

LIN, Meifeng............................. Many-flavor Domain Wall Fermions and Fixed Topology

LIU, Keh-Fei ................................ Progress with Overlap Fermions

MAWHINNEY, Robert .......... Decaying Pseudoscalars from DWF LQCD

MEINEL, Stefan ...................... Axial Couplings and Strong Decay Widths of Heavy Hadrons 
MISUMI, Tatsuhiro New Fermion Discretizations and their Application

MUKHERJEE, Swagato.......... Thermodynamics with Domain Wall Fermions and Anomaly in Hot QCD

NARAYANAN, Rajamani........Large N Gauge Theories with Adjoint Fermions

NEUBERGER, Herbert........... Lattice Radial Quantization

ORGINOS, Kostas................... Lattice Gauge Theory and Applications to Nuclear Physics

PERIS, Santiago..........................The Anomaly Triangle and g-2

ROBERTS, Lee ..........................The New Muon g-2 Experiment at Fermilab: A Challenge for Theorists and Experimentalists SACHRAJDA, Christopher..... Prospects for the Computation of Rare Kaon Decay Amplitudes

SAKAI, Yoshihide..................... Physics Prospects and Status of SuperKEKB/Belle II

SCHAEFER, Stefan.................. Lattice QCD with Open Boundary Conditions

SEMERTZIDIS, Yannis ..........Storage Ring Electric Dipole Moment Experiment for the Proton

SHAMIR, Yigal...........................SU(N) Gauge Theories with Symmetric-representation Fermions

SHINTANI, Eigo........................ Nucleon EDM from Lattice QCD

WITZEL, Oliver........................ B-physics with Domain-wall Light and Relativistic Heavy Quarks

YAMADA, Norikazu................. Exploring Many Flavor QCD with Wilson Fermion

YAMAZAKI, Takeshi ............... Light Nuclei from Quenched Lattice QCD

YU, Jianglei..................................Computing the K_L - K_S Mass Difference in Lattice QCD

ZHOU, Ran................................ B to Kll and B K \gamma Decay Form Factors from Three-flavor Lattice QCD

\section{Agenda}

\section{Registered List of Participants}

\section{Additional RIKEN BNL Research Center Proceeding Volumes}




\title{
Chiral Symmetry Restoration
} and

\section{Eigenvalue Density of Dirac Operator at Finite Temperature}

\author{
Sinya AOKI \\ University of Tsukuba \\ with N. Fukaya and Y. Taniguchi for JLQCD Collaboration
}

"New Horizon for Lattice Gauge Theory Computations with Chiral Fermions ", 2012.05.14-16, BNL, NY, USA 


\section{Introduction}

\section{Flavor-Chiral Symmetries of QCD at low T}

$U\left(N_{f}\right)_{L} \otimes U\left(N_{f}\right)_{R}$

flavor

chiral anomaly

(explicit breaking)

QCD at high $\mathrm{T}$

deconfinement (QGP)

restoration of chiral symmetry

low T $U(1)_{B} \otimes S\left(N_{f}\right)_{V}$ phase transition

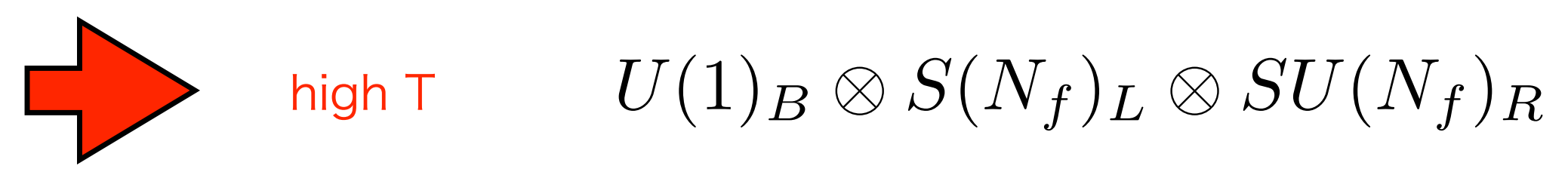

Questions in this study

1. Constraints to eigenvalue density: $\quad \rho(\lambda)=\lim _{V \rightarrow \infty} \frac{1}{V} \sum_{n} \delta\left(\lambda-\lambda_{n}\right)$

2. Constraint to singlet susceptibility: $\quad \chi_{\text {singlet }}=\int d^{4} x[\pi(x) \pi(0)-\eta(x) \eta(0)]$

spontaneous breaking of chiral symmetry 


\section{Previous studies}

$$
\rho(\lambda)=\lim _{V \rightarrow \infty} \frac{1}{V} \sum_{n} \delta\left(\lambda-\lambda_{n}\right)
$$

\section{Lin (HotQCD11), DW}

\section{Cossu et al. (JLQCD11), Overlap}
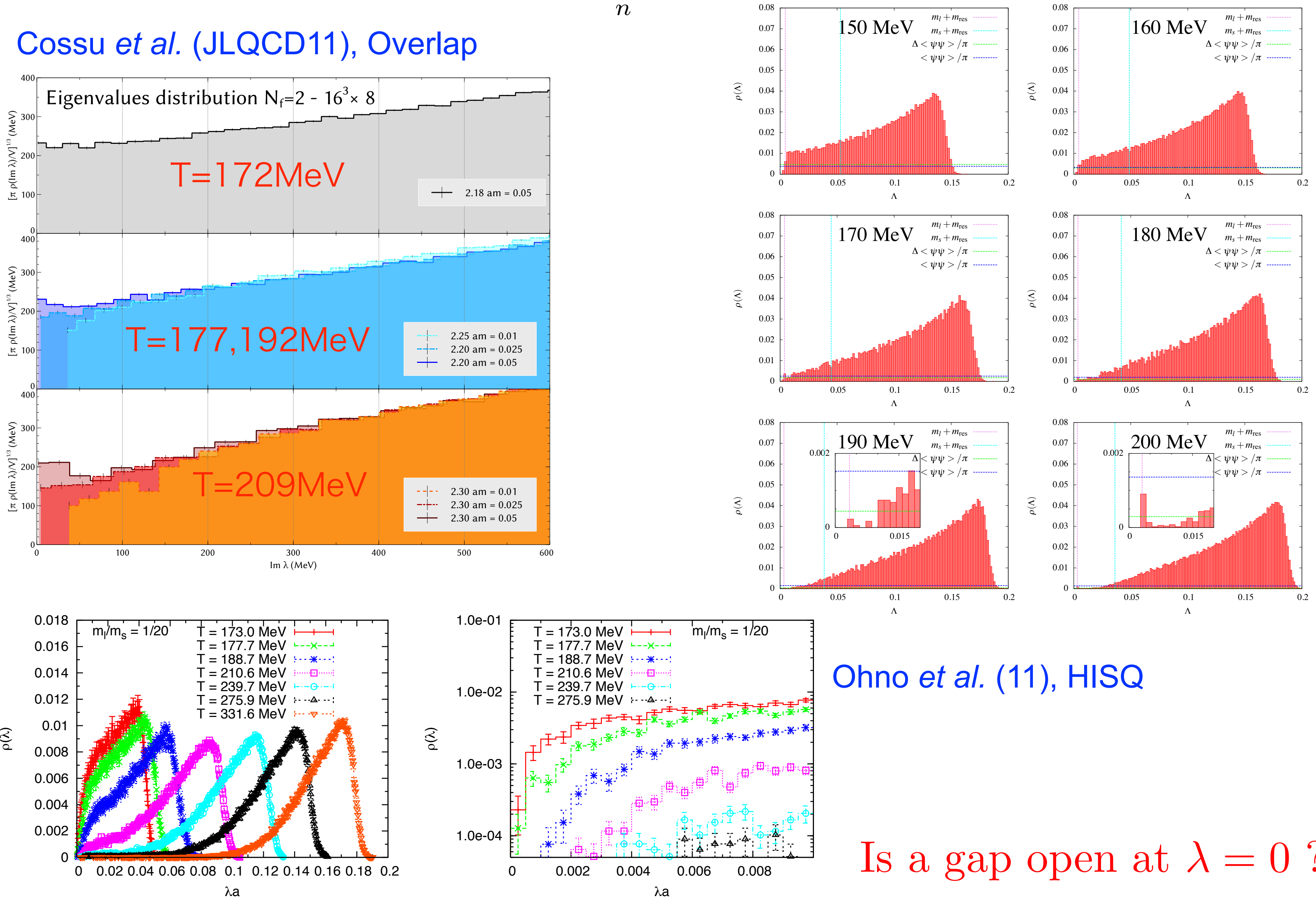

Ohno et al. (11), HISQ

Is a gap open at $\lambda=0 ?$ 
Chandrasekharan-Christ(95), KS

Bernald, et al. (96), KS
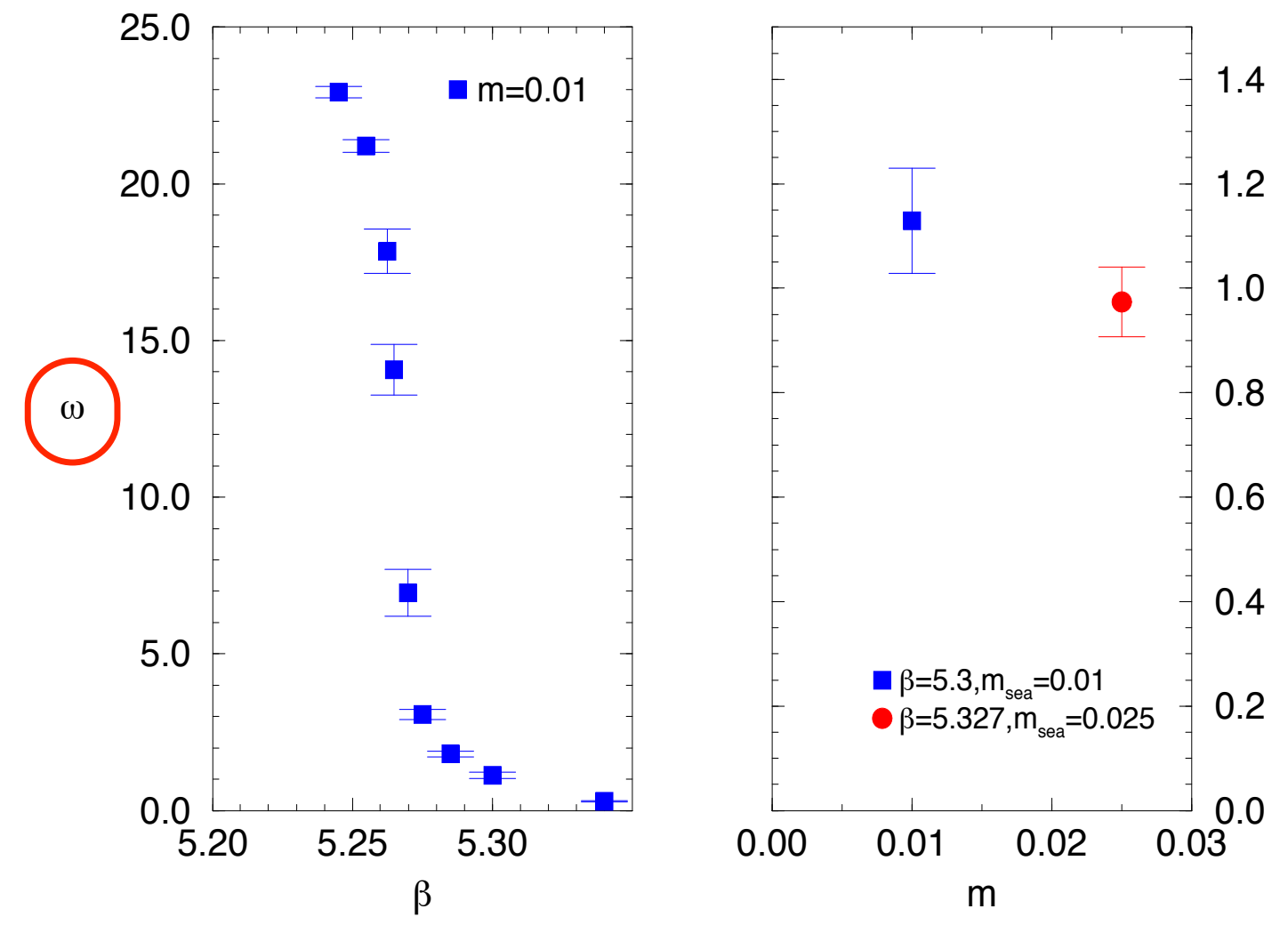

Chandrasekharan et al., (98), KS
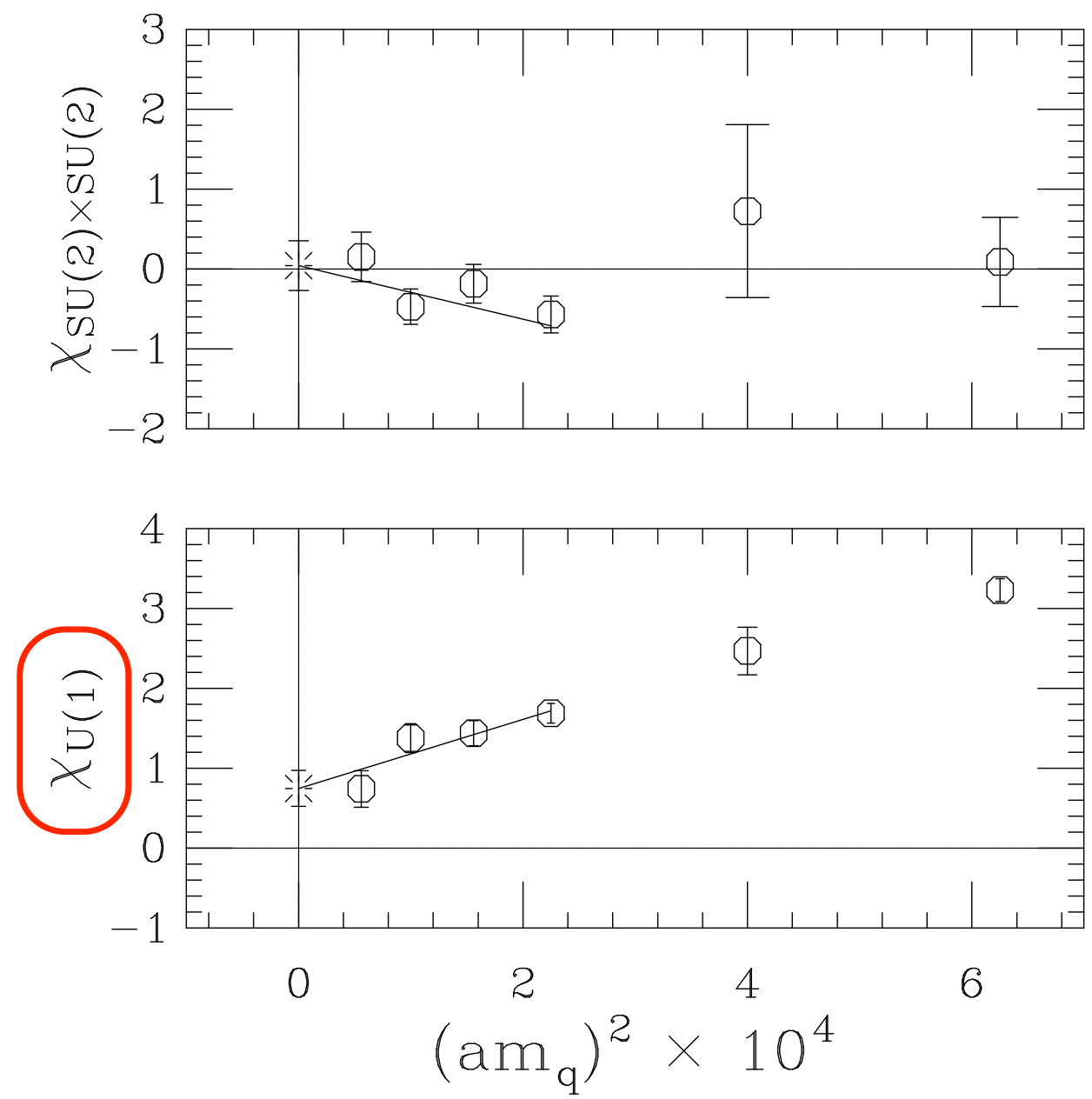

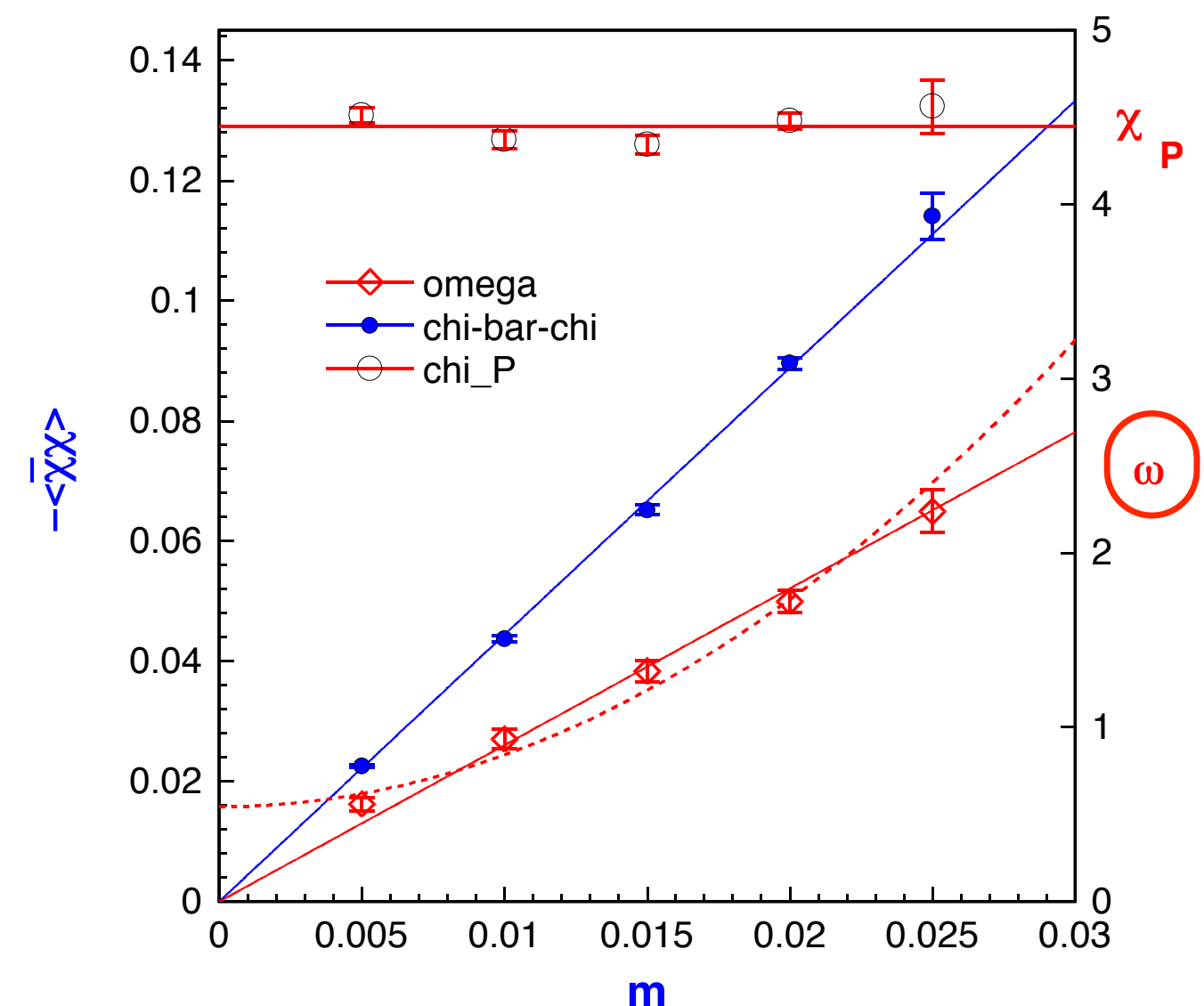

Hegde (HotQCD11), DW

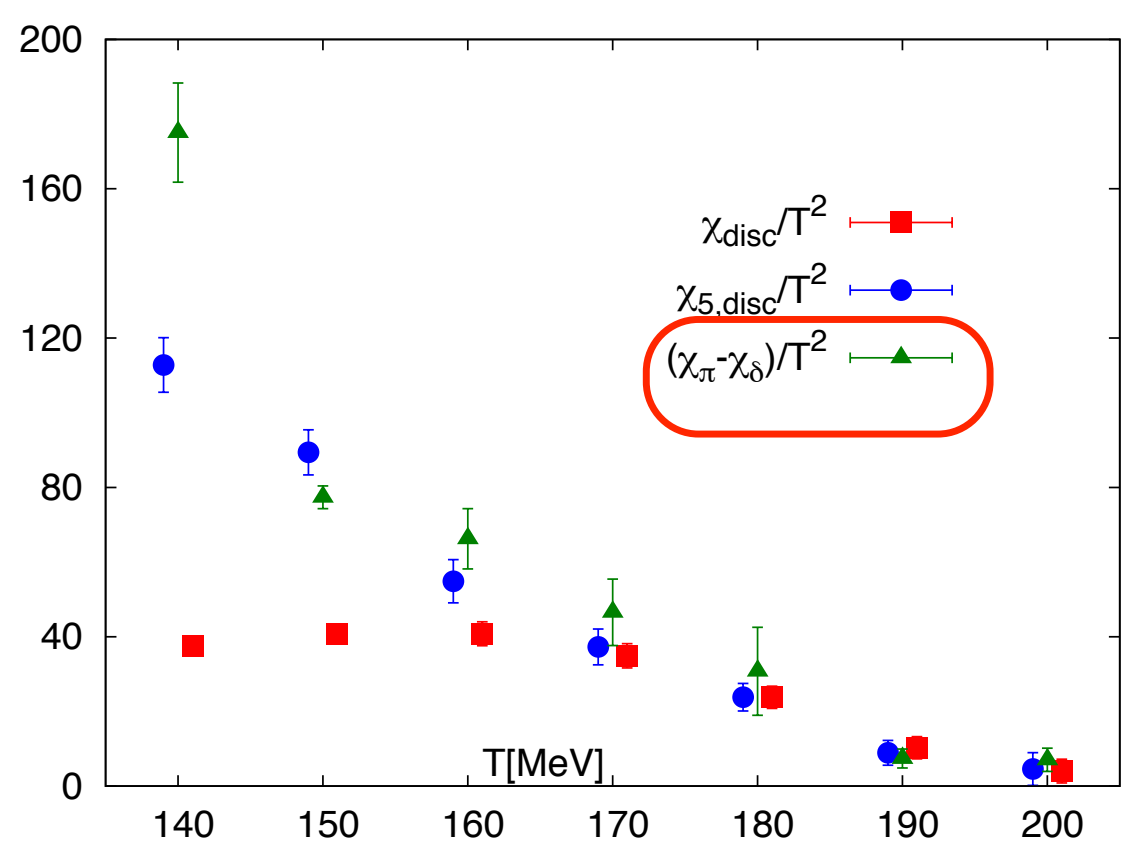


give constraints to eigenvalue densities of 2-flavor overlap fermions, if chiral symmetry in QCD is restored at finite temperature. discuss a behavior of singlet susceptibility using the constraints.

\section{Content}

1. Introduction

2. Overlap fermions

3. Constraints to eigenvalue densities

4. Discussions: singlet susceptibility 


\section{Overlap fermions}

Action $\quad S=\bar{\psi}[D-m F(D)] \psi, \quad F(D)=1-\frac{R a}{2} D$

Ginsparg-Wilson relation $\quad D \gamma_{5}+\gamma_{5} D=a D R \gamma_{5} D$

Eigenvalue spectrum

$$
\lambda_{n}^{A}+\bar{\lambda}_{n}^{A}=a R \bar{\lambda}_{n}^{A} \lambda_{n}^{A}
$$

$$
D(A) \phi_{n}^{A}=\lambda_{n}^{A} \phi_{n}^{A}
$$

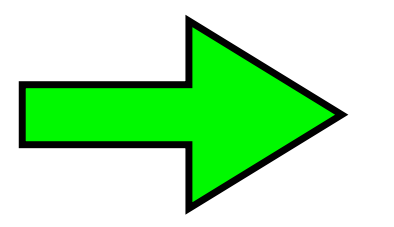

$D(A) \gamma_{5} \phi_{n}^{A}=\bar{\lambda}_{n}^{A} \gamma_{5} \phi_{n}^{A}$

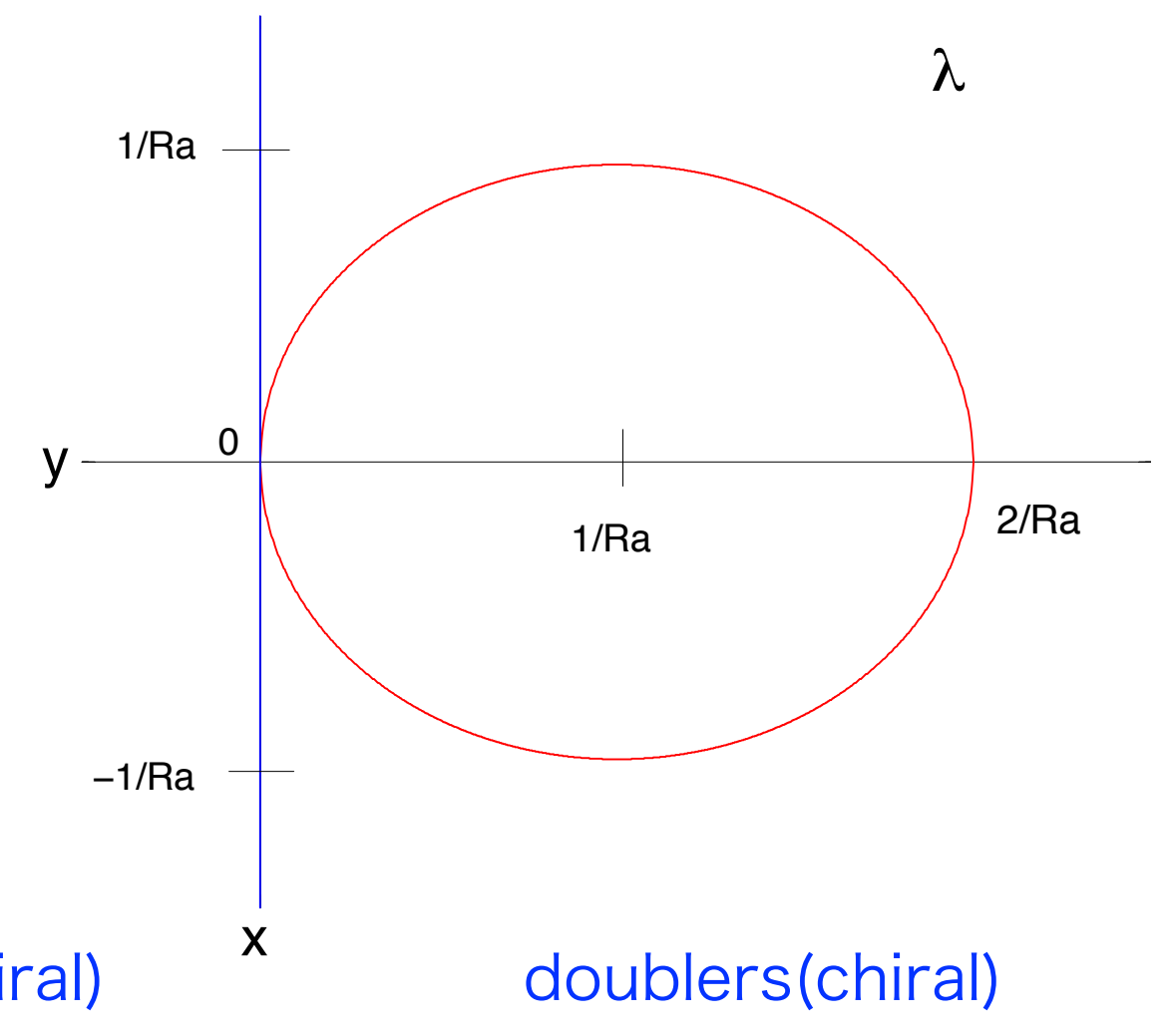

\section{Propagator bulk modes(non-chiral)}

zero modes (chiral)

doublers(chiral)

$$
\begin{gathered}
S(x, y)=\sum_{n}\left[\frac{\phi_{n}(x) \phi_{n}^{\dagger}(y)}{f_{m} \lambda_{n}-m}+\frac{\gamma_{5} \phi_{n}(x) \phi_{n}^{\dagger}(y) \gamma_{5}}{f_{m} \bar{\lambda}_{n}-m}\right]-\sum_{k=1}^{N_{R+L}} \frac{1}{m} \phi_{k}(x) \phi_{k}^{\dagger}(y)+\sum_{K=1}^{N_{D}} \frac{R a}{2} \phi_{K}(x) \phi_{K}^{\dagger}(y) \\
f_{m}=1+\frac{R m a}{2}
\end{gathered}
$$

Measure $\quad P_{m}(A)=e^{-S_{Y M}(A)}(-m)^{N_{f} N_{R+L}^{A}}\left(\frac{2}{R a}\right)^{N_{f} N_{D}^{A}} \prod_{\Im \lambda_{n}^{A}>0}\left(Z_{m}^{2} \bar{\lambda}_{n}^{A} \lambda_{n}^{A}+m^{2}\right)$ $Z_{m}^{2}=1-(m a)^{2} \frac{R^{2}}{4}$

positive definite and even function of $m \neq 0$ for even $N_{f}$ 
$\theta^{a}(x) \delta_{x}^{a} \psi(x)=i \theta^{a}(x) T^{a} \gamma_{5}(1-R a D) \psi(x)$, $\theta^{a}(x) \delta_{x}^{a} \bar{\psi}(x)=i \bar{\psi}(x) \theta^{a}(x) T^{a} \gamma_{5}$,

$$
\begin{aligned}
& \left\langle\left(J_{x}^{a}-\delta_{x}^{a} S\right) O+\delta_{x}^{a} \mathcal{O}\right\rangle=0 \\
& \int d^{4} x\left\langle\left\{J_{x}^{a}+2 m P^{a}(x)\right\} \mathcal{O}+\delta_{x}^{a} \mathcal{O}\right\rangle=0 . \quad \text { integrated WTI }
\end{aligned}
$$

"measure" term (anomaly)

$$
J_{x}^{a}=-2 i \operatorname{tr} T^{a} \gamma_{5}\left(1-\frac{R}{2} a D\right)(x, x)=-\delta^{a 0} 2 i N_{f} \operatorname{tr} \gamma_{5}\left(1-\frac{R}{2} a D\right)(x, x)
$$

Operators

$$
\begin{aligned}
& S^{a}(x)=\bar{\psi}(x) T^{a} F(D) \psi(x), \quad \text { scalar } \\
& P^{a}(x)=\bar{\psi}(x) T^{a} i \gamma_{5} F(D) \psi(x) \text {, pseudo-scalar } \\
& S^{a}=\int d^{4} x S^{a}(x), \quad P^{a}=\int d^{4} x P^{a}(x)
\end{aligned}
$$

chiral rotation at $\mathrm{N} \_\mathrm{f}=2$

$$
\begin{aligned}
\delta^{b} S^{a} & =2 \delta^{a b} P^{a}, \quad \delta^{b} P^{a}=-2 \delta^{a b} S^{a} \\
\delta^{0} S^{a} & =\delta^{a} S^{0}=2 P^{a}, \quad \delta^{0} P^{a}=\delta^{a} P^{0}=-2 S^{a}
\end{aligned}
$$

$$
\mathcal{O}_{n_{1}, n_{2}, n_{3}, n_{4}}=\left(P^{a}\right)^{n_{1}}\left(S^{a}\right)^{n_{2}}\left(P^{0}\right)^{n_{3}}\left(S^{0}\right)^{n_{4}} \quad N=\sum_{i} n_{i}, \quad n_{1}+n_{2}=\text { odd, } n_{1}+n_{3}=\text { odd }
$$

$$
\lim _{m \rightarrow 0}\left\langle\delta^{a} \mathcal{O}_{n_{1}, n_{2}, n_{3}, n_{4}}\right\rangle_{m}=0
$$

$\frac{\delta^{a}}{2} \mathcal{O}_{n_{1}, n_{2}, n_{3}, n_{4}}=-n_{1} \mathcal{O}_{n_{1}-1, n_{2}, n_{3}, n_{4}+1}+n_{2} \mathcal{O}_{n_{1}, n_{2}-1, n_{3}+1, n_{4}}-n_{3} \mathcal{O}_{n_{1}, n_{2}+1, n_{3}-1, n_{4}}+n_{4} \mathcal{O}_{n_{1}+1, n_{2}, n_{3}, n_{4}-1}$ 


\section{Constraints to eigenvalue densities}

$\mathrm{N}=1 \quad \mathcal{O}_{1,0,0,0}=P^{a}, \delta^{a} \mathcal{O}_{1,0,0,0}=-2 S^{0}$

$$
\begin{aligned}
& \frac{1}{V}\left\langle S^{0}\right\rangle_{m}=\underline{\frac{N_{f}}{m V}\left\langle N_{R+L}^{A}\right\rangle_{m}}+N_{f}\left\langle I_{1}\right\rangle_{m} \quad\left\langle\mathcal{O}^{A}\right\rangle_{m}=\frac{1}{Z} \int \mathcal{D} A P_{m}(A) \mathcal{O}^{A} \\
& \rightarrow 0 \text { as } V \rightarrow \infty \\
& I_{1} \rightarrow \frac{1}{Z_{m}} \int_{0}^{\Lambda_{R}} d \lambda \underline{\rho^{A}(\lambda)} \frac{2 m_{R}}{\lambda^{2}+m_{R}^{2}} g_{0}(\lambda) \\
& m_{R}=\frac{m}{Z_{m}}, g(\lambda)=1-\frac{\lambda^{2}}{\Lambda_{R}^{2}} \\
& \Lambda_{R}=\frac{2}{R a}: \text { cut-off } \\
& \rho^{A}(\lambda) \equiv \lim _{V \rightarrow \infty} \frac{1}{V} \sum_{n} \delta\left(\lambda-\sqrt{\bar{\lambda}_{n}^{A} \lambda_{n}^{A}}\right)=\sum_{k=0}^{\infty} \rho_{k}^{A} \frac{|\lambda|^{k}}{k !}=\rho_{0}^{A}+\rho_{1}^{A}|\lambda|+\cdots \\
& \Longrightarrow I_{1}=\pi \rho_{0}^{A}+O(m)
\end{aligned}
$$

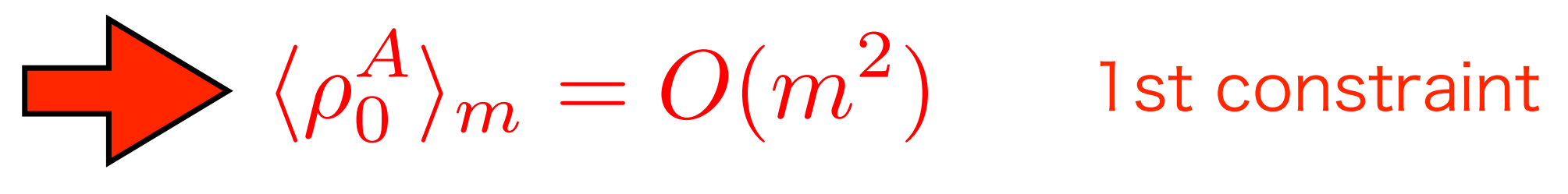


$\mathrm{N}=2 \quad \chi^{\sigma-\pi}=\frac{1}{V^{2}}\left\langle S_{0}^{2}-P_{a}^{2}\right\rangle_{m}, \quad \chi^{\eta-\delta}=\frac{1}{V}\left\langle P_{0}^{2}-S_{a}^{2}\right\rangle_{m}$

$$
\begin{aligned}
& \chi^{\sigma-\pi}=\left\langle N_{f}^{2}\left(\frac{1}{m V} N_{R+L}+I_{1}\right)^{2}\right\rangle_{m}+O\left(\frac{1}{V}\right) \\
& \lim _{m \rightarrow 0} \lim _{V \rightarrow \infty} \frac{1}{m^{2} V^{2}} \frac{\left\langle\left(N_{R+L}^{A}\right)^{2}\right\rangle_{m}}{O\left(m^{N_{f} V}\right)}=\lim _{m \rightarrow 0} \lim _{V \rightarrow \infty} \frac{1}{m V} \frac{\left\langle N_{R+L}^{A} \rho_{0}^{A}\right\rangle_{m}}{O\left(m^{N_{f} V}\right)}=\lim _{m \rightarrow 0} \frac{\left\langle\left(\rho_{0}^{A}\right)^{2}\right\rangle_{m}}{O\left(m^{2}\right)}=0
\end{aligned}
$$

These conditions are automatically satisfied.

$$
\begin{gathered}
\chi^{\eta-\delta}=N_{f}\left\langle\frac{1}{m^{2} V}\left\{2 N_{R+L}-N_{f} Q(A)^{2}\right\}+\frac{1}{Z_{m}}\left(\frac{I_{1}}{m_{R}}+I_{2}\right)\right\rangle_{m} \\
\frac{I_{1}}{m_{R}}+I_{2}=\rho_{0}^{A}\left(\frac{\pi_{m}}{m}+\frac{2}{\Lambda_{R}}\right)+2 \rho_{1}^{A}+O(m), \quad N_{2}^{A}-\frac{2}{Z_{m}} \int_{0}^{\Lambda_{R}} d \lambda \rho^{A}(\lambda) \frac{m_{R}^{2}-\lambda^{2} g_{0}\left(\lambda^{2}\right) g_{m}}{\left(\lambda^{2}+m_{R}^{2}\right)^{2}}, \quad g_{m}=\frac{1}{Z_{m}^{2}}\left(1+\frac{m^{2}}{2 \Lambda_{R}^{2}}\right)
\end{gathered}
$$

$$
\lim _{m \rightarrow 0} \chi^{\eta-\delta}=0 \Longrightarrow \lim _{m \rightarrow 0} \frac{N_{f}^{2}\left\langle Q(A)^{2}\right\rangle_{m}}{\frac{m^{2} V}{O\left(m^{N_{f} \sqrt{V}-2}\right)}}=2 \lim _{m \rightarrow 0}\left\langle\rho_{1}^{A}\right\rangle_{m}
$$

$\checkmark\left\langle\rho_{1}^{A}\right\rangle_{m}=O\left(m^{2}\right) \quad$ 2nd constraint 
$\mathrm{N}=3$

$\left\langle\mathcal{O}_{2001}\right\rangle_{m} \rightarrow 0, \quad\left\langle-\mathcal{O}_{0201}+2 \mathcal{O}_{1110}\right\rangle_{m} \rightarrow 0, \quad\left\langle\mathcal{O}_{0021}+2 \mathcal{O}_{1110}\right\rangle_{m}=0$

$\left\langle-\mathcal{O}_{0003}+2 \mathcal{O}_{2001}\right\rangle_{m} \rightarrow 0, \quad\left\langle\mathcal{O}_{0021}-\mathcal{O}_{0201}+\mathcal{O}_{1110}\right\rangle_{m} \rightarrow 0$,

+ positivity

$$
\lim _{m \rightarrow 0} \lim _{V \rightarrow \infty} \frac{\chi_{3}}{V^{4}}=\pi N_{f} \lim _{m \rightarrow 0}\left[\frac{\left\langle\rho_{0}^{A}\right\rangle_{m}}{m^{2}}+\frac{2 \bar{\rho}_{1}}{m \pi}+\frac{\left\langle\rho_{2}^{A}\right\rangle_{m}}{2}\right],=0
$$

$\checkmark\left\langle\rho_{0}^{A}\right\rangle_{m}=O\left(m^{4}\right),\left\langle\rho_{2}^{A}\right\rangle_{m}=O\left(m^{2}\right)$

3rd constraints

$\mathrm{N}=4 \quad$ No additional constraints

Final results

$$
\lim _{m \rightarrow 0}\left\langle\rho^{A}(\lambda)\right\rangle_{m}=\lim _{m \rightarrow 0}\left\langle\rho_{3}^{A}\right\rangle_{m} \frac{|\lambda|^{3}}{3 !}+O\left(\lambda^{4}\right)
$$




\section{Discussion:Singlet susceptbility}

\section{Singlet susceptibility}

$$
\begin{gathered}
\chi^{\pi-\eta}=\lim _{V \rightarrow \infty} \frac{N_{f}^{2}}{m^{2} V}\left\langle Q(A)^{2}\right\rangle_{m}=\left(m^{N_{f} \sqrt{V}-2}\right) \\
\lim _{m \rightarrow} \chi^{\pi-\eta}=0
\end{gathered}
$$

singlet susceptibility becomes zero if the chiral symmetry is recovered at hight $\mathrm{T}$.

This, however, does not mean U(1)_A symmetry is recovered at high T.

$\lim \chi^{\pi-\eta}=0$ is necessary but NOT sufficient for the recovery of U(1)_A. $m \rightarrow$

Future new constraints at $\mathrm{N}>4$ ?

eigenvalues of Dirac operator have a gap near zero ?

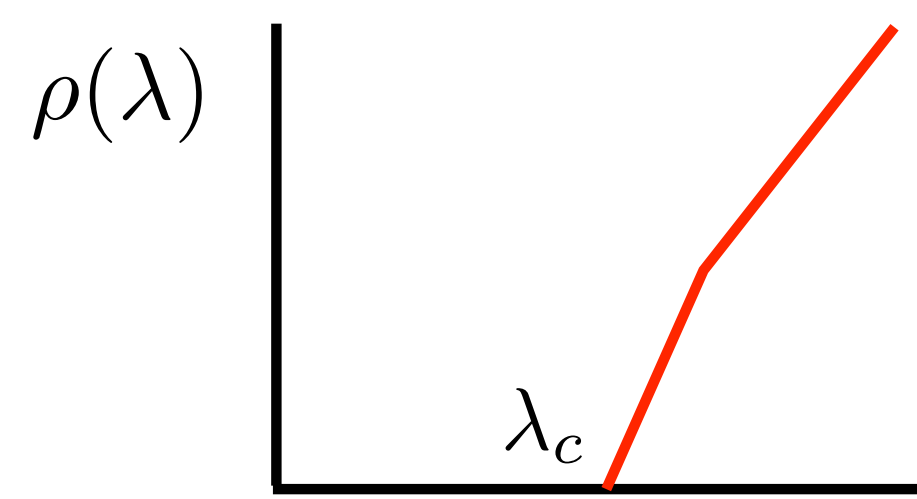




\section{Exploring technicolor from QCD}

Yasumichi Aoki [Koboyashi-Maskawa institute, Nagoya University] for the LatKMI collaboration

- RBRC workshop: New Horizons for Lattice Gauge Theory Computations -

图而名古屋大学 May 16, 2012 \begin{tabular}{|l|l|l|}
\hline $\mathrm{K}$ & $\mathrm{M}$ & $i$ \\
$\mathrm{I}$ & $\mathrm{M}$ & $\mathrm{X}$ \\
$\mathrm{K}$ & $\mathrm{M}$ & $\mathrm{I}$ \\
\hline
\end{tabular} 


\section{LatKMl collaboration}

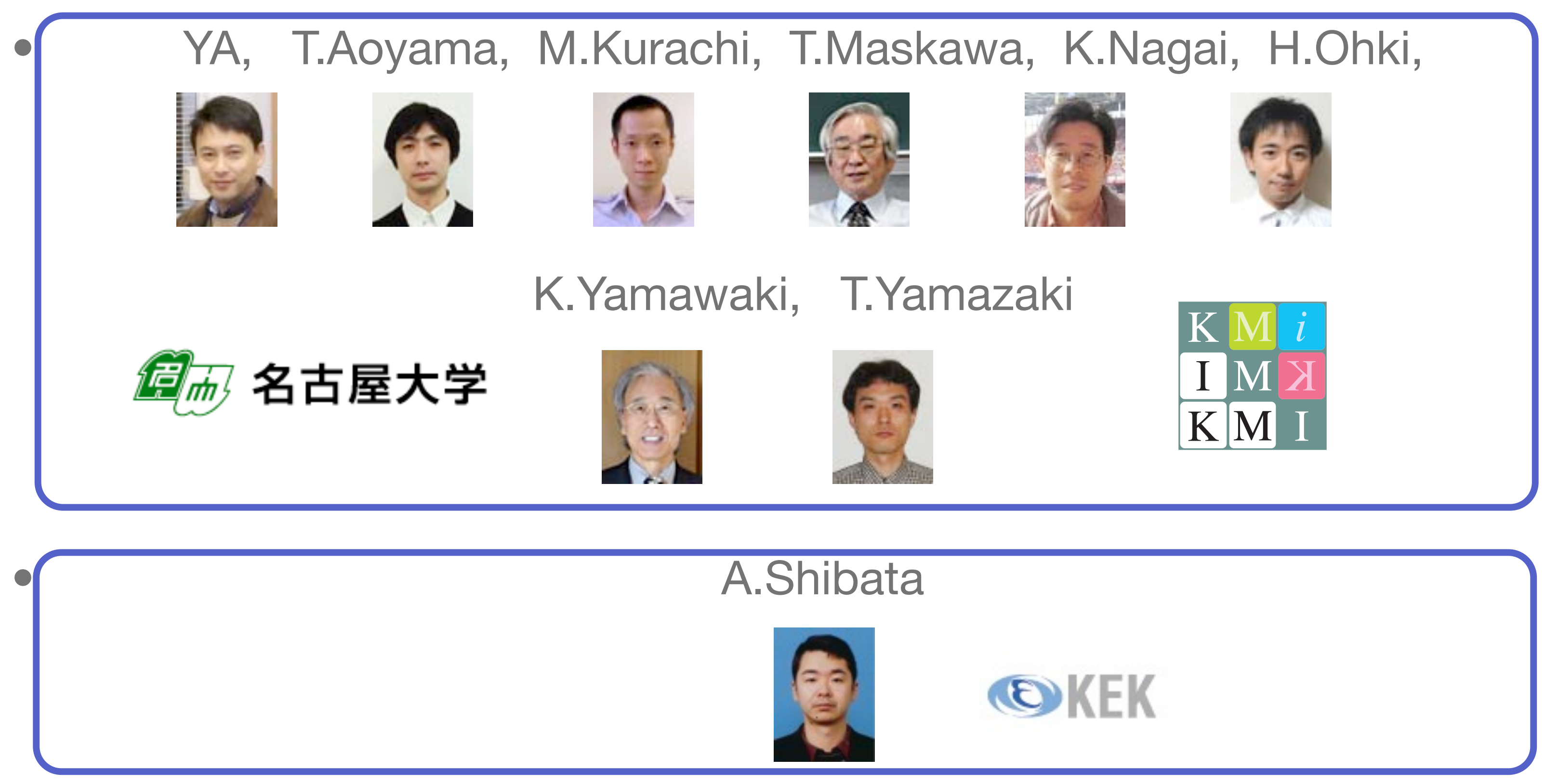


conformal window and walking coupling

- non-Abelian gauge theory with $\mathrm{N}_{f}$ massless fermions -
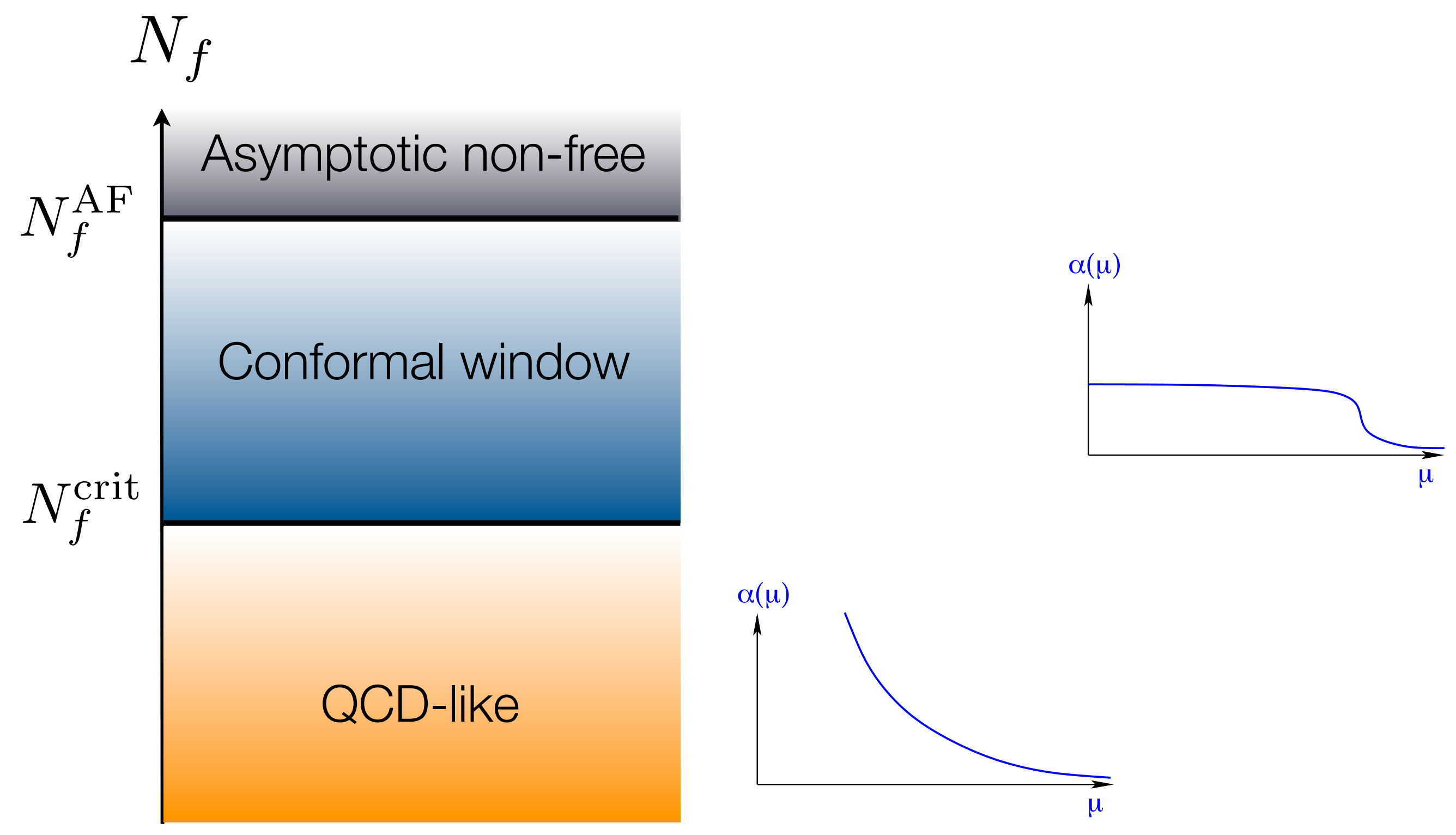


\section{conformal window and walking coupling}

- non-Abelian gauge theory with $\mathrm{N}_{f}$ massless fermions -
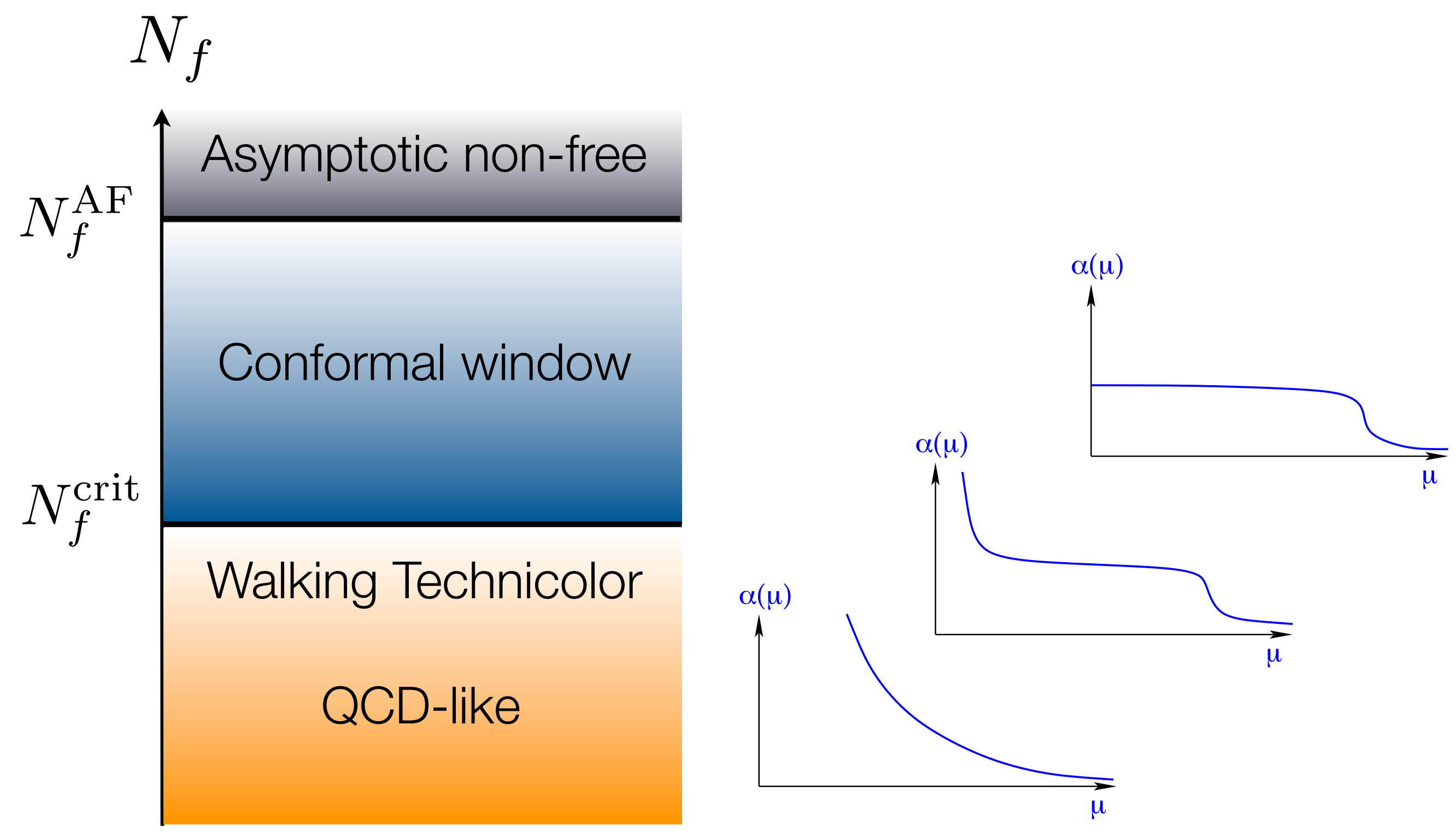


\section{conformal window and walking coupling}

- non-Abelian gauge theory with $\mathrm{N}_{\mathrm{f}}$ massless fermions -

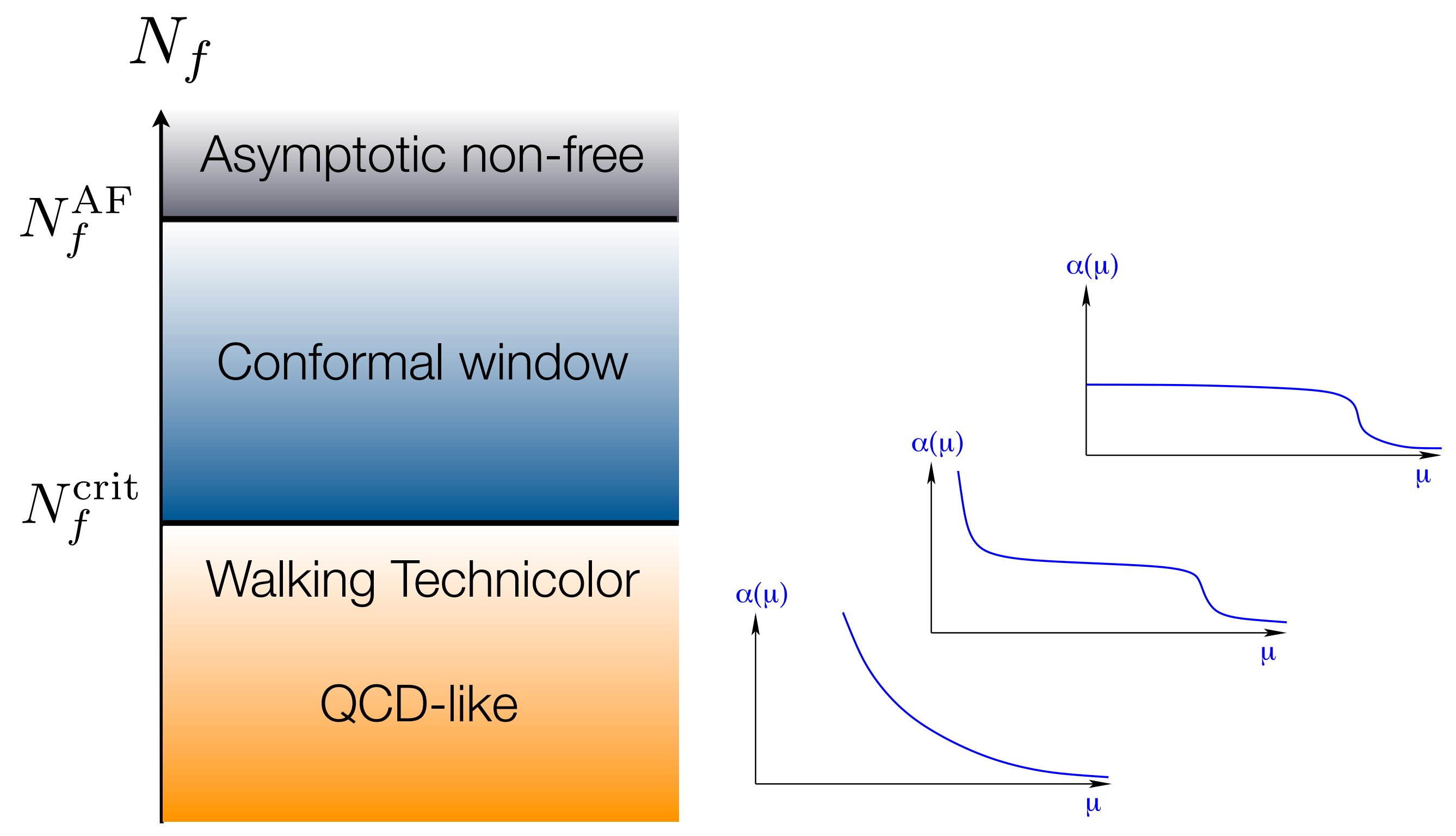

- Walking Techinicolor could be realized just below the conformal window 


\section{conformal window and walking coupling}

- non-Abelian gauge theory with $\mathrm{N}_{\mathrm{f}}$ massless fermions -

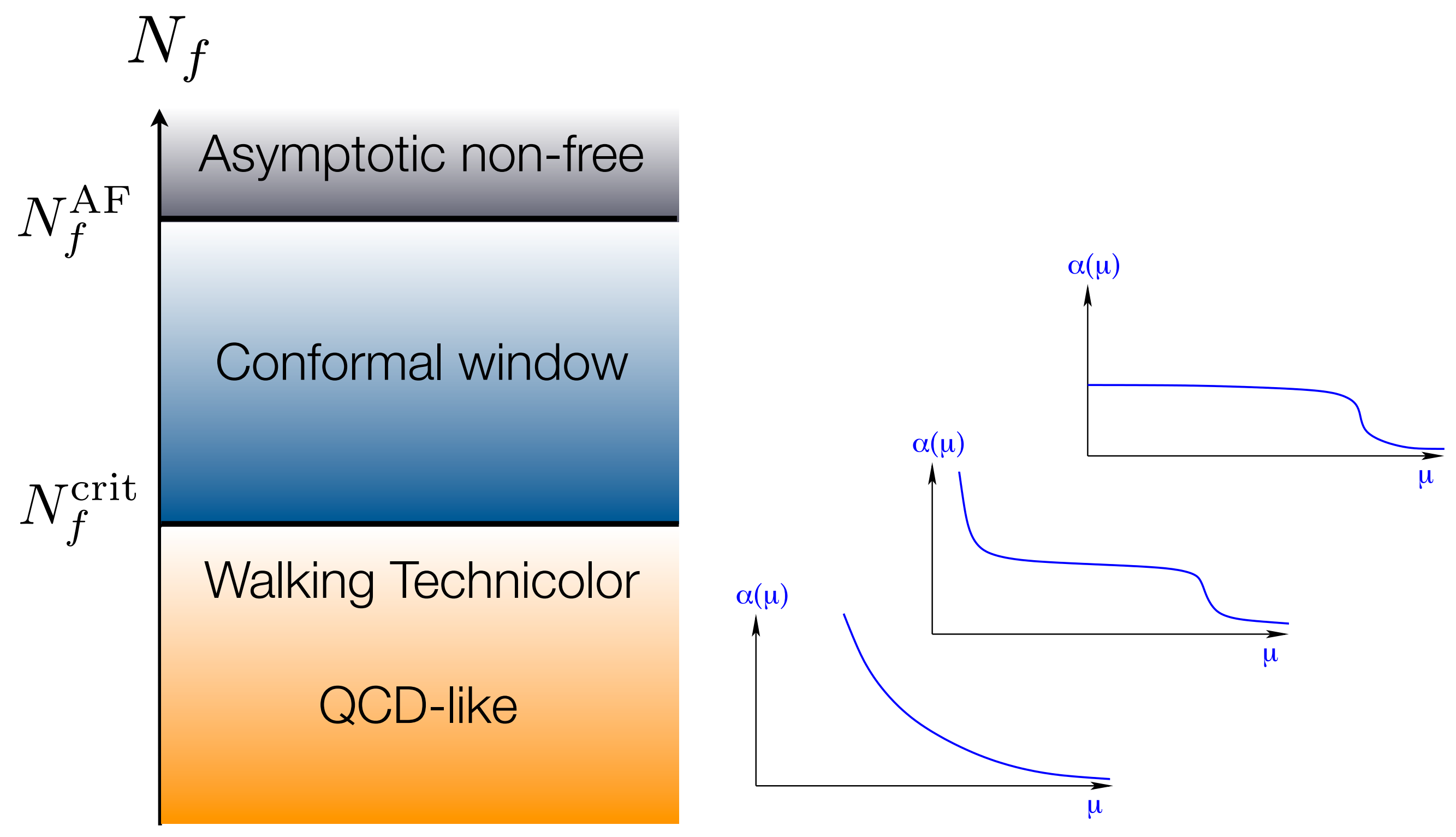

- Walking Techinicolor could be realized just below the conformal window

- crucial information: $\mathrm{N}_{\mathrm{f}}$ crit \& mass anomalous dimension around $\mathrm{N}_{\mathrm{f}}$ crit 


\section{models being studied:}

- $\mathrm{SU}(3)$

- fundamental: $\mathrm{Nf}=6,8,10,12,16$

- sextet: $\mathrm{Nf}=2$

- $S U(2)$

- adjoint: $\mathrm{Nf}=2$

- fundamental: $\mathrm{Nf}=8$

- $S U(4)$

- decuplet: $\mathrm{Nf}=2$

\section{SU(N) Phase Diagram}

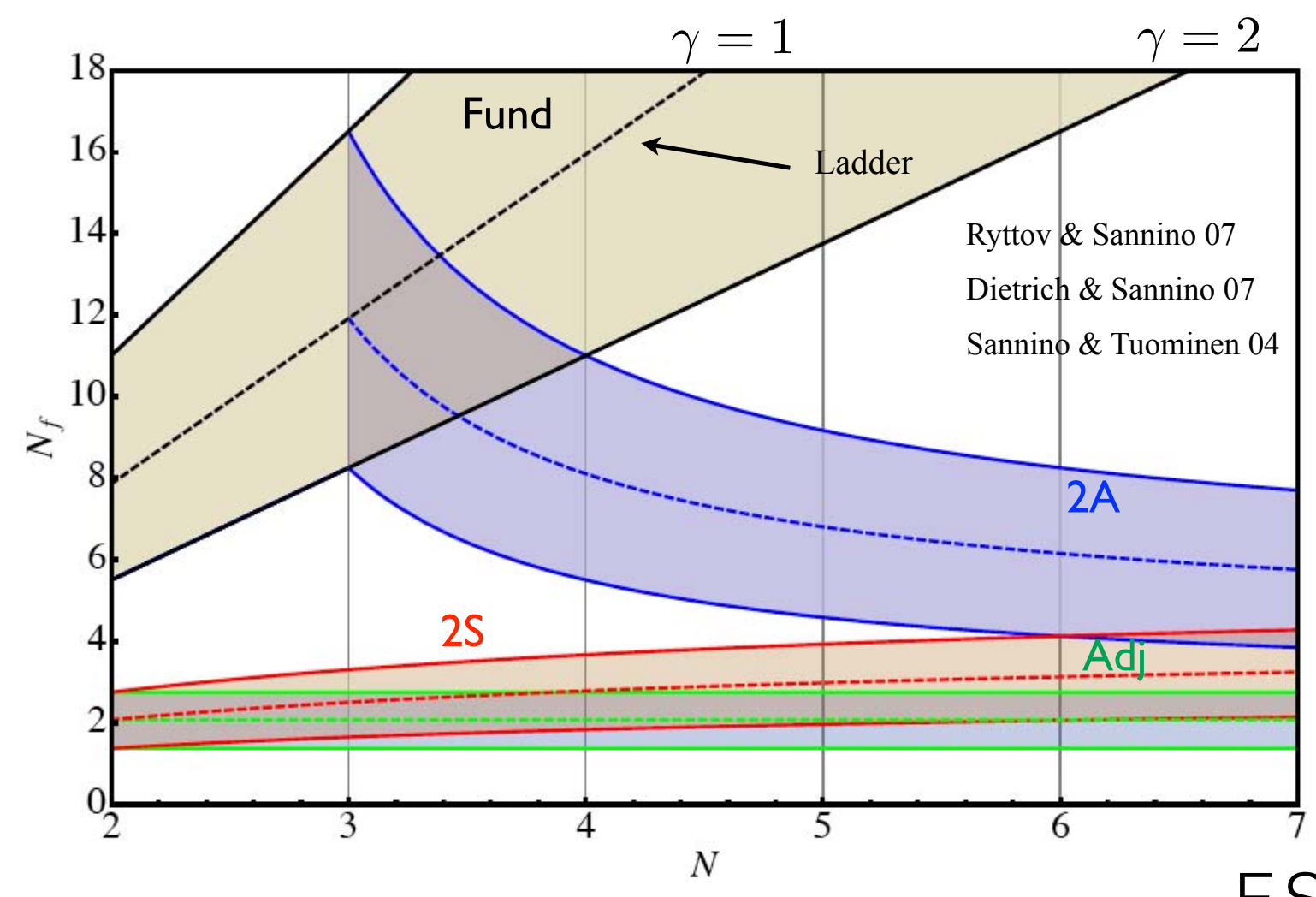

F.Sanino 


\section{models being studied:}

- $\mathrm{SU}(3)$

- fundamental: $\mathrm{Nf}=6,(8,10,12$, (16)

- sextet: $\mathrm{Nf}=2$

- $S U(2)$

- adjoint: $\mathrm{Nf}=2$

- fundamental: $\mathrm{Nf}=8$

- $S U(4)$

- decuplet: $\mathrm{Nf}=2$

\section{SU(N) Phase Diagram}

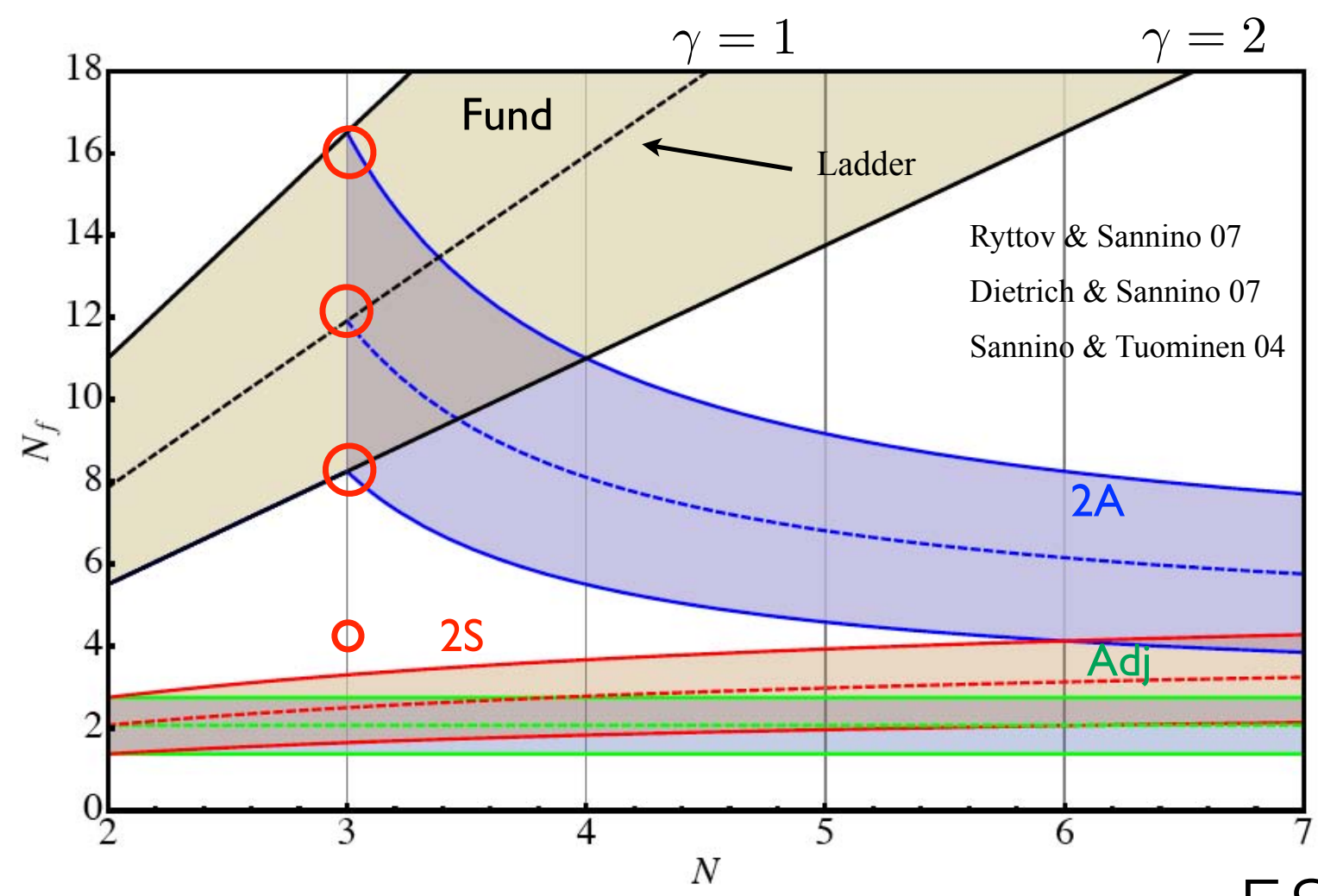

F.Sanino 


\section{models being studied:}

- $\mathrm{SU}(3)$

- fundamental: Nf=6,8, 10, (12) (16)

- sextet: $\mathrm{Nf}=2$

- $S U(2)$

- adjoint: $\mathrm{Nf}=2$

- fundamental: $\mathrm{Nf}=8$

- $S U(4)$

- decuplet: $\mathrm{Nf}=2$

\section{SU(N) Phase Diagram}

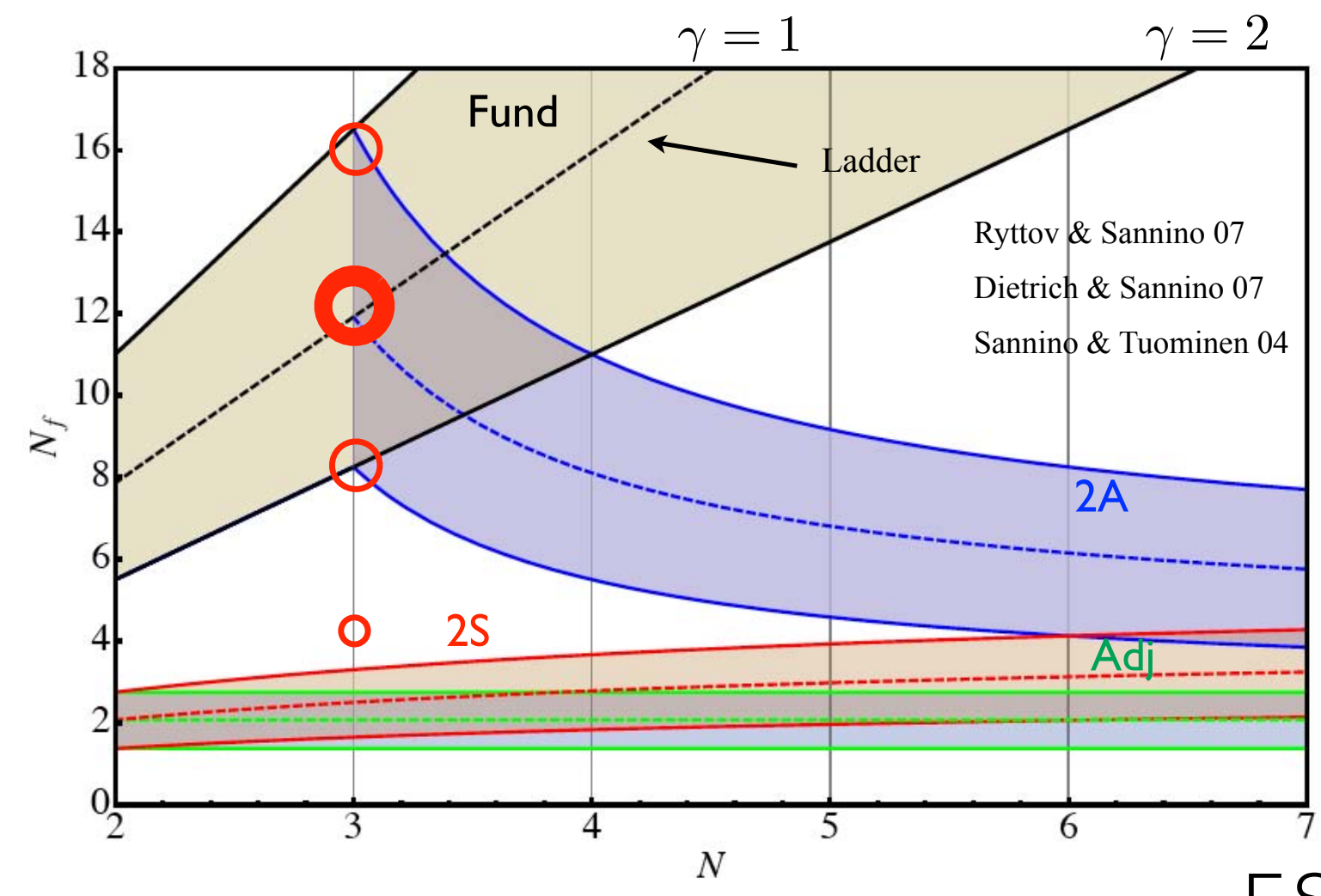

F.Sanino 
$\mathrm{SU}(3)+\mathrm{N}_{\mathrm{f}}=12 \quad$ [fundamental] 


\section{Hadron spectrum: $\mathrm{m}_{\mathrm{f}}$-response in mass deformed theory}

- IR conformal phase:

- coupling runs below $\mu=m_{f}$ : like $n_{f}=0$ QCD with $\Lambda_{Q C D} \sim m_{f}$

- multi particle / glueball state : $\left.\left.M_{H} \propto m_{f}^{1 /(1+\gamma} m^{*}\right) ; \quad F_{\pi} \propto m_{f}^{1 /(1+\gamma} m^{*}\right)$

- S $\chi$ SB phase:

- ChPT (but, large $N_{f}$, small $F \quad \Leftrightarrow$ real QCD)

- hard to get to the chiral regime

- at leading: $M_{\pi}^{2} \propto m_{f}, ; F_{\pi}=F+c m_{f}$

- so far no chiral logs are observed $\rightarrow$ polynomial in $\mathrm{m}_{\mathrm{f}}$ 


\section{Simulation}

- $\mathrm{N}_{\mathrm{f}}=12$ HISQ (Highly Improved Staggered Quarks)

- tree level Symanzik gauge

- $\beta=6 / g^{2}=3.7, \quad V=L^{3} x T: L / T=3 / 4 ; L=18,24,30, \quad 0.04 \leqq m_{f} \leqq 0.2$

- $\beta=6 / g^{2}=4.0, \quad V=L^{3} x T: L / T=3 / 4 ; L=18,24,30, \quad 0.05 \leqq m_{f} \leqq 0.24$

- $\mathrm{N}_{\mathrm{f}}=4 \mathrm{HISQ}$ for the reference of $\mathrm{S} \chi \mathrm{SB}$ for comparison

- using MILC code v7 with some modifications 


\section{staggered flavor symmetry for $\mathrm{N}_{\mathrm{f}}=12 \mathrm{HISQ}$}

- comparing mesonic mass with local PS and V operators for $\beta=3.7$

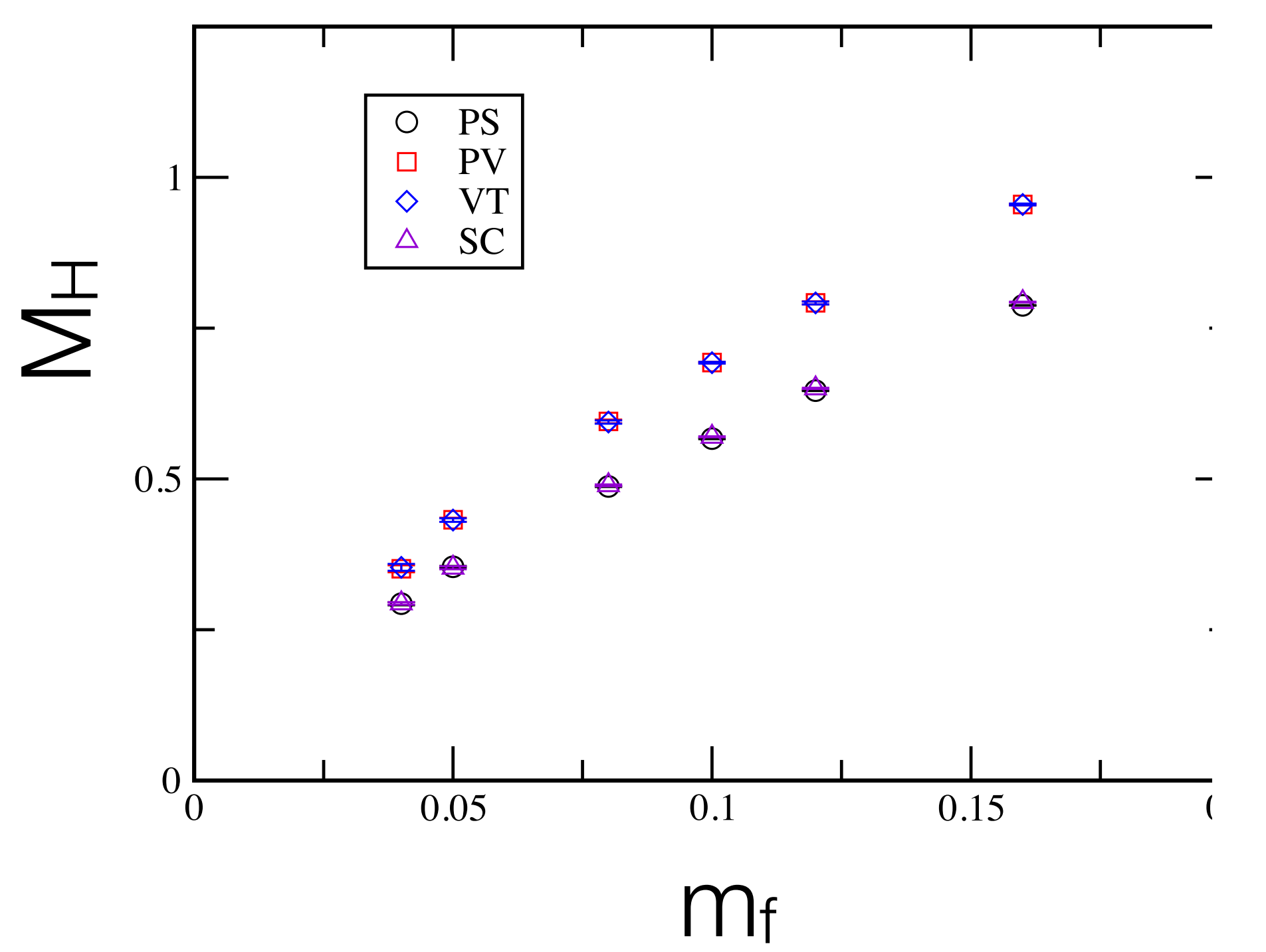


a crude analysis: $F_{\pi} / M_{\pi} v s M_{\pi}$

$N_{f}=12: H I S Q$

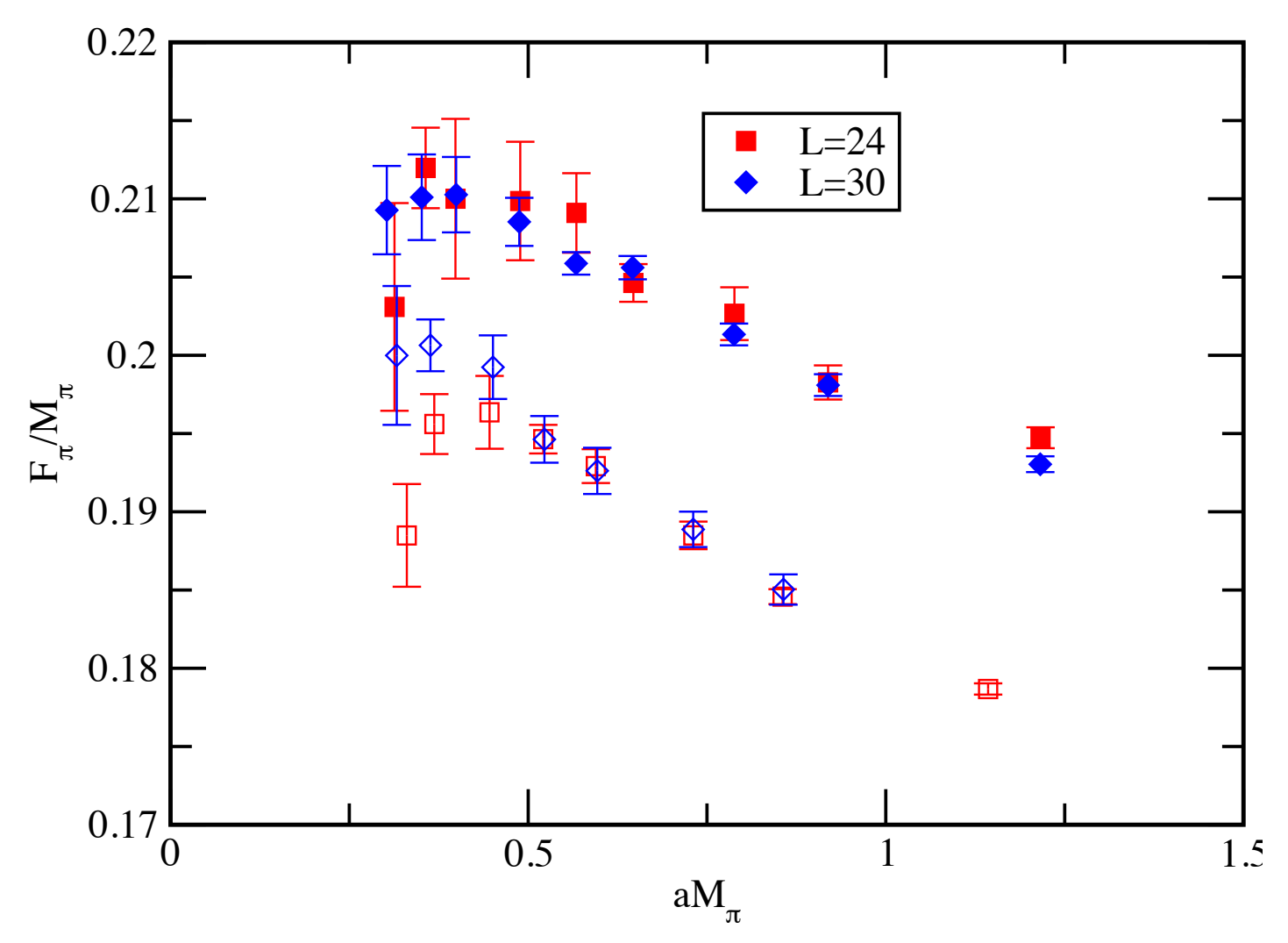

$N_{\mathrm{f}}=4: \mathrm{HISQ} \quad \beta=3.7$

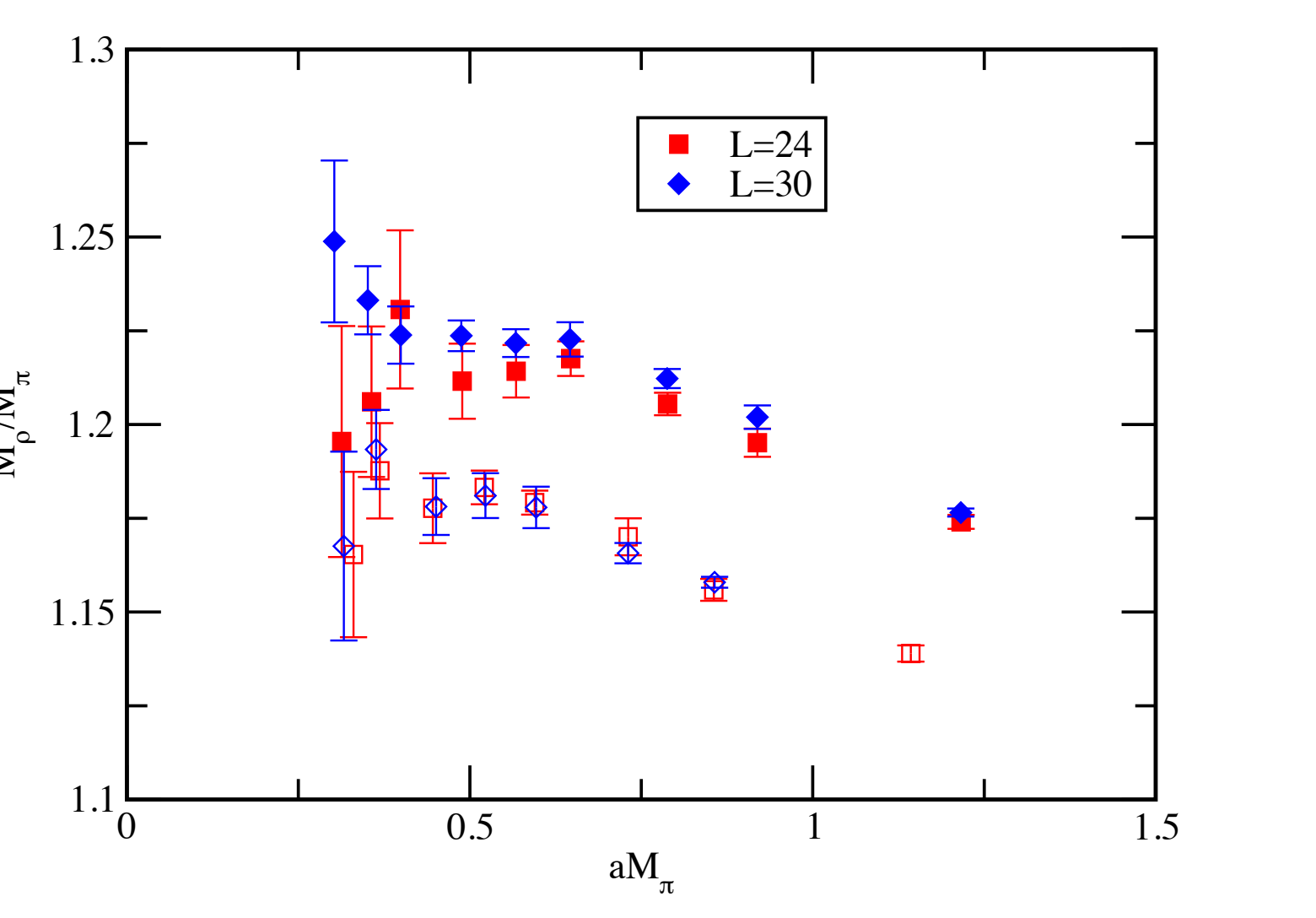


a crude analysis: $F_{\pi} / M_{\pi} v s M_{\pi}$

$\mathrm{N}_{\mathrm{f}}=12: \mathrm{HISQ}$

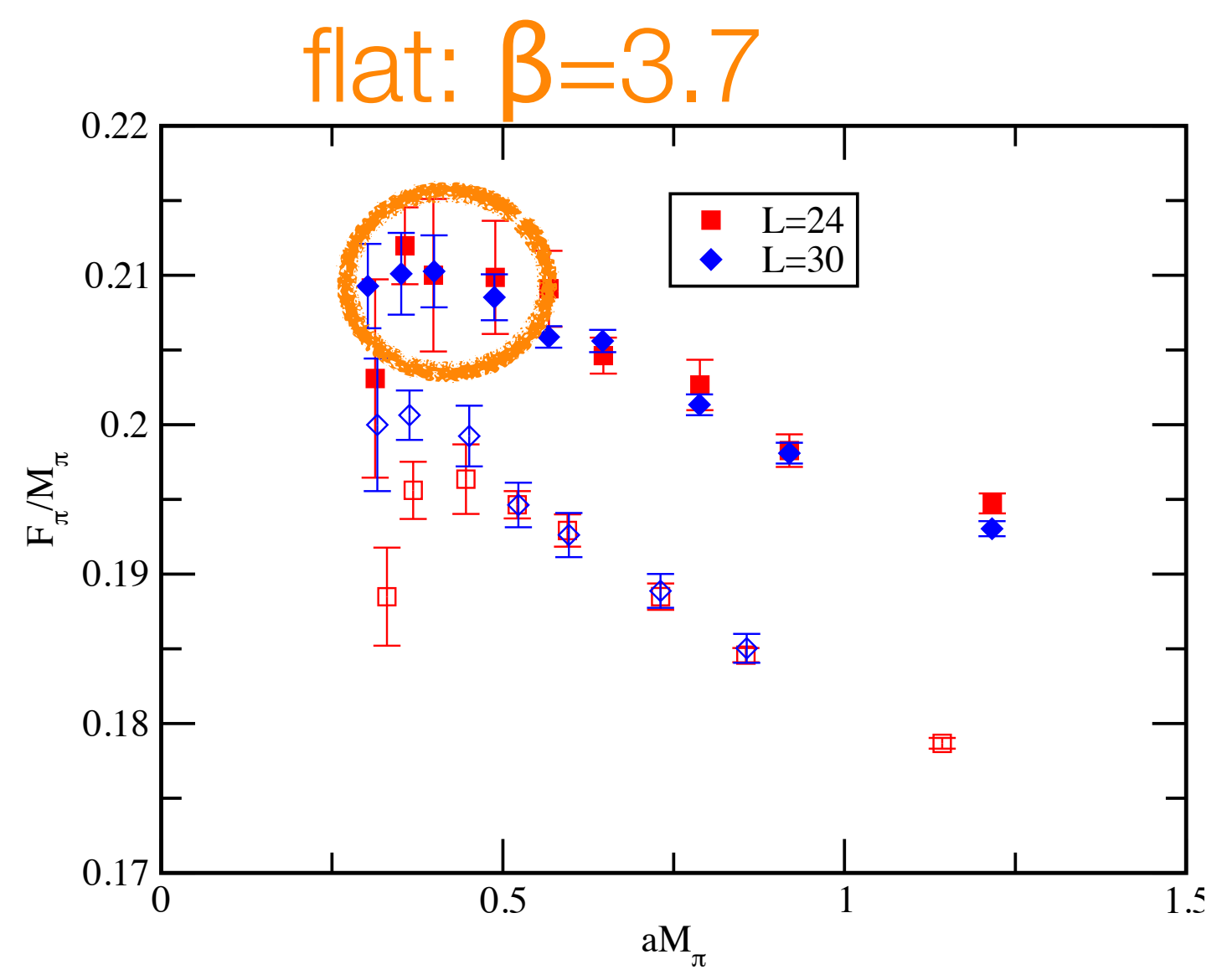

$N_{\mathrm{f}}=4: \mathrm{HISQ} \quad \beta=3.7$

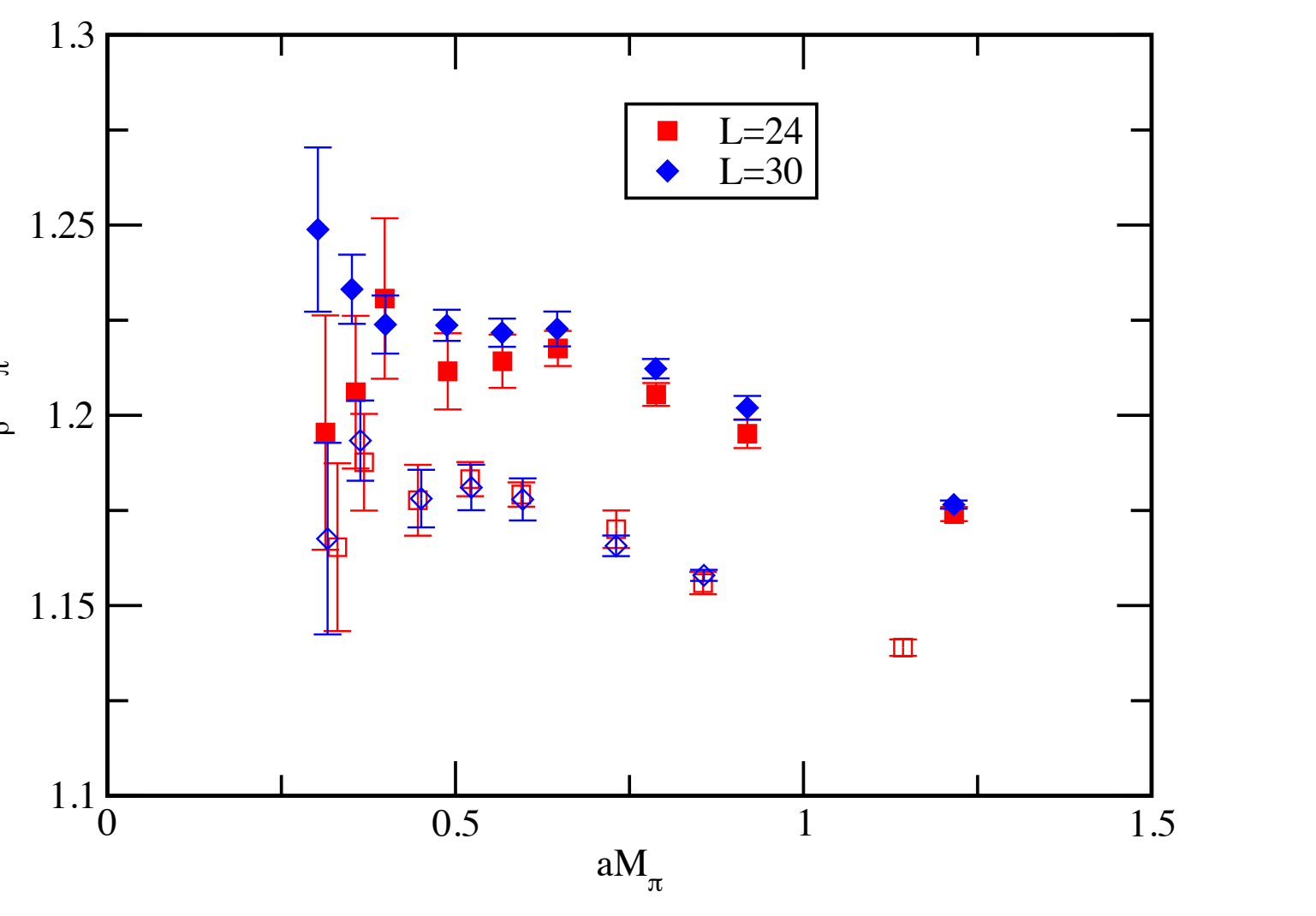

- $\beta=3.7$ : small mass: consistent with hyper-scaling 


\section{a crude analysis: $F_{\Pi} / M_{\pi} v s M_{\pi}$}

$$
N_{f}=12: H I S Q
$$

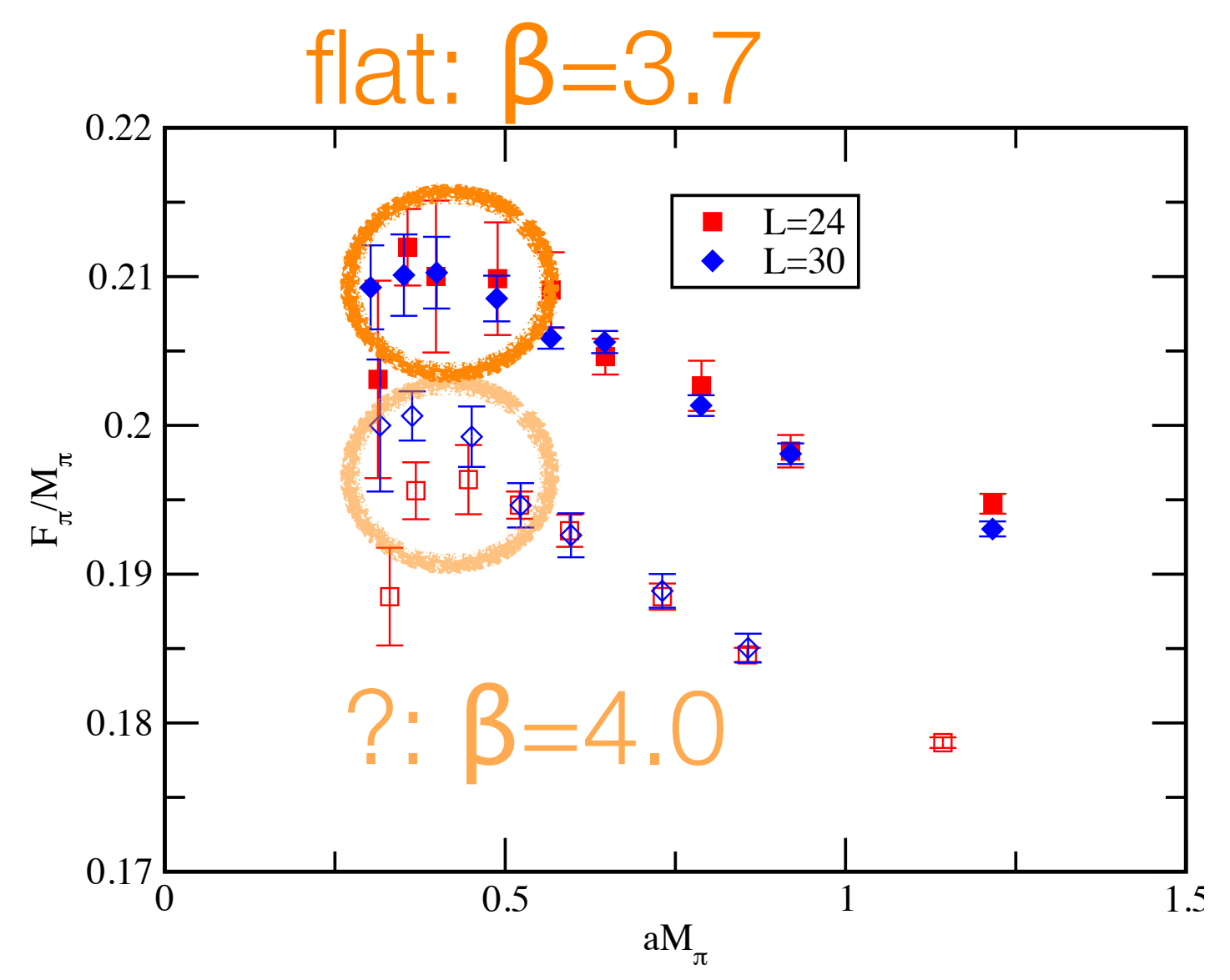

$\mathrm{N}_{f}=4: \mathrm{HISQ} \quad \beta=3.7$

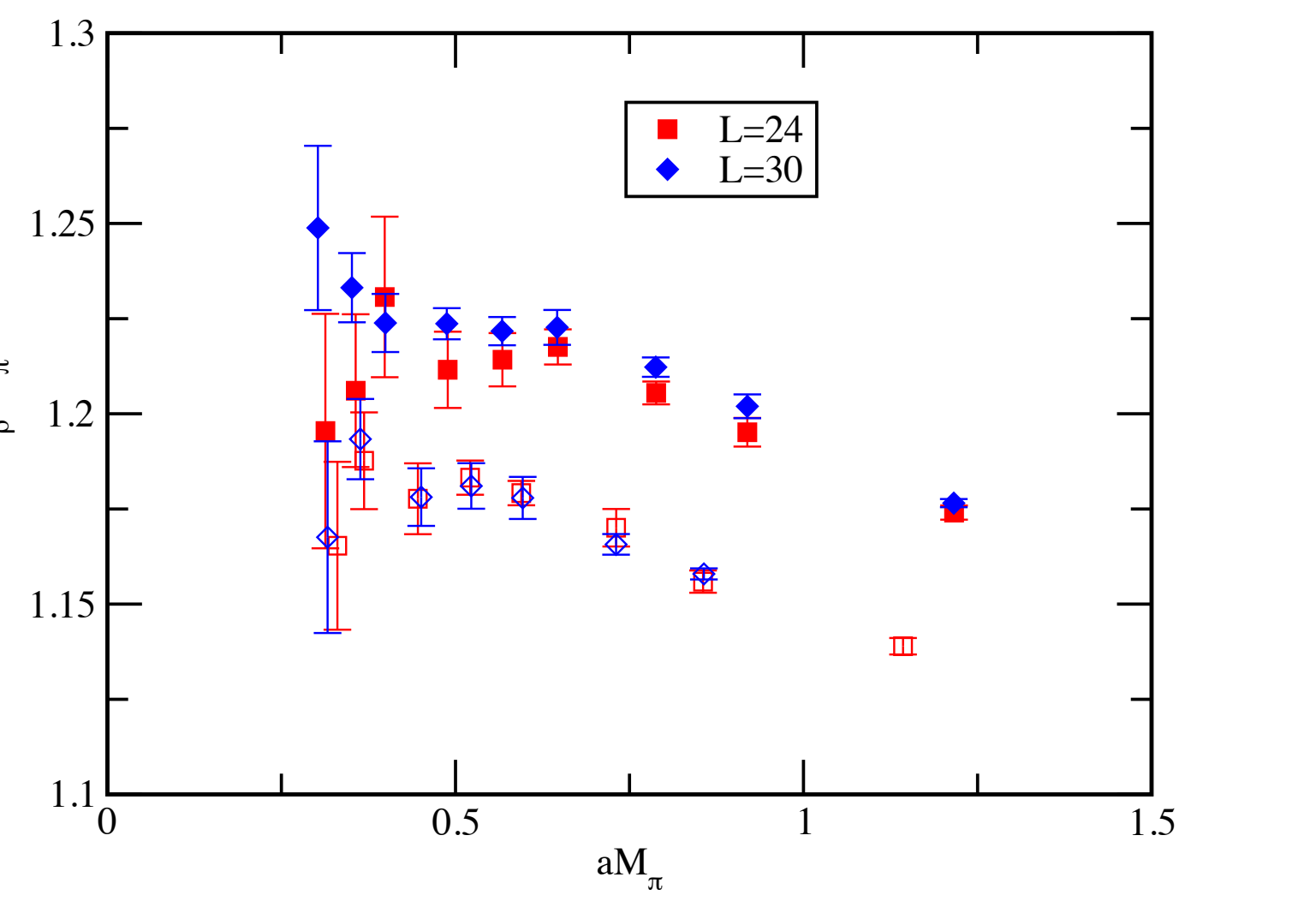

- $\beta=3.7$ : small mass: consistent with hyper-scaling

- $\beta=4.0$ : mass too heavy ? inconsistent with being in the hyper-scaling region 
a crude analysis: $M_{\rho} / M_{\pi}$ vs $M_{\pi}$

$$
N_{i}=12: H I S Q
$$

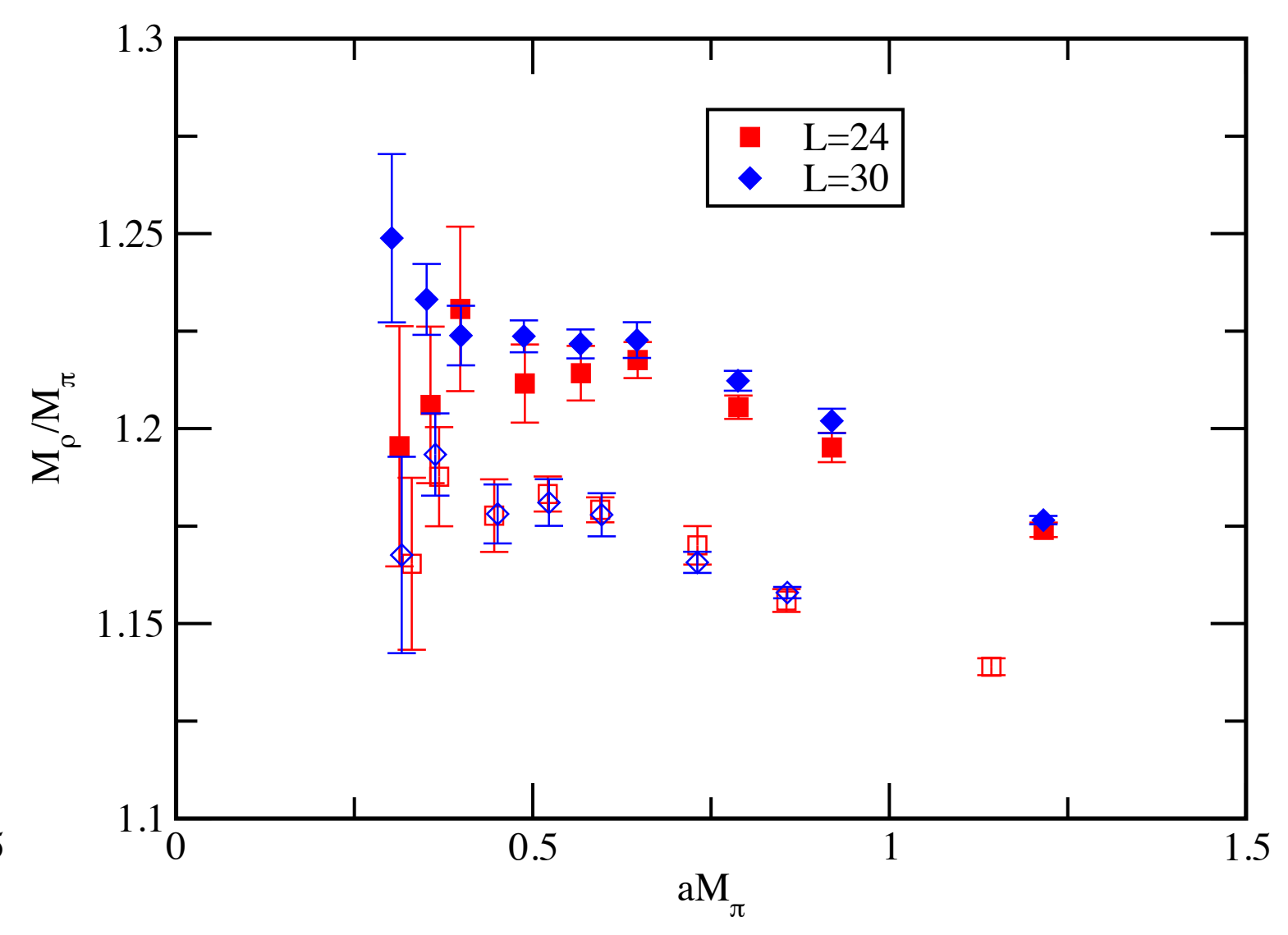


a crude analysis: $M_{\rho} / M_{\pi}$ vs $M_{\pi}$

$$
\mathrm{N}_{\mathrm{i}}=12: \mathrm{HISQ}
$$

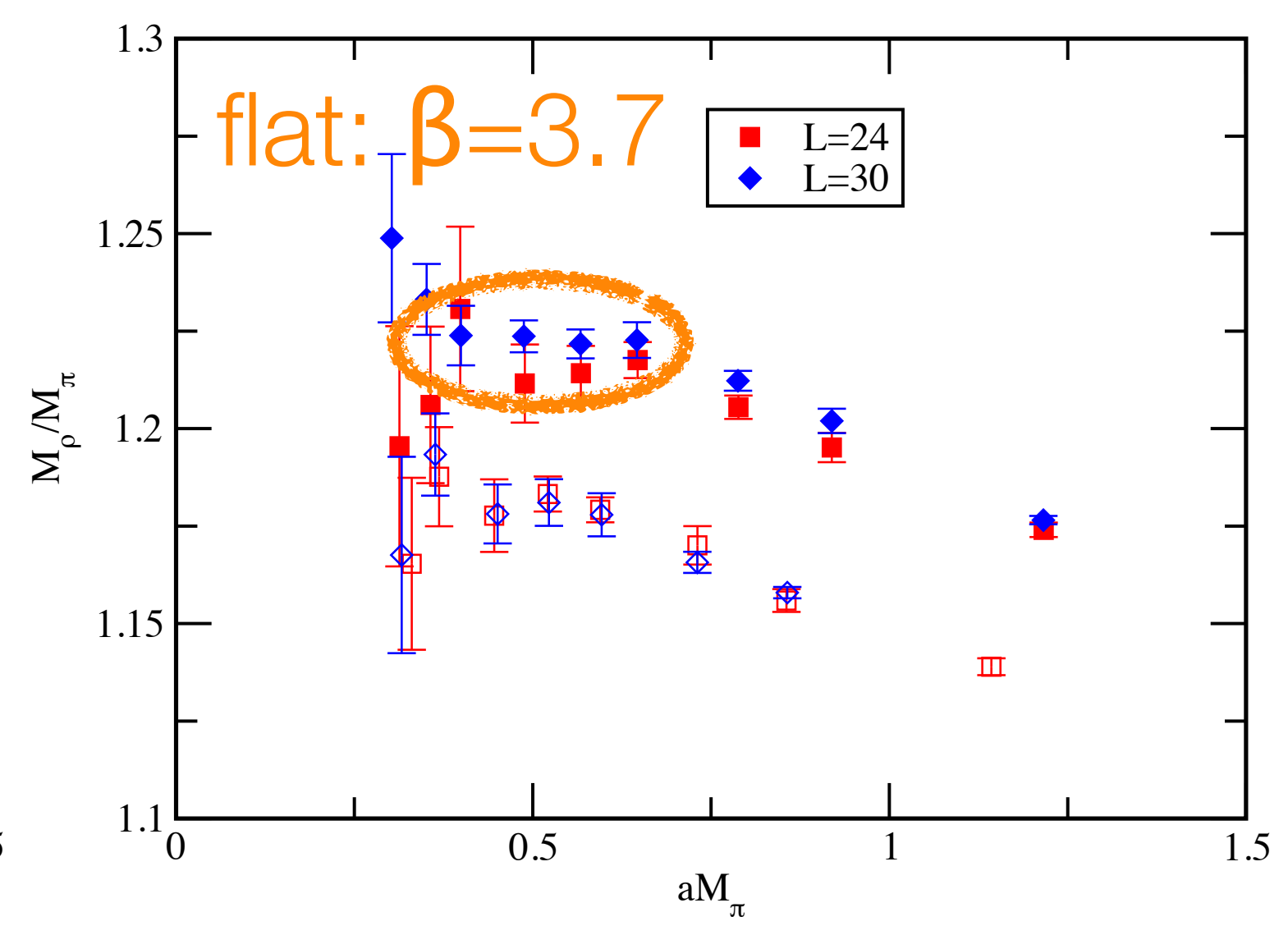


a crude analysis: $M_{\rho} / M_{\pi}$ vs $M_{\Pi}$

$$
\mathrm{N}_{\mathrm{i}}=12: \mathrm{HISQ}
$$

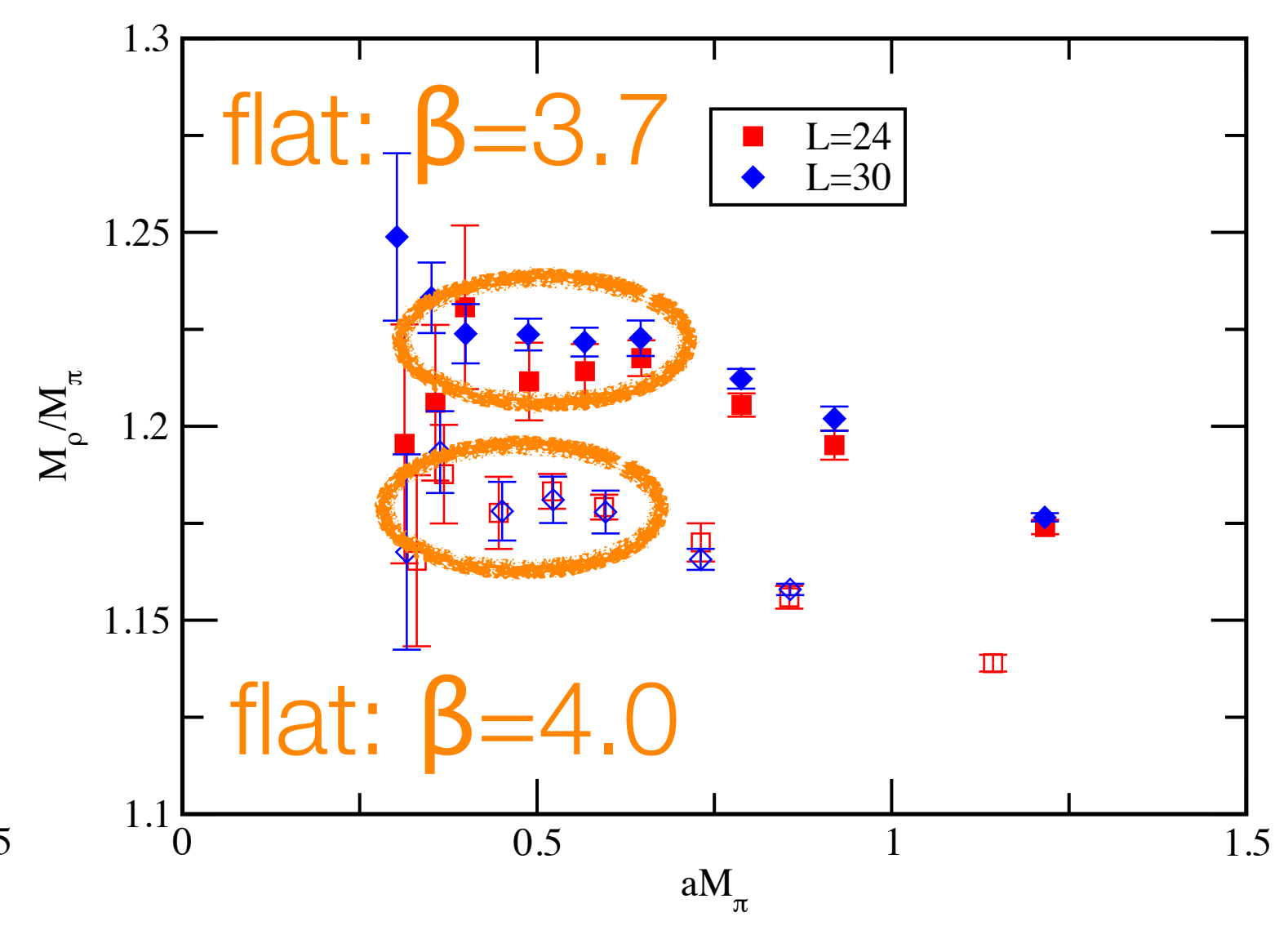

- $\beta=3.7$ \& 4.0: small mass (wider than $F_{\pi}$ ): consistent with hyper scaling (HS) 


\section{a crude analysis: $M_{\rho} / M_{\pi}$ vs $M_{\pi}$}

$$
\mathrm{N}_{\mathrm{i}}=12: \mathrm{HISQ}
$$

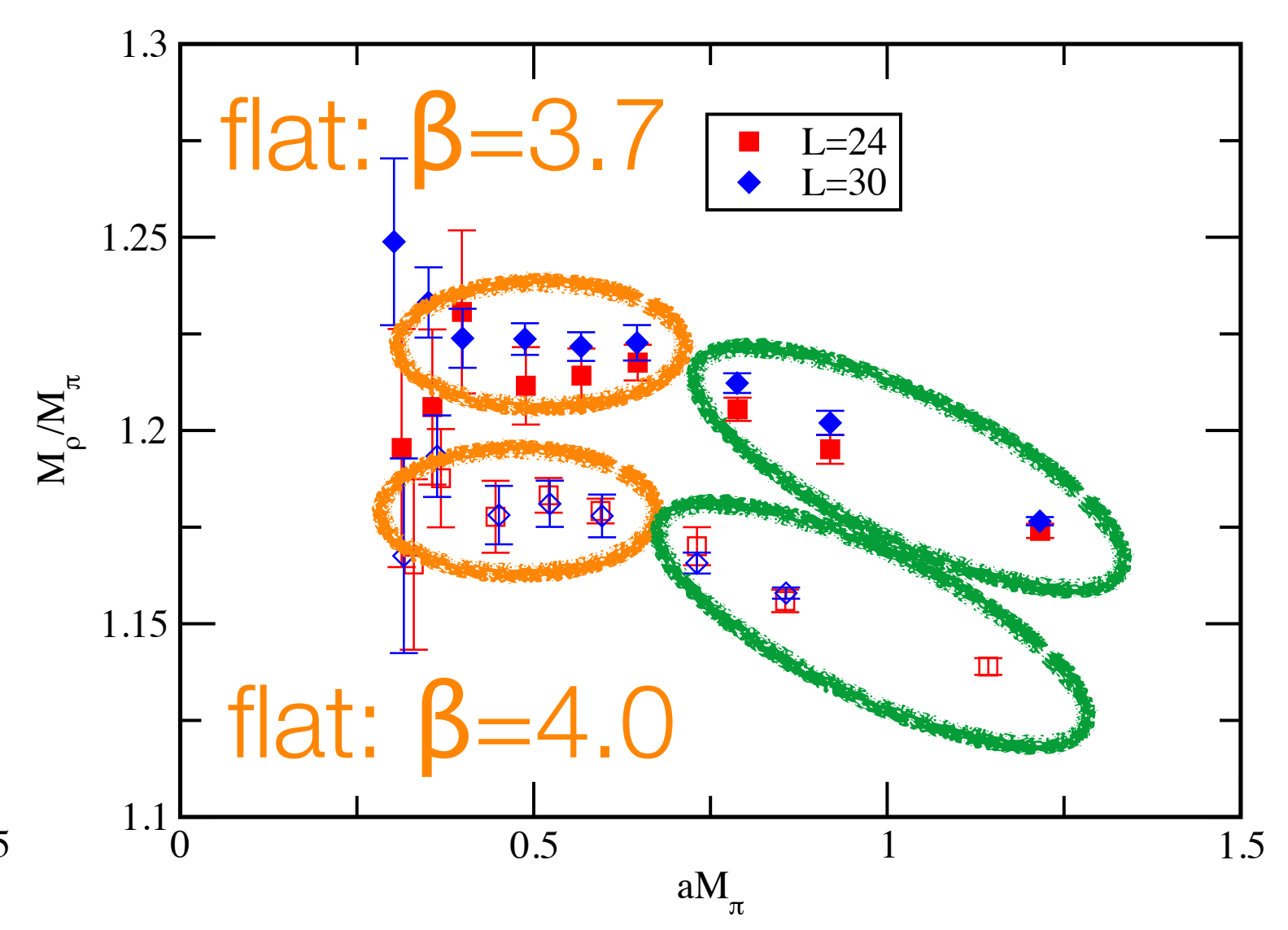

- $\beta=3.7$ \& 4.0: small mass (wider than $F_{\pi}$ ): consistent with hyper scaling (HS)

- mass dependence at the tail is due to non-universal mass correction to HS 


\section{a crude analysis: $M_{\rho} / M_{\pi} v s M_{\pi}$}

$$
\mathrm{N}_{\mathrm{i}}=12: \mathrm{HISQ}
$$

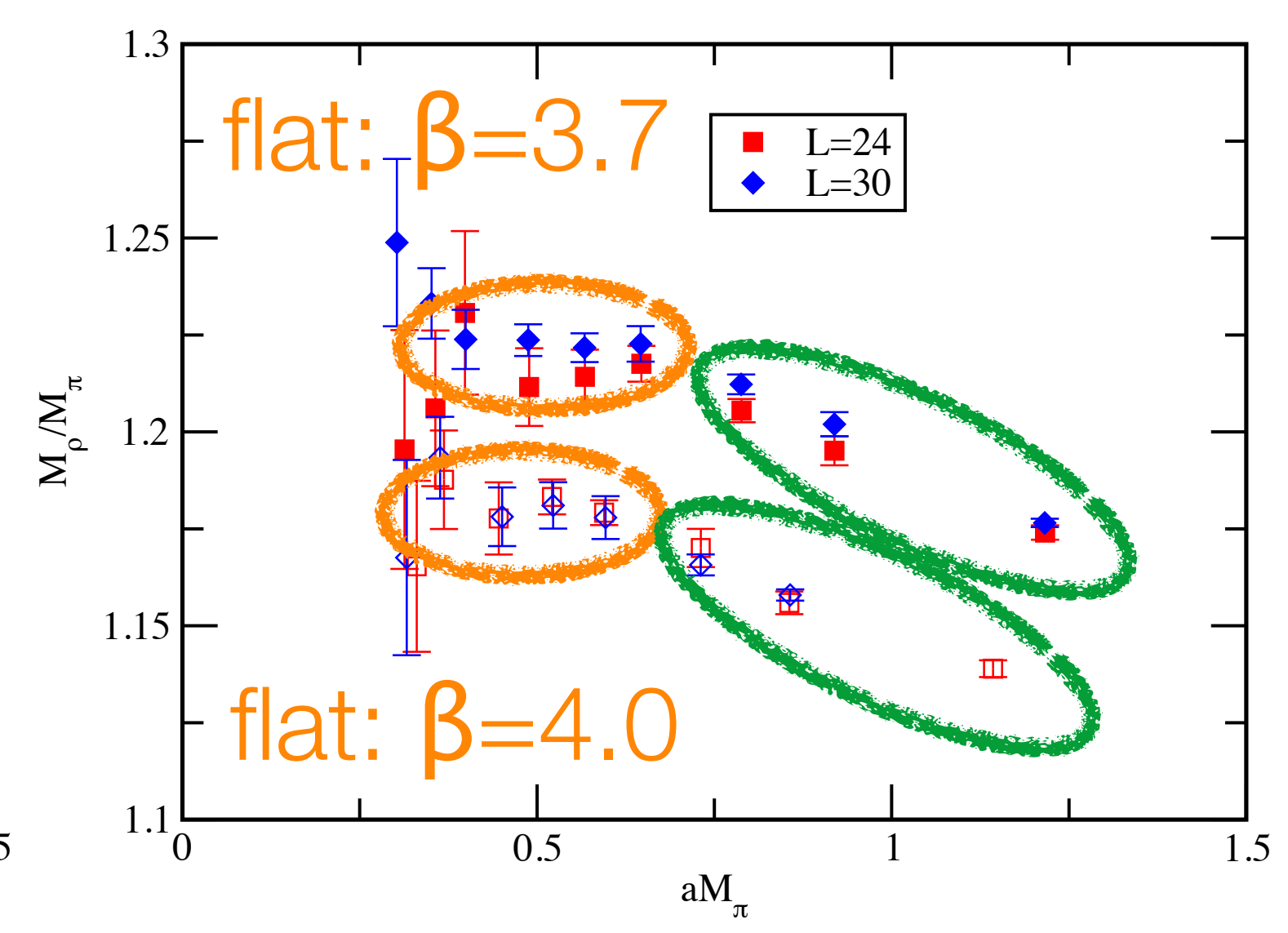

- one can attempt to perform a matching

- $\beta=3.7$ \& 4.0: small mass (wider than $F_{\pi}$ ): consistent with hyper scaling (HS)

- mass dependence at the tail is due to non-universal mass correction to HS 


\section{a crude analysis: $M_{\rho} / M_{\pi}$ vs $M_{\pi}$}

$$
\mathrm{N}_{\mathrm{i}}=12: \mathrm{HISQ}
$$

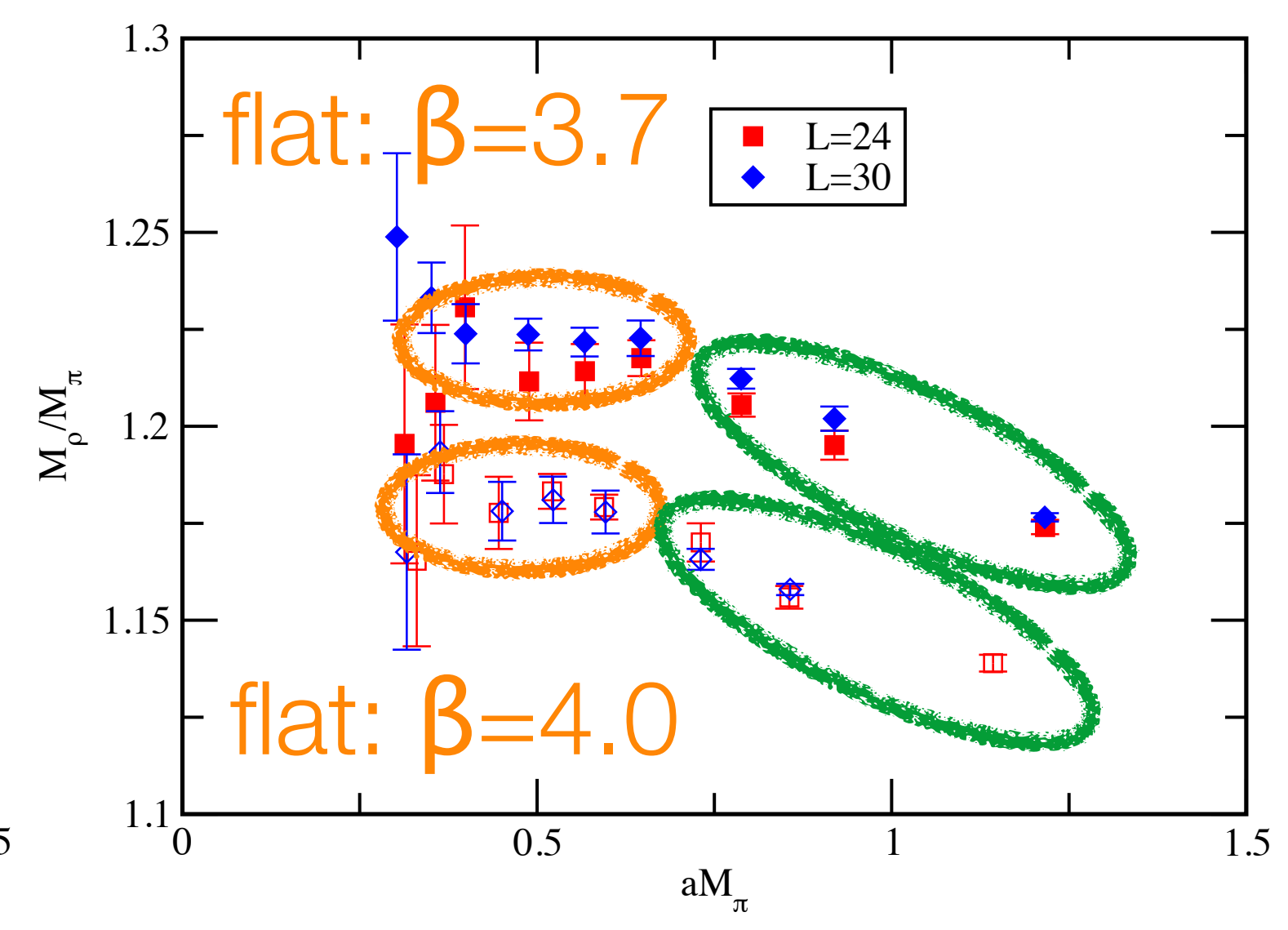

- one can attempt to perform a matching

- $a(\beta=3.7)>a(\beta=4.0)$

- $\beta=3.7$ \& 4.0: small mass (wider than $F_{\pi}$ ): consistent with hyper scaling (HS)

- mass dependence at the tail is due to non-universal mass correction to HS 


\section{a crude analysis: $M_{\rho} / M_{\pi} v s M_{\pi}$}

$$
\mathrm{N}_{\mathrm{i}}=12: \mathrm{HISQ}
$$

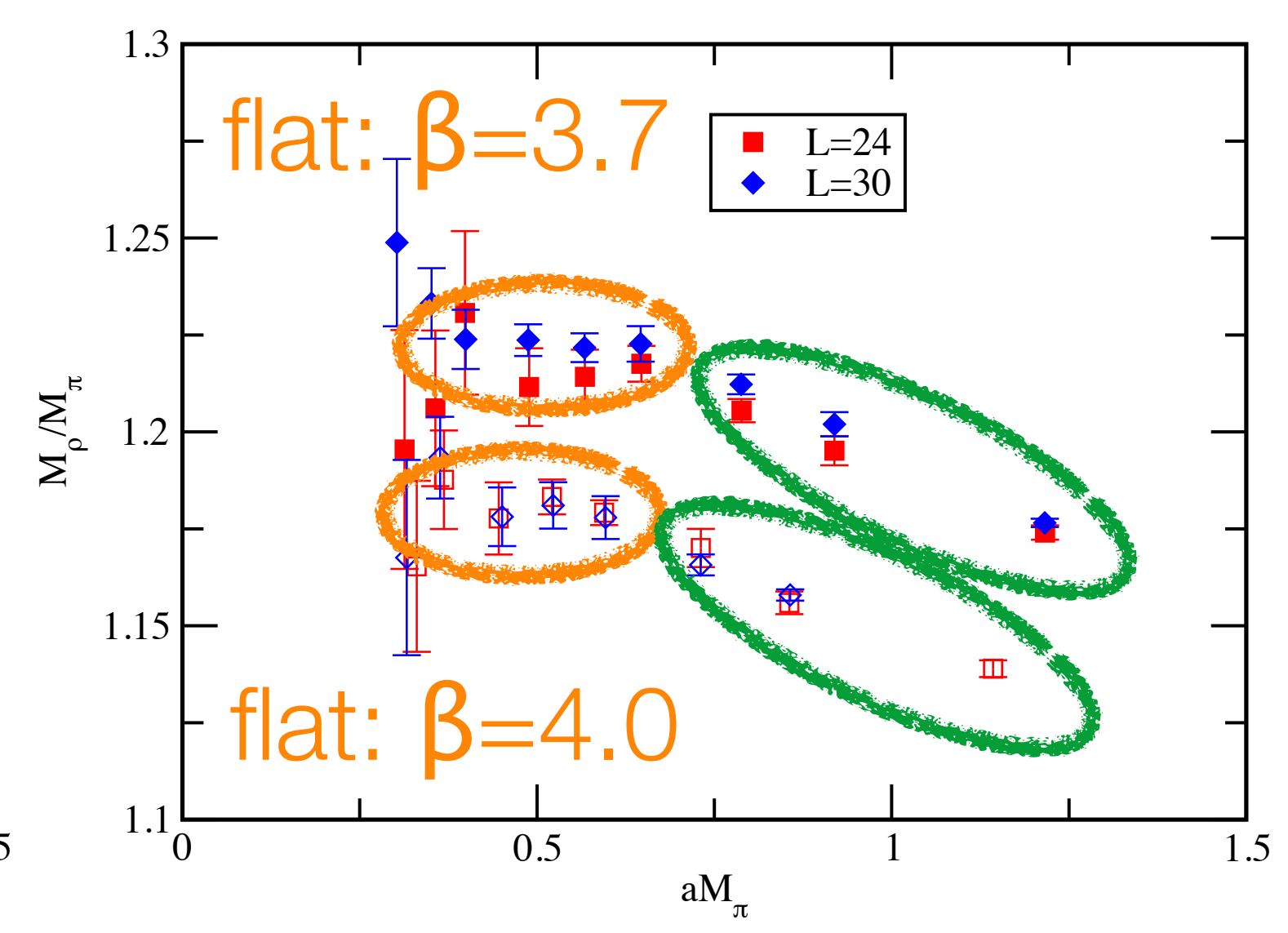

- one can attempt to perform a matching

- $a(\beta=3.7)>a(\beta=4.0)$

- movement: correct direction in asymptotically free domain!

- $\beta=3.7$ \& 4.0: small mass (wider than $F_{\pi}$ ): consistent with hyper scaling $(H S)$

- mass dependence at the tail is due to non-universal mass correction to HS 


\section{conformal (finite size) scaling}

- Scaling dimension at IR fixed point [Wilson-Fisher]; Hyper Scaling [Miransky]

- mass dependence is described by anomalous dimensions at IRFP

- quark mass anomalous dimension $\gamma^{*}$

- operator anomalous dimension

- meson mass and pion decay constant obey same scaling

$$
m_{\pi}=c_{m} m_{f}^{\frac{1}{1+\gamma^{*}}} \quad f_{\pi}=c_{f} m_{f}^{\frac{1}{1+\gamma^{*}}}
$$

- finite size scaling in a $L^{4}$ box (DeGrand; Del Debbio et al)

- scaling variable: $\quad x=L m_{f}^{\frac{1}{1+\gamma^{*}}}$

$$
L f_{\pi}=F(x) \quad L m_{\pi}=G(x)
$$


$N_{f}=4$ see if data align at some $\gamma$
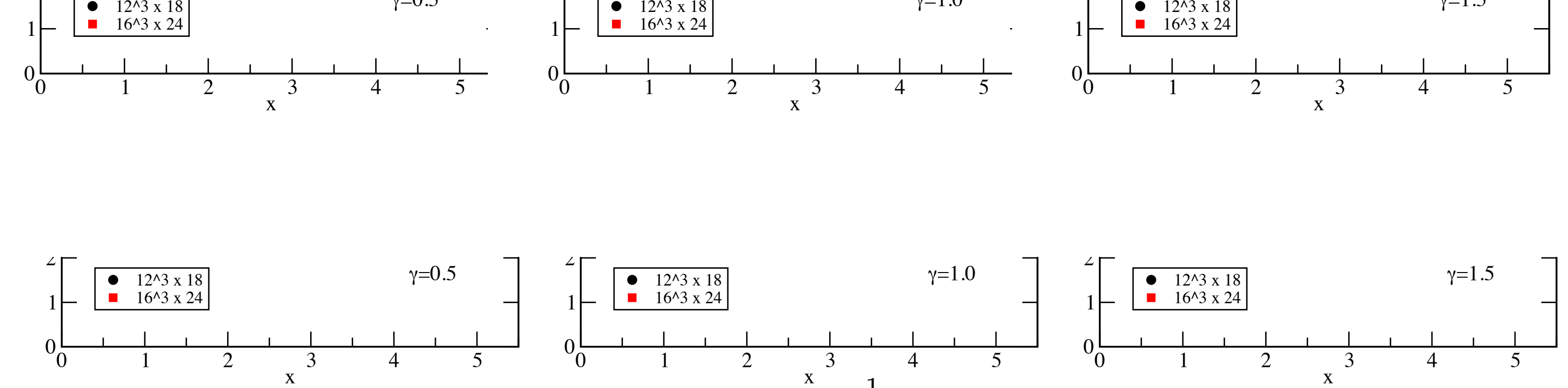

$$
x=L m_{f}^{\frac{1}{1+\gamma}}
$$
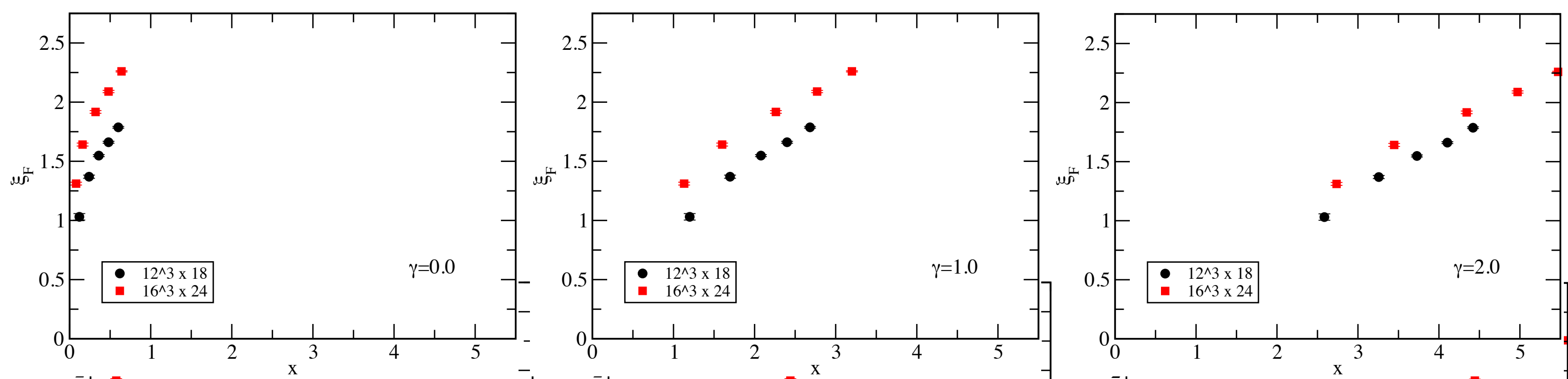
$\mathrm{N}_{\mathrm{f}}=4$ see if data align at some $\gamma$
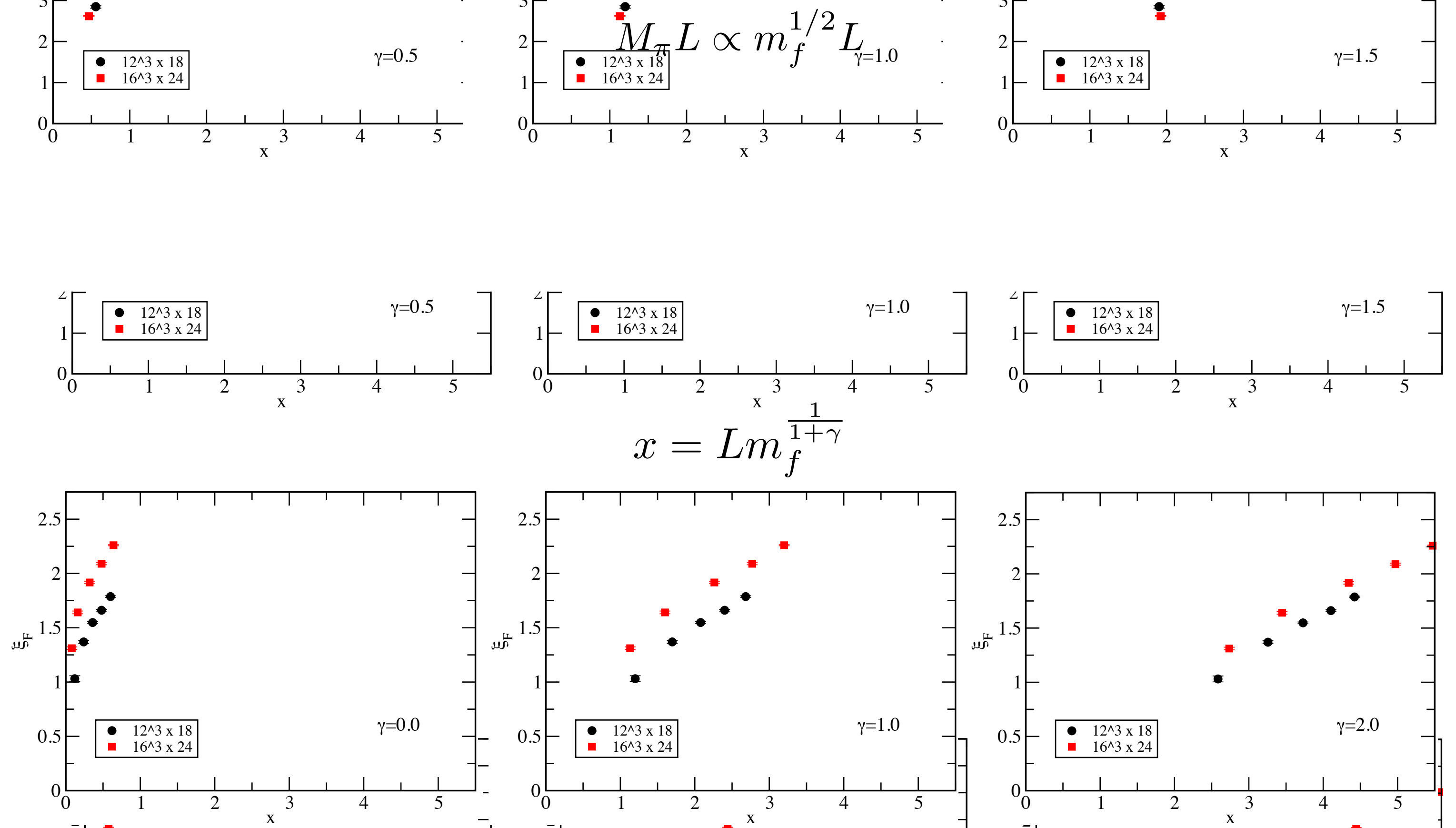
$N_{f}=4$ see if data align at some $\gamma$
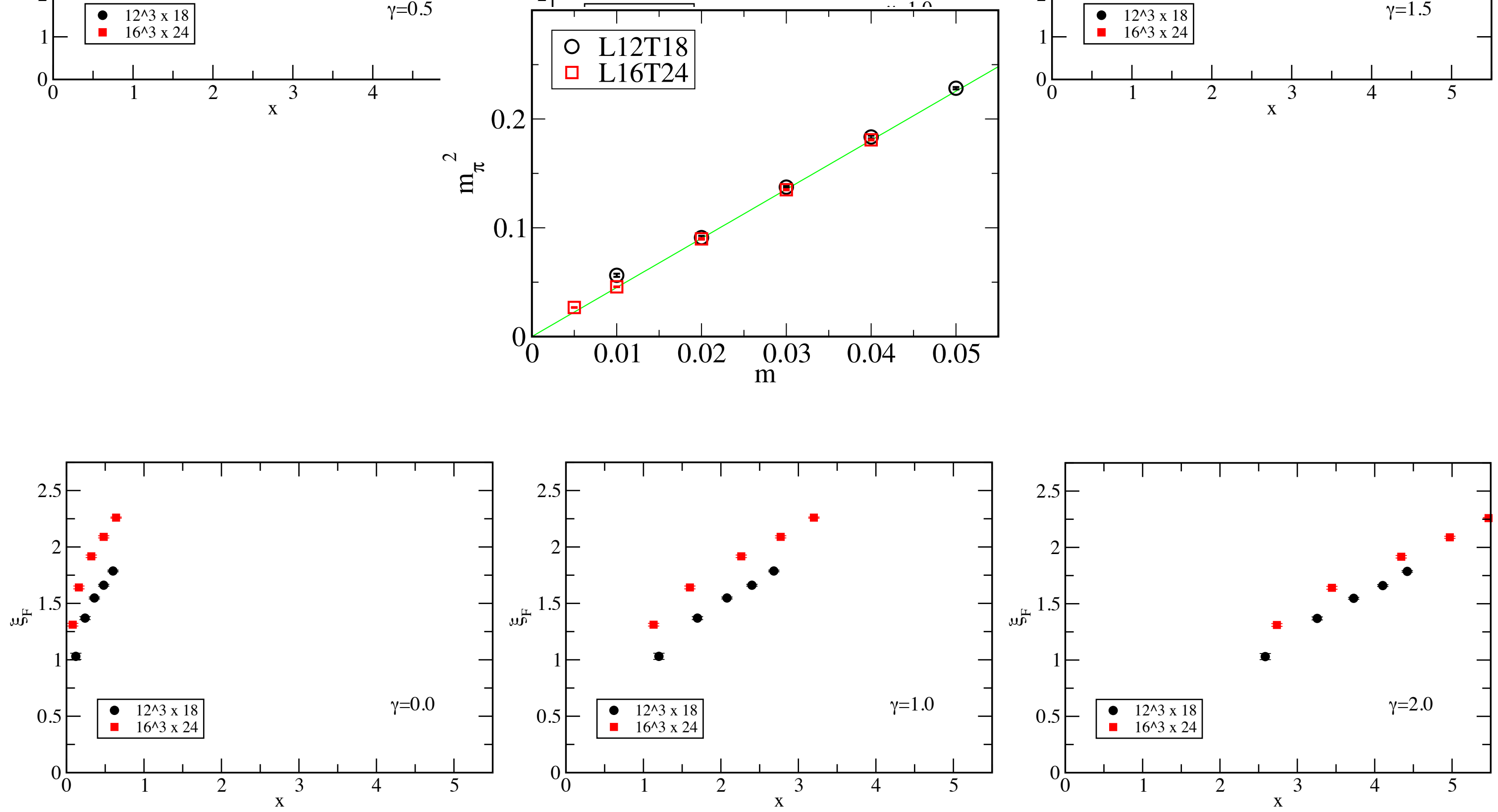
$\mathrm{N}_{\mathrm{f}}=4$ see if data align at some $\gamma$
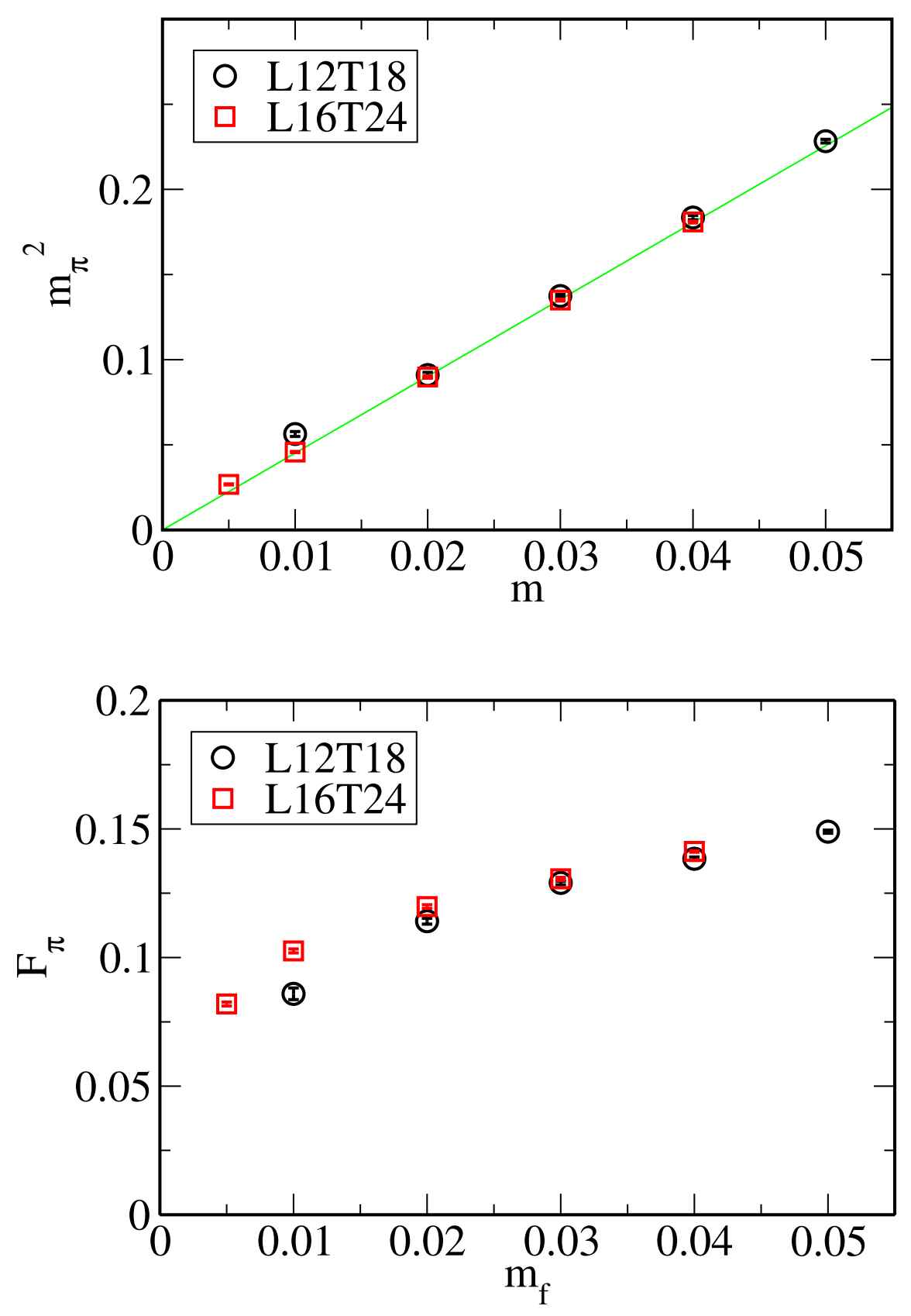
$N_{f}=12$ see if data align at some $\gamma$
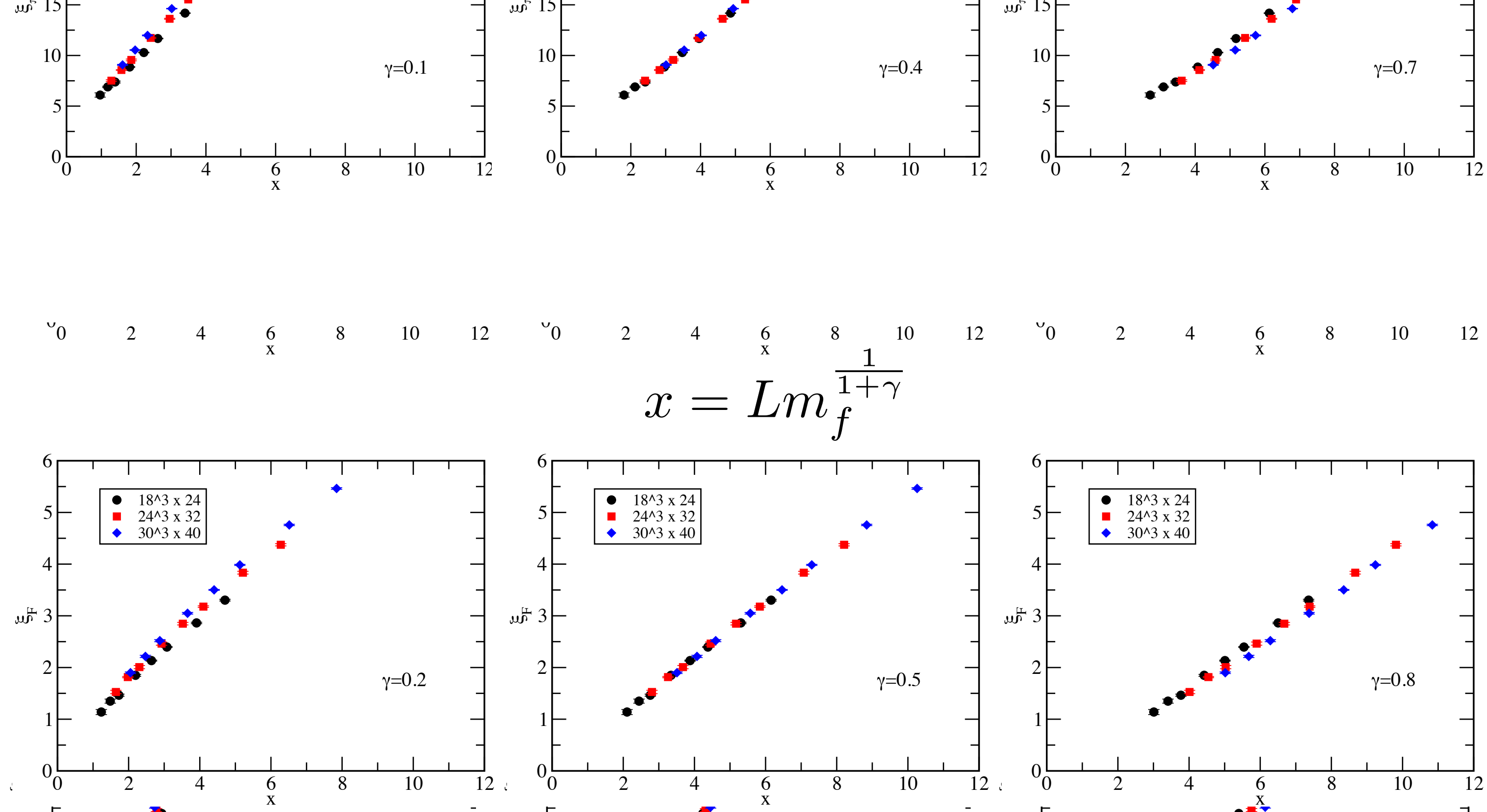
to quantify the "alignment" without resorting to a model 


\section{to quantify the "alignment" without resorting to a model}

- $\gamma$ of optimal alignment will minimize:

$$
P_{p}(\gamma)=\frac{1}{\mathcal{N}} \sum_{K} \sum_{j \notin K} \frac{\left|\xi_{p}^{j}-f_{p}^{(K)}\left(x_{j}\right)\right|^{2}}{\delta^{2} \xi_{p}^{j}}
$$




\section{to quantify the "alignment" without resorting to a model}

- $\gamma$ of optimal alignment will minimize:

$$
P_{p}(\gamma)=\frac{1}{\mathcal{N}} \sum_{K} \sum_{j \notin K} \frac{\left|\xi_{p}^{j}-f_{p}^{(K)}\left(x_{j}\right)\right|^{2}}{\delta^{2} \xi_{p}^{j}}
$$

- $\xi_{p}=L M_{p}$ for $p=\pi, \rho ; \quad \xi_{F}=L F_{\pi}$ 


\section{to quantify the "alignment" without resorting to a model}

- $\gamma$ of optimal alignment will minimize:

$$
P_{p}(\gamma)=\frac{1}{\mathcal{N}} \sum_{K} \sum_{j \notin K} \frac{\left|\xi_{p}^{j}-f_{p}^{(K)}\left(x_{j}\right)\right|^{2}}{\delta^{2} \xi_{p}^{j}}
$$

- $\xi_{p}=L M_{p}$ for $p=\pi, \rho ; \quad \xi_{F}=L F_{\pi}$

- $f_{p}(x)$ : interpolation .... linear 


\section{to quantify the "alignment" without resorting to a model}

- $\gamma$ of optimal alignment will minimize:

$$
P_{p}(\gamma)=\frac{1}{\mathcal{N}} \sum_{K} \sum_{j \notin K} \frac{\left|\xi_{p}^{j}-f_{p}^{(K)}\left(x_{j}\right)\right|^{2}}{\delta^{2} \xi_{p}^{j}}
$$

- $\xi_{p}=L M_{p}$ for $p=\pi, \rho ; \quad \xi_{F}=L F_{\pi}$

- $f_{p}(x)$ : interpolation .... linear

- (quadratic for a systematic error) 


\section{to quantify the "alignment" without resorting to a model}

- $\gamma$ of optimal alignment will minimize:

$$
P_{p}(\gamma)=\frac{1}{\mathcal{N}} \sum_{K} \sum_{j \notin K} \frac{\left|\xi_{p}^{j}-f_{p}^{(K)}\left(x_{j}\right)\right|^{2}}{\delta^{2} \xi_{p}^{j}}
$$

- $\xi_{p}=L M_{p}$ for $p=\pi, \rho ; \quad \xi_{F}=L_{\pi}$

- $f_{p}(x)$ : interpolation .... linear

- (quadratic for a systematic error)

- if $\xi^{j}$ is away from $f\left(x_{i}\right)$ by $\delta \xi^{j}$ as average $\rightarrow P=1$ 


\section{to quantify the "alignment" without resorting to a model}

- $\gamma$ of optimal alignment will minimize:

$$
P_{p}(\gamma)=\frac{1}{\mathcal{N}} \sum_{K} \sum_{j \notin K} \frac{\left|\xi_{p}^{j}-f_{p}^{(K)}\left(x_{j}\right)\right|^{2}}{\delta^{2} \xi_{p}^{j}}
$$

- $\xi_{\mathrm{p}}=\mathrm{LM} \mathrm{M}_{\mathrm{p}}$ for $\mathrm{p}=\pi, \rho ; \quad \xi_{\mathrm{F}}=\mathrm{LF} \pi$

- $f_{p}(x)$ : interpolation .... linear

- (quadratic for a systematic error)

- if $\xi^{j}$ is away from $f\left(x_{i}\right)$ by $\delta \xi^{j}$ as average $\rightarrow P=1$

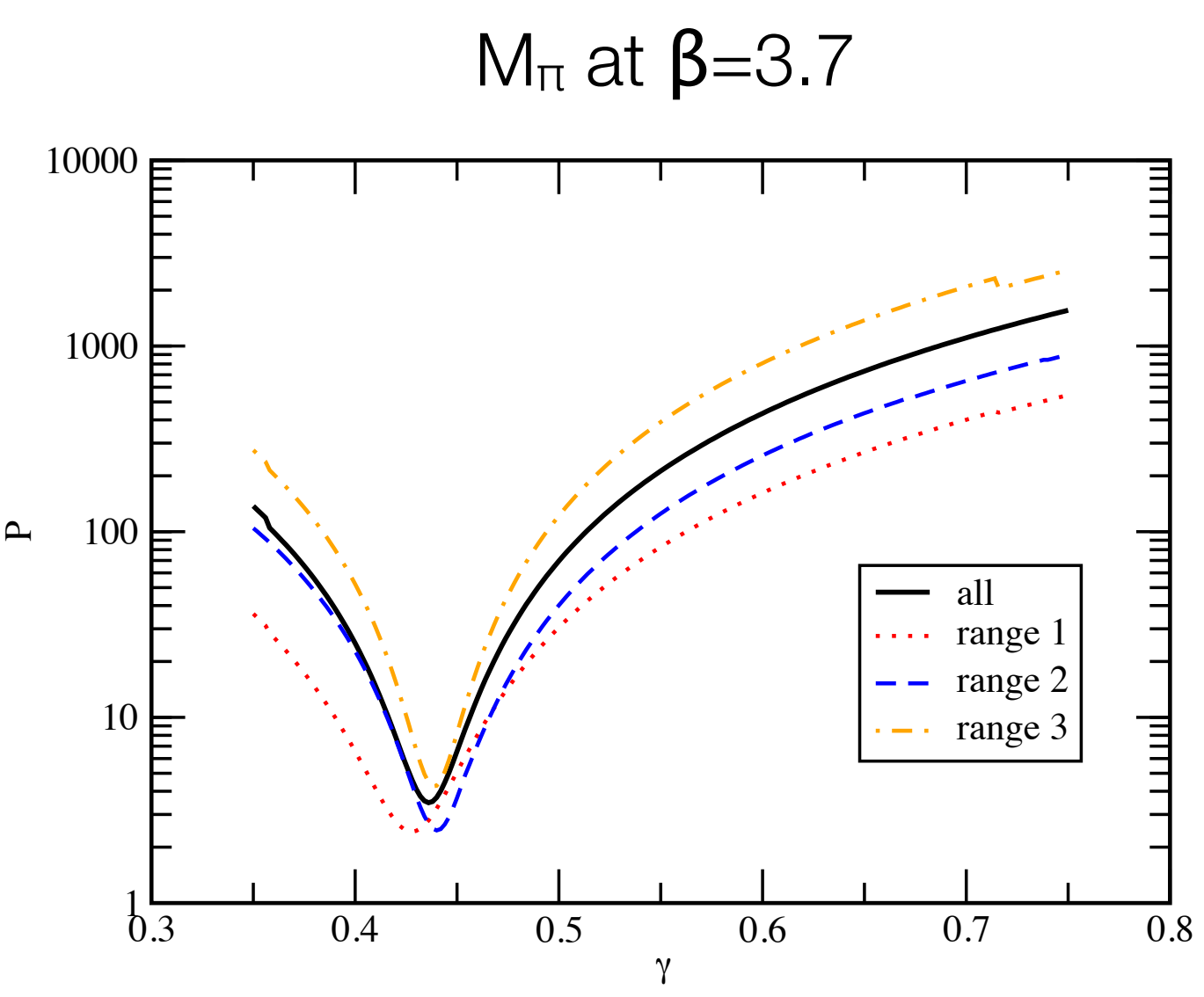




\section{to quantify the "alignment" without resorting to a model}

- $\gamma$ of optimal alignment will minimize:

$$
P_{p}(\gamma)=\frac{1}{\mathcal{N}} \sum_{K} \sum_{j \notin K} \frac{\left|\xi_{p}^{j}-f_{p}^{(K)}\left(x_{j}\right)\right|^{2}}{\delta^{2} \xi_{p}^{j}}
$$

- $\xi_{\mathrm{p}}=\mathrm{LM}$ for $\mathrm{p}=\pi, \rho ; \quad \xi_{\mathrm{F}}=\mathrm{LF}_{\pi}$

- $f_{p}(x)$ : interpolation .... linear

- (quadratic for a systematic error)

- if $\xi^{j}$ is away from $f\left(x_{i}\right)$ by $\delta \xi^{j}$ as average $\rightarrow P=1$

- optimal $Y$ from the minimum of $P$

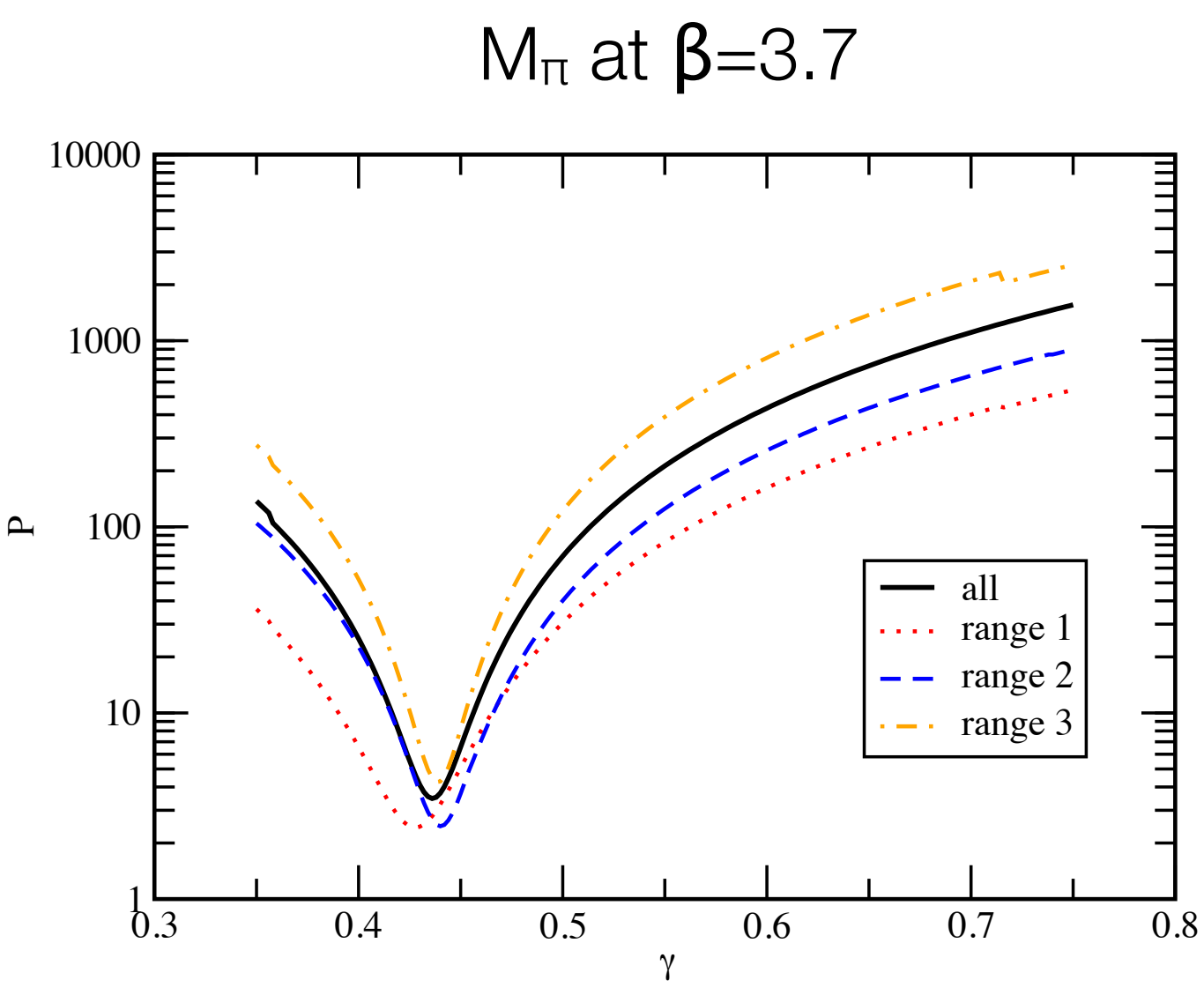




\section{to quantify the "alignment" without resorting to a model}

- $\gamma$ of optimal alignment will minimize:

$$
P_{p}(\gamma)=\frac{1}{\mathcal{N}} \sum_{K} \sum_{j \notin K} \frac{\left|\xi_{p}^{j}-f_{p}^{(K)}\left(x_{j}\right)\right|^{2}}{\delta^{2} \xi_{p}^{j}}
$$

- $\xi_{\mathrm{p}}=\mathrm{LM} \mathrm{M}_{\mathrm{p}}$ for $\mathrm{p}=\pi, \rho ; \quad \xi_{\mathrm{F}}=\mathrm{LF}_{\pi}$

- $f_{p}(x)$ : interpolation .... linear

- (quadratic for a systematic error)

- if $\xi^{j}$ is away from $f\left(x_{i}\right)$ by $\delta \xi^{j}$ as average $\rightarrow P=1$

- optimal $\gamma$ from the minimum of $P$

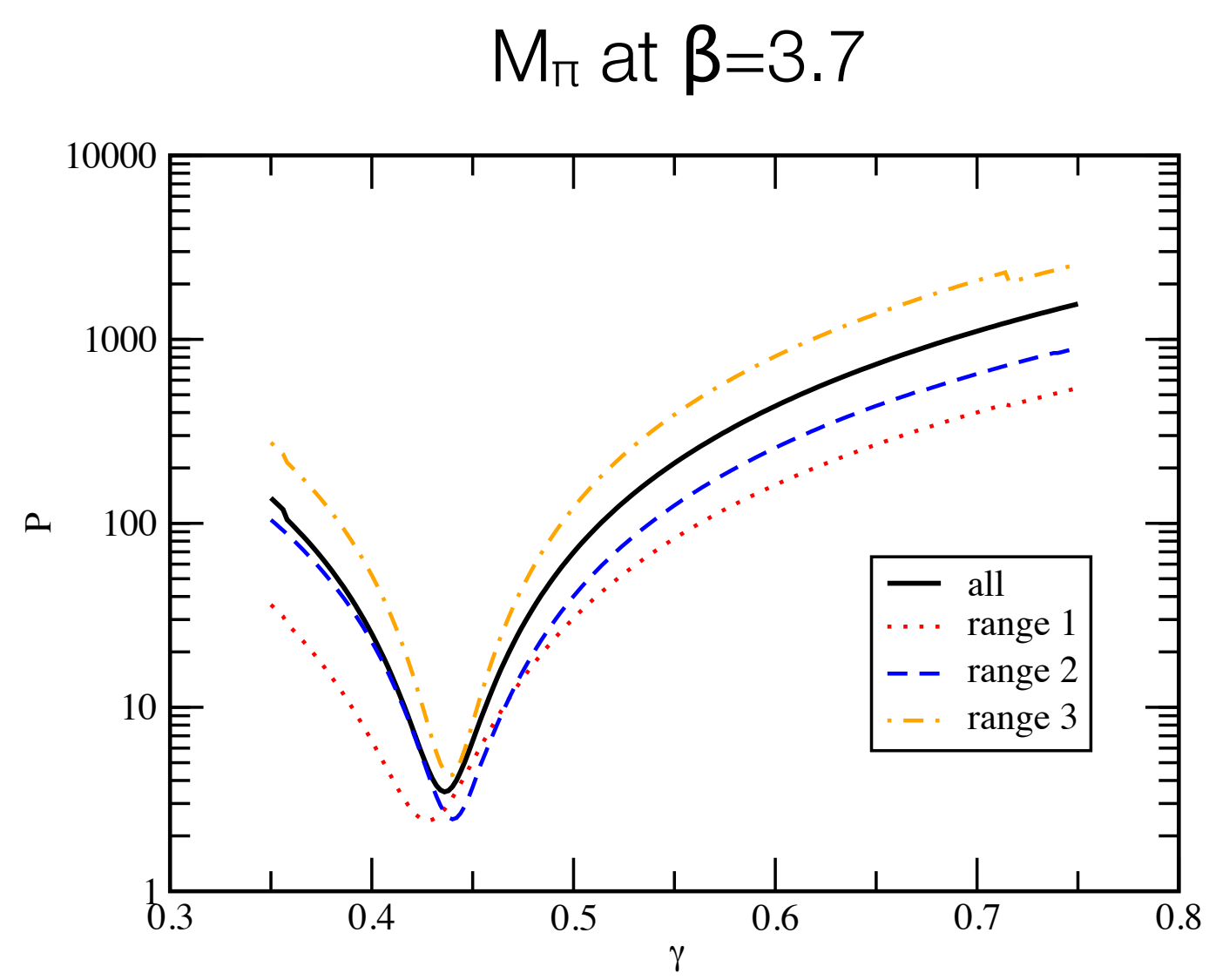

- systematic error due to small $L$, large $m$ estimated by examining the $x$ and $L$ range dependence 


\section{summary of $\gamma$ obtained by minimizing $P(\gamma)$}

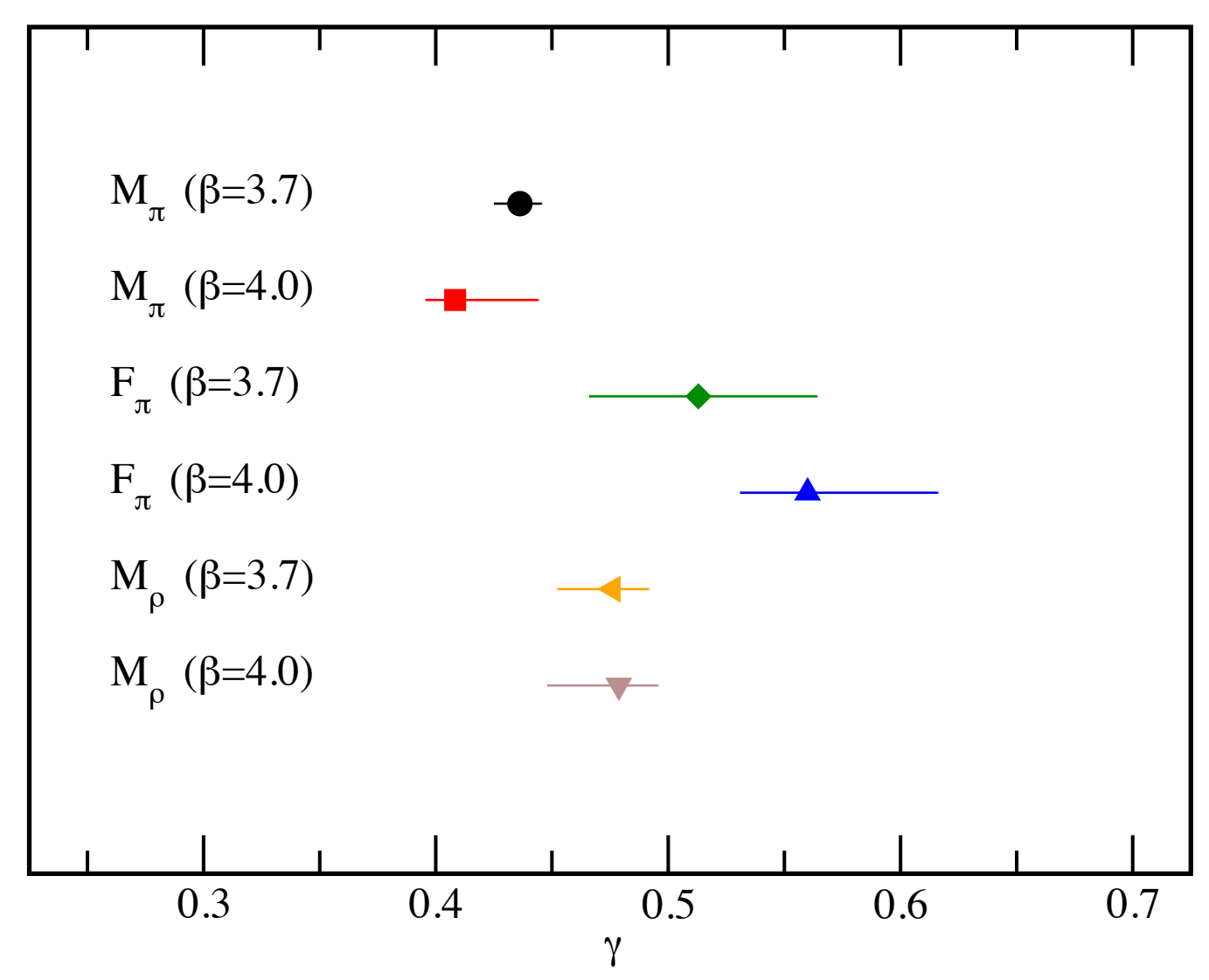




\section{summary of $\gamma$ obtained by minimizing $P(\gamma)$}

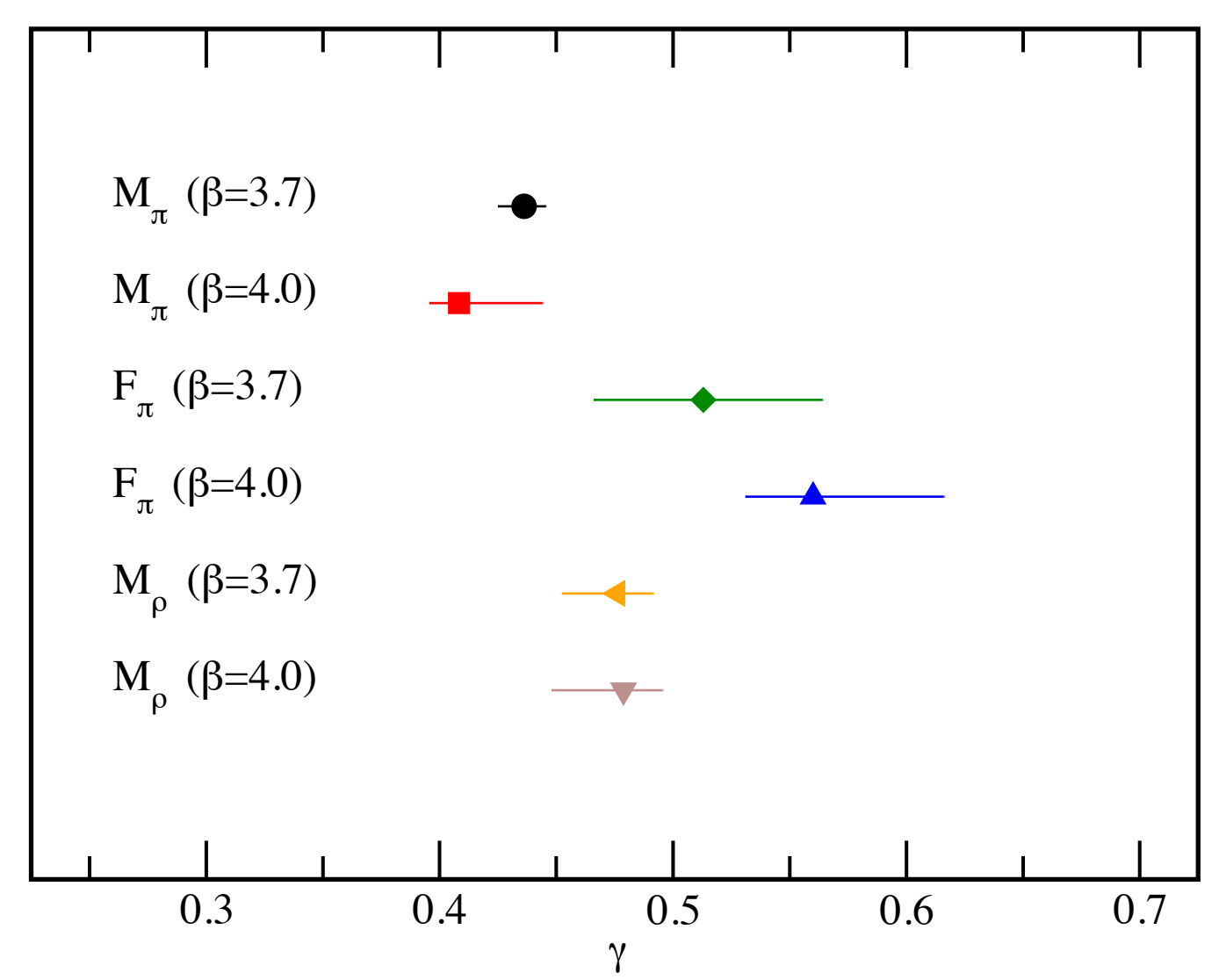

- consistent $\gamma$ by $1.5 \sigma$ level except for $F_{\pi}$ at $\beta=4.0$ 


\section{summary of $\gamma$ obtained by minimizing $P(\gamma)$}

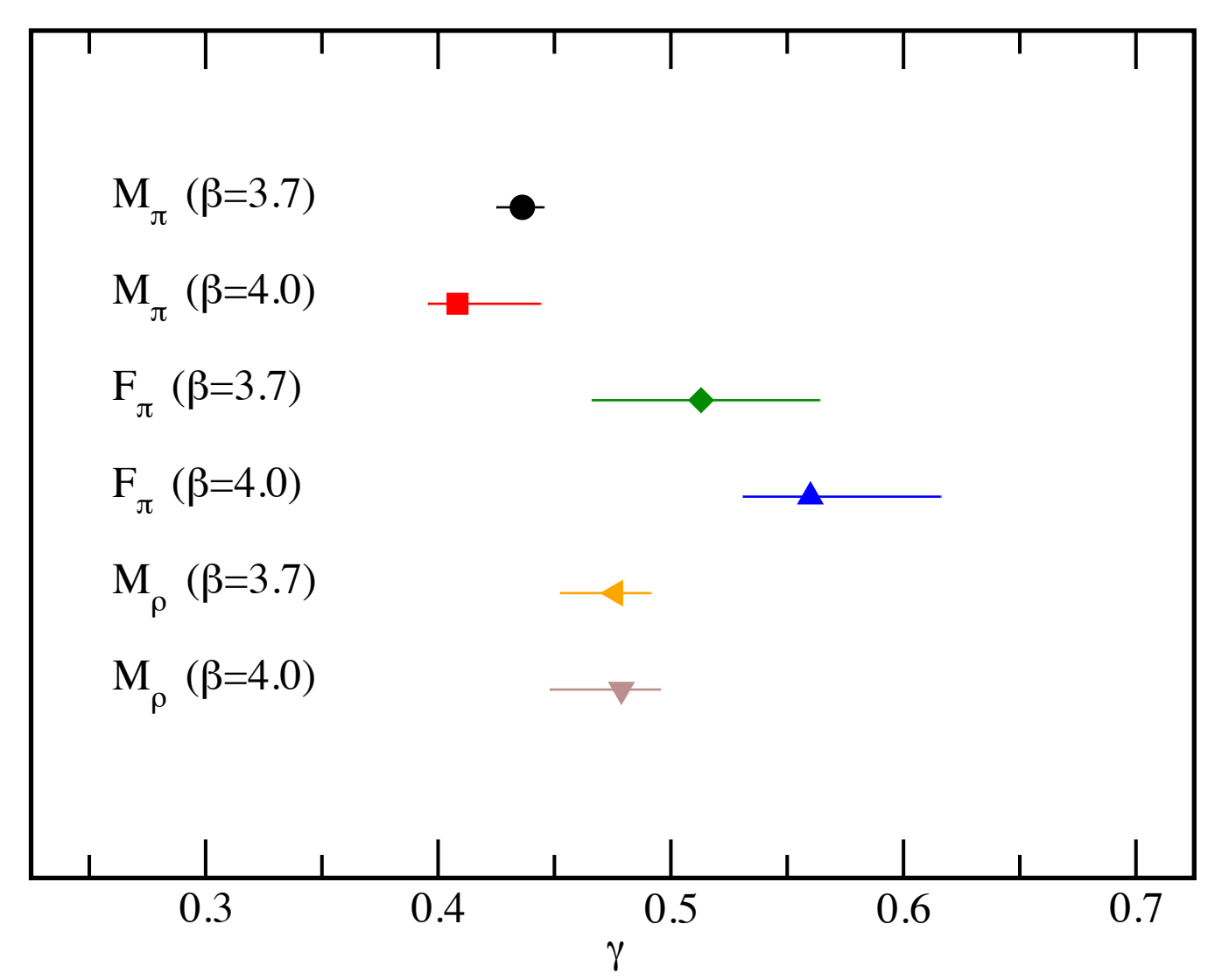

- consistent $\gamma$ by $1.5 \sigma$ level except for $F_{\pi}$ at $\beta=4.0$

- remember: $F_{\pi}$ at $\beta=4.0$ speculated to be out of the scaling region 


\section{summary of $\gamma$ obtained by minimizing $P(\gamma)$}

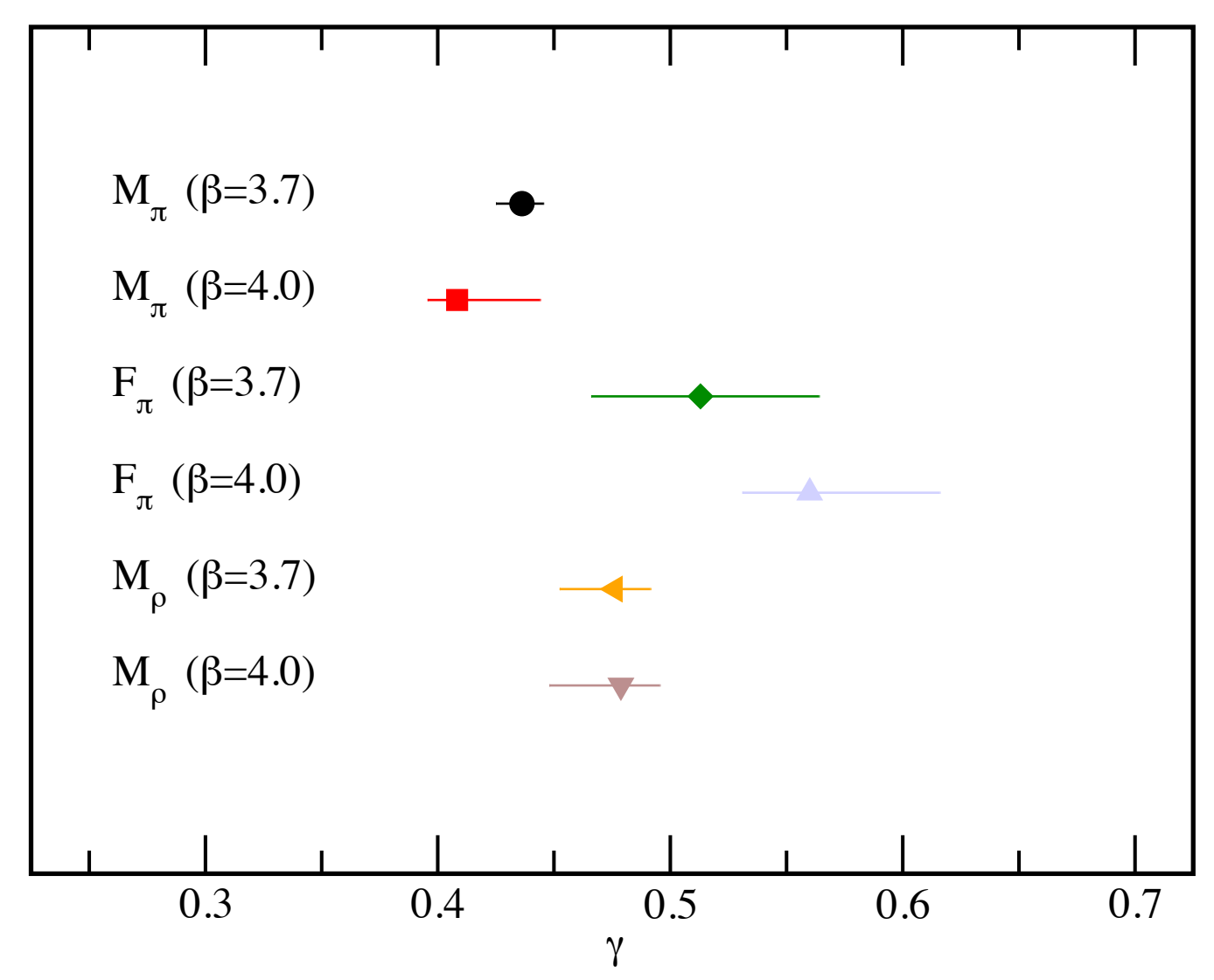

- consistent $\gamma$ by $1.5 \sigma$ level except for $F_{\pi}$ at $\beta=4.0$

- remember: $F_{\pi}$ at $\beta=4.0$ speculated to be out of the scaling region

- universal low energy behavior: good with $0.4<\gamma^{*}<0.5$ 


\section{Conformal type fit with finite volume correction}

$$
\begin{aligned}
& \xi=L M_{\pi}, L F_{\pi}, L M_{\rho} \\
& \xi=c_{0}+c_{1} L m_{f}^{1 /(1+\gamma)} \cdots \text { fit a, } \\
& \xi=c_{0}+c_{1} L m_{f}^{1 /(1+\gamma)}+c_{2} L m_{f}^{\alpha} \cdots \text { fit b. }
\end{aligned}
$$

\begin{tabular}{c|ccc} 
& $\gamma$ & $\alpha$ & $\chi^{2} /$ dof \\
\hline fit a & $0.455(3)$ & - & 5.43 \\
\hline fit b-1 & $0.417(10)$ & $\frac{(3-2 \gamma)}{(1+\gamma)}$ & 1.88 \\
fit b-2 & $0.431(8)$ & {$[2]$} & 1.83 \\
\hline
\end{tabular}
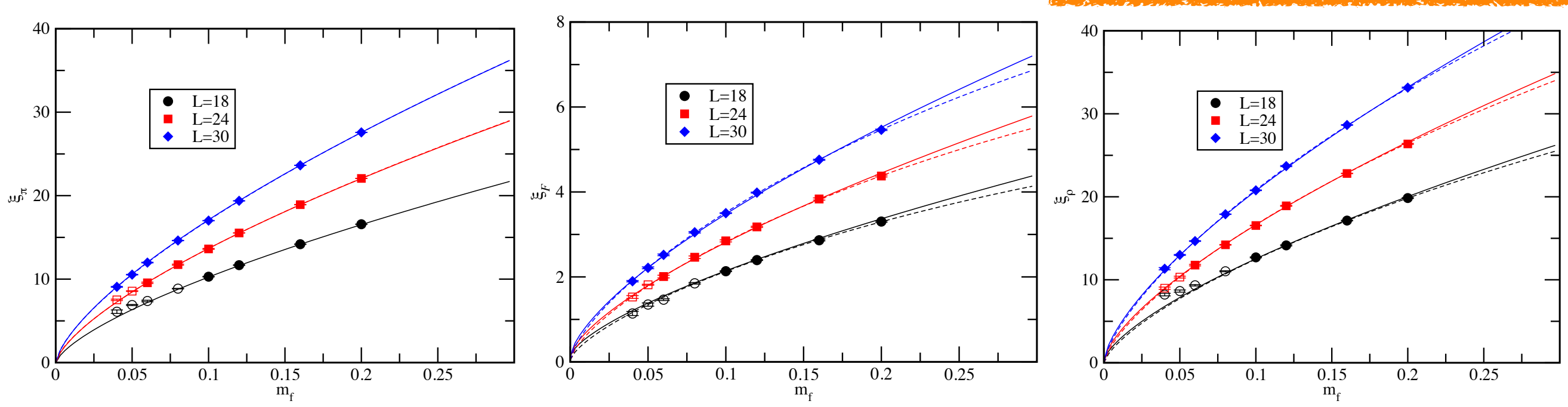

- simultaneous fit it with a leading mass dependent correction is not bad

- b-1: Ladder Schwinger-Dyson, b-2: $(a m)^{2}$ lattice artifact

- resulting $\mathrm{\gamma}$ is consistent with the model independent analysis 


\section{ChPT fit (after infinite volume extrapolation)}

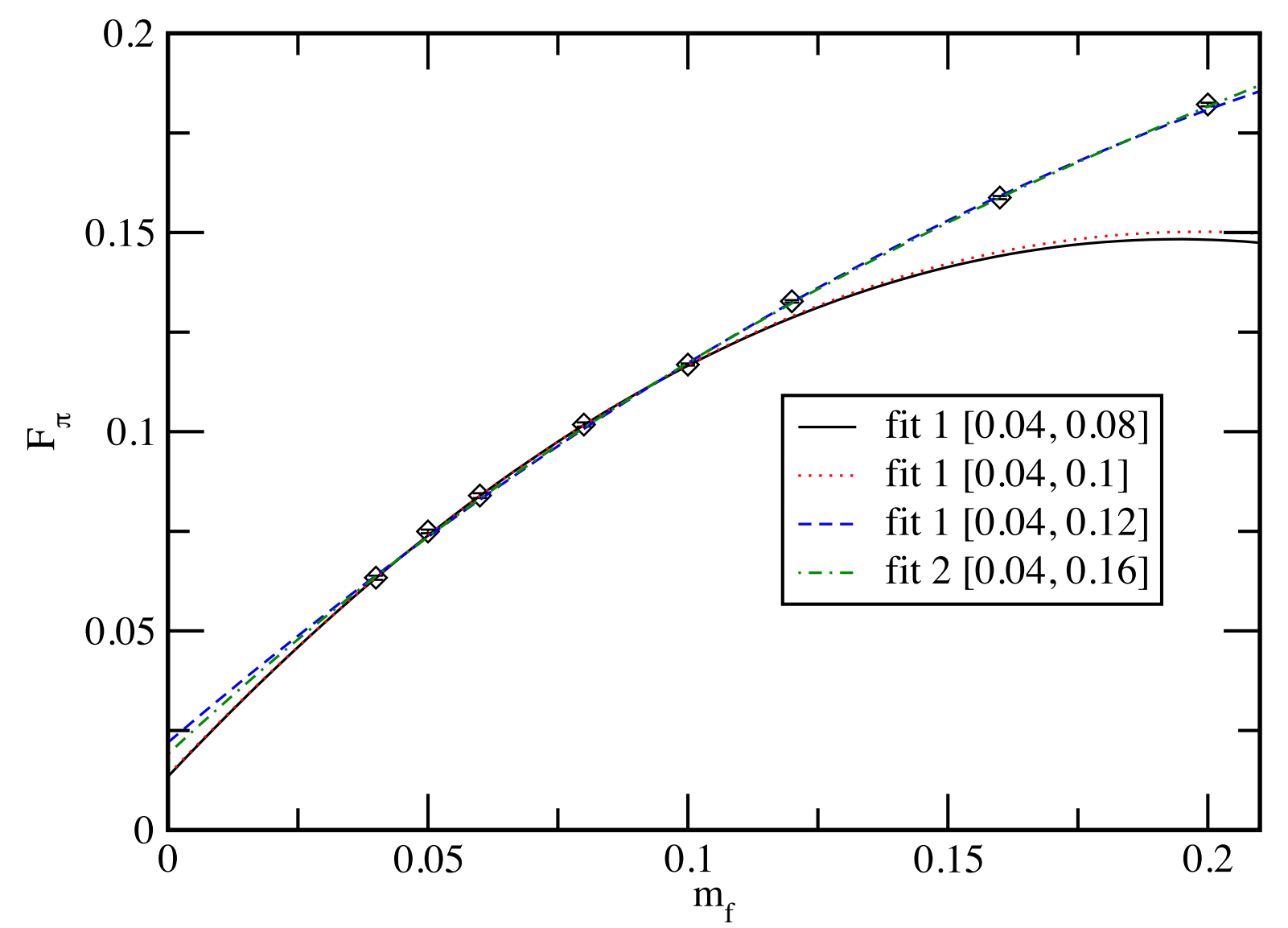

$$
h\left(m_{f}\right)= \begin{cases}c_{0}+c_{1} m_{f}+c_{2} m_{f}^{2} & \cdots \text { fit } 1 \\ c_{0}+c_{1} m_{f}+c_{2} m_{f}^{2}+c_{3} m_{f}^{3} & \cdots \text { fit } 2\end{cases}
$$

\begin{tabular}{c|ccccc} 
fit range & $c_{0}$ & $c_{1}$ & $c_{2}$ & $c_{3}$ & $\chi^{2} /$ dof \\
\hline fit $1:[0.04,0.08]$ & $0.0101(54)$ & $1.53(19)$ & $-4.8(1.5)$ & - & 2.09 \\
fit $1:[0.04,0.1]$ & $0.0138(29)$ & $1.39(88)$ & $-3.62(61)$ & - & 1.39 \\
fit $1:[0.04,0.12]$ & $0.0226(17)$ & $1.113(45)-1.64(27)$ & - & 5.42 \\
fit $2:[0.04,0.16]$ & $0.0182(34)$ & $1.28(13)$ & $-3.4(1.3)$ & $6.0(4.2)$ & 4.75
\end{tabular}

- 2 nd order polynomial fit is reasonably good for small mass range \& $c_{0}>0$ 


\section{ChPT fit (after infinite volume extrapolation)}

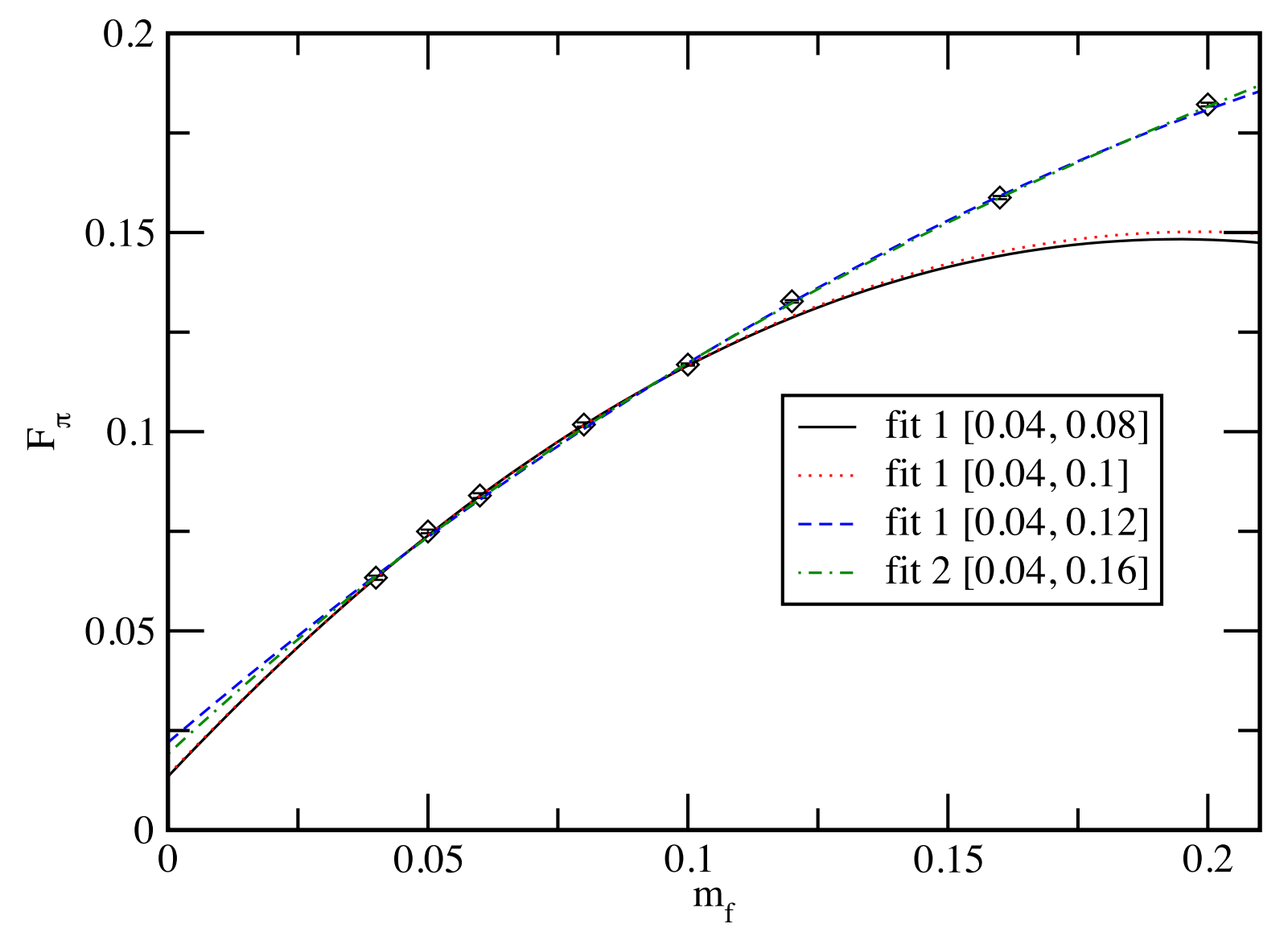

$$
h\left(m_{f}\right)= \begin{cases}c_{0}+c_{1} m_{f}+c_{2} m_{f}^{2} & \cdots \text { fit } 1 \\ c_{0}+c_{1} m_{f}+c_{2} m_{f}^{2}+c_{3} m_{f}^{3} & \cdots \text { fit } 2\end{cases}
$$

\begin{tabular}{c|ccccc} 
fit range & $c_{0}$ & $c_{1}$ & $c_{2}$ & $c_{3}$ & $\chi^{2} /$ dof \\
\hline fit $1:[0.04,0.08]$ & $0.0101(54)$ & $1.53(19)$ & $-4.8(1.5)$ & - & 2.09 \\
fit $1:[0.04,0.1]$ & $0.0138(29)$ & $1.39(88)$ & $-3.62(61)$ & - & 1.39 \\
fit $1:[0.04,0.12]$ & $0.0226(17)$ & $1.113(45)$ & $-1.64(27)$ & - & 5.42 \\
fit $2:[0.04,0.16]$ & $0.0182(34)$ & $1.28(13)$ & $-3.4(1.3)$ & $6.0(4.2)$ & 4.75
\end{tabular}

- 2 nd order polynomial fit is reasonably good for small mass range \& $c_{0}>0$ 


\section{ChPT fit (after infinite volume extrapolation)}

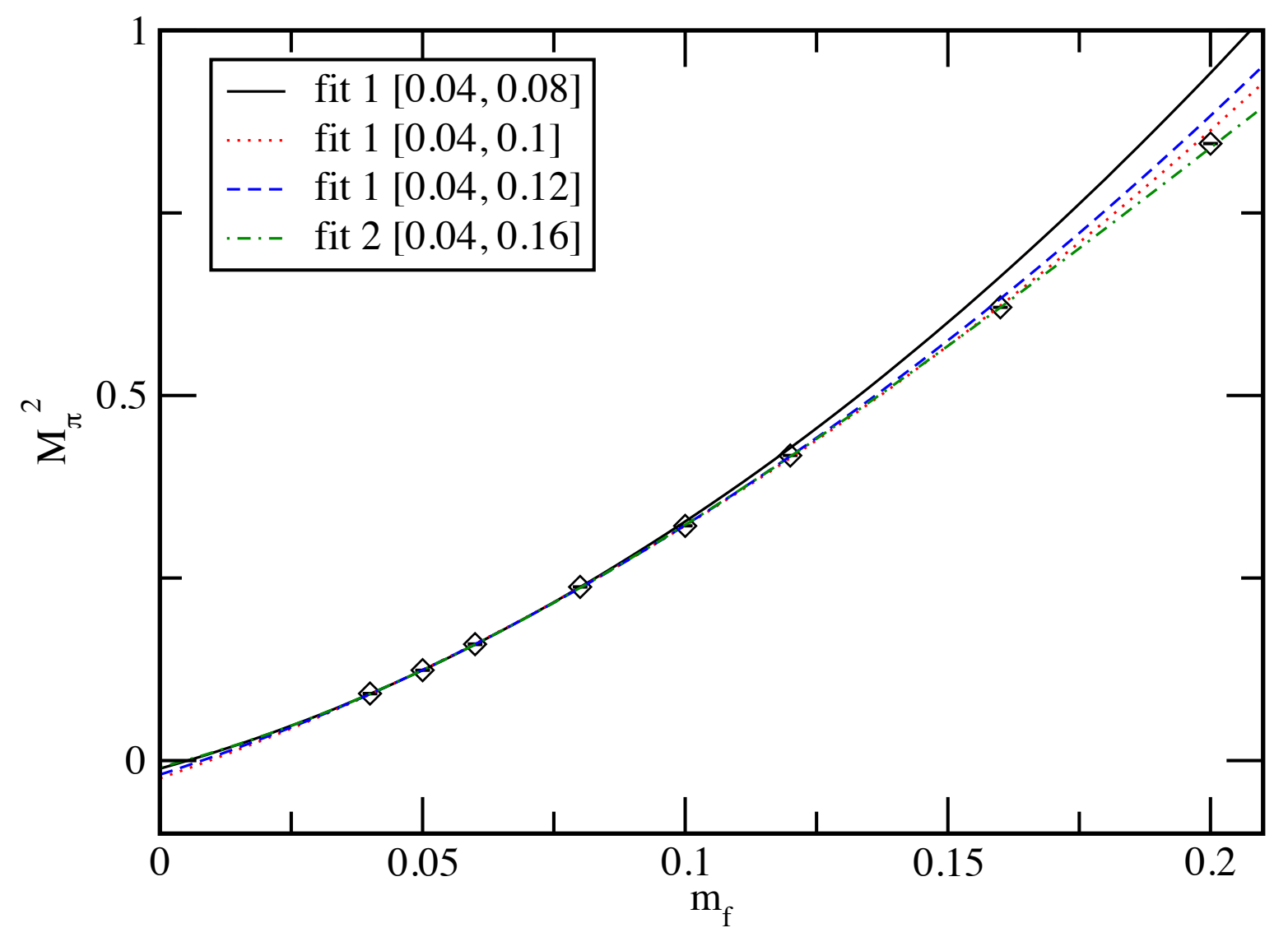

$$
\begin{aligned}
& M_{\pi}^{2}=h\left(m_{f}\right)= \begin{cases}c_{0}+c_{1} m_{f}+c_{2} m_{f}^{2} & \cdots \text { fit } 1 \\
c_{0}+c_{1} m_{f}+c_{2} m_{f}^{2}+c_{3} m_{f}^{3} & \cdots \text { fit } 2\end{cases} \\
& \begin{array}{c|ccccc}
\text { fit range } & c_{0} & c_{1} & c_{2} & c_{3} & \chi^{2} / \mathrm{dof} \\
\hline \text { fit } 1:[0.04,0.08] & -0.0090(93) & 1.95(32) & 14.2(2.6) & - & 0.16
\end{array} \\
& \text { [0] } \quad 1.640(31) 16.68(47) \quad-\quad 0.56
\end{aligned}
$$

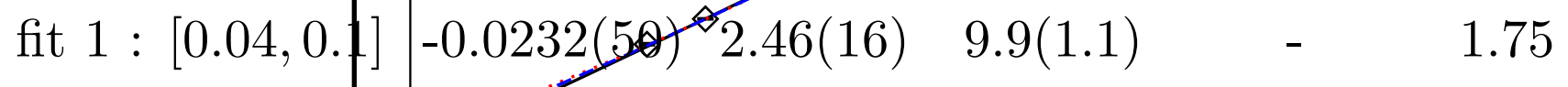

$$
\begin{aligned}
& \text { [0] } \quad 1.754(21) 14.73(25) \quad-\quad 8.54 \\
& \text { fit } 1:[0.04,0.12][-0.0174(31) 2.27(85) \quad 11.32(52) \quad-\quad 1.93 \\
& \text { [0] } 1.801(16) 14.09(16) \quad-\quad 9.36 \\
& \text { fit } 2:[0.04,0.16] \mid-0.0044(61) \quad 1.69(22) \quad 19.1(2.4)-32.9(7.6) \quad 3.28 \\
& \text { [0] } \quad 1.537(29) 20.76(53)-38.2(2.3) \quad 2.59
\end{aligned}
$$




\section{ChPT fit (after infinite volume extrapolation)}

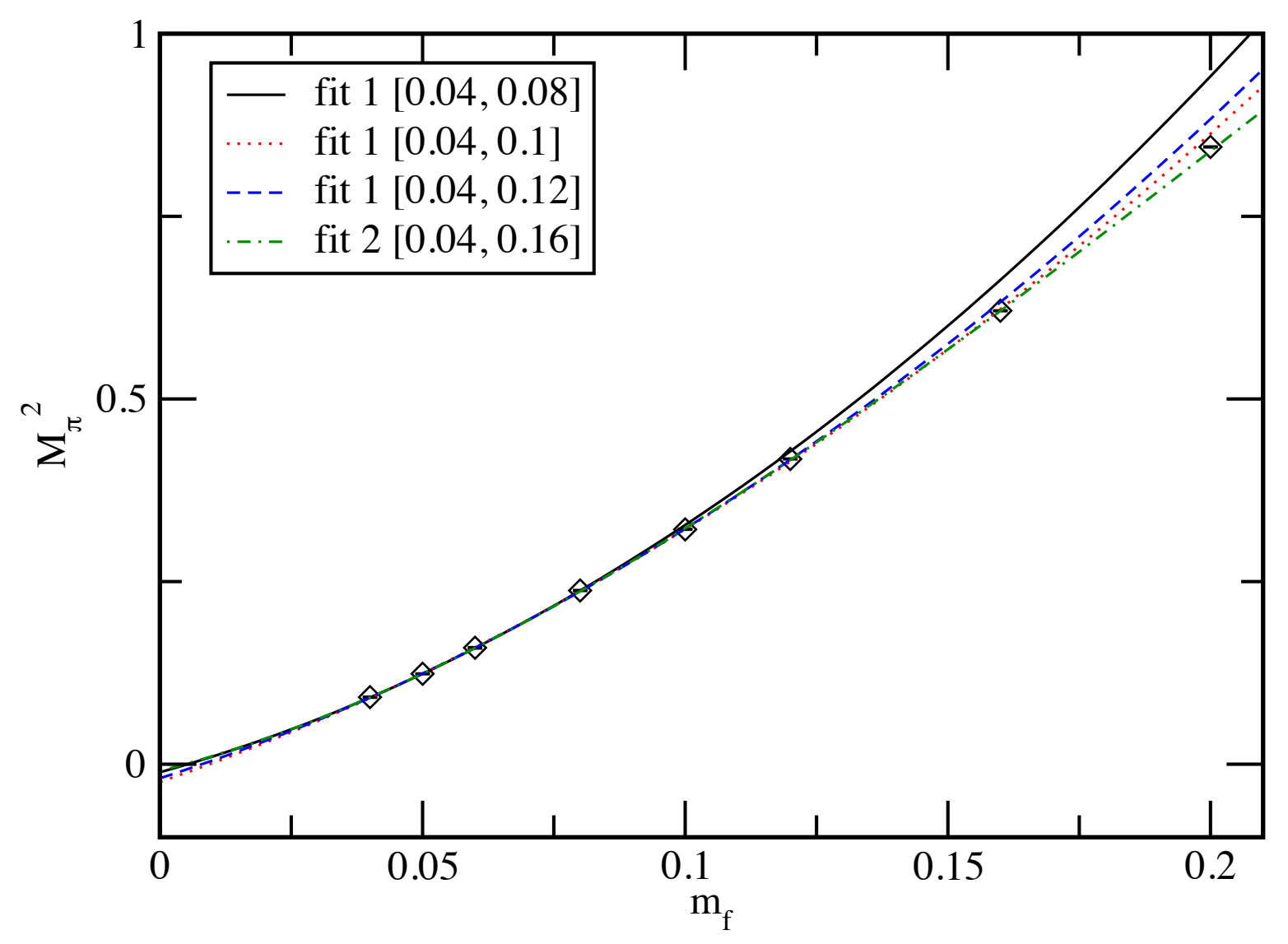

- wide range fit ends up $\mathrm{c}_{0}<0$

\begin{tabular}{|c|c|c|c|c|c|}
\hline \multicolumn{2}{|c|}{$M_{\pi}^{2}=h\left(m_{f}\right)=\{$} & \multicolumn{4}{|c|}{$\begin{array}{ll}c_{0}+c_{1} m_{f}+c_{2} m_{f}^{2} & \cdots \text { fit } 1 \\
c_{0}+c_{1} m_{f}+c_{2} m_{f}^{2}+c_{3} m_{f}^{3} & \cdots \text { fit } 2\end{array}$} \\
\hline fit range & $c_{0}$ & $c_{1}$ & $c_{2}$ & $c_{3}$ & $\chi^{2} /$ dof \\
\hline \multirow[t]{2}{*}{ fit $1:[0.04,0.08]$} & $-0.0090(93)$ & $1.95(32)$ & $14.2(2.6)$ & - & 0.16 \\
\hline & {$[0]$} & $1.640(31)$ & $16.68(47)$ & - & 0.56 \\
\hline \multirow[t]{2}{*}{ fit $1:[0.04,0.1]$} & $-0.0232(50)$ & $2.46(16)$ & $9.9(1.1)$ & - & 1.75 \\
\hline & {$[0]$} & $1.754(21)$ & $14.73(25)$ & - & 8.54 \\
\hline \multirow[t]{2}{*}{ fit $1:[0.04,0.12]$} & $-0.0174(31)$ & $2.27(85)$ & $11.32(52)$ & - & 1.93 \\
\hline & {$[0]$} & $1.801(16)$ & $14.09(16)$ & - & 9.36 \\
\hline \multirow[t]{2}{*}{ fit $2:[0.04,0.16]$} & $-0.0044(61)$ & $1.69(22)$ & $19.1(2.4)$ & $-32.9(7.6)$ & 3.28 \\
\hline & {$[0]$} & $1.537(29)$ & $20.76(53)$ & $-38.2(2.3)$ & 2.59 \\
\hline
\end{tabular}




\section{ChPT fit (after infinite volume extrapolation)}

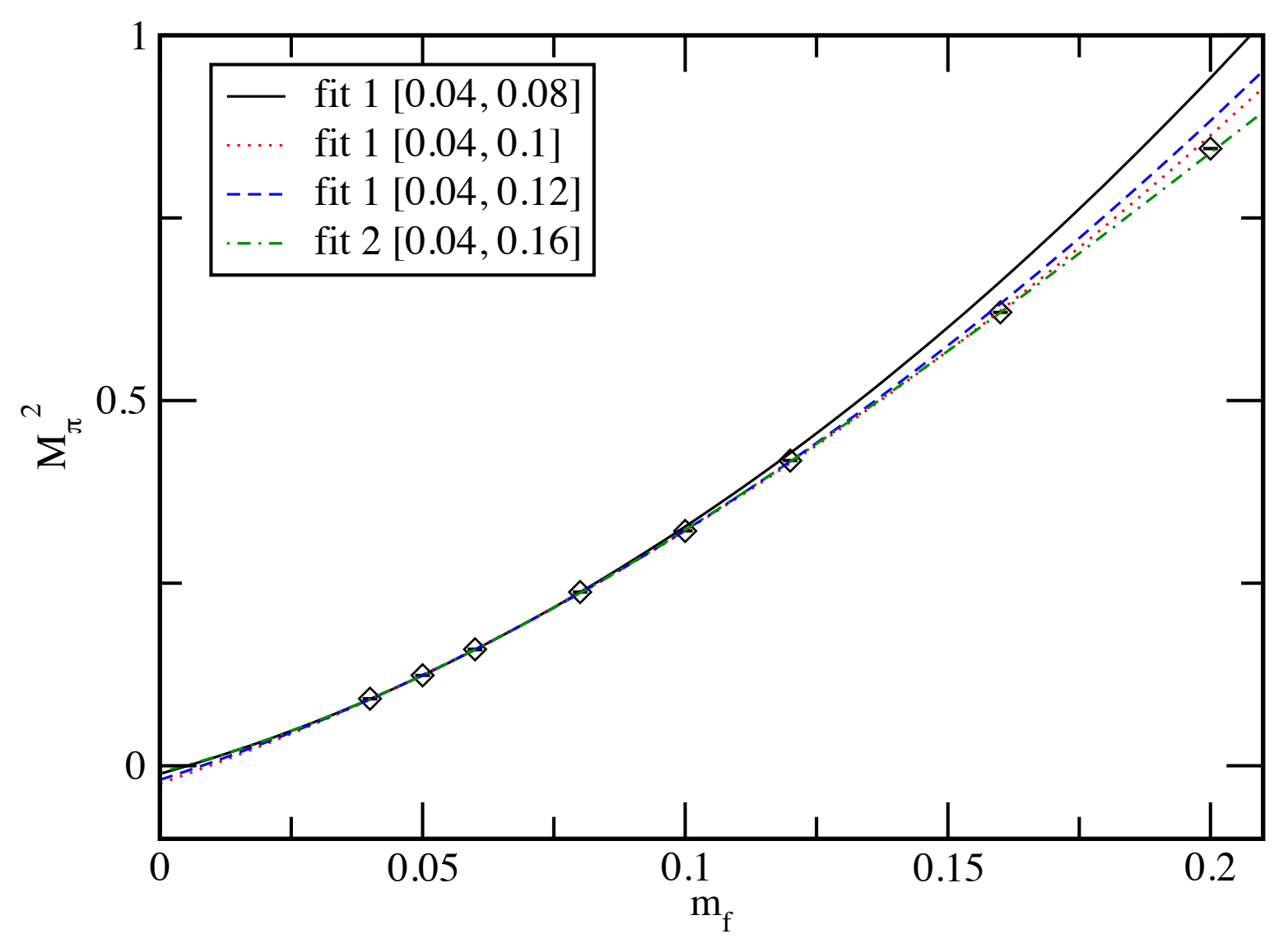

- wide range fit ends up $\mathrm{c}_{0}<0$
$M_{\pi}^{2}=h\left(m_{f}\right)= \begin{cases}c_{0}+c_{1} m_{f}+c_{2} m_{f}^{2} & \cdots \text { fit } 1 \\ c_{0}+c_{1} m_{f}+c_{2} m_{f}^{2}+c_{3} m_{f}^{3} & \cdots \text { fit2 }\end{cases}$

\begin{tabular}{|c|c|c|c|c|c|}
\hline fit range & $c_{0}$ & $c_{1}$ & $c_{2}$ & $c_{3}$ & $\chi^{2} / \operatorname{dof}$ \\
\hline \multirow[t]{2}{*}{ fit $1:[0.04,0.08]$} & $-0.0090(93)$ & $1.95(32)$ & $14.2(2.6)$ & - & 0.16 \\
\hline & {$[0]$} & $1.640(31)$ & $16.68(47)$ & - & 0.56 \\
\hline \multirow[t]{2}{*}{ fit $1:[0.04,0.1]$} & $-0.0232(50)$ & $2.46(16)$ & $9.9(1.1)$ & - & 1.75 \\
\hline & {$[0]$} & $1.754(21)$ & $14.73(25)$ & - & 8.54 \\
\hline fit $1:[0.04,0.12]$ & $-0.0174(31)$ & $2.27(85)$ & $11.32(52)$ & - & 1.93 \\
\hline
\end{tabular}

$[0] \quad 1.801(16) 14.09(16) \quad-\quad 9.36$

fit $2:[0.04,0.16]-0.0044(61) \quad 1.69(22) \quad 19.1(2.4) \quad-32.9(7.6) \quad 3.28$

[0] $\quad 1.537(29) 20.76(53)-38.2(2.3) \quad 2.59$

- consistent with $\mathrm{c}_{0}=0$ for small mass range 


\section{ChPT fit (after infinite volume extrapolation)}

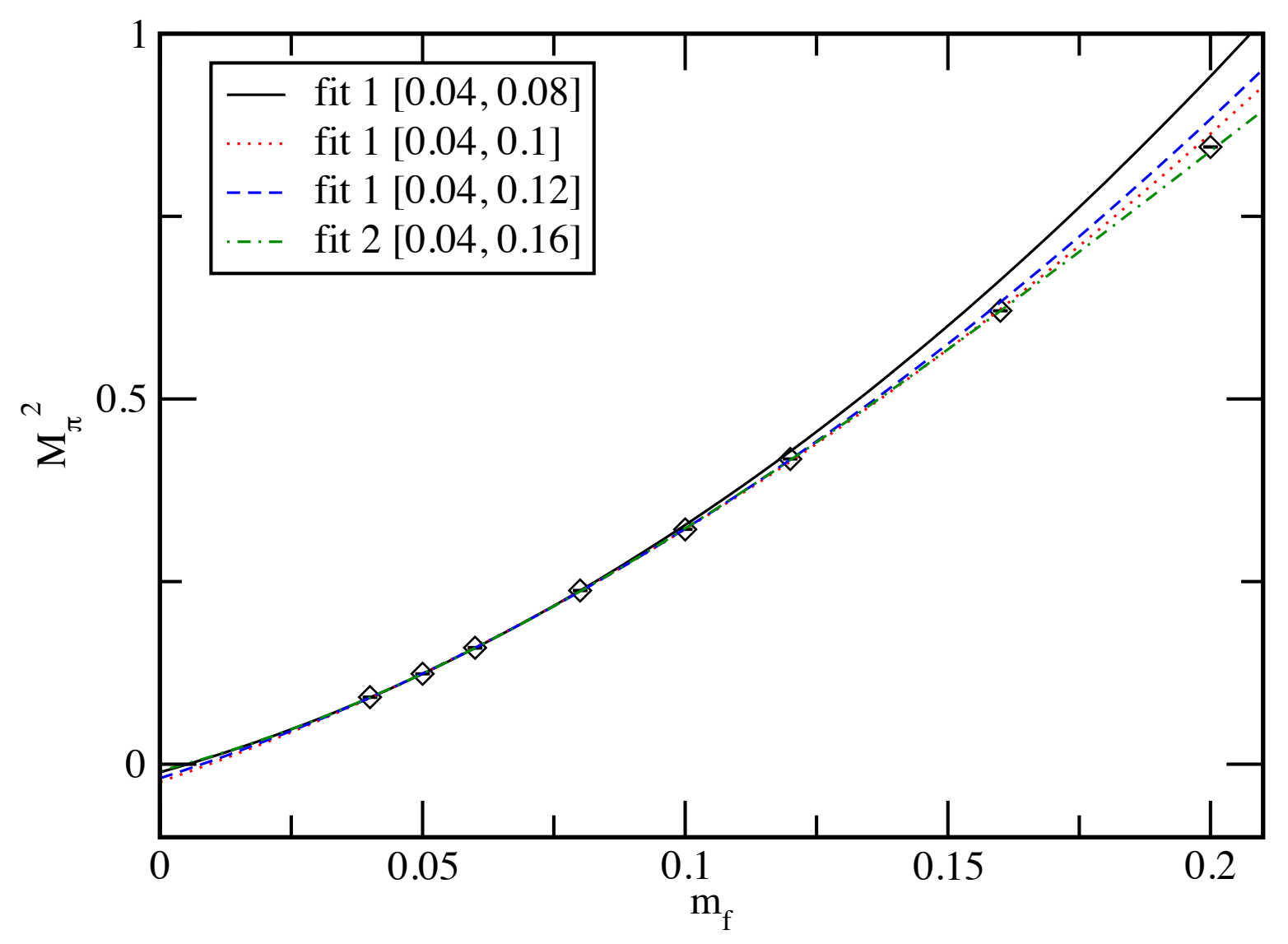

- wide range fit ends up $c_{0}<0$

$$
M_{\pi}^{2}=h\left(m_{f}\right)= \begin{cases}c_{0}+c_{1} m_{f}+c_{2} m_{f}^{2} & \cdots \text { fit } 1 \\ c_{0}+c_{1} m_{f}+c_{2} m_{f}^{2}+c_{3} m_{f}^{3} & \cdots \text { fit2 }\end{cases}
$$

\begin{tabular}{c|ccccc} 
fit range & $c_{0}$ & $c_{1}$ & $c_{2}$ & $c_{3}$ & $\chi^{2} / \mathrm{dof}$ \\
\hline fit $1:[0.04,0.08]$ & $-0.0090(93)$ & $1.95(32)$ & $14.2(2.6)$ & - & 0.16 \\
& {$[0]$} & $1.640(31)$ & $16.68(47)$ & - & 0.56 \\
fit $1:[0.04,0.1]$ & $-0.0232(50)$ & $2.46(16)$ & $9.9(1.1)$ & - & 1.75 \\
& {$[0]$} & $1.754(21)$ & $14.73(25)$ & - & 8.54 \\
fit $1:[0.04,0.12]$ & $-0.0174(31)$ & $2.27(85)$ & $11.32(52)$ & - & 1.93
\end{tabular}

[0] $1.801(16) 14.09(16) \quad-\quad 9.36$

fit $2:[0.04,0.16] \mid-0.0044(61) \quad 1.69(22) \quad 19.1(2.4)-32.9(7.6) \quad 3.28$ [0] $\quad 1.537(29) 20.76(53)-38.2(2.3) \quad 2.59$

- consistent with $\mathrm{c}_{0}=0$ for small mass range

- But: $M_{\pi} /(4 \pi F) \sim 2$ at lightest point $\rightarrow$ difficult to tell real chiral behavior 


\section{Summary:}

$\mathrm{SU}(3)$ gauge theory with $\mathrm{N}_{\mathrm{f}}=12$ fundamental fermion simulation with $\mathrm{HISQ}$

- $\beta=3.7,4.0$ : consistent with being in the asymptotically free regime

- $M_{\pi}, F_{\pi}, M_{\rho}$ : consistent with the finite size hyper scaling for conformal theory

- resulting $\mathrm{Y}^{\star}$ from different quantities, lattice spacings are consistent except

- $F_{\pi}$ at $\beta=4.0$ ( $m_{\mathrm{f}}$ likely too heavy for universal mass dep. to dominate)

- need careful continuum scaling needed to get more accurate than $0.4<\gamma^{*}<0.5$

- real / remnant (approximate) conformal property is definitely there

- could not exclude S $\chi$ SB with very small breaking scale

- even if $\mathrm{S} \chi \mathrm{SB}, \gamma_{\mathrm{m}}$ too small for walking theory of phenomenological interest

- $\mathrm{N}_{\mathrm{f}}=8$ theory is interesting \& under investigation with same lattice set up 
Thank you for your attention 


\section{ChPT inspired infinite volume limit $(\beta=3.7)$}

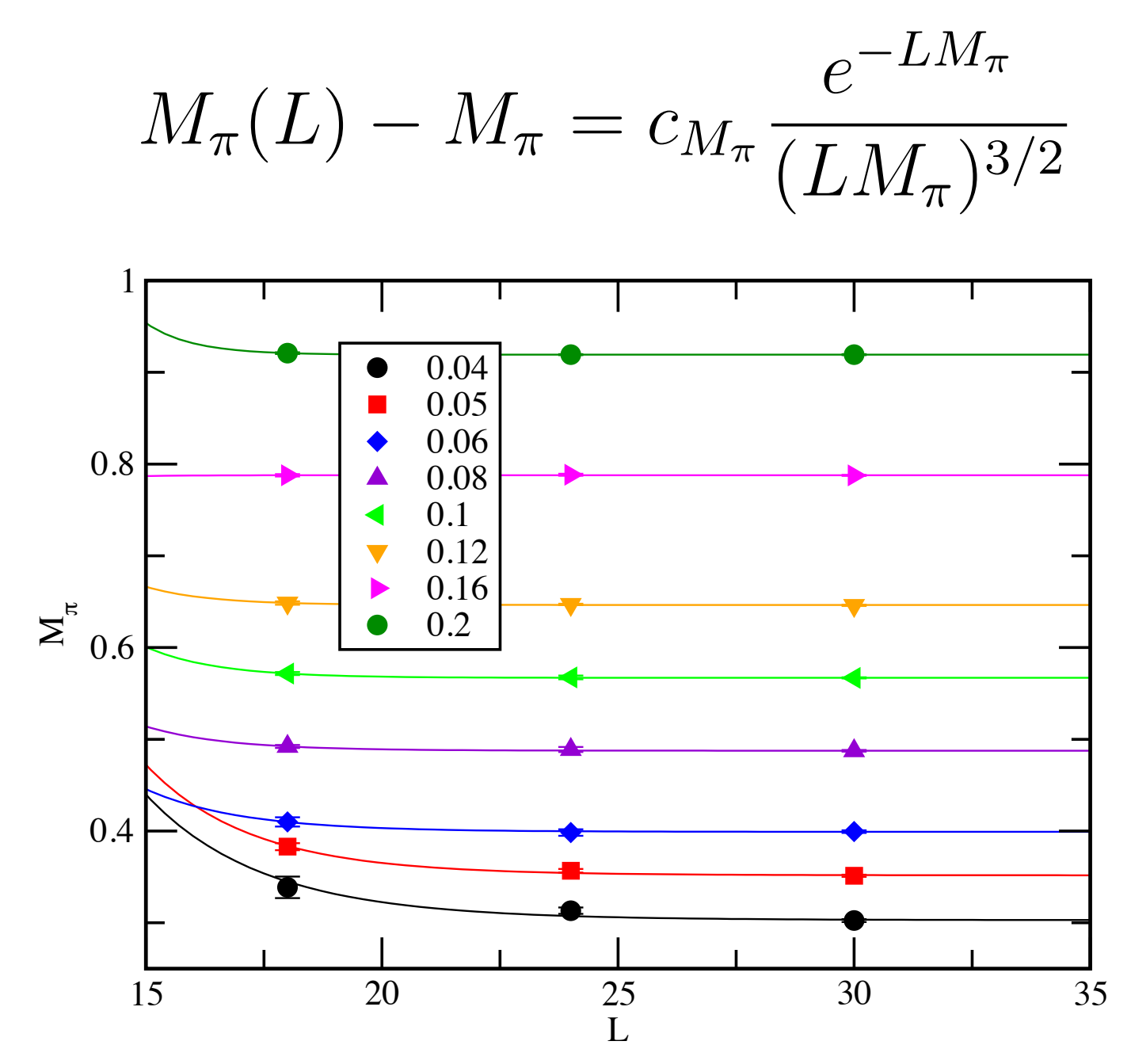

- ChPT type finite volume effect $\rightarrow$ chiral fit results not inconsistent with $\mathrm{S} \chi \mathrm{SB}$ 


\section{HISQ action}

- proposed by HPQCD collaboration for

- smaller taste violation than other approaches

- better handling of heavy quarks

- being used in simulations

- MILC: $\mathrm{Nf}=2+1+1 \mathrm{QCD}$

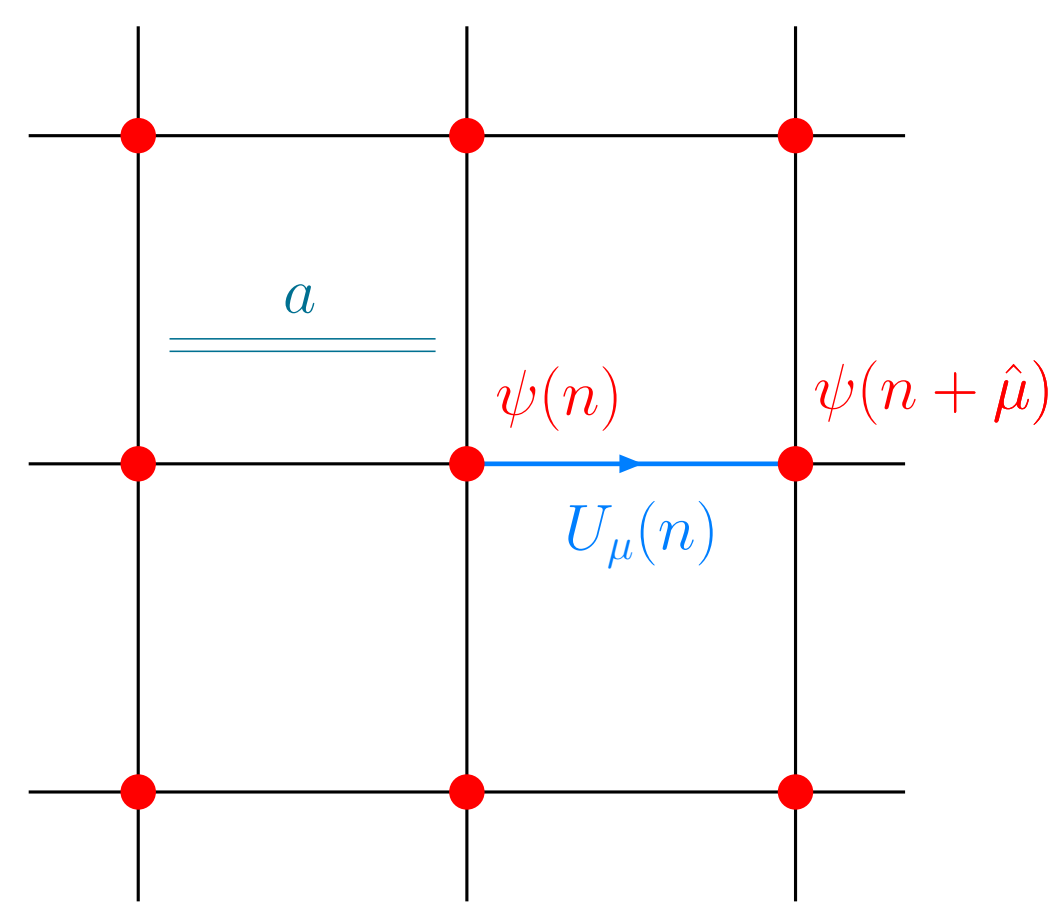

- HOTQCD: QCD thermodynamics: Bazavov-Petreczky (Lat'10 proceedings)

- HISQ/tree is best of [HISQ/tree, Asqtad, stout] for flavor (taste) symmetry, dispersion relation 


\section{HISQ action}

- proposed by HPQCD collaboration for

- smaller taste violation than other approaches

- better handling of heavy quarks

- being used in simulations

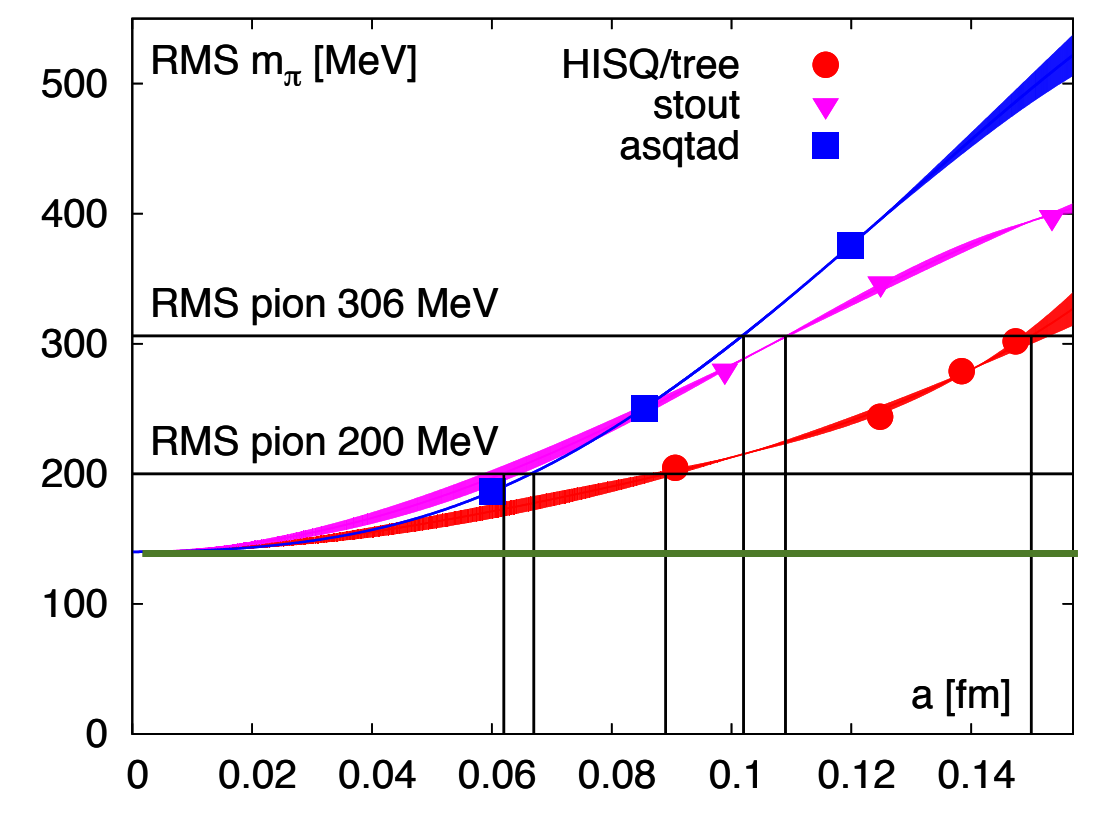

- MILC: $\mathrm{Nf}=2+1+1 \mathrm{QCD}$

Figure 2: RMS pion mass when $m_{\gamma_{5}}=140 \mathrm{MeV}$. See details in the text.

- HOTQCD: QCD thermodynamics: Bazavov-Petreczky (Lat'10 proceedings)

- HISQ/tree is best of [HISQ/tree, Asqtad, stout] for flavor (taste) symmetry, dispersion relation 


\section{LHC (Large Hadron Collider)}

- excess @ 125 GeV

- $1 \sigma$ level (look elsewhere)

- larger when ATLAS \& CMS results are combined?

- $\mathrm{M}_{\mathrm{w}}=\mathrm{Mz}_{\mathrm{z}} \cos \theta_{\mathrm{w}}=\mathrm{gF} / 2\left(\mathrm{~F}_{\mathrm{\pi}}=\mathrm{V}_{\mathrm{weak}}=246 \mathrm{GeV}\right)$

- $\mathrm{M}_{\mathrm{H}} \sim 500 \mathrm{GeV}$ : problem ?

- even if scalar is fund at $\sim 125 \mathrm{GeV}$

- possible techni-dilaton (Matsuzaki-Yamawaki,,)

- $0++$ glueball tends to be much lighter than techni-hadrons

- Cf. SU(2) lattice work by Del Debbio et al

- important to investigate glueball for SU(3) as well !!! 


\title{
An approach to non-leptonic B-decays on the lattice
}

\author{
Christopher Aubin \\ May 15, 2012
}

New Horizons for Lattice Computations with Chiral Fermions

with

\section{David Lin \& Amarjit Soni}

\section{Phys. Let. B7I0 164}




\section{Outline}

Nonleptonic $B$ decays (CKM)

Weak operators

Four-point functions on the lattice

Starting points:

Chiral Perturbation Theory

Resonance contribution

Hard pion ChPT 
$B^{-} \rightarrow D^{0} P^{-}$

$$
B^{-} \rightarrow \bar{D}^{0} P^{-}
$$

On the lattice, define a reduced ratio:

$$
r_{B P}^{\text {red }} \equiv \frac{r_{B P}}{V_{C K M}^{\text {combo }}}=r_{B P} \frac{\left|V_{b b}^{*} V_{u q}\right|^{2}}{\left|V_{u b}^{*} V_{c q}\right|^{2}}
$$

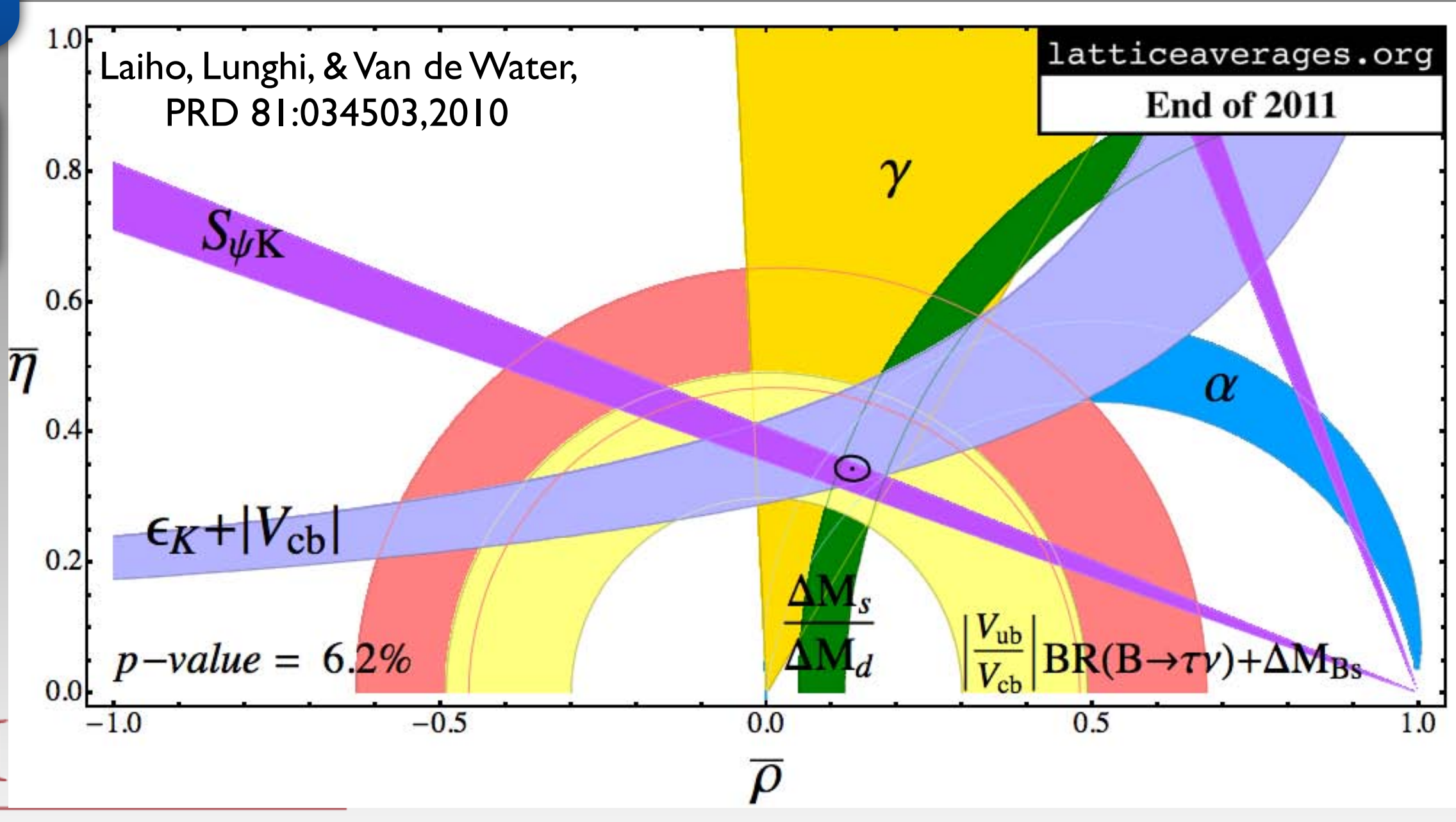

experimentally accessible

\section{The ratio}

$$
r_{B P}=\frac{\operatorname{Br}\left[B^{-} \rightarrow \bar{D}^{0} P^{-}\right]}{\operatorname{Br}\left[B^{-} \rightarrow D^{0} P^{-}\right]}
$$

can give insight into $\gamma$

(HFAG, arXiv: I0 I0.1589)

\section{$\frac{\text { FORDHAM UNI }}{\text { THE JESUIT UNIVERSITY OF NEW YORK }}$}


$B^{-} \rightarrow D^{0} P^{-} \quad B^{-} \rightarrow \bar{D}^{0} P^{-}$

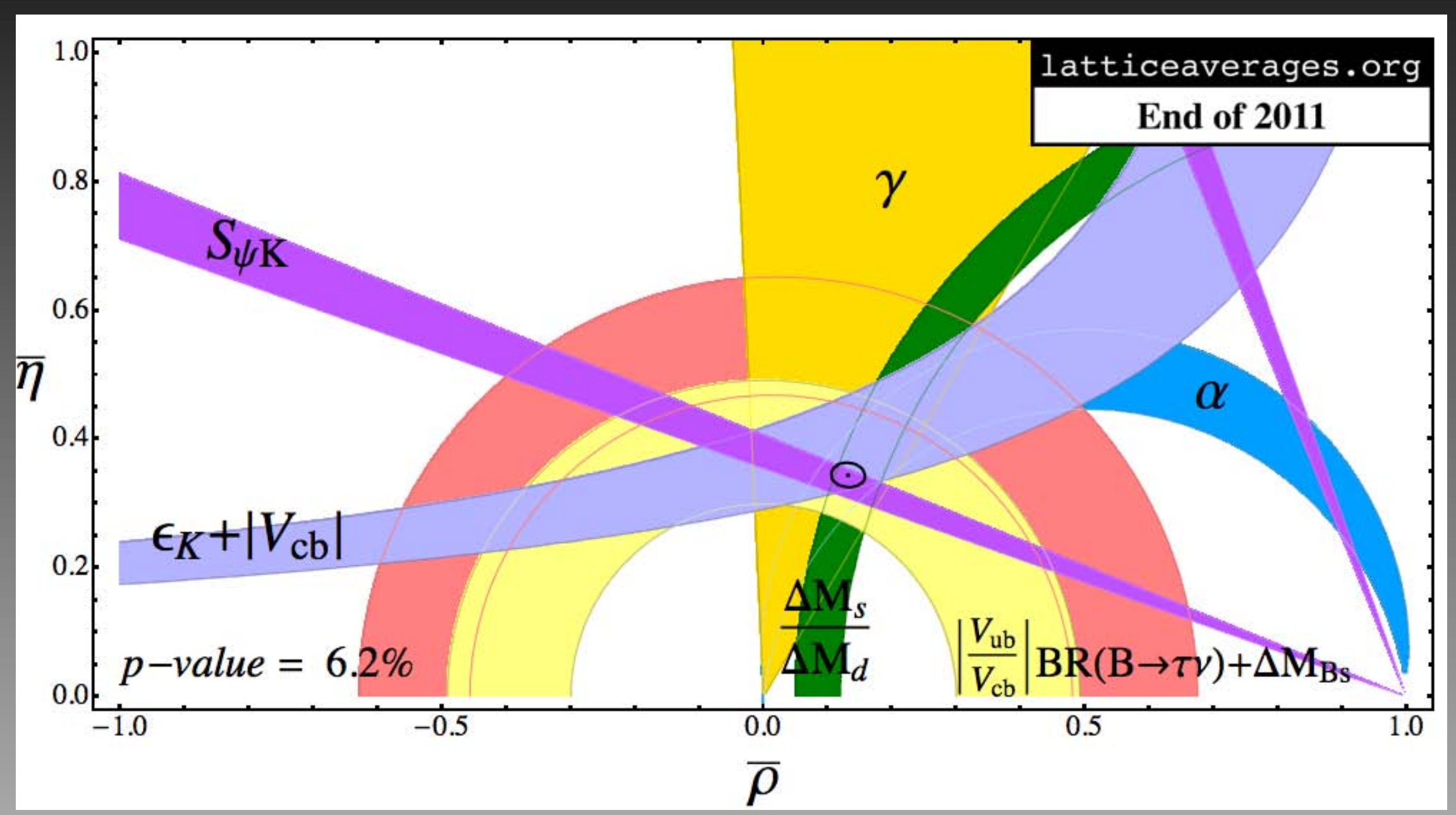

$\gamma \quad O(25 \%)$

$\alpha \quad O(5 \%)$

$\beta \quad O(3 \%)$

\section{Hope:}

\section{Determination of the real part} of these amplitudes to $15-20 \%$ [ $\sim 5$ years?]

$$
\Rightarrow \gamma \sim 10 \%
$$


Weak operators

$$
\begin{aligned}
Q_{1}^{b \rightarrow c, i} & =\left(\bar{q}_{\alpha}^{i} \gamma^{\mu}\left(1-\gamma_{5}\right) b_{\alpha}\right)\left(\bar{c}_{\beta} \gamma_{\mu}\left(1-\gamma_{5}\right) u_{\beta}\right) \\
Q_{2}^{b \rightarrow c, i} & =\left(\bar{q}_{\alpha}^{i} \gamma^{\mu}\left(1-\gamma_{5}\right) b_{\beta}\right)\left(\bar{c}_{\beta} \gamma_{\mu}\left(1-\gamma_{5}\right) u_{\alpha}\right) \\
Q_{1}^{b \rightarrow \bar{c}, i} & =\left(\bar{q}_{\alpha}^{i} \gamma^{\mu}\left(1-\gamma_{5}\right) b_{\alpha}\right)\left(\bar{u}_{\beta} \gamma_{\mu}\left(1-\gamma_{5}\right) c_{\beta}\right) \\
Q_{2}^{b \rightarrow \bar{c}, i} & =\left(\bar{q}_{\alpha}^{i} \gamma^{\mu}\left(1-\gamma_{5}\right) b_{\beta}\right)\left(\bar{u}_{\beta} \gamma_{\mu}\left(1-\gamma_{5}\right) c_{\alpha}\right)
\end{aligned}
$$

\section{Maiani-Testa theorem}

$$
\left\langle 0\left|\pi D \mathcal{O}_{\text {weak }} B\right| 0\right\rangle
$$

4 pt functions only yields real part (no strong phase)

\section{Can be circumvented \\ (Lellouch-Lüscher, RBC/UKQCD) e.g., $K \rightarrow 2 \pi$}




\section{Heavy-light meson ChPT}

$$
\mathcal{L}_{\mathrm{G}}=\frac{f^{2}}{8} \operatorname{Tr}\left(\partial_{\mu} \Sigma \partial^{\mu} \Sigma^{\dagger}\right)+\frac{1}{4} \mu f^{2} \operatorname{Tr}\left(\mathcal{M} \Sigma+\mathcal{M} \Sigma^{\dagger}\right)
$$$$
\Sigma \longrightarrow L \Sigma R^{\dagger}, \text { where } L \in \mathrm{SU}(3)_{\mathrm{L}}, \text { and } R \in \mathrm{SU}(3)_{\mathrm{R}}
$$

$$
\begin{aligned}
& H_{v, a}^{(\bar{Q})}=\left(\gamma^{\mu} \mathcal{V}_{\mu, a}^{*}(\bar{Q})-\gamma_{5} \mathcal{P}_{a}^{(\bar{Q})}\right)\left(\frac{1-\not}{2}\right) \\
& H_{v, a}^{(Q)}=\left(\frac{1+\not}{2}\right)\left(\gamma^{\mu} \mathcal{\nu}_{\mu_{, a}^{*}}^{*(Q)}-\gamma_{5} \mathcal{P}_{a}^{(Q)}\right)
\end{aligned}
$$

$$
\begin{aligned}
& \mathbb{V}_{\mu}=\frac{i}{2}\left[\sigma^{\dagger} \partial_{\mu} \sigma+\sigma \partial_{\mu} \sigma^{\dagger}\right] \\
& \mathbb{A}_{\mu}=\frac{i}{2}\left[\sigma^{\dagger} \partial_{\mu} \sigma-\sigma \partial_{\mu} \sigma^{\dagger}\right]
\end{aligned} \quad \begin{aligned}
& H_{Q}(x) \rightarrow S H_{Q}(x) \mathbb{U}^{\dagger}(x), \bar{H}_{Q}(x) \rightarrow \mathbb{U}(x) \bar{H}_{Q}(x) S^{\dagger} \\
& H_{\bar{Q}}(x) \rightarrow \mathbb{U}(x) H_{\bar{Q}}(x) S^{\dagger}, \bar{H}_{\bar{Q}}(x) \rightarrow S \bar{H}_{\bar{Q}}(x) \mathbb{U}^{\dagger}(x)
\end{aligned}
$$

$$
\sigma=\sqrt{\Sigma}=\mathrm{e}^{i \Phi / f}
$$

$$
\sigma(x) \rightarrow L \sigma(x) \mathbb{U}^{\dagger}(x)=\mathbb{U}(x) \sigma(x) R^{\dagger}
$$

$$
\mathcal{L}_{\mathrm{HL}, 1}=-i \operatorname{Tr}(\bar{H} H v \cdot \overleftarrow{D})+g_{\pi} \operatorname{Tr}\left(\bar{H} H \gamma^{\mu} \gamma_{5} \mathbb{A}_{\mu}\right)
$$

\section{FORDHAM UNIVERSITY}




\section{Heavy-light meson ChPT}

\section{Chiral-level Weak operators:}

$b \rightarrow c$ operators

$\mathcal{O}_{\chi, i}=\sum_{x}\left\{\alpha_{1, x} \operatorname{Tr}_{\mathrm{D}}\left[\left(\sigma_{1 k} \bar{H}_{v^{\prime}, k}^{(c)}\right) \Gamma_{2} \Xi_{x}^{\prime} \Xi_{x} \Gamma_{1}\left(H_{v, l}^{(b)} \sigma_{l i}^{\dagger}\right)\right]+\alpha_{2, x} \operatorname{Tr}_{\mathrm{D}}\left[\left(\sigma_{1 k} \bar{H}_{v^{\prime}, k}^{(c)}\right) \Gamma_{2} \Xi_{x}^{\prime}\right] \operatorname{Tr}_{\mathrm{D}}\left[\Xi_{x} \Gamma_{1}\left(H_{v, l}^{(b)} \sigma_{l i}^{\dagger}\right)\right]\right\}$

$b \rightarrow \bar{c}$ operators

$\overline{\mathcal{O}}_{x, i}=\sum_{x}\left\{\bar{\alpha}_{1, x} \operatorname{Tr}_{\mathrm{D}}\left[\Xi_{x}^{\prime} \Gamma_{2}\left(\bar{H}_{v^{\prime}, k}^{(c)} \sigma_{k 1}^{\dagger}\right) \Xi_{x} \Gamma_{1}\left(H_{v, l}^{(b)} \sigma_{l i}^{\dagger}\right)\right]+\bar{\alpha}_{2, x} \operatorname{Tr}_{\mathrm{D}}\left[\Xi_{x}^{\prime} \Gamma_{2}\left(\bar{H}_{v, k}^{(c)} \sigma_{k 1}^{\dagger} \sigma_{k 1}^{\dagger}\right)\right] \operatorname{Tr}_{\mathrm{D}}\left[\Xi_{x} \Gamma_{1}\left(H_{v, l}^{(b)} \sigma_{l i}^{\dagger}\right)\right]\right\}$

$$
\begin{aligned}
\left\{\Xi_{x}^{\prime}, \Xi_{x}\right\}=\{ & \{1,1\},\left\{\gamma_{\nu}, \gamma^{\mu}\right\},\left\{\phi^{\prime}, \phi\right\},\left\{\psi^{\prime}, 1\right\},\{1, \phi\},\left\{\sigma_{\mu \nu}, \sigma^{\mu \nu}\right\}, \\
& \left.\left\{\gamma_{5}, \gamma_{5}\right\},\left\{\gamma_{\mu} \gamma_{5}, \gamma^{\mu} \gamma_{5}\right\},\left\{\psi^{\prime} \gamma_{5}, \phi \gamma_{5}\right\},\left\{p^{\prime} \gamma_{5}, \gamma_{5}\right\},\left\{\gamma_{5}, \phi \gamma_{5}\right\}\right\}
\end{aligned}
$$

\section{FORDHAM UNIVERSITY}

THE JESUIT UNIVERSITY OF NEW YORK 


\section{Heavy-light meson ChPT}

$$
\begin{aligned}
\mathcal{O}_{\chi, i}= & {\left[\beta_{1}+\left(\beta_{1}+\beta_{2}\right)\left(v^{\prime} \cdot v\right)\right]\left[\left(\sigma_{1 k} \mathcal{P}_{k}^{(c) \dagger}\right)\left(\mathcal{P}_{l}^{(b)} \sigma_{l i}^{\dagger}\right)\right] } \\
+ & {\left[\left(\beta_{1}-\beta_{2}\right) v^{\prime \mu}-\beta_{1} v^{\mu}\right]\left[\left(\sigma_{1 k} \mathcal{P}_{k}^{(c) \dagger}\right)\left(\mathcal{V}_{\mu, l}^{*(b)} \sigma_{l i}^{\dagger}\right)\right] } \\
+ & {\left[\beta_{1} v^{\prime \mu}-\left(\beta_{1}+\beta_{2}\right) v^{\mu}\right]\left[\left(\sigma_{1 k} \mathcal{V}_{\mu, k}^{*(c) \dagger}\right)\left(\mathcal{P}_{l}^{(b)} \sigma_{l i}^{\dagger}\right)\right] } \\
- & 4\left[\left(\beta_{1}-\beta_{2}\right)+\beta_{1}\left(v^{\prime} \cdot v\right)\right]\left[\left(\sigma_{1 k} \mathcal{V}_{\mu, k}^{*(c) \dagger}\right)\left(\mathcal{V}_{l}^{*(b) \mu} \sigma_{l i}^{\dagger}\right)\right], \\
\overline{\mathcal{O}}_{\chi, i}= & {\left[\bar{\beta}_{1}+\bar{\beta}_{2}\left(v^{\prime} \cdot v\right)\right]\left[\left(\mathcal{P}_{k}^{(\bar{c}) \dagger} \sigma_{k 1}^{\dagger}\right)\left(\mathcal{P}_{l}^{(b)} \sigma_{l i}^{\dagger}\right)\right] } \\
- & {\left[\bar{\beta}_{2} v^{\prime \mu}-\left(\bar{\beta}_{1}+\bar{\beta}_{5}\right) v^{\mu}-\bar{\beta}_{3}\left(v^{\prime} \cdot v\right) v^{\mu}\right]\left[\left(\mathcal{P}_{k}^{(\bar{c} \dagger} \sigma_{k 1}^{\dagger}\right)\left(\mathcal{V}_{\mu, l}^{*(b)} \sigma_{l i}^{\dagger}\right)\right] } \\
+ & {\left[\bar{\beta}_{1} v^{\prime \mu}-\bar{\beta}_{2} v^{\mu}\right]\left[\left(\mathcal{V}_{\mu, k}^{*(\bar{c} \dagger} \sigma_{k 1}^{\dagger}\right)\left(\mathcal{P}_{l}^{(b)} \sigma_{l i}^{\dagger}\right)\right] } \\
+ & {\left[4 \bar{\beta}_{2}-\bar{\beta}_{3}-2\left(\bar{\beta}_{1}+\bar{\beta}_{4}+\bar{\beta}_{5}\right)\left(v^{\prime} \cdot v\right)\right]\left[\left(\mathcal{V}_{\mu, k}^{*(\bar{c}) \dagger} \sigma_{k 1}^{\dagger}\right)\left(\mathcal{V}_{l}^{*(b) \mu} \sigma_{l i}^{\dagger}\right)\right], }
\end{aligned}
$$




\section{Leading order}

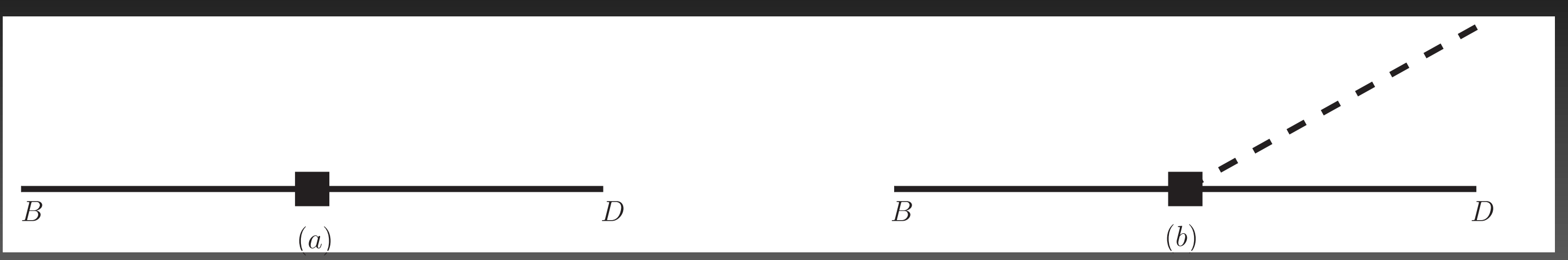

$$
\begin{aligned}
& \left\langle D^{0} K^{-}\left|\mathcal{O}_{\chi, s}\right| B^{-}\right\rangle=\left\langle D^{0} \pi^{-}\left|\mathcal{O}_{\chi, d}\right| B^{-}\right\rangle=\frac{i}{f}\left\langle D^{-}\left|\mathcal{O}_{\chi, s}\right| B^{-}\right\rangle \\
& \left\langle\bar{D}^{0} K^{-}\left|\overline{\mathcal{O}}_{\chi, s}\right| B^{-}\right\rangle=\left\langle\bar{D}^{0} \pi^{-}\left|\overline{\mathcal{O}}_{\chi, d}\right| B^{-}\right\rangle=\frac{i}{f}\left\langle D^{-}\left|\overline{\mathcal{O}}_{\chi, s}\right| B^{-}\right\rangle
\end{aligned}
$$

\section{similar expressions for $K \rightarrow 2 \pi$ [Bernard et al, PRD32 2343]}

Can also include resonances: FORDHAM UNIVERSITY

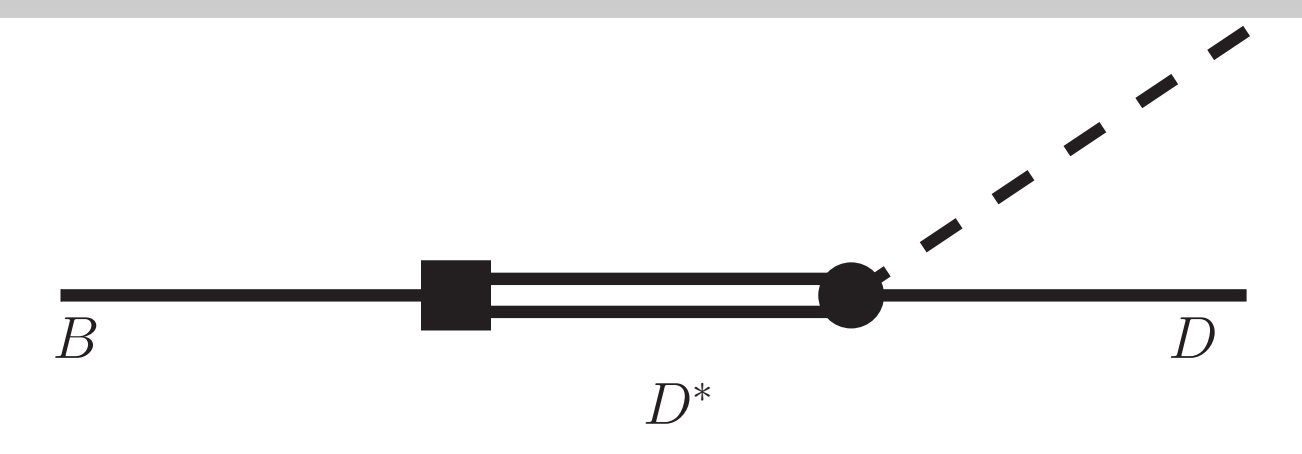

THE JESUIT UNIVERSITY OF NEW YORK 
Of course here the physical point has

$$
\mathbf{p}_{\pi}=\mathbf{p}_{D} \approx 2 \mathrm{GeV}
$$

Not appropriate for chiral expansion unless one takes the unphysical point

$$
m_{B} \approx m_{D}
$$

But then we're far from the point of interest.

\section{How to go beyond tree-level?}




\section{Beyond tree-level:}

\section{Hard-pion ChPT (HPChPT)}

Exploit the fact that hard scales can be absorbed into LEC's

Flynn \& Sachrajda, Nucl.Phys. B8I2, $64 K_{\ell 3}$ Bijnens \& Celis, Phys.Lett. B680, $466 K \rightarrow 2 \pi$ Bijnens \& Jemos, Nucl.Phys. B840, $54 \quad B \rightarrow \pi$ 


\section{HPChPT}

\section{In the SU(2) chiral theory, we have the generic one-loop form}

tree-level amplitude

$$
\mathcal{M}=\mathcal{M}^{\text {tree }}\left[1+a \frac{m_{\pi}^{2}}{16 \pi^{2} f^{2}} \ln \left(\frac{m_{\pi}^{2}}{\Lambda^{2}}\right)+L m_{\pi}^{2}\right]
$$

\section{$a$ and $L$ are LEC's that depend on the hard scales}

\section{FORDHAM UNIVERSITY}




\section{HPChPT example}

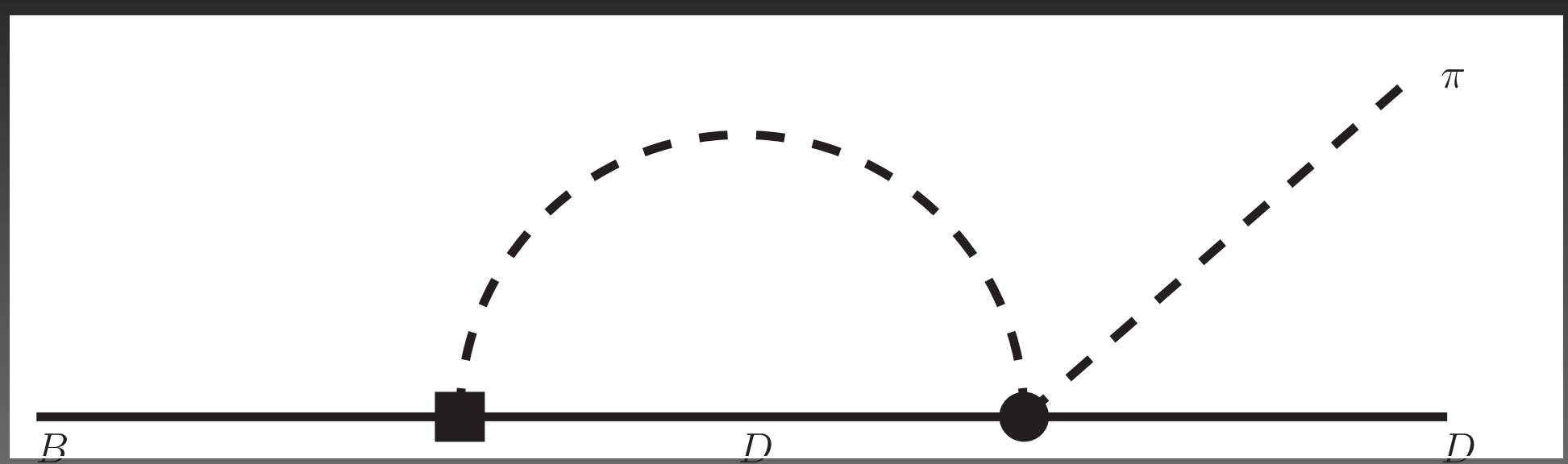

$$
\begin{aligned}
& \frac{\left\langle D^{0} \pi^{-}\left|\mathcal{O}_{\chi, d}\right| B^{-}\right\rangle^{\text {tree }}}{8 f^{2}} \int \frac{d^{d} \ell}{(2 \pi)^{d}} \frac{i}{\ell^{2}-m_{\pi}^{2}+i \epsilon} \frac{i v^{\prime} \cdot\left(\ell-p_{\pi}\right)}{v^{\prime} \cdot\left(\ell-k-p_{\pi}\right)-\Delta+i \epsilon} \\
& \frac{\left\langle D^{0} \pi^{-}\left|\mathcal{O}_{\chi, d}\right| B^{-}\right\rangle^{\text {tree }}}{8} I
\end{aligned}
$$

$$
I=\frac{1}{16 \pi^{2} f^{2}}\left[\frac{v^{\prime} \cdot k+\Delta}{v^{\prime} \cdot\left(k+p_{\pi}\right)+\Delta+i \epsilon} I_{2}\left(m_{\pi}, v^{\prime} \cdot\left(k+p_{\pi}\right)+\Delta+i \epsilon\right)-m_{\pi}^{2} \ln \left(\frac{m_{\pi}^{2}}{\Lambda^{2}}\right)\right]
$$

\section{FORDHAM UNIVERSITY}

THE JESUIT UNIVERSITY OF NEW YORK 


$$
I=\frac{1}{16 \pi^{2} f^{2}}\left[\frac{v^{\prime} \cdot k+\Delta}{v^{\prime} \cdot\left(k+p_{\pi}\right)+\Delta+i \epsilon} I_{2}\left(m_{\pi}, v^{\prime} \cdot\left(k+p_{\pi}\right)+\Delta+i \epsilon\right)-m_{\pi}^{2} \ln \left(\frac{m_{\pi}^{2}}{\Lambda^{2}}\right)\right]
$$

$$
\begin{gathered}
\text { in the limit } v^{\prime} \cdot k \gg m_{\pi} \\
I_{2}\left(m_{\pi}, v^{\prime} \cdot\left(k+p_{\pi}\right)+\Delta\right) \approx-m_{\pi}^{2} \ln \left(\frac{m_{\pi}^{2}}{\Lambda^{2}}\right)
\end{gathered}
$$

\section{Inject momentum into weak vertex so that...}

\section{FORDHAM UNIVERSITY}




$$
\begin{aligned}
& p_{\pi} \approx 0 \quad I\left(p_{\pi} \approx 0\right) \rightarrow-2 \frac{m_{\pi}^{2}}{16 \pi^{2} f^{2}} \ln \left(\frac{m_{\pi}^{2}}{\Lambda^{2}}\right) \\
& p_{\pi} \approx k \quad I\left(p_{\pi} \approx k\right) \rightarrow-\frac{3}{2} \frac{m_{\pi}^{2}}{16 \pi^{2} f^{2}} \ln \left(\frac{m_{\pi}^{2}}{\Lambda^{2}}\right)
\end{aligned}
$$

\section{Different contributions to $a, L$ in above expression}

Both $a$ and $L$ have unknown dependence on kinematics, but pion mass dependence is known. 


\section{Conclusion}

Problem is far from solved

Need full one-loop calculation

Need lattice calculations (takers?)

Key: Feasible problem for lattice to tackle 


\title{
Status and Prospects for some MILC and Fermilab/MILC Projects
}

\author{
Claude Bernard \\ Washington University \\ St. Louis \\ (MILC \& Fermilab Lattice/MILC Collaborations)
}

New Horizons for Lattice Computations with Chiral Fermions Brookhaven, May 14-16, 2012 


\section{Heavy-light Decay Constants}

$\downarrow$ Fermilab heavy quarks with MILC 2+1 Asqtad staggered light quarks

- "Old data" project

- $0.15 \mathrm{fm} \leq \mathrm{a} \leq 0.09 \mathrm{fm}$

- 4 sources per configuration

- renormalization mostly non-perturbative; 1-loop perturbation theory for remainder

- to appear in PRD shortly

- "New data" project: similar to above, but:

- $0.15 \mathrm{fm} \leq a \leq 0.045 \mathrm{fm}$

- 2 to 5 times more configurations/ensemble

- in progress

$\downarrow$ For D system, ongoing HISQ project with MILC 2+1+1 HISQ quarks: more later. 


\section{Heavy-light Decay Constants}

$\downarrow$ Fermilab heavy quarks with MILC 2+1 Asqtad staggered light quarks

- "Old data" project

- $0.15 \mathrm{fm} \leq \mathrm{a} \leq 0.09 \mathrm{fm}$

- 4 sources per configuration

- renormalization mostly non-perturbative; 1-loop perturbation theory for remainder

- to appear in PRD shortly

- "New data" project: similar to above, but:

J. Simone, D. Toussaint,

- $0.15 \mathrm{fm} \leq a \leq 0.045 \mathrm{fm}$

- 2 to 5 times more configurations/ensemble

- in progress

$\downarrow$ For D system, ongoing HISQ project with MILC 2+1+1 HISQ quarks: more later. 


\section{Asqtad Ensembles}

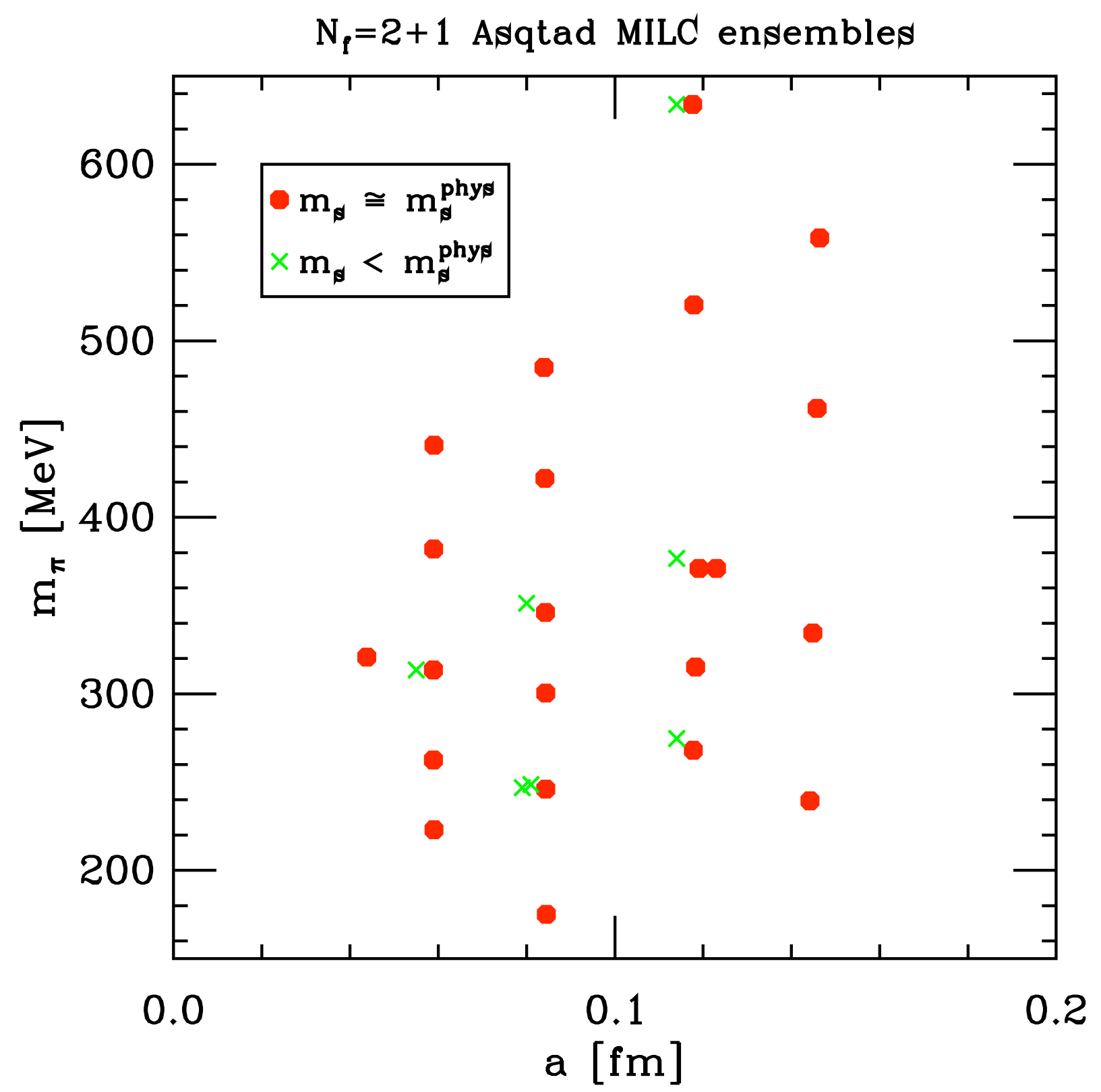




\section{Asqtad Ensembles}

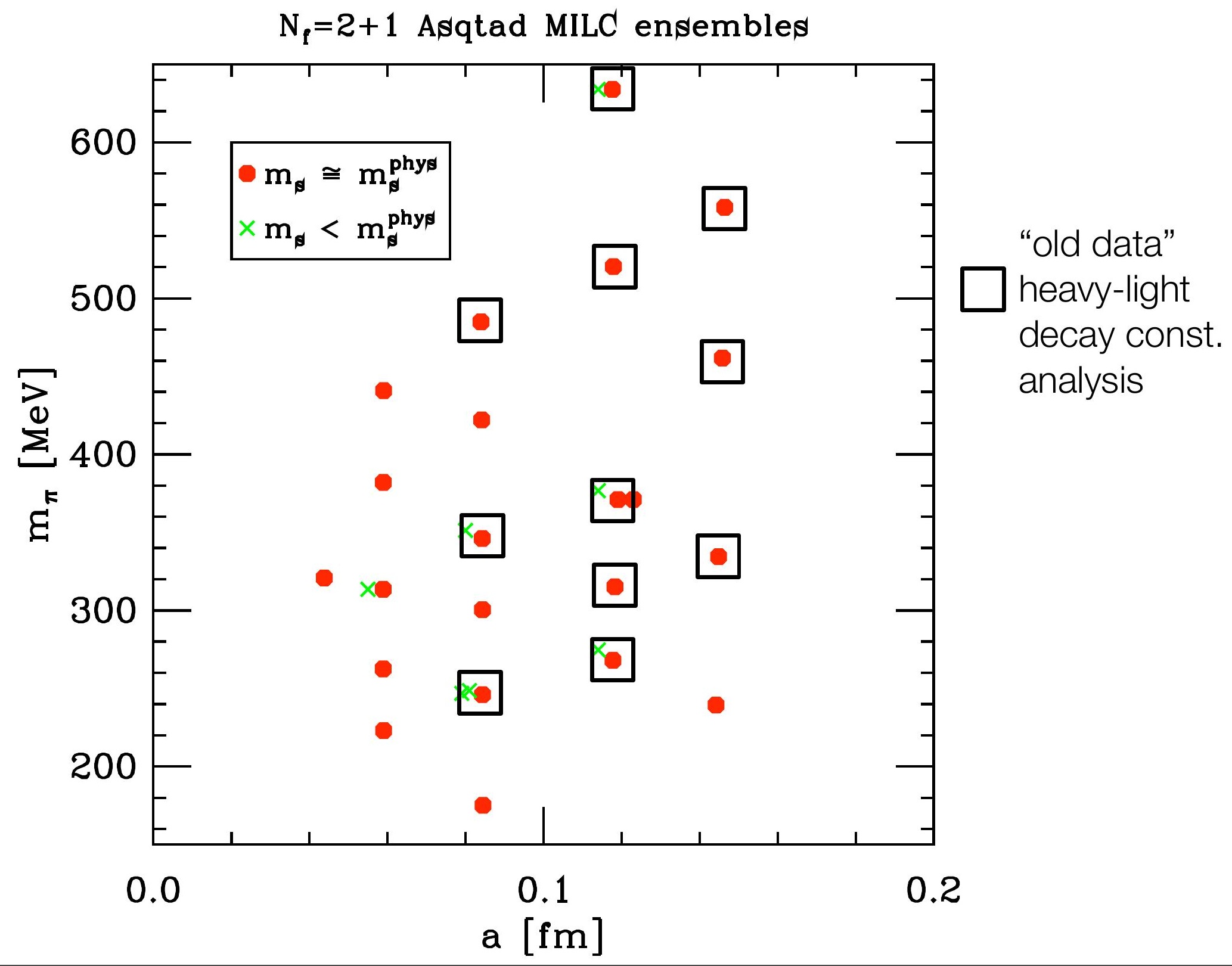




\section{Asqtad Ensembles}

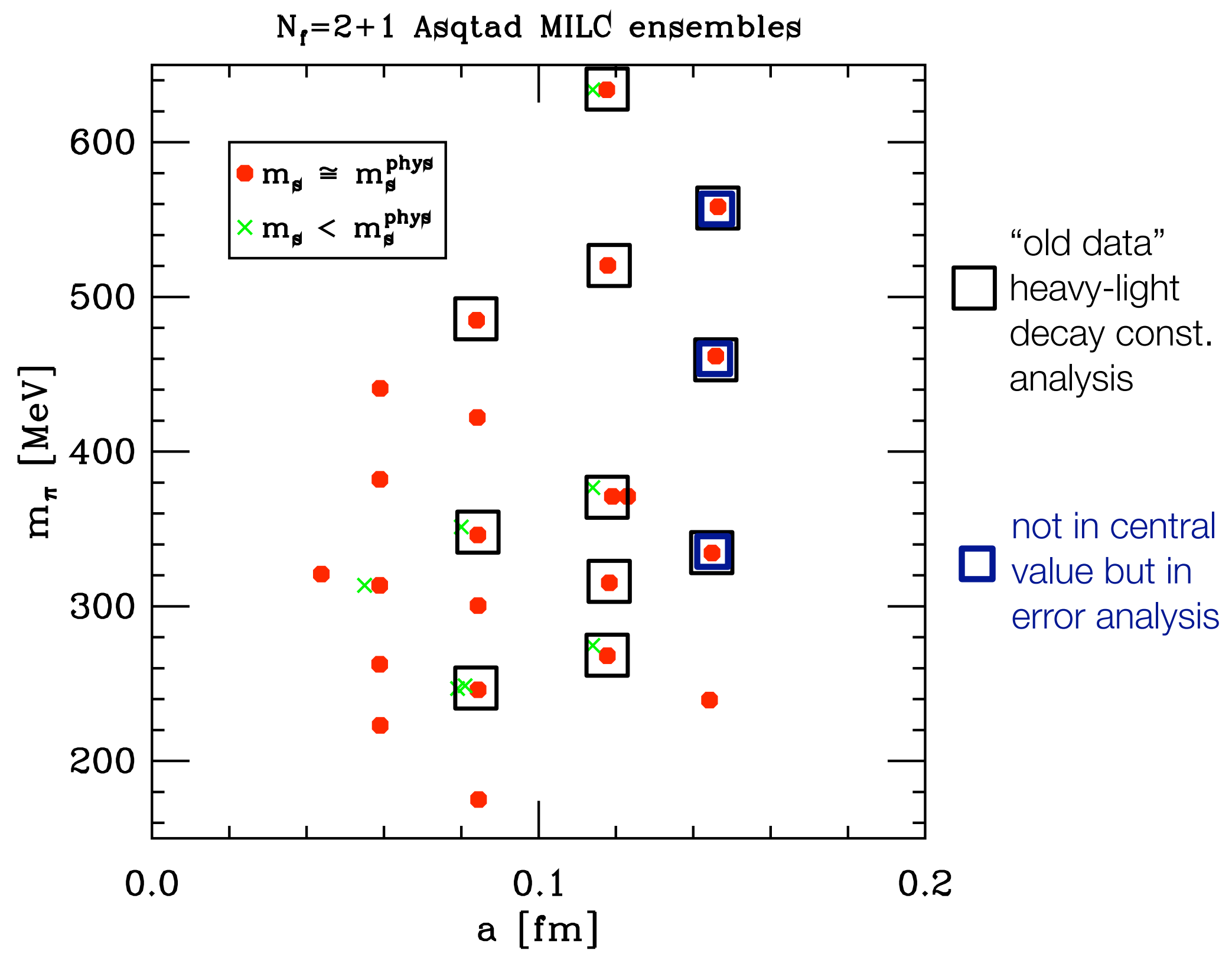




\section{D system}

$\uparrow f_{D}$ as function of light

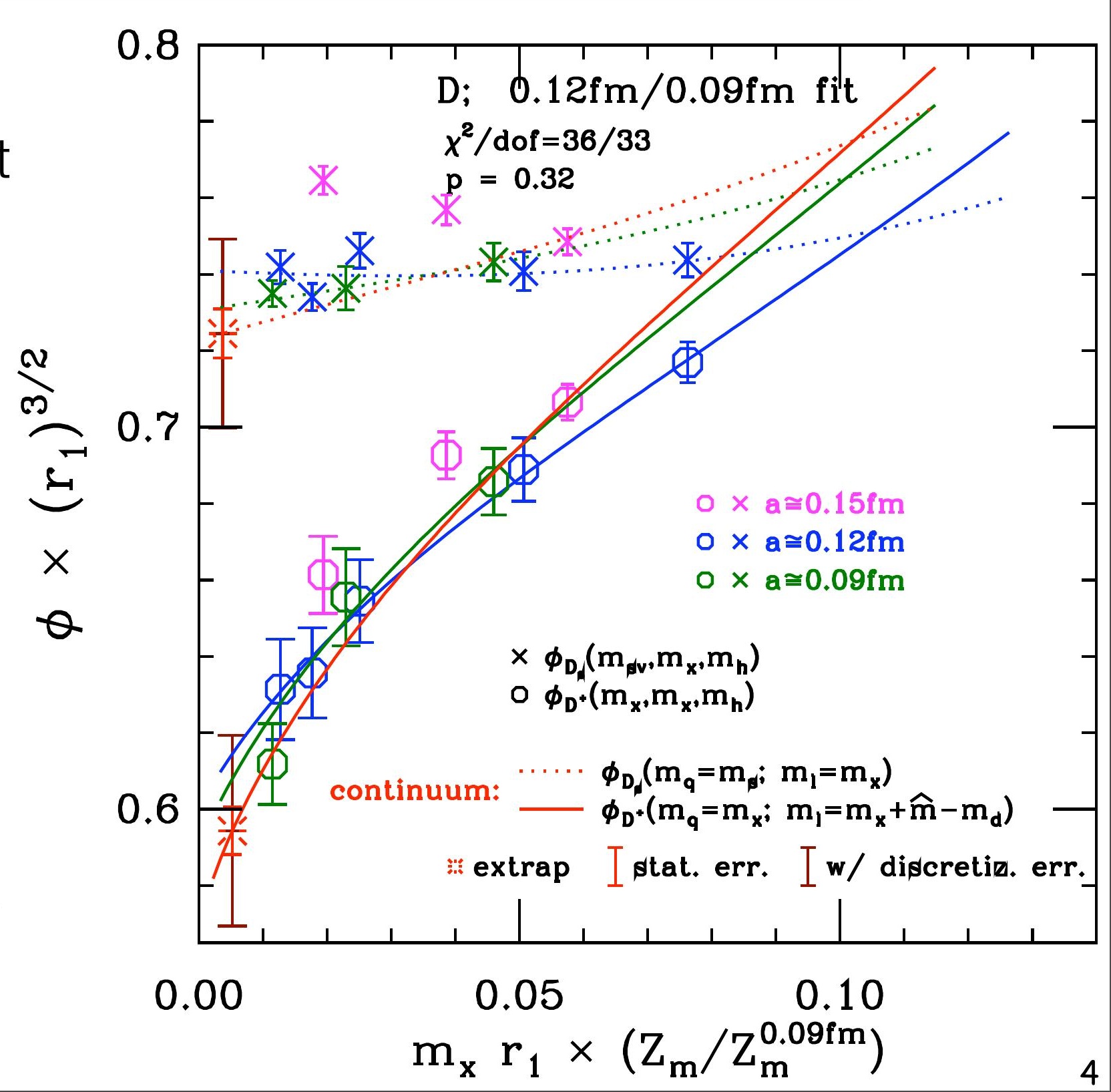

\section{$\downarrow f_{D s}$ as function of} light sea mass $\mathrm{m}$.

- valence mass held fixed $\approx \mathrm{m}_{\mathrm{s}}$.

$\downarrow \mathrm{a} \cong 0.15 \mathrm{fm}$ points not included in fit.

- note qualitatively different behavior 


\section{B system}

$\downarrow f_{B}$ as function of light

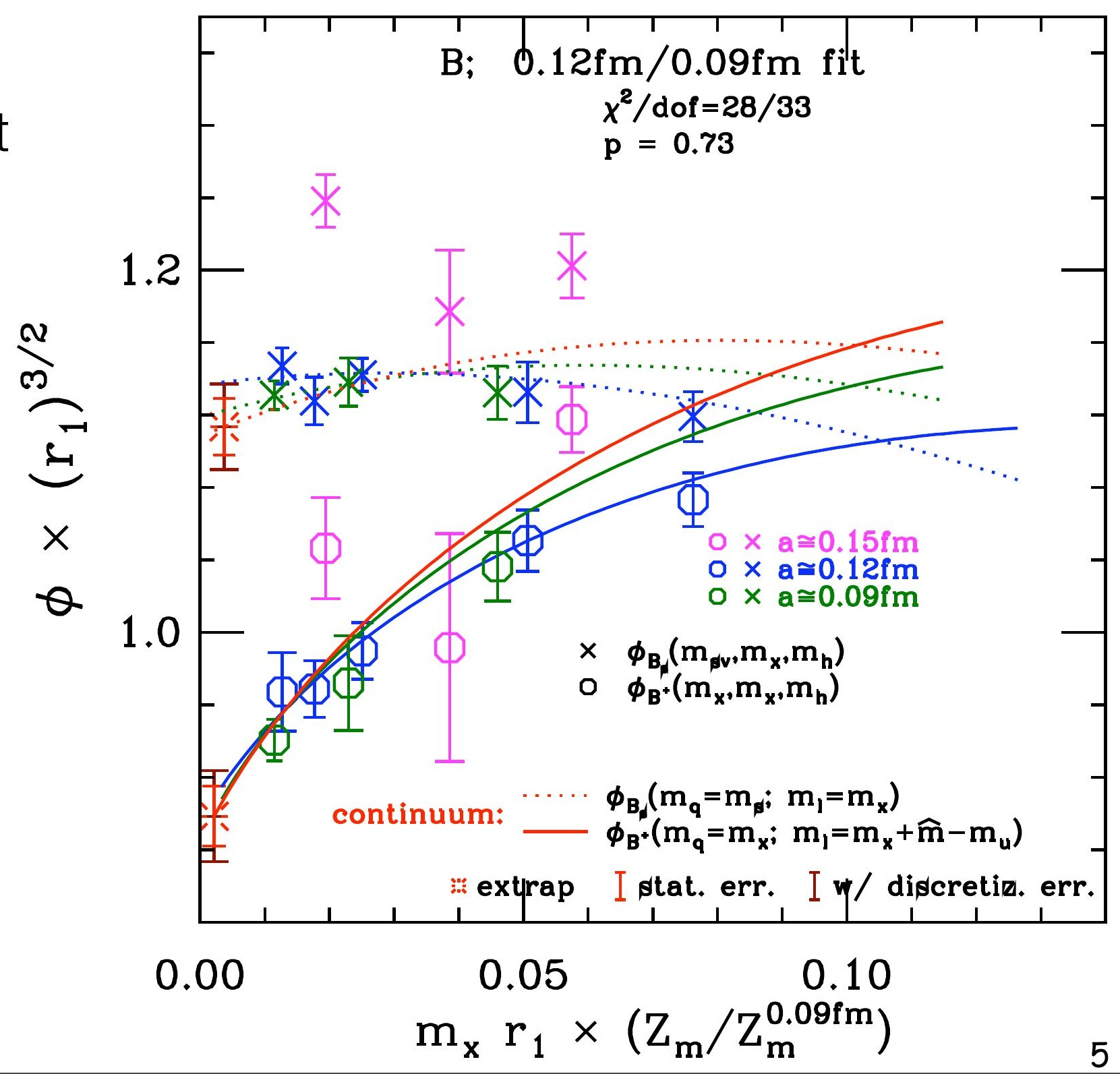

$\downarrow f_{B s}$ as function of light sea mass $\mathrm{m}$.

- valence mass held fixed $\approx \mathrm{m}_{\mathrm{s}}$.

$+\mathrm{a} \cong 0.15 \mathrm{fm}$ points not included in fit.

- qual. different behavior + large stat. errors

$$
\mathrm{m}_{\mathrm{x}} \mathrm{r}_{1} \times\left(\mathrm{Z}_{\mathrm{m}} / \mathrm{Z}_{\mathrm{m}}^{0.09 \mathrm{fm}}\right)
$$




\section{Fermilab/MILC Results}

$$
\begin{aligned}
f_{D_{s}} & =260.1 \pm 10.8 \mathrm{MeV} \\
f_{D^{+}} & =218.9 \pm 11.3 \mathrm{MeV} \\
f_{D_{s}} / f_{D^{+}} & =1.188 \pm 0.025 \\
f_{B_{s}} & =242.0 \pm 9.5 \mathrm{MeV} \\
f_{B^{+}} & =196.9 \pm 8.9 \mathrm{MeV} \\
f_{B_{s}} / f_{B^{+}} & =1.229 \pm 0.026
\end{aligned}
$$

- errors include statistics and systematic errors

- discretization errors for heavy \& light quarks automatically included with statistics errors by our Bayesian procedure

- have added on other systematics in quadrature 


\section{Heavy-light Decay Constants}

$\uparrow$ Fermilab heavy quarks with MILC 2+1 asqtad staggered light quarks

- "Old data" project

- $0.15 \mathrm{fm} \leq a \leq 0.09 \mathrm{fm}$

- 4 sources per configuration

- renormalization mostly non-perturbative; 1-loop perturbation theory for remainder

- to appear in PRD shortly

- "New data" project: similar to above, but:

- $0.15 \mathrm{fm} \leq a \leq 0.045 \mathrm{fm}$

- 2 to 5 times more configurations/ensemble

- in progress

$\uparrow$ For D system, ongoing HISQ project with MILC 2+1+1 HISQ quarks: more later. 


\section{Asqtad Ensembles}

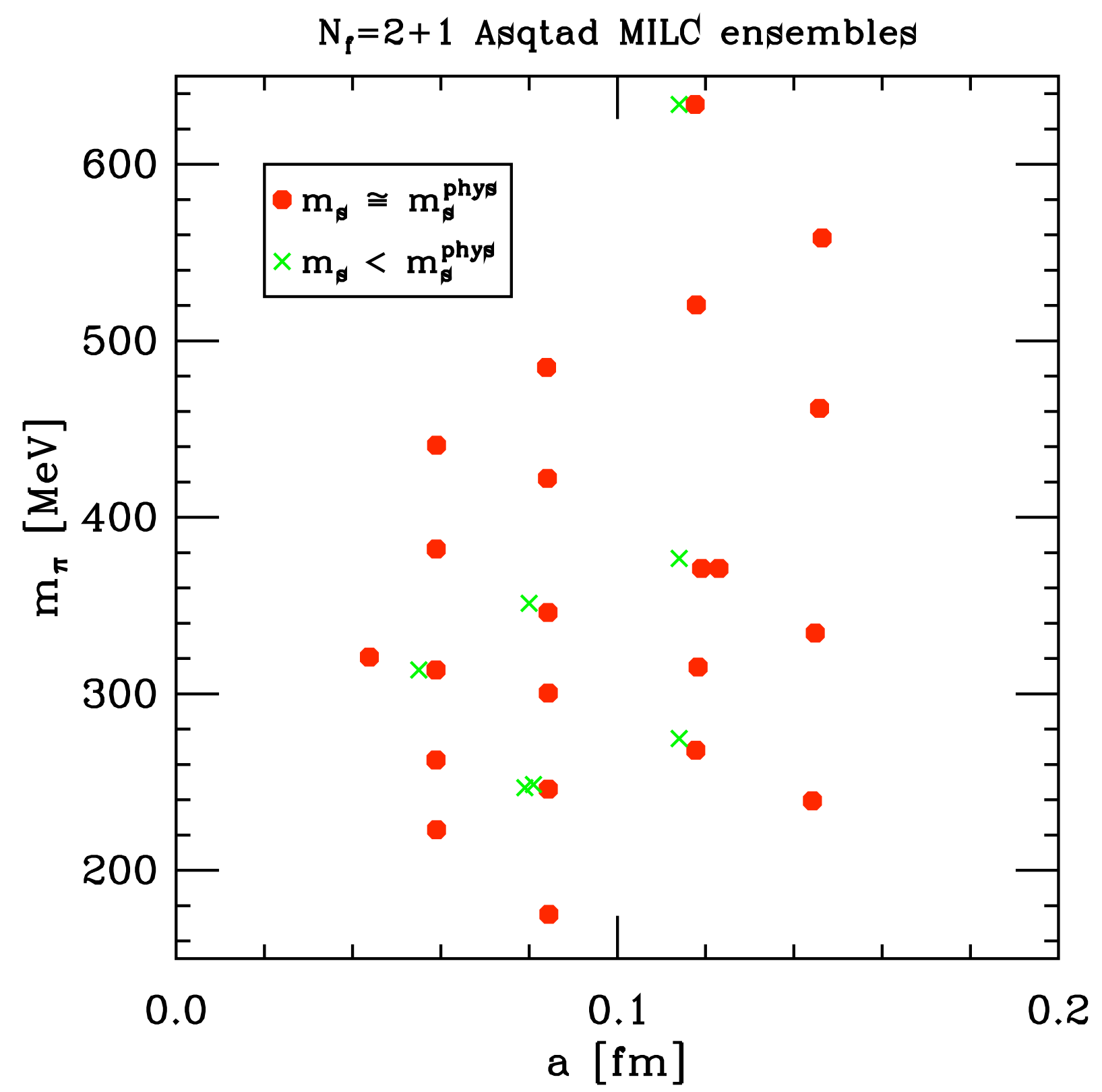




\section{Asqtad Ensembles}

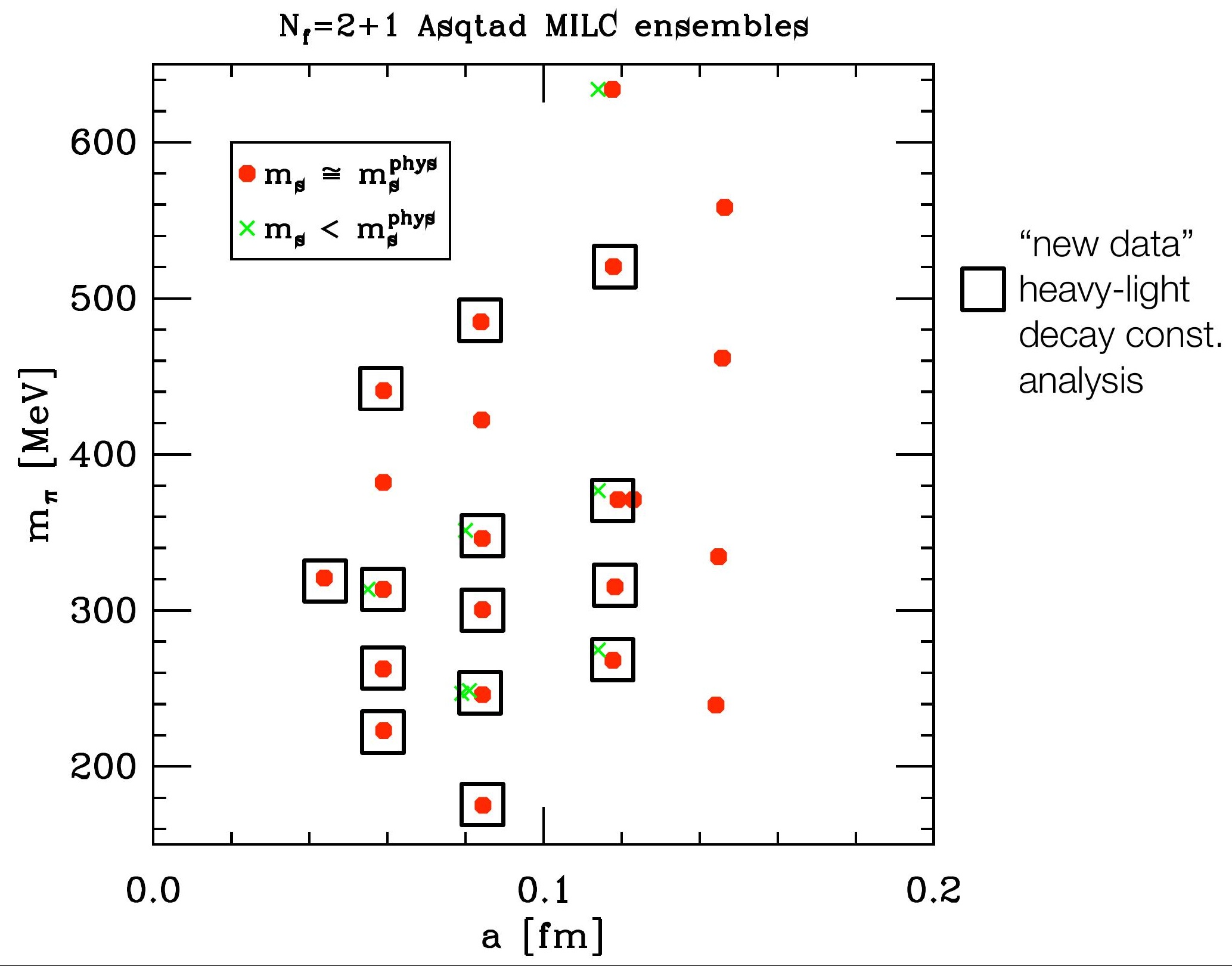




\section{Asqtad Ensembles}

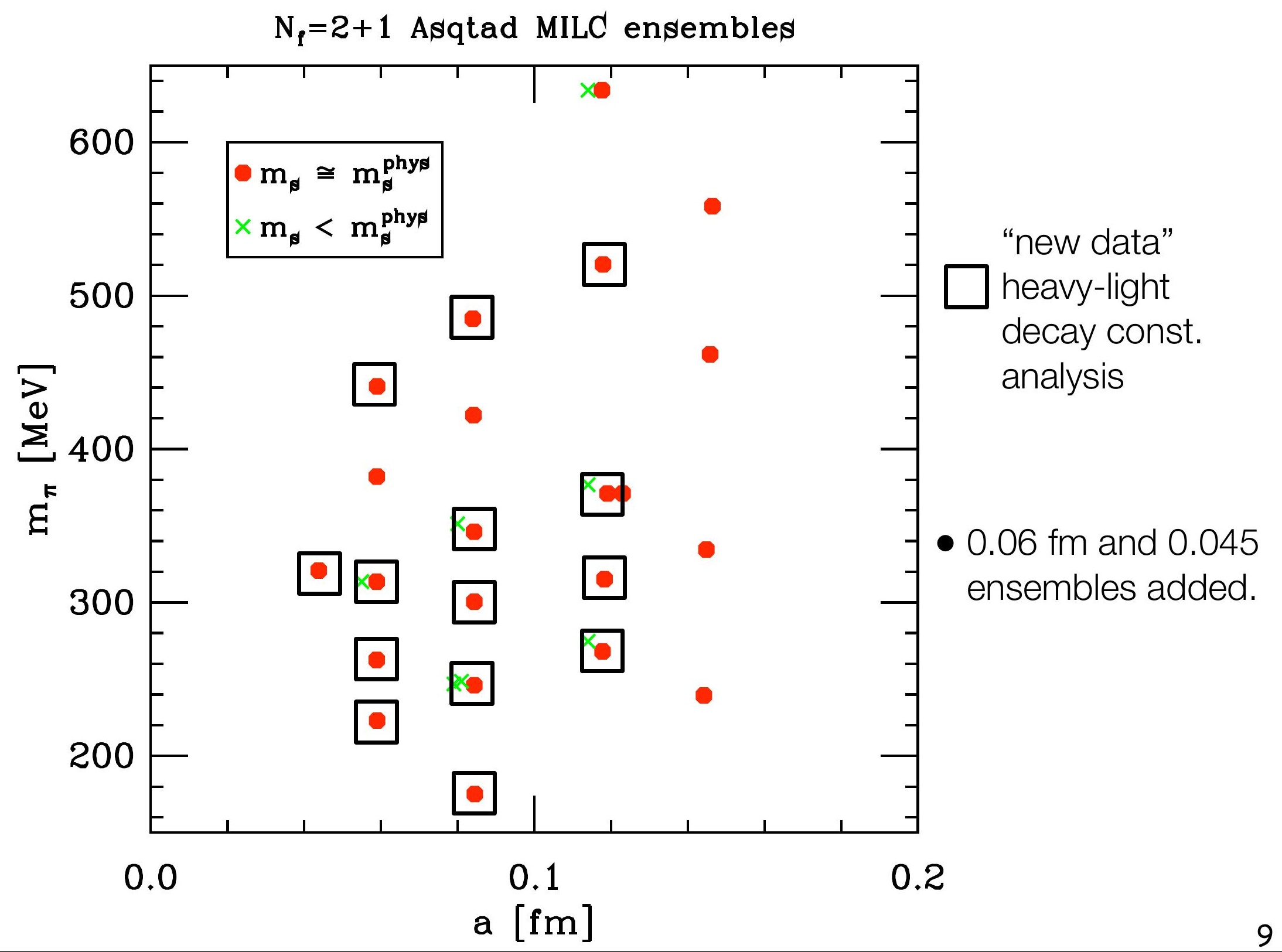




\section{Asqtad Ensembles}

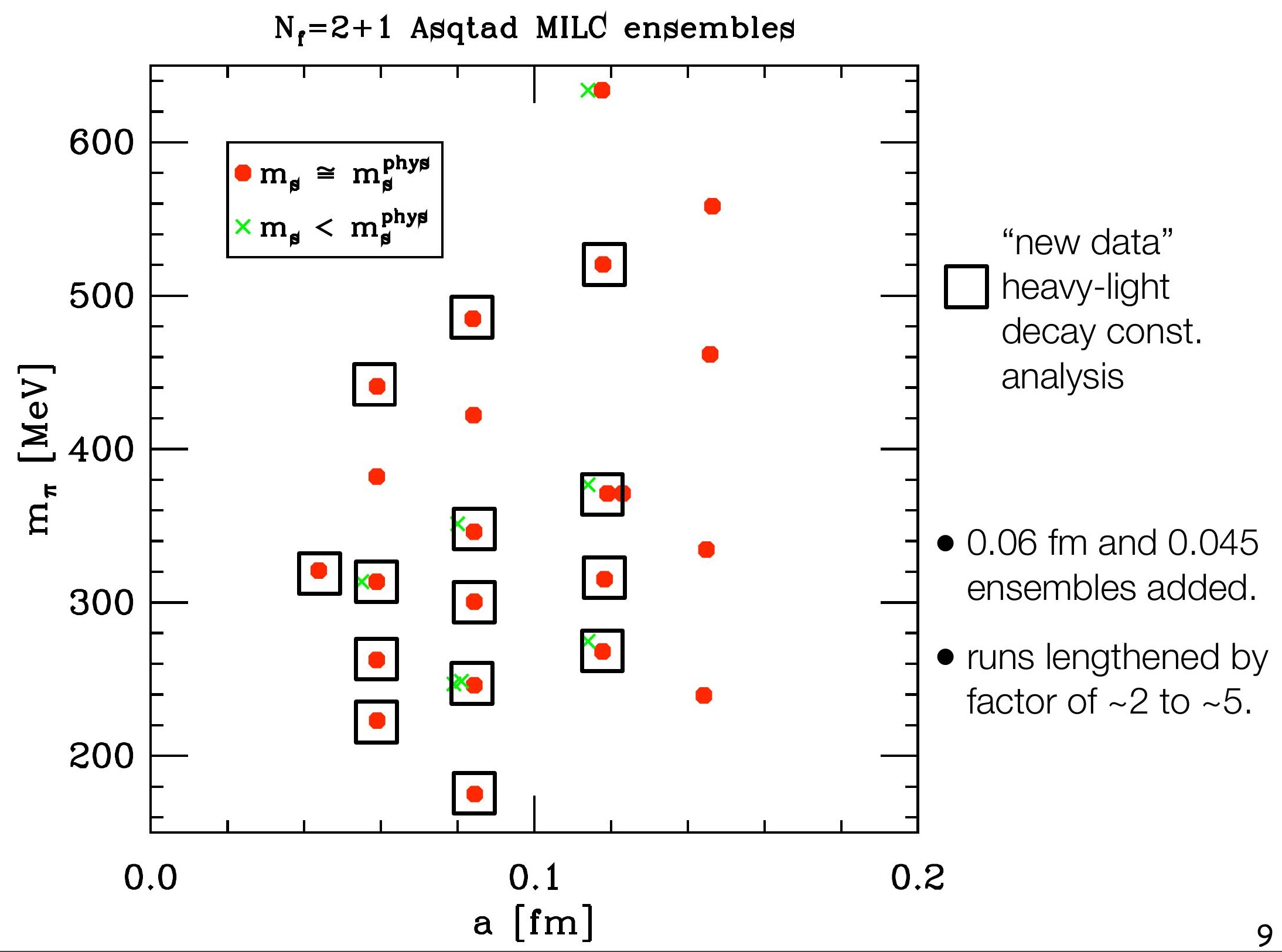




\section{"New Data" fo}

- statistical errors reduced as expected.

- correlator fits still need work; chiral fits are in progress.

- trend: $f_{D}$ and $f_{D s} \downarrow$ as $a \downarrow$

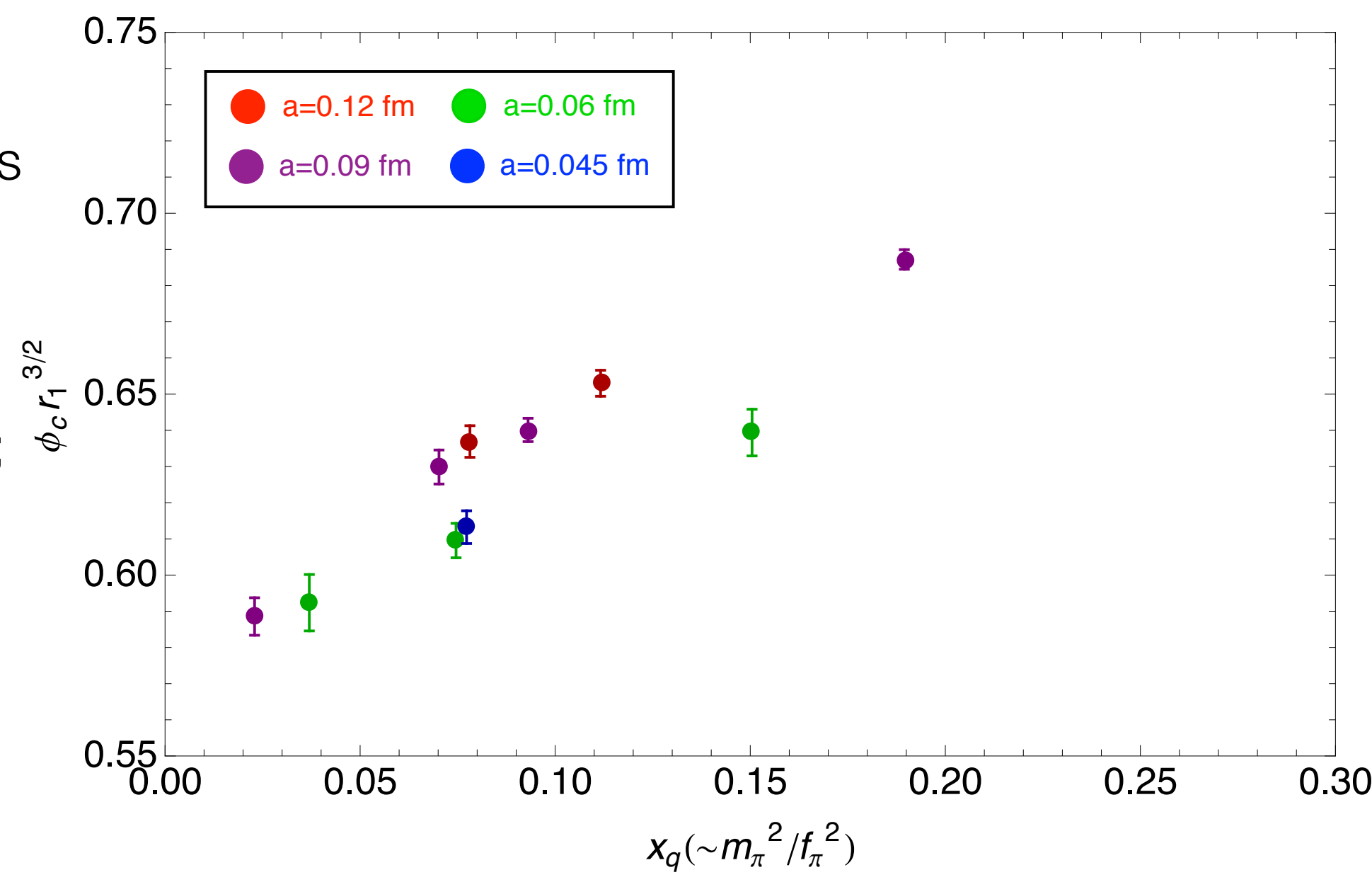




\section{Outlook: Fermilab/MILC}

\begin{tabular}{|c|c|c|}
\multicolumn{1}{c|}{} & \multicolumn{2}{|c|}{$\%$ Errors } \\
\hline Quantity & $\begin{array}{c}\text { "Old data" } \\
\text { arXiv:1112.3051 }\end{array}$ & $\begin{array}{c}\text { "New data" } \\
\text { (in progress) }\end{array}$ \\
\hline$f_{D_{s}}$ & 4.2 & 2.2 \\
\hline$f_{D}$ & 5.2 & 2.8 \\
\hline$f_{D_{s}} / f_{D}$ & 2.1 & 1.1 \\
\hline$f_{B_{S}}$ & 3.9 & 2.6 \\
\hline$f_{B}$ & 4.5 & 2.8 \\
\hline$f_{B_{S}} / f_{B}$ & 2.1 & 1.2 \\
\hline
\end{tabular}




\section{B Mixing}

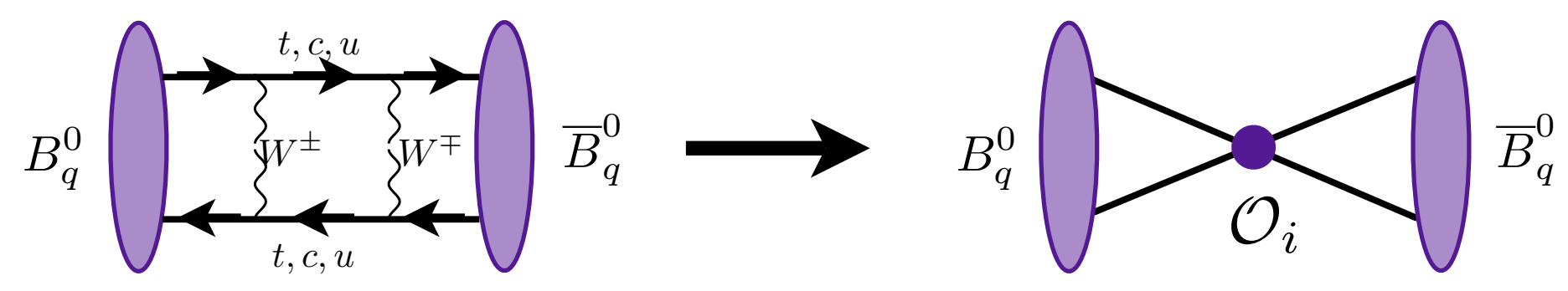

Operators

$$
\mathcal{H}_{\text {eff }}=\sum_{i=1}^{5} C_{i} \mathcal{O}_{i}
$$

are

$$
\mathcal{O}_{1}=\left(\bar{b}^{\alpha} \gamma_{\mu} L q^{\alpha}\right)\left(\bar{b}^{\beta} \gamma_{\mu} L q^{\beta}\right)
$$

SM

$\mathcal{O}_{2}=\left(\bar{b}^{\alpha} L q^{\alpha}\right)\left(\bar{b}^{\beta} L q^{\beta}\right)$

$\mathcal{O}_{3}=\left(\bar{b}^{\alpha} L q^{\beta}\right)\left(\bar{b}^{\beta} L q^{\alpha}\right)$

$\mathrm{BSM}$
Common parametrization

$\left\langle\bar{B}_{q}^{0}\left|\mathcal{O}_{i}(\mu)\right| B_{q}^{0}\right\rangle \propto f_{B_{q}}^{2} B_{i}(\mu)$ 


\section{B Mixing}

$\downarrow$ Fermilab heavy quarks with MILC 2+1 asqtad staggered light quarks

- "Old data" project

- $0.12 \mathrm{fm} \leq a \leq 0.09 \mathrm{fm}$

- focus on SM operators, and in particular on $0_{1}$, which gives

$$
\xi=f_{B_{s}} \sqrt{\hat{B}_{B_{s}}} / f_{B_{d}} \sqrt{\hat{B}_{B_{d}}}
$$

- construct operators from Fermilab quark + naive quark (made from staggered).

- drop NLO "wrong spin" terms [ $\Rightarrow$ systematic error estimate].

-1-loop perturbation theory for mixing.

- to be posted in next month or so.

- "New data" project: similar to above, but:

- $0.15 \mathrm{fm} \leq a \leq 0.045 \mathrm{fm}$.

- complete set of SM and BSM operators.

- all wrong spin terms included correctly in ChPT.

- in progress. 


\section{B Mixing}

$\downarrow$ Fermilab heavy quarks with MILC 2+1 asqtad staggered light quarks

- "Old data" project

- $0.12 \mathrm{fm} \leq a \leq 0.09 \mathrm{fm}$

- focus on SM operators, and in particular on $0_{1}$, which gives

$$
\xi=f_{B_{s}} \sqrt{\hat{B}_{B_{s}}} / f_{B_{d}} \sqrt{\hat{B}_{B_{d}}}
$$

- construct operators from Fermilab quark + naive quark (made from staggered).

- drop NLO "wrong spin" terms [ $\Rightarrow$ systematic error estimate].

- 1-loop perturbation theory for mixing.

- to be posted in next month or so.

- "New data" project: similar to above, but:

- $0.15 \mathrm{fm} \leq a \leq 0.045 \mathrm{fm}$.

- complete set of SM and BSM operators.

- all wrong spin terms included correctly in ChPT.

- in progress.

R.T. Evans,

E. Gámiz 


\section{Asqtad Ensembles}

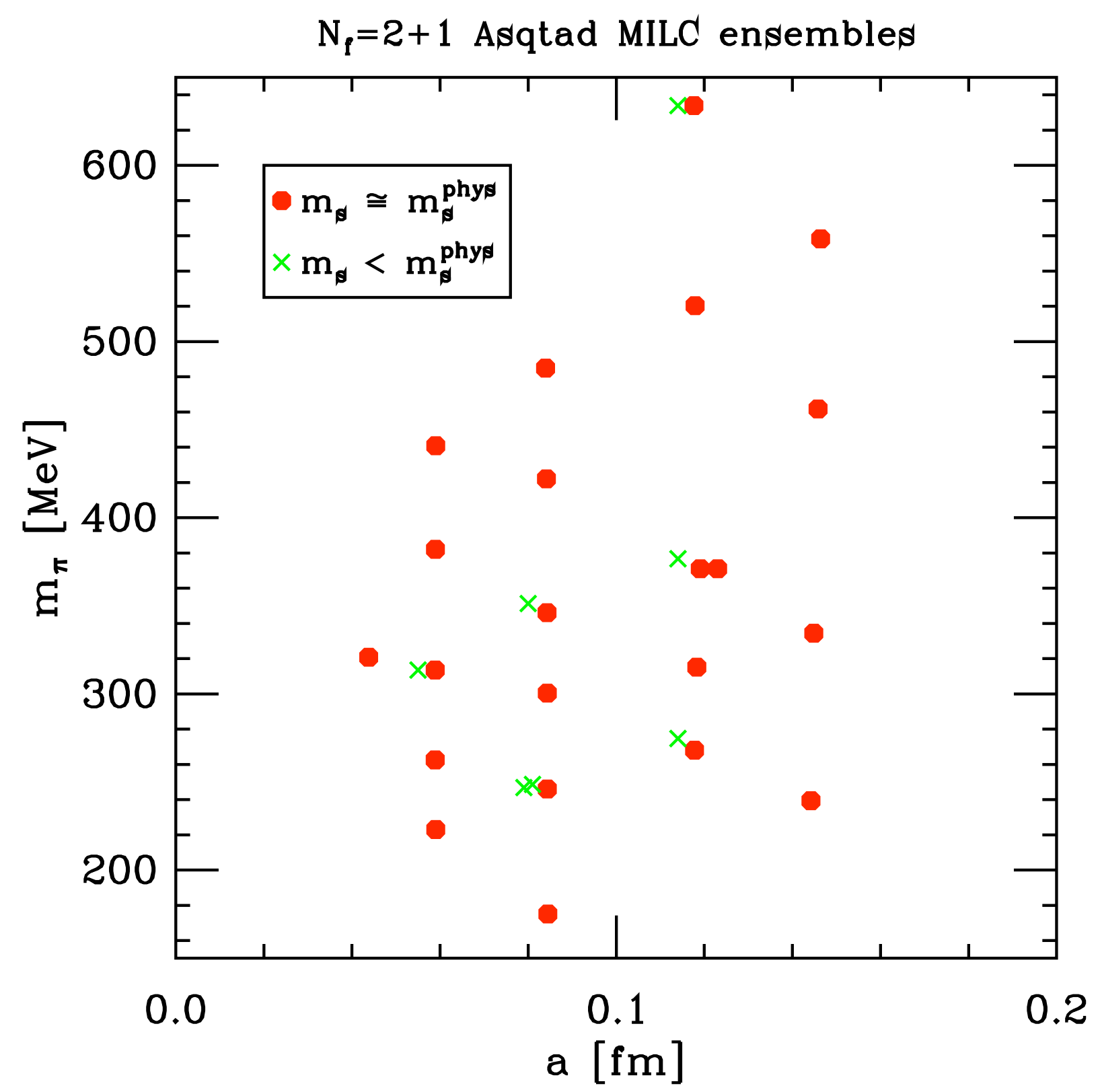




\section{Asqtad Ensembles}

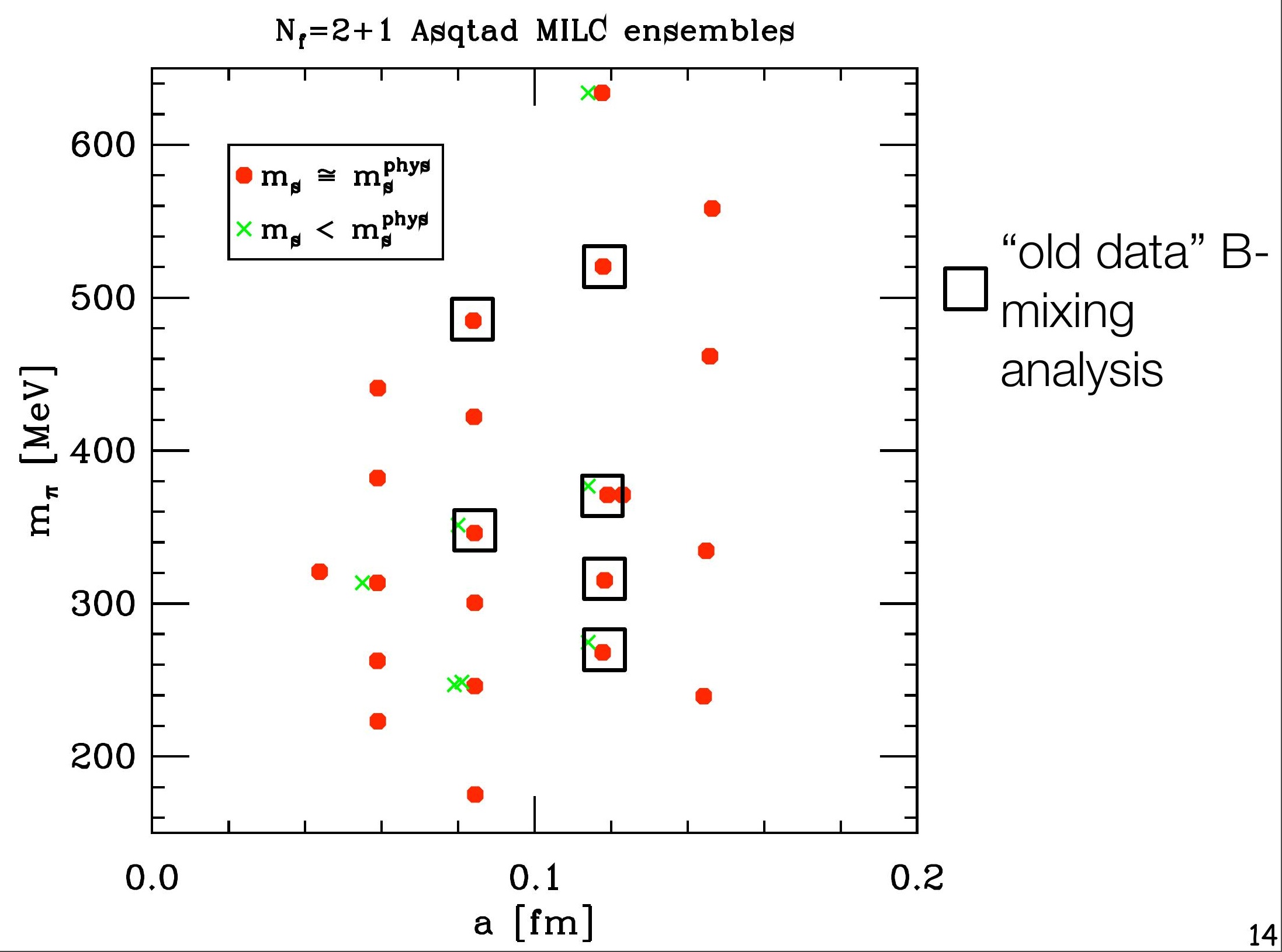




\section{"Wrong spin" Issue}

^ Four quark operators as in [HPQCD, PRD 80 ('09)014503].

- local products of bilinears of heavy quark fields $\bar{Q}(x)$ and naive quarks $\Psi(x)$ (made from staggered):

$$
\bar{Q}(x) \Gamma \Psi(x) \bar{Q}(x) \Gamma^{\prime} \Psi(x)
$$

$\downarrow$ Desired spin-taste of staggered quarks not constructed by separately summing each bilinear over hypercube

$\Rightarrow$ contributions from unwanted spin-tastes.

- vanish in continuum limit by taste conservation.

- but will appear in staggered ChPT at some order.

- we had an argument (in a collaboration note) that chiral logs from wrong-spin taste first appear at NNLO. [Was used in HPQCD paper.]

- in writing up our B mixing computation, found flaw in previous argument: such terms appear at NLO and need to be included. 


\section{"Wrong-spin" and Chiral/Continuum Errors}

$\downarrow$ Effects of wrong spin ops have now been calculated to 1loop in staggered ChPT [CB].

- don't have all needed matrix elements in old-data calc, but can estimate effect by sample new-data calc.

$<O_{1}>$ versus $\left(r_{1} m_{\pi}\right)^{2}$ Wrong Spin Included

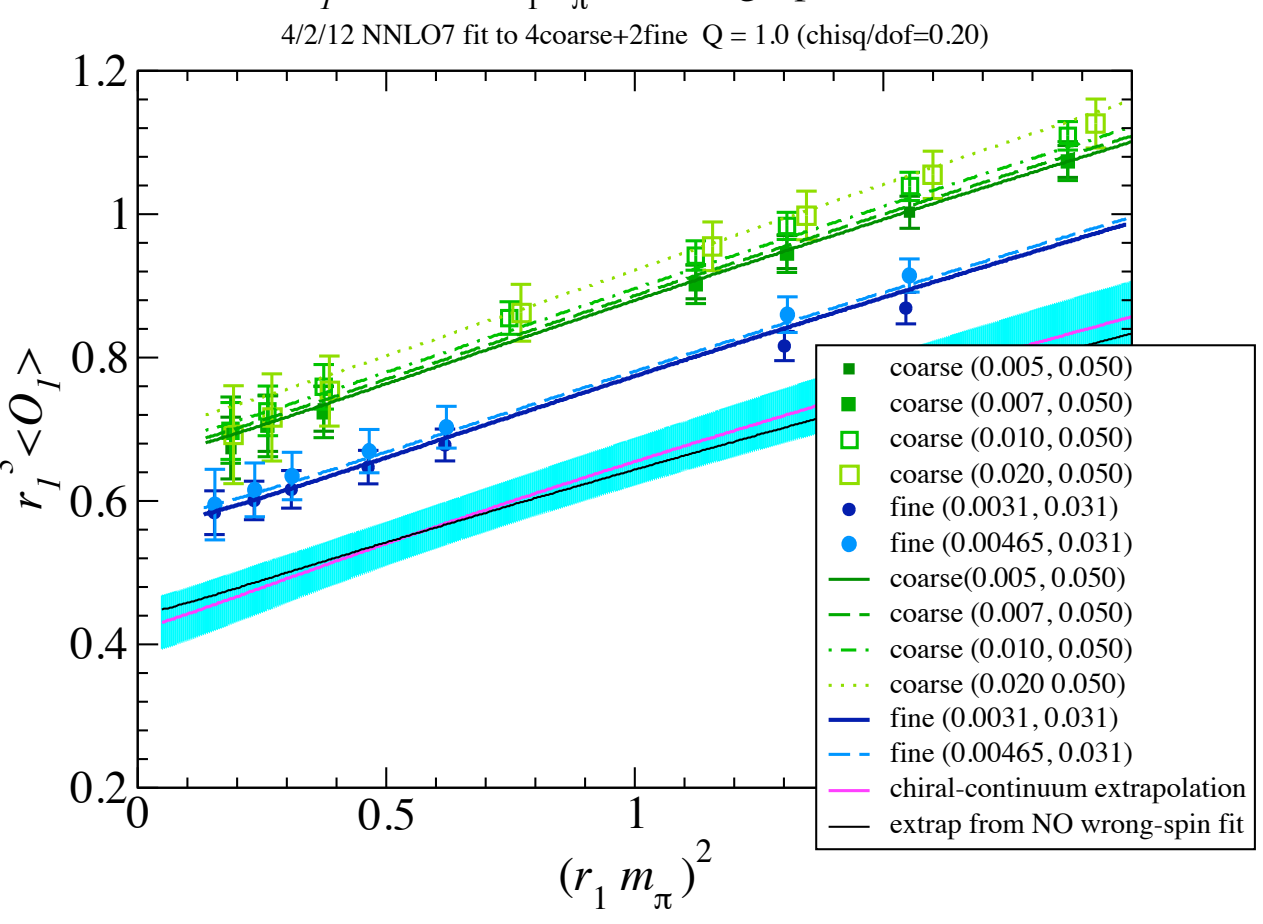

$<O_{1}>$ versus $\left(r_{1} m_{\pi}\right)^{2}$ Wrong Spin Included

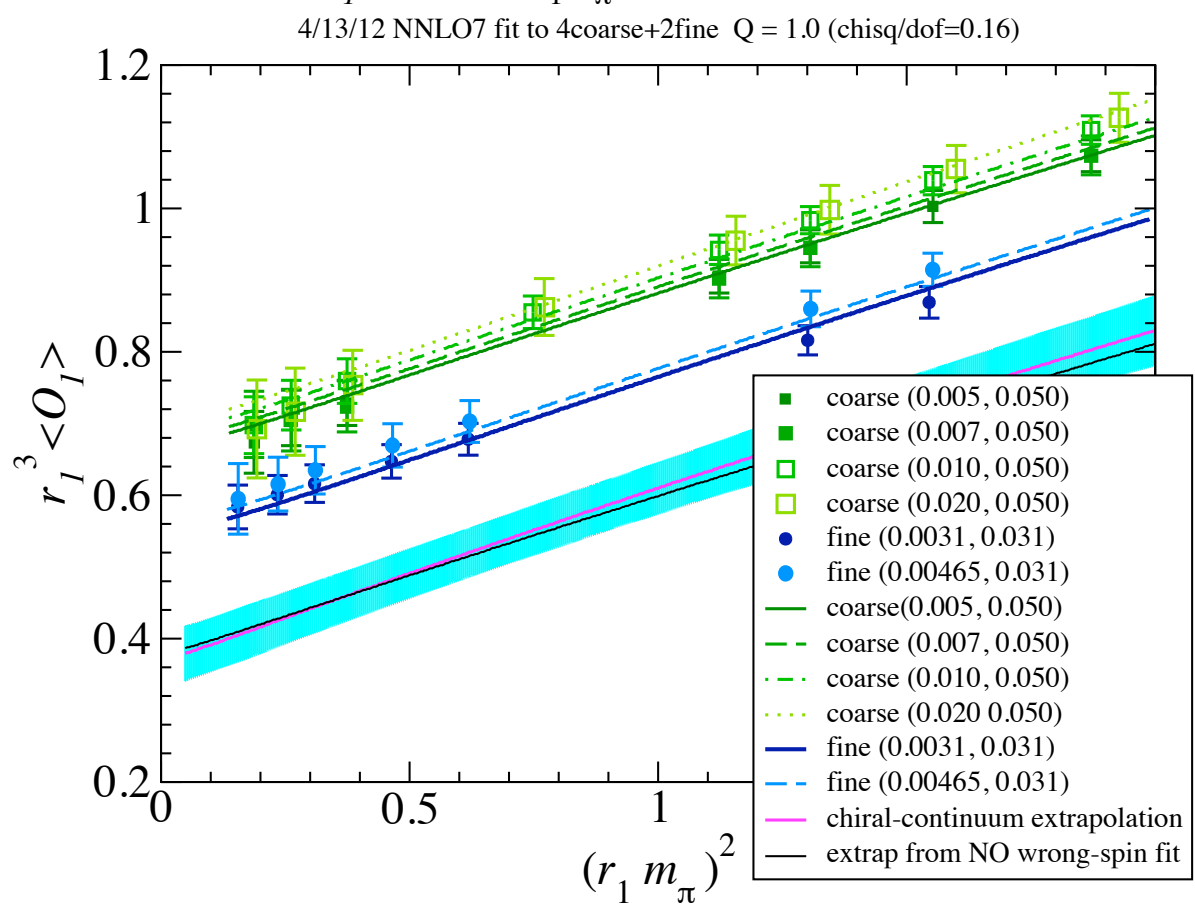

- wrong-spin contrib < stat + other chiral/continuum errors, but effect on slope seems significant $\Rightarrow$ tends to increase $\xi$. 


\section{Final "Old-Data" Results}

TABLE IX. Complete error budget and total error for the $B^{0}$ mixing parameter $\xi$. All errors are given in \%.

\begin{tabular}{lc}
\hline \hline Source of uncertainty & Error $(\%)$ \\
\hline Statistics $\oplus$ light-quark disc. $\oplus$ chiral extrapolation & 3.7 \\
Mixing with wrong-spin operators & 3.2 \\
Heavy-quark discretization & 0.3 \\
Scale uncertainty $\left(r_{1}\right)$ & 0.2 \\
$g_{B B^{*} \pi}$ & 0.7 \\
Light-quark masses & 0.5 \\
One-loop matching & 0.5 \\
Tuning $\kappa_{b}$ & 0.4 \\
Finite volume & 0.1 \\
Mistuned coarse $u_{0}$ & 0.1 \\
\hline Total Error & 5.0 \\
\hline \hline
\end{tabular}




\section{Final "Old-Data" Results}

TABLE IX. Complete error budget and total error for the $B^{0}$ mixing parameter $\xi$. All errors are given in \%.

\begin{tabular}{lcc}
\hline \hline Source of uncertainty & Error $(\%)$ & \\
\cline { 1 - 2 } Statistics $\oplus$ light-quark disc. $\oplus$ chiral extrapolation & 3.7 & \\
Mixing with wrong-spin operators & 3.2 & \\
Heavy-quark discretization & 0.3 & \\
Scale uncertainty $\left(r_{1}\right)$ & 0.2 & \\
$g_{B B^{*} \pi}$ & 0.7 & \\
Light-quark masses & 0.5 & small \\
One-loop matching & 0.5 & because \\
Tuning $\kappa_{b}$ & 0.4 & ratio \\
Finite volume & 0.1 & \\
Mistuned coarse $u_{0}$ & 0.1 & \\
Total Error & 5.0 &
\end{tabular}




\section{Final "Old-Data" Results}

TABLE IX. Complete error budget and total error for the $B^{0}$ mixing parameter $\xi$. All errors are given in \%.

\begin{tabular}{lc}
\hline \hline Source of uncertainty & Error $(\%)$ \\
\hline Statistics $\oplus$ light-quark disc. $\oplus$ chiral extrapolation & 3.7 \\
Mixing with wrong-spin operators & 3.2 \\
Heavy-quark discretization & 0.3 \\
Scale uncertainty $\left(r_{1}\right)$ & 0.2 \\
$g_{B B^{*} \pi}$ & 0.7 \\
Light-quark masses & 0.5 \\
One-loop matching & 0.5 \\
Tuning $\kappa_{b}$ & 0.4 \\
Finite volume & 0.1 \\
Mistuned coarse $u_{0}$ & 0.1 \\
\hline Total Error & 5.0 \\
\hline \hline
\end{tabular}

Result: $\quad \xi=1.268(63) \quad$ (nearly final) 


\section{B Mixing}

$\downarrow$ Fermilab heavy quarks with MILC 2+1 asqtad staggered light quarks

- "Old data" project

- $0.12 \mathrm{fm} \leq a \leq 0.09 \mathrm{fm}$

- focus on SM operators, and in particular on $0_{1}$, which gives

$$
\xi=f_{B_{s}} \sqrt{\hat{B}_{B_{s}}} / f_{B_{d}} \sqrt{\hat{B}_{B_{d}}}
$$

- construct operators from Fermilab quark + naive quark (made from staggered).

- drop NLO "wrong spin" terms [ $\Rightarrow$ systematic error estimate].

-1-loop perturbation theory for mixing.

- to be posted in next month or so.

- "New data" project: similar to above, but:

- $0.15 \mathrm{fm} \leq a \leq 0.045 \mathrm{fm}$.

- complete set of SM and BSM operators.

C. Bouchard,

E. Freeland

- all wrong spin terms included correctly in ChPT.

- in progress. 


\section{Asqtad Ensembles}

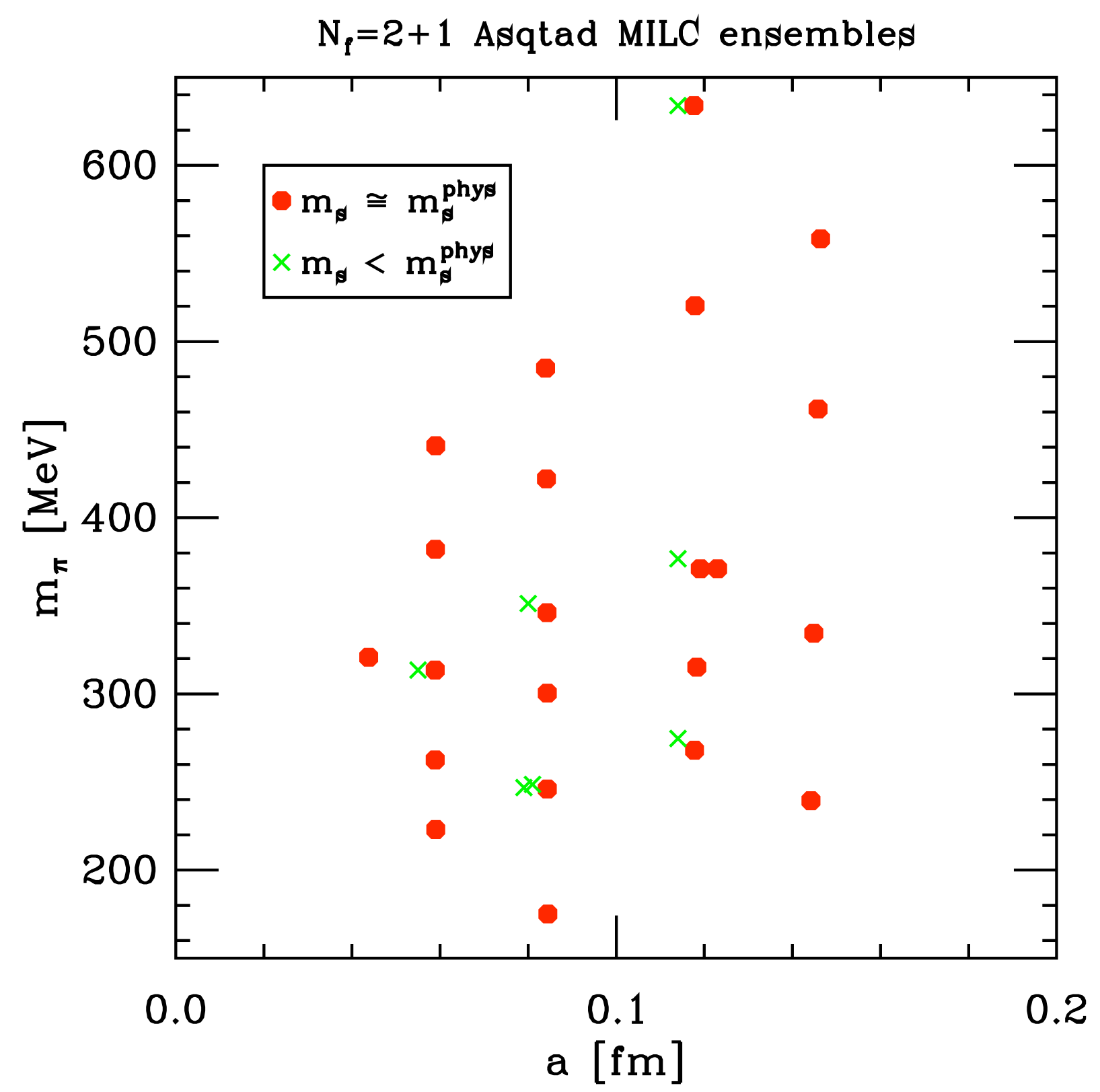




\section{Asqtad Ensembles}

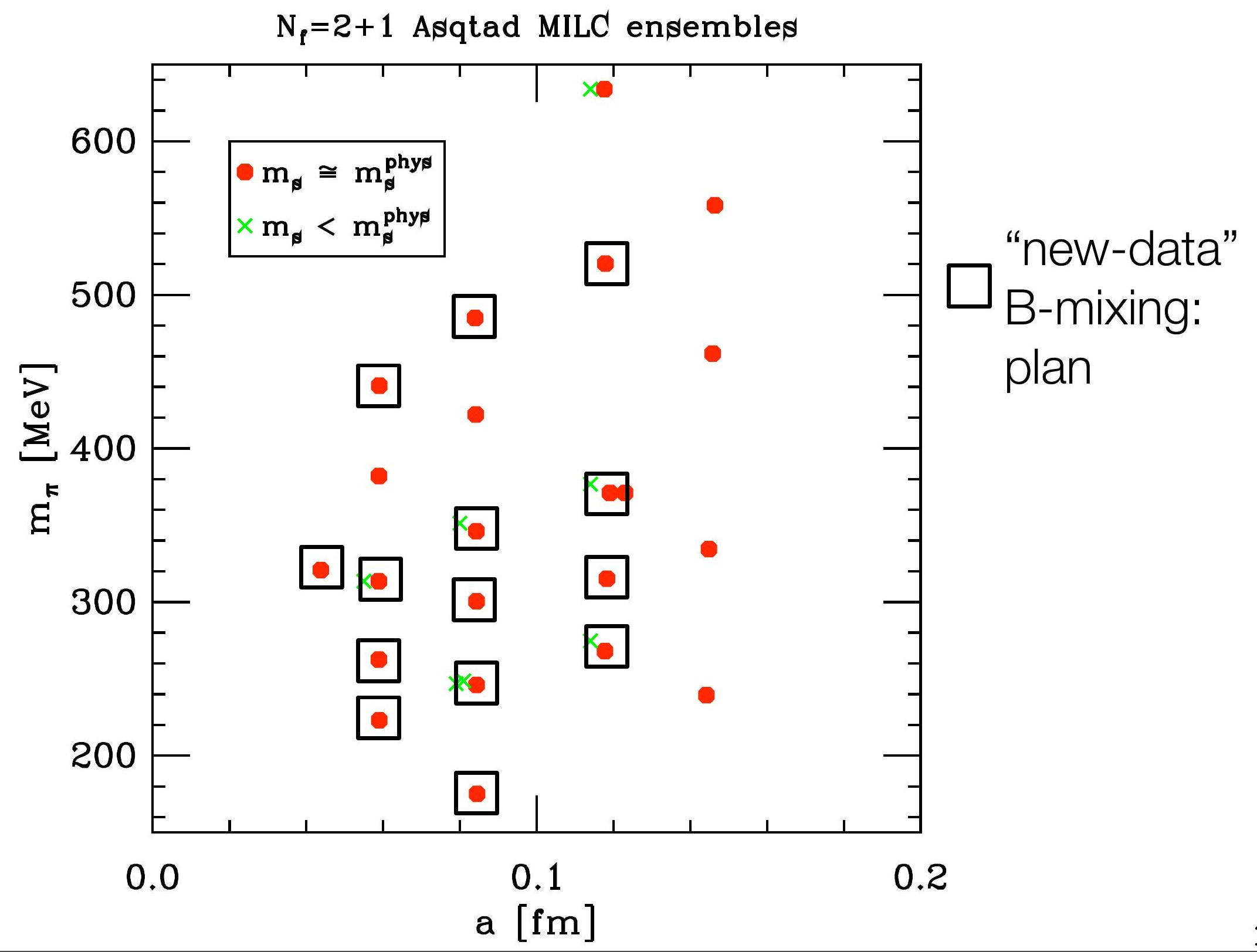




\section{Asqtad Ensembles}

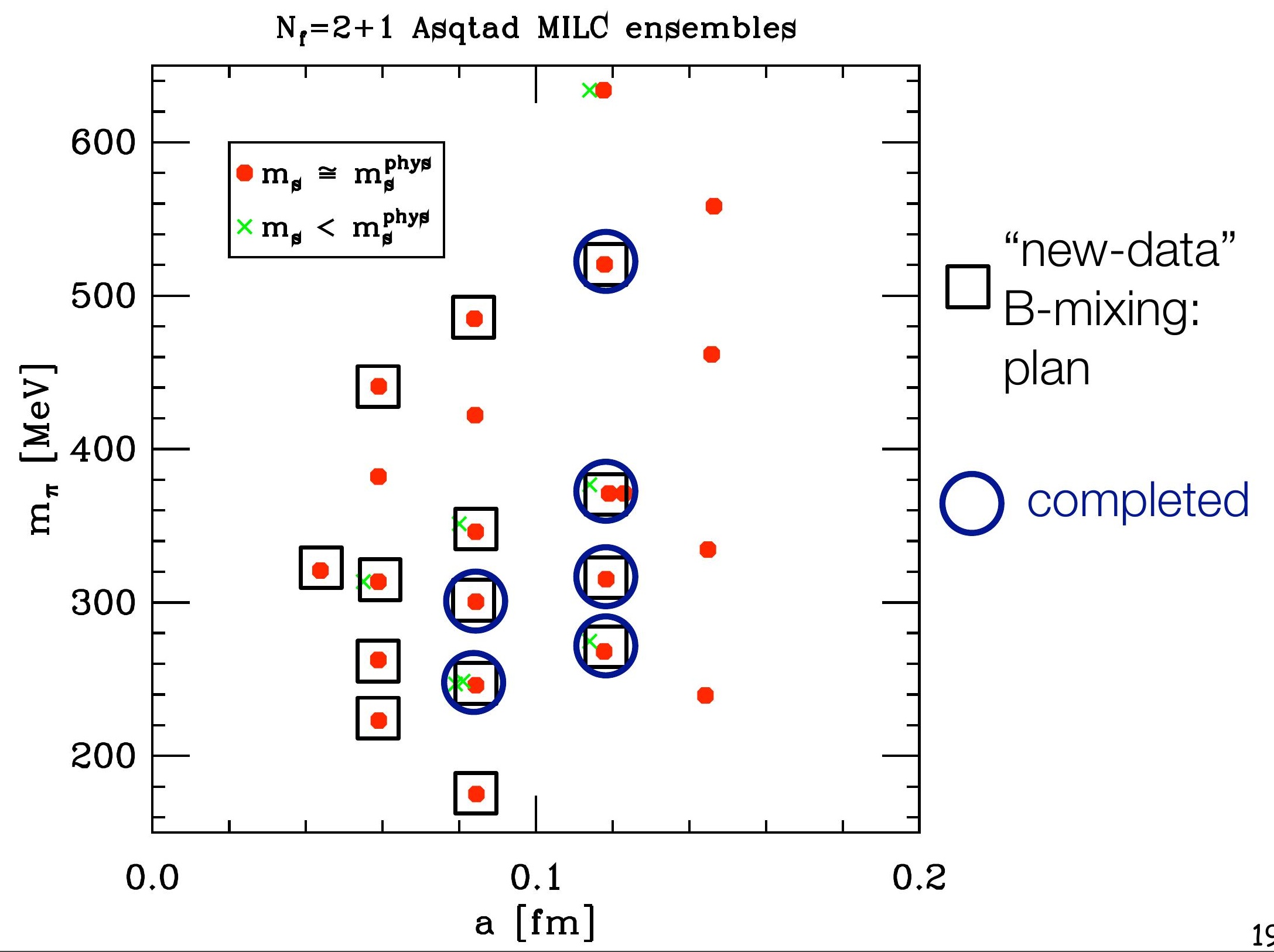




\section{Asqtad Ensembles}

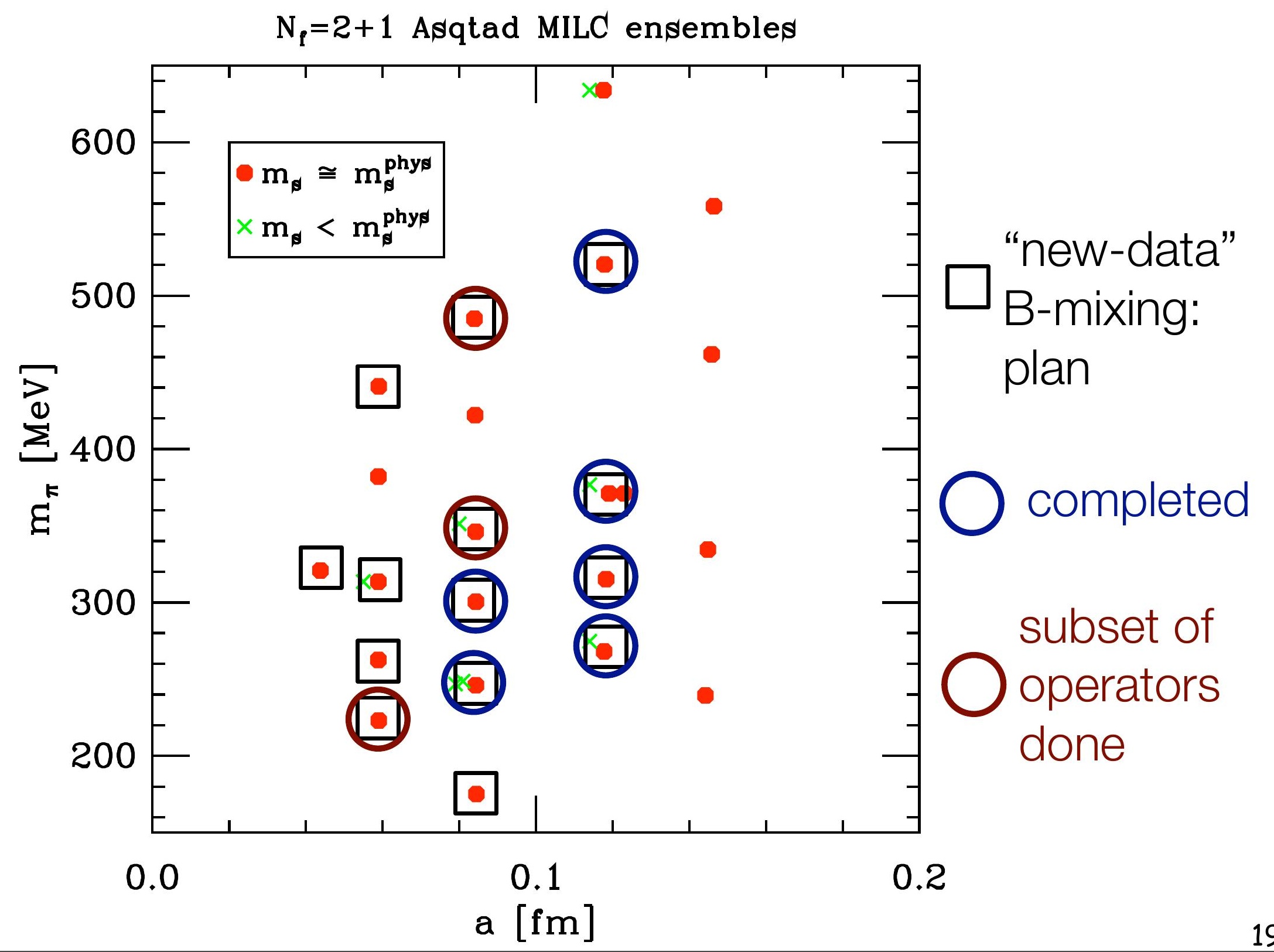




\section{Matrix element of $\mathrm{O}_{2}$}

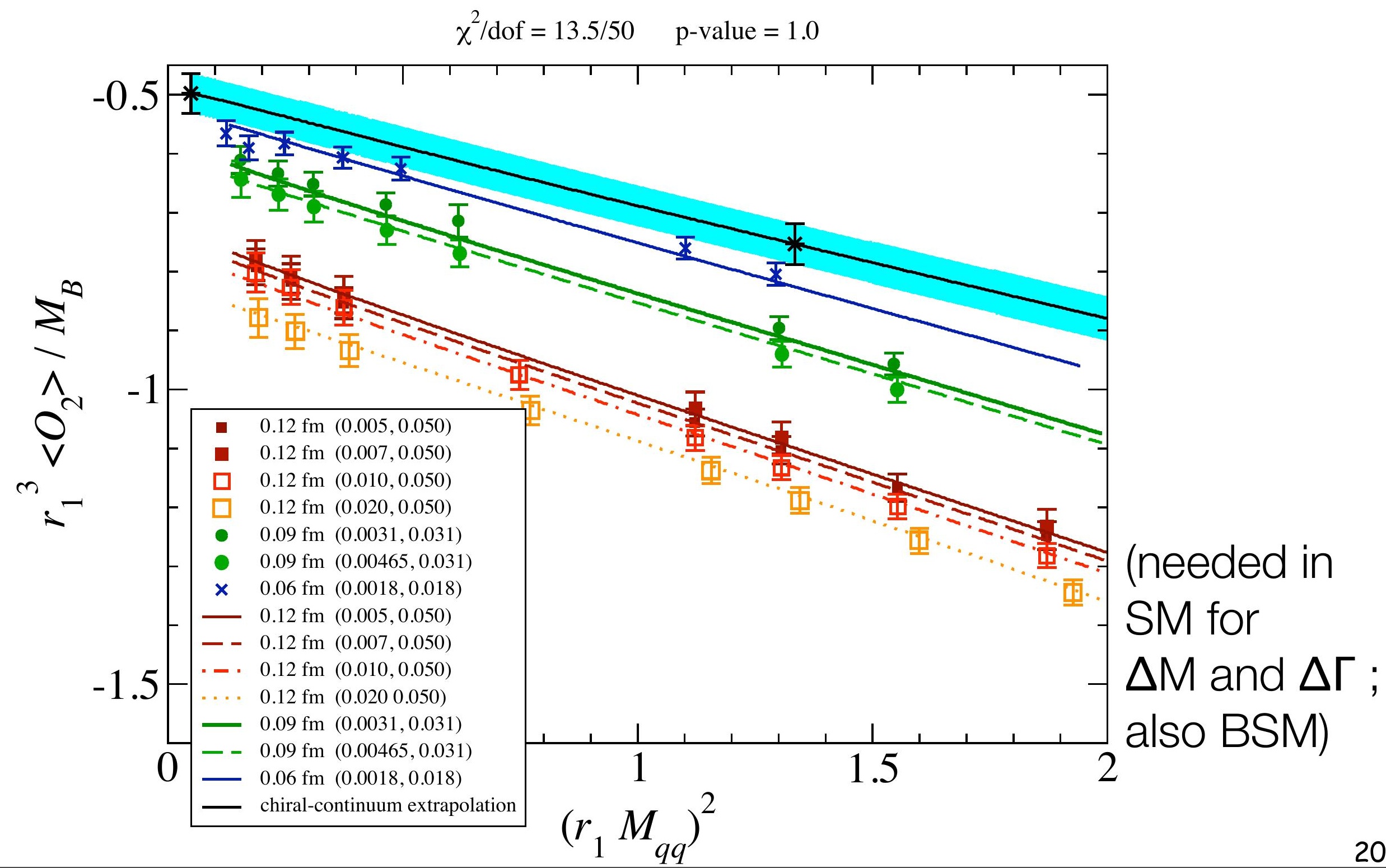


Anticipated Error Budget 


\section{Anticipated Error Budget}

$\downarrow$ Snapshot of statistical and chiral-continuum extrap errors at current state of running:

\begin{tabular}{llccccc}
\hline & Source of Error [\%] & $\left\langle\mathscr{O}_{1}\right\rangle$ & $\left\langle\mathscr{O}_{2}\right\rangle$ & $\left\langle\mathscr{O}_{3}\right\rangle$ & $\left\langle\mathscr{O}_{4}\right\rangle$ & $\left\langle\mathscr{O}_{5}\right\rangle$ \\
\hline \multirow{2}{*}{$B_{d}^{0}$} & statistical & 8.6 & 6.8 & 16 & 4.3 & 5.5 \\
& chiral-continuum systematic & 12 & 11 & 3.3 & 0.2 & 4.4 \\
\hline \multirow{2}{*}{$B_{s}^{0}$} & statistical & 6.7 & 4.6 & 10 & 2.5 & 3.4 \\
& chiral-continuum systematic & 1.8 & 6.6 & 4.5 & 1.6 & 3.7 \\
\hline
\end{tabular}




\section{Anticipated Error Budget}

$\downarrow$ Snapshot of statistical and chiral-continuum extrap errors at current state of running:

\begin{tabular}{llccccc}
\hline & Source of Error [\%] & $\left\langle\mathscr{O}_{1}\right\rangle$ & $\left\langle\mathscr{O}_{2}\right\rangle$ & $\left\langle\mathscr{O}_{3}\right\rangle$ & $\left\langle\mathscr{O}_{4}\right\rangle$ & $\left\langle\mathscr{O}_{5}\right\rangle$ \\
\hline \multirow{2}{*}{$B_{d}^{0}$} & statistical & 8.6 & 6.8 & 16 & 4.3 & 5.5 \\
& chiral-continuum systematic & 12 & 11 & 3.3 & 0.2 & 4.4 \\
\hline \multirow{2}{*}{$B_{s}^{0}$} & statistical & 6.7 & 4.6 & 10 & 2.5 & 3.4 \\
& chiral-continuum systematic & 1.8 & 6.6 & 4.5 & 1.6 & 3.7 \\
\hline
\end{tabular}

- Should decrease significantly as we add finer ensembles. 


\section{Anticipated Error Budget}

$\downarrow$ Snapshot of statistical and chiral-continuum extrap errors at current state of running:

\begin{tabular}{llccccc}
\hline & Source of Error [\%] & $\left\langle\mathscr{O}_{1}\right\rangle$ & $\left\langle\mathscr{O}_{2}\right\rangle$ & $\left\langle\mathscr{O}_{3}\right\rangle$ & $\left\langle\mathscr{O}_{4}\right\rangle$ & $\left\langle\mathscr{O}_{5}\right\rangle$ \\
\hline \multirow{2}{*}{$B_{d}^{0}$} & statistical & 8.6 & 6.8 & 16 & 4.3 & 5.5 \\
& chiral-continuum systematic & 12 & 11 & 3.3 & 0.2 & 4.4 \\
\hline \multirow{2}{*}{$B_{s}^{0}$} & statistical & 6.7 & 4.6 & 10 & 2.5 & 3.4 \\
& chiral-continuum systematic & 1.8 & 6.6 & 4.5 & 1.6 & 3.7 \\
\hline
\end{tabular}

- Should decrease significantly as we add finer ensembles.

$\downarrow$ Some other expected errors:

\begin{tabular}{lc}
\hline \hline Source of Error [\%] & $\left\langle\mathscr{O}_{i}\right\rangle$ \\
\hline scale $\left(r_{1}\right)$ & 3 \\
$\kappa_{b}$ tuning & 4 \\
light-quark masses & 1 \\
heavy-quark discretization & 4 \\
one-loop matching & 8 \\
finite-volume effects & 1 \\
\hline subtotal & 10
\end{tabular}




\section{Anticipated Error Budget}

$\downarrow$ Snapshot of statistical and chiral-continuum extrap errors at current state of running:

\begin{tabular}{llccccc}
\hline & Source of Error [\%] & $\left\langle\mathscr{O}_{1}\right\rangle$ & $\left\langle\mathscr{O}_{2}\right\rangle$ & $\left\langle\mathscr{O}_{3}\right\rangle$ & $\left\langle\mathscr{O}_{4}\right\rangle$ & $\left\langle\mathscr{O}_{5}\right\rangle$ \\
\hline \multirow{2}{*}{$B_{d}^{0}$} & statistical & 8.6 & 6.8 & 16 & 4.3 & 5.5 \\
& chiral-continuum systematic & 12 & 11 & 3.3 & 0.2 & 4.4 \\
\hline \multirow{2}{*}{$B_{s}^{0}$} & statistical & 6.7 & 4.6 & 10 & 2.5 & 3.4 \\
& chiral-continuum systematic & 1.8 & 6.6 & 4.5 & 1.6 & 3.7 \\
\hline
\end{tabular}

- Should decrease significantly as we add finer ensembles.

$\downarrow$ Some other expected errors:

\begin{tabular}{lc}
\hline Source of Error [\%] & $\left\langle\mathscr{O}_{i}\right\rangle$ \\
\hline scale $\left(r_{1}\right)$ & 3
\end{tabular}

- limit to precision.

$\kappa_{b}$ tuning

light-quark masses $\quad 1$

\begin{tabular}{lc}
$\begin{array}{l}\text { light-quark masses } \\
\text { heavy-quark discretization }\end{array}$ & 1 \\
one-loop matching & 8 \\
\hline finite-volume effects & 1 \\
\hline subtotal & 10
\end{tabular}




\section{Anticipated Error Budget}

$\downarrow$ Snapshot of statistical and chiral-continuum extrap errors at current state of running:

\begin{tabular}{llccccc}
\hline & Source of Error [\%] & $\left\langle\mathscr{O}_{1}\right\rangle$ & $\left\langle\mathscr{O}_{2}\right\rangle$ & $\left\langle\mathscr{O}_{3}\right\rangle$ & $\left\langle\mathscr{O}_{4}\right\rangle$ & $\left\langle\mathscr{O}_{5}\right\rangle$ \\
\hline \multirow{2}{*}{$B_{d}^{0}$} & statistical & 8.6 & 6.8 & 16 & 4.3 & 5.5 \\
& chiral-continuum systematic & 12 & 11 & 3.3 & 0.2 & 4.4 \\
\hline \multirow{2}{*}{$B_{s}^{0}$} & statistical & 6.7 & 4.6 & 10 & 2.5 & 3.4 \\
& chiral-continuum systematic & 1.8 & 6.6 & 4.5 & 1.6 & 3.7 \\
\hline
\end{tabular}

- Should decrease significantly as we add finer ensembles.

$\downarrow$ Some other expected errors:

\begin{tabular}{lc}
\hline \hline Source of Error [\%] & $\left\langle\mathscr{O}_{i}\right\rangle$ \\
\hline scale $\left(r_{1}\right)$ & 3 \\
$\kappa_{b}$ tuning & 4 \\
light-quark masses & 1 \\
heavy-quark discretization & 4 \\
one-loop matching & 8 \\
finite-volume effects & 1 \\
\hline subtotal & 10
\end{tabular}




\section{MILC HISQ 2+1+1 Ensembles}

$\downarrow$ Asqtad ensembles are complete; though there is more physics still to extract.

$\downarrow$ For higher precision, have moved to HISQ [Follana et al. [HPQCD], PRD 75 (2007) 054502].

- Reduced $O\left(\alpha_{s} a^{2}\right)$ and $O\left(\alpha_{s}^{2} a^{2}\right)$ errors with respect to Asqtad.

- $\left(a m_{c}\right)^{4}$ errors reduced $\Rightarrow$ treat charm with same relativistic action as light quarks.

- Ensembles include charm sea quarks

- (although error of quenching charm is probably negligible in most cases, it doesn't cost much to include it in sea.) 


\section{HISQ Ensembles}

$\mathrm{N}_{\mathrm{f}}=2+1+1$ Hisq MILC ensembles

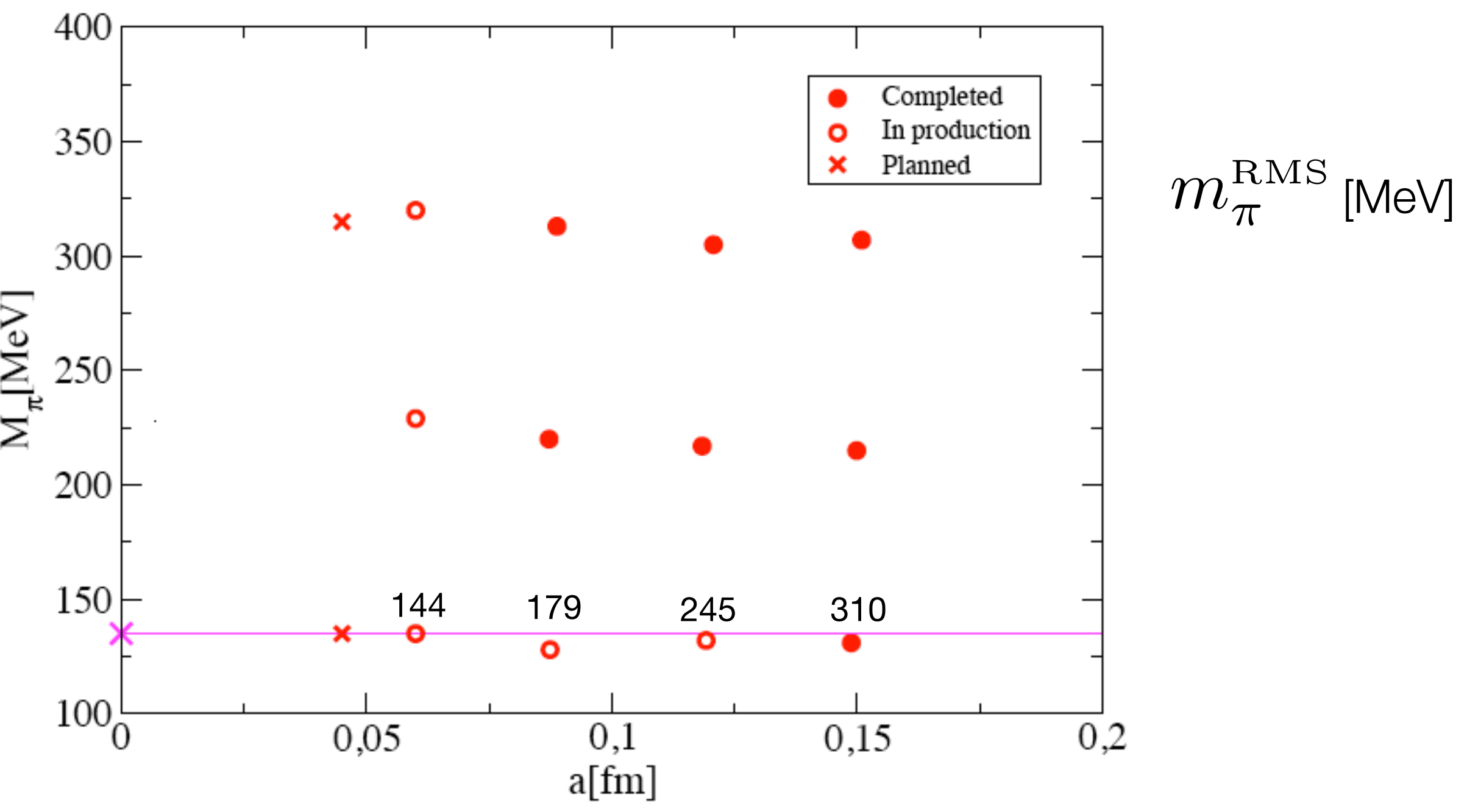




\section{HISQ Ensembles}

$\mathrm{N}_{\mathrm{f}}=2+1+1$ Hisq MILC ensembles

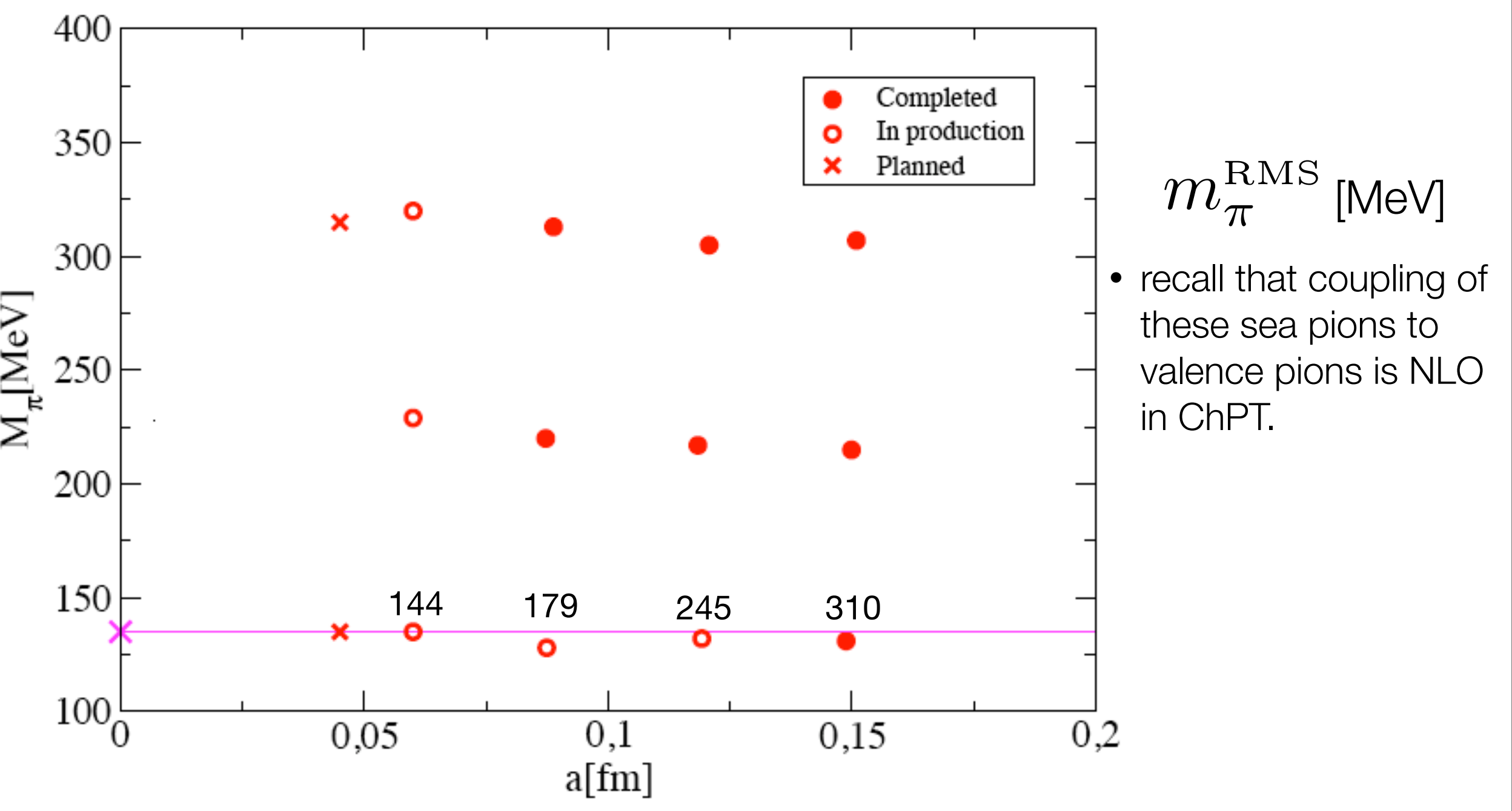




\section{HISQ Ensembles}

$\mathrm{N}_{\mathrm{f}}=2+1+1$ Hisq MILC ensembles

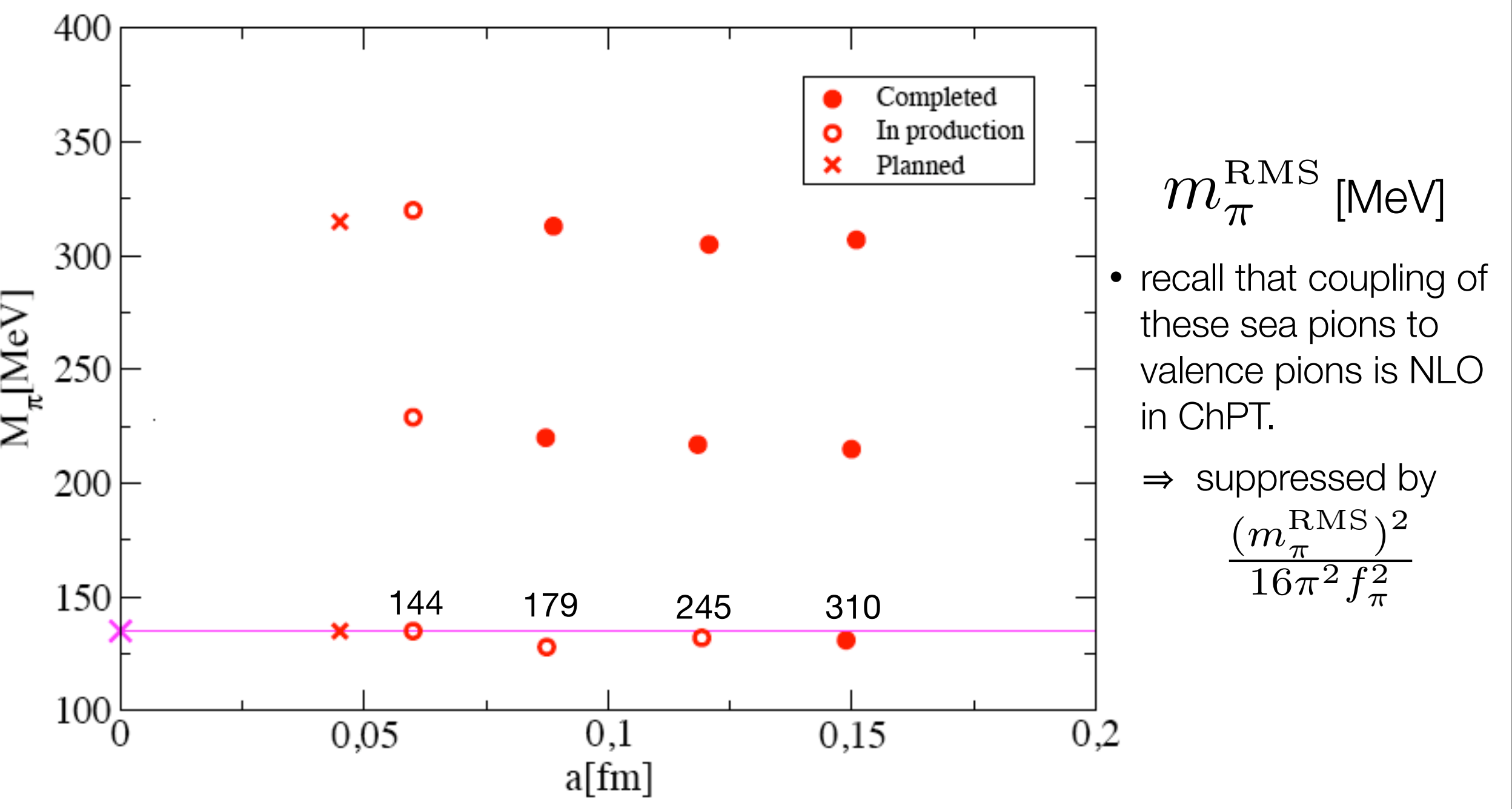




\section{Light decay constants $w / 2+1+1 \mathrm{HISQ}$}

$\downarrow$ In Asqtad case, needed ensembles with $m_{s}$ lighter than physical to control SU(3) chiral extrapolation.

- In HISQ case, such ensembles have not been available (but are coming on line now...), so SU(3) fits have not yet been very successful.

^ "Heavy kaon" SU(2) chiral perturbation theory [à la RBC/UKQCD and PACS-CS], has been recently worked out for staggered case [CB, Du, and Lightman], but not yet tried.

$\downarrow$ So focus for now on physical-mass HISQ ensembles, where ChPT not needed. 


\section{$\operatorname{HISQ} f_{\pi}, f_{k}$}

$\downarrow$ Suggests following interpolating forms:

$\left(a m_{x y}\right)^{2}=A_{1}+B_{1}\left(a m_{x}+a m_{y}\right)$

$a f_{x y}= \begin{cases}A_{2}+B_{2}\left(a m_{x}+a m_{y}\right), & \text { for } m_{x} \sim m_{l} \text { and } m_{y} \sim m_{l} \\ A_{3}+B_{3} a m_{x}+C_{3} a m_{y}, & \text { for } m_{x} \sim m_{l} \text { and } m_{y} \sim m_{s}\end{cases}$

$\downarrow$ On each ensemble, do linear interpolations of this form between $\left(m_{x}, m_{y}\right)=\left(m_{l}, m_{1}\right)$ and its nearest neighbor, and between $\left(m_{x}, m_{y}\right)=\left(m_{l}, m_{s}\right)$ and its nearest neighbors.

$\downarrow$ Require that $\left(f_{x x} / m_{x x}\right)^{2}=\left(f_{\Pi} / m_{\pi}\right)^{2}$, solve for $m_{x}$ to determine physical light mass mphys

- (Checked that quadratic interpolation with 3 points makes little difference)

$\downarrow$ Require that $\left(m_{x y} / m_{x x}\right)^{2}=\left(m_{k} / m_{\pi}\right)^{2}$, solve for $m_{y}$ to determine physical strange mass $m_{s}$ phys

- (Checked that interpolating $2\left(m_{x y}\right)^{2}-\left(m_{x x}\right)^{2}$ to find $m_{s}{ }^{\text {phys }}$ with makes little difference) 


\section{$\operatorname{HISQ} f_{\pi}, f_{K}$}

$\downarrow$ Then linearly interpolate $f$ and $f$ to physical masses.

- for the moment focus on $\mathrm{f}_{\mathrm{k}} / \mathrm{f}_{\pi}$; compute it for each ensemble.

- then fit it as function of $\mathrm{a}^{2}$.

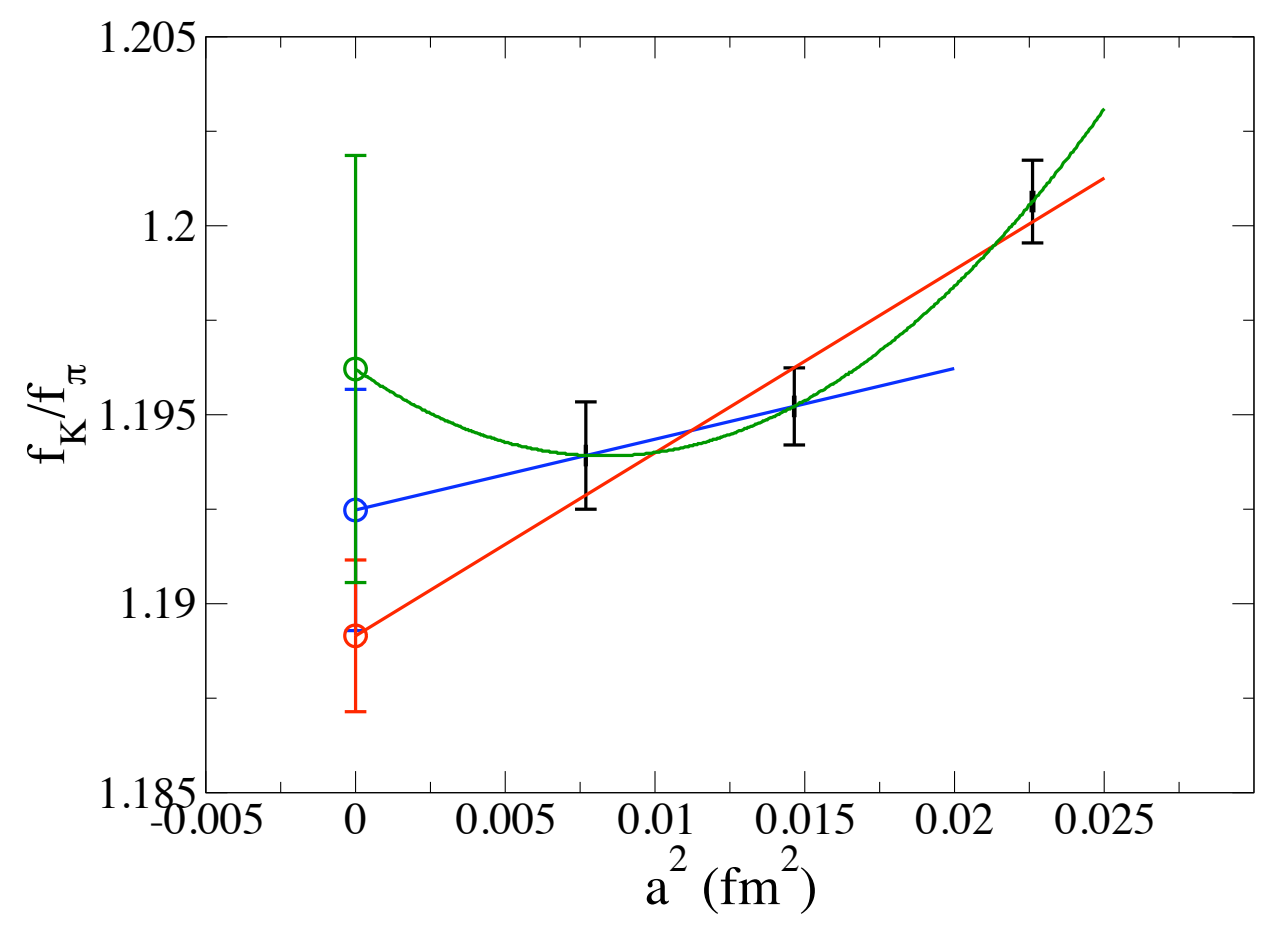

- two finest points in linear fit: $\left(f_{K} / f_{\pi}\right)_{\text {continuum }}=1.1925(32)$

- all 3 points in linear fit: $\left(\mathrm{f}_{K} / \mathrm{f}_{\pi}\right)_{\text {continuum }}=1.1892(20)$

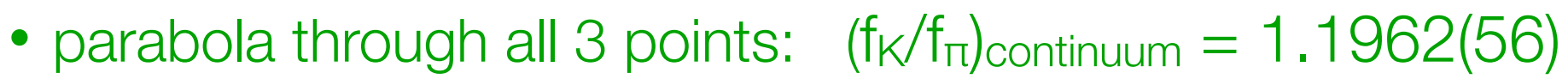




\section{HISQ $f_{\pi}, f_{K}:$ systematic errors}

$\downarrow$ Half the largest difference between continuum extraps to estimate that error.

- Finite volume effects from ensembles with $L=24,32$, and 40 with $a=0.12 \mathrm{fm}$ and $m_{l}=0.1 m_{s}$.

Volume dependence of $\mathrm{am}_{\mathrm{xx}}$

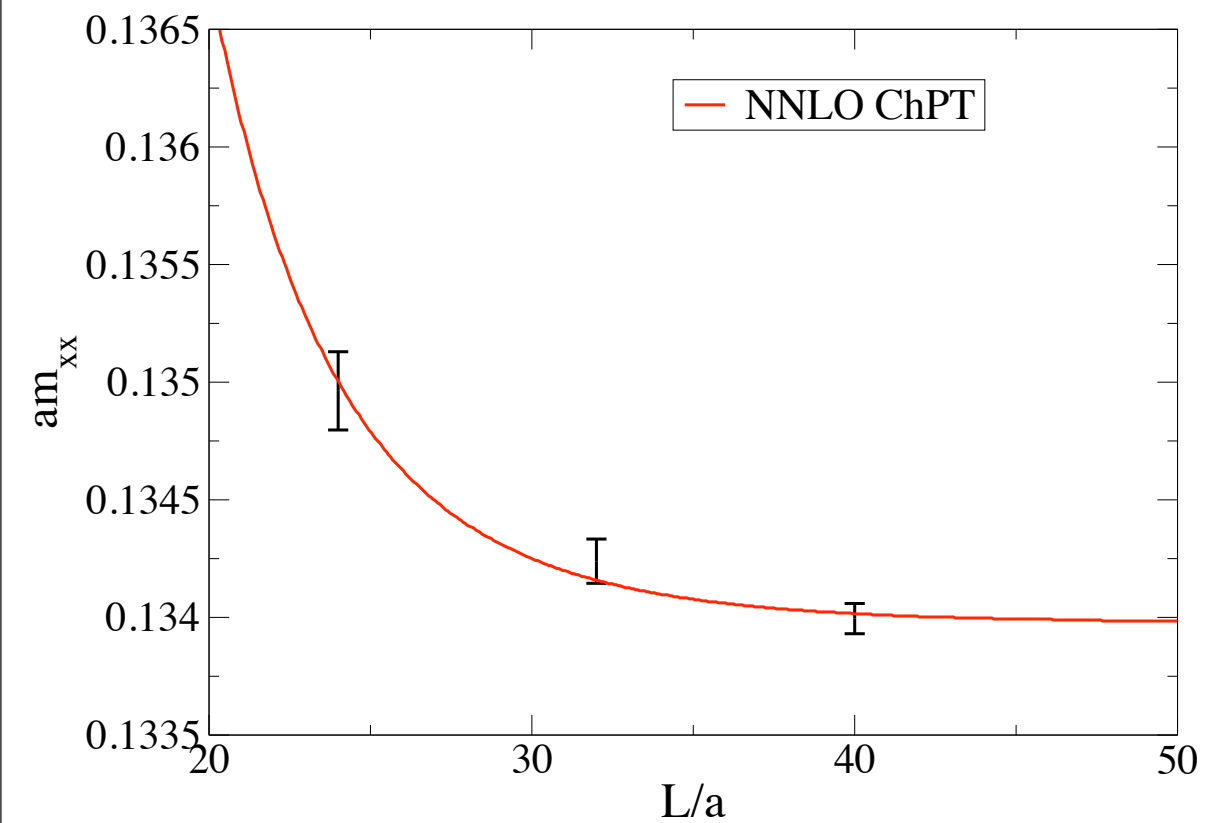

Volume dependence of $\mathrm{af}_{\mathrm{xx}}$

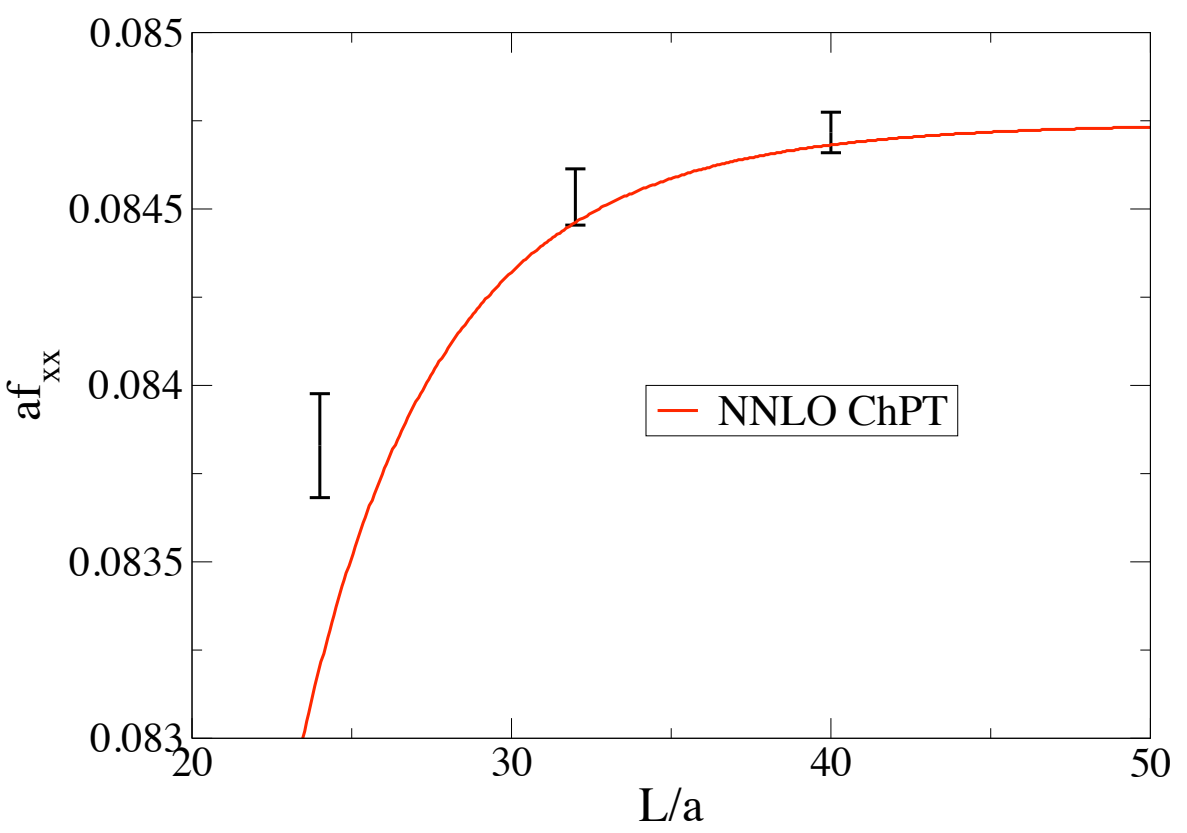

- "NNLO ChPT" means using Colangelo, Dürr, Haefeli, NPB 721 ('05) 136] to terms of $\mathcal{O}\left(\left[m_{\pi}^{2} / 16 \pi^{2} f^{2}\right]^{2}\right)$. 


\section{HISQ $f_{\pi}, f_{K}:$ preliminary result}

\ Find: $f_{K} / f_{\Pi}=1.1925(32)_{\text {stat }}(36)_{\text {continuum(32) finite volume }}$

$\downarrow$ Effect still to investigate: tuning error in sea quark masses.

$\downarrow$ More study of interpolating fits also needed.

- A systematic way will be to use the SU(2) staggered ChPT; will also allow us to get information out of the ensembles with $\mathrm{u}, \mathrm{d}$ mass heavier than physical, and find LECs.

- Some ensembles with $\mathrm{m}_{\mathrm{s}}$ lighter than physical have recently been completed; SU(3) fits should now also be possible. 


\section{D decay constants $w / 2+1+1 \mathrm{HISQ}$}

^ advantage of HISQ: discretization errors sufficiently reduced (both $\mathrm{a}^{2}$ and $(\mathrm{ma})^{4}$ ) that charm may be treated with same action as light quarks.

- avoid renormalization errors and many tuning issues.

- shares to some degree the small statistical errors of staggered light pseudoscalars. 


\section{HISQ Ensembles}

\section{$\mathrm{N}_{\mathrm{f}}=2+1+1$ Hisq MILC ensembles}

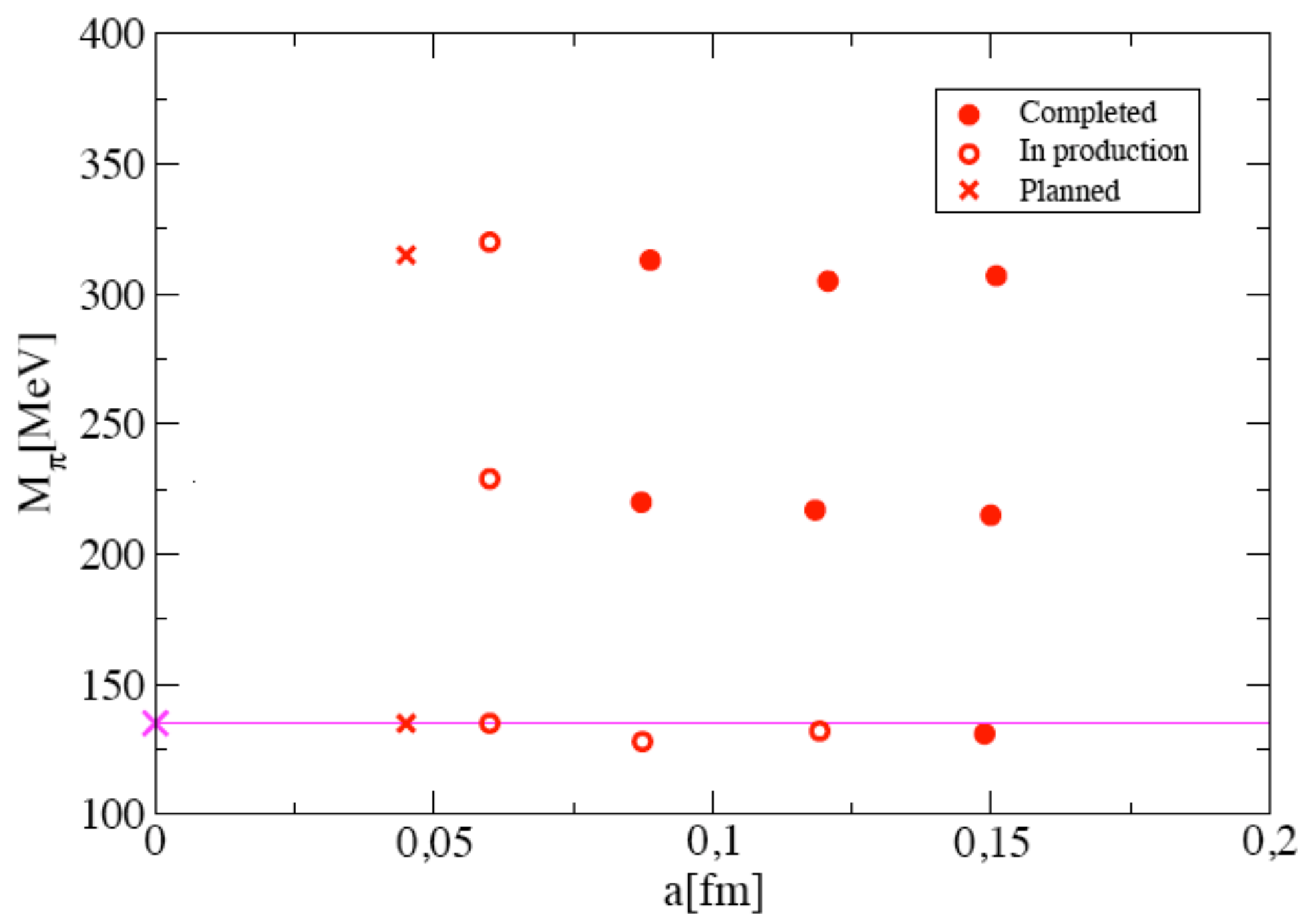




\section{HISQ Ensembles}

\section{$\mathrm{N}_{\mathrm{f}}=2+1+1$ Hisq MILC ensembles}

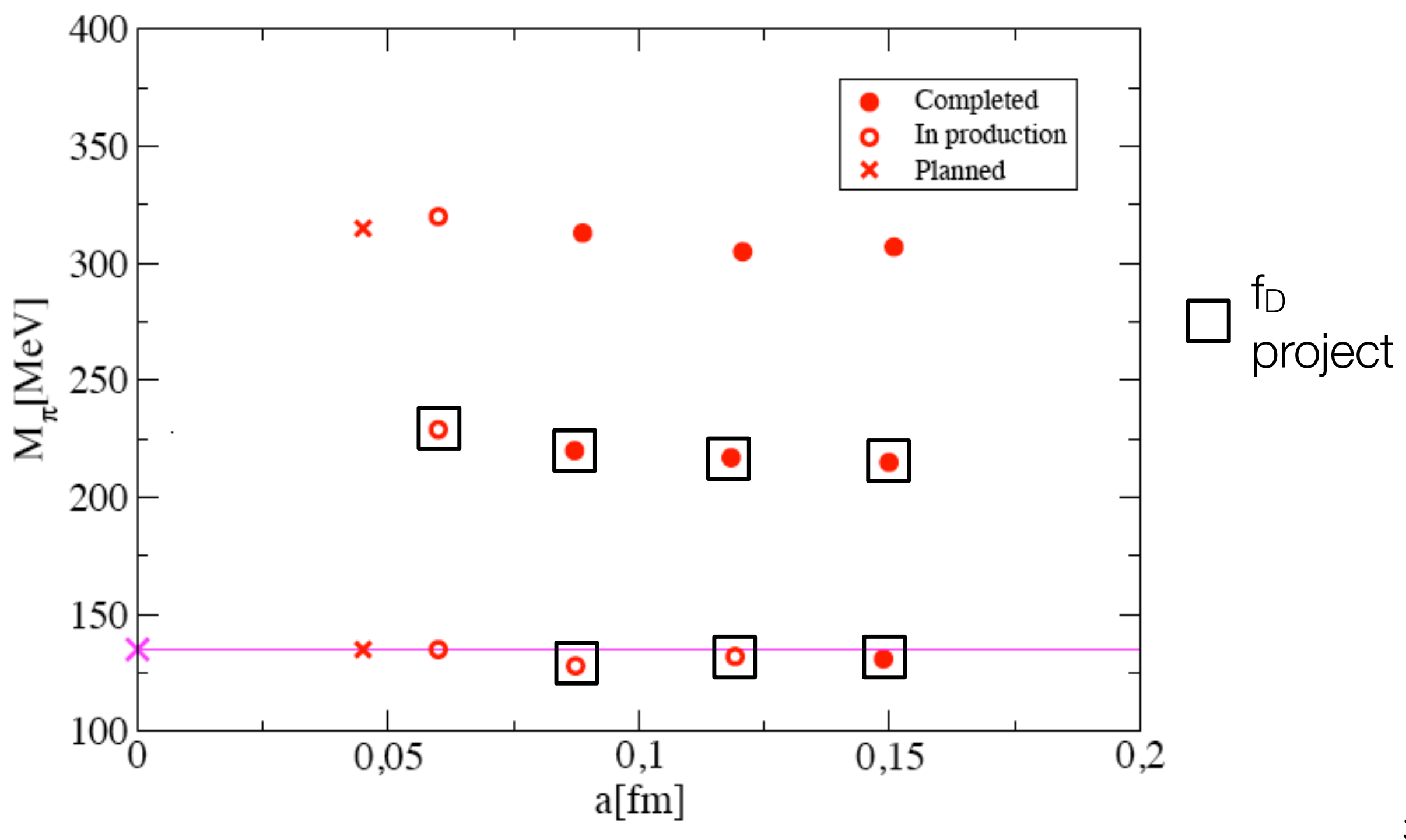




\section{HISQ Ensembles}

$$
\mathrm{N}_{\mathrm{f}}=2+1+1 \text { Hisq MILC ensembles }
$$

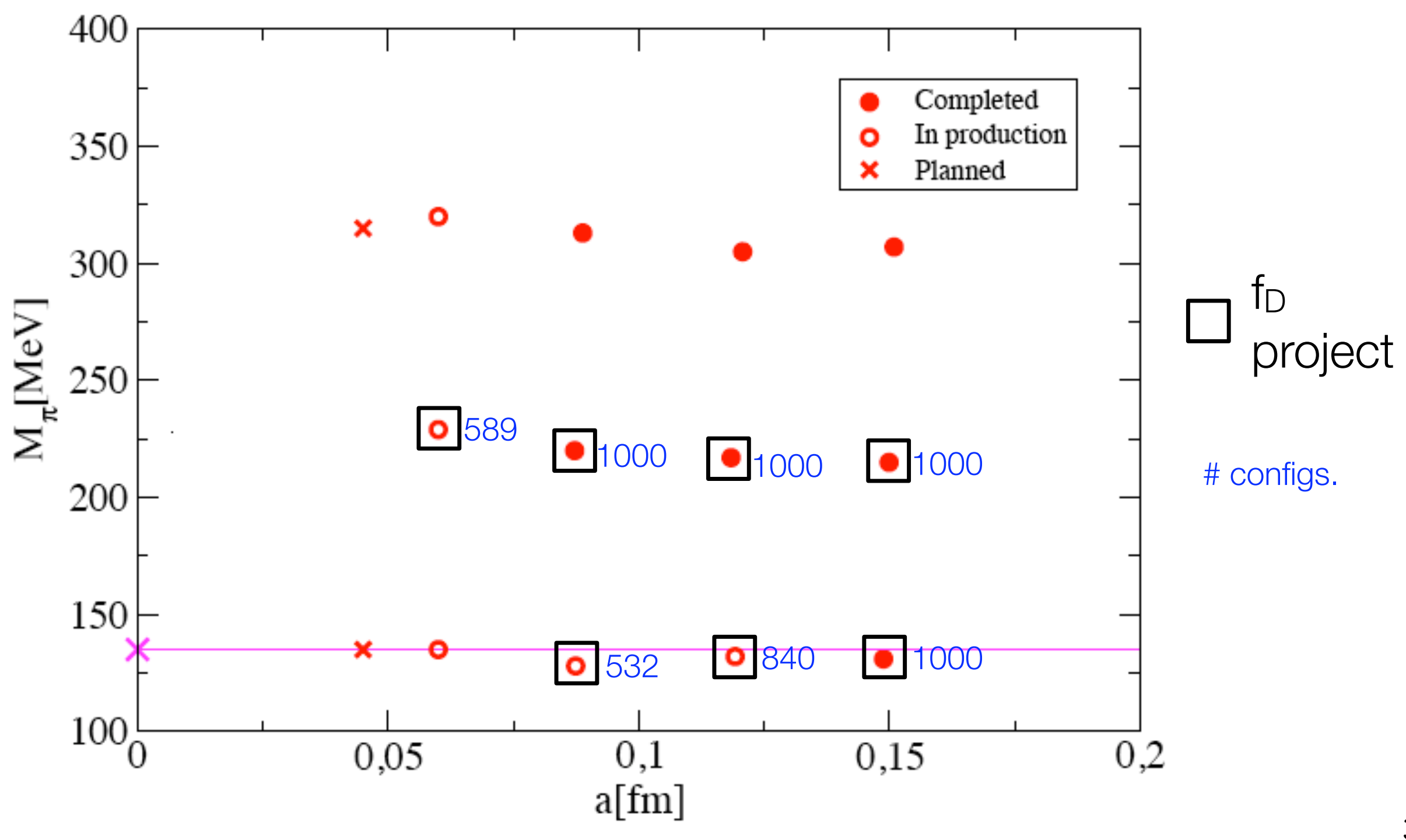




\section{$f_{D}, f_{D s}$ procedure}

$\downarrow$ On each ensemble:

- $\left(M_{\pi} / f_{\pi}\right)^{2} \rightarrow a m_{u, d}^{\text {phys }}$, and then $\rightarrow a$ (cubic interpolation through 3 light valence masses)

- $2 M_{K}^{2}-M_{\pi} \rightarrow a m_{s}^{\text {phys }}$ (linear interpolation/extrapolation through 2 strange valence masses)

- $M_{D_{s}} \rightarrow a m_{c}^{\mathrm{phys}}$ (linear interpolation/extrapolation through 2 charm valence masses)

- $f \rightarrow f_{D}, f \rightarrow f_{D_{s}}$ at proper adjusted masses (linear interpolation in light, strange and charm masses) 


\section{HISQ $f_{D}, f_{D s}$}

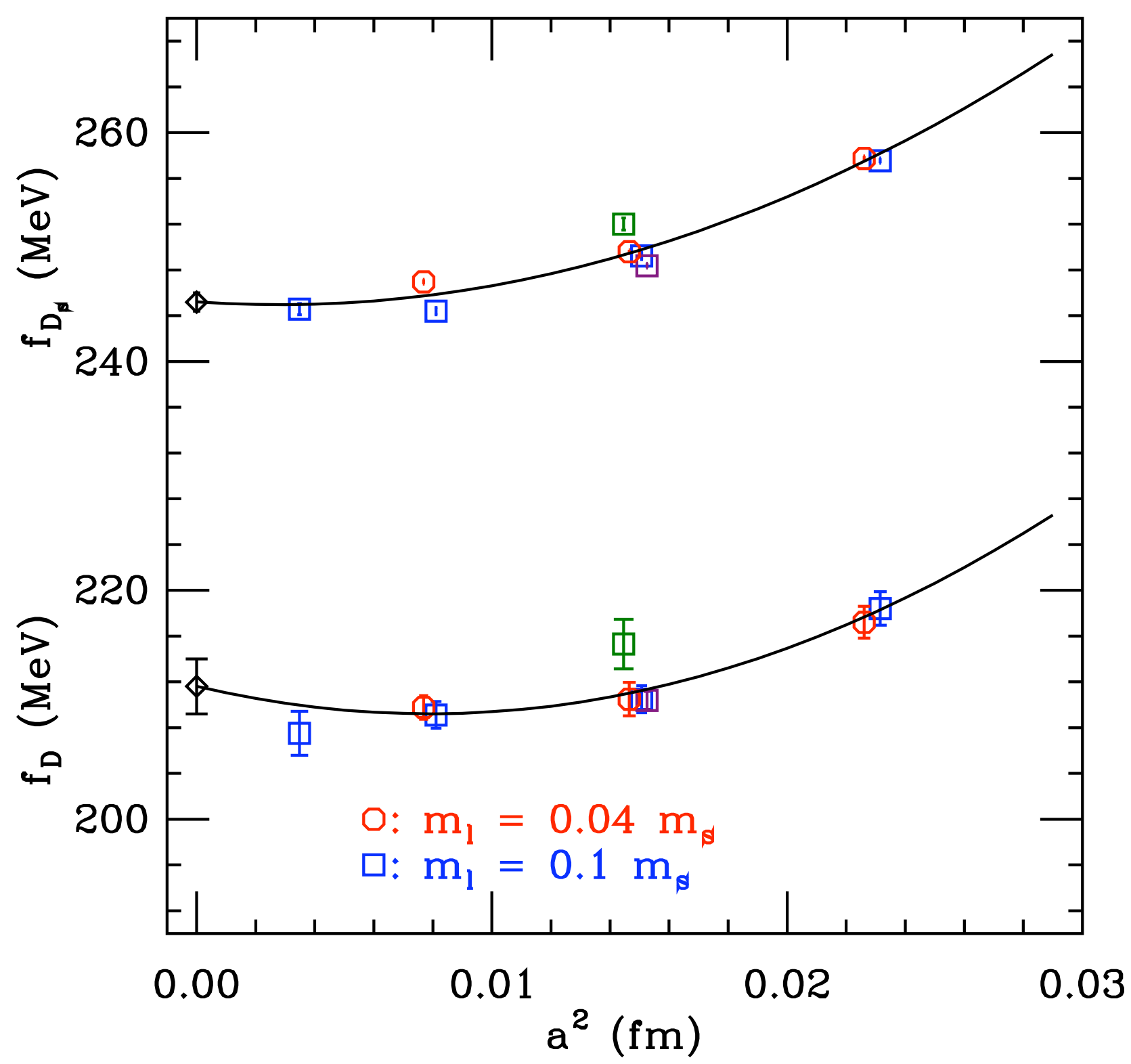




\section{HISQ $f_{D}, f_{D s}$}

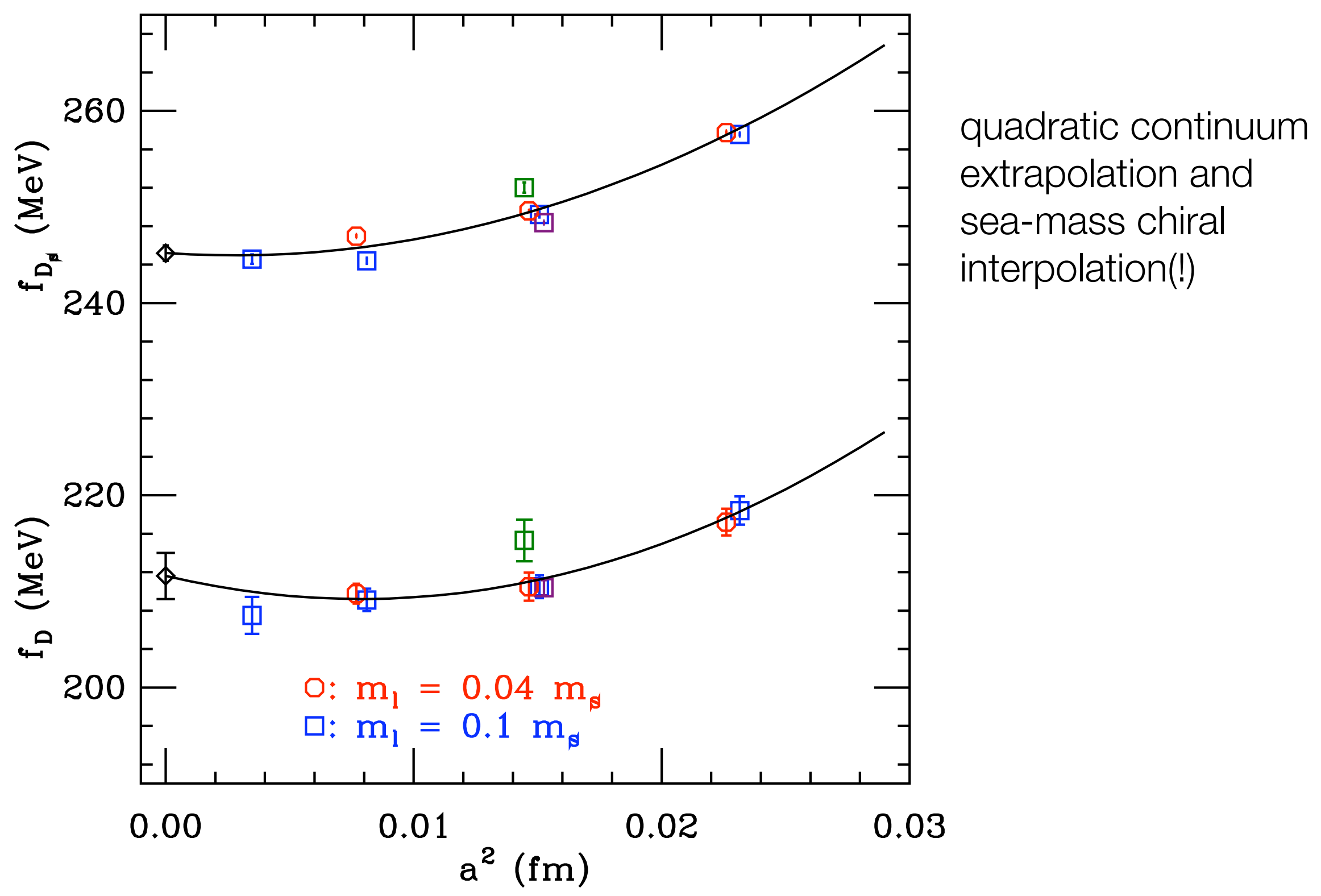




\section{HISQ $f_{D}, f_{D s}$}

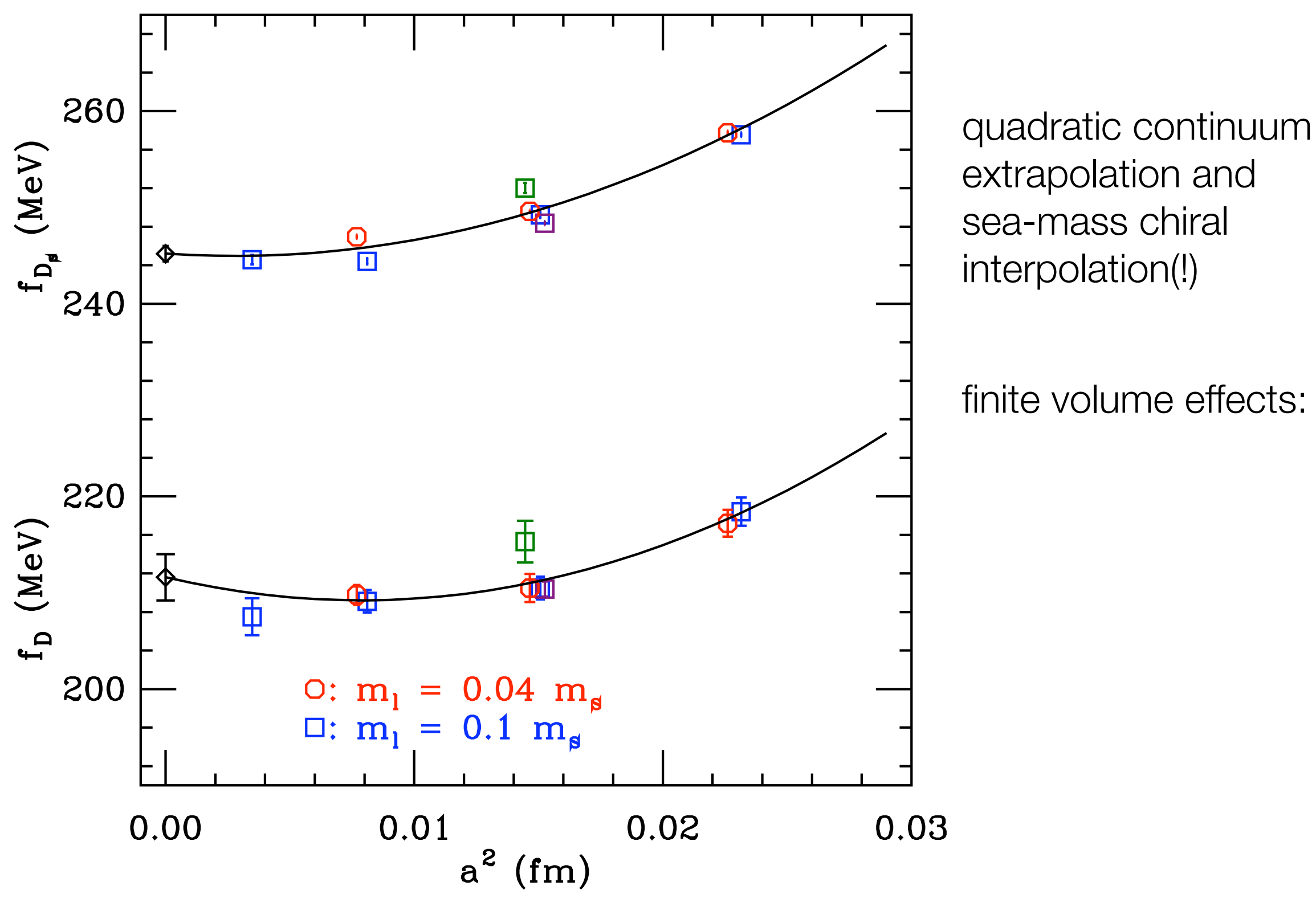




\section{HISQ $f_{D}, f_{D s}$}

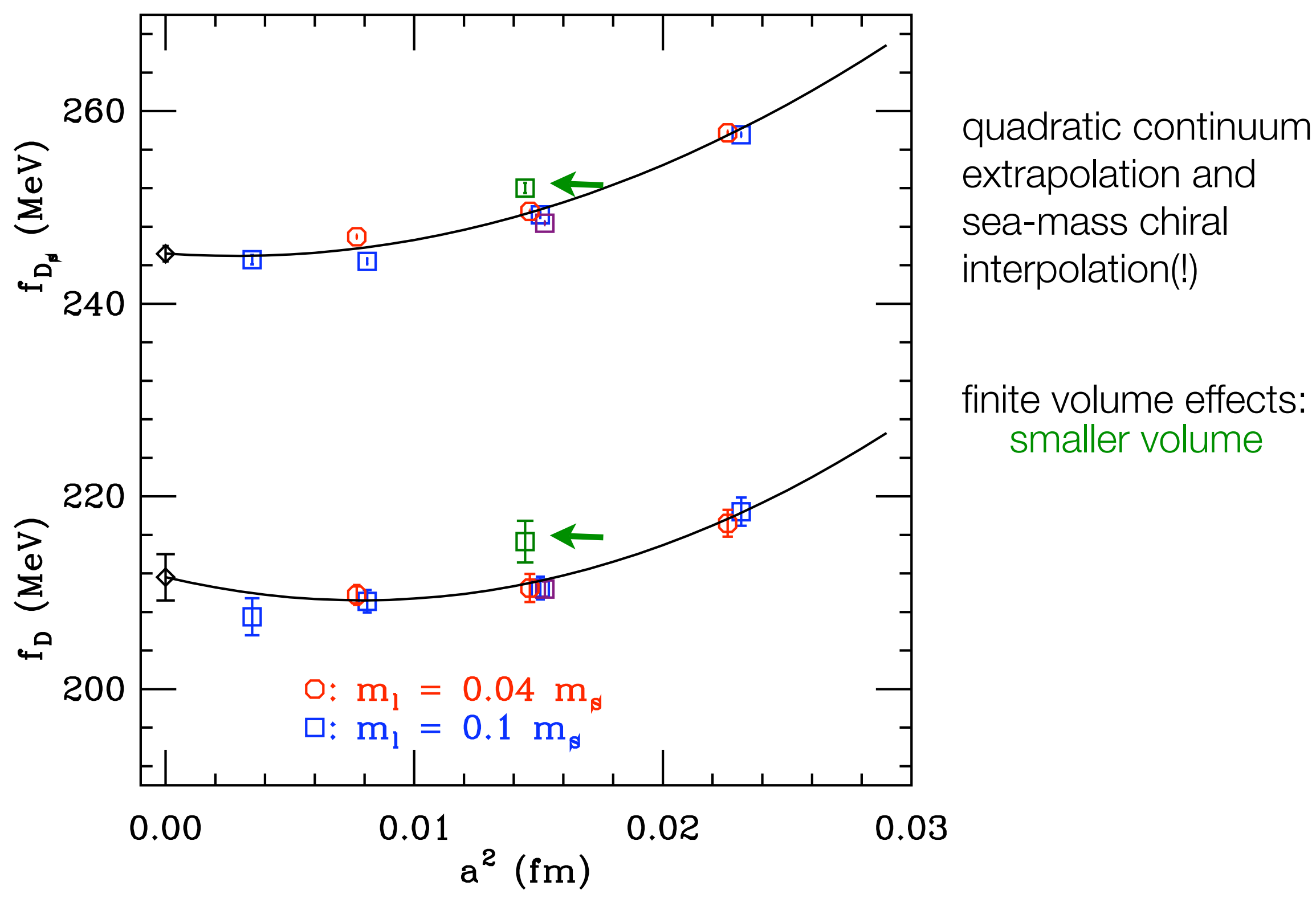




\section{HISQ $f_{D}, f_{D s}$}

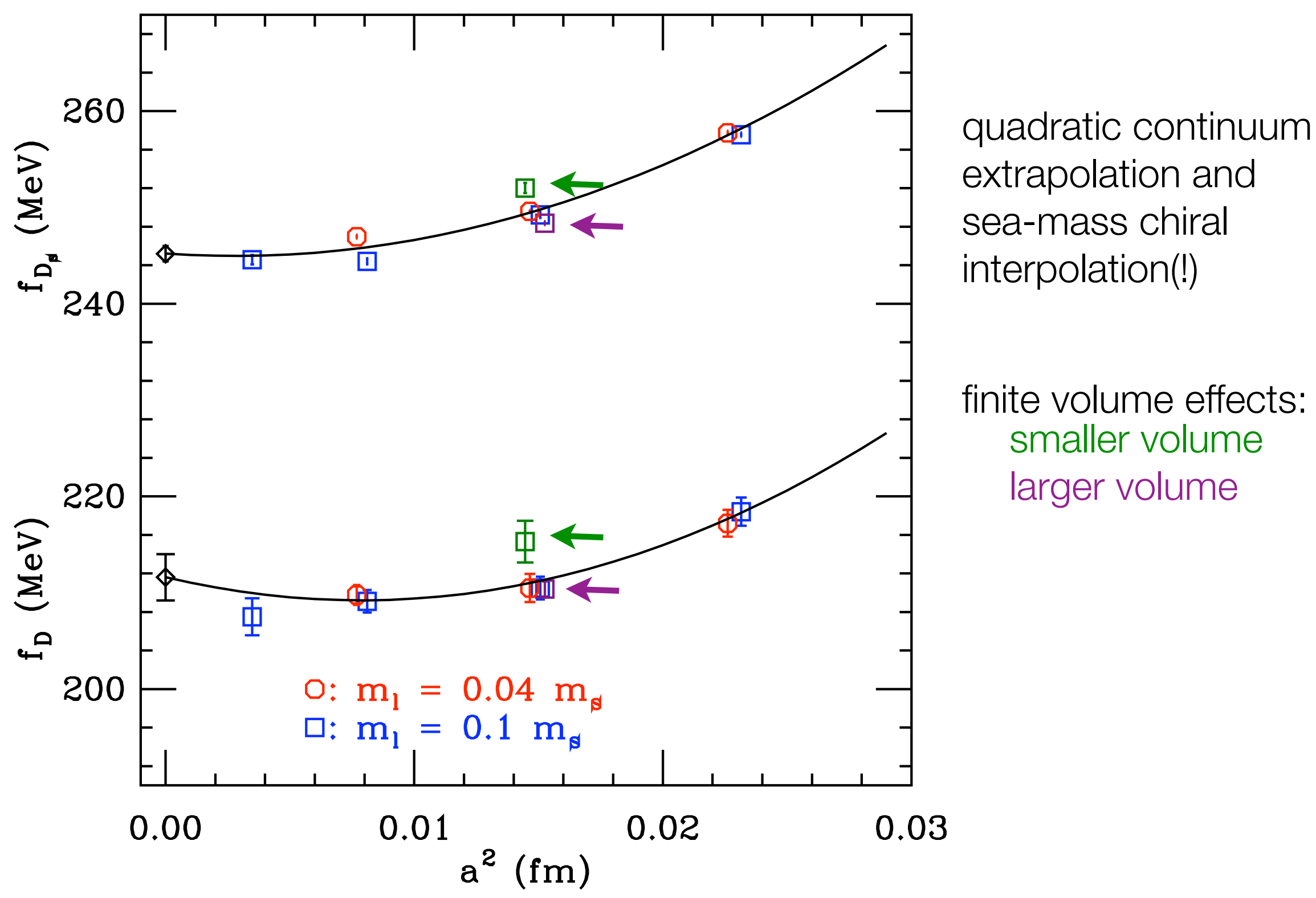




\section{HISQ $f_{D}, f_{D s}$}

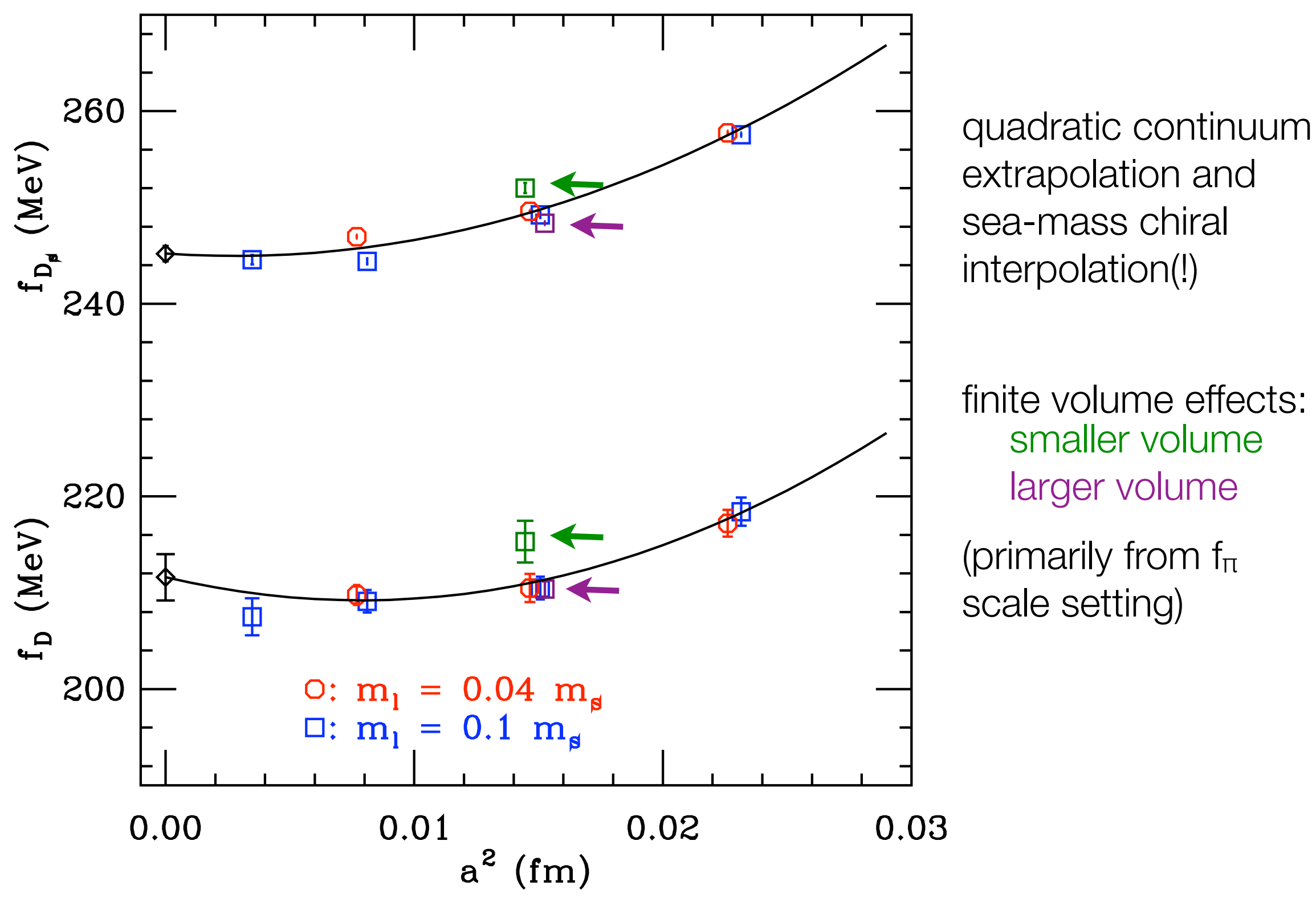




\section{Finite size effects}
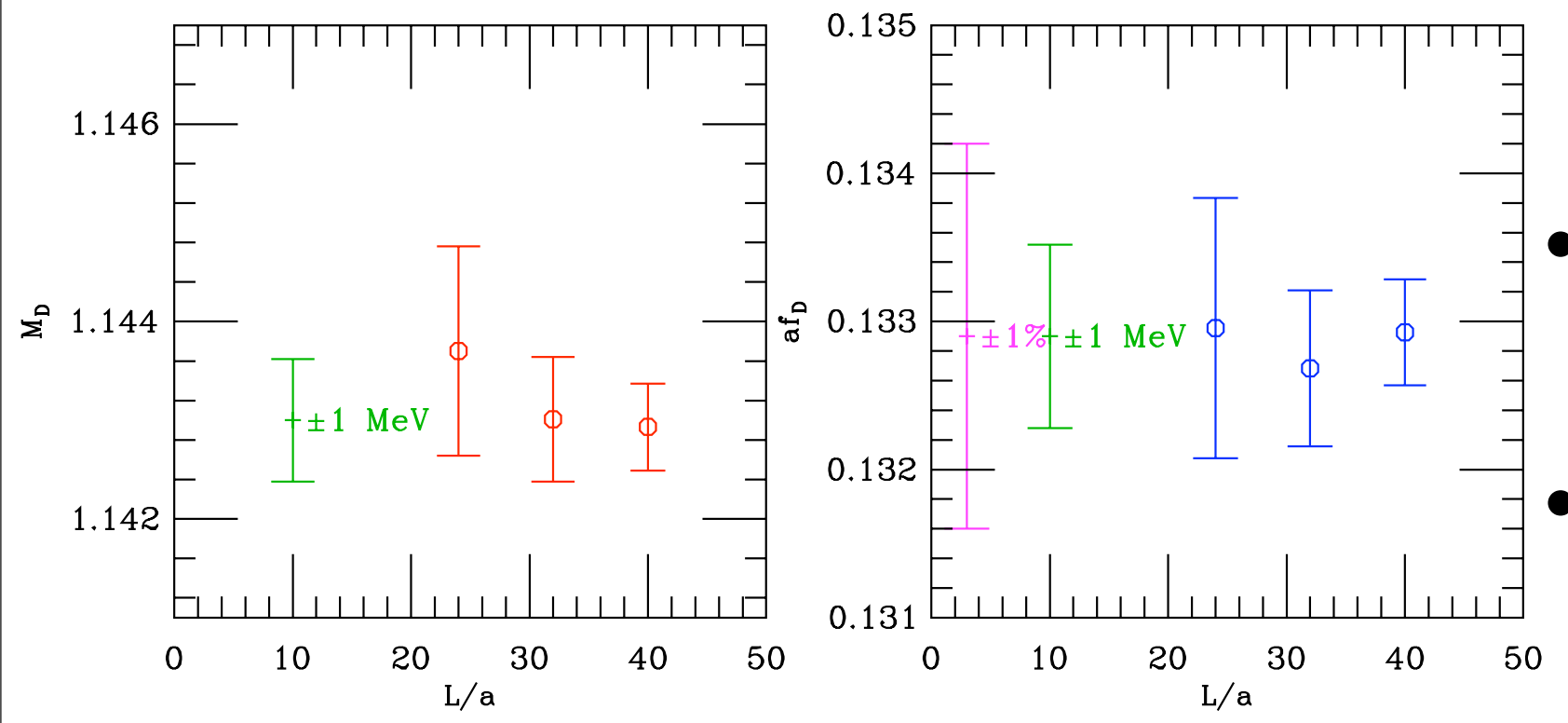

- Not much evidence for finite size effects.

- Here, results are in lattice units.

- Finite-volume effects
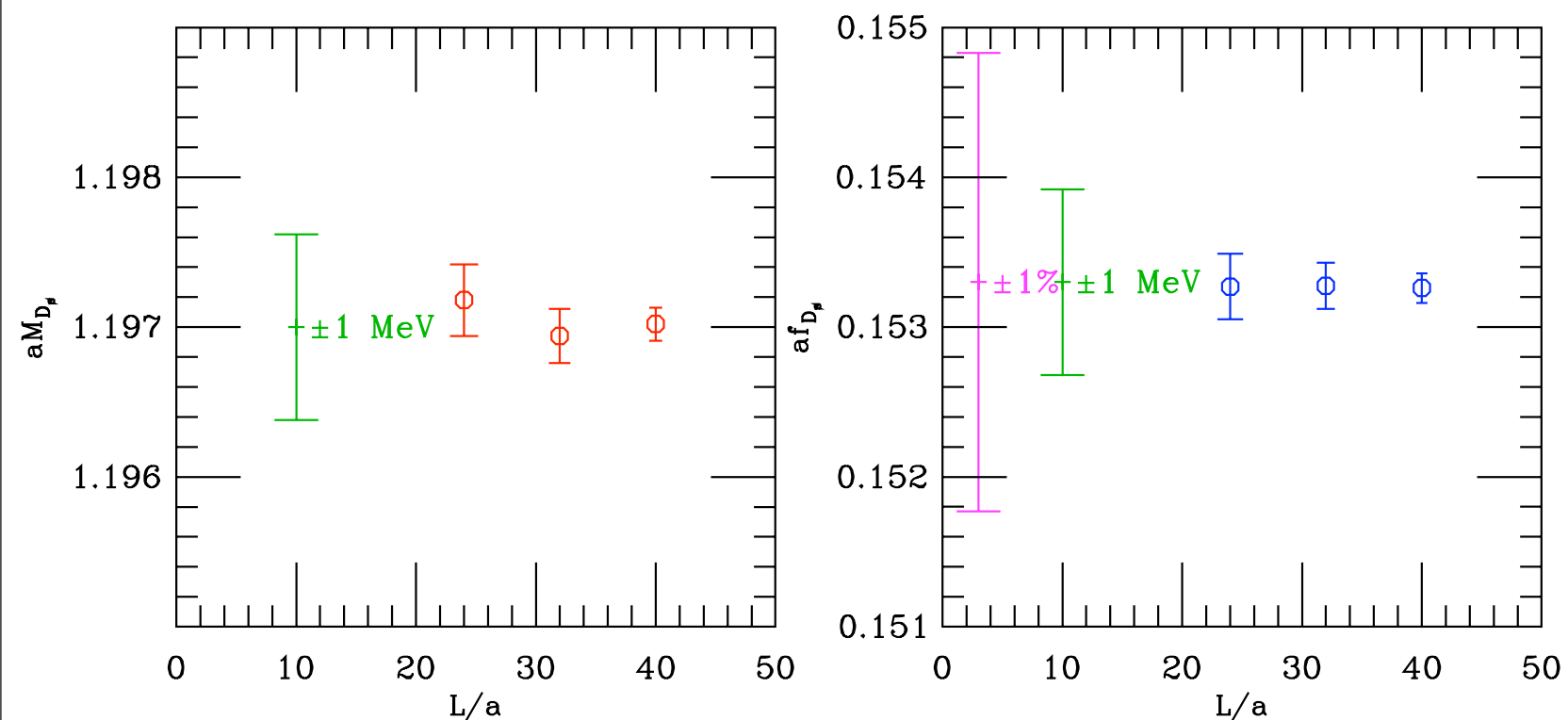
can enter if scale is set in finite-volumedependent way, e.g. $\mathrm{f}_{\Pi}$. 


\section{HISQ $f_{D}, f_{D s}$}

$\downarrow$ Very preliminary results:

$$
\begin{gathered}
f_{D}=211.6 \pm 2.4 \pm ? ? ? \mathrm{MeV} \\
f_{D_{s}}=245.2 \pm 0.8 \pm ? ? ? \mathrm{MeV}
\end{gathered}
$$

- ??? are systematic errors, including:

- continuum extrapolation/chiral interpolation

- staggered ChPT has been worked out [CB and J. Komijani] and may help to control continuum extrapolation.

- finite volume.

- isospin: easy to determine valence isospin breaking, e.g., $f_{D^{+}}$vs. generic $f_{D}$.

- EM effects: from Gläßle and Bali, arXiv:1111.3958 and Davies, et al., PRD 82 (2010) 114504, expect $<0.5 \%$

- ultimately plan to check with our EM code.... 


\section{Outlook: Fermilab/MILC}

\section{\% Errors}

Quantity

"Old data"

Fermilab $(c, b) \quad$ Fermilab $(c, b)$ arXiv:1112.3051
"New data"

(in progress)
HISQ (c) /

Fermilab (b)

(in progress)

\begin{tabular}{|c|c|c|c|}
\hline$f_{D_{S}}$ & 4.2 & 2.2 & 1.0 \\
\hline$f_{D}$ & 5.2 & 2.8 & 1.5 \\
\hline$f_{D_{s}} / f_{D}$ & 2.1 & 1.1 & 0.5 \\
\hline$f_{B S}$ & 3.9 & 2.6 & $\sim 1.5 ?$ \\
\hline$f_{B}$ & 4.5 & 2.8 & $\sim 2.0 ?$ \\
\hline$f_{B_{S}} / f_{B}$ & 2.1 & 1.2 & $\sim 0.8 ?$ \\
\hline
\end{tabular}




\section{Outlook: Fermilab/MILC}

\section{\% Errors}

Quantity "Old data" "New data" HISQ (c) / Fermilab (c,b) Fermilab (c,b) Fermilab (b) arXiv:1112.3051 (in progress)

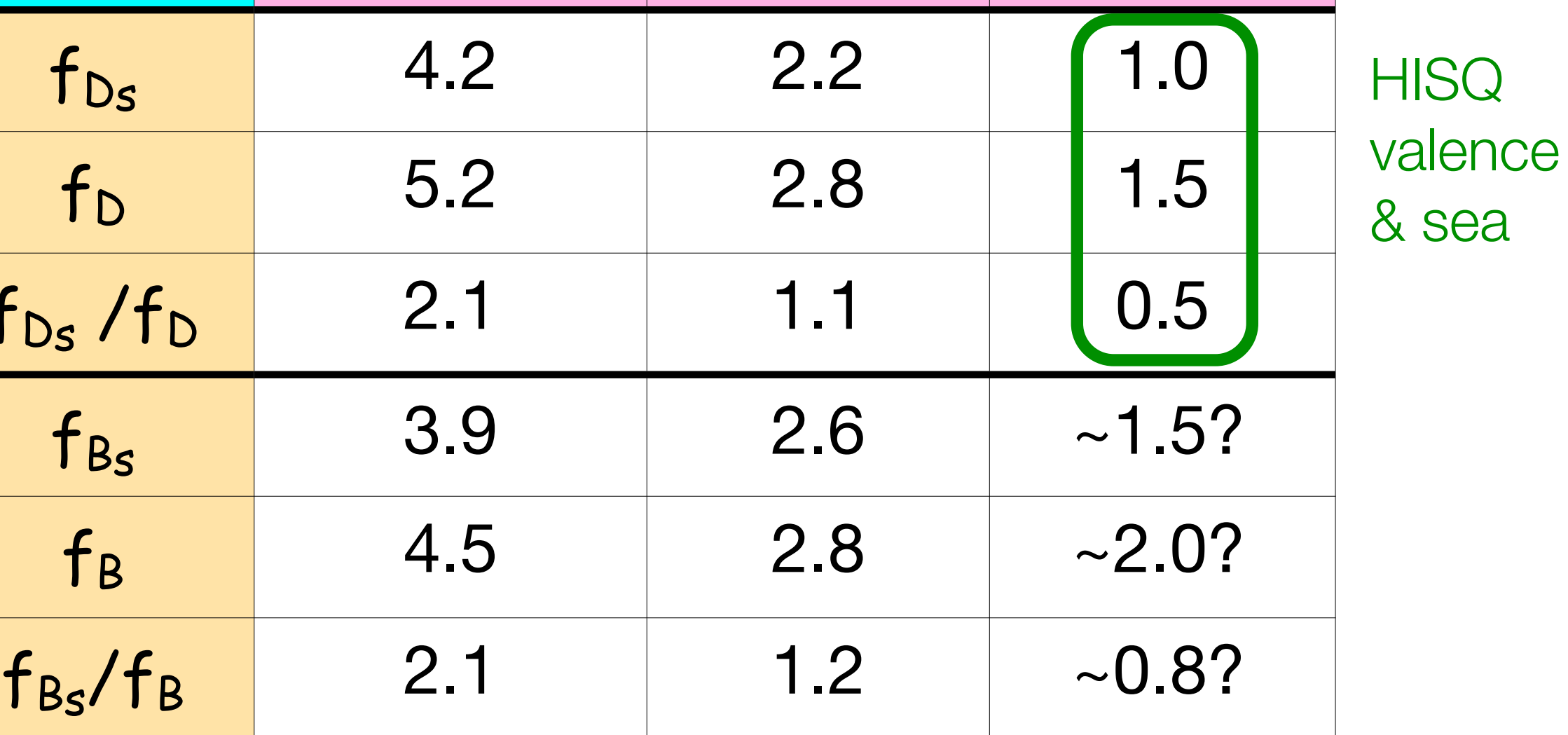




\section{Outlook: Fermilab/MILC}

\section{\% Errors}

Quantity

"Old data"

Fermilab (c,b) arXiv:1112.3051
"New data"

Fermilab $(c, b)$ (in progress)
HISQ (c) /

Fermilab (b) (in progress)

\begin{tabular}{|c|c|c|c|c|}
\hline$f_{D s}$ & 4.2 & 2.2 & 1.0 & HISQ \\
\hline$f_{D}$ & 5.2 & 2.8 & 1.5 & $\begin{array}{l}\text { valence } \\
\& \text { sea }\end{array}$ \\
\hline$f_{D_{s}} / f_{D}$ & 2.1 & 1.1 & 0.5 & \\
\hline$f_{B_{S}}$ & 3.9 & 2.6 & $\sim 1.5 ?$ & Fermilab \\
\hline$f_{B}$ & 4.5 & 2.8 & $\sim 2.0 ?$ & + HISQ D \\
\hline$f_{B_{S}} / f_{B}$ & 2.1 & 1.2 & $\sim 0.8 ?$ & $\begin{array}{l}\text { (valence \& } \\
\text { sea) }\end{array}$ \\
\hline
\end{tabular}




\section{Comment}

$\downarrow$ Best direction for us for B physics not obvious.

- Use Fermilab or Oktay-Kronfeld (improved Fermilab) b quarks?

- Push/extrapolate HISQ up to the b [HPQCD]?

- Leverage HISQ data for D (or heavier D) by using Fermilab quarks for $\mathrm{B} / \mathrm{D}$ ratios?

- In any case, will eventually need non-perturbative or 2-loop matching for many quantities to match other systematic improvements. 


\section{$\mathrm{K} \rightarrow \pi$ semileptonic decay}

$\downarrow$ Focus at $\mathrm{q}^{2}=0$, where we can use the method HPQCD proposed for semileptonic D decay:

- Full matrix element of vector current $V_{\mu}$ is hard because conserved current is complicated and local current needs renormalization.

- Instead use $\partial^{\mu} V_{\mu}=\left(m_{b}-m_{a}\right) S$

- $S$ is local, and product $\left(m_{b}-m_{a}\right) S$ not renormalized.

- This is sufficient for $f_{+}\left(q^{2}=0\right)=f_{0}\left(q^{2}=0\right)$.

$\downarrow$ Two-part program:

- HISQ valence on 2+1 Asqtad ensembles (close to completion).

- HISQ valence on $2+1+1 \mathrm{HISQ}$ ensembles (early stage).

- ultimately to include $\mathrm{D} \rightarrow \mathrm{K}$, and $\mathrm{q}^{2} \neq 0$ 


\section{$\mathrm{K} \rightarrow \pi$ semileptonic decay}

$\downarrow$ Focus at $\mathrm{q}^{2}=0$, where we can use the method HPQCD proposed for semileptonic $D$ decay:

- Full matrix element of vector current $V_{\mu}$ is hard because conserved current is complicated and local current needs renormalization.

- Instead use $\partial^{\mu} V_{\mu}=\left(m_{b}-m_{a}\right) S$

- $S$ is local, and product $\left(m_{b}-m_{a}\right) S$ not renormalized.

- This is sufficient for $f_{+}\left(q^{2}=0\right)=f_{0}\left(q^{2}=0\right)$.

$\downarrow$ Two-part program:

- HISQ valence on 2+1 Asqtad ensembles (close to completion).

- HISQ valence on $2+1+1 \mathrm{HISQ}$ ensembles (early stage).

- ultimately to include $\mathrm{D} \rightarrow \mathrm{K}$, and $\mathrm{q}^{2} \neq 0$ 


\section{Asqtad Ensembles}

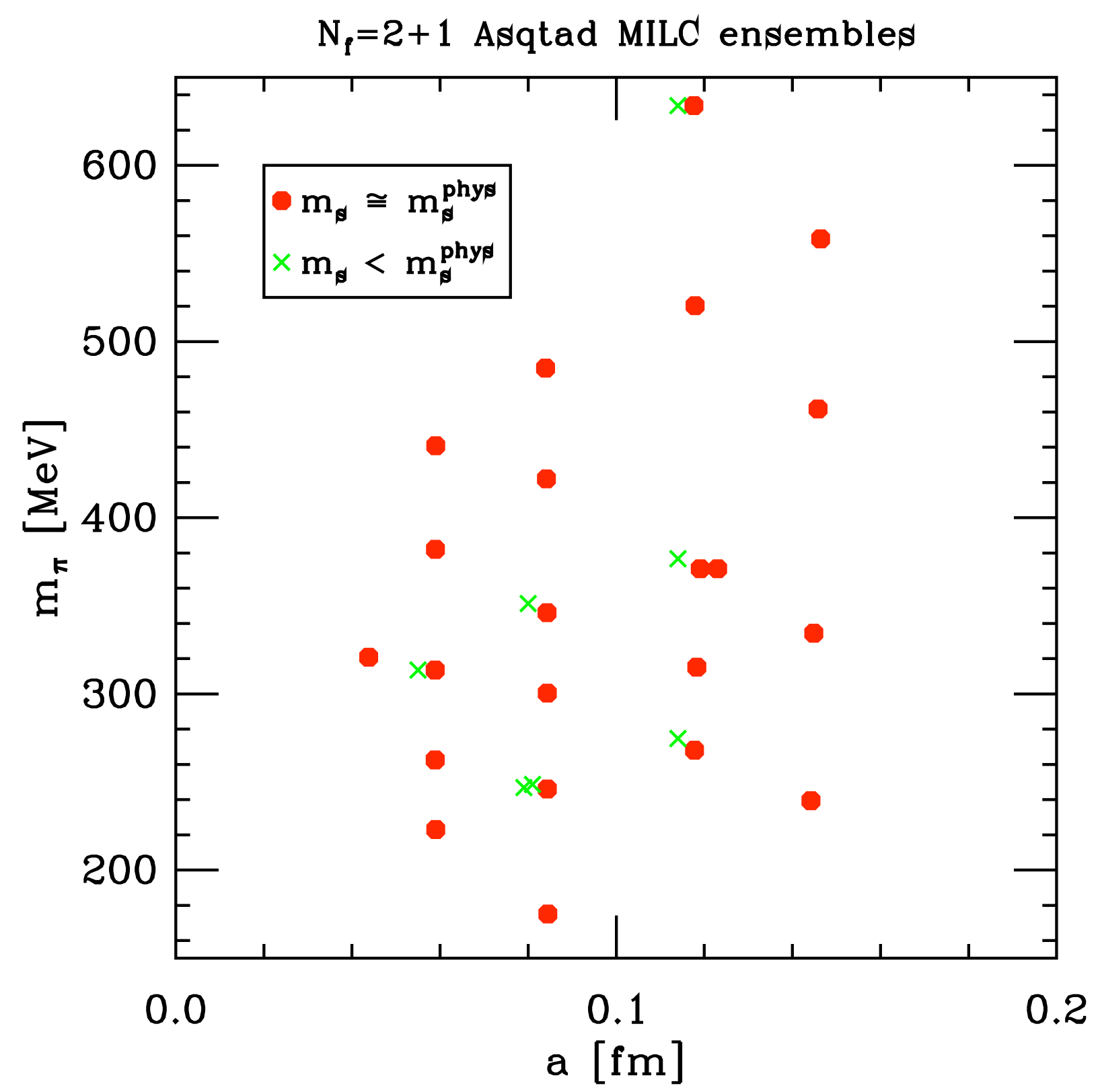




\section{Asqtad Ensembles}

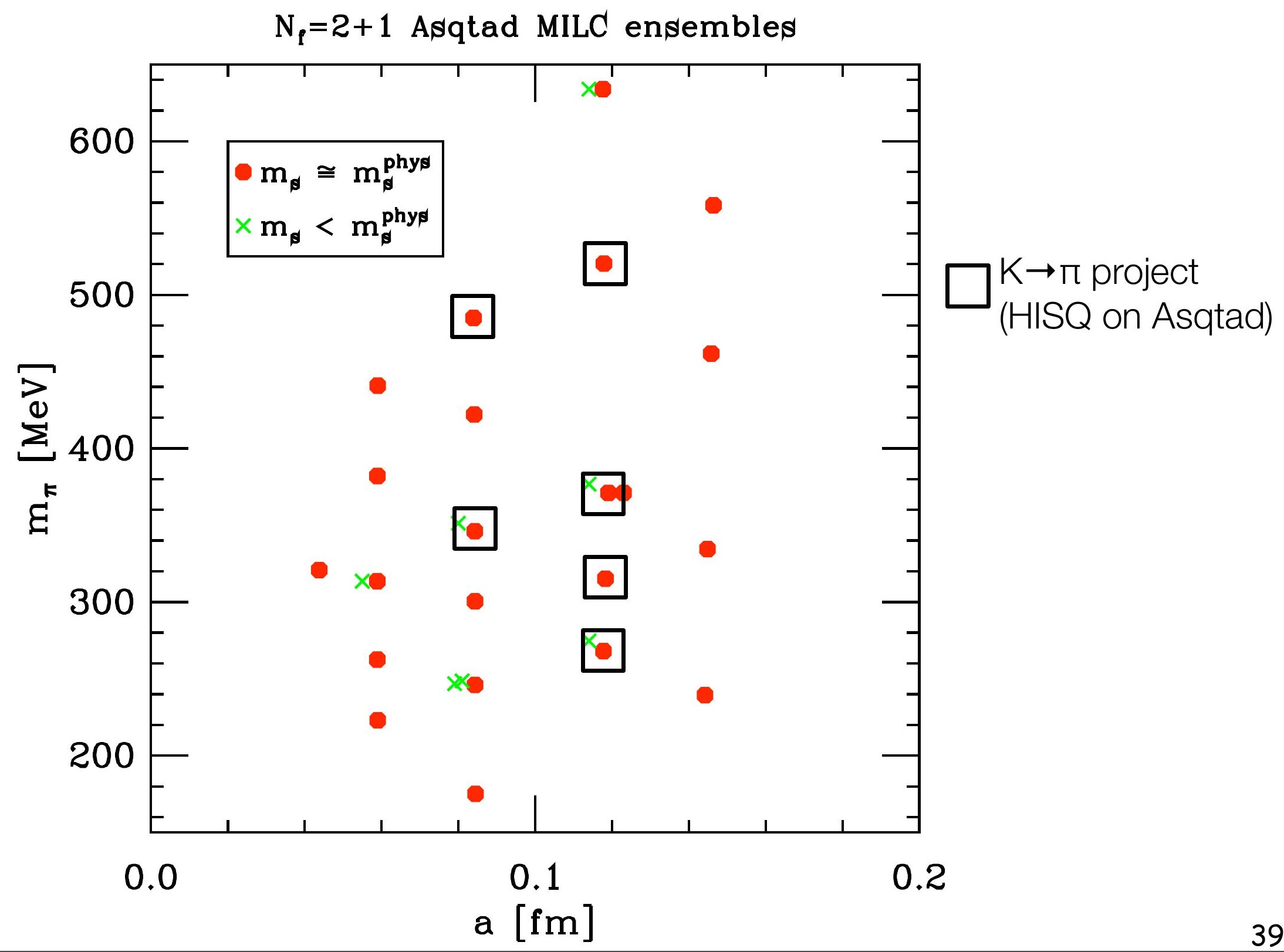




\section{$\mathrm{K} \rightarrow \pi$; HISQ on Asqtad}

- Strange HISQ valence mass tuned to its physical value [from Davies, et al, PRD 81 (2010) 034506, using the " $n_{s}$ "].

- Light HISQ valence mass tuned to Asqtad sea by:

$$
\frac{m_{l}^{\text {val }}(\text { Hisq })}{m_{s}^{\text {phys }}(\text { Hisq })}=\frac{m_{l}^{\text {sea }}(\text { Asqtad })}{m_{s}^{\text {phys }}(\text { Asqtad })}
$$

- So as close to "unitary" as possible for mı in this mixed-action theory.

- Mixed-action SChPT at 1-loop has been calculated [E. Gámiz and $\mathrm{CB}]$, but still needs checking. 


\section{$\mathrm{K} \rightarrow \pi$; HISQ on Asqtad}

$\downarrow$ Expected error budget:

- Statistical: 0.2--0.3\%

- Chiral extrapolation, fitting function: 0.1\%

- Discretization: 0.15\%

- Mistuning of $\mathrm{m}_{\mathrm{s}}$ in the sea: $0.2 \%$

^ Total: $0.35 \%--0.5 \%$, should be competitive with state of the art: RBC/UKQCD. 


\section{$\mathrm{K} \rightarrow \pi$ semileptonic decay}

$\downarrow$ Focus at $\mathrm{q}^{2}=0$, where we can use the method HPQCD proposed for semileptonic $D$ decay:

- Full matrix element of vector current $V_{\mu}$ is hard because conserved current is complicated and local current needs renormalization.

- Instead use $\partial^{\mu} V_{\mu}=\left(m_{b}-m_{a}\right) S$

- $S$ is local, and product $\left(m_{b}-m_{a}\right) S$ not renormalized.

- This is sufficient for $f_{+}\left(q^{2}=0\right)=f_{0}\left(q^{2}=0\right)$.

$\downarrow$ Two-part program:

- HISQ valence on 2+1 Asqtad ensembles (close to completion).

- HISQ valence on 2+1+1 HISQ ensembles (early stage).

- ultimately to include $\mathrm{D} \rightarrow \mathrm{K}$, and $\mathrm{q}^{2} \neq 0$ 


\section{HISQ Ensembles}

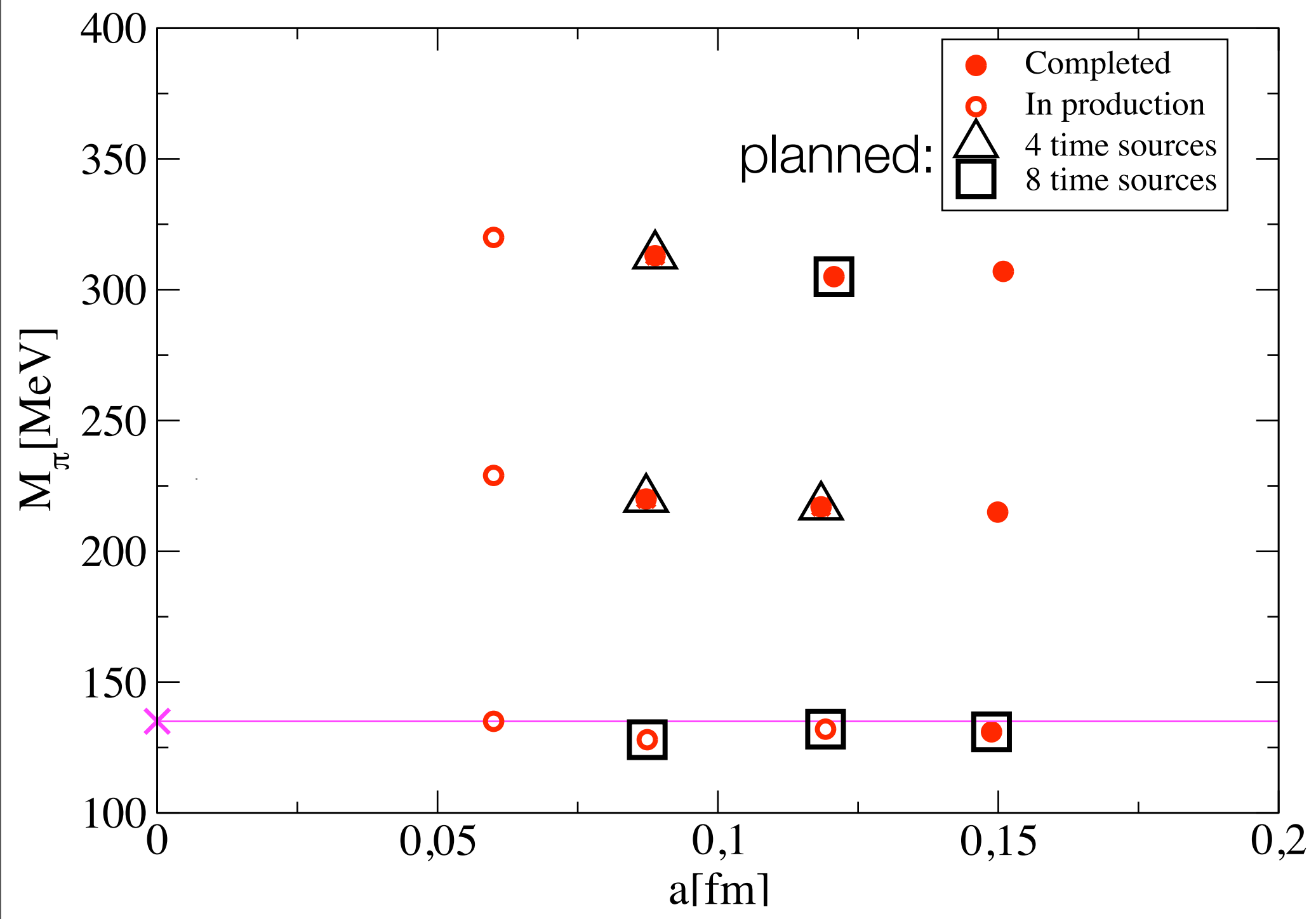




\section{HISQ Ensembles}

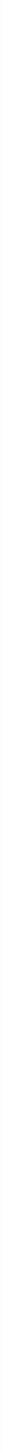




\section{$\mathrm{K} \rightarrow \pi$ : including HISQ on HISQ}

\section{Sample Chiral Fit}

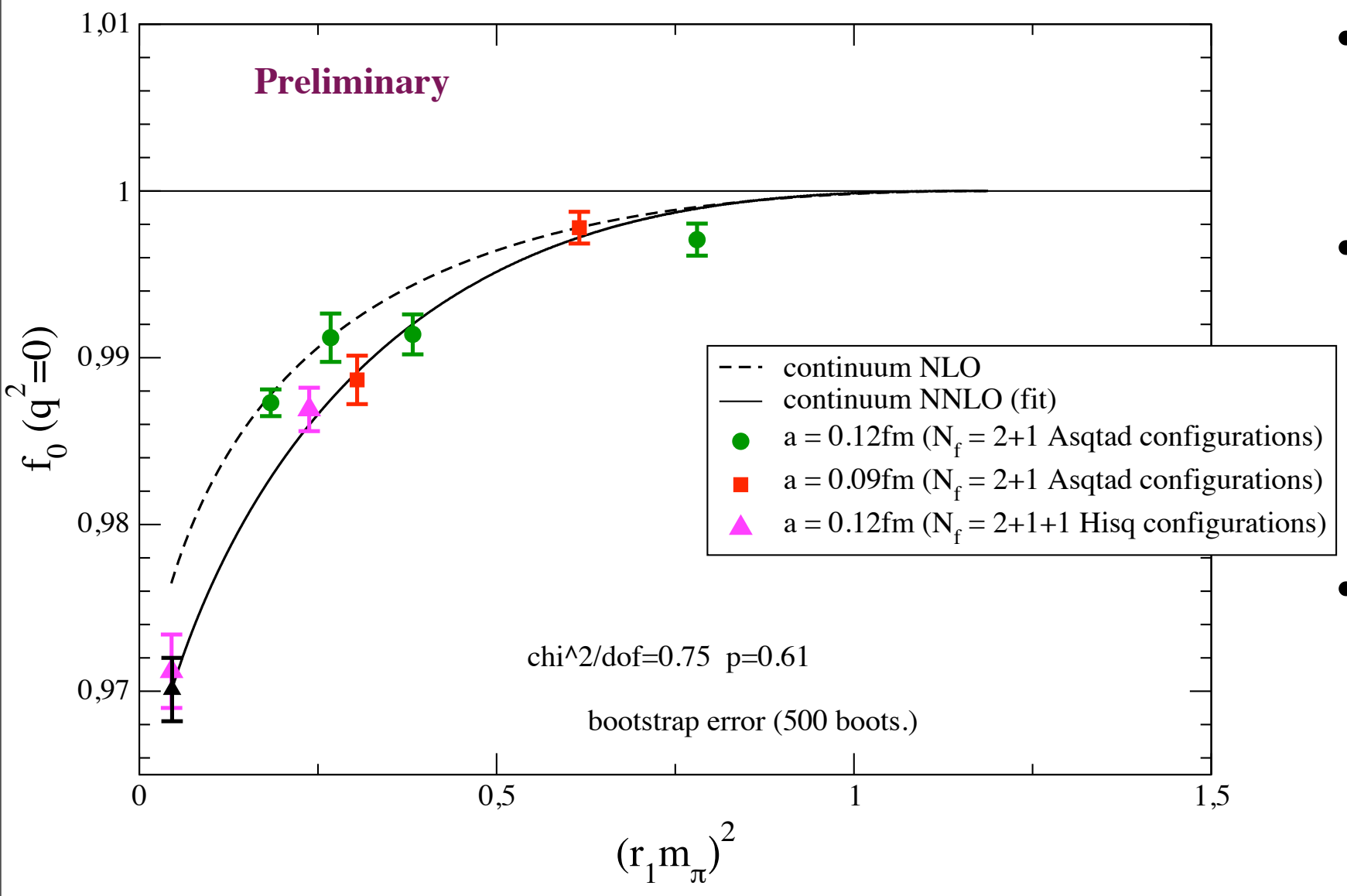

- Consistency with extrapolated HISQ on Asqtad results.

- Stat. errors larger on physical mass ensemble; momentum needed for $\mathrm{q}=0$ is larger.

- Ensembles with heavier-than-physical u,d mass important for reducing final error.

- $\mathrm{D} \rightarrow \mathrm{K}$ being done in parallel, but fits not analyzed yet... 


\section{Some projects I didn't talk about:}

Heavy-Quark Semileptonic Decays [Fermilab/MILC]

$\downarrow$ B $\rightarrow D^{\star}$ lv [arXiv:0808.2519, arXiv:1011.2166] (J. Laiho)

$\downarrow \mathrm{B} \rightarrow \mathrm{D} \ell \mathrm{v}$ [arXiv:1111.0677] (S. Qiu)

$\downarrow \mathrm{B} \rightarrow \mathrm{K} \ell \ell ; \mathrm{B} \rightarrow \mathrm{K}^{*} \mathrm{\gamma}$ [arXiv:1111.0677] [R. Zhou; see his talk]

$\downarrow B \rightarrow \pi \ell v$ [arXiv:0811.3640] (R. Van de Water)

$\downarrow B_{s} \rightarrow \mu^{+} \mu^{-}$[using $f$ for $\left(B_{s} \rightarrow D_{s}\right) /(B \rightarrow D)$; arXiv:1202.6346] (D. Du)

$\hookrightarrow \mathrm{D} \rightarrow \pi$ I v, D $\rightarrow$ K I v [arXiv:0811.3640] (J. Bailey)

Quarkonia [Fermilab/MILC]

$\downarrow$ [arXiv, 0912.2701, arXiv:1012.1837] (L. Levkova, C. DeTar, A. El-Khadra, E. Freeland,S. Gottlieb, A. Kronfeld,...) 


\section{Some projects I didn't talk about:}

Electromagnetic Effects [MILC]

- Pseudoscalar mesons [arXiv:0812.4486, arXiv: 1011.3994, PoS(Lat10) 127] (S. Basak, A. Torok, S. Gottlieb, L. Levkova, E.

Freeland, CB)

$\uparrow$ Baryons (S. Gottlieb \& students)

Strangeness content of the nucleon, etc. [MILC]

$\downarrow$ Nucleon strangeness [arXiv:0905.2432, arXiv:1011.5271]

(D. Toussaint, W. Freeman)

$\uparrow$ Nucleon charm [arXiv:1204.3866] (D. Toussaint, W. Freeman)

$\downarrow \sigma_{\Pi \mathrm{N}}$ (D. Toussaint, W. Freeman) 


\section{Fermilab Lattice/MILC Collaboration}

J. Bailey

A. Bazavov

C. Bernard

C. Bouchard

C. DeTar

A.X. El-Khadra

R.T. Evans

E.D. Freeland

W. Freeman

E. Gamiz

S. Gottlieb

J. Komijani

U.M. Heller

J.E. Hetrick

J. Kim

A.S. Kronfeld

J. Laiho

L. Levkova

M. Lightman

P.B. Mackenzie

E. Neil

M.B. Oktay

J. Simone

R. Sugar

D. Toussaint
Seule U.

U. of Arizona

Washington U.

Ohio State

U. of Utah

$U$. of Illinois

U. of Illinois, North Carolina State U.

$U$. of Illinois, Benedictine U.

George Washington U.

Fermilab, U. de Granada

Indiana U.

Washington U.

APS

$U$. of the Pacific

U. of Arizona

Fermilab

U. of Glasgow

U. of Utah

Washington U.

Fermilab

Fermilab

U. of Utah

Fermilab

U.C. Santa Barbara

U. of Arizona

R.S. Van de Water BNL 


\title{
Chiral Fermions via Energy Level Splitting
}

\author{
Artan Boriçi \\ University of Tirana
}

borici@fshn.edu.al 


\section{Outline of the talk}

- Overlap/Domain Wall and Minimally Doubled fermions

- Crank-Nicolson discretisation scheme

- Applications: I. Crank-Nicolson-Wilson operator

- Applications: II. Crank-Nicolson chiral operator 


\section{Chiral Fermions and the Lattice}

- Lorentz invariance broken to hypercubic symmetry:

- there is no such a thing like spin on the lattice;

- at least in the sense of $\mathrm{SU}(2)$ representations;

- Dirac field does not fit the lattice.

- $\Rightarrow$ Requiring emerging spin on the lattice brings undesirable results;

- Theorem (Nielsen-Ninomiya). There are no local chiral fermions on the lattice.

- Broken Lorentz invariance + emerging spin makes a "choose-only-oneitem" menu:

- Many chirality pairs of flavours (naive, minimally doubled,...);

- One flavour of explicitely broken chirality (Wilson,... ). 


\section{Overlap/Domain Wall Fermions}

Adding a new item in the menu:

\section{On shell chirality but an extra dimension.}

- Satisfy Ginsparg-Wilson relation: on shell chiral symmetry;

- Expensive to compute: involves nested Krylov subspaces;

- Large density of near zero modes of the kernel;

- Topology stalling simulation algorithms; Solution:

- Fixed topology overlap: unitarity violations

- Fat links: lattice spacing of smoothed configuration? 


\section{Minimally Doubled Fermions}

- Reduce the number of doubler to two: 25 years old idea revived 5 years ago with new actions;

- That is nice but in expense of hypercubic symmetry:

- Symmetry restoration requires fine tuning i.e. non-perturbative renormalization;

- Simulation of one flavour needs rooting.

Lesson: lose symmetry in favour of less doublers.

Question: How far can one push it?

One can isolate a single flavour compensated by ghost particles, i.e. no Dirac/Weyl content:

Weyl and ghost fermions on the lattice, A.B. ArXiv:1010.5156

Nielsen-Ninomiya theorem can be genralized, but the result is essentially the same: the ghosts remain in the continuum limit.

Wilson strategy: distribute doublers in the real axis, revived recently by Creutz and collaborators.

A. Boriçi New Horizons for Lattice Computations with Chiral Fermions BNL, 14-16 May $2012 \quad 0-4$ 


\section{Crank-Nicolson discretization}

- Example: Schrödinger equation in Euclidean space:

$$
\partial_{t} \psi(t, x)=H \psi(t, x), \quad \psi(0, x)=\psi_{o}(x) .
$$

- Solution $\psi(t, x)=e^{t H} \psi(0, x)$.

- Numerical problem: exp approximation.

- Problem: non-local grid in $t$;

- Requirement: stay with nearest neighbours, i.e. Euler scheme: $\Rightarrow$ order $O(a)$ errors.

- Crank-Nicholson scheme:

$$
\begin{aligned}
& \partial \psi(t, x) \rightarrow \frac{1}{a}[\psi(t+a, x)-\psi(t, x)], \quad H \psi(t, x) \rightarrow \frac{H}{2}[\psi(t, x)+\psi(t+a, x)] \\
& \quad \Rightarrow \psi(t+a, x)=\frac{1+\frac{a}{2} H}{11-\frac{a}{2} H} \psi(t, x)=\left(\mathbb{1}+a H+\frac{a^{2}}{2} H^{2}+O\left(a^{3}\right)\right) \psi(t, x) \\
& \quad \Rightarrow \text { order } O\left(a^{2}\right) \text { errors in expense of solving linear systems. }
\end{aligned}
$$




\section{Crank-Nicolson and Dirac operator}

- Momentum space spin-1/2 Hamiltonian on the lattice: $H=\vec{\sigma} \sin \vec{p}$;

- $\Rightarrow$ Crank-Nicolson time discretised operator:

$$
D(p)=e^{i p_{4}}-1+\frac{1}{2} \vec{\sigma} \sin \vec{p}\left(e^{i p_{4}}+1\right) .
$$

- Particle content:

$$
D(p)=0 \Leftrightarrow 4 \sin ^{2} \frac{p_{4}}{2}+\sin ^{2} \vec{p} \cos ^{2} \frac{p_{4}}{2}=0 .
$$

- $\Rightarrow 8$ particles located at the edges of the $3 \mathrm{~d}$ Brillouin zone.

- Result:

The number of doublers is reduced by a factor of two! 


\section{Crank-Nicolson-Wilson operator}

- Reduce the number of doublers using the Wilson approach:

$$
D_{C N W}(p)=\gamma_{4}\left(e^{i p_{4}}-1\right)+\frac{1}{2}\left[i \vec{\gamma} \sin \vec{p}+\sum_{k}\left(1-\cos p_{k}\right)\right]\left(e^{i p_{4}}+1\right) \text {. }
$$

- $D_{C N W}(p)=0 \Leftrightarrow$

$$
4 \sin ^{2} \frac{p_{4}}{2}+\left\{\sin ^{2} \vec{p}+\left[\sum_{k}\left(1-\cos p_{k}\right)\right]^{2}\right\} \cos ^{2} \frac{p_{4}}{2}=0 .
$$

- Result:

- Second order accurate in time;

- Smaller additive mass renormalisation;

- Hypercubic symmetry broken to cubic symmetry. 


\section{Degenerate doublet of Crank-Nicolson-Wilson fermions}

Restore hybercubic symmetry defining a doublet of Crank-Nicolson-Wilson fermions:

$$
\mathcal{D}_{C N W}=\left(\begin{array}{cc}
D_{C N W}^{(+)}(m) & 0 \\
0 & D_{C N W}^{(-)}(m)
\end{array}\right)
$$

where:

$$
\begin{aligned}
& D_{C N W}^{(+)}(m)=m \mathbb{1}+\gamma_{4}\left(e^{i p_{4}}-1\right)+\frac{1}{2}\left[i \vec{\gamma} \sin \vec{p}+\sum_{k}\left(1-\cos p_{k}\right)\right]\left(e^{i p_{4}}+1\right) \\
& D_{C N W}^{(-)}(m)=m \mathbb{1}+\gamma_{4}\left(1-e^{-i p_{4}}\right)+\frac{1}{2}\left[i \vec{\gamma} \sin \vec{p}+\sum_{k}\left(1-\cos p_{k}\right)\right]\left(e^{-i p_{4}}+1\right)
\end{aligned}
$$

- Hypercubic symmetry restored under flavour exchanging operation;

- Result: $O\left(a_{t}^{2}\right)$ errors theory without fine tuning +3 -space Wilson. 


\section{A chiral theory with doublers}

- Start with a general theory of many chirality pairs flavours:

$$
E_{n}(\vec{p})=\alpha_{n} \vec{\sigma} \vec{p}, \quad n=1,2, \ldots, m,
$$

- For example naive fermions have this continuum limit with $\alpha_{n}= \pm 1$;

- We are seeking a theory with a singlet chirality ground state:

- the opposite chirality counterpart should occupy a different level;

- the theory has a energy gap $\Delta(\vec{p})=E_{1}(\vec{p})-E_{0}(\vec{p})$.

- Energy gap increases with momenta:

- A non-uniform gap;

- Maximal gap at cuttoff.

- $\Rightarrow$ A chiral theory of free fermions with doublers. 


\section{Lattice implementation}

- Start from Crank-Nicolson discretisation in time and naive discretisation in 3-space:

$$
D(p)^{\prime}=e^{i p_{4}}-1+\frac{1}{2} \vec{\sigma} \sin \vec{p}\left(e^{i p_{4}}+1\right) ;
$$

- Add a pure imaginary operator of the Wilson type:

$$
D(p)=e^{i p_{4}}-1+\frac{1}{2}\left[\vec{\sigma} \sin \vec{p}+i r \sum_{k}\left(1-\cos p_{k}\right)\right]\left(e^{i p_{4}}+1\right) .
$$

- $D(p)=0 \Leftrightarrow$

$$
\left\{2 \sin \frac{p_{4}}{2}+\left[r \sum_{k}\left(1-\cos p_{k}\right)\right] \cos \frac{p_{4}}{2}\right\}^{2}+\sin ^{2} \vec{p} \cos ^{2} \frac{p_{4}}{2}=0 .
$$




\section{Particle spectra}

- 8 zeros in the edges of 3d Brillouin zone:

$$
\vec{p}^{*} \in\{(0,0,0),(0,0, \pi),(0, \pi, 0),(\pi, 0,0),(0, \pi, \pi),(\pi, 0, \pi),(\pi, \pi, 0),(\pi, \pi, \pi)\}
$$

- Define $n\left(\vec{p}^{*}\right)=\frac{1}{2} \sum_{k}\left(1-\cos p_{k}^{*}\right)$

- $\Rightarrow D(p)=0 \Leftrightarrow \tan \frac{p_{4}^{*}}{2}=-r n\left(\vec{p}^{*}\right)$.

- Define chirality $\chi\left(\vec{p}^{*}\right)=\cos p_{1}^{*} \cos p_{2}^{*} \cos p_{3}^{*} \Rightarrow$

\begin{tabular}{l|l|l|l}
$\vec{p}^{*}$ & $n\left(\vec{p}^{*}\right)$ & $\chi\left(\vec{p}^{*}\right)$ & Degeneracy \\
\hline$(0,0,0)$ & 0 & 1 & 1 \\
$(0,0, \pi),(0, \pi, 0),(\pi, 0,0)$ & 1 & -1 & 3 \\
$(0, \pi, \pi),(\pi, 0, \pi),(\pi, \pi, 0)$ & 2 & 1 & 3 \\
$(\pi, \pi, \pi)$ & 3 & -1 & 1
\end{tabular}

- Continuum limit dispersion relation: $E_{n}(\vec{p}) \rightarrow \frac{\vec{\sigma} \vec{p}}{1+r^{2} n^{2}}, \quad n=0,1,2,3$. 


\section{Broken hypercubic symmetry}

- Restore hypercubic symmetry defining a quartet of such fermions:

$$
\mathcal{D}=\left(\begin{array}{cccc}
0 & 0 & 0 & D_{r}^{(+)} \\
0 & 0 & D_{-r}^{(+)} & 0 \\
0 & D_{r}^{(-)} & 0 & 0 \\
D_{-r}^{(-)} & 0 & 0 & 0
\end{array}\right)
$$

- $\Rightarrow$ symmetry is restored under $(+)$ and $(-)$ flavour exchange as well as under $r$ and $-r$ flovour exchange.

- Result: a free theory of four flavours with definity chirality.

- Interacting theory: close to continumm theory should be Ok;

- Strong coupling: doubler mixing. 


\section{Conclusions}

- Crank-Nicolson discretisation scheme offers new definitions of fermions on the lattice:

I. Crank-Nicolson-Wilson operator is a one flavour theory with broken chiral symmetry and second order discretisation errors in $a_{t}$.

II. A free fermion chiral theory via doubler level splitting. 


\section{Prospects of Multi-grid and}

Domain Decomposition Dirac Solvers on GPU

Richard C. Brower ( with SciDAC collaborators

Ron Babich, Mike Clark, James Osborn et al!)

New Horizons for Lattice Computations with Chiral Fermions BNL May 15, 2012 


\section{Algorithms are increasingly Essential and increasingly Complex.}

- Causes:

- Higher resolution physics.

- Hierarchical Petascale hardware.

- Long multi-year cycle:

1. Physical Insight

2. Mathematics of Complexity (flops \& comms)

3. Coding prototype on New Hardware

4. Testing and Integration into Applications 


\section{Outline: \\ A few future (spectacular?) examples.}

I. Moebius DW Fermions (retrospective!)

II. Multigrid Wilson-clover Dirac Solver (at last!)

III. GPUs with Domain Decomposition

IV. The Future: MG/GPU/DD for HMC

- (MG for Domain Wall: next talk by Saul Cohen) 


\section{Moebius as Scaled Shamir DW}




\section{$\epsilon_{L_{s}}[\lambda]$ for $1 / L_{s}<|\lambda|<L_{s}$}

Good to $O\left(L_{s}\right)$

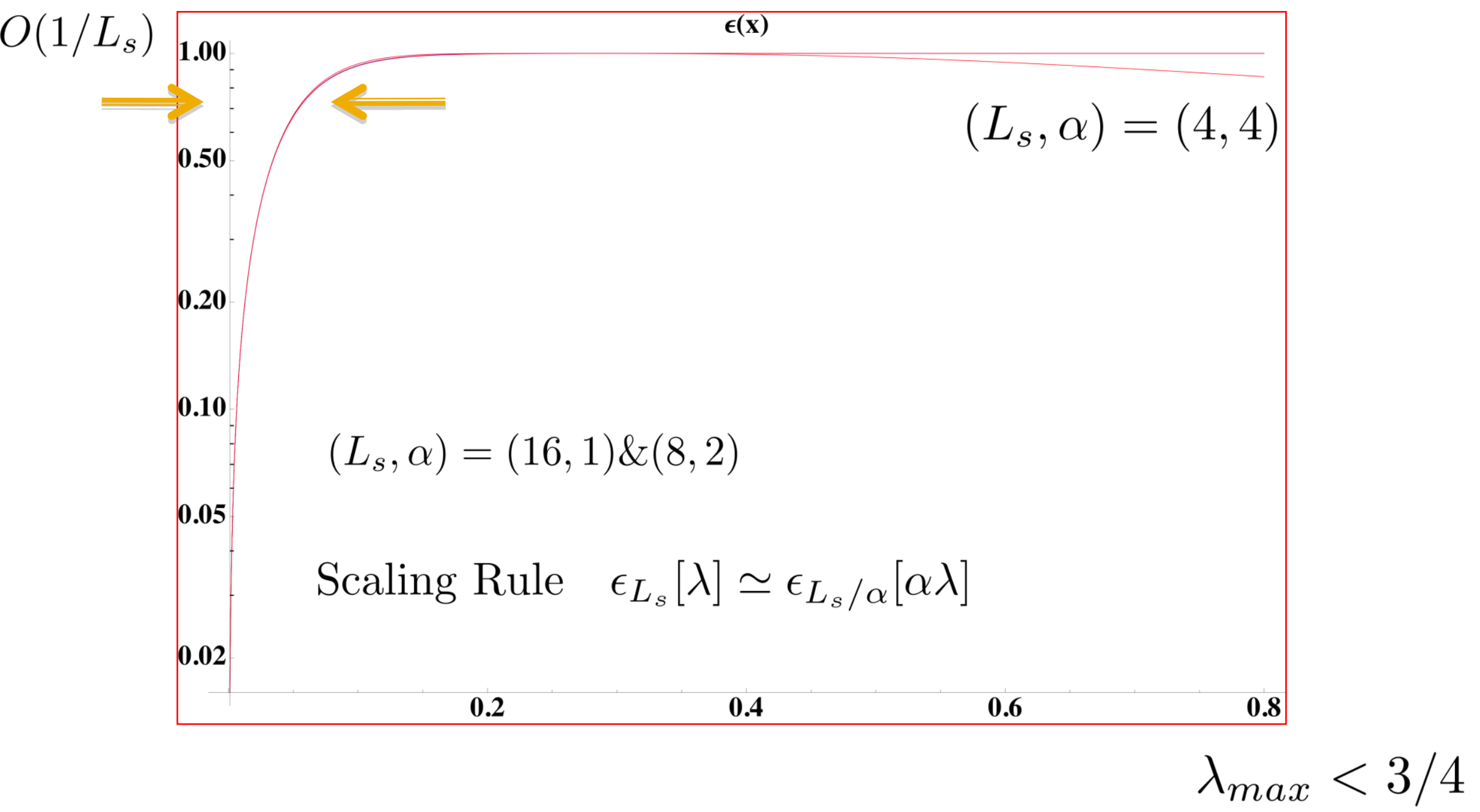




\section{Estimate of Mres}

$$
\begin{aligned}
& \bar{m}_{r e s}=\frac{\operatorname{Tr}\left[D_{o v}^{\dagger-1} \Delta_{L_{s}}\left(H_{5}\right) D_{o v}^{-1}\right]}{\operatorname{Tr}\left[D_{o v}^{-1} D_{o v}^{\dagger-1}\right]}=\sum_{\lambda} w_{\pi}(\lambda) \Delta_{L_{s}}(\lambda) \\
& \Delta_{L_{s}}(\alpha \lambda)=\frac{1}{4}\left(1-\epsilon_{L}^{2}[\alpha \lambda]\right) \\
& \rightarrow e^{-L_{s}|\log (1+\alpha \lambda)-\log (1-\alpha \lambda)|} \simeq e^{-2 L_{s} \alpha \lambda}
\end{aligned}
$$

Error from $|\lambda|<1 / 2 \alpha L s \sim 1 / L_{s}^{2}$

Small density of "topological defects"

Thus Moebius for $L s=32 \rightarrow$ Shamir at $L s=1000 ! ! ! ! ! !$ 


\section{Multigrid for Wilson clover Solver}




\section{Multigrid Premier:}

\section{Classic example - PDE with NO}

No change when $\quad x \rightarrow \lambda x, y \rightarrow \lambda y, z \rightarrow \lambda z$

- Laplace: $\phi \rightarrow \lambda^{-1} \phi$

$$
-\nabla^{2} \phi(x, y, z)=-\left[\frac{\partial^{2}}{\partial x^{2}}+\frac{\partial^{2}}{\partial y^{2}}+\frac{\partial^{2}}{\partial z^{2}}\right] \phi=e^{2} \delta(x) \delta(y) \delta(z)
$$

- Solution (Green's function)

$$
\phi=\frac{1}{4 \pi} \frac{e^{2}}{\sqrt{x^{2}+y^{2}+z^{2}}}=\frac{e^{2}}{4 \pi r}
$$




\section{Precondition operator on $\mathrm{a}=\mathrm{h}$}

\section{by "renormalized" operator on $\mathrm{a}=2 \mathrm{~h}$}

$$
A \phi=b \Longrightarrow \phi(x+h)-2 \phi(x)+\phi(x-h)+h^{2} m^{2} \phi(x)=b(x)
$$

$\mathrm{h} \rightarrow 2 \mathrm{~h}$ Restriction $\mathrm{R}=\mathrm{P}^{\dagger}$

$2 \mathrm{~h} \rightarrow \mathrm{h}$ Prologation $\mathrm{P}$
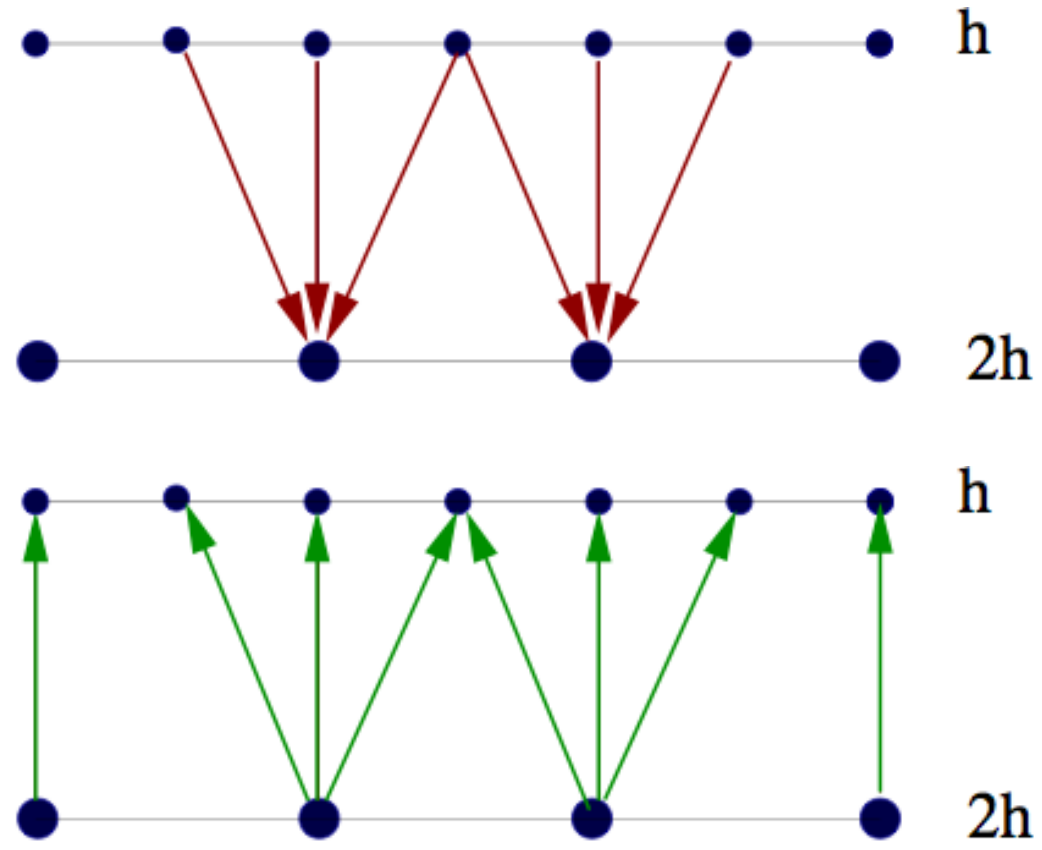

(1) Restriction must preserves the scale invariant solutions

(2) Coarse operator is renormalized: $m \rightarrow 2 m$ (in units $h=1$ ) 


\section{Multi-grid V-Cycle}

Restrict until exact solve possible

Interpolate back to fine grid Relax

$O(N)$ to $O(N \log N)$ scaling

Memory $\mathrm{O}(\mathrm{N})$

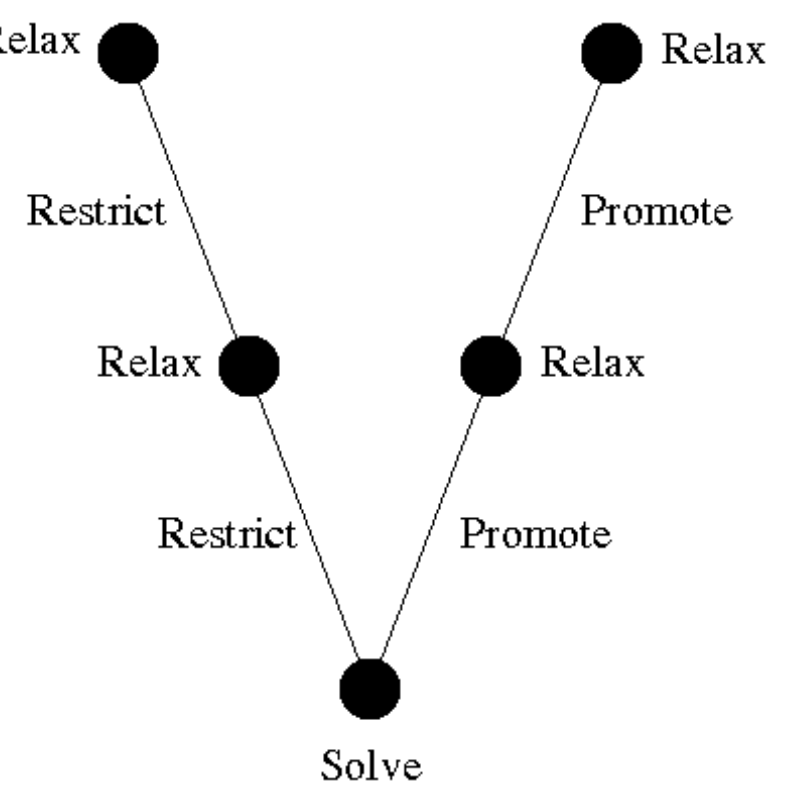

Extra Work: $1+w+w^{*} w+. .<1 /(1-w)$ 


\section{Ren Group motivated attempts in 1990's:}

See Thomas Kalkretuer hep-lat/9409008 review on "MG Methods for Propagators in LGT".

Israel: Ben-Av, M. Harmatz, P.G. Lauwers \& S.Solomon

Boston: Brower, Edwards, Rebbi \& Vicari

Amsterdam: A. Hulsebos, J Smit J. C. Vick

Hamburg: T. Kalkreuter, G. Mack \& M. Speh

\begin{tabular}{|c|c|c|c|}
\hline group & operator to be inverted & gauge field & lattice sizes \\
\hline $\begin{array}{c}\text { "Israel } \\
{[3,13, \text { and references therein }]} \\
\text { 1989-ongoing }\end{array}$ & $\begin{array}{c}\not \not D+m \\
\text { staggered fermions }\end{array}$ & $\begin{array}{l}\text { 2-d } U(1) \\
\text { 2-d } S U(2) \\
\text { 2-d } S U(3)\end{array}$ & $\begin{array}{l}\leq 256^{2} \\
\leq 256^{2} \\
\leq 128^{2}\end{array}$ \\
\hline $\begin{array}{c}\text { "Amsterdam" } \\
{[14, \text { and references therein }]} \\
1990-1992\end{array}$ & $\begin{array}{l}\qquad-\not D^{2}+m^{2} \\
\text { staggered fermions } \\
\text { staggered fermions } \\
\text { and Wilson fermions }\end{array}$ & 2-d $S U(2)$ & $\begin{array}{l}\leq 128^{2} \\
\leq 128^{2}\end{array}$ \\
\hline $\begin{array}{c}\text { "Boston" } \\
{[7, \text { and references therein }]} \\
1990-1991\end{array}$ & $\begin{array}{l}\left(\gamma_{\mu}+1\right) D_{\mu}+m \\
\text { Wilson fermions } \\
\left(\gamma_{\mu}+1\right) D_{\mu}+m \\
\text { Wilson fermions }\end{array}$ & $\begin{array}{l}2-d U(1) \\
4-d U(1) \\
2-d S U(2) \\
2-d U(1)\end{array}$ & $\begin{array}{l}\leq 64^{2} \\
\leq 16^{4} \\
\leq 32^{2} \\
64^{2}\end{array}$ \\
\hline $\begin{array}{c}\text { "Hamburg" } \\
{[21,18,22,23,1,17,19,20,2,24]} \\
1990 \text {-ongoing }\end{array}$ & $\begin{array}{c}-\not D^{2}+m^{2} \\
\text { staggered fermions }\end{array}$ & $\begin{array}{l}\text { 2-d } S U(2) \\
\text { 4-d } S U(2) \\
\text { 2-d } S U(2) \\
\text { 4-d } S U(2)\end{array}$ & $\begin{array}{l}\leq 128^{2} \\
\leq 18^{4} \\
\leq 162^{2} \\
\leq 18^{4}\end{array}$ \\
\hline
\end{tabular}

Table 1: Overview of works on MG methods for propagators in lattice gauge theories. 


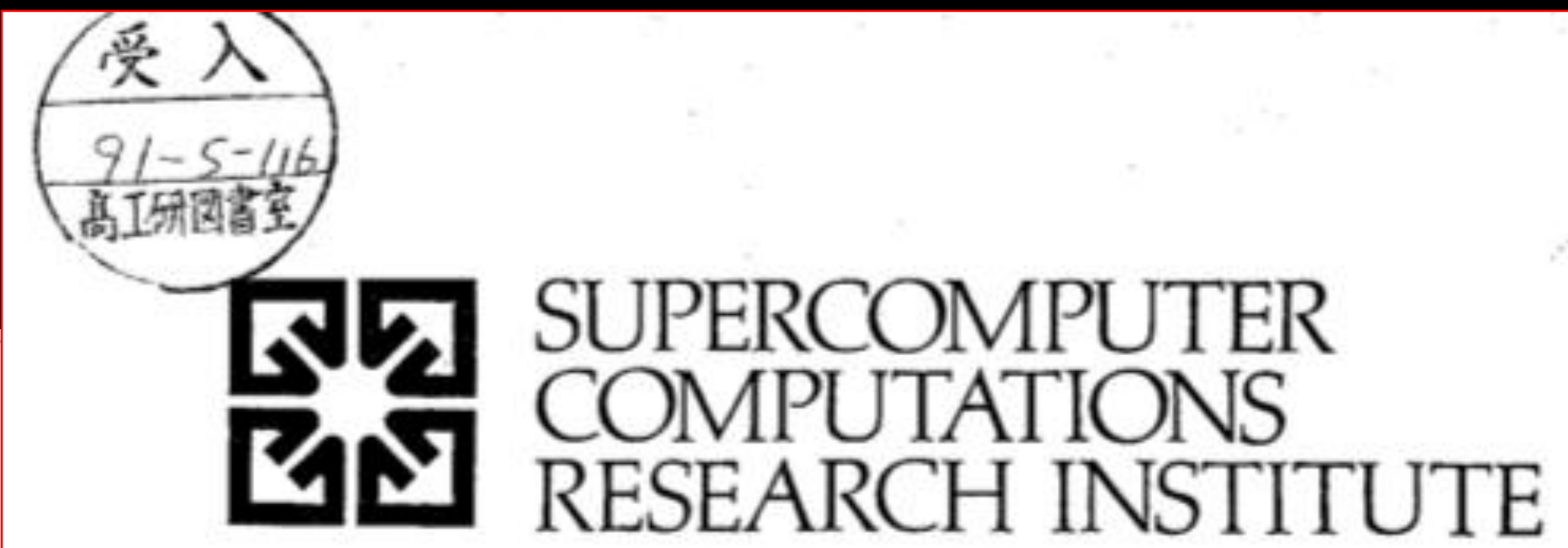

\author{
PROJECTIVE MULTIGRID FOR \\ WILSON FERMIONS
}

by

Richard C. Brower, Robert G. Edwards,

Claudio Rebbi, and Ettore Vicari 


\section{2×2 Blocks for 2-d U(1) Dirac (Naive scaling of free problem)}

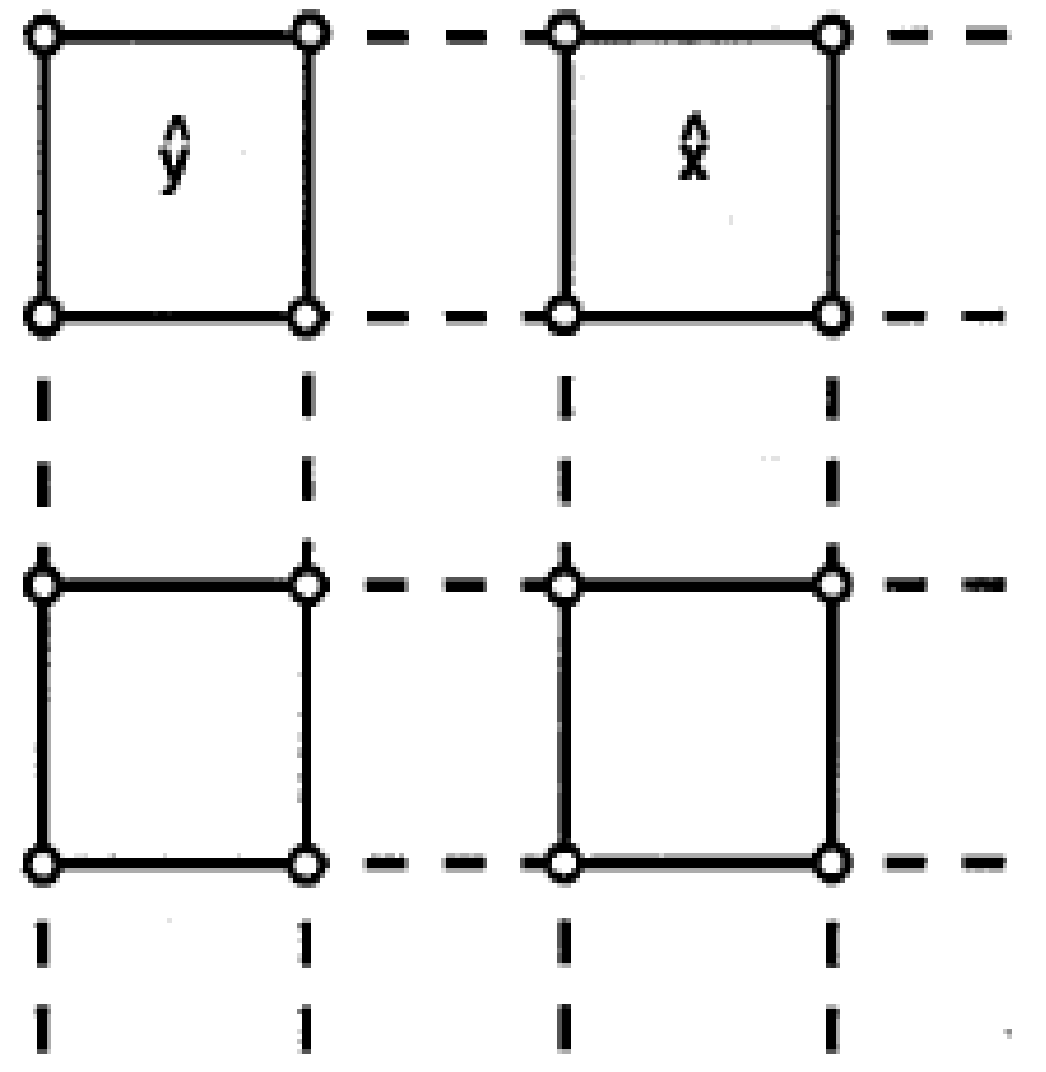

2-d Lattice, $U_{\mu}(x)$ on links $\Psi(x)$ on sites

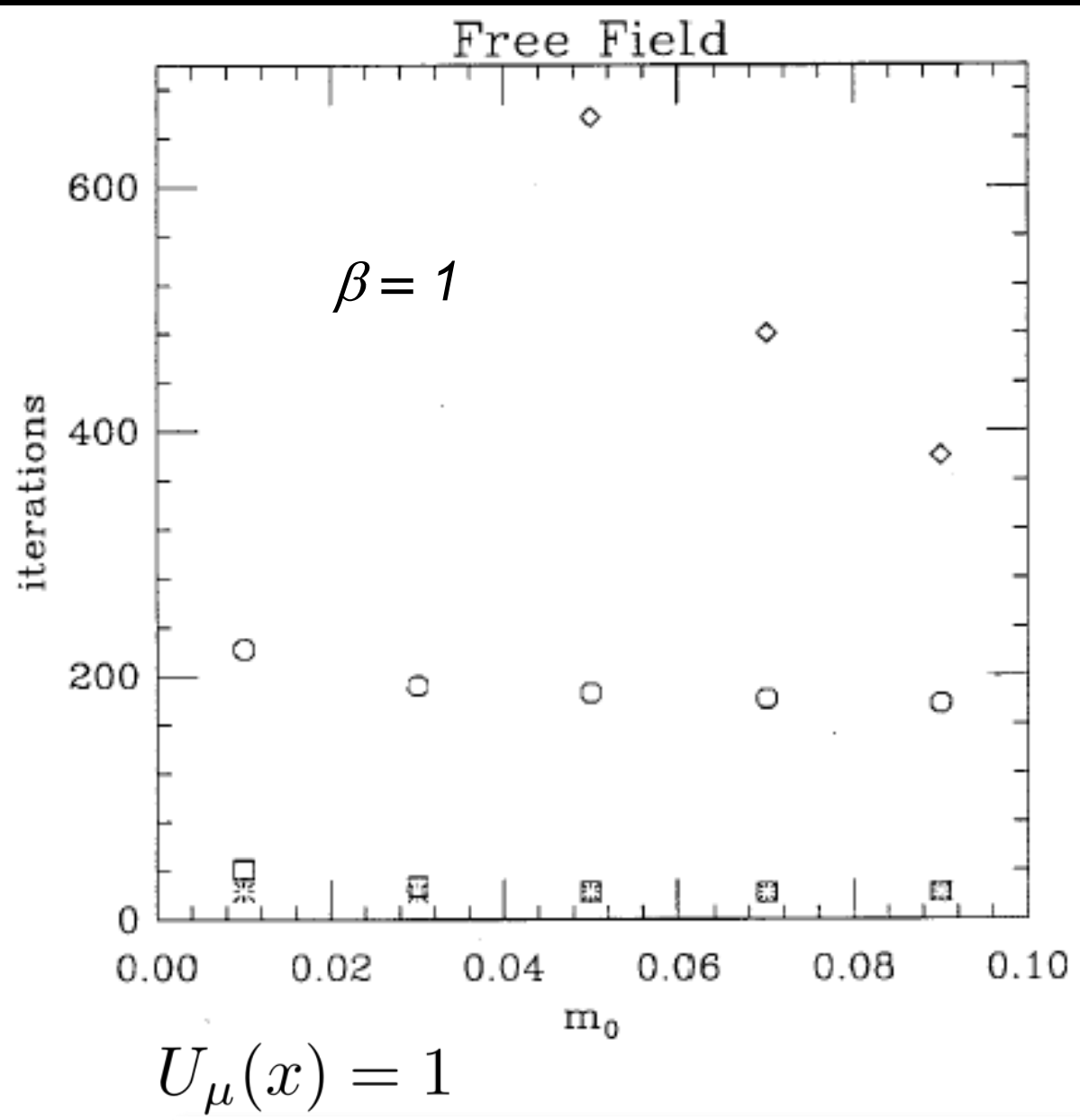

Gauss-Jacobi (Diamond), CG (circle), $\checkmark$ cycle (square), $W$ cycle (star) 


\section{Universal critical slowing: $\tau=F\left(\mathrm{~m} \mathrm{I}_{\sigma}\right)$}
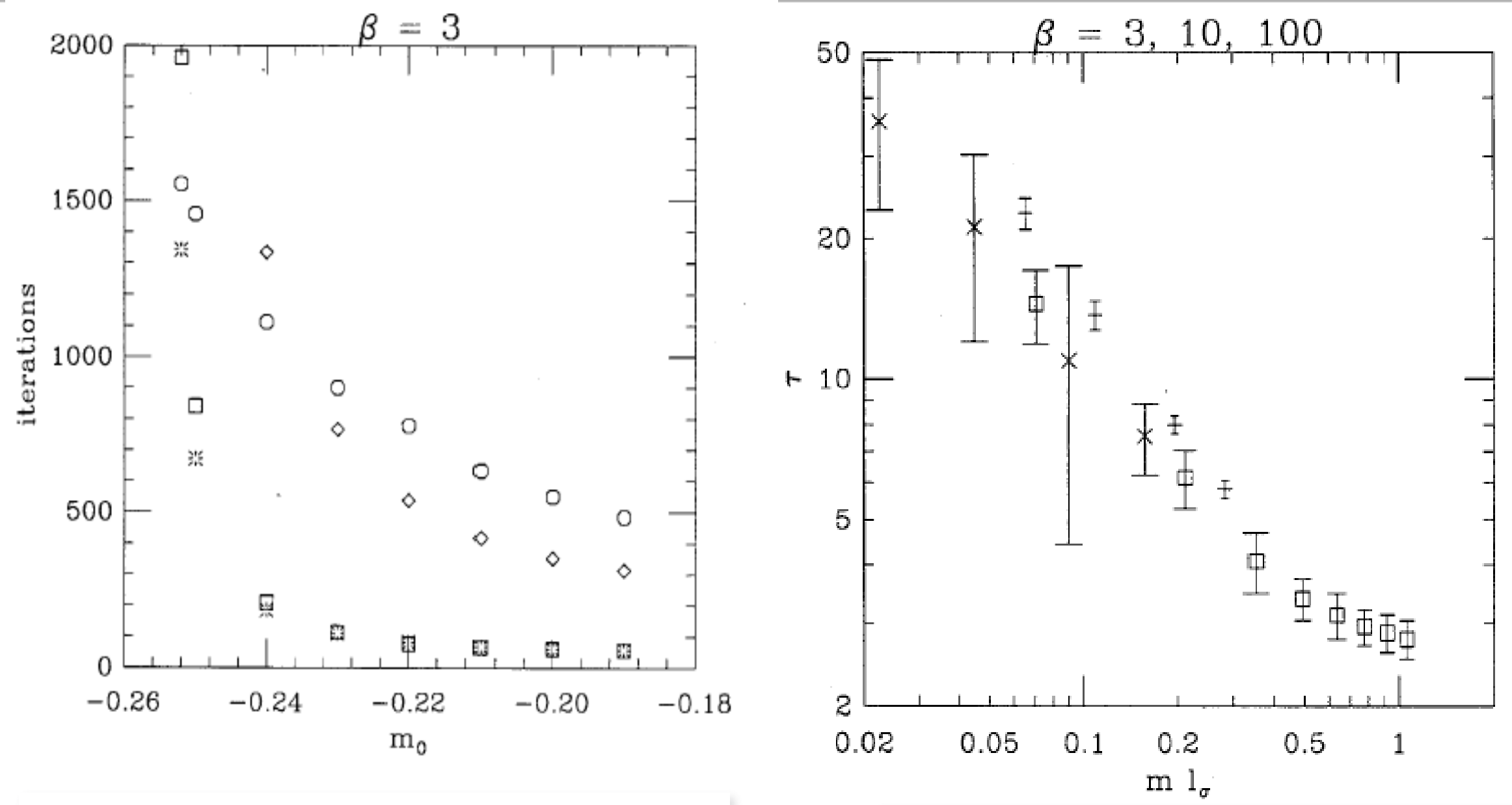

Gauss-Jacobi (Diamond), CG(circle), 3 level (square \& star)

$$
\beta=3 \text { (cross) 10(plus) 100( square) }
$$




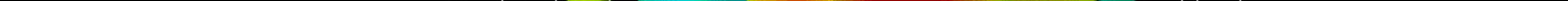




\section{QCD Multigrid Applied Math/Physics Collaboration}

\section{Many different people (TOPS, QCD) and institutions involved in the collaboration}

- CU Boulder

- Tom Manteuffel

- Steve McCormick

- Marian Brezina

- John Ruge

- James Brannick

- Christian Ketelsen

- Scott MacLachlan

- Lawrence Livermore

- Rob Falgout

- Columbia

- David Keyes
- Boston University

- Rich Brower

- Claudio Rebbi

- Mike Clark

- James Osborn

- Ron Babich

- Saul Cohen

Penn State

- James Brannick

- Ludmil Zikatanov

Tufts

- Scott MacLachlan

- Argonne

- James Osborn
Harvard U

Mike Clark

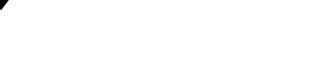

- INT Seattle

- Saul Cohen 


\section{Adaptive Smooth Aggregation Algebraic Multigrid}
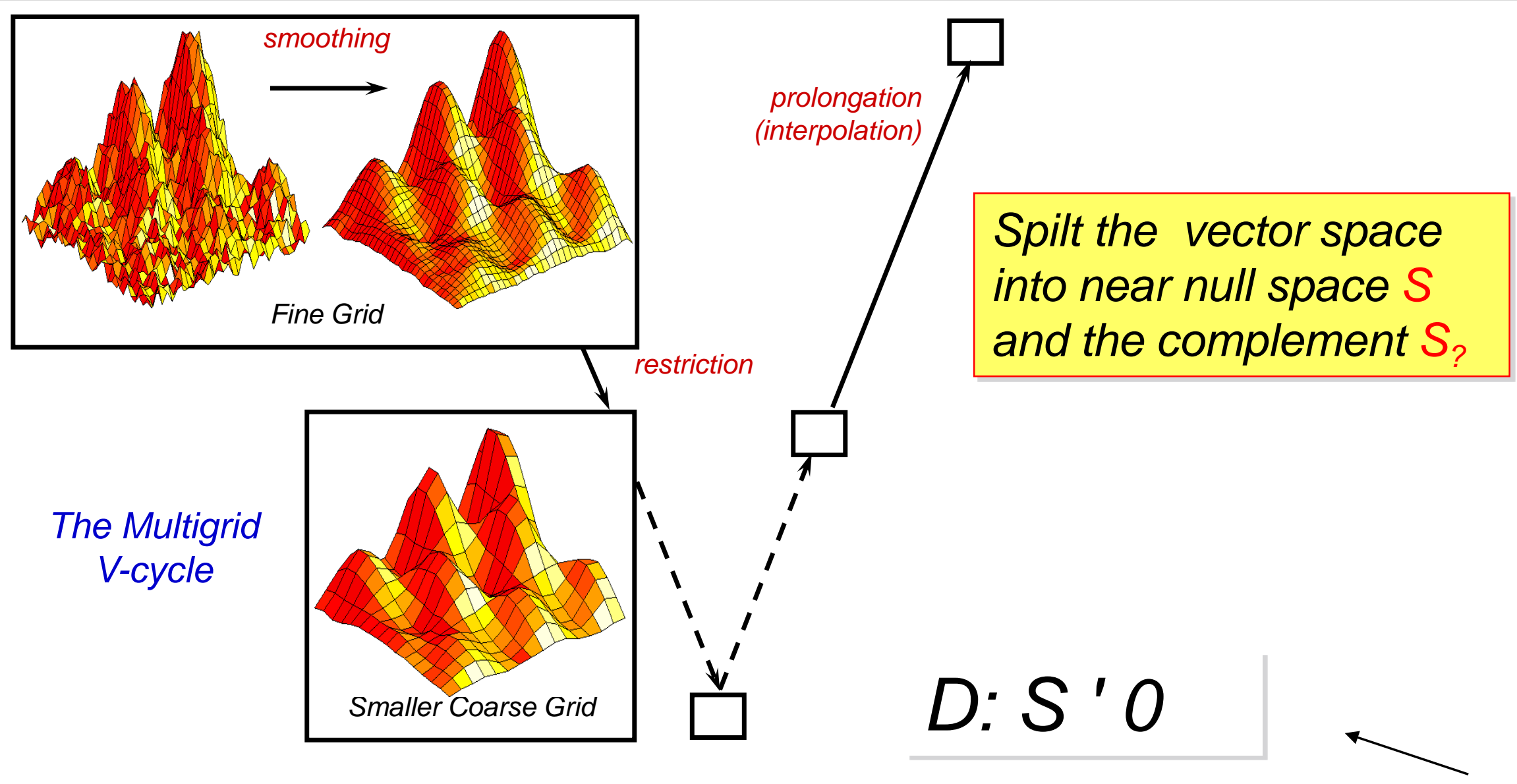

Slow convergence of Dirac solver is due to small eigenvalues for vectors in near null subspace: $S$. 


\section{Physical Requirements}

- Partial success (RG) weak coupling

- Maintain Gauge invariance

- Maintain ${ }_{5}^{\circ}$ Hermiticity

- Local adaptive blocking: Projective MG

- Chiral Symmetry (density of small e.v.)

- Null vector: Atya- Singer Index Theorem

$$
H=\gamma_{5} D=D^{\dagger} \gamma_{5}
$$

Prolongator $P \Longrightarrow$ Restrictor $R=P^{\dagger} \gamma_{5}$ 


\section{Py:fine ! coarse ( non-square matrix ${ }^{y}$ )}

(fine lattice vector space)

(coarse lattice vector space)

$\operatorname{ker}(P y)$

UV

But $P y P=1_{c c}$ so $\operatorname{Ker}(P)=0$
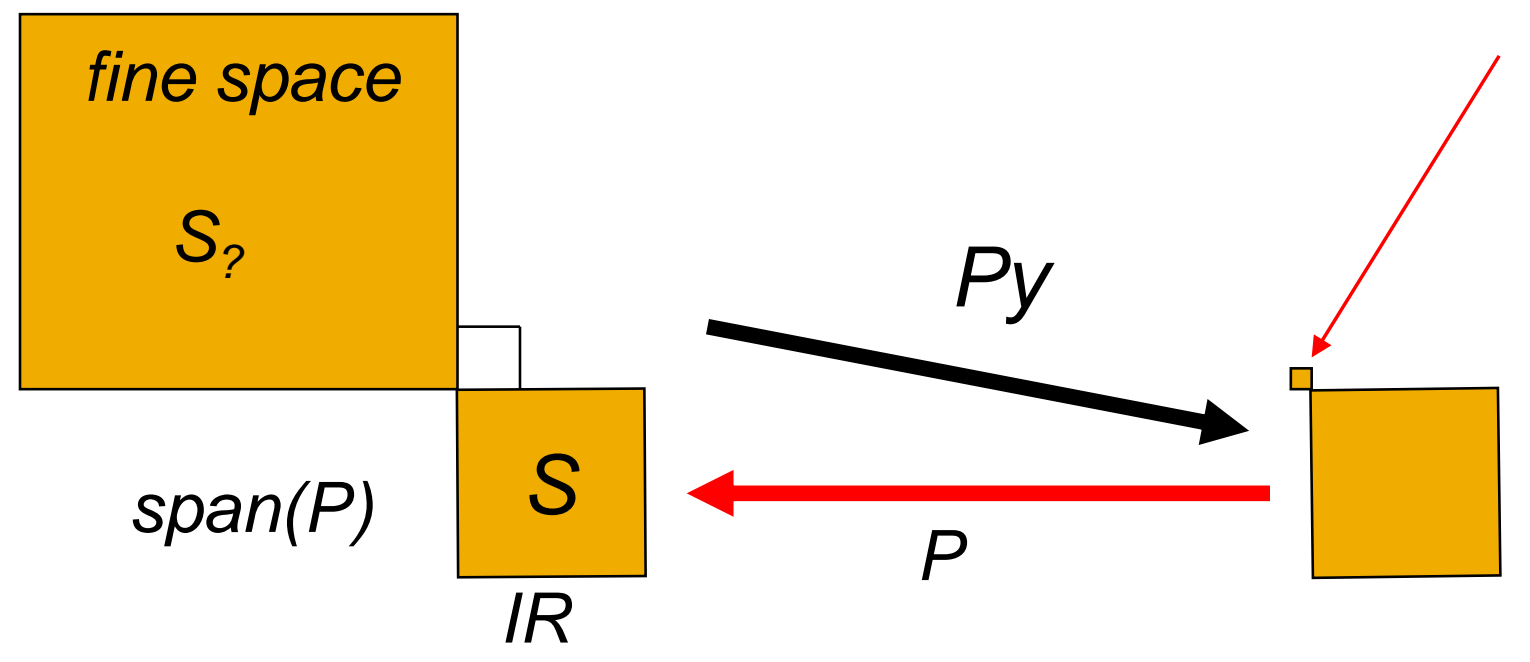

$\operatorname{span}(P y)$

$$
S=\operatorname{span}(P)=\operatorname{Image}(P y)
$$

$\operatorname{rank}(P)=\operatorname{rank}(P y)=\operatorname{dim}(S)=N_{o} N_{B}=2 N_{o} L 4 / 44$

y See Front cover of Gilbert Strang's undergraduate text! 


\section{General Problem: D $\tilde{\mathbf{A}}=\mathbf{b}$}

- "split" vector space into:

- near null DS 'o \& Complement $S_{\text {? }}$

- Schur decomposition (of course) does this!

- Coarse = near null (IR), Fine = complement (UV)

Splitting is essential the idea of the Schur complement:

$\left[\begin{array}{ll}D_{c c} & D_{c f} \\ D_{f c} & D_{f f}\end{array}\right]=\left[\begin{array}{cc}1 & 0 \\ D_{f c} D_{c c}^{-1} & 1\end{array}\right]\left[\begin{array}{cc}D_{c c} & 0 \\ 0 & D_{f f}-D_{f c} D_{c c}^{-1} D_{c f}\end{array}\right]\left[\begin{array}{cc}1 & D_{c c}^{-1} D_{c f} \\ 0 & 1\end{array}\right]$

Implies $\quad D^{-1}=\left[\begin{array}{cc}1 & -D_{f c} D_{c c}^{-1} \\ 0 & 1\end{array}\right]\left[\begin{array}{cc}D_{c c}^{-1} & 0 \\ 0 & D_{\text {Schur }}^{-1}\end{array}\right]\left[\begin{array}{cc}1 & 0 \\ -D_{c c}^{-1} D_{c f} & 1\end{array}\right]$

with $D_{S c h u r} \equiv D_{f c} D_{c c}^{-1} D_{c f}$ 


\section{Petrov-Galerkin oblique projection}

$$
\begin{gathered}
D_{\text {Schur }}=\left[D-D P \frac{1}{P^{\dagger} D P} P D\right]=\left[1-D P \frac{1}{P^{\dagger} D P} P\right] D \\
P^{\dagger} D_{S c h u r}=D_{S c h u r} P=0
\end{gathered}
$$

The coarse operator : $\quad \hat{D}=D_{c c}=P^{\dagger} D P$

The projection op to coarse space : $P P^{\dagger}$

But the Schur complement use "oblique projects:

$$
\begin{gathered}
\Pi_{L}^{\dagger}=D P \frac{1}{P^{\dagger} D P} P^{\dagger} \quad, \quad \Pi_{R}=P \frac{1}{P^{\dagger} D P} P^{\dagger} D \\
D_{\text {Schur }}=D-\Pi_{L}^{\dagger} D \Pi_{R}=\left(1-\Pi_{L}^{\dagger}\right) D\left(1-\Pi_{R}\right)
\end{gathered}
$$




\section{2-level Multigrid Cycle (simplified)}

- Smooth $\quad x=(1-D) x^{0}+b \quad \Longrightarrow \quad r=(1-D) r^{0}$

- Coarsen $\quad D_{c}=P^{\dagger} D P \quad \& \quad r_{c}=P^{\dagger} r$

- Solver

$$
A_{c} e_{c}=r_{c} \quad \Longrightarrow e_{c}=A_{c}^{-1} P^{\dagger} r
$$

- Interpolate

$$
e=P e_{c}
$$

- Update

$$
x^{\prime}=x+e \quad \Longrightarrow \quad r^{\prime}=b-D(x+e)
$$

- Hence

$$
r^{\prime}=\left[1-D P \frac{1}{P^{\dagger} D P} P^{\dagger}\right] r \quad \Longrightarrow \quad P^{\dagger} r^{\prime} \equiv 0
$$

Exact null space "deflation" by right "preconditioner:

$$
M^{-1}=P \frac{1}{P^{\dagger} D P} P^{\dagger}
$$




\section{AMG on Wilson-clover Dirac Operator}

- Devil is in the details!

- Rigorous MG proofs for normal equation (Dy D $\tilde{A}=b$ )

- But would like to project $D$ to avoid higher complexity.

- Multigrid is recursive to multi-levels.

- Must preserves Gauge invariance and ${ }_{5}^{\circ}\left(\left[{ }_{5}^{0}, \mathrm{P}\right]=0\right)$

- First benchmarks for Wilson-Dirac Operator:

- Asym $V=16^{3} \times 64,24^{3} \times 64,32^{3} \times 96\left(N_{f}=2,400 \mathrm{MeV}\right.$ pion $)$

- $N_{o}=20$ null vectors $\tilde{A} s^{x}$ with 4th order MR with subset refinement.

- MG Blocks $=4^{4} \times N_{c} \times 2$ and 3 level V MG cycle

- pre and post-smoothing is done by 4 iteration GCR (later GMRES)

- Extend to Red/Black preconditioning

(James Osborn implement on BG/P in SciDAC-2 API

Future SciDAC-3 develop in HYPER framework/GPUs etc). 
$32^{3} \times 256$ aniso clover on $1024 \mathrm{BG} / \mathrm{P}$ cores

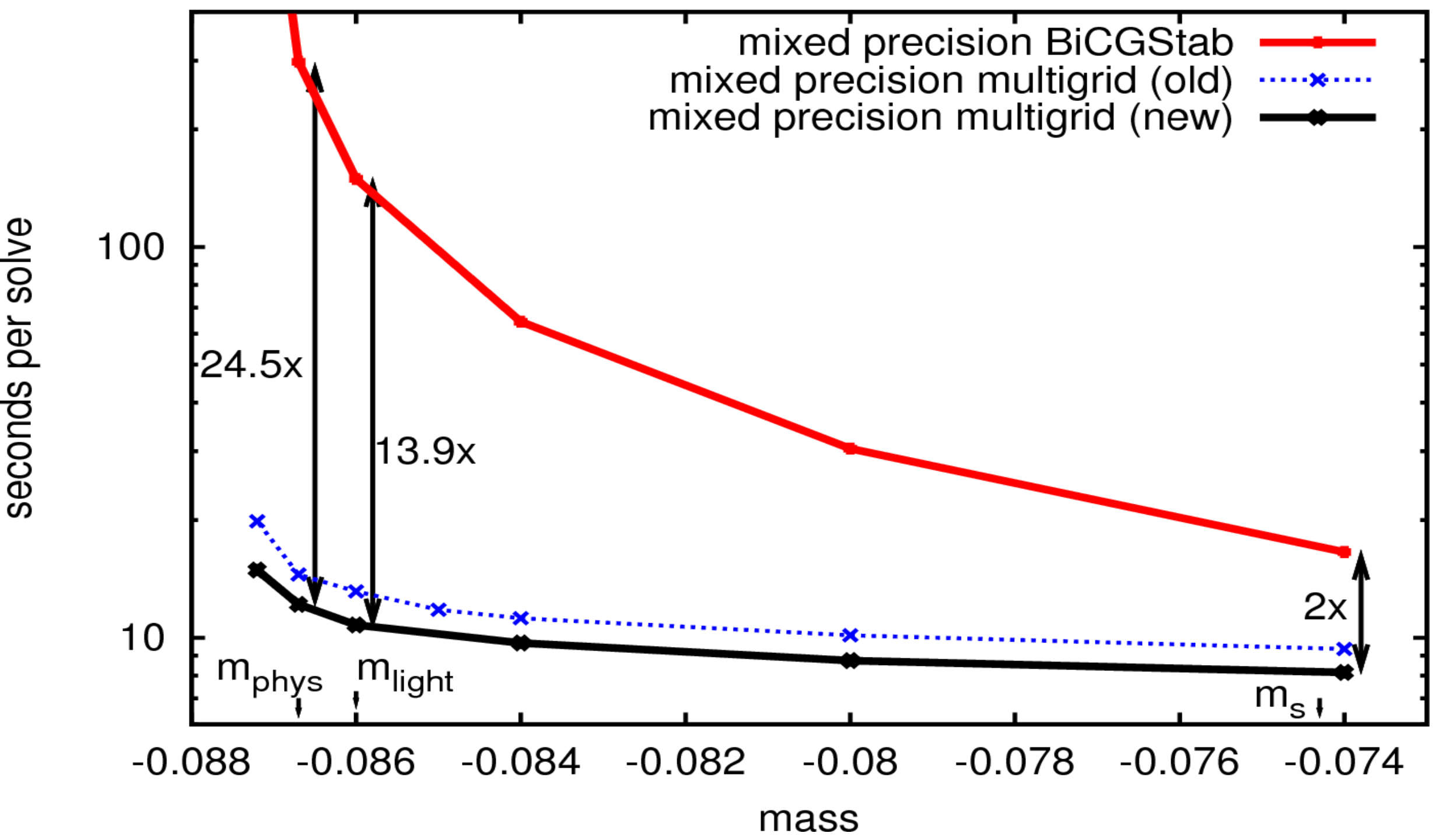

"Adaptive multigrid algorithm for the lattice Wilson-Dirac operator" R. Babich, J. Brannick, R. C. Brower, M. A. Clark, T. Manteuffel, S. McCormick, J. C. Osborn, and C. Rebbi, Phys. Rev. Lett. 105, 201602 (2010). (Latest Result: Finite T lattice $128^{\wedge} 3 \times 96 \rightarrow 32 \times$ speed up! James Osborn.) 


\section{Total cost $32^{3} \times 256$}

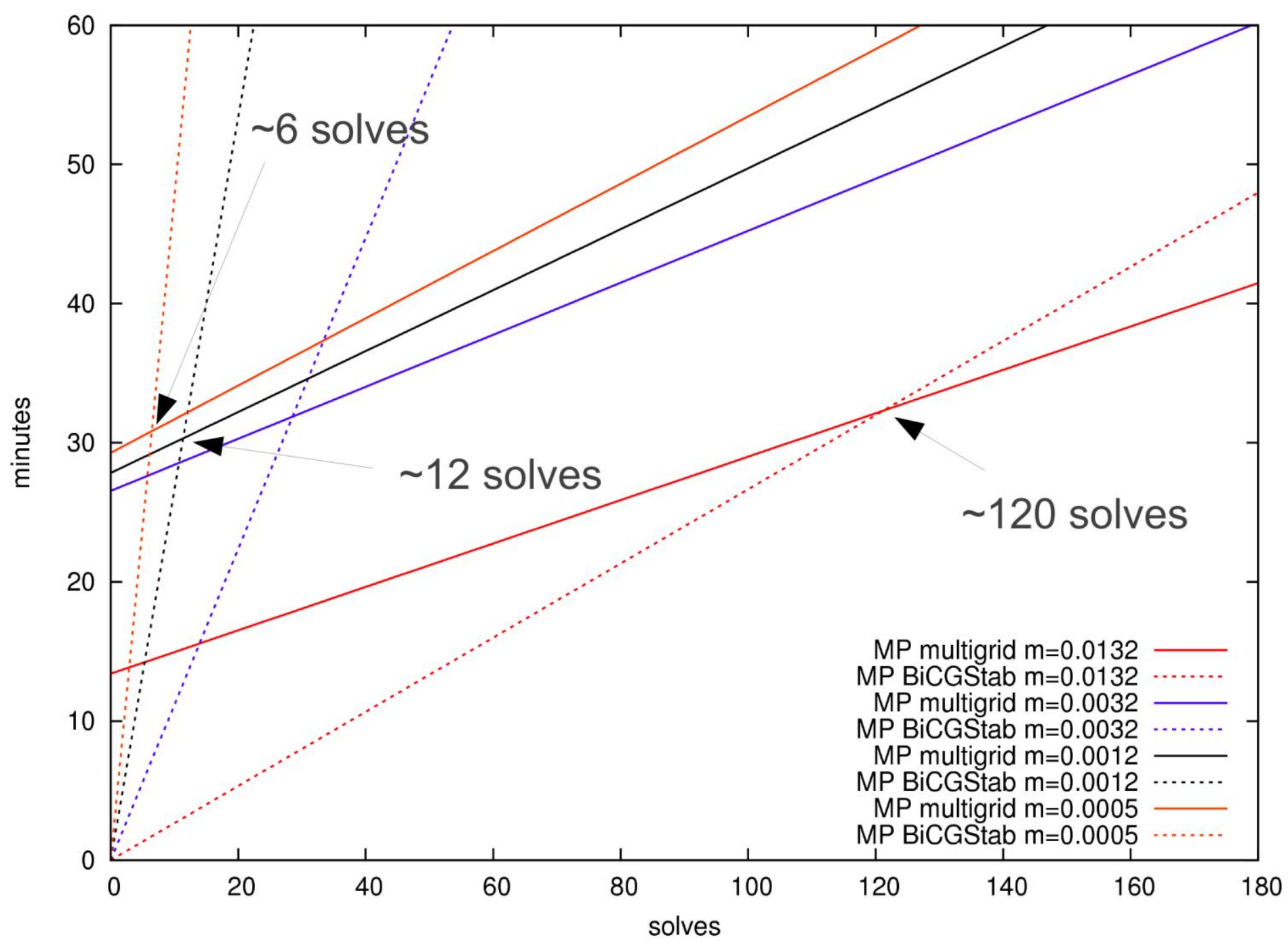




\section{Error vs residual}

- Error:

$$
e=x^{*}-x
$$

- Residual:

$$
r=b-A x
$$

$$
=\mathrm{Ae}
$$

- Residual not as sensitive to low modes

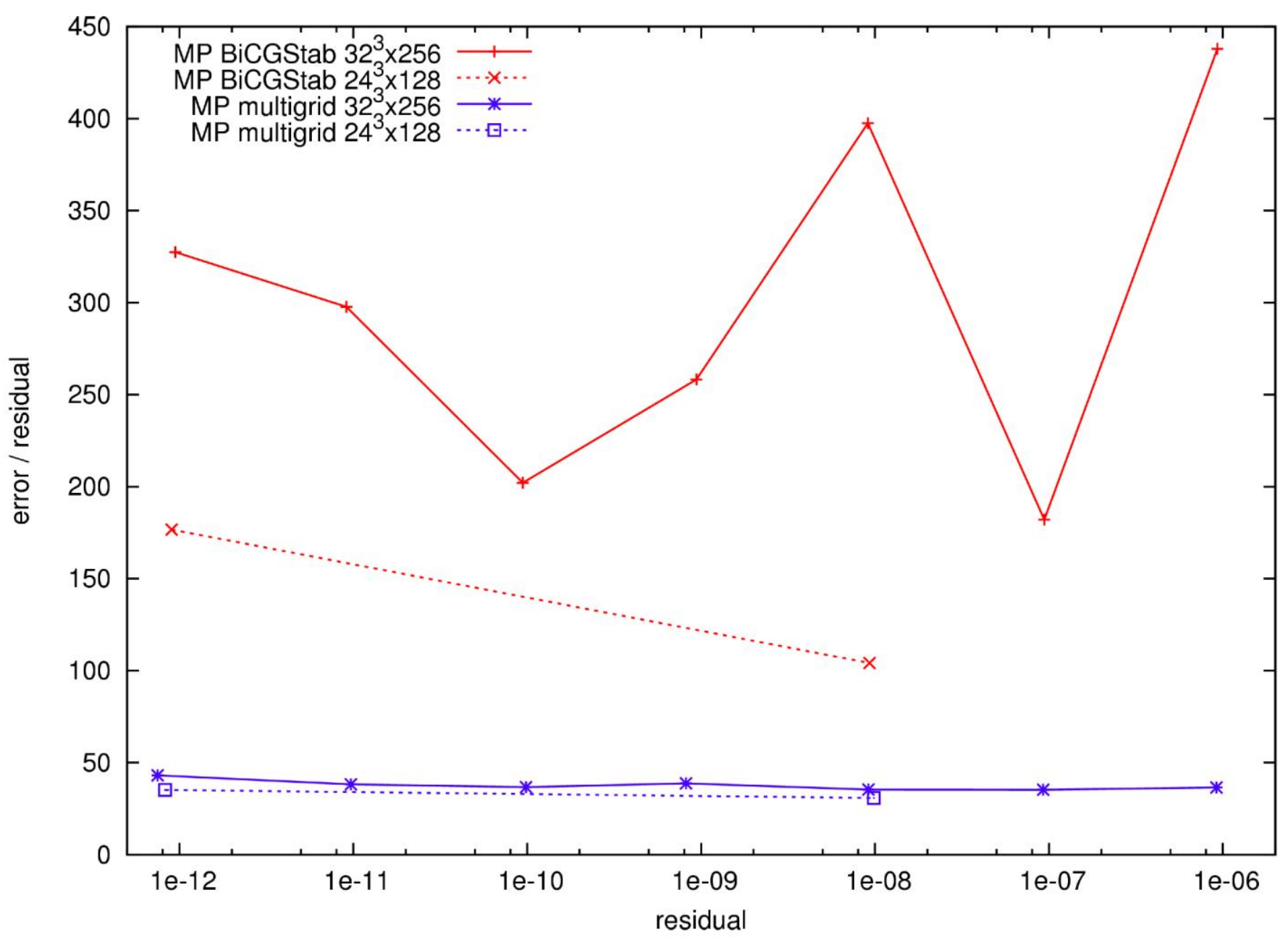

Speed up is even better at fixed error. 


\section{QUDA: QCD in CUDA for GPUs}




\section{Disruptive many-core Architectures}

- $1 / 4 \mathrm{CM}-2$

Nvidia FERMI chip

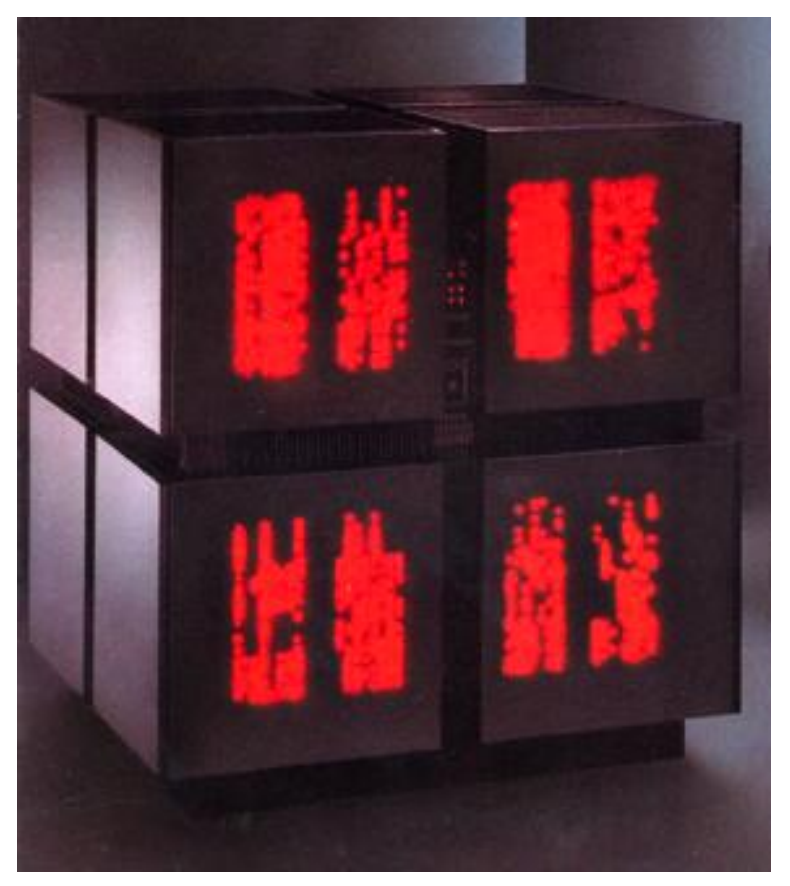

$16 \mathrm{~K}$ bit serial $\mathrm{PE}$.)

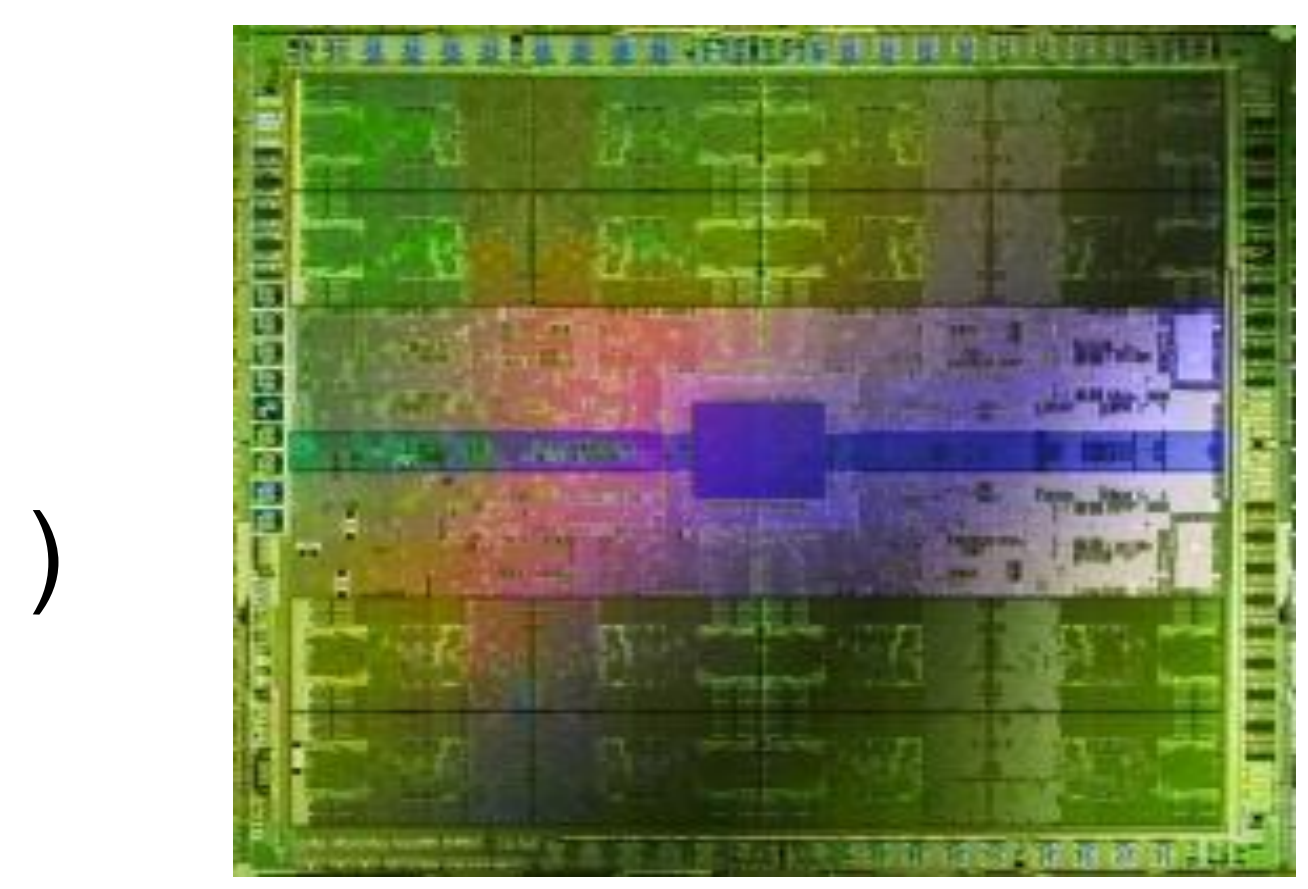

$512 \times 32$ bit $\mathrm{PE}=16 \mathrm{~K}$ bits

Nvidia Kepler has 1536 Floating point cores! Maxwell ??? Next ??? 


\title{
2005-2006
}

Available online at www.sciencedirect.com

\section{$\because \because$ ScienceDirect}

Computer Physics

Communications

Computer Physics Communications 177 (2007) 631-639

www.elsevier.com/locate/cpc

\section{Lattice $\mathrm{QCD}$ as a video game}

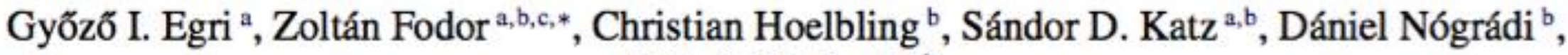 \\ Kálmán K. Szabó ${ }^{\text {b }}$ \\ a Institute for Theoretical Physics, Eotwös University, Budapest, Hungary \\ ${ }^{\mathrm{b}}$ Department of Physics, University of Wuppertal, Germany \\ ' Department of Physics, University of California, San Diego, USA
}

Received 2 February 2007; received in revised form 29 May 2007; accepted 7 June 2007

Available online 15 June 2007

\section{Abstract}

The speed, bandwidth and cost characteristics of today's PC graphics cards make them an attractive target as general purpose computational platforms. High performance can be achieved also for lattice simulations but the actual implementation can be cumbersome. This paper outlines the architecture and programming model of modern graphics cards for the lattice practitioner with the goal of exploiting these chips for Monte Carlo simulations. Sample code is also given.

() 2007 Elsevier B.V. All rights reserved. 


\section{QUDA (QCD/CUDA) collaborators}

- Ronald Babich (BU $\rightarrow$ PSC $\rightarrow$ NVIDIA)*

- Kipton Barros (BU $\rightarrow$ LANL)

- Michael Clark (BU $\rightarrow$ Harvard $\rightarrow$ NVIDIA)*

- Justin Foley (Utah)

- Joel Giedt (Rensselear)

- Steven Gottlieb (Indiana)

- Balint Joo (Jefferson Lab)

- James Osbone (Utah $\rightarrow$ BU $\rightarrow$ Argonne)

- Claudio Rebbi (BU)

- Gouchin Shi (NCSA)

- et al 


\section{How to get QUDA \& join the Fun}

\section{github}

: lattice / quda

Source Commits Network

Pull Requests (0)

Fork Queue

Issues (3)

f Admin

$\odot$ Unwatch

6. Fork

ثิ Pull Request

$\odot 7$ / 2

Browse Issues

Milestones

\section{Everyone's Issues}

Assigned to you

Mentioning you

Milestone: Adaptive Multigrid

3 open issues

LABELS

clean-up

feature
3

3

0

\$

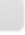

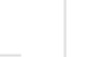

(1)

\section{https://github.com/lattice/quda}




\section{GPU memory hierarchy}

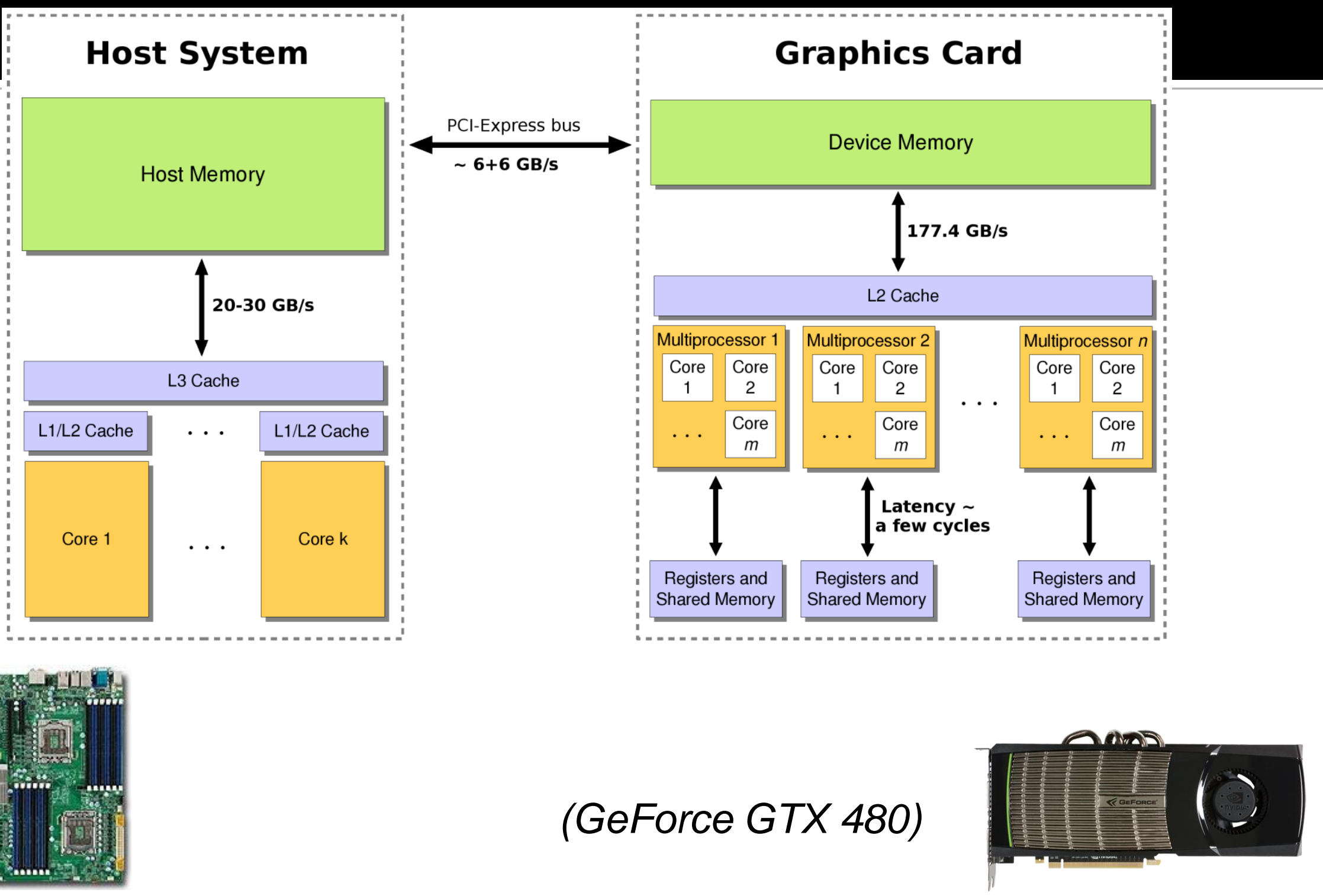




\section{Tricks to reduce memory traffic}

Reconstruct SU(3) matrices from 8 or 12 real numbers on the fly, e.g.,

$$
\left(\begin{array}{l}
\mathbf{a} \\
\mathbf{b} \\
\mathbf{c}
\end{array}\right)=\left(\begin{array}{ccc}
a_{1} & a_{2} & a_{3} \\
b_{1} & b_{2} & b_{3} \\
c_{1} & c_{2} & c_{3}
\end{array}\right) \quad \begin{array}{r}
\mathbf{c}=(\mathbf{a} \times \mathbf{b})^{*} \\
\quad S U(2): U=a_{0} \sigma_{0}+\vec{a} \cdot \sigma \\
a_{0}^{2}+\vec{a} \cdot \vec{a}=1 \Longrightarrow S_{3} \text { sphere }
\end{array}
$$

Better still $\mathrm{SU}(3)$ has 8 parameters on $\mathrm{S}_{3} \times \mathrm{S}_{5}$

- Choose a gamma basis with $\square_{4}$ diagonal.

$$
P^{ \pm 4}=\left(\begin{array}{rrrr}
1 & 0 & \pm 1 & 0 \\
0 & 1 & 0 & \pm 1 \\
\pm 1 & 0 & 1 & 0 \\
0 & \pm 1 & 0 & 1
\end{array}\right) \quad \longleftrightarrow\left\{\begin{array}{l}
P^{+4}=\left(\begin{array}{llll}
2 & 0 & 0 & 0 \\
0 & 2 & 0 & 0 \\
0 & 0 & 0 & 0 \\
0 & 0 & 0 & 0
\end{array}\right) \\
P^{-4}=\left(\begin{array}{llll}
0 & 0 & 0 & 0 \\
0 & 0 & 0 & 0 \\
0 & 0 & 2 & 0 \\
0 & 0 & 0 & 2
\end{array}\right)
\end{array}\right\} \begin{aligned}
& \text { similarity } \\
& \text { transforms on } \\
& D
\end{aligned}
$$

- Fix to the temporal gauge (setting gauge links in the $t$-direction to the identity). 


\section{Mixed precision with reliable updates}

- Using a mixed-precision solver incorporating "reliable updates" (Clark et al., arXiv:0911.3191) with half precision greatly reduces time-to-solution while maintaining double precision accuracy.
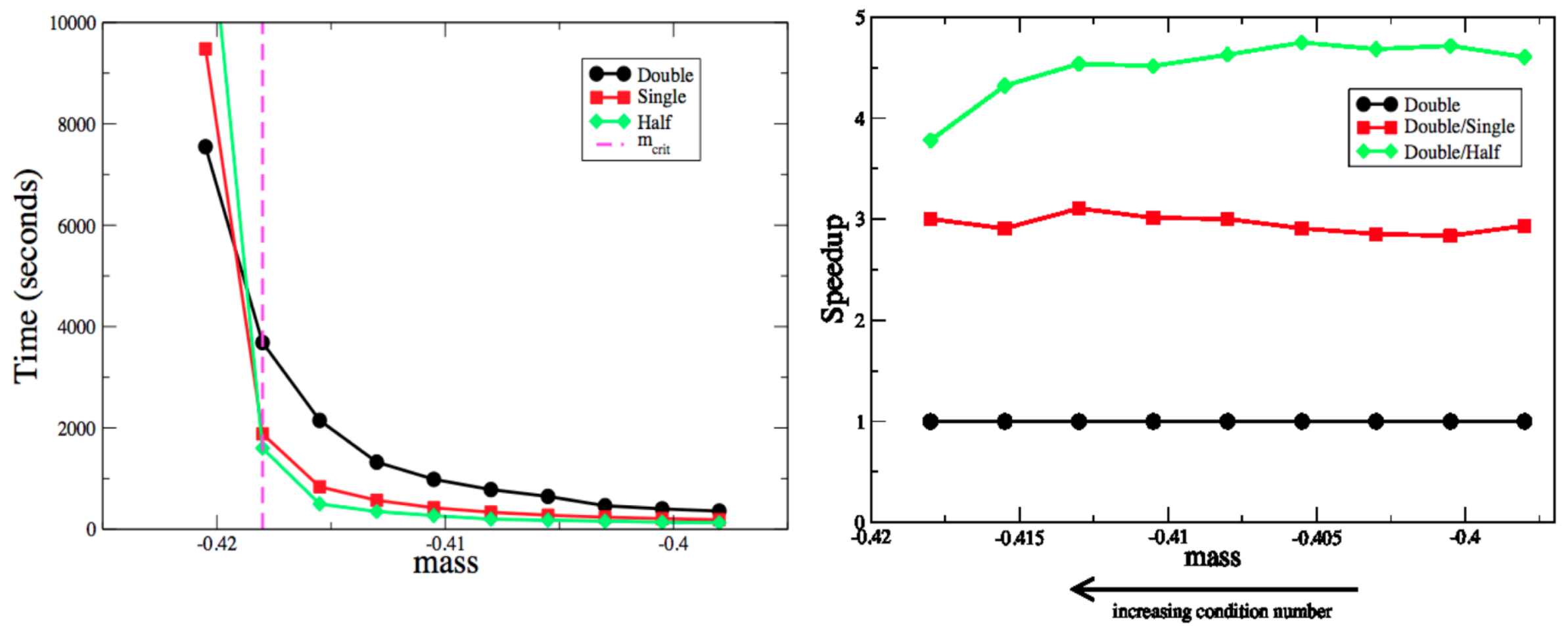


\section{Big Cray/GPU machines are coming.}

\section{Cray XK6 Compute Node}

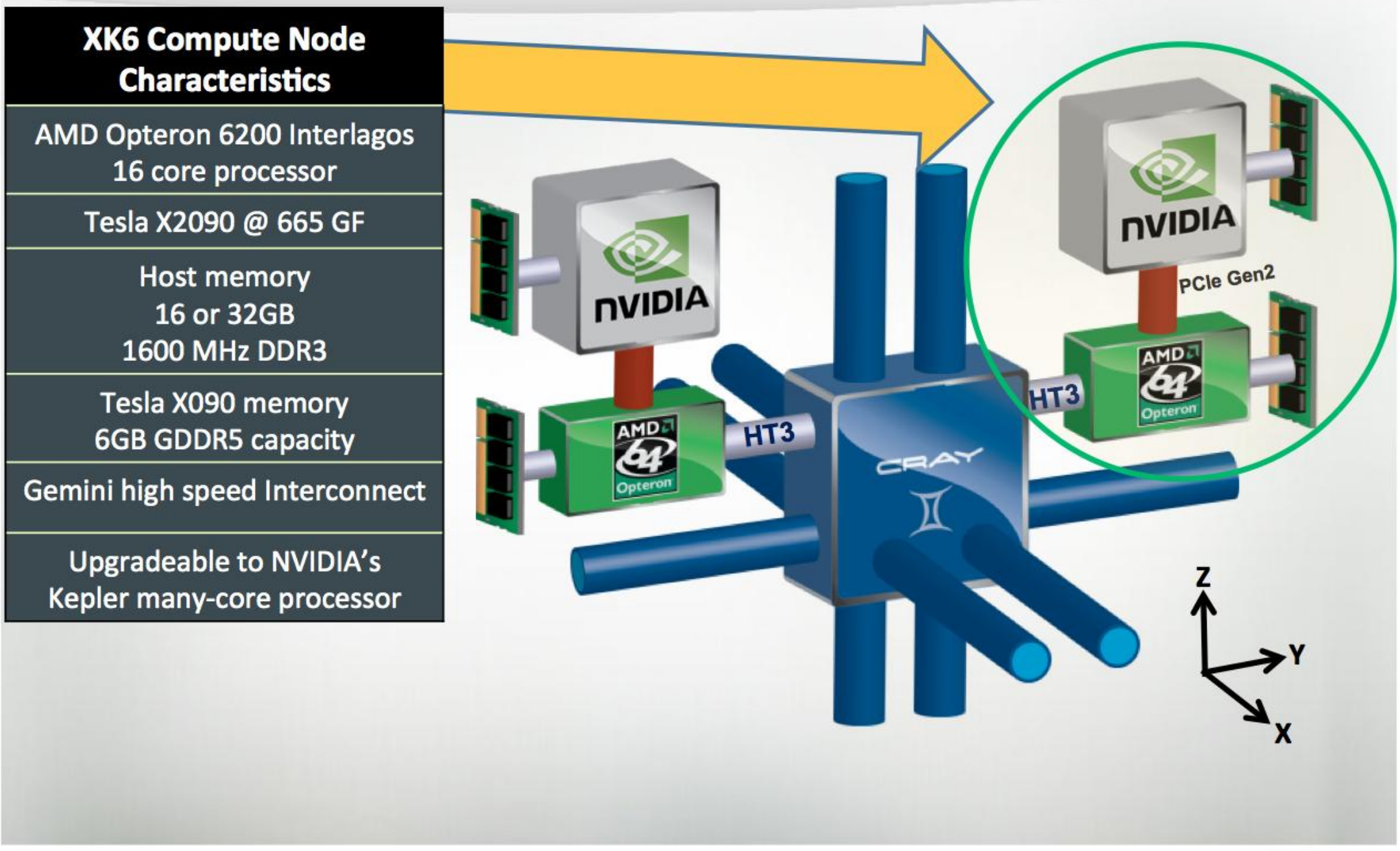




\section{LOCATION LOCATION LOCATION!}

TO GET STRONG SCALING NEED TO MANAGE A MULTILEVEL MEMORY HEIRARCHY

THIS IS THE CHALLENGE OF HETEROGENOUS ARCHITECTURE OF THE PRESENT AND THE FUTURE!
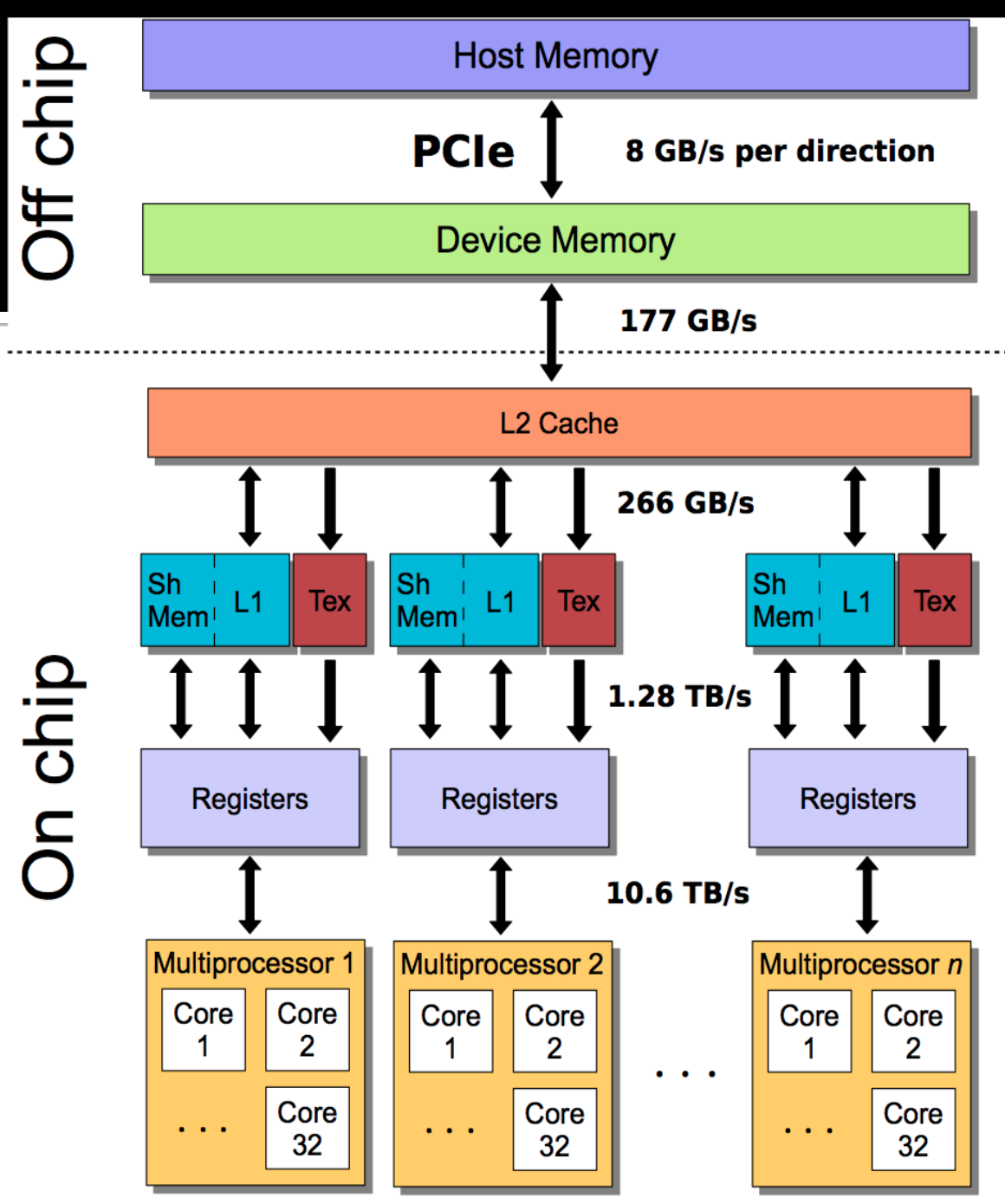

Figure 1: Peak bi-directional bandwidth between various levels of the memory hierarchy on a Tesla M2090. 


\section{Additive Schwarz}

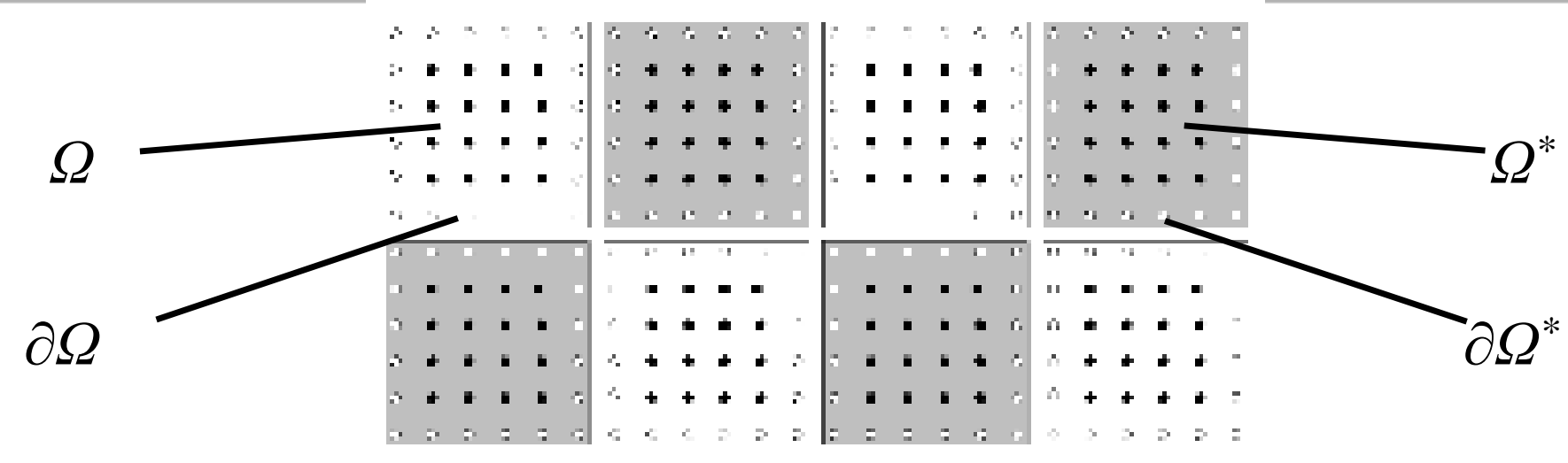

- Update domains simultaneously Block Jacobi

- Expect higher iteration count than multiplicative

- Algorithm is trivially parallel

- Well suited to GPU architecture 


\section{Block Jacobi "DD" on LLNL Edge}

\section{Flops versus speedup}
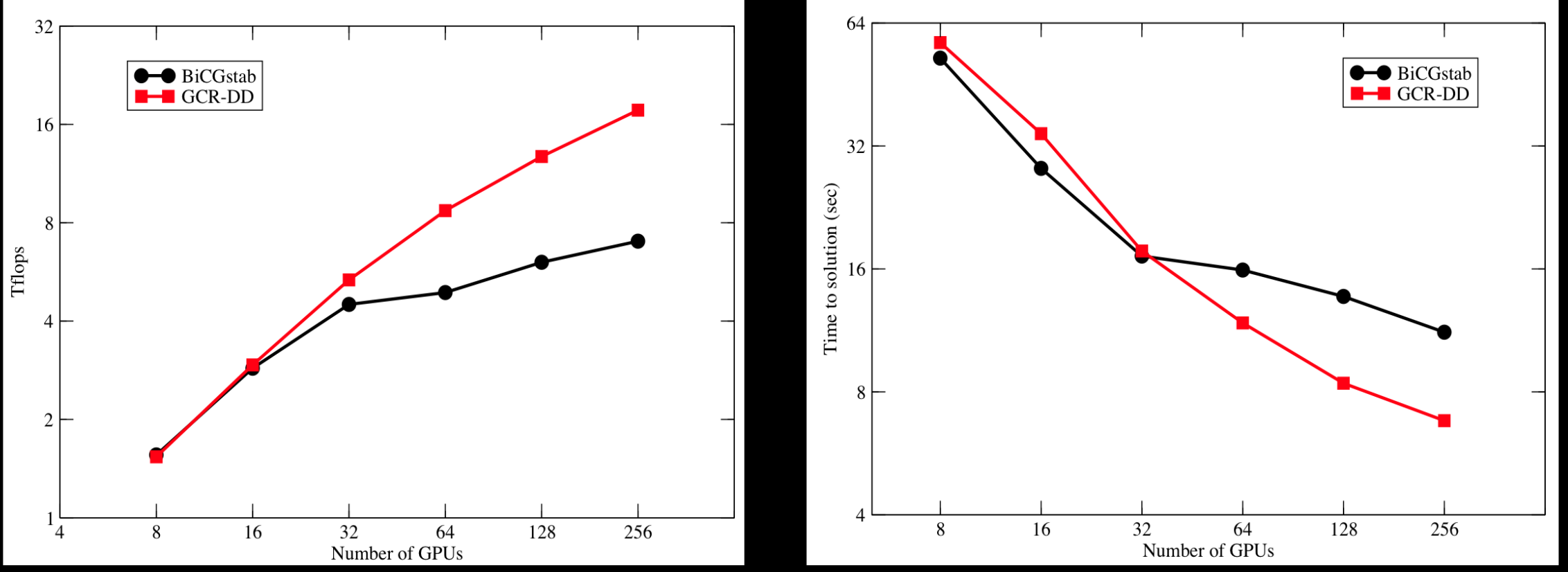

Babich, Clark, Joo, Shi, Brower, Gottlieb, “Scaling Lattice QCD beyond 100 GPUs," SC'11 


\section{Compare with Cray: $32 \mathrm{~K}=64 \mathrm{GPUs}$}

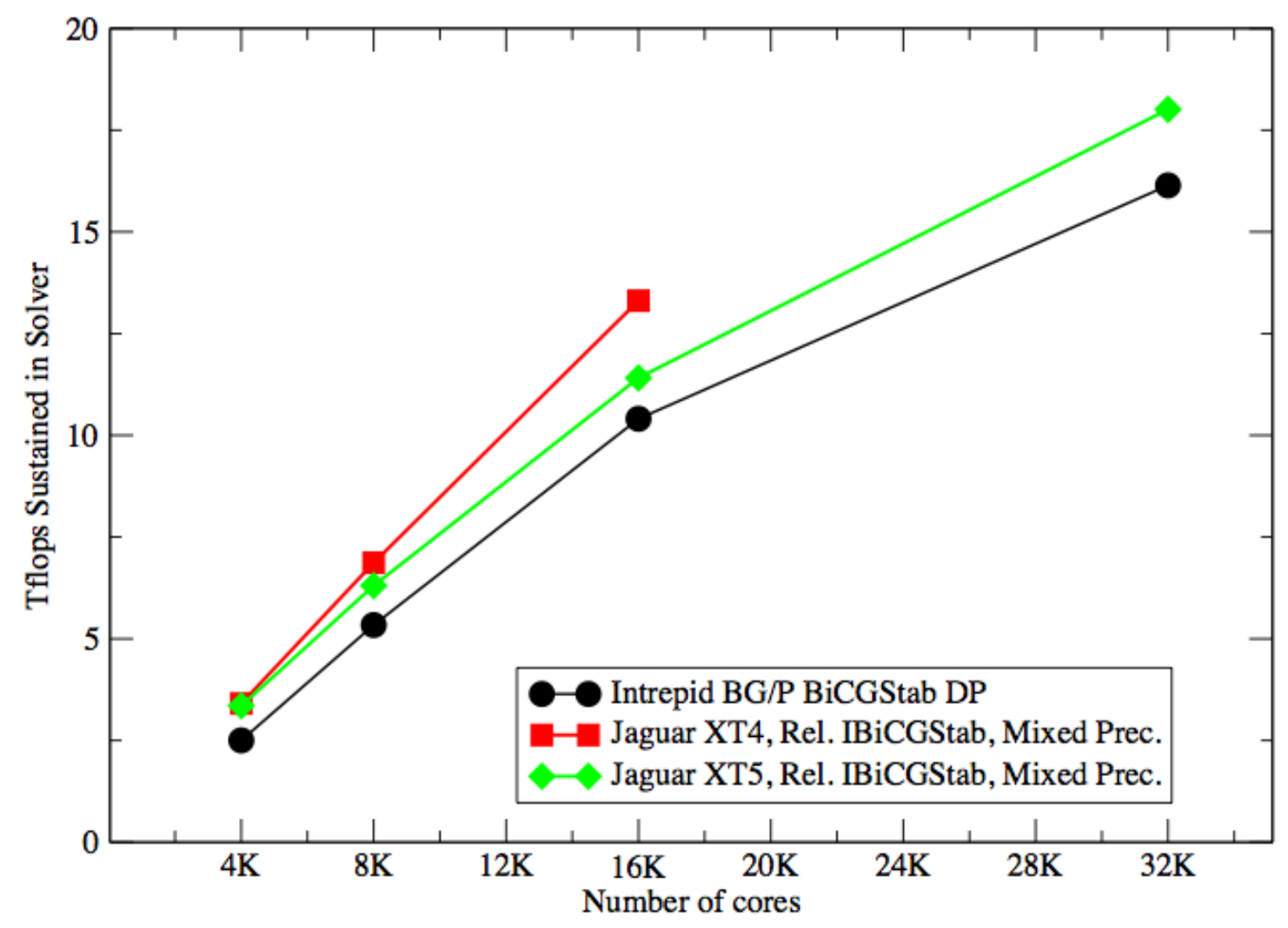




\section{Mike Clark and Balint Joo's Mixed DD GCR Algorithm}

\section{Mixed-Precision GCR-DD solver}

\section{Generate Subspace}

Apply Preconditioner: reduced precision inner solve

$$
\begin{gathered}
r_{0}=b-M x \\
\hat{r}_{0}=r_{0} \\
\hat{x}_{0}=0 \\
k=0
\end{gathered}
$$

$$
\begin{aligned}
& \hat{p}_{k}=\hat{K}^{-1} \hat{r}_{k} \\
& \hat{z}_{k}=\hat{M} \hat{p}_{k}
\end{aligned}
$$$$
\beta_{i, k}=\left(\hat{z}_{i}, \hat{z}_{k}\right)
$$$$
\text { Orthogonalize } \hat{z}-s
$$$$
\gamma_{k}=\left\|\hat{z}_{k}\right\|
$$$$
\text { normalize } \hat{z}_{k}
$$$$
\alpha_{k}=\left(\hat{z}_{k}, \hat{r}_{k}\right)
$$$$
\hat{r}_{k+1}=\hat{r}_{k}-\alpha_{k} \hat{z}_{k}
$$$$
k=k+1
$$

\section{Reduced Precision}

$\mathrm{M}$ v

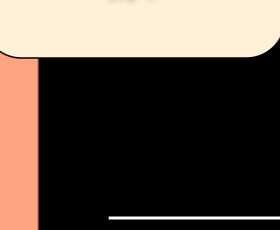

\section{Update Solution}

$$
\begin{array}{r}
\text { Solve for } \chi_{l} l=k, k-1, \ldots, 0 \text { : } \\
\gamma_{l} \chi_{l} \sum_{i=l+1}^{k} \beta_{l, i} \chi_{l}=\alpha_{l}
\end{array}
$$

Compute correction for $x$ :

$$
\begin{aligned}
& \hat{x}=\sum_{i=0}^{k-1} \chi_{i} p_{i} \\
& x=x+\hat{x}
\end{aligned}
$$




\section{TitanDev (Balint and Clark)}

\section{Benchmarks: + GPU (DD+GCR)}

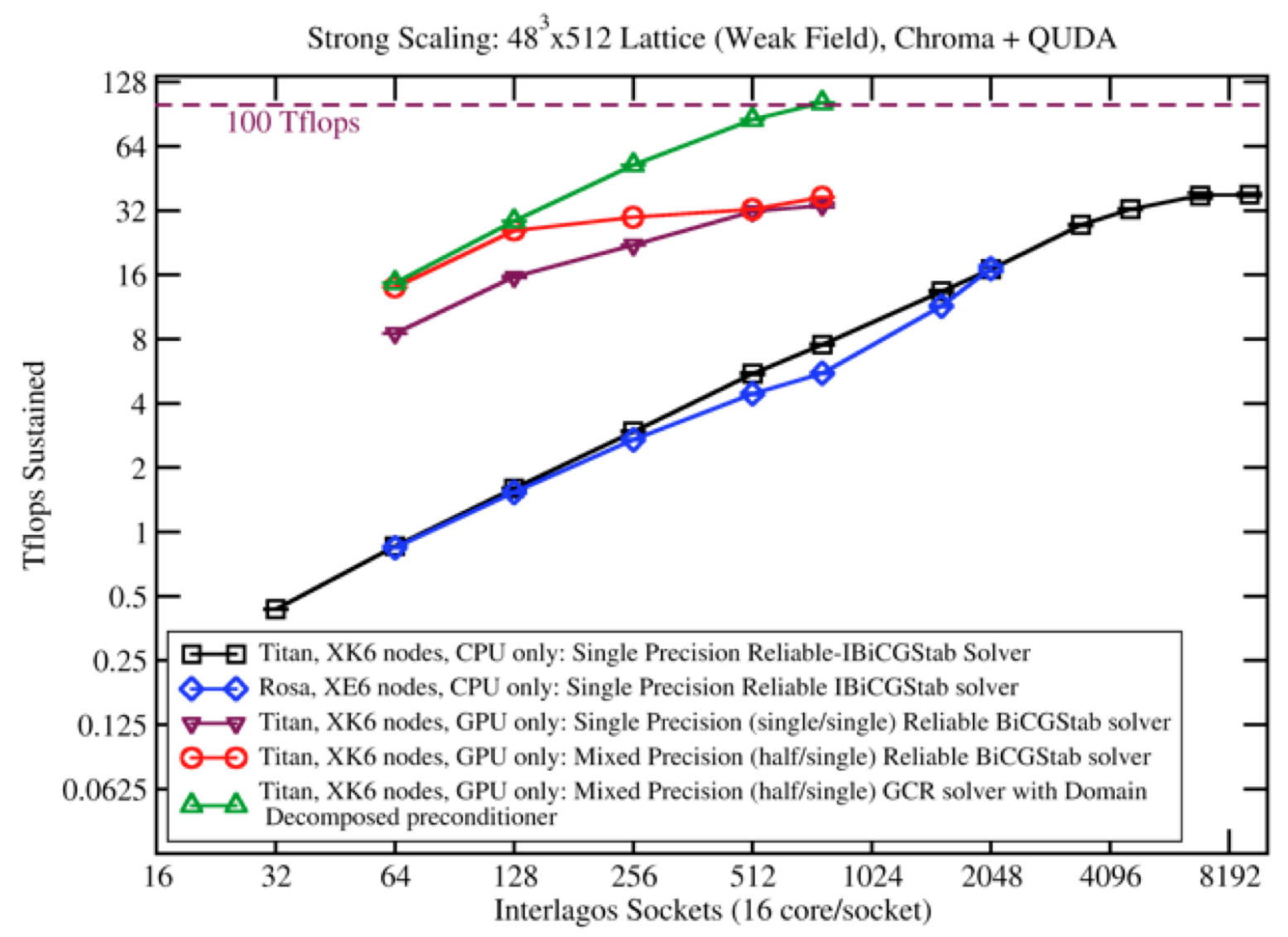




\section{Next Project: MG on GPU}

Cost in $\$$ s reduced by a at least factor of

\section{X \\ GPU O(10x) \\ MG O(10x)}
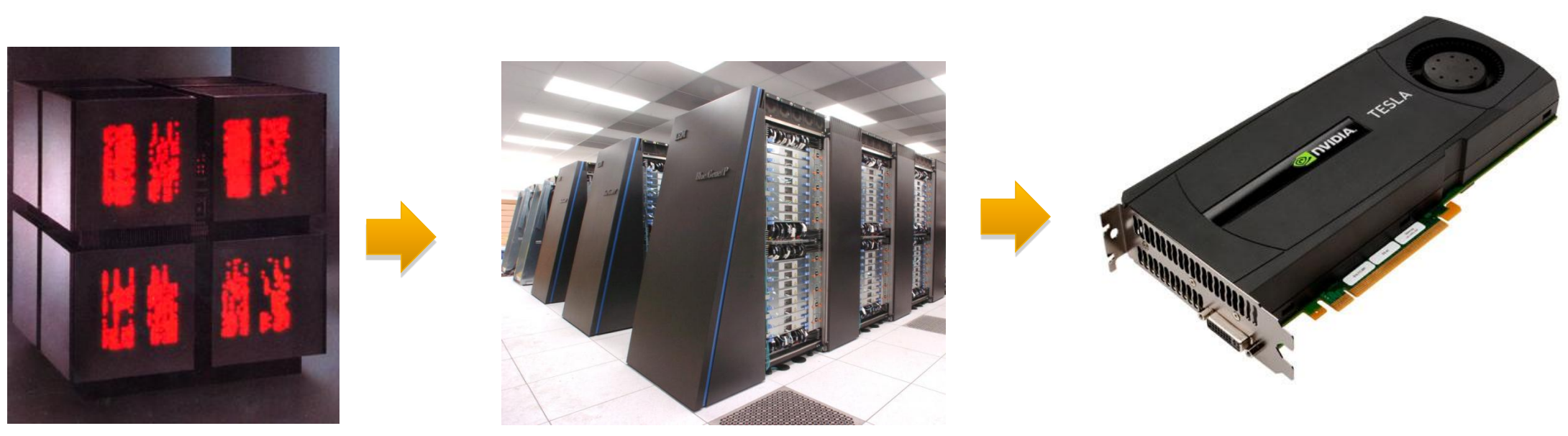
IV. The Future: MG/GPU/DD for HMC 


\section{Multigrid for HMC?}

- Parallel MG works: When size is too small the common practice is to copy lower levels to all nodes and solver with zero comm.

- Set up can be amortized by refining near null vectors from past or intermittent refresh

- Good for BSM Technicolor HMC! Faster solver encourage optimization with more solves. 


\section{Monte Carlo Evolution: Lüscher's intermittently update of S subspace(0710.6417V1)}

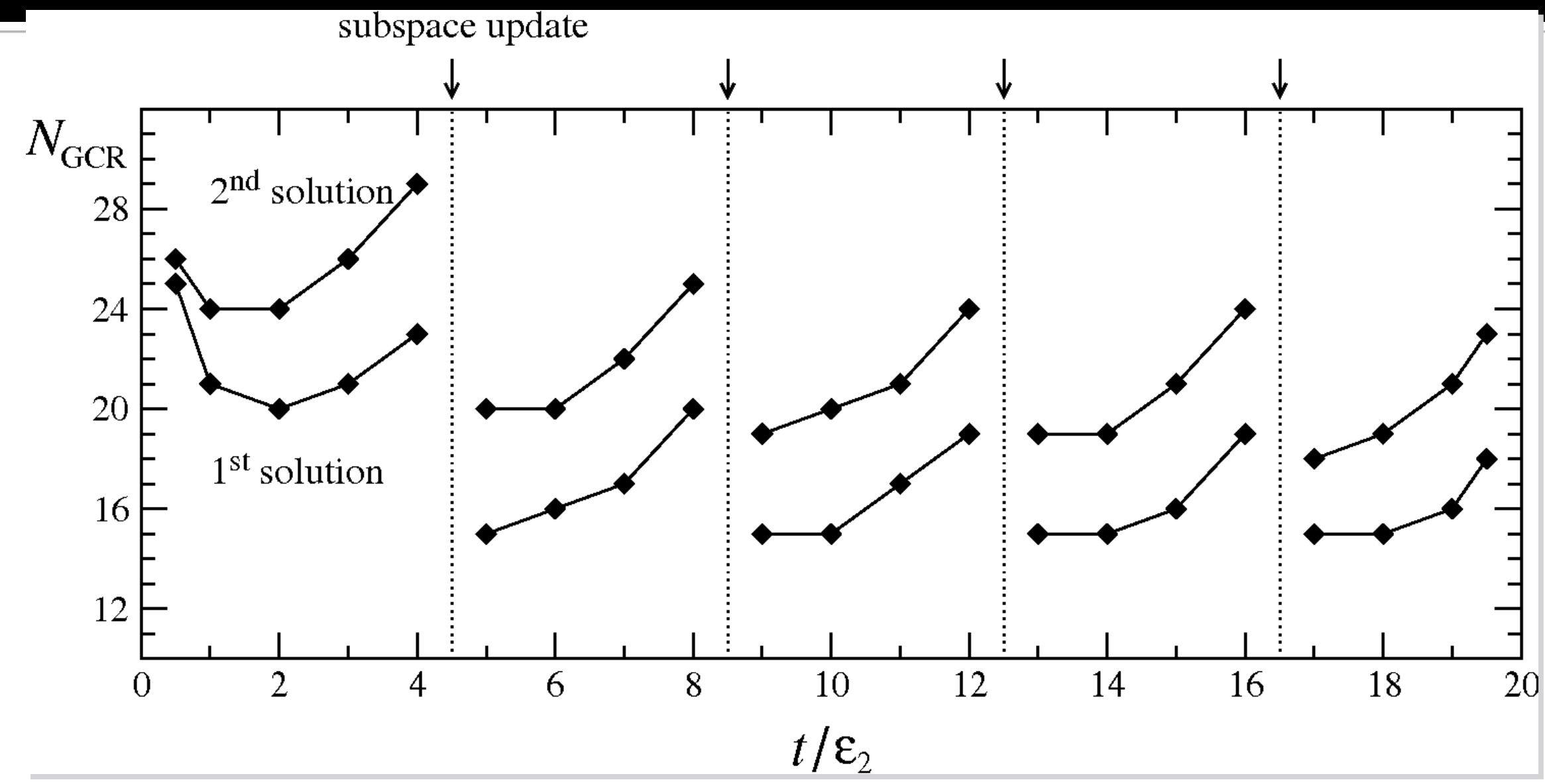

y Combined with "Chronological Inverter" Brower, Ivaneko, Levi, Orginos 


\section{At Conformal FT: Radial Quantization CFT}

- Exact:

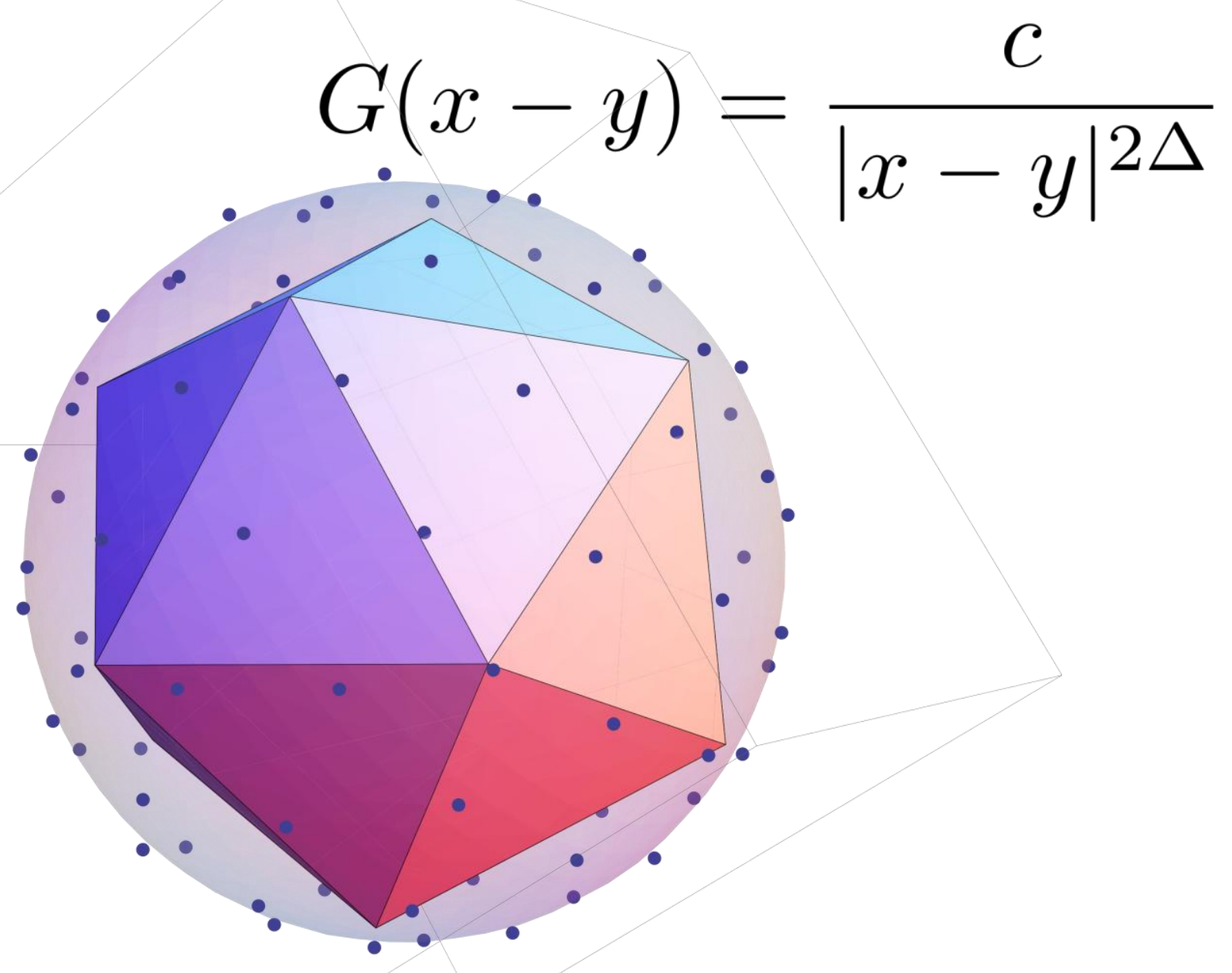

See Herbert Neuberger on Wednesday! 


\section{Summary: Multiscale Narrative for Lattice Field Theories}

- Past: 1990-2010

The good olde days of "MPI Data Parallel code" for SPMD algorithms "easily" mapped on to uniform architecture of Beowulf clusters.

- Present 2010-2012

Transition to high resolution multi-scale QCD and MPI network of threaded multi-core micros

- Future: 2012-2018

Profound challenges to adapt efficient multi-scale algorithm to a complex heterogeneous architecture with deep memory hierarchies. 


\section{Extra Slide!}




\section{NEXT TALK: Domain Wall Multigrid}

- 5-d Wilson with "negative mass" and Dirichlet B.C. in $5^{\text {th }}$ "time" interval $[0$, Ls]

- New Gamma 5: $\quad \Gamma_{5}=\gamma_{5} \mathcal{R}\left(s \leftrightarrow L_{s}-s\right)$

- $D$ is now violently not normal: $D^{\dagger} D \neq D D^{\dagger}$

- Physical long wavelength mode are low singular (eigen)vectors NOT eigenvectors of D! 


\section{A Chiral Fermion}

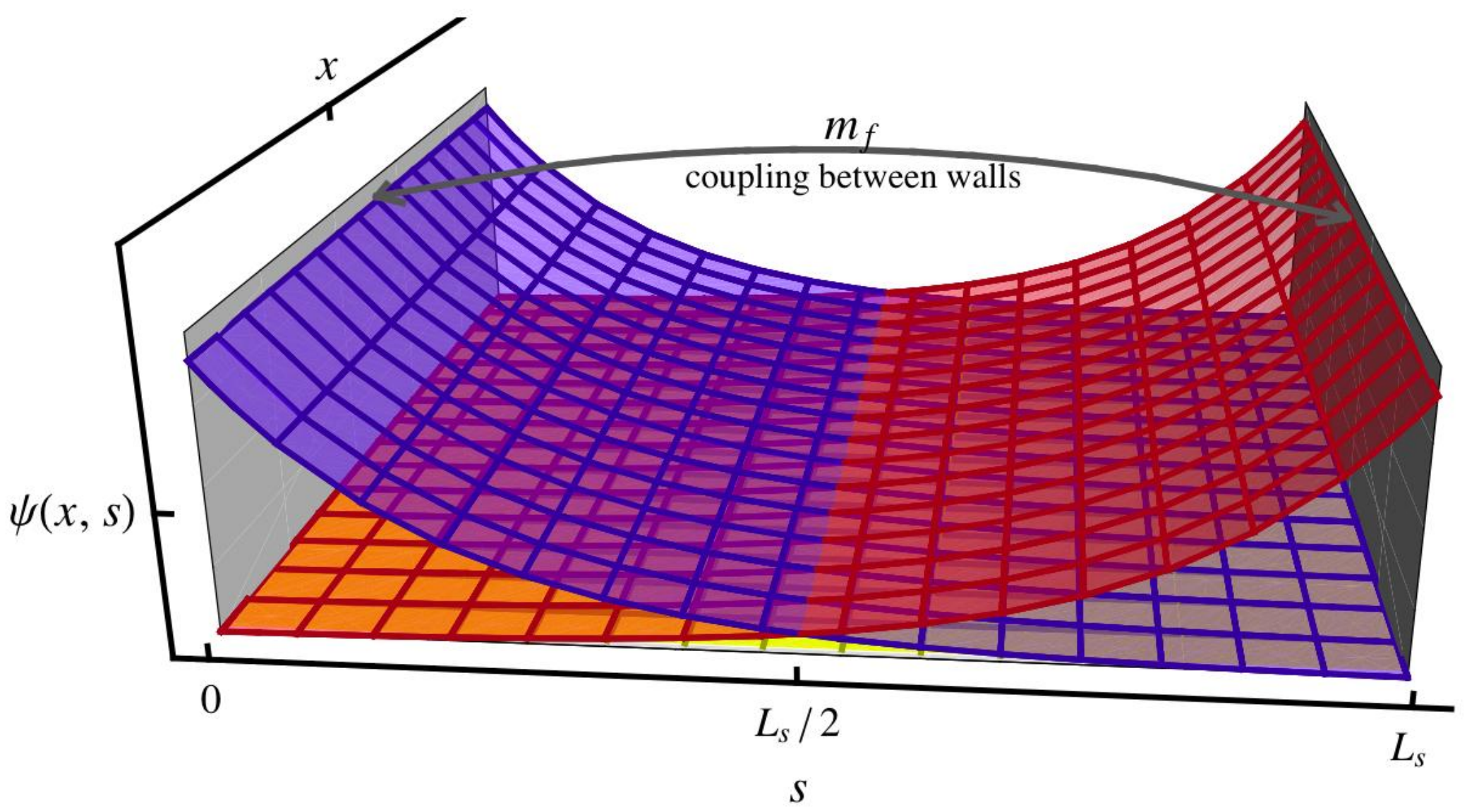




\section{Domain-Wall Operator}

No zero e.v.

\section{Spectrum}

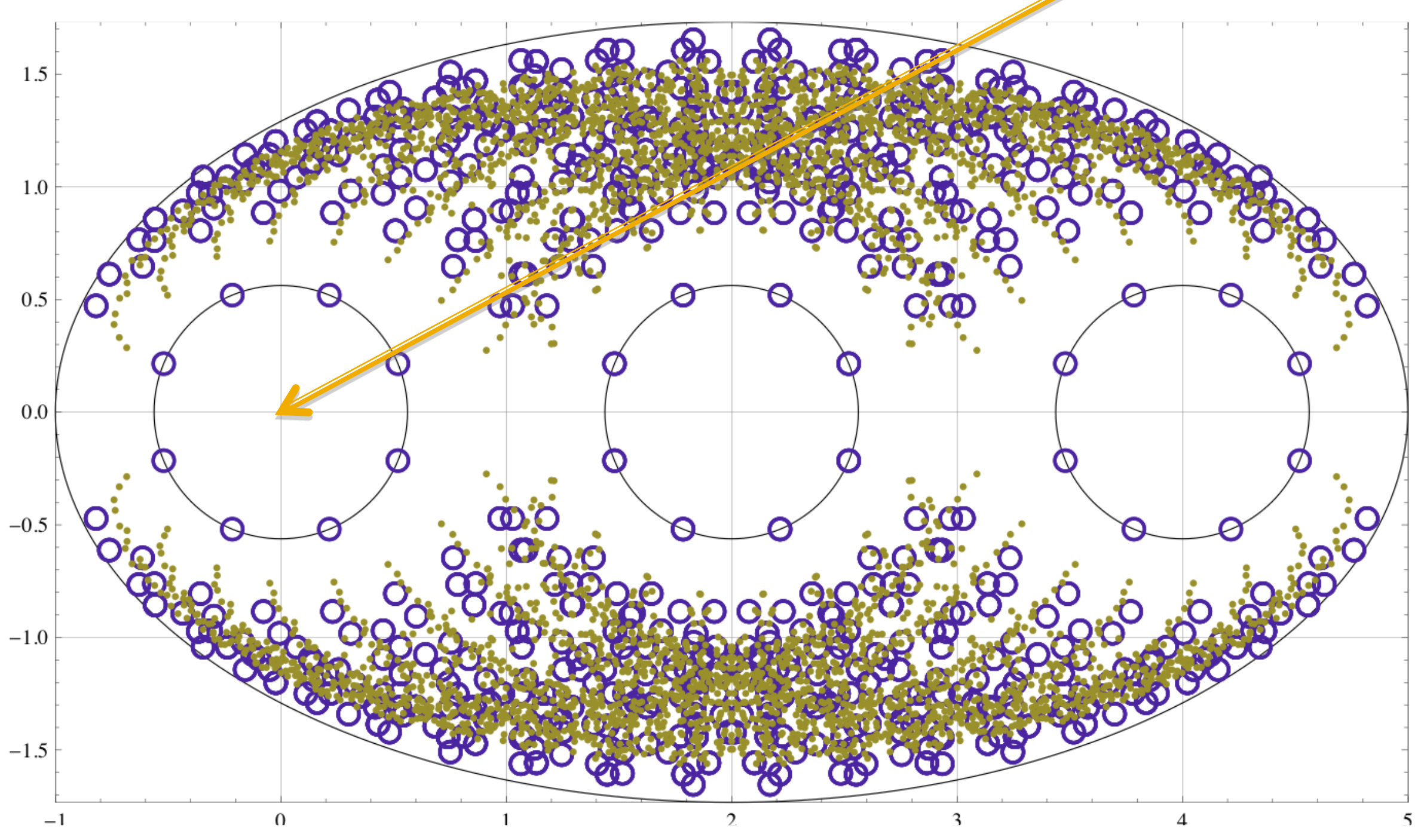

$2+1 d$ but $4+1 d$ would have 5 eyes 


\section{Some References}

- "Adaptive multigrid algorithm for the lattice Wilson-Dirac operator" R. Babich, J. Brannick, R. C. Brower, M. A. Clark, T. Manteuffel, S. McCormick, J. C. Osborn, and C. Rebbi, arXiv:1005.3043V2 [hep-lat], Phys. Rev. Lett. 105, 201602 (2010).

- "Adaptive Multigrid Algorithm for Lattice QCD" J. Brannick, R. C. Brower, M. A. Clark, J. C. Osborn, C. Rebbi, arXiv:0707.4018 Phys. Rev. Lett. 100, 041601 (2008)

- "Multigrid solver for clover fermions", R. Babich, J. Brannick, R. Brower, M. Clark, S. Cohen, J. Os- born and C. Rebbi, Lattice 2010, Villasimius, Sardinia, Italy (2010).

- "The role of multigrid algorithms for LQCD", R. Babich, J. Brannick, R. C. Brower, M. A. Clark, S. D. Cohen, J. C. Osborn and C. Rebbi, PoS LAT2009, 031 (2009) [arXiv:0912.2186 [hep-lat]].

- "The removal of critical slowing down", J. Brannick, R. C. Brower, M. A. Clark, S. F. McCormick, T. A. Manteuffel, J. C. Osborn and C. Rebbi, PoS LATTICE2008, 035 (2008) [arXiv:0811.4331 [hep-lat]]. 
- "Algorithms for Lattice Field Theory at Extreme Scales", Richard C. Brower, Ronald , Anthony

Kennedy, James Osborn and Claudio Rebbi, SciDAC 2010, Chattanooga, TN (2010).

- "Hadronic Physics using Lattice OCD and GPUs" B. Joo, R. Babich, R. C. Brower, M. A . Clark, J. Chen, J. Dudek, R. G. Edwards, M. J. Peardon, C. Rebbi, D. G. Richards, G. Shi, C. Thomas, W. Watson, USOCD Collaboration and Hadron Spectrum Collaboration, SciDAC 2010, Chattanooga, TN (2010).

- "Solving Lattice QCD systems of equations using mixed precision solvers on GPUs", M. A. Clark, R. Babich, K. Barros, R. C. Brower and C. Rebbi, Comput. Phys. Commun. 181, 1517-1528 (2010) [arXiv:0911.3191 [hep-lat]].

- "Blasting through lattice calculations using CUDA", K. Barros, R. Babich, R. Brower, M. A. Clark and C. Rebbi, PoS LATTICE2008, 045 (2008) [arXiv:0810.5365 [heplat].

- "Moebius Algorithm for Domain Wall and GapDW Fermions", R. Brower, R. Babich, K. Orginos, C. Rebbi, D. Schaich and P. Vranas, PoS LATTICE2008, 034 (2008) [arXiv:0906.2813 [hep-lat]]. 


\section{QUDA: QCD on CUDA}

- OUDA library http://lattice.bu.edu/quda

- CG and BiCGstab solvers for Wilson and cloverimproved Wilson, mixed precision .

- Release 0.3 staggered fermions, contributed by Steve Gottlieb, Guochun Shi, ...

- Domain wall (Joel Giedt), twisted mass (contributed by Alexei Strelchenko), and multi-GPU ...

- Interfaced to Chroma/QDP++, QDP/C, CPS, etc. 


\section{MG Dirac Solvers for fun and profit.}

Lattice QCD/CFT

Graphene
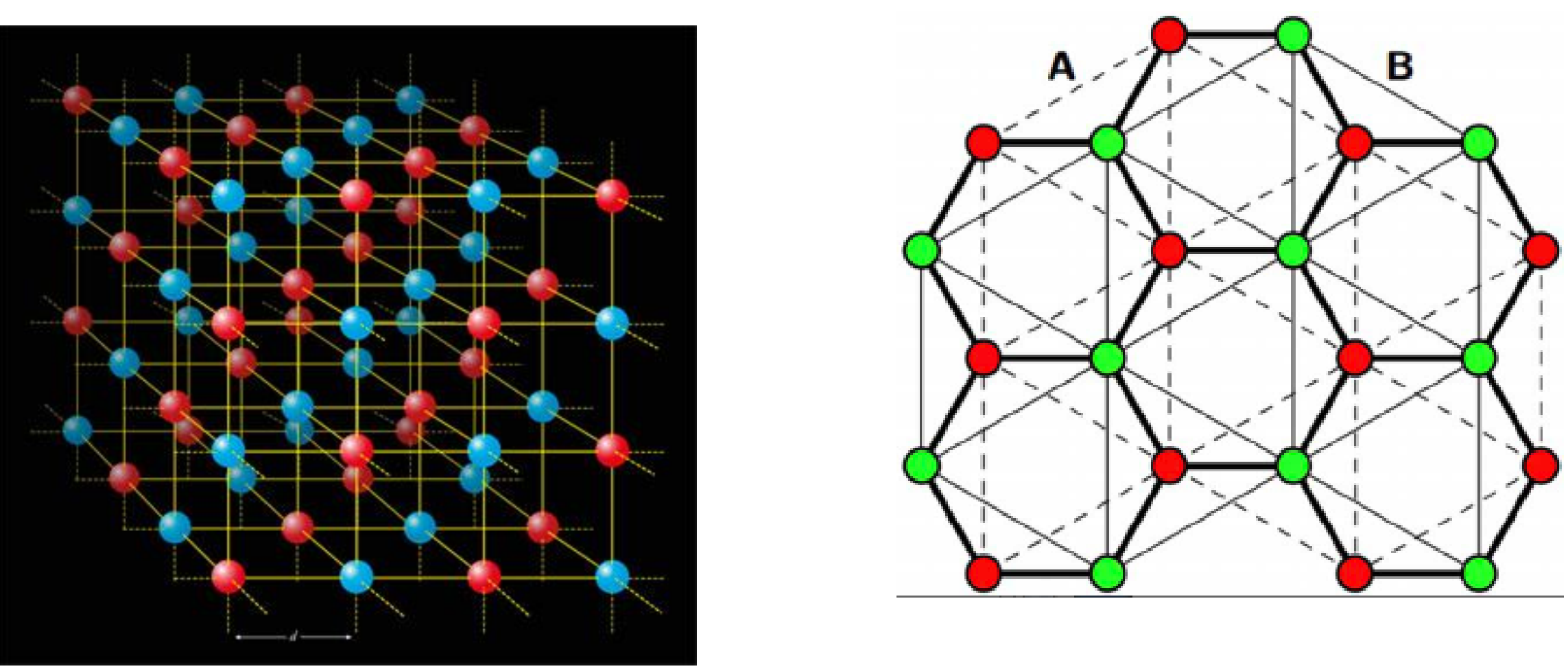


\section{Lack of Critical slowing down:}

\section{CG iteration count is insensitive to quark mass and lattice volume!}

\begin{tabular}{|c|c|c|c|c|}
\hline & \multicolumn{3}{|c|}{ Lattice volumes } & $m_{s}(-0.38922)$ \\
\hline Mass: & $16^{3} \times 64$ & $24^{3} \times 64$ & $32^{3} \times 96$ & \\
\hline-.3980 & 40 & 40 & 41 & \\
\hline-.4005 & 41 & 41 & 42 & \\
\hline-.4030 & 42 & 42 & 43 & \\
\hline-.4055 & 42 & 43 & 43 & Small increase is probabl \\
\hline-.4080 & 43 & 44 & 45 & not significant? \\
\hline-.4105 & 44 & 46 & 49 & \\
\hline-.4130 & 45 & 49 & 52 & \\
\hline-.4155 & 47 & 54 & 57 & physical $m 2_{1 / 4}$ \\
\hline-.4180 & 50 & 62 & 89 & Chiral limit: $m 2_{1 / 4}=0$ \\
\hline
\end{tabular}

"Adaptive Multigrid Algorithm for Lattice QCD" J. Brannick, R. C. Brower, M. A. Clark, J. C. Osborn, C. Rebbi, Phys. Rev. Lett. 100, 041601 (2008) 


\section{Clover performance (singleprecision)}

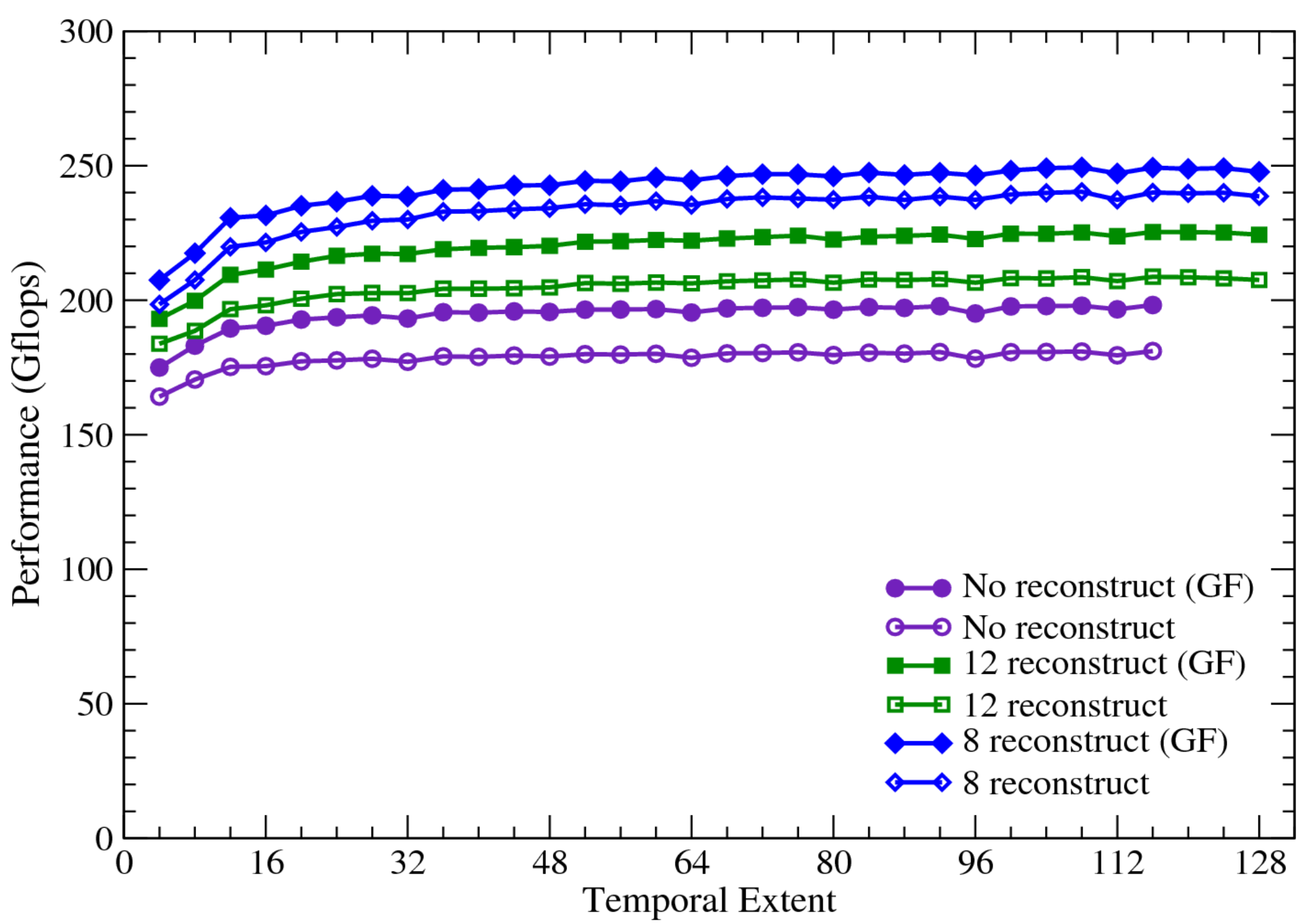




\section{Dslash performance (half precision)}

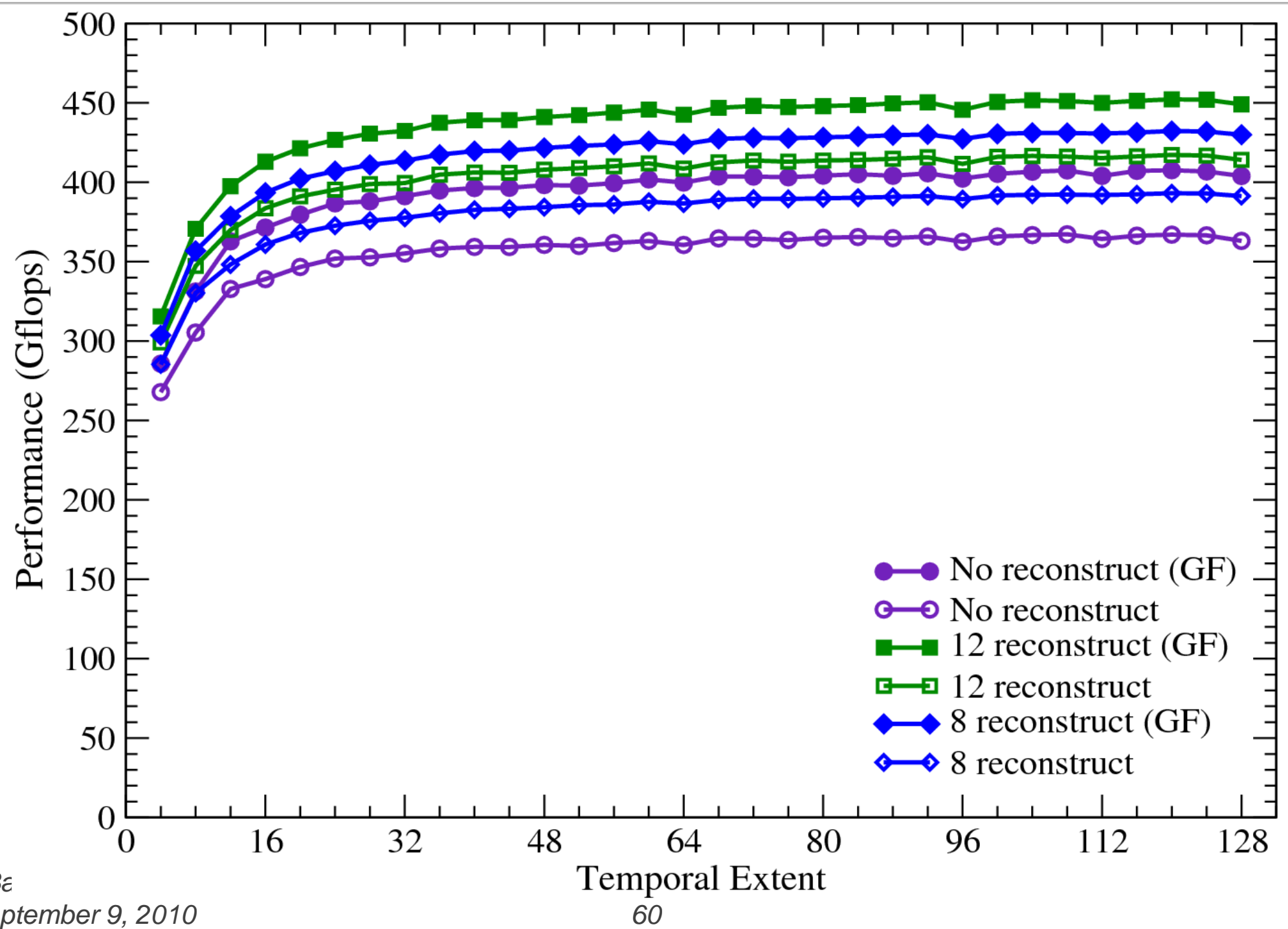




\section{Dslash performance (double precision)}

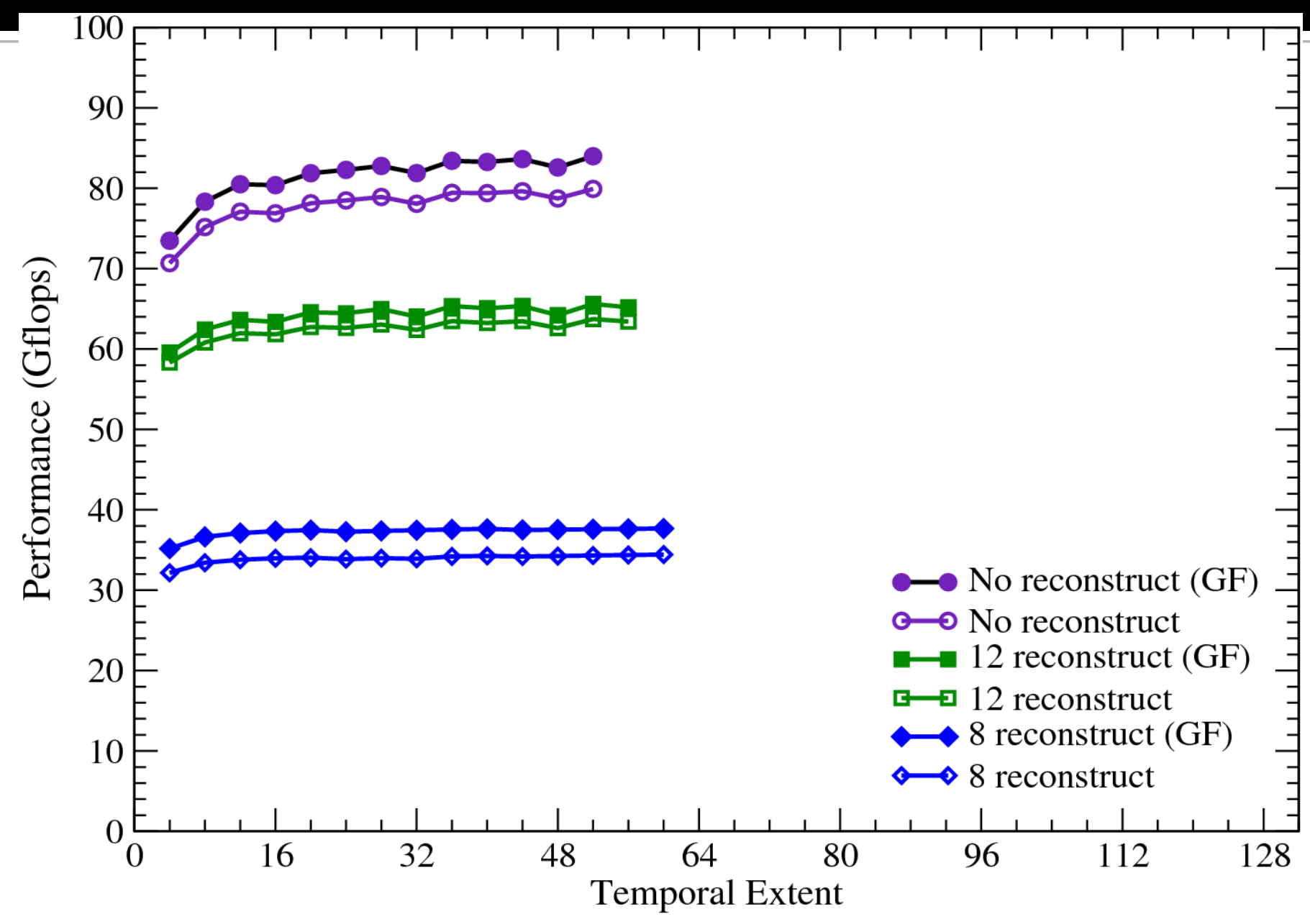




\section{GPUs are in serious use for "analysis"}

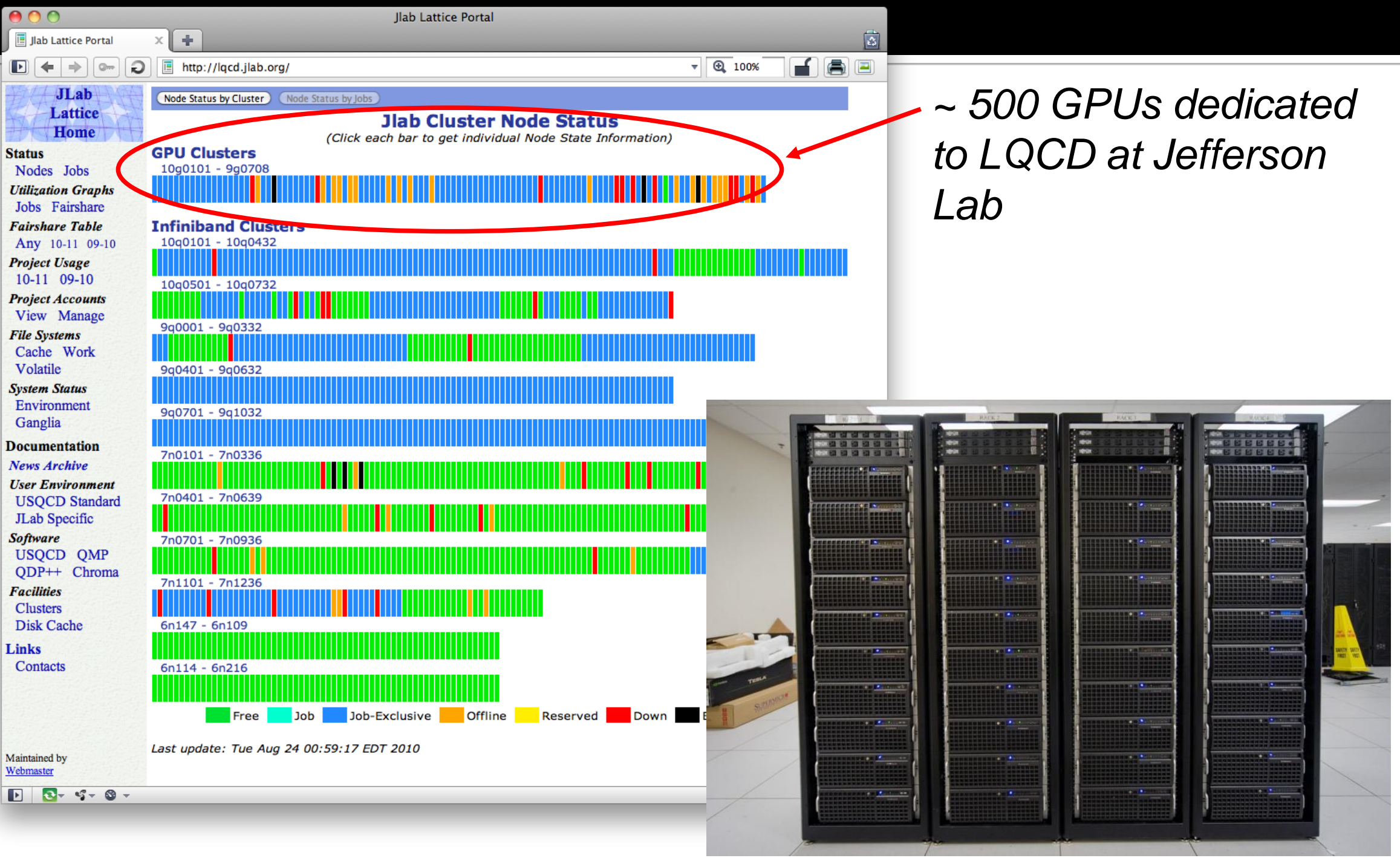

Ron Babich (BU) - QCDNA

VI, September 9, 2010 


\section{Can they also make a dent here?}

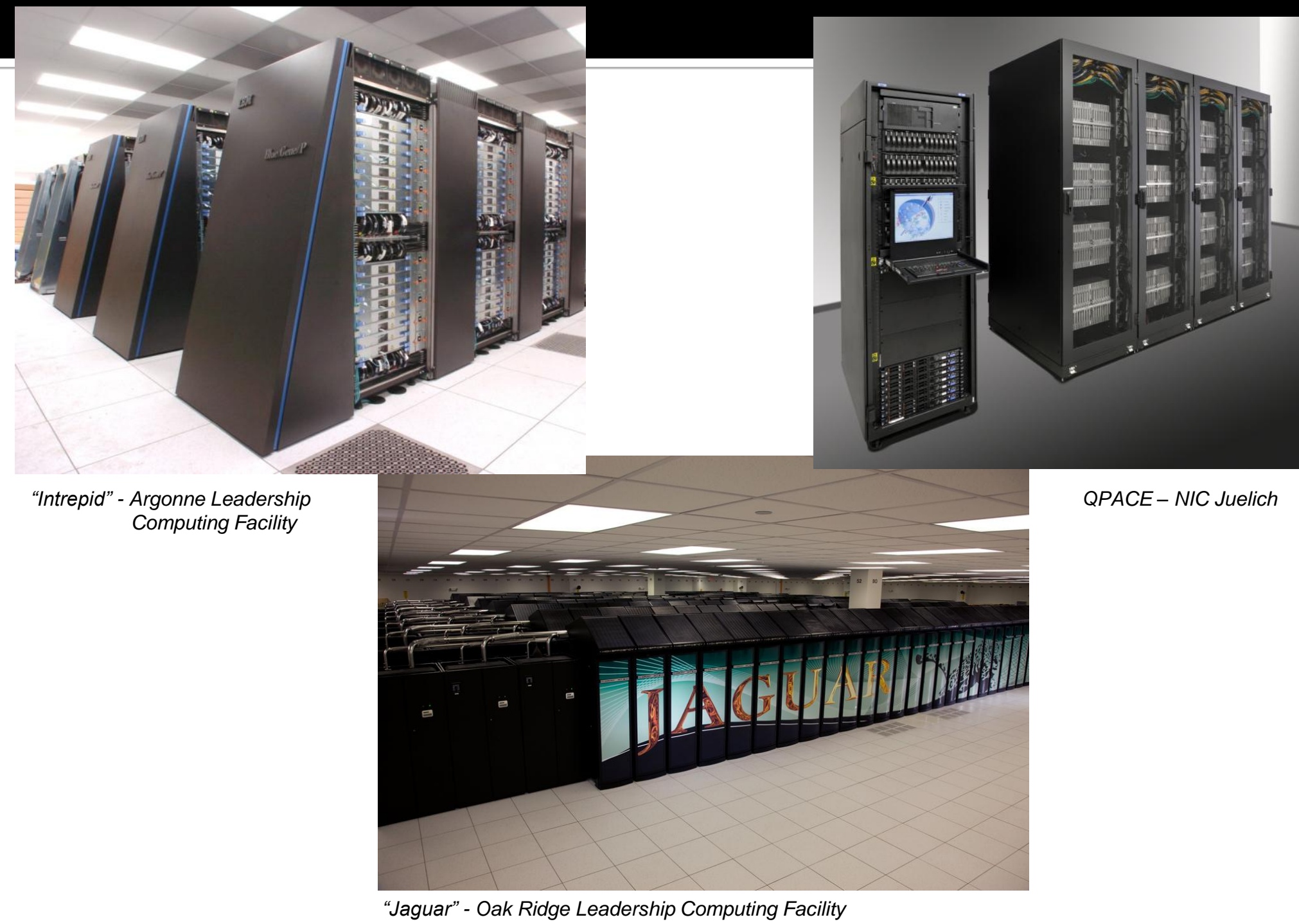




\section{ORNL's "Titan" 20 PF System Goals}

- Initial 1 PF delivery in 2011, final 20 PF system in 2012

- Designed for science from the ground up

- Similar number of cabinets, cabinet design, and cooling as Jaguar

- Operating system upgrade of today's Linux Operating System

- Gemini interconnect

- 3-D Torus

- Globally addressable memory

- Advanced synchronization features

- New accelerated node design using GPUs

- 20 PF peak performance

- 9x performance of today's XT5

- Larger memory

- 3x larger and $4 x$ faster file system 


\section{Part III: Future Challenges}

- Fermions PDEs are ubiquitous in Quantum Field theories an Nano materials. Lattice geometries and boundary condition present new fun challenges.

- Finite T lattice (e.g. $32 \times$ speed up on $128^{3} \times 96$ lattice*)

- Old/New RG Geometric/Adaptive Hybrid MG

- Monte Carlo Evolution of OCD: Sim implicit integrator

- Conformal Theories for LHC Higgsless models

- Radial lattices for conform/string duals

- Domain Wall/Overlap 5-d fore EXACT chirality

- Conformal Graphene Lagrangian

- $\quad$ etc etc ...

* has $4.8 \times 10 * * 9$ d.o.f. 


\section{NCSA: Blue Waters_2 (post IBM)}

- Blue Waters will be composed of more than 235 Cray XE6 cabinets based on the recently announced AMD Opteron ${ }^{\mathrm{TM}} 6200$ Series processor (formerly code-named "Interlagos") and more than 30 cabinets of a future version of the recently announced Cray XK6 supercomputer with NVIDIA ${ }^{\circledR}$ Tesla $^{\mathrm{TM}}$ GPU computing capability. 


\section{Potential Exascale Architecture Swimlanes}

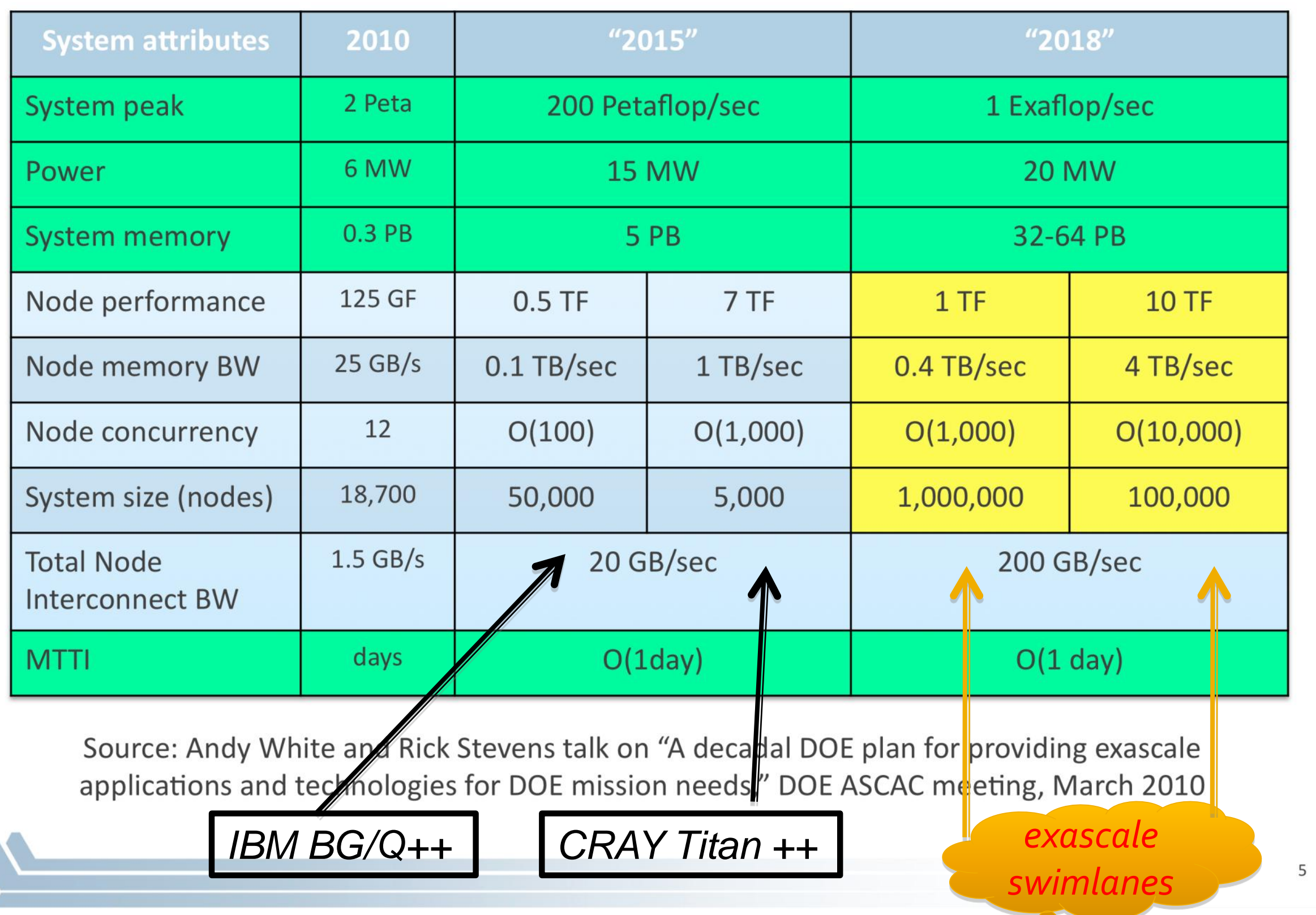




\section{-Stronger scaling on the EDGE}

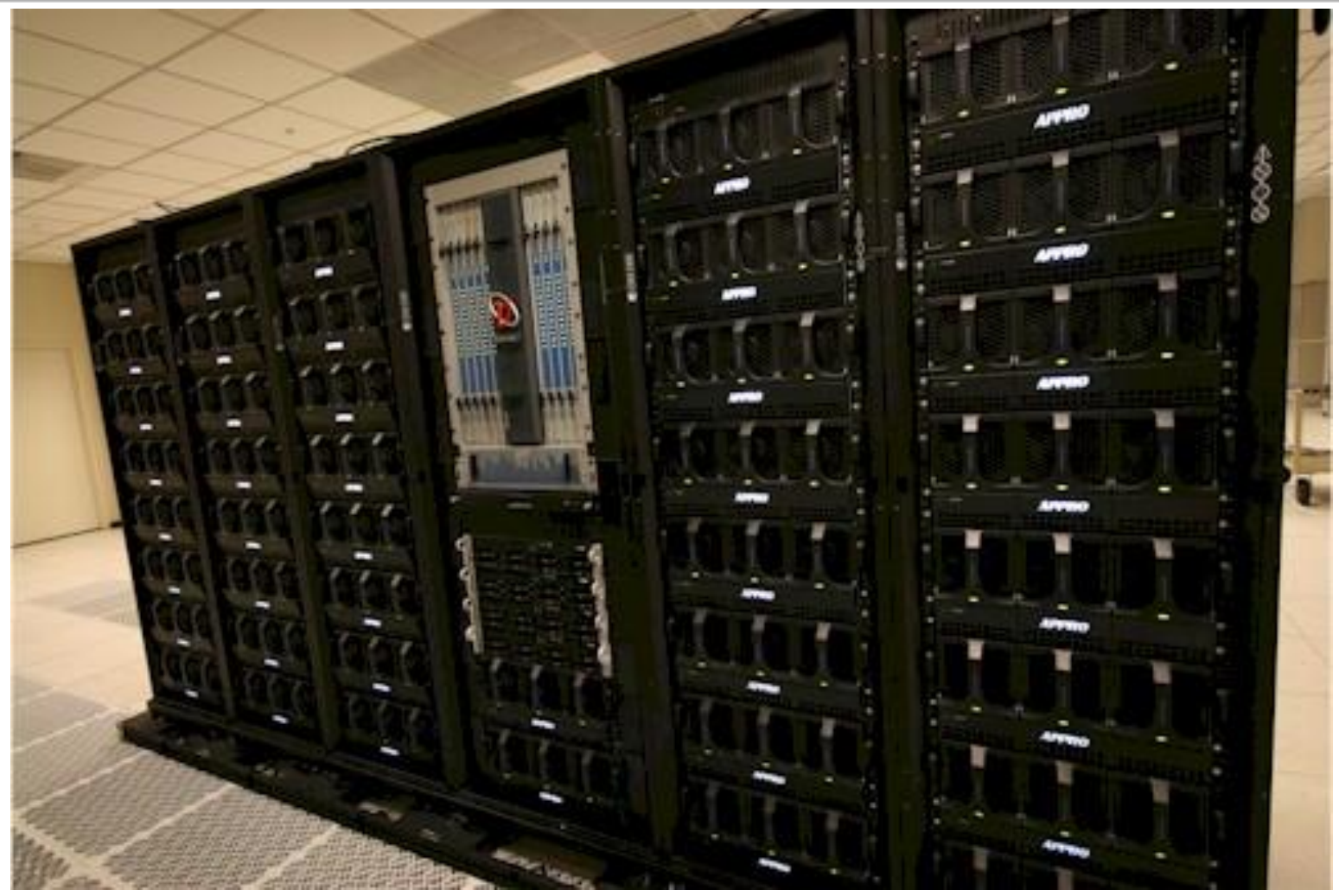

Scaling Lattice QCD beyond 100 GPUs

Authors: R. Babich, M. A. Clark, B. Joó, G. Shi, R. C. Brower, S. Gottlieb arXiv:1109.2935v1 [hep-lat] and SuperComputing 2011 


\section{Byte/flop ratios}

bandwidth to memory, rather than raw floating point throughput.

- We've seen that applying the Wilson Dirac operator (in single precision) requires that we move $\mathbf{1 4 4 0}$ bytes for every 1320 flops.

- Clover-improved Wilson (Sheikholeslami-Wohlert) is a bit "better" (less memory-bound), at 1728 bytes and $\quad 1824$ flops.

- Asqtad and HISO are a bit worse, at 1560 bytes and 1146 flops.

- Other operations (beyond the matrix-vector product) are often much worse. For example, adding two vectors of length $N$ involves reading/writing a total of $12 N$ bytes but consists of only $\mathbf{N}$ flops. 


\section{CUDA programming model}

Kernel launched by host

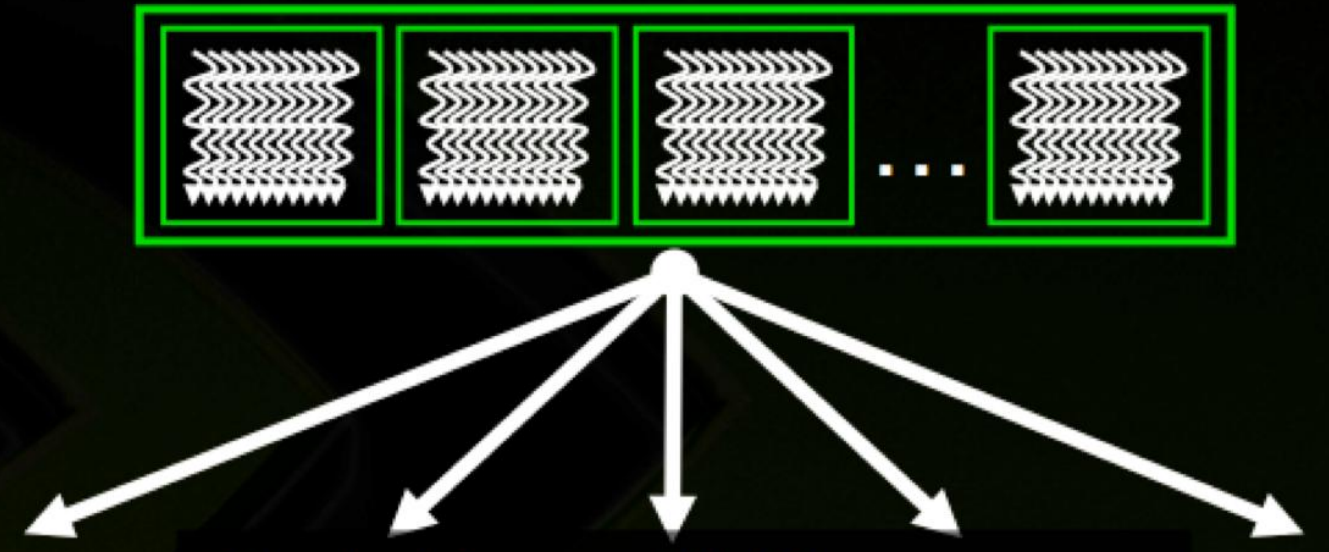

Blocks Run on Multiprocessors

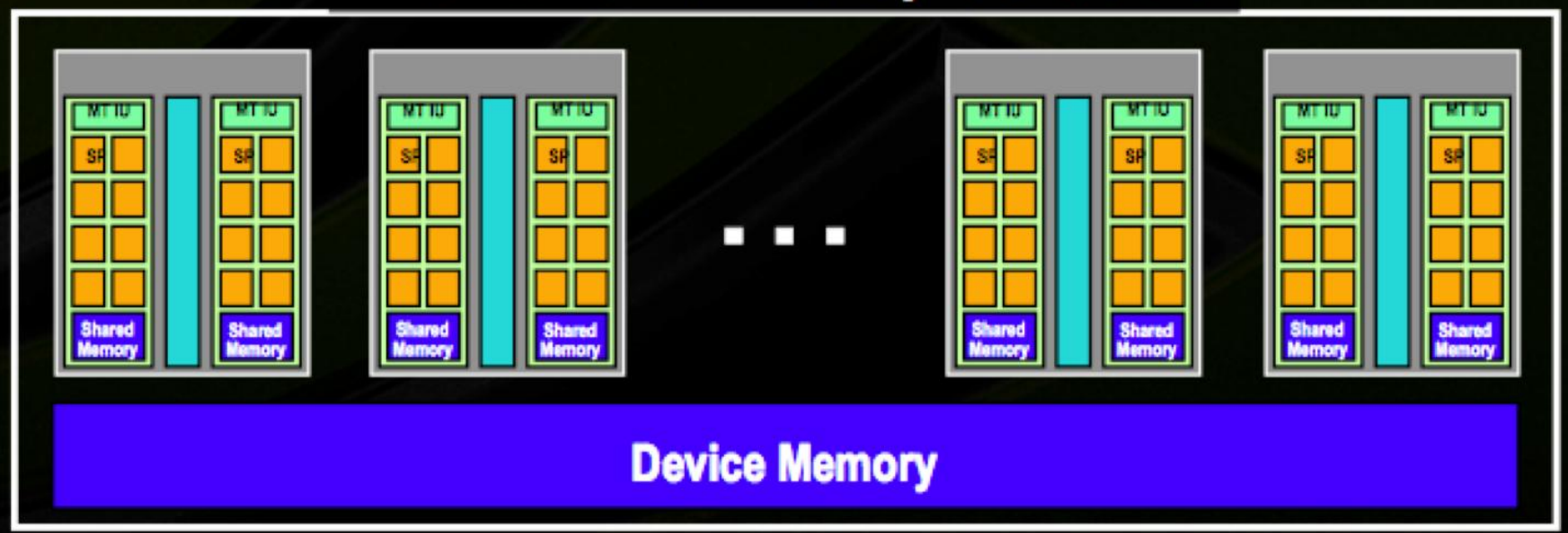




\section{CUDA PROGRAMMING}

void saxpy_serial (int $n$, float $a$, float ${ }^{*} x$, float ${ }^{*} y$ )

\{

for (int $i=0$; $\mathbf{i}<\mathbf{n} ;++\mathbf{i}$ )

$y[i]=a^{*} x[i]+y[i]$

\}

// Invoke serial SAXPY kerne1

saxpy_serial $(n, 2.0, x, y)$;

Standard C Code

_global_ void saxpy_parallel(int $n$, float a, float *x, float *y) \{

int $\mathbf{i}=$ blockIdx. $\mathbf{x}^{*}$ blockDim.x + threadIdx.x;

if $(i<n) \quad y[i]=a^{*} x[i]+y[i]$;

\}

// Invoke paralle1 SAXPY kerne1 with 256 threads/block int nblocks $=(n+255) / 256$;

saxpy_para11e1 $<<<$ nblocks, 256 $>(n, 2.0, x, y)$; 


\section{A tale of two processors}

\section{"Gulftown"}

- Intel Xeon X5680

- 6 cores (each with 4-wide SSE unit)

- 1.17 billion transistors

- Shared L3 Cache: $12 \mathrm{MB}$

- L1+L2: $6 \times(320 \mathrm{~KB})=1920 \mathrm{~KB}$

- 160 Gflops (SP)

- $\quad 32 \mathrm{~GB} / \mathrm{s}$ memory bandwidth

- up to $288 \mathrm{~GB}$ ( $96 \mathrm{~GB}$ is realistic)

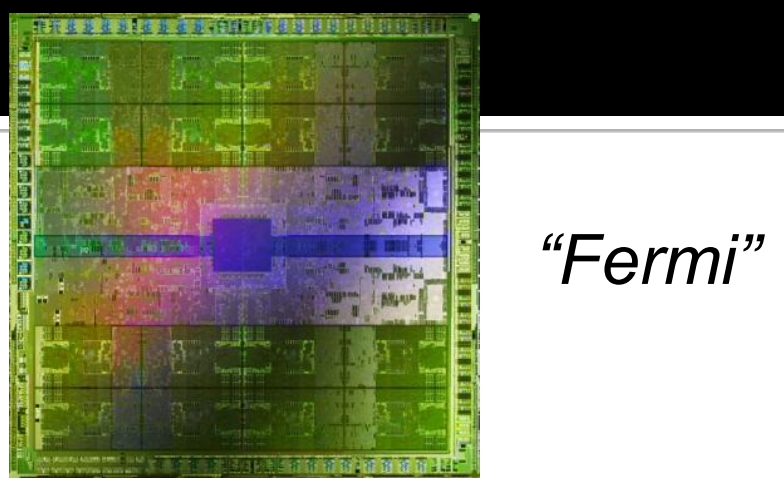

- NVIDIA GeForce GTX 480

- 480 cores

- 3.0 billion transistors

- Shared L2 Cache: 768 KB

- L1+SM+Reg: $15 \times 192 \mathrm{~KB}=2880 \mathrm{~KB}$

- 1345 Gflops (SP)

- $177 \mathrm{~GB} / \mathrm{s}$ memory bandwidth

- $1.5 \mathrm{~GB}$ (up to $6 \mathrm{~GB}$ in Tesla variant) 


\section{Mixed precision with reliable updates}

- As discussed yesterday, in the usual method of iterative refinement (or "defect correction"), the Krylov subspace is thrown away at every restart:

$$
\begin{aligned}
& r_{0}=b-A x_{0} ; \\
& k=0 ; \\
& \text { while }\left\|r_{k}\right\|>\epsilon \text { do } \\
& \qquad \begin{array}{l}
\text { Solve } A p_{k+1}=r_{k} \text { to precision } \epsilon^{i n} \\
x_{k+1}=x_{k}+p_{k+1} \\
r_{k+1}=b-A x_{k+1} \\
k=k+1
\end{array} \\
& \text { end }
\end{aligned}
$$

An alternative is "reliable updates," originally introduced to combat residual drift caused by the erratic convergence of BiCGstab: G. L. G. Sleijpen, and H. A. van der Vorst, "Reliable updated residuals in hybrid Bi-CG methods," Computing 56, 141-164 (1996). 


\title{
Chiral Symmetry and Residual Mass in Lattice QCD with Domain-Wall Fermions
}

\author{
Ting-Wai Chiu (趙挺偉) \\ Physics Department \\ National Taiwan University
}

"New Horizons for Lattice Computations with Chiral Fermions" Brookhaven National Laboratory, USA May 14-18, 2012 


\section{Outline}

- Introduction

- Axial Ward Idenity in Lattice QCD with ODWF

- Generating Functional for n-point Green's Function

- A New Formula for the Residual Mass

- An Upper Bound for the Residual Mass

- Concluding Remarks

Reference:

Yu-Chih Chen, TWC, "Chiral Symmetry and Residual Mass in Lattice QCD with Optimal Domain-Wall Fermion", in preparation 


\section{Domain-Wall Fermions [Kaplan, 1992]}

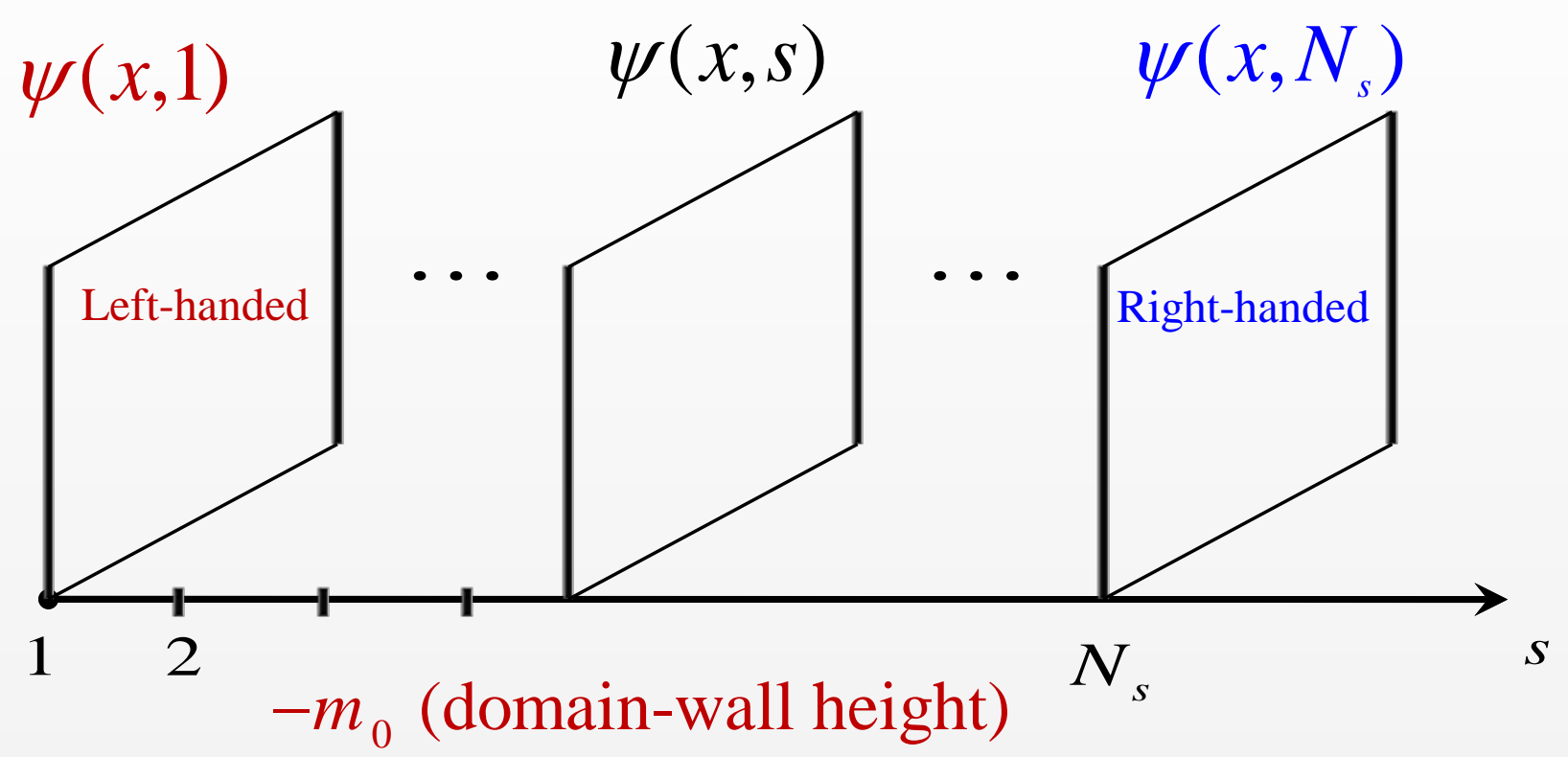

$D_{\text {dwf }}$ is a local op. with the nearest neighbor coupling along $\hat{s}$

$$
\int[d \bar{\psi}][d \psi] \exp \left(-\bar{\Psi} D_{\mathrm{dwf}} \Psi\right)=\operatorname{det} D_{c} \quad D_{c}=\frac{1+\gamma_{5} S}{1-\gamma_{5} S}
$$

$N_{s} \rightarrow \infty, S \rightarrow \frac{H}{\sqrt{H^{2}}}, \quad D_{c} \gamma_{5}+\gamma_{5} D_{c}=0$, Exact Chiral Sym.

At finite $N_{s}, S$ is not equal to the optimal rational approx. 


\section{Optimal Rational Approximation for Square Root}

For the inverse square root function, the optimal rational approx. was obtained by Zolotarev in 1877.

$$
\begin{aligned}
& \frac{1}{\sqrt{x}}, x \in[1, b] \\
& R_{Z}^{(n-1, n)}(x)=\frac{2 \Lambda}{1+\Lambda} \frac{1}{M} \frac{\prod_{l=1}^{n-1}\left(1+x / C_{2 l}\right)}{\prod_{l=1}^{n}\left(1+x / C_{2 l-1}\right)} \\
& R_{Z}^{(n, n)}(x)=\frac{2 \lambda}{1+\lambda} \frac{1}{m} \frac{\prod_{l=1}^{n}\left(1+x / c_{2 l}\right)}{\prod_{l=1}^{n}\left(1+x / c_{2 l-1}\right)}
\end{aligned}
$$

where $\lambda, \Lambda, m, M, C_{2 l}, C_{2 l-1}, C_{2 l}, C_{2 l-1}$

are expressed in terms of the

Jacobian Elliptic functions.

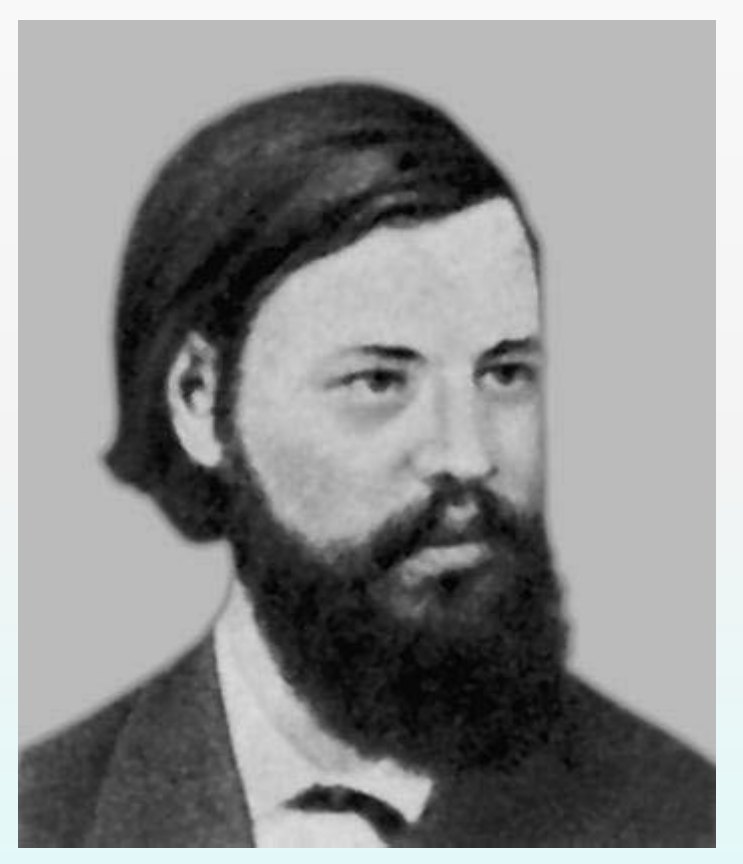

Yegor Ivanovich Zolotarev (1847-1878) 


\section{Salient Feature of Optimal Rational Approximation}

$$
1-\sqrt{x} R_{Z}^{(n, m)}(x)
$$

Has $(n+m+2)$ alternate change of sign in $\left[x_{\min }, x_{\max }\right]$, and attains its max. and min. (all with equal magnitude)

In the figure, $n=m=6$ it has 14 alternate change of sign in $[1,1000]$

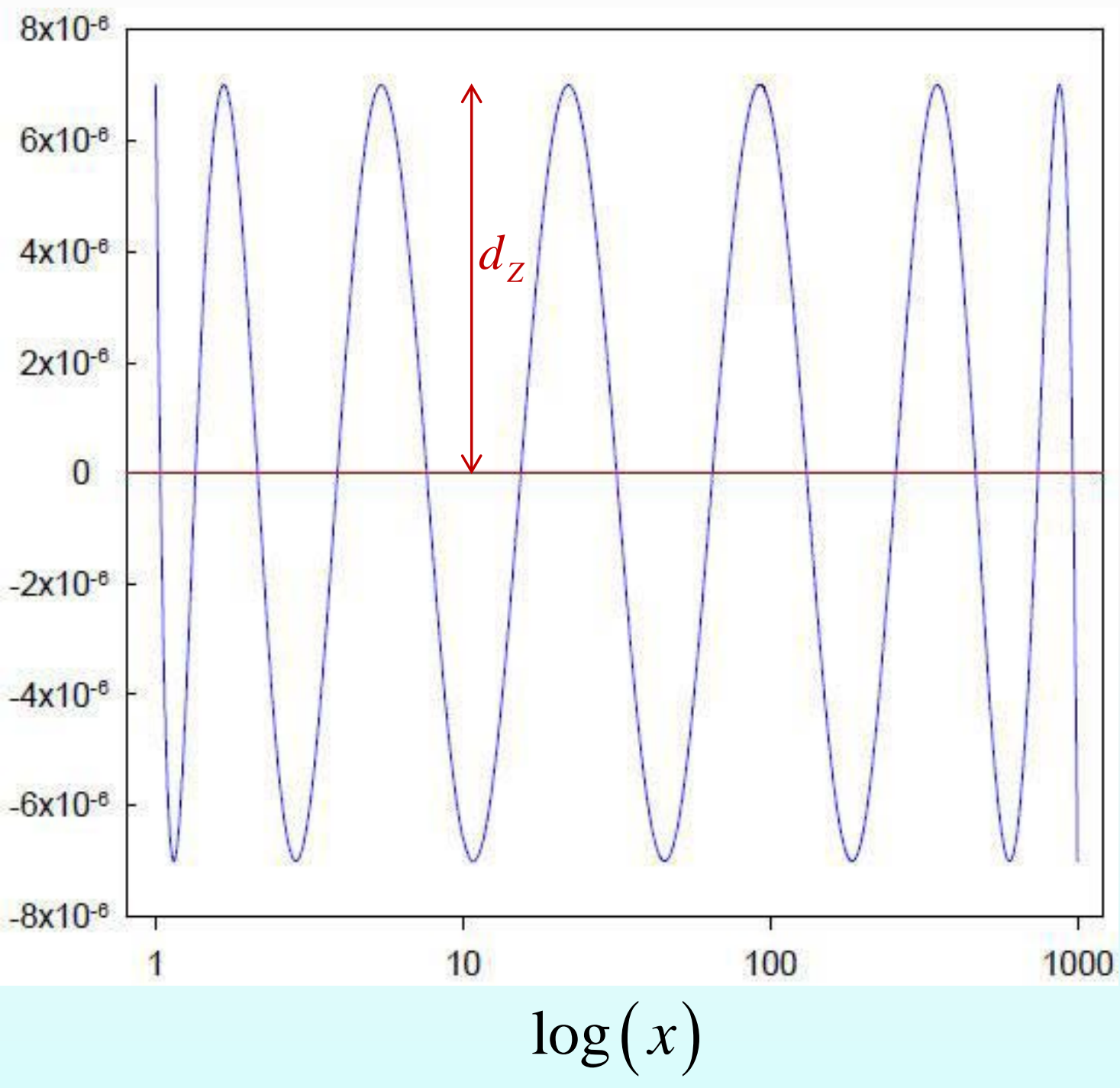




\section{Optimal Domain-Wall Fermion}

[ TWC, Phys. Rev. Lett. 90 (2003) 071601 ]

$$
\begin{aligned}
A_{\mathrm{odwf}}=\sum_{s, s^{\prime}=1}^{N_{s}} \sum_{x, x^{\prime}} \bar{\psi}_{x, s}\left[\left(I+\rho_{s} D_{w}\right)_{x, x^{\prime}} \delta_{s, s^{\prime}}-\left(I-\sigma_{s} D_{w}\right)_{x, x^{\prime}}\left(P_{-} \delta_{s^{\prime}, s+1}+P_{+} \delta_{s^{\prime}, s-1}\right)\right] \psi_{x^{\prime}, s^{\prime}} \\
\equiv \bar{\Psi} D_{\mathrm{odwf}} \Psi \quad m_{0} \in(0,2) \\
D_{w}=\sum_{\mu=1}^{4} \gamma_{\mu} t_{\mu}+W-m_{0}, \quad m_{\mu}\left(x, x^{\prime}\right)=\frac{1}{2}\left[U_{\mu}(x) \delta_{x^{\prime}, x+\mu}-U_{\mu}^{\dagger}\left(x^{\prime}\right) \delta_{x^{\prime}, x-\mu}\right] \\
W\left(x, x^{\prime}\right)=\sum_{\mu=1}^{4} \frac{1}{2}\left[2 \delta_{x, x^{\prime}}-U_{\mu}(x) \delta_{x^{\prime}, x+\mu}-U_{\mu}^{\dagger}\left(x^{\prime}\right) \delta_{x^{\prime}, x-\mu}\right]
\end{aligned}
$$

with boundary conditions

$$
\begin{aligned}
P_{+} \psi(x, 0)=-r m_{q} P_{+} \psi\left(x, N_{s}\right), & m_{q} \text { : bare quark mass } \\
P_{-} \psi\left(x, N_{s}+1\right)=-r m_{q} P_{-} \psi(x, 1), & P_{ \pm}=\frac{1}{2}\left(1 \pm \gamma_{5}\right)
\end{aligned}
$$




\section{Optimal Domain-Wall Fermion (cont.)}

The action for Pauli-Villars fields is similar to $A_{\text {odwf }}$

$A_{P V}=\sum_{s, s^{\prime}=1}^{N_{s}} \sum_{x, \chi^{\prime}} \bar{\phi}_{x, s}\left[\left(I+\rho_{s} D_{w}\right)_{x, x^{\prime}} \delta_{s, s^{\prime}}-\left(I-\sigma_{s} D_{w}\right)_{x, x^{\prime}}\left(P_{-} \delta_{s^{\prime}, s+1}+P_{+} \delta_{s^{\prime}, s-1}\right)\right] \phi_{x^{\prime}, s^{\prime}}$

but with boundary conditions: $P_{+} \phi(x, 0)=-P_{+} \phi\left(x, N_{s}\right)$,

$$
P_{-} \phi\left(x, N_{s}+1\right)=-P_{-} \phi(x, 1)
$$

$>$ In the original formulation of ODWF, $\rho_{s}=\sigma_{s}=\omega_{s}$

$$
\omega_{s}=\frac{1}{\lambda_{\min }} \sqrt{1-\kappa^{\prime 2} s n^{2}\left(v_{s} ; \kappa^{\prime}\right)}, \quad s=1, \cdots, N_{s}
$$

where $\operatorname{sn}\left(v_{s} ; \kappa^{\prime}\right)$ is the Jacobian elliptic function with argument $v_{s}$ and modulus $\kappa^{\prime}=\sqrt{1-\lambda_{\min }^{2} / \lambda_{\max }^{2}}$,

$\lambda_{\min }^{2}$ and $\lambda_{\max }^{2}$ are lower and upper bounds of the eigenvalues of $H_{w}^{2}$ 


\section{Optimal Domain-Wall Fermion (cont.)}

$\int[d \bar{\psi}][d \psi][d \bar{\phi}][d \phi] \exp \left(-A_{\mathrm{odwf}}-A_{\mathrm{PV}}\right)=\operatorname{det} D\left(m_{q}\right)$

The effective 4D Dirac operator

$$
\begin{aligned}
D\left(m_{q}\right)= & m_{q}+\left(m_{0}-m_{q} / 2\right)\left[1+\gamma_{5} S_{o p t}\left(H_{w}\right)\right] \\
S_{o p t}\left(H_{w}\right) & =\frac{1-\prod_{s=1}^{N_{s}} T_{s}}{1+\prod_{s=1}^{N_{s}} T_{s}}, \quad T_{s}=\frac{1-\omega_{s} H_{w}}{1+\omega_{s} H_{w}} \\
& = \begin{cases}H_{w} R_{Z}^{(n-1, n)}\left(H_{w}^{2}\right), & N_{s}=2 n \\
H_{w} R_{Z}^{(n, n)}\left(H_{w}^{2}\right), & N_{s}=2 n+1\end{cases}
\end{aligned}
$$

Zolotarev optimal rational approximation for $\frac{1}{\sqrt{H_{w}^{2}}}$ 


\section{Optimal Domain-Wall Fermion (cont.)}

$>$ For $\rho_{s}=c \omega_{s}+d, \sigma_{s}=c \omega_{s}-d, \quad c, d$ (constants)

The effective 4D Dirac operator becomes

$$
\begin{aligned}
D\left(m_{q}\right)=m_{q} & +\left(m_{0}\left(1-d m_{0}\right)-\frac{m_{q}}{2}\right)\left[1+\gamma_{5} S_{\text {opt }}(H)\right], H=\frac{c H_{w}}{1+d \gamma_{5} H_{w}} \\
S_{\text {opt }}(H) & =\frac{1-\prod_{s=1}^{N_{s}} T_{s}}{1+\prod_{s=1}^{N_{s}} T_{s}}, \quad T_{s}=\frac{1-\omega_{s} H}{1+\omega_{s} H} \quad \begin{array}{l}
\text { only } d=0 \text { is good } \\
\text { for the projection of } \\
\text { low-modes of } D(0)
\end{array} \\
& = \begin{cases}H R_{Z}^{(n-1, n)}\left(H^{2}\right), \quad N_{s}=2 n \\
H R_{Z}^{(n, n)}\left(H^{2}\right), N_{s}=2 n+1\end{cases}
\end{aligned}
$$




\section{Optimal Domain-Wall Fermion (cont.)}

$>$ For the special case $\rho_{s}=1, \sigma_{s}=0$

It reduces to the conventional DWF which does not have the optimal chiral symmetry.

$$
\begin{aligned}
& \begin{array}{l}
D\left(m_{q}\right)=m_{q}+\left(\frac{m_{0}}{2}\left(2-m_{0}\right)-\frac{m_{q}}{2}\right)\left[1+\gamma_{5} S_{\text {polar }}(H)\right], \quad H=\frac{H_{w}}{2+\gamma_{5} H_{w}} \\
S_{\text {polar }}(H)=\frac{1-T^{N_{s}}}{1+T^{N_{s}}}, \quad T=\frac{1-H}{1+H}
\end{array} \\
& b_{l}=\sec ^{2}\left[\frac{\pi}{N_{s}}\left(l-\frac{1}{2}\right)\right] \quad=\left\{\begin{array}{l}
H\left(\frac{2}{N_{s}} \sum_{l=1}^{n} \frac{b_{l}}{H^{2}+d_{l}}\right), \quad N_{s}=2 n \\
H\left(\frac{1}{N_{s}}+\frac{2}{N_{s}} \sum_{l=1}^{n} \frac{b_{l}}{H^{2}+d_{l}}\right), N_{s}=2 n+1
\end{array}\right. \\
& \quad \begin{array}{l}
\text { Polar approximation } \\
d_{l}=\tan ^{2}\left[\frac{\pi}{N_{s}}\left(l-\frac{1}{2}\right)\right]
\end{array}
\end{aligned}
$$




\section{Axial Ward Idenity in Lattice QCD with ODWF}

$$
\mathcal{A}_{f}=\sum_{s, s^{\prime}=0}^{N_{s}+3} \sum_{x, x^{\prime}} \bar{\psi}_{s}(x)\left\{\left(\rho_{s} D_{w}+\mathbb{I}\right)_{x, x^{\prime}} \delta_{s_{q}, s^{\prime}}+\left(\sigma_{s} D_{w}-\mathbb{I}\right)_{x, x^{\prime}}\left(P_{-} \delta_{s^{\prime}, s+1}+P_{+} \delta_{s^{\prime}, s-1}\right)\right\} \psi_{s^{\prime}}\left(x^{\prime}\right)
$$

with boundary conditions

$$
\begin{aligned}
P_{+} \psi(x,-1) & =-r m_{q} P_{+} \psi\left(x, N_{s}+3\right), \\
P_{-} \psi\left(x, N_{s}+4\right) & =-r m_{q} P_{-} \psi(x, 0)
\end{aligned}
$$

Transparent layers: $\quad \rho_{0}=\rho_{N_{s}+3}=\sigma_{0}=\sigma_{N_{s}+3}=0$

[TWC, hep-lat/0303008]

$$
\rho_{n}=\rho_{n+1}=\sigma_{n}=\sigma_{n+1}=0 \quad n=\left[N_{s} / 2\right]
$$

The quark fields are defined in terms of the boundary modes

$$
\begin{aligned}
& q(x)=\sqrt{r}\left[P_{-} \psi_{0}(x)+P_{+} \psi_{N_{s}+3}(x)\right] \\
& \bar{q}(x)=\sqrt{r}\left[\bar{\psi}_{0}(x) P_{+}+\bar{\psi}_{N_{s}+3}(x) P_{-}\right]
\end{aligned}
$$




\section{Axial Ward Idenity (cont.)}

Now we consider $N_{f}$ flavors of quarks with degenerate mass $m_{q}$, and the infinitesimal flavor non-singlet transformation

$$
\begin{gathered}
\delta \psi_{s}(x)=i \theta_{s}(x) \lambda^{a} \psi_{s}(x) \\
\delta \bar{\psi}_{s}(x)=-i \theta_{s}(x) \lambda^{a} \bar{\psi}_{s}(x) \\
\theta_{s}(x)= \begin{cases}\theta(x), & 0 \leq s \leq n, n \equiv\left[\frac{N_{s}}{2}\right], \\
-\theta(x), & n+1 \leq s \leq N_{s}+3 .\end{cases}
\end{gathered}
$$

Here $\lambda^{a}$ is one of the flavor group generators in the fundamental representation

$$
\delta \sum_{s=0}^{N_{s}+3} \sum_{x, u} \rho_{s}\left[\bar{\psi}_{s}(x) \lambda^{a} D_{w}(x, y) \psi_{s}(y)\right]=\sum_{x} i \theta(x) \sum_{\mu} \Delta_{\mu} \hat{j}_{\mu}^{a}(x)
$$

$$
\begin{gathered}
\delta \sum_{s=0}^{N_{s}+3}\left[-\bar{\psi}_{s}(x) \lambda^{a} P_{-} \psi_{s+1}(x)-\bar{\psi}_{s}(x) \lambda^{a} P_{+} \psi_{s-1}(x)\right]=-\sum_{x} 2 i \theta(x)\left[J_{5}^{a}(x, n)+m_{q} \bar{q}(x) \lambda^{a} \gamma_{5} q(x)\right] \\
\delta \sum_{s=0}^{N_{s}+3} \sum_{x, y} \sigma_{s}\left\{\bar{\psi}_{s}(x) \lambda^{a} D_{w}(x, y)\left[P_{-} \psi_{s+1}(y)+P_{+} \psi_{s-1}(y)\right]\right\}=\sum_{x} i \theta(x) \sum_{\mu} \Delta_{\mu} \hat{k}_{\mu}^{a}(x)
\end{gathered}
$$




\section{Axial Ward Idenity (cont.)}

$$
\begin{aligned}
& J_{5}^{a}(x, n)=-\bar{\psi}_{n}(x) \lambda^{a} P_{-} \psi_{n+1}(x)+\bar{\psi}_{n+1}(x) \lambda^{a} P_{+} \psi_{n}(x) \\
& \hat{j}_{\mu}^{a}(x) \equiv \sum_{s=1}^{N_{s}+2} \operatorname{sign}\left(n-s+\frac{1}{2}\right) j_{\mu}^{a}(x, s) \\
& j_{\mu}^{a}(x, s)=\frac{\rho_{s}}{2}\left[\bar{\psi}_{s}(x) \lambda^{a}\left(1-\gamma_{\mu}\right) U_{\mu}(x) \psi_{s}(x+\mu)-\bar{\psi}_{s}(x+\mu) \lambda^{a}\left(1+\gamma_{\mu}\right) U_{\mu}^{\dagger}(x) \psi_{s}(x)\right] \\
& \hat{k}_{\mu}^{a}(x) \equiv \hat{k}_{\mu}^{a+}(x)+\hat{k}_{\mu}^{a-}(x), \\
& \hat{k}_{\mu}^{a \pm}(x) \equiv \sum_{s=1}^{N_{s}+2} \operatorname{sign}\left(n-s+\frac{1}{2}\right) k_{\mu}^{a \pm}\left(x, s^{\prime}\right. \\
& k_{\mu}^{a \pm}(x, s)=\frac{\sigma_{s}}{2}\left[\bar{\psi}_{s}(x) \lambda^{a}\left(1-\gamma_{\mu}\right) U_{\mu}(x) P_{ \pm} \psi_{s \mp 1}(x+\mu)-\bar{\psi}_{s}(x+\mu) \lambda^{a}\left(1+\gamma_{\mu}\right) U_{\mu}^{\dagger}(x) P_{ \pm} \psi_{s \mp 1}(x)\right] \\
& J_{\mu}^{a}(x) \equiv \hat{k}_{\mu}^{a}(x)+\hat{j}_{\mu}^{a}(x) \\
& \Delta_{\mu} f(x, s) \equiv f(x, s)-f(x-\mu, s)
\end{aligned}
$$




\section{Axial Ward Idenity (cont.)}

For any observable $\mathcal{O}, \delta^{a}\langle\mathcal{O}\rangle=0$, which gives the axial Ward identity

$$
\Delta_{\mu}\left\langle J_{\mu}^{a}(x) \mathcal{O}(y)\right\rangle=2 m_{q}\left\langle\bar{q}(x) \lambda^{a} \gamma_{5} q(x) \mathcal{O}(y)\right\rangle+2\left\langle J_{5}^{a}(x, n) \mathcal{O}(y)\right\rangle+i\left\langle\delta^{a} \mathcal{O}(y)\right\rangle
$$

As $N_{s} \rightarrow \infty$, the anomalous term $\left\langle J_{5}^{a}(x, n) \mathcal{O}(y)\right\rangle$ vanishes if

$\mathcal{O}(y)$ only involves the quark fields [Furman \& Shamir, 1995]

After summing over all sites $x$

$$
-i \sum_{x}\left\langle\delta^{a} \mathcal{O}(y)\right\rangle=2 m_{q} \sum_{x}\left\langle\bar{q}(x) \lambda^{a} \gamma_{5} q(x) \mathcal{O}(y)\right\rangle+2 \sum_{x}\left\langle J_{5}^{a}(x, n) \mathcal{O}(y)\right\rangle
$$

Thus, the effect of chiral symmetry breaking due to finite $N_{s}$ can be regarded as an additive mass to the bare quark mass $m_{q}$, the so-called residual mass

$$
m_{\text {res }}[\mathcal{O}(y)]=\frac{\sum_{x}\left\langle J_{5}^{a}(x, n) \mathcal{O}(y)\right\rangle}{\sum_{x}\left\langle\bar{q}(x) \lambda^{a} \gamma_{5} q(x) \mathcal{O}(y)\right\rangle} \quad \text { dependent on } \mathrm{O} \text {, and } \mathrm{y}
$$




\section{Axial Ward Idenity (cont.)}

Summing over all sites $y$ in the AWI gives the global residual mass

$$
M_{\text {res }}[\mathcal{O}]=\frac{\sum_{x, y}\left\langle J_{5}^{a}(x, n) \mathcal{O}(y)\right\rangle}{\sum_{x, y}\left\langle\bar{q}(x) \lambda^{a} \gamma_{5} q(x) \mathcal{O}(y)\right\rangle}
$$

For $\mathcal{O}(y)=\bar{q}(y) \lambda^{b} \gamma_{5} q(y) \quad$ pseudoscalar

$$
\begin{array}{rlr}
m_{\text {res }}(y) & =\frac{\sum_{x}\left\langle J_{5}^{a}(x, n) \bar{q}(y) \lambda^{b} \gamma_{5} q(y)\right\rangle}{\sum_{x}\left\langle\bar{q}(x) \lambda^{a} \gamma_{5} q(x) \bar{q}(y) \lambda^{b} \gamma_{5} q(y)\right\rangle} \quad \text { local } \\
M_{\text {res }} & =\frac{\sum_{x, y}\left\langle J_{5}^{a}(x, n) \bar{q}(y) \lambda^{b} \gamma_{5} q(y)\right\rangle}{\sum_{x, y}\left\langle\bar{q}(x) \lambda^{a} \gamma_{5} q(x) \bar{q}(y) \lambda^{b} \gamma_{5} q(y)\right\rangle} \quad \text { global }
\end{array}
$$

which are usually used as a measure of the chiral symmetry breaking due to finite $N_{s}$ 


\section{Generating Functional for n-point Green's Function}

Use the transformation

$$
\begin{aligned}
\eta_{s} & =\left(P_{-} \delta_{s^{\prime}, s}+P_{+} \delta_{s^{\prime}, s-1}\right) \psi_{s^{\prime}} \Leftrightarrow \psi_{s}=\left(P_{-} \delta_{s^{\prime}, s}+P_{+} \delta_{s^{\prime}, s+1}\right) \eta_{s^{\prime}} \\
\bar{\eta}_{s} & =\bar{\psi}_{s} \gamma_{5} Q_{-}^{s} \Leftrightarrow \bar{\psi}_{s}=\bar{\eta}_{s}\left(Q_{-}^{s}\right)^{-1} \gamma_{5} \\
Q_{ \pm}^{s} & \equiv \rho_{s} H_{w} P_{ \pm}+\sigma_{s} H_{w} P_{\mp} \pm 1 \\
T_{s} & =-\left(Q_{+}^{s}\right)^{-1} Q_{-}^{s}=\frac{1-H_{s}}{1+H_{s}} \\
H_{s} & =\left(\rho_{s}+\sigma_{s}\right) H_{w}\left[2+\left(\rho_{s}-\sigma_{s}\right) \gamma_{5} H_{w}\right]^{-1} \\
\mathcal{A}_{f}= & \bar{\eta}_{0}\left(P_{-}-r m_{q} P_{+}\right) \eta_{0}-\bar{\eta}_{0} \eta_{0}+\sum_{s=1}^{N_{s}+2}\left[\bar{\eta}_{s} \eta_{s}-\bar{\eta}_{s} T_{s}^{-1} \eta_{s+1}\right] \\
& +\bar{\eta}_{N_{s}+3} \eta_{N_{s}+3}-\bar{\eta}_{N_{s}+3}\left(P_{+}-r m_{q} P_{-}\right) \eta_{0},
\end{aligned}
$$




\section{Generating Functional (cont.)}

Including external sources

$$
\begin{array}{cc}
\bar{\eta}_{n} J_{n}+\bar{J}_{n+1} \eta_{n+1}+\bar{\eta}_{n+1} J_{n+1}, & J \equiv \sqrt{r} J_{q} \\
\bar{J}_{q} q+\bar{q} J_{q}=\bar{J} \eta_{0}-\bar{\eta}_{0} P_{+} J+\bar{\eta}_{N_{s}+3} P_{-} J, & \bar{J} \equiv \sqrt{r} \bar{J}_{q} \\
Z\left[J_{q}, \bar{J}_{q}, J_{n}, J_{n+1}, \bar{J}_{n+1}\right]=\mathcal{J} \int[d \bar{\eta}][d \eta] e^{-S_{J}} & \\
S_{J}=\mathcal{A}_{f}-\bar{J}_{\eta_{0}}+\bar{\eta}_{0} P_{+} J-\bar{\eta}_{n} J_{n}-\bar{J}_{n+1} \eta_{n+1}-\bar{\eta}_{n+1} J_{n+1}-\bar{\eta}_{N_{s}+3} P_{-} J \\
\mathcal{J} \text { is the Jacobian of the transformation } \\
\mathcal{J}=\prod_{s=0}^{N_{s}+3} \operatorname{det}\left[\gamma\left(\rho_{s} H_{w} P_{-}+\sigma_{s} H_{w} P_{+}-1\right)\right]
\end{array}
$$




\section{Generating Functional (cont.)}

Integrating $\left(\eta_{s}, \bar{\eta}_{s}\right)$ successively from $s=N_{s}+3$ to $s=0$ :

$Z\left[J_{q}, \bar{J}_{q}, J_{n}, J_{n+1}, \bar{J}_{n+1}\right]$

$=K \operatorname{det}\left[r\left(D_{c}+m_{q}\right)\right] \exp \left\{\bar{J}_{n+1} T_{U}^{-1} P_{-} J+\bar{J}_{n+1} J_{n+1}+\right.$

$$
\left.+\left[\bar{J}+\bar{J}_{n+1} T_{U}^{-1}\left(P_{+}-r m_{q} P_{-}\right)\right]\left(r D_{c}+r m_{q}\right)^{-1}\left[J+\widehat{T}^{-1} J_{n}+\widehat{T}^{-1} J_{n+1}\right]\right\}
$$

$T_{L}^{-1} \equiv \prod_{s=1}^{n} T_{s}^{-1}$

$D_{c}=\frac{1}{r} \frac{1+\gamma_{5} S}{1-\gamma_{5} S}$

$T_{U}^{-1} \equiv \prod_{\substack{s=n+1 \\ N_{s}+2}}^{N_{s}+2} T_{s}^{-1}$

$S=\frac{1-\prod_{s=0}^{N_{s}+3} T_{s}}{1+\prod_{s=0}^{N_{s}+3} T_{s}}$

$T^{-1} \equiv \prod_{s=1} T_{s}^{-1}=T_{L}^{-1} T_{U}^{-1}$

$T_{s}=\frac{1-H_{s}}{1+H_{s}}$

$\widehat{T}^{-1} \equiv\left(-P_{+}+T^{-1} P_{-}\right)^{-1} T_{L}^{-1}$

$H_{s}=\left(\rho_{s}+\sigma_{s}\right) H_{w}\left[2+\left(\rho_{s}-\sigma_{s}\right) \gamma_{5} H_{w}\right]^{-1}$

$K \equiv \mathcal{J} \operatorname{det}\left[-P_{+}+T^{-1} P_{-}\right]$ 


\section{Generating Functional (cont.)}

(I) The valence quark propagator

$$
\langle q(x) \bar{q}(y)\rangle=-\left.\frac{1}{Z} \frac{\delta^{2} Z}{\delta \bar{J}_{q}(x) \delta J_{q}(y)}\right|_{0}=\left(D_{c}+m_{q}\right)^{-1}(x, y)
$$

(II) The mixed correlator of the first kind

$$
\begin{aligned}
& \qquad \begin{aligned}
\left\langle q(x) \bar{\eta}_{n}(y)\right\rangle & =-\left.\frac{1}{Z} \frac{\delta^{2} Z}{\delta \bar{J}_{q}(x) \delta J_{n}(y)}\right|_{0} \\
& =\sqrt{r}\left(r D_{c}+r m_{q}\right)^{-1}\left(-P_{+}+T^{-1} P_{-}\right)^{-1} T_{L}^{-1} \\
& =-\frac{1}{\sqrt{r}} D^{-1}\left(m_{q}\right) \gamma_{5} \frac{T_{L}^{-1}}{T^{-1}+1}
\end{aligned} \\
& D^{-1}\left(m_{q}\right)=\left(1+r D_{c}\right)\left(D_{c}+m_{q}\right)^{-1}=r+\left(1-r m_{q}\right)\left(D_{c}+m_{q}\right)^{-1}
\end{aligned}
$$




\section{Generating Functional (cont.)}

(III) The mixed correlator of the second kind

$$
\begin{aligned}
\left\langle q(x) \bar{\eta}_{n+1}(y)\right\rangle & =-\left.\frac{1}{Z} \frac{\delta^{2} Z}{\delta \bar{J}_{q}(x) \delta J_{n+1}(y)}\right|_{0} \\
& =\sqrt{r}\left(r D_{c}+r m_{q}\right)^{-1}\left(-P_{+}+T^{-1} P_{-}\right)^{-1} T_{L}^{-1} \\
& =-\frac{1}{\sqrt{r}} D^{-1}\left(m_{q}\right) \gamma_{5} \frac{T_{L}^{-1}}{T^{-1}+1}=\left\langle q(x) \bar{\eta}_{n}(y)\right\rangle .
\end{aligned}
$$

(IV) The mixed correlator of the third kind

$$
\begin{aligned}
\left\langle\eta_{n+1}(x) \bar{q}(y)\right\rangle & =-\left.\frac{1}{Z} \frac{\delta^{2} Z}{\delta \bar{J}_{n+1}(x) \delta J_{q}(y)}\right|_{0} \\
& =T_{U}^{-1}\left(-r m_{q} P_{-}+P_{+}\right)\left(r D_{c}+r m_{q}\right)^{-1} \sqrt{r}+T_{U}^{-1} P_{-} \sqrt{r} \\
& =T_{U}^{-1} \frac{1}{2 \sqrt{r}}\left(1+\frac{1+r m_{q}}{1-r m_{q}} \gamma_{5}\right) D^{-1}\left(m_{q}\right)-\frac{\sqrt{r}}{1-r m_{q}} T_{U}^{-1} \gamma_{5}
\end{aligned}
$$




\section{A New Formula for the Residual Mass}

$$
m_{\text {res }}(y)=\frac{\sum_{x}\left\langle J_{5}^{a}(x, n) \bar{q}(y) \lambda^{b} \gamma_{5} q(y)\right\rangle}{\sum_{x}\left\langle\bar{q}(x) \lambda^{a} \gamma_{5} q(x) \bar{q}(y) \lambda^{b} \gamma_{5} q(y)\right\rangle}
$$

$$
\begin{aligned}
& \sum_{x}\left\langle\bar{q}(x) \lambda^{a} \gamma_{5} q(x) \bar{q}(y) \lambda^{b} \gamma_{5} q(y)\right\rangle \\
= & -\operatorname{tr}_{F}\left(\lambda^{a} \lambda^{b}\right) \operatorname{tr}_{D C}\left\{\left[\left(D_{c}+m_{q}\right)^{-1}\right]^{\dagger}\left(D_{c}+m_{q}\right)^{-1}\right\}(y, y) \\
& \sum_{x}\left\langle J_{5}^{a}(x, n) \bar{q}(y) \lambda^{b} \gamma_{5} q(y)\right\rangle \quad J_{5}^{a}(x, n)=-\bar{\psi}_{n}(x) \lambda^{a} P_{-} \psi_{n+1}(x)+\bar{\psi}_{n+1}(x) \lambda^{a} P_{+} \psi_{n}(x) \\
= & \operatorname{tr}\left(\lambda^{a} \lambda^{b}\right)\left\{\sum_{x} \operatorname{tr}\left[\left\langle q(y) \bar{\psi}_{n}(x)\right\rangle P_{-}\left\langle\psi_{n+1}(x) \bar{q}(y)\right\rangle \gamma_{5}\right]-\sum_{x} \operatorname{tr}\left[\left\langle q(y) \bar{\psi}_{n+1}(x)\right\rangle P_{+}\left\langle\psi_{n}(x) \bar{q}(y)\right\rangle \gamma_{5}\right]\right\} \\
= & \operatorname{tr}\left(\lambda^{a} \lambda^{b}\right) \sum_{x} \operatorname{tr}\left[\left\langle q(y) \bar{\eta}_{n}(x)\right\rangle\left\langle\eta_{n+1}(x) \bar{q}(y) \gamma_{5}\right\rangle\right] \\
= & -\operatorname{tr}\left(\lambda^{a} \lambda^{b}\right) \frac{1}{4 r} \operatorname{tr}\left\{\left[D^{-1}\left(m_{q}\right)\right]^{\dagger}\left(1-S^{2}\right) D^{-1}\left(m_{q}\right)\right](y, y)
\end{aligned}
$$




\section{A New Formula for the Residual Mass}

$$
\begin{aligned}
m_{r e s}(y) & =\frac{\sum_{x}\left\langle J_{5}^{a}(x, n) \bar{q}(y) \lambda^{b} \gamma_{5} q(y)\right\rangle}{\sum_{x}\left\langle\bar{q}(x) \lambda^{a} \gamma_{5} q(x) \bar{q}(y) \lambda^{b} \gamma_{5} q(y)\right\rangle} \\
& =\frac{1}{4 r} \frac{\operatorname{tr}\left\{\left[D^{-1}\left(m_{q}\right)\right]^{\dagger}\left(1-S^{2}\right) D^{-1}\left(m_{q}\right)\right\}(y, y)}{\operatorname{tr}\left\{\left[\left(D_{c}+m_{q}\right)^{-1}\right]^{\dagger}\left(D_{c}+m_{q}\right)^{-1}\right\}(y, y)} \\
D^{-1}\left(m_{q}\right) & =r+\left(1-r m_{q}\right)\left(D_{c}+m_{q}\right)^{-1} \text { : sea quark propagator } \\
\left(D_{c}+m_{q}\right)^{-1}: \text { valence quark propagator } & \\
M_{\text {res }} & =\frac{1}{4 r} \frac{\operatorname{Tr}\left\{\left[D^{-1}\left(m_{q}\right)\right]^{\dagger}\left(1-S^{2}\right) D^{-1}\left(m_{q}\right)\right\}}{\operatorname{Tr}\left\{\left[\left(D_{c}+m_{q}\right)^{-1}\right]^{\dagger}\left(D_{c}+m_{q}\right)^{-1}\right\}}
\end{aligned}
$$

However, it is tedious to use these formulas to compute the residual mass since they involve the multiplication of

$$
S=\left(1-\prod_{s} T_{s}\right)\left(1+\prod_{s} T_{s}\right)^{-1}=H \sum_{i=1}^{n} b_{i}\left(H^{2}+d_{i}\right)^{-1}
$$

to the columns of the sea-quark propagator, requiring multi-shift CG. 


\section{A New Formula for the Residual Mass (cont.)}

$$
\begin{aligned}
& \operatorname{tr}\left\{\left[D^{-1}\left(m_{q}\right)\right]^{\dagger}\left(1-S^{2}\right) D^{-1}\left(m_{q}\right)\right\}(y, y) \\
= & \operatorname{tr}\left\{\left[D^{-1}\left(m_{q}\right)\right]^{\dagger} D^{-1}\left(m_{q}\right)\right\}(y, y)-\operatorname{tr}\left\{\left[S D^{-1}\left(m_{q}\right)\right]^{\dagger}\left(S D^{-1}\left(m_{q}\right)\right)\right\}(y, y)
\end{aligned}
$$

Using

$$
\begin{aligned}
& S=\gamma_{5}\left[2 r \frac{D\left(m_{q}\right)-m_{q}}{1-r m_{q}}-1\right] \\
& S D\left(m_{q}\right)^{-1}=\gamma_{5}\left[\frac{2 r}{1-r m_{q}}-\frac{1+r m_{q}}{1-r m_{q}} D\left(m_{q}\right)^{-1}\right] \\
& =\gamma_{5}\left[r-\left(1+r m_{q}\right)\left(D_{c}+m_{q}\right)^{-1}\right] .
\end{aligned}
$$

$$
\begin{aligned}
& \operatorname{tr}\left\{\left[S D^{-1}\left(m_{q}\right)\right)\right]^{\dagger}\left(S D^{-1}\left(m_{q}\right)\right\}(y, y) \\
& =r^{2} \operatorname{tr} \mathbb{I}-2 r\left(1+r m_{q}\right) \operatorname{Re} \operatorname{tr}\left(D_{c}+m_{q}\right)^{-1}(y, y)
\end{aligned}
$$

$$
+\left(1+r m_{q}\right)^{2} \operatorname{tr}\left\{\left[\left(D_{c}+m_{q}\right)^{-1}\right]^{\dagger}\left(D_{c}+m_{q}\right)^{-1}\right\}(y, y) .
$$




\section{A New Formula for the Residual Mass (cont.)}

Using

$$
\begin{aligned}
& \quad D^{-1}\left(m_{q}\right)=\left(1+r D_{c}\right)\left(D_{c}+m_{q}\right)^{-1}=r+\left(1-r m_{q}\right)\left(D_{c}+m_{q}\right)^{-1} \\
& \operatorname{tr}\left\{\left[D^{-1}\left(m_{q}\right)\right]^{\dagger} D^{-1}\left(m_{q}\right)\right\}(y, y) \\
& =r^{2} \operatorname{tr} \mathbb{I}+2 r\left(1-r m_{q}\right) \operatorname{Re} \operatorname{tr}\left(D_{c}+m_{q}\right)^{-1}(y, y) \\
& \quad+\left(1-r m_{q}\right)^{2} \operatorname{tr}\left\{\left[\left(D_{c}+m_{q}\right)^{-1}\right]^{\dagger}\left(D_{c}+m_{q}\right)^{-1}\right\}(y, y)
\end{aligned}
$$

Thus

$$
\operatorname{tr}\left\{\left[D^{-1}\left(m_{q}\right)\right]^{\dagger}\left(1-S^{2}\right) D^{-1}\left(m_{q}\right)\right\}(y, y)
$$$$
=4 \mathrm{r} \operatorname{Re} \operatorname{tr}\left\{\left(D_{c}+m_{q}\right)^{-1}(y, y)\right\}-4 r m_{q} \operatorname{tr}\left\{\left[\left(D_{c}+m_{q}\right)^{-1}\right]^{\dagger}\left(D_{c}+m_{q}\right)^{-1}\right\}(y, y)
$$

$$
m_{\text {res }}(y)=\frac{\operatorname{Re} \operatorname{tr}\left\{\left(D_{c}+m_{q}\right)^{-1}(y, y)\right\}}{\operatorname{tr}\left\{\left[\left(D_{c}+m_{q}\right)^{\dagger}\left(D_{c}+m_{q}\right)\right]^{-1}(y, y)\right\}}-m_{q}
$$

which immediately gives the residual mass once the 12 columns of $\left(D_{c}+m_{q}\right)^{-1}(x, y)$ have been computed. 


\section{An Upper Bound for the Global Residual Mass}

$$
\begin{aligned}
M_{\text {res }} & =\frac{1}{4 r} \frac{\operatorname{Tr}\left\{\left[D^{-1}\left(m_{q}\right)\right]^{\dagger}\left(1-S^{2}\right) D^{-1}\left(m_{q}\right)\right\}}{\operatorname{Tr}\left\{\left[\left(D_{c}+m_{q}\right)^{-1}\right]^{\dagger}\left(D_{c}+m_{q}\right)^{-1}\right\}} \\
& =\frac{\operatorname{Re} \operatorname{Tr}\left\{\left(D_{c}+m_{q}\right)^{-1}\right\}}{\operatorname{Tr}\left\{\left[\left(D_{c}+m_{q}\right)^{\dagger}\left(D_{c}+m_{q}\right)\right]^{-1}\right\}}-m_{q}
\end{aligned}
$$

$\operatorname{Tr}\left\{\left[D\left(m_{q}\right)^{-1}\right]^{\dagger}\left(1-S^{2}\right) D^{-1}\left(m_{q}\right)\right\}=\operatorname{Tr}\left\{\left(1-S^{2}\right)\left(D^{\dagger} D\right)^{-1}\left(m_{q}\right)\right\} \leq \sum_{j} \alpha_{j} \beta_{j}$ von Neumann's trace inequality

$\alpha_{j}$ and $\beta_{j}$ are the eigenvalues of $\left(1-S^{2}\right)$ and $\left(D^{\dagger} D\right)^{-1}\left(m_{q}\right)$ respectively For ODWF, $S=S_{\text {opt }},\left|\alpha_{j}\right| \leq 2 d_{z}$. Thus

$$
\operatorname{Tr}\left\{\left[D\left(m_{q}\right)^{-1}\right]^{\dagger}\left(1-S_{\text {opt }}^{2}\right) D^{-1}\left(m_{q}\right)\right\} \leq 2 d_{Z} \operatorname{Tr}\left\{\left(D^{\dagger} D\right)^{-1}\left(m_{q}\right)\right\}
$$




\section{An Upper Bound for the Residual Mass (cont.)}

$$
\begin{array}{cc} 
& M_{\text {res }} \leq \frac{d_{Z}}{2 r} \frac{\operatorname{Tr}\left\{\left[D\left(m_{q}\right)^{-1}\right]^{\dagger} D^{-1}\left(m_{q}\right)\right\}}{\operatorname{Tr}\left\{\left[\left(D_{c}+m_{q}\right)^{-1}\right]^{\dagger}\left(D_{c}+m_{q}\right)^{-1}\right\}} \\
\because \quad & D^{-1}\left(m_{q}\right)=\left(1+r D_{c}\right)\left(D_{c}+m_{q}\right)^{-1}=r+\left(1-r m_{q}\right)\left(D_{c}+m_{q}\right)^{-1} \\
\therefore \quad & \lambda_{j}=r+\left(1-r m_{q}\right) \xi_{j}
\end{array}
$$

$\xi_{j}$ is a singular value of $\left(D_{c}+m_{q}\right)^{-1}$

$\lambda_{j}$ is the corresponding singular value of $D^{-1}\left(m_{q}\right)$

$\therefore \quad \frac{\sum_{j}\left|\lambda_{j}\right|^{2}}{\sum_{j}\left|\xi_{j}\right|^{2}}=\left(1-r m_{q}\right)^{2}+2 r\left(1-r m_{q}\right) \frac{\langle\operatorname{Re}(\xi)\rangle}{\left\langle|\xi|^{2}\right\rangle}+\frac{r^{2}}{\left\langle|\xi|^{2}\right\rangle}$

$$
\left\langle|\xi|^{2}\right\rangle=\frac{1}{N} \sum_{j=1}^{N}\left|\xi_{j}\right|^{2}, \quad\langle\operatorname{Re}(\xi)\rangle=\frac{1}{N} \sum_{j=1}^{N} \operatorname{Re}\left(\xi_{j}\right)
$$

$N$ is the total number of singular values of $\left(D_{c}+m_{q}\right)^{-1}$ 


\section{An Upper Bound for the Residual Mass (cont.)}

$\therefore \frac{\operatorname{Tr}\left\{\left[D\left(m_{q}\right)^{-1}\right]^{\dagger} D^{-1}\left(m_{q}\right)\right\}}{\operatorname{Tr}\left\{\left[\left(D_{c}+m_{q}\right)^{-1}\right]^{\dagger}\left(D_{c}+m_{q}\right)^{-1}\right\}}=\left(1-r m_{q}\right)^{2}+2 r\left(1-r m_{q}\right) \frac{\langle\operatorname{Re}(\xi)\rangle}{\left\langle|\xi|^{2}\right\rangle}+\frac{r^{2}}{\left\langle|\xi|^{2}\right\rangle}$

$$
M_{\text {res }} \leq \frac{d_{Z}}{2 r}\left[\left(1-r m_{q}\right)^{2}+2 r\left(1-r m_{q}\right) \frac{\langle\operatorname{Re}(\xi)\rangle}{\left\langle|\xi|^{2}\right\rangle}+\frac{r^{2}}{\left\langle|\xi|^{2}\right\rangle}\right]
$$

Thus, to obtain the upper bound of $M_{\text {res }}$ amounts to working out

(i) an upper bound for $\langle\operatorname{Re}(\xi)\rangle /\left\langle|\xi|^{2}\right\rangle$

(ii) a lower bound for $\left\langle|\xi|^{2}\right\rangle$

It can be shown that

$$
\begin{gathered}
\frac{\langle\operatorname{Re}(\xi)\rangle}{\left\langle|\xi|^{2}\right\rangle} \leq m+\frac{(1+m) d_{Z}}{2-(3-m) d_{Z}} \\
\left\langle|\xi|^{2}\right\rangle \geq \frac{r^{2}}{1+m}\left[\frac{2-(3-m) d_{Z}}{2 m+(1-m)^{2} d_{Z}}\right]
\end{gathered}
$$




\section{An Upper Bound for the Residual Mass (cont.)}

$$
\begin{aligned}
M_{r e s} \leq \frac{d_{Z}}{2 r}\left\{\left(1-r m_{q}\right)^{2}+2 r\left(1-r m_{q}\right)\left[r m_{q}\right.\right. & \left.+\frac{\left(1+r m_{q}\right) d_{Z}}{2-\left(3-r m_{q}\right) d_{Z}}\right] \\
& \left.+\left(1+r m_{q}\right)\left[\frac{2 r m_{q}+\left(1-r m_{q}\right)^{2} d_{Z}}{2-\left(3-r m_{q}\right) d_{Z}}\right]\right\}
\end{aligned}
$$

For light quarks, $r m_{q} \ll 1$

$$
M_{\text {res }} \leq \frac{d_{Z}}{2 r}
$$

For $\mathrm{ODWF}, d_{Z} \ll 1$ in most cases

$$
M_{\text {res }} \leq \frac{d_{Z}}{2 r}\left[\left(1+2 r^{2} m_{q}\right)^{2}\left(1-r m_{q}\right)+2 r^{2} m_{q}^{2}\right] \equiv \frac{d_{Z}}{2 r} F\left(r, m_{q}\right)
$$

$$
M_{r e s}\left[d_{Z} /(2 r)\right]^{-1}=F\left(r, m_{q}\right)
$$




\section{An Upper Bound for the Residual Mass (cont.)}

It is clear that the derivation also goes through for the conventional DWF with nonzero weights $\rho_{\mathrm{s}}=c_{1}$ (constant) and $\sigma_{\mathrm{s}}=c_{2}$ (constant)

by replacing $\left\|1-S_{\text {opt }}\right\| \leq d_{Z}$ with $\left\|1-S_{\text {polar }}\right\|<\Delta_{\max }$

$\Delta_{\max }$ can be determined for given $\lambda_{\min }$ and $\lambda_{\max }$

$$
\begin{aligned}
& M_{\text {res }} \leq \frac{\Delta_{\max }}{2 r}\left\{\left(1-r m_{q}\right)^{2}+2 r\right.\left(1-r m_{q}\right)\left[r m_{q}+\frac{\left(1+r m_{q}\right) \Delta_{\max }}{2-\left(3-r m_{q}\right) \Delta_{\max }}\right] \\
&\left.+\left(1+r m_{q}\right)\left[\frac{2 r m_{q}+\left(1-r m_{q}\right)^{2} \Delta_{\max }}{2-\left(3-r m_{q}\right) \Delta_{\max }}\right]\right\}
\end{aligned}
$$

if $\Delta_{\max }$ is sufficiently small

$$
M_{\text {res }}\left[\Delta_{\max } /(2 r)\right]^{-1} \approx F\left(r, m_{q}\right) \quad \text { universal for any DWF }
$$




\section{An Upper Bound for the Residual Mass (cont.)}

How does the residual mass depend on the quark mass?

Consider $\rho_{\mathrm{s}}=c \omega_{s}+d, \sigma_{\mathrm{s}}=c \omega_{\mathrm{s}}-d$, with

$$
H=c H_{w}\left(1+d \gamma_{5} H_{w}\right)^{-1}, r=\left[2 m_{0}\left(1-d m_{0}\right)\right]^{-1}
$$

(i) $c=1, d=0, H=H_{w}, r=1 /\left(2 m_{0}\right)$

(ii) $c=1 / 2, d=1 / 2, H=H_{w}\left(2+\gamma_{5} H_{w}\right)^{-1}, r=\left[m_{0}\left(2-m_{0}\right)\right]^{-1}$ 


\section{An Upper Bound for the Residual Mass (cont.)}

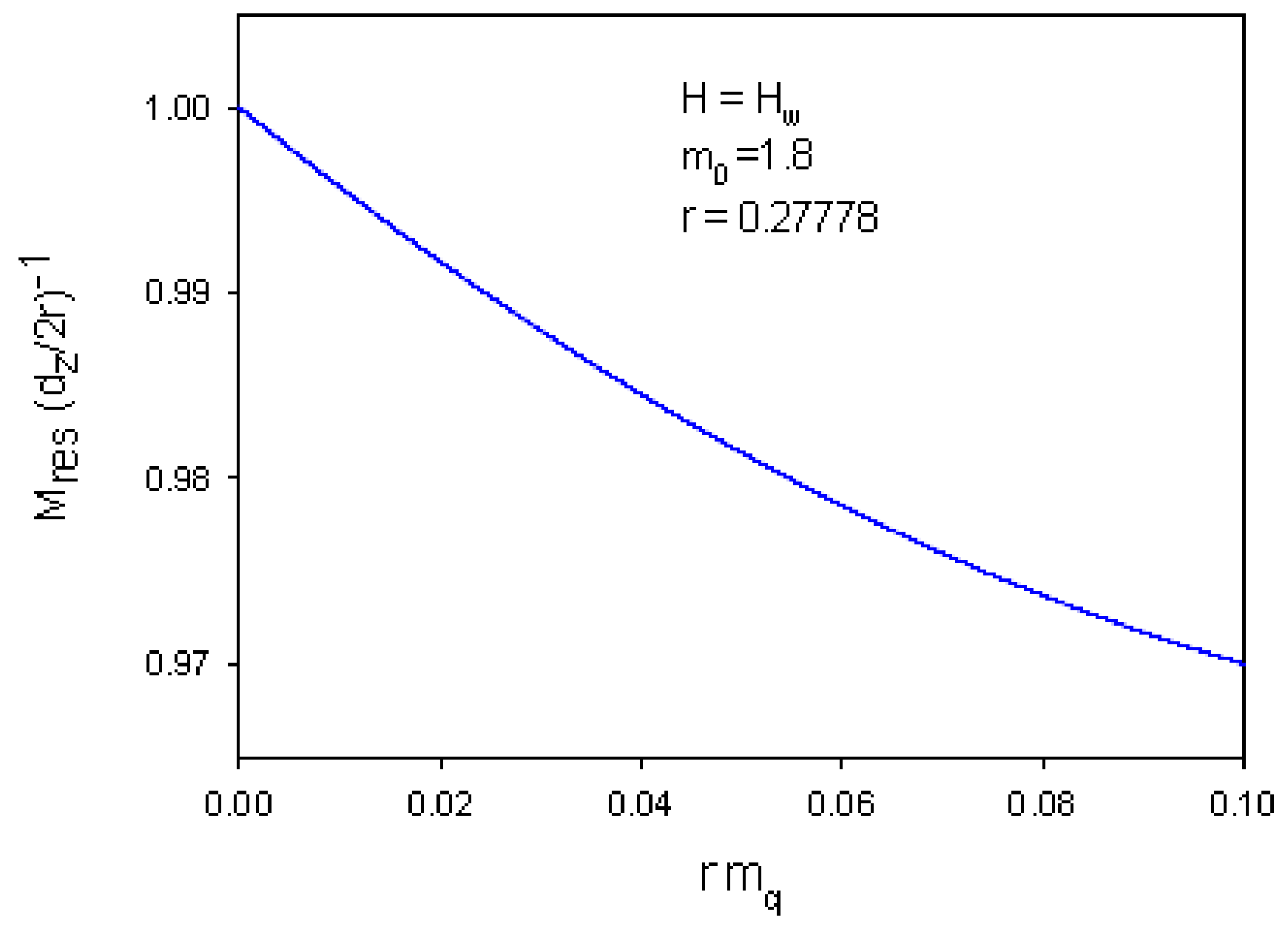




\section{An Upper Bound for the Residual Mass (cont.)}

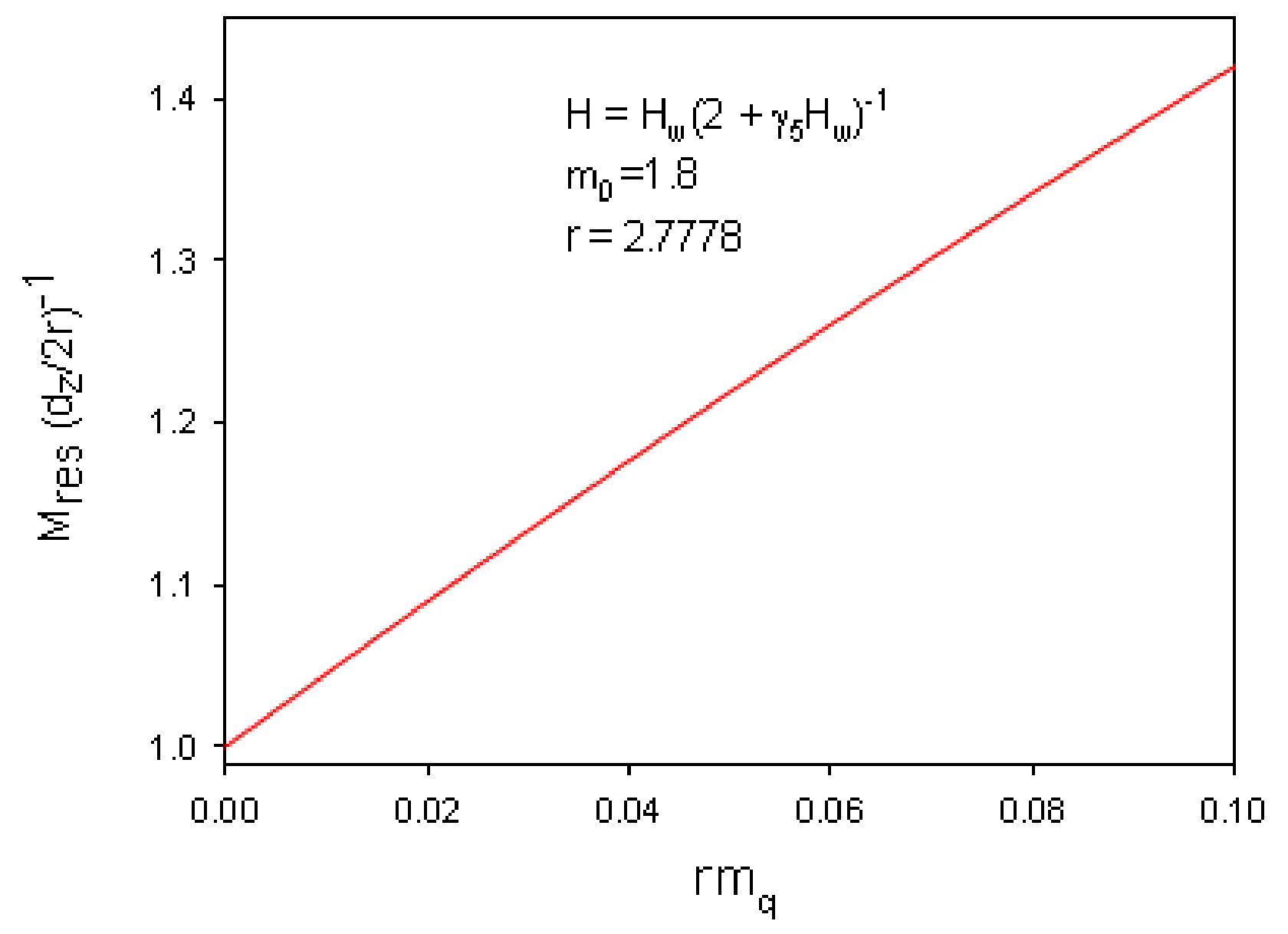




\section{Concluding Remarks}

> Axial Ward identity is derived for LQCD with ODWF

> Z[J] for n-point Green's function is obtained

$>$ A practical formula for the residual mass is obtained

$>$ An upper bound for the residual mass is obtained

- It provides a guideline for designing simulations with DWF

- The normalized residual mass is expressed as

$$
F\left(r, m_{q}\right)=\left(1+2 r^{2} m_{q}\right)\left(1-r m_{q}\right)+2 r^{2} m_{q}^{2}
$$

which is universal for any DWF with sufficiently small $\Delta_{\max }$

$>$ The residual mass of quark for any observable $\mathrm{O}$ should be measured respectively. 


\title{
Multigrid Acceleration for Domain-Wall Fermion Inversion
}

\author{
Saul D. Cohen
}

\section{W UNIVERSITY of WASHINGTON}

New Horizons for Lattice Computations with Chiral Fermions 2012 May 15 


\section{Observables on the Lattice}

\section{...That Are Exceedingly Difficult}

- Disconnected diagrams (strangeness of the proton, dark matter couplings)

- Zero-flavor physics ( $\eta^{\prime}, a_{0}$ properties)

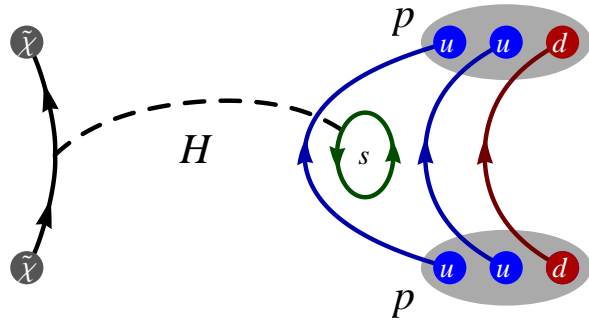

- Precision physics (nuclear binding energies, NPLQCD use 100 s of sources)

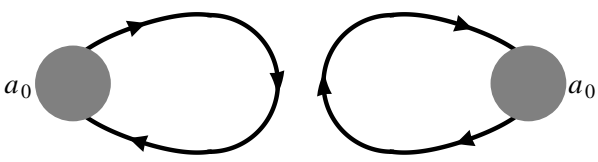




\section{A Chiral Fermion}

Domain-Wall Fermions

- Low-lying eigenstates: chiral modes bound to the edges of $5^{\text {th }}$ dimension

- Very helpful for weak matrix elements $\left(B_{K}\right)$, $N P R$, operator mixing

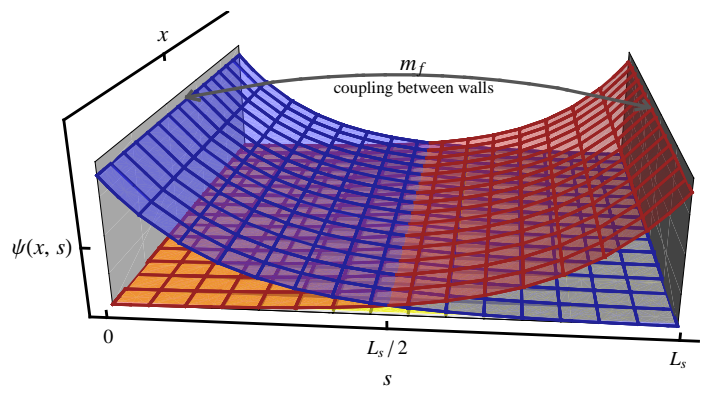




\section{Domain-Wall Operator}

\section{Spectrum}

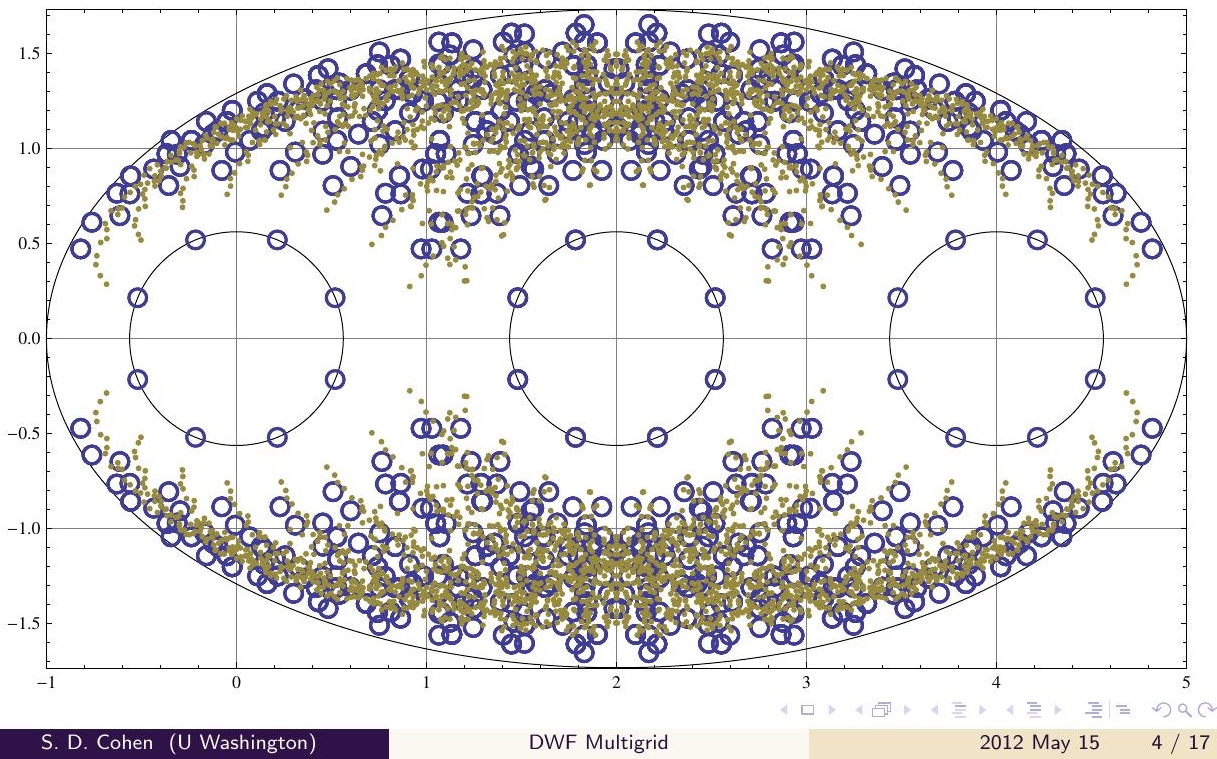




\section{Krylov Subspace Solvers}

Krylov solvers spend $50-90 \%$ of a lattice calculation's computer power

- Includes the very common conjugate-gradients (CG) algorithm

- Constructs the Krylov subspace $\mathcal{K}_{n}=\left\{r, A r, A^{2} r, \ldots, A^{n} r\right\}$

- Number of iterations scales like the square-root of the matrix's condition-number: $\sqrt{\kappa}$

- For the Dirac operator, $\kappa \propto 1 / m=$ "critical slowing"

- Very efficient at eliminating modes with large eigenvalues 


\section{Algorithm Study}

Due to the indefinite spectrum of the DWF operator, none of the standard Krylov solvers is guaranteed to work.

Most solvers, including BiCGStab, fail to converge.

TFQMR and QMR work, but very slowly.

CGNR is by far the fastest.

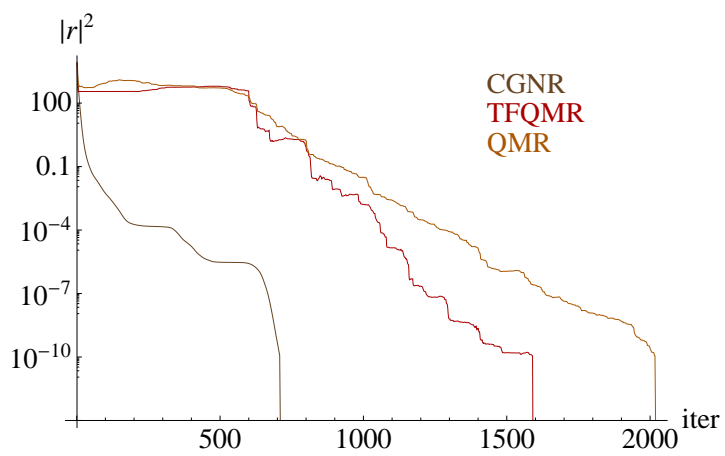




\section{Normal-Equation Solvers}

If we accept that we must use the normal equation, most Krylov solvers work.

CG remains fastest.

However, it cannot be preconditioned in a flexible way.

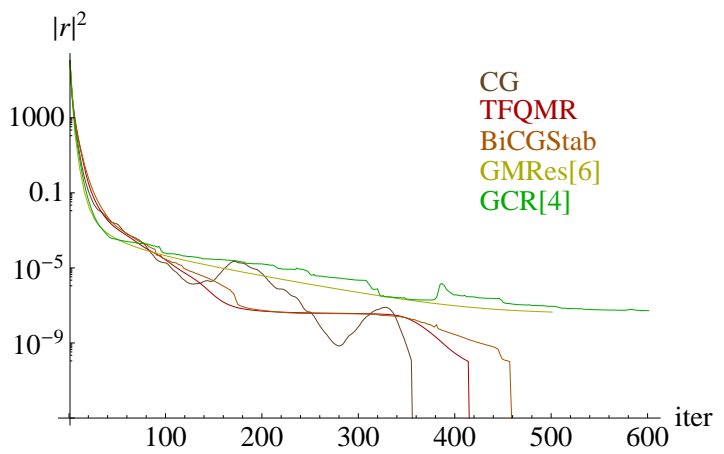




\section{Sketch of Multigrid Algorithm}

\section{$\checkmark$-Cycling}

$$
\begin{aligned}
& D x=b \\
& x_{1}=S^{\nu_{1}}\left(D^{\dagger} D, D^{+} b\right) \quad x=x_{1}+P x_{2}+S^{\nu_{3}}\left(D^{\dagger} D, D^{\dagger} b_{3}\right. \\
& \begin{array}{|ll|ll|ll|ll|}
\hline \bullet & \bullet & \bullet & \bullet & \bullet & \bullet & \bullet & \bullet \\
\bullet & \bullet & \bullet & \bullet & \bullet & \bullet & \bullet & \bullet \\
\hline \bullet & \bullet & \bullet & \bullet & \bullet & \bullet & \bullet & \bullet \\
\bullet & \bullet & \bullet & \bullet & \bullet & \bullet & \bullet & \bullet \\
\hline \bullet & \bullet & \bullet & \bullet & \bullet & \bullet & \bullet & \bullet \\
\bullet & \bullet & \bullet & \bullet & \bullet & \bullet & \bullet & \bullet \\
\hline \bullet & \bullet & \bullet & \bullet & \bullet & \bullet & \bullet & \bullet \\
\bullet & \bullet & \bullet & \bullet & \bullet & \bullet & \bullet & \bullet \\
\hline
\end{array} \\
& \begin{array}{|ll|ll|ll|ll|}
\hline \bullet & \bullet & \bullet & \bullet & \bullet & \bullet & \bullet & \bullet \\
\bullet & \bullet & \bullet & \bullet & \bullet & \bullet & \bullet & \bullet \\
\hline \bullet & \bullet & \bullet & \bullet & \bullet & \bullet & \bullet & \bullet \\
\bullet & \bullet & \bullet & \bullet & \bullet & \bullet & \bullet & \bullet \\
\hline \bullet & \bullet & \bullet & \bullet & \bullet & \bullet & \bullet & \bullet \\
\bullet & \bullet & \bullet & \bullet & \bullet & \bullet & \bullet & \bullet \\
\hline \bullet & \bullet & \bullet & \bullet & \bullet & \bullet & \bullet & \bullet \\
\bullet & \bullet & \bullet & \bullet & \bullet & \bullet & \bullet & \bullet \\
\hline
\end{array} \\
& x_{2}=S^{v_{2}}\left(P^{+} D^{+} D P, b_{2}\right)
\end{aligned}
$$

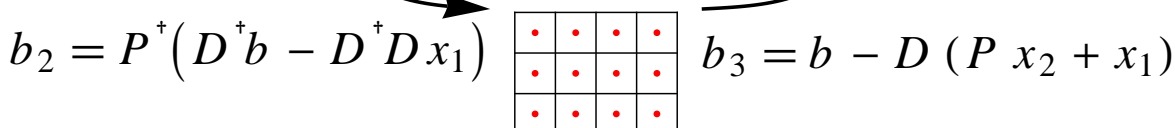




\section{Sketch of Multigrid Algorithm}

\section{Constructing the Coarse Space}

$$
\begin{aligned}
& V=\left(\begin{array}{cccc|cccc|c}
v_{1}^{(1)} & v_{2}^{(1)} & v_{3}^{(1)} & v_{4}^{(1)} & v_{5}^{(1)} & v_{6}^{(1)} & v_{7}^{(1)} & v_{8}^{(1)} & \cdots \\
v_{1}^{(2)} & v_{2}^{(2)} & v_{3}^{(2)} & v_{4}^{(2)} & v_{5}^{(2)} & v_{6}^{(2)} & v_{7}^{(2)} & v_{8}^{(2)} & \cdots \\
\vdots & \vdots & \vdots & \vdots & \vdots & \vdots & \vdots & \vdots & \ddots
\end{array}\right) \\
& P=\left(\begin{array}{cccc|cccc|c}
v_{1}^{(1)} & v_{2}^{(1)} & v_{3}^{(1)} & v_{4}^{(1)} & 0 & 0 & 0 & 0 & \cdots \\
0 & 0 & 0 & 0 & v_{5}^{(1)} & v_{6}^{(1)} & v_{7}^{(1)} & v_{8}^{(1)} & \cdots \\
\vdots & \vdots & \vdots & \vdots & \vdots & \vdots & \vdots & \vdots & \cdots \\
v_{1}^{(2)} & v_{2}^{(2)} & v_{3}^{(2)} & v_{4}^{(2)} & 0 & 0 & 0 & 0 & \cdots \\
0 & 0 & 0 & 0 & v_{5}^{(2)} & v_{6}^{(2)} & v_{7}^{(2)} & v_{8}^{(2)} & \cdots \\
\vdots & \vdots & \vdots & \vdots & \vdots & \vdots & \vdots & \vdots & \ddots
\end{array}\right)
\end{aligned}
$$




\section{Sketch of Multigrid Algorithm}

Visualizing the Subspaces

$$
r^{\prime}=\left[1-A P \frac{1}{P^{\dagger} A P} P^{\dagger}\right] r
$$

\section{Multigrid}

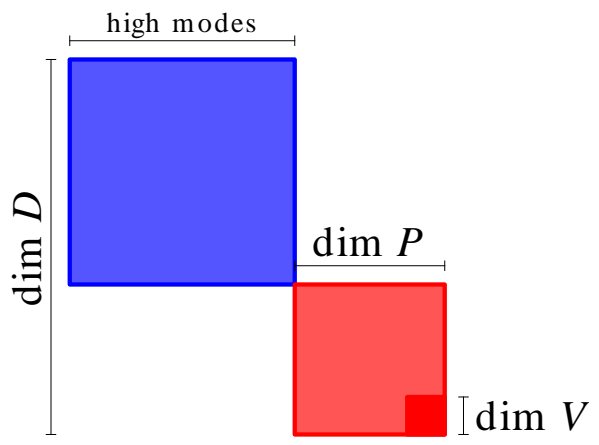

\section{Deflation}

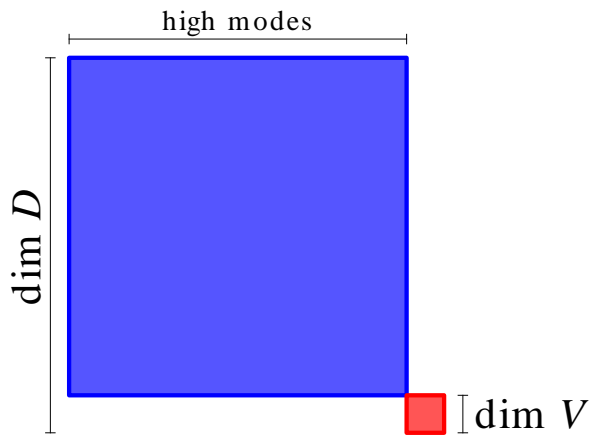




\section{Sketch of Multigrid Algorithm}

Visualizing the Subspaces

$\psi_{n} \cdot\left(P\left(R \psi_{n}\right)\right)$

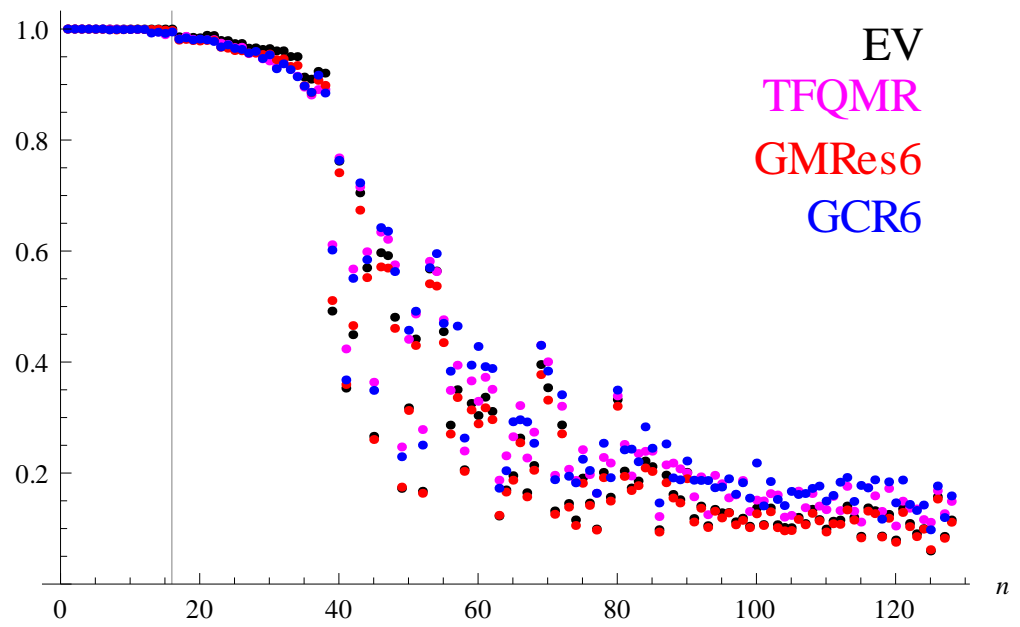




\section{Adaptive Smoothed Algebraic Multigrid}

(1) Find a set of near-null vectors $V$ for which $\psi^{\dagger} D^{\dagger} D \psi \approx 0$ for all $\psi \in V$.

The method by which $V$ is constructed does not matter much. Using a solver that will be reused later is convenient, but not necessary. 


\section{Adaptive Smoothed Algebraic Multigrid}

(1) Find a set of near-null vectors $V$ for which $\psi^{\dagger} D^{\dagger} D \psi \approx 0$ for all $\psi \in V$.

(2) Block the vectors to form the prolongator $P$. Let the unprolongator be $P^{\dagger}$.

(- Construct the coarse operator $P^{\dagger} D^{\dagger} D P$. Use a V-cycle with Krylov smoother and Krylov iteration on coarse operator as a preconditioner

Since $D^{\dagger} D$ is normal, its left and right eigenvectors are the same. $P$ and $R$ can be constructed from the same set of near-null vectors. 


\section{Adaptive Smoothed Algebraic Multigrid}

(2) Find a set of near-null vectors $V$ for which $\psi^{\dagger} D^{\dagger} D \psi \approx 0$ for all $\psi \in V$.

(2) Block the vectors to form the prolongator $P$. Let the unprolongator be $P^{\dagger}$.

(3) Construct the coarse operator $P^{\dagger} D^{\dagger} D P$. Use a $\mathrm{V}$-cycle with Krylov smoother and Krylov iteration on coarse operator as a preconditioner to an outer Krylov solver.

The outer solver must be flexibly preconditioned. The selection of the smoother has some effect, but the coarse-level solver can be anything. 


\section{Adaptive Smoothed Algebraic Multigrid}

(1) Find a set of near-null vectors $V$ using the previously described algorithm for which $\psi^{\dagger} D^{\dagger} D \psi \approx 0$ for all $\psi \in V$.

(2) Block the vectors to form the prolongator $P$. Let the unprolongator be $P^{\dagger}$.

(3) Construct the coarse operator $P^{\dagger} D^{\dagger} D P$. Use a $\mathrm{V}$-cycle with Krylov smoother and Krylov iteration on coarse operator as a preconditioner to an outer Krylov solver.

How is this "adaptive"? We can select new near-null vectors from those that are poorly converged by our current algorithm. Repeat as necessary. 


\section{Applied ASAM on DWF Normal Equation}

\section{$20^{2} \times 8, U(1)$, quenched}

This algorithm seems to work well on the $2 \mathrm{~d} U(1)$ DWF normal equation, removing the slowing at small masses.

$20^{2} \times 8 \mathrm{U}(1)$ lattice

$4^{2} \times 8$ blocks

$N_{v}=16$

Outer solver: CG Smoother: GMRes(6) Coarse solver: $\mathrm{CG}$ Coarse $r^{2}: 10^{-3}$

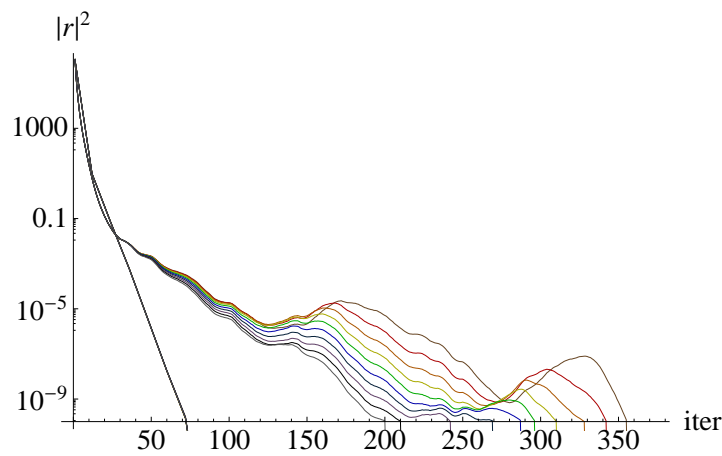




\section{Applied ASAM on DWF Normal Equation}

\section{$20^{2} \times 8, U(1)$, quenched}

This algorithm seems to work well on the 2d U(1) DWF normal equation, removing the slowing at small masses.

$20^{2} \times 8 \mathrm{U}(1)$ lattice

$4^{2} \times 8$ blocks

$N_{v}=16$

Outer solver: CG Smoother: $\operatorname{GMRes}(6)$ Coarse solver: CG Coarse $r^{2}: 10^{-3}$

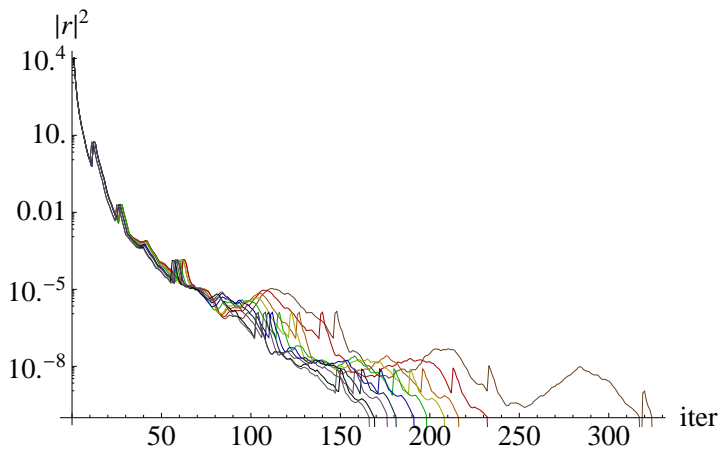




\section{Applied ASAM on DWF Normal Equation \\ $16^{3} \times 32 \times 16$, SU(3), 6-flavor}

The effect is equally impressive on a moderately-sized production lattice.

$16^{3} \times 32 \times 16$ Technicolor lattice

$4^{4} \times 16$ blocks

$N_{v}=24$

Outer solver: GCR(12)

Smoother: $12 \times \mathrm{GCR}(12)$

Coarse solver: $\mathrm{CG}$

Coarse $r^{2}$ : to $10^{-4}$ then $10^{-2} /$ cycle

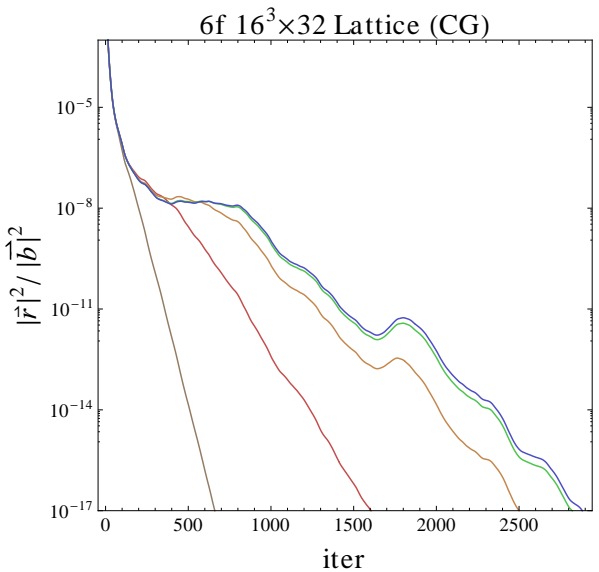




\section{Applied ASAM on DWF Normal Equation \\ $16^{3} \times 32 \times 16$, SU(3), 6-flavor}

The effect is equally impressive on a moderately-sized production lattice.

$16^{3} \times 32 \times 16$ Technicolor lattice

$4^{4} \times 16$ blocks

$N_{v}=24$

Outer solver: GCR(12)

Smoother: $12 \times \mathrm{GCR}(12)$

Coarse solver: $\mathrm{CG}$

Coarse $r^{2}$ : to $10^{-4}$ then $10^{-2} /$ cycle

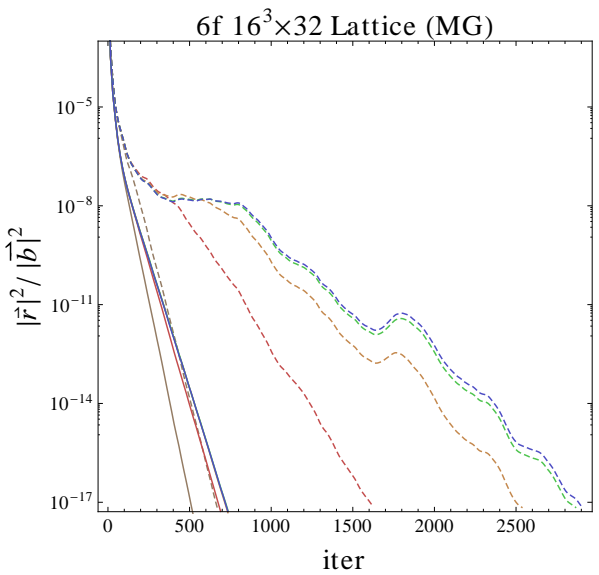




\section{Applied ASAM on DWF Normal Equation \\ $16^{3} \times 32 \times 16$, SU(3), 6-flavor}

The effect is equally impressive on a moderately-sized production lattice.

$16^{3} \times 32 \times 16$ Technicolor lattice

$4^{4} \times 16$ blocks

$N_{v}=24$

Outer solver: $\mathrm{GCR}(12)$

Smoother: $12 \times \mathrm{GCR}(12)$

Coarse solver: $\mathrm{CG}$

Coarse $r^{2}$ : to $10^{-4}$ then $10^{-2} /$ cycle

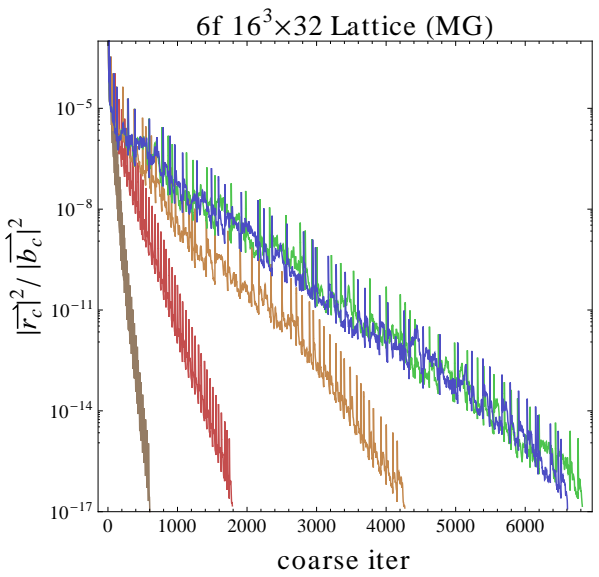




\section{Applied ASAM on DWF Normal Equation}

\section{$16^{3} \times 32 \times 16, \mathrm{SU}(3), 6$-flavor}

The effect is equally impressive on a moderately-sized production lattice.

$16^{3} \times 32 \times 16$ Technicolor lattice

$4^{4} \times 16$ blocks

$N_{v}=24$

Outer solver: $\mathrm{GCR}(12)$

Smoother: $12 \times \operatorname{GCR}(12)$

Coarse solver: $\mathrm{CG}$

Coarse $r^{2}$ : to $10^{-4}$ then $10^{-2} /$ cycle

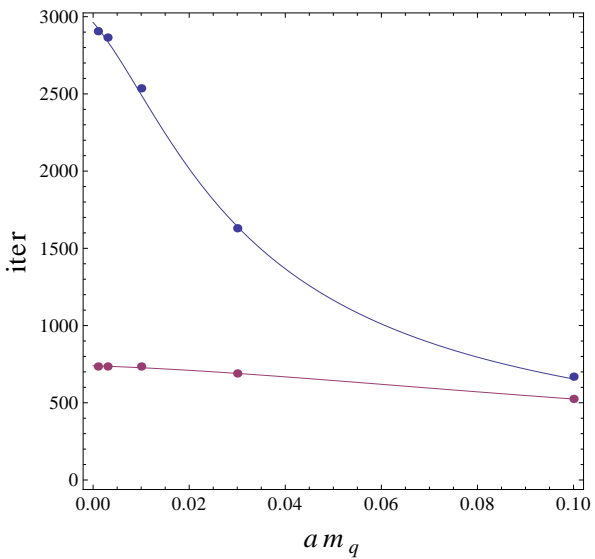




\section{Applied ASAM on Clover-Wilson Fermions}

Graphics courtesy of J. Osborn

Multigrid works even better on clover fermions.

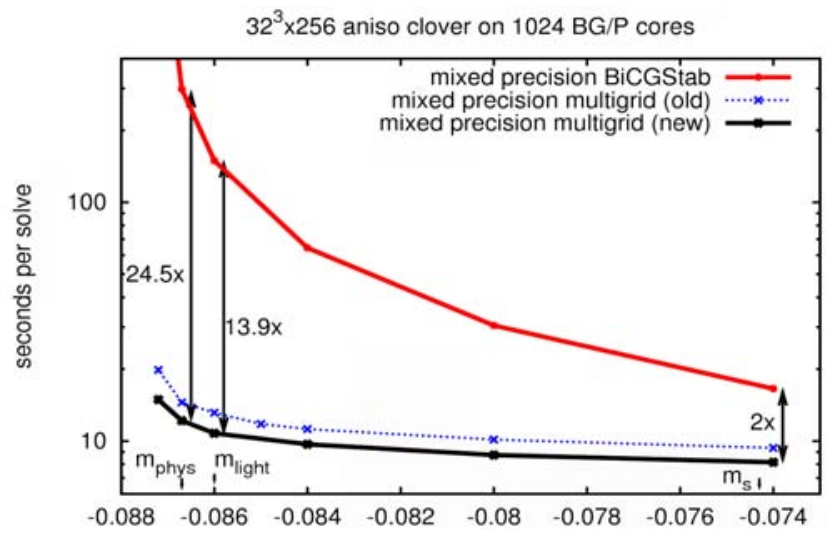




\section{Applied ASAM on Clover-Wilson Fermions}

Graphics courtesy of J. Osborn

If the 2-level multigrid starts to slow at very low mass, add more levels.

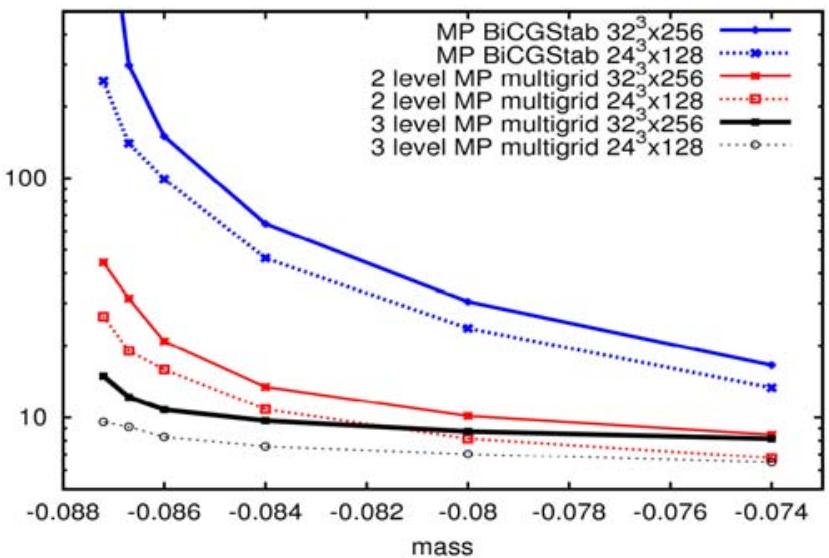




\section{Improved Error for Fixed Residual}

Graphics courtesy of J. Osborn

Since multigrid works efficiently on relevant eigenmodes, the quality of the solution is better than standard solvers.

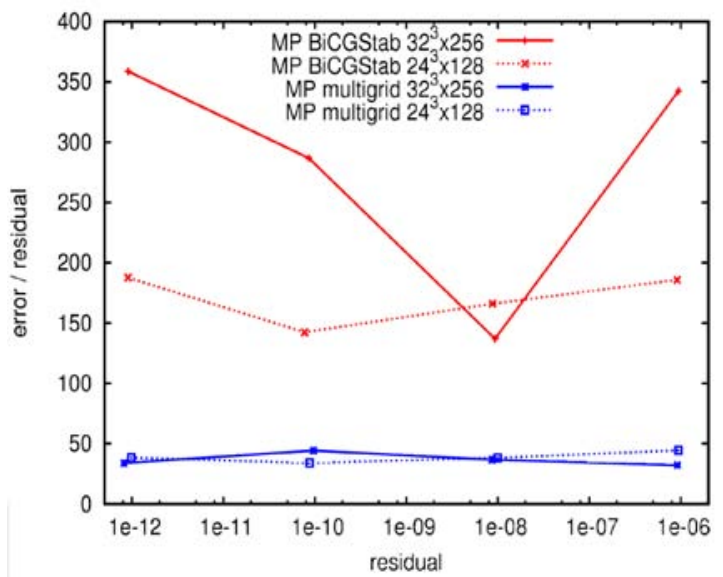




\section{Summary}

- Conclusions

- Multigrid greatly speeds calculation of propagators

- For clover fermions at the physical mass: $20 \times$ faster

- Works for DWF normal equation; improved by factor of 4-8

- Multigrid works even better on larger lattices

- Recent advances

- Integrated as a user-friendly module in Chroma

- Scaling tests for DWF on large lattices (running on Kraken)

- Future Work

- Port to GPUs 


\section{Is This Algorithm Good Enough?}

- Advantages

- Blocks away the entire $5^{\text {th }}$ dimension.

- Factor of 4-8 decrease in fine-operator applications.

- Removes the critical slowing at small quark masses.

- Should have excellent scaling with the lattice volume.

- Disadvantages

- Not a nearest-neighbor operator; poor parallelization.

- Are there more vectors in $P$ (and $R$ ) than we need? 


\section{Constructing a 1-Hop Coarse Operator}

Can we use $\left(P^{\dagger} D^{\dagger} P\right)\left(P^{\dagger} D P\right)$ as a coarse operator?

- No, the DWF operator is not normal: left-vectors are not right-vectors (conjugated).

Can we use $\left(P^{\dagger} \Gamma_{5} D P\right)^{2}$ as a coarse operator?

- $\Gamma_{5}=\gamma_{5} \mathcal{R}$, where $\mathcal{R}$ reverses the $5^{\text {th }}$ dimension

- Sounds promising, but empirical tests show no convergence

We know $\left(P^{\dagger} D^{\dagger} 1\right)(1 D P)$ works; how do we get closer to that? 


\section{Constructing a 1-Hop Coarse Operator}

Can we use $\left(P^{\dagger} D^{\dagger} P\right)\left(P^{\dagger} D P\right)$ as a coarse operator?

- No, the DWF operator is not normal: left-vectors are not right-vectors (conjugated).

Can we use $\left(P^{\dagger} \Gamma_{5} D P\right)^{2}$ as a coarse operator?

- $\Gamma_{5}=\gamma_{5} \mathcal{R}$, where $\mathcal{R}$ reverses the $5^{\text {th }}$ dimension

- Sounds promising, but empirical tests show no convergence 


\section{Constructing a 1-Hop Coarse Operator}

Can we use $\left(P^{\dagger} D^{\dagger} P\right)\left(P^{\dagger} D P\right)$ as a coarse operator?

- No, the DWF operator is not normal: left-vectors are not right-vectors (conjugated).

Can we use $\left(P^{\dagger} \Gamma_{5} D P\right)^{2}$ as a coarse operator?

- $\Gamma_{5}=\gamma_{5} \mathcal{R}$, where $\mathcal{R}$ reverses the $5^{\text {th }}$ dimension

- Sounds promising, but empirical tests show no convergence

We know $\left(P^{\dagger} D^{\dagger} 1\right)(1 D P)$ works; how do we get closer to that? 


\section{Constructing a 1-Hop Coarse Operator}

Try $\left(P^{\dagger} D^{\dagger} R^{\dagger}\right)(R D P)$ where $\operatorname{dim} R>\operatorname{dim} P$.

In the limit where $\operatorname{dim} R \rightarrow \operatorname{dim} D$, this is the known-good algorithm.

$16^{3} \times 32 \times 16$ Technicolor lattice

$4^{4} \times 16$ blocks

$N_{v}(R)=48$

$N_{v}(P)=12-48$

$m_{q}=0.01$

Requires fast implementation of rectangular-matrix linear algebra

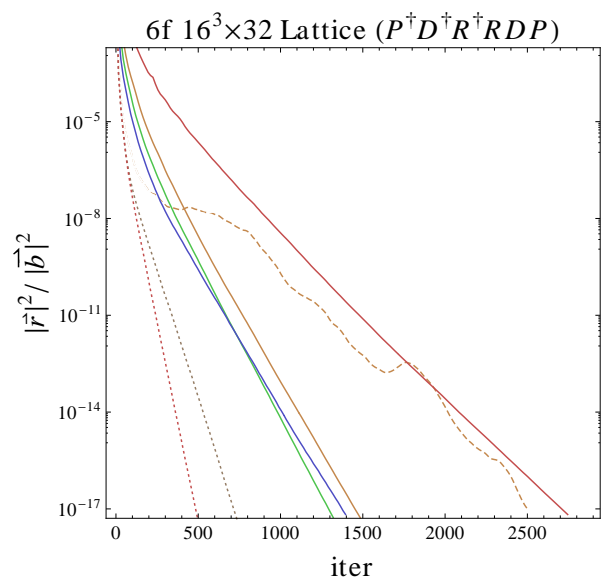




\section{The axial symmetry at finite temperature in Latitice QCD with Overlap fermions}

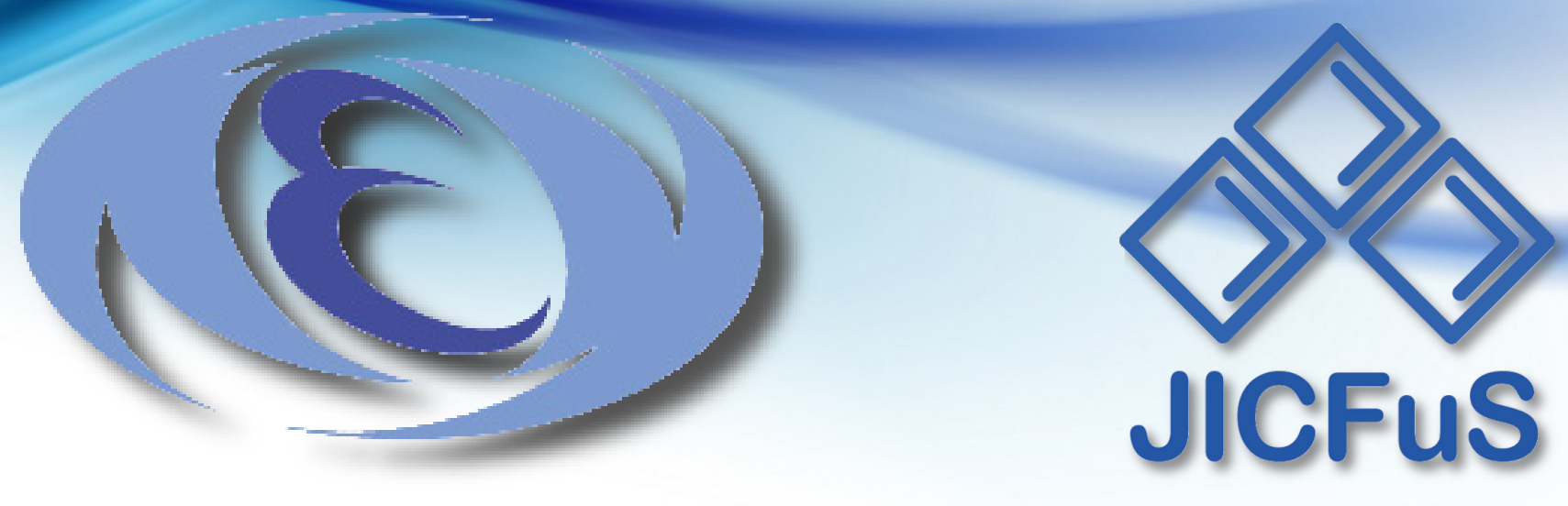

Guido Cossu, KEK

$$
\text { 高工ネルギー加速器研究榢構 }
$$

New Horizons for Lattice Computations with Chiral Fermions RIKEN BNL Research center workshop - May 14, 2012 


\section{高正齐ルギ一加速器研究機棈}

Axiaj symsmetry at finite temp. with overlap fermions

\section{Summary:}

$\checkmark$ Motivation

$\checkmark$ Chiral phase transition at finite temperature and axial symmetry

$\checkmark$ Simulating dynamical (overlap) fermions

$\checkmark$ Topology fixing and friends

$\checkmark$ Methodology and results (quenched and $\mathrm{N}_{\mathrm{f}}=2$ )

$\checkmark$ Discussion and conclusions

People involved in this project

JLQCD group: S. Hashimoto, S. Aoki, T. Kaneko, H. Matsufuru,

J. Noaki, E. Shintani

See for example POS(Lattice2010)174 (arXiv:1011.0257),

PoS(Lattice 2011)188 (arXiv:1204.4519), article in prep. 


\section{高工齐ルギ一加速器研究機棈}

Axial symmsnetry at finite temp. with overlap fermions

\section{Motivation}

Pattern of chiral symmetry breaking at low temperature QCD

$S U\left(N_{f}\right)_{V} \times S U\left(N_{f}\right)_{A} \times U(1)_{V} \times U(1)_{A} \rightarrow S U\left(N_{f}\right)_{V} \times U(1)_{V}$

Well known facts about axial symmetry

- Axial anomaly - Non vanishing topological susceptibility

- Mass splitting of the $\eta^{\prime}(958 \mathrm{Mev})$ with respect to the lighter Goldsone bosons

What is the fate of the axial $\mathrm{U}_{A}(1)$ symmetry at finite temperature $\left(T \geqslant T_{c}\right)$ ?

Dirac Overlap operator, retaining the maximal amount of chiral symmetry on the lattice is, theoretically, the best way to answer this question.

- Experimental data: PHENIX(BNL) Phys.Rev. C83 (2011) 054903

"the mass of the eta' meson is reduced by Delta-m $>200 \mathrm{MeV}$, at the $99.9 \%$ confidence level, in the considered model class" 


\section{高工齐ルギ一加速器研究機棈} Axiaj symsmetry at finite temp. with overlap fermions

\section{Goal of the current project}

Check restoration of axial $\mathrm{U}_{A}(1)$ symmetry by measuring (spatial) meson correlators at finite temperature in full QCD with overlap

Degeneracy of correlators is the signal that we are looking for

$$
\begin{aligned}
& \sigma\left(1_{4} \otimes 1_{2}\right) \triangle \text { Chiral sym. }-\pi\left(i \gamma_{5} \otimes \tau^{a}\right) \\
& U(1)_{A} \\
& \eta\left(i \gamma_{5} \stackrel{\vee}{\otimes} 1_{2}\right), \text { Chiral sym. } \delta\left(1_{4} \otimes^{\boldsymbol{\nabla}} \tau^{a}\right)
\end{aligned}
$$

As I will show in this talk,

there is one issue to solve before attacking the real problem... 


\section{高壬齐ルギ一加速器研究機楎}

Axiaj symsmetry at finite temp. with overlap fermions

\section{Simulating dynamical overlap fermions}

In order to avoid expensive tricks to handle the zero modes of the Hermitian Wilson operator JLQCD simulations use (JLQCD 2006):

- Iwasaki action (suppresses Wilson operator near zero modes)

- Extra Wilson fermions and twisted mass ghosts to rule out the zero modes

Topology is thus fixed throughout the HMC trajectory.

The effect of fixing topology is expected to be a Finite Size Effect (actually $\mathrm{O}(1 / \mathrm{V})$ ), next slides 


\section{高工齐ルギ一加速器研究機棈} Axial symsmsetry at finite temp. with overlap fermions

Fixing topology: how to deal with it at

Partition function at fixed topology

$Z_{Q}=\frac{1}{2 \pi} \int_{-\pi}^{\pi} d \theta \exp (-V F(\theta)) \quad F(\theta) \equiv E(\theta)-i \theta Q / V$

where the energy can be $E(\theta)=\sum_{n=1}^{\infty} \frac{c_{2 n}}{(2 n) !} \theta^{2 n}=\frac{\chi_{t}}{2} \theta^{2}+O\left(\theta^{4}\right)$
expanded

Using saddle point expansion around $\theta_{c}=i \frac{Q}{\chi_{t} V}\left(1+O\left(\delta^{2}\right)\right)$ one obtains the Gaussian distribution

$$
Z_{Q}=\frac{1}{\sqrt{2 \pi \chi_{t} V}} \exp \left[-\frac{Q^{2}}{2 \chi_{t} V}\right]\left[1-\frac{c_{4}}{8 V \chi_{t}^{2}}+O\left(\frac{1}{V^{2}}, \delta^{2}\right)\right]
$$




\section{高工齐ルギ一加速器研究機棈}

Axiaj symsmetry at finite temp. with overlap fermions

Fixing topology: how to deal with it at

From the previous partition function we can extract the relation between correlators at fixed $\boldsymbol{\theta}$ and correlators at fixed $\mathrm{Q}$

In particular for the topological susceptibility and using the Axial Ward Identity we obtain a relation involving fermionic quantities:

$\lim _{x \mid \rightarrow \operatorname{large}}\langle m P(x) m P(0)\rangle_{Q}^{\operatorname{disc}} \equiv \frac{1}{V}\left(\frac{Q^{2}}{V}-\chi_{t}-\frac{c_{4}}{2 \chi_{t} V}\right)+O\left(e^{-m_{\pi}|x|}\right)$

$\mathrm{P}(\mathrm{x})$ is the flavor singlet pseudo scalar density operator Aoki et al. PRD76,054508 (2007)

What is the effect of fixing $Q$ at finite temperature? 


\section{高壬齐ルギ一加速器研究機構}

Axial symmmetry at finite temp. with overlap fermions

\section{Results}

$\checkmark$ Simulation details

$\checkmark$ Eigenvalues distribution

$\checkmark$ Finite temperature quenched SU(3) at fixed topology Hitachi SR16K

$\checkmark$ Meson correlators in two flavors QCD

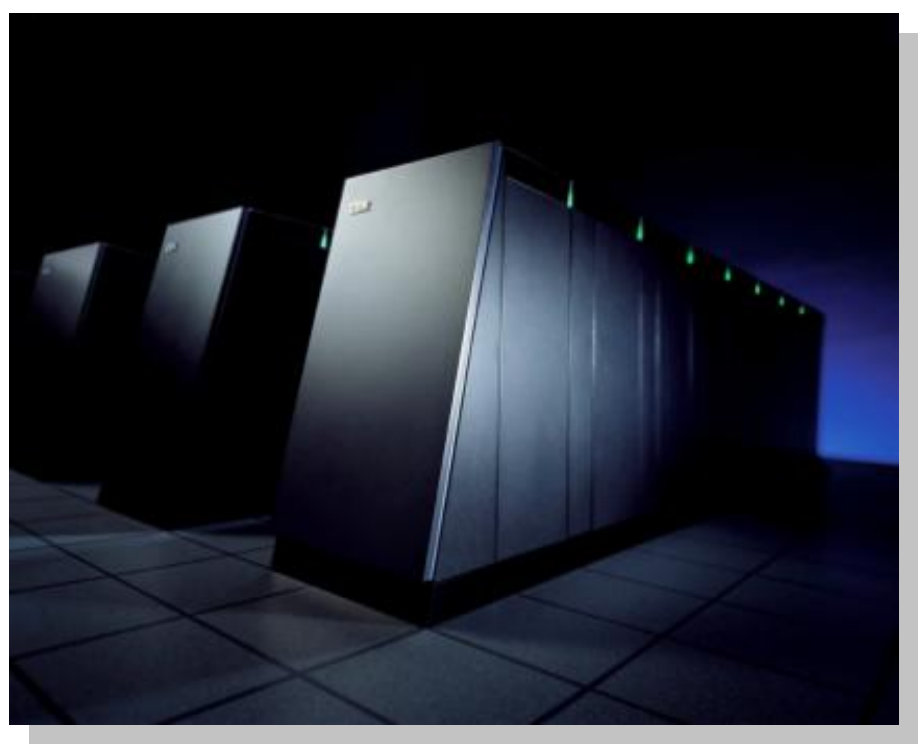




\section{高工齐ルギ一加速器研究機棈}

Axial symmmetry at finite temp. with overlap fermions

\section{Simulation details}

Pure gauge $\left(16^{3} \times 6,24^{3} \times 6\right)$ :

Iwasaki action + top. fixing term

\begin{tabular}{|l|l|l|l|}
\hline$\beta$ & \multicolumn{1}{|c|}{$a(\mathrm{fm})$} & \multicolumn{1}{|c|}{$T(\mathrm{MeV})$} & \multicolumn{1}{|c|}{$T / \mathrm{Tc}$} \\
\hline 2.35 & 0.132 & 249.1 & 0.86 \\
\hline 2.40 & 0.123 & 268.1 & 0.93 \\
\hline 2.43 & 0.117 & 280.9 & 0.97 \\
\hline 2.44 & 0.115 & 285.7 & 0.992 \\
\hline 2.445 & 0.114 & 288 & 1.0 \\
\hline 2.45 & 0.1133 & 290.2 & 1.01 \\
\hline 2.46 & 0.111 & 295.1 & 1.02 \\
\hline 2.48 & 0.107 & 305.6 & 1.06 \\
\hline 2.50 & 0.104 & 316.2 & 1.10 \\
\hline 2.55 & 0.094 & 347.6 & 1.20 \\
\hline
\end{tabular}

Two flavors QCD $\left(16^{3} \times 8\right)$

Iwasaki + Overlap + top. Fix

$\mathrm{O}(300)$ trajectories per $\mathrm{T}$ $a m=0.05,0.025,0.01$

\begin{tabular}{|c|c|c|c|}
\hline$\beta$ & $a(f m)$ & $T(\mathrm{MeV})$ & $T / T c$ \\
\hline 2.18 & 0.1438 & 171.5 & 0.95 \\
\hline 2.20 & 0.1391 & 177.3 & 0.985 \\
\hline 2.25 & 0.12818 & 192.2 & 1.06 \\
\hline 2.30 & 0.1183 & 208.5 & 1.15 \\
\hline 2.40 & 0.1013 & 243.5 & 1.35 \\
\hline 2.45 & 0.0940 & 262.4 & 1.45 \\
\hline
\end{tabular}


(f1) 高正永ルギ二加速器研究機構 Axial symmmetry at finite temp. with overlap fermions

Eigenvalue distribution

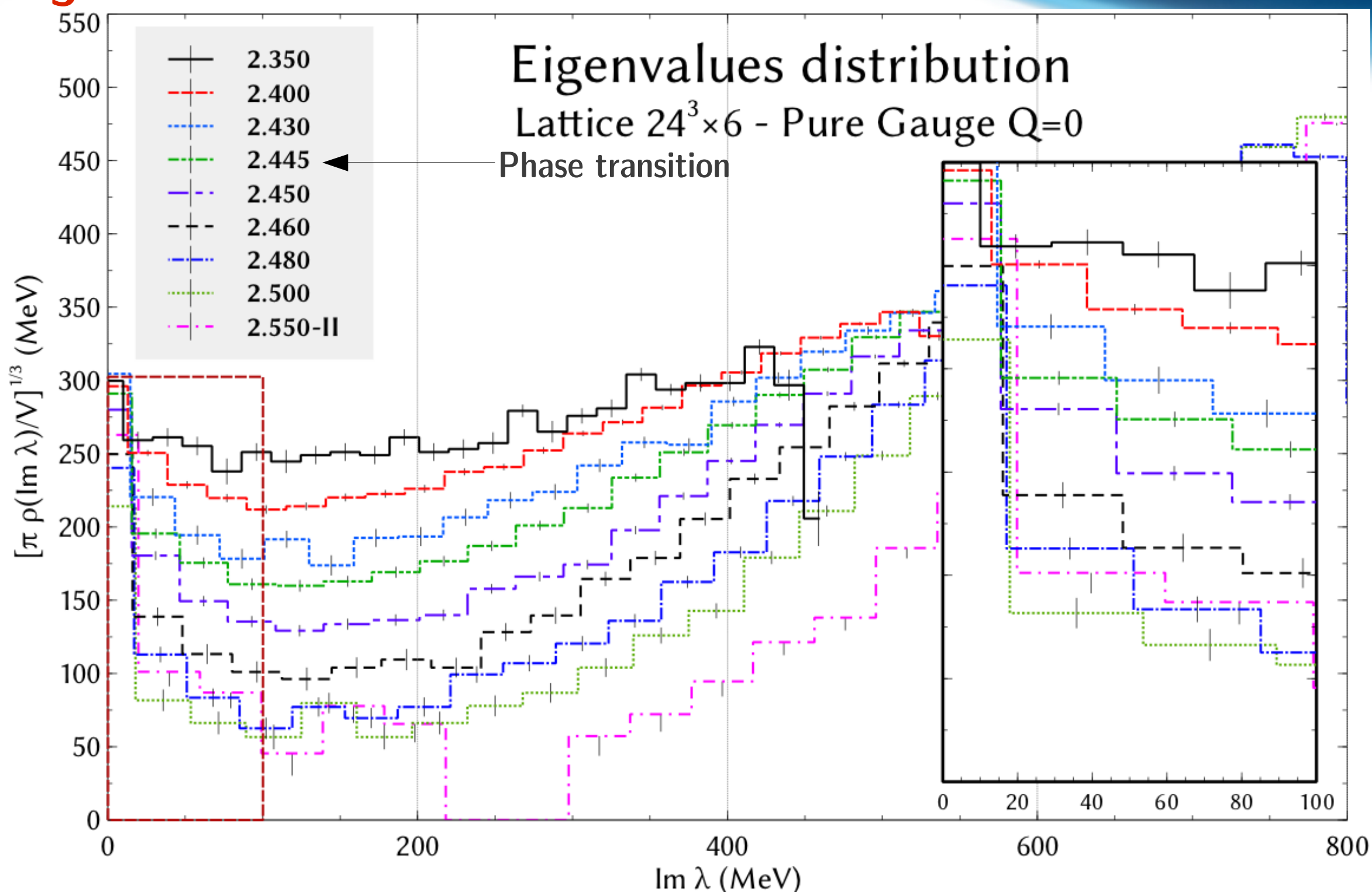




\section{高壬齐ルギ一加速器研究機楎}

Axiaj symsmetry at finite temp. with overlap fermions

Topological susceptibility in pure gaugesth

$\lim _{|x| \rightarrow \operatorname{large}}\langle m P(x) m P(0)\rangle_{Q}^{\text {disc }} \equiv \frac{1}{V}\left(\frac{Q^{2}}{V}-\chi_{t}-\frac{c_{4}}{2 \chi_{t} V}\right)+O\left(e^{-m_{\pi}|x|}\right)$

-(Spatial) Correlators are always approximated by the first 50 eigenvalues

-Pure gauge: double pole formula for disconnected diagram

-Topological susceptibility estimated by a joint fit of connected and disconnected contribution. 
(E)高エネルギ一加速器研究機構 Axial symsmsetry at finite temp. with overlap fermions

\section{Topological susceptibility in pure gauge the}

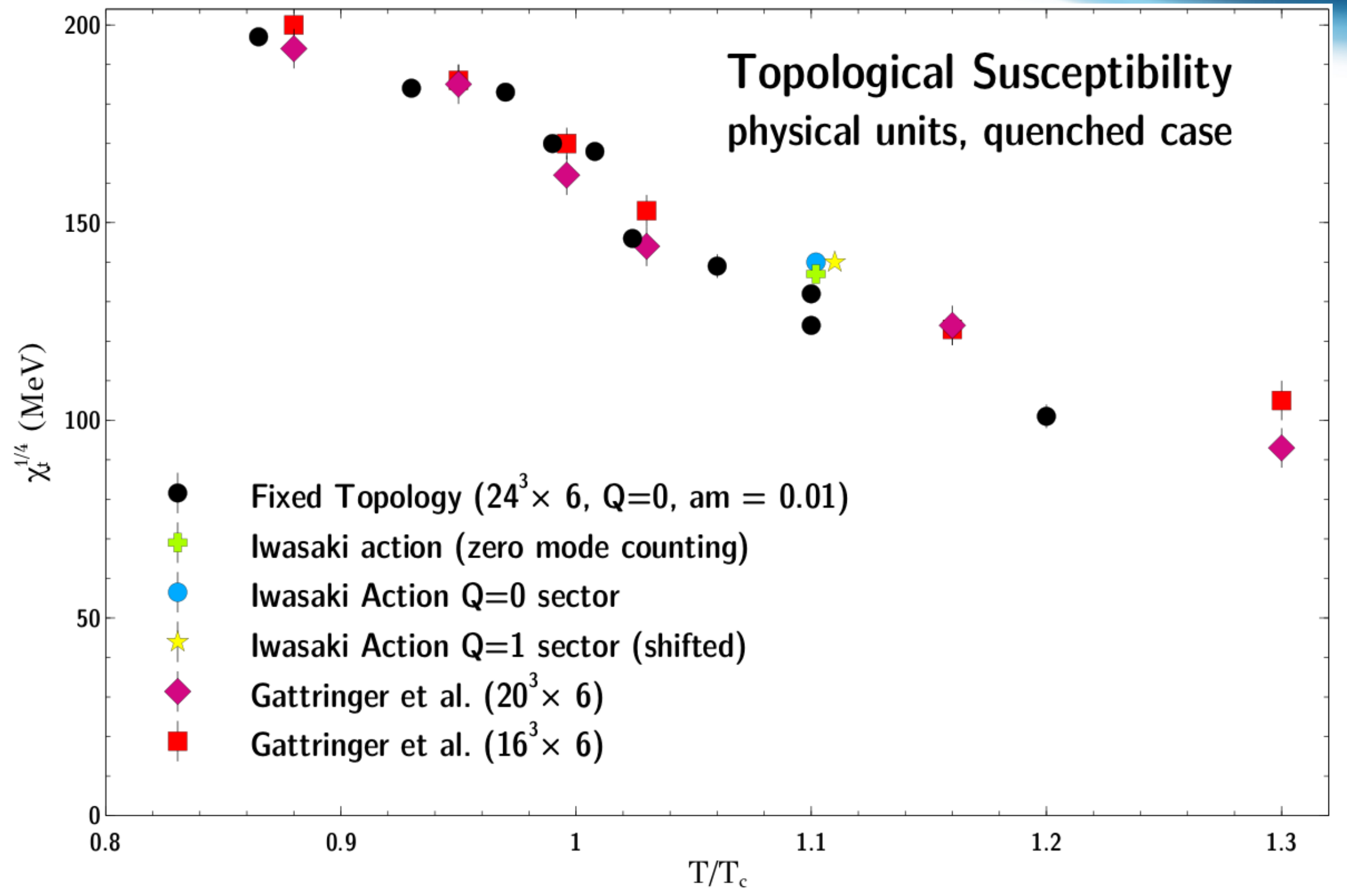




\section{高工齐ルギ一加速器研究機棈}

Axial symmetry at finite temp. with overlap fermions

\section{Full QCD - Eigenvalues}

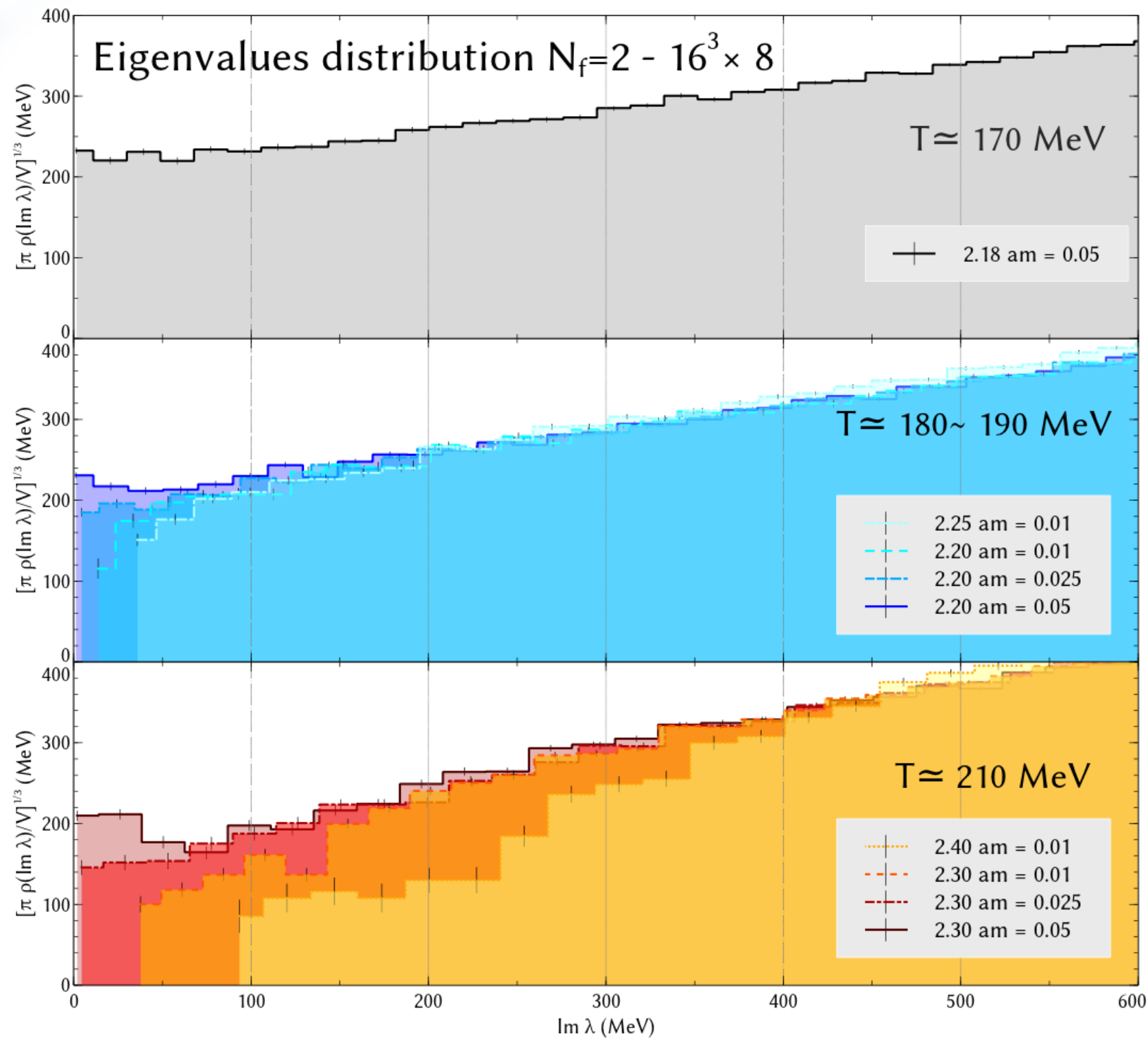

Effect of axial symmetry on the Dirac sperctrum

$\chi^{\pi-\delta}=\int d \lambda \rho_{m}(\lambda) \frac{4 m^{2}}{\lambda^{2}+m^{2}}$

If axial symmetry is restored we can conclude that

$\lim _{\lambda \rightarrow 0} \lim _{m \rightarrow 0} \frac{\rho_{m}(\lambda)}{\lambda}=0$

Ref: S. Aoki, internal note 


\section{(C)高工衣ルギ一加速器研究機構}

Axial symmsnetry at finite temp. with overlap fermions

Full QCD - Meson correlators

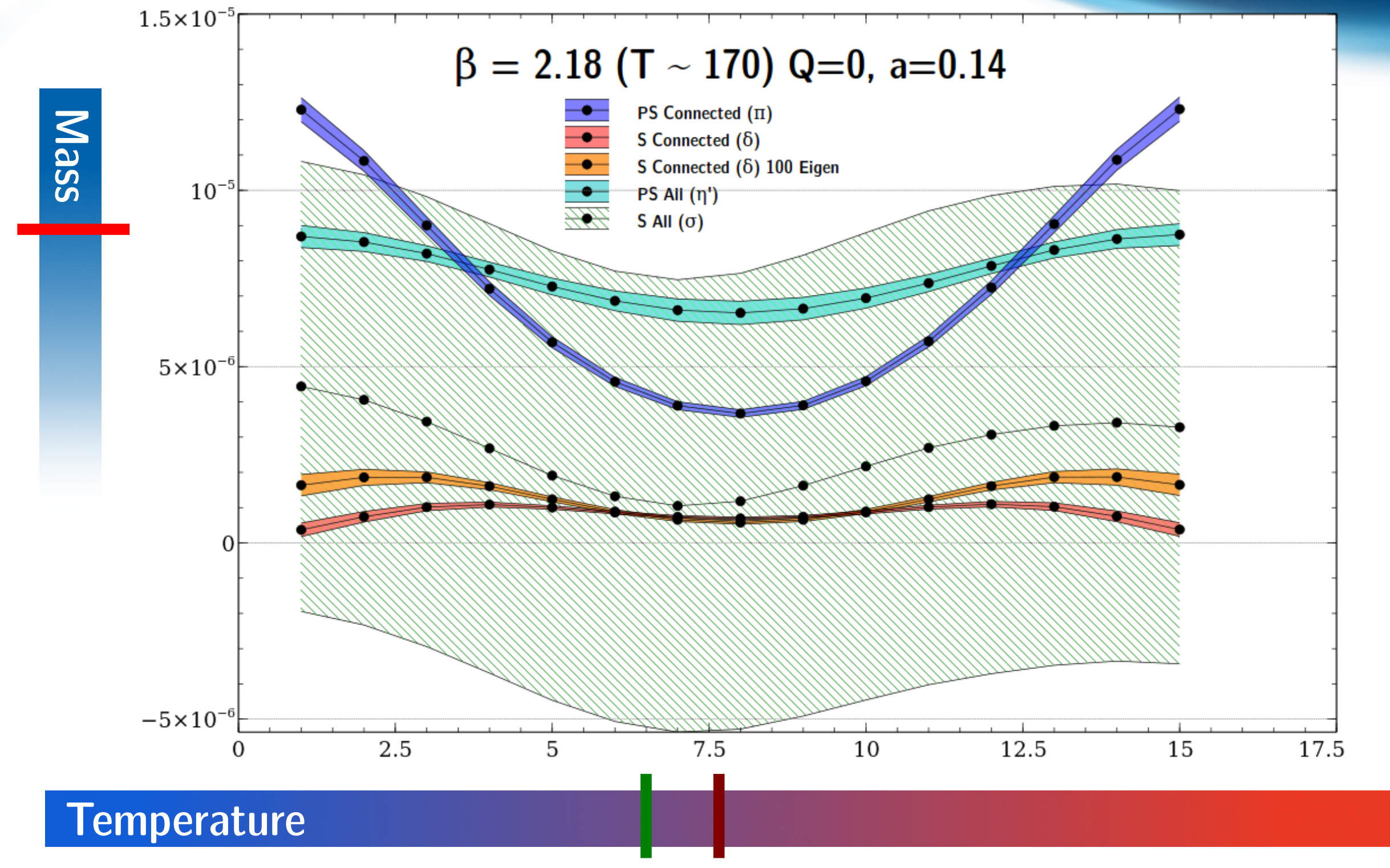




\section{(C) 高工齐ルギ一加速器研究機構}

Axial symsmetry at finite temp. with overlap fermions

Full QCD - Meson correlators

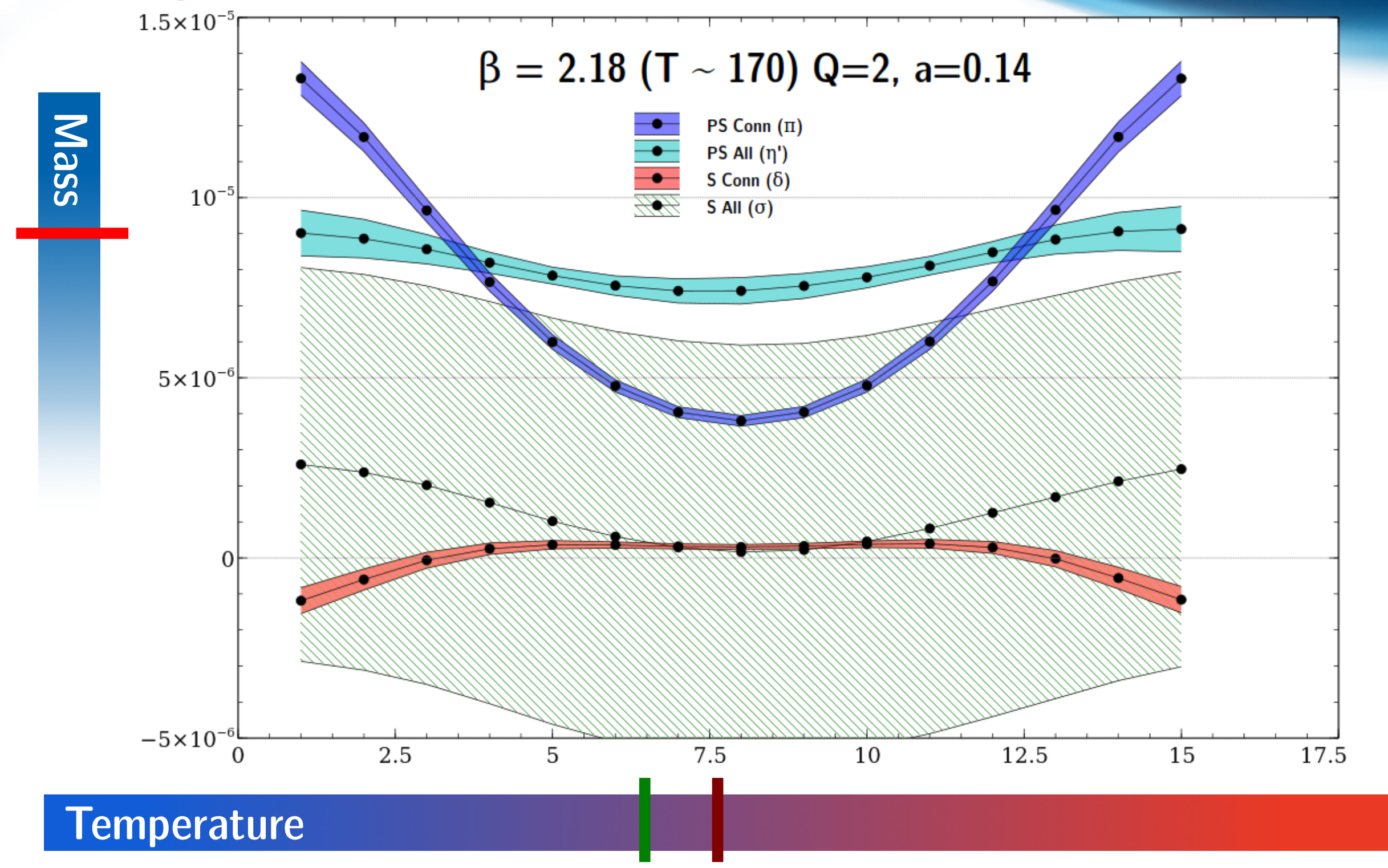




\section{(E) 高正齐ルギ一加速器研究機棈}

Axial symmsnetry at finite temp. with overlap fermions

Full QCD - Meson correlators

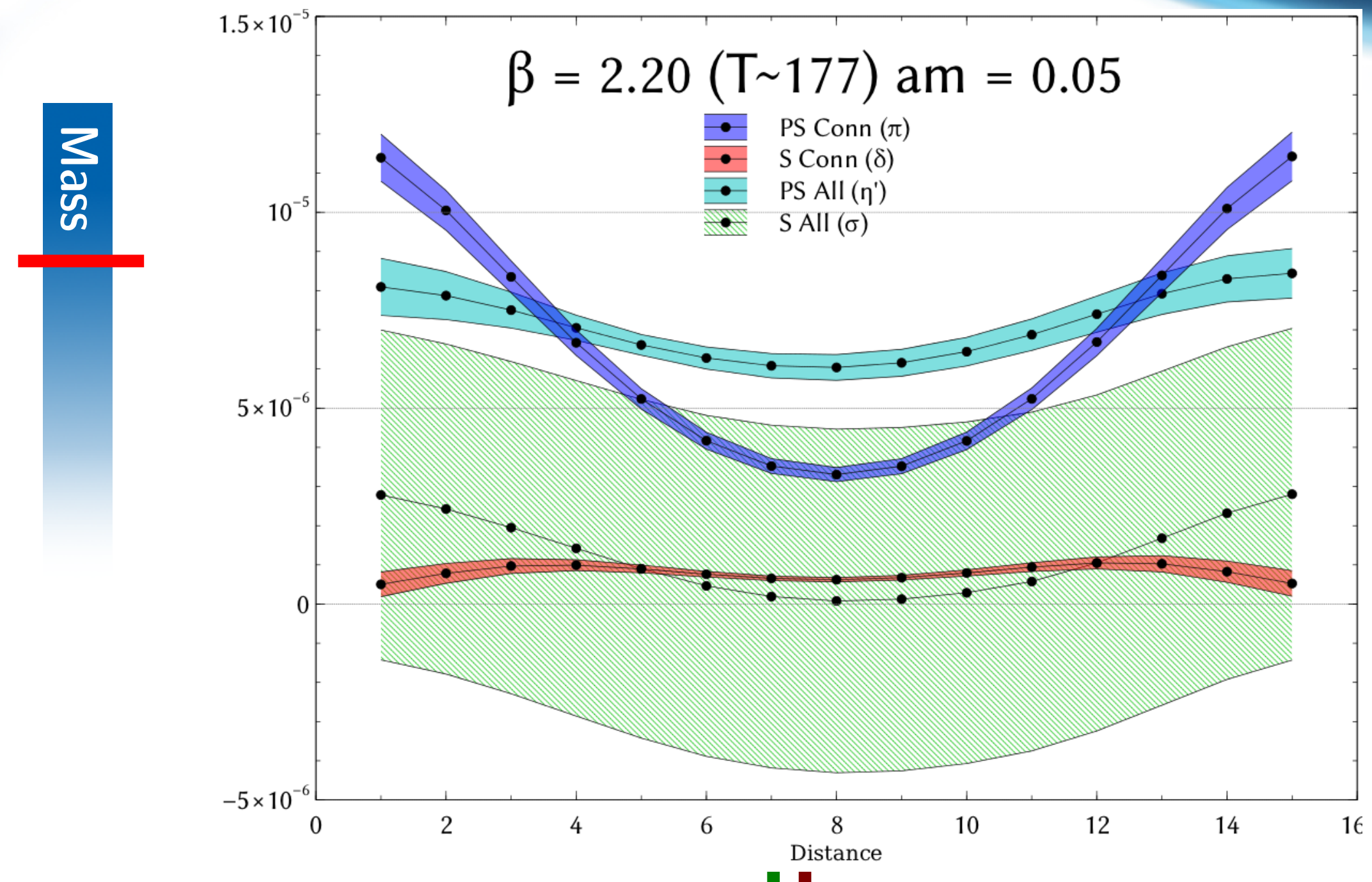




\section{(E) 高正齐ルギ一加速器研究機棈}

Axial symmsnetry at finite temp. with overlap fermions

Full QCD - Meson correlators

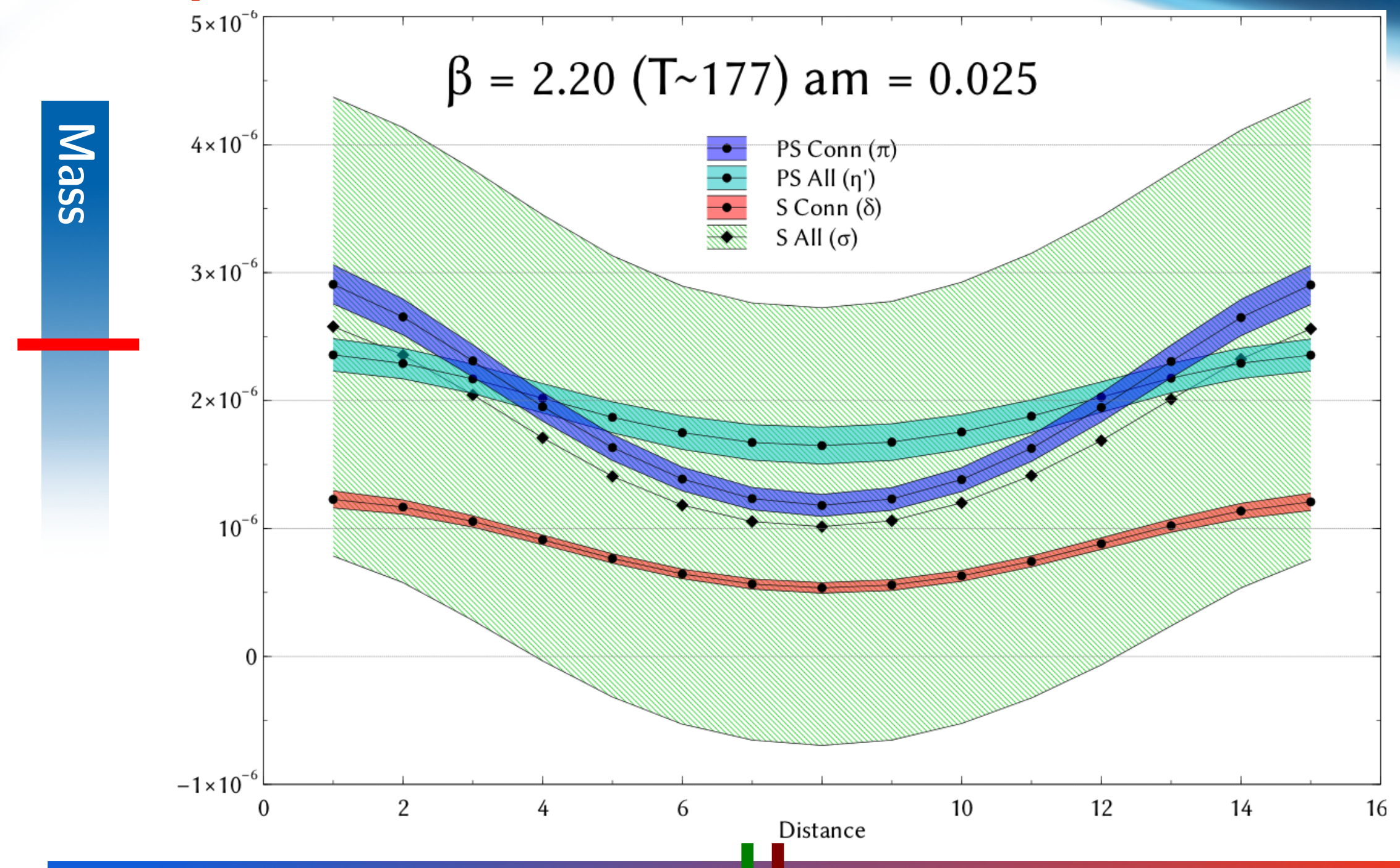

Temperature 


\section{(£)高壬齐ルギ一加速器研究機棈}

Axiaj symsmetry at finite temp. with overlap fermions

Full QCD - Meson correlators

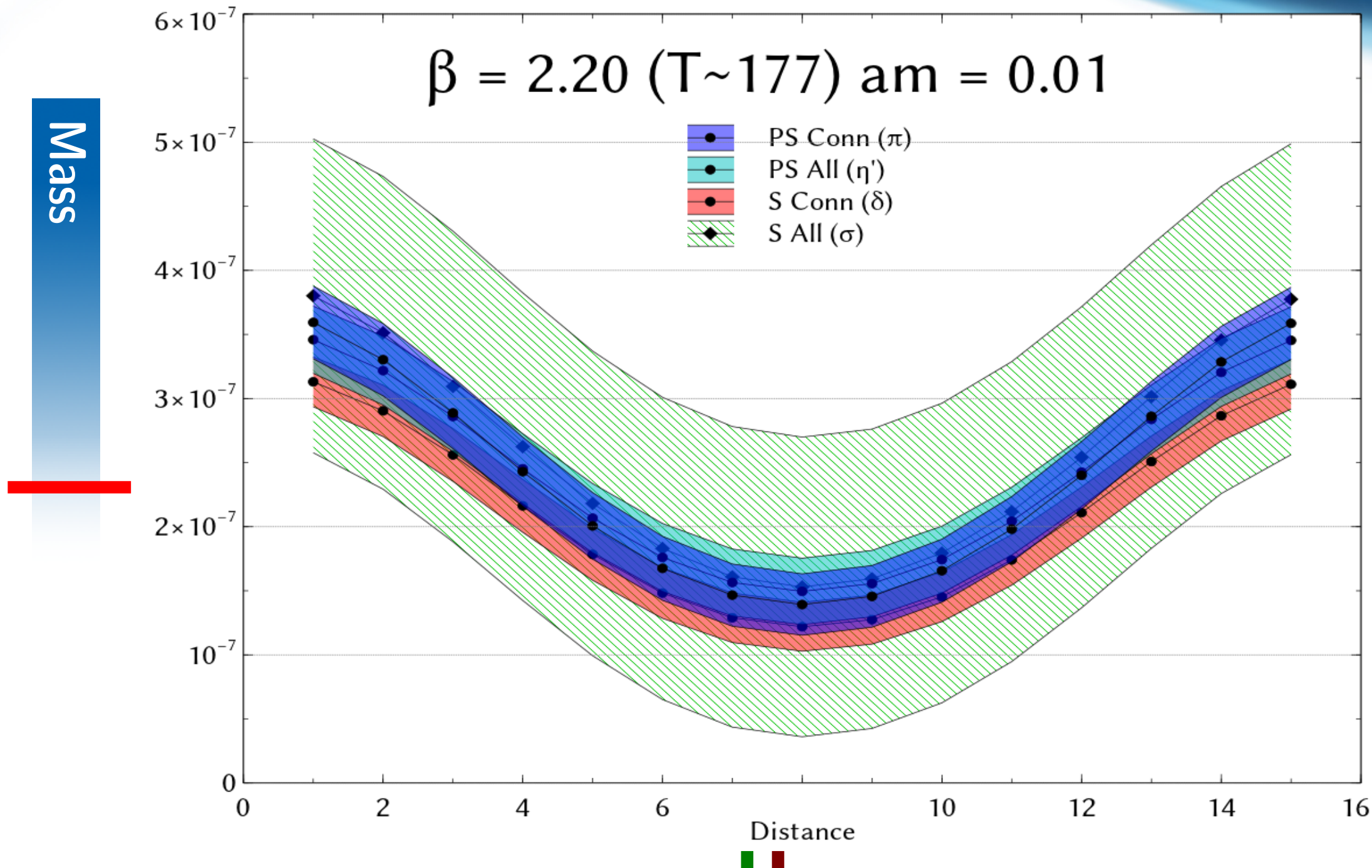


(E) 高壬齐ルギ一加速器研究機棈 Axial symmmetry at finite temp. with overlap fermions

\section{Full QCD - Meson correlators}

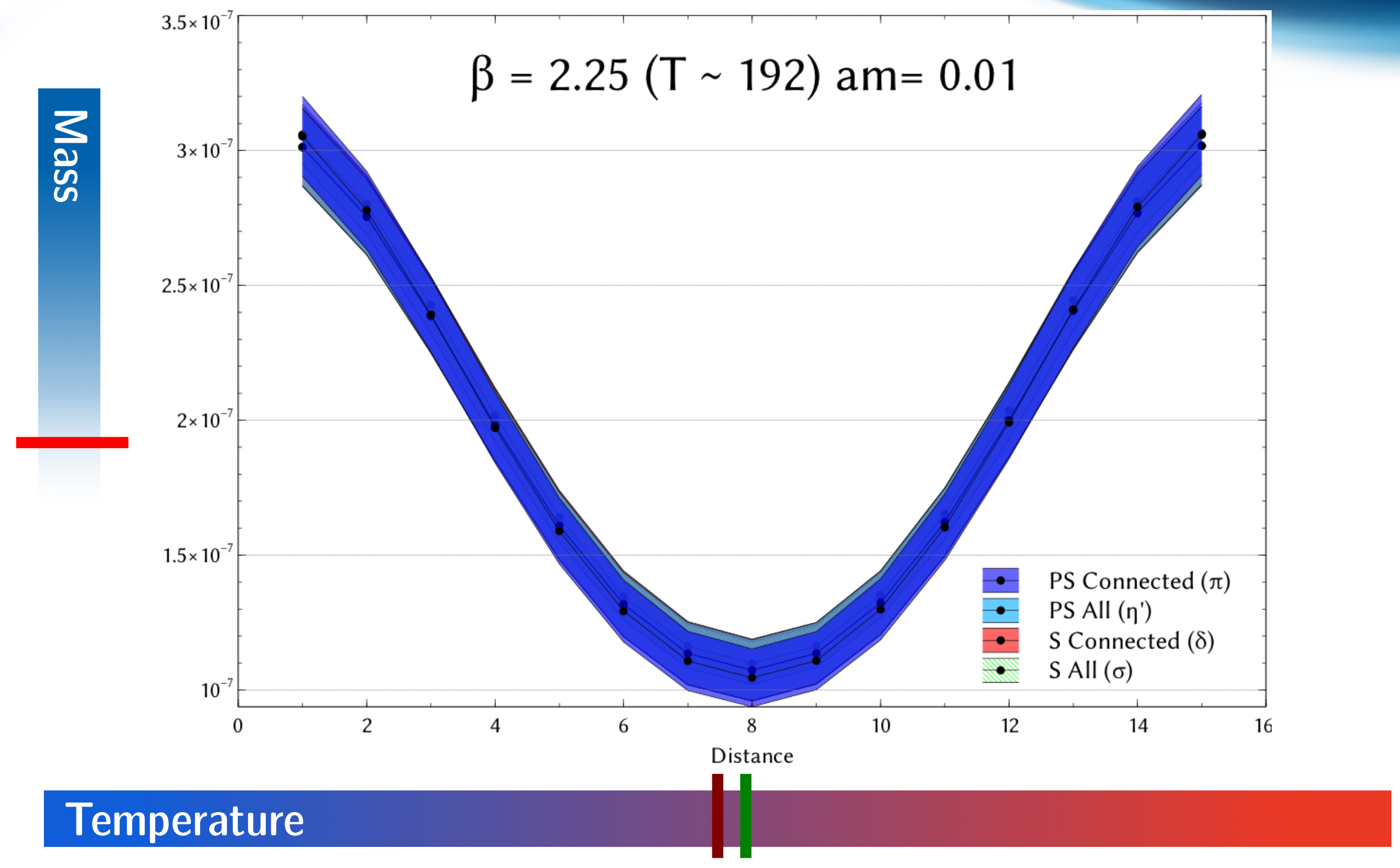




\section{(£)高壬齐ルギ一加速器研究機棈}

Axial symmsnetry at finite temp. with overlap fermions

Full QCD - Meson correlators

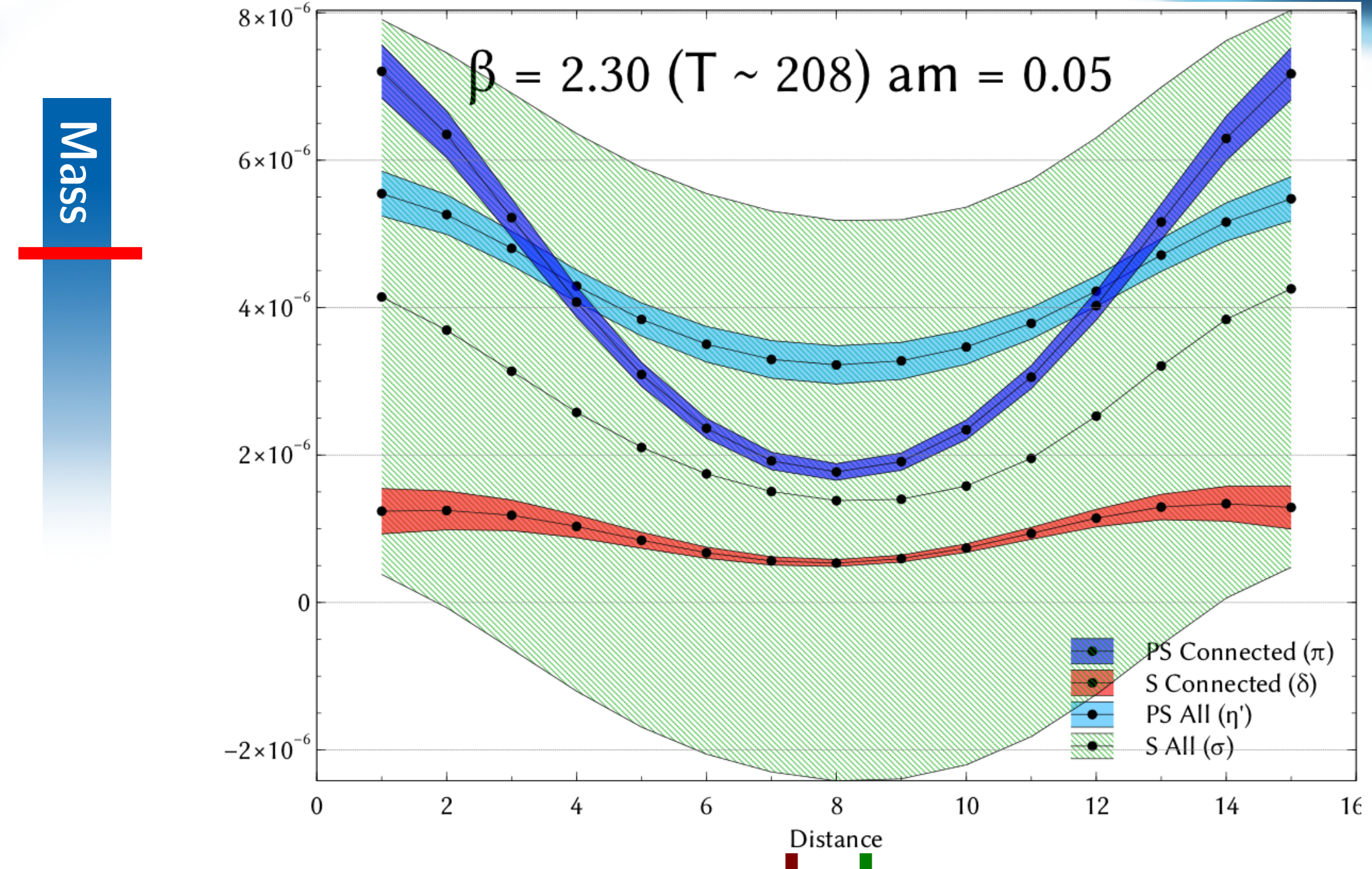

Temperature 


\section{(£) 高王亦ルギ一加速器研究機棈}

Axial symmsnetry at finite temp. with overlap fermions

Full QCD - Meson correlators

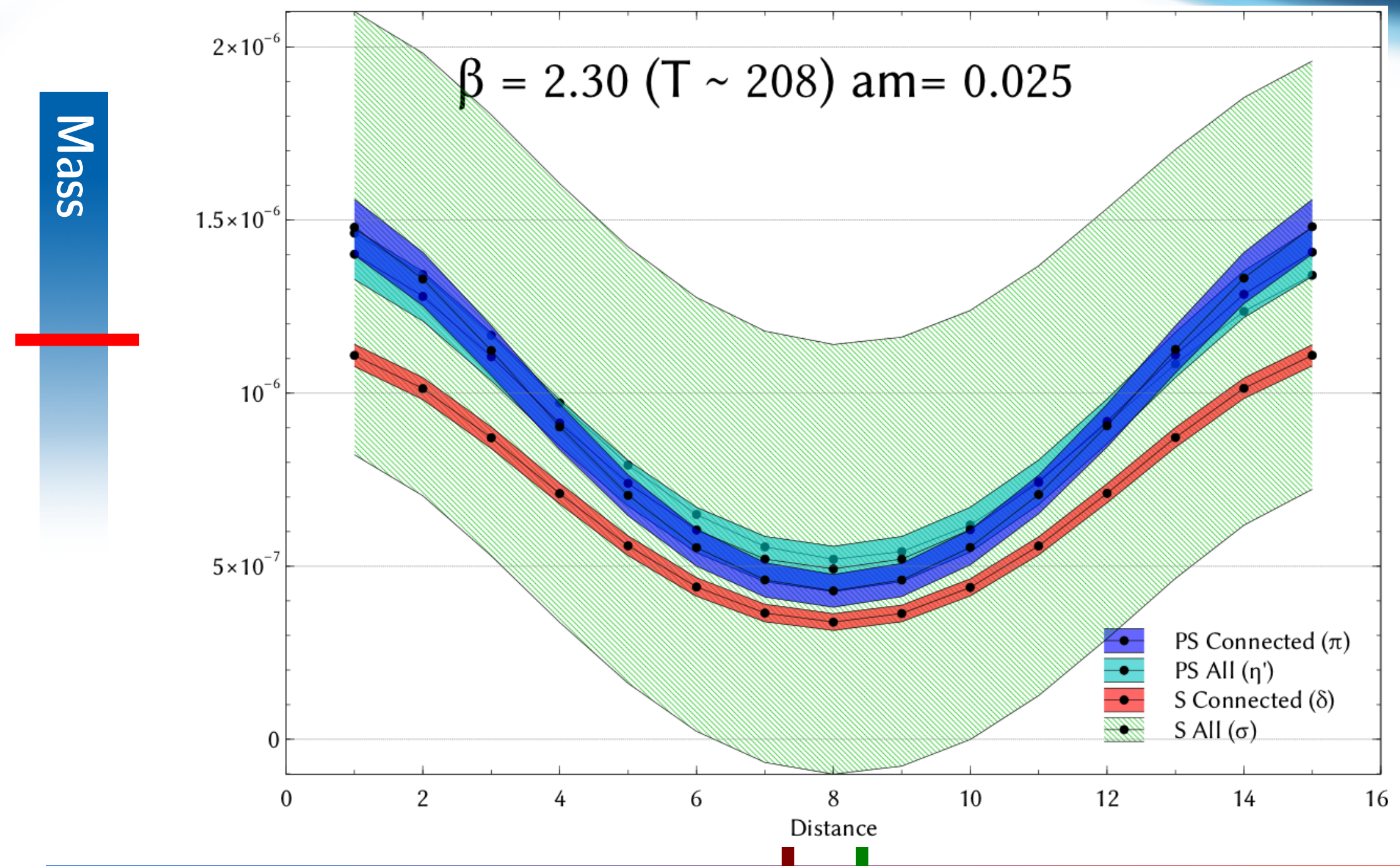




\section{(£) 高正亦ルギ一加速器研究機棈}

Axial symmmetry at finite temp. with overlap fermions

Full QCD - Meson correlators

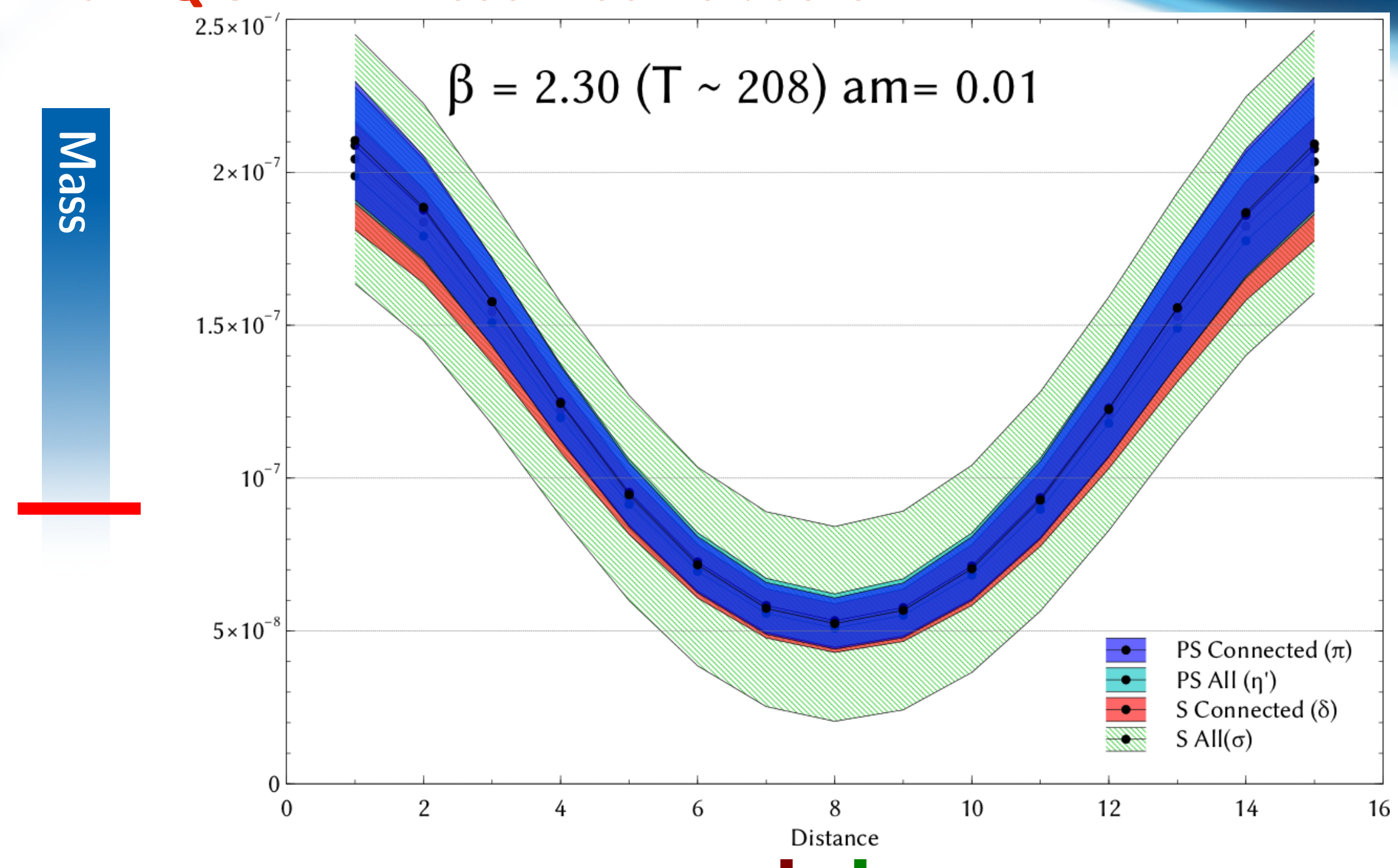

Temperature 


\section{(£) 高王齐ルギ一加速器研究機棈}

Axial symsmetry at finite temp. with overlap fermions

\section{Full QCD - Meson correlators}
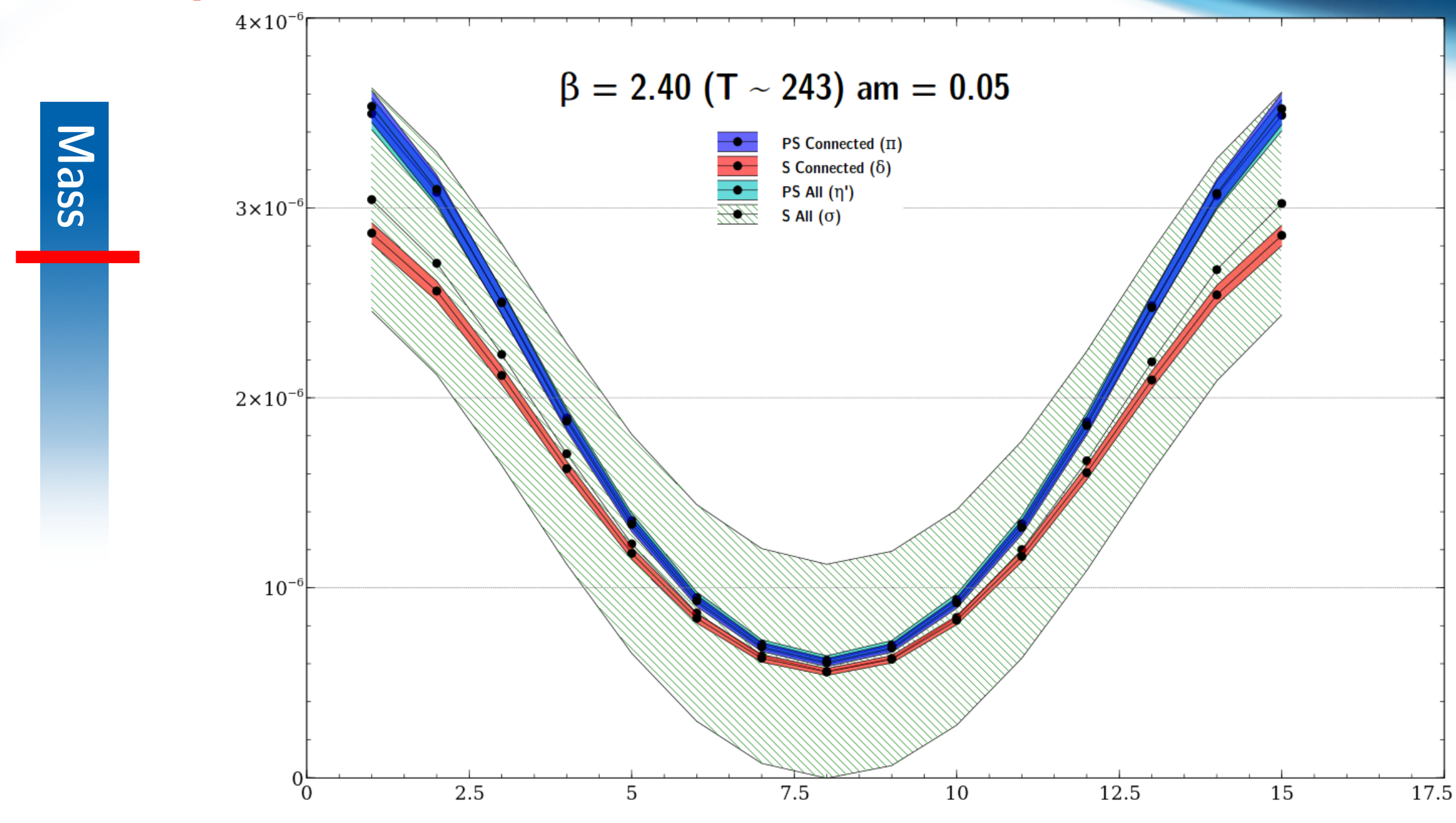


\section{高工齐ルギ一加速器研究機棈}

Axiaj symsmetry at finite temp. with overlap fermions

\section{Summary}

- Overlap fermions are the best choice to check axial anomaly at finite temperature

- Current machine and algorithms permit now realistic simulations...

- ....at the cost of fixing topology

- We checked feasibility of finite $t$. sim. by test runs in pure gauge theory

- In pure gauge systematic errors are under control.

- Results in Full QCD show signal of restoration of axial $\mathrm{U}_{A}(1)$ symmetry

- We need 1-2 points more in the chiral limit 
(C) 高王ルギ一加速器研究機棈 Axial symmsnetry at finite temp. with overlap fermions

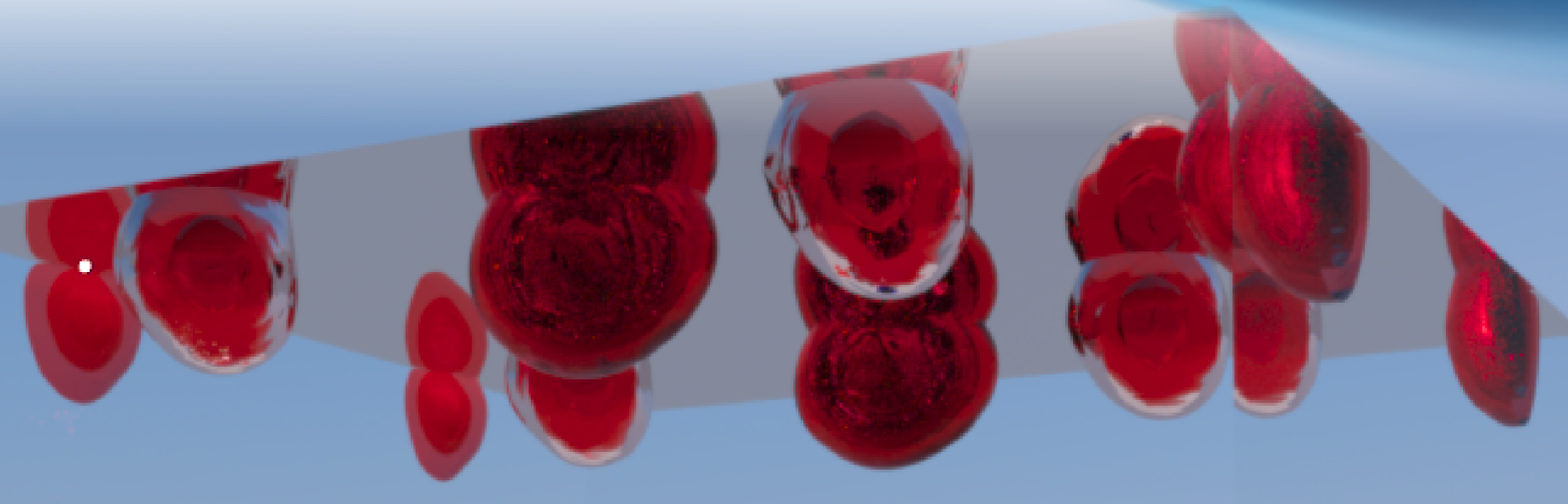

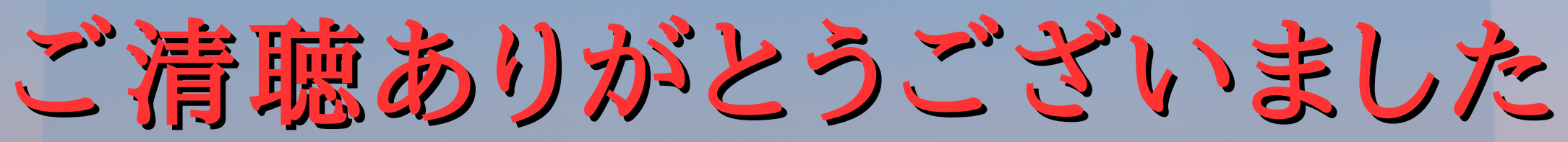
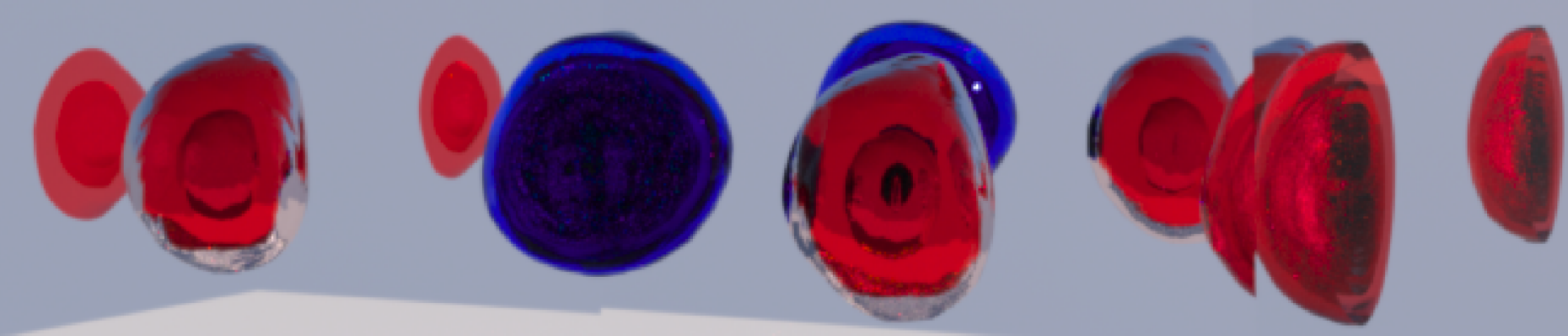

LuxRay Artistic Rendering of Lowest Eigenmode 
高壬ネルギ一加速器研究機楎 Axial symmsmetry at finite temp. with overlap fermions

\section{Backup slides}




\section{高亦ルギ一加速器研究機棈}

Axiaj symsmetry at finite temp. with overlap fermions

Previous analyses

Vranas (Nucl.Phys.Proc.Suppl. 83 (2000) 414-416)

Measured the difference of susceptibilities $\chi$ of $\pi$ and $\delta$.

- Action: DWF, $\mathrm{L}_{s}=24$

- Found a very small relative difference just above $T_{c}$ in the chiral limit

- Residual mass effects?

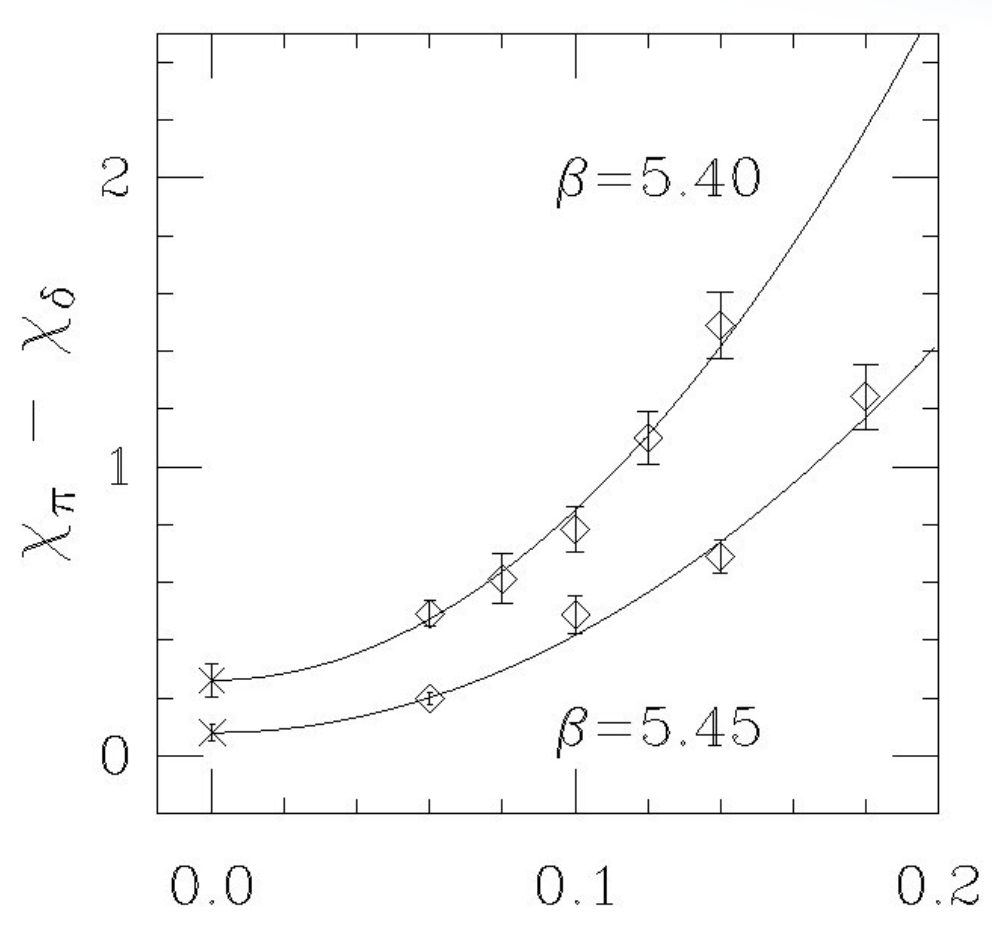

Other works with staggered fermions established a restoration at $\mathbf{T}>\mathbf{T}_{c}$ Even bigger problems due to breaking of $\mathbf{U}_{A}(1)$ by staggered fermions. 
(E) 高互弈ルギー加速器研究機棈 Axial symsmsetry at finite temp. with overlap fermions

Topological susceptibility in
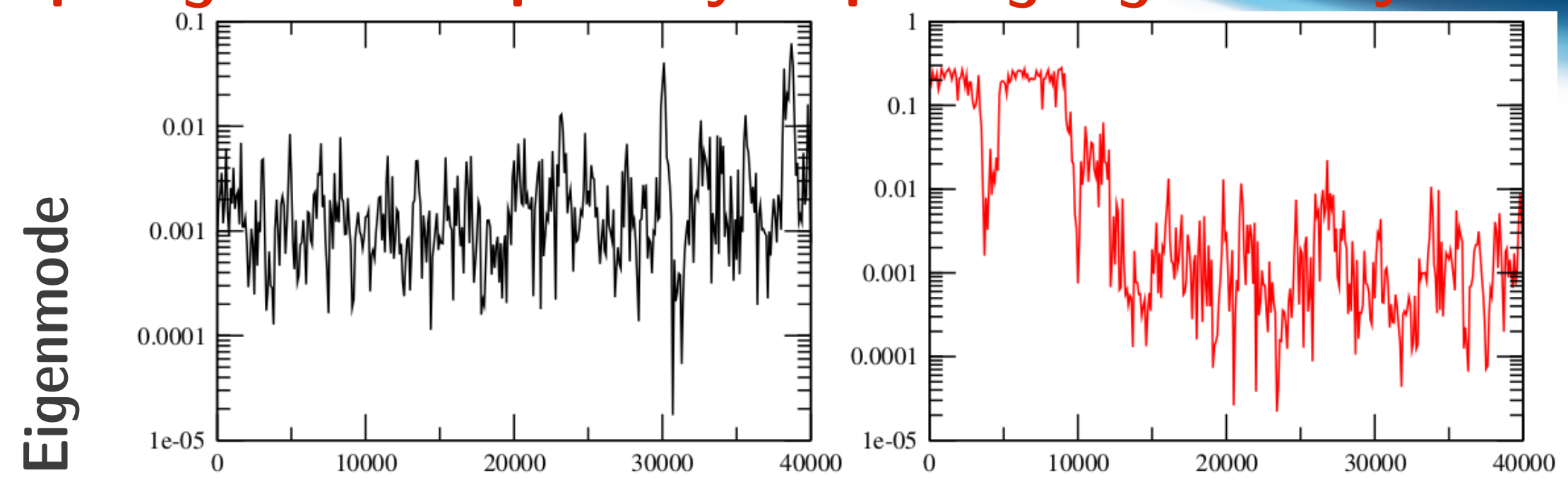

苛
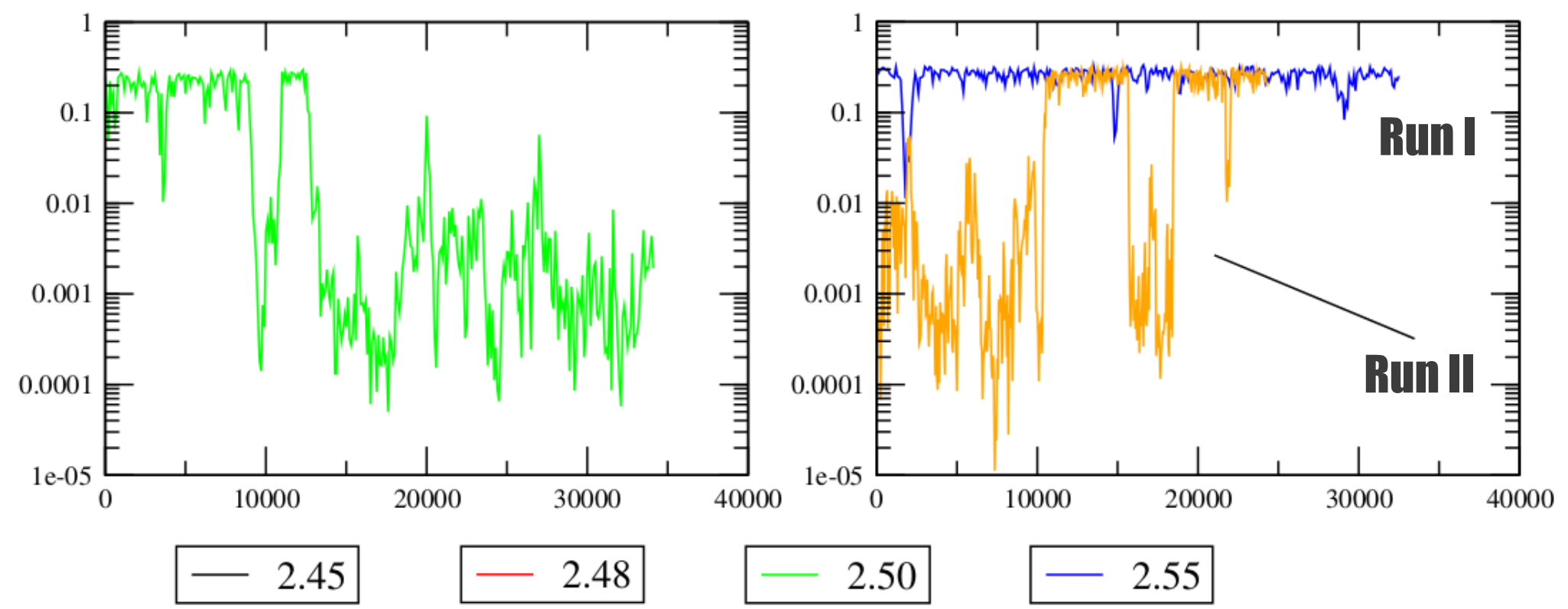

Axial symmsnetry at finite temp. with overlap fermions

Topological susceptibility in pure gauge theory

$\beta=2.50$

Eigenvalues Distribution Comparison, $\mathrm{Q}=0$ sector

- $\quad 2.50$ Iwasaki + Top. Fix

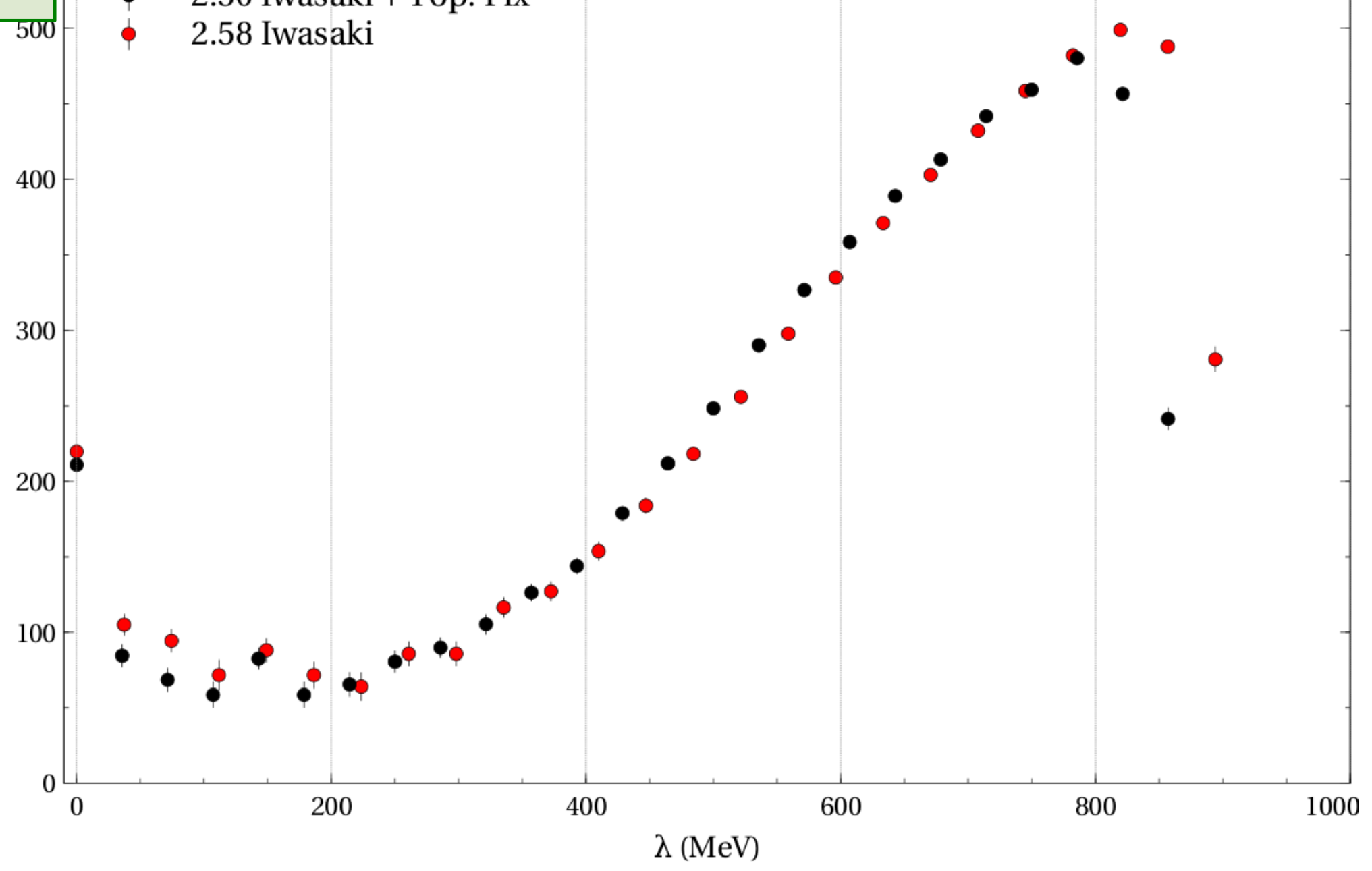




\section{(E) 高正齐ルギ一加速器研究機棈}

Axial symmmetry at finite temp. with overlap fermions

Topological susceptibility in pure gauge Inverse Participation Ratio

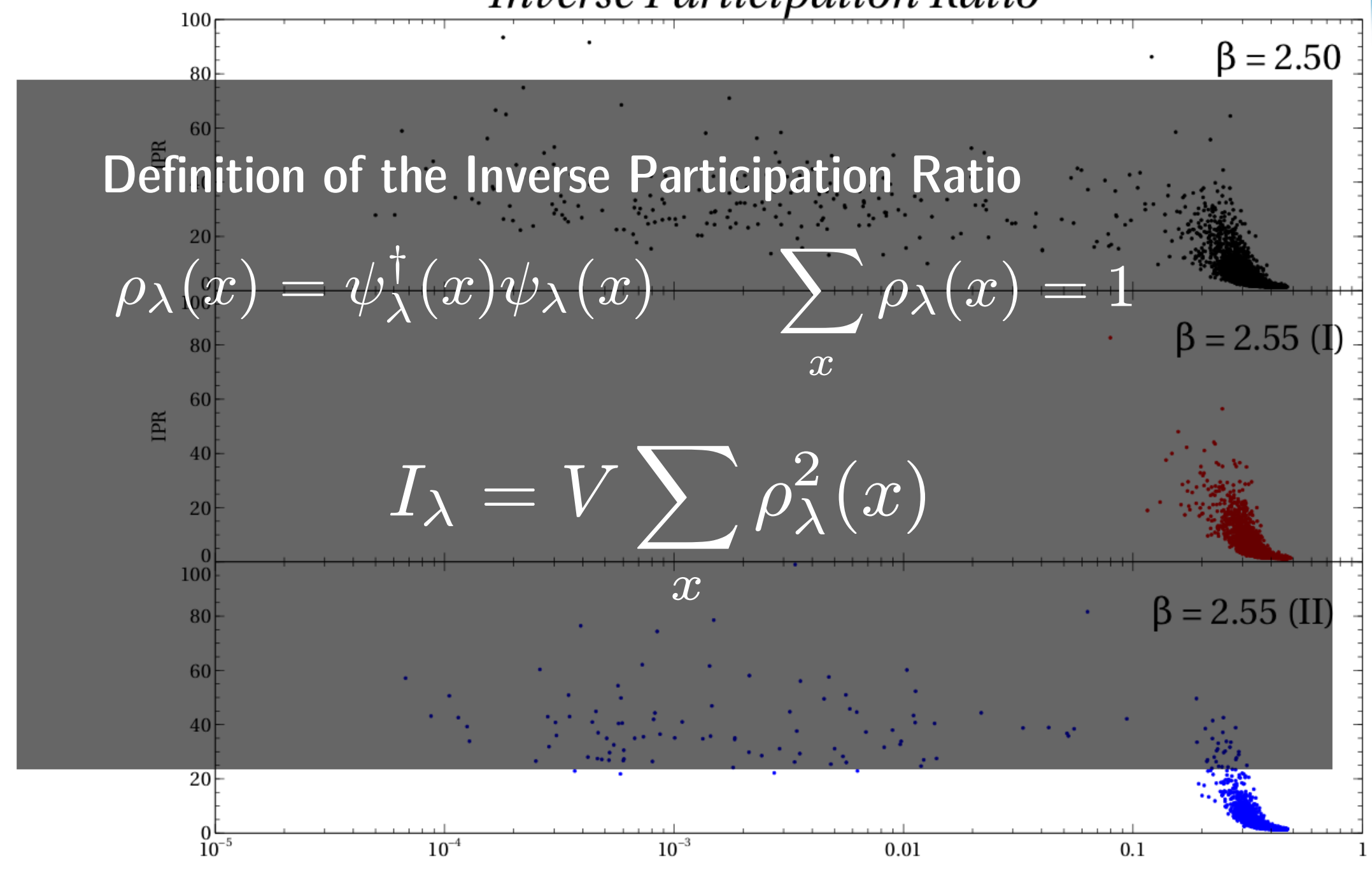




\section{高工齐ルギ一加速器研究機棈}

Axiaj symsmetry at finite temp. with overlap fermions

Topological susceptibility in pure gauge theory

Inverse Participation Ratio

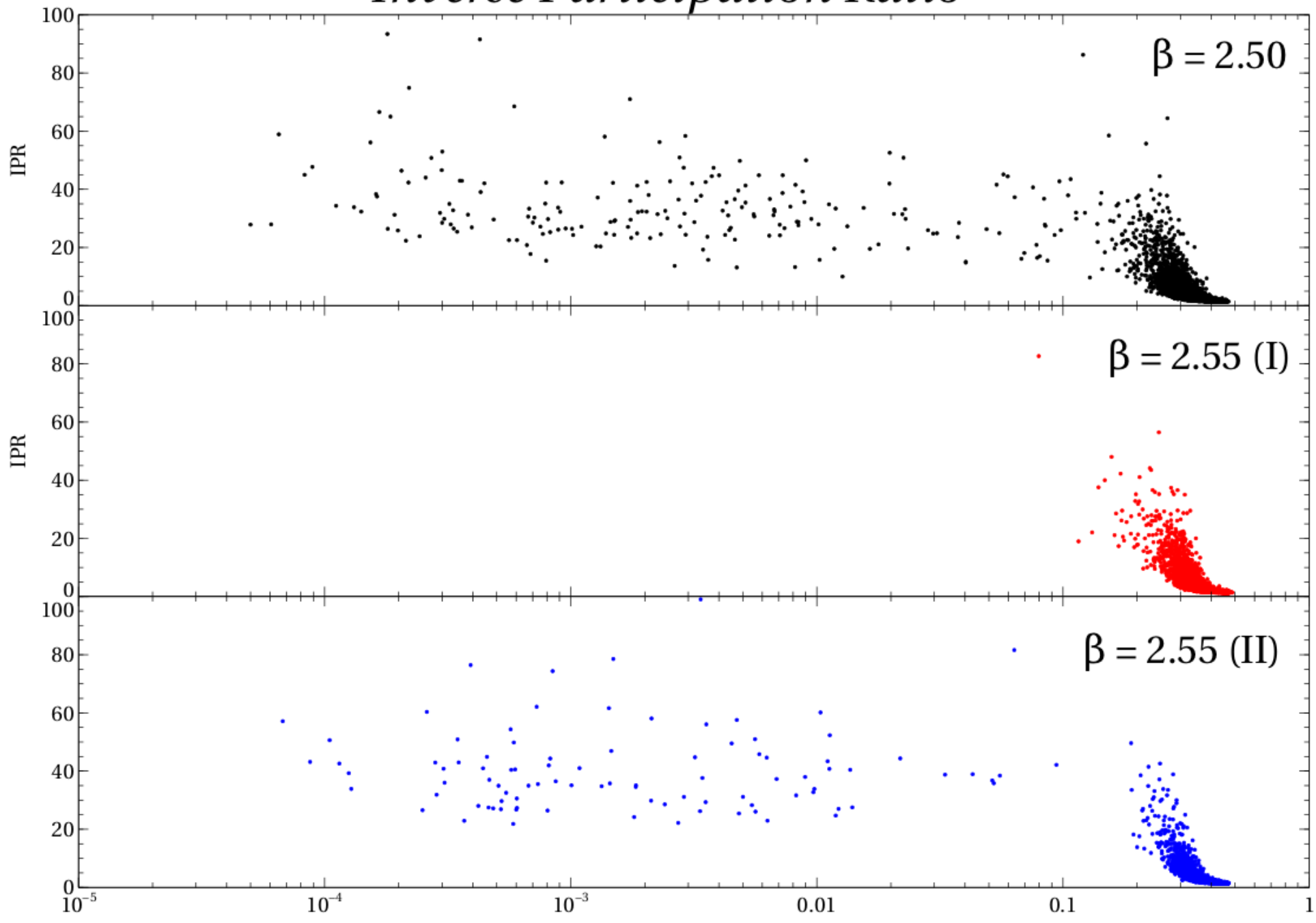




\section{高工齐ルギ一加速器研究機棈}

\section{Axial symsmetry at finite temp. with overlap fermions}
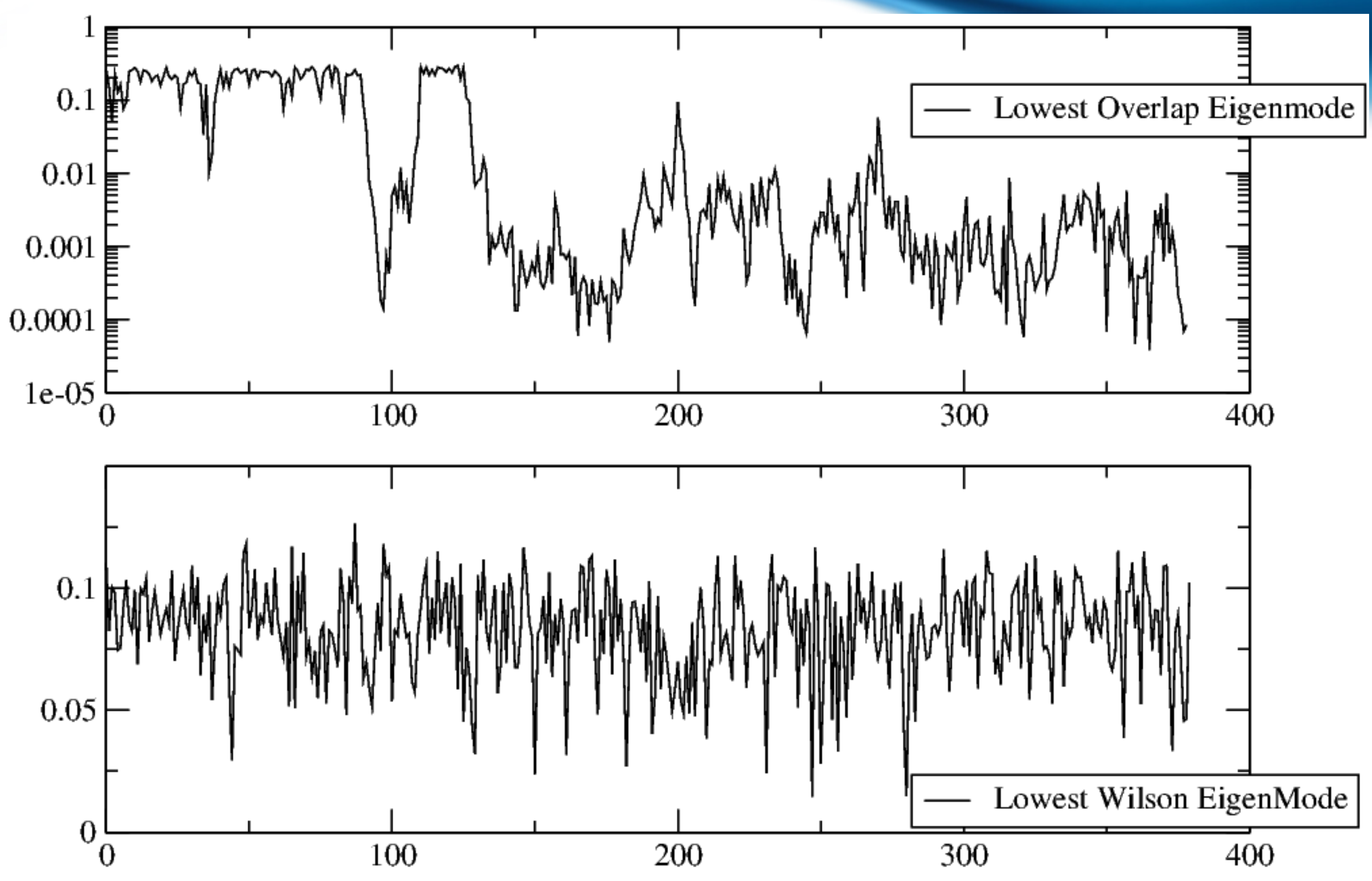

Axial symsmetry at finite temp. with overlap fermions
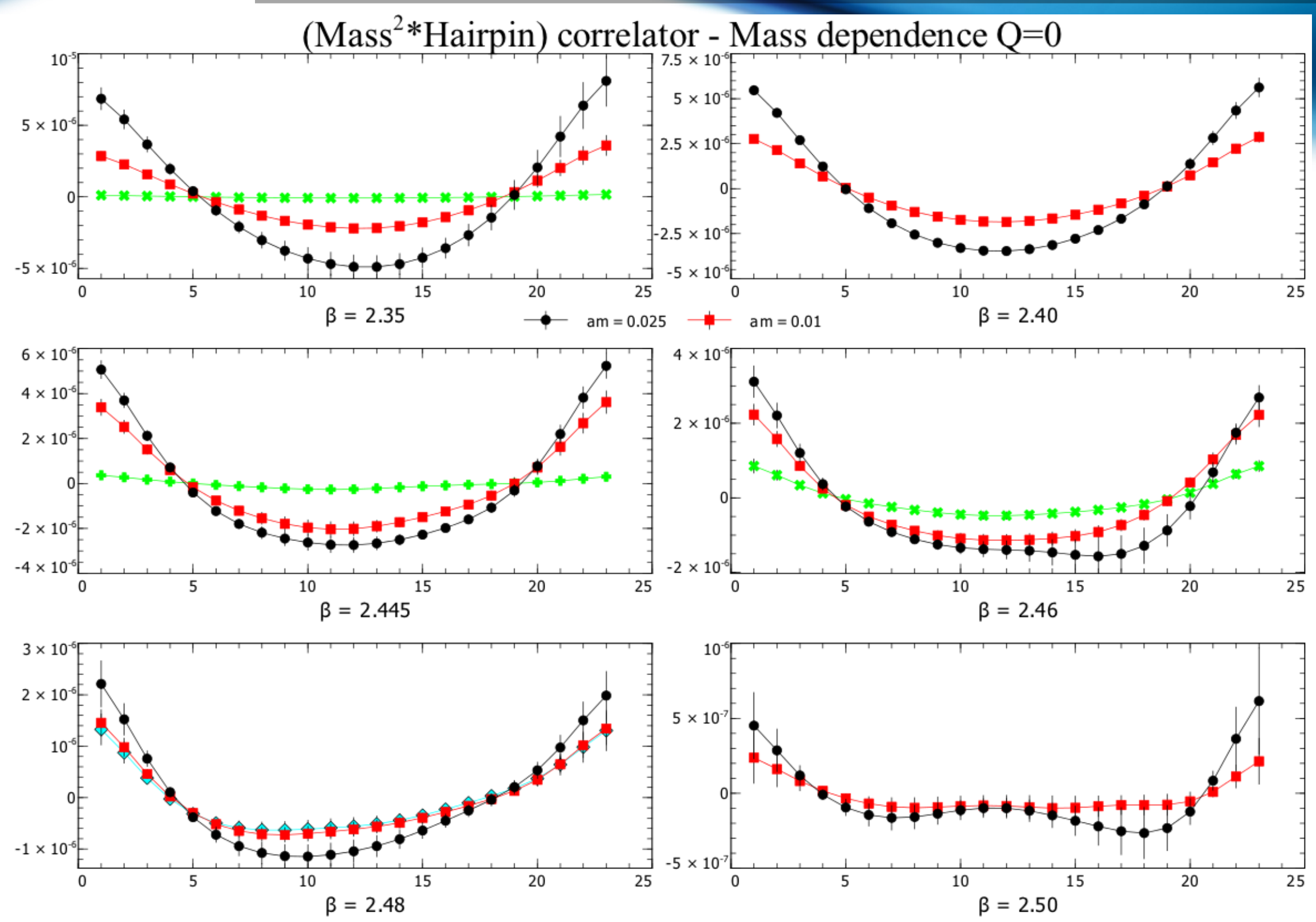
(E) 高工ネルギ一加速器研究機棈 Axial symsmetry at finite temp. with overlap fermions
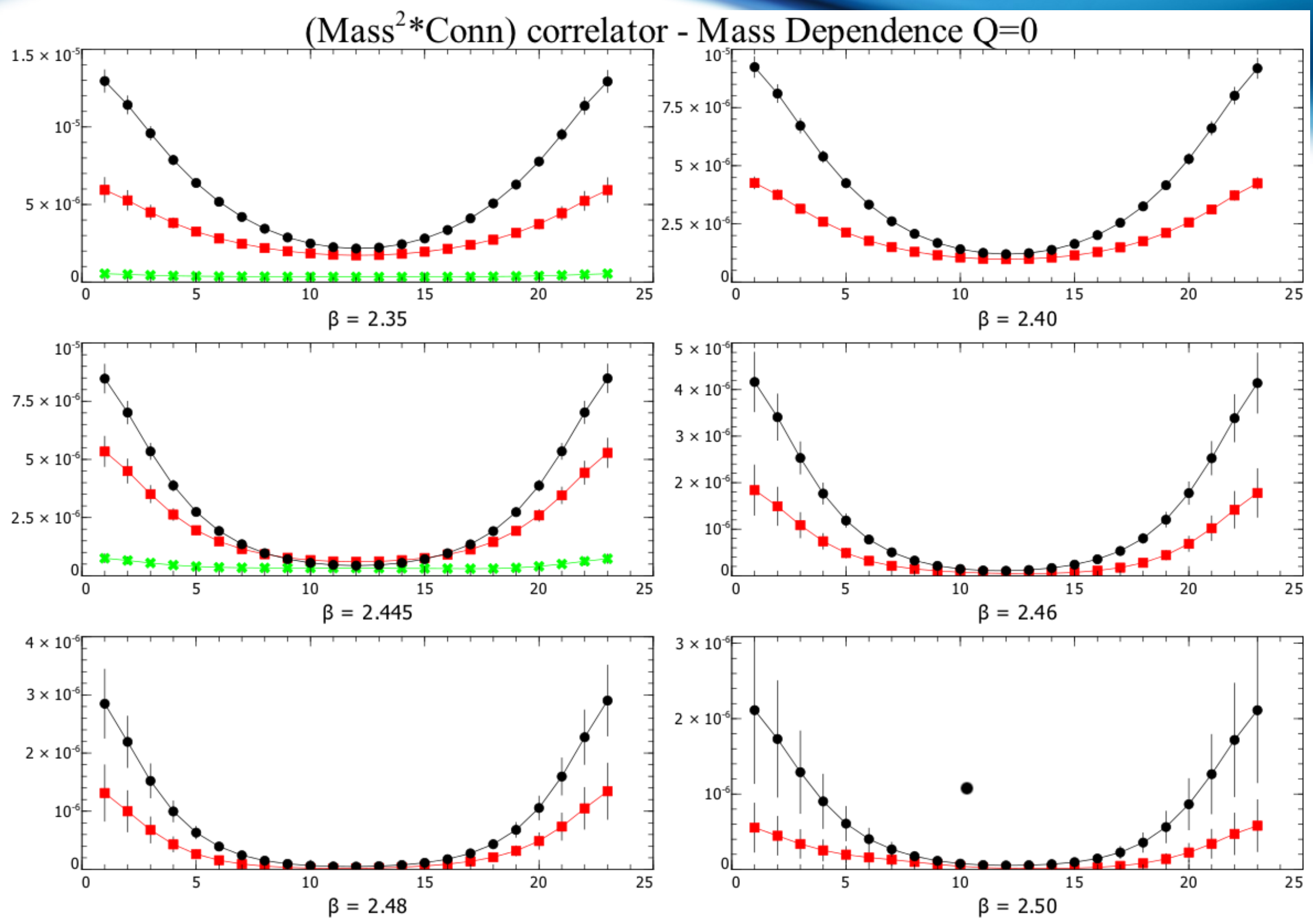


\section{高壬齐ルギ一加速器研究機楎}

Axial symmmetry at finite temp. with overlap fermions

History of Topological Charge

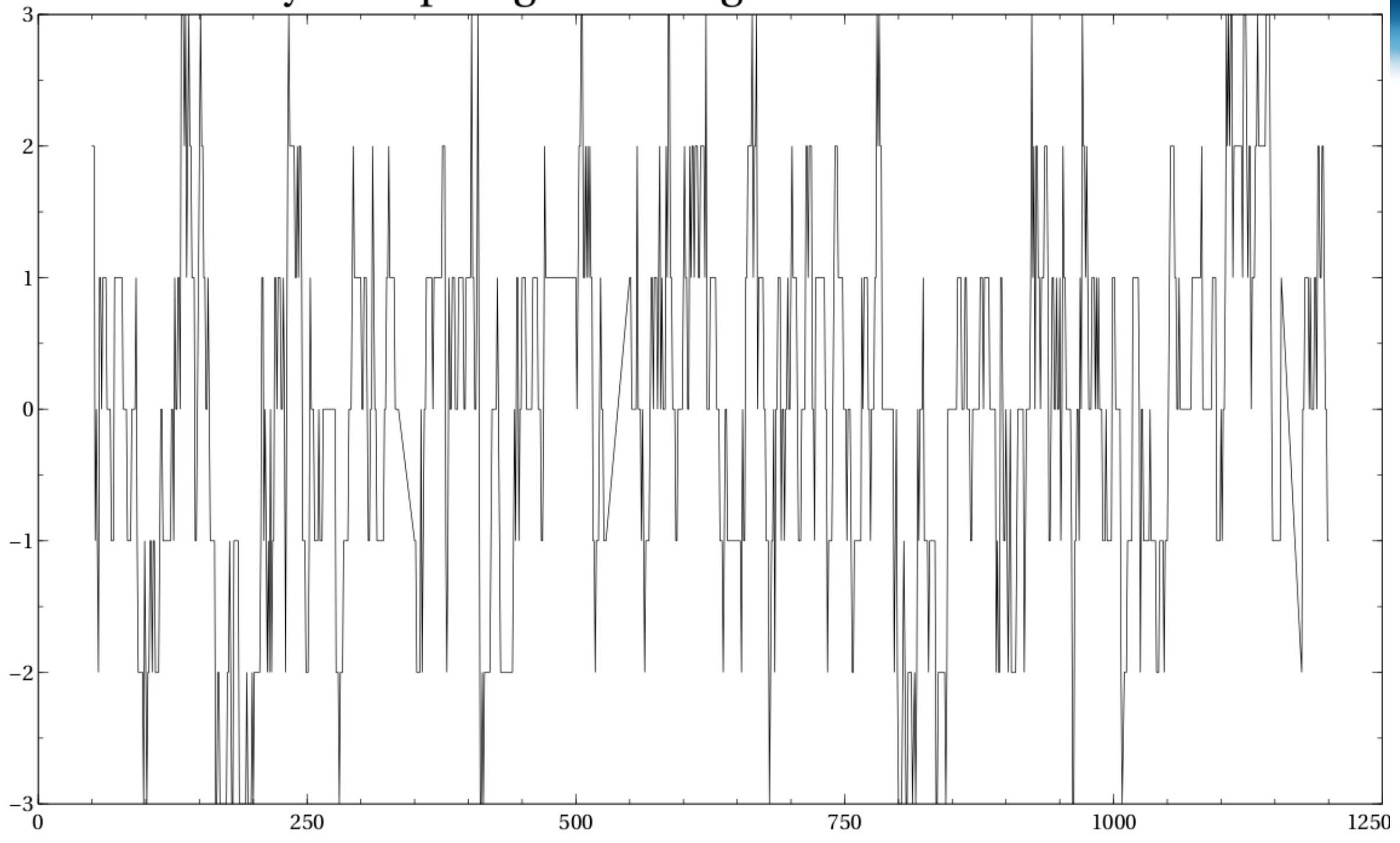




\title{
On quark masses
}

\author{
Michael Creutz
}

BNL

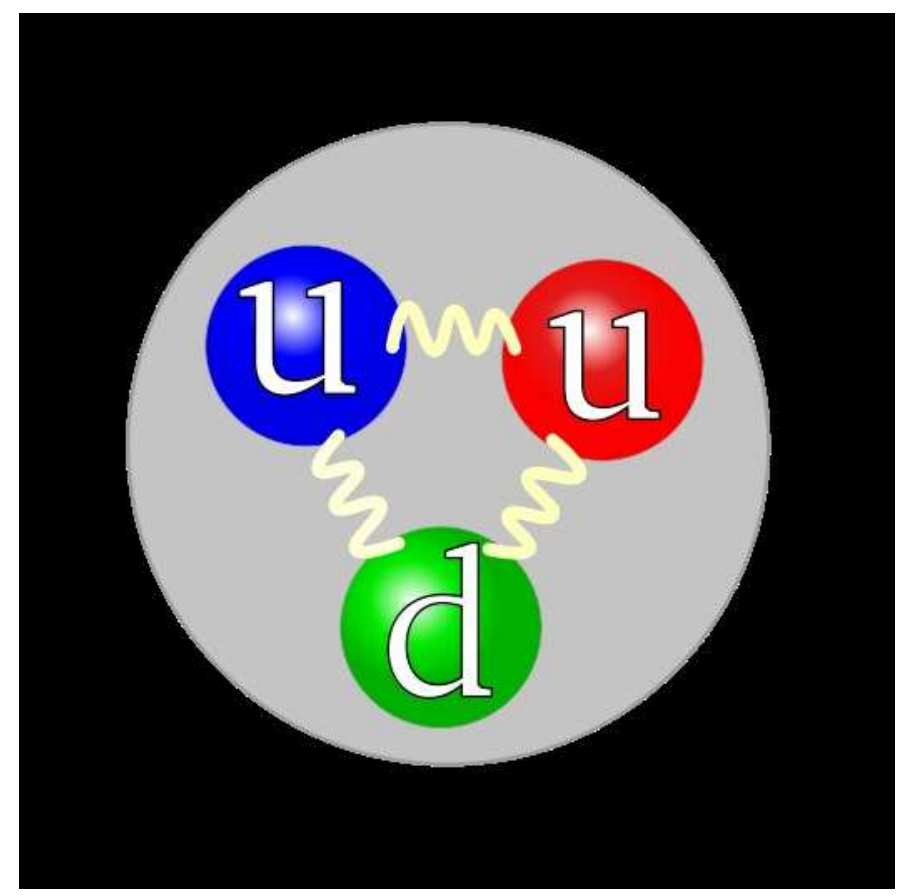




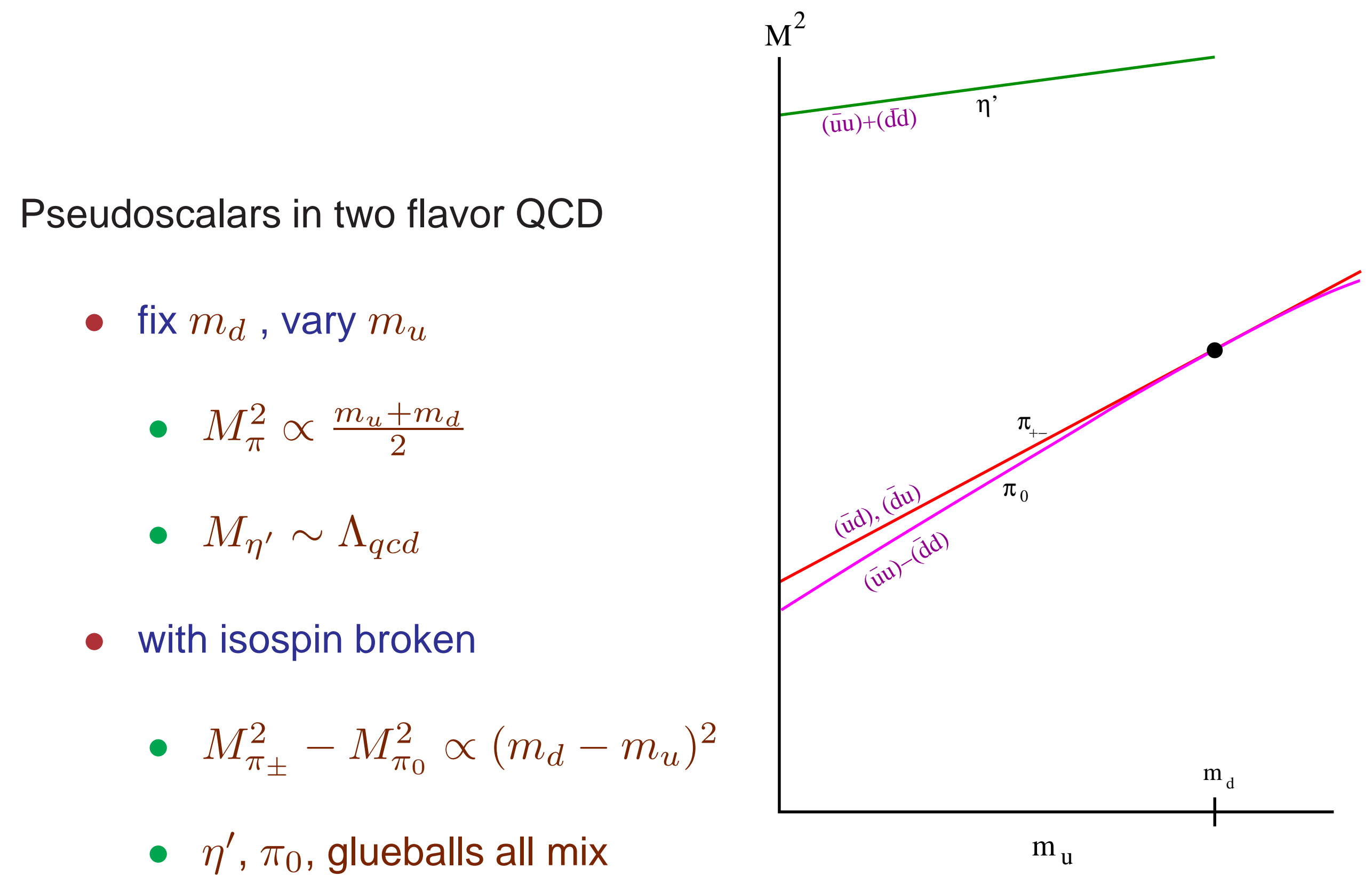


No singularity at $m_{u}=0$

- extrapolate to negative $m_{u}$

- $M_{\pi_{0}}^{2}$ can go negative

- pion condensate forms

- $\left\langle\pi_{0}\right\rangle \neq 0$

- CP broken

- occurs at $\Theta=\pi$

- $\prod_{q} m_{q}<0$

Dashen 1971

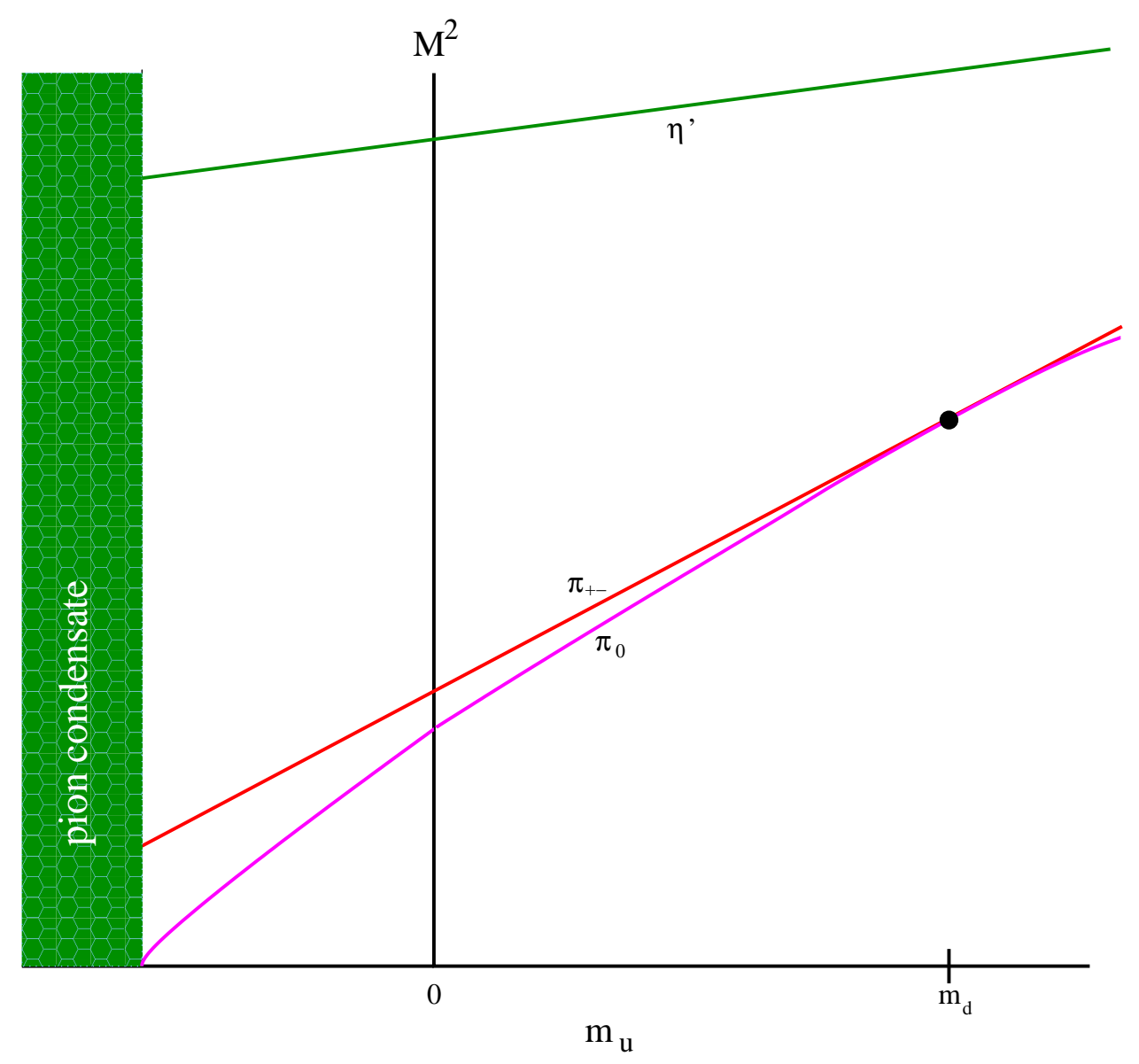

Manifested in both "linear" and "nonlinear" sigma models 
Second order transition at non-vanishing $m_{u}$ and $m_{d}$ of opposite sign

- long distance physics without small Dirac eigenvalues

No structure at $m_{u}=0$ when $m_{d} \neq 0$

- no long distance physics despite possible small Dirac eigenvalues

At the heart of several frustrating and bitter controversies

- Does $m_{u}=0$ have any fundamental meaning?

- Do rooted staggered fermions make sense?

- Is topological susceptibility a physical observable? 
Two flavors in the massless limit: $m_{u}=m_{d}=0$

- massive proton, neutron, eta prime, glueballs

- 3 massless Goldstone pions

Eta prime and neutral pion: distinct mixtures of $\bar{u} u, \bar{d} d$, and glue

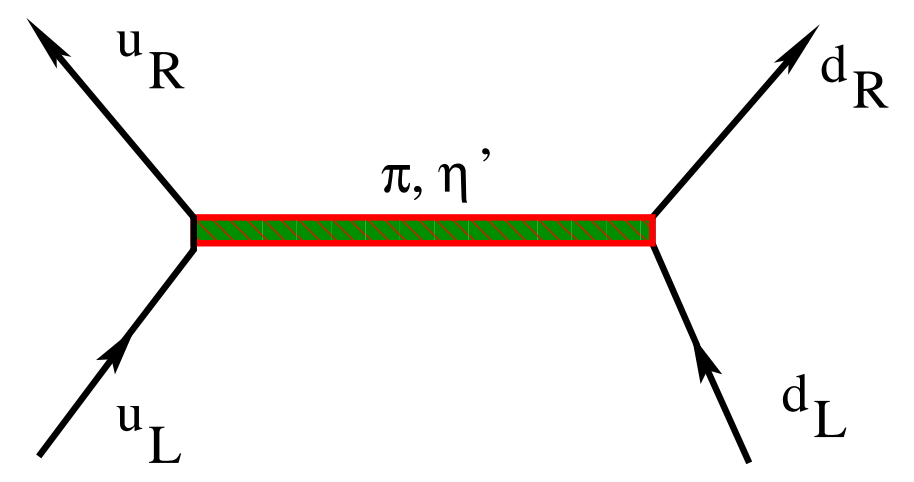

- anomaly: $\pi_{0}$ and $\eta^{\prime}$ not degenerate

- four point vertex $\left\langle\bar{u}_{L} u_{R} \bar{d}_{L} d_{R}\right\rangle$ does not vanish

Helicity-flip quark-quark scattering does not vanish in the chiral limit 
Now turn on a small $d$ quark mass

- closing $d$ loop induces $u_{L} u_{R}$ mixing

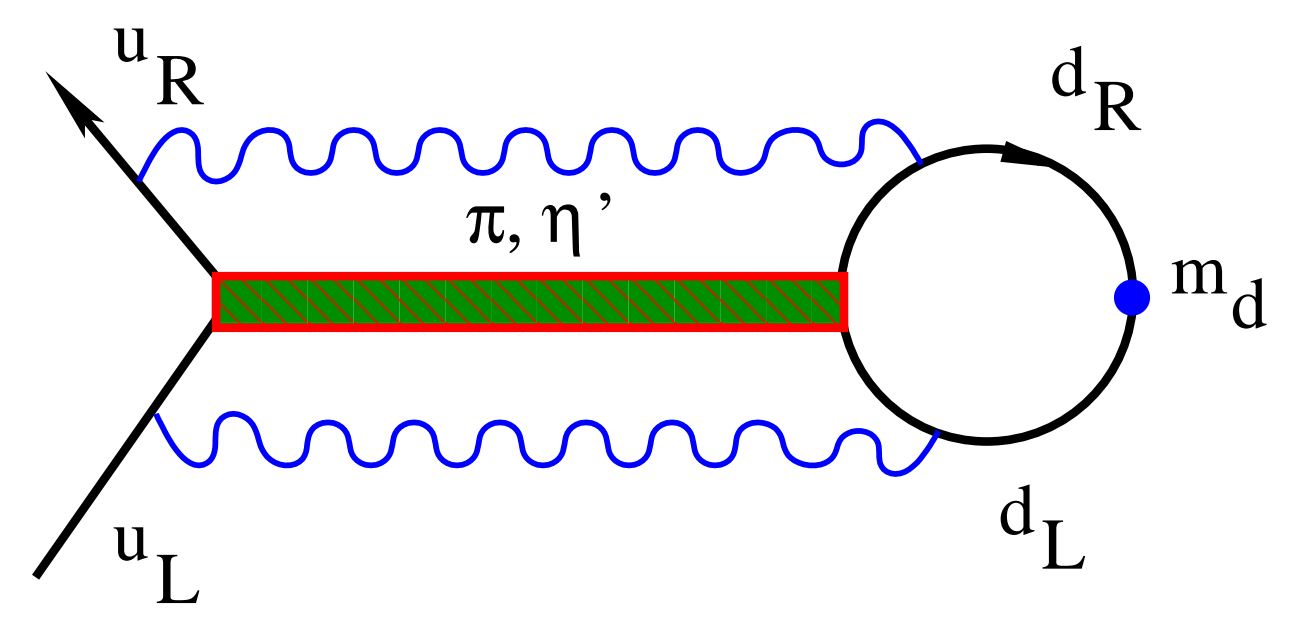

- gluons inserted to compensate for odd meson parity

Non-zero $d$ quark mass induces an effective mass for the $u$ quark 
Non-perturbative effects

- renormalize $\frac{m_{u}}{m_{d}}$

- quark mass ratios not renormalization group invariant

- (except in isospin limit)

Effect automatically included in lattice simulations

Old point

- Georgi, McArthur, 1981 (unpublished)

- Banks, Nir, Seiberg, 1994 (conference proceedings)

- MC, 2003 (unpublished)

- MC, 2004 (PRL) 
Intense consternation from the perturbative community

- effect not seen perturbatively, i.e. in the $\overline{M S}$ scheme

- consequences

- mass renormalization is not flavor blind

- mass independent regularization problematic

- inherent ambiguities defining $m_{u}=0$

$\overline{M S}$ is only a perturbative regulator

- when $m_{u} \neq m_{d}$

Matching lattice masses to $\overline{M S}$ is not appropriate! 


\section{Specific critiques}

Complaint 1:

- Use a mass independent regularization

- $a \frac{d m_{i}}{d a}=\gamma(g) m_{i} \Rightarrow \frac{m_{i}}{m_{j}}=$ constant

Response:

- allowed, but obscures above off-diagonal $m_{d}$ effect on $m_{u}$

- no guarantee that $\frac{m_{i}}{m_{j}}$ universal between schemes

- lattice is not a mass independent scheme

- unclear how to do matching 
When $m_{u} \neq m_{d}$

- isospin broken

$$
\text { - } \frac{M_{\pi^{0}}^{2}}{M_{\pi^{ \pm}}^{2}}=1-O\left(\frac{\left(m_{u}-m_{d}\right)^{2}}{\left(m_{u}+m_{d}\right) \Lambda_{q c d}}\right)
$$

Holding quark mass ratios fixed

- hadronic mass ratios scale dependent

Holding hadronic mass ratios fixed

- quark mass ratios scale dependent 
Complaint 2:

- Do matching at $100 \mathrm{GeV}$

- instantons exponentially suppressed and irrelevant

\section{Response:}

- the lattice simulations are not done at miniscule scales

- instanton effects must be included

- $1 / g^{2} \sim \log (\mu) \sim \log (1 / a)$

- exponential suppression in $1 / g^{2} \rightarrow$ power in scale $\mu$ 
Effect controlled by

- $M_{\eta^{\prime}}-M_{\pi_{0}} \propto \mu g^{-\beta_{1} / \beta_{0}^{2}} e^{-1 /\left(2 \beta_{0} g^{2}\right)} \not \supset 0$

- $\beta_{0}=\frac{1}{16 \pi^{2}}\left(11-2 N_{f} / 3\right)$

- $\beta_{1}=\left(\frac{1}{16 \pi^{2}}\right)^{2}\left(102-38 N_{f} / 3\right)$

- also proportional to $m_{d}-m_{u}$

- estimate at scale $\mu=2 \mathrm{GeV}$

- $\Delta m_{u}(\mu) \sim \frac{\left(M_{\eta^{\prime}}-M_{\pi_{0}}\right)\left(m_{d}-m_{u}\right)}{\mu}=O(1 \mathrm{MeV})$

- same magnitude as quoted "results" 
Note

- $M_{\eta^{\prime}} \propto \mu g^{-\beta_{1} / \beta_{0}^{2}} e^{-1 /\left(2 \beta_{0} g^{2}\right)}$

- exponential behavior controlled by

- $\frac{1}{2 \beta_{0} g^{2}}=\frac{8 \pi^{2}}{\left(11-2 n_{f} / 3\right) g^{2}}<<\frac{8 \pi^{2}}{g^{2}}=$ classical instanton action

- topological excitations above quantum, not classical, vacuum

- classical instanton action strongly overestimates suppression 
Rooted staggered quarks

- tastes: $\left(S U(4)_{u}, S U(4)_{d}\right)$

- well separated spurious states

- not only in chiral limit

- one massless at $m_{u}=0$

- required by symmetry

Can multiple artifacts cancel?

- requires unitarity violation

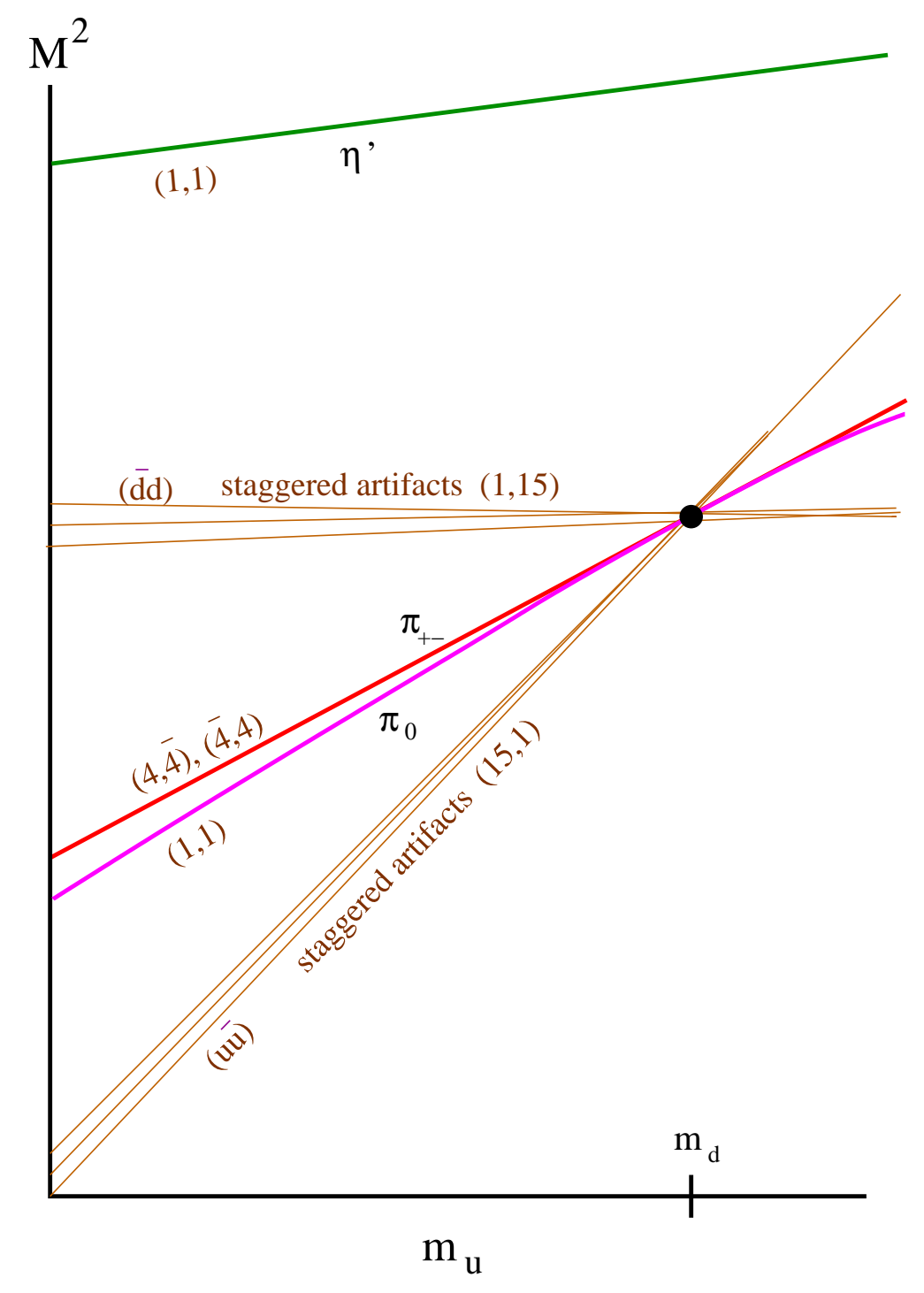

Plausible??? 


\section{Summary}

Non-perturbative effects mix mass terms for different species

- effect absent in perturbation theory

- inappropriate to match lattice and perturbative masses

Interesting phase structure with negative mass quarks

- CP violating pion condensation

- no structure at $m_{u}=0$ when $m_{d} \neq 0$

Crucial to resolving many controversies

- $m_{u}=0$, topological susceptibility, rooting

Review: Acta Physica Slovaca 61, 1 (2011), arXiv:1103.3304

free download at http://www.physics.sk/aps/ 


\section{Extra Slides}


Ising-like transition at $m_{u}<0$

- order parameter $\left\langle\pi_{0}\right\rangle \neq 0$

- breaks CP spontaneously

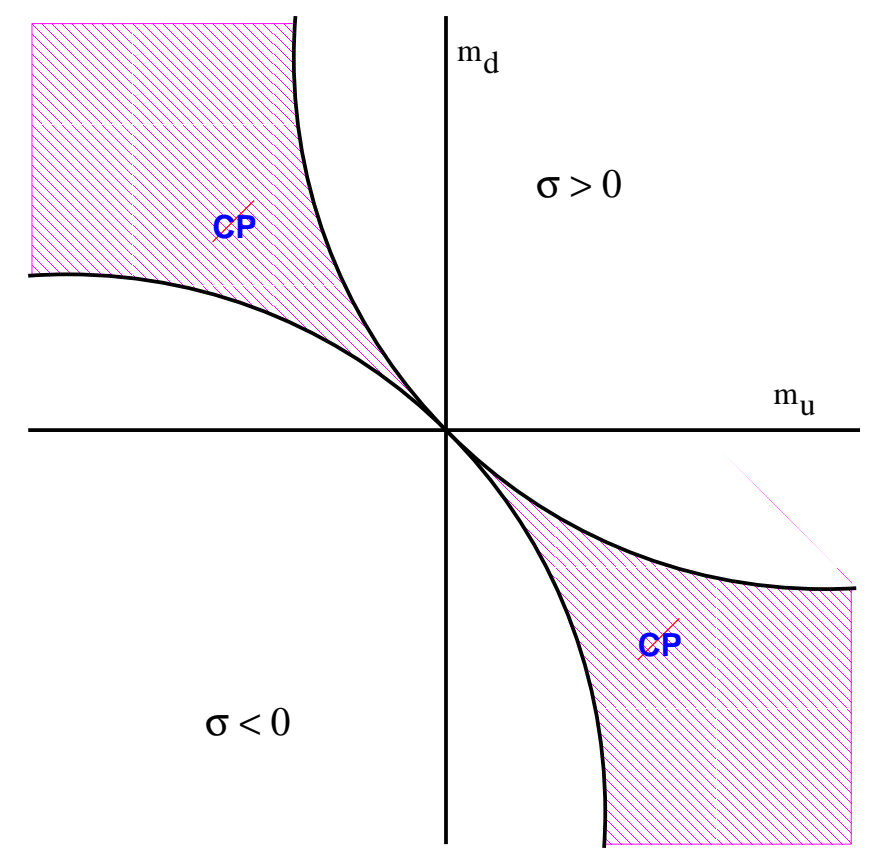

Connected with the anomaly and $M_{\eta^{\prime}} \sim \Lambda_{q c d}$

- non-perturbative 
General mass term $m_{1} \bar{\psi} \psi+m_{2} \bar{\psi} \tau_{3} \psi+i m_{3} \bar{\psi} \gamma_{5} \psi$

- average quark mass, quark mass difference, CP violation from Theta

Two intersecting first order surfaces

- $\left(m_{1}=0, m_{3} \neq 0\right) \quad$ and $\quad\left(m_{1}<m_{2}, m_{3}=0\right)$

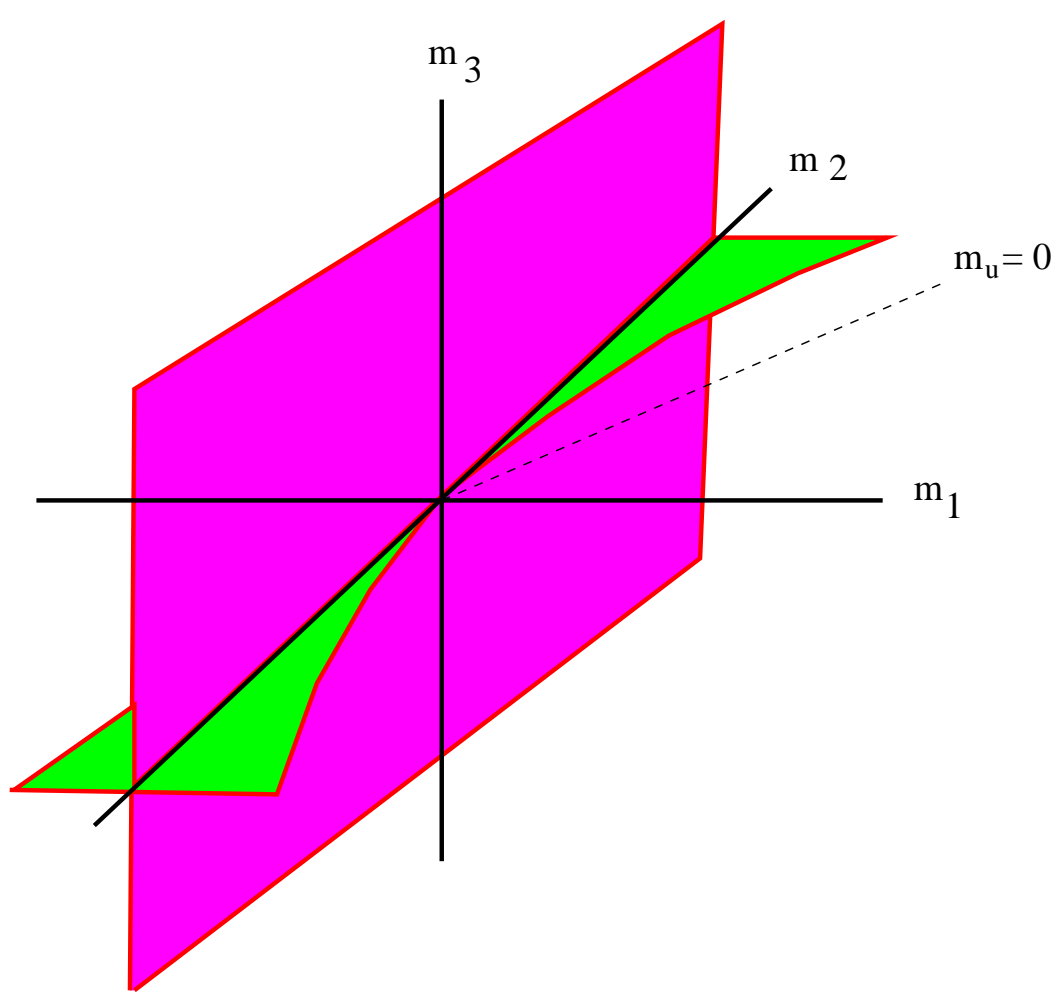

Second order edge at $m_{3}=0,0<\left|m_{1}\right|<\left|m_{2}\right|$ 
CP breaking related to the Aoki phase

- Wilson fermion lattice artifacts

- phase persists in isospin limit

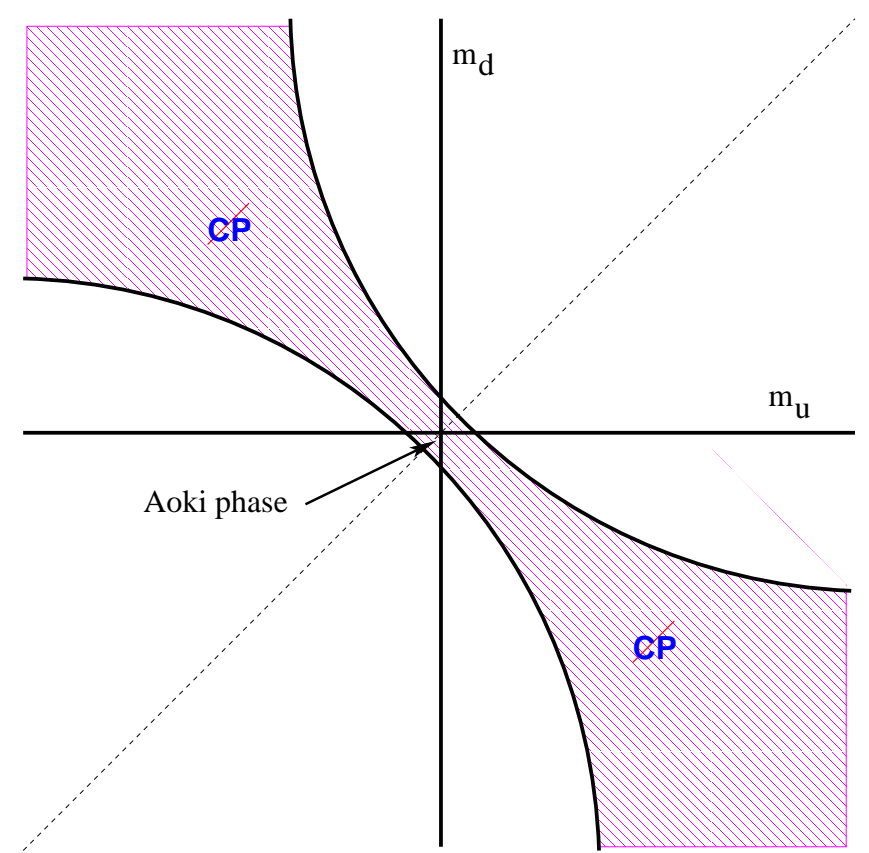

Which alternative remains controversial

- can depend on lattice action
- First order alternative

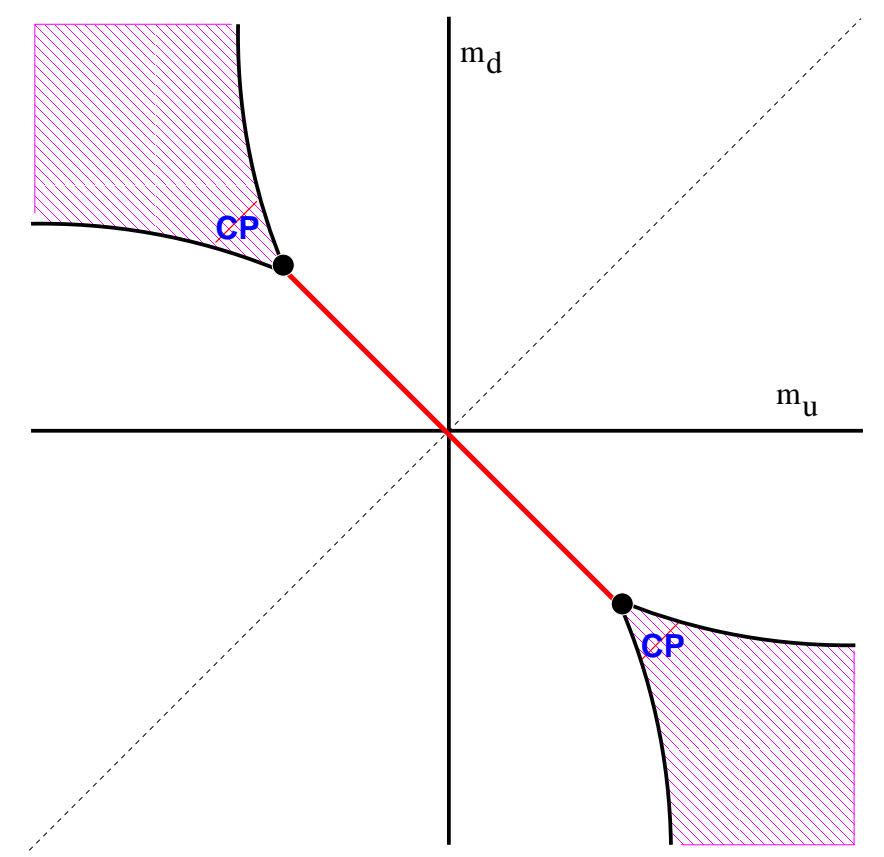




\section{Exploring Three-Nucleon Forces in Lattice QCD}

\section{Takumi Doi}

(Nishina Center, RIKEN)

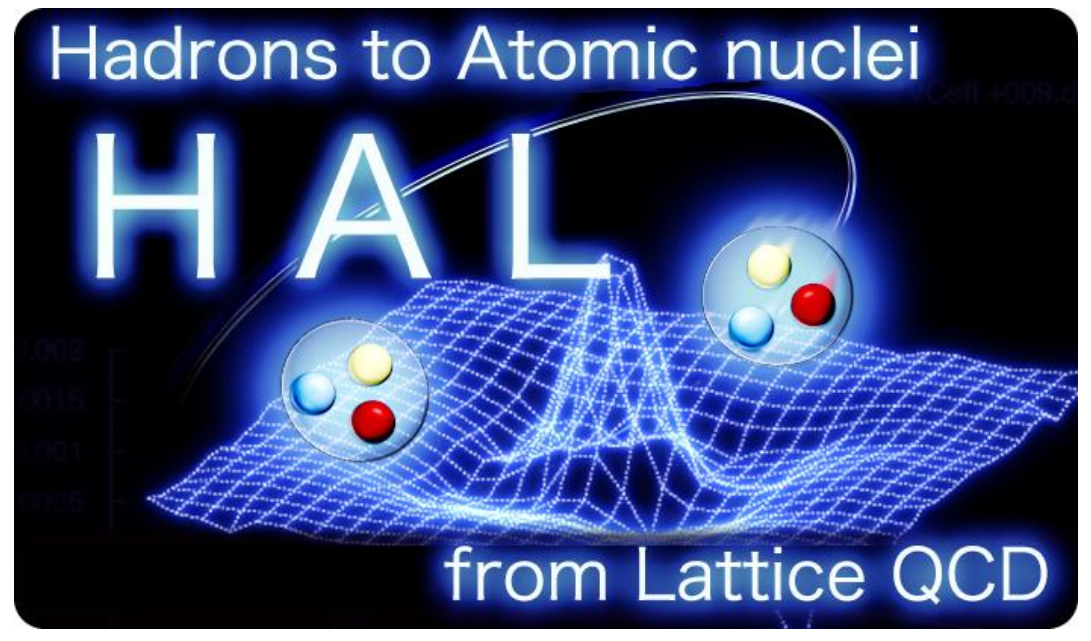

for HAL QCD Collaboration

S. Aoki, N. Ishii, H. Nemura, K. Sasaki, M. Yamada (Univ. of Tsukuba)

B. Charron (Univ. of Tokyo)

T. Hatsuda (RIKEN)

Y. Ikeda (Tokyo Inst. Tech.)

T. Inoue (Nihon Univ.)

K. Murano (RIKEN) 


\section{Nuclear Physics on the Lattice}

- Frontier in the (coming) physical point Lattice era

- Nuclear Physics directly from Lattice QCD (+QED)

- (Many other interesting topics in this workshop...)

- Traditional Nuclear Physics

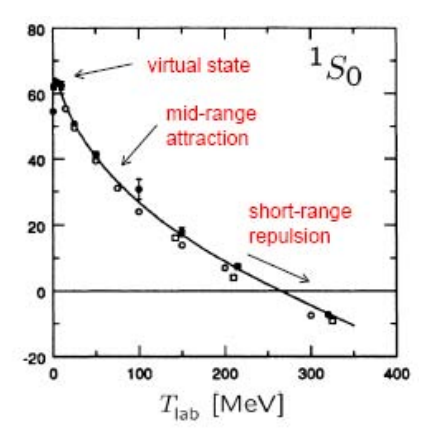

NN phase shifts from experiments

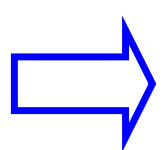

Phenomenological Nuclear Forces

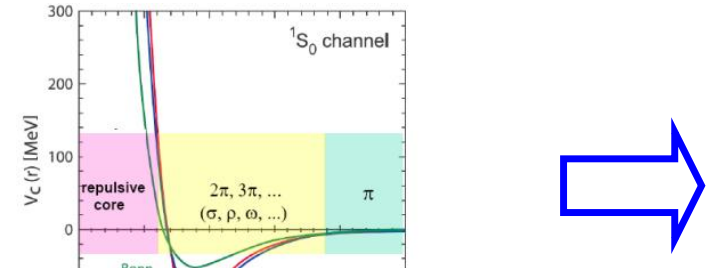

Various applications

- No clear connection to QCD so far

- $\rightarrow$ Lattice QCD can establish firm foundations for Nuclear Physics 


\section{Nuclear Physics and Astrophysics from Lattice QCD}

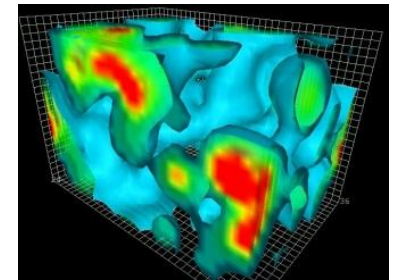

QCD Vacuum

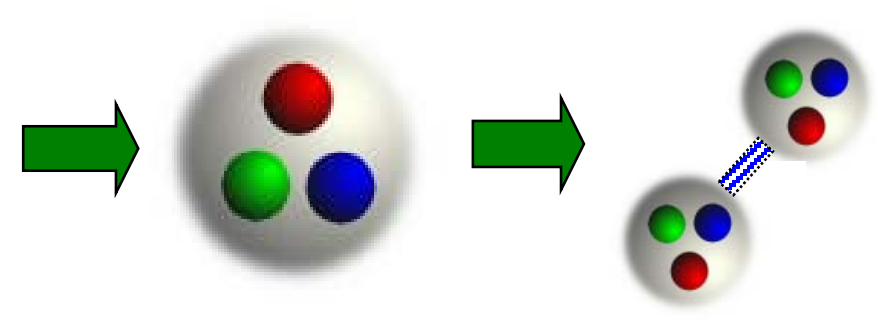

Baryon

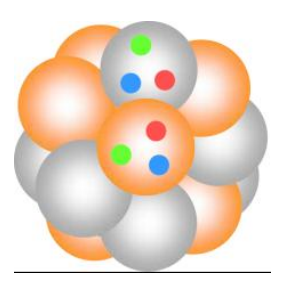

Few-Body / Light Nuclei

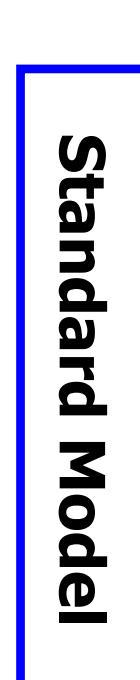

05/16/2012 1st-principle lat calc.
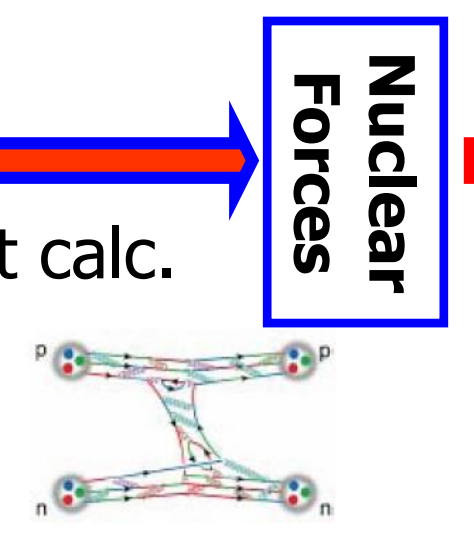

Ab-initio nuclear calc.

Neutron Star / Supernova

Nuclear and Astro Physicists thirst for Lattice QCD predictions !

1st-principle lat calc. (Yamazaki's talk)

(Another talk by Orginos) 
Frontier in Hadron-Hadron Interactions $\Rightarrow$ Three-Nucleon Forces (3NF)

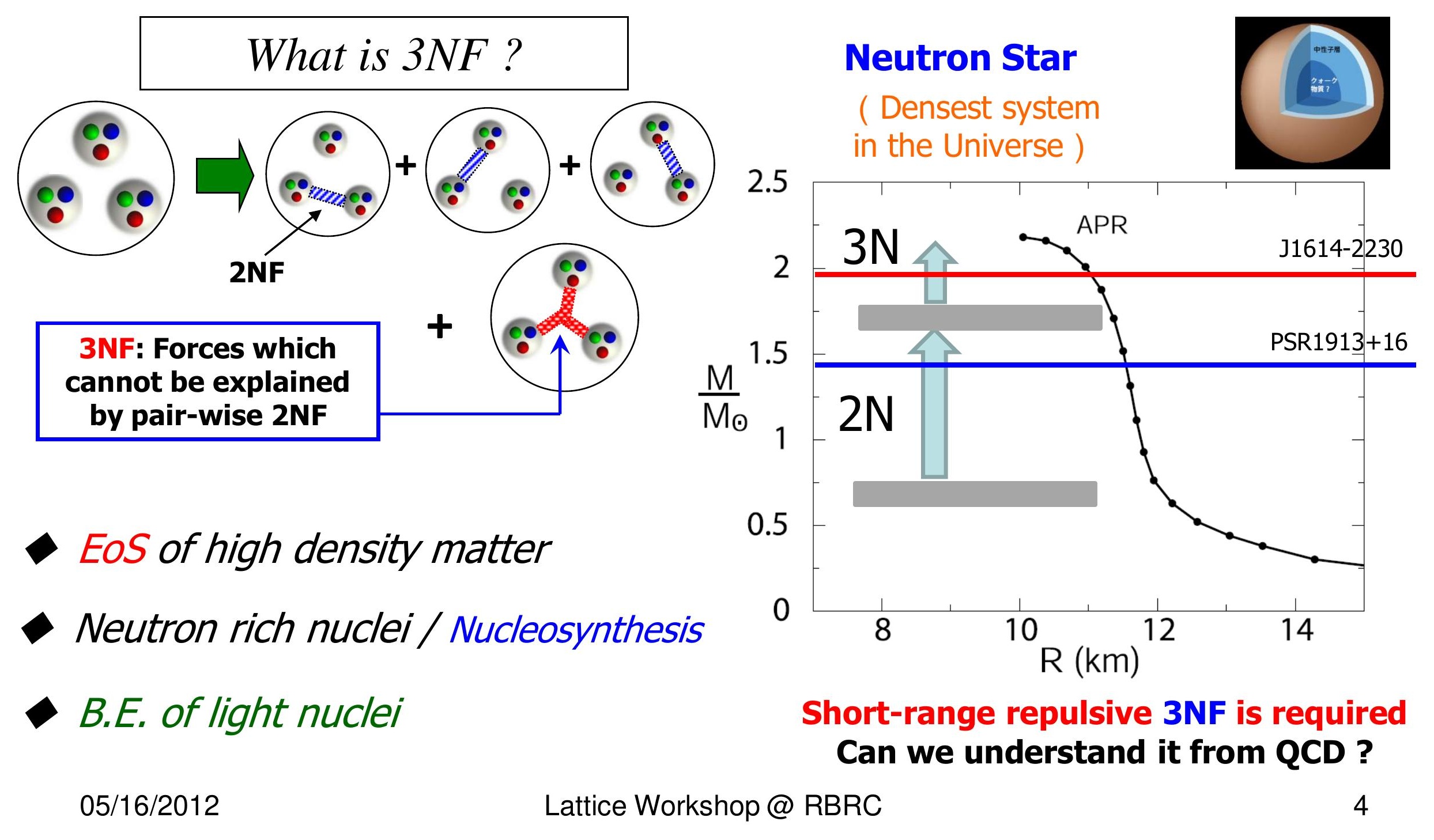




\section{Nuclear Force from Lattice}

QCD

\section{[HAL QCD strategy]}

- Potential is constructed so as to reproduce the NN phase shift (or, S-matrix)

- Nambu-Bethe-Salpeter(NBS) wave function

$$
\psi(\vec{r})=\langle 0|N(\vec{x}+\vec{r}, t) N(\vec{x}, t)| 2 N\rangle
$$

- Key concept: asymptotic region $\leftrightarrow$ phase shift

$$
\left(\nabla^{2}+k_{\delta}^{2}\right) \psi(\vec{r})=0, \quad r>R
$$

Luscher, NPB354(1991)531

C.-J.Lin et al., NPB619(2001)467 CP-PACS Coll., PRD71(2005)094504

- Define the potential at interaction region

$$
\left(\nabla^{2}+k_{\delta}^{2}\right) \psi(\vec{r})=\int d \vec{r}^{\prime} U\left(\vec{r}, \vec{r}^{\prime}\right) \psi\left(\overrightarrow{r^{\prime}}\right), \quad r<R
$$

Ishii-Aoki-Hatsuda PRL99(2007)

Aoki-Hatsuda-Ishii PTP123(2010)89

- Non-local, but E-independent potential

- The combination of (2NF, 3NF) $\rightarrow$ observables

- $\leftarrow$ systematic determination by Lat QCD

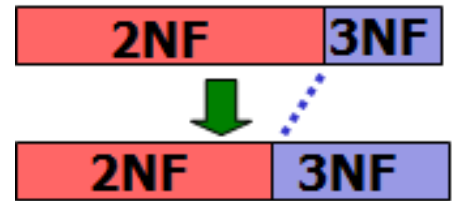




\section{Challenges in multi-baryons on the lattice}

- $\mathrm{S} / \mathrm{N}$ issue in the system of \#baryon = A

$$
S / N \sim \exp \left[-A\left(m_{N}-3 m_{\pi} / 2\right) t\right] \quad \text { Lepage(1989) }
$$

- small energy splitting by scatt. $\quad \Delta E \simeq \frac{\vec{p}^{2}}{m_{N}} \simeq \frac{1}{m_{N}} \frac{(2 \pi)^{2}}{L^{2}}$

- $\rightarrow$ requires larger $t$...

- Enormous computational cost for correlator

- \# of Wick contraction $\sim\left[\left(\frac{3}{2} A\right) !\right]^{2}$

- \# of color/spinor contractions $\underset{\text { (color) (spinor) }}{\sim 6^{A} \cdot 4^{A}}$ 


\section{$\mathrm{S} / \mathrm{N}$ issue $\rightarrow \mathrm{New}$ algorithm (t-dep Schrodinger eq)}

Elastic scatt states share the same potential (E-indep of potential $U\left(r, r^{\prime}\right)$ is the key)

$$
\begin{gathered}
\left(k^{2} / m_{N}-H_{0}\right) \psi_{E}(\vec{r})=\int d \vec{r}^{\prime} U\left(\vec{r}, \vec{r}^{\prime}\right) \psi_{E}\left(\vec{r}^{\prime}\right) \\
\left(-\frac{\partial}{\partial t}-H_{0}\right) R(\vec{r}, t)=\int d \vec{r}^{\prime} U\left(\vec{r}, \vec{r}^{\prime}\right) R\left(\vec{r}^{\prime}, t\right)
\end{gathered}
$$

\section{Grand state saturation is NOT necessary!}

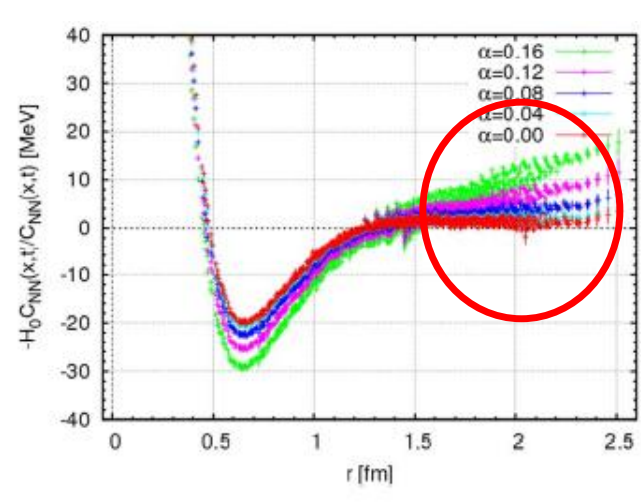

Poor Convergence

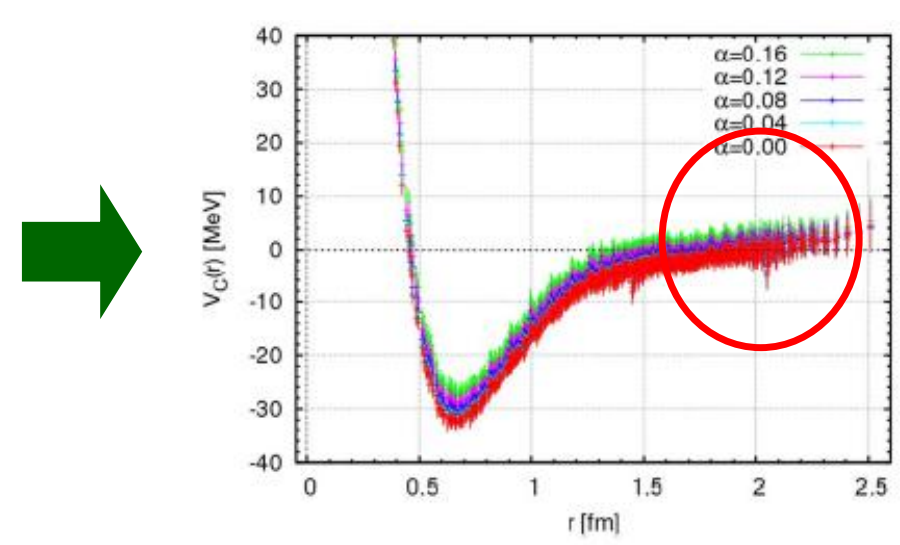

Good Convergence !

Lattice Workshop @ RBRC

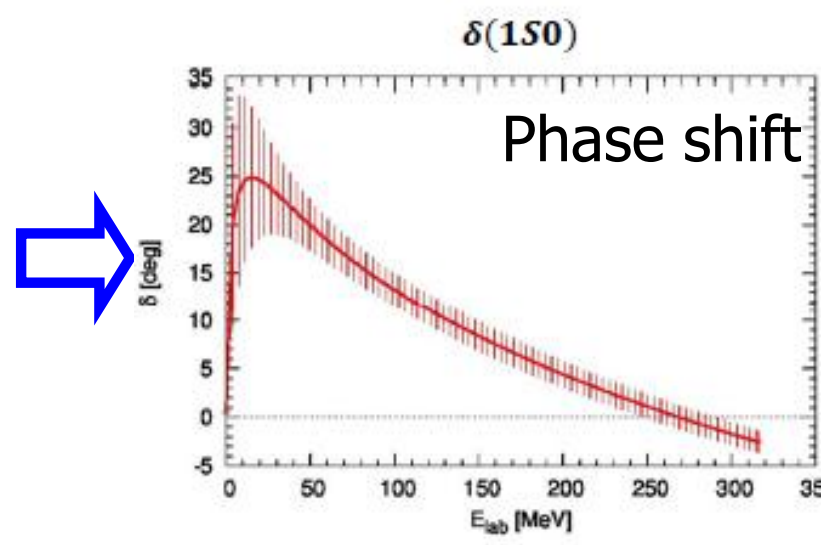

$a_{0}\left({ }^{1} S_{0}\right)=1.6(11) \mathrm{fm}$

N.Ishii et al. (HAL QCD Coll. \} arXiv:1203.3642, PLB in press 


\section{Computational cost issue $\rightarrow$ Unified contraction algorithm}

color/spinor contractions

- Traditional algorithm $\Pi^{2 N} \simeq \frac{\langle q q q q q q(t)}{4} \frac{\left.\bar{q}\left(\xi_{1}^{\prime}\right) \bar{q}\left(\xi_{2}^{\prime}\right) \bar{q}\left(\xi_{3}^{\prime}\right) \bar{q}\left(\xi_{4}^{\prime}\right) \bar{q}\left(\xi_{5}^{\prime}\right) \bar{q}\left(\xi_{6}^{\prime}\right)\left(t_{0}\right)\right\rangle}{{ }_{\text {Permutations }}} \times \frac{\operatorname{Coeff}^{2 N}\left(\xi_{1}^{\prime}, \cdots, \xi_{6}^{\prime}\right)}{\text { color } \epsilon_{a b c}, \text { spinor }\left(C \gamma_{5}\right), \text { etc. }}$

- New algorithm

(Wick contractions)

- For each flavor, we impose same spacial label at source quark

- $\rightarrow$ Permutation applies to color/spinor indices at "Coeff"

\section{Permuted Sum 7}

$\Pi^{2 N} \simeq\left\langle q q q q q q(t) \bar{q}\left(\xi_{1}^{\prime}\right) \bar{q}\left(\xi_{2}^{\prime}\right) \bar{q}\left(\xi_{3}^{\prime}\right) \bar{q}\left(\xi_{4}^{\prime}\right) \bar{q}\left(\xi_{5}^{\prime}\right) \bar{q}\left(\xi_{6}^{\prime}\right)\left(t_{0}\right)\right\rangle \times \overline{\operatorname{Coeff}^{2 N}\left(\xi_{1}^{\prime}, \cdots, \xi_{6}^{\prime}\right)}$

- Construct a list for color/spinor sum Sum over color/spinor unified list

- $\leftarrow$ Wick contraction and color/spinor contractions are unified

- Significant improvement

$\times 192$ for ${ }^{3} \mathrm{H} /{ }^{3} \mathrm{He}, \times 20736$ for ${ }^{4} \mathrm{He}$ ( $x$ add'l. speedup)

TD, Endres, arXiv:1205.0585 


\section{Lattice QCD Calculations}

\section{Numerical Setup \& Results}

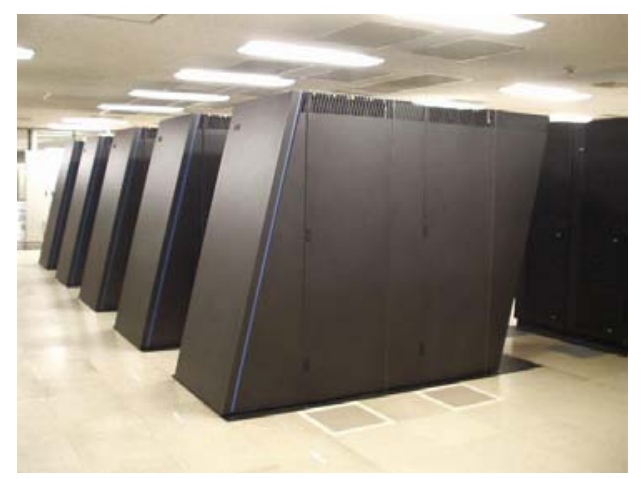

BG/L@KEK

\section{T2K@Tsukuba}

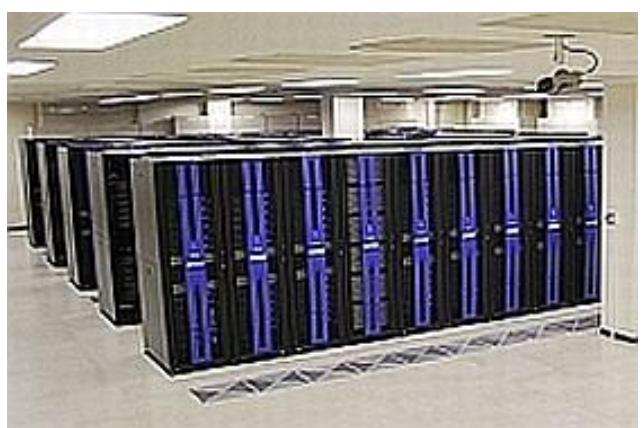

SR16000 @YITP, KEK
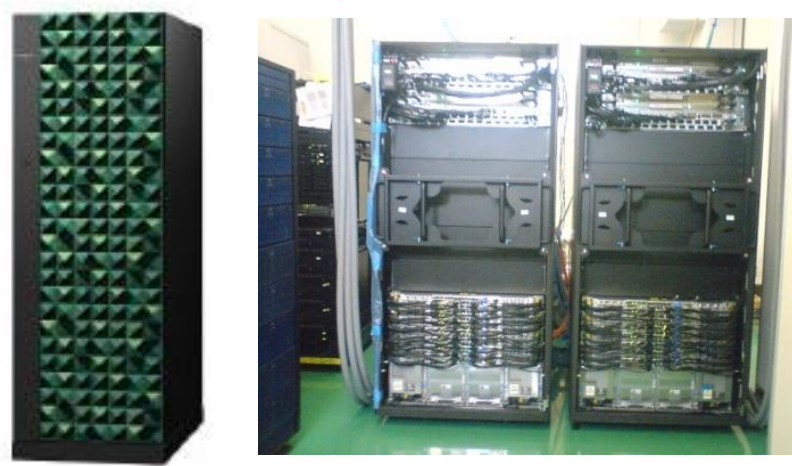

「SR16000 モデルXM1」 


\section{Lattice calculation setup}

$\mathrm{Nf}=2$ clover fermion $+\mathrm{RG}$ improved gauge action (CP-PACS)

- 598 configs $\times 32$ measurements

- beta=1.95, $\left(\mathrm{a}^{-1}=1.27 \mathrm{GeV}, \mathrm{a}=0.156 \mathrm{fm}\right)$

CP-PACS Coll. S. Aoki et al., Phys. Rev. D65 (2002) 054505

- $16^{3} \times 32$ lattice, $L=2.5 \mathrm{fm}$

- $\mathrm{M}(\pi)=1.13 \mathrm{GeV}$

- $M(N)=2.15 \mathrm{GeV}$

- $\mathrm{M}(\Delta)=2.31 \mathrm{GeV}$

$$
(\kappa(\mathrm{ud})=0.13750)
$$$$
(M \pi L=14)
$$

- Correlators

- Standard nucleon op to define the wave function / potential at sink

$$
N=\epsilon_{a b c}\left(q_{a}^{T} C \gamma_{5} q_{b}\right) q_{c}
$$

- Non-rela limit op is used to create $3 \mathrm{~N}$ state at source

$$
G\left(\vec{r}_{2}, t-t_{0}\right)=\sum_{\vec{x}}\langle 0| N \frac{\left(\vec{x}+\vec{r}_{2}, t\right) N\left(\vec{x}-\vec{r}_{2}, t\right) N(\vec{x}, t)}{\text { sink }} \frac{\overline{N N N}\left(t_{0}\right)|0\rangle}{\text { source }}
$$




\section{NF calculation in Lat QCD}

- We fix the geometry of $3 \mathrm{~N}$ to extract the genuine 3NF - We study linear setup $\rightarrow$ reduced to $3 \times 3$ coupled channel
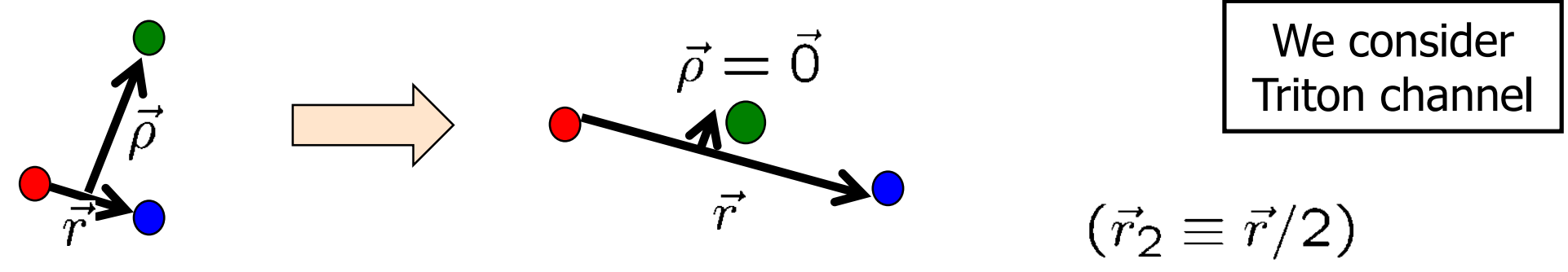

- Linear setup with various distance " $\mathbf{r}_{\mathbf{2}}$ "

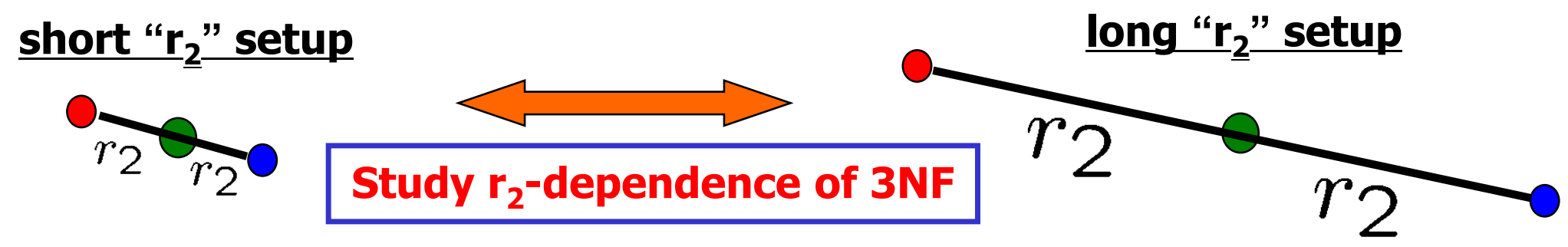




\section{Results for wave functions}

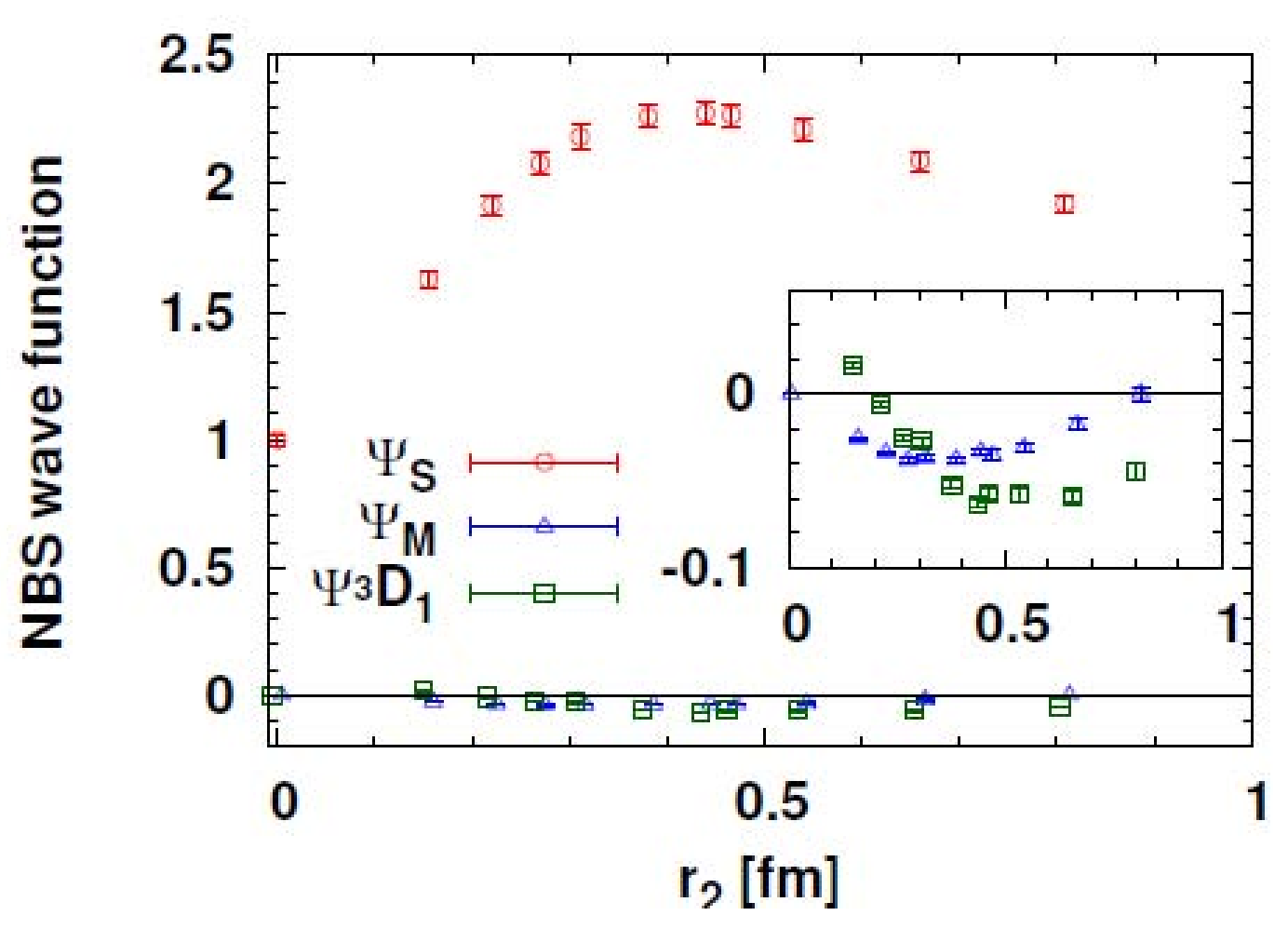

T.D. et al. (HAL QCD) PTP127(2012)723

$05 / 16 / 2012$

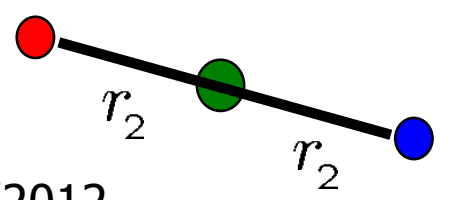

2

\section{Red: $\Psi_{S}$ \\ Blue: $\Psi_{M}$ \\ Green: $\Psi_{3 D 1}$}

\section{$\Psi_{s}$ overwhelms the wave function:}

$\rightarrow$ Indication of the dominance of all S-wave component, higher waves suppressed 


\section{Genuine 3NF from Lat QCD}
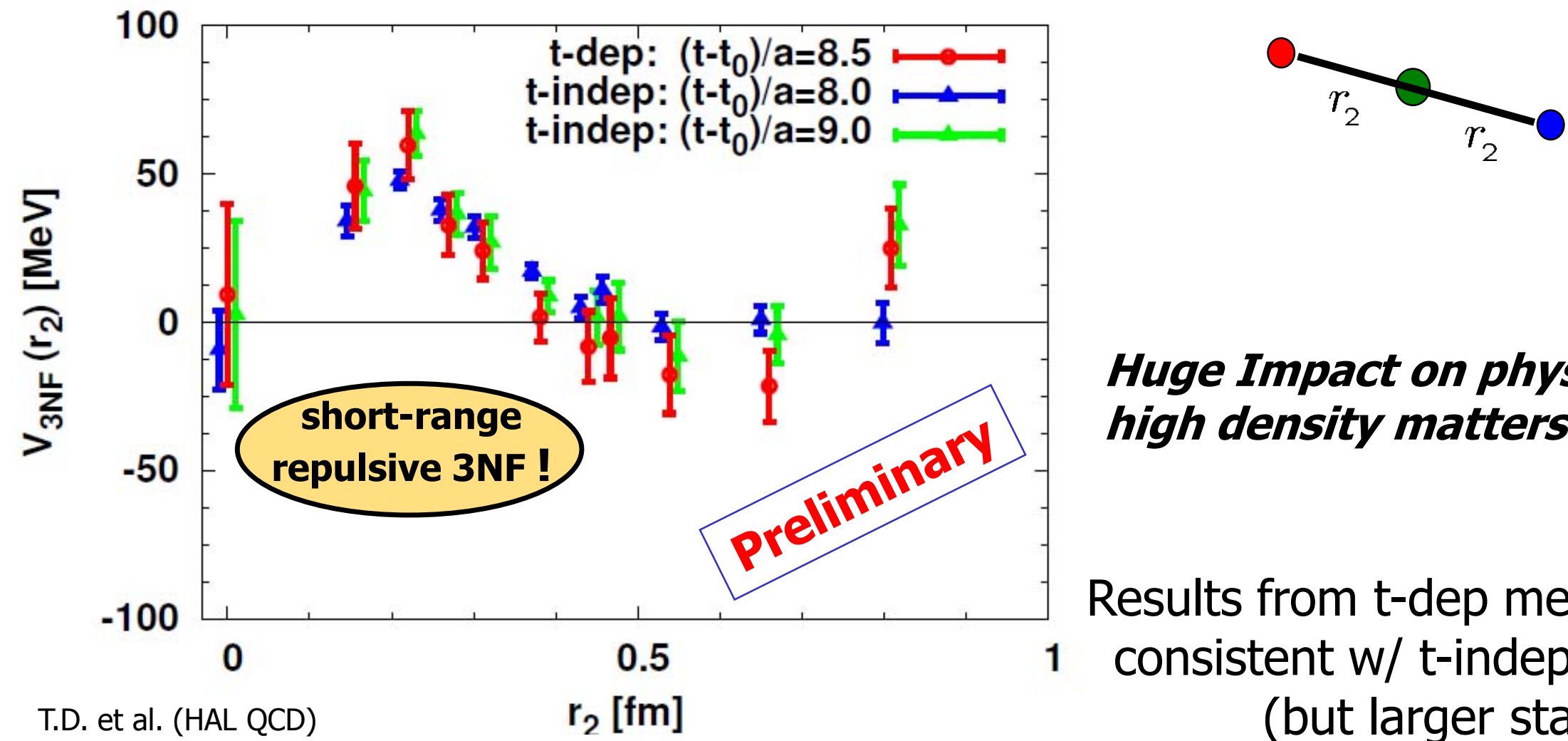

Huge Impact on physics of high density matters !

Results from t-dep method are 1 consistent $\mathrm{w} / \mathrm{t}$-indep method (but larger stat errors) PTP127(2012)723 


\section{Summary/Outlook}

- Nuclear Physics on the Lattice

- New Horizons for Lattice Computations

- Lattice Predictions are highly awaited

- Huge impact on astrophysics as well

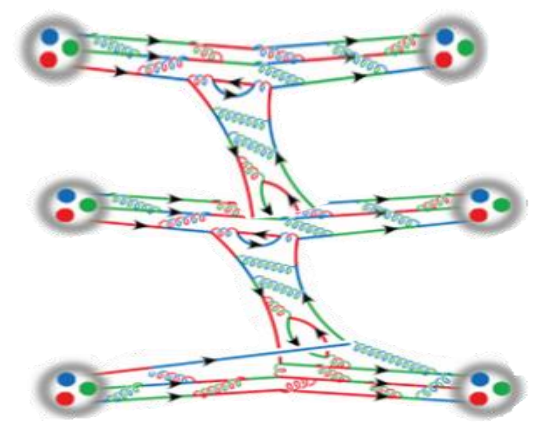

- Three-Nucleon Force (3NF) from Lattice QCD

- Repulsive 3NF at short distance observed

- Outlook

- Realistic potentials with physically light masses w/ large volumes

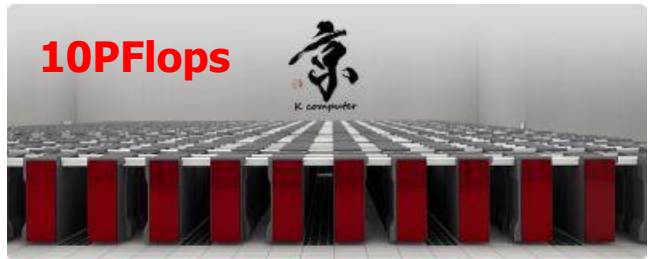

Ab-initio calculation using 2 NF, 3NF as well as hyperon forces from Lat QCD 


\section{Backup Slides}




\section{Parity-even $2 \mathrm{~N}$ potentials (input)}

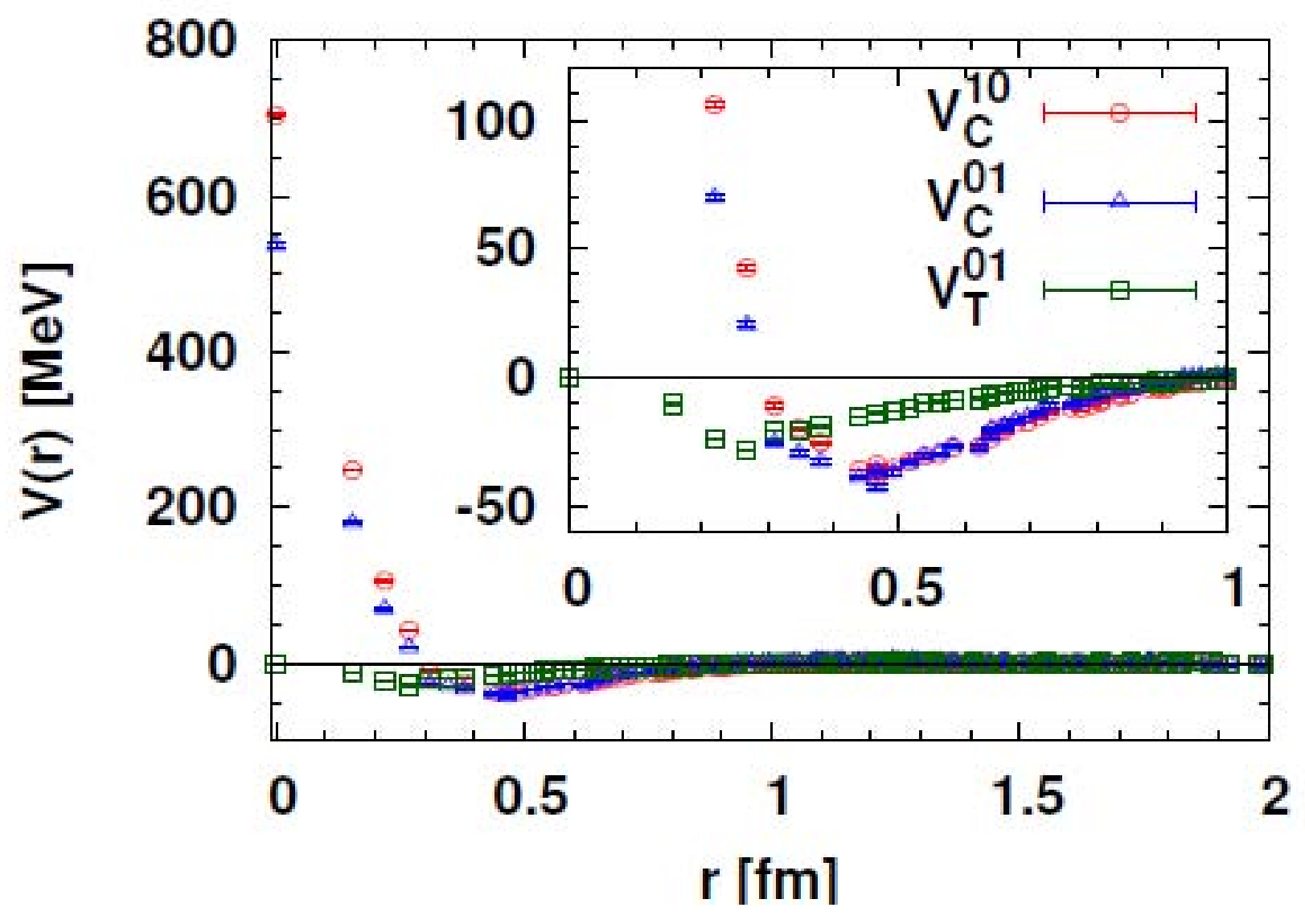

$05 / 16 / 2012$

Lattice Workshop @ RBRC

$M(\pi)=1.13 \mathrm{GeV}$ 


\section{Extension to}

\section{Three-Nucleon Systems}

- We extend the HAL QCD Strategy to $3 \mathrm{~N}$ system to directly determine $3 \mathrm{NF}$

- c.f. Current status of 3N (3B, 4N) Lattice calc

- T. Yamazaki et al. [PACS-CS Coll.] PRD81(2010)111504

- Quenched clover, $\mathrm{m}(\mathrm{pi})=0.8 \mathrm{GeV}, \mathrm{L}=3-12 \mathrm{fm}$

- B.E. : ${ }^{3} \mathrm{He}\left(={ }^{3} \mathrm{H}\right)=18.2(3.5)(2.9) \mathrm{MeV},{ }^{4} \mathrm{He}=27.7(7.8)(5.5) \mathrm{MeV}$

- S. Beane et al. [NPLQCD Coll.] PRD80(2009)074501

- $\mathrm{Nf}=2+1$ clover, $\mathrm{m}(\mathrm{pi})=0.39 \mathrm{GeV}, \mathrm{L}=2.5 \mathrm{fm}$

- $\left(\Xi^{0} \Xi^{0} \mathrm{n}\right)$ and (pnn) are explored, $E_{3 B}-E_{t h} \gtrsim 0$

- Only (binding) energies have been calculated in these studies 


\section{How can we tackle 3NF in Lattice QCD?}

- In the case of $\mathbf{2 N}$ system...

- Calc 4pt func $\rightarrow$ NBS amp.

$$
\psi(\vec{r})=\langle 0|N(\vec{x}+\vec{r} ; t) N(\vec{x} ; t)| 2 N\rangle
$$

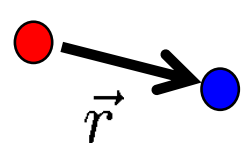

\section{Extention to $3 \mathrm{~N}$ system}

- Calc 6pt func $\rightarrow$ NBS amp. of 3N

- Obtain 3NF through

$$
\psi(\vec{r}, \vec{\rho})=\langle 0 N(\vec{x}+\vec{r}] N(\vec{x})|N(\vec{x}+\vec{r} / 2+\vec{\rho})| 3 N\rangle
$$

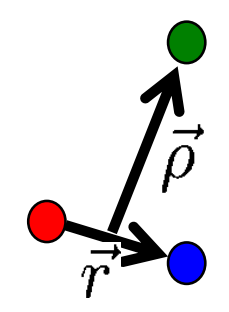

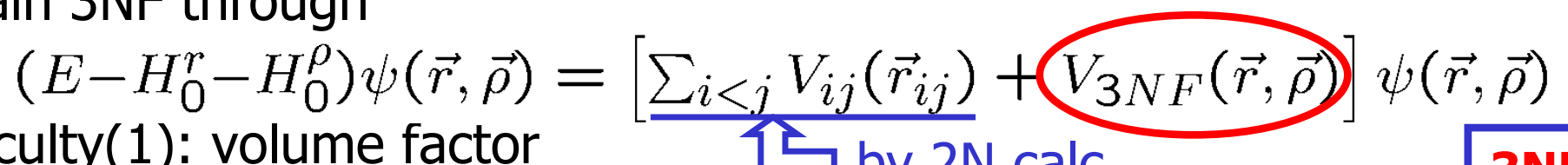

- Difficulty(1): volume factor 它 by $2 \mathrm{~N}$ calc

- $2 \mathrm{~N}$ : naïve $\mathrm{O}\left(\mathrm{L}^{6}\right)$ calc $\rightarrow O\left(\mathrm{~L}^{3} \log \mathrm{L}^{3}\right)$

- $3 N$ : naïve $O\left(L^{9}\right)$ calc $\rightarrow O\left(L^{6} \log L^{6}\right)>\mathbf{O}\left(\mathbf{1 0}^{4}-\mathbf{1 0}^{5}\right)$ factor

- Difficulty(2): naïve calc of quark dof grows in factorial $\left(\sim N_{u}\right.$ ! $N_{d}$ !)

\section{$3 N F$ is} exceptionally challenging problem !

- 2N: $\mathrm{O}\left(\mathrm{L}^{3}\right) \times \mathrm{N}_{\text {wick }} \times$ color/spinor loops

- 3N: $\mathrm{O}\left(\mathrm{L}^{6}\right) \times \mathrm{N}_{\text {wick }} X$ color/spinor loops $O\left(L^{3}\right) \times O(4000)=O\left(10^{7}-10^{8}\right)$ factor 


\section{Features of Linear setup for ${ }^{3} \mathrm{H}$}

- Simplified coupled channel analysis possible

- The vector to 3rd particle $\vec{\rho}=\overrightarrow{0}$

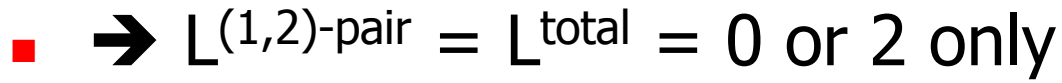

(1) $\quad \vec{\rho}=\overrightarrow{0}$

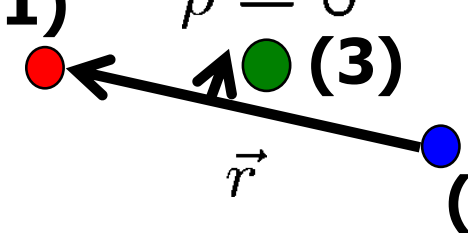

(2)

- $\rightarrow$ Possible bases are only three, which can be labeled by ${ }^{1} \mathrm{~S}_{0},{ }^{3} \mathrm{~S}_{1},{ }^{3} \mathrm{D}_{1}$ for $(1,2)$-pair

Schrodinger Eq.

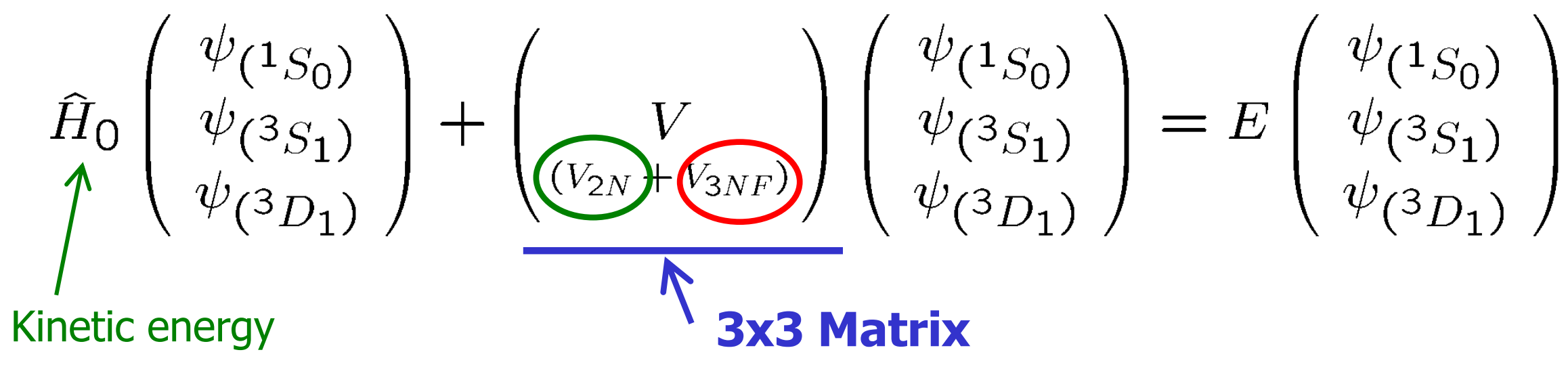




\section{Parity-odd potential Issue}

- However, in order to determine 3NF in $3 \times 3$ coupled channel, we need information of parity-odd potential

- Although $(1,2)$-pair is $L=$ even, $(3,1),(2,3)$-pair have $L=$ odd components

- Parity-odd potential from lattice QCD is under R\&D now (K.Murano et al.)

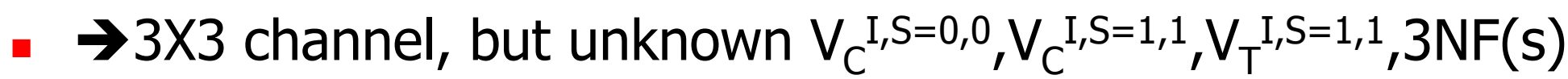

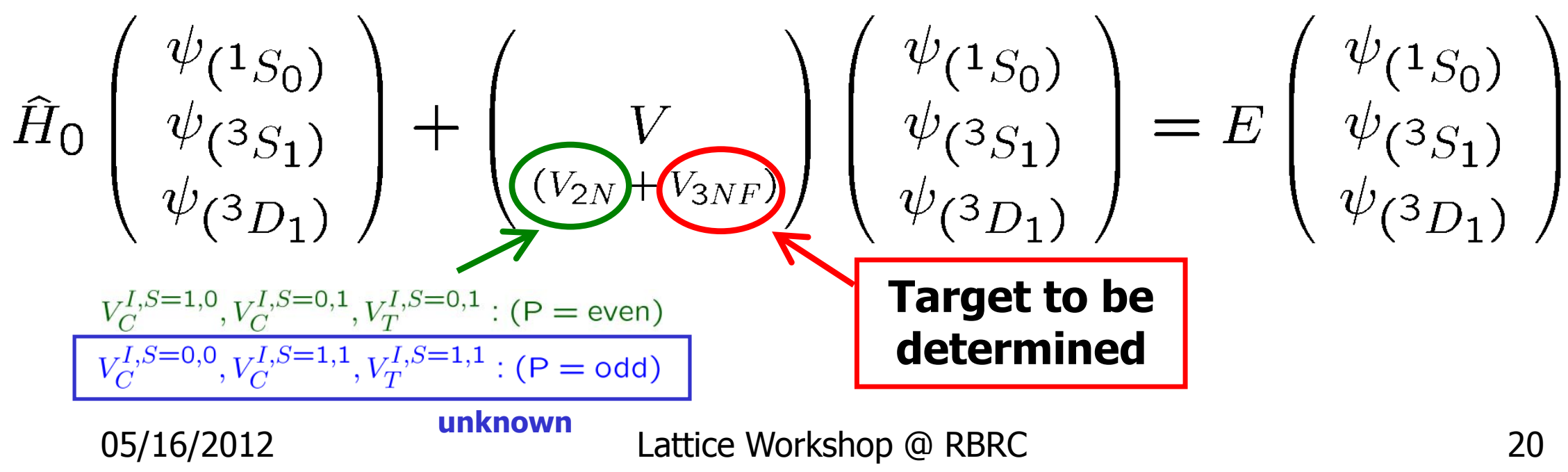




\section{Solution using}

\section{"symmetric" wave function}

- We can construct the wave function in which any $2 \mathrm{~N}$ pair is spin/isospin anti-symmetric
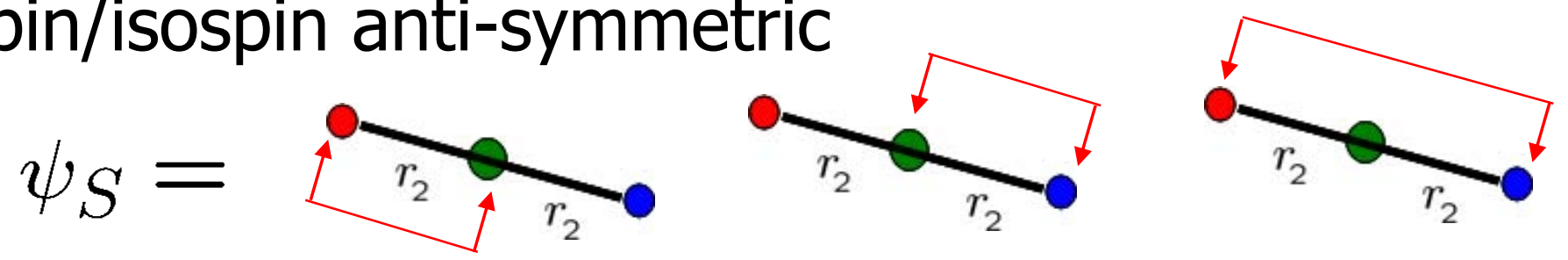

- $\rightarrow \underline{L}=$ even for any $\mathbf{2 N}$ pair automatically guaranteed

- Bases are rotated as $\left.\left.\left|\psi_{S_{S_{0}}}\right\rangle\left|\left\langle\psi_{S_{S_{1}}}\right\rangle\right| \psi_{3_{D_{1}}}\right\rangle \rightarrow\left|\psi_{S}\right\rangle|| \psi_{M}\right\rangle\left|\psi_{3_{D_{1}}}\right\rangle$

$$
\begin{aligned}
& \left|\psi_{S}\right\rangle=1 / \sqrt{2}\left(-\left|\psi_{S_{0}}\right\rangle+\left|\psi_{S_{S_{1}}}\right\rangle\right) \quad\left|\psi_{M}\right\rangle=1 / \sqrt{2}\left(+\left|\psi_{1_{S_{0}}}\right\rangle+\left|\psi_{S_{S_{1}}}\right\rangle\right)
\end{aligned}
$$

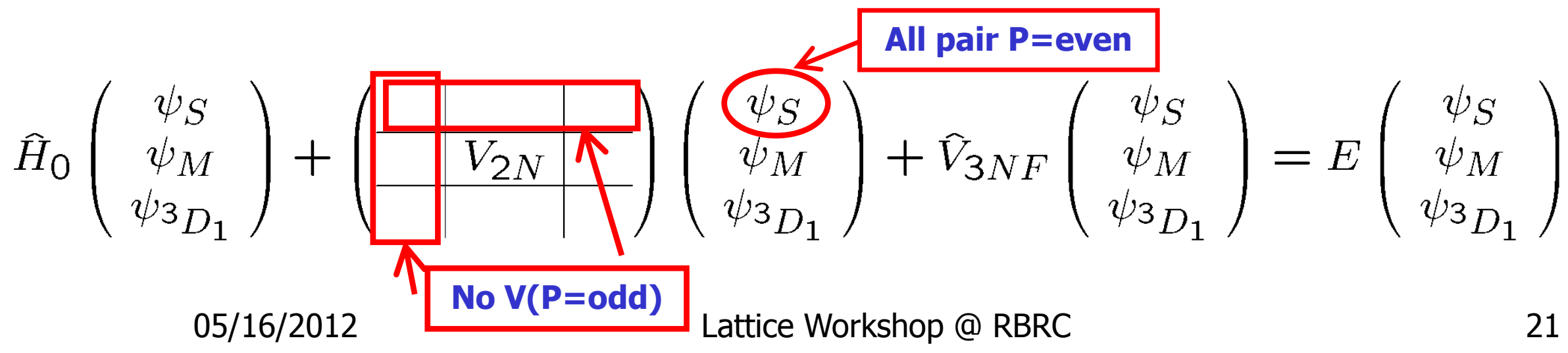




\section{Solution using}

\section{"symmetric" wave function}

We can construct the wave function in which any $2 \mathrm{~N}$ pair is spin/isospin anti-symmetric

- $\rightarrow \underline{L}=$ even for any $2 \mathrm{~N}$ pair automatically guaranteed

- 3x3 coupled channel is reduced to

- one channel with only 3NF unknown

- two channels with $\mathrm{V}_{C} \mathrm{I}, \mathrm{S}=0,0, \mathrm{~V}_{\mathrm{C}}^{\mathrm{I}, \mathrm{S}=1,1}, \mathrm{~V}_{\mathrm{T}}^{\mathrm{I}, \mathrm{S}=1,1}{ }_{1}(3 \mathrm{NF})$ unknown

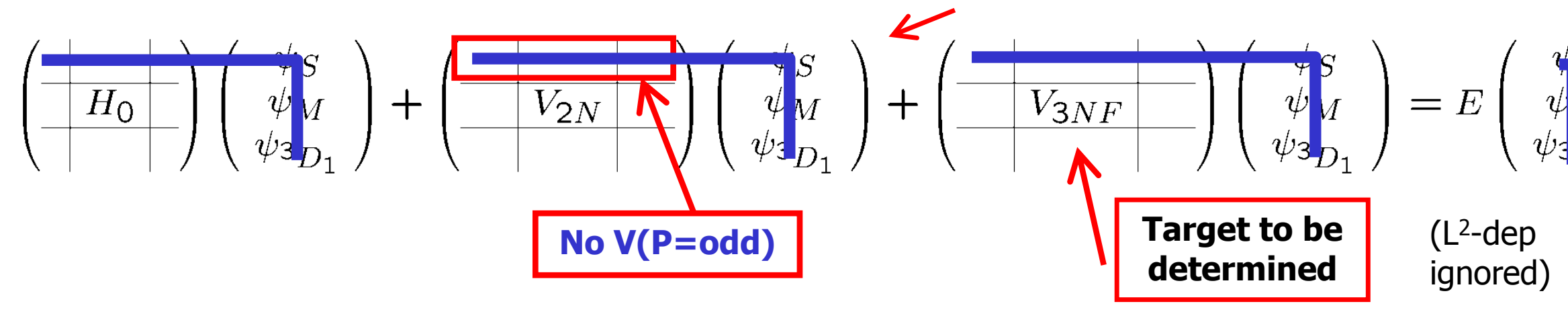

- $\rightarrow$ Even without parity-odd V, we can determine one 3NF

- This method works for any fixed 3D-geometry other than linear $05 / 16 / 2012$ 


\section{Effective Schrodinger equation with E-independent potential}

\section{$K(\vec{x} ; E) \equiv \vec{\nabla}^{2}+k^{2} \psi(\vec{x} ; E) \quad$ [START] local but E-dep pot. ( $\mathbf{L}^{\mathbf{3}} \mathbf{x L}^{\mathbf{3}}$ dof)}

(1) We assume $\psi(x ; E)$ for different $E$ is linearly independent with each other.

(2) $\Psi(x ; E)$ has a "left inverse" as an integration operator as

$$
E \equiv 2 \sqrt{m_{N}^{2}+k^{2}}
$$

$$
\int d^{3} x \tilde{\psi}\left(\vec{x} ; E^{\prime}\right) \psi(\vec{x} ; E)=2 \pi \delta\left(E-E^{\prime}\right)
$$

(3) $\mathrm{K}(\mathrm{x} ; \mathrm{E})$ can be factorized as

$$
\begin{aligned}
K(\vec{x} ; E) & =\int \frac{d E^{\prime}}{2 \pi} K\left(\vec{x} ; E^{\prime}\right) \times \int d_{k}^{3}, \mathscr{y}_{\tilde{\psi}}\left(\vec{y} ; E^{\prime}\right) \psi(\vec{y} ; E) \\
& =\int d^{3} y\left\{\sum_{\alpha} \int \frac{d E^{\prime}}{2 \pi} K\left(\vec{x} ; E^{\prime}\right) \tilde{\psi}\left(\vec{y} ; E^{\prime}\right)\right\} \psi(\vec{y} ; E)
\end{aligned}
$$

(4) We are left with an effective Schrodinger equation with an E-independent popential

U.

$$
\vec{\nabla}^{2}+k^{2} \psi(\vec{x} ; E)=m_{N} \int d^{3} y U(\vec{x}, \vec{y}) \psi(\vec{y} ; E)
$$

[GOAL] non-local but E-indep pot. ( $L^{3} \times L^{3}$ dof) 


\title{
Two-Photon Decay of Neutral Pion from Lattice QCD
}

\author{
Xu Feng (KEK)
}

work with S. Aoki, H. Fukaya, S. Hashimoto, T. Kaneko, J. Noaki and $\mathrm{E}$. Shintani on behalf of JLQCD collaboration

BNL, New York, 2012/05/14 


\section{Motivation}

- PrimEx@JLab: $\Gamma_{\pi^{0} \gamma \gamma}=7.82(22)$ eV [PrimEx, PRL106, 2011]

- Precision: $2.8 \% \rightarrow 1.4 \%$ (projected goal)

- Benchmark test in the chiral anomaly sector of QCD

- $\Gamma_{\pi^{0} \gamma \gamma}=(\pi / 4) \alpha_{e}^{2} m_{\pi}^{3} \mathcal{F}_{\pi^{0} \gamma \gamma}^{2}\left(m_{\pi}^{2}, 0,0\right)$

- $\mathcal{F}_{\pi^{0} \gamma \gamma}\left(m_{\pi}^{2}, p_{1}^{2}, p_{2}^{2}\right): \pi^{0} \rightarrow \gamma^{*} \gamma^{*}$ transition form factor

- $m_{\pi}: \pi^{0}$ mass; $p_{1}, p_{2}:$ photon 4-momentum 


\section{Lattice setup}

- overlap fermion: exact chiral symmetry $\Rightarrow$ test chiral anomaly

- all-to-all propagators $\Rightarrow C\left(t_{1}, t_{2}, t_{3}\right)$

- low mode: low-lying eigenvalues and eigenvectors

- high mode: stochastic propagators

- calculate correlator at any time slice of $t_{1}, t_{2}, t_{3}$

- disconnected diagram 


\section{Ensemble information}

- four $m_{u, d}: m_{\pi}=540 \rightarrow 290 \mathrm{MeV} \Rightarrow$ chiral exapolation

- $m_{s}$ fixed to be close to its physical value $\Rightarrow$ dynamical $s$-quark effects

- $L / a=16$ and $24 \Rightarrow$ finite-size effects

- $Q=0$ and $1 \Rightarrow$ fixing-topology effects

- $a=0.11 \mathrm{fm} \Rightarrow$ study possible lattice artifacts 


\section{Theoretical setup}

- starting point: S-matrix

$$
\left\langle\gamma\left(p_{1}, \lambda_{1}\right) \gamma\left(p_{2}, \lambda_{2}\right) \mid \pi^{0}(q)\right\rangle
$$

- transition form factor is defined by matrix element

$$
\underbrace{\int d^{4} x e^{i p_{1} x}\left\langle\Omega\left|T\left\{J_{\mu}(x) J_{\nu}(0)\right\}\right| \pi^{0}(q)\right\rangle}_{\mathcal{M}_{\mu \nu}}=\epsilon_{\mu \nu \alpha \beta} p_{1}^{\alpha} p_{2}^{\beta} F_{\pi^{0} \gamma \gamma}\left(m_{\pi}^{2}, p_{1}^{2}, p_{2}^{2}\right)
$$

- $\epsilon_{\mu \nu \alpha \beta} p_{1}^{\alpha} p_{2}^{\beta}$ : induced by the negative parity of the $\pi^{0}$

- chiral limit, photon on-shell, ABJ anmoaly:

$$
\mathcal{F}_{\pi^{0} \gamma \gamma}(0,0,0)=\mathcal{F}_{\pi^{0} \gamma \gamma}^{\mathrm{ABJ}}=\frac{1}{4 \pi^{2} F_{\pi}}
$$




\section{Method}

- matrix element in the Minkowski space-time

$$
\mathcal{M}_{\mu \nu}=\int d^{4} x e^{i p_{1} x}\left\langle\Omega\left|T\left\{J_{\mu}(x) J_{\nu}(0)\right\}\right| \pi^{0}(q)\right\rangle
$$

- analytical continuation from Minkowski to Euclidean space-time [Ji, Jung, 2001; Dudek, Edwards, 2006]

$$
\mathcal{M}_{\mu \nu}=\int d t e^{\omega t} \int d^{3} \vec{x} e^{-i \vec{p}_{1} \cdot \vec{x}}\left\langle\Omega\left|T\left\{J_{\mu}(x) J_{\nu}(0)\right\}\right| \pi^{0}(q)\right\rangle
$$

- pion is on-shell: $q=\left(E_{\pi}, \vec{q}\right) \Rightarrow q^{2}=m_{\pi}^{2}$

- photon 4-momentum: $p_{1}=\left(\omega, \vec{p}_{1}\right)$ and $p_{2}=\left(E_{\pi}-\omega, \vec{q}-\vec{p}_{1}\right)$

- requirement: $p_{1,2}^{2}<m_{\rho}^{2}$ or $E_{\pi \pi}^{2}$ (hadron production threshold) 


\section{Amplitude $A_{\pi}(\tau)$}

- observable:

$$
\mathcal{M}_{\mu \nu}=\int d t e^{\omega t} \int d^{3} \vec{x} e^{-i \vec{p}_{1} \cdot \vec{x}}\left\langle\Omega\left|T\left\{J_{\mu}(x) J_{\nu}(0)\right\}\right| \pi^{0}(q)\right\rangle
$$

- correlation function:

$$
C_{\mu \nu}=\left\langle\Omega\left|J_{\mu}\left(\vec{p}_{1}, t_{1}\right) J_{\nu}\left(\vec{p}_{2}, t_{2}\right) \pi^{0}\left(-\vec{q}, t_{\pi}\right)\right| \Omega\right\rangle
$$

- set $\tau=t_{1}-t_{2}$ and $t=\min \left\{t_{1}, t_{2}\right\}$

$$
A_{\pi}(\tau) \equiv \lim _{t-t_{\pi} \rightarrow \infty} C_{\mu \nu}\left(t_{1}, t_{2}, t_{\pi}\right) / e^{-E_{\pi}\left(t-t_{\pi}\right)}
$$

- matrix element $\mathcal{M}_{\mu \nu}$ is given by

$$
\mathcal{M}_{\mu \nu}=\frac{2 E_{\pi}}{\phi_{\pi}}\left(\int_{0}^{\infty} d \tau e^{\omega \tau} A_{\pi}(\tau)+\int_{-\infty}^{0} d \tau e^{\left(\omega-E_{\pi, \vec{q}}\right) \tau} A_{\pi}(\tau)\right)
$$




\section{Distribution of $A_{\pi}(\tau)$}

- $\left\langle J_{\mu}\left(\vec{p}_{1}, t_{1}\right) J_{\nu}\left(\vec{p}_{2}, t_{2}\right) \pi^{0}\left(\vec{q}, t_{\pi}\right)\right\rangle: \mu=1, \nu=2$

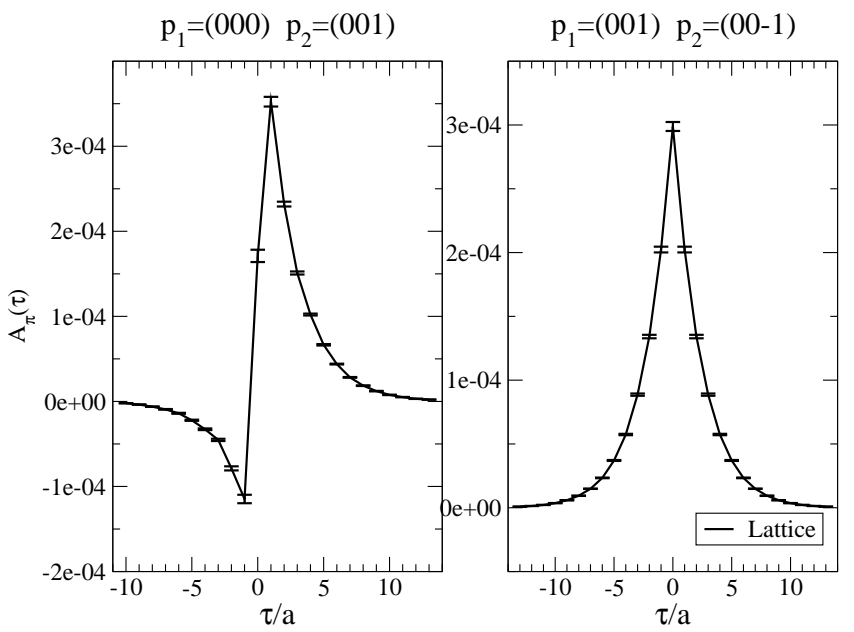




\section{Time dependence of $A_{\pi, 1,2}$}

- VMD model: $\mathcal{F}_{\pi^{0} \gamma \gamma}^{\mathrm{VMD}}\left(m_{\pi}^{2}, p_{1}^{2}, p_{2}^{2}\right)=c_{V} G_{V}\left(p_{1}^{2}\right) G_{V}\left(p_{2}^{2}\right)$

- $G_{V}\left(p^{2}\right)=M_{V}^{2} /\left(M_{V}^{2}-p^{2}\right)$ is the vector meson propagator, $M_{V}=m_{\rho}$

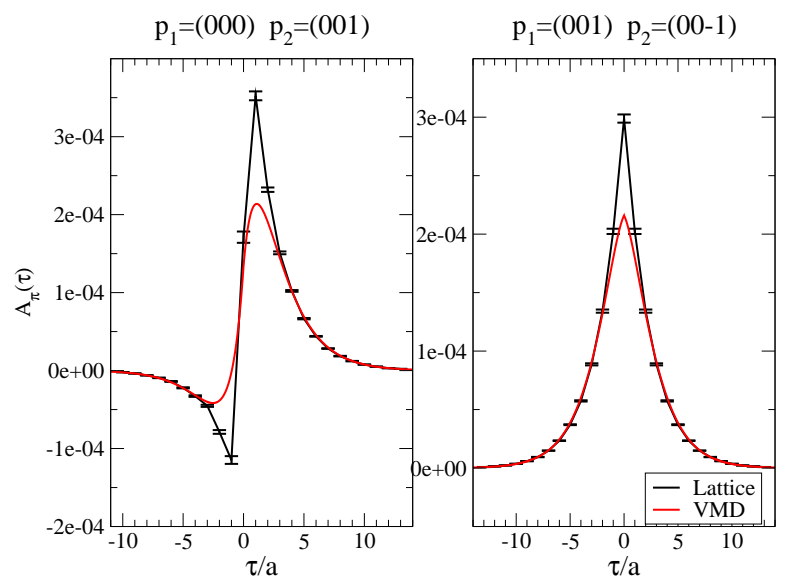




\section{Fit ansatz}

- lowest vector meson effects should be accounted for first

- corrected by including excited-state effects

$$
\begin{aligned}
\mathcal{F}_{\pi^{0} \gamma \gamma}\left(m_{\pi}^{2}, p_{1}^{2}, p_{2}^{2}\right) & =c_{V} G_{V}\left(p_{1}^{2}\right) G_{V}\left(p_{2}^{2}\right) \\
& +\sum_{V^{\prime}} c_{V^{\prime}}\left(G_{V}\left(p_{1}^{2}\right) G_{V^{\prime}}\left(p_{2}^{2}\right)+G_{V^{\prime}}\left(p_{1}^{2}\right) G_{V}\left(p_{2}^{2}\right)\right) \\
& +\sum_{V^{\prime}, V^{\prime \prime}} c_{V^{\prime} V^{\prime \prime}} G_{V^{\prime}}\left(p_{1}^{2}\right) G_{V^{\prime \prime}}\left(p_{2}^{2}\right)
\end{aligned}
$$

- replace $G_{V^{\prime}}\left(p^{2}\right)$ by a basis of polynomial function

$$
\begin{aligned}
\mathcal{F}_{\pi^{0} \gamma \gamma}\left(m_{\pi}^{2}, p_{1}^{2}, p_{2}^{2}\right) & =c_{V} G_{V}\left(p_{1}^{2}\right) G_{V}\left(p_{2}^{2}\right) \\
& +\sum_{m} c_{m}\left(\left(p_{2}^{2}\right)^{m} G_{V}\left(p_{1}^{2}\right)+\left(p_{1}^{2}\right)^{m} G_{V}\left(p_{2}^{2}\right)\right) \\
& +\sum_{m, n} c_{m, n}\left(p_{1}^{2}\right)^{m}\left(p_{2}^{2}\right)^{n}
\end{aligned}
$$




\section{Form factor}

- $\mathcal{F}_{\pi^{0} \gamma \gamma}=\int d t e^{\omega t}\left\langle\Omega\left|T\left\{J_{\mu}\left(\vec{p}_{1}, t\right) J_{\nu}\left(\vec{p}_{2}, 0\right)\right\}\right| \pi^{0}(q)\right\rangle / \epsilon_{\mu \nu \alpha \beta} p_{1}^{\alpha} p_{2}^{\beta}$

- tunning $\omega: p_{1}^{2}=\omega^{2}-\vec{p}_{1}^{2} \quad p_{2}^{2}=\left(E_{\pi}-\omega\right)^{2}-\vec{p}_{2}^{2}$
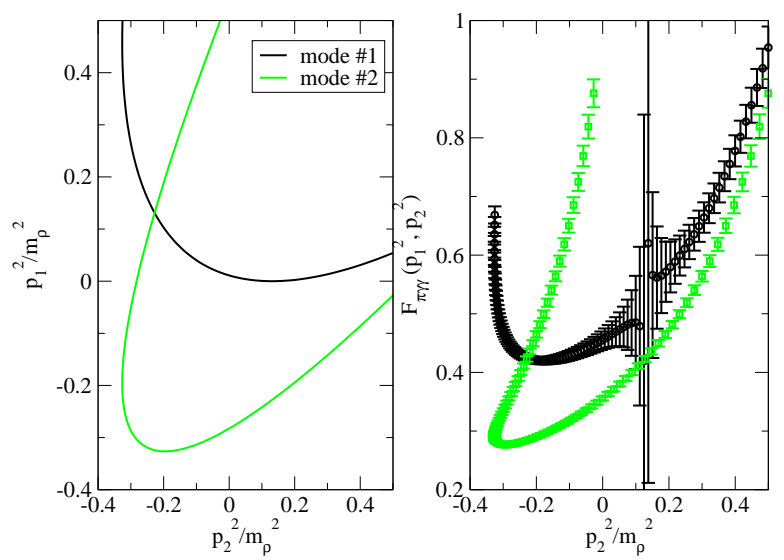


\section{Combined fit}

- fit ansatz $\mathcal{F}_{\pi^{0} \gamma \gamma}\left(m_{\pi}^{2}, p_{1}^{2}, p_{2}^{2}\right)=c_{V} G_{V}\left(p_{1}^{2}\right) G_{V}\left(p_{2}^{2}\right)+$

$$
\sum_{m} c_{m}\left(\left(p_{2}^{2}\right)^{m} G_{V}\left(p_{1}^{2}\right)+\left(p_{1}^{2}\right)^{m} G_{V}\left(p_{2}^{2}\right)\right)+\sum_{m, n} c_{m, n}\left(p_{1}^{2}\right)^{m}\left(p_{2}^{2}\right)^{n}
$$
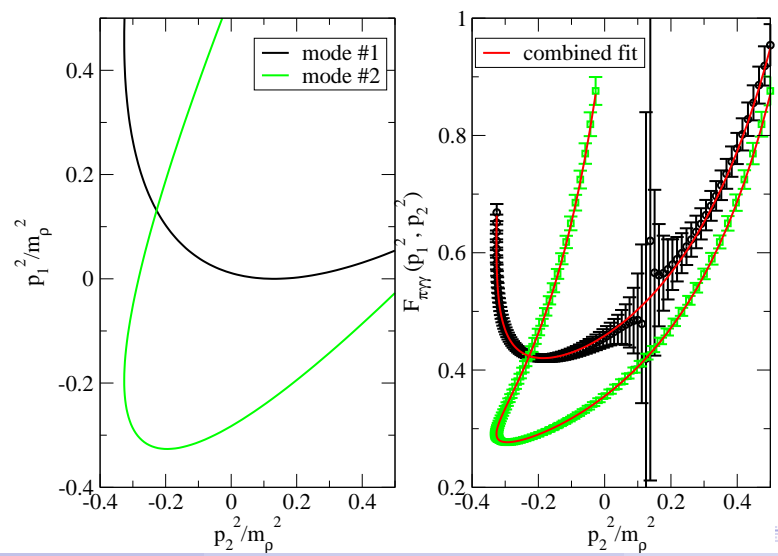


\section{On-shell photon limit}

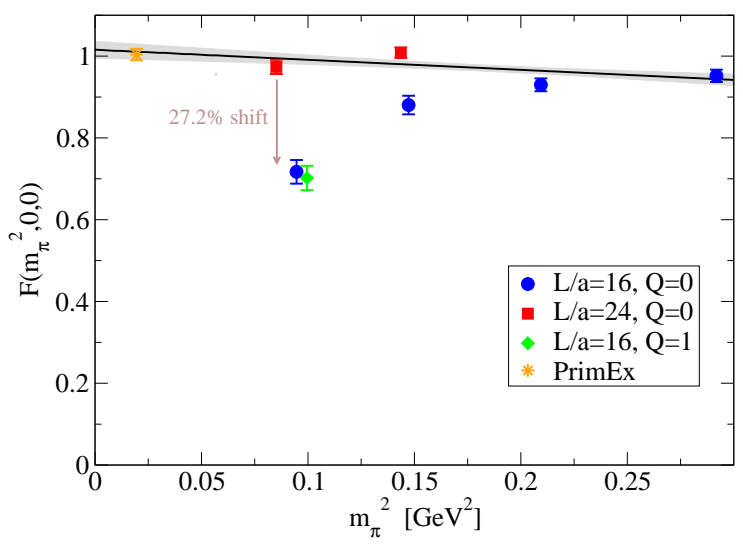

- $F\left(m_{\pi}^{2}, 0,0\right) \equiv \mathcal{F}_{\pi^{0} \gamma \gamma}\left(m_{\pi}^{2}, 0,0\right) / \mathcal{F}_{\pi^{0} \gamma \gamma}^{\mathrm{ABJ}}$

- data with $m_{\pi} L \geq 4$ : consistent with $\mathrm{ABJ}$ and PrimEx

- $L=16$ : smallest two quark mass, $m_{\pi} L<4$, big FS effects

- FS effects checked at topological sector $Q=0$ and 1 


\section{Expansion of matrix element}

- expand the correlator into three hadronic matrix elements:

$$
\left\langle J_{\mu} J_{\nu} \pi^{0}\right\rangle \rightarrow\left\langle\Omega\left|J_{\mu}\right| V\right\rangle\left\langle V\left|J_{\nu}\right| \pi^{0}\right\rangle\left\langle\pi^{0}\left|\pi^{0}\right| \Omega\right\rangle
$$

- vector-meson electromagnetic coupling $g_{V}$

$$
\left\langle\Omega\left|J_{\mu}\right| V, p, \varepsilon\right\rangle=M_{V} F_{V \varepsilon_{\mu}}(p)=M_{V}^{2} g_{V} \varepsilon_{\mu}(p)
$$

- $V \pi \gamma$ coupling $g_{V \pi \gamma}, V \rightarrow \pi \gamma$ decay

$$
\left\langle V, \varepsilon_{\mu}\left(p_{1}\right)\left|j_{\nu}(0)\right| \pi^{0}(q)\right\rangle=\frac{g_{V \pi \gamma}}{M_{V}} \varepsilon_{\mu \nu \alpha \beta} p_{1 \alpha} p_{2 \beta} K_{V}\left(p_{2}^{2}\right), \quad p_{2}=q-p_{1}
$$

- pion decay constant $F_{\pi}$

$$
\left\langle\pi^{0}\left|\pi^{0}\right| \Omega\right\rangle=\frac{m_{\pi}^{2}}{2 m_{u, d}} F_{\pi}
$$




\section{Finite-size effects}

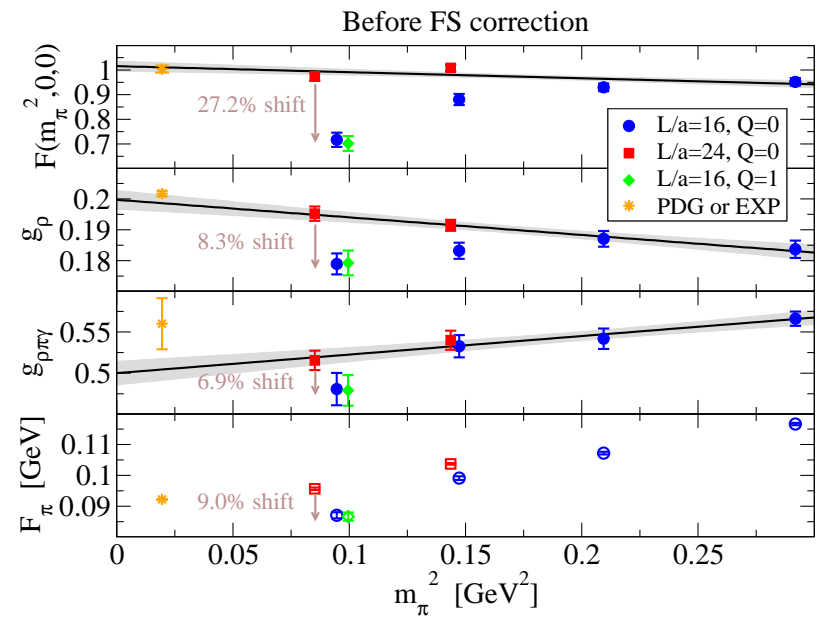

- FS effects accumulate and add up to a large effect 


\section{Finite-size corrections}

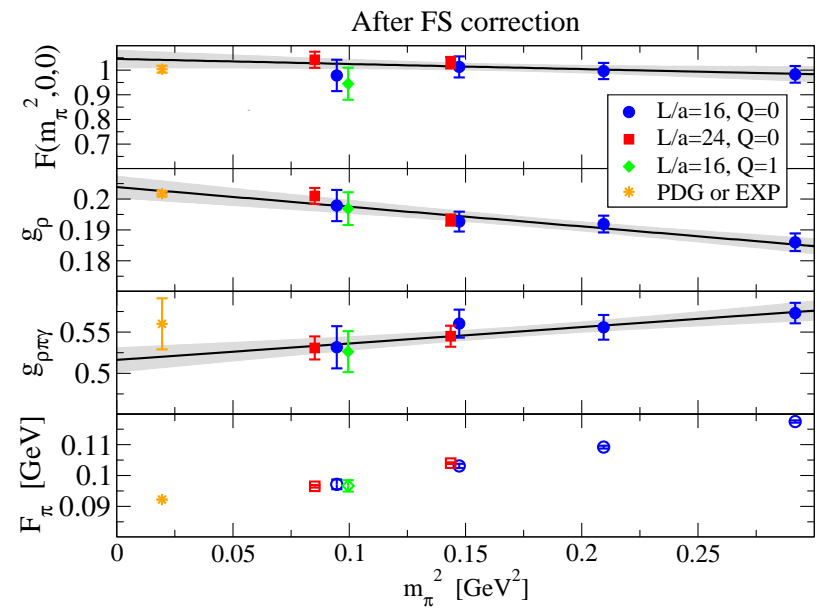

- FS corrections $R_{\mathcal{O}} \equiv \mathcal{O}(\infty) / \mathcal{O}(L)$ with $\mathcal{O}=g_{\rho}, g_{\rho \pi \gamma}$ and $F_{\pi}$ - assume that $R_{F\left(m_{\pi}^{2}, 0,0\right)}=R_{g_{\rho}} R_{g_{\rho \pi \gamma}} R_{F_{\pi}}$ 


\section{Disconnected-diagram effects}

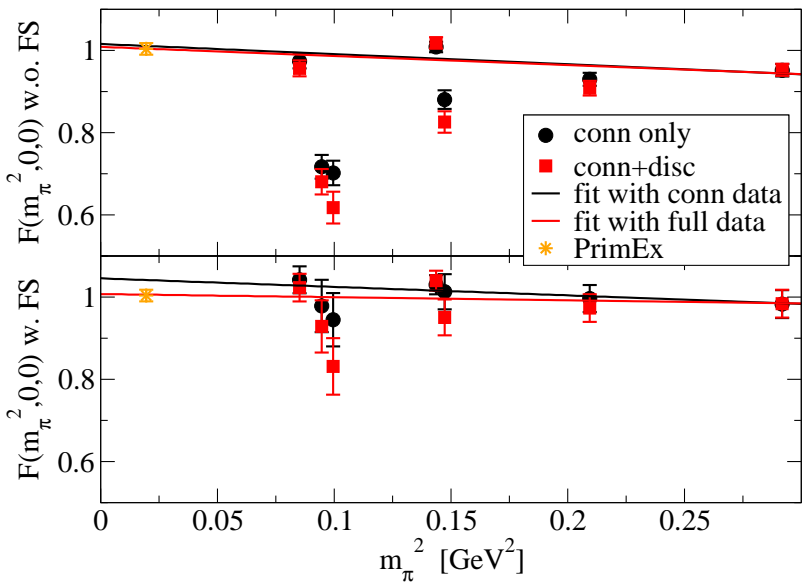

- all-to-all propagator: control error of disc. contribution

- although not significant, conn+disc systematically shift down

- precision level ( $2 \%$ for form factor): disc. diagram should be included 


\section{Lattice artifacts}

- discrete data v.s. continuum case?

- right fig: turnover obtained; left fig: not obtained?

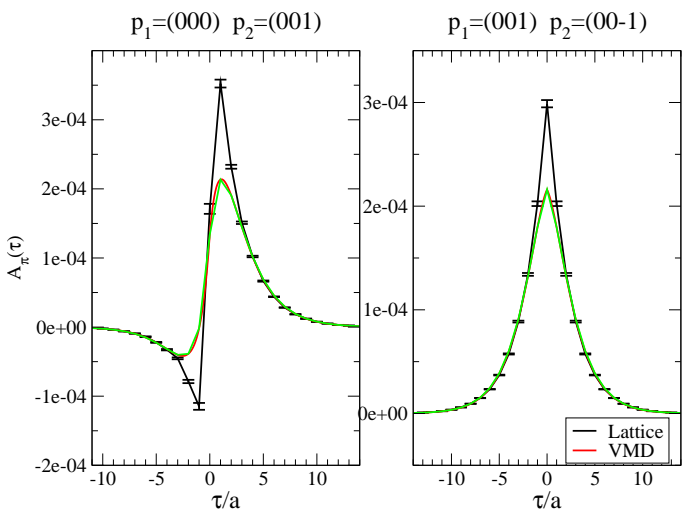

- disc. effects in VMD model: less than $5 \times 10^{-4}$, neglegiable

- turnover contributed by ground and excited states, not very=large 


\section{Conclusion}

- after examining possible systematic effects

$$
\begin{aligned}
F(0,0,0) & =1.009(22)(29) \\
F\left(m_{\pi, \text { phy }}^{2}, 0,0\right) & =1.005(20)(30) \\
\Gamma_{\pi^{0} \gamma \gamma} & =7.93(29)(43) \mathrm{eV}
\end{aligned}
$$

- ABJ anomaly and PrimEx measurement

$$
\begin{aligned}
F(0,0,0) & =1 \\
F\left(m_{\pi, \text { phy }}^{2}, 0,0\right) & =1.004(14) \\
\Gamma_{\pi^{0} \gamma \gamma} & =7.82(22) \mathrm{eV}
\end{aligned}
$$

- $m_{u} \neq m_{d}$, effects mixing of $m_{\pi}$ with $\eta$ and $\eta^{\prime}$ are not yet studied 


\section{Motivated by muon $g_{\mu}-2$}

- Exp. determination of $g_{\mu}-2$ to 0.54 ppm [E821@BNL, PRD73, 2006]

- S.M. prediction of $g_{\mu}-2$ to $0.51 \mathrm{ppm}$ [Jegerlehner, EPJC71, 2011]

- Discrepancy: $3.3 \sigma \Rightarrow$ New Physics ??

- HLbL is predicted to be dominant error in the next round

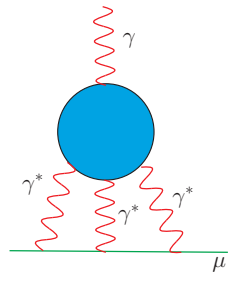

(a)

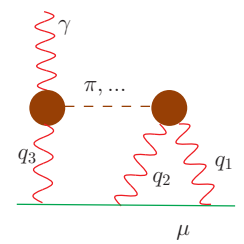

(b)

- Difficult: HLbL involves $\left\langle J_{\mu} J_{\nu} J_{\rho} J_{\sigma}\right\rangle$

- Better to start with $\pi^{0}\left(\eta, \eta^{\prime}\right) \rightarrow \gamma^{*} \gamma^{*}$ 


\section{$\pi^{0}$ contribution}

\begin{tabular}{cccccccc}
\hline Contribution & BPP & HKS & KN & MV & BP & PdRV & N/JN \\
\hline$\pi^{0}, \eta, \eta^{\prime}$ & $85 \pm 13$ & $82.7 \pm 6.4$ & $83 \pm 12$ & $114 \pm 10$ & - & $114 \pm 13$ & $99 \pm 16$ \\
$\pi, K$ loops & $-19 \pm 13$ & $-4.5 \pm 8.1$ & - & - & - & $-19 \pm 19$ & $-19 \pm 13$ \\
$\pi, K$ loops + other subleading in $N_{c}$ & - & - & - & $0 \pm 10$ & - & - & - \\
axial vectors & $2.5 \pm 1.0$ & $1.7 \pm 1.7$ & - & $22 \pm 5$ & - & $15 \pm 10$ & $22 \pm 5$ \\
scalars & $-6.8 \pm 2.0$ & - & - & - & - & $-7 \pm 7$ & $-7 \pm 2$ \\
quark loops & $21 \pm 3$ & $9.7 \pm 11.1$ & - & - & - & 2.3 & $21 \pm 3$ \\
\hline total & $83 \pm 32$ & $89.6 \pm 15.4$ & $80 \pm 40$ & $136 \pm 25$ & $110 \pm 40$ & $105 \pm 26$ & $116 \pm 39$
\end{tabular}

- summary table [Jegerlehner, Nyffeler, Phys.Rept.477:1-110,2009]

- $\pi^{0}\left(\eta, \eta^{\prime}\right) \rightarrow \gamma^{*} \gamma^{*}$ are consistent to total contributions

- Among three PS mesons, $\pi^{0}$ takes about $70 \%$

- calulation on the $\pi^{0} \rightarrow \gamma^{*} \gamma^{*}$ can be duplicated to the $\eta, \eta^{\prime}$ sector 


\section{Non-perturbative nature}

- Invariant mass spectrum for two-photon

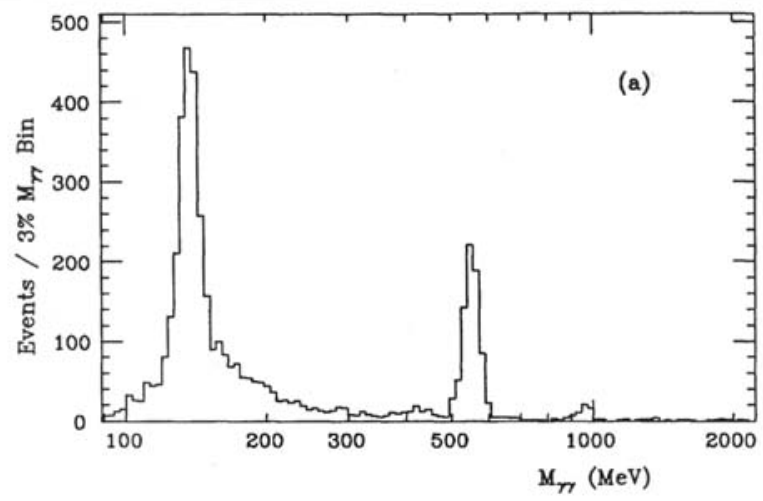

- Three spikes presents three bound states: $\pi^{0}, \eta, \eta^{\prime}$

- Bound states $\rightarrow$ confinement $\rightarrow$ LQCD 


\section{Rho mass}

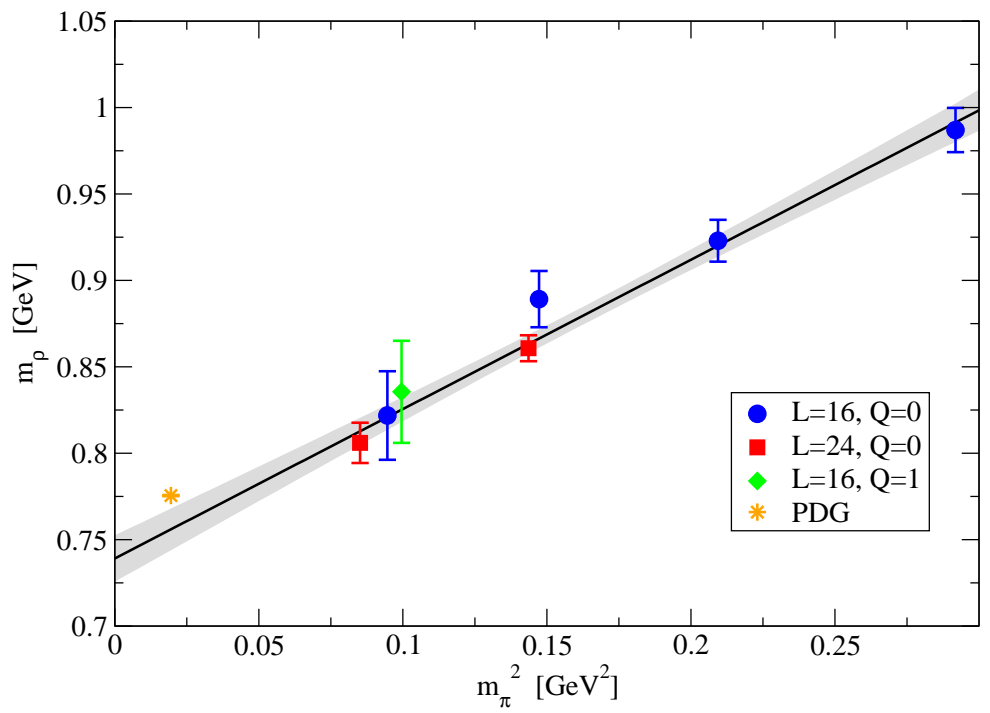




\section{High precision scale setting}

\section{Z. Fodor}

University of Wuppertal \& Budapest, UCSD, FZJ

Budapest-Marseille-Wuppertal Collaboration, 1203.4469*

\section{BNL, May 14, 2012}




\section{Outline}

(1) Scale setting

(2) Flow of the gauge field

(3) Pure gauge

(4) Full QCD 


\section{Scale settings and the static potential}

raw output of lattice QCD: physical quantities in lattice unit

$\Rightarrow$ measure a dimensionful quantity $Q\left(M_{\Omega}\right.$ or $\left.f_{K}\right)$

the lattice spacing is given by $a=\left(a Q^{\text {lat }}\right) / Q^{\exp }$

today erros below $2 \%$ for several lattice predictions

it depends crucially on the error of the lattice spacing

need for a controlled/small error lattice spacing determination

not necessarily directly accesable for experiments e.g. potential popular choices are:

string tension (strictly speaking doesn't exist: string breaking)

the Sommer-scale $r_{x}^{2} \cdot d V / d r=C_{X}$

originally $r_{0}$ with $C_{0}=1.65$ or MILC choice $r_{1}$ with $C_{1}=1$ 


\section{Sommer-scale, Omega mass, $f_{\pi}$ and $f_{K}$}

unfortunately, the calculations of $r_{0} \& r_{1}$ are quite involved far more complicated than fitting the masses of particles

complications are reflected in the literature MILC: $r_{1}=0.3117(22) \mathrm{fm}$ (less than $1 \%$ accuracy)

RBC/UKQCD: $r_{1}=0.3333(93)(1)(2) \mathrm{fm}$

$7 \%$ difference and $2.3 \sigma$ tension between them

another popular way is to use the Omega baryon mass the experimental value of $M_{\Omega}$ is well known more CPU demanding \& sensititve to the strange quark mass mismatched strange quark mass leads to a mismatched scale difficulties with $f_{\pi}$ (chiral extrapolation) \& $f_{K}$ (mismatched $m_{s}$ ) suggestion of M. Luscher: use the Wilson flow to set the scale 


\section{Definition of the flow of the gauge field}

consider the flow: $B_{\mu}(t, x)$ for $t>0$ with $B_{\mu}(0, x)=A_{\mu}(x)$ flow equation: $\partial_{t} B_{\mu}=D_{\nu} G_{\mu \nu}$ with $G_{\mu \nu}=\partial_{\mu} B_{\nu}-\partial_{\nu} B_{\mu}+\left[B_{\mu}, B_{\nu}\right]$ the evolution in thas a smoothing effect:

$\partial_{t} B_{\mu}=\Delta B_{\mu}-\partial_{\mu} \partial_{\nu} B_{\nu}+$ non-linear terms the first term is the same as in the heat-equation $B_{\mu}(t, x)=\int d^{4} x K_{t}(x-y) A_{\mu}(y)+\ldots$

$K_{t}$ four dimensional heat kernel $K_{t}(r)=\exp \left(-r^{2} / 4 t\right) /(4 \pi t)^{2}$ smoothing effect with $\sqrt{8 t}$ smoothing range

on the lattice regularize it: $V_{t}(x, \mu)$ for $t>0$ with $V_{0}(x, \mu)=U(x, \mu)$ flow equation with $(Z)$ staples: $\partial_{t} V_{t}(x, \mu)=Z\left(V_{t}(x, \mu)\right) \cdot V_{t}(x, \mu)$ 


\section{Wilson flow: technical realization}

flow equation: $\dot{V}_{t}=Z\left(V_{t}\right) V_{t}$, where $Z$ is the staple equivalent to a series of infinitesimal stout smearing steps in our case it is integrated with 4th-order Runge-Kutta scheme M. Luscher, JHEP 1008 (2010) 071

evolution from time $t$ to time $t+\epsilon$ is given by $Z_{i}=\epsilon Z\left(W_{i}\right)$

$$
\begin{aligned}
W_{0} & =V_{t} \\
W_{1} & =\exp \left(\frac{1}{4} Z_{0}\right) W_{0}, \\
W_{2} & =\exp \left(\frac{8}{9} Z_{1}-\frac{17}{36} Z_{0}\right) W_{1}, \\
V_{t+\epsilon} & =\exp \left(\frac{3}{4} Z_{2}-\frac{8}{9} Z_{1}+\frac{17}{36} Z_{0}\right) W_{2}
\end{aligned}
$$




\section{Wilson flow and the coupling}

\section{Luscher, JHEP 1008 (2010) 071}

as a representative example $E=G_{\mu \nu}^{a} G_{\mu \nu}^{a} / 4$ is considered lattice: $E(t)$ can be defined by the (1-plaquette) or clover terms they only differ by discretization effects lattice: we expect $\langle E\rangle \propto(1$-plaquette $) \cdot t^{2}$ behavior

very important results about the renormalization of the Wilson flow

calculation of $\langle E\rangle$ up to $\alpha_{S}^{2}(q)$ with $q=(8 t)^{-1 / 2}$ (result has been obtained in the continuum $\overline{M S}$ scheme)

$$
\langle E\rangle=\frac{3}{4 \pi t^{2}} \alpha(q)\left\{1+k_{1} \alpha(q)+\mathcal{O}\left(\alpha^{2}\right)\right\}, \quad k_{1}=1.0978+0.0075 N_{f}
$$

above the cut-off (small $t$ ): lattice and continuum quite different 


\section{Lattice study of the Wilson flow (pure gauge)}

the perturbation QCD expansion works for small $t(\ll 1 \mathrm{fm})$ for large $t$ one uses numerical lattice simulations $\mathrm{SU}(3)$ pure gauge theory with lattice spacing $\mathrm{a}=0.05 \mathrm{fm}$

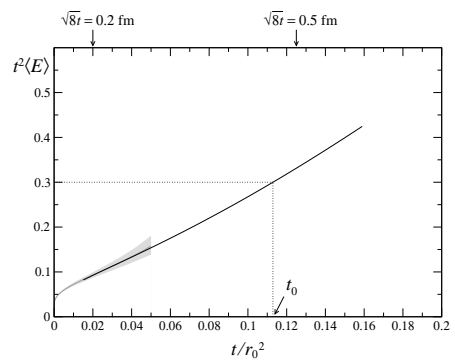

statistical error: smaller than the thickness of the (linear) line lattice: expect (1-plaquette) $\cdot t^{2}$ behavior for small $t$ perturbation theory is given by the band (uncertainty on $\Lambda$ ) 


\section{Wilson flow for scale setting: quenched}

$\langle E\rangle$ is physical: approaches its continuum limit with $a^{2}$ test it with the reference scale $t_{0}$ given by

$$
\left\{t^{2}\langle E\rangle\right\}_{t=t_{0}}=0.3
$$

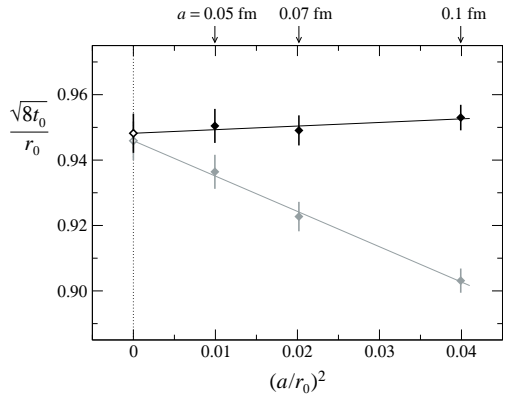

scaling violation increases toward smaller reference scales for which the smoothing range is only $2-3$ times the lattice spacing 


\section{Gauge flow for dynamical fermions $\& w_{0}$}

one can determine the gauge flow also for the dynamical case use the Wilson flow or the gauge flow defined by the action

$t^{2}\langle E(t)\rangle$ incorporates informations from all $t>\mathcal{O}(1 / \sqrt{t})$

its derivative (almost constant) mostly from scales around $\mathcal{O}(1 / \sqrt{t})$ advantage: flow at small $t \sim a^{2}$ is a subject of cutoff effects

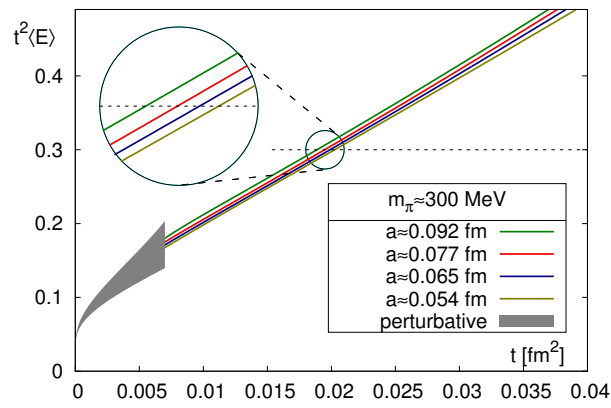

observed "linearity" for $t^{2}\langle E\rangle$ one can extract it by $t \cdot d t^{2}\langle E\rangle / d t$ instead $t^{2}\langle E\rangle=0.3$ (M. Luscher) $t \cdot d t^{2}\langle E\rangle / d t=0.3$ ( $w_{0}$ scale) $a \rightarrow 0$ : non-universal part shrinks $w_{0}$ has less cutoff effects than $t_{0}$ 


\section{Continuum limit is the same}

different definitions should have the same continuum limit one can use the Wilson flow or the Symanzik flow: $M_{\pi}=135 \mathrm{MeV}$

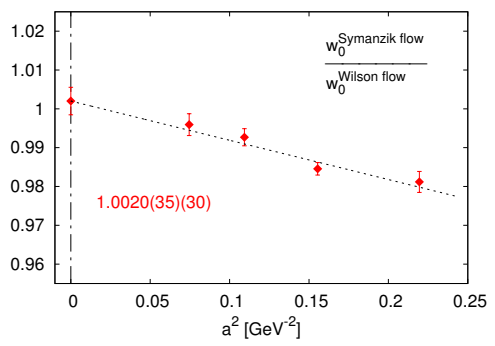

original definition of Luscher has the largest cut-off effect various definitions of $w_{0}$ have tiny ones (a few $\%$ or less) (statistical errors are neglible, good for scale setting) 


\section{Finite volume effects}

how sensitive is $w_{0}$ to the size of the system only for boxes $<2 \mathrm{fm}: M_{\pi} L \approx 1.35$ instead of 4 $\Longrightarrow$ finite volume effects are tiny, far below the $1 \%$ level

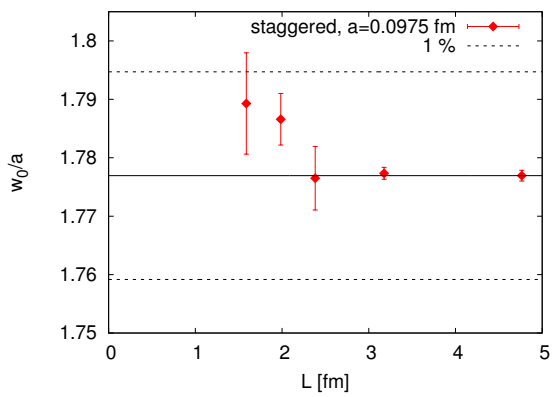

robust and stable method for determining the scale 


\section{$a \rightarrow 0:$ Wilson \& staggered $w_{0}$ Budapest:Marselle-Wuppertal Collaboration, 1203.4469-}
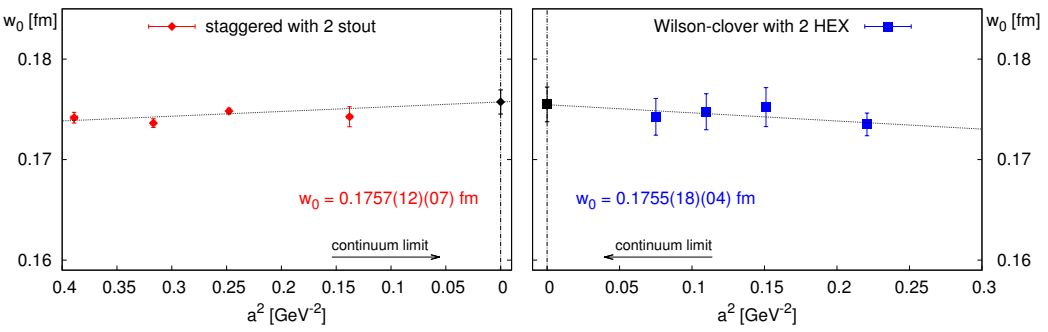

the physical scale was obtained by the Omega baryon mass

our final result is the Wilson result (staggered is a cross check) (no rooting $\Longrightarrow$ theoretically cleaner)

$$
w_{0}=0.1755(18)(04) \mathrm{fm}
$$

error (dominantly statistical) is $1 \%$ (and comes not from the gauge flow itself, but from $M_{\Omega}$ ) 


\section{$a \rightarrow 0$ : Wilson \& staggered $w_{0}$ Budapest-Marsenle-Wuppertal Collaboration, 1203.4469-}

Staggered with 2 stout

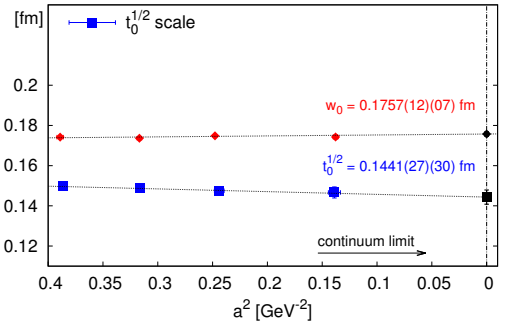

Wilson-clover with 2 HEX

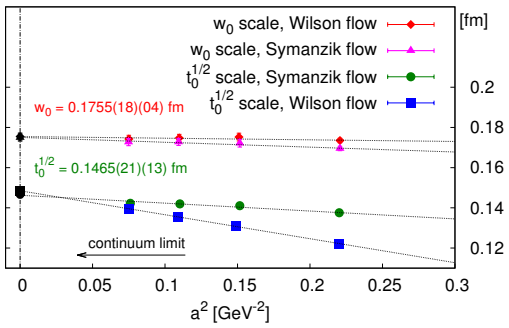

the physical scale was obtained by the Omega baryon mass our final result is the Wilson result (staggered is a cross check) (no rooting $\Longrightarrow$ theoretically cleaner)

$$
w_{0}=0.1755(18)(04) \mathrm{fm}
$$

error (dominantly statistical) is $1 \%$ (and comes not from the gauge flow itself, but from $M_{\Omega}$ ) 


\section{Scale off the physical point}

usually runs aren't at physical masses: what is the scale there measure $M_{\pi}, M_{K}$ and $w_{0}: x=w_{0}^{2} M_{\pi}^{2}$ and $y=w_{0}^{2}\left(M_{K}^{2}-M_{\pi}^{2} / 2\right)$ $w_{0}=0.18515-0.5885 x^{2}-0.0497 y-0.11 x y-1.476 x^{3} \pm 18 \cdot 10^{-3} \pm 4 \cdot 10^{-3}[\mathrm{fm}]$

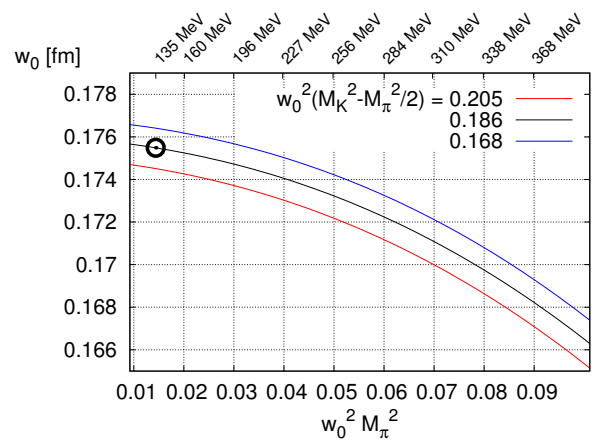

change $M_{\pi}$ from 135 to $350 \mathrm{MeV}$ $4 \%$ change in the lattice spacing (same size as cutoff effects) change $m_{s}$ by $10 \%$ $0.5 \%$ change in the lattice spacing error is $1 \%$ in the continuum limit 


\section{Error analysis: $2 \mathrm{HEX}$ data set}

histogram method to give statistical and systematic errors 64 possible results ( $m_{q}$ interpolation, $M_{\pi}$ cut, $\mathrm{a} \rightarrow 0$, fit range, scale)

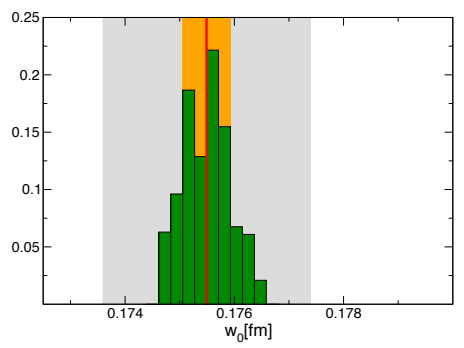

orange/gray bands: systematic/full error; red line: result

\begin{tabular}{|l|l|l|l|l|}
\hline interpolation & $M_{\pi}$-cut & $\mathrm{a} \rightarrow 0$ & fit range & scale \\
\hline $15 \%$ & $40 \%$ & $55 \%$ & $55 \%$ & $45 \%$ \\
\hline
\end{tabular}




\section{Outline}

(1) Scale setting

(2) Flow of the gauge field

(3) Pure gauge

(4) Full QCD 
Thermodynamic potentials from shifted boundary conditions

\section{Leonardo Giusti}

University of Milano-Bicocca

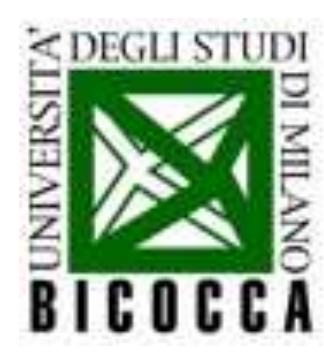

Based on: L. G. and H. B. Meyer PRL 106 (2011) 131601, arXiv:1110.3136 and in preparation 
Relation between the entropy density and the response of the system to the shift

$$
s=-\left.\frac{1}{T^{2}} \lim _{V \rightarrow \infty} \frac{1}{V} \frac{d^{2}}{d z^{2}} \ln Z(\{0,0, z\})\right|_{z=0}
$$

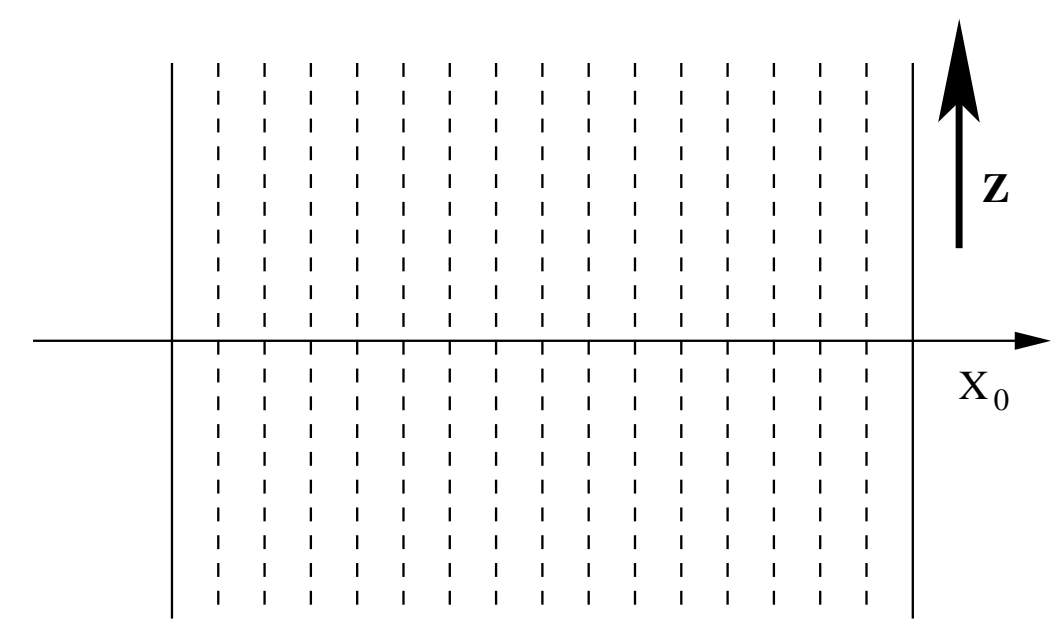

- Generalization to the specific heat

๑ Finite-size effects

- Extension to the lattice (and exploratory numerical study for $S U(3)$ Yang-Mills)

- Conclusions and outlook 
The relative contribution to the partition function of states with momentum $\mathbf{p}$ is $\left[T=1 / L_{0}\right]$

$$
\frac{R(\mathbf{p})}{V}=\frac{\operatorname{Tr}\left\{e^{-L_{0} \hat{\mathrm{H}}} \hat{\mathrm{P}}^{(\mathbf{p})}\right\}}{\operatorname{Tr}\left\{e^{-L_{0} \hat{\mathrm{H}}}\right\}}
$$

where

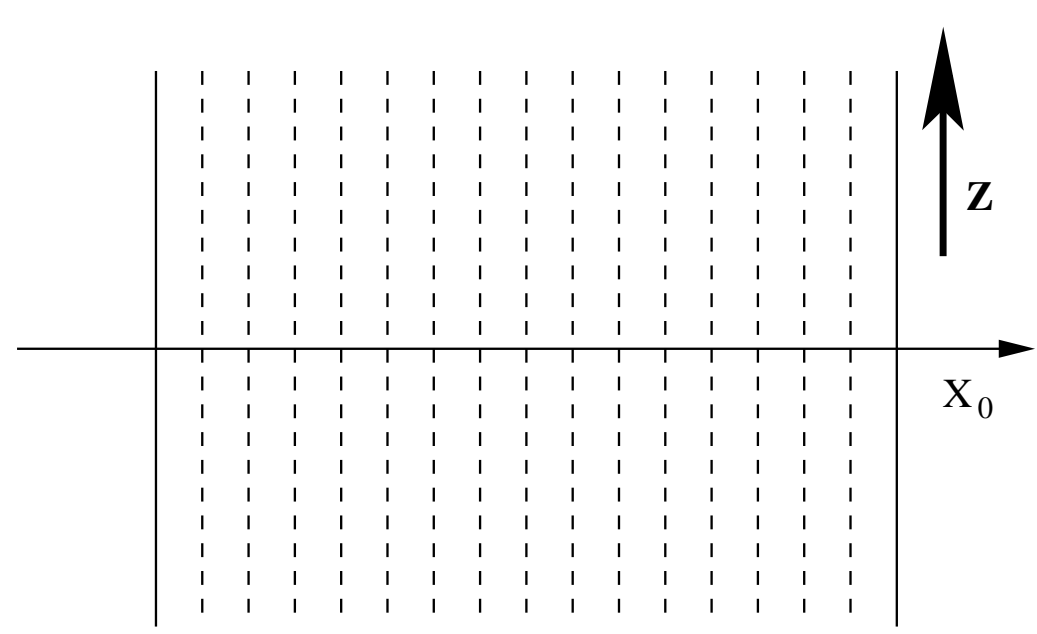

$$
\hat{\mathrm{P}}^{(\mathbf{p})}=\frac{1}{V} \int d^{3} \mathbf{z} e^{-i \mathbf{p} \cdot \mathbf{z}} e^{i \hat{\mathbf{p}} \cdot \mathbf{z}}, \quad e^{i \hat{\mathbf{p}} \mathbf{z}}|\phi\rangle=\left|\phi^{\mathbf{z}}\right\rangle
$$

- The momentum distribution can then be written as

$$
\frac{R(\mathbf{p})}{V}=\frac{1}{V} \int d^{3} \mathbf{z} e^{-i \mathbf{p} \cdot \mathbf{z}} \frac{Z(\mathbf{z})}{Z}, \quad \phi\left(L_{0}, \mathbf{x}\right)=\phi(0, \mathbf{x}+\mathbf{z})
$$

where $Z(\mathbf{z})$ is the usual path integral but with shifted boundary conditions in time direction 
- As usual the generator of its cumulants is defined as

$$
e^{-K(\mathbf{z})}=\frac{1}{V} \sum_{\mathbf{p}} e^{i \mathbf{p} \cdot z} R(\mathbf{p}) \quad \Longrightarrow \quad e^{-K(\mathbf{z})}=\frac{Z(\mathbf{z})}{Z}
$$

- The momentum cumulants can then be written as

$$
\frac{\left\langle\hat{p}_{1}^{2 n_{1}} \hat{p}_{2}^{2 n_{2}} \hat{p}_{3}^{2 n_{3}}\right\rangle_{c}}{V}=\frac{(-1)^{n_{1}+n_{2}+n_{3}}}{V} \frac{\partial^{2 n_{1}}}{\partial z_{1}^{2 n_{1}}} \frac{\partial^{2 n_{2}}}{\partial z_{2}^{2 n_{2}}} \frac{\partial^{2 n_{3}}}{\partial z_{3}^{2 n_{3}}} \ln \left[\frac{Z(\mathbf{z})}{Z}\right]_{\mathbf{z}=\mathbf{0}}
$$

In the continuum they equal the standard definition

$$
\left\langle\hat{p}^{2 n_{1}} \hat{p}^{2 n_{2}} \hat{p}^{\left.2 n_{3}\right\rangle_{c}}=(-1)^{n_{1}+n_{2}+n_{3}}\left\langle\bar{T}_{01} \cdots \bar{T}_{03}\right\rangle_{c}, \quad \bar{T}_{0 k}\left(x_{0}\right)=\int d^{3} x T_{0 k}(x)\right.
$$

and, being conn. corr. functions of the momentum charge, they are finite as they stand. The generator $K(\mathbf{z})$ and the distribution $R(\mathbf{p})$ are thus expected to be finite as well 
- On the lattice a theory is invariant under a discrete group of translations only. It is still possible, however, to factorize the Hilbert space in sectors with definite conserved total momentum

The momentum distribution is given by

$$
\frac{\mathcal{R}(\mathbf{p})}{V}=\frac{a^{3}}{V} \sum_{\mathbf{z}} e^{-i \mathbf{p} \cdot \mathbf{z}} \frac{\mathcal{Z}(\mathbf{z})}{\mathcal{Z}}
$$

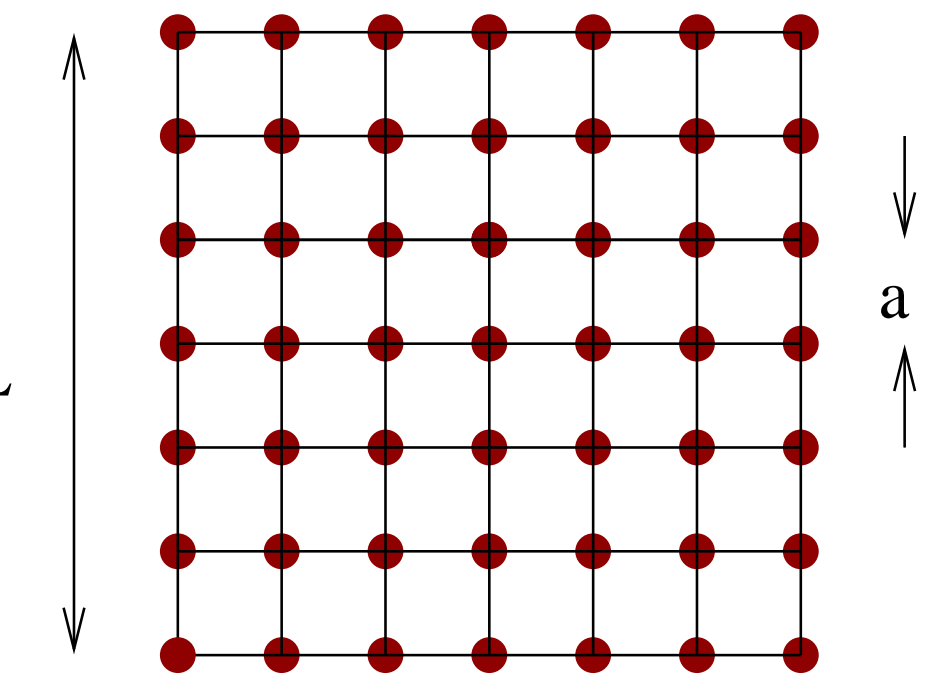

where $\mathcal{Z}(\mathbf{z})$ is the usual PI but with (discrete) shifted boundary conditions.

- Since only physical states contribuite to it, $\mathcal{R}(\mathbf{p})$ is expected to converge to the continuum universal value without need for UV renormalization

- The shifted boundary conditions allow us to define connected correlation functions of the momentum which do not require any UV renormalization

- Their continuum limit satisfies the standard EMT WIs, which can be used to interpret the cumulants in terms of basic thermodynamic potentials 
In the continuum a judicious combination of Wls associated with translational invariance

$$
\partial_{\mu}\left\langle T_{\mu \nu}(x) O_{1} \ldots O_{n}\right\rangle=-\sum_{i=1}^{n}\left\langle O_{1} \ldots \delta_{x} O_{i} \ldots O_{n}\right\rangle
$$

leads to $\left(x_{0} \neq y_{0}, w_{k} \neq z_{k}\right)$

$$
L_{0}\left\langle\bar{T}_{0 k}\left(x_{0}\right) T_{0 k}(y)\right\rangle-L_{k}\left\langle\widetilde{T}_{0 k}\left(w_{k}\right) T_{0 k}(z)\right\rangle=\left\langle T_{00}\right\rangle-\left\langle T_{k k}\right\rangle
$$

where

$$
\bar{T}_{0 k}\left(x_{0}\right)=\int d^{3} x T_{0 k}(x), \quad \widetilde{T}_{0 k}\left(x_{k}\right)=\int\left[\prod_{\nu \neq k} d x_{\nu}\right] T_{0 k}(x)
$$

- Note that:

* All operators at non-zero distance

* Number of EMT on the two sides different

* Trace component of EMT does not contribute

* On the lattice it can be imposed to fix the renormalization of $T_{0 k}$ 
Ward identities for two-point correlators of $\bar{T}_{0 k}$ (II)

Ward

The commutator of boost with momentum

$$
\left[\hat{K}_{k}, \hat{p}_{k}\right]=i \hat{H}
$$

is expressed in the Euclidean by the WIs

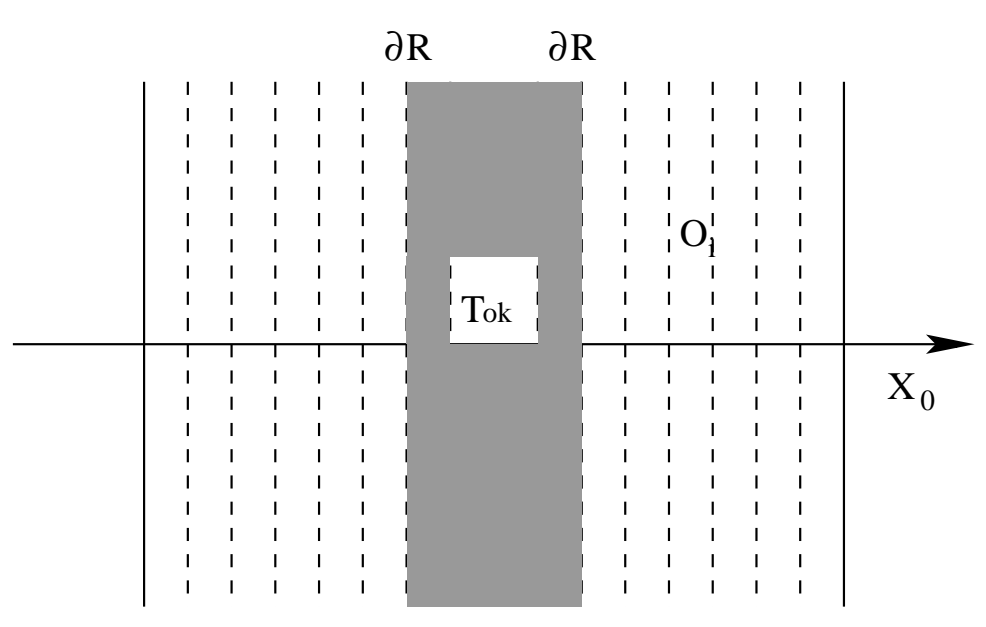

$$
\int_{\partial R} d \sigma_{\mu}(x)\left\langle K_{\mu ; 0 k}(x) \bar{T}_{0 k}\left(y_{0}\right) O_{1} \ldots O_{n}\right\rangle_{c}=\left\langle\bar{T}_{00}\left(y_{0}\right) O_{1} \ldots O_{n}\right\rangle_{c}
$$

when the $O_{i}$ are localized external fields.

- In a 4D box boost transformations are incompatible with (periodic) boundary conditions. Wls associated with $S O(4)$ rotations must be modified by finite-size contributions

- The finite-volume theory is translational invariant, and it has a conserved $T_{\mu \nu}$. Modified WIs associated to boosts constructed from those associated to translational invariance

$$
L_{0}\left\langle\bar{T}_{0 k}\left(x_{0}\right) T_{0 k}(y)\right\rangle-L_{k}\left\langle\widetilde{T}_{0 k}\left(w_{k}\right) T_{0 k}(z)\right\rangle=\left\langle T_{00}\right\rangle-\left\langle T_{k k}\right\rangle
$$


Ward identities for two-point correlators of $\bar{T}_{0 k}$ (III)

In the thermodynamic limit the WI reads $\left(y_{0} \neq x_{0}\right)$

$$
L_{0}\left\langle\bar{T}_{0 k}\left(x_{0}\right) T_{0 k}(y)\right\rangle=\left\langle T_{00}\right\rangle-\left\langle T_{k k}\right\rangle
$$

B By remembering that in the Euclidean

$$
\hat{p}_{k} \leftrightarrow-i \bar{T}_{0 k}, \quad e=-\left\langle T_{00}\right\rangle, \quad p=\left\langle T_{k k}\right\rangle \quad \Longrightarrow \quad \frac{\left\langle\hat{p}_{k}^{2}\right\rangle}{V}=T\{e+p\}=T^{2} s
$$

In a finite box and for $M \neq 0$ ( $M$ lightest screening mass)

$$
\left\langle\bar{T}_{0 k}\left(x_{0}\right) T_{0 k}(y)\right\rangle=-T\{e+p\}+\frac{\nu M T^{2}}{2 \pi L}\left[M+3 T \frac{\partial M}{\partial T}\right] e^{-M L}+\ldots
$$

i.e. leading finite-size effects are known functions of $M$, and are exponentially small in $M L$ 
By putting together the two formulas

$$
s=-\left.\frac{1}{T^{2}} \lim _{V \rightarrow \infty} \frac{1}{V} \frac{d^{2}}{d z^{2}} \ln Z(\{0,0, z\})\right|_{z=0}
$$

- On the lattice the only difference is the discrete derivative

$$
s=-\frac{1}{T^{2}} \lim _{V \rightarrow \infty} \lim _{a \rightarrow 0} \frac{2}{n_{z}^{2} a^{2} V} \ln \left[\frac{\mathcal{Z}\left(\left\{0,0, n_{z} a\right\}\right)}{\mathcal{Z}}\right]
$$

with $n_{z}$ being kept fixed when $a \rightarrow 0$

Note that:

* No ultraviolet renormalization

* Finite volume effects exponentially small

* Discretization effects $\mathcal{O}\left(a^{2}\right)$ once action improved 
- There is more information in $K(\mathbf{z})$. Again a judicious combination of Wls leads to

$$
\left\langle\bar{T}_{0 k}\left(x_{0}^{1}\right) \bar{T}_{0 k}\left(x_{0}^{2}\right) \ldots \bar{T}_{0 k}\left(x_{0}^{2 n}\right)\right\rangle_{c}=\left\langle\bar{T}_{00}\left(x_{0}^{1}\right) \bar{T}_{k k}\left(x_{0}^{2}\right) \ldots \bar{T}_{0 k}\left(x_{0}^{2 n}\right)\right\rangle_{c}+\text { f.s.c. }
$$

ـ At finite temperature and volume

$$
\begin{aligned}
L_{0}\left\langle\bar{T}_{00}\left(y_{0}\right) \bar{T}_{0 k}\left(x_{0}^{1}\right) \ldots \bar{T}_{0 k}\left(x_{0}^{2 n}\right)\right\rangle_{c} & =L_{0} \frac{\partial}{\partial L_{0}}\left\langle\bar{T}_{0 k}\left(x_{0}^{1}\right) \ldots \bar{T}_{0 k}\left(x_{0}^{2 n}\right)\right\rangle_{c} \\
L_{0}\left\langle\bar{T}_{k k}\left(y_{0}\right) \bar{T}_{0 k}\left(x_{0}^{1}\right) \ldots \bar{T}_{0 k}\left(x_{0}^{2 n}\right)\right\rangle_{c} & =\left\{L_{k} \frac{\partial}{\partial L_{k}}+2 n\right\}\left\langle\bar{T}_{0 k}\left(x_{0}^{1}\right) \ldots \bar{T}_{0 k}\left(x_{0}^{2 n}\right)\right\rangle_{c}
\end{aligned}
$$

By combining these relations, and by taking the infinite volume limit

$$
\left\langle\bar{T}_{0 k}\left(x_{0}^{1}\right) \ldots \bar{T}_{0 k}\left(x_{0}^{2 n}\right)\right\rangle_{c}=(2 n-1) \frac{\partial}{\partial L_{0}}\left\{\frac{1}{L_{0}}\left\langle\bar{T}_{0 k}\left(x_{0}^{1}\right) \ldots \bar{T}_{0 k}\left(x_{0}^{2 n-2}\right)\right\rangle_{c}\right\}
$$

- Expression of finite-size corrections similar to the one of two-point corr. functions 
- Written for cumulants the recursive relation reads

$$
\left\langle\hat{p}_{z}^{2 n}\right\rangle_{c}=(2 n-1) T^{2} \frac{\partial}{\partial T}\left\{T\left\langle\hat{p}_{z}^{2 n-2}\right\rangle_{c}\right\}
$$

and analogously for mixed ones

? The definition of the specific heat implies

$$
c_{v}=T \frac{\partial}{\partial T} s \quad \Longrightarrow \quad c_{v}=\frac{1}{V}\left[\frac{\left\langle\hat{p}_{z}^{4}\right\rangle_{c}}{3 T^{4}}-3 \frac{\left\langle\hat{p}_{z}^{2}\right\rangle_{c}}{T^{2}}\right]
$$

and therefore

$$
c_{v}=\left.\lim _{V \rightarrow \infty} \frac{1}{V}\left[\frac{1}{3 T^{4}} \frac{d^{4}}{d z^{4}}+\frac{3}{T^{2}} \frac{d^{2}}{d z^{2}}\right] \ln Z(\{0,0, z\})\right|_{z=0}
$$

- On the lattice the only difference are the discrete derivatives. Finite-size corrections are again known, and are exponentially suppressed 
- If $T$ is the only dimensionful parameter in the problem, the recursive relation implies

$$
\frac{\left\langle\hat{p}_{z}^{2 n}\right\rangle_{c}}{V}=c_{2 n} T^{2 n+3} \quad \Longrightarrow \quad c_{2 n}=\frac{(n+1)}{4}(2 n !) c_{2}
$$

By using the moment-cumulant transformation, the generator of the cumulants reads

$$
K(\{0,0, z\})=\sum_{n=1}^{\infty}(-1)^{n+1} \frac{\left\langle\hat{p}_{z}^{2 n}\right\rangle_{c}}{2 n !} z^{2 n}
$$

- The series can be re-summed to obtain

$$
\frac{K(\{0,0, z\})}{V}=\frac{s}{4}\left\{1-\frac{1}{\left(1+z^{2} T^{2}\right)^{2}}\right\}
$$

i.e. the entropy determines all the cumulants. The combination of scale and relativistic invariance fixes the functional form to be the one of the free case. 
The most straightforward way for computing the cumulant generator is to rewrite it as

$$
\frac{\mathcal{Z}(\mathbf{z})}{\mathcal{Z}}=\prod_{i=0}^{n-1} \frac{\mathcal{Z}\left(\mathbf{z}, r_{i}\right)}{\mathcal{Z}\left(\mathbf{z}, r_{i+1}\right)}
$$

where a set of $(n+1)$ systems is designed so that the relevant phase spaces of successive path integrals overlap and that $\mathcal{Z}\left(\mathbf{z}, r_{0}\right)=\mathcal{Z}(\mathbf{z})$ and

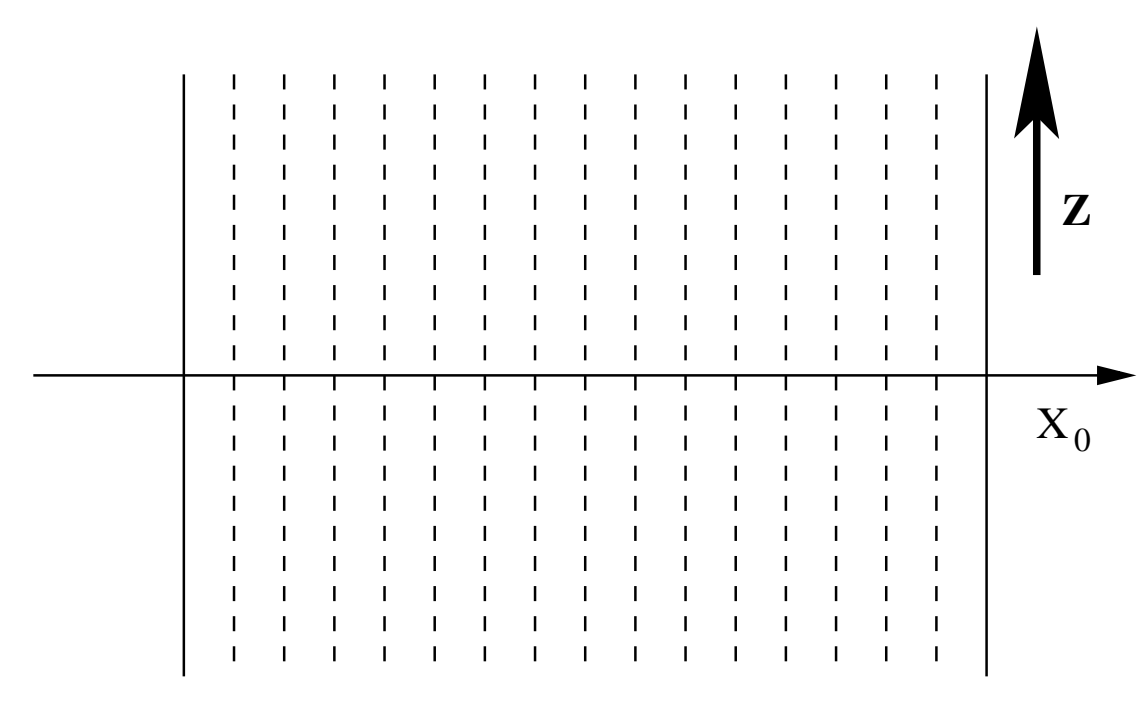
$\mathcal{Z}\left(\mathbf{z}, r_{n}\right)=\mathcal{Z}$

๑ The path integrals of the interpolating systems are defined as

$$
\mathcal{Z}(\mathbf{z}, r)=\int D U D U_{4, L_{0} / a-1} e^{-\bar{S}_{G}\left[U, U_{4}, r\right]}
$$

where $U_{4, L_{0} / a-1}$ is an extra $\left(5^{t h}\right)$ temporal link assigned to each point of last time-slice 
- The action of the interpolating systems is

$$
\bar{S}_{G}\left[U, U_{4}, r\right]=S_{G}[U]+\frac{\beta}{3}(1-r) \sum_{\mathbf{x}, k} \operatorname{Re} \operatorname{Tr}\left\{U_{0 k}\left(L_{0} / a-1, \mathbf{x}\right)-U_{4 k}\left(L_{0} / a-1, \mathbf{x}\right)\right\}
$$

with the extra space-time plaquette given by

$$
U_{4 k}\left(L_{0} / a-1, \mathbf{x}\right)=U_{4}\left(L_{0} / a-1, \mathbf{x}\right) U_{k}(0, \mathbf{x}+\mathbf{z}) U_{4}^{\dagger}\left(L_{0} / a-1, \mathbf{x}+\hat{k}\right) U_{k}^{\dagger}\left(L_{0} / a-1, \mathbf{x}\right)
$$

- If we define the "reweighting" observable as

$$
O\left[U, r_{i+1}\right]=e^{\bar{S}_{G}\left[U, U_{4}, r_{i+1}\right]-\bar{S}_{G}\left[U, U_{4}, r_{i}\right]}
$$

then

$$
\frac{\mathcal{Z}\left(\mathbf{z}, r_{i}\right)}{\mathcal{Z}\left(\mathbf{z}, r_{i+1}\right)}=\left\langle O\left[U, r_{i+1}\right]\right\rangle_{r_{i+1}}
$$


- The action of the interpolating systems is

$$
\bar{S}_{G}\left[U, U_{4}, r\right]=S_{G}[U]+\frac{\beta}{3}(1-r) \sum_{\mathbf{x}, k} \operatorname{Re} \operatorname{Tr}\left\{U_{0 k}\left(L_{0} / a-1, \mathbf{x}\right)-U_{4 k}\left(L_{0} / a-1, \mathbf{x}\right)\right\}
$$

with the extra space-time plaquette given by

$$
U_{4 k}\left(L_{0} / a-1, \mathbf{x}\right)=U_{4}\left(L_{0} / a-1, \mathbf{x}\right) U_{k}(0, \mathbf{x}+\mathbf{z}) U_{4}^{\dagger}\left(L_{0} / a-1, \mathbf{x}+\hat{k}\right) U_{k}^{\dagger}\left(L_{0} / a-1, \mathbf{x}\right)
$$

- On each lattice the entropy is finally given by

$$
s=-\frac{2}{\mathbf{z}^{2} T^{2} V} \sum_{i=0}^{n-1} \ln \left[\frac{\mathcal{Z}\left(\mathbf{z}, r_{i}\right)}{\mathcal{Z}\left(\mathbf{z}, r_{i+1}\right)}\right]
$$




\section{Numerical results for the entropy}

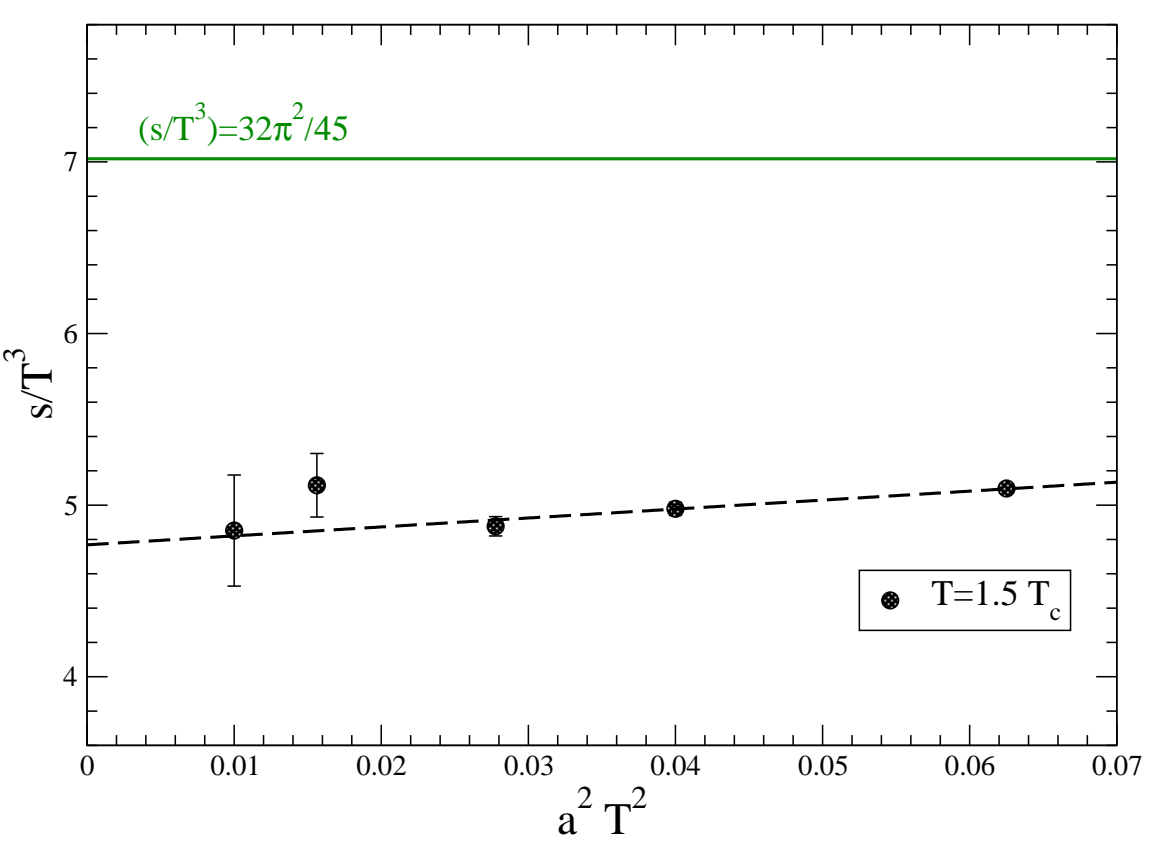

Lat $\quad 6 / g_{0}^{2} \quad L_{0} / a L / a \quad K(\mathbf{z}, a) \quad \frac{2 K(\mathbf{z}, a)}{|\mathbf{z}|^{2} T^{5} V}$

$\begin{array}{llllll}\mathrm{A}_{1} & 5.9 & 4 & 12 & 17.20(11) & 5.10(3)\end{array}$

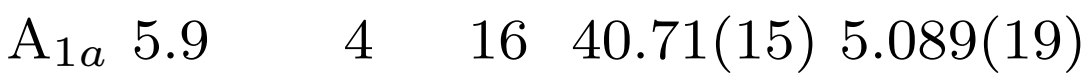

$\begin{array}{llllll}\mathrm{A}_{2} & 6.024 & 5 & 16 & 13.05(10) & 4.98(4)\end{array}$

$\begin{array}{llllll}\mathrm{A}_{3} & 6.137 & 6 & 18 & 7.32(8) & 4.88(6)\end{array}$

$\begin{array}{llllll}\mathrm{A}_{4} & 6.337 & 8 & 24 & 4.32(16) & 5.12(19)\end{array}$

$\begin{array}{llllll}\mathrm{A}_{5} & 6.507 & 10 & 30 & 2.62(17) & 4.9(3)\end{array}$ 


\section{Numerical results for the entropy}

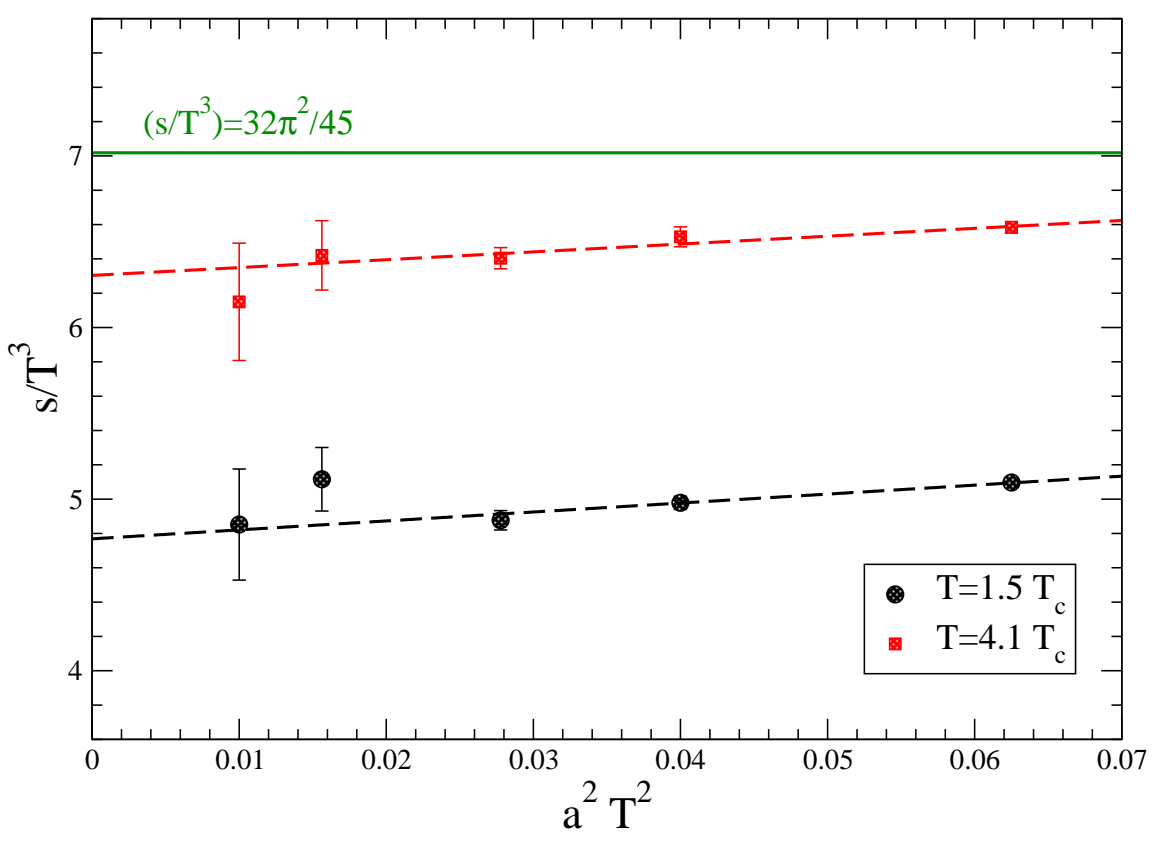

- A linear extrapolation in $a^{2}$ gives

$$
\begin{array}{rlrl}
\frac{s}{T^{3}} & =4.77 \pm 0.08 \pm ? ? & T & =1.5 T_{c} \\
\frac{s}{T^{3}} & =6.30 \pm 0.09 \pm ? ? & T & =4.1 T_{c}
\end{array}
$$

- Compatible with previous computations, but continuum extrapolation must be improved

[Boyd et al. 96; Namekawa et al. 01]
Lat $\quad 6 / g_{0}^{2} \quad L_{0} / a L / a \quad K(\mathbf{z}, a) \quad \frac{2 K(\mathbf{z}, a)}{|\mathbf{z}|^{2} T^{5} V}$

$\begin{array}{llllll}\mathrm{A}_{1} & 5.9 & 4 & 12 & 17.20(11) & 5.10(3)\end{array}$

$\begin{array}{lllll}\mathrm{A}_{1 a} & 5.9 \quad 4 \quad 16 & 40.71(15) & 5.089(19)\end{array}$

$\begin{array}{llllll}\mathrm{A}_{2} & 6.024 & 5 & 16 & 13.05(10) & 4.98(4)\end{array}$

$\begin{array}{llllll}\mathrm{A}_{3} & 6.137 & 6 & 18 & 7.32(8) & 4.88(6)\end{array}$

$\begin{array}{llllll}\mathrm{A}_{4} & 6.337 & 8 & 24 & 4.32(16) & 5.12(19)\end{array}$

$\begin{array}{llllll}\mathrm{A}_{5} & 6.507 & 10 & 30 & 2.62(17) & 4.9(3)\end{array}$

Lat $6 / g_{0}^{2} \quad L_{0} / a L / a \quad K(\mathbf{z}, a) \quad \frac{2 K(\mathbf{z}, a)}{|\mathbf{z}|^{2} T^{5} V}$

$\begin{array}{llllll}\mathrm{B}_{1} & 6.572 & 4 & 12 & 22.22(11) & 6.58(3)\end{array}$

$\mathrm{B}_{1 a} 6.572 \quad 4 \quad 16 \quad 53.47(16) 6.684(20)$

$\begin{array}{llllll}\mathrm{B}_{2} & 6.747 & 5 & 16 & 17.11(15) & 6.53(6)\end{array}$

$\begin{array}{llllll}\mathrm{B}_{3} & 6.883 & 6 & 18 & 9.61(9) & 6.40(6)\end{array}$

$\begin{array}{llllll}\mathrm{B}_{4} & 7.135 & 8 & 24 & 5.42(17) & 6.42(20)\end{array}$

$\begin{array}{llllll}\mathrm{B}_{5} & 7.325 & 10 & 30 & 3.32(18) & 6.1(3)\end{array}$ 


\section{Numerical results for the entropy}

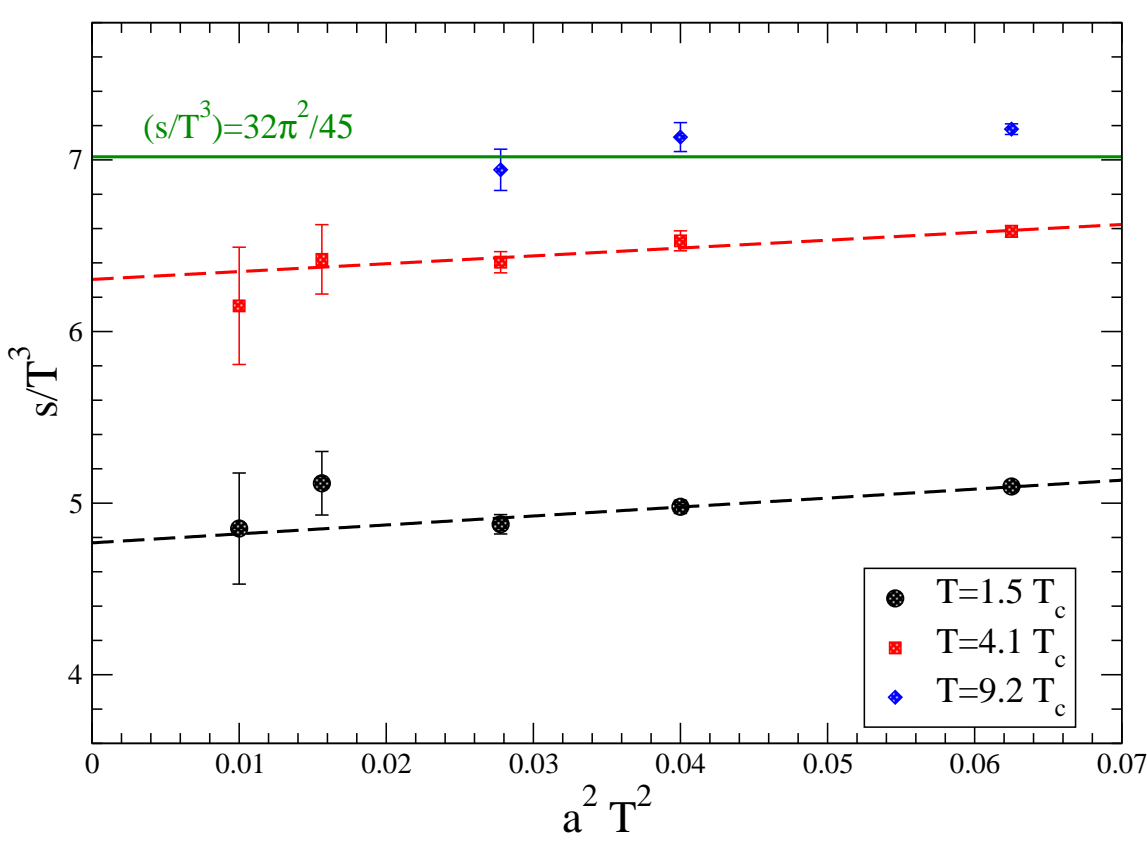

๑ A linear extrapolation in $a^{2}$ gives

$$
\begin{array}{rlrl}
\frac{s}{T^{3}} & =4.77 \pm 0.08 \pm ? ? & T & =1.5 T_{c} \\
\frac{s}{T^{3}} & =6.30 \pm 0.09 \pm ? ? & T & =4.1 T_{c}
\end{array}
$$

- Compatible with previous computations, but continuum extrapolation must be improved

[Boyd et al. 96; Namekawa et al. 01]
Lat $\quad 6 / g_{0}^{2} \quad L_{0} / a L / a \quad K(\mathbf{z}, a) \quad \frac{2 K(\mathbf{z}, a)}{|\mathbf{z}|^{2} T^{5} V}$

$\begin{array}{llllll}\mathrm{A}_{1} & 5.9 & 4 & 12 & 17.20(11) & 5.10(3)\end{array}$

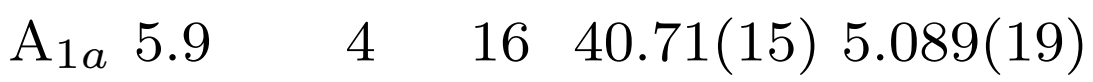

$\begin{array}{llllll}\mathrm{A}_{2} & 6.024 & 5 & 16 & 13.05(10) & 4.98(4)\end{array}$

$\begin{array}{llllll}\mathrm{A}_{3} & 6.137 & 6 & 18 & 7.32(8) & 4.88(6)\end{array}$

$\begin{array}{llllll}\mathrm{A}_{4} & 6.337 & 8 & 24 & 4.32(16) & 5.12(19)\end{array}$

$\begin{array}{llllll}\mathrm{A}_{5} & 6.507 & 10 & 30 & 2.62(17) & 4.9(3)\end{array}$

Lat $6 / g_{0}^{2} \quad L_{0} / a L / a \quad K(\mathbf{z}, a) \quad \frac{2 K(\mathbf{z}, a)}{|\mathbf{z}|^{2} T^{5} V^{3}}$

$\begin{array}{llllll}\mathrm{C}_{1} & 7.234 & 4 & 16 & 57.44(25) & 7.18(3)\end{array}$

$\begin{array}{llllll}\mathrm{C}_{2} & 7.426 & 5 & 20 & 36.5(4) & 7.13(8)\end{array}$

$\begin{array}{llllll}\mathrm{C}_{3} & 7.584 & 6 & 24 & 24.7(4) & 6.94(12)\end{array}$ 
- Correlation functions of total momentum fields can be related to derivatives of path integrals with shifted boundary conditions

- One of the applications is the computation of thermodynamic potentials, which can be connected to the cumulants via Ward identities of EMT. The entropy, for instance, is

$$
s=-\left.\frac{1}{T^{2}} \lim _{V \rightarrow \infty} \frac{1}{V} \frac{d^{2}}{d z^{2}} \ln Z(\{0,0, z\})\right|_{z=0}
$$

If lightest screening mass $M \neq 0$, leading finite-size corrections exponentially small in $M L$

- On the lattice these formulas apply once the derivative is discretized and the continuum limit is taken. No additive (vac. subtraction) or multiplicative UV renormalization is needed

- Same Wls allow for a non-perturbative renormalization of $T_{0 k}$

๑ Feasibility study very promising even with a very simple-minded (expensive) algorithm 


\title{
Padé approximants and g-2 for the muon
}

Christopher Aubin, Tom Blum, Maarten Golterman, Santi Peris

\author{
New Horizons for Lattice \\ Computations with Chiral Fermions \\ BNL, May 14-16, 2012
}


Contribution from lowest-order hadronic vacuum polarization (HLO)
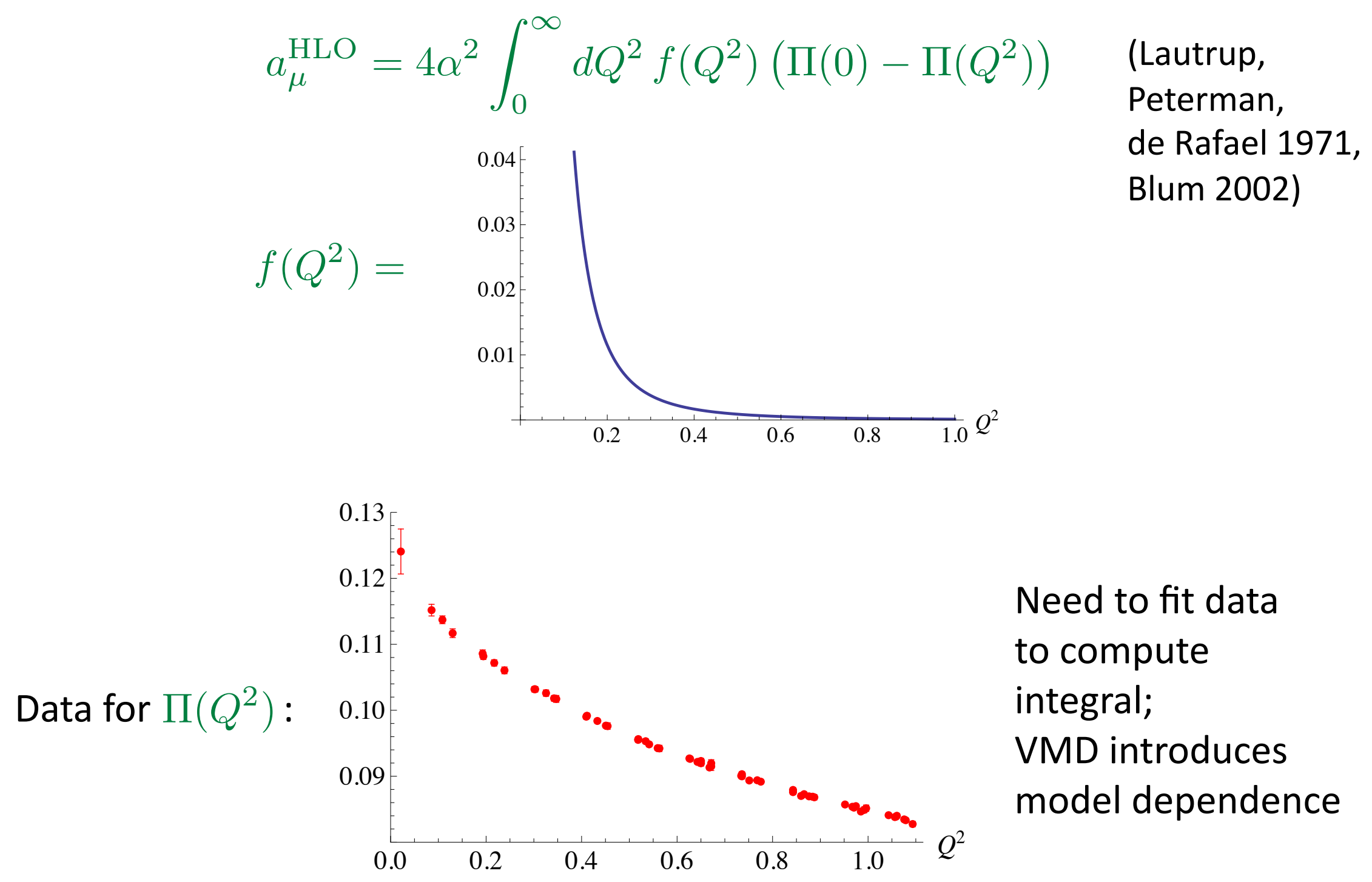
Multi-point Padé approximants:

Write $\quad\left(\Pi(0)-\Pi\left(Q^{2}\right)\right) / Q^{2}=\int_{4 m_{\pi}^{2}}^{\infty} d t \frac{\rho(t)}{t\left(t+Q^{2}\right)} \equiv \Phi\left(Q^{2}\right), \quad \rho(t) \geq 0$

This integral is a Stieltjes function, analytic everywhere except cut $\left(-\infty,-4 m_{\pi}^{2}\right]$.

Theorem: Given $P$ points $\left(Q_{i}^{2}, \Phi\left(Q_{i}^{2}\right)\right)$ a sequence of PAs can be constructed which converge to $\Phi\left(Q^{2}\right)$ on any closed, bounded region of the complex place excluding the cut, in the limit $P \rightarrow \infty$. (Baker 1969, Barnsley 1973)

Construction: $\Phi\left(Q^{2}\right)=\frac{\Psi_{0}}{1+\frac{\left(Q^{2}-Q_{1}^{2}\right) \Psi_{1}}{1+\cdot \frac{\left(Q^{2}-Q_{P-2}^{2}\right) \Psi_{P-2}}{1+\left(Q^{2}-Q_{P-1}^{2}\right) \Psi_{-1}}}}$

with $\Psi_{i}$ related to $\Phi\left(Q_{j \leq i+1}^{2}\right)$ ( $\Psi_{0}=\Phi\left(Q_{1}^{2}\right)$, etc.), yields a [[(P -1$\left.\left.) / 2\right],[P / 2]\right]$ PA. 


\section{Parametrization and strategy}

Furthermore, can prove that (Baker, Barnsley)

$$
\Pi\left(Q^{2}\right)=\Pi(0)-Q^{2}\left(a_{0}+\sum_{n=1}^{[P / 2]} \frac{a_{n}}{b_{n}+Q^{2}}\right)
$$

with $a_{n}>0$ (positive residues) and $b_{[P / 2]}>\cdots>b_{1}>4 m_{\pi}^{2}$ (all poles on cut), $a_{0}=0$ for $P$ even .

Fit this form for $P=2,3,4,5$; yields $[0,1],[1,1],[1,2],[2,2]$ PAs.

Compute $\quad a_{\mu}^{\mathrm{HLO}, Q^{2} \leq 1}=4 \alpha^{2} \int_{0}^{1 \mathrm{GeV}^{2}} d Q^{2} f\left(Q^{2}\right)\left(\Pi(0)-\Pi\left(Q^{2}\right)\right)$

Note: VMD is same as $[0,1]$ PA with $b_{1}=m_{\rho}^{2}$ fixed; NOT a (valid) PA! 
Test on MILC lattices with $a=0.09 \mathrm{fm}, m_{\pi}=480 \mathrm{MeV}$

\begin{tabular}{|c|c|c|c|c|c|}
\hline \multirow[b]{2}{*}{$\mathrm{PA}$} & \multirow[b]{2}{*}{ \# parameters } & \multicolumn{2}{|c|}{$\begin{array}{c}\text { correlated } \\
\text { interval } 0<Q^{2} \leq 0.6 \mathrm{GeV}^{2}\end{array}$} & \multicolumn{2}{|c|}{$\begin{array}{c}\text { uncorrelated } \\
\text { interval } 0<Q^{2} \leq 1 \mathrm{GeV}^{2}\end{array}$} \\
\hline & & $\chi^{2} /$ dof & $10^{10} a_{\mu}^{\mathrm{HLO}, Q^{2} \leq 1}$ & $\chi^{2} /$ dof & $10^{10} a_{\mu}^{\mathrm{HLO}, Q^{2} \leq 1}$ \\
\hline VMD & 2 & $5.86 / 3^{*}$ & $363(7)$ & $4.37 / 18$ & $413(8)$ \\
\hline$[0,1]$ & 3 & $11.4 / 8$ & $338(6)$ & $3.58 / 17$ & $373(37)$ \\
\hline$[1,1]$ & 4 & $7.49 / 7$ & $350(8)$ & $3.36 / 16$ & $424(116)$ \\
\hline$[1,2]$ & 5 & $7.49 / 6$ & $350(8)$ & $3.35 / 15$ & $443(293)$ \\
\hline$[2,2]$ & 6 & $7.49 / 5$ & $350(7)$ & $3.35 / 14$ & $445(432)$ \\
\hline
\end{tabular}

* interval $0<Q^{2} \leq 0.35 \mathrm{GeV}^{2}$

uncorrelated VMD fit agrees with Aubin and Blum, 2007

- Correlated: VMD bad, clear improvement with addition of parameters

- Difficult to determine $2^{\text {nd }}$ pole, but $a_{\mu}$ insensitive to higher poles

- Internal consistency, except uncorr. VMD (unknown systematic error!) and correlated PAs 

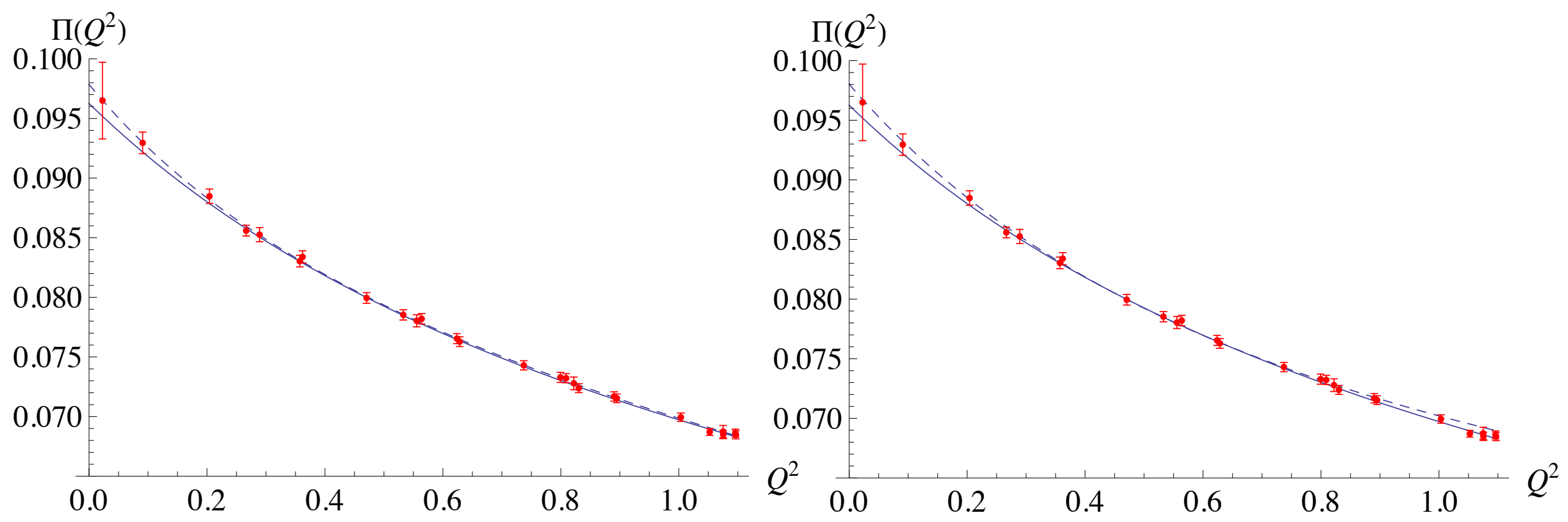

$[1,1]$ corr. (solid) and uncorr. (dashed) $\quad[1,1]$ corr. (solid) and VMD uncorr. (dashed)

- uncorrelated fits look better at small $Q^{2}$

- also considered MILC lattices with $a=0.06 \mathrm{fm}, m_{\pi}=220 \mathrm{MeV}$ - similar $a_{\mu}^{\mathrm{HLO}, Q^{2} \leq 1}=572(41) \times 10^{10}[1,1]$ corr.,$a_{\mu}^{\mathrm{HLO}, Q^{2} \leq 1}=646(8) \times 10^{10}$ VMD uncorr.

- not possible to decide which fit is best, based on current data 


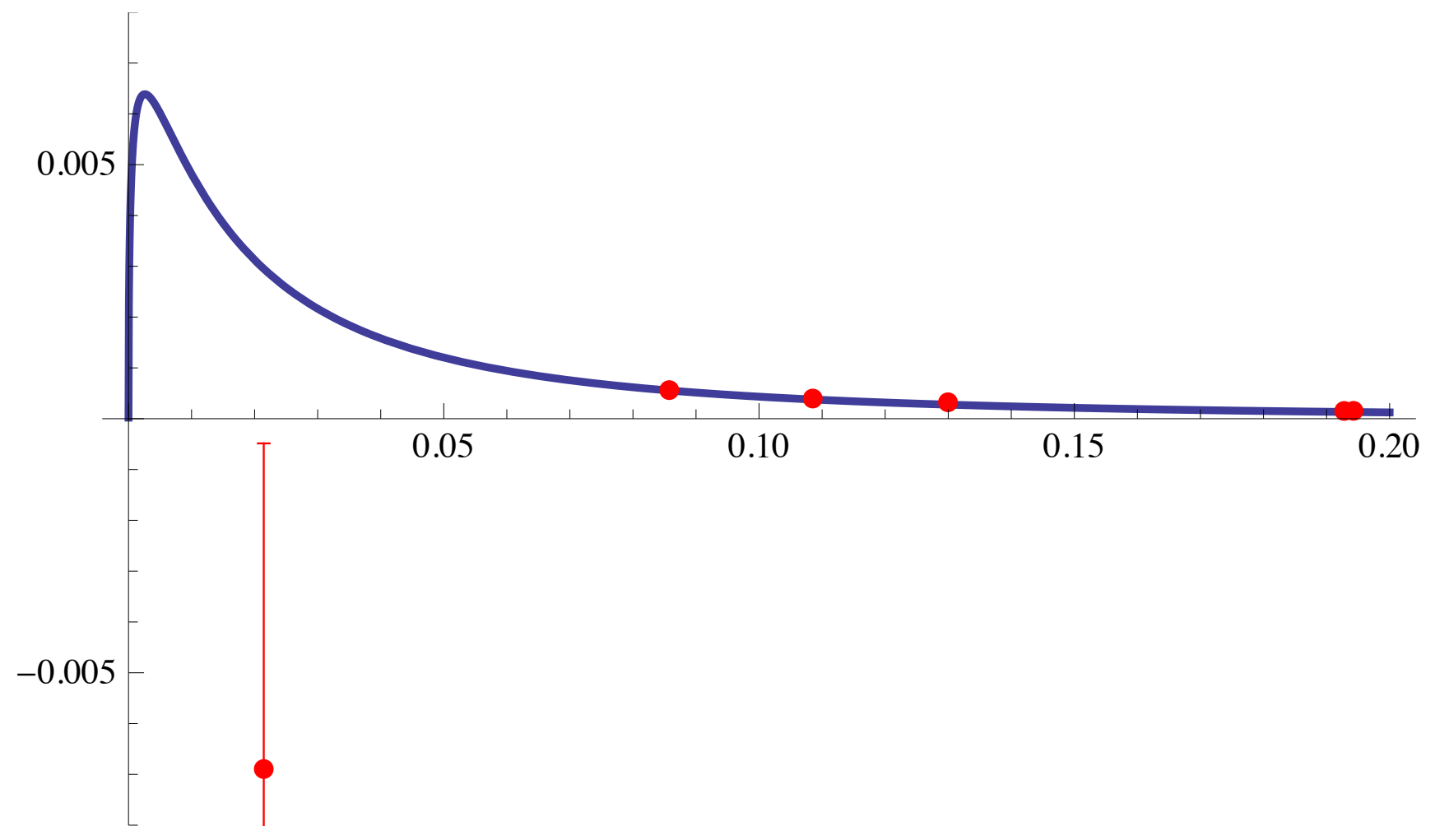

Integrand of $a_{\mu}^{\mathrm{HLO}} /\left(4 \alpha^{2}\right)$ compared with data (MILC, $a=0.06 \mathrm{fm}, m_{\pi}=220 \mathrm{MeV}$ )

$\Rightarrow$ need more data at low $Q^{2}$ with smaller errors! In progress... 


\section{Conclusions}

- New method to parametrize hadronic vacuum polarization; avoid model dependence of vector meson dominance. Based on representation of vacuum polarization in terms of Stieltjes function.

- Tested on two examples of numerical data for vacuum polarization. Padé approximant fits can lead to larger statistical errors, but avoid unknown systematic error associated with VMD.

- Method looks promising, but data at lower momenta and smaller errors are indispensible (difference between $a_{\mu}(V M D)$ and $a_{\mu}([1,1])$ is about $17 \%$ ).

- Note: long chiral extrapolation - also need data with small pion mass! 
Backup slide 1: comparison with polynomial fits

\begin{tabular}{|c|c|c|c|c|c|c|c|c|}
\hline & \multicolumn{2}{|c|}{ Poly 3} & \multicolumn{2}{c|}{ Poly 4} & \multicolumn{2}{c|}{ PA $[1,1]$} & \multicolumn{2}{c|}{ PA $[1,2]$} \\
\hline \hline \# points & $\chi^{2} /$ dof & $a_{\mu}^{(1)}$ & $\chi^{2} /$ dof & $a_{\mu}^{(1)}$ & $\chi^{2} /$ dof & $a_{\mu}^{(1)}$ & $\chi^{2} /$ dof & $a_{\mu}^{(1)}$ \\
\hline 16 & $9.6 / 12$ & 543 & $9.5 / 11$ & 483 & $9.7 / 12$ & 564 & $9.7 / 11$ & 565 \\
18 & $11.4 / 14$ & 526 & $10.5 / 13$ & 596 & $11.2 / 14$ & 541 & $11.5 / 13$ & 561 \\
20 & $13.1 / 16$ & 536 & $13.1 / 15$ & 535 & $13.9 / 16$ & 572 & $13.9 / 15$ & 572 \\
22 & $16.5 / 18$ & 541 & $15.9 / 17$ & 513 & $18.5 / 18$ & 566 & $18.5 / 17$ & 566 \\
24 & $16.6 / 20$ & 537 & $16.4 / 19$ & 521 & $19.4 / 20$ & 583 & $19.4 / 19$ & 583 \\
26 & $30.7 / 22$ & 505 & $23.6 / 21$ & 580 & $26.8 / 22$ & 557 & $26.7 / 21$ & 560 \\
\hline
\end{tabular}

- Poly 3, PA [1,1] and PA [1,2] correlated fits all good, not so Poly 4.

- Stability from PA $[1,1]$ to PA $[1,2]$, not from Poly 3 to Poly 4. 
Backup slide 2: chiral extrapolation

Assume VMD, and approximate $\Pi\left(Q^{2}\right)_{\text {subtr }}=g_{\rho}^{2} Q^{2} / m_{\rho}^{2}$ (continuum)

$$
\Pi\left(Q^{2}\right)_{\text {subtr }}=g_{V}^{2} Q^{2} / m_{V}^{2} \text { (lattice) }
$$

Define $I_{H}=\alpha^{2} \int_{0}^{\infty} \frac{d Q^{2}}{Q^{2}} w\left(\left(Q^{2} / m_{\mu}^{2}\right)\left(H_{\text {phys }}^{2} / H_{\text {latt }}^{2}\right)\right) \Pi\left(Q^{2}\right)_{\text {subtr }}$

(Feng et al. 2011)

Then $I_{H}=\left(\frac{H_{\text {latt }}}{H_{\text {phys }}}\right)^{2} \frac{g_{V}^{2}}{g_{\rho}^{2}} \frac{m_{\rho}^{2}}{m_{V}^{2}} I_{\text {model }}$ hence choose $H_{\text {latt }}=m_{V} / g_{V}$

Whatever choice: model dependent! $\quad 1^{\text {st }}$ PA pole not equal to $m_{V}^{2}$

Cannot avoid small pion masses (much smaller than $300 \mathrm{MeV}$ ) 


\title{
A novel phase in SU(3) gauge theory with many light fermions
}

\author{
Anna Hasenfratz \\ University of Colorado
}

New Horizons for Lattice Computations Workshop BNL

May 162012

In collaboration with A. Cheng, D. Schaich and G. Petropoulos ArXiv: 1111:2317 


\section{The many faces of gauge-fermion systems}

Renormalization group $\beta$ function at 2 loops

$$
\begin{aligned}
& \beta\left(g^{2}\right)=\frac{d g^{2}}{d \log \left(\mu^{2}\right)}=\frac{b_{1}}{16 \pi^{2}} g^{4}+\frac{b_{2}}{\left(16 \pi^{2}\right)^{2}} g^{6}+\ldots \quad b_{1}=-11+\frac{2}{3} N_{f}, \\
& b_{2}=-102+\frac{38}{3} N_{f}
\end{aligned}
$$
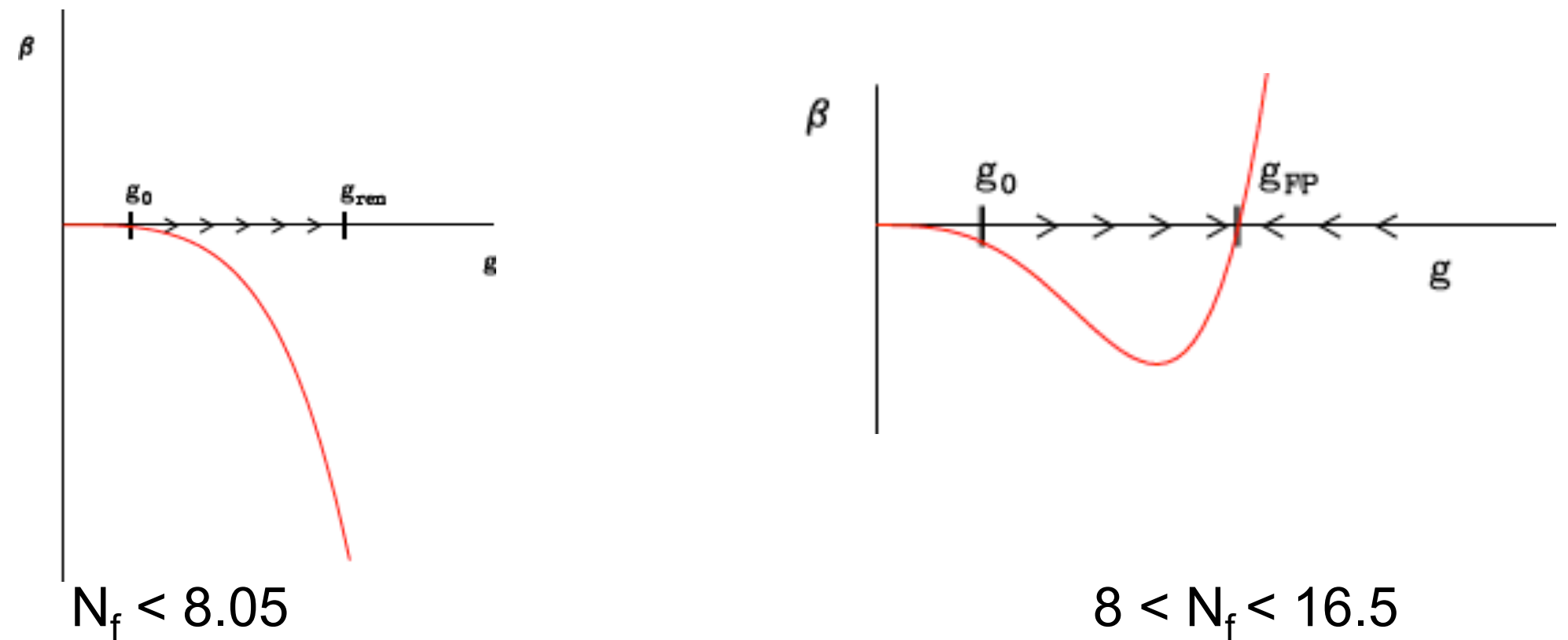

$8<N_{f}<16.5$ 


\section{The many faces of gauge-fermion systems}

Renormalization group $\beta$ function at 2 loops

$$
\begin{aligned}
& \beta\left(g^{2}\right)=\frac{d g^{2}}{d \log \left(\mu^{2}\right)}=\frac{b_{1}}{16 \pi^{2}} g^{4}+\frac{b_{2}}{\left(16 \pi^{2}\right)^{2}} g^{6}+\ldots \\
& b_{1}=-11+\frac{2}{3} N_{f}, \\
& b_{2}=-102+\frac{38}{3} N_{f}
\end{aligned}
$$

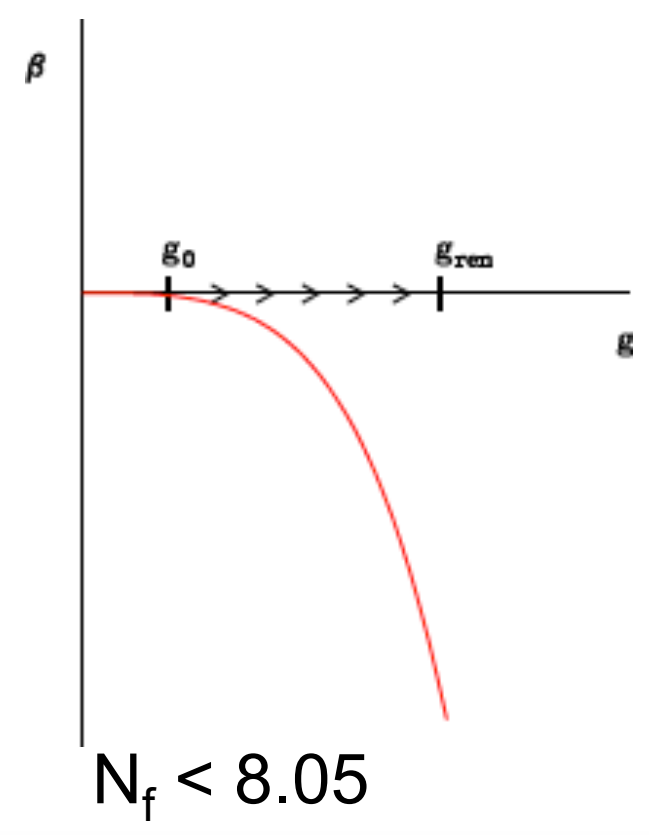

Chirally broken \& confining

- QCD with 2+1 (+1) flavors

- Original technicolor candidates 


\section{The many faces of gauge-fermion systems}

Renormalization group $\beta$ function at 2 loops

$$
\begin{aligned}
& \beta\left(g^{2}\right)=\frac{d g^{2}}{d \log \left(\mu^{2}\right)}=\frac{b_{1}}{16 \pi^{2}} g^{4}+\frac{b_{2}}{\left(16 \pi^{2}\right)^{2}} g^{6}+\ldots \quad b_{1}=-11+\frac{2}{3} N_{f}, \\
& b_{2}=-102+\frac{38}{3} N_{f}
\end{aligned}
$$

Conformal

- IRFP where $g_{0}$ is irrelevant

- universal anomalous dimension

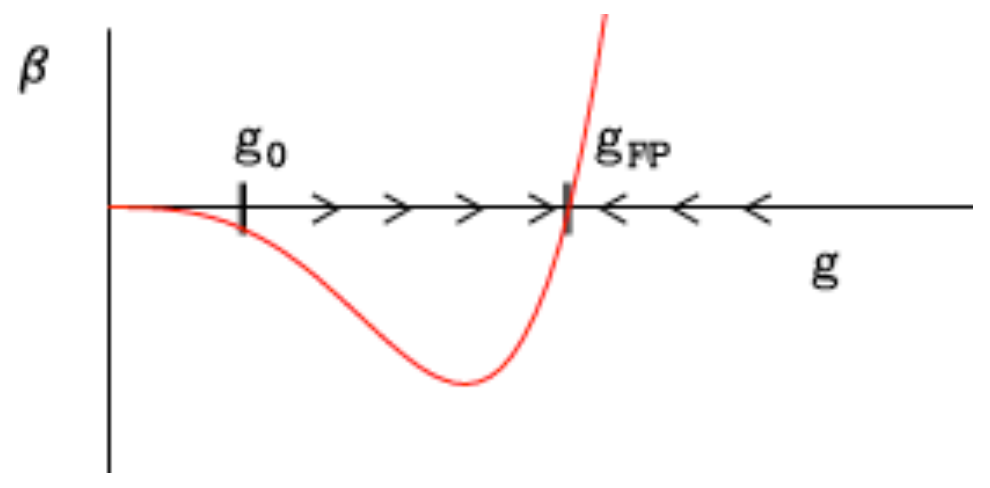

$$
8<N_{f}<16.5
$$




\section{The many faces of gauge-fermion systems}

Renormalization group $\beta$ function at 2 loops

$$
\begin{aligned}
& \beta\left(g^{2}\right)=\frac{d g^{2}}{d \log \left(\mu^{2}\right)}=\frac{b_{1}}{16 \pi^{2}} g^{4}+\frac{b_{2}}{\left(16 \pi^{2}\right)^{2}} g^{6}+\ldots \\
& b_{1}=-11+\frac{2}{3} N_{f}, \\
& b_{2}=-102+\frac{38}{3} N_{f}
\end{aligned}
$$

What we really want is walking

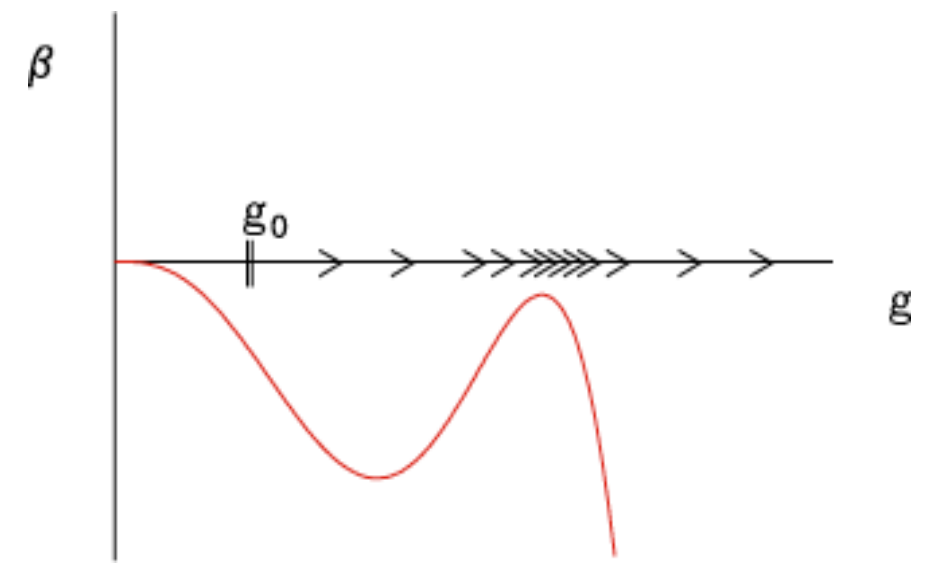

The gauge coupling changes slowly and the anomalous mass dimension remains large across an extended energy scale 


\section{Roadmap for the conformal window}

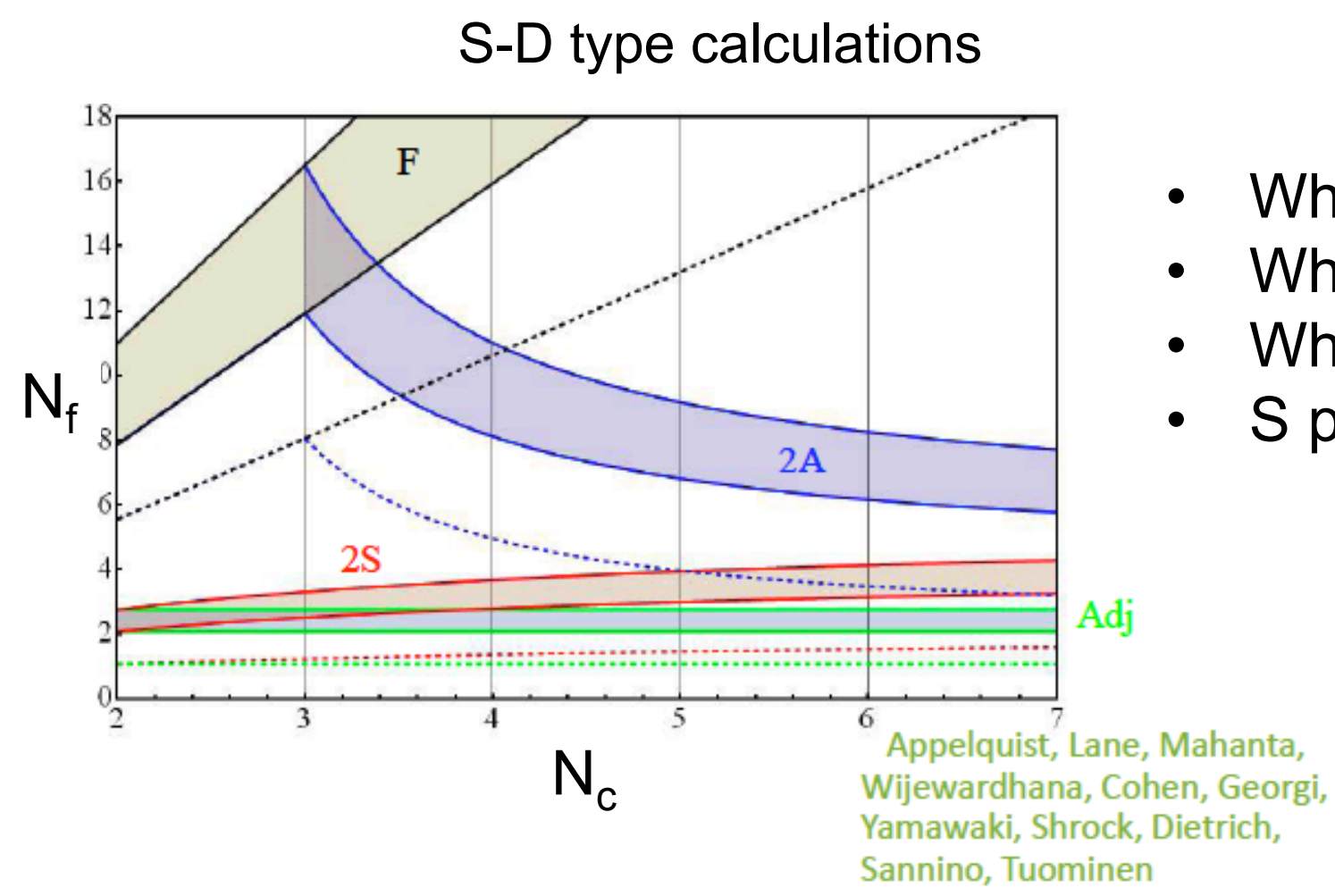




\section{Roadmap for the conformal window}

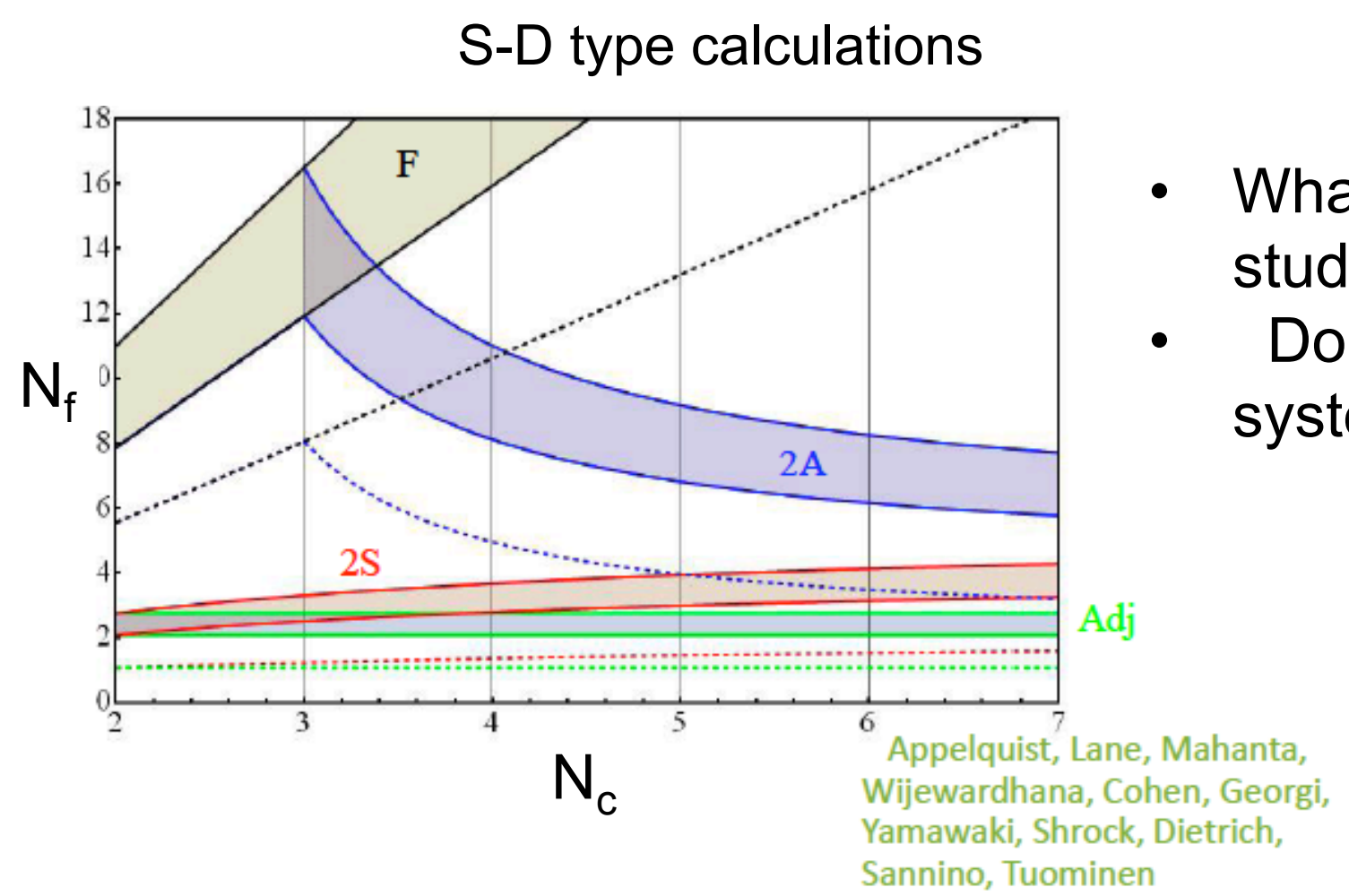

Don't be unpatient : QCD has a 25 year head start 
A phase with novel symmetry breaking pattern

Strongly coupled systems can lead to unexpected phenomena:

$\mathrm{N}_{\mathrm{f}}=12$ and 8 flavors $\mathrm{SU}(3)$ with staggered fermions

- 8 flavors: most likely chirally broken

- 12 flavors: still controversial

Goal: study the phase diagram both at zero and at finite temperature and contrast the two systems

(Lattice action: it's a good one) 
Why Finite temperature?

QCD like

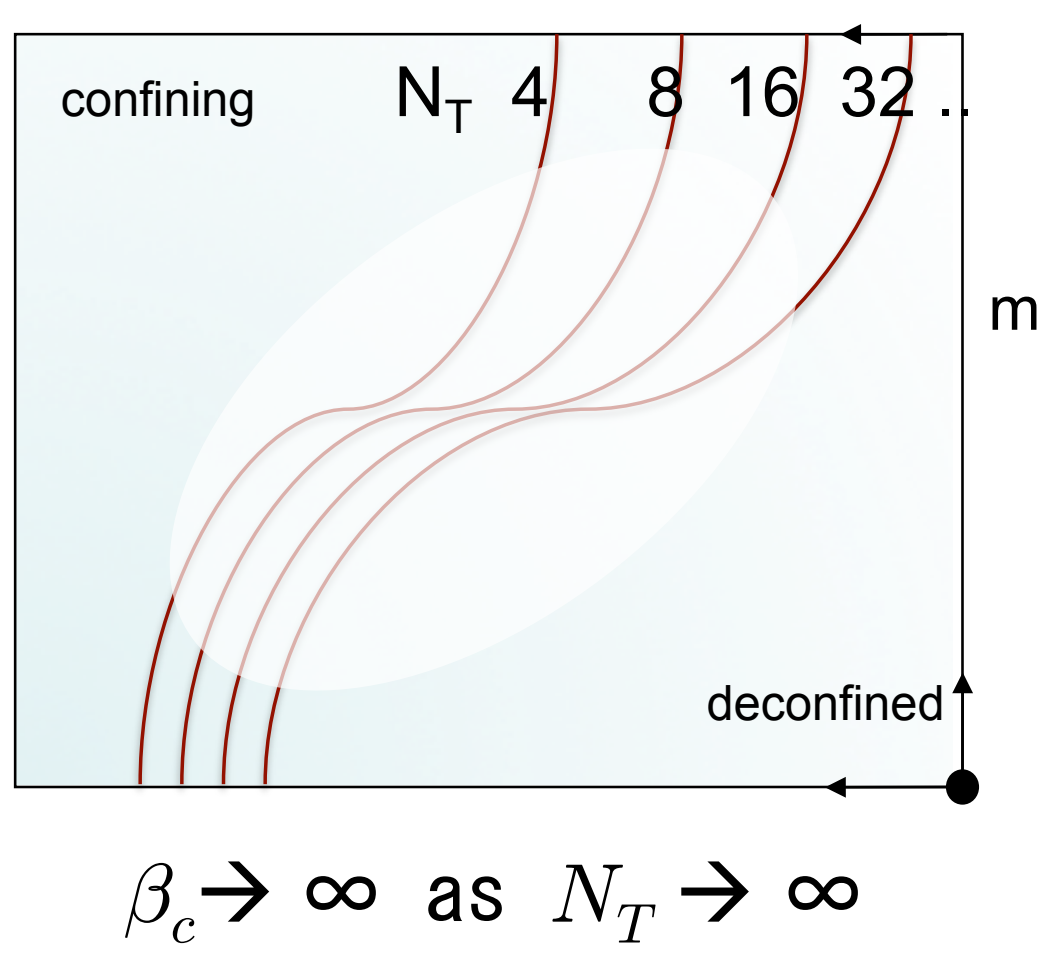

Conformal

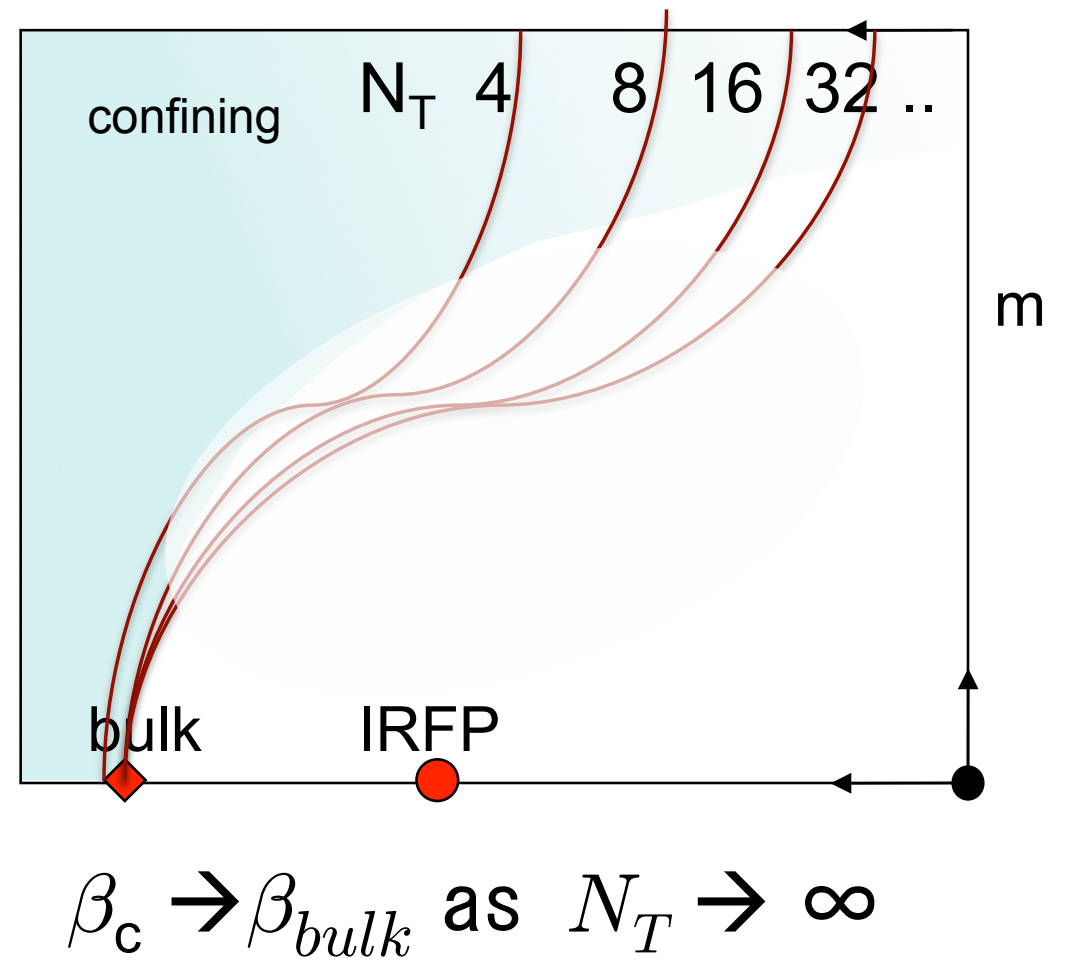

The scaling of $\beta_{c}$ is a good test of conformality (possibly) 


\section{The phase stucture of $\mathrm{N}_{\mathrm{f}}=12$}
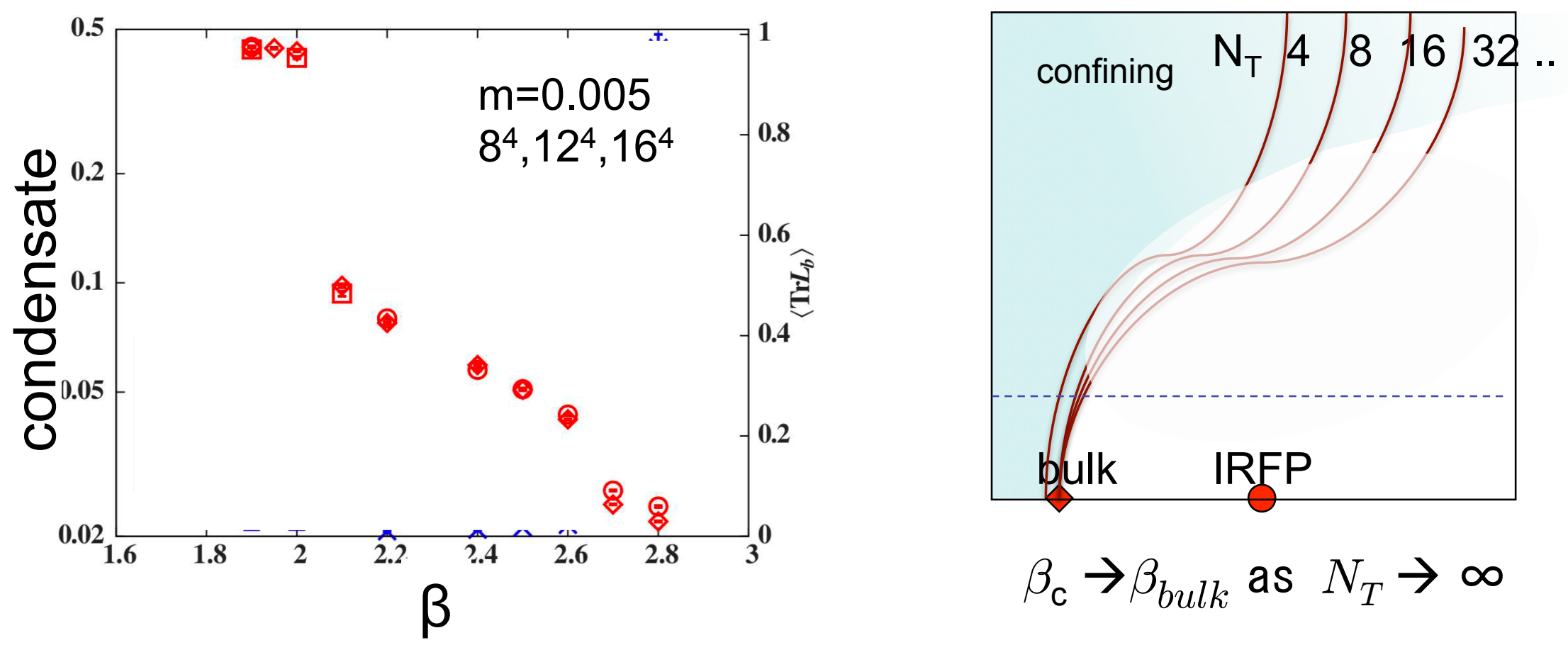


\section{The phase stucture of $\mathrm{N}_{\mathrm{f}}=12$}
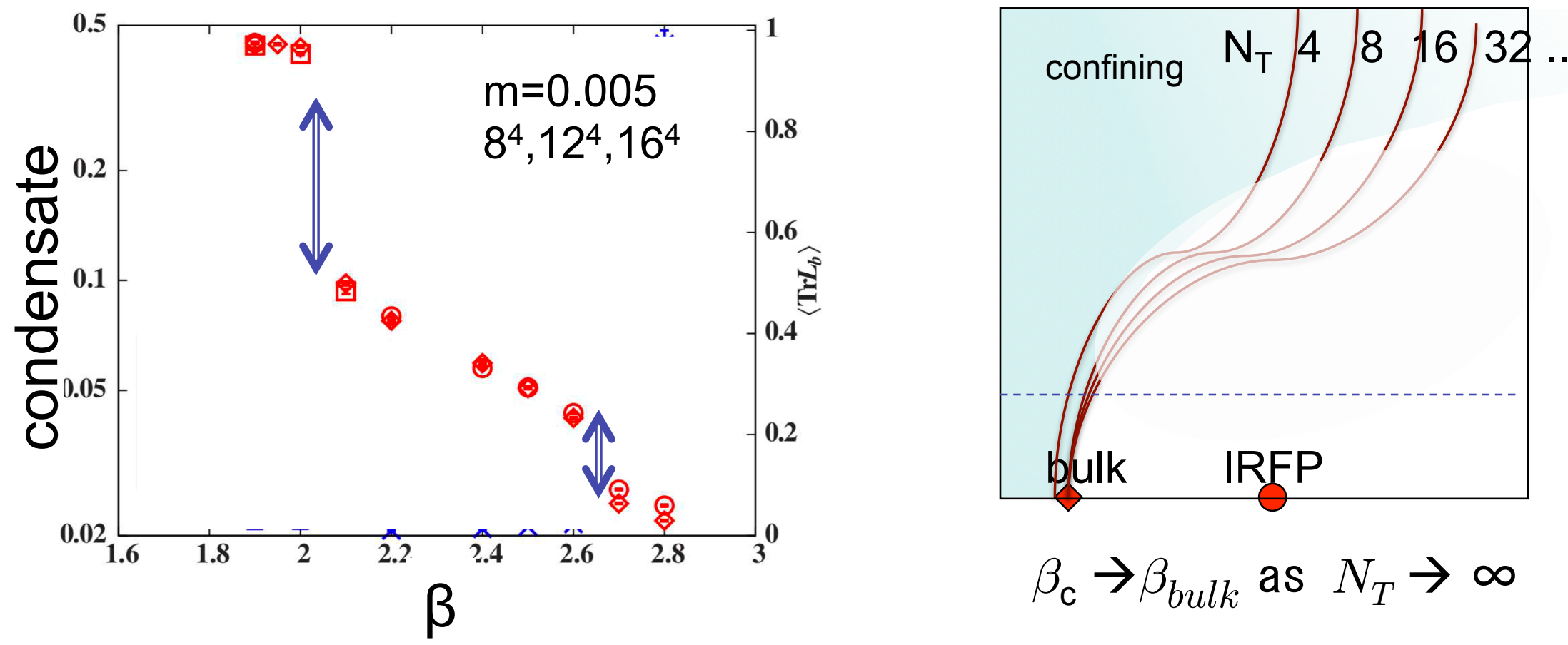

Similar transitions were observed previously by INFN/Groeningen group and LHC collaboration 


\section{Phase diagram $\beta$-m plane $N_{f}=12$}

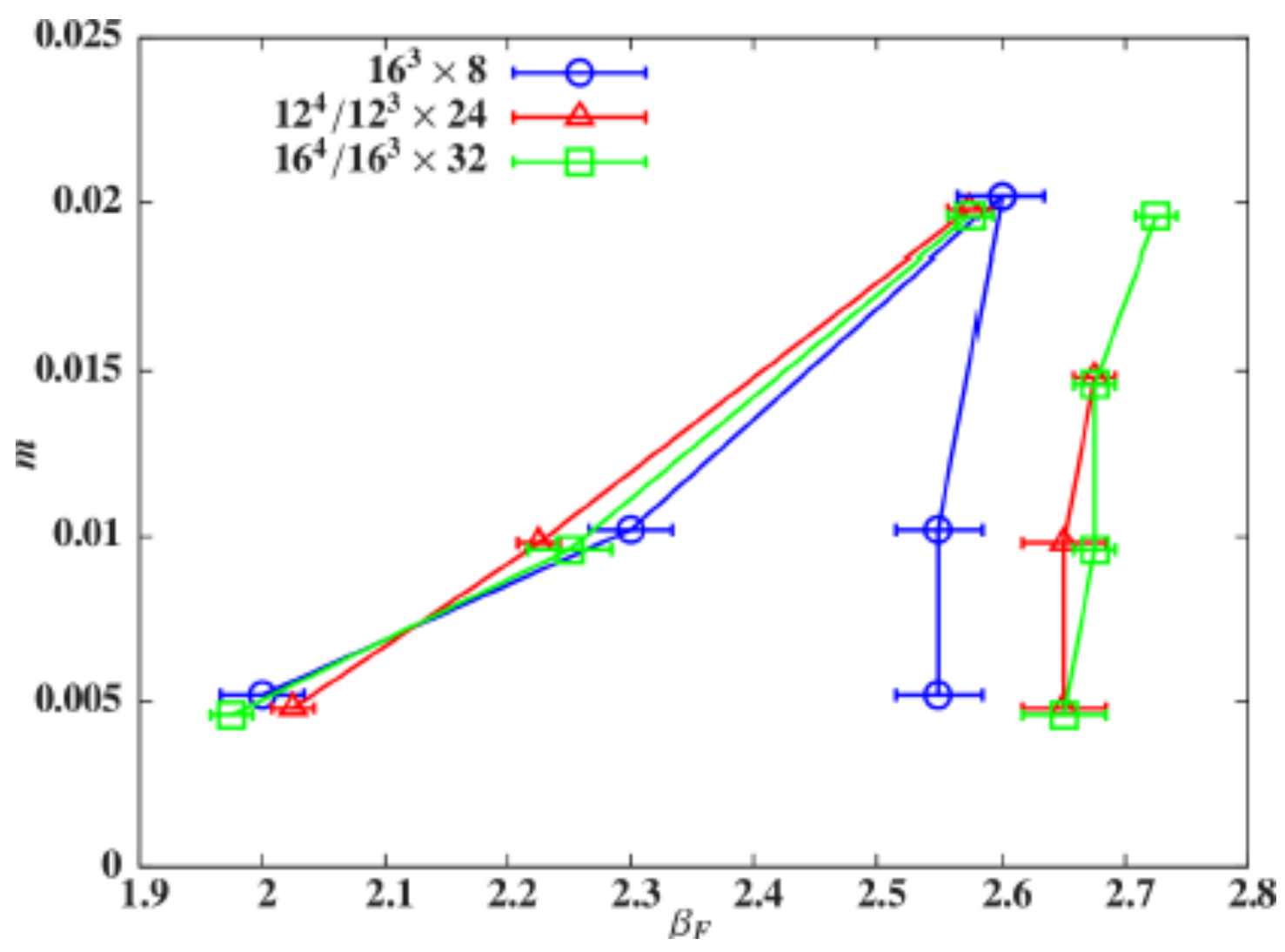

Intermediate phase bordered by bulk $1^{\text {st }}$ order transitions 


\section{Phase diagram $\beta$-m plane $N_{f}=12$}

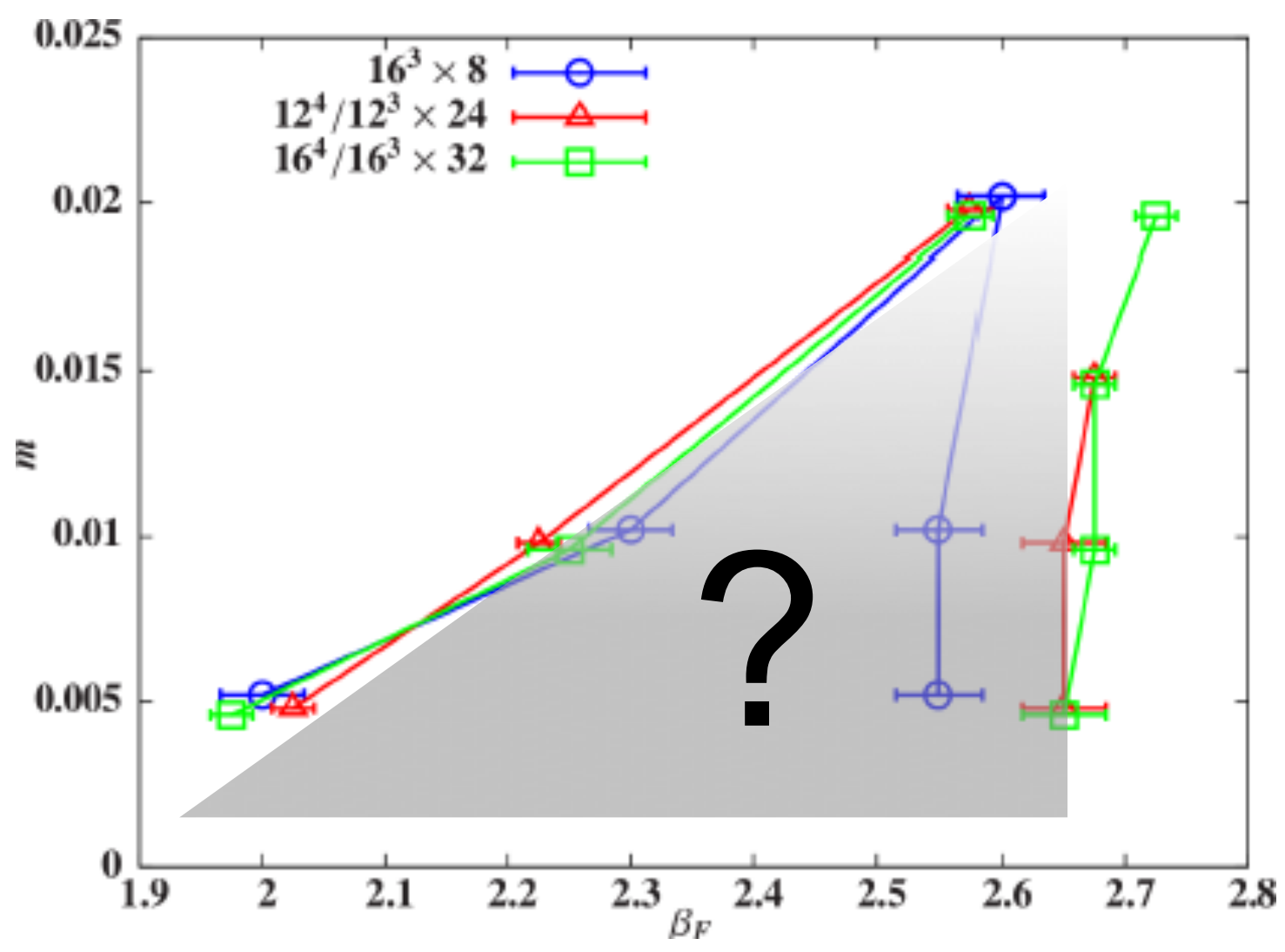

Intermediate phase bordered by bulk $1^{\text {st }}$ order transitions 
New symmetry breaking pattern in the IM phase

Single-site shift symmetry $\left(\mathrm{S}^{4}\right)$ of the staggered action

$$
\begin{aligned}
\chi(n) & \rightarrow \xi_{\mu}(n) \chi(n+\mu), \quad \xi_{\mu}(n)=(-1)^{\sum_{\nu>\mu} n_{\nu}} \\
U_{\mu}(n) & \rightarrow U_{\mu}(n+\mu),
\end{aligned}
$$

is broken $\rightarrow$ plaquette expectation value is "striped"
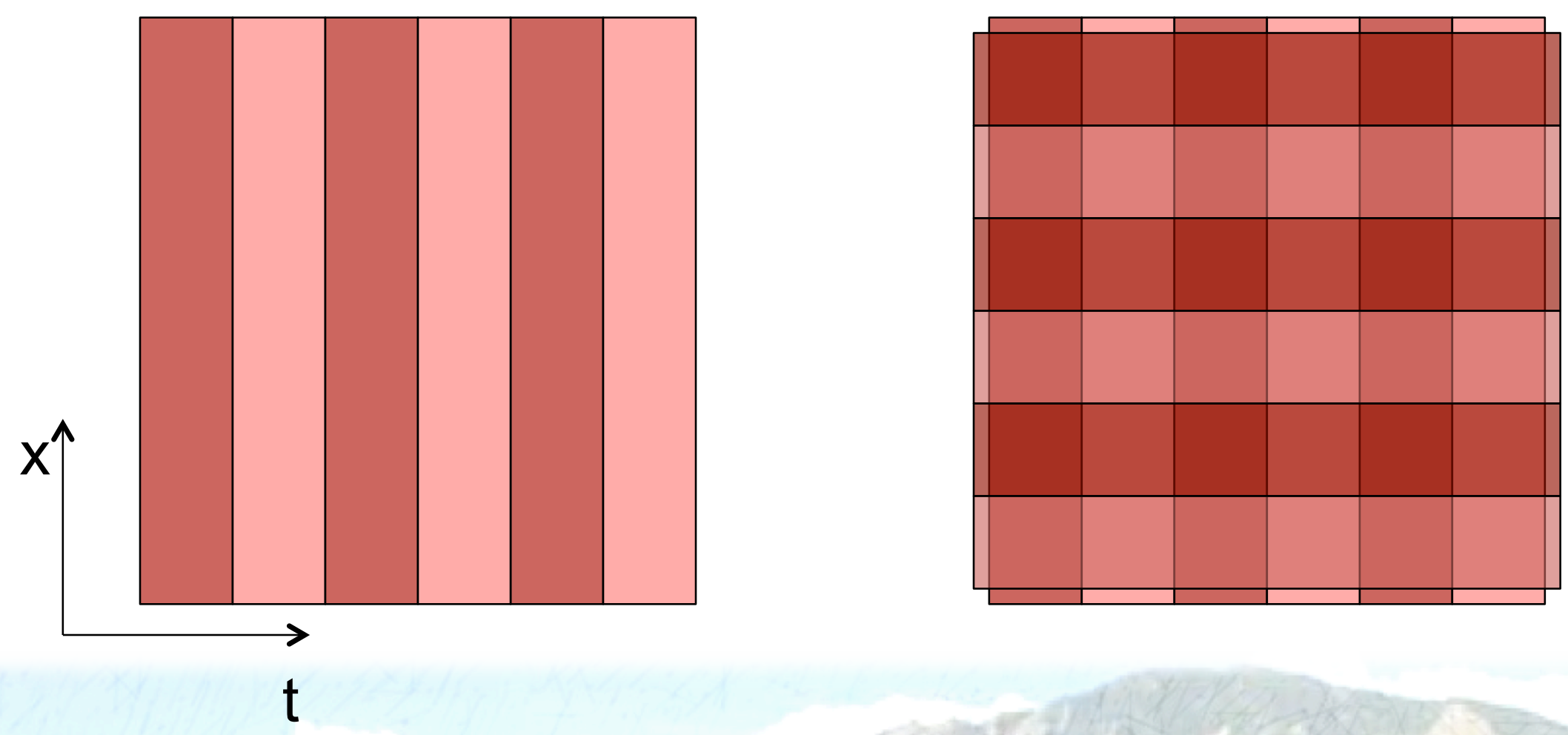


\section{New symmetry breaking pattern}

\section{Order parameter I:}

$$
\Delta P_{\mu}=\left\langle\operatorname{Re} \operatorname{Tr} \square_{n}-\operatorname{Re} \operatorname{Tr} \square_{n+\mu}\right\rangle_{n_{\mu} \text { even }}
$$

Plaquette on even \& odd time slices are different - this is on the background gauge configuration!

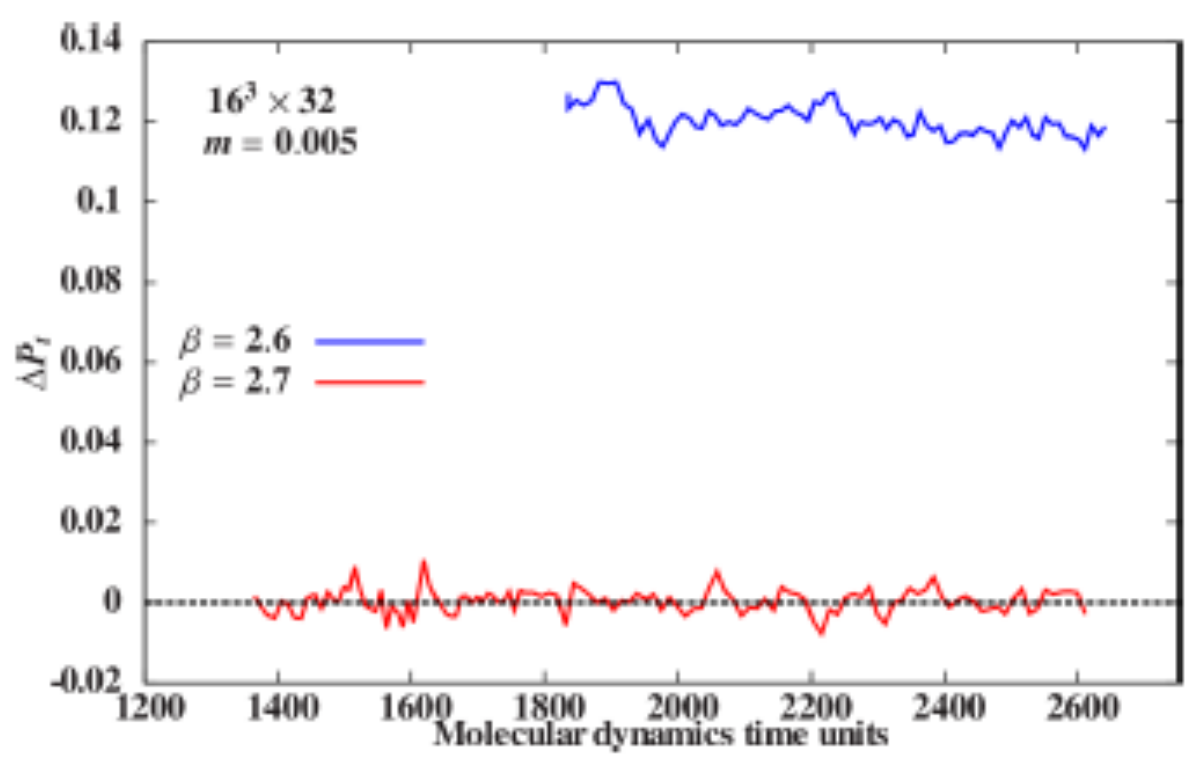

$\beta=2.6 \mathrm{IM}$ phase

$\beta=2.7$ weak coupling phase 


\section{New symmetry breaking pattern}

$\mathrm{S}^{4}$ breaking occurs at the fermionic level:

Order parameter -II: link difference

$$
\begin{aligned}
\Delta L_{\mu}= & \left\langle\alpha_{\mu}(n) \bar{\chi}(n) U_{\mu}(n) \chi(n+\mu)\right. \\
& \left.-\alpha_{\mu}(n+\mu) \bar{\chi}(n+\mu) U_{\mu}(n+\mu) \chi(n+2 \mu)\right\rangle_{n_{\mu} \mathrm{ev}}
\end{aligned}
$$
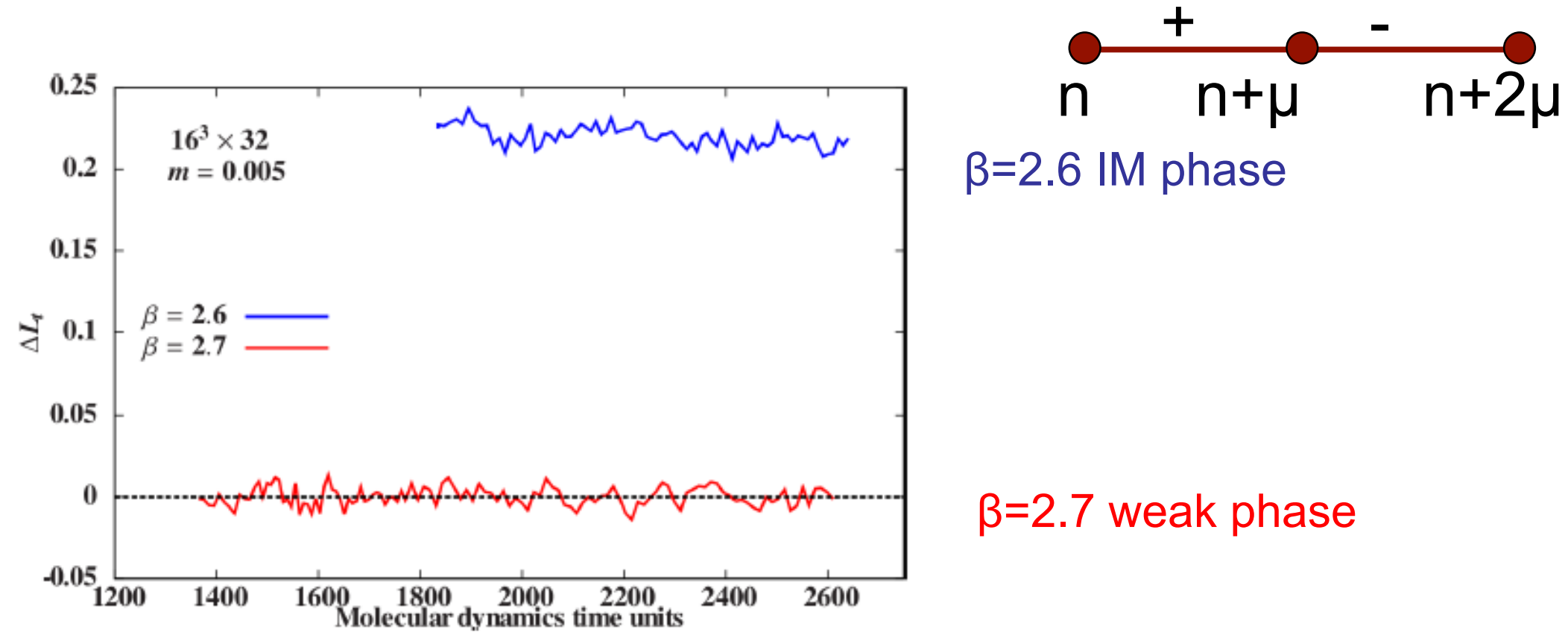

$\beta=2.6 \mathrm{IM}$ phase

$\beta=2.7$ weak phase 


\section{New symmetry breaking pattern}

- Single-site shift symmetry is exact in the action.

- Both $\Delta \mathrm{P}$ and $\Delta \mathrm{L}$ are order parameters of $\mathrm{S}^{4}$

- When $S^{4}$ is broken, the phase has to be separated by a "real" phase transition

- The $S^{4}$ broken $\left(g^{4}\right)$ phase cannot go away with the volume

- $S^{4}$ is related to taste so this could be a special taste breaking

What are the physical properties of the $S^{4} b$ phase? 


\section{The Polyakov line}

Is it deconfining? Polyakov line is very noisy but the blocked Poly line is sensitive:

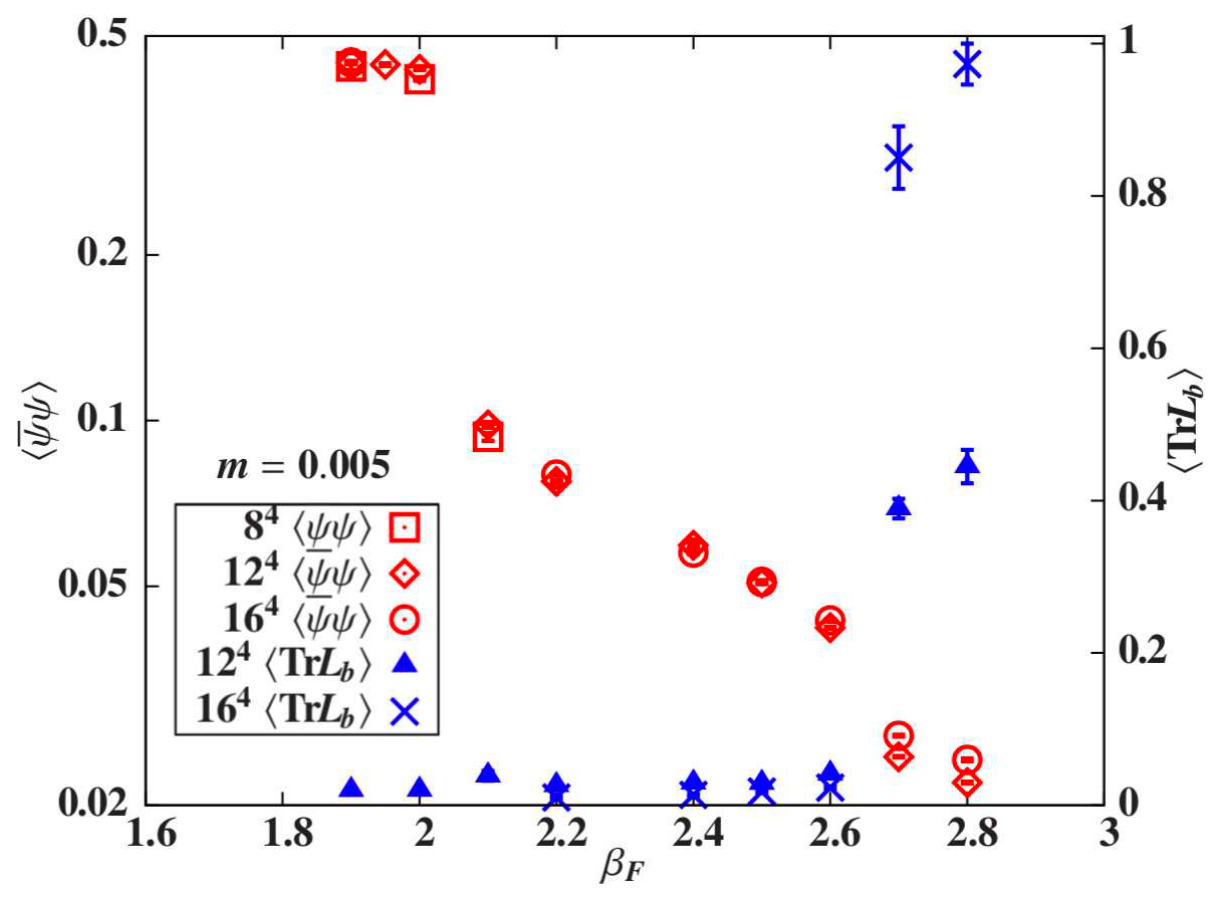

Blocked Poly line is measured on RG blocked lattices:

- improved Poly line

or

- Poly line on renormalized trajectory, after removing UV fluctuations 


\section{The static potential $\rightarrow$ Confinement!}

Static potential on $12^{3}, 16^{3}$ volumes (no volume dependence!) shows a linear term: $\quad r_{0}=2.1-2.7, \sqrt{ } \sigma=0.40--0.48$

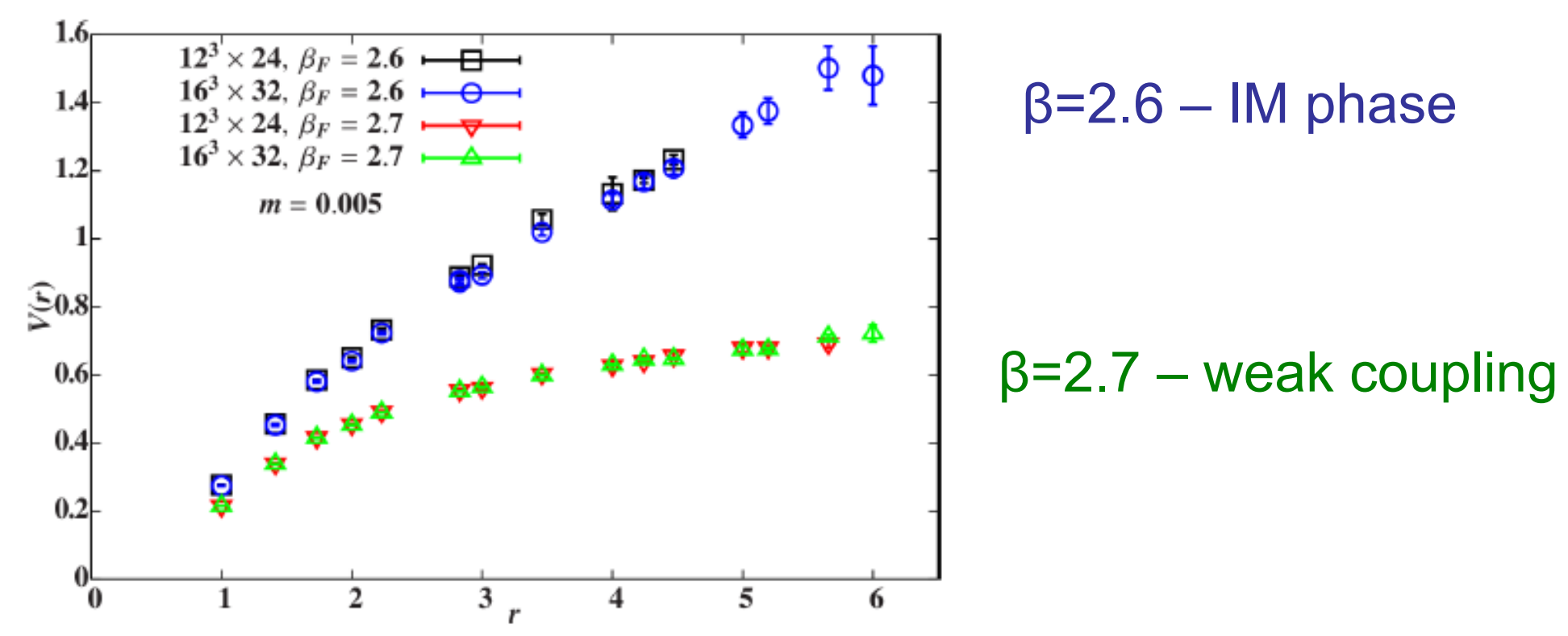

No comment about the weak coupling side from the potential - too small volume 


\section{Chiral properties : Dirac eigenvalue spectrum}

4 different volumes, 12 eigenvalues each:

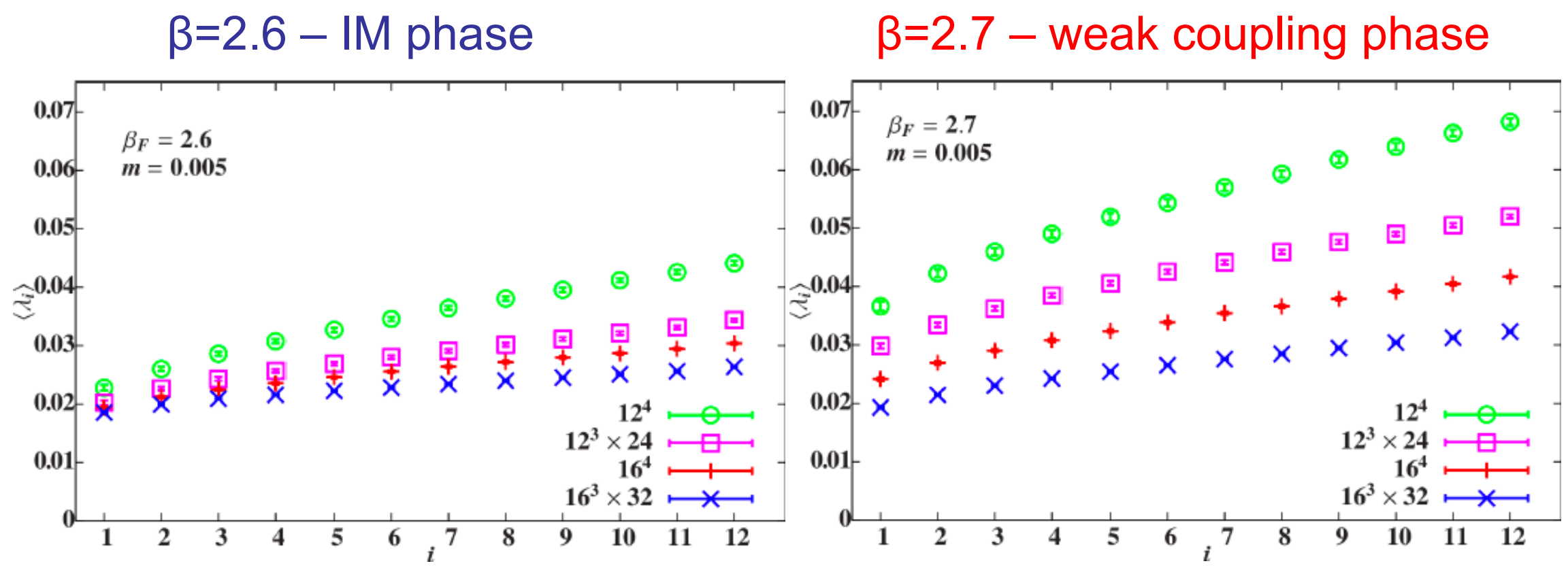

Qualitatively different - quantitative description? 


\section{Dirac eigenvalue spectrum}

- RMT predictions require knowledge of the dynamics

- Simple volume scaling is more general. In the chiral limit

$$
\begin{aligned}
& \rho(\lambda) \sim\left(\lambda-\lambda_{0}\right)^{\alpha} \quad \lambda_{0}: \text { soft edge } \\
& \int_{m}^{n} \rho(\lambda) d \lambda=\frac{n-m}{V}+\mathrm{O}\left(1 / V^{2}\right) \\
& \lambda_{n}-\lambda_{0} \sim\left(\frac{n-x_{0}}{V}\right)^{1 /(\alpha+1)}, \quad \frac{D}{\alpha+1}=y_{m} \\
& \text { - Conformal IRFP : } \mathrm{y}_{\mathrm{m}}=1+\mathrm{Y}^{*},: \text { independent of } \mathrm{V}, \beta \\
& \text { - Confining system : } \mathrm{y}_{\mathrm{m}}=1+\mathrm{Y}(\mathrm{L}): \text { in volumes smaller than the } \\
& \text { confinement scale }
\end{aligned}
$$




\section{Dirac eigenvalue spectrum}

Fit in $S^{4} b$ phase $(\beta=2.6)$ :

Needs soft edge $\lambda_{0}=0.0175$ with $\alpha=0.6(1)$ (RMT prediction: $\alpha=1 / 2$ )

Very chiral symmetric! (Even $\mathrm{U}(1)_{\mathrm{A}}$ restoring )
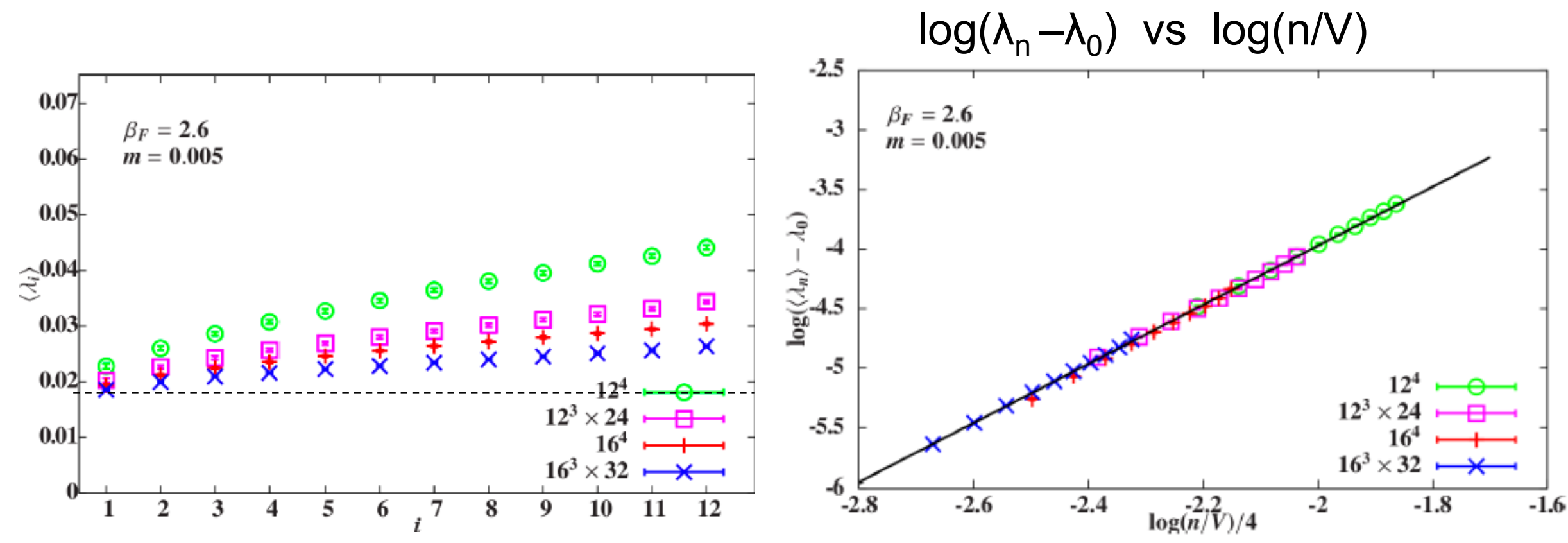
Scaling of Dirac eigenmodes, $N_{f}=12$, weak coupling side

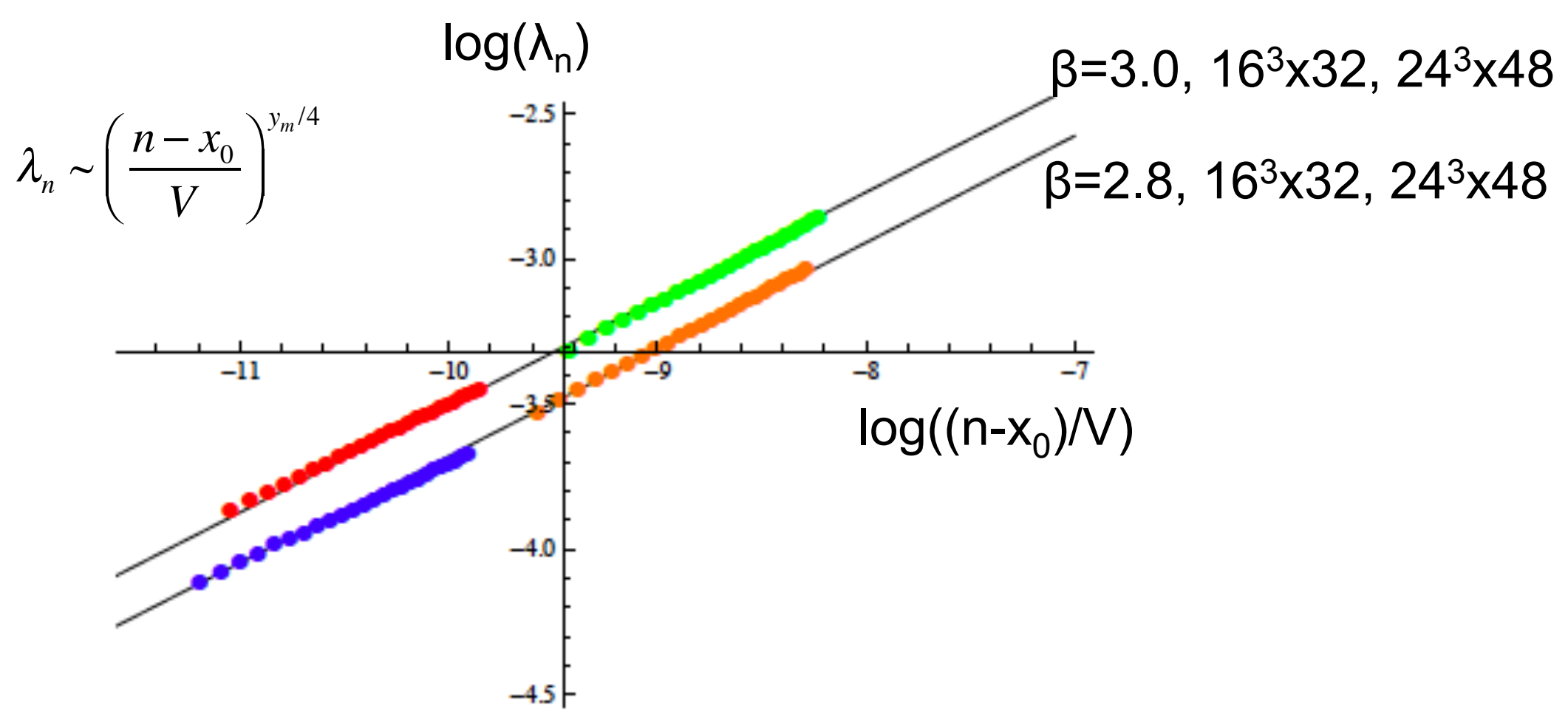

Two coupling values, four volumes with 30 eigenmodes each are consistent, predict $\gamma_{m}=0.47(2)$

Very efficient method to determine the anomalous dimension (we need larger volumes, other $\beta$, more eigenmodes to reduce error) 


\section{The $S^{4} b$ phase}

Is most likely a lattice artifact

- Breaks single-site translational symmetry

- It is confining with small correlation length

- It is chirally symmetric

- (yes, it would violate the 't Hooft anomaly matching in the continuum)

What happens at finite temperature? 


\section{Phase structure at finite $T, N_{f}=12$}

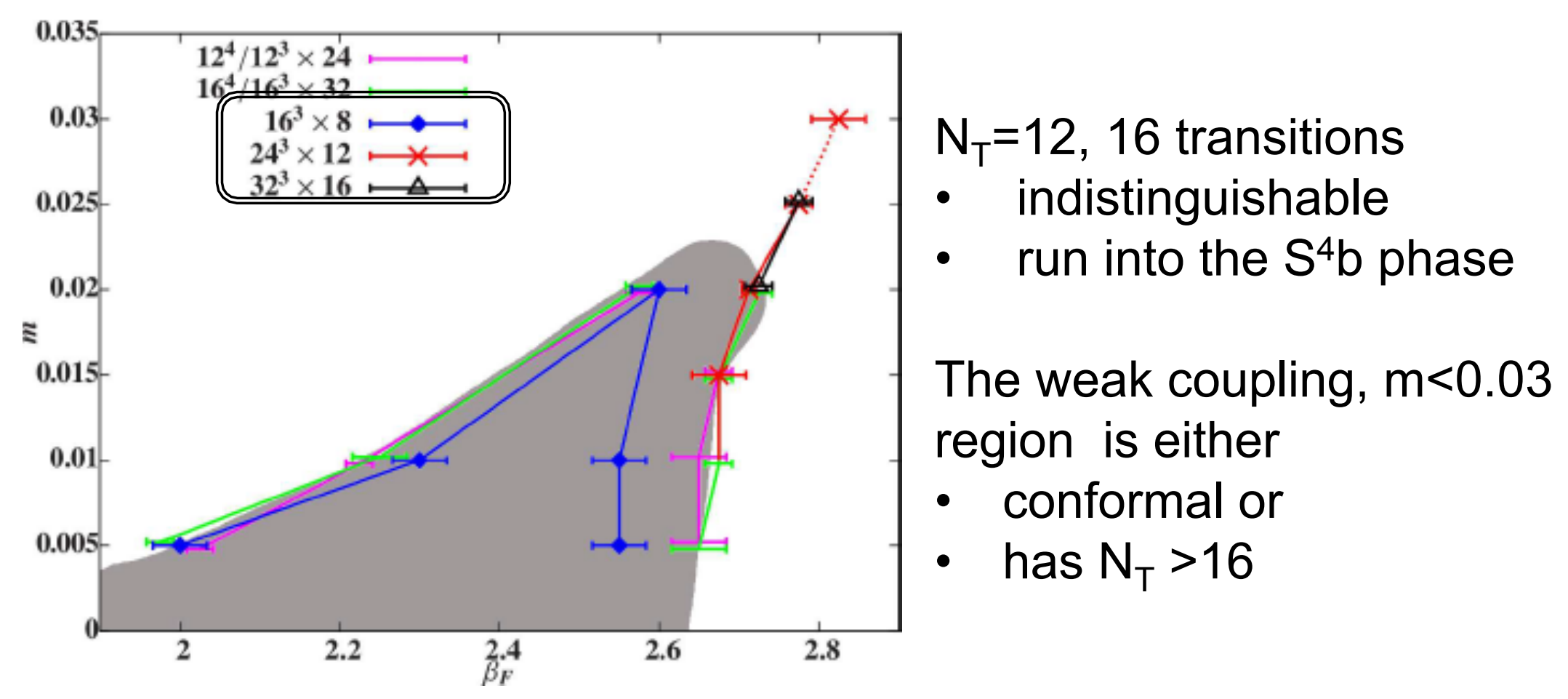

Preliminary; more data in progress 


\section{Improved Polyakov line}

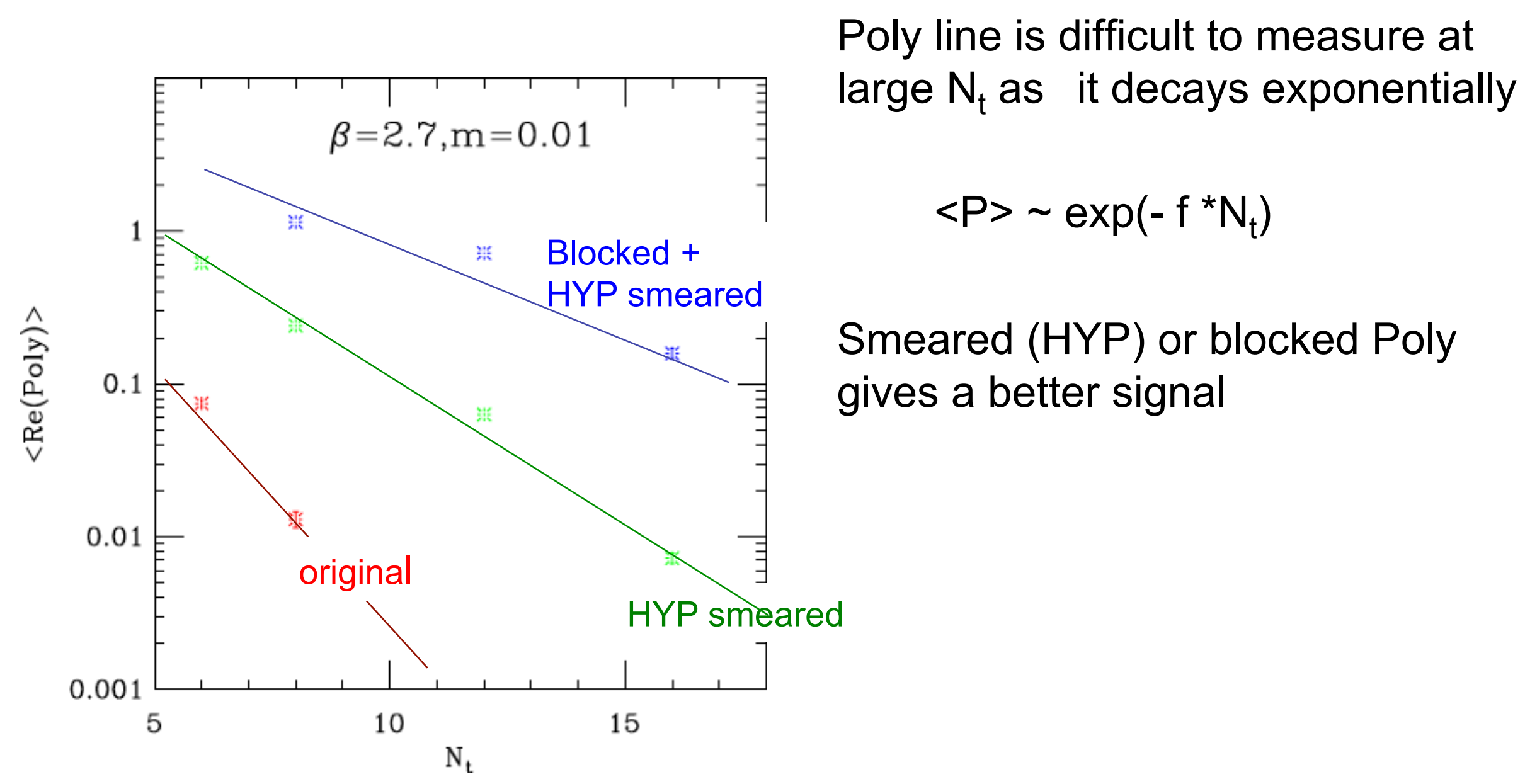




\section{$\mathrm{S}^{4} \mathrm{~b}$ phase in other systems}

There are signs of the intermediate phase with other $\mathrm{N}_{\mathrm{f}}=12$ actions

Is it unique to $\mathrm{N}_{\mathrm{f}}=12$ ?

No. 
Phase structure with $\mathrm{N}_{\mathrm{f}}=8$

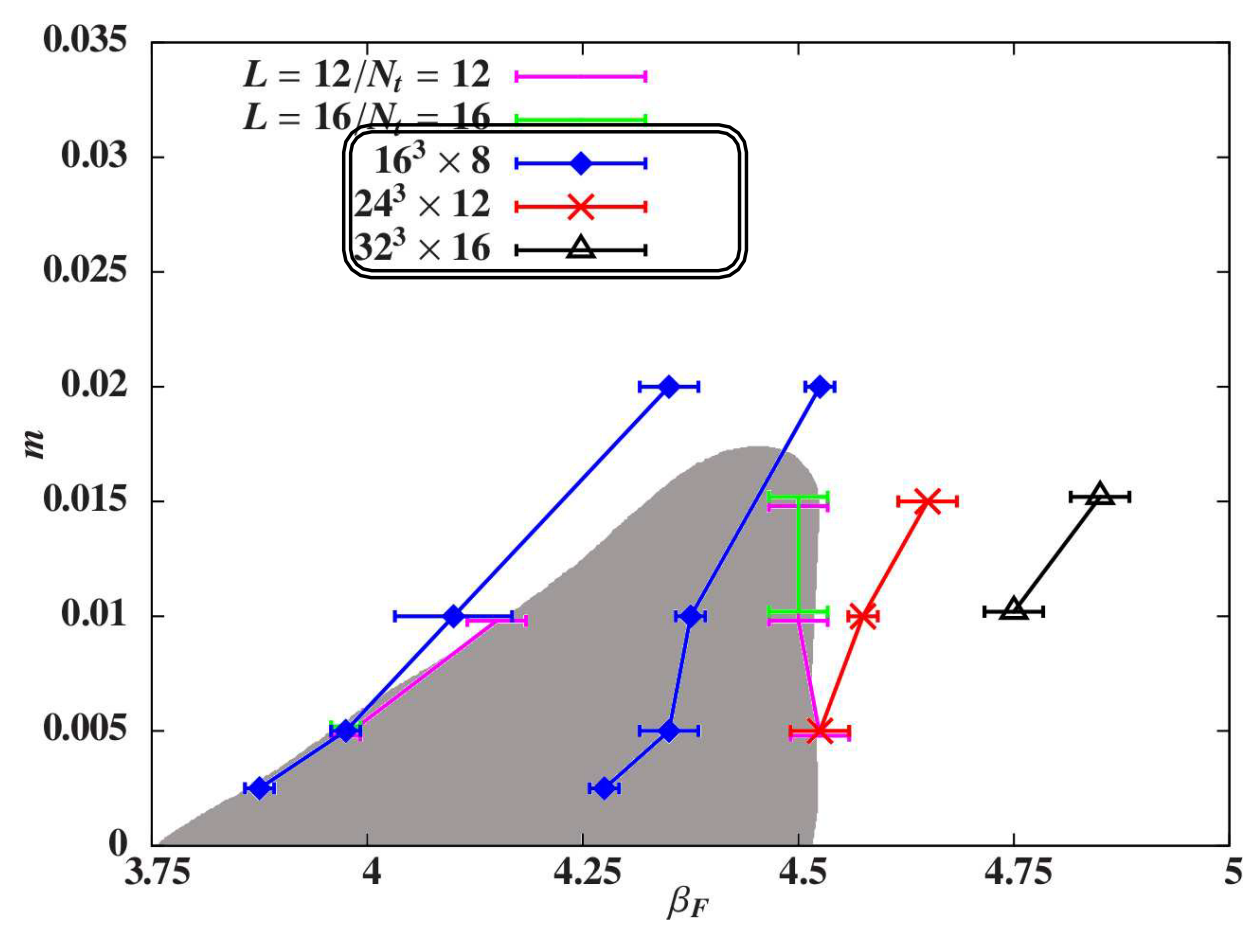

The $S^{4} b$ phase, bordered by bulk transitions, is present $\rightarrow \mathrm{S}^{4} \mathrm{~b}$ does not imply conformality

But $\mathrm{N}_{\mathrm{T}}=12,16$ transitions behave differently: move toward $g^{2}=0$ as $\mathrm{N}_{\mathrm{T}}$ increases

This is consistent with confinement

Preliminary; more data in progress 


\section{Summary \& Outlook}

These systems are complicated and have strange (strong coupling) lattice artifacts

Progress is steady but we need better understanding

The $S^{4} b$ phase is present with 2 sets of staggered fermions. Could it show up in $2+1$ flavor simulations? 


\title{
Four flavour simulations with maximally twisted mass QCD
}

\author{
Karl Jansen
}

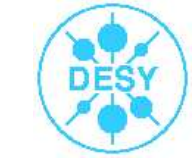

NIC

for the European Twisted Mass Collaboration

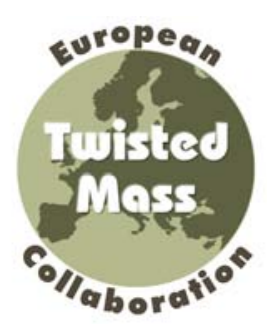

- Twisted mass fermions

- Selected results for $N_{f}=2+1+1$ flavours

- A glimpse at our next plans 
- Cyprus (Nicosia)

- France (Orsay, Grenoble)

- Italy (Rome I,II,III, Trento)

- Netherlands (Groningen)

- Poland (Poznan)

- Spain (Huelva, Madrid, Valencia)

- Switzerland (Bern)

- United Kingdom (Glasgow, Liverpool)

- Germany (Berlin/Zeuthen, Bonn, Frankfurt, Hamburg) 
Collaboration performed very successful $N_{f}=2$ research programme

Simulation results versus PDG

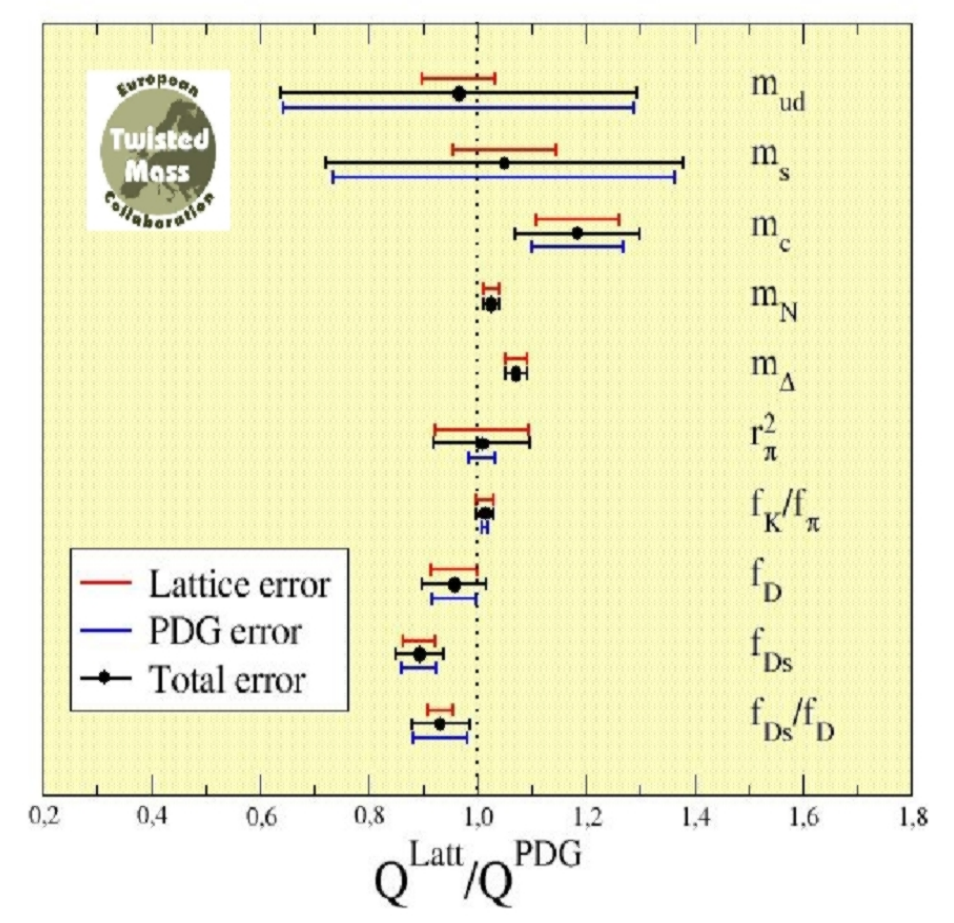

Low energy constants

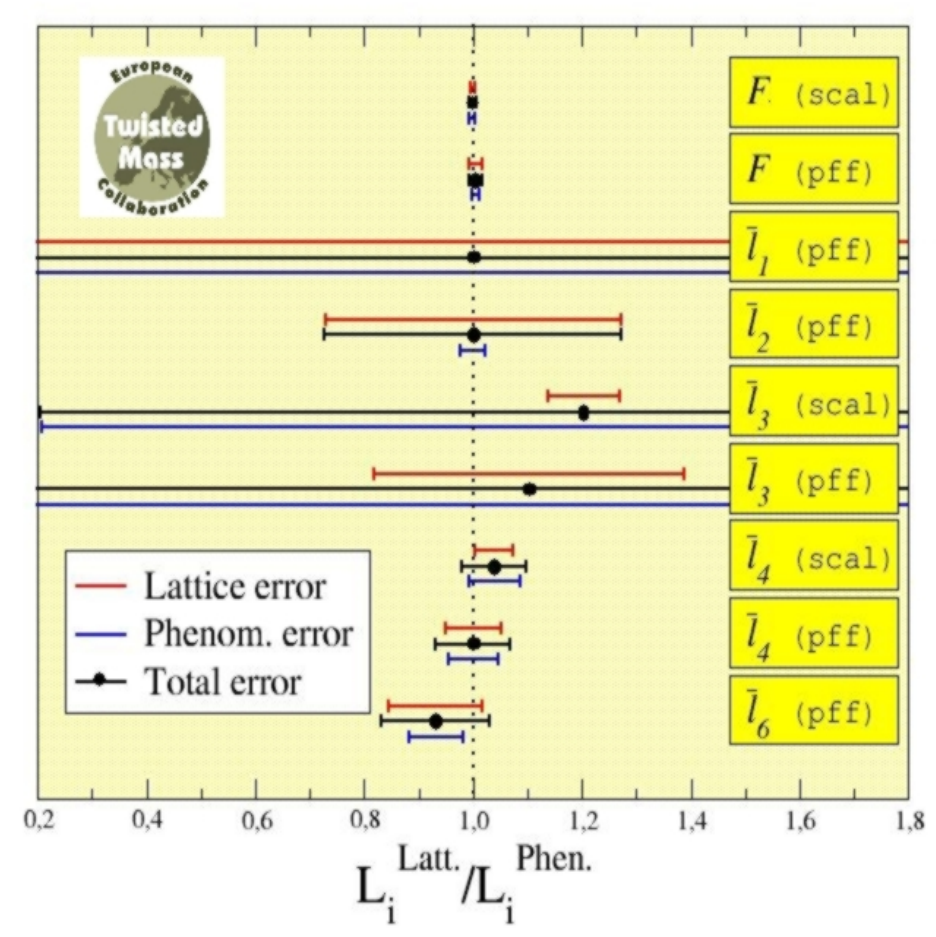




\section{The $\rho$-meson resonance: dynamical quarks at work}

(X. Feng, D. Renner, K.J.)

- usage of three Lorentz frames
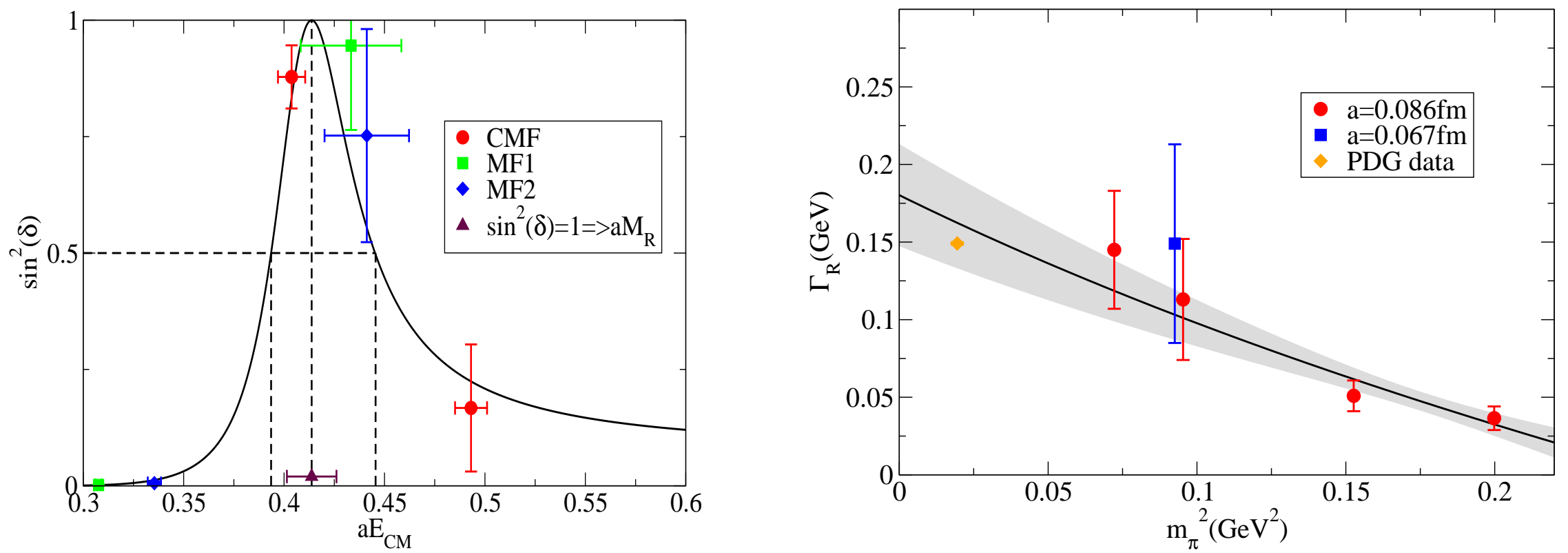

$m_{\pi^{+}}=330 \mathrm{MeV}, a=0.079 \mathrm{fm}, L / a=32$

fitting $z=\left(M_{\rho}+i \frac{1}{2} \Gamma_{\rho}\right)^{2}$

$m_{\rho}=1033(31) \mathrm{MeV}, \Gamma_{\rho}=123(43) \mathrm{MeV}$ 


\section{Natural $^{2}$ next step: move to four flavours}

- twisted mass: works with quark doublets

- maximal twist: automatic $O(a)$-improvement

- infrared cut-off due to twisted quark mass

- simplification in renormalization $\left(f_{\pi},\langle N|\bar{q} q| N\rangle\right)$

need to control isospin splitting effects

- strange and charm quarks needed for physical quantities

- mesons: $m_{\text {strange }}, m_{\text {charm }}, f_{D}, f_{D_{s}}, \eta^{\prime}, \eta_{c}$

- baryons: spectrum, $\langle N|\bar{q} q| N\rangle$

- $g_{\mu}-2 \leftarrow$ can unambiguously compare to experimental value

- running of $\alpha_{\text {strong }}(\mu)$ with four flavours 


\section{Wilson (Frezzotti, Rossi) twisted mass QCD (Frezzotti, Grassi, Sint, Weisz)}

Fermion action of twisted mass fermions

$$
\begin{gathered}
S_{l}=\sum_{x} \bar{\chi}_{x}^{l}\left[m_{q}+\frac{1}{2} \gamma_{\mu}\left[\nabla_{\mu}+\nabla_{\mu}^{*}\right]-\operatorname{ar} \frac{1}{2} \nabla_{\mu}^{*} \nabla_{\mu}+i \mu_{\mathrm{tm}} \tau_{3} \gamma_{5}\right] \chi_{x}^{l} \\
S_{h}=\sum_{x} \bar{\chi}_{x}^{h}\left[m_{q}+\frac{1}{2} \gamma_{\mu}\left[\nabla_{\mu}+\nabla_{\mu}^{*}\right]-\operatorname{ar} \frac{1}{2} \nabla_{\mu}^{*} \nabla_{\mu} i \gamma_{5} \tau_{1} \mu_{\sigma}+\tau_{3} \mu_{\delta}\right] \chi_{x}^{h}
\end{gathered}
$$

- quark mass parameter $m_{q}$, twisted mass parameter $\mu_{\mathrm{tm}}$

- strange and charm quark masses

$$
\begin{aligned}
& m_{s}=Z_{P}^{-1} \mu_{\sigma}-Z_{S}^{-1} \mu_{\delta} \\
& m_{c}=Z_{P}^{-1} \mu_{\sigma}+Z_{S}^{-1} \mu_{\delta}
\end{aligned}
$$

- note, $m_{q}$ the same in $S_{l}$ and $S_{h}$ 


\section{A word on flavour breaking}

- isospin (flavour symmetry) violation at any $a \neq 0: \quad m_{\pi}^{ \pm}-m_{\pi}^{0} \neq 0$

- observe large $O\left(a^{2}\right)$ effect in neutral pion mass

- effect visible both for $N_{f}=2$ and $N_{f}=2+1+1$

- theoretical understanding (Dimopoulos et.al., Phys. Rev. D81, 034509 (2010))

- $\chi \mathrm{PT}$ analysis of meson sector including pion mass splitting $a^{2}$ effect (O. Bär, Phys. Rev. D82 (2010) 094505)
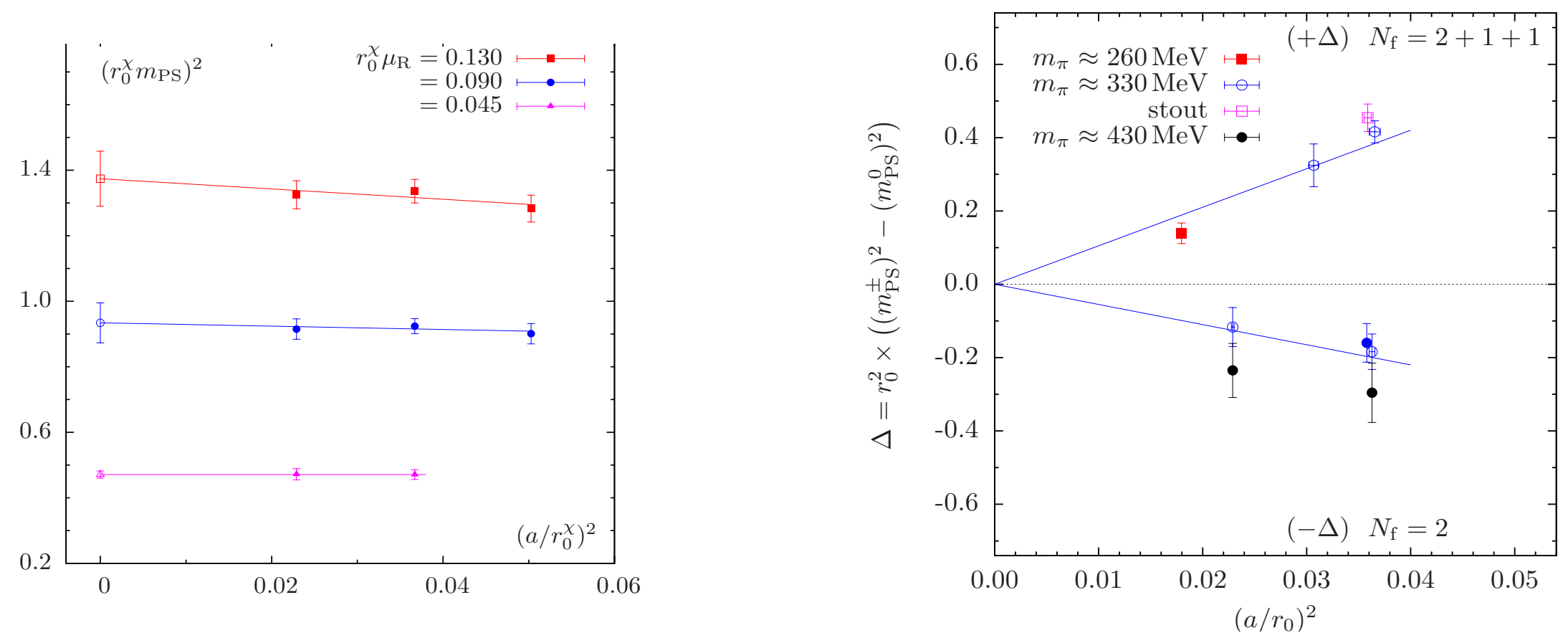


\section{Tuning to maximal twist}

Maximal twist: tune $m_{q}$ such that

$$
m_{\mathrm{PCAC}}=\frac{\sum_{\mathbf{x}}\left\langle\partial_{0} A_{0}^{a}(x) P^{a}(0)\right\rangle}{2 \sum_{\mathbf{x}}\left\langle P^{a}(x) P^{a}(0)\right\rangle}=0
$$

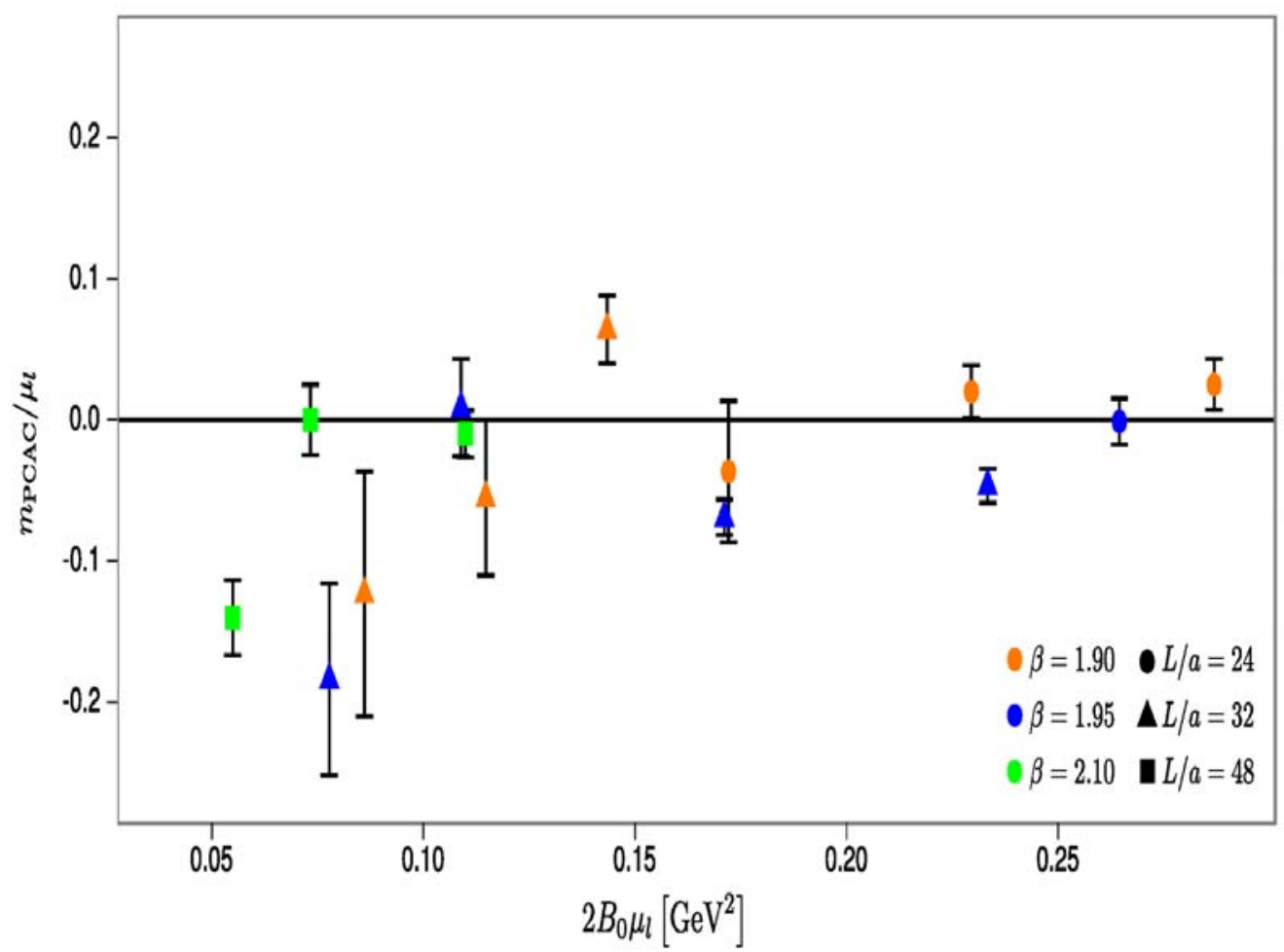

- tuning of $m_{q}$ at each $\mu_{\mathrm{tm}}$ used

- demand $m_{\mathrm{PCAC}} \lesssim 0.1 \mu_{\mathrm{tm}}$

- demand $\Delta\left(m_{\mathrm{PCAC}}\right) \lesssim 0.1 \mu_{\mathrm{tm}}$ 


\section{Autocorrelations from gradient flow}

(Deuzemann, Wenger) see also talk by Z. Fodor

- flow of the gauge field $U(x, \mu)$ according to the flow equation

$$
\begin{aligned}
& \frac{\partial}{\partial t} V_{t}(x, \mu)=-g_{0}^{2}\left\{\partial_{x, \mu} S_{\text {latt }} W\left(V_{t}\right)\right\} V_{t}(x, \mu) \\
& V_{t=0}(x, \mu)=U(x, \mu)
\end{aligned}
$$

- lattice: discrete integration scheme with finite step size $\epsilon$ for integration

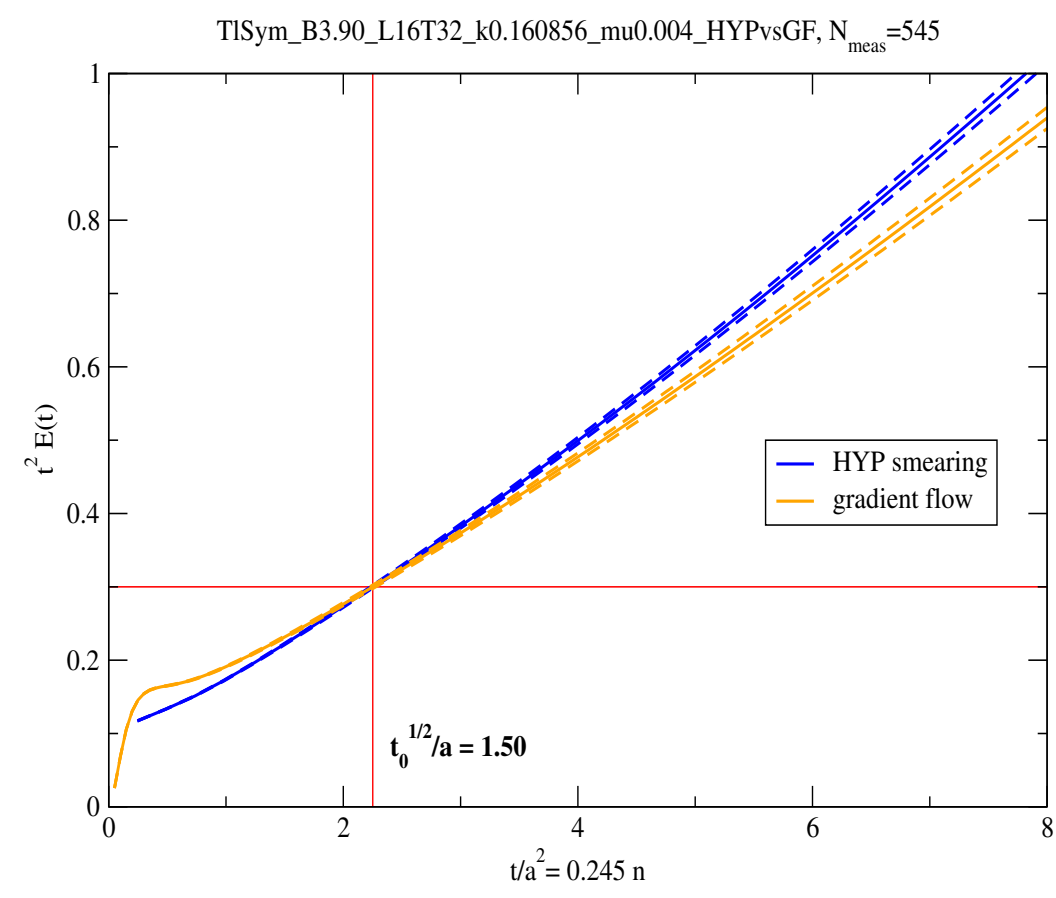

$a \approx 0.08 \mathrm{fm} m_{\pi} \approx 300 \mathrm{MeV}$

energy density:

$$
E(t)=2 \sum_{\text {plaq }} \operatorname{Retr}\left[1-V_{t}(\text { plaq })\right]
$$




\section{Topology and autocorrelations}

- topological charge well defined on smooth configurations $V_{t}$

- use standard field theoretic definition $F_{\mu \nu} \tilde{F}_{\mu \nu}\left(V_{t}\right)$

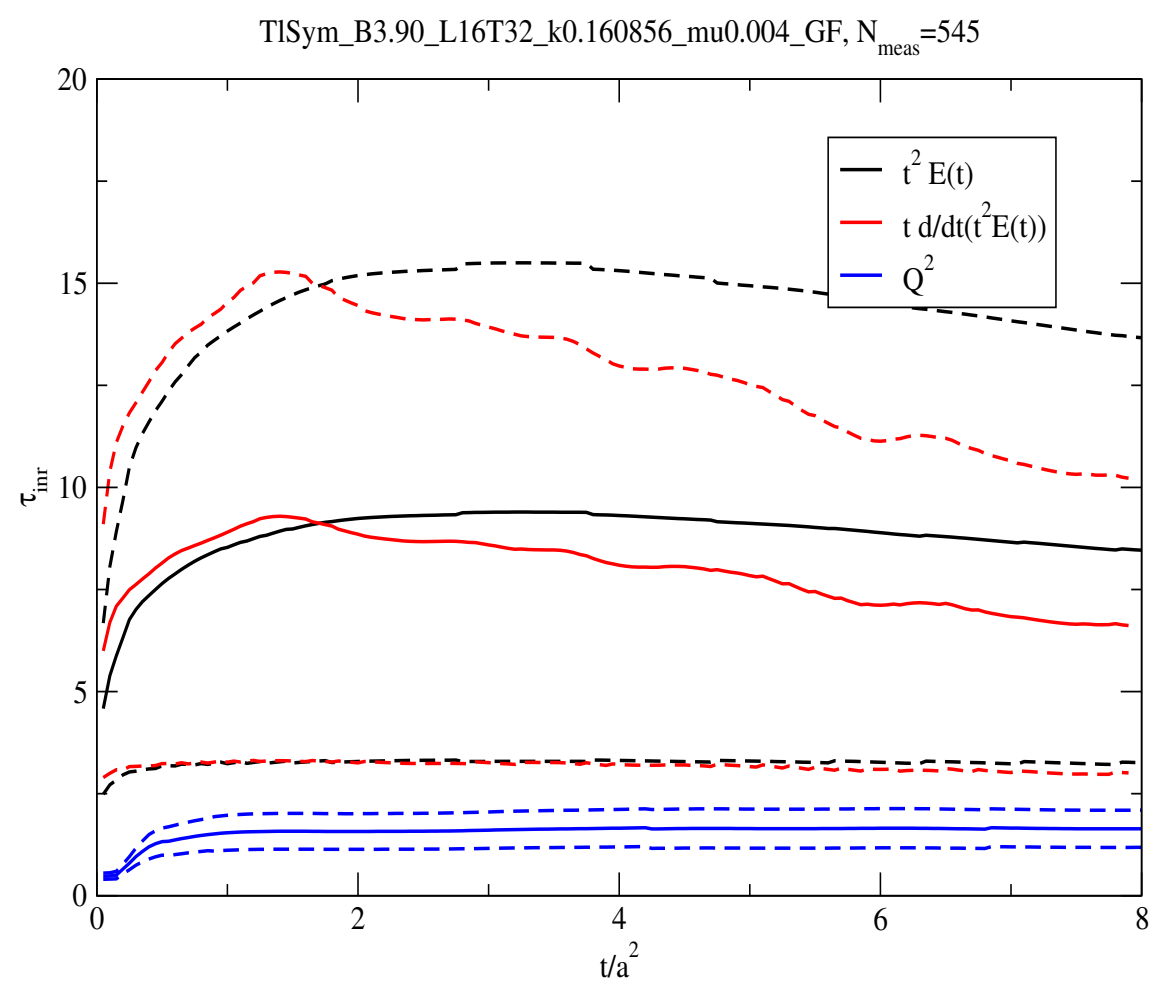

gradient flow gives largest autocorrelation

autocorrelation from topological susceptibility significantly smaller

warning 


\section{$N_{f}=2+1+1$ light quark sector: scaling}

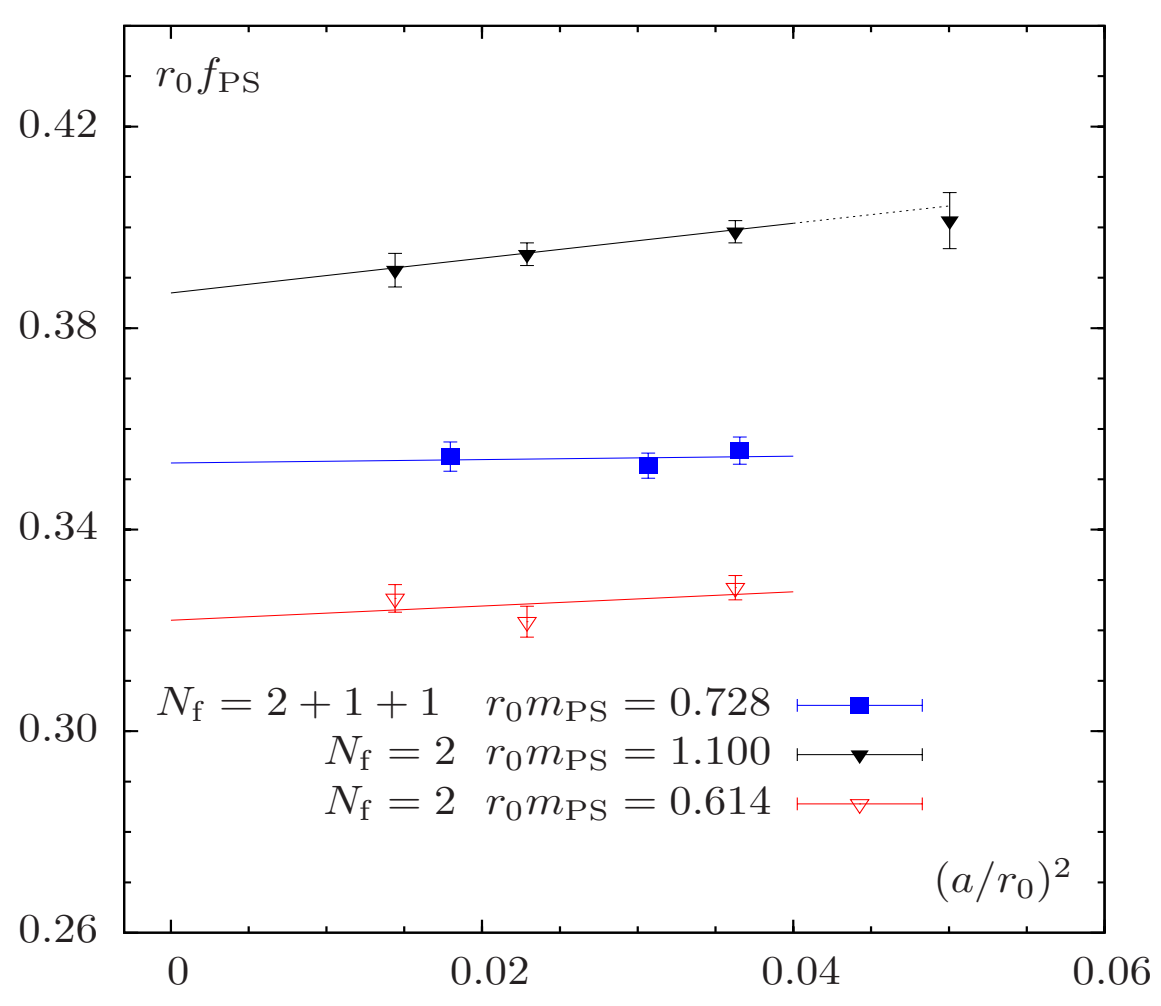

pseudoscalar decay constant $f_{\mathrm{PS}}$

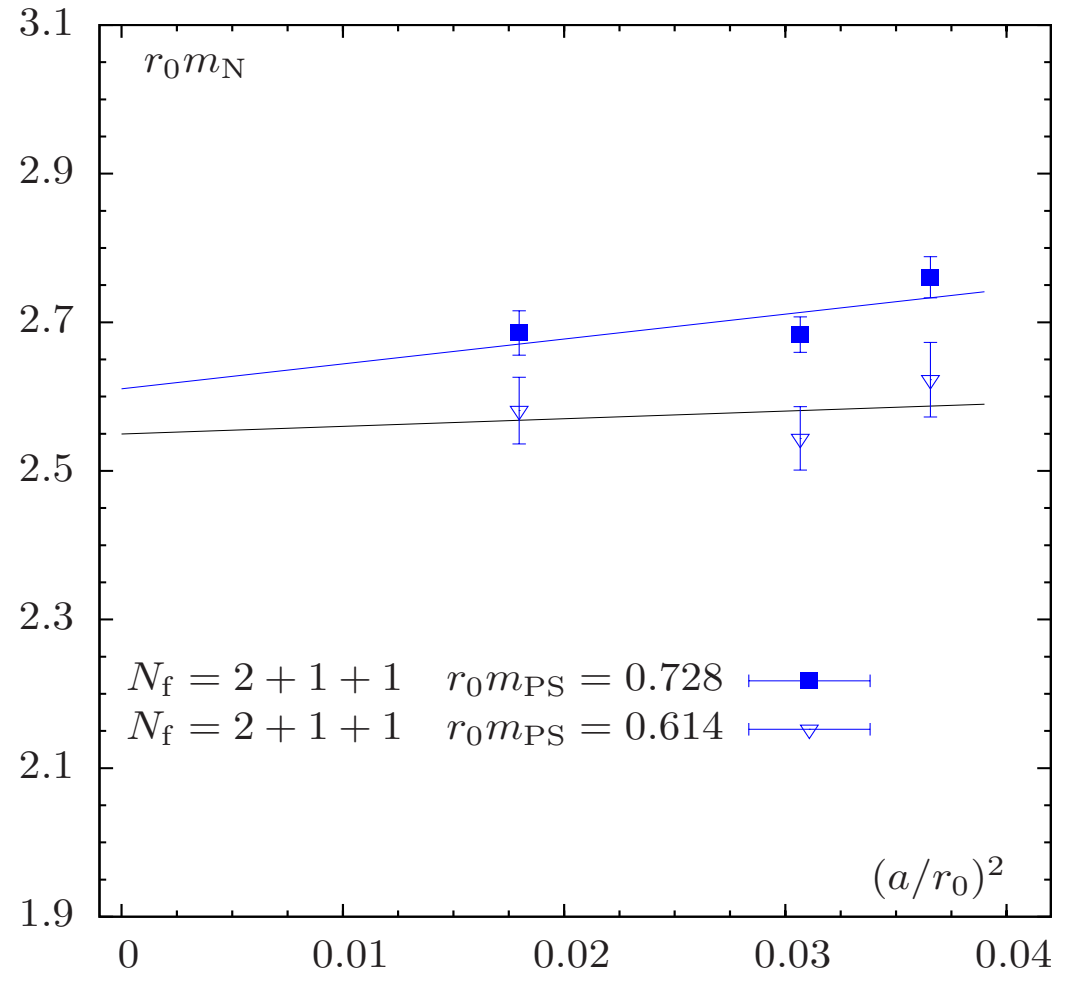

nucleon mass 


\section{$N_{f}=2+1+1$ light quark sector: $\chi \mathbf{P T}$ fit}

- basic formulae: $N_{f}=2$ continuum $\chi \mathrm{PT}$ at NLO

$$
\begin{array}{r}
m_{\mathrm{PS}}^{2}=\chi_{\mu}\left[1+\xi \log \left(\chi_{\mu} / \Lambda_{3}^{2}\right)\right] \quad K_{m}^{2}(L) \\
f_{\mathrm{PS}}=f_{0}\left[1-2 \xi \log \left(\chi_{\mu} / \Lambda_{4}^{2}\right)\right] K_{f}(L) \\
\chi_{\mu}=2 \hat{B}_{0} Z_{\mu} \mu_{q}, \quad \xi=\chi_{\mu} /\left(2 \pi f_{0}\right)^{2}
\end{array}
$$

- finite size corrections $K_{m}(L), K_{f}(L)$ (Gasser, Leutwyler, 1987, 1988; Colangelo, Dürr, Haefeli, 2005) in preparation: analysis a la Colangelo, Wenger, Wu; Bär; Ueda, Aoki

- fit simultaneously to our data at all $\beta$-values

- fit includes renormalisation constant $Z_{\mu}=1 / Z_{P}$ 


\section{$N_{f}=2+1+1$ light quark sector: $\chi \mathbf{P T}$ fit}

- estimate systematic effects : lattice artifacts, FSE
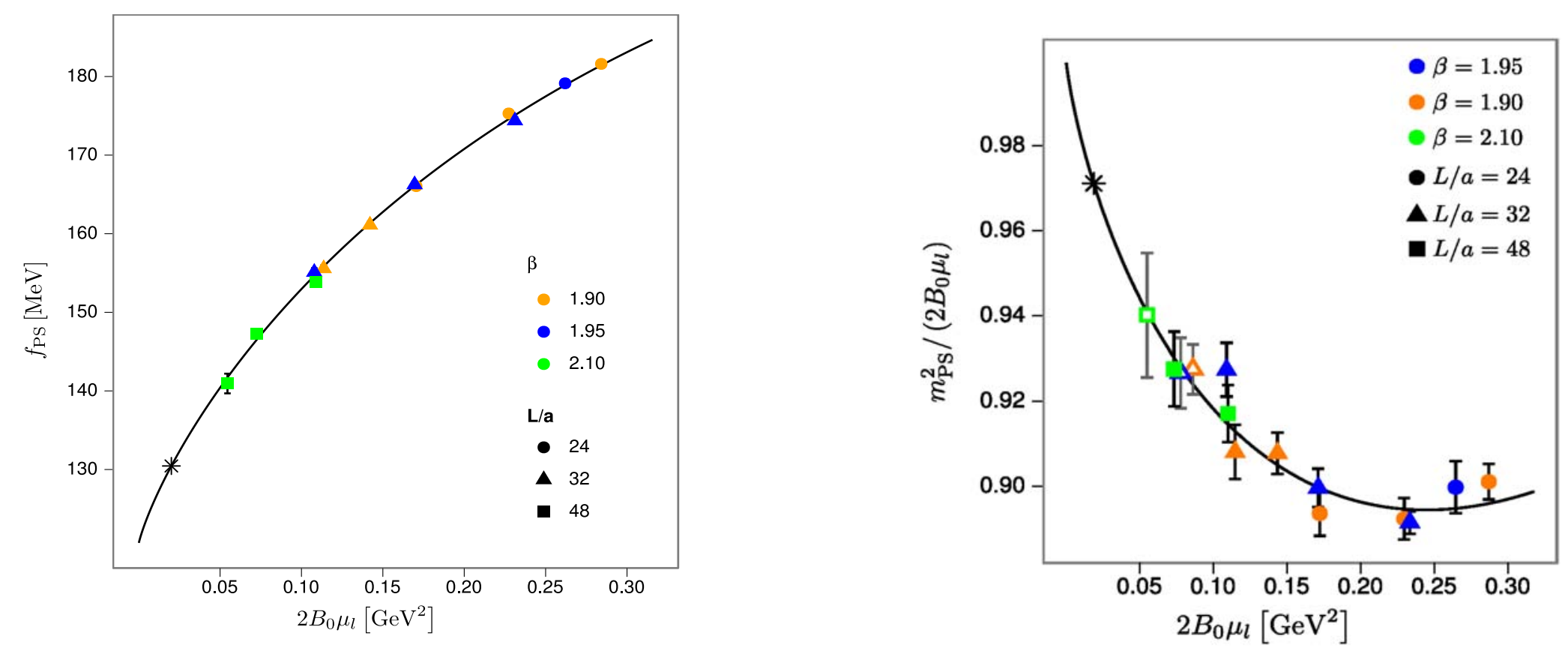
Comparison of low energy constants

\begin{tabular}{lrr}
\hline \hline & $N_{f}=2$ & $N_{f}=2+1+1$ \\
\hline \hline$\ell_{3}$ & $3.70(27)$ & $3.50(31)$ \\
$\bar{\ell}_{4}$ & $4.67(10)$ & $4.66(33)$ \\
$f_{\pi} / f_{0}$ & $1.076(3)$ & $1.076(9)$ \\
$B_{0}[\mathrm{MeV}]$ & $2437(120)$ & $2638(200)$ \\
$\left\langle r^{2}\right\rangle_{s}^{\mathrm{NLO}}\left[\mathrm{fm}^{2}\right]$ & $0.710(28)$ & $0.715(77)$ \\
\hline
\end{tabular}




\section{$N_{f}=2+1+1$ adding strange quark: fit formulae}

- $S U(2) \chi \mathrm{PT}$ Fit Formulae for $f_{K}$ and $f_{\pi}$ :

$$
\begin{gathered}
f_{\mathrm{PS}}\left(\mu_{\ell}, \mu_{\ell}, \mu_{\ell}\right)=f_{0} \cdot\left(1-2 \xi_{\ell \ell} \ln \xi_{\ell \ell}+b \xi_{\ell \ell}\right) \\
f_{\mathrm{PS}}\left(\mu_{\ell}, \mu_{\ell}, \mu_{s}\right)=\left(f_{0}^{(K)}+f_{m}^{(K)} \xi_{s s}\right) \cdot\left[1-\frac{3}{4} \xi_{\ell \ell} \ln \xi_{\ell \ell}+\left(b_{0}^{(K)}+b_{m}^{(K)} \xi_{s s}\right) \xi_{\ell \ell}\right] \\
\xi_{X Y}=\frac{m_{\mathrm{PS}}^{2}\left(\mu_{\ell}, \mu_{X}, \mu_{Y}\right)}{\left(4 \pi f_{0}\right)^{2}}
\end{gathered}
$$

(Gasser, Leutwyler (1984); Allton et al (2008); ETMC, Blossier et al. (2010))

- correct for finite size effects using NLO $\chi \mathrm{PT}$ (Gasser, Leutwyler (1987); Becirevic, Villadoro (2004)) 


\section{$N_{f}=2+1+1$ light quark sector: fit results}

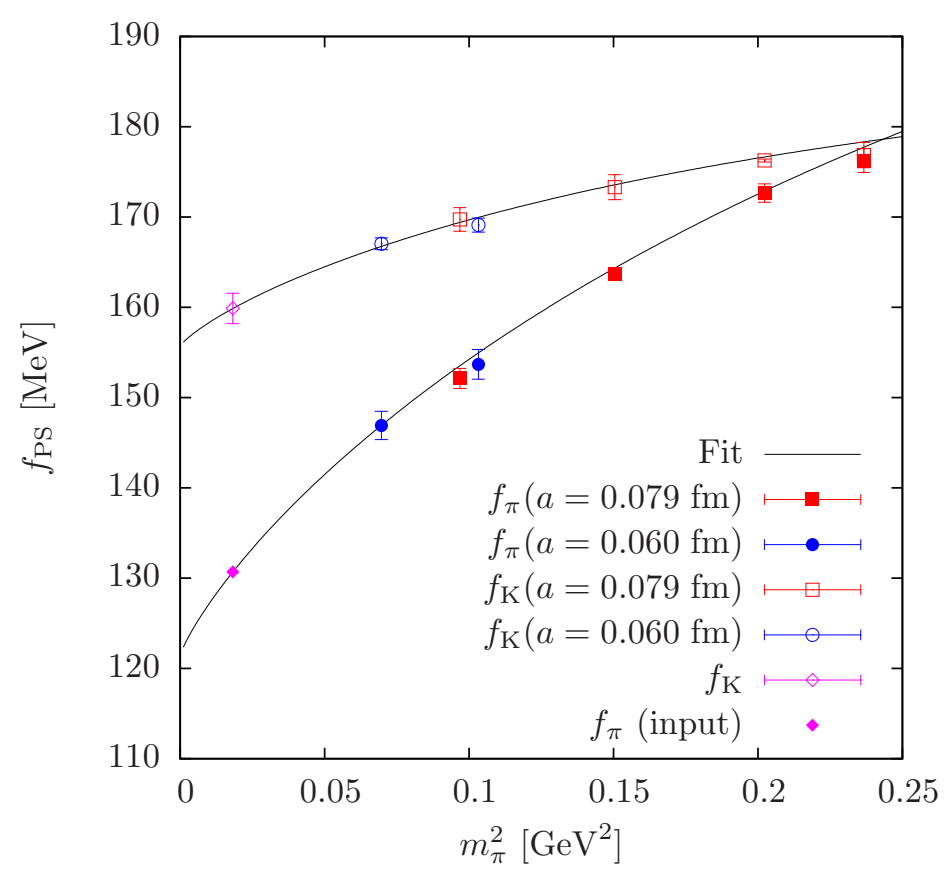

- fit $\beta=1.95(a=0.079 \mathrm{fm})$ and $\beta=2.10(a=0.06 \mathrm{fm})$ simultaneously

- from setting $m_{\mathrm{PS}}^{2}\left(\mu_{\ell}, \mu_{s}, \mu_{s}\right)=2 m_{K}^{2}-m_{\pi}^{2}$

- $m_{\pi}=135 \mathrm{MeV}, \quad f_{\pi}=130.7 \mathrm{MeV}$, $m_{K}=497.7 \mathrm{MeV}$

preliminary fit results:

- $f_{K} / f_{\pi}=1.224(13), f_{K}=160(2) \mathrm{MeV}, \bar{\ell}_{4}=4.78(2),\left|V_{\mathrm{us}}\right|=0.220(2)$

- errors statistical only 


\section{$N_{f}=2+1+1$ heavy quark sector}

Wilson twisted mass Dirac operator for $(c, s)$ pair:

$D_{h}=\left(\begin{array}{cc}\gamma_{\mu} \tilde{\nabla}_{\mu}+\mu_{\sigma}+\mu_{\delta} & i \gamma_{5}\left(\frac{a}{2} \nabla_{\mu}^{*} \nabla_{\mu}-m_{q}\right) \\ i \gamma_{5}\left(\frac{a}{2} \nabla_{\mu}^{*} \nabla_{\mu}-m_{q}\right) & \gamma_{\mu} \tilde{\nabla}_{\mu}+\mu_{\sigma}-\mu_{\delta}\end{array}\right)$

- mixing of $c$ and $s$ flavour and of parity

- Kaon is the ground state : good precision

- D meson appears as an excited state

- three independent methods:

- generalised eigenvalue problem

- multi-exponential fits

- imposing parity and flavour restoration at finite $a$

- they provide consistent results for $m_{D}$

- overcome mixing of flavour $\leadsto$ mixed action
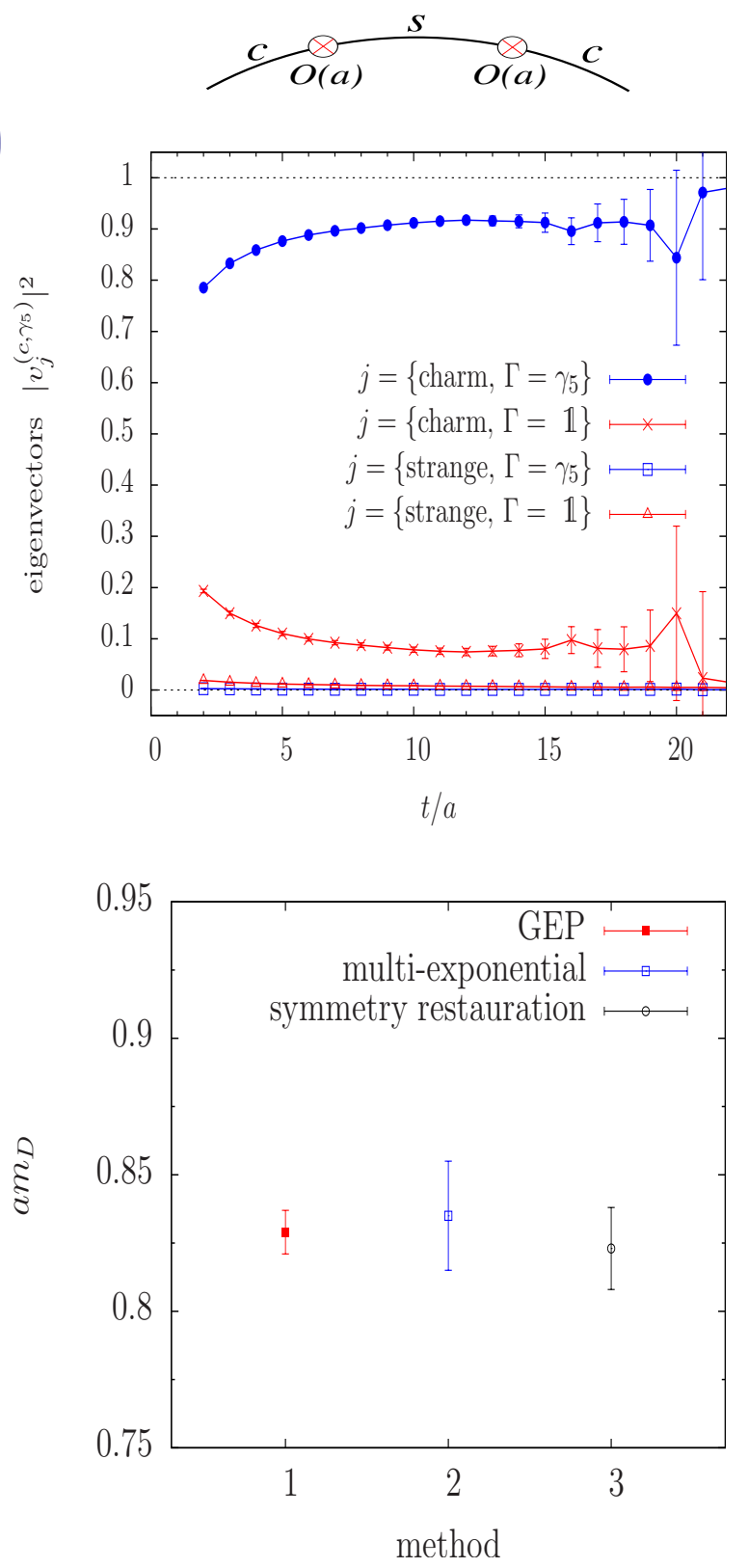


\section{$N_{f}=2+1+1$ approaching the charm quark}

- introduce Wilson twisted mass doublets in the valence sector

$$
D_{\text {tm }}\left(\mu_{\text {val }}\right)=D+m_{\text {crit }}+i \mu_{\text {val }} \gamma_{5} \tau^{3}
$$

(Osterwalder, Seiler (1990), Pena et al. (2004); Frezzotti, Rossi (2004))

- $m_{\text {crit }}$ from unitary set-up

- $4-6$ values for $\mu_{v a l}$ in the strange $\mu_{s}$ and the charm $\mu_{c}$ region inversions with multi-mass solver

- matching to unitary set-up using $m_{K}$ and $m_{D}$

- $\Rightarrow$ avoid flavour breaking
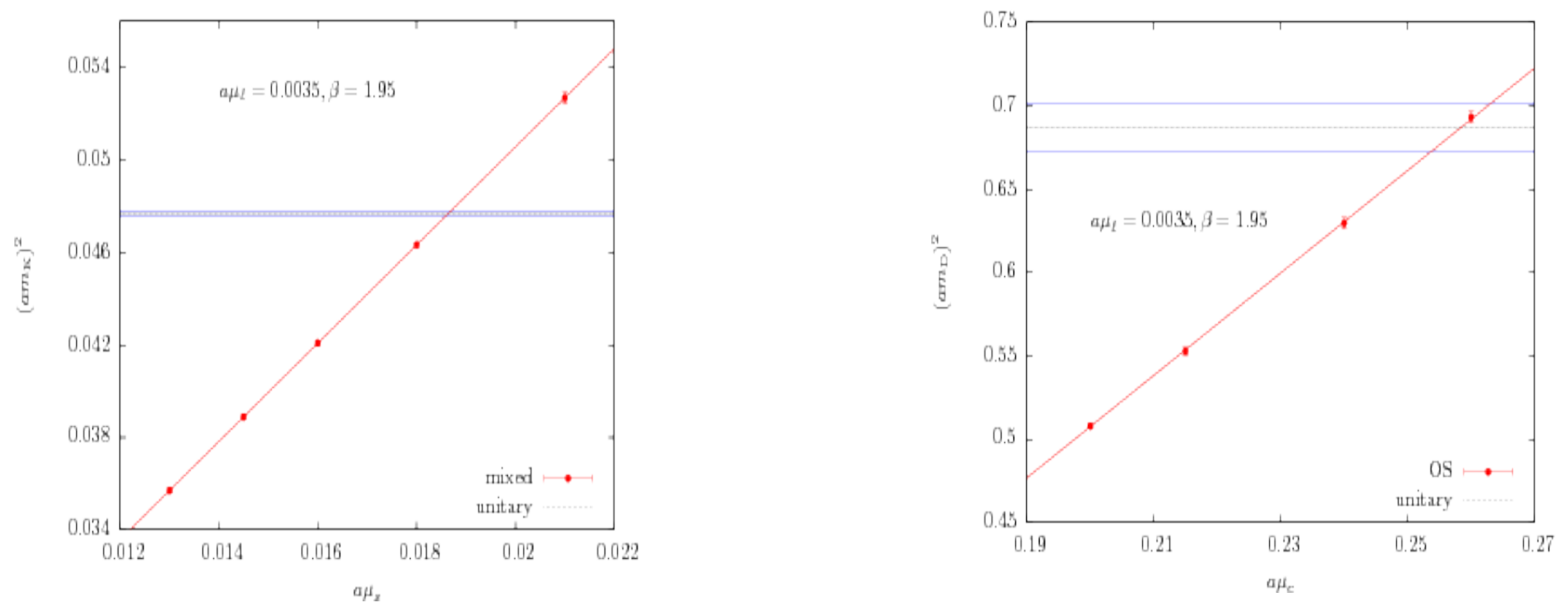


\section{Unitary versus Osterwalder-Seiler: $f_{K}$}

- the unitary $f_{K}$ can be computed from: $f_{K}=\left(m_{\ell}+m_{s}\right) \frac{\left\langle 0\left|P_{K}\right| K\right\rangle}{m_{K}^{2}}$ with $m_{s}=\mu_{\sigma}-\left(Z_{\mathrm{P}} / Z_{\mathrm{S}}\right) \mu_{\delta}$

- similar formula for $f_{D}$

- $P_{K}$ is the physical Kaon projecting operator

- the mixed action $f_{K}$ computed from: $f_{\mathrm{PS}}=\left(\mu_{v a l}^{(1)}+\mu_{v a l}^{(2)}\right) \frac{|\langle 0|P| P S\rangle|}{m_{\mathrm{PS}} \sinh m_{\mathrm{PS}}}$,

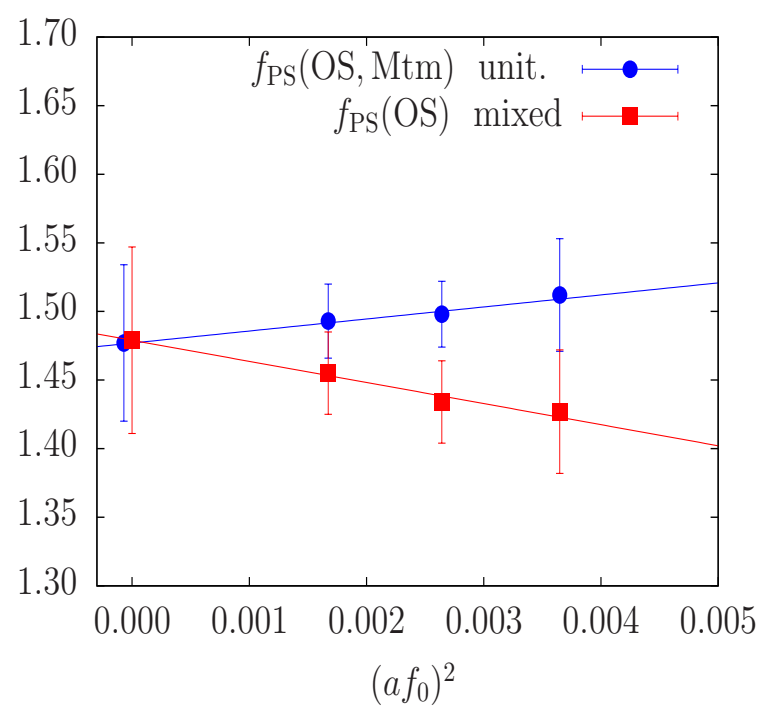

Test for $N_{f}=2$

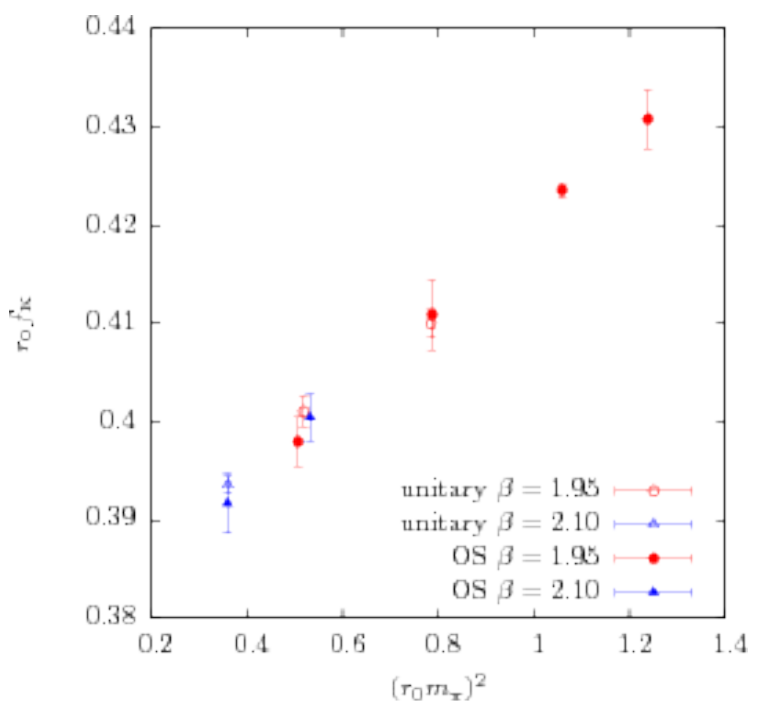

situation for $N_{f}=2+1+1$ 
Decay constants, $F_{D}, F_{D_{s}}$

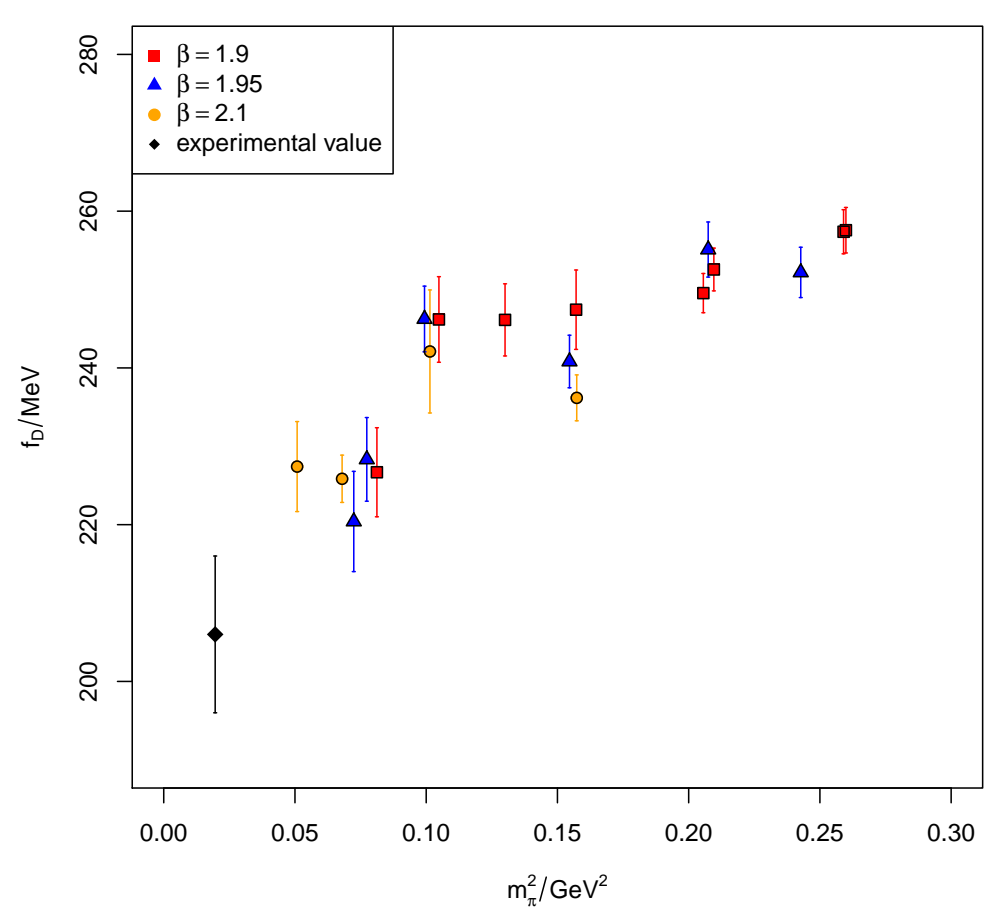

- quark mass dependence of $f_{D}$

- input: $m_{\pi}=135 \mathrm{MeV}, \quad f_{\pi}=130.7 \mathrm{MeV}$, $m_{K}=497.7 \mathrm{MeV}$

- all results preliminary

- results very encouraging $f_{D_{s}}=250(3) \mathrm{MeV}, \quad f_{D}=204(3) \mathrm{MeV}, \quad f_{D_{s}} / f_{D}=1.230(6)$

- very preliminary but very first results from $N_{f}=2+1+1$ ! 


\section{The strange quark content of the nucleon}

(Drach, Dinter, Frezzotti, Herdoiza, Rossi, K.J.)

- neutralino in supersymmetric models candidate for dark matter

- interaction with nucleon most strongly through the strange quark content via the Higgs boson exchange diagram

spin independend cross section:

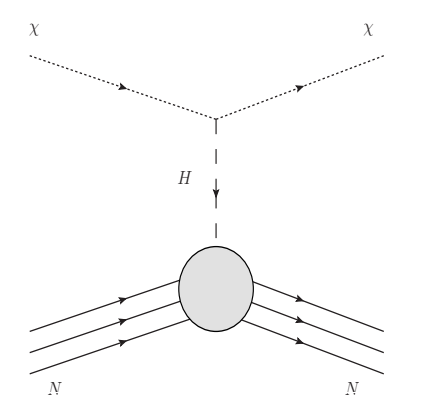

$$
\begin{gathered}
\sigma_{\mathrm{SI}} \propto \sum_{q} \frac{f_{T_{q}}}{m_{q}} ; q=u, d, s, c \\
f_{T_{q}}=\frac{\left\langle N\left|m_{q} \bar{q} q\right| N\right\rangle}{m_{N}} \equiv m_{q} B_{q}
\end{gathered}
$$

$\Rightarrow$ cross section proportional to quark content; independent from quark mass

Study here: $\langle N|\bar{s} s| N\rangle=m_{N} B_{s} ;\langle N|\bar{c} c| N\rangle=m_{N} B_{c}$ 


\section{The problem}

spin independent cross section strongly dependend on pion-nucleon sigma term $\Sigma_{\pi N}$

Varying $48 \mathrm{MeV}<\Sigma_{\pi N}<80 \mathrm{MeV}$

$\Rightarrow$ cross section changes by an order of magnitude

$\Sigma_{\pi N}$ connected to $y_{N}$ parameter

$$
y_{N}=\frac{2 B_{s}}{B_{u}+B_{d}} \quad ; B_{q}=\frac{\langle N|\bar{q} q| N\rangle}{m_{N}}
$$

relation: $y_{N}=1-\sigma_{0} / \Sigma_{\pi N}$

$$
\sigma_{0}=m_{q}\langle N|\bar{u} u+\bar{d} d-2 \bar{s} s| N\rangle, \quad m_{q}=\left(m_{u}+m_{d}\right) / 2
$$

from $\chi \mathrm{PT}: y_{N}=0.44(13) \rightarrow$ quite large

want: a first principle, non-perturbative computation of $f_{T_{s}}, f_{T_{c}}$ and $y_{N}$ 


\section{Twisted mass fermions: special noise reduction technique}

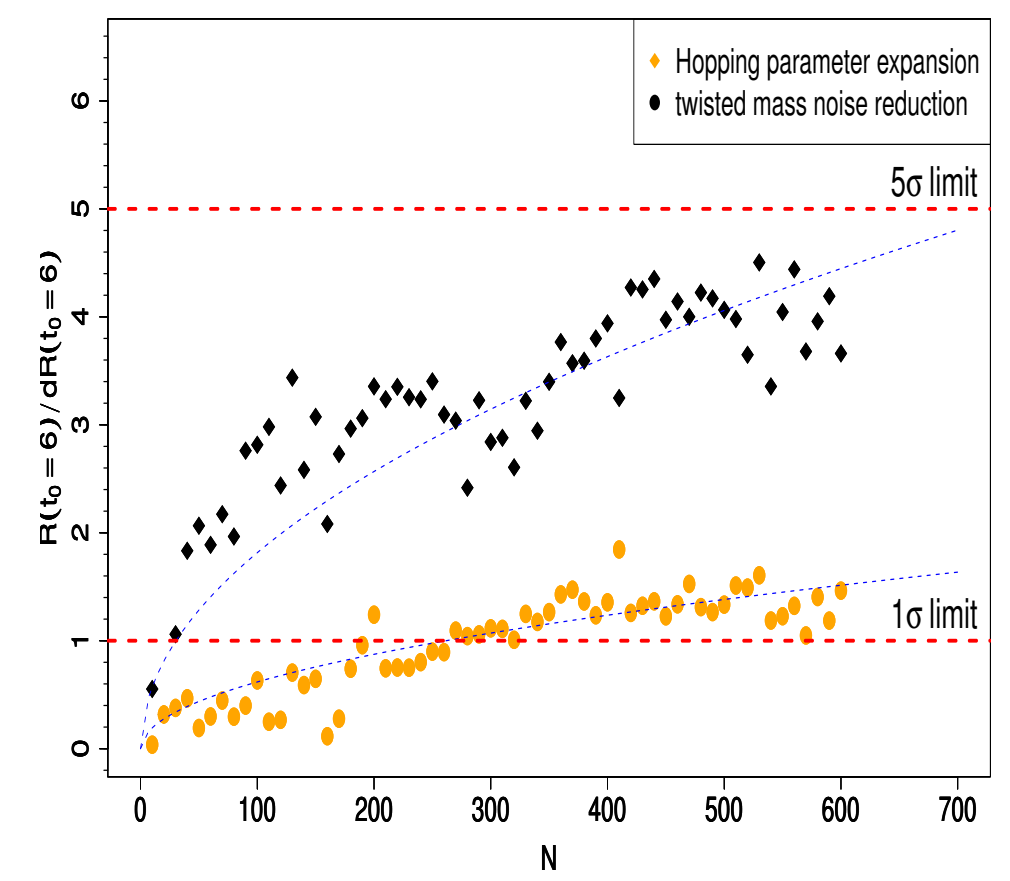

- standard calculation very (too?) difficult

- using techniques for twisted mass fermions

$\rightarrow$ can obtain a signal with reasonable statistics

- remark: calculation of dis-connected contribution to neutral pion mass much easier 


\section{Twisted mass fermions: strange and charm: $\langle N|\bar{s} s| N\rangle,\langle N|\bar{c} c| N\rangle$,}

- charm quark content

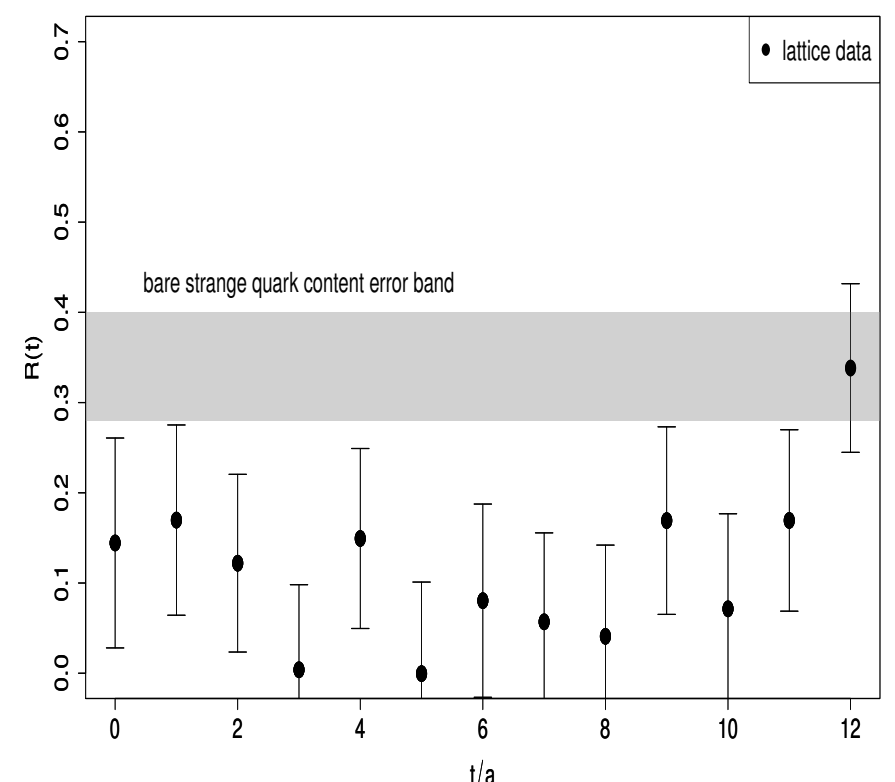

band: plateau value for strange quark content

- $\langle N|\bar{c} c| N\rangle\langle\langle N|\bar{s} s| N\rangle$ (note: still need mulitplication with quark mass)

- $f_{T_{s}}=0.011(2)(1), \sigma_{0}=0.137(2)(3) \mathrm{MeV}, y_{N}=0.065(12)(2)$ (note: all results still need extrapolation to physical point)

- still $y_{N}^{\text {latt }} \ll y_{N}^{\mathrm{XPT}} \rightarrow$ bad news for experiments if value persists in chiral limit

- for twisted mass: no mixing effects in renormalization 


\section{Spectral projectors: $\Sigma$ and $\chi_{\text {topo }}$}

(Giusti, Lüscher; Lüscher, Palombi; Giusti, Rossi, Testa)

- In the continum:

$$
\begin{gathered}
\frac{\Sigma}{\pi}=\lim _{\lambda \rightarrow 0} \lim _{m \rightarrow 0} \lim _{V \rightarrow \infty} \rho(\lambda, m) \\
\rho(\lambda, m)=\frac{1}{V} \sum_{k=1}^{\infty}\left\langle\delta\left(\lambda-\lambda_{k}\right)\right\rangle, \quad \Sigma=-\lim _{m \rightarrow 0} \lim _{V \rightarrow \infty}\langle\bar{u} u\rangle
\end{gathered}
$$

- mode number $\nu \leadsto$ average number of eigenmodes of $D_{m}^{\dagger} D_{m}$ with $\lambda \leq M^{2}$

$$
\nu(M, m)=V \int_{-\Lambda}^{\Lambda} d \lambda \rho(\lambda, m), \quad \Lambda=\sqrt{M^{2}-m^{2}}
$$

$\nu(M, m)=\nu_{R}\left(M_{R}, m_{R}\right) \leadsto$ renormalization-group invariant

- $\Sigma_{R} \propto \frac{\partial}{\partial M_{R}} \nu_{R}$ 


\section{Condensate}

- Spectral Projector $\mathbb{P}_{M}$ to compute $\nu(M, m)$

$$
\nu\left(M, m_{q}\right)=\left\langle\operatorname{Tr}\left\{\mathbb{P}_{M}\right\}\right\rangle
$$

- Approximation of $\mathbb{P}_{M}$ :

$$
\begin{aligned}
\mathbb{P}_{M} & \approx h(\mathbb{K})^{4}, \\
\mathbb{X} & =\frac{2 M^{2}}{D_{m}^{\dagger} D_{m}+M^{2}}-1
\end{aligned}
$$

- $h(x)$ is an approximation to the step function $\theta(x)$ in the interval $[-1,1]$

$$
h(x)=\frac{1}{2}\left\{1-x P\left(x^{2}\right)\right\}
$$

where $P(x)$ is the polynomial which minimizes $\delta=\max _{\epsilon \leq y \leq 1}\|1-\sqrt{y} P(y)\|$

- $\nu\left(M, m_{q}\right)=\left\langle\mathcal{O}_{N}\right\rangle, \quad \mathcal{O}_{N}=\frac{1}{N} \sum_{k=1}^{N}\left(\eta_{k}, \mathbb{P}_{M} \eta_{k}\right)$

$\eta_{k}$ sources generated randomly 


\section{Chiral limit of scalar condensate}

(Cichy, Garcia Ramos, K.J.)

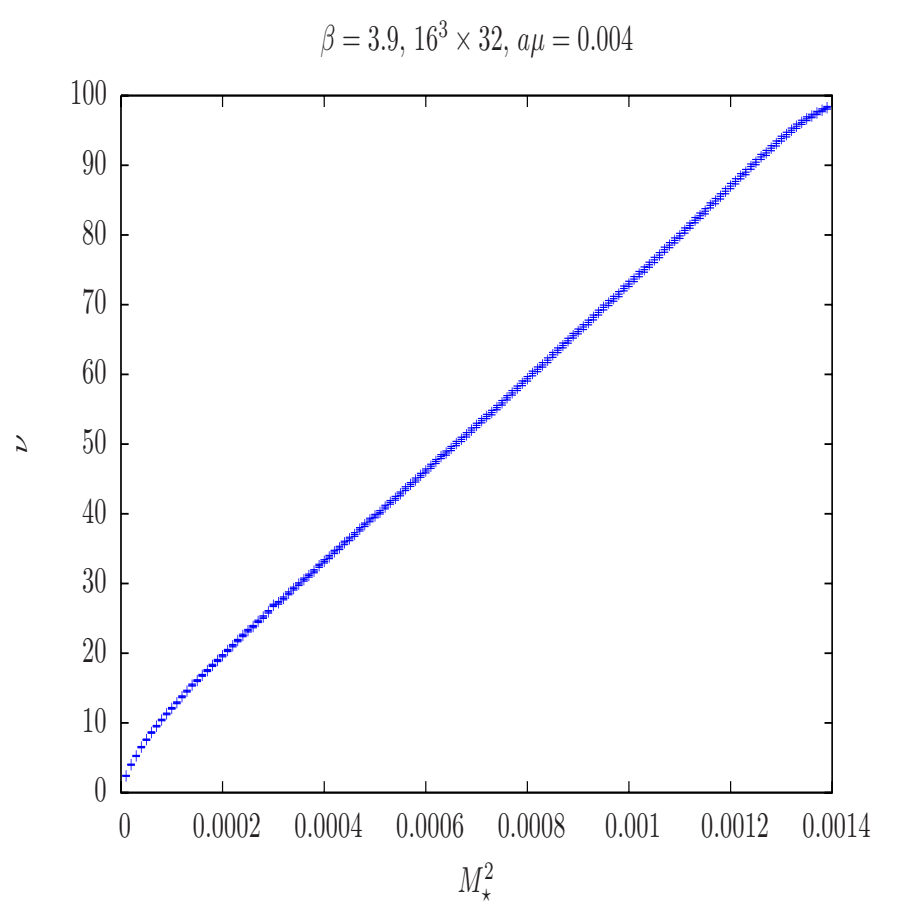

linear behaviour of mode number

slope: $\Sigma_{\text {spectral }}^{\text {ren }}$

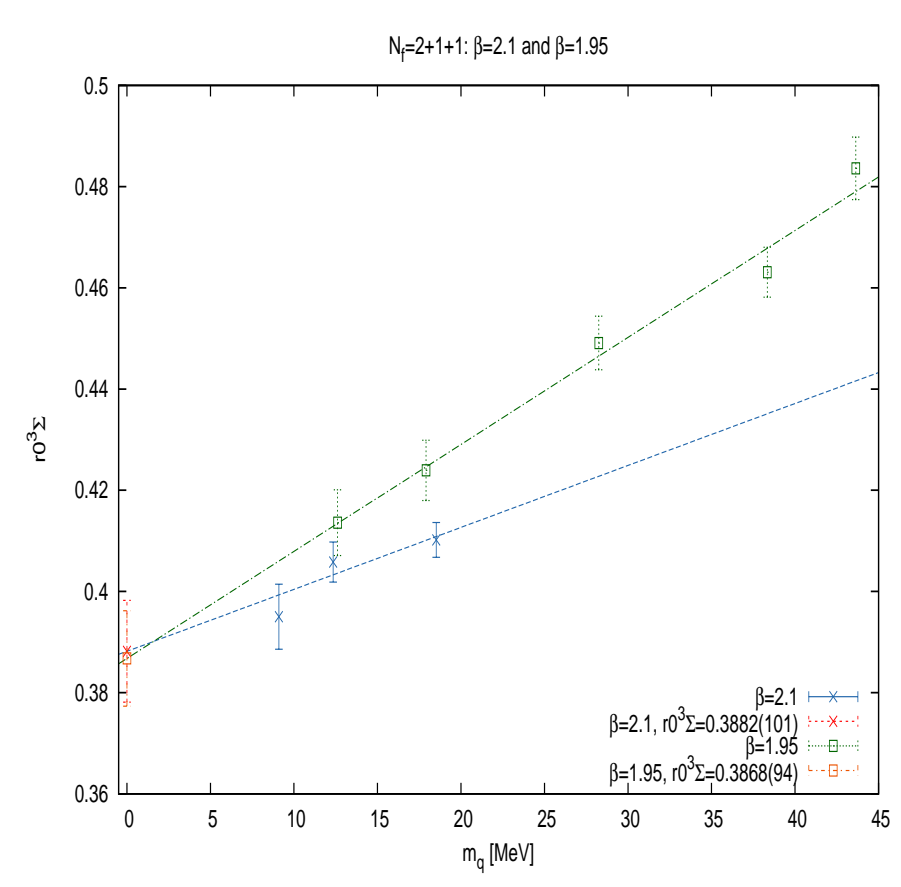

chiral extrapolation of condensate two lattice spacings 


\section{Non-perturbative renormalization for $N_{f}=2+1+1$}

renormalisation factors computed from dedicated $N_{f}=4$ flavour simulations of Wilson fermions

- RI-MOM scheme at non zero values of both the standard and twisted mass parameters

$$
M_{R}=\frac{1}{Z_{\mathrm{P}}} \sqrt{\left(Z_{\mathrm{A}} m_{\mathrm{PCAC}}\right)^{2}+\mu_{q}^{2}} \rightarrow 0
$$

- $O(a)$ improvement via average of simulations with $+m_{\mathrm{PCAC}}$ and $-m_{\mathrm{PCAC}}$

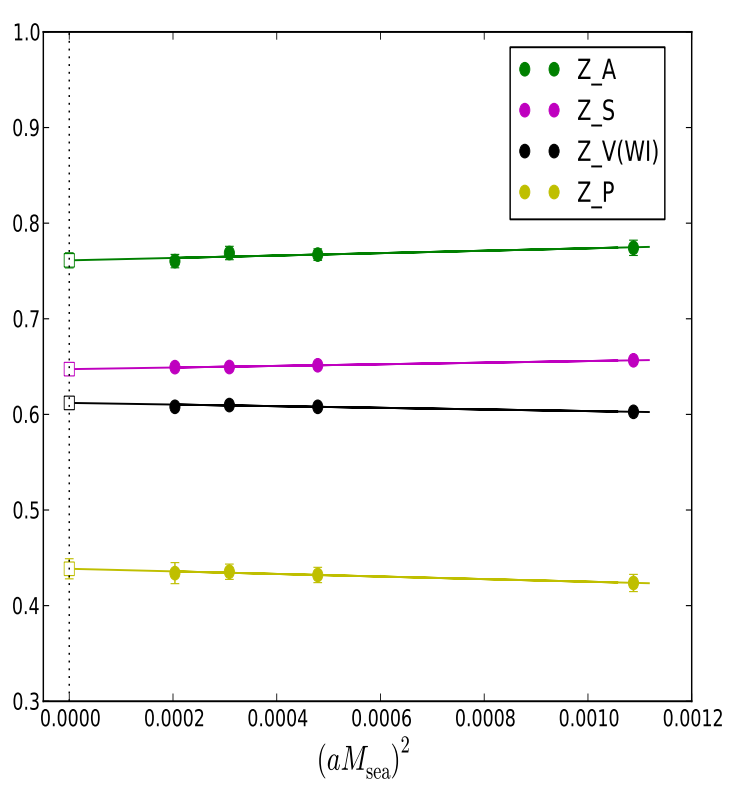

$$
\begin{aligned}
& \text { study at } \beta=1.95 \\
& a=0.08 \mathrm{fm}, L=1.9 \mathrm{fm} \\
& \text { - linear mass dependence } \\
& \text { - allows for chiral extrapolation }
\end{aligned}
$$


Simulation setup for $N_{f}=2+1+1$

\section{Configurations available through ILDG}

\begin{tabular}{lrrrr}
\hline \hline$\beta$ & $a[\mathrm{fm}]$ & $L^{3} T / a^{4}$ & $m_{\pi}[\mathrm{MeV}]$ & therm/production \\
\hline \hline 1.9 & $\approx 0.085$ & $24^{3} 48$ & $300-500$ & $1500 / 5000$ \\
1.95 & $\approx 0.075$ & $32^{3} 64$ & $300-500$ & $1500 / 5000$ \\
2.0 & $\approx 0.065$ & $32^{3} 64$ & 300 & $1500 / 5000$ \\
2.1 & $\approx 0.055$ & $48^{3} 96$ & $230-500$ & $1500 / 5000$ \\
\hline
\end{tabular}

- can go down to $m_{\pi} \approx 230 \mathrm{MeV}$

- see only small lattice spacing scaling

- can perform rich physics programme

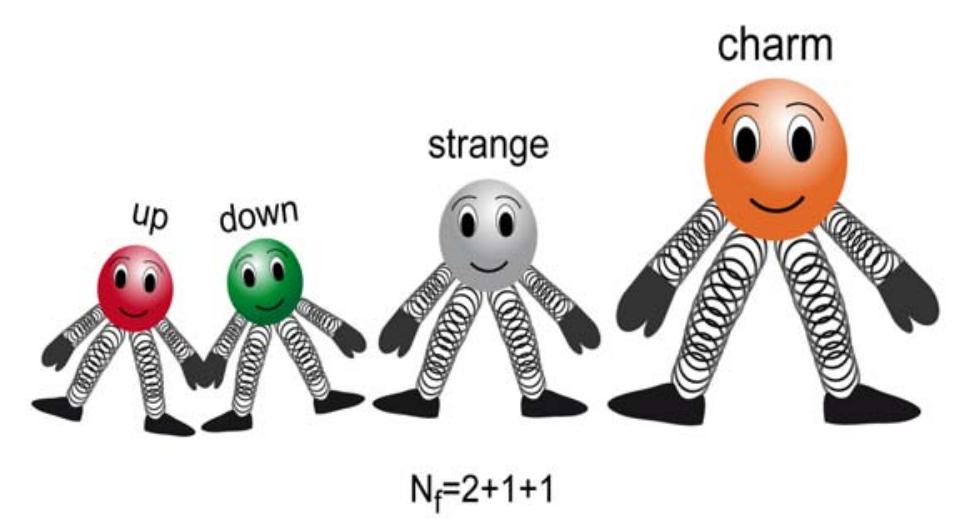




\section{Size of cut-off effects: pion mass splitting}

- question: how fast can we reach the physical point?

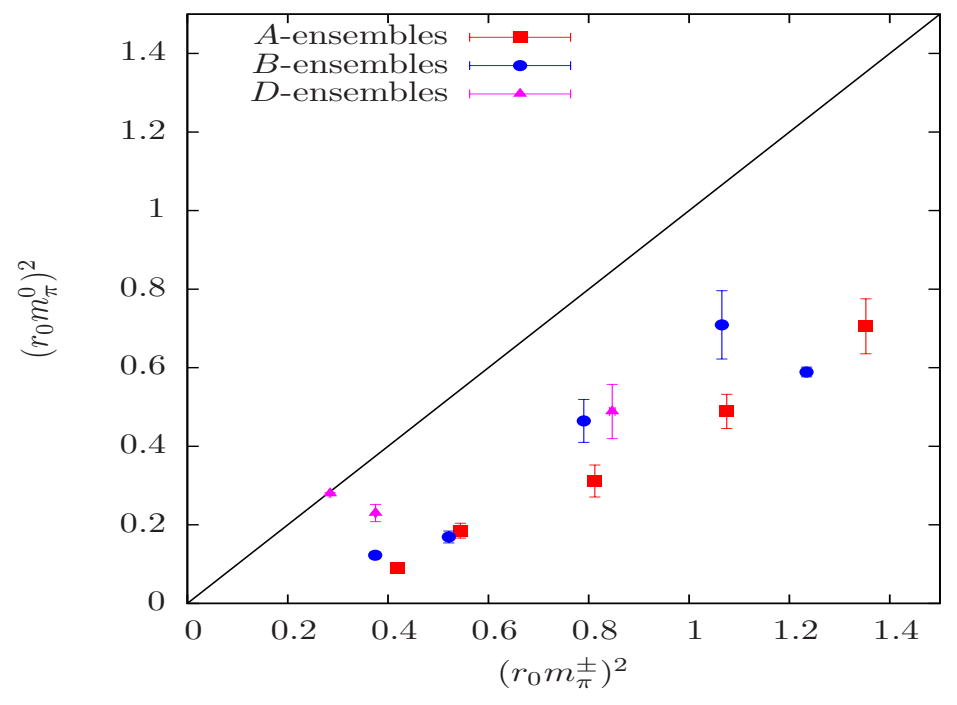

- answer: not fast enough!
- Preliminary!

- $m_{\pi}^{0}<m_{\pi}^{ \pm} \rightarrow c_{2}<0$

significant flavour violations 
Effects of smearing for $N_{f}=2+1+1$

\begin{tabular}{lcccc}
\hline action & $a \mu_{\ell}$ & $\kappa_{\text {crit }}$ & $Z_{V}[\mathrm{WI}]$ & $Z_{P} / Z_{S}$ \\
\hline 0-stout & 0.0060 & 0.163265 & 0.60 & 0.67 \\
& 0.0080 & 0.163260 & & \\
& 0.0080 & 0.163204 & & 0.77 \\
1-stout & 0.0060 & 0.145511 & 0.73 & \\
& 0.0080 & 0.145510 & & 0.86 \\
\hline 4-stout & 0.0015 & 0.136720 & 0.82 & \\
\hline
\end{tabular}

- $\kappa_{\text {crit }} \rightarrow$ moves closer to $1 / 8$

- $Z$ factors $\rightarrow$ move closer to 1

- plaquette behaves much smoother:

$$
\left.\frac{\Delta[\langle P\rangle]}{\Delta\left[(2 \kappa)^{-1}\right]}\right|_{\kappa_{\text {crit }}}=11.4(\text { no stout }) \rightarrow-3.4 \text { (stout) }
$$

- proof-tested that simulations at $m_{\pi} / f_{\pi}=$ physical feasible at lattice spacing $a \approx 0.1 \mathrm{fm}$ 


\section{Summary}

- ETMC successful simulations with $N_{f}=2$ and $N_{f}=2+1+1$ quark flavour

- found good scaling behaviour

- could achieve accurate results for many quantities

- performed dedicated renormalization programme

- simulation at physical point difficult with present action

- developed new (maximally) twisted mass action

- reach $m_{\pi} / f_{\pi}=$ physical at lattice spacing $a \approx 0.1 \mathrm{fm}$

- $m_{\pi}^{0} \approx m_{\pi}^{ \pm}$

- plan new simulations at physical point (+isospin + electromagnetism) 


\title{
Exploring the phases of QCD with many flavors
}

\author{
Xiao-Yong Jin
}

RIKEN Advanced Institute for Computational Science

New Horizons for Lattice Computations with Chiral Fermions

with Robert Mawhinney (Columbia University) 


\section{Introduction}

- Detailed data in:

- My dissertation (2011):

http://academiccommons. columbia. edu/catalog/ac: 137844

- Lattice conference proceedings (2008-2011).

- Goal: Identify the phase (walking or conformal or ...)

- Hadronic observables from zero temperature simulations, including meson propagators and masses, $\pi$ decay constant, Quark potential and string tension...

- Zero temperature and finite temperature

- DBW2 gauge with naïve staggered fermion action 


\section{To see the whole picture}

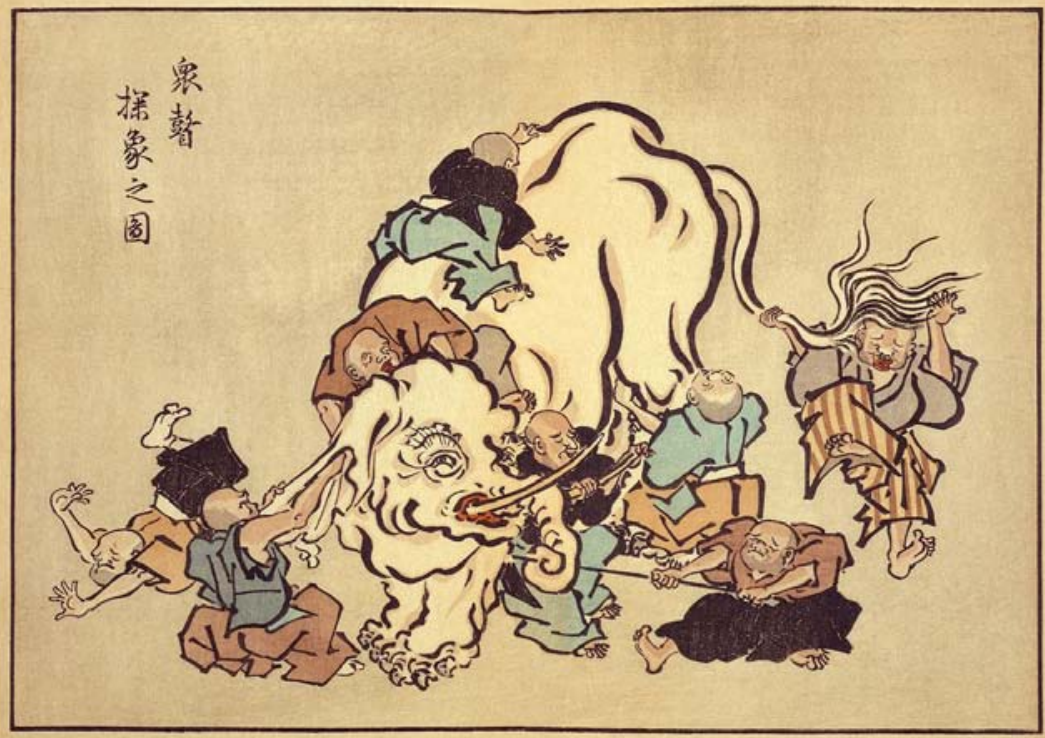




\section{The subject of this talk}

- Understand the direct implication of our data

- Carefully speculate about our blind spots 


\section{Summary of data}

\section{8 flavors}
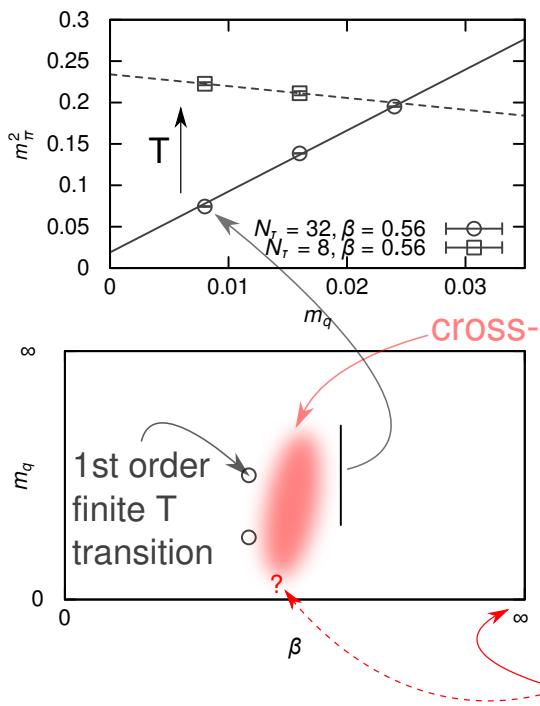

\section{2 flavors}

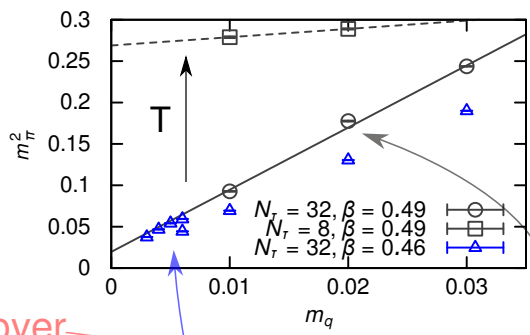




\section{Zero temperature simulations}

\section{8 flavors}

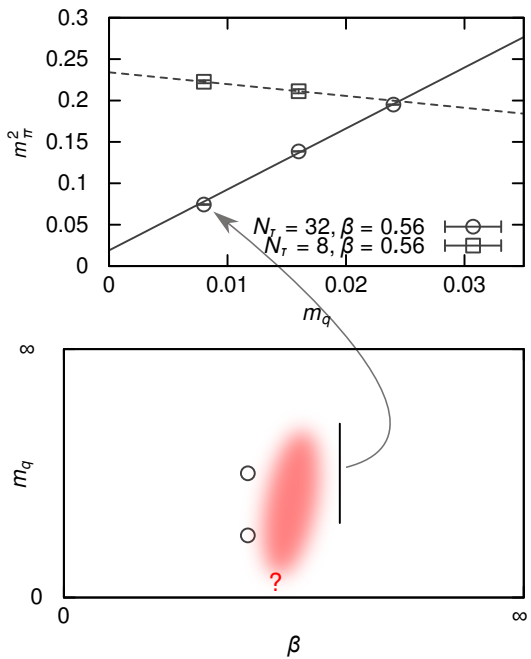

12 flavors

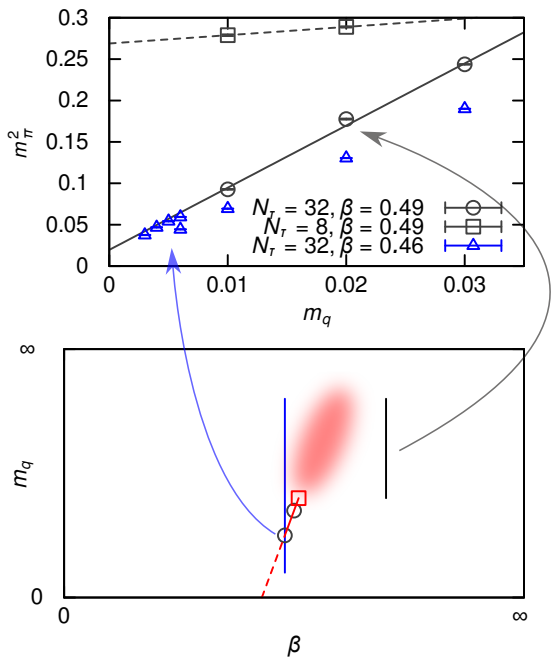




\section{Finite temperature}

\section{8 flavors}

\section{2 flavors}
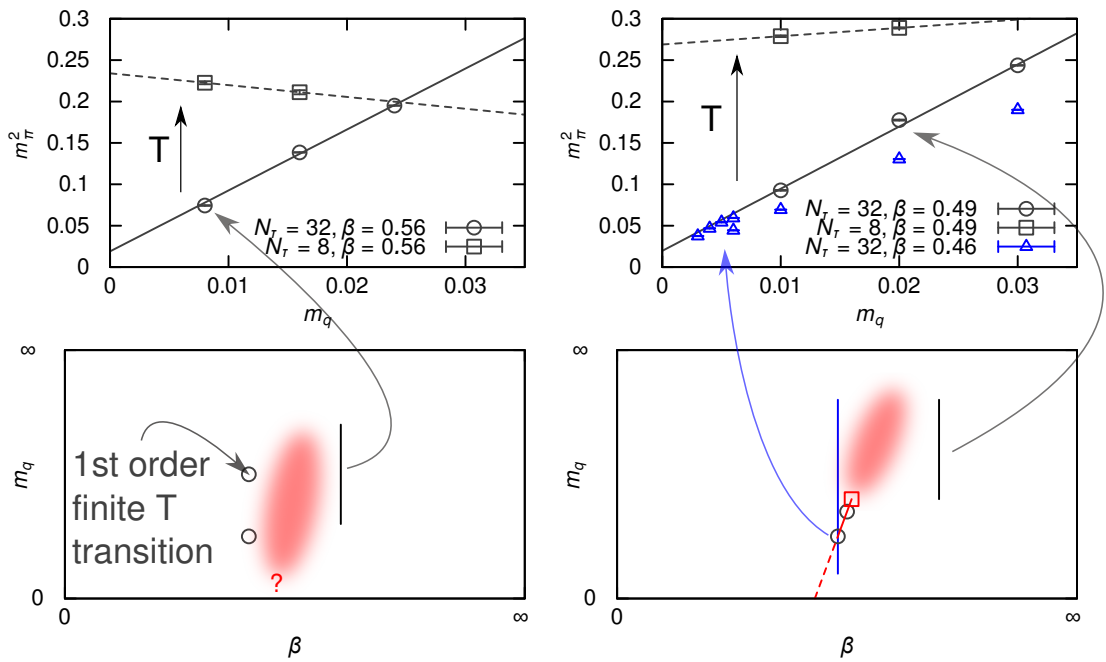


\section{The bulk transition}

\section{8 flavors}

\section{2 flavors}
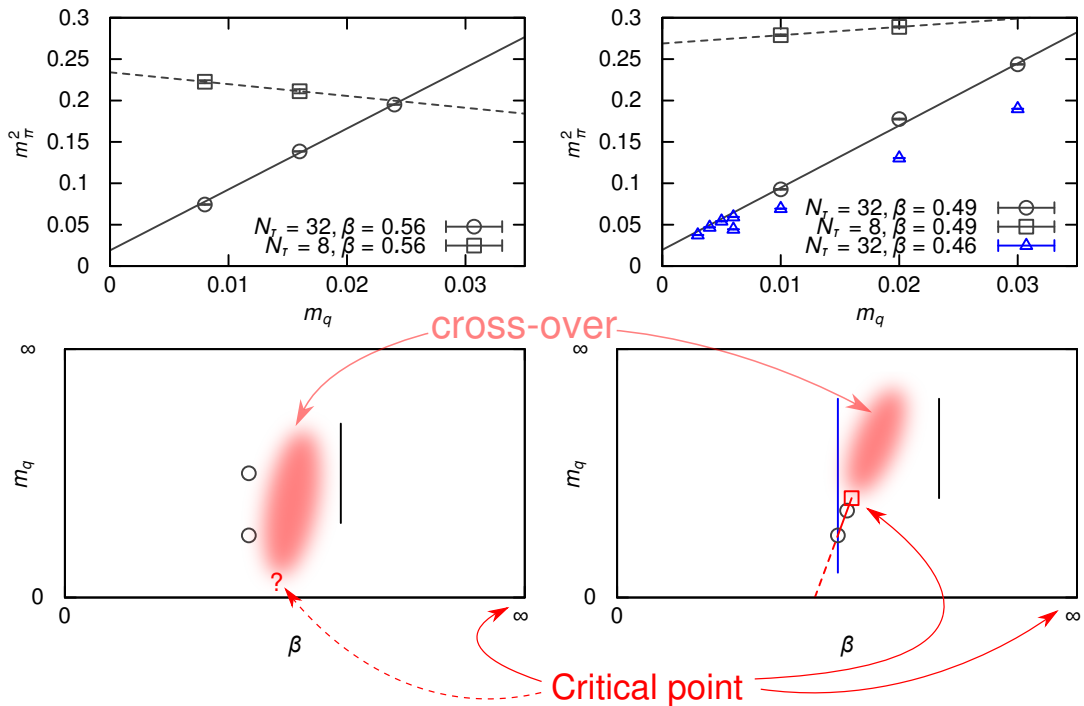


\section{The bulk transition}

- 8 flavors:

- Bulk transition appears (strengthens) when simple plaquette action is used instead of DBW2.

- 12 flavors:

- Volume independent $\rightarrow$ finite temperature effect less likely.

- For most channels, the difference in particle masses on the two sides of the bulk transition become smaller and vanish when quark mass is increased.

- The scalar singlet is found to be special. 


\section{Scalar singlet meson}

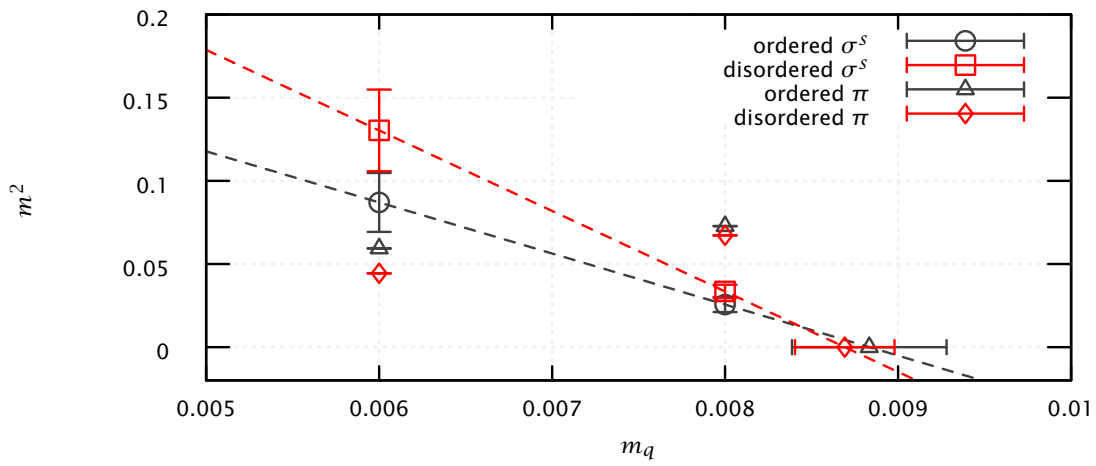

- The scalar singlet meson becomes lighter along the 1 st order bulk transition line approaching the 2 nd order critical end point.

- Critical exponent is consistent with Ginzburg-Landau theory.

- Continuum limit of the lattice theory at second order critical point is likely a free scalar field theory. 


\section{Consequences of a light scalar singlet meson}

\section{Chiral perturbation theory is not applicable.}




\section{Scaling of $m_{\rho_{2}} / f_{\pi}$ versus quark mass}

\section{8 flavors}

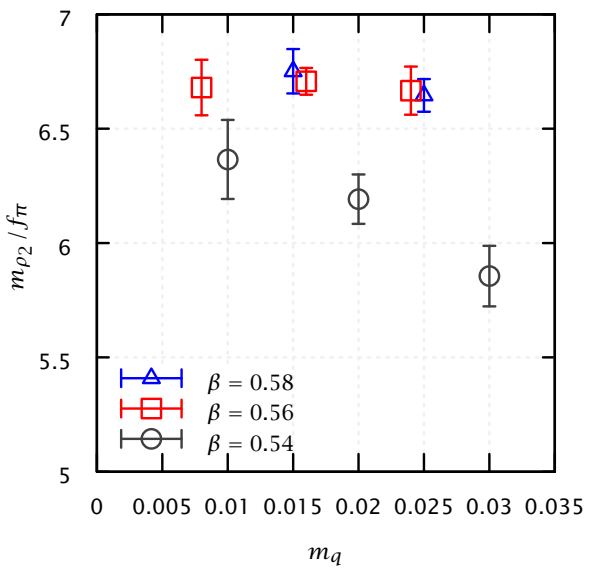

12 flavors

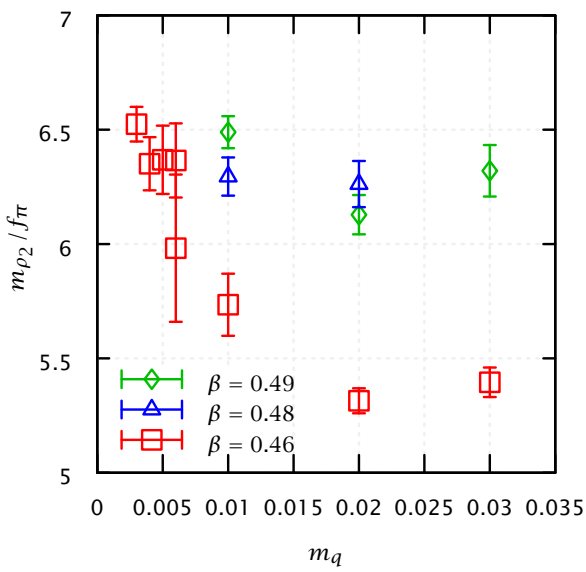

- Approaches to a constant at weak couplings

- Other ratios behave the same, except for pion masses 


\section{Scaling of $m_{\pi} / f_{\pi}$ versus quark mass} 8 flavors

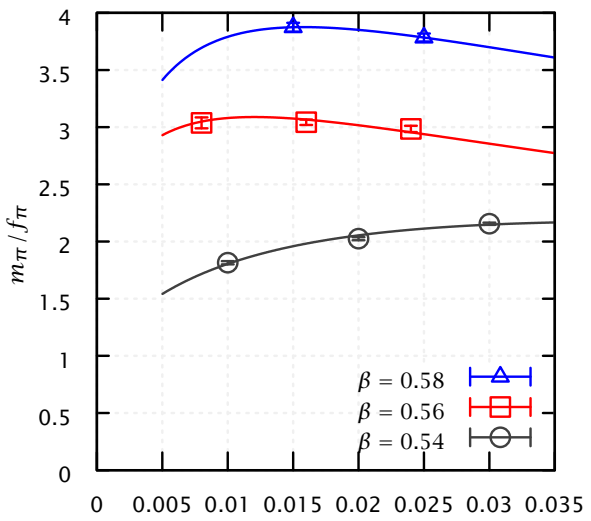

$m_{q}$
12 flavors

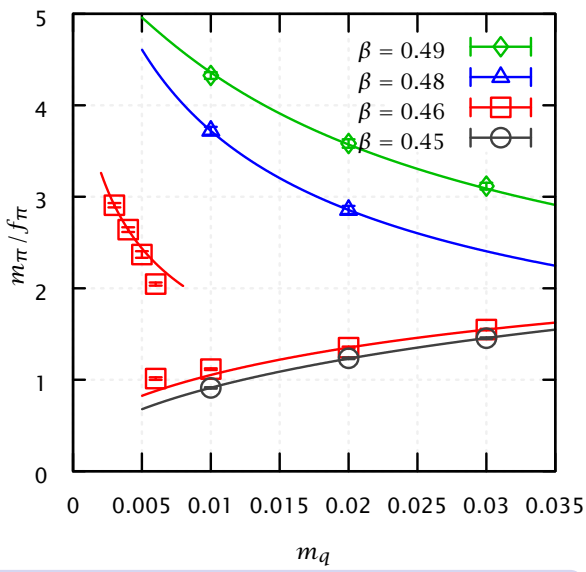

Warnings:

- Backward flow of constant $m_{\pi} / f_{\pi}$ in weaker couplings.

- Misleading conclusions at large $m_{q}$. 


\section{The flow of constant physics $\left(m_{\pi} / f_{\pi}\right)$}

A sketch of $m_{\pi} / f_{\pi}$ surface parameterized by $m$ and $\beta$.

Strong coupling side

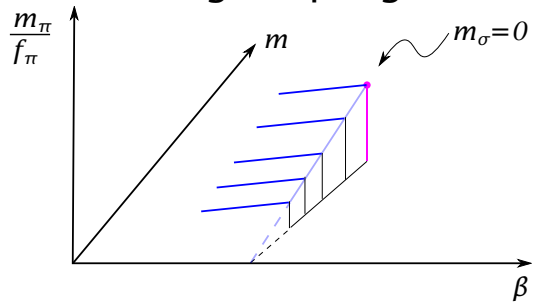

Same as QCD except the $\beta \rightarrow \infty$ cannot be reached, because the bulk transition blocks the way.
Weak coupling side

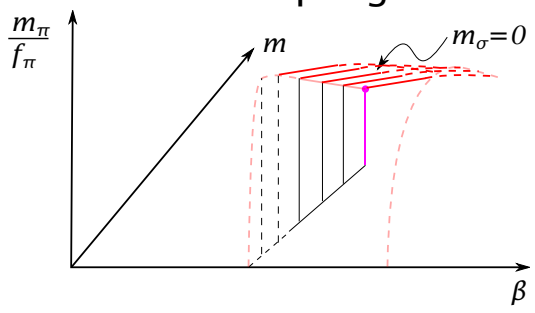

Comparing with 8-flavor data, we conjecture that only when $m_{q}$ is much smaller and $\beta$ is much larger than the bulk transition, can we recover QCD behavior. 


\section{Conjectured lines of constant physics $\left(m_{\pi} / f_{\pi}\right)$}

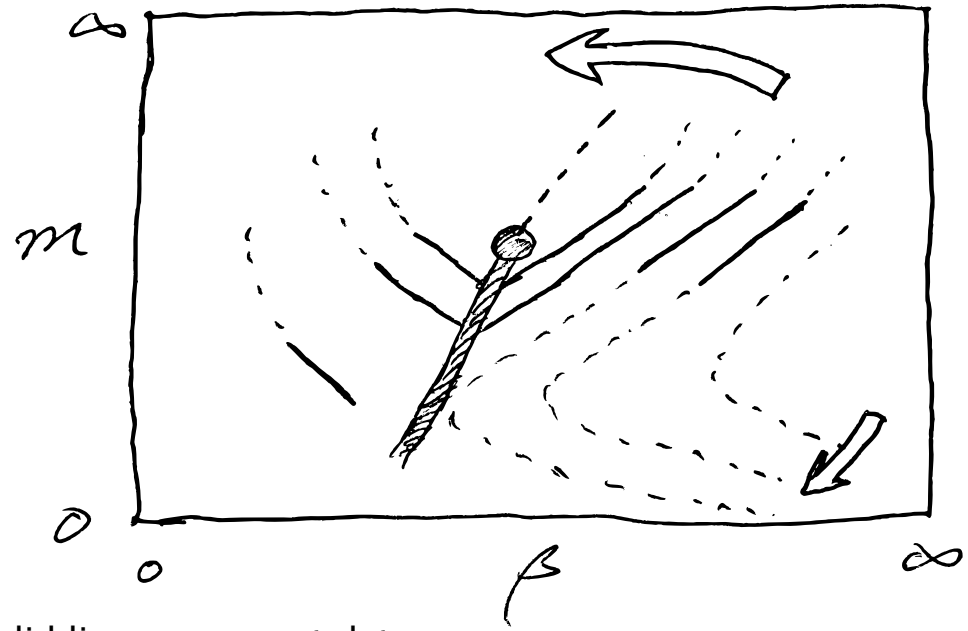

- Solid lines represent data.

- Dashed lines are our conjecture.

- Arrows denotes the direction of $m_{\pi} / f_{\pi}$ going smaller. 


\section{Conclusion}

- Our data show:

- 12-flavor has an additional critical point where scalar correlation length diverges.

- 12-flavor show the same behavior as 8-flavor at larger $m_{q}$.

- We conjecture:

- Possible bulk transition with 8-flavor at smaller $m_{q}$.

- For 12 flavors, much smaller $m_{q}$ and/or much weaker coupling is required to move away from the additional critical point and recover the physics at $\beta \rightarrow \infty$. 


\title{
Kaon semileptonic decays \\ in lattice QCD with exact chiral symmetry
}

\section{T. Kaneko for JLQCD collaboration}

\author{
KEK Theory Center
}

RIKEN BNL Research Center Workshop, May 15, 2012 


\section{1. introduction}

$\underline{K \rightarrow \pi \text { decay form factors }}$

$$
\begin{aligned}
& \left\langle\pi^{+}\left(p^{\prime}\right)\left|V_{\mu}\right| K^{0}(p)\right\rangle=\left(p+p^{\prime}\right)_{\mu} f_{+}\left(q^{2}\right)+\left(p-p^{\prime}\right)_{\mu} f_{-}\left(q^{2}\right), \\
& f_{0}\left(q^{2}\right)=f_{+}\left(q^{2}\right)+\frac{q^{2}}{M_{K}^{2}-M_{\pi}^{2}} f_{-}\left(q^{2}\right), \quad \xi\left(q^{2}\right)=\frac{f_{-}\left(q^{2}\right)}{f_{+}\left(q^{2}\right)}
\end{aligned}
$$

- vector form factor at $q^{2}=0: f_{+}(0) \Leftrightarrow$ determination of $\left|V_{u s}\right|$

- $M_{\pi, \operatorname{sim}} \lesssim M_{K}: \quad N_{f}=3: R B C / U K Q C D, 2008,2010, \quad N_{f}=2: E T M, 2009$ (see also MILC/FNAL/HPQCD, 2005; FNAL/MILC @ Lat'11)

- $\Gamma \Rightarrow\left|V_{u s}\right| f_{+}(0)=0.2163(5) \quad\left(\right.$ FlaviaNet, 2010) $\Leftrightarrow f_{+}(0) \mathrm{w} / \lesssim 1 \%$ accuracy

other information of ME : $f_{-}(0), \quad \lambda_{+, 0}^{\prime \prime \prime} \quad \Leftrightarrow$ consistency with ChPT, exp't 


\section{1. introduction}

\section{this talk}

report on JLQCD's calculation of $K \rightarrow \pi$ form factors in $N_{f}=2+1$ QCD

- overlap quarks $\Rightarrow$ straightforward comparison w/ ChPT $(a=0)$

- all-to-all quark prop. $\Rightarrow$ precise calculation of relevant meson correlators

$\underline{\text { outline }}$

- simulation method

- extraction of form factors

- $q^{2}$ interpolation

- chiral extrapolation 


\section{2. simulation method}

$\underline{\text { configurations }}$

- $N_{f}=2+1 \mathrm{QCD}$

- Iwasaki gauge + overlap quarks $+\operatorname{det}\left[H_{W}^{2}\right] / \operatorname{det}\left[H_{W}^{2}+\mu^{2}\right] \Rightarrow$ speed up, fix $Q$

○ $a=0.1120(5)(3) \mathrm{fm}\left(M_{\Omega}\right.$ as input $) \Leftrightarrow O\left(\left(a \Lambda_{\mathrm{QCD}}\right)^{2}\right) \approx 8 \%$ error 


\section{2. simulation method}

\section{configurations}

- $N_{f}=2+1 \mathrm{QCD}$

- Iwasaki gauge + overlap quarks $+\operatorname{det}\left[H_{W}^{2}\right] / \operatorname{det}\left[H_{W}^{2}+\mu^{2}\right] \Rightarrow$ speed up, fix $Q$

○ $a=0.1120(5)(3) \mathrm{fm}\left(M_{\Omega}\right.$ as input $) \Leftrightarrow O\left(\left(a \Lambda_{\mathrm{QCD}}\right)^{2}\right) \approx 8 \%$ error

\section{$\underline{\text { measurements of meson correlators }}$}

- $4 m_{u d}$ 's $\Rightarrow M_{\pi} \simeq 290-540 \mathrm{MeV} ; \quad m_{s}=0.080 \Leftrightarrow m_{s, \text { phys }}=0.081$

- $16^{3} \times 48$ or $24^{3} \times 48$ (depending on $m_{u d}$ ) $\Rightarrow M_{\pi} L \gtrsim 4$

○ in $Q=0$ sector $\Rightarrow$ fixed $Q$ effects $\propto V^{-1} ;$ sub-\% for $M_{\{\pi, K\}}, f_{\{\pi, K\}}$

- 50 conf $\times 50$ HMC traj. for each $\left(m_{u d}, m_{s}\right)$ 


\section{2. simulation method}

\section{configurations}

- $N_{f}=2+1 \mathrm{QCD}$

- Iwasaki gauge + overlap quarks $+\operatorname{det}\left[H_{W}^{2}\right] / \operatorname{det}\left[H_{W}^{2}+\mu^{2}\right] \Rightarrow$ speed up, fix $Q$

○ $a=0.1120(5)(3) \mathrm{fm}\left(M_{\Omega}\right.$ as input $) \Leftrightarrow O\left(\left(a \Lambda_{\mathrm{QCD}}\right)^{2}\right) \approx 8 \%$ error

\section{$\underline{\text { measurements of meson correlators }}$}

- $4 m_{u d}$ 's $\Rightarrow M_{\pi} \simeq 290-540 \mathrm{MeV} ; \quad m_{s}=0.080 \Leftrightarrow m_{s, \text { phys }}=0.081$

- $16^{3} \times 48$ or $24^{3} \times 48$ (depending on $m_{u d}$ ) $\Rightarrow M_{\pi} L \gtrsim 4$

○ in $Q=0$ sector $\Rightarrow$ fixed $Q$ effects $\propto V^{-1} ;$ sub-\% for $M_{\{\pi, K\}}, f_{\{\pi, K\}}$

- 50 conf $\times 50$ HMC traj. for each $\left(m_{u d}, m_{s}\right)$

o w/ all-to-all propagator $\Rightarrow 160-240$ low-modes + noise method

- twisted boundary conditions (TBCs) $\Rightarrow q^{2} \in\left[-0.1 \mathrm{GeV}^{2}, q_{\max }^{2}\right]$

o reweighting $\Rightarrow$ additional $m_{s}=0.060$ 


\section{1 ratio method}

$$
\begin{aligned}
& C_{V_{\mu}}^{P Q}\left(\mathbf{p}, \mathbf{p}^{\prime}\right)=\left\langle\mathcal{O}_{Q}\left(\mathbf{p}^{\prime}\right) V_{\mu, \text { lat }} \mathcal{O}_{P}^{\dagger}(\mathbf{p})\right\rangle \sim \frac{\sqrt{Z_{Q}\left(\mathbf{p}^{\prime}\right) Z_{P}(\mathbf{p})}}{4 E_{P} E_{Q} Z_{V}}\left\langle Q\left(\mathbf{p}^{\prime}\right)\left|V_{\mu}\right| P(\mathbf{p})\right\rangle e^{-E_{Q} \Delta t^{\prime}-E_{P} \Delta t} \\
& C^{P}(\mathbf{p})=\left\langle\mathcal{O}_{P}\left(\mathbf{p}^{\prime}\right) \mathcal{O}_{P}^{\dagger}(\mathbf{p})\right\rangle \sim \frac{Z_{P}}{2 E_{P}} e^{-E_{Q} \Delta t^{\prime}} \quad(P, Q=K \text { or } \pi)
\end{aligned}
$$

ratio method (Hashimoto et al., 1999)

(partially) cancel $Z_{V}, \exp \left[-E_{\pi(K)} \Delta t\right]$, fluctuation in $C_{V_{\mu}}^{P Q}\left(\mathbf{p}, \mathbf{p}^{\prime}\right)$ and $C^{P}(\mathbf{p})$

$$
\begin{aligned}
& R=\frac{C_{V_{4}}^{K \pi}(\mathbf{0}, \mathbf{0}) C_{V_{4}}^{\pi K}(\mathbf{0}, \mathbf{0})}{C_{V_{4}}^{K K}(\mathbf{0}, \mathbf{0}) C_{V_{4}}^{\pi \pi}(\mathbf{0}, \mathbf{0})} \rightarrow \frac{\left(M_{K}+M_{\pi}\right)^{2}}{4 M_{K} M_{\pi}} f_{0}\left(q_{\max }^{2}\right)^{2} \quad\left(q_{\max }^{2}=\left(M_{K}-M_{\pi}\right)^{2}\right) \\
& \tilde{R}=\frac{C_{V_{4}}^{K \pi}\left(\mathbf{p}, \mathbf{p}^{\prime}\right) C^{K}(\mathbf{0}) C^{\pi}(\mathbf{0})}{C_{V_{4}}^{K \pi}(\mathbf{0}, \mathbf{0}) C^{K}(\mathbf{p}) C^{\pi}\left(\mathbf{p}^{\prime}\right)} \rightarrow\left\{1+\frac{E_{K}(\mathbf{p})-E_{\pi}\left(\mathbf{p}^{\prime}\right)}{E_{K}(\mathbf{p})+E_{\pi}\left(\mathbf{p}^{\prime}\right)} \xi\left(q^{2}\right)\right\} \frac{f_{+}\left(q^{2}\right)}{f_{0}\left(q_{\max }^{2}\right)} \\
& R_{k}=\frac{C_{V_{k}}^{K \pi}\left(\mathbf{p}, \mathbf{p}^{\prime}\right) C_{V_{4}}^{K K}\left(\mathbf{p}, \mathbf{p}^{\prime}\right)}{C_{V_{4}}^{K \pi}\left(\mathbf{p}, \mathbf{p}^{\prime}\right) C_{V_{k}}^{K K}\left(\mathbf{p}, \mathbf{p}^{\prime}\right)} \rightarrow \text { a function of } \xi\left(q^{2}\right)
\end{aligned}
$$

$\Rightarrow$ can construct $f_{+}\left(q^{2}\right), f_{0}\left(q^{2}\right), \xi\left(q^{2}\right)$ 


\section{2 extraction of form factors : statistical accuracy}

all-to-all propagator $\Rightarrow$ can improve stat. accuracy

$C_{V_{\mu}}^{K \pi}$ at each jackknife sample

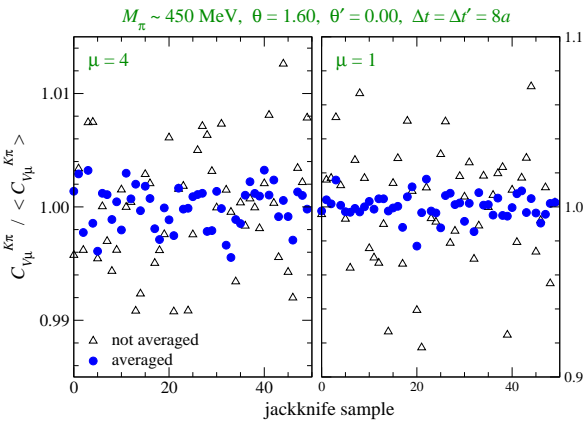

$\xi\left(q^{2} ; \Delta t, \Delta t^{\prime}\right)$ vs $\Delta t$

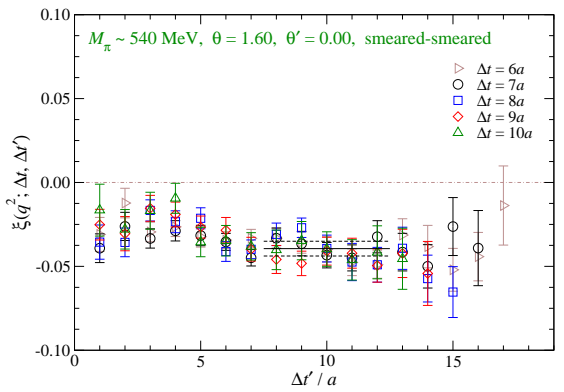

- all-to-all $\Rightarrow$ average over location of meson source op.

$\Leftrightarrow$ a factor of 3 (4) improvement for $\mu=4$ (1)

- clear signal for $\xi\left(q^{2}\right): 10-30 \%$ (comparison w/ exp't (later))

- $f_{\{+, 0\}}\left(q^{2}\right)$ : statistical accuracy sub-\% level

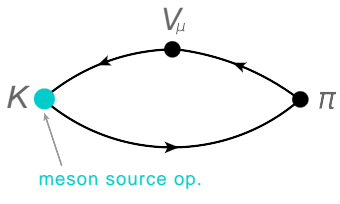




\section{2 extraction of form factors : statistical accuracy}

reweighting $\Rightarrow$ can impair stat. accuracy

$$
\begin{gathered}
\left\langle C_{V_{\mu}}^{P Q}\right\rangle_{m_{s}^{\prime}}=\left\langle C_{V_{\mu}}^{P Q} \tilde{w}\left(m_{s}^{\prime}, m_{s}\right)\right\rangle_{m_{s}} \\
\tilde{w}\left(m_{s}^{\prime}, m_{s}\right)=\frac{w\left(m_{s}^{\prime}, m_{s}\right)}{\left\langle w\left(m_{s}^{\prime}, m_{s}\right)\right\rangle_{m_{s}}} \\
w\left(m_{s}^{\prime}, m_{s}\right)=\operatorname{det}\left[D\left(m_{s}^{\prime}\right)\right] / \operatorname{det}\left[D\left(m_{s}\right)\right] \\
=\frac{\prod_{k=1}^{N_{e}} \lambda_{k}\left(m_{s}^{\prime}\right)}{\prod_{k=1}^{N_{e}} \lambda_{k}\left(m_{s}\right)} \times \frac{1}{N_{r}} \sum_{r=1}^{N_{r}} e^{-\frac{1}{2} \xi_{r}^{\dagger} \frac{D\left(m_{s}^{\prime}\right)}{D\left(m_{s}\right)} \xi_{r}}
\end{gathered}
$$

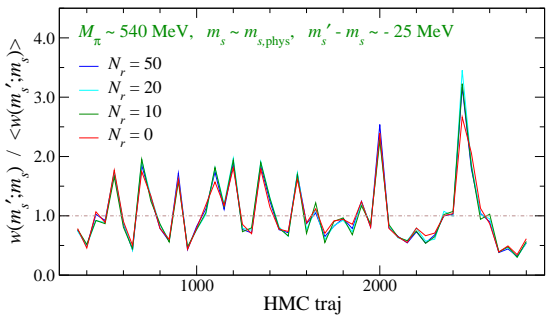

- $24^{3}\left(16^{3}\right) \times 48, \approx 200$ low-modes

$\Rightarrow$ do not need large $N_{r}$

- largely increase $\Delta\left[C_{V_{\mu}}^{P Q}\right]$

o at most $\times 2$ for ratios

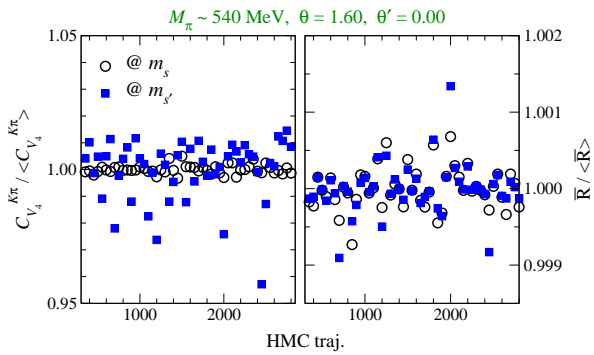

$$
\text { all-to-all + ratio + reweight } \Rightarrow \Delta\left[f_{+, 0}\left(q^{2}\right)\right] \lesssim 1.5 \%, \quad \Delta\left[\xi\left(q^{2}\right)\right] \sim 20-40 \%
$$




\section{4. $q^{2}$-dependence}

\section{$f_{0}\left(q^{2}\right)$ vs $q^{2}$}
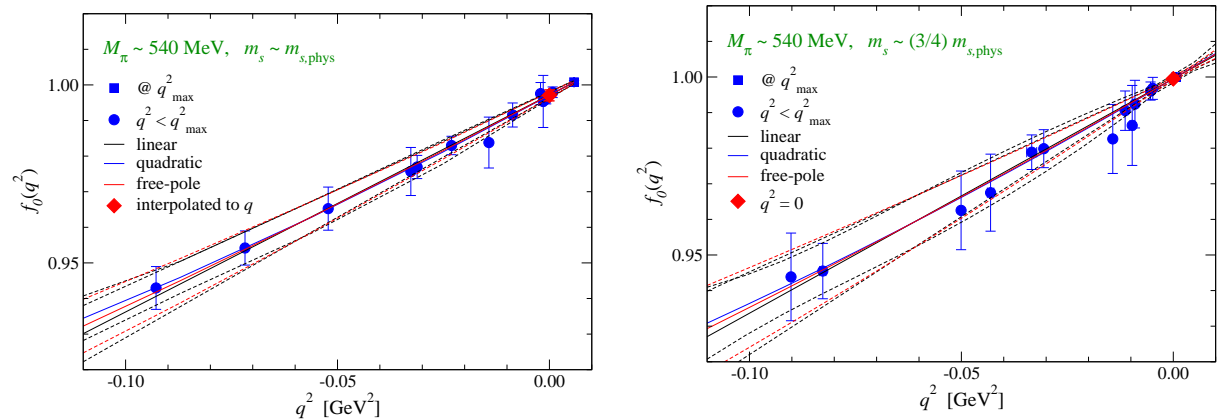

- reweighting $\Rightarrow$ slightly larger error

- small curvature in simulated region of $q^{2}$

$\Leftrightarrow M_{\text {pole }}=1.2-1.3 \mathrm{GeV} @ m_{q, \text { phys }}(P D G, 2010) \Rightarrow q^{4} / M_{\text {pole }}^{4} \lesssim 0.6 \%$

- well described by any of

$$
f_{0}\left(q^{2}\right)=f_{0}(0)\left(1+a_{1} q^{2}\right), \quad f_{0}(0)\left(1+a_{1} q^{2}+a_{2} q^{4}\right), \quad \frac{f_{0}(0)}{1-q^{2} / M_{\text {pole }}^{2}}
$$




\section{4. $q^{2}$-dependence}

\section{$f_{0}\left(q^{2}\right)$ vs $q^{2}$}

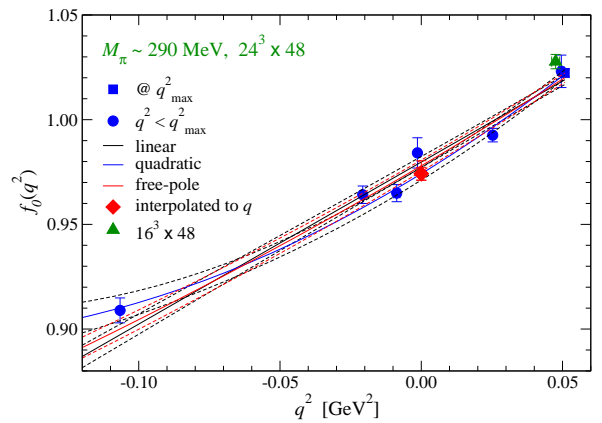

fit results for $f_{+}(0)$

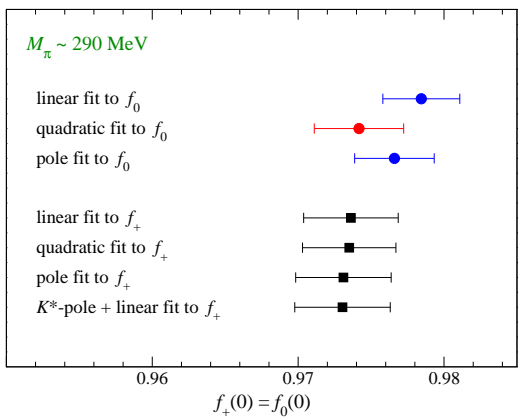

- $f_{+}(0) \quad\left(=f_{0}(0)\right), \quad d f_{+, 0}(0) /\left.d q^{2}\right|_{q^{2}=0}:$ stable against choice of fit form

- small finite volume effects $\Leftrightarrow$ lattice boundaries + fixed $Q$ $16^{3} \times 48 \Leftrightarrow 24^{3} \times 48: \quad 0.4-0.7 \%(\lesssim 2.2-2.5 \sigma) \quad \Rightarrow$ even smaller on $24^{3} \times 48(?)$

- this talk: quadratic fit $\Rightarrow f_{+}(0), d f_{+}\left(q^{2}\right) /\left.d q^{2}\right|_{q^{2}=0}$ 


\section{1 chiral fit : $f_{+}(0)$}

chiral expansions (cf. JLQCD, 2008)

$$
f_{+}(0)=1+f_{2}+f_{4}+\cdots=1+f_{2}+\Delta f
$$

- $x_{\pi(K)} \equiv M_{\pi(K)}^{2} /\left(4 \pi F_{0}\right)^{2} \quad$ (“x-expansion”) small $F_{0}=52.5(5.1) \mathrm{MeV} \ll F_{\pi} \Rightarrow$ enhanced chiral corrections $\mathcal{O}_{2 n}=\left(M_{\pi} / F_{0}\right)^{2 n}$

- $\xi_{\pi(K)} \equiv M_{\pi(K)}^{2} /\left(4 \pi F_{\pi}\right)^{2} \quad$ (“ $\xi$-expansion”) better convergence for $M_{\{\pi, K\}}, F_{\{\pi, K\}} \quad$ (JLQCD, 2008 $\left.\left(N_{f}=2\right) ; 2009\left(N_{f}=2+1\right)\right)$ 


\section{1 chiral fit : $f_{+}(0)$}

\section{chiral expansions (cf. JLQCD, 2008)}

$$
f_{+}(0)=1+f_{2}+f_{4}+\cdots=1+f_{2}+\Delta f
$$

- $x_{\pi(K)} \equiv M_{\pi(K)}^{2} /\left(4 \pi F_{0}\right)^{2} \quad$ (“ $x$-expansion")

small $F_{0}=52.5(5.1) \mathrm{MeV} \ll F_{\pi} \Rightarrow$ enhanced chiral corrections $\mathcal{O}_{2 n}=\left(M_{\pi} / F_{0}\right)^{2 n}$

- $\xi_{\pi(K)} \equiv M_{\pi(K)}^{2} /\left(4 \pi F_{\pi}\right)^{2} \quad$ (“ $\xi$-expansion”)

better convergence for $M_{\{\pi, K\}}, F_{\{\pi, K\}} \quad$ (JLQCD, 2008 $\left.\left(N_{f}=2\right) ; 2009\left(N_{f}=2+1\right)\right)$

\section{fitting w/ -expansion}

- NLO (Gasser-Leutwyler, 1985)

Ademollo-Gatto $\Rightarrow f_{2}\left(F_{\pi}, M_{\{\pi, K, \eta\}}\right)$

- NNLO and higher orders

(Post-Schilcher, 2001; Bijnens-Talavera, 2003)

$$
\Delta f\left(F_{\pi},\left\{L_{i}\right\},\left\{C_{i}\right\}, M_{\{\pi, K, \eta\}}\right)
$$

- modeling $\Delta f$ (as in previous studies)

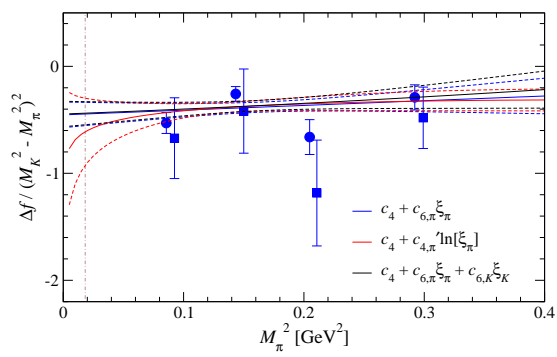
$\Delta f=\left(M_{K}^{2}-M_{\pi}^{2}\right)^{2}\left(c_{4}\right.$
$+c_{4}^{\prime} \ln \left[\xi_{\pi}\right]$,
$c_{4}^{\prime \prime} \ln ^{2}\left[\xi_{\pi}\right]$,
$c_{6, \pi} \xi_{\pi}$,
$\left.c_{6, K} \xi_{K}\right)$ 


\section{1 chiral fit : $f_{+}(0)$}

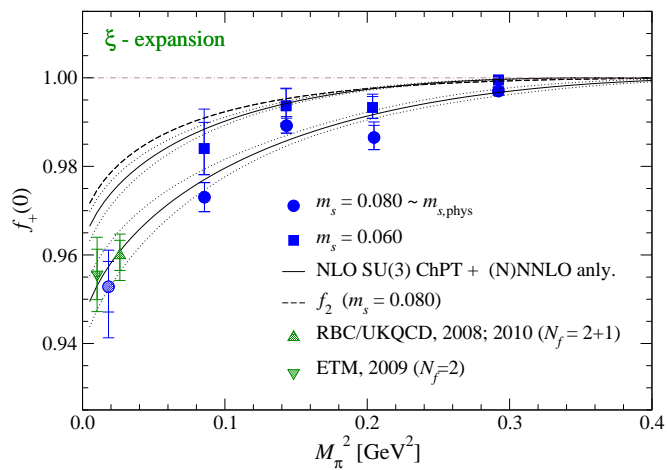

\section{fit results}

- test parameterizations of $\Delta f$

- $c_{4}+c_{6, \pi} \xi_{\pi} \Rightarrow$ central value

- $c_{4}$

- $c_{4}+c_{6, \pi} \xi_{\pi}+c_{6, K} \xi_{K}$

- $c_{4}+c_{4, \pi}^{\prime} \ln \left[\xi_{\pi}\right]$ (ill-determined $c_{X}$ )

- fit excluding largest $m_{u d}$

- assume $O\left((a \Lambda)^{2}\right)$ error in $f_{2}+\Delta f$ $f_{+}(0)=0.953(6)_{\text {stat }}\left(\begin{array}{l}+4 \\ -9\end{array}\right)_{\text {chiral }}(4)_{a \neq 0}$ 


\section{1 chiral fit : $f_{+}(0)$}

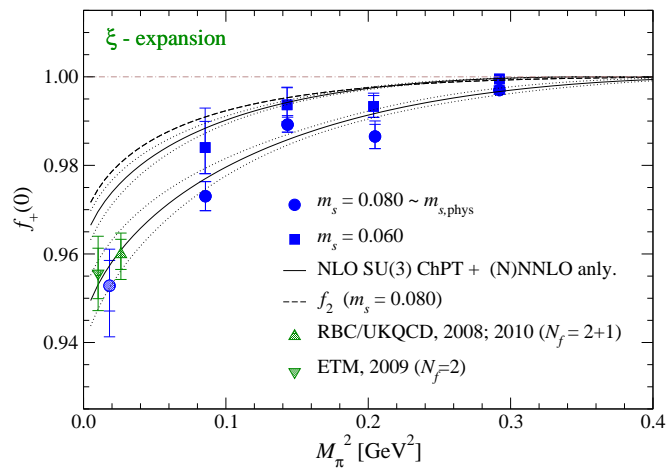

\section{fit results}

- test parameterizations of $\Delta f$

- $c_{4}+c_{6, \pi} \xi_{\pi} \Rightarrow$ central value

- $c_{4}$

- $c_{4}+c_{6, \pi} \xi_{\pi}+c_{6, K} \xi_{K}$

- $c_{4}+c_{4, \pi}^{\prime} \ln \left[\xi_{\pi}\right]$ (ill-determined $c_{X}$ )

- fit excluding largest $m_{u d}$

- assume $O\left((a \Lambda)^{2}\right)$ error in $f_{2}+\Delta f$

$$
f_{+}(0)=0.953(6)_{\text {stat }}\left({ }_{-9}^{+4}\right)_{\text {chiral }}(4)_{a \neq 0}
$$

CKM unitarity

$\left|V_{u s}\right| f_{+}(0)=0.2163(5) \quad\left(\right.$ FlaviaNet, 2010) $\quad \Rightarrow \quad\left|V_{u s}\right|=0.2270(+20 /-27)$

$\left|V_{u d}\right|=0.9743(2), \quad\left|V_{u b}\right| \sim 10^{-3} \quad$ (ICHEP'10)

$\Delta_{\mathrm{CKM}}=\left|V_{u d}\right|^{2}+\left|V_{u s}\right|^{2}+\left|V_{u b}\right|^{2}-1=0.0007(+9 /-13)$ 


\section{2 chiral fit : $\left\langle r^{2}\right\rangle_{V}^{K \pi}$}

$\underline{\text { slope of } f_{+}\left(q^{2}\right)}$

- $f_{+}\left(q^{2}\right)=f_{+}(0)\left\{1+(1 / 6)\left\langle r^{2}\right\rangle_{V}^{K \pi} q^{2}+\cdots\right\}$

- SU(3) NLO ChPT (Gasser-Leutwyler, 1985) + NNLO analy., $\xi$-expansion, $\left\langle r^{2}\right\rangle_{V}^{K \pi}=12 L_{9}^{r} / F_{\pi}^{2}-\left(1 / F_{\pi}^{2}\right)$ "logs" $+c_{\pi} \xi_{\pi}+c_{K} \xi_{K}$

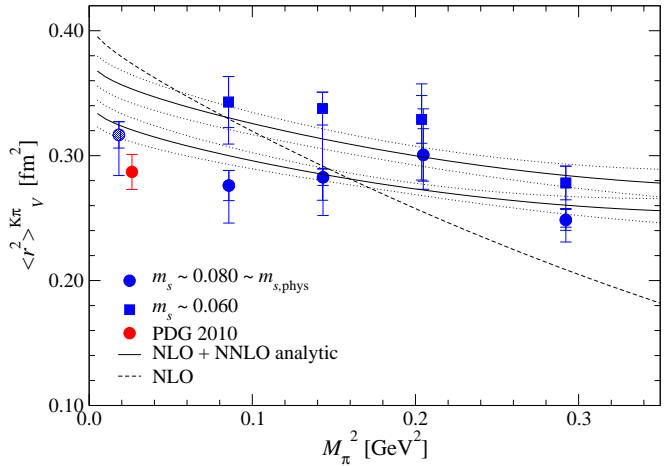

T. Kaneko
- significant NNLO @ $m_{u d(s), s i m}$

$\Rightarrow$ milder $m_{u d}$-dependence

$\Leftrightarrow\left\langle r^{2}\right\rangle_{V}^{\pi},\left\langle r^{2}\right\rangle_{V}^{K} \quad\left(J L Q C D, N_{f}=2,3\right)$

- $L_{9}^{r}\left(M_{\rho}\right)$ : consistent $\mathrm{w} /$ pheno.

- $L_{9}^{r}=6.2(0.5)(2.7) \times 10^{-3}$

o pheno. : $5.91(4) \times 10^{-3}$

(Bijnens, 2007, $\xi$-expansion)

- $\left\langle r^{2}\right\rangle_{V}^{K \pi}$ : consistent w/ exp't 
5.3 chiral fit : $\xi(0)=f_{-}(0) / f_{+}(0)$

\section{$\xi\left(q^{2}\right)$ vs $q^{2}$}

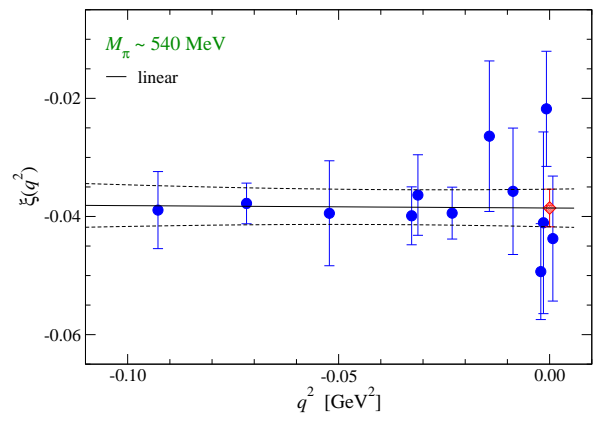

$\underline{\xi(0) \text { vs } M_{K}^{2}-M_{\pi}^{2}}$

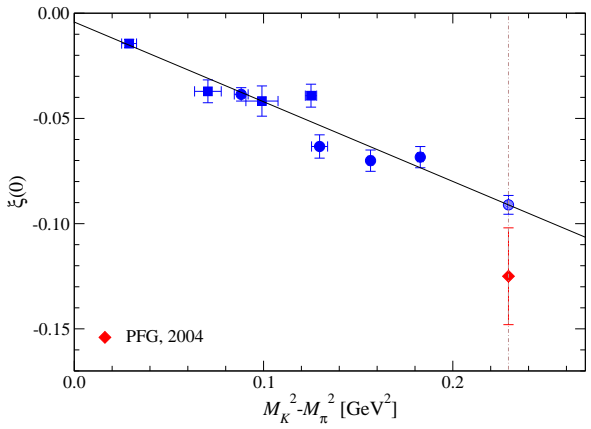

- mild $q^{2}$ dependence $\mathrm{w} /$ our statistical accuracy

cf. $f_{-, \text {analy }}\left(q^{2}\right)=\left(M_{K}^{2}-M_{\pi}^{2}\right)\left(4 L_{5}^{r} / F_{\pi}^{2}-2 L_{9}^{r} / F_{\pi}^{2}\right) \quad$ (Bijnens-Talavera 2003)

- (for simplicity) fit form linear in $\left(M_{K}^{2}-M_{\pi}^{2}\right): \xi(0)=c_{0}+c_{1}\left(M_{K}^{2}-M_{\pi}^{2}\right)$

- vanish in SU(3) limit as expected : $c_{0}=-0.0017(9)$

- consistent $\mathrm{w} /$ experiment : $\xi(0)=-0.125(23)$ (PDG 2004, $K_{l 3}^{+}$)

- one-loop chiral log.s (and two-loops?) should be included (Bijnens-Talavera 2003) 


\section{6. summary}

kaon semileptonic form factors in $N_{f}=2+1 \mathrm{QCD}$ with overlap quarks

- techniques

- all-to-all propagators $\Rightarrow$ precise determination of $f_{+, 0}\left(q^{2}\right)$

- TBCs $\Rightarrow$ precise determination of $f_{+}(0), \xi(0),\left\langle r^{2}\right\rangle_{V, S}^{K \pi}$

- reweighting $\Rightarrow$ additional $m_{s}$

o chiral fits

- $f_{+}(0)=0.953(6)_{\text {stat }}\left({ }_{-9}^{+4}\right)_{\text {chiral }}(4)_{\mathrm{a} \neq 0}, \quad\left|V_{u s}\right|=0.2270\left({ }_{-26}^{+17}\right)$, $\Delta_{\mathrm{CKM}}=\left|V_{u d}\right|^{2}+\left|V_{u s}\right|^{2}+\left|V_{u b}\right|^{2}-1=7\left(_{-13}^{+9}\right) \times 10^{-4}$

- $\left\langle r^{2}\right\rangle_{V}^{K \pi}, \xi(0)$ : reasonably consistent $\mathrm{w} /$ exp't

- future directions

- further refinements $\Leftrightarrow$ more rigorous treatment of NNLO $\Leftrightarrow$ overlap quarks

- $\pi, K$ EM form factors $\Leftrightarrow$ test of ChPT

- $D, B$ meson decays $\Leftrightarrow$ flavor physics 


\section{1 chiral fit : $f_{+}(0)$}

$\underline{F_{0}}$

- phenomenological estimates

(Bijnens@ Lattice'07; $\mu=0.77 \mathrm{GeV}$ )

\begin{tabular}{l|l|l|l}
\hline $10^{3} L_{4}^{r}$ & $\equiv 0$ & $\equiv 0.5$ & $\equiv 0.2$ \\
\hline $10^{3} L_{6}^{r}$ & $\equiv 0$ & $\equiv 0.1$ & $\equiv 0$ \\
\hline$F_{0}[\mathrm{MeV}]$ & 87.7 & 70.4 & 80.4 \\
\hline
\end{tabular}

- JLQCD’s analysis (Noaki@ Lattice'10)

- NNLO fit to $M_{\{\pi, K\}}, F_{\{\pi, K\}}$

- chiral expansion $\mathrm{w} / M_{\pi}^{2} / F_{\pi}^{2}$ instead of $M_{\pi}^{2} / F_{0}$

$\Rightarrow$ better convergence

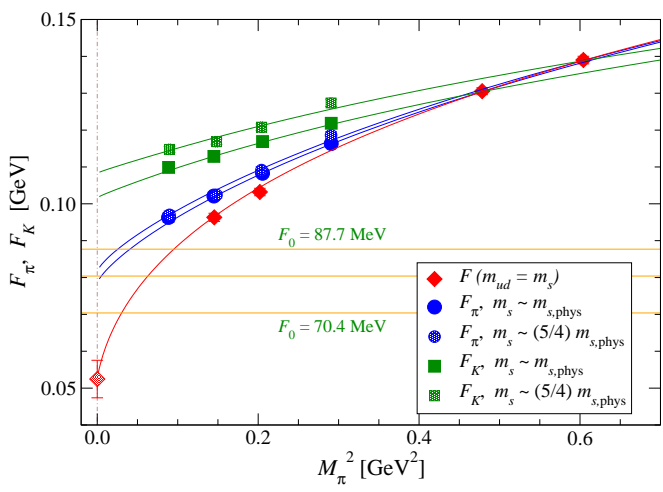

- single lattice spacing $\sim 0.11 \mathrm{fm}$

- $F_{0}=52.5(5.1) \mathrm{MeV}$

small $F_{0} \Rightarrow$ enhance chiral corrections : NLO $\propto 1 / F_{0}^{2} ;$ NNLO $\propto 1 / F_{0}^{4}$ 
Progress Towards $\Delta \mathrm{I}=1 / 2 \mathrm{~K} \rightarrow \pi \pi$ Decays with G-parity Boundary Conditions

\section{Christopher Kelly Columbia University}




\section{Outline}

- Motivation

- Challenges

- G-Parity Implementation

- G-Parity Contractions

- The Strange Quark

- Conclusions and Outlook 
Motivation 


\section{$\mathbf{K} \rightarrow \pi \pi$ Decays}

- Direct CP-violation first observed in $K \rightarrow \pi \pi$ decays.

- Two types of decay:

$$
\begin{aligned}
& \Delta I=3 / 2: K^{+} \rightarrow\left(\pi^{+} \pi^{0}\right)_{I=2} \text { with amplitude } A_{2}
\end{aligned}
$$

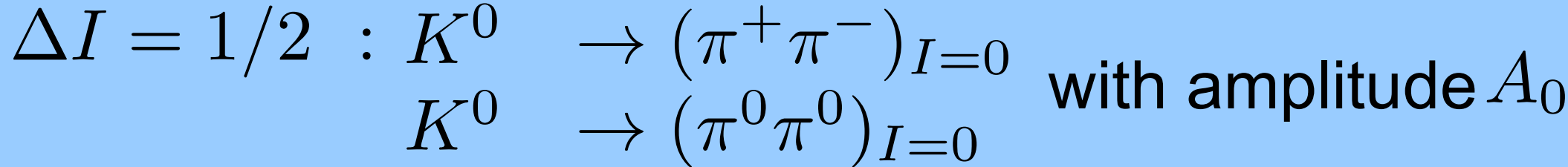

Direct CP-violation: $\epsilon^{\prime}=\frac{i \omega e^{i\left(\delta_{2}-\delta_{0}\right)}}{\sqrt{2}}\left(\frac{\operatorname{Im} A_{2}}{\operatorname{Re} A_{2}}-\frac{\operatorname{Im} A_{0}}{\operatorname{Re} A_{0}}\right)$
where $\omega=\operatorname{Re} A_{2} / \operatorname{Re} A_{0}$ and $\delta_{I}$ are strong rescattering phase shifts.

- Strong interactions very important - origin of the so-called $\Delta I=1 / 2$ rule: preference to decay to $I=0$ final state. Mechanism for this is not yet understood. 


\section{$\mathbf{K} \rightarrow \pi \pi$ Decays on the Lattice.}

- Direct calculation of $K \rightarrow \pi \pi$ decays essential to understanding $\Delta I=1 / 2$ rule and in the search for BSM physics.

- Lattice computation of realistic decays has only recently become possible.

- RBC \& UKQCD recently published (arXiv:1111.1699) calculation of $\Delta I=3 / 2$ decay using:

- $2+1 f$ domain wall fermions on a $32^{3} \times 64 \times 32$ lattice with $a^{-1}=1.37(1) \mathrm{GeV}$.

- Near physical pions: $m_{\pi}^{P Q} \sim 140 \mathrm{MeV}, m_{\pi}^{\text {uni }} \sim 170 \mathrm{MeV}$

- Energy conserving decays

- Determined $\operatorname{Re} A_{2}$ and $\operatorname{Im} A_{2}$. 


\section{$\mathbf{K} \rightarrow \pi \pi$ Decays on the Lattice.}

- Combining results of $\Delta I=3 / 2$ calculation with experimental value for $\epsilon^{\prime} / \epsilon$, we obtained first value for $\operatorname{Im} A_{0}$

- Calculation of $A_{0}$ from first principles is much more difficult. 
Challenges 


\section{Forming the $\pi \pi$ Propagator}

- $\pi \pi$ state has vacuum quantum numbers, hence there are disconnected diagrams:
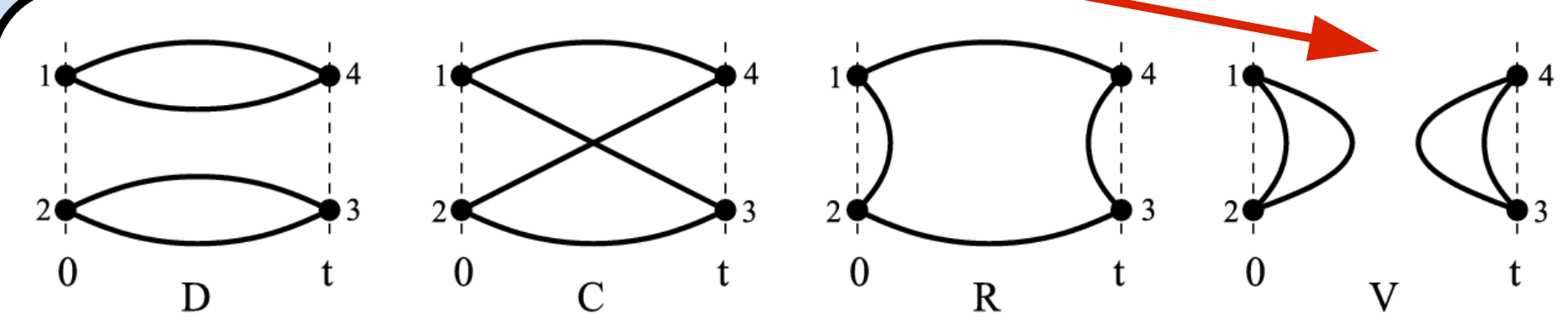

- Need large statistics and many source positions (or A2A/AMA propagators) to resolve.

- With Blue Gene/Q resources we can now perform such calculations with large enough physical volumes. 


\section{Physical Kinematics}

- Best method is to use a stationary kaon and pions moving with equal momentum in opposite directions.

- This is an excited state of the $\pi \pi$ system - normally require very large statistics for decent signal.

- Instead impose antiperiodic BCs on d-quark propagator. $\pi^{ \pm}$gains momentum $\pi / L$, but for $\pi^{0}$ the p's cancel.

- This breaks isospin symmetry! However for $\Delta I=3 / 2$ we can use Wigner-Eckart theorem and isospin to relate $\Delta I_{z}=1 / 2 K^{+} \rightarrow \pi^{+} \pi^{0}$ to $\Delta I_{z}=3 / 2 K^{+} \rightarrow \pi^{+} \pi^{+}$.

- As $\pi^{+} \pi^{+}$is only charge-2 final state (q-conservation still true), isospin breaking becomes unimportant as this state cannot mix with $I=0$ states.

- Note: In practise we needed APBC in 2 dirs for physical kinematics. 


\section{Physical Kinematics}

- For $\Delta I=1 / 2$ the Wigner-Eckart trick cannot be used.

- Isospin-breaking BCs would allow mixing between $I=0$ and $I=2$ final states. Separation would be difficult.

- Need to apply BCs that commute with isospin.

- Also, for $\Delta I=1 / 2$ the vacuum plays a role, so the BCs must be applied to both the valence and sea quarks. 


\section{G-Parity Boundary Conditions}

- G-parity is a charge conjugation followed by a 180 degree isospin rotation about the $y$-axis:

$$
\begin{array}{rlrl}
\hat{G}=\hat{C} e^{i \pi \hat{I}_{y}} & : \quad \hat{G}\left|\pi^{ \pm}\right\rangle & =-\left|\pi^{ \pm}\right\rangle \\
\hat{G}\left|\pi^{0}\right\rangle & =-\left|\pi^{0}\right\rangle
\end{array}
$$

- At the quark level:

$$
\hat{G}\left(\begin{array}{c}
u \\
d
\end{array}\right)=\left(\begin{array}{c}
-C \bar{d}^{T} \\
C \bar{u}^{T}
\end{array}\right) \text { where } C=\gamma^{2} \gamma^{4} .
$$

- G-parity commutes with isospin.

- Pions are all eigenstates with e-val -1, hence G-parity BCs make pion wavefunctions antiperiodic, with minimum momentum $\pi / L$. 


\section{G-Parity Implementation}




\section{Gauge Field Boundary Conditions}

- $d$-field becomes $C \bar{u}^{T}$ across the boundary. Consider a bilinear on the boundary under a gauge transformation $V$ :

$$
\begin{aligned}
& \bar{d}(L-1) U_{y}(L-1) C \bar{u}^{T}(0) \\
& \quad \longrightarrow \bar{d}(L-1) V^{\dagger}(L-1) U_{y}(L-1) V^{*}(0) C \bar{u}^{T}(0) .
\end{aligned}
$$

- Link must transform as

$$
U_{y}(L-1) \rightarrow V(L-1) U_{y}(L-1) V^{T}(0)
$$

- Link parallel to boundary on on other side $(y \geq L)$ must then transform as:

- $U_{x}(x, y, ..) \rightarrow V^{*}(x, y, ..) U_{x}(x, y, ..) V^{T}(x+1, y, .$.

- Gauge fields therefore obey complex-conjugate BCs. 


\section{The Two-Flavor Method}

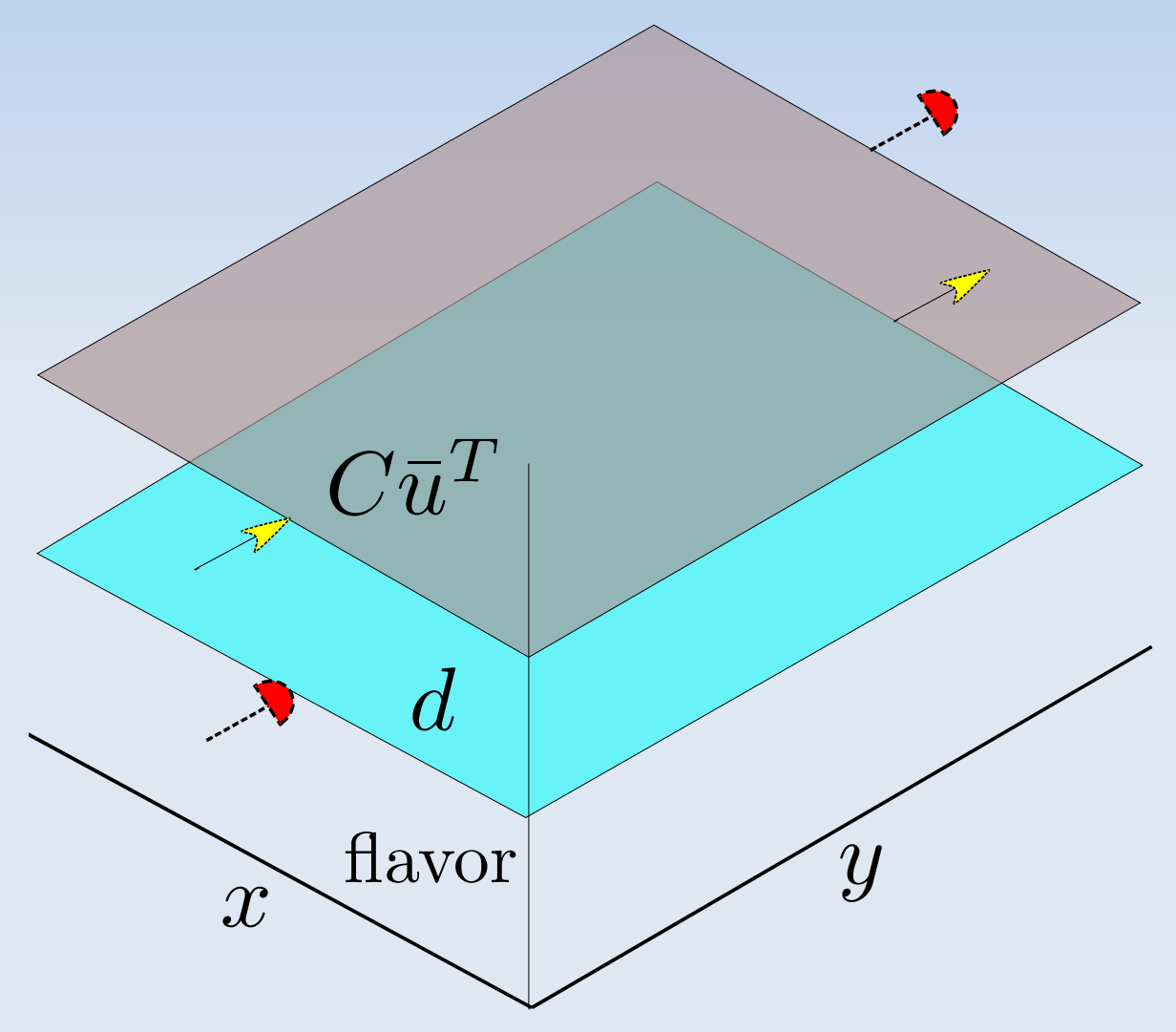

- Two fermion fields on each site indexed by flavor index:

$\psi^{(1)}(x)=d(x), \psi^{(2)}(x)=C \bar{u}^{T}(x)$

- BCs are:

$$
\begin{aligned}
& \psi^{(1)}(x+L \hat{y})=\psi^{(2)}(x), \\
& \psi^{(2)}(x+L \hat{y})=-\psi^{(1)}(x),
\end{aligned}
$$

- Periodic BCs in other dirs.

- Single U-field shared by both flavors, with complex conj BCs.

- Dirac op for $\psi^{(2)}$ uses $U_{\mu}^{*}$. 


\section{The One-Flavor Method}

- Obtain equivalent formulation by unwrapping flavor indices onto two halves of doubled lattice:

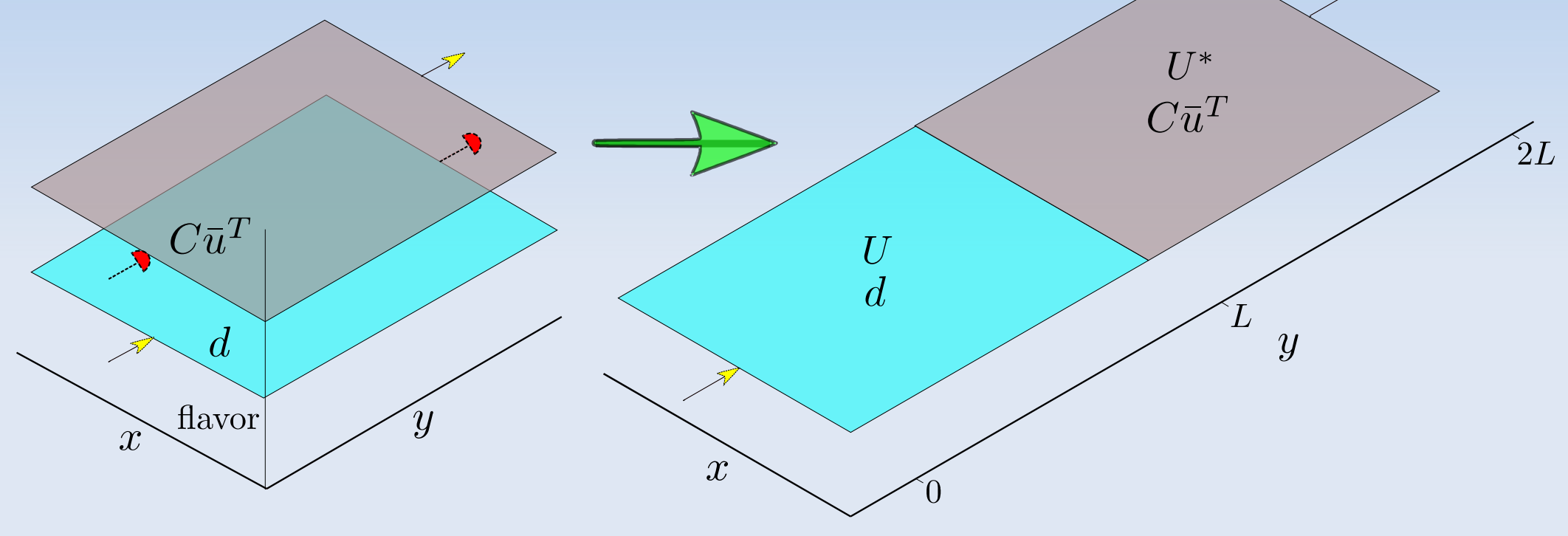

- Antiperiodic boundary conditions in G-parity direction.

- $U$-field on first half and $U^{*}$-field on second half. 


\section{Choosing an Approach}

- One flavor setup is much easier to implement.

- However recall that we needed APBC in 2 directions for physical kinematics in $\Delta I=3 / 2$ calculation.

- G-parity in >1 dir using one-flavor method requires doubling the lattice again, which is highly inefficient.

- A second approach requires non-nearest neighbour communication:

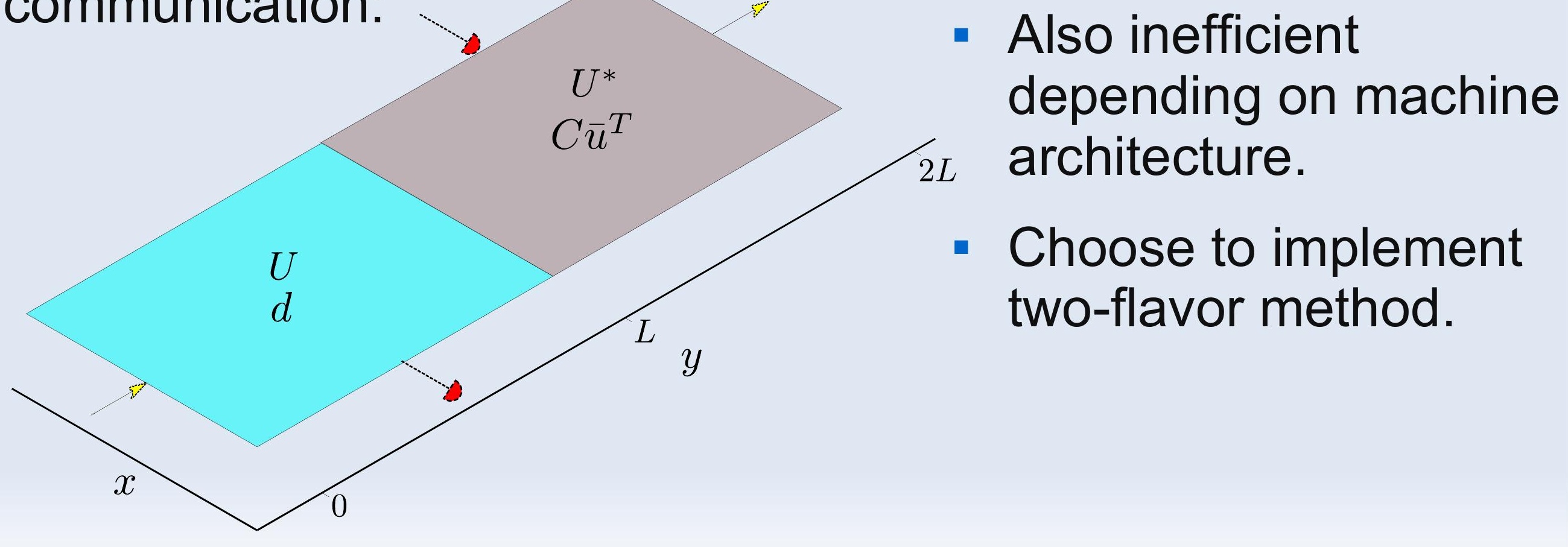




\section{Unusual Contractions}

- Flavor mixing at boundary allows contraction of up and down fields:

$$
\begin{aligned}
& \bar{\psi}_{x}^{(2)} \bar{\psi}_{y}^{(1)}=\mathcal{G}_{x, y}^{(2,1)}=C \overline{\bar{u}}_{x}^{T} \bar{d}_{y}, \\
& \bar{\psi}_{y}^{(1)} \bar{\psi}_{x}^{(2)}=\mathcal{G}_{y, x}^{(1,2)}=-\bar{d}_{y} u_{x}^{T} C^{T}
\end{aligned}
$$

- Interpret as boundary creating/destroying flavor (violating baryon number):

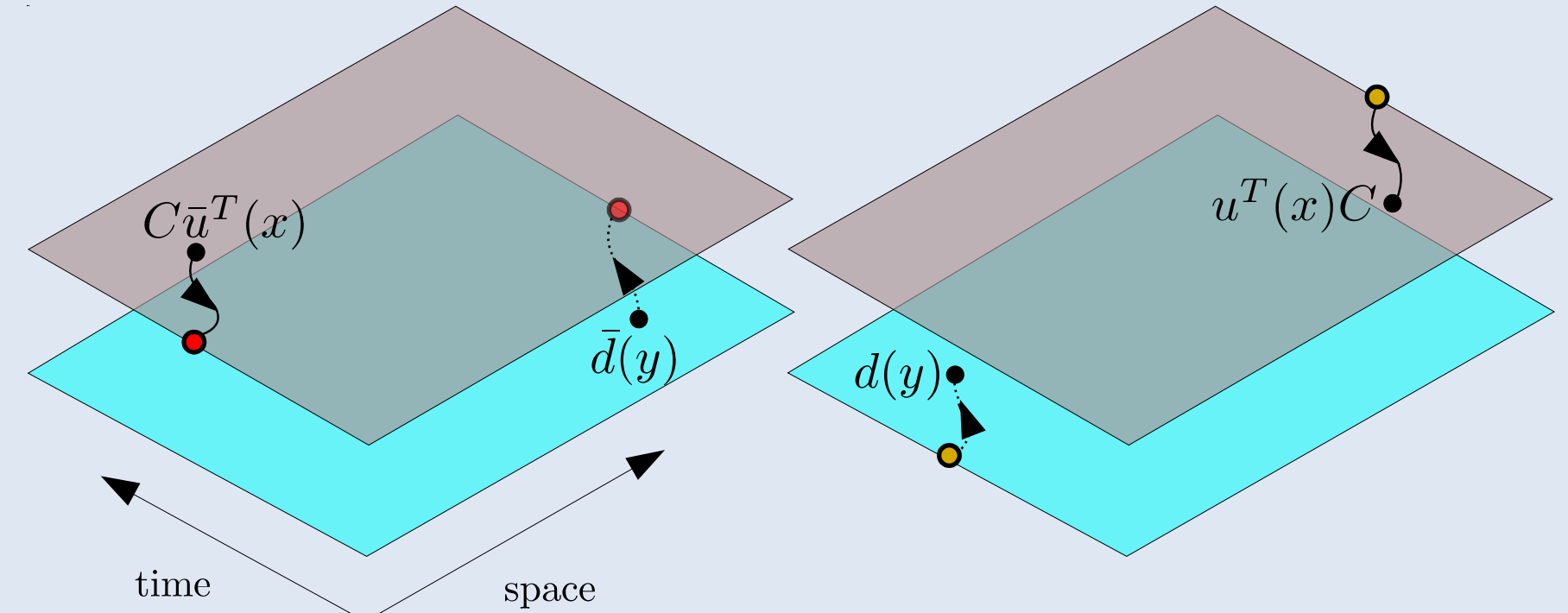

- Also have $\gamma^{5}$-hermiticity: $\left[\gamma^{5} \mathcal{G}_{x, y}^{(2,1)} \gamma^{5}\right]^{\dagger}=\mathcal{G}_{y, x}^{(1,2)}$ 


\section{Pion Correlation Functions}

- $\pi^{+}$correlation function

$$
\left\langle\bar{d}_{x} \gamma^{5} u_{x} \bar{u}_{y} \gamma^{5} d_{y}\right\rangle=\left\langle\bar{\psi}_{x}^{(1)}\left[\gamma^{5} C\right] \bar{\psi}_{x}^{(2) T} \psi_{y}^{(2) T}\left[C \gamma^{5}\right] \psi_{y}^{(1)}\right\rangle
$$

- Now has two contractions:

$\operatorname{tr}\left\{\mathcal{G}_{x, y}^{(1,1) T} C \gamma^{5} \mathcal{G}_{x, y}^{(2,2)} \gamma^{5} C\right\}+\operatorname{tr}\left\{\mathcal{G}_{x, y}^{(1,2) T} C \gamma^{5} \mathcal{G}_{x, y}^{(2,1)} \gamma^{5} C\right\}$

arrows point from source to sink

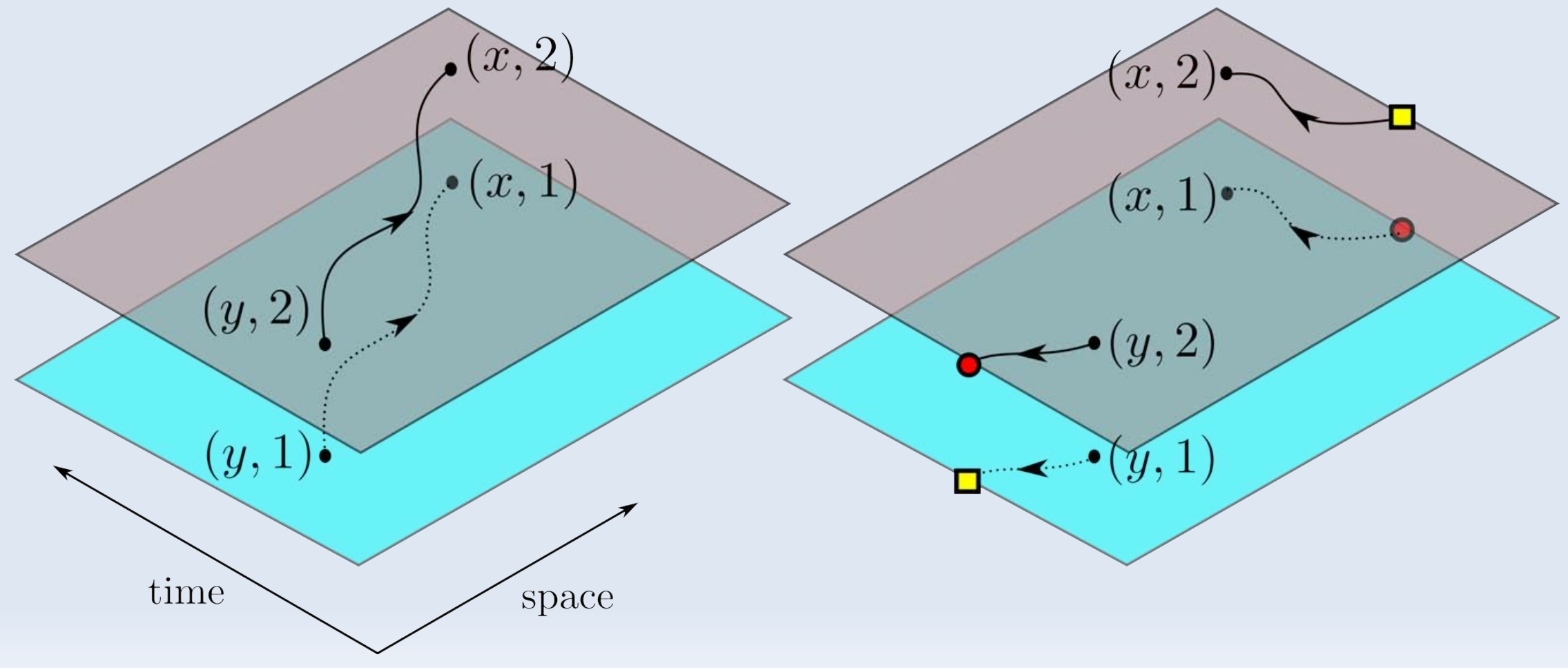




\section{Results: Pion Correlator}

- Generated an $8^{3} \times 32 \times 10$ DWF quenched ensemble.

- 150 configs (20 MD tu's sep) with G-parity in 0,1,2,3 dirs.

- Coulomb-gauge fixed wall source propagators.

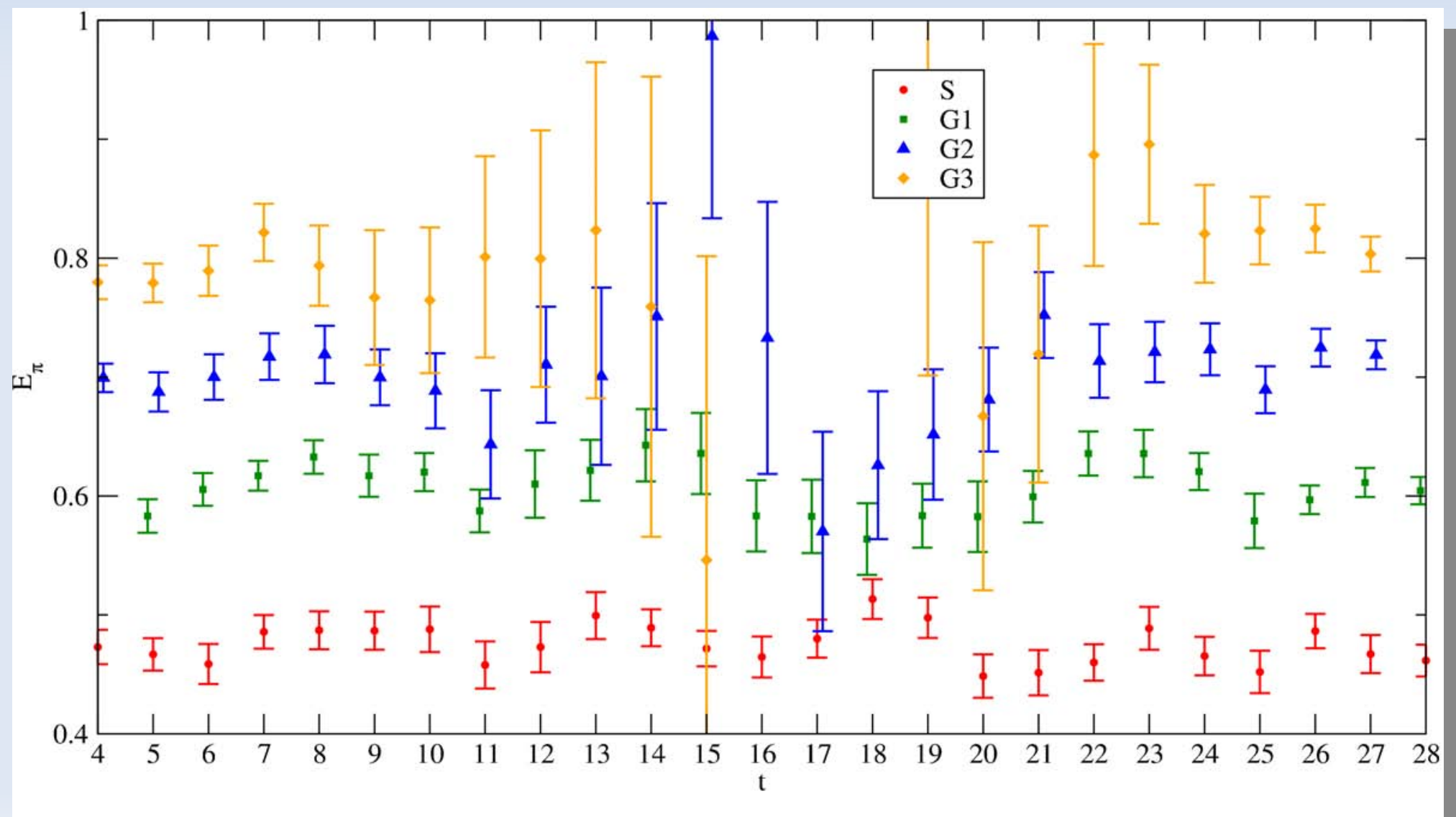




\section{Results: Pion Dispersion Relation}

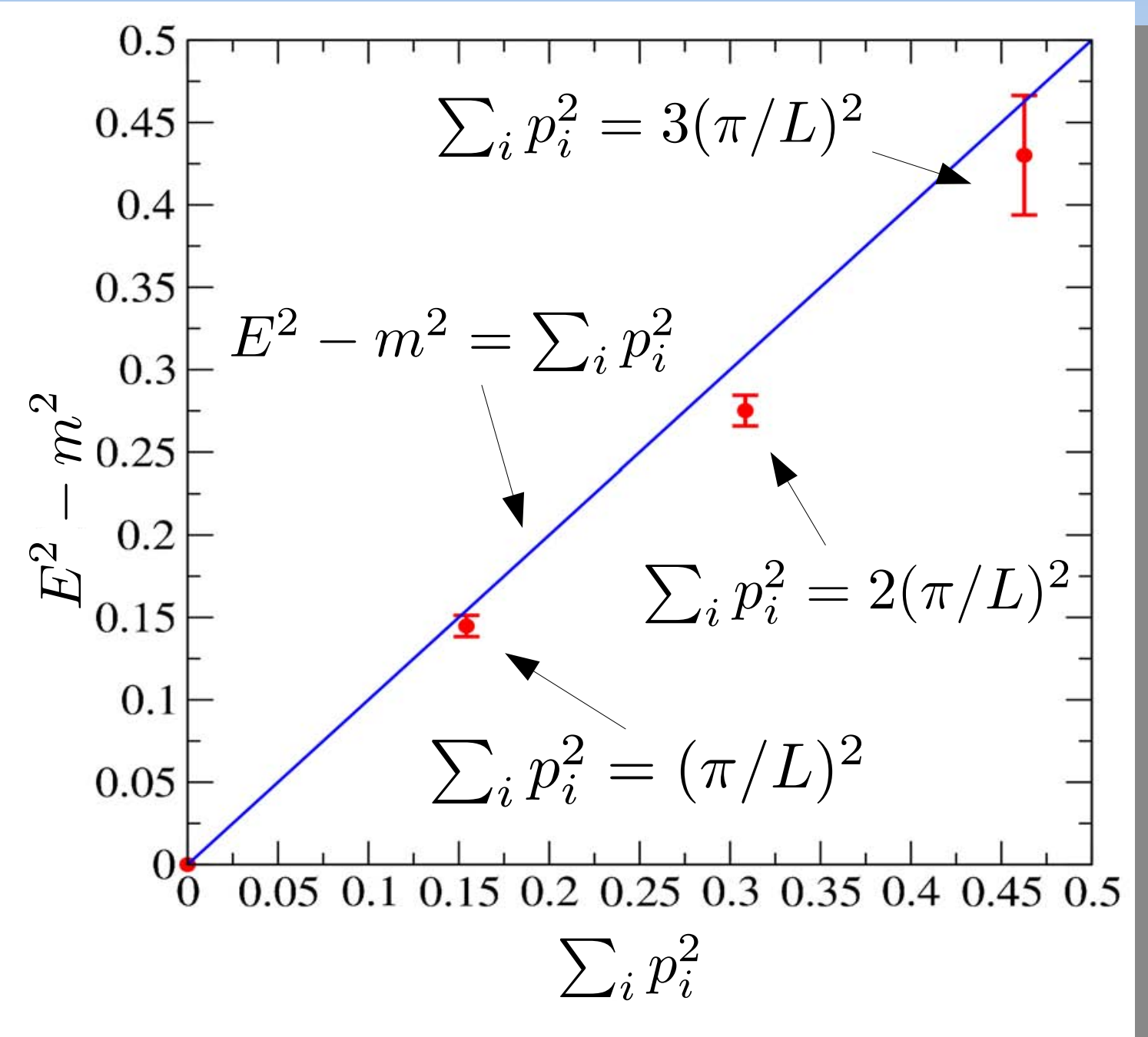

- Deviations from continuum disp. reln. expected on lattice. e.g. free-field: $\quad E^{2}-m^{2}=\sum_{i} \sin ^{2}\left(p_{i}\right)$ 
The Strange Quark 


\section{Kaons}

- $K \rightarrow \pi \pi$ calculation needs stationary $K^{0}$.

- $\frac{1}{\sqrt{2}}(\bar{s} d+\bar{d} s)$ not a G-parity eigenstate.

- Need an eigenstate with e-val +1 for periodic BCs and hence $p_{\min }=0$.

- Introduce 'strange isospin' $\left(I^{\prime}\right)$ : s-quark in doublet $\left(\begin{array}{c}s^{\prime} \\ s\end{array}\right)$

- A neutral kaon-like state:

$$
K_{0}^{\prime}=\frac{1}{2}\left(\bar{s} d+\bar{d} s+\bar{s}^{\prime} u+\bar{u} s^{\prime}\right)
$$

is an eigenstate of 'modified G-parity': $\hat{G}=\hat{C} e^{i \pi \hat{I}_{y}} e^{i \pi \hat{I}_{y}^{\prime}}$ with e-val +1 .

- Need factor of 2 in decay calc as only $1 / 2$ of components of initial kaon couple to $\pi \pi$. 
- Theory has one too many flavors. Must take square-root of $s^{\prime} / s$ determinant in evolution to revert to 3 flavors.

- Determinant becomes non-local.

- Non-locality is however only a boundary effect that vanishes as $L \rightarrow \infty$. With sufficiently large volumes the effect should be minimal. 


\section{Charged Kaon Correlator}

- $K^{+}$analogue: $\left|K^{+\prime}\right\rangle=\left(\bar{u} \gamma^{5} s-\bar{s}^{\prime} \gamma^{5} d\right)|0\rangle$

- 2-point function has 4 contractions:

$$
\begin{aligned}
& \operatorname{tr}\left\{\mathcal{G}_{x, y}^{(2,2) T} \gamma^{5} C \mathcal{G}_{x, y}^{(3,3)} \gamma^{5} C\right\}+\operatorname{tr}\left\{\mathcal{G}_{x, y}^{(4,4) T} \gamma^{5} C \mathcal{G}_{x, y}^{(1,1)} \gamma^{5} C\right\} \\
& -\operatorname{tr}\left\{\mathcal{G}_{x, y}^{(1,2) T} \gamma^{5} C \mathcal{G}_{x, y}^{(4,3)} \gamma^{5} C\right\}-\operatorname{tr}\left\{\mathcal{G}_{x, y}^{(3,4) T} \gamma^{5} C \mathcal{G}_{x, y}^{(2,1)} \gamma^{5} C\right\}
\end{aligned}
$$

- If we make the masses of the $\left(s^{\prime}, s\right)$ and $(u, d)$ doublets the same this reduces to:

$$
2 \operatorname{tr}\left\{\mathcal{G}_{x, y}^{(1,1) T} C \gamma^{5} \mathcal{G}_{x, y}^{(2,2)} \gamma^{5} C\right\}-2 \operatorname{tr}\left\{\mathcal{G}_{x, y}^{(1,2) T} C \gamma^{5} \mathcal{G}_{x, y}^{(2,1)} \gamma^{5} C\right\}
$$

- This is just twice the $\pi^{+}$correlation function but with the opposite sign between the 2 contractions.

- Periodicity of spatial dependence appears to arise due to some cancellation between the two contractions. 


\section{Results: Degenerate $K^{+\prime}$ Dispersion Relation}

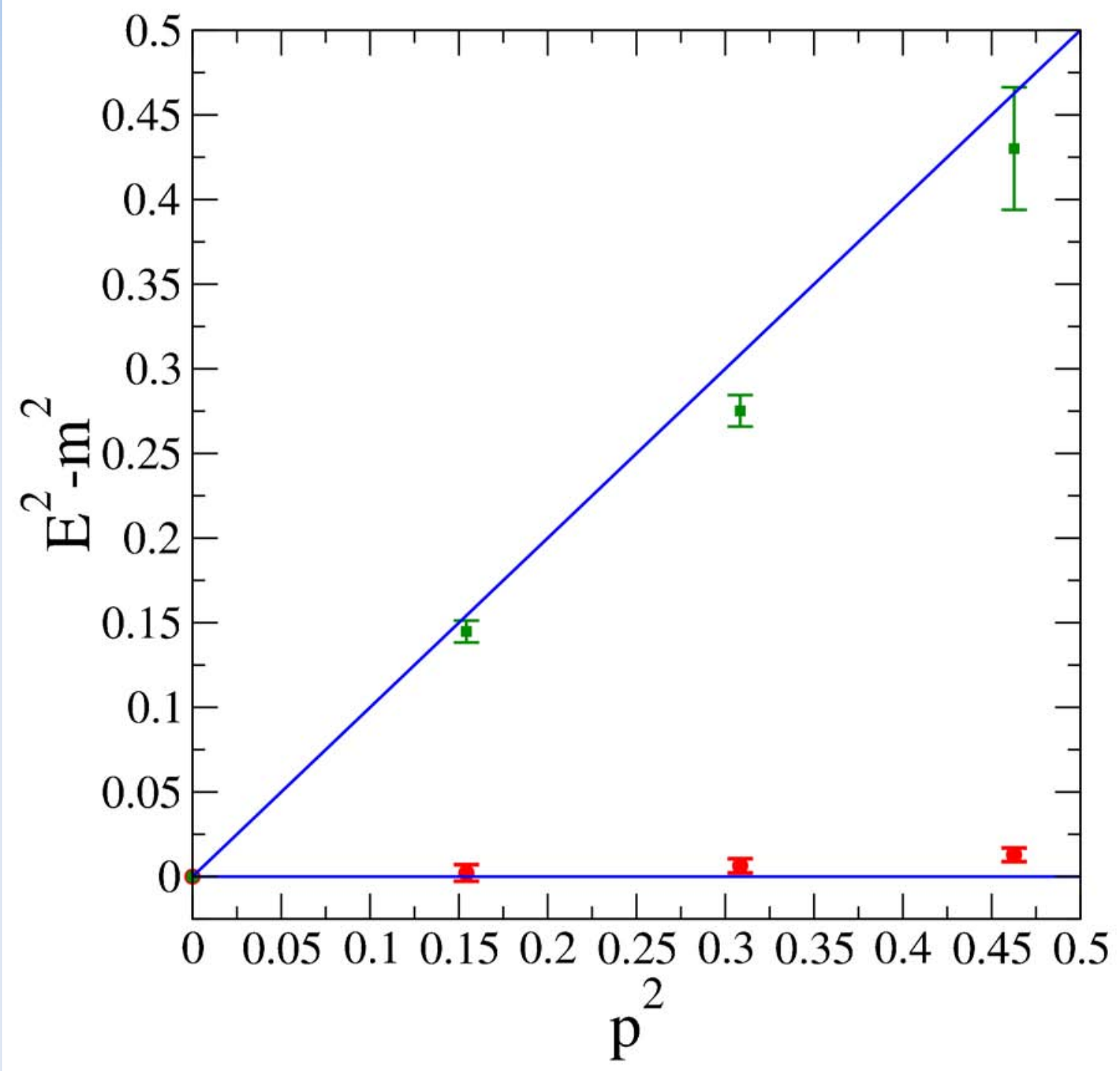




\section{Conclusions and Outlook}

- G-parity boundary conditions look to be very promising for realising $\Delta I=1 / 2 K \rightarrow \pi \pi$ decays with physical kinematics.

- Direct calculation of $A_{0}$ is essential for understanding $\Delta I=1 / 2$ rule and in the search for BSM physics.

- Coding two-flavor method is challenging but significant progress has been made.

- Aim to dedicate significant BlueGene/Q resources towards generating $32^{3} \times 64 \times 32$ DWF Iwasaki+DSDR ensembles with G-parity BCs and physical pions. 


\section{Recent progress in multi GPU conjugate gradient convertor with staggerêd fermion}

- New Horizons for Lattice Gauge Theory Computations at BNL 2012 


\section{Introduction}

1. GPU computation for HPC

- GPGPU: General Purpose computation using GPU.

- CUDA : C extension(API) for GPGPU by nVIDIA

- Open CL : Standard C extension for GPGPU

2. Conjugate Gradient algorithm

Iterative method for solving linear algebraic equations : $b=A x$

3. CG implementation on CUDA 


\section{Why use the GPU ?}

High performance computing

1. If money is not a problem ?

- Just buy a good super computer

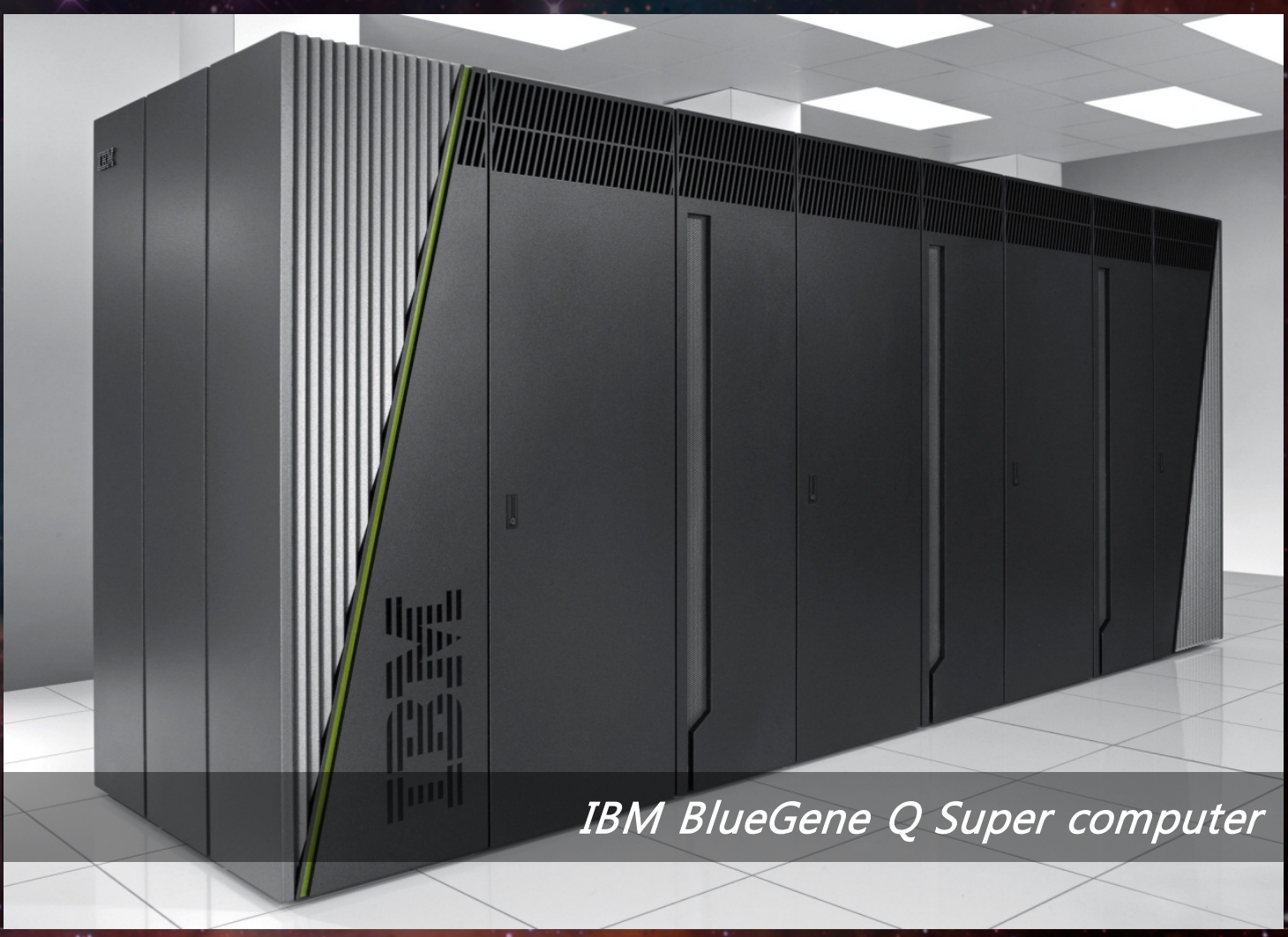




\section{Why use the GPU ?}

High performance computing

2. Physicist in Korea?

- No money.

x - Find alternative way

- GPU Supercomputing !

- Cost \& energy effective

- Hard to develop
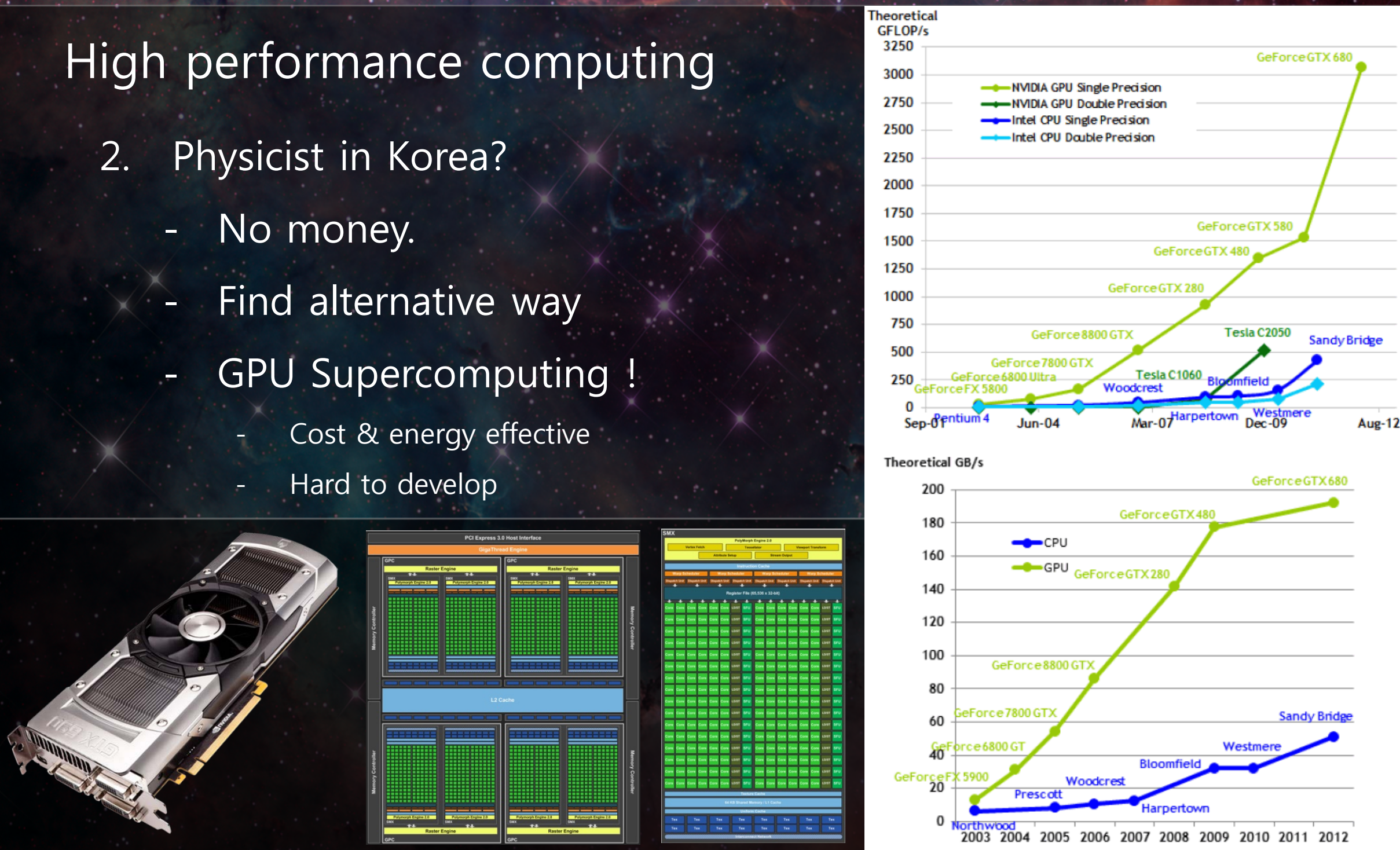

Theoretical GB/s

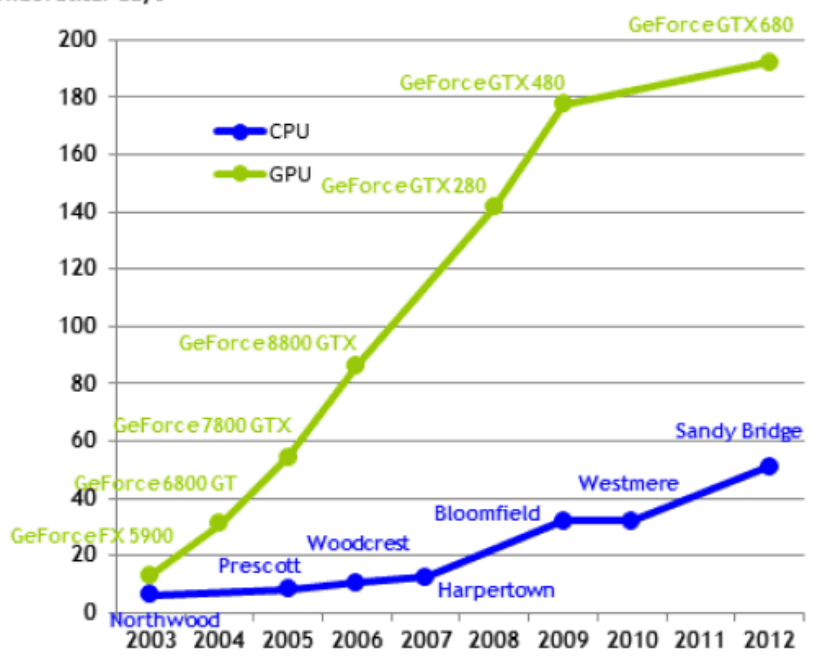

Hyung-Jin Kim, New.Horizons for Lattice Gauge Theory Computations at'BNL.2012 


\section{Machine Environment in SNU}

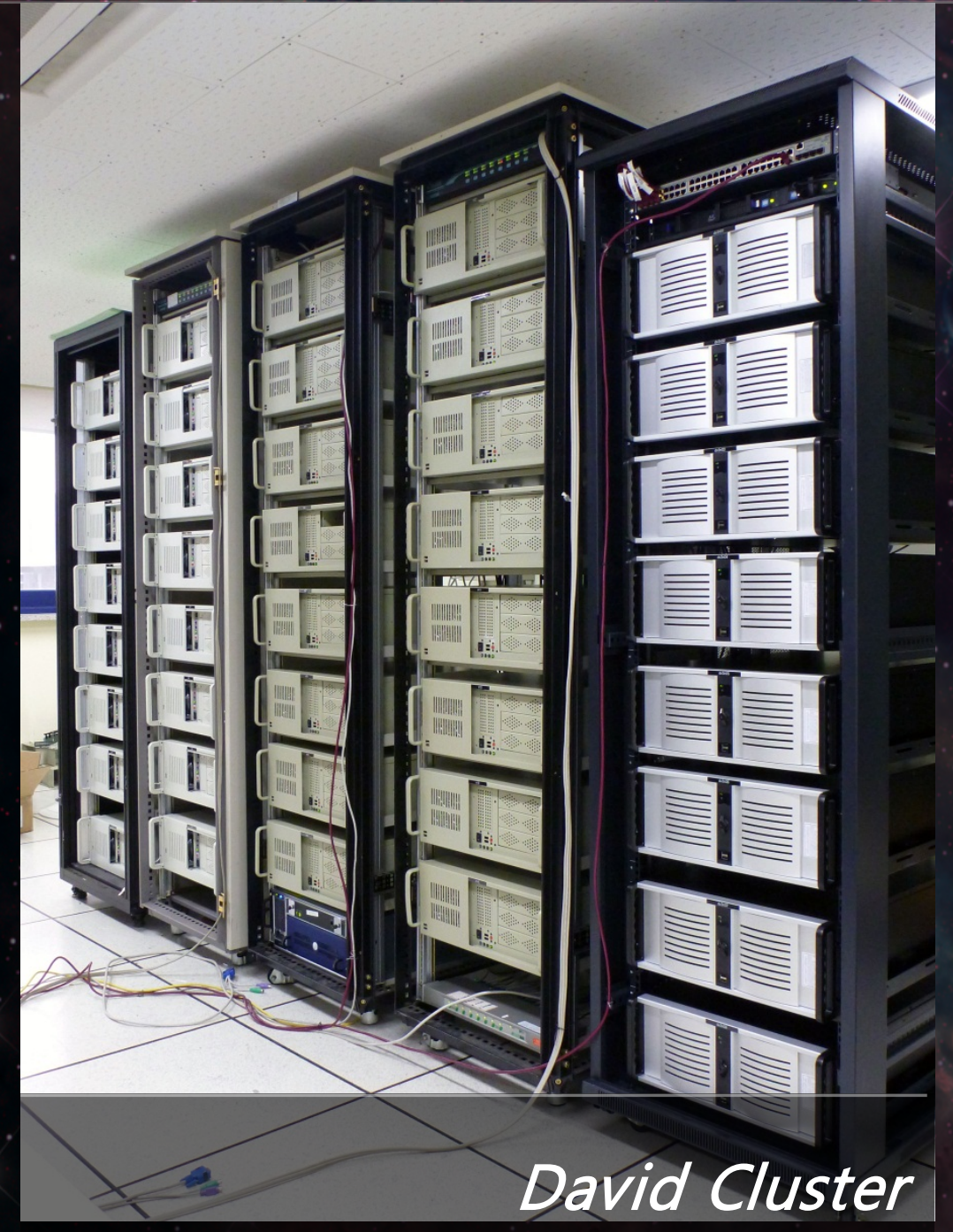

64x GTX 480 GPUs + 16x GTX 590 GPUs $20 G b$ 32nodes $+40 G b 8$ nodes Infiniband
- David 1, 2 GPU cluster

- Intel core i7 920, 32 node

- $32 \times 2 \times$ GTX 480 GPUs

- 20Gbps infiniband network

- Theoretical performance

SP : 1.34 TFLOPS / GPU

DP : 168 GFLOPS / GPU

Bandwidth : 177.4 GB / sec / GPU

- David 3 GPU cluster

- Intel core i7 950, 8 node

- $8 \times 2$ x GTX 590 GPUs.

- 40Gbps infiniband network 


\section{CG Algorithm}

- Conjugate gradient operation

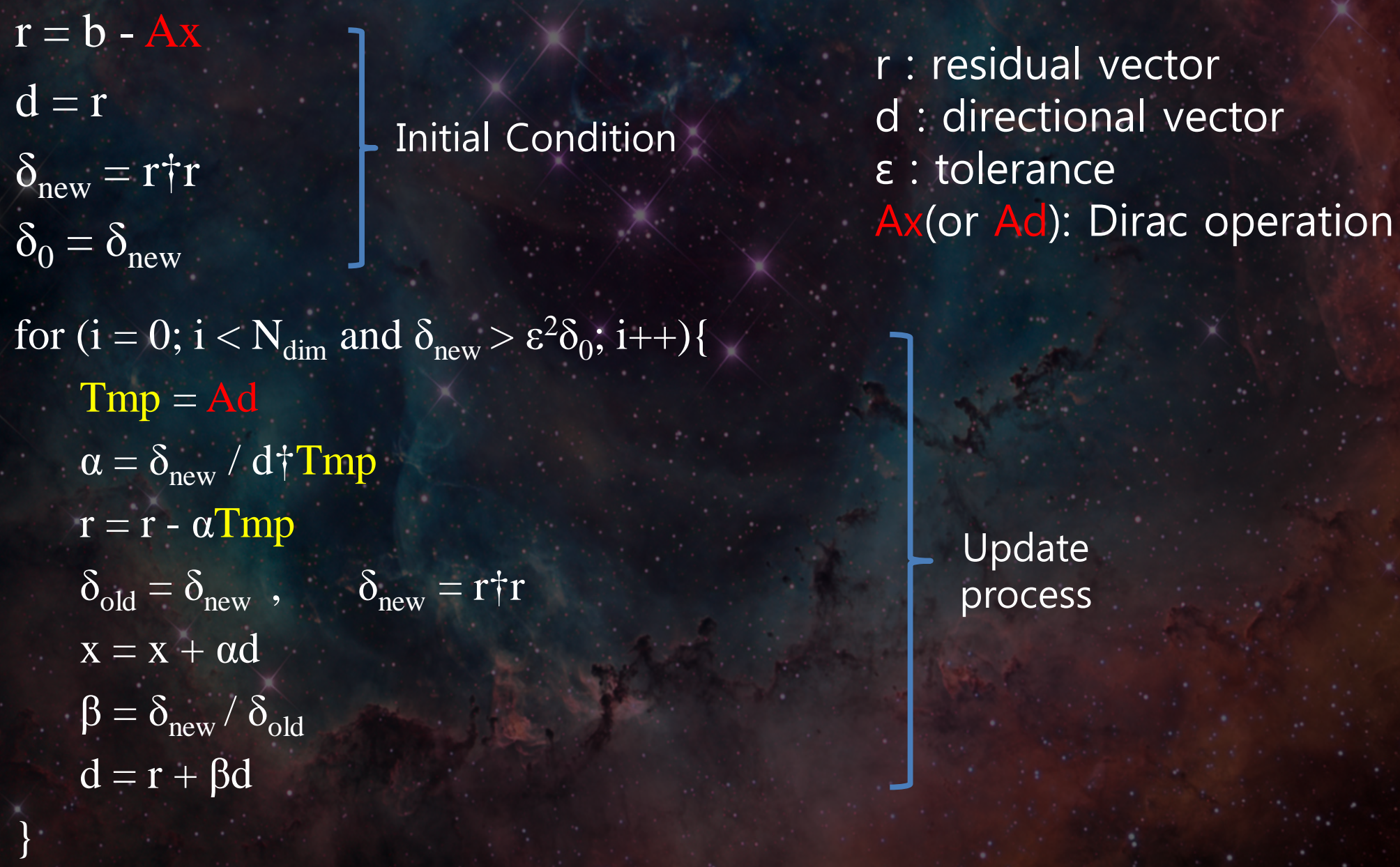

$r$ : residual vector

d : directional vector

$\varepsilon$ : tolerance

Ax(or Ad): Dirac operation 


\section{Staggered Dirac Operator}

\section{Dirac operation}

- Dirac equation $\mathrm{h}=\mathrm{A} \chi ; \quad \mathrm{A} \equiv-D^{2}+m^{2}$

$$
\begin{aligned}
D_{x, y} & =U_{\mu}(x) \delta_{y, x+\mu}-U_{\mu}(x-\mu) \delta_{y, x-\mu} \\
\Rightarrow & D \chi(\mathrm{x})=\Sigma \mathrm{U}_{\mu}(\mathrm{x}) \chi(\mathrm{x}+\mu)-\mathrm{U}_{\mu}(\mathrm{x}-\mu) \chi(\mathrm{x}-\mu) \\
& \left(\begin{array}{l}
x^{\prime \prime} 1 \\
x^{\prime \prime} 2 \\
x^{\prime \prime} 3
\end{array}\right)=\left(\begin{array}{lll}
a 1 & a 2 & a 3 \\
b 1 & b 2 & b 3 \\
c 1 & c 2 & c 3
\end{array}\right)\left(\begin{array}{l}
x 1 \\
x 2 \\
x 3
\end{array}\right)-\left(\begin{array}{lll}
a^{\prime} 1 & a^{\prime} 2 & a^{\prime} 3 \\
b^{\prime} 1 & b^{\prime} 2 & b^{\prime} 3 \\
c^{\prime} 1 & c^{\prime} 2 & c^{\prime} 3
\end{array}\right)\left(\begin{array}{l}
x^{\prime} 1 \\
x^{\prime} 2 \\
x^{\prime} 3
\end{array}\right)
\end{aligned}
$$

- $6[\chi(\mathrm{x})]+8 \times 6[\chi(\mathrm{x} \pm \mu)]+8 \times 18\left[\mathrm{U}_{\mu}(\mathrm{x})\right]=198: 792$ bytes(SP), 1584 bytes(DP)

- $\mathrm{U}_{\mu}(\mathrm{x}) \mathrm{X}(\mathrm{x}+\mu)$ part has 72 floating point calculations

$\rightarrow 8 \times 72=576$ floating point calculations per site

- For $28 \wedge 3 \times 96$ lattice, there are $10^{6}$ of lattice even(or odd) sites

$\rightarrow 0.61$ Giga floating point calculations (Total 1 CG iteration $\doteq 0.624 \times 10^{9}$ )

$\rightarrow 1.55$ Giga bytes(DP) of data transfer 


\section{C \& CUDA Code Comparison}

\section{CPU code(CPS Lib.)}

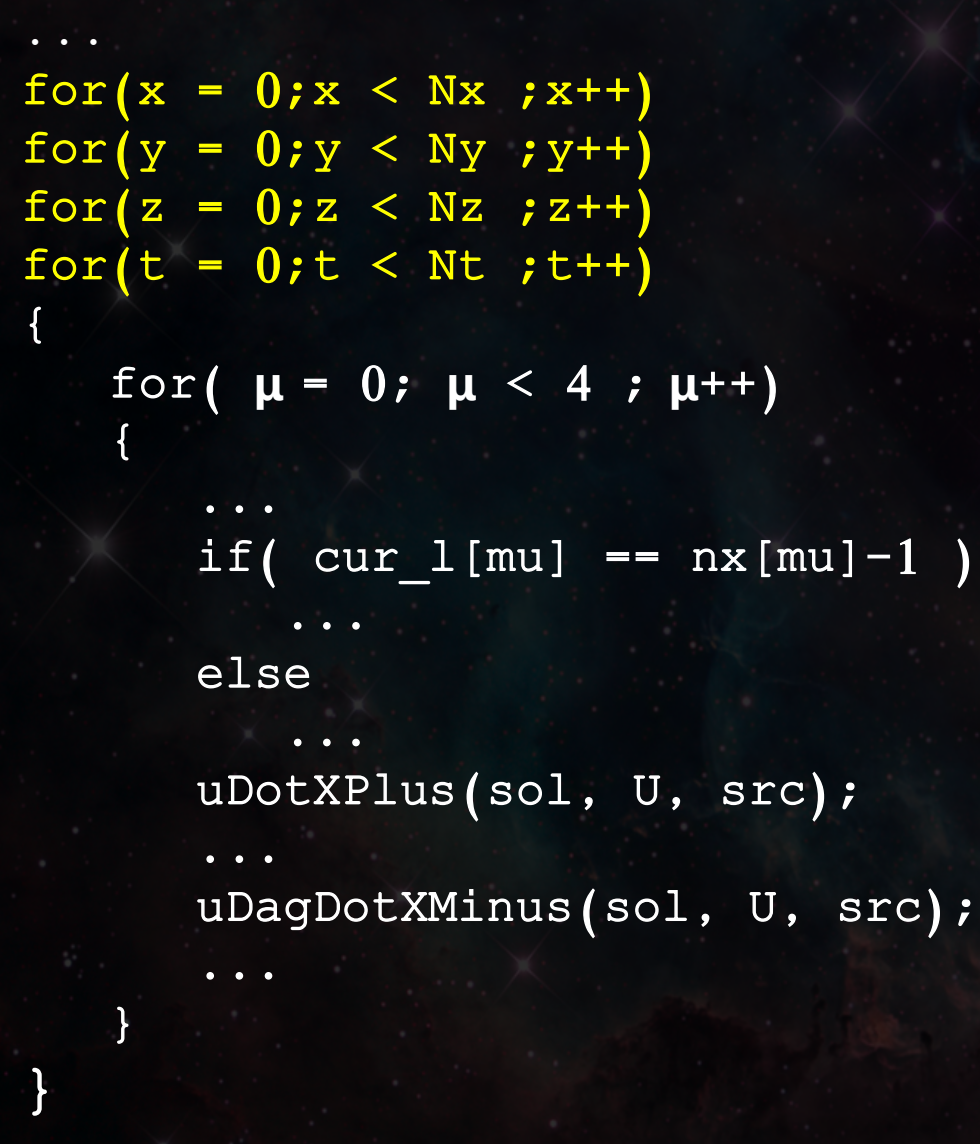

\section{CUDA code}

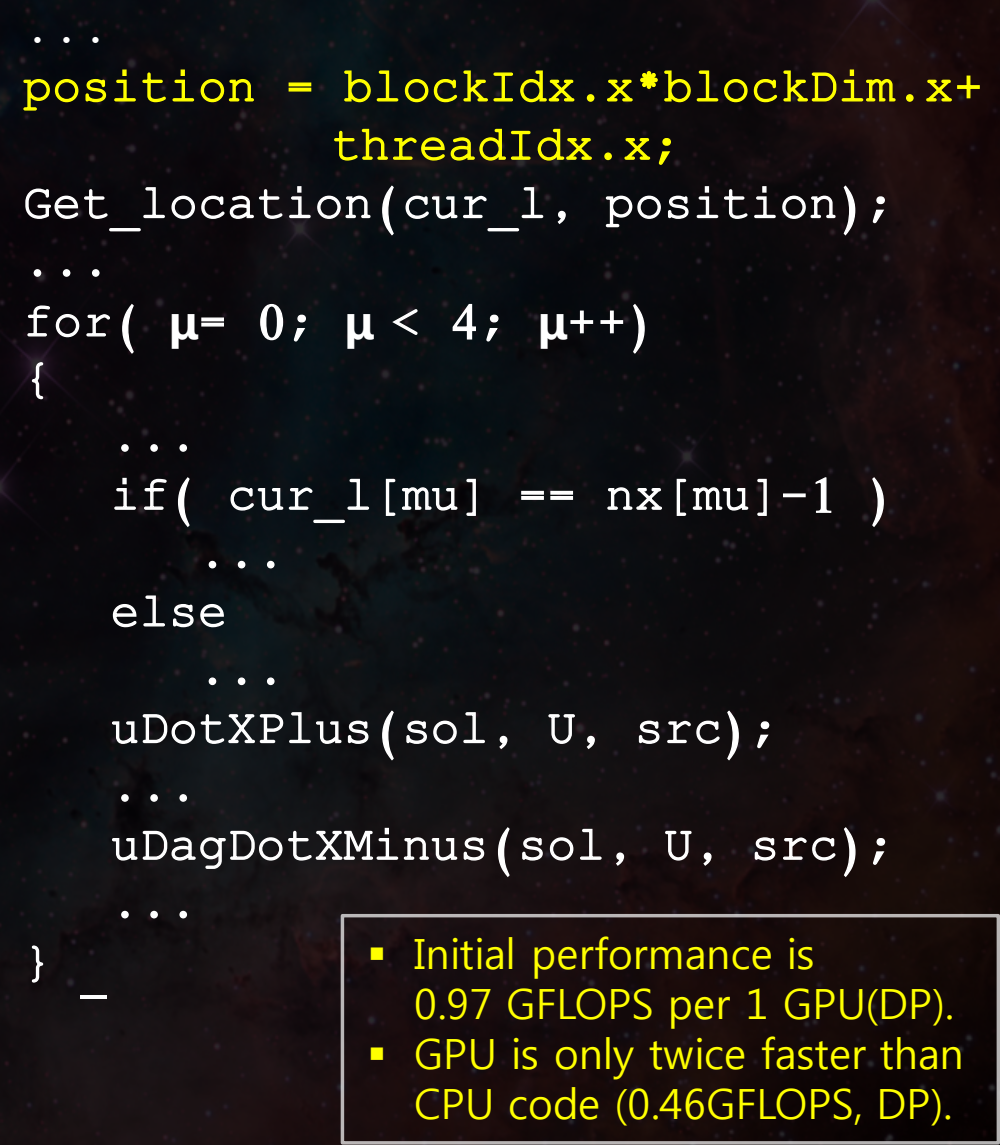




\section{CUDA Optimization(1)}

1. Coalesced memory access

2. Using fast on-chip memory(register \& shared memory)

- Increase data reusability

3. Adjust the code for CUDA architecture

- Higher CUDA occupancy.

- Reduce the branch code

4. Data compression

- SÚ(3) data reconstruction(8, 10,12 parameter)

5. Using mixed precision

- Load balancing between single precision and double precision calculation 


\section{CUDA Optimization(2)}

- Coalesced Memory Access

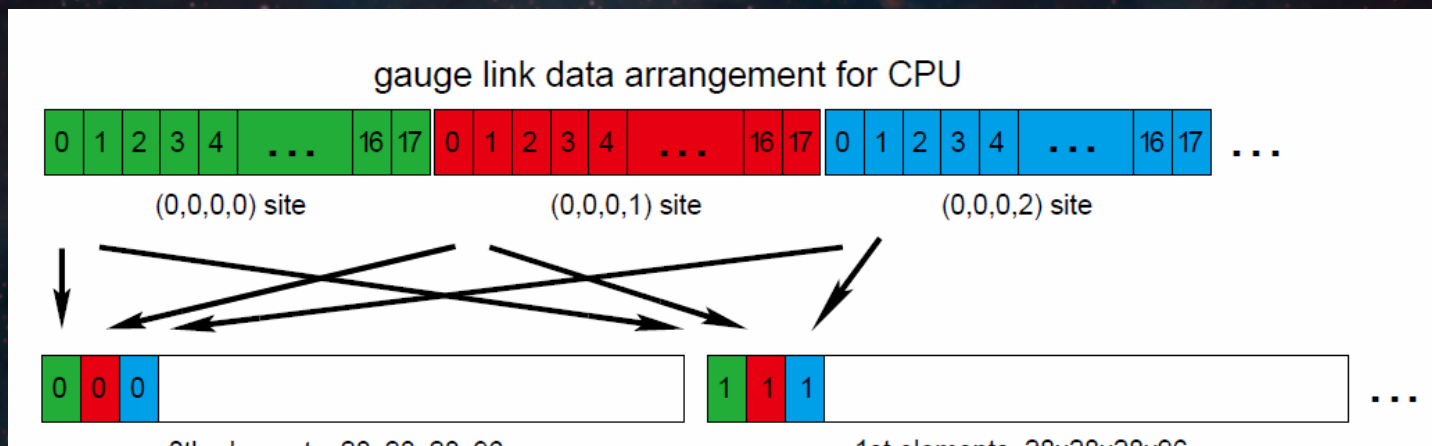

Oth elements, $28 \times 28 \times 28 \times 96$

1st elements, $28 \times 28 \times 28 \times 96$

gauge link data rearrangement for GPU

- 64 bit memory access channel

- Multiple data accesses by Warp(32 threads)

- Using Fast On-chip Memory ( register \& shared memory )

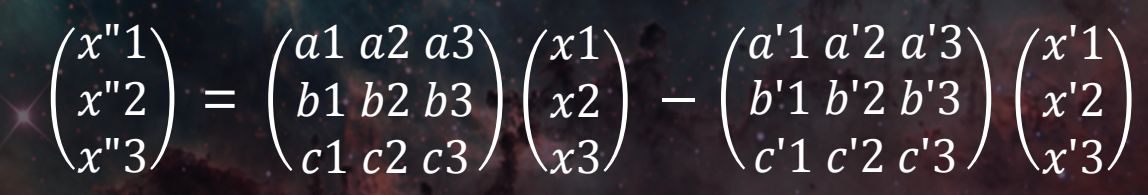

- Output vector is accessed 6 times

- ' Input vectors are accessed 2 times 


\section{CUDA Optimization(3)}

- Data Reconstruction ( Data Compression )

- In most application, The performance of GPU application is bounded to memory bandwidth

- Need to load balancing between calculation and data accessing

- Data has SU(3) symmetry, so this property can be used for data compression

- $8,10,12$ parameter SU(3) reconstructions

- Each method needs different optimization

- 10 parameter method is chosen to production 


\section{CUDA Optimization(ᄂ)}

- Using Mixed Precision

- Performance gap between Single Precision and Double Precision in GPU

- Tesla : DP / SP $=1 / 2, \quad$ Geforce : DP $/ \mathrm{SP}=1 / 8,1 / 12$ or $1 / 24$ !

- To retrieve this performance discrepancy, mixed precision method can be used

- The main idea of iterative refinement is using two types of iterative loops to get the true solution value.

- At first, by using the single precision iteration, we can approach fast to the roughly estimated solution

- Next, double precision or more precise iteration can be used to get the more accurate solution 


\section{IIPI Communication}

\section{- Practical multi GPU performance}

- Network IO (include PCI-E bus IO) : bottle-neck

- MPI(mvapich) is used for multi GPU implementing

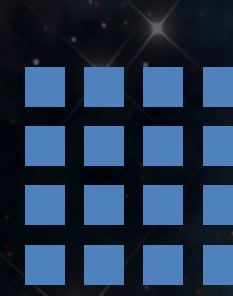

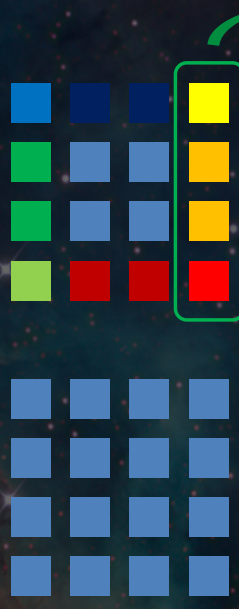

Lattice data at each node

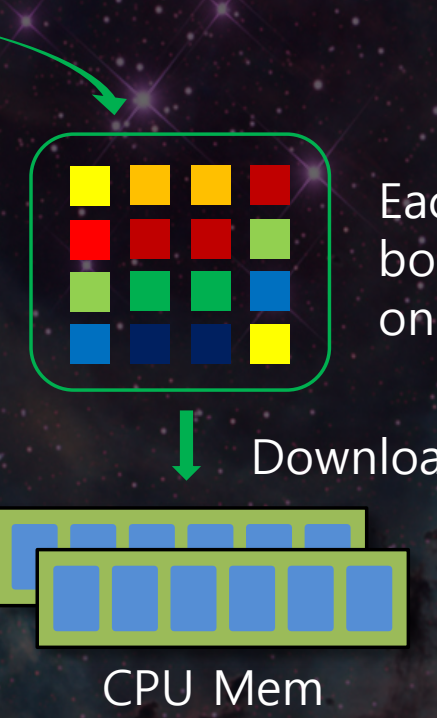

Each node collects boundary data on GPU Memory.
Upload the transferred Data from host to device

MPI data transfer Data send and receive are done concurrently

by 4 direction
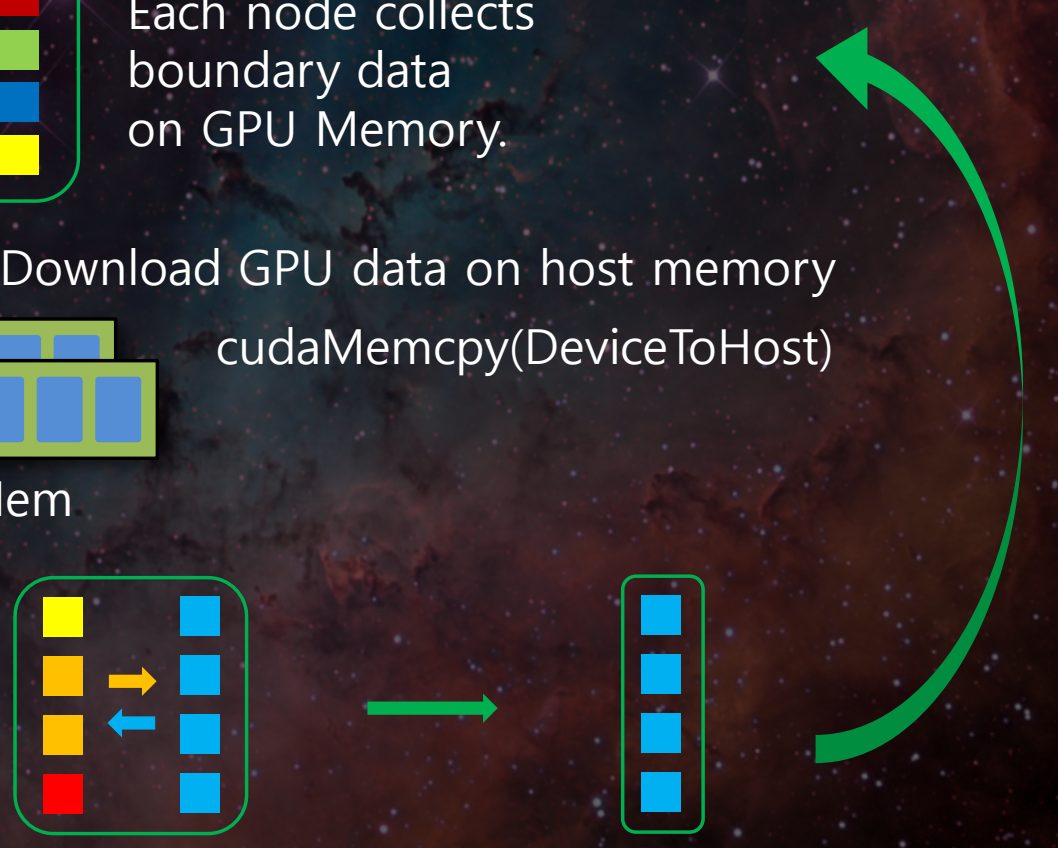


\section{Communication Optimization(1)}

1. Reduce the size of surface data

\section{Lattice Data}

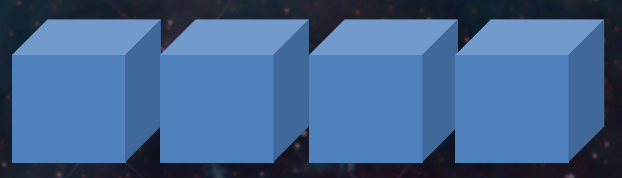

then

2. Reduce the unnecessary data transfer

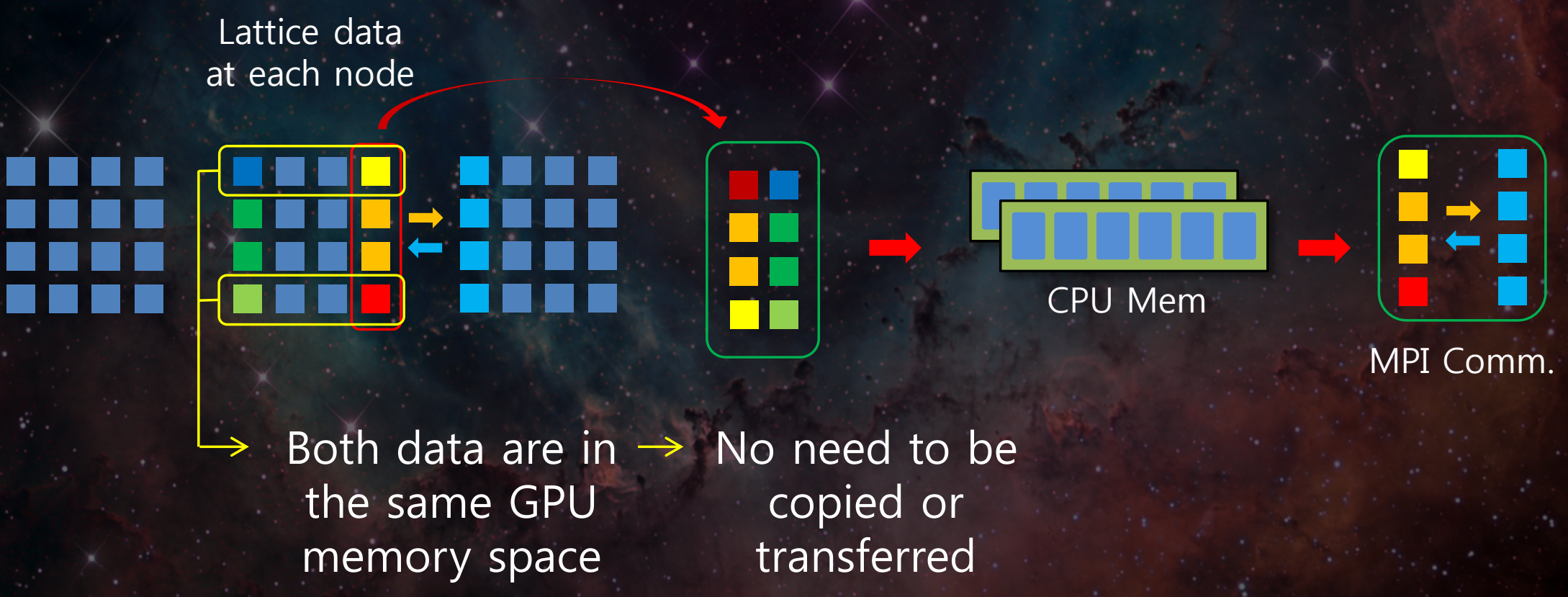




\section{Communication Optimization(2)}

3. Use GPU direct technology GPU direct 1.0

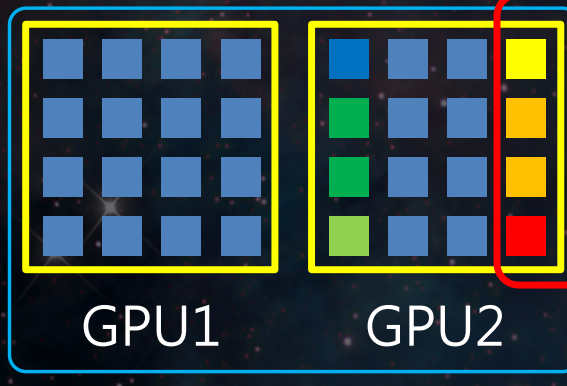

Node1

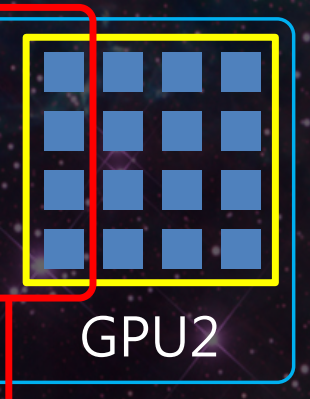

GPU direct 2.0
- GPU direct 1.0

- GPU direct Access

Possible only in Tesla GPU

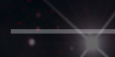

GPU direct 2.0

Key features

- GPU direct Transfer

is now implementing

- It may give $20 \%$

enhancement on network

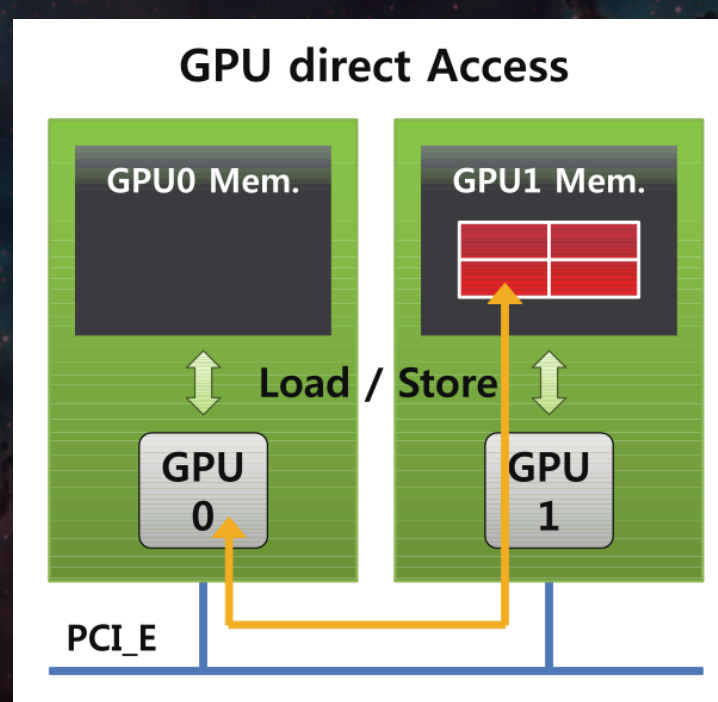

Node2 


\section{Performance (1)}

- Total CG Performance(exçlude network, used 4 GPU, 2896

lattice)

- Used GTX 480 : bandwidth =118GBytes/sec (measured),

. SP FLOPS $=1344$ GF (ideal),. DP. FLOPS $=168$ GF (ideal)

- For 1 Dirac operation,

Double Precision

Total $2.77 \mathrm{~ms}$ (measured)

In double precision, it takes $2 \times 2.77 \mathrm{~ms}$

$\rightarrow 576 \times 28 \times 28 \times 28 \times 96 / 4 / 2 / 0.00277$

\section{$=55$ GFLOPS}

In single precision, it takes $2 \times 1.04 \mathrm{~ms}$

$\rightarrow 576 \times 28 \times 28 \times 28 \times 96 / 4 / 2 / 0.00104$

\section{= 145 GFLOPS}

- Total mixed iteration $=30468(S P)+52(D P)$

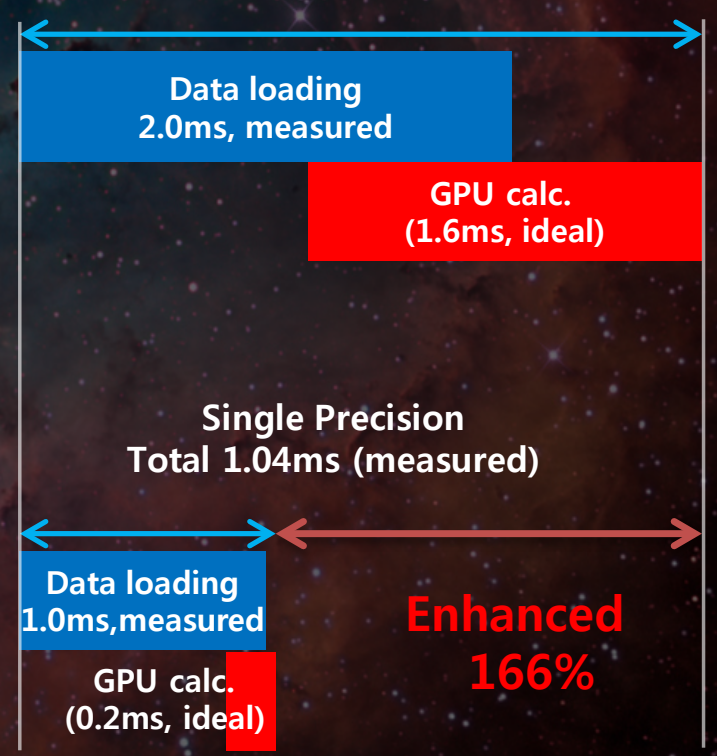




\section{Performance (2)}

- Time table of total sequence : For 1D $D(x)$ operation,

\begin{tabular}{|c|c|c|}
$\begin{array}{c}\text { GTX 480, 590 } \\
\text { Optimized Result }\end{array}$ & $\begin{array}{c}\text { Double } \\
\text { Precision } \\
\text { (ms) }\end{array}$ & $\begin{array}{c}\text { Single } \\
\text { Precision } \\
\text { (ms) }\end{array}$ \\
\hline GPU calculation time & 2.77 & 1.04 \\
\hline boundary data collect & 0.5 & 0.38 \\
\hline Memcpy GPU to CPU & 1.3 & 0.25 \\
\hline MPI communication & 2.1 & 0.6 \\
Memcpy CPU to GPU & 1.3 & 0.26 \\
\hline Total Comm. time & $\sim 5.5$ & $\sim 1.5$ \\
\hline Total time & $\sim 8.3$ & 2.6 \\
\hline GFLOPS(measured) & $\mathbf{1 8 . 8}$ & $\mathbf{4 4}$ \\
\hline
\end{tabular}




\section{HISO action}

- We are also implemented HISQ action to our CG invertor

- by Boram Yoon(in SNU)

- In single precision, for 1 Dirac operation, it takes $2 \times 1.98 \mathrm{~ms}$

× $2 \times 576 \times 28 \times 28 \times 28 \times 96 / 6 / 2 / 0.00198=102$ GFLOPS

- Most of times are also used for data loading

- Include network comm., real performance is 18.2GFLOPS

- Boundary data size is doubled!

- Optimization is still in progress 


\section{Summary}

- CUDA version of CG invertor is successfully implemented on CPS library

- For better performance, many optimization method were applied

- Network communication can be optimized a little bit more

- In mixed precision, CUDA version of staggered CG invertor shows 44 GFLOPS / GPU of performance.

- We are also preparing HISQ CG invertor 


\title{
1+1+1 Flavor QCD+QED Simulation at the Physical Point
}

\author{
Y.Kuramashi (U. of Tsukuba/RIKEN AICS) \\ for PACS-CS Collaboration
}

May 16, $2012 @ B N L$ 


\section{Collaboration members}

- Physicists:

S.Aoki, N.Ishizuka, K.Kanaya, Y.Kuramashi, Y.Namekawa, Tsukuba

Y.Taniguchi, A.Ukawa, N.Ukita, T.Yamazaki, T.Yoshie

K.-I.Ishikawa, M.Okawa

Hiroshima

D.Kadoh, Y.Nakamura, T.Izubuchi

RIKEN

- Computer scientists:

T.Boku, M.Sato, D.Takahashi, O.Tatebe

Tsukuba

T.Sakurai, H.Tadano 


\section{Plan of talk}

$\S 1$. Introduction

$\S 2$. Previous works

§3. Method

$\S 4$. Parameters and solver

$\S 5$. Results

$\S 6$. Summary 


\section{$\S 1$. Introduction}

Isospin symmetry breaking

- Quark mass difference:

$$
\mathrm{m}_{\mathrm{u}} \neq \mathrm{m}_{\mathrm{d}}
$$

- Electric charge difference $e_{p h} Q_{u} \neq e_{p h} Q_{d}, e_{p h}^{2}=4 \pi / 137$

\section{Mass splittings among} isospin multiplets

$m_{\pi 0}-m_{\pi \pm}, m_{K 0}-m_{K \pm}, m_{n}-m_{p}$

1+1+1 flavor QCD+QED simulation is a straightforward way

$$
\text { isospin multiplets } \Leftrightarrow \mathrm{u}, \mathrm{d}, \mathrm{s} \text { quark masses }
$$




\section{§2. Previous works}

\section{Group}

Duncan et al.

Blum et al.

Blum et al.

MILC

BMW

Duncan et al.

Ishikawa et al.
PRD71(2005)094509

Ref

PRL76(1996)3896

PRD76(2007)114508

PRD82(2010)094508

Lat08,Lat10

Lat10

arXiv:1202.6018
Action

qQED+qQCD Wilson

$q Q E D+N f=2 Q C D D W$

$q Q E D+N f=2+1$ QCD DW

$q Q E D+N f=2+1$ QCD Stag

$q Q E D+N f=2+1$ QCD Clover

Test of RW for fQED DW

$\mathrm{fQED}+\mathrm{Nf}=2+1 \mathrm{QCD} D W$ RW for fQED 


\section{§3. Method}

Reweighting from $\mathrm{Nf}=2+1 \mathrm{QCD}$ to $\mathrm{Nf}=1+1+1 \mathrm{QCD}+\mathrm{QED}$

$$
\left\langle\mathcal{O}[U]\left(\kappa_{\mathrm{u}}^{*}, \kappa_{\mathrm{d}}^{*}, \kappa_{\mathrm{s}}^{*}\right)\right\rangle_{\left(\kappa_{\mathrm{u}}^{*}, \kappa_{\mathrm{d}}^{*}, \kappa_{\mathrm{s}}^{*}\right), \mathrm{fQED}}=\frac{\left\langle\mathcal{O}[U]\left(\kappa_{\mathrm{u}}^{*}, \kappa_{\mathrm{d}}^{*}, \kappa_{\mathrm{s}}^{*}\right) \operatorname{det}\left[W_{\mathrm{uds}}[U]\right]\right\rangle_{\left(\kappa_{\mathrm{ud}}, \kappa_{\mathrm{s}}\right), \mathrm{qQED}}}{\left\langle\operatorname{det}\left[W_{\mathrm{uds}}[U]\right]\right\rangle_{\left(\kappa_{\mathrm{ud}}, \kappa_{\mathrm{s}}\right), \mathrm{qQED}}}
$$

with $\left(\mathrm{K}_{\mathrm{u}}{ }^{*}, \mathrm{~K}_{\mathrm{d}}{ }^{*}, \mathrm{~K}_{\mathrm{s}}{ }^{*}\right)$ hopping parameters at the physical point

$$
W_{\mathrm{uds}}[U]=\prod_{q=\mathrm{u}, \mathrm{d}, \mathrm{s}} \frac{D\left(e_{\mathrm{ph}} Q_{q}, \kappa_{q}^{*}\right)}{D\left(0, \kappa_{q}\right)}
$$




\section{Evaluation of $\operatorname{det}\left[\mathrm{W}_{\mathrm{uds}}[\mathrm{U}]\right]$}

Introduce a complex bosonic field $\eta$

$$
\left|\operatorname{det}\left[W_{\text {uds }}\right]\right|^{2}=\left\langle\mathrm{e}^{-\left|W_{\text {uds }}^{-1} \eta\right|^{2}+|\eta|^{2}}\right\rangle_{\eta}
$$

Given a set of $\eta_{i}\left(i=1, \ldots, N_{\eta}\right)$ with Gaussian distribution

$$
\operatorname{det}\left[W_{\mathrm{uds}}[U]\right]=\left[\lim _{N_{\eta} \rightarrow \infty} \frac{1}{N_{\eta}} \sum_{i=1}^{N_{\eta}} \mathrm{e}^{-\left|W_{\mathrm{uds}}^{-1}[U] \eta_{i}\right|^{2}+\left|\eta_{i}\right|^{2}}\right]^{\frac{1}{2}}
$$


- Determinant breakup

Hasenfratz et al.,PRD78(2008)014515

divide the reweighting path $\left(\mathrm{e}=0 ; \mathrm{K}_{\mathrm{ud}}, \mathrm{K}_{\mathrm{s}}\right) \Rightarrow\left(\mathrm{e}=\mathrm{e}_{\mathrm{ph}} ; \mathrm{K}_{\mathrm{u}}{ }^{*}, \mathrm{~K}_{\mathrm{d}}{ }^{*}, \mathrm{~K}_{\mathrm{s}}{ }^{*}\right)$

in the parameter space into $\mathrm{N}_{B}$ subintervals

$$
\operatorname{det}\left[W_{\mathrm{uds}}\right]=\operatorname{det}\left[W_{\mathrm{uds}}^{(1)}\right] \times \operatorname{det}\left[W_{\mathrm{uds}}^{(2)}\right] \times \cdots \times \operatorname{det}\left[W_{\mathrm{uds}}^{\left(N_{B}\right)}\right]
$$

- Combined reweighting factor

-Averaged photon field

$$
\text { explained in next slides }
$$




\section{Combined reweighting factor}

Ishikawa et al., arXiv:1202.6018

- Combined reweighting factor for $u, d, s$ quarks with a single set of noise

$$
\begin{aligned}
& \operatorname{det}\left[W_{\text {uds }}[U]\right]=\left[\lim _{N_{\eta} \rightarrow \infty} \frac{1}{N_{\eta}} \sum_{i=1}^{N_{\eta}} \mathrm{e}^{-\left|W_{\mathrm{uds}}^{-1}[U] \eta_{i}\right|^{2}+\left|\eta_{i}\right|^{2}}\right]^{\frac{1}{2}} \\
& \operatorname{det}\left[\mathbf{W}_{\text {uds }}\right] \text { rather than } \operatorname{det}\left[\mathbf{W}_{\mathrm{u}}\right] \times \operatorname{det}\left[\mathbf{W}_{\mathrm{d}}\right] \times \operatorname{det}\left[\mathbf{W}_{\mathrm{s}}\right]
\end{aligned}
$$

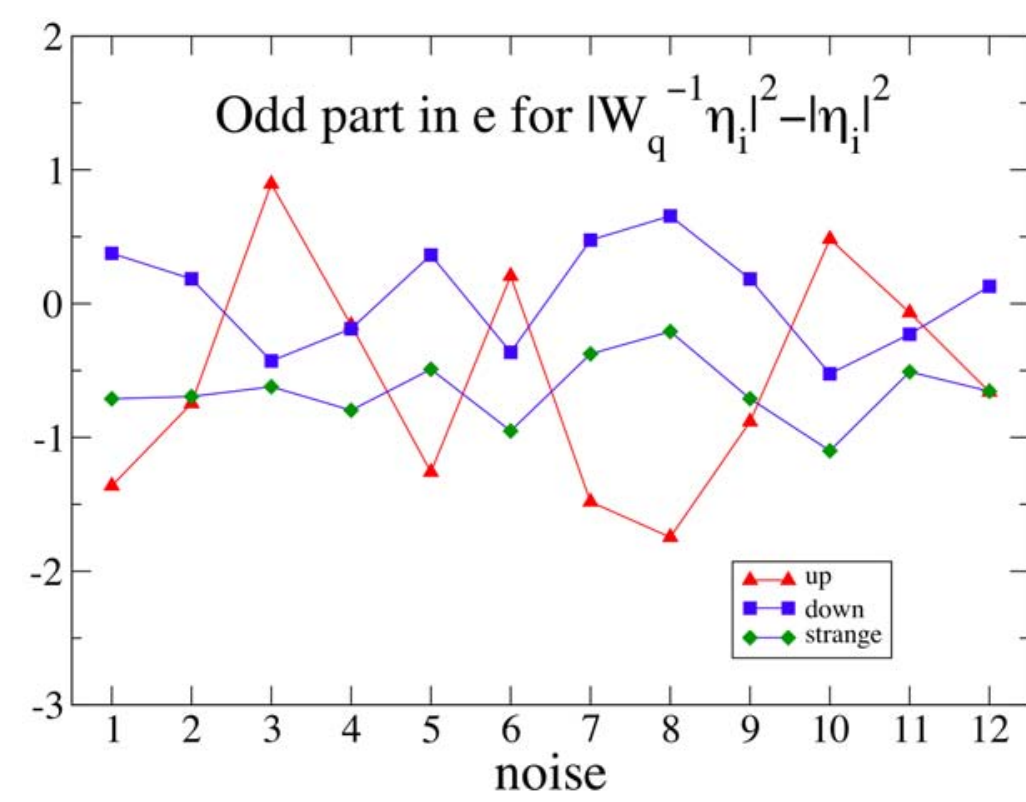

Odd part is extracted from \pm e calculations

Leading contribution of $O(e)$ is partially cancelled due to $Q_{u}+Q_{d}+Q_{s}=0$ 


\section{Averaged photon field (1)}

- generate photon field $b_{\mu}$ on $64^{3} \times 128$ lattice $\left(\Leftrightarrow 32^{3} \times 64\right.$ QCD lattice) with non-compact pure gauge action

$$
S_{b}=\sum_{n, \mu, \nu} \frac{1}{4}\left(\partial_{\mu} b_{\nu}(n)-\partial_{\nu} b_{\mu}(n)\right)^{2}+\sum_{n, \mu, \nu} c_{1}\left(\partial_{\mu}\left(\partial_{\mu} b_{\nu}(n)-\partial_{\nu} b_{\mu}(n)\right)\right)^{2}
$$

$c_{1}=-0.646$ is chosen such that

$$
\left.S_{A}=\sum_{N, \mu, \nu} \frac{1}{4}\left(\nabla_{\mu} A_{\nu}(N)-\nabla_{\nu} A_{\mu}(N)\right)^{2}\right\rangle=\left\langle\sum_{N, \mu, \nu} \frac{1}{4}\left(\nabla_{\mu} B_{\nu}(N)-\nabla_{\nu} B_{\mu}(N)\right)^{2}\right\rangle
$$

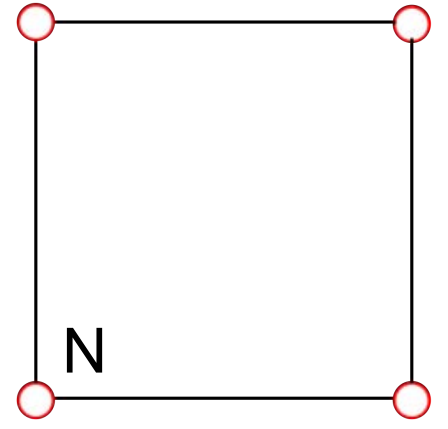




\section{Averaged photon field (2)}

- averaged photon fields over independent paths inside the $2^{4}$ hypercube on the QED lattice

$$
\begin{aligned}
& \bar{B}_{\mu}(N=n / 2)= \frac{1}{27}\left[\left(b_{\mu}(n)+b_{\mu}(n+\hat{\mu} / 2)\right)\right. \\
&+\sum_{\nu \neq \mu} \sum_{\nu_{s}= \pm \nu}\left(b_{\nu_{s}}(n)+b_{\mu}\left(n+\hat{\nu}_{s} / 2\right)+b_{\mu}\left(n+\hat{\nu}_{s} / 2+\hat{\mu} / 2\right)-b_{\nu_{s}}(n+\hat{\mu})\right) \\
&+\frac{1}{2} \sum_{\rho \neq \nu \neq \mu} \sum_{\rho_{s}= \pm \rho \nu_{s}= \pm \nu} \sum_{\rho_{s}}(n)+b_{\nu_{s}}\left(n+\hat{\rho}_{s} / 2\right) \\
&+b_{\mu}\left(n+\hat{\rho}_{s} / 2+\hat{\nu}_{s} / 2\right)+b_{\mu}\left(n+\hat{\rho}_{s} / 2+\hat{\nu}_{s} / 2+\hat{\mu} / 2\right) \\
&\left.\quad-b_{\rho_{s}}(n+\hat{\mu})-b_{\nu_{s}}\left(n+\hat{\rho}_{s} / 2+\hat{\mu}\right)\right) \\
&+\frac{1}{6} \sum_{\sigma \neq \rho \neq \nu \neq \mu} \sum_{\sigma_{s}= \pm \sigma} \sum_{\rho_{s}= \pm \rho \nu_{s}= \pm \nu}\left(b_{\sigma_{s}}(n)+b_{\rho_{s}}\left(n+\hat{\sigma}_{s} / 2\right)+b_{\nu_{s}}\left(n+\hat{\sigma}_{s} / 2+\hat{\rho}_{s} / 2\right)\right. \\
& \quad+b_{\mu}\left(n+\hat{\sigma}_{s} / 2+\hat{\rho}_{s} / 2+\hat{\nu}_{s} / 2\right)+b_{\mu}\left(n+\hat{\sigma}_{s} / 2+\hat{\rho}_{s} / 2+\hat{\nu}_{s} / 2+\hat{\mu} / 2\right) \\
&\left.\left.\quad-b_{\sigma_{s}}(n+\hat{\mu})-b_{\rho_{s}}\left(n+\hat{\sigma}_{s} / 2+\hat{\mu}\right)-b_{\nu_{s}}\left(n+\hat{\sigma}_{s} / 2+\hat{\rho}_{s} / 2+\hat{\mu}\right)\right)\right]
\end{aligned}
$$

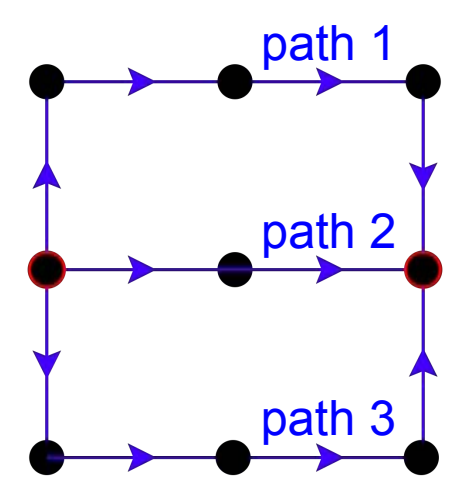

- construct $U(1)$ link variable in terms of the averaged photon field

- numerically checked that the averaged and conventional photon fields give consistent results for $\mathrm{m}_{\mathrm{PSO}}-\mathrm{m}_{\mathrm{PS} \pm}$ 


\section{§4. Parameters and solver}

- Configuration parameters PACS-CS,PRD81(2010)074503

$\mathrm{Nf}=2+1$ flavor $\mathrm{QCD}$ near the physical point

NP O(a)-improved Wilson-clover quark and Iwasaki gauge action

$\beta=1.9,32^{3} \times 64, a \sim 0.1 \mathrm{fm},\left(\mathrm{K}_{\mathrm{ud}}, \mathrm{K}_{\mathrm{s}}\right)=(0.137785,0.13660), 2000 \mathrm{MD}$ time

- Reweighting parameters

physical point: $\left(\mathrm{K}_{\mathrm{u}}{ }^{*}, \mathrm{~K}_{\mathrm{d}}{ }^{*}, \mathrm{~K}_{\mathrm{s}}{ }^{*}\right)=(0.13787014,0.13779700,0.13669510)$

determined from $\pi^{+}, \mathrm{K}^{+}, \mathrm{K}^{0}, \Omega^{-}$masses

$\mathrm{e}_{\mathrm{ph}}{ }^{2}=4 \pi / 137$

$N_{B}=426, N_{n}=12$ for each piece of divided determinant

- Modified block BiCGStab

Nakamura et al.,CPC183(2012)34

quark matrix inversions for $\mathrm{N}_{\mathrm{n}}=12$ noises

a factor of 3 4 cost reduction compared with non-block solvers 


\section{Modified block BiCGStab (1)}

A solver algorithm for linear eqs with multiple right-hand sides $D x^{(i)}=b^{(i)}(i=1, \ldots, L) \Rightarrow D X=B$

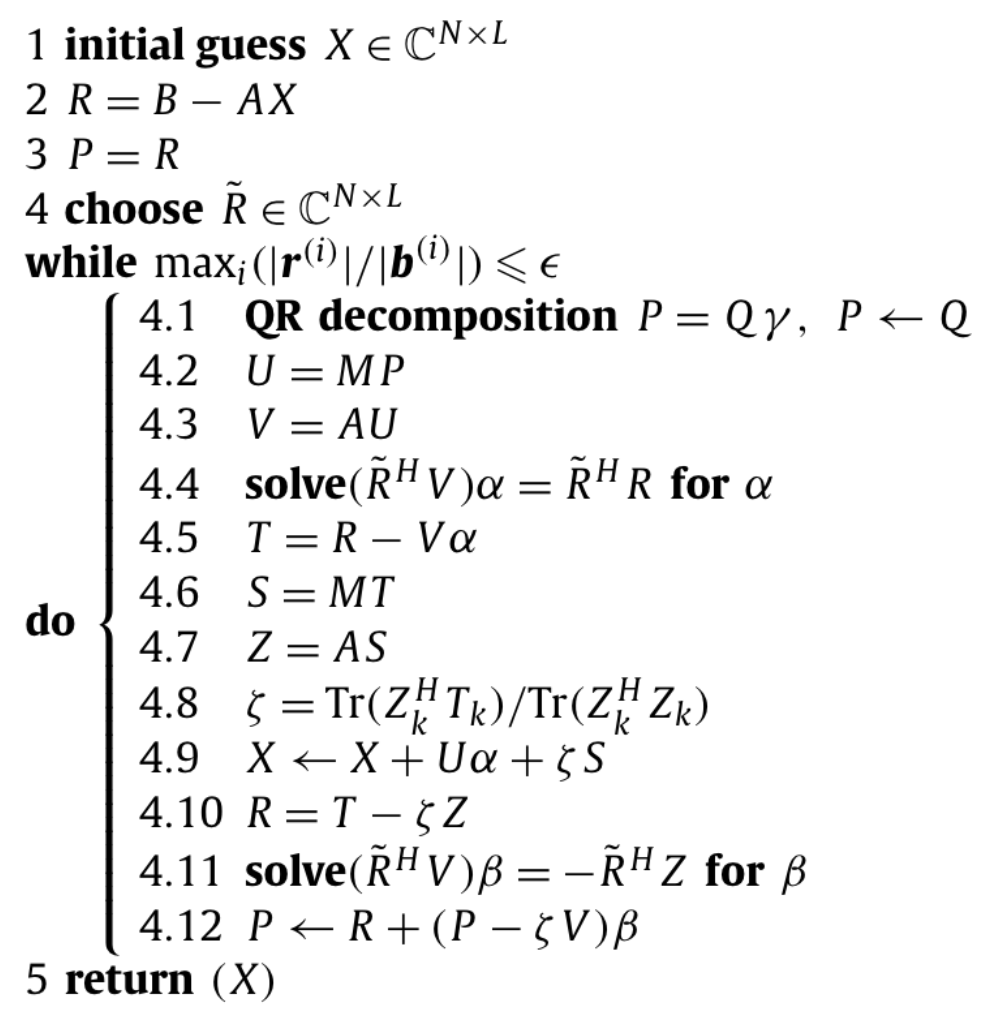

Basic idea: blocked version searches the solution vectors with the enlarged Krylov subspace 


\section{Modified block BiCGStab (2)}

\section{a representative case}

$\mathrm{Nf}=2+1 \mathrm{QCD}, 32^{3} \times 64, \mathrm{a} \sim 0.1 \mathrm{fm}$, $\left(K_{\mathrm{ud}}, \mathrm{K}_{\mathrm{s}}\right)=(0.137785,0.13660)$, point source

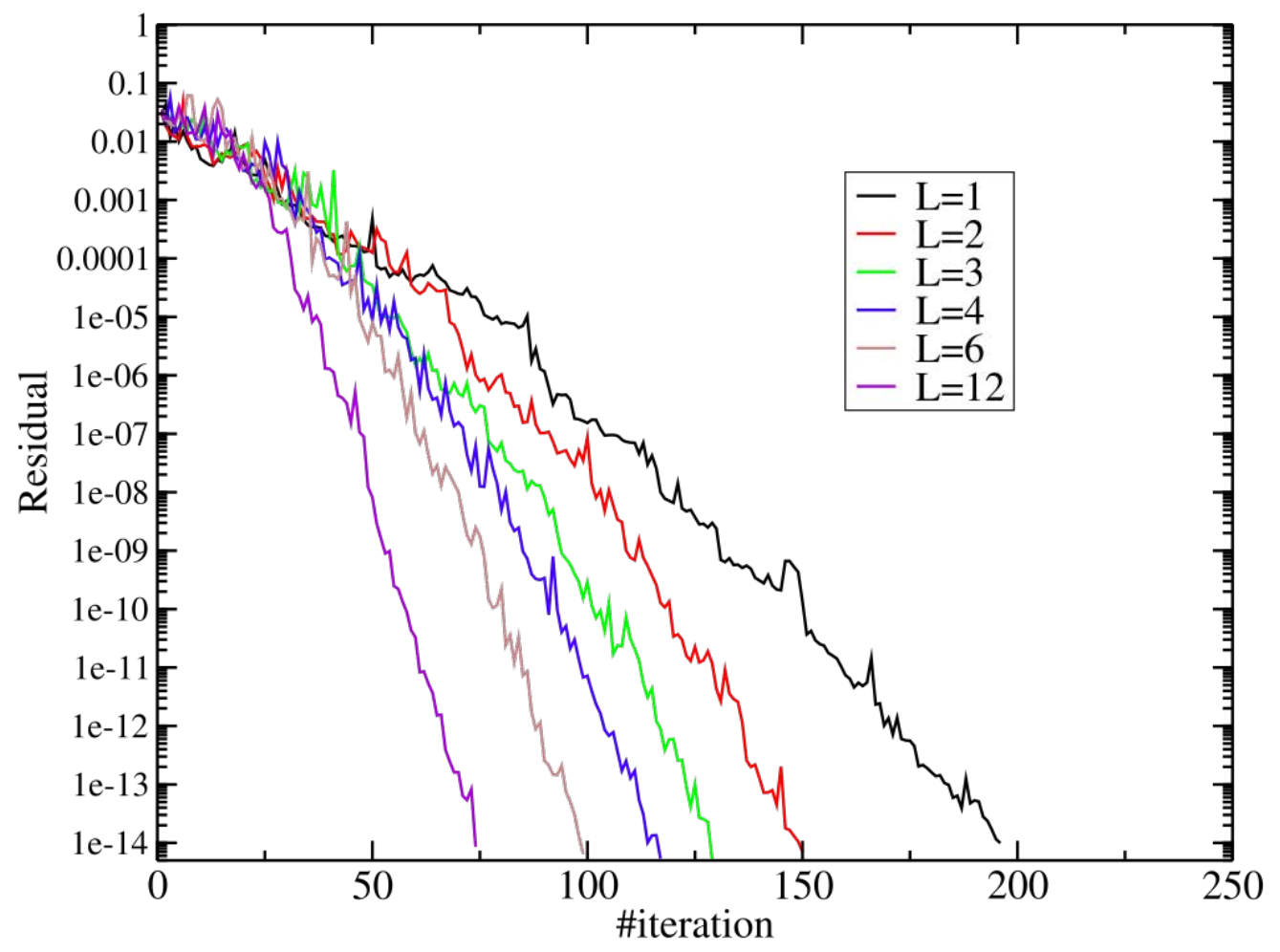




\section{Modified block BiCGStab (3)}

Performance test averaged over 10 configs

$$
D x^{(i)}=b^{(i)}(i=1, \ldots, 12)
$$

\begin{tabular}{lllrl}
\hline$L \times 12 / L$ & Time $[\mathrm{s}]$ & $T$ (gain) & \multicolumn{1}{l}{ NMVM } & NM (gain) \\
\hline $1 \times 12$ & $3827(755)$ & 1 & $17146(3326)$ & 1 \\
$2 \times 6$ & $2066(224)$ & 1.9 & $12942(1379)$ & 1.3 \\
$3 \times 4$ & $1619(129)$ & 2.4 & $10652(832)$ & 1.6 \\
$4 \times 3$ & $1145(99)$ & 3.3 & $9343(835)$ & 1.8 \\
$6 \times 2$ & $1040(87)$ & 3.7 & $7888(663)$ & 2.2 \\
$12 \times 1$ & $705(70)$ & 5.4 & $6106(633)$ & 2.8 \\
\hline
\end{tabular}

$T$ (gain) $>N M($ gain) is thanks to effective usage of cache 


\section{$\S 5$. Results}

Configuration dependence of reweighting factor

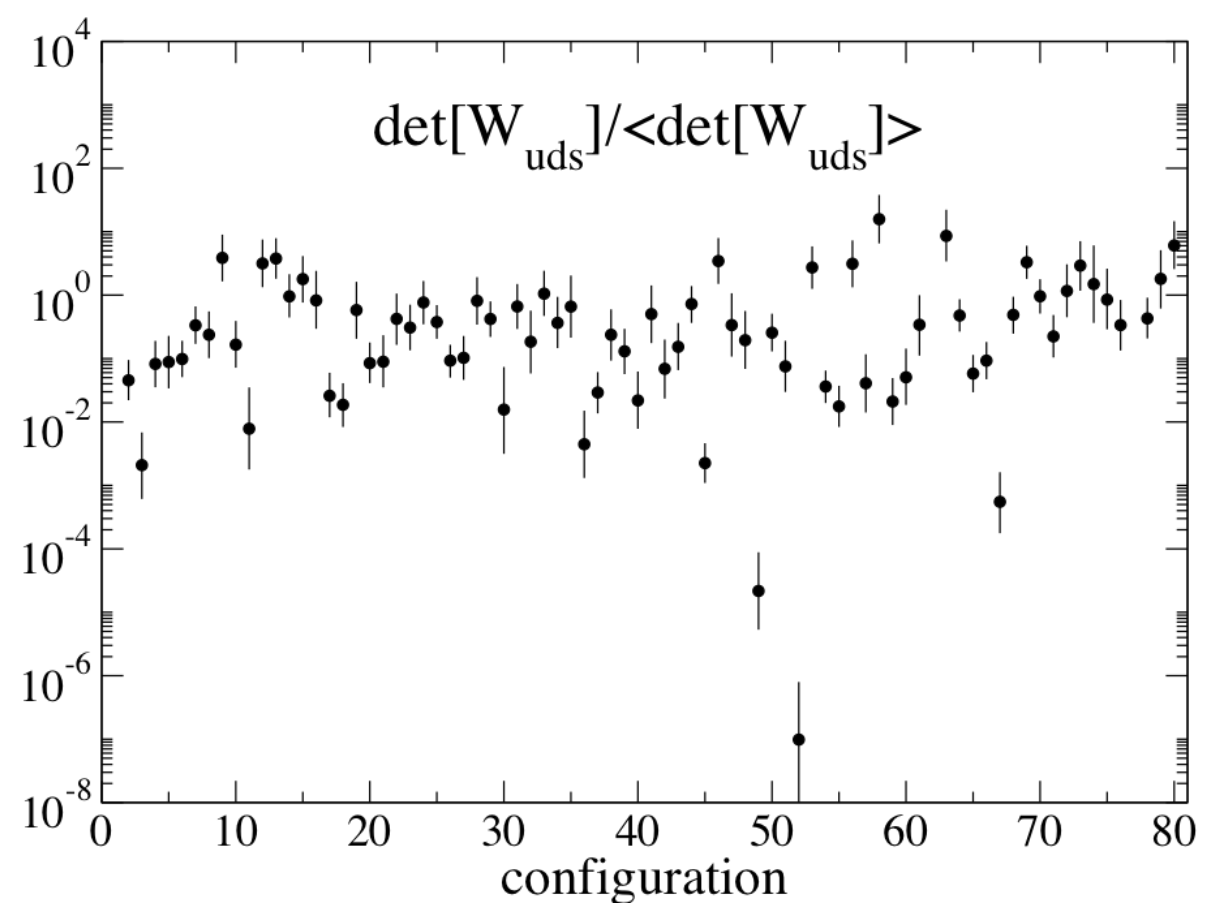

Normalized by <det[$\left[\mathrm{W}_{\text {uds }}\right]>$

$$
\left\langle\mathcal{O}[U]\left(\kappa_{\mathrm{u}}^{*}, \kappa_{\mathrm{d}}^{*}, \kappa_{\mathrm{s}}^{*}\right)\right\rangle_{\left(\kappa_{\mathrm{u}}^{*}, \kappa_{\mathrm{d}}^{*}, \kappa_{\mathrm{s}}^{*}\right), \mathrm{fQED}}=\frac{\left\langle\mathcal{O}[U]\left(\kappa_{\mathrm{u}}^{*}, \kappa_{\mathrm{d}}^{*}, \kappa_{\mathrm{s}}^{*}\right) \operatorname{det}\left[W_{\mathrm{uds}}[U]\right]\right\rangle_{\left(\kappa_{\mathrm{ud}}, \kappa_{\mathrm{s}}\right), \mathrm{qQED}}}{\left\langle\operatorname{det}\left[W_{\mathrm{uds}}[U]\right]\right\rangle_{\left(\kappa_{\mathrm{ud}}, \kappa_{\mathrm{s}}\right), \mathrm{qQED}}}
$$




\section{Effective masses for $\pi^{+}, \mathrm{K}^{+}, \mathrm{K}^{0}, \Omega^{-}$}

Smear-local propagators, $\mathrm{N}_{\mathrm{n}}=12$
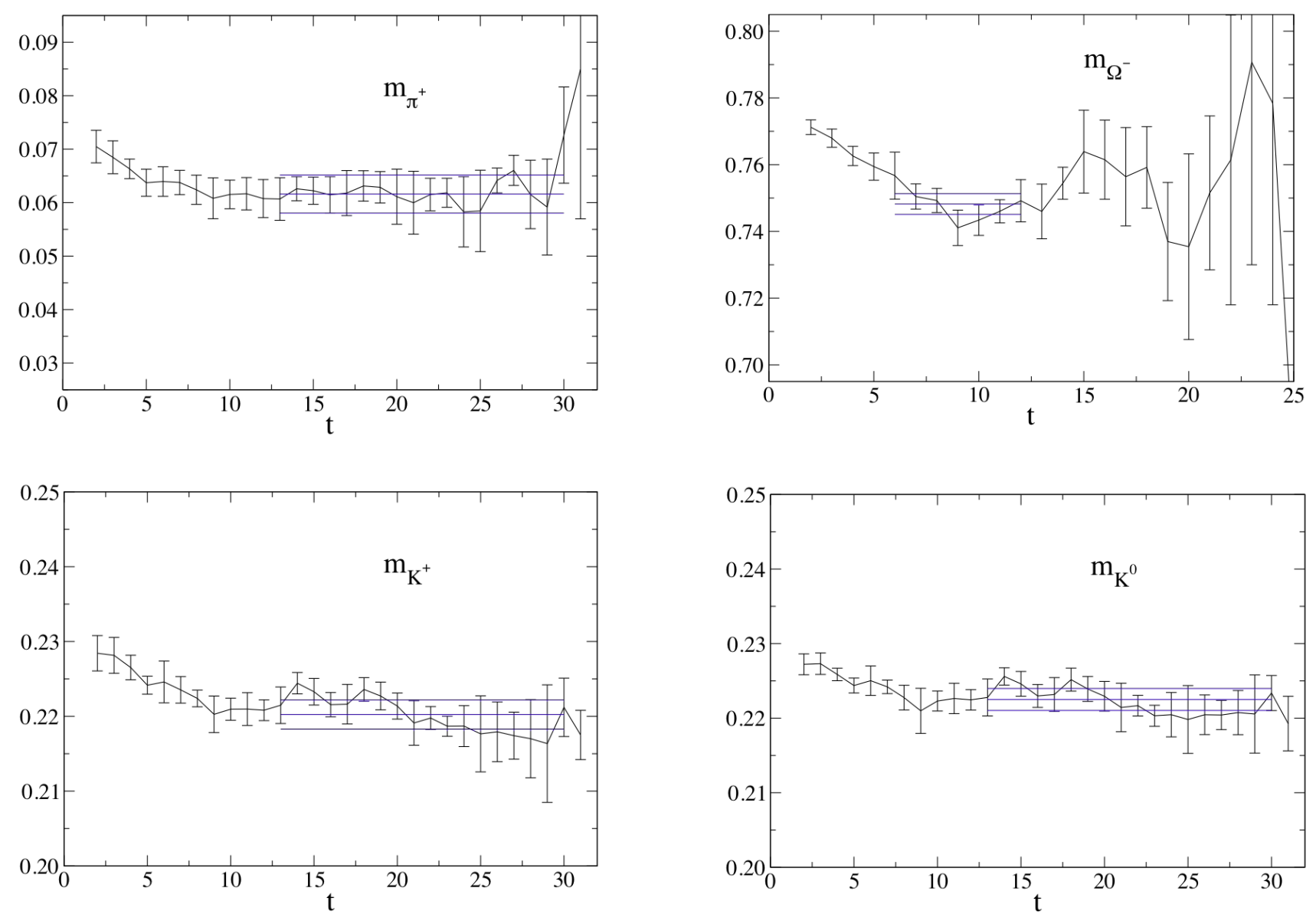


\section{$N_{\eta}$ dependence of hadron mass ratios}

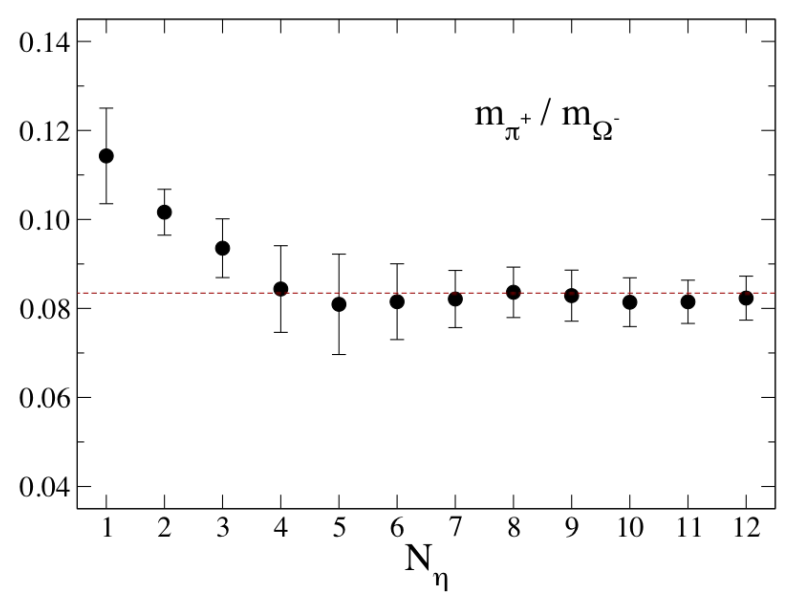

\begin{tabular}{lcc}
\hline \hline & Our results $[\mathrm{MeV}]$ & Experiment $[\mathrm{MeV}]$ \\
\hline$m_{\pi^{+}}$ & $137.7(8.0)$ & $139.57018(35)$ \\
$m_{K^{+}}$ & $492.3(4.7)$ & $493.677(16)$ \\
$m_{K^{0}}$ & $497.4(3.7)$ & $497.614(24)$ \\
$m_{\Omega^{-}}$ & input & $1672.45(29)$ \\
\hline \hline
\end{tabular}
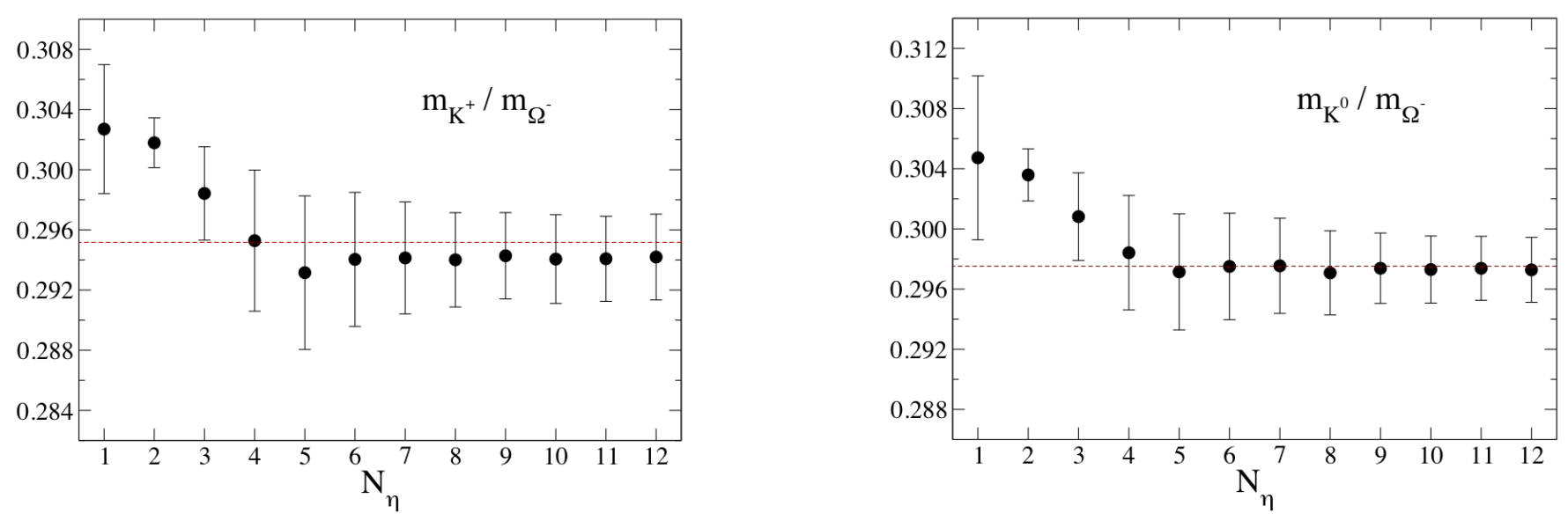

plateau close to the experimental value for $N_{\eta} \gtrsim 4$ 


\section{Ratio of $\mathrm{K}^{0}$ to $\mathrm{K}^{+}$propagators}

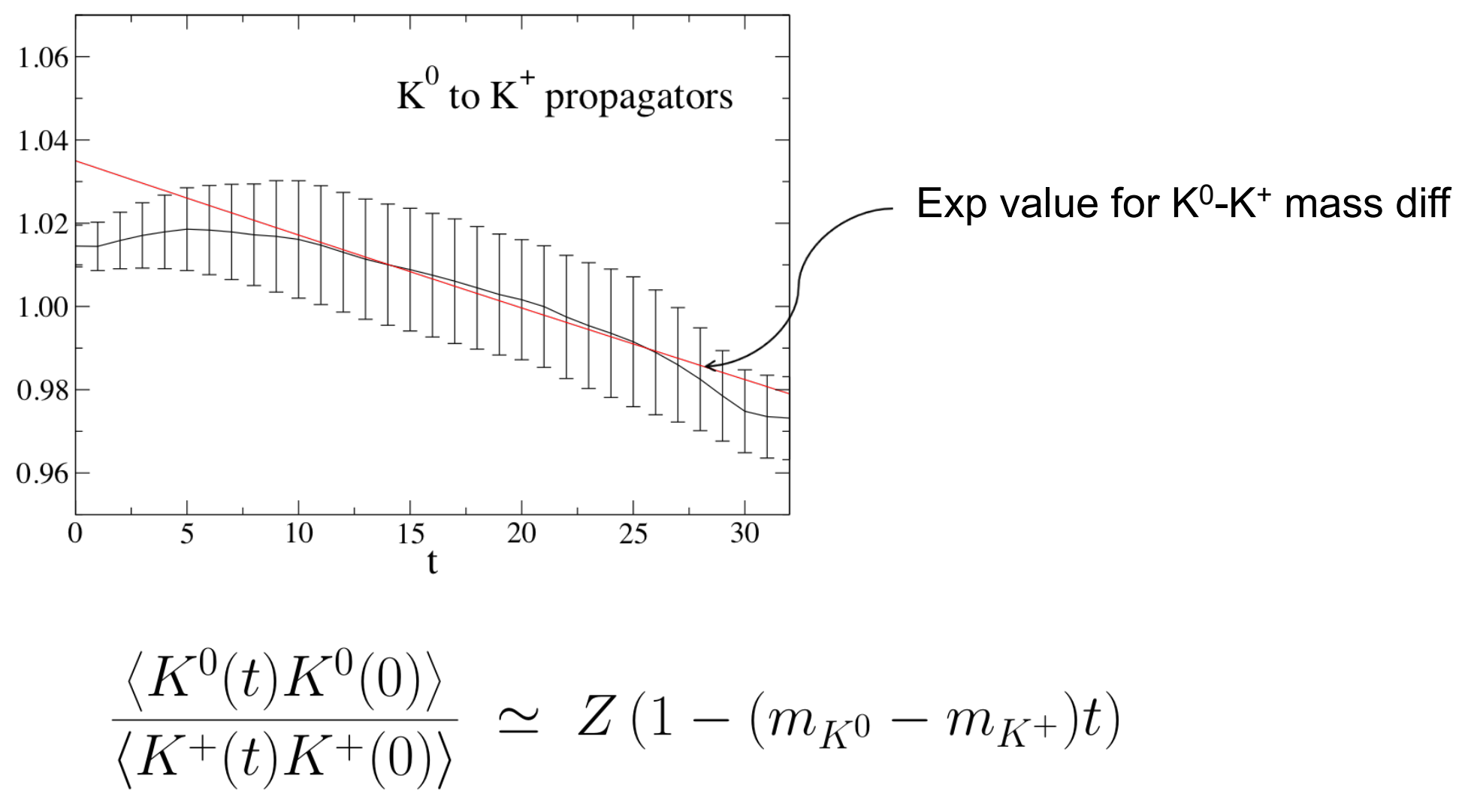

much smaller than 1

Fit result 4.54(1.09) MeV is consistent with exp value 3.937(28) MeV 


\section{Quark masses with NP renormalization factor}

- NP renormalization factor with Schrödinger functional scheme

PACS-CS, JHEP1008(2010)101

$$
\begin{aligned}
& \text { PRD81(2010)074503 [2+1f QCD (1)] } \\
& \text { PRD79(2009)034503 [2+1f QCD (2)] }
\end{aligned}
$$

- neglect QED corrections to the renormalization factor

- physical inputs: $\mathrm{m}_{\pi^{+}}, \mathrm{m}_{\mathrm{K} 0}, \mathrm{~m}_{\mathrm{K}+}, \mathrm{m}_{\Omega^{-}}$

- MS-bar scheme at $\mu=2 \mathrm{GeV}$

\section{This work}

2+1f QCD (1) 2+1f QCD (2)

$\mathrm{m}_{\mathrm{u}}[\mathrm{MeV}]$ 2.57(26)(07)

$\mathrm{m}_{\mathrm{d}}[\mathrm{MeV}] \quad 3.68(29)(10)$

$\mathrm{m}_{\mathrm{s}}[\mathrm{MeV}] \quad 83.60(58)(2.23) \quad 86.7(2.3)$

87.7(3.1)

$\mathrm{m}_{\mathrm{ud}}[\mathrm{MeV}] \quad 3.12(24)(08)$

$2.78(27)$

$3.05(12)$

$m_{u} / m_{d}$

$0.698(51)$

$\mathrm{m}_{\mathrm{s}} / \mathrm{m}_{\mathrm{ud}} \quad 26.8(2.0)$

$31.2(2.7)$

$28.78(40)$

- possible QED finite size effects: $-13.50 \%$ for $u,+2.48 \%$ for $d,-0.07 \%$ for $s$ 


\section{§6. Summary}

-1+1+1 flavor QCD+QED simulation at the physical point

- Dynamical quark effects in QED and u-d quark mass difference are incorporated by reweighting technique

$\cdot \mathrm{u}, \mathrm{d}, \mathrm{s}$ quark masses are determined with $\mathrm{m}_{\pi^{+}}, \mathrm{m}_{\mathrm{K} 0}, \mathrm{~m}_{\mathrm{K}_{+},} \mathrm{m}_{\Omega^{-}}$as physical inputs

- Direct investigation of finite size effects due to QED is left as a future work 


\section{The Composite Higgs Mechanism and the Conformal Window}

\section{Lattice Higgs Collaboration (LHC)}

USQCD BSM

BNL workshop 2012 with Zoltan Fodor, Kieran Holland, Daniel Nogradi, Chris Schroeder, Chik Him Wong

\section{Julius Kuti}

\section{University of California, San Diego}

New Horizons for Lattice Computations with Chiral Fermions BNL May 14, 2012 


\section{Outline}

- LHC Higgs search and BSM implications focus on SU(3) fermion representation with two flavors

- Two RG based strategies

- New results on the $\mathrm{Nf}=2$ sextet model in the $\mathrm{SU}(3)$ color rep

- Cosmology connection

(dark baryon matter and EW phase transition)

- Conformal FSS method

- New results on FSS in the $\mathrm{Nf}=12$ model in the fundamental $\mathrm{SU}(3)$ rep

- Outlook 


\section{Atlas and CMS compared (from Vivek Sharma)}

For low Higgs mass hypothesis both CMS \& ATLAS see an excess in event yield over expected background

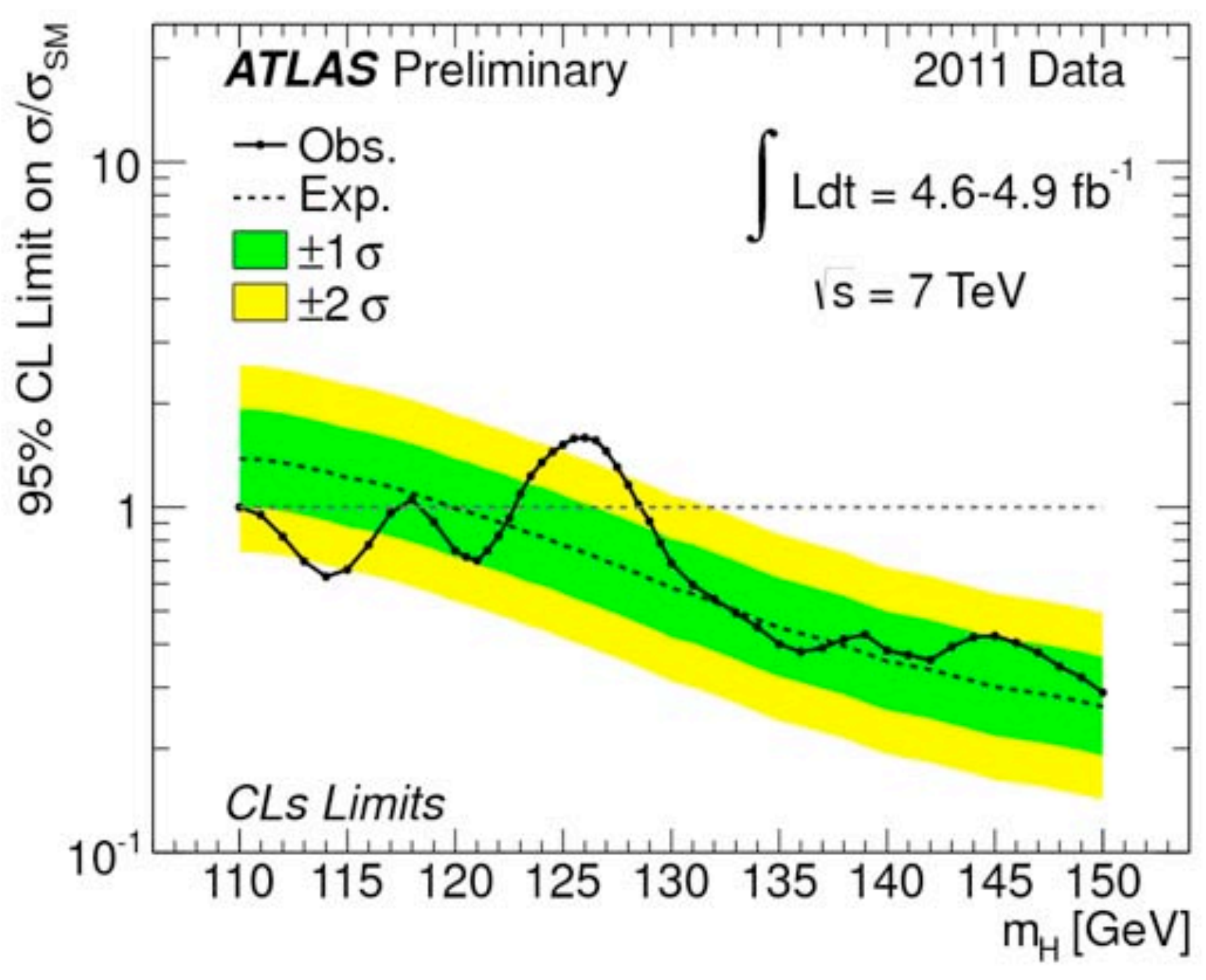

ATLAS excess at $\mathrm{M} \approx 126 \mathrm{GeV}$

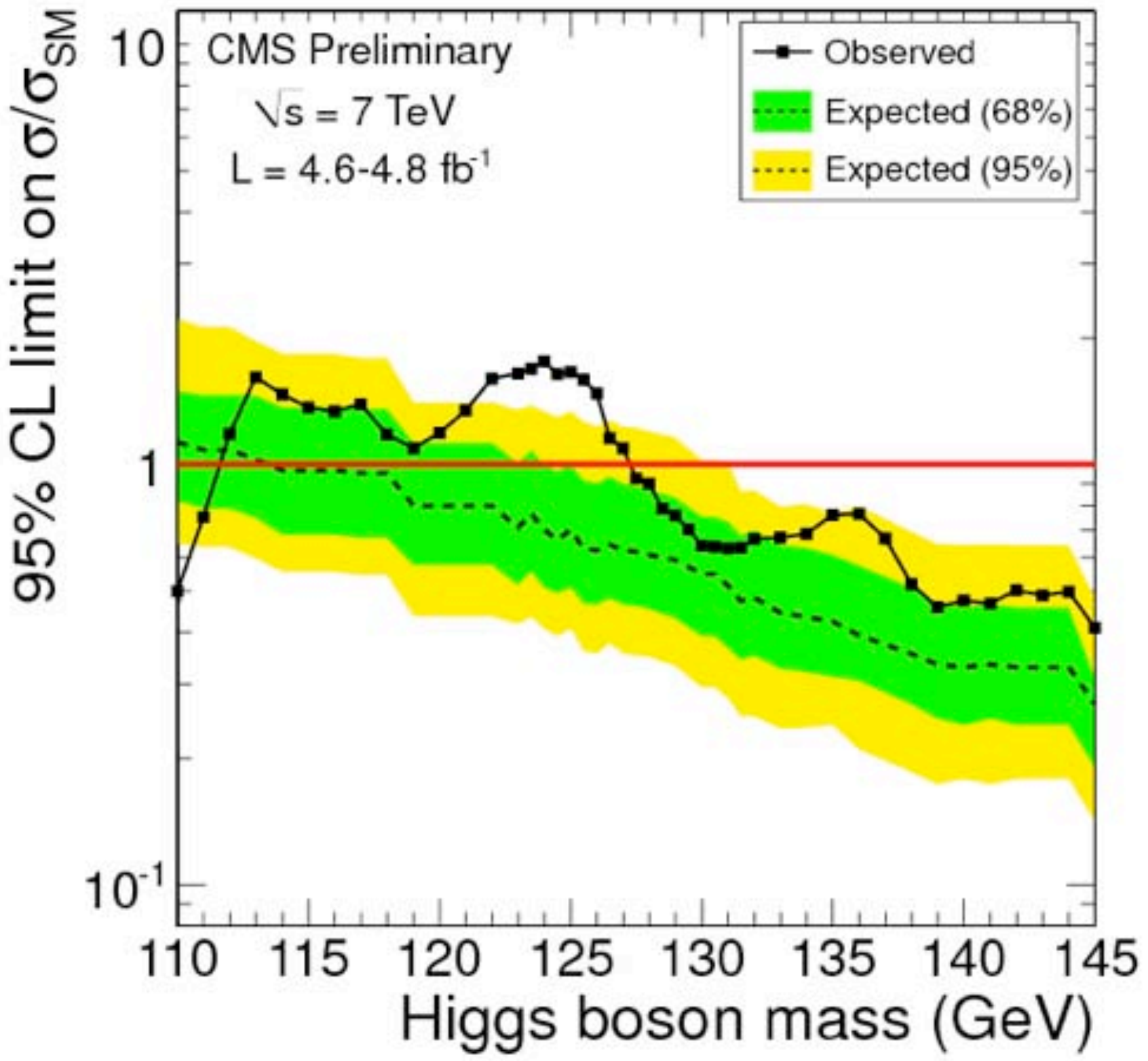

CMS excess at $\mathrm{M} \approx 125 \mathrm{GeV}$ 
What comes at the end of the LHC run?

- light Higgs with non-SM couplings (dilaton?)

- Heavy Higgs, or Higgsless

- SM Higgs (SUSY symmetry breaking?)

- USQCD composite Higgs and SUSY - timely efforts 
What comes at the end of the LHC run?

- light Higgs with non-SM couplings (dilaton?)

- Heavy Higgs, or Higgsless

- SM Higgs (SUSY symmetry breaking?)

- USQCD composite Higgs and SUSY - timely efforts

- Composite Higgs mechanism

- The paradigm is important again

- Higgsless QCD-like (cutoff $\Lambda$ to $3 \mathrm{TeV}$ )

- changes close to conformal windo

- non-perturbative lattice studies needed

- USQCD effort: 
What comes at the end of the LHC run?

- light Higgs with non-SM couplings (dilaton?)

- Heavy Higgs, or Higgsless

- SM Higgs (SUSY symmetry breaking?)

- USQCD composite Higgs and SUSY - timely efforts

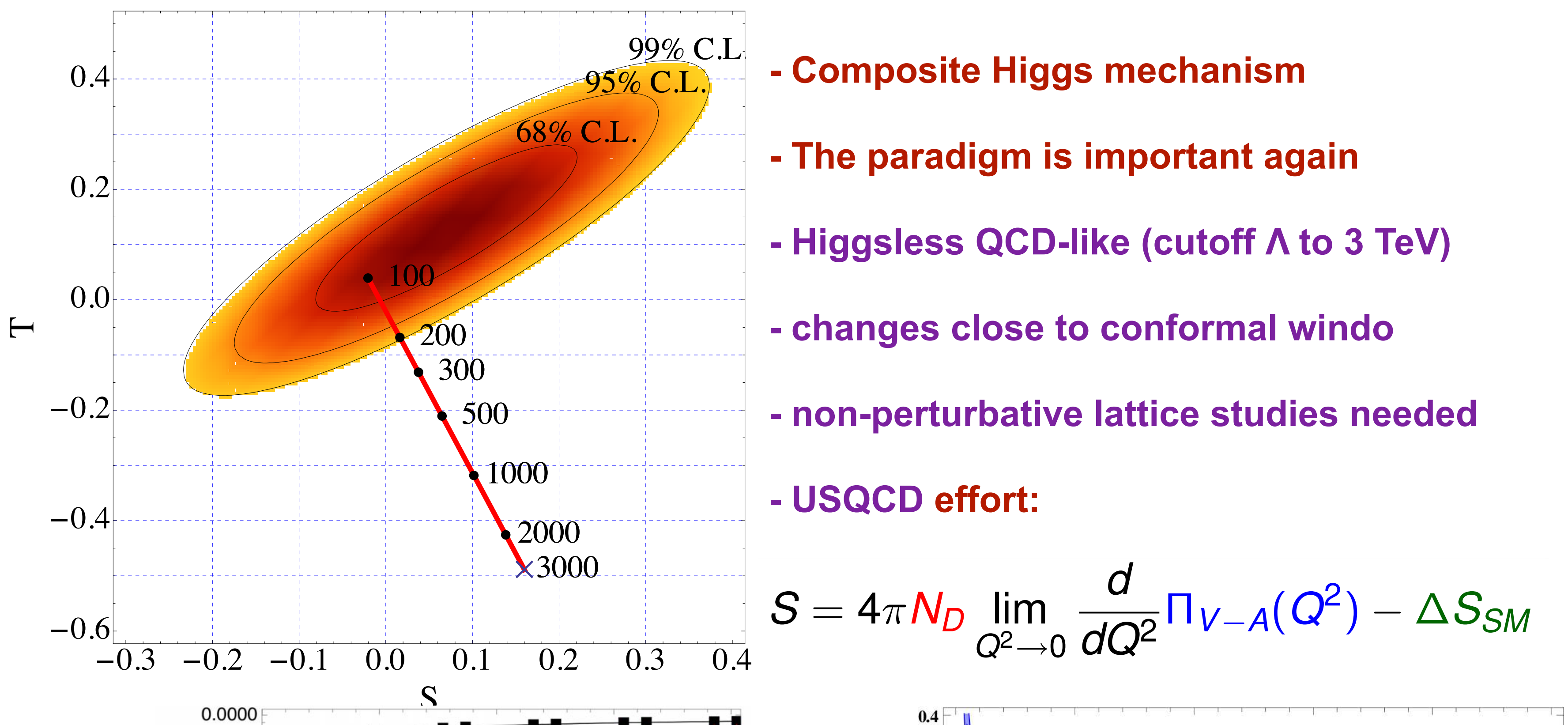



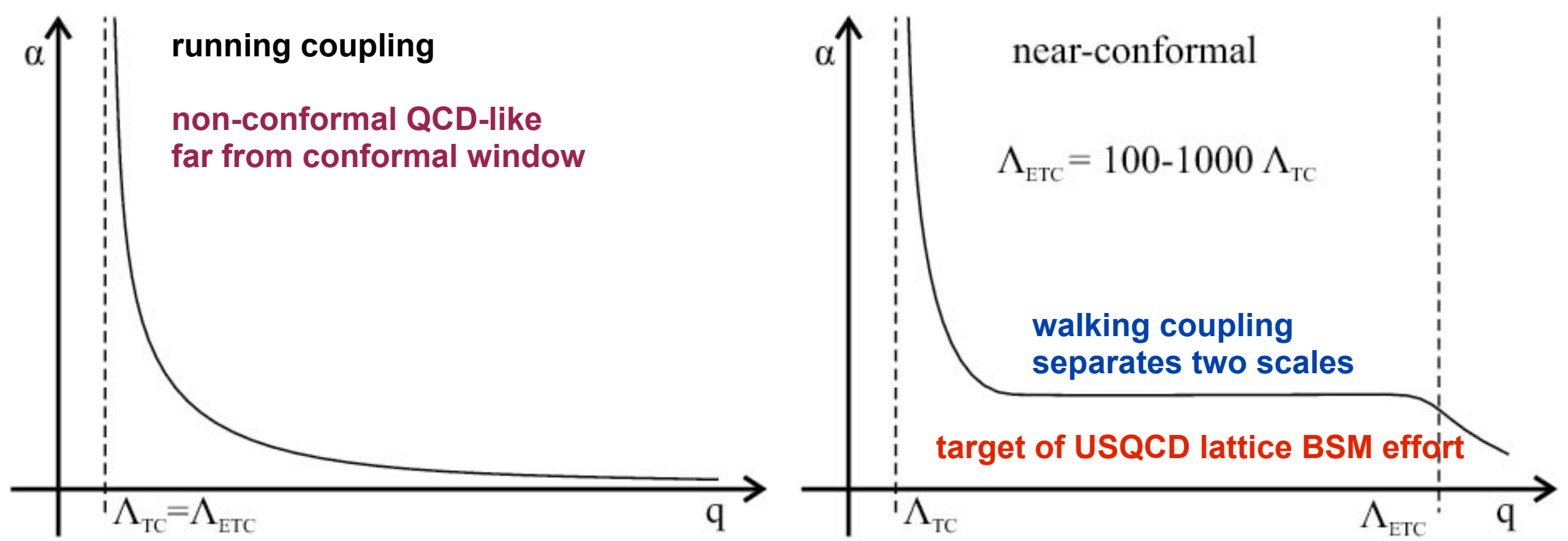

original Technicolor paradigm replaced with sextet SU(3) color rep:

- one massless fermion doublet chiral SB

- three Goldstone pions

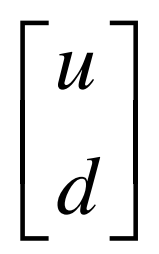

- become longitudinal

components of weak bosons

$\Lambda_{T C} \sim T e V$

- composite Higgs mechanism

scale of Higgs condensate $\sim \mathrm{F}=250 \mathrm{GeV}$

- flavor changing currents and fermion mass generation would be problems

- conflicts with EW precision constraints?

\section{Extended Technicolor paradigm:}

- requires walking gauge coupling chiral SB on $\Lambda_{T C} \sim T e V$ scale

- fermion mass generation from scale at $\Lambda_{E T C} \sim 100-1000 \Lambda_{T C}$

- can solve problem of flavor changing currents

- composite Higgs mechanism

- broken scale invariance (Dilaton) $\rightarrow$ light non-SM composite Higgs particle?

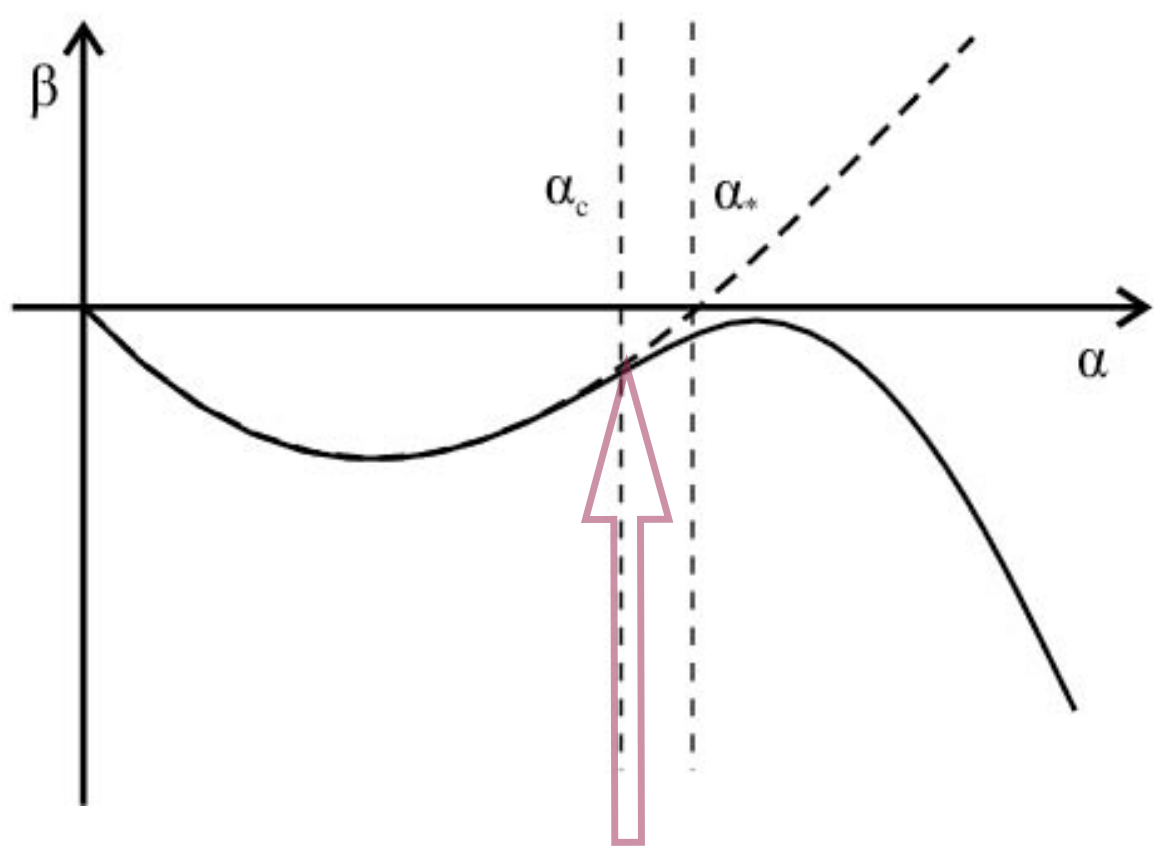

- can avoid conflict with EW precision constraints

- candidate models require nonperturbative lattice studies

- focus is on composite Higgs mechanism

Chiral symmetry breaking turns conformal FP into walking

important for lattice studies in BSM theory space 


\section{theory space and conformal window} critically important for composite Higgs space of color, flavor, and massless fermion representation

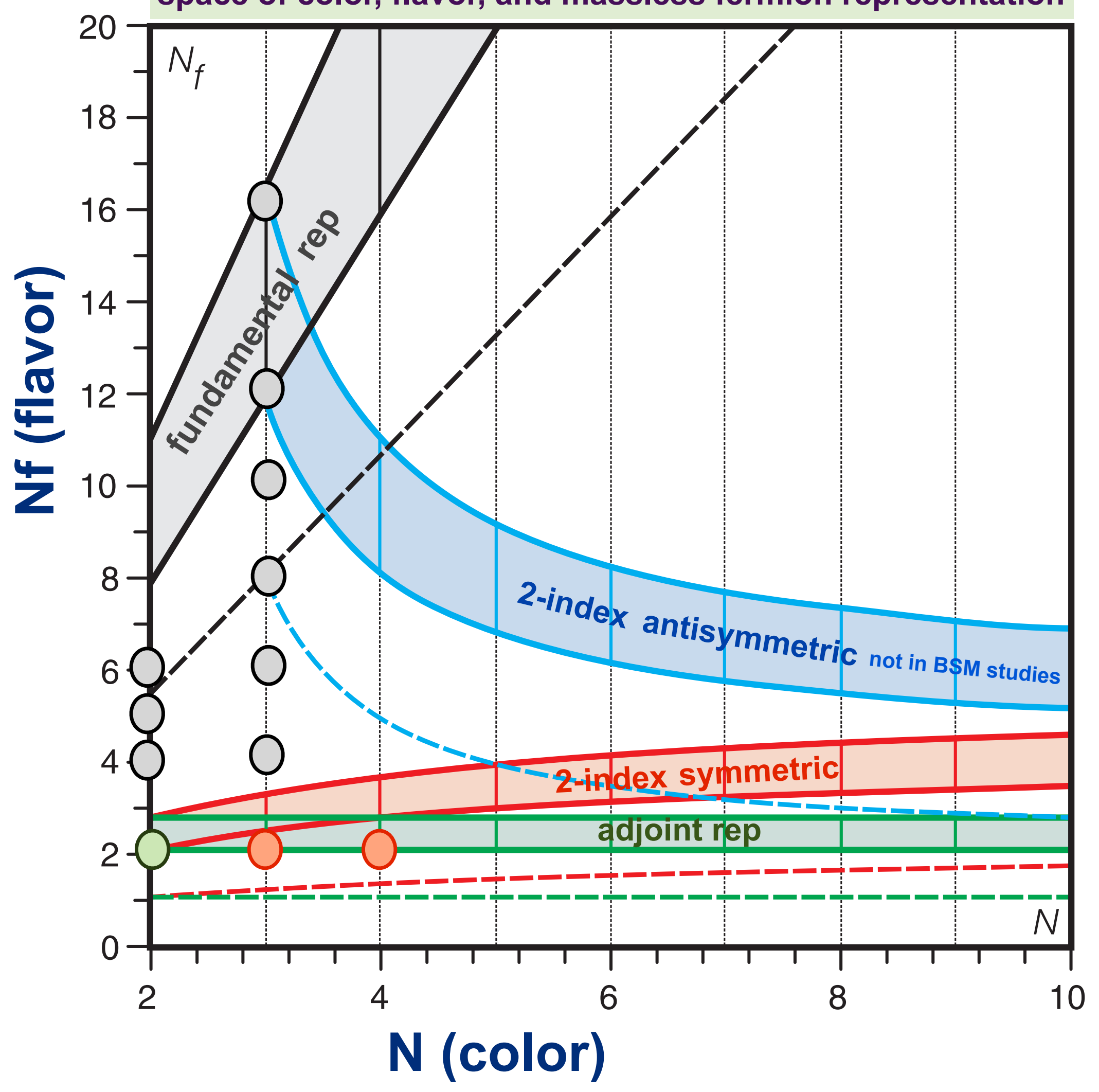

for each rep BSM interest is below conformal window but close to it:

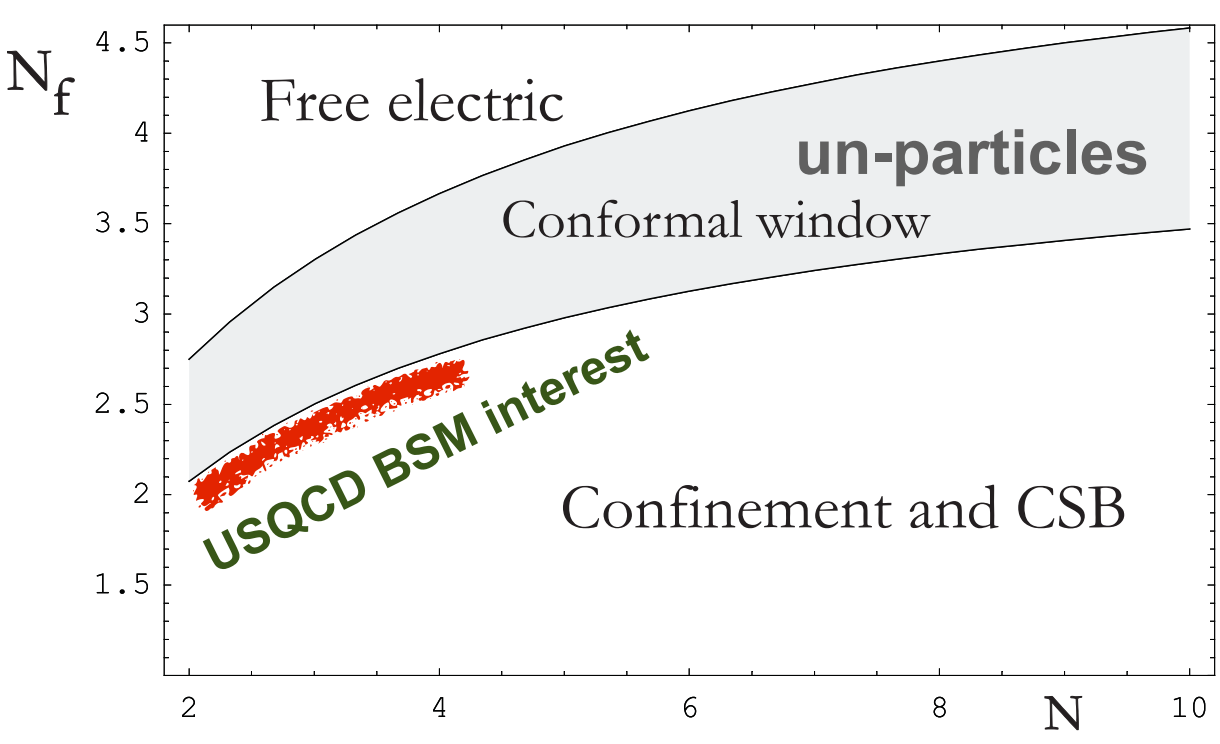

USQCD BSM results of last 12

0 months in 3 reps including

new projects just starting 


\title{
Lattice navigation map
}

\author{
critical surface \\ massless fermions (staggered)
}

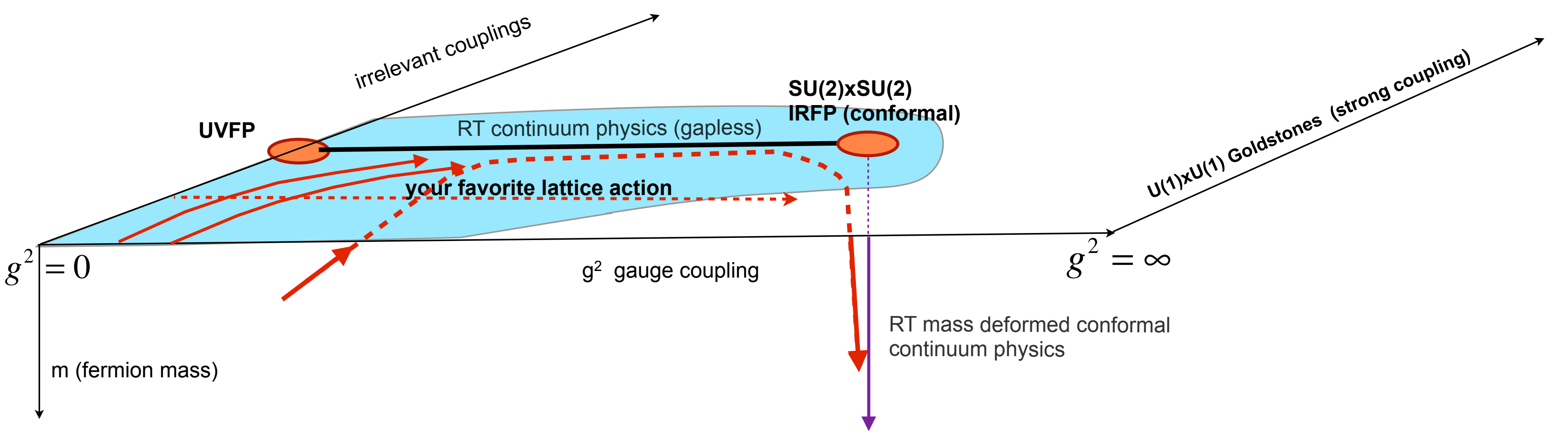




\section{Lattice navigation map}

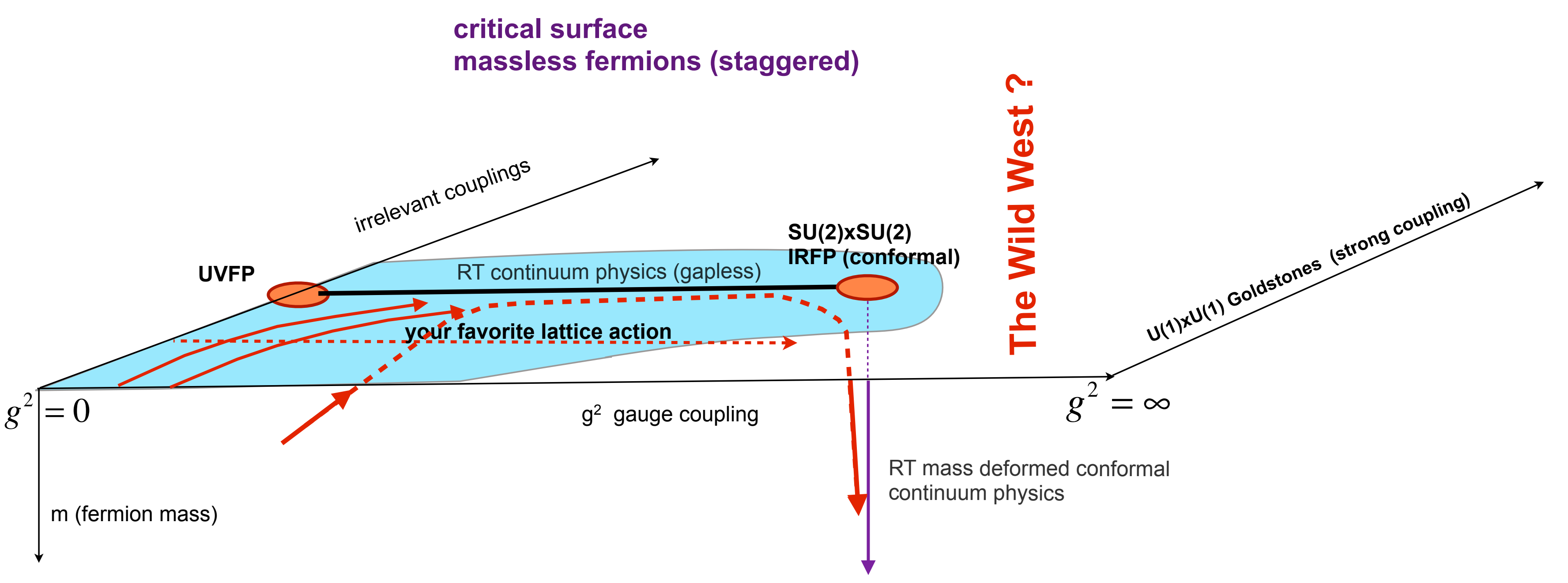




\section{status of $\mathrm{SU}(3) \mathrm{Nf}=2$ sextet model minimal composite Higgs model?}

our group: mass-deformed theory close to $m=0$ critical surface and $m->0$ limit:

- two strategies complement: (1) inf volume conform scaling

(2) mass-deformed FSS

(1) is used in sextet model

- direct access to effective anomalous dimension $\gamma$

- similar to tests of RG scaling laws of moments of current correlator functions (in progress) 
$M_{\pi}(L)$ sextet rep with finite volume fit $(m=0.003)$

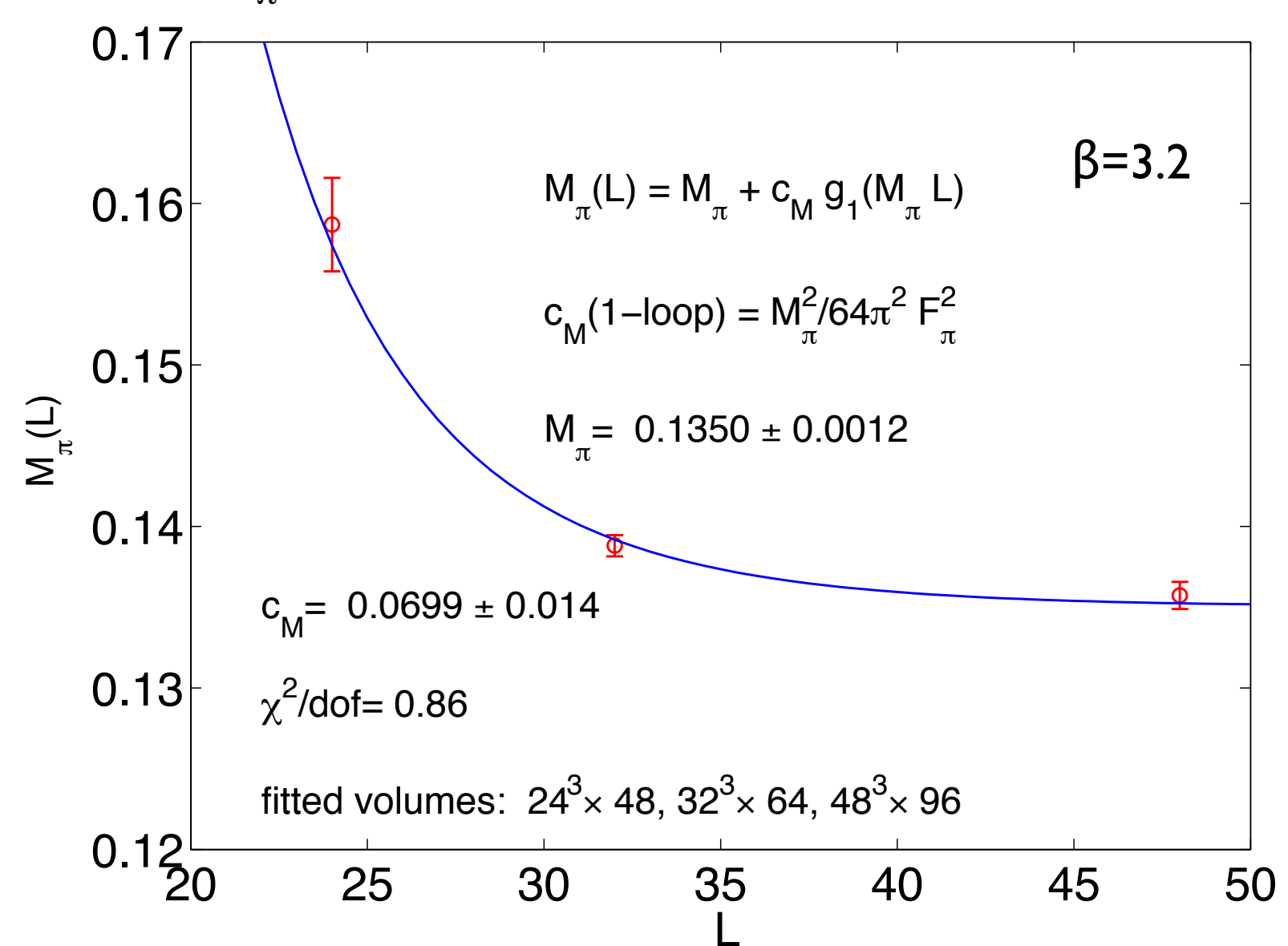

\section{mass-deformed theory}

close to $m=0$ critical surface inf volume extrapolated chiral and conform scaling tests in sextet model

for $L \cdot M \pi>5$ one percent level reached
$F_{\pi}(L)$ sextet rep with finite volume fit $(m=0.003)$
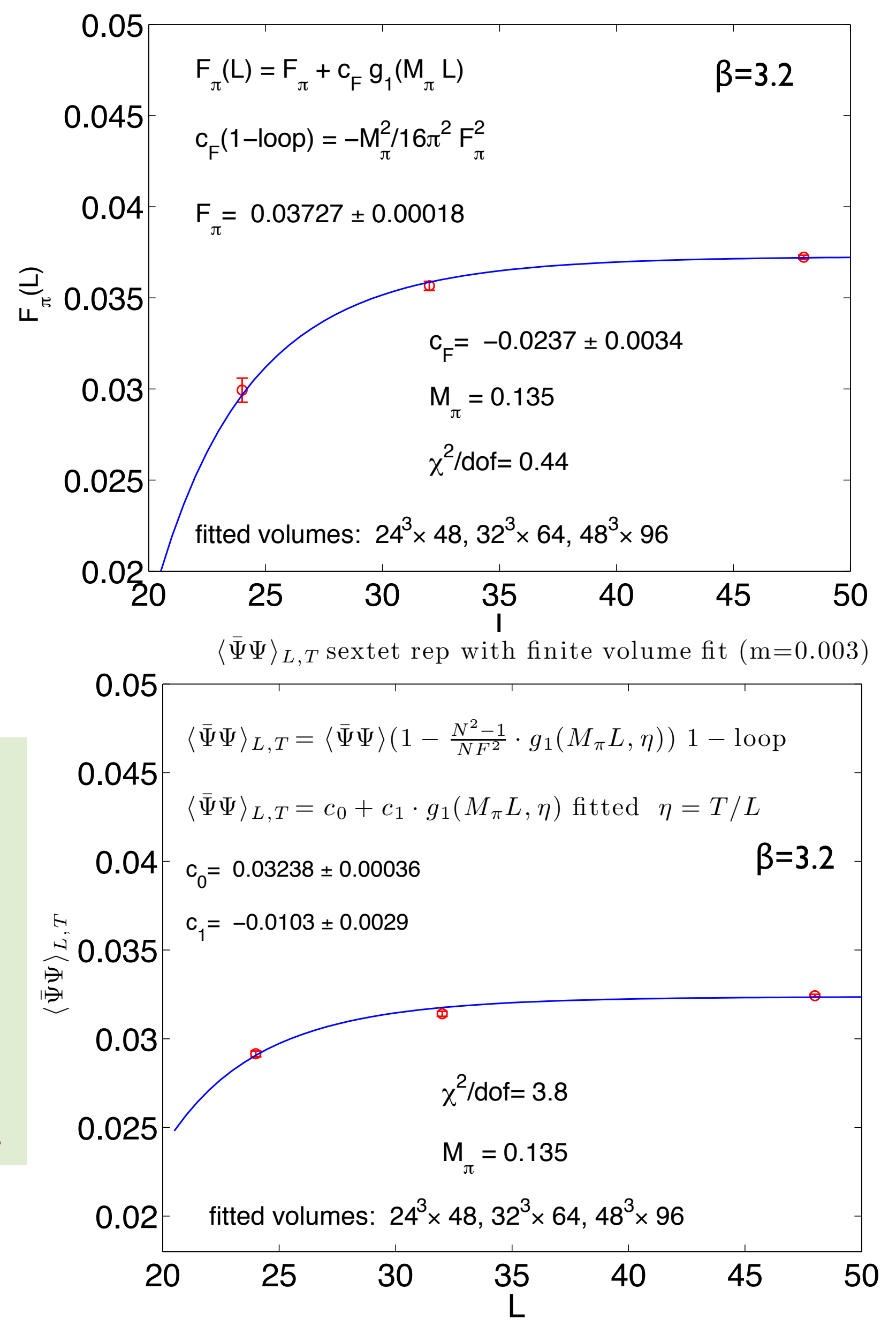


\section{$\mathrm{Nf}=2 \mathrm{SU}(3)$ sextet chiral condensate}
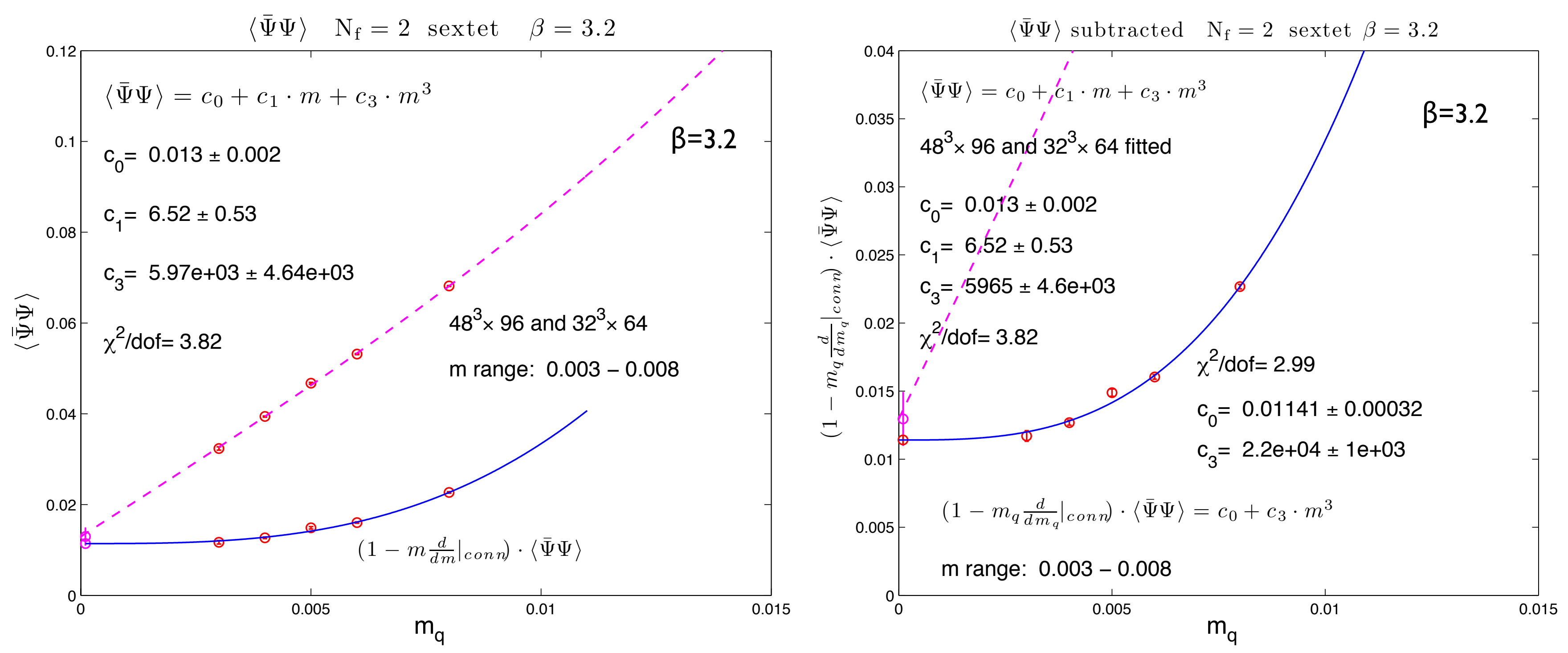

- two independent determinations of the chiral condensate

- consistently non-vanishing in chiral limit

- all sextet results are treated as inf volume (only $m=0.003$ is truly extrapolated) 


\section{$N f=2 S U(3)$ sextet chiral fits $M_{\pi}$ and $F_{\pi}$}
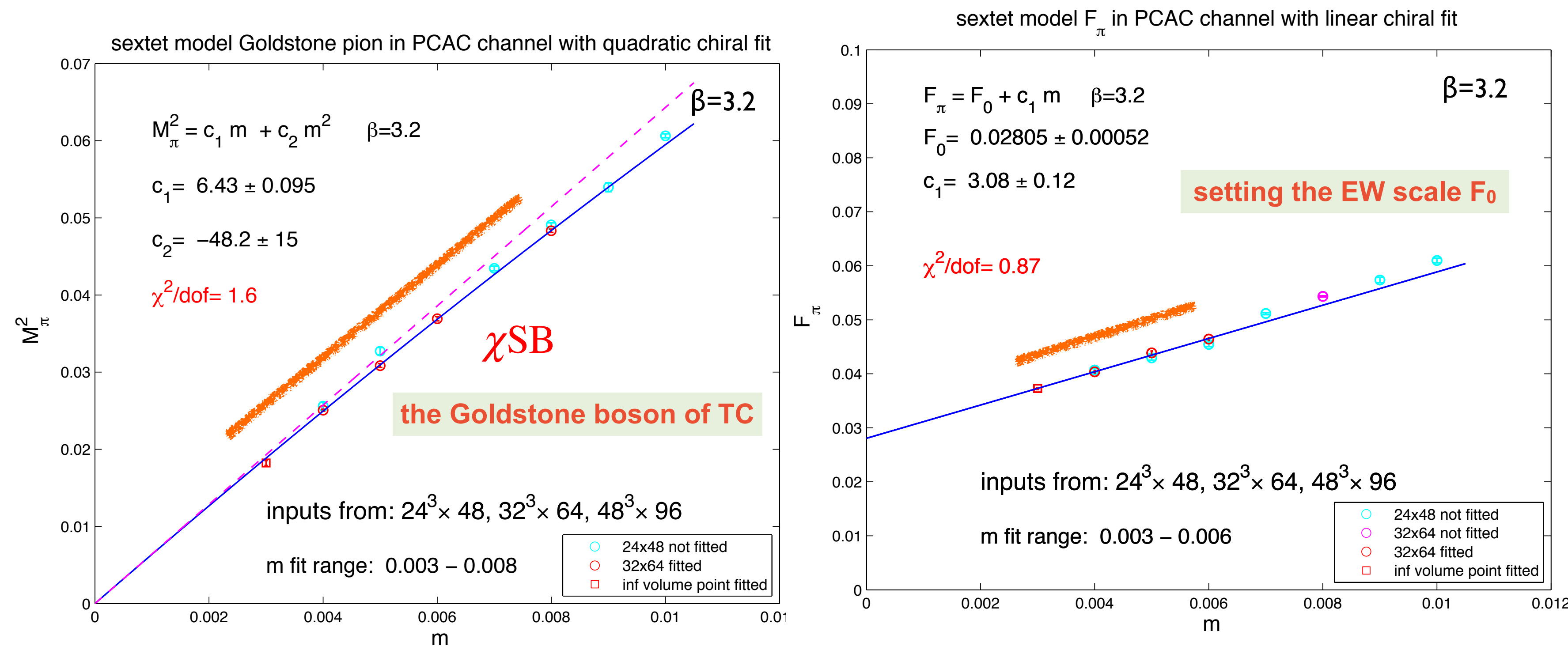

$m=0.003-0.006$ range close to chiral log regime? $\quad \mathrm{Nf}=2$ helps! log detection will require even more precise data 


\section{$N f=2 S U(3)$ sextet chiral fits $M_{\rho}$ and $M_{H}$}
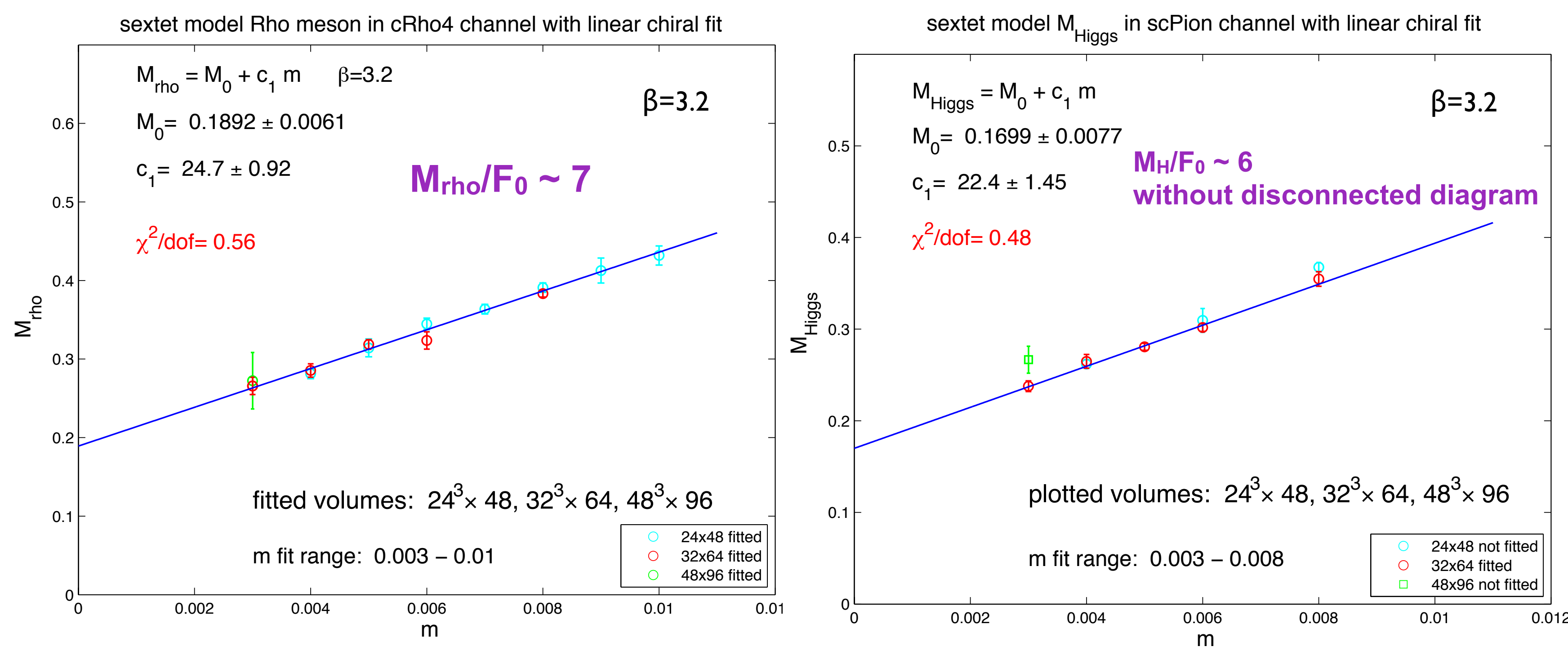

$m=0.003-0.006$ range close to chiral log regime? $\quad N f=2$ helps! log detection will require even more precise data 


\section{conformal hypothesis breaks down in global fits:}

sextet model Goldstone pion in PCAC channel with conformal fit

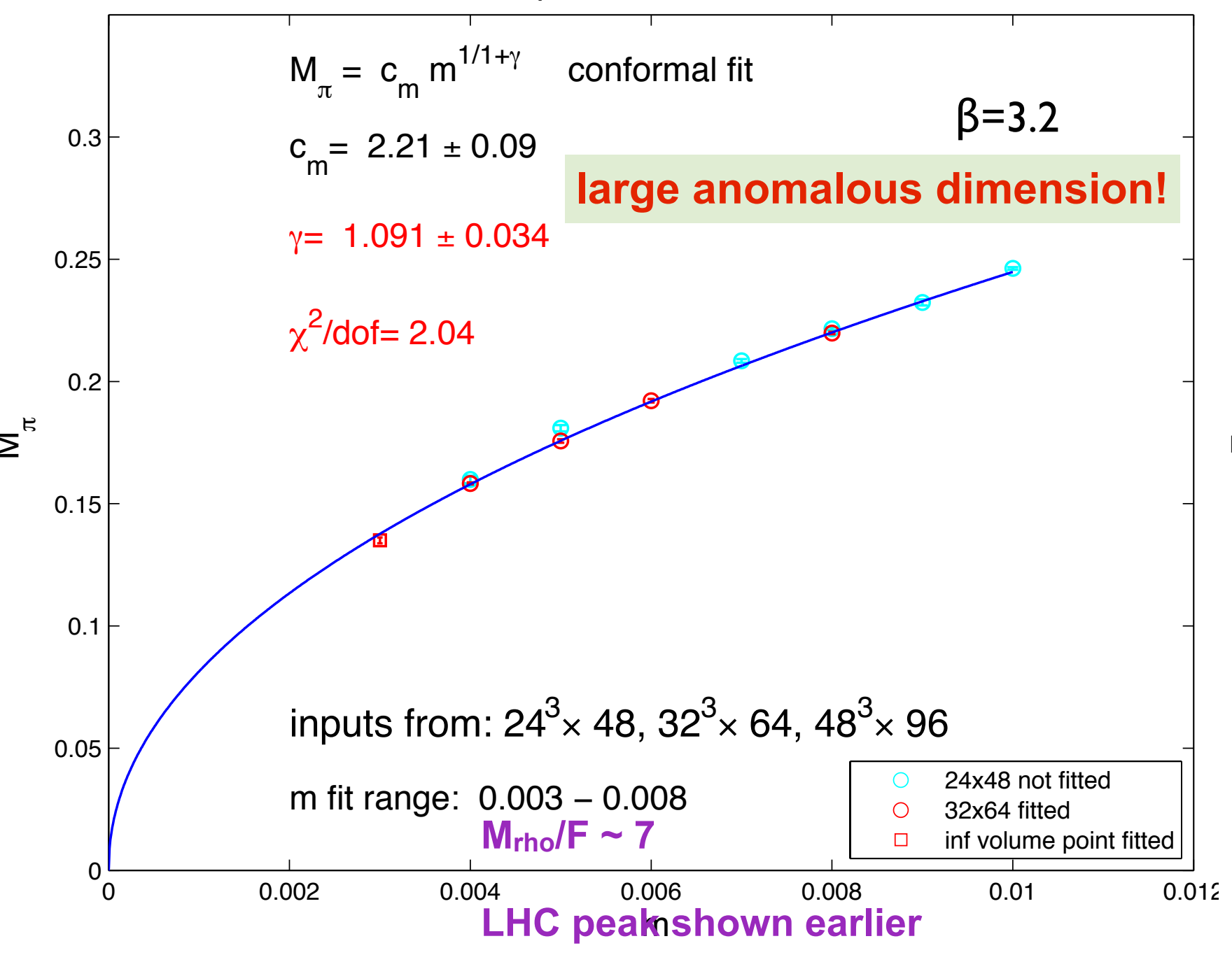

sextet model $\mathrm{F}_{\pi}$ in PCAC channel with conformal fit

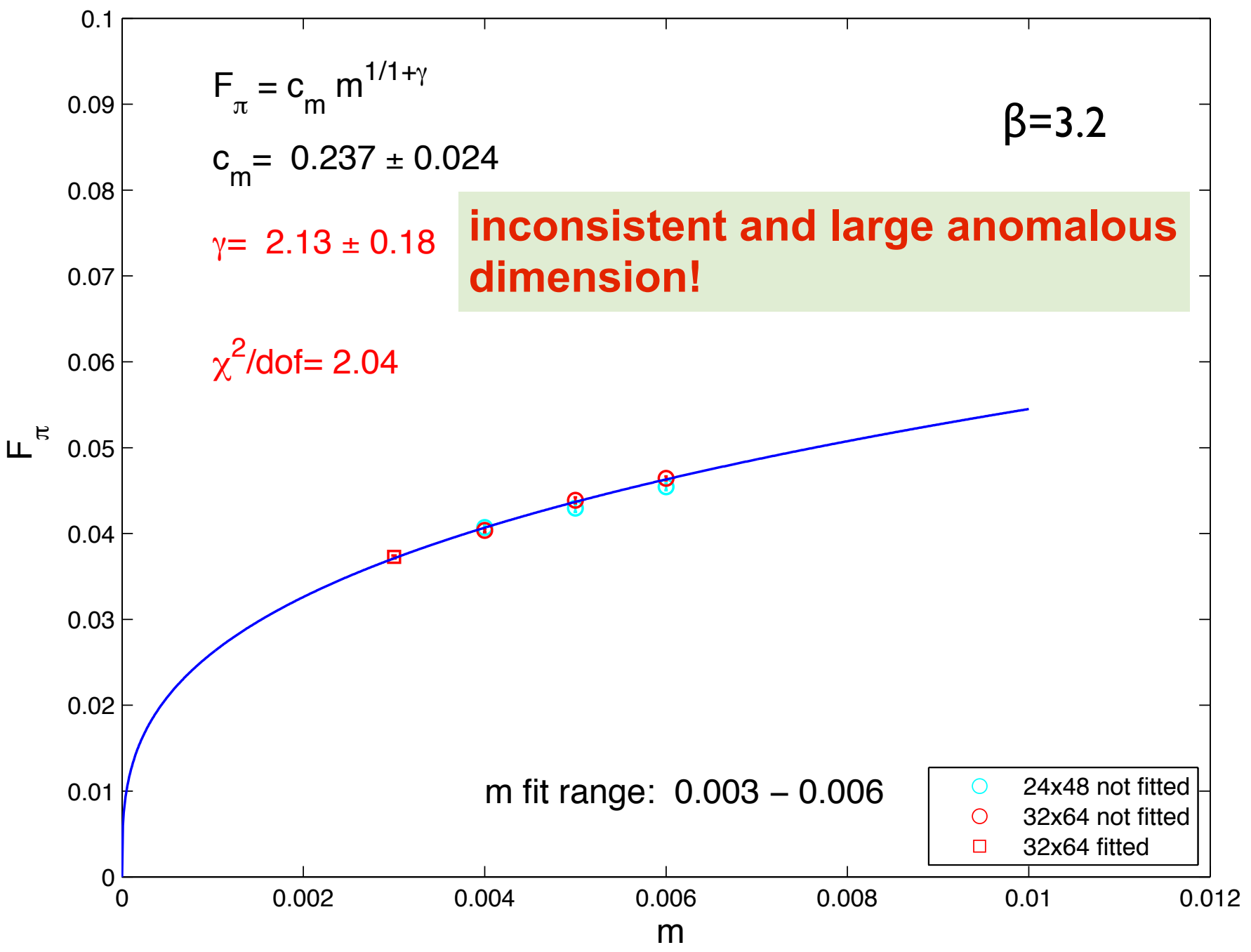

\section{inconsistent large critical exponents $\gamma$}




\section{conformal hypothesis breaks down in global fits:}
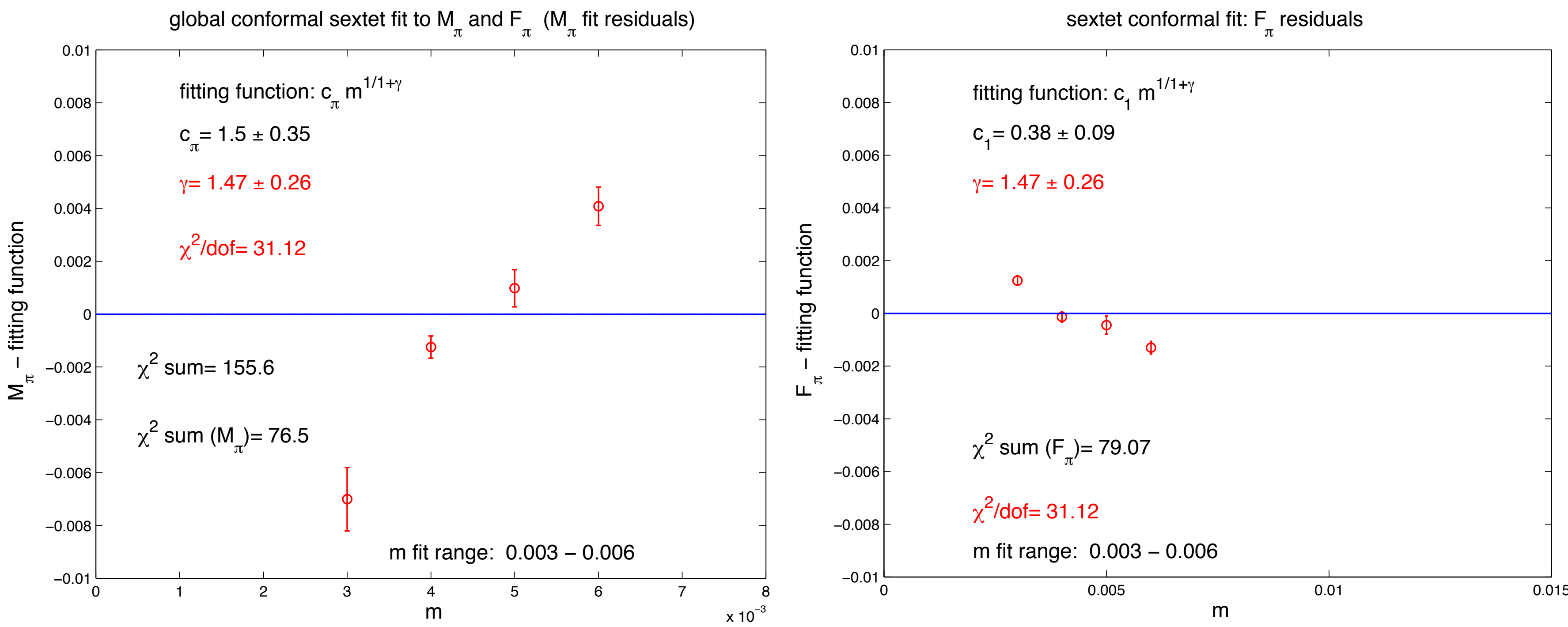

large and inconsistent critical exponents $\gamma$
are we close enough to the critical surface? 


\section{working on baryon spectrum}

\section{dark matter candidate}

- lightest technibaryon can be stable by analog of $\mathrm{U}(1)_{\mathrm{B}}$

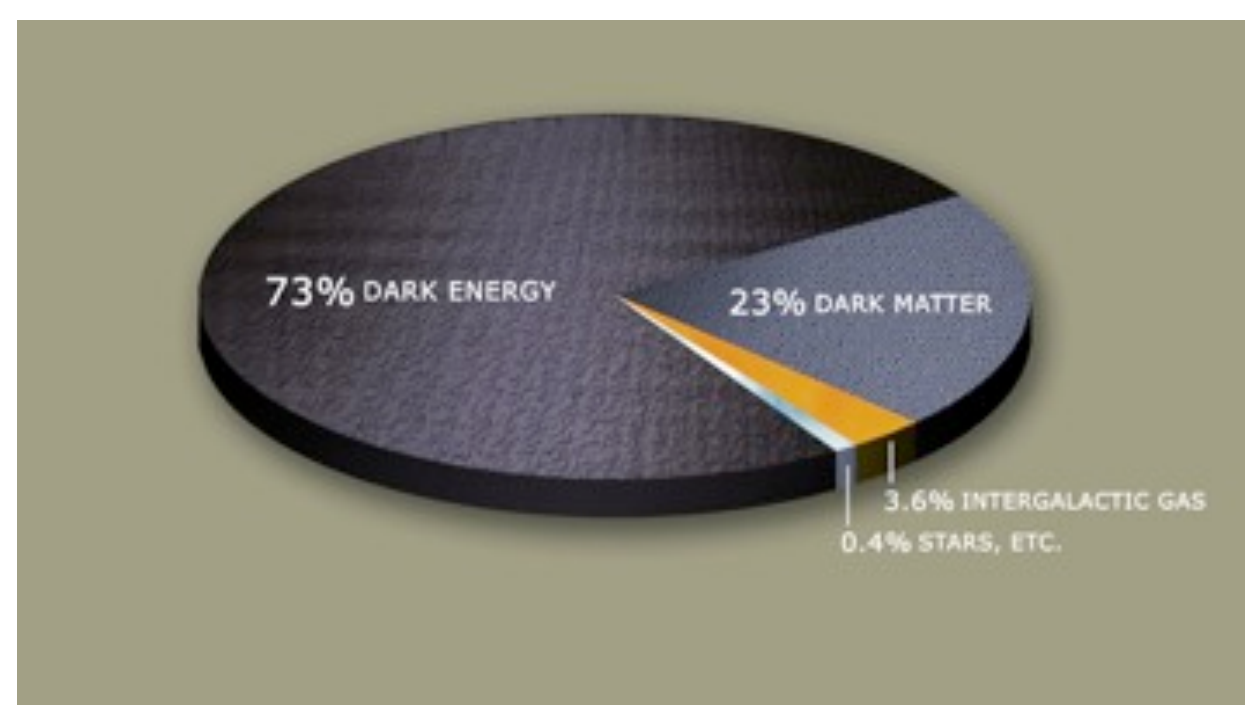

- an initial matter/anti-matter asymmetry gets shared among baryons, leptons, technibaryons via sphalerons

- can get observed $\Omega_{\mathrm{DM}} / \Omega_{\mathrm{B}}$ easily for $\sim$ TeV scale DM must be electrically neutral, EW singlets to avoid direct detection Then leading operators are charge radius and polarizability:

$$
\text { ex.) } \frac{B^{*} B v_{\mu} \partial_{\nu} F^{\mu \nu}}{\Lambda_{T C}^{2}}, \frac{B^{*} B F_{\mu \nu} F^{\mu \nu}}{\Lambda_{T C}^{3}}
$$




\title{
EW phase transition in sextet Higgs model - early universe
}

\author{
sextet model (Kogut-Sinclair)
}

potential implications in early cosmology
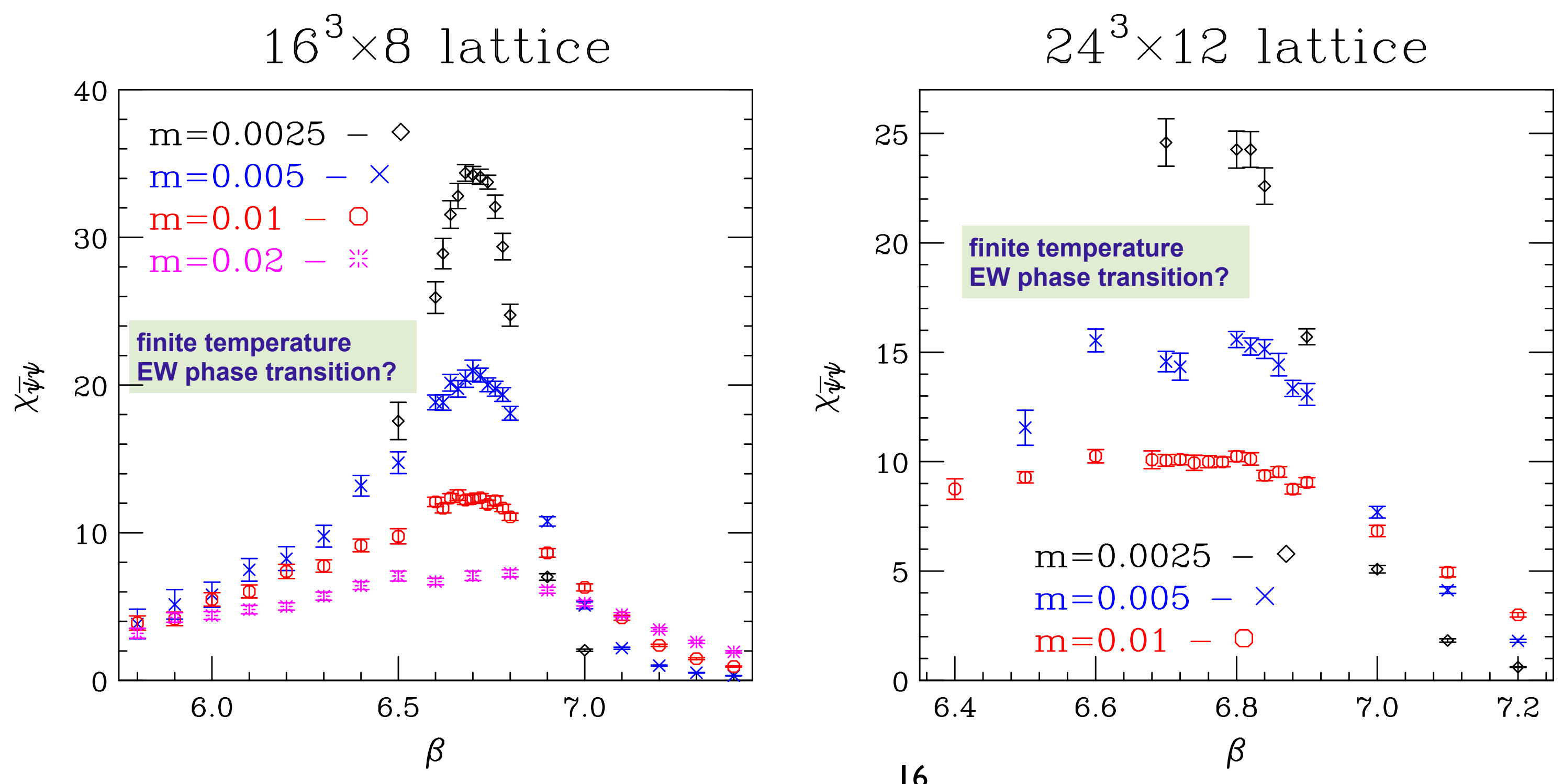
$N f=2 S U(3)$ sextet rep summary: 
- No inconsistency with $\chi S B$ in $\mathrm{Nf}=2 \mathrm{SU}(3)$ sextet model

- We find inconsistency with conformal symmetry in all tests

- The effective anomalous dimension is inconsistent and large $y$ is in 1-2 range

- Kogut and Sinclair: looking for finite temperature $\chi S B$ phase transition 
- No inconsistency with $\chi S B$ in $\mathrm{Nf}=2 \mathrm{SU}(3)$ sextet model

- We find inconsistency with conformal symmetry in all tests

- The effective anomalous dimension is inconsistent and large $y$ is in 1-2 range

- Kogut and Sinclair: looking for finite temperature $\chi S B$ phase transition

- DeGrand et al. find: $\mathrm{Nf}=2$ sextet beta function might have an IRFP zero?

- uninteresting model with small anomalous dimension?

$Y<0.45$ controversy is not resolved
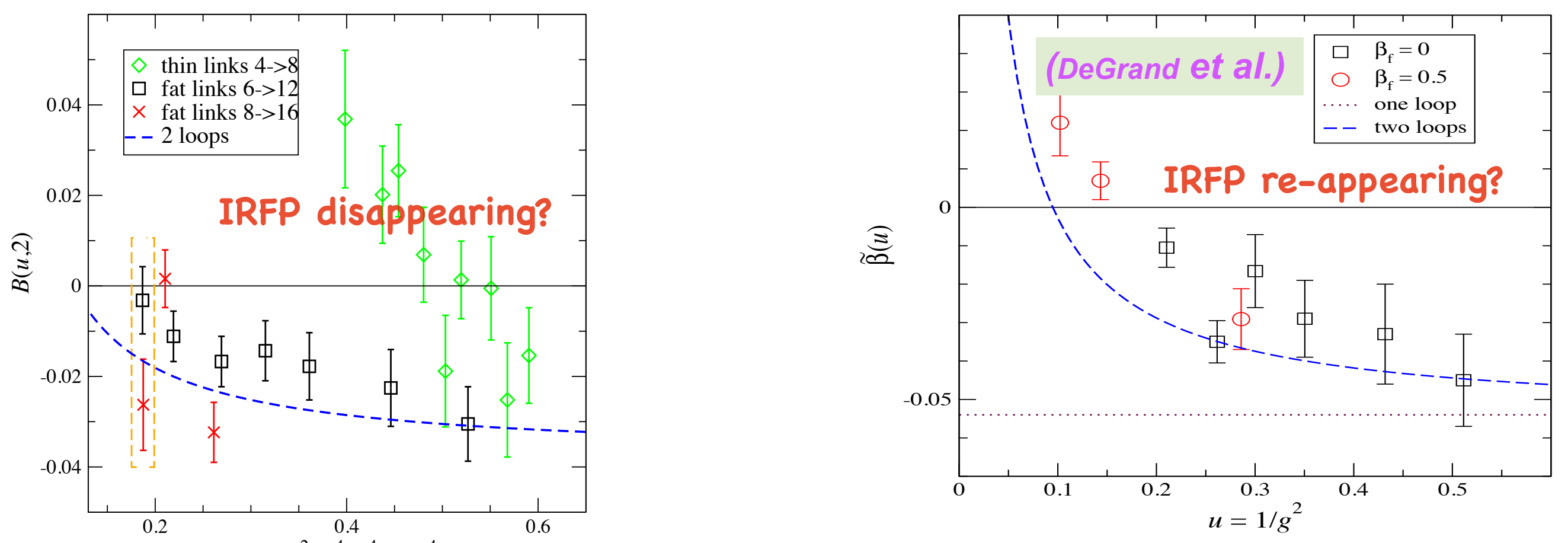

$u=1 / g^{2}\left(4^{4}, 6^{4}\right.$, or $\left.8^{4}\right)$ 
- No inconsistency with $\chi S B$ in $\mathrm{Nf}=2 \mathrm{SU}(3)$ sextet model

- We find inconsistency with conformal symmetry in all tests

- The effective anomalous dimension is inconsistent and large $y$ is in 1-2 range

- Kogut and Sinclair: looking for finite temperature $\chi S B$ phase transition

- DeGrand et al. find: $\mathrm{Nf}=2$ sextet beta function might have an IRFP zero?

- uninteresting model with small anomalous dimension?

$Y<0.45$ controversy is not resolved

- We expect: the $N f=2$ sextet model with $S U(3)$ color is an interesting candidate for the composite Higgs mechanism

- But viability requires confirmation, studies of the running coupling, the S-parameter, and composite Higgs physics 


\section{status of SU(3) Nf=12 fundamental rep}

our group: mass-deformed theory close to $m=0$ critical surface and $m->0$ limit:

- two strategies complement: (1) inf volume conform scaling

(2) mass-deformed FSS

(2) is used in Nf=12 fundamental SU(3) rep

- direct access to some effective anomalous dimension

- similar to tests of RG scaling laws of moments of correlator functions (in progress) 


\section{bulk transition? (Schroeder, Latt 2011)}

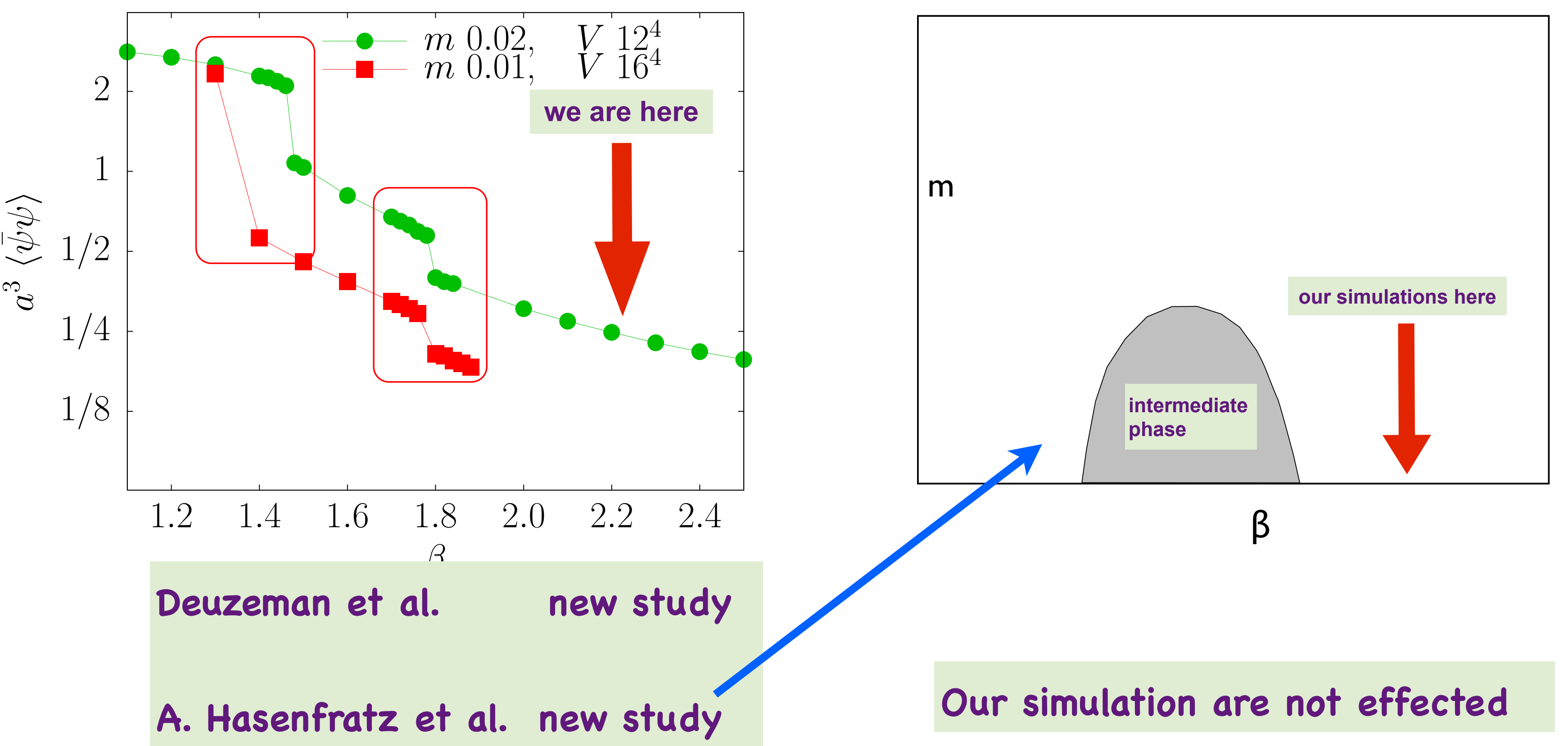




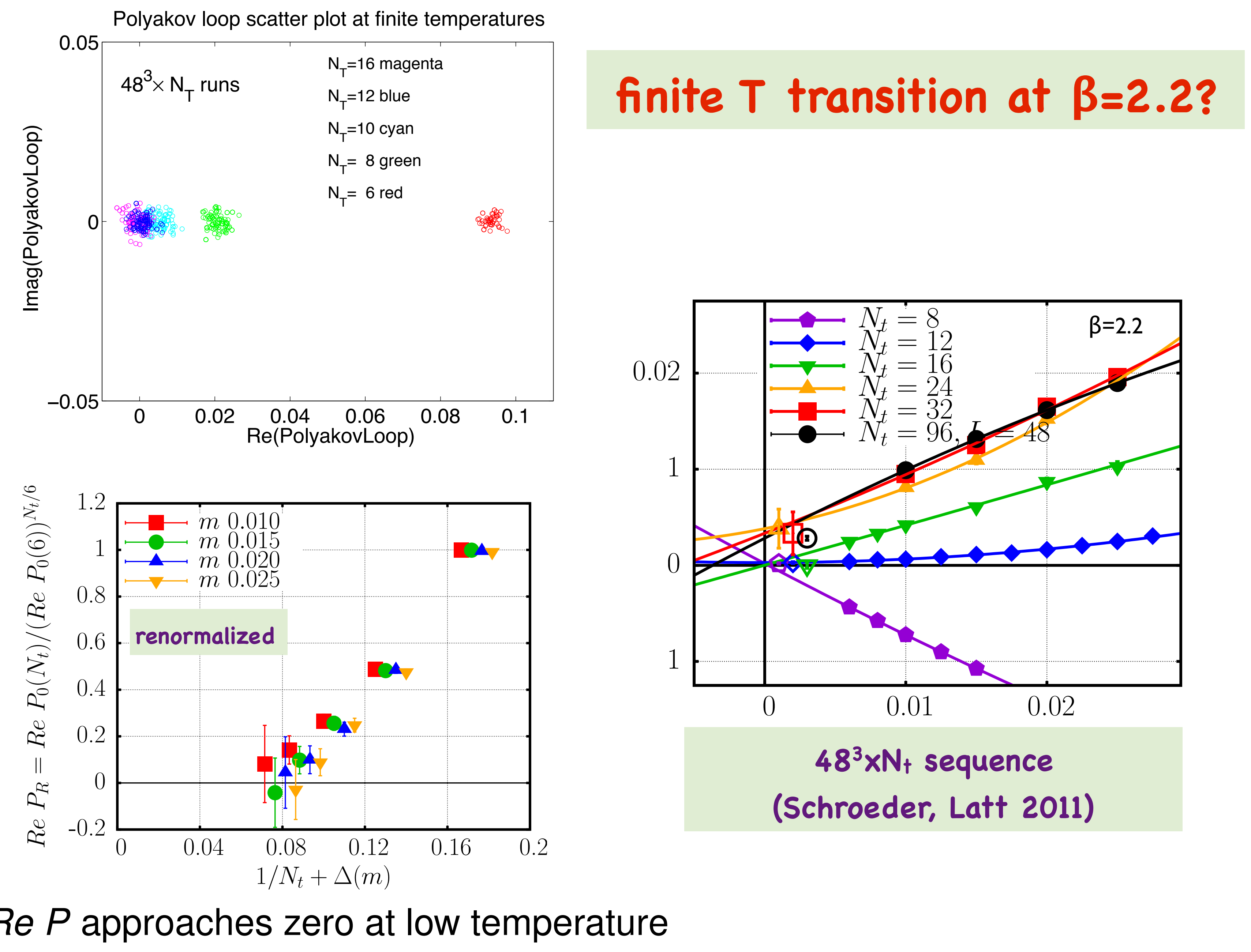




\section{zero temp confinement - running coupling without IRFP?}
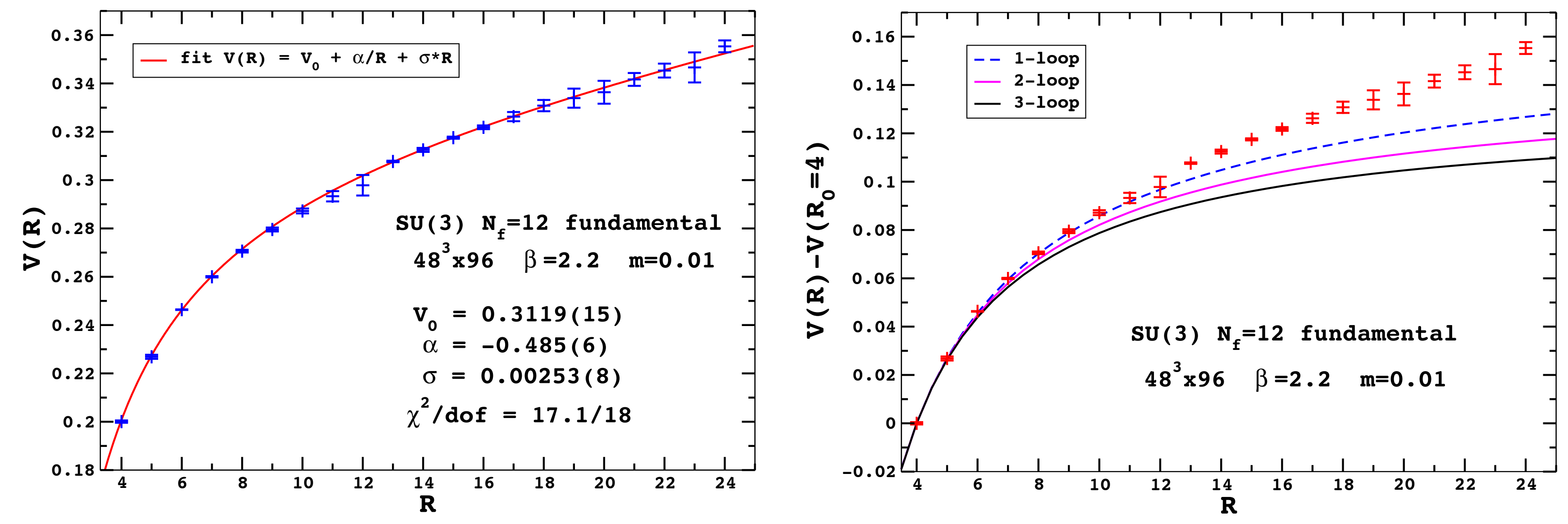

$m \rightarrow 0$ and $a \rightarrow 0$ limits? finite volume effects?

\section{useful to compare with other methods:}

- SF?

- MCRG?

- two new methods we are developing: current correlator moments and wilson flow 


\section{small chiral symmetry breaking condensate}
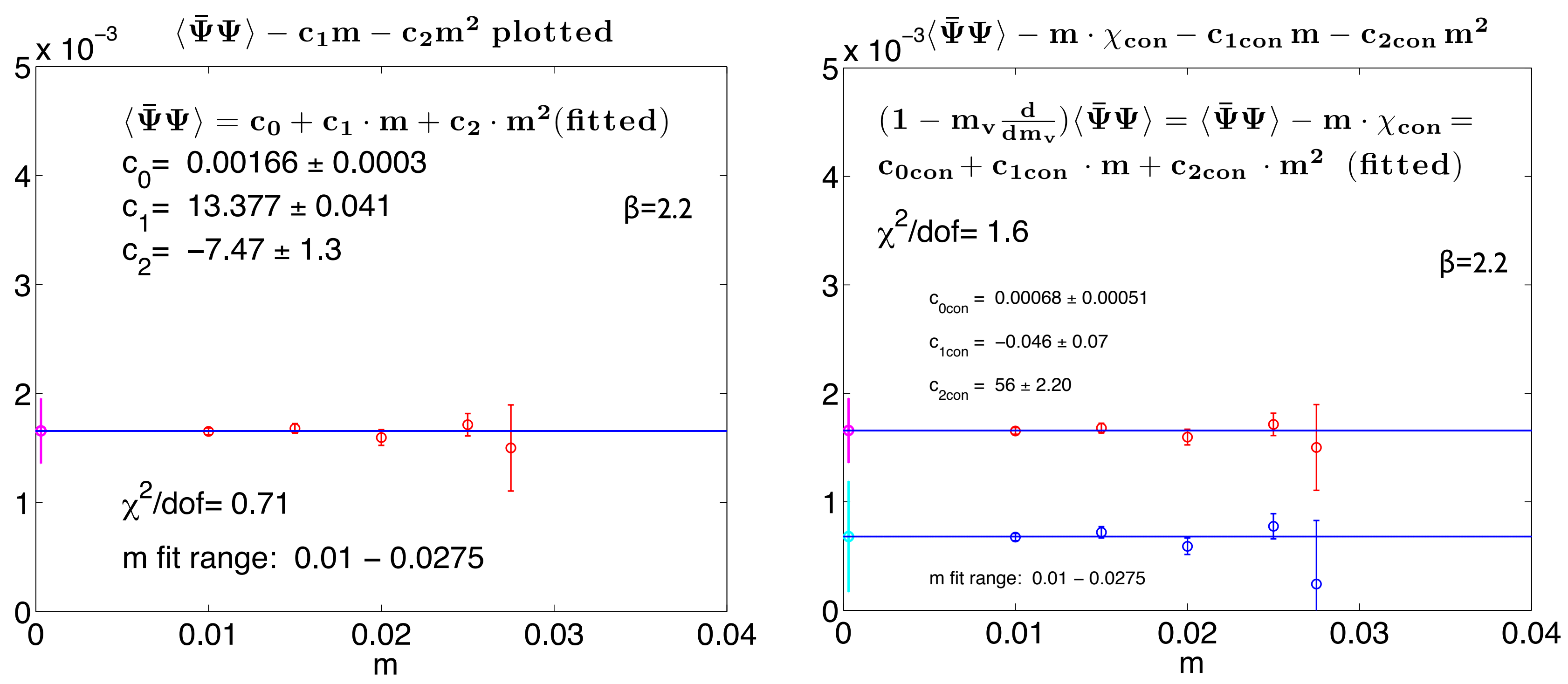

- two independent extrapolation

- condensate very small

- would not be decisive without other tests 


\section{conformal scaling test with FSS heavy use of RG theory}

$$
\begin{aligned}
& L M=f(x)+L^{-\omega} g(x) \\
& x=m^{1 / 1+\gamma} L \\
& \omega=\beta^{\prime}\left(g^{*}\right)
\end{aligned}
$$




\section{conformal scaling - spline B-form fits}

conformal FSS fit with spline based B-form (Goldstone pion)

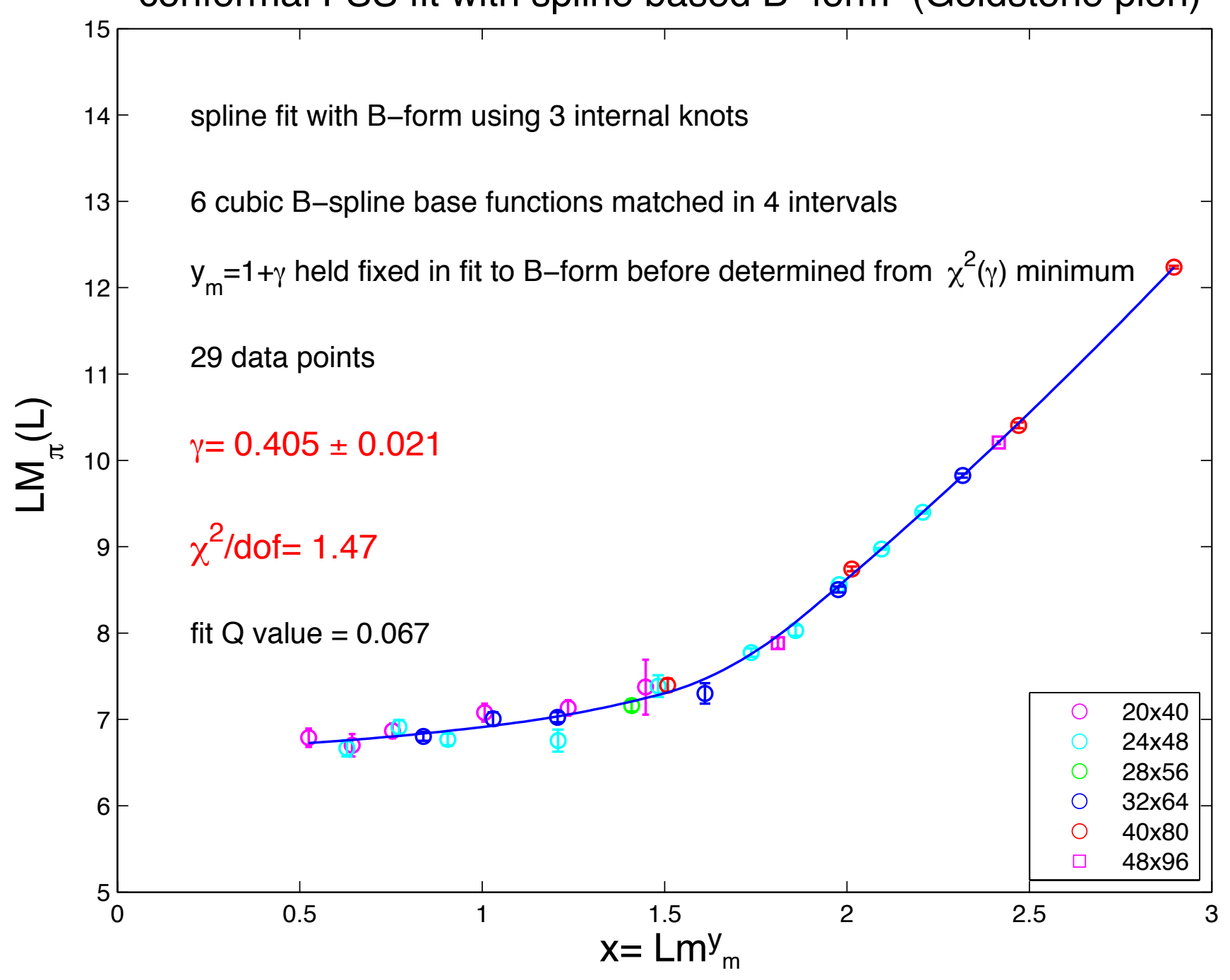

conformal FSS fit with spline based B-form ( $F_{\pi}$ in PCAC channel)

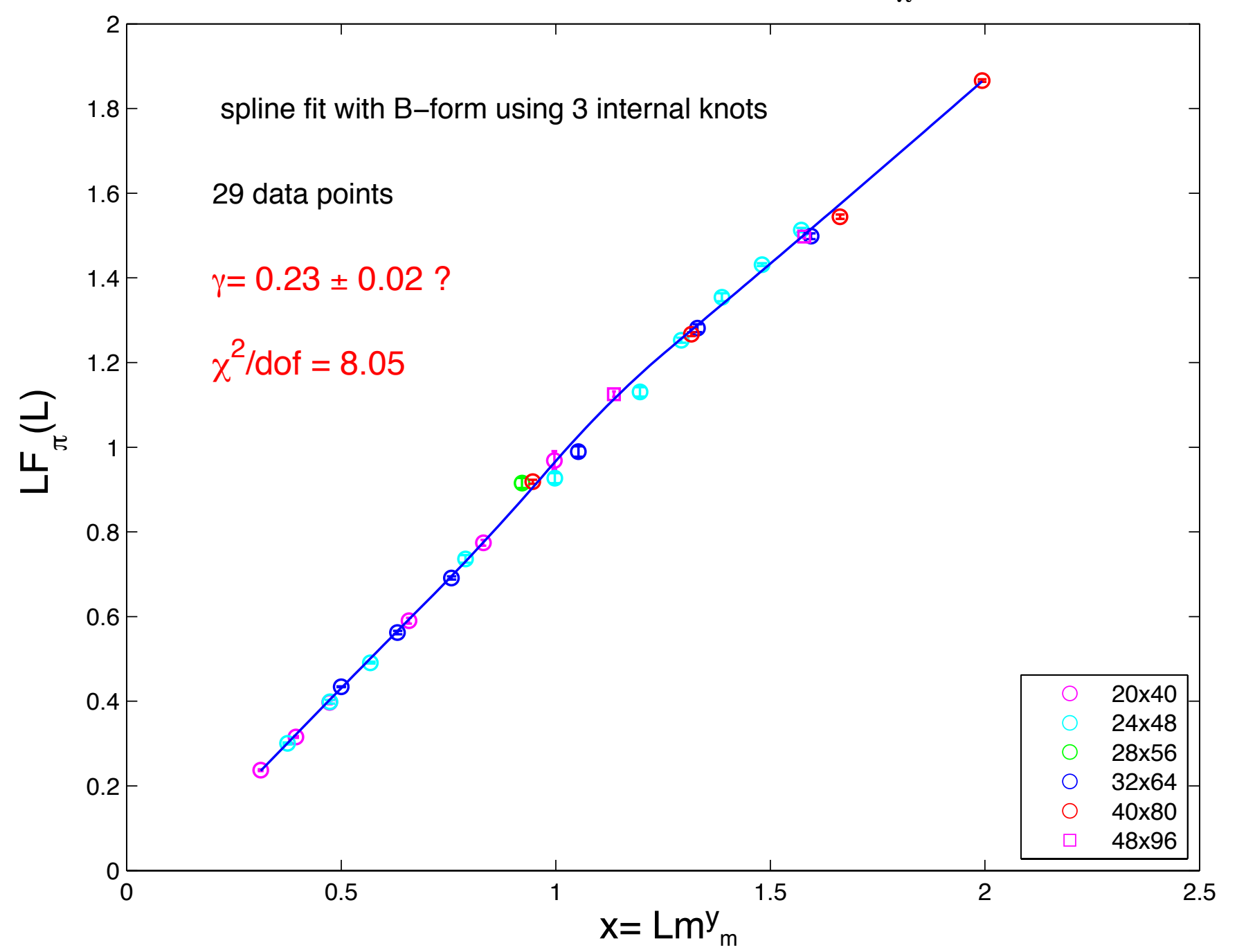

is this glass half full or half empty?

can chiral symmetry breaking fake scaling form? 


\section{Deceptions of $\chi \mathrm{SB}$ FSS behavior:}

large volume

hadrons point-like

$\pi$ exchange $\sim \exp (-\mathrm{mL})$

$\delta E=\sum_{\vec{n}} V(\vec{n} L)$

$\delta E=\frac{1}{L^{3}} \sum_{\vec{n}} \hat{V}\left(\vec{n} \frac{2 \pi}{L}\right) \quad$ Poisson resummation, $\hat{V}(\vec{k})$ is the Fourier transform

$\hat{V}(\vec{k})=\frac{1}{\vec{k}^{2}+m^{2}} \Rightarrow \mathrm{V}(\mathrm{r})=\frac{e^{-m r}}{r}$ for large $\mathrm{r}$ in point-like approximation

$\delta E \approx V(0)+6 V(L) \quad \delta E \approx \frac{e^{-m L}}{L}$ point-like interaction for large L (non-relativistic)
$\hat{V}(\vec{k})=\frac{F(\vec{k})^{2}}{\vec{k}^{2}+m^{2}} \quad$ extended hadron with form factor

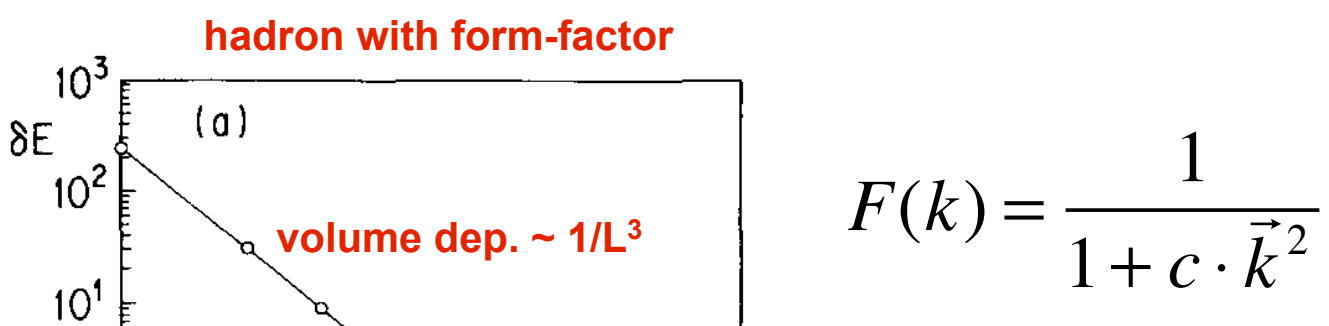

\section{Text}

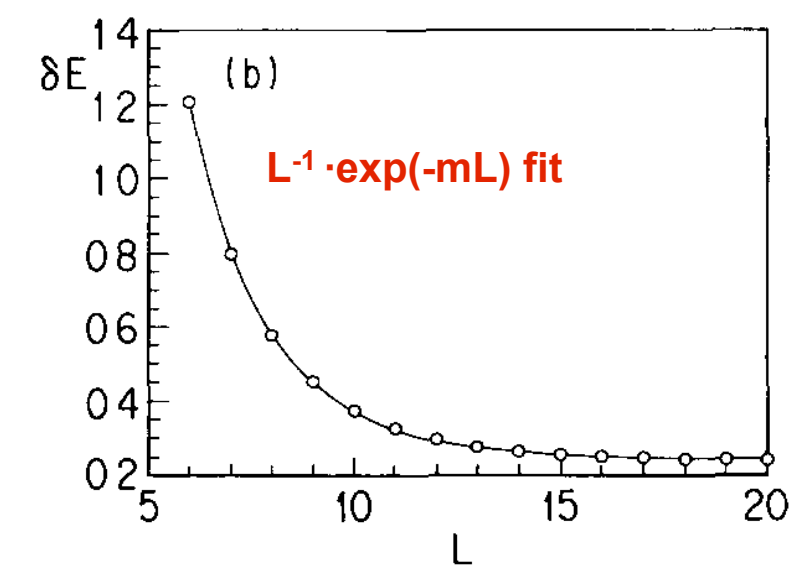

Lüscher made it relativistic using field theory

Leutwyler put in the chiral vertices, hence the $\tilde{g}(m L)$ form in chiral PT the size where the $1 / L^{3}$ correction to the masses disappears and the exponential behavior sets in depends on the behavior of the hadron form factor

the characteristic inverse power vs. exponential behavior can frustrate at limited lattice sizes the analysis of chiral vs. conformal hypotheses 
$N f=12 S U(3)$ fundamental rep summary: 
- We run in the weak coupling phase

- At fixed coupling and very small fermion mass we see confining potential and finite temp $\chi S B$ transition

- The effective anomalous dimension $Y$ is not consistent across channels can be explaind by scaling violation effects or underestimated errors? 
- We run in the weak coupling phase

- At fixed coupling and very small fermion mass we see confining potential and finite temp $\chi S B$ transition

- The effective anomalous dimension $Y$ is not consistent across channels can be explaind by scaling violation effects or underestimated errors?

- Running coupling methods like TPL and SF have problems to control systematics no comment on MCRG 
- We run in the weak coupling phase

- At fixed coupling and very small fermion mass we see confining potential and finite temp $\chi S B$ transition

- The effective anomalous dimension $\gamma$ is not consistent across channels can be explaind by scaling violation effects or underestimated errors?

- Running coupling methods like TPL and SF have problems to control systematics no comment on MCRG

Running coupling problems in TPL and SF schemes: 
- We run in the weak coupling phase

- At fixed coupling and very small fermion mass we see confining potential and finite temp $\chi S B$ transition

- The effective anomalous dimension $Y$ is not consistent across channels can be explaind by scaling violation effects or underestimated errors?

- Running coupling methods like TPL and SF have problems to control systematics no comment on MCRG

Running coupling problems in TPL and SF schemes:
Continuum extrapolation

on TPL scheme

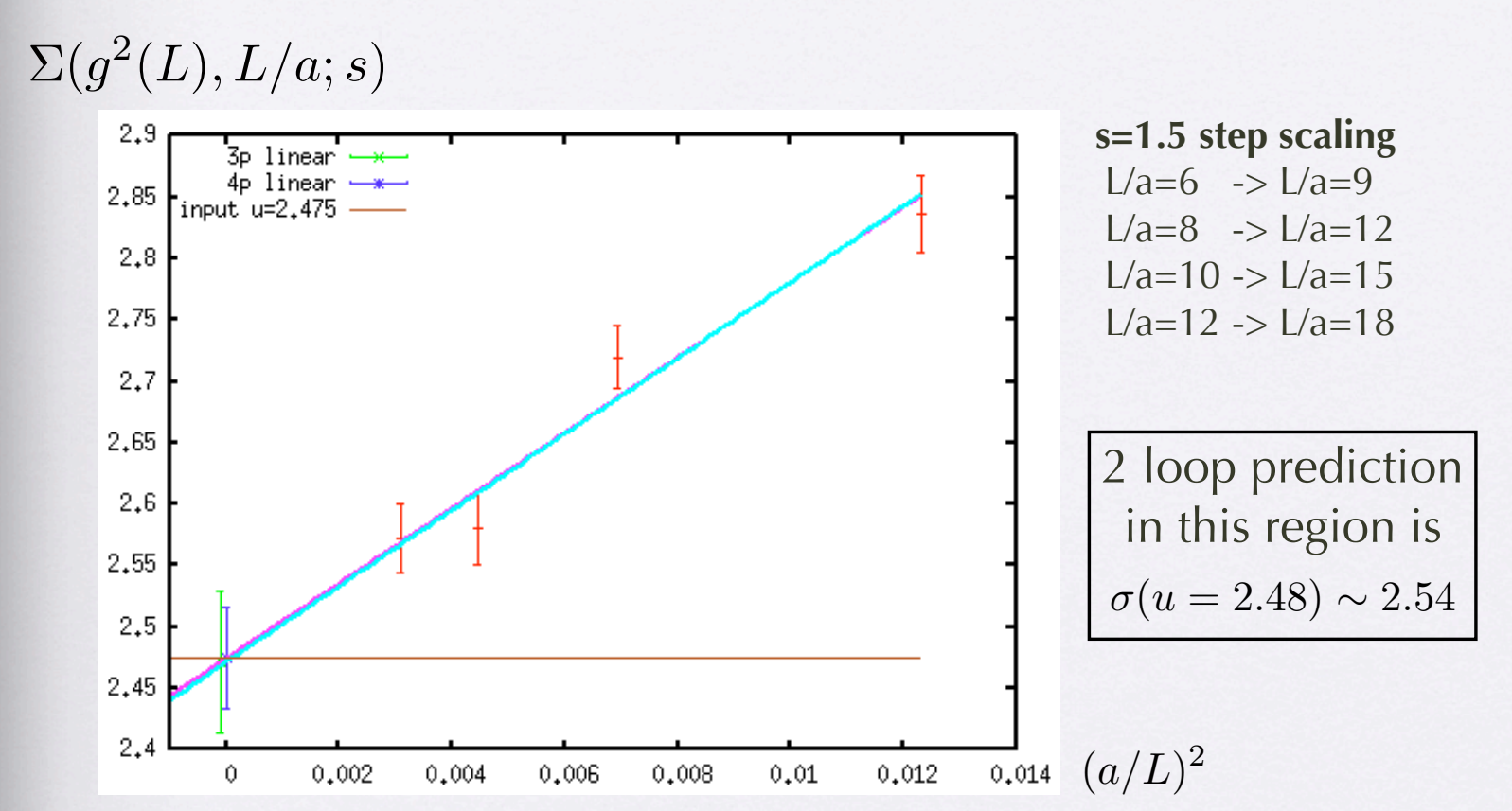

The systematic error is small in the strong coupling region. 
- We run in the weak coupling phase

- At fixed coupling and very small fermion mass we see confining potential and finite temp $\chi S B$ transition

- The effective anomalous dimension $Y$ is not consistent across channels can be explaind by scaling violation effects or underestimated errors?

- Running coupling methods like TPL and SF have problems to control systematics no comment on MCRG

Running coupling problems in TPL and SF schemes:
Continuum extrapolation

on TPL scheme

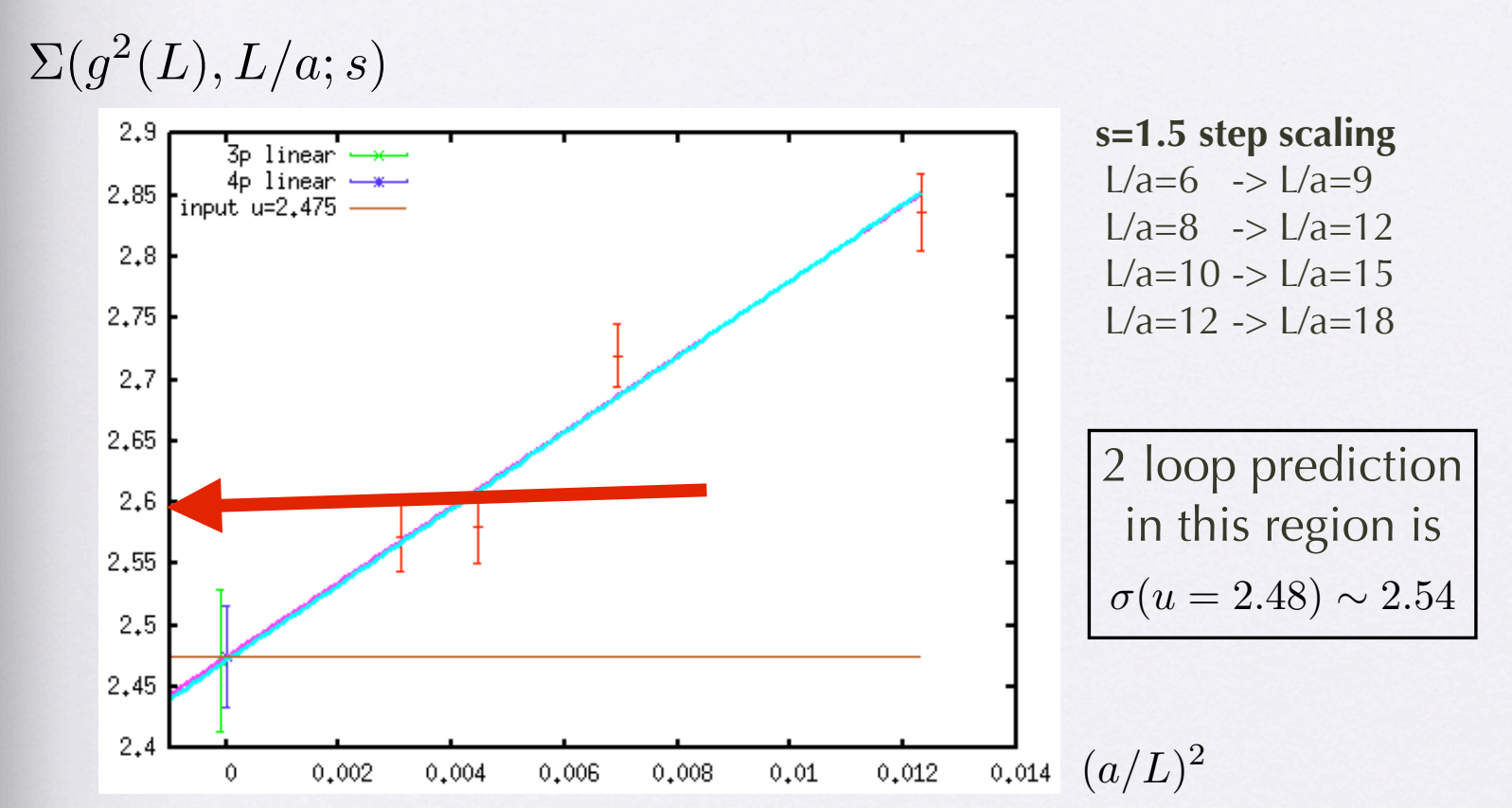

The systematic error is small in the strong coupling region. 
- We run in the weak coupling phase

- At fixed coupling and very small fermion mass we see confining potential and finite temp $\chi S B$ transition

- The effective anomalous dimension $Y$ is not consistent across channels can be explaind by scaling violation effects or underestimated errors?

- Running coupling methods like TPL and SF have problems to control systematics

no comment on MCRG

Continuum extrapolation

on TPL scheme

Running coupling problems in TPL and SF schemes:
$\Sigma\left(g^{2}(L), L / a ; s\right)$

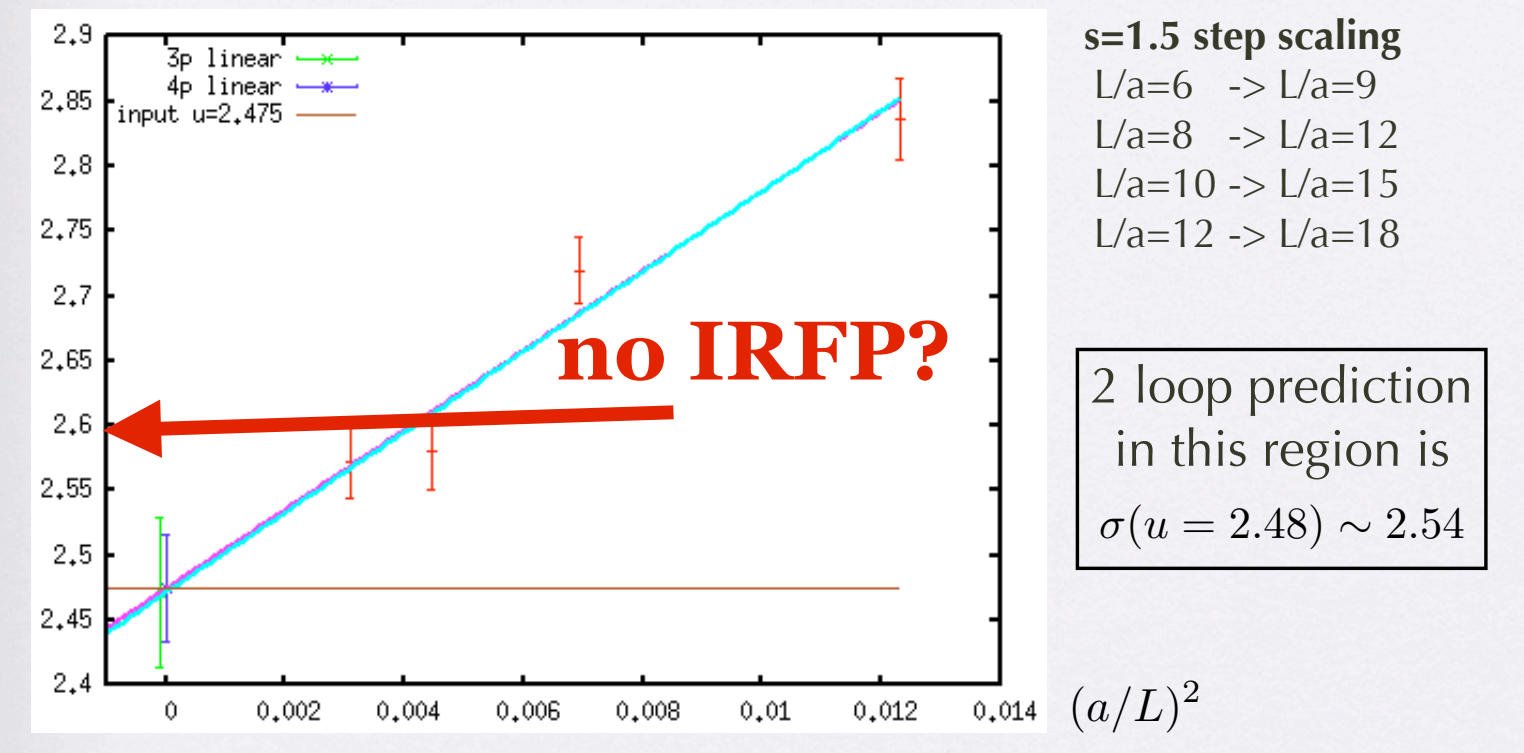

The systematic error is small in the strong coupling region. 
- We run in the weak coupling phase

- At fixed coupling and very small fermion mass we see confining potential and finite temp $\chi S B$ transition

- The effective anomalous dimension $Y$ is not consistent across channels can be explaind by scaling violation effects or underestimated errors?

- Running coupling methods like TPL and SF have problems to control systematics

no comment on MCRG

- We are working on two new running coupling methods with massless fermions using moments of current correlators and wilson flows both are $g(L)$

- Difficult decision on the $\mathrm{Nf}=12$ model: we would probably prefer to put all the resources into the sextet model without the community controversies at $\mathrm{Nf}=12$

- But sextet project benefitted directly from what we have learned from $N f=12$ 


\section{backup slides}




\section{conformal scaling and scaling violations}

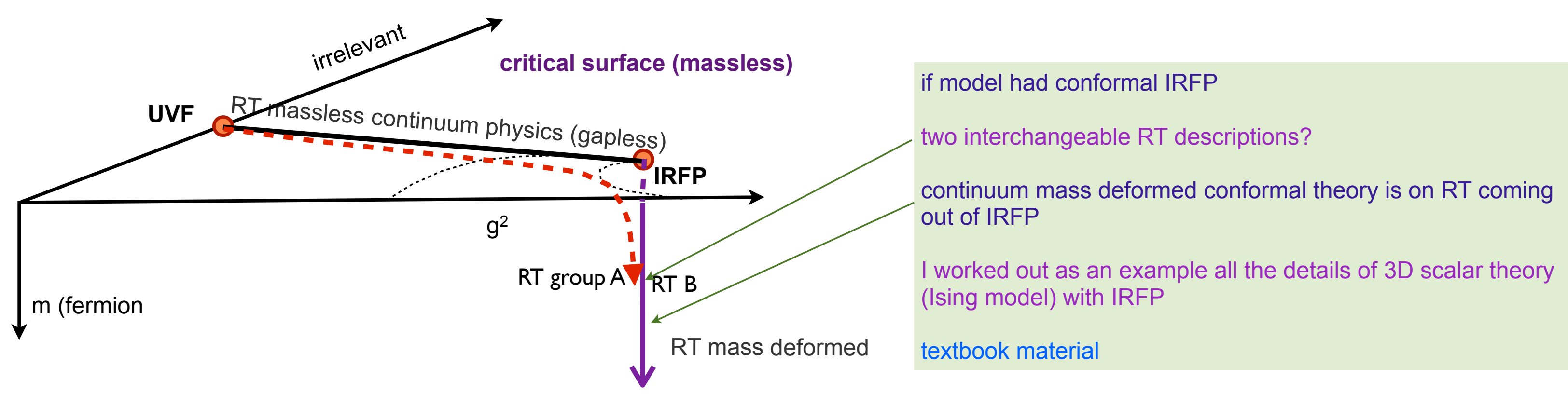

free energy on RT:

$$
f\left(u_{1}, u_{2}, \ldots\right)=\underset{\text { analytic }}{g\left(u_{1}, u_{2}, \ldots\right)+b^{-d} f_{s}\left(b^{y_{1}} u_{1}, b^{y_{2}} u_{2} \ldots\right)}
$$

$\mathrm{y}_{1}>0$ only relevant exponent in our case

$\mathrm{u}_{1}=\mathrm{t} \sim \mathrm{m}$ identified, $\mathrm{y}_{1}=\mathrm{y}_{\mathrm{m}}$ in Technicolor notation

y2 controls scaling violations, leading correction term

analytic function which can have terms like $\sim \mathrm{m}^{\mathrm{k}}$ are typically sub-leading

Fisher and Brezin worked out most of what we know!

similarly, in conformal finite size scaling analysis:

$\xi / L=f_{1}(x)+L^{-\omega} f_{2}(x)$ with $x=L m^{1 / y_{m}}$

correlation length measured in L units
RG scaling of 2-point function:

Del Debbio and collaborators early conform apps $G^{(2)}\left(r, m, u_{2}, \ldots\right)=b^{-2 d} G\left(r / b, b^{y_{m}} m, b^{y_{2}} u_{2}, \ldots\right)$ from $G^{(2)}\left(r, m, u_{2}, \ldots\right) \sim e^{-M r}$ asymptotics with $M \sim m^{1 / y_{m}}$ scaling follows leading correction to the scaling term should be $\sim m^{\omega}$ where $\omega=\beta^{\prime}\left(g^{*}\right)$ analysis would change with second relevant operator at IRFP!

- analytic terms exists, but no reason to be leading conformal scaling correction

- correlators of composite operators require inhomogeneous RG! 


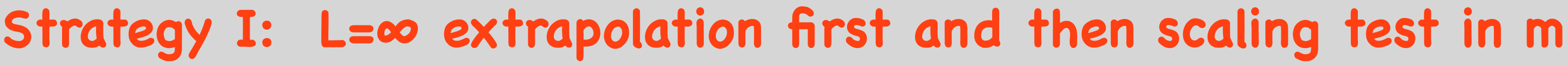

\section{Chiral hypothesis \\ (in)complete analysis on both sides \\ Conformal hypothesis}

chiral logs not reached yet!

$\left(N_{f}=8\right.$, or $\left.N_{f}=12\right) \quad N_{f}=2$ sextet easier reach

$\begin{aligned}\left(M_{\pi}^{2}\right)_{N L O}=\left(M_{\pi}^{2}\right)_{L O}+\left(\delta M_{\pi}^{2}\right)_{1-l o o p}+ & \left(\delta M_{\pi}^{2}\right)_{m^{2}} \\ & +\left(\delta M_{\pi}^{2}\right)_{a^{2} m}+\left(\delta M_{\pi}^{2}\right)_{a^{4}} \\ & \sim a^{2} m \sim a^{4}\end{aligned}$

$\left(M_{\pi}^{2}\right)_{L O}=2 B \cdot m+a^{2} \Delta_{B} \quad$ kept cutoff term in B see LO a a $^{2}$ term would require more data

$\left(\delta M_{\pi}^{2}\right)_{1-\text { loop }}=\left[\left(M_{\pi}^{2}\right)_{L O}+a^{2}\right]^{2} \ln \left(M_{\pi}^{2}\right)_{L O}$

$M_{\pi}^{2}=c_{1} m+c_{2} m^{2}+\operatorname{logs}$ fitted function for all Goldstones

$M_{n u c}=c_{0}+c_{1} m+\operatorname{logs} \quad$ nucleon states, rho, a l, higgs, ...

$\left(F_{\pi}\right)_{L O}=F, \quad\left(\delta F_{\pi}\right)_{1-\text { loop }}=\left[\left(M_{\pi}^{2}\right)_{L O}+a^{2}\right] \ln \left(M_{\pi}^{2}\right)_{L O}$

chiral log regime was not reached in fermion mass range

$\left(\delta F_{\pi}\right)_{m^{2}} \sim m, \quad\left(\delta F_{\pi}\right)_{a^{2} m}=a^{2}$

kept cutoff term in $\mathrm{F}$

$F_{\pi}=F+c_{1} m+\log \mathrm{s} \quad$ fitted function

$\langle\bar{\psi} \psi\rangle=\langle\bar{\psi} \psi\rangle_{0}+c_{1} m+c_{2} m^{2}+\operatorname{logs}$ chiral condensate

$$
M_{\pi}=c_{\pi} \cdot m^{1 / y_{m}}, \quad y_{m}=1+\gamma
$$

leading conformal scaling

functional form for all hadron masses

$$
F_{\pi}=c_{F} \cdot m^{1 / y_{m}}, \quad y_{m}=1+\gamma
$$

same critical exponent

$\langle\bar{\psi} \psi\rangle=c_{\gamma} \cdot m^{(3-\gamma) / y_{m}}+c_{1} m \quad$ Del Debbio and Zwicky

Asymptotic infinite volume limit has not been reached yet in important candidate models for conformal window

infinite volume conformal scaling violation analysis?

conformal finite size scaling analysis and its scaling violations? 



\title{
Recent progress in calculating $B_{K}$ using staggered fermions
}

\author{
Weonjong Lee
}

Lattice Gauge Theory Research Center

Department of Physics and Astronomy

Seoul National University

BNL Workshop, 05/15/2012 


\section{SWME Collaboration \\ Staggered $\varepsilon^{\prime} / \varepsilon$ Project 1998 - Present}




\section{SWME Collaboration}

- Seoul National University (SNU):

Prof. Weonjong Lee

Jon Bailey and Nigel Cundy (Research Assistant Prof's)

10 graduate students.

- Brookhaven National Laboratory (BNL):

Dr. Chulwoo Jung.

Dr. Hyung-Jin Kim (Postdoc).

- University of Washington, Seattle (UW):

Prof. Stephen R. Sharpe.

- KISTI: Dr. Taegil Bae (Postdoc).

- University of Arizona: Dr. Jongjeong Kim (Postdoc). 


\section{Indirect $\mathrm{CP}$ Violation and $B_{K}$}




\section{$\varepsilon$ and $\hat{B}_{K}$}

- $\varepsilon=(2.228 \pm 0.011) \times 10^{-3} \times e^{i \pi / 4}$ in experiment. 


\section{$\varepsilon$ and $\hat{B}_{K}$}

- $\varepsilon=(2.228 \pm 0.011) \times 10^{-3} \times e^{i \pi / 4}$ in experiment.

- Relation between $\varepsilon$ and $\hat{B}_{K}$ in standard model.

$$
\begin{aligned}
\varepsilon & =\exp \left(i \phi_{\varepsilon}\right) \sqrt{2} \sin \left(\phi_{\varepsilon}\right) C_{\varepsilon} \operatorname{Im} \lambda_{t} X \hat{B}_{K}+\xi \\
X & =\operatorname{Re} \lambda_{c}\left[\eta_{1} S_{0}\left(x_{c}\right)-\eta_{3} S_{3}\left(x_{c}, x_{t}\right)\right]-\operatorname{Re} \lambda_{t} \eta_{2} S_{0}\left(x_{t}\right) \\
\lambda_{i} & =V_{i s}^{*} V_{i d}, \quad x_{i}=m_{i}^{2} / M_{W}^{2}, \quad C_{\varepsilon}=\frac{G_{F}^{2} F_{K}^{2} m_{K} M_{W}^{2}}{6 \sqrt{2} \pi^{2} \Delta M_{K}} \\
\xi & =\exp \left(i \phi_{\varepsilon}\right) \sin \left(\phi_{\varepsilon}\right) \frac{\operatorname{Im} A_{0}}{\operatorname{Re} A_{0}}
\end{aligned}
$$




\section{$\varepsilon$ and $\hat{B}_{K}$}

- $\varepsilon=(2.228 \pm 0.011) \times 10^{-3} \times e^{i \pi / 4}$ in experiment.

- Relation between $\varepsilon$ and $\hat{B}_{K}$ in standard model.

$$
\begin{aligned}
\varepsilon & =\exp \left(i \phi_{\varepsilon}\right) \sqrt{2} \sin \left(\phi_{\varepsilon}\right) C_{\varepsilon} \operatorname{Im} \lambda_{t} X \hat{B}_{K}+\xi \\
X & =\operatorname{Re} \lambda_{c}\left[\eta_{1} S_{0}\left(x_{c}\right)-\eta_{3} S_{3}\left(x_{c}, x_{t}\right)\right]-\operatorname{Re} \lambda_{t} \eta_{2} S_{0}\left(x_{t}\right) \\
\lambda_{i} & =V_{i s}^{*} V_{i d}, \quad x_{i}=m_{i}^{2} / M_{W}^{2}, \quad C_{\varepsilon}=\frac{G_{F}^{2} F_{K}^{2} m_{K} M_{W}^{2}}{6 \sqrt{2} \pi^{2} \Delta M_{K}} \\
\xi & =\exp \left(i \phi_{\varepsilon}\right) \sin \left(\phi_{\varepsilon}\right) \frac{\operatorname{Im} A_{0}}{\operatorname{Re} A_{0}}
\end{aligned}
$$

- Definition of $B_{K}$ in standard model.

$$
\begin{aligned}
B_{K} & =\frac{\left\langle\bar{K}_{0}\left|\left[\bar{s} \gamma_{\mu}\left(1-\gamma_{5}\right) d\right]\left[\bar{s} \gamma_{\mu}\left(1-\gamma_{5}\right) d\right]\right| K_{0}\right\rangle}{\frac{8}{3}\left\langle\bar{K}_{0}\left|\bar{s} \gamma_{\mu} \gamma_{5} d\right| 0\right\rangle\left\langle 0\left|\bar{s} \gamma_{\mu} \gamma_{5} d\right| K_{0}\right\rangle} \\
\hat{B}_{K} & =C(\mu) B_{K}(\mu), \quad C(\mu)=\alpha_{s}(\mu)^{-\frac{\gamma_{0}}{2 b_{0}}}\left[1+\alpha_{s}(\mu) J_{3}\right]
\end{aligned}
$$




\section{$B_{K}$ on the lattice}




\section{$B_{K}$ definition in standard model}

$$
\begin{aligned}
B_{K} & =\frac{\left\langle\bar{K}_{0}\left|\left[\bar{s} \gamma_{\mu}\left(1-\gamma_{5}\right) d\right]\left[\bar{s} \gamma_{\mu}\left(1-\gamma_{5}\right) d\right]\right| K_{0}\right\rangle}{\frac{8}{3}\left\langle\bar{K}_{0}\left|\bar{s} \gamma_{\mu} \gamma_{5} d\right| 0\right\rangle\left\langle 0\left|\bar{s} \gamma_{\mu} \gamma_{5} d\right| K_{0}\right\rangle} \\
\hat{B}_{K} & =C(\mu) B_{K}(\mu) \\
C(\mu) & =\alpha_{s}(\mu)^{-\frac{\gamma_{0}}{2 b_{0}}}\left[1+\alpha_{s}(\mu) J_{3}\right]
\end{aligned}
$$


What do we calculate on the lattice?

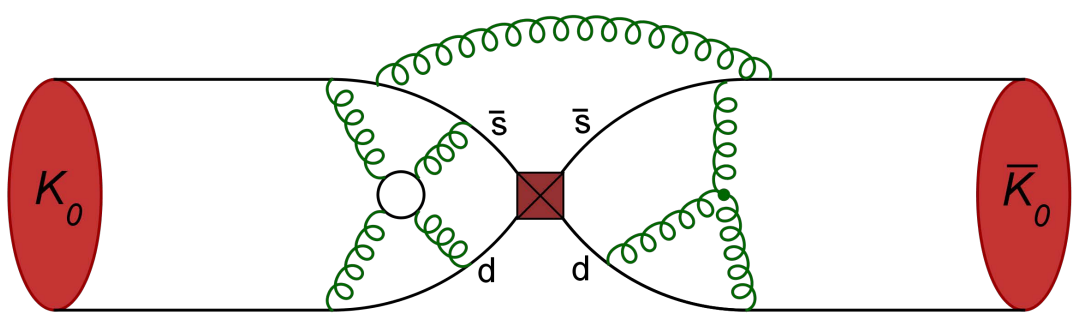




\section{Data Analysis for $B_{K}$}




\section{Data for $B_{K}$ with $a m_{d}=a m_{s}=0.025\left(20^{3} \times 64\right)$}

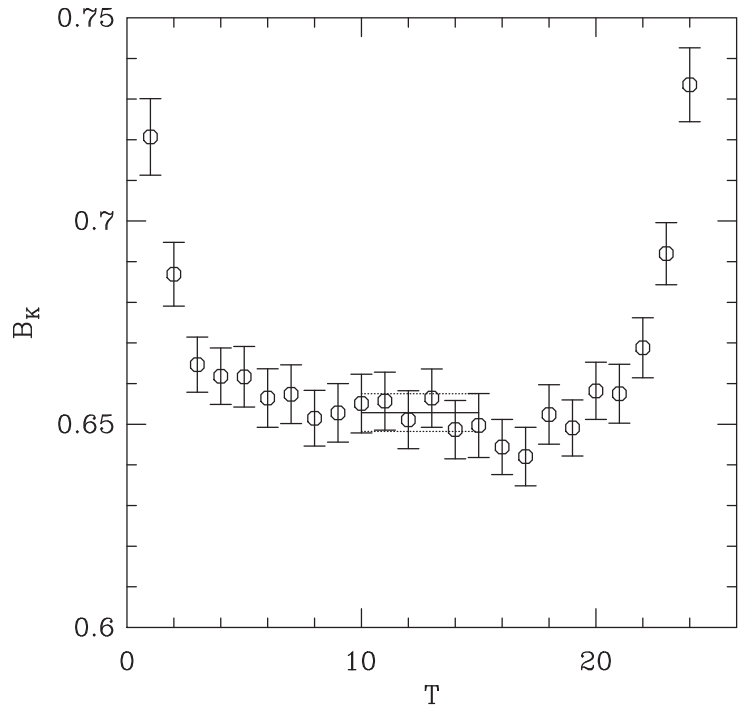




\section{$B_{K}$ in $N_{f}=2+1 \mathrm{QCD}$}

\begin{tabular}{l|l|l|l|l|l}
\hline$a(\mathrm{fm})$ & $a m_{l} / a m_{s}$ & geometry & ens $\times$ meas & round & production \\
\hline 0.12 & $0.03 / 0.05$ & $20^{3} \times 64$ & $564 \times 9$ & 2nd & done (SNU) \\
0.12 & $0.02 / 0.05$ & $20^{3} \times 64$ & $486 \times 9$ & 2nd & done (SNU) \\
0.12 & $0.01 / 0.05$ & $20^{3} \times 64$ & $671 \times 9$ & 2nd & done (SNU) \\
0.12 & $0.01 / 0.05$ & $28^{3} \times 64$ & $274 \times 8$ & 2nd & done (BNL) \\
0.12 & $0.007 / 0.05$ & $20^{3} \times 64$ & $651 \times 10$ & 2nd & done (SNU) \\
0.12 & $0.005 / 0.05$ & $24^{3} \times 64$ & $509 \times 9$ & 2nd & done (SNU) \\
\hline $0.09(\mathrm{~F} 1)$ & $0.0062 / 0.031$ & $28^{3} \times 96$ & $995 \times 9$ & 2nd & done (SNU) \\
0.09 & $0.0031 / 0.031$ & $40^{3} \times 96$ & $805 \times 1$ & 2nd & SNU $\left(^{*}\right)$ \\
\hline 0.06 & $0.0036 / 0.018$ & $48^{3} \times 144$ & $744 \times 2$ & 2nd & SNU $/ J \mathrm{JAB}($ \\
0.06 & $0.0025 / 0.018$ & $56^{3} \times 144$ & $700 \times 1$ & 1 st & $\mathrm{KISTI}\left({ }^{*}\right)$ \\
0.06 & $0.0018 / 0.018$ & $64^{3} \times 144$ & $700 \times-$ & - & - \\
\hline $0.045(\mathrm{U} 1)$ & $0.0030 / 0.015$ & $64^{3} \times 192$ & $705 \times 1$ & 2nd & $\mathrm{SNU}\left(^{*}\right)$ \\
\hline
\end{tabular}




\section{SU(2) SChPT Fitting for $B_{K}$}

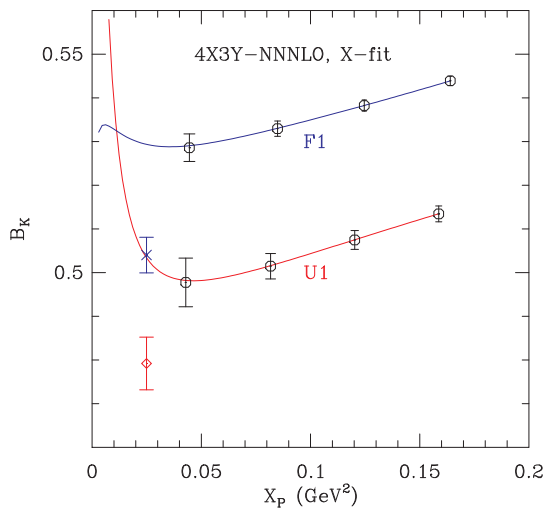

(a) X-fit

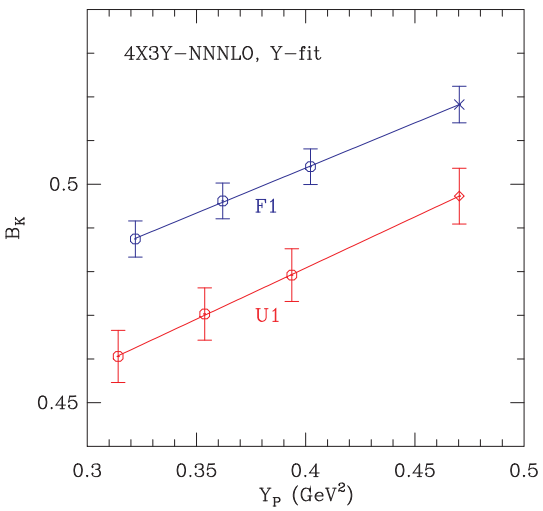

(b) Y-fit

- Fit type $=\mathrm{SU}(2) \mathrm{S} \chi \mathrm{PT}, 4 \mathrm{X} 3 \mathrm{Y}, \mathrm{NNNLO}$, Bayesian, FV 


\section{$B_{K}$ vs. $a^{2}$ : Discretization Error}

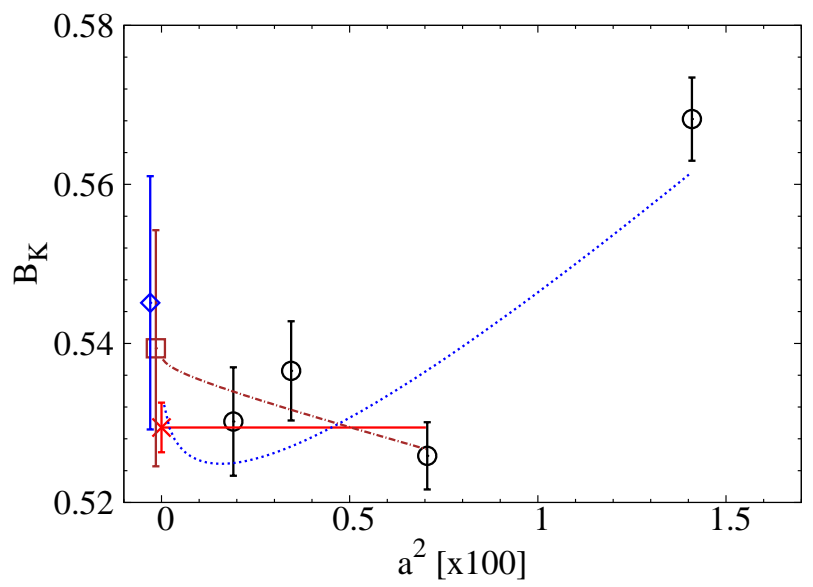

- Fit type = SU(2) SChPT; const, 3pt:g4-a2g2-a4, 4pt:g4-a2g2-a4 fits 


\section{Error Budget of $B_{K}$ [ SU(2)-SChPT, 4X3Y, NNNLO]}

\begin{tabular}{|c|c|c|}
\hline cause & error $(\%)$ & memo \\
\hline statistics & 0.59 & 4X3Y-NNNLO-BAYES + const \\
\hline matching factor & 4.4 & $\Delta B_{K}^{(2)}(\mathrm{U} 1)$ \\
\hline discretization & 1.9 & diff. of const and constrained fits \\
\hline X-fits & 0.33 & varying Bayesian priors (S1) \\
\hline Y-fits & 0.07 & diff. of linear and quadratic (C3) \\
\hline$a m_{l}$ extrap & 1.5 & diff. of (C3) and linear extrap \\
\hline$a m_{s}$ extrap & 1.3 & diff. of (C3) and linear extrap \\
\hline finite volume & 0.5 & diff. of $V=\infty$ and FV fits \\
\hline$r_{1}$ & 0.14 & $r_{1}$ error propagation (C3) \\
\hline$f_{\pi}$ & 0.4 & $132 \mathrm{MeV}$ vs. $124.4 \mathrm{MeV}$ \\
\hline
\end{tabular}




\section{Current Status of $B_{K}(1)$}

- Lattice QCD (SWME):

$$
B_{K}(\mathrm{RGI})=\hat{B}_{K}=0.727 \pm 0.004 \text { (stat) } \pm 0.038 \text { (sys) }
$$

- Experiments: (most updated version in 2012)

$$
B_{K}(\mathrm{RGI})=\left\{\begin{array}{cl}
1.01 \pm 0.11 & \text { for exclusive } V_{c b} \\
0.824 \pm 0.060 & \text { for inclusive } V_{c b}
\end{array}\right.
$$

- Hence, we observe 2.6 $\sigma$ difference between the SM theory and experiments (exclusive $V_{c b}$ ) and $1.6 \sigma$ difference (inclusive $V_{c b}$ ). Is this substantial? 


\section{Current Status of $B_{K}(2)$}

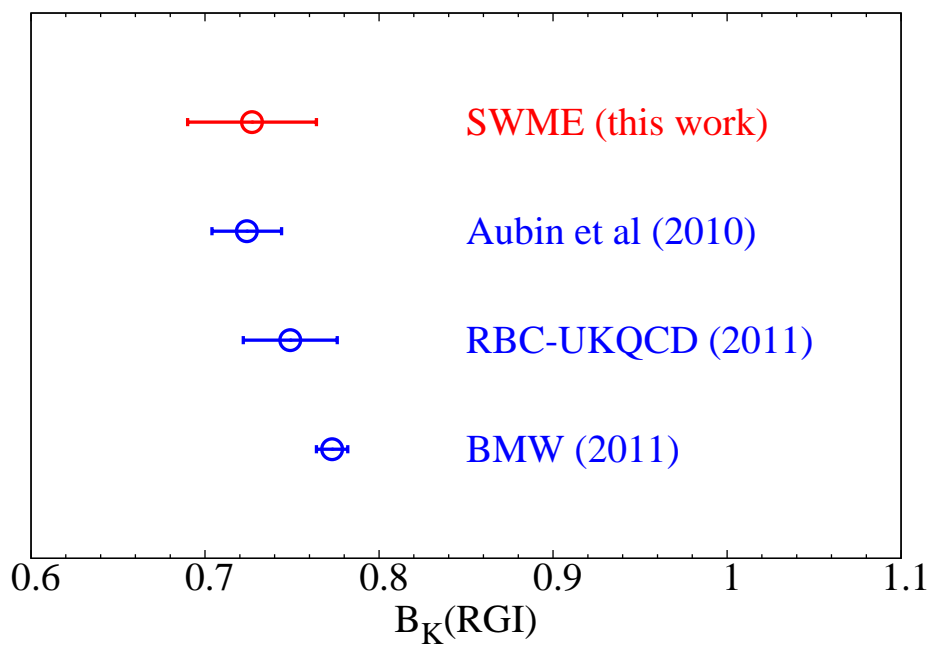




\section{Current Status of $B_{K}(3)$}

- SWME (Stag):

$$
\hat{B}_{K}=0.727 \pm 0.004 \text { (stat) } \pm 0.038 \text { (sys) }
$$

- Laiho / Van de Water (DWF + Stag):

$$
\hat{B}_{K}=0.724 \pm 0.008(\text { stat }) \pm 0.028 \text { (sys) }
$$

- RBC / UKQCD (DWF):

$$
\hat{B}_{K}=0.749 \pm 0.007(\text { stat }) \pm 0.026(\text { sys })
$$

- BMW (Wilson):

$$
\hat{B}_{K}=0.773 \pm 0.008(\text { stat }) \pm 0.009(\text { sys })
$$




\section{Preliminary Theoretical Expectation for $\varepsilon_{K}$}

- Inclusive $V_{c b}$ :

$$
\varepsilon_{K}= \begin{cases}2.07 \pm 0.16 & (\text { Lat Avg) } \\ 1.95 \pm 0.19 & \text { (SWME) }\end{cases}
$$

- Exclusive $V_{c b}$ :

$$
\varepsilon_{K}= \begin{cases}1.66 \pm 0.21 & (\text { Lat Avg) } \\ 1.56 \pm 0.22 & (\text { SWME) }\end{cases}
$$

- Experiment:

$$
\varepsilon_{K}=2.228 \pm 0.011 \quad \text { (PDG) }
$$

- We observe about $3.0 \sigma$ tension in the exclusive $V_{c b}$ channel of $\varepsilon_{K}$. 


\section{Preliminary check for CKM unitarity}

- CKM unitarity:

$$
\left[V V^{\dagger}\right]_{c t}=0
$$

- Exclusive $V_{c b}$ : Lattice 2012 (Yong-Chull Jang)

$$
\left[V V^{\dagger}\right]_{c t}=0.00153(48) \quad \text { (preliminary) }
$$

- Inclusive $V_{c b}$ : Lattice 2012 (Yong-Chull Jang)

$$
\left[V V^{\dagger}\right]_{c t}=0.00128(47) \sim 0.0084(47) \quad \text { (preliminary) }
$$

- We observe about $3.2 \sigma$ tension in the exclusive $V_{c b}$ channel of the CKM unitarity. 


\section{Preliminary error analysis with inclusive $V_{c b}$}

- Lattice 2012: Yong-Chull Jang

- Lat. Avg.:

$$
\left\{\begin{array}{cc}
\bar{\eta} & 27 \% \\
\eta_{3} & 19 \% \\
m_{c} & 16 \% \\
\cdots & \cdots
\end{array}\right.
$$

- SWME:

$$
\left\{\begin{array}{cc}
\hat{B}_{K} & 34 \% \\
\bar{\eta} & 18 \% \\
\eta_{3} & 12 \% \\
m_{c} & 11 \% \\
\cdots & \cdots
\end{array}\right.
$$




\section{Preliminary error analysis with exclusive $V_{c b}$}

- Lattice 2012: Yong-Chull Jang

- Lat. Avg.:

$$
\left\{\begin{array}{cc}
V_{c b} & 58 \% \\
\bar{\eta} & 11 \% \\
\eta_{3} & 9 \% \\
m_{c} & 8 \% \\
\cdots & \cdots
\end{array}\right.
$$

- SWME:

$$
\left\{\begin{array}{cc}
V_{c b} & 49 \% \\
\hat{B}_{K} & 18 \% \\
\bar{\eta} & 9 \% \\
\eta_{3} & 8 \% \\
m_{c} & 7 \% \\
\cdots & \cdots
\end{array}\right.
$$




\section{BSM corrections to $B_{K}$ on the lattice}




\section{BSM operators for $B_{K}$}

- BSM operators:

$$
\begin{aligned}
& O_{2}=\bar{s}_{a}\left(1-\gamma_{5}\right) d_{a} \bar{s}_{b}\left(1-\gamma_{5}\right) d_{b} \\
& O_{3}=\bar{s}_{a}\left(1-\gamma_{5}\right) d_{b} \bar{s}_{b}\left(1-\gamma_{5}\right) d_{a} \\
& O_{4}=\bar{s}_{a}\left(1-\gamma_{5}\right) d_{a} \bar{s}_{b}\left(1+\gamma_{5}\right) d_{b} \\
& O_{5}=\bar{s}_{a}\left(1-\gamma_{5}\right) d_{b} \bar{s}_{b}\left(1+\gamma_{5}\right) d_{a}
\end{aligned}
$$

- B parameters:

$$
B_{j}(\mu)=\frac{\left\langle\bar{K}_{0}\left|O_{j}(\mu)\right| K_{0}\right\rangle}{N_{j}\left\langle\bar{K}_{0}|P(\mu)| 0\right\rangle\left\langle 0|P(\mu)| K_{0}\right\rangle}
$$




\section{What do we calculate on the lattice?}

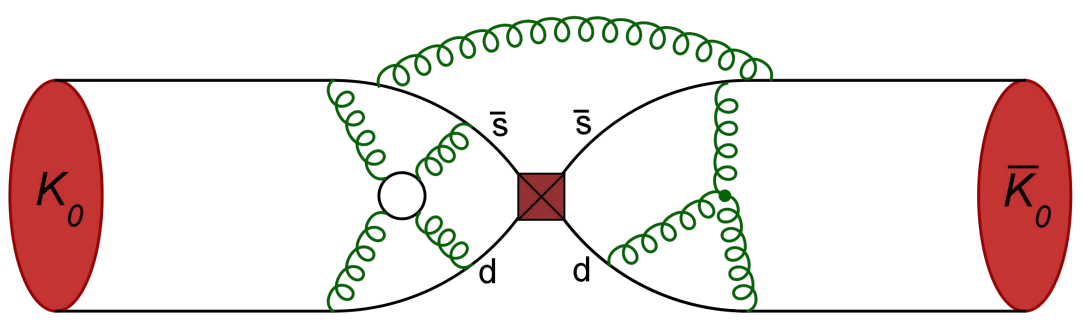




\section{Data Analysis for BSM corrections to $B_{K}$}




\section{Data analysis for $B_{2}$ with $a m_{d}=a m_{s}=0.025\left(20^{3} \times 64\right)$}

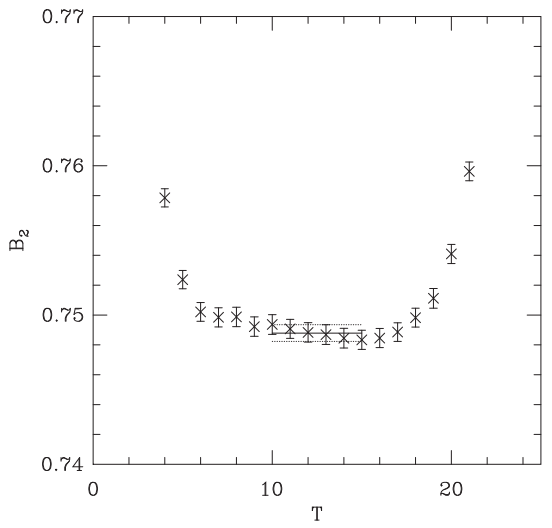

(c) raw data

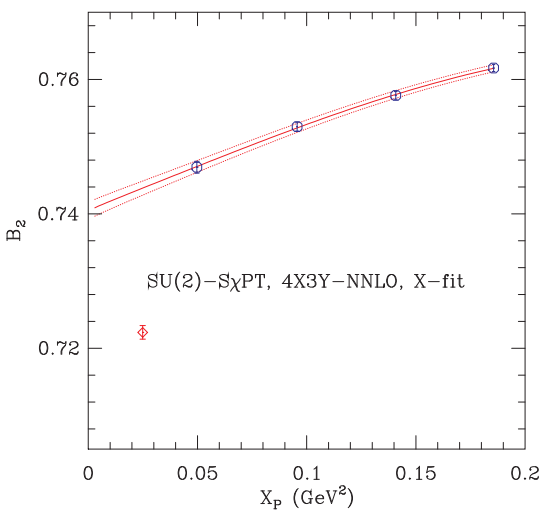

(d) X-fit

Preliminary !!! 


\section{Data analysis for $B_{3}$ with $a m_{d}=a m_{s}=0.025\left(20^{3} \times 64\right)$}

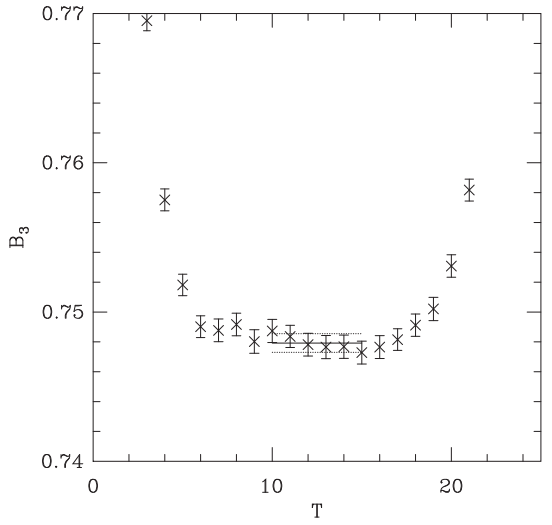

(e) raw data

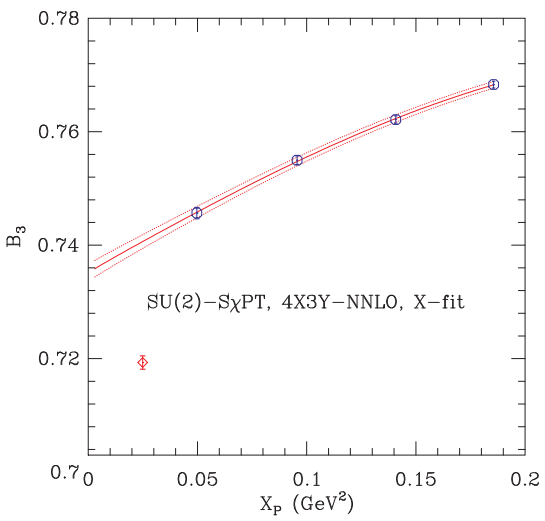

(f) $X$-fit

\section{Preliminary !!!}




\section{Data analysis for $B_{4}$ with $a m_{d}=a m_{s}=0.025\left(20^{3} \times 64\right)$}

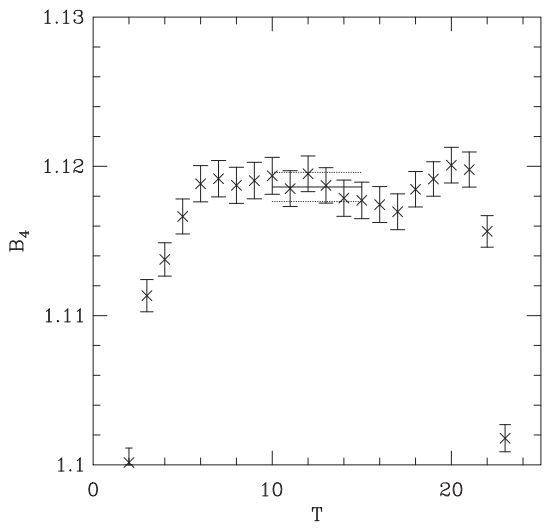

(g) raw data

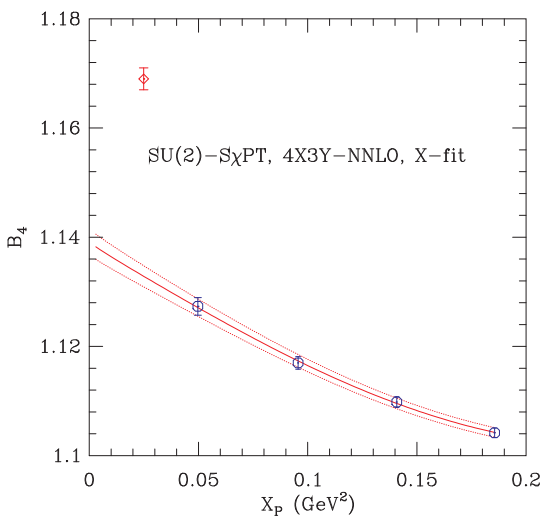

(h) X-fit

\section{Preliminary !!!}




\section{Data analysis for $B_{5}$ with $a m_{d}=a m_{s}=0.025\left(20^{3} \times 64\right)$}

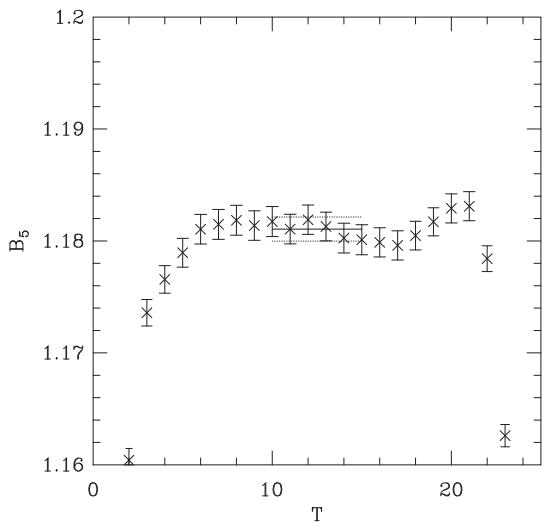

(i) raw data

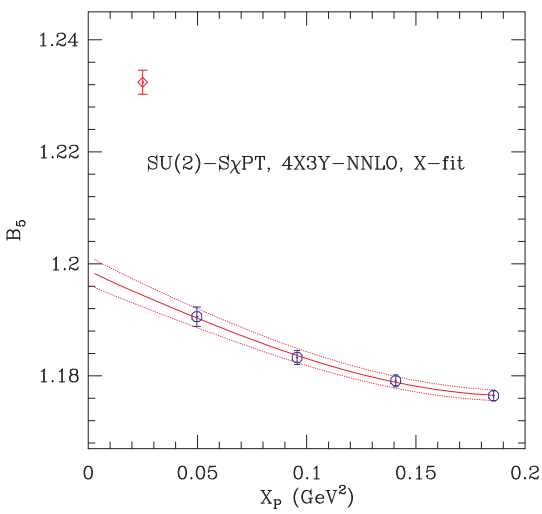

(j) X-fit

Preliminary !!! 


\section{Current Status of BSM corrections to $B_{K}$}

- We are in the middle of data analysis at the tree level. Hence the results are very preliminary.

- We plan to complete the first round data analysis at the one-loop level by Lattice 2012. We will present the preliminary results in Lattice 2012. [Dr. Hyung-Jin Kim]

- We plan to complete the second round data analysis using the NPR matching by the end of 2012 . 


\section{Summary}

- We plan to reduce the overall error of $B_{K}$ below the $2 \%$ level. (at least for SWME). We have to reduce the overall error of exclusive $V_{c b}$ below the $0.5 \%$ level.

- How?

Answer: NPR, or two-loop matching.

- Lattice 2012: Jangho Kim (NPR).

- Lattice 2012: Kwangwoo Kim (two-loop matching ???). 


\section{Future Theoretical Perspectives (1)}

- Hansen \& Sharpe (2012): multiple-channel scattering phase shift formula

- This directly applied to staggered $\pi-\pi$ scattering case: $N=5$ channels.

- There is a remaining difficulty in the unitarity ansatz of the S-matrix.

- We will overcome this difficulty using the guidance of staggered chiral perturbation theory (SChPT).

- It could be very likely to calculate the $\pi-\pi$ scattering phase shift with the systematic errors under control in near future.

- This will make it possible to calculate the $K \rightarrow \pi \pi$ decay amplitude using staggered fermions. 


\section{Future Theoretical Perspectives (2)}

- We have extended the SChPT calculation to non-Goldstone pion sectors for pion mass and decay constants $f_{\pi}, f_{K}$.

- We plan to extend the horizon to the mixed action case (HYP stag/asqtad or HYP/HISQ) in 2012.

- We plan to find the best channels to calculate the $\pi-\pi$ scattering phase shift using staggered fermions. The SChPT will be the main tool for this mission. 


\title{
Lattice study of the phase structure
}

\section{of the strong Yukawa model}

\author{
arXiv:1111.4544 (with updates)
}

$$
\text { C.-J. David Lin }
$$

National Chiao-Tung University

\author{
BNL \\ $14 / 05 / 2012$
}




\section{The collaboration}

- Germany

- NIC, DESY Zeuthen and Humboldt University Berlin John Bulava (CERN), Philipp Gerhold ( $\rightarrow$ d-Fine), Karl Jansen, Attila Nagy.

- Japan

- Kobayashi-Maskawa Institute, Nagoya University Kei-Ichi Nagai.

- Taiwan

- National Chiao-Tung University, Hsinchu C.-J.David Lin, Kenji Ogawa.

- National Taiwan University, Taipei George W.-S. Hou, Bastian Knippschild, Brian Smigielski ( $\rightarrow$ U.S.). 


\section{Motivation Heavy fermions beyond SM3?}

- Not much is known for strong (non-perturbative) Yukawa theory.

- Heavy extra generation of fermions may

- enhance CP violation.

G.W.S. Hou, 2008

- offer an alternative way to break EW symmetry dynamically and induces bound states to unitarise $W W$ scattering.

B. Holdom, 2007

- UV stablise the SM. 


\section{Outline}

- Goals, general issues and recent developments.

- Simultation setup.

- The phase structure.

- Exploratory numerical studies.

- VEV.

- Susceptibility and critical exponents.

- Binder's cumulant.

- Future plan. 


\section{Targets for the bare strong-Yukawa regime}

- The nature of the phase transitions.

$\Rightarrow$ Connection to the continuum world (next slide).

- Possible bound states.

$\Rightarrow$ Computation of the spectrum.

- Possible new mechanism for dynamical symmetry breaking.

$\Rightarrow$ Heavy scalar with fermion condensate? 


\section{General issues and strategy}

- The triviality (Landau-pole) problem.

$\Rightarrow$ Non-trivial to take the lattice spacing to zero.

- Look for 2nd-order phase transitions via "scanning simulations".

$\Rightarrow \xi \rightarrow \infty$

- Problem: Finite-volume effects.

$\Rightarrow$ Phase transitions are washed out.

$\Rightarrow$ Severe near the critical points since $L=\widehat{L} a$.

- Chiral fermions required. 


\section{New ingredients in current work}

- Previous studies (circa 1990):

Lee, Shigemitsu, Shrock; Bock et al.,...

- Use fermions without exact chiral symmetry.

$\Rightarrow$ Ambiguity in defining chiral fermions.

- Small $\left(\sim 8^{3} \times 16\right)$ volumes and no $L \rightarrow \infty$ limit taken.

- Current new-generation simulations:

- Use the overlap fermion (exact chiral symmetry).

- Several large volumes and $L \rightarrow \infty$ limit taken.

$\Rightarrow$ Test finite-size scaling behaviour.

$\Rightarrow$ Determine the order of the phase transition. 


\section{Reminder: Notaion for scalar field theory}

- The discretised Euclidean scalar action $(a=1)$

$$
S_{\varphi}=-\sum_{x, \mu} \varphi_{x}^{\alpha} \varphi_{x+\widehat{\mu}}^{\alpha}+\sum_{x}\left[\frac{1}{2}\left(2 d+m_{0}^{2}\right) \varphi_{x}^{\alpha} \varphi_{x}^{\alpha}+\frac{1}{4} \lambda_{0}\left(\varphi_{x}^{\alpha} \varphi_{x}^{\alpha}\right)^{2}\right] .
$$

- $\varphi=\sqrt{2 \kappa} \phi, \quad m_{0}^{2}=\frac{1-2 \AA}{\kappa}-2 d, \quad \lambda_{0}=\frac{\widehat{\lambda}}{\kappa^{2}}$

$$
\begin{aligned}
& S_{\phi}=-2 \kappa \sum_{x, \mu} \phi_{x}^{\alpha} \phi_{x+\hat{\mu}}^{\alpha}+\sum_{x}\left[\phi_{x}^{\alpha} \phi_{x}^{\alpha}+\hat{\lambda}\left(\phi_{x}^{\alpha} \phi_{x}^{\alpha}-1\right)^{2}\right], \\
& Z_{\phi}=\int \prod_{x, \alpha} d \phi_{x}^{\alpha} \exp \left(-S_{\phi}\right)=\int \prod_{x, \alpha} d \mu\left(\phi_{x}^{\alpha}\right) \exp \left(2 \kappa \sum_{x, \mu} \phi_{x}^{\alpha} \phi_{x+\hat{\mu}}^{\alpha}\right), \\
& d \mu\left(\phi_{x}^{\alpha}\right)=d \phi_{x}^{\alpha} \exp \left[-\phi_{x}^{\alpha} \phi_{x}^{\alpha}-\widehat{\lambda}\left(\phi_{x}^{\alpha} \phi_{x}^{\alpha}-1\right)^{2}\right] .
\end{aligned}
$$

- "staggered symmetry" : $\kappa \rightarrow-\kappa$ and $\phi_{x}^{\alpha} \rightarrow(-1)^{x_{1}+x_{2}+\ldots+x_{d}} \phi_{x}^{\alpha}$. 


\section{Fermions and the Yukawa couplings}

- Use the overlap Dirac operator with exact lattice chiral symmetry.

- The Yukawa terms $S_{H Y}=\sum_{x} y\left(\bar{t}_{x}, \bar{b}_{x}\right)_{L} \Phi_{x} b_{x, R}+y\left(\bar{t}_{x}, \bar{b}_{x}\right)_{L} \widetilde{\Phi}_{x} t_{x, R}+$ h.c..

- $\Phi$ is a complex scalar doublet and $\tilde{\Phi}=i \tau_{2} \Phi^{*}$.

- Results presented in this talk are from $8^{3} \times 16,12^{3} \times 24$ and $16^{3} \times 32$. 


\section{Phase diagram of the $\mathrm{H}-\mathrm{Y}$ model (qualitative)}

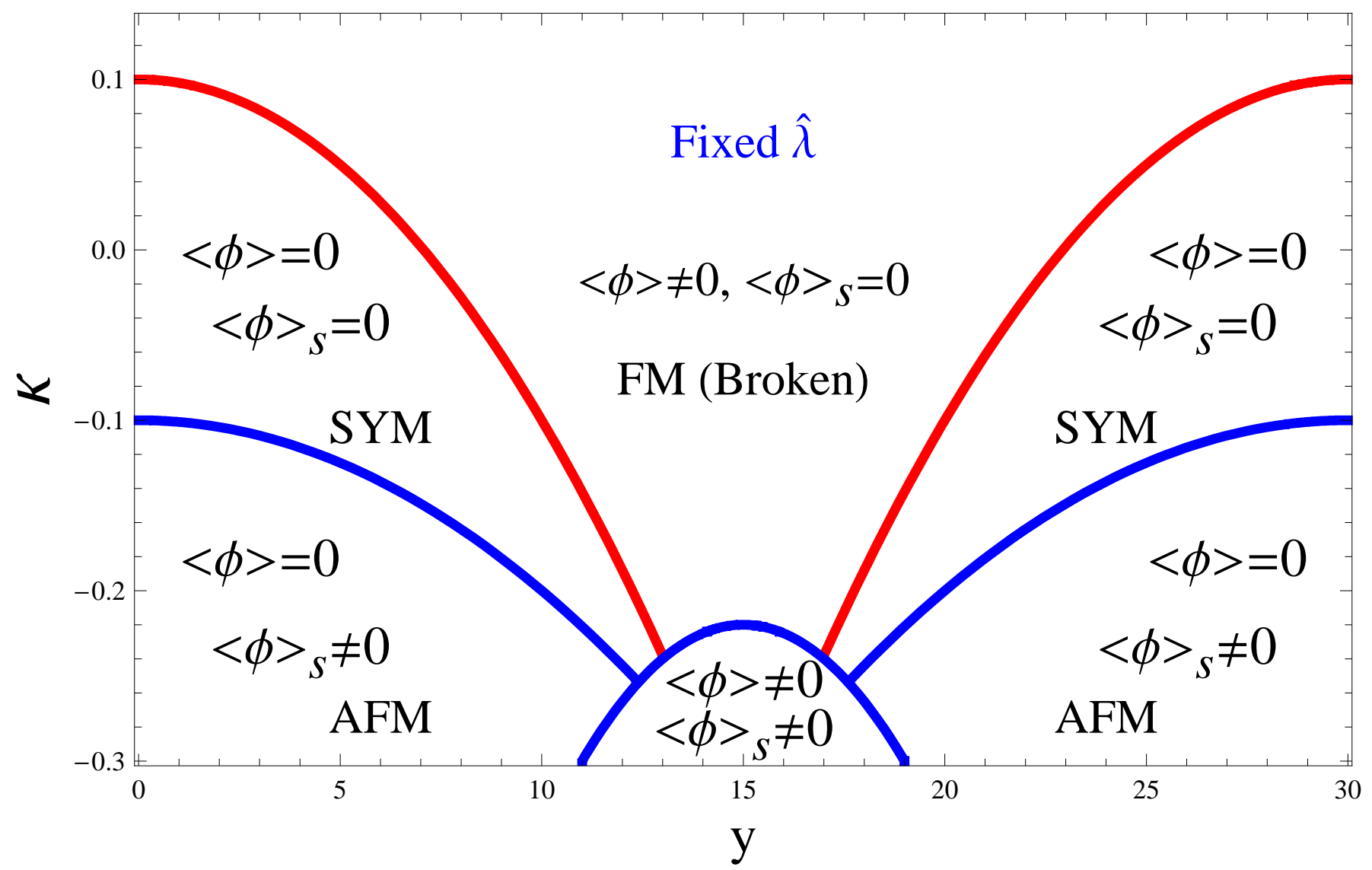

* From earlier work using Wilson fermions.

$\Rightarrow$ Controversy from staggered-fermion calculations. 


\section{Evidence of a symmetric phase at large $y$}

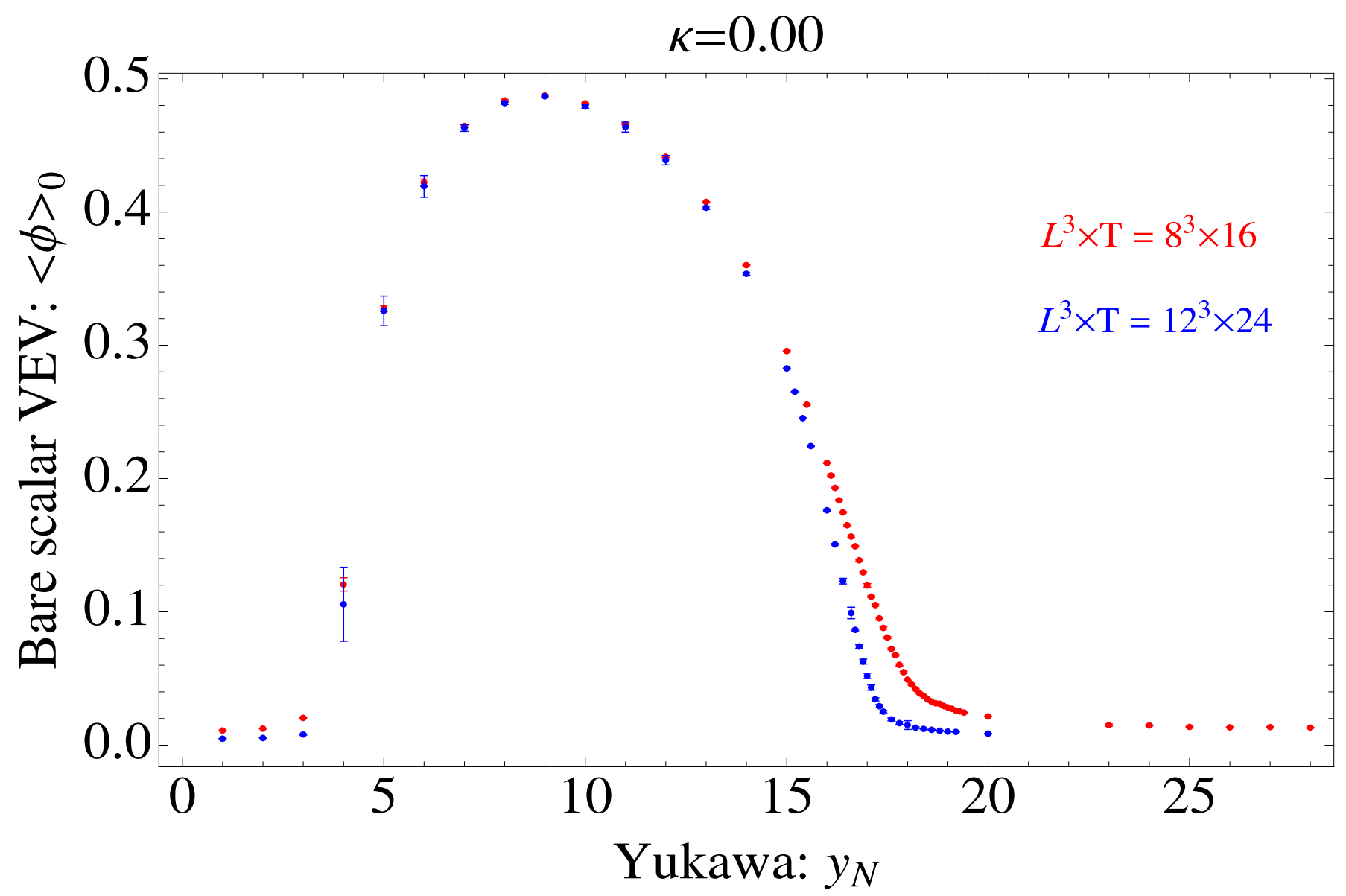

Consistent with recent results in P. Gerhold and K. Jansen, 2007. 


\section{The bare scalar vev at large $Y$}

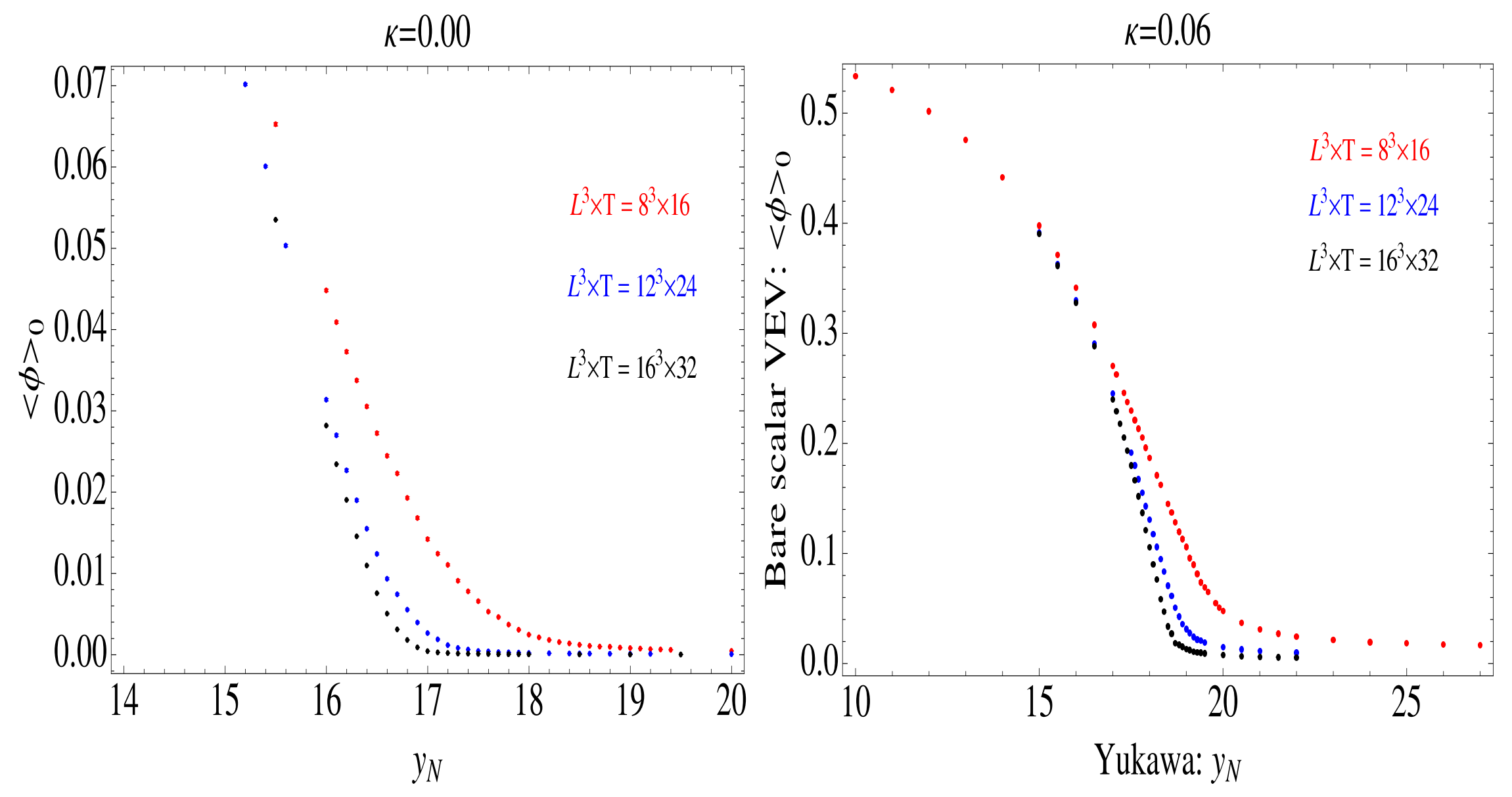




\section{Finite-size scaling of susceptibility}

- Susceptibility: $\chi=V_{4}\left(\left\langle\phi^{2}\right\rangle-\langle\phi\rangle\langle\phi\rangle\right)$.

- The scaling behaviour from solving the RGE,

- Universal function $\chi L_{s}^{-\gamma / \nu} \sim g\left(\tilde{t} L_{s}^{1 / \nu}\right)$, where $\tilde{t}=\left(y / y_{\text {crit }}-1\right)$.

- critical exponents $\gamma$ and $\nu$.

- Modelling the scaling violation from

M. Fisher and M. Barber, 1972

$\Rightarrow \chi L_{s}^{-\gamma / \nu} \sim g\left(t L_{s}^{1 / \nu}\right)$, where $t=\left(y /\left(y_{\text {crit }}-A_{4} / L_{s}^{b}\right)-1\right)$.

- Fit all the data to the (partly empirical) function at fixed $\kappa$

K. Jansen and P. Seuferling, 1990

$$
\chi=A_{1}\left\{L_{s}^{-2 / \nu}+A_{2,3}\left(y-y_{\text {crit }}-A_{4} / L_{s}^{b}\right)^{2}\right\}^{-\gamma / 2} .
$$




\section{Finite-size fit of susceptibility}
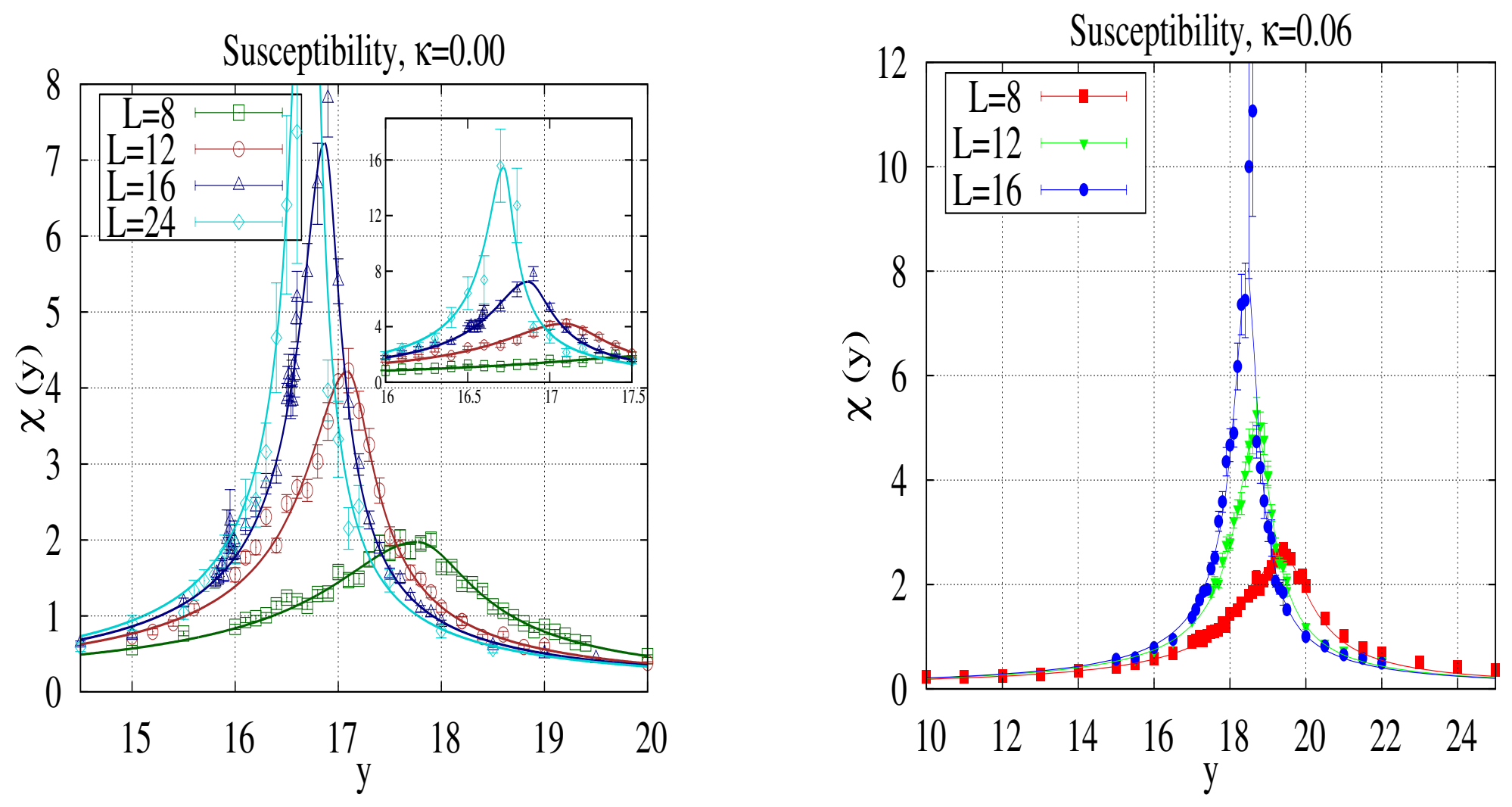

Fit range: $\quad y=15.0 \sim 20.0$

$$
y=14.0 \sim 22.0
$$




\section{Finite-size scaling of susceptibility}
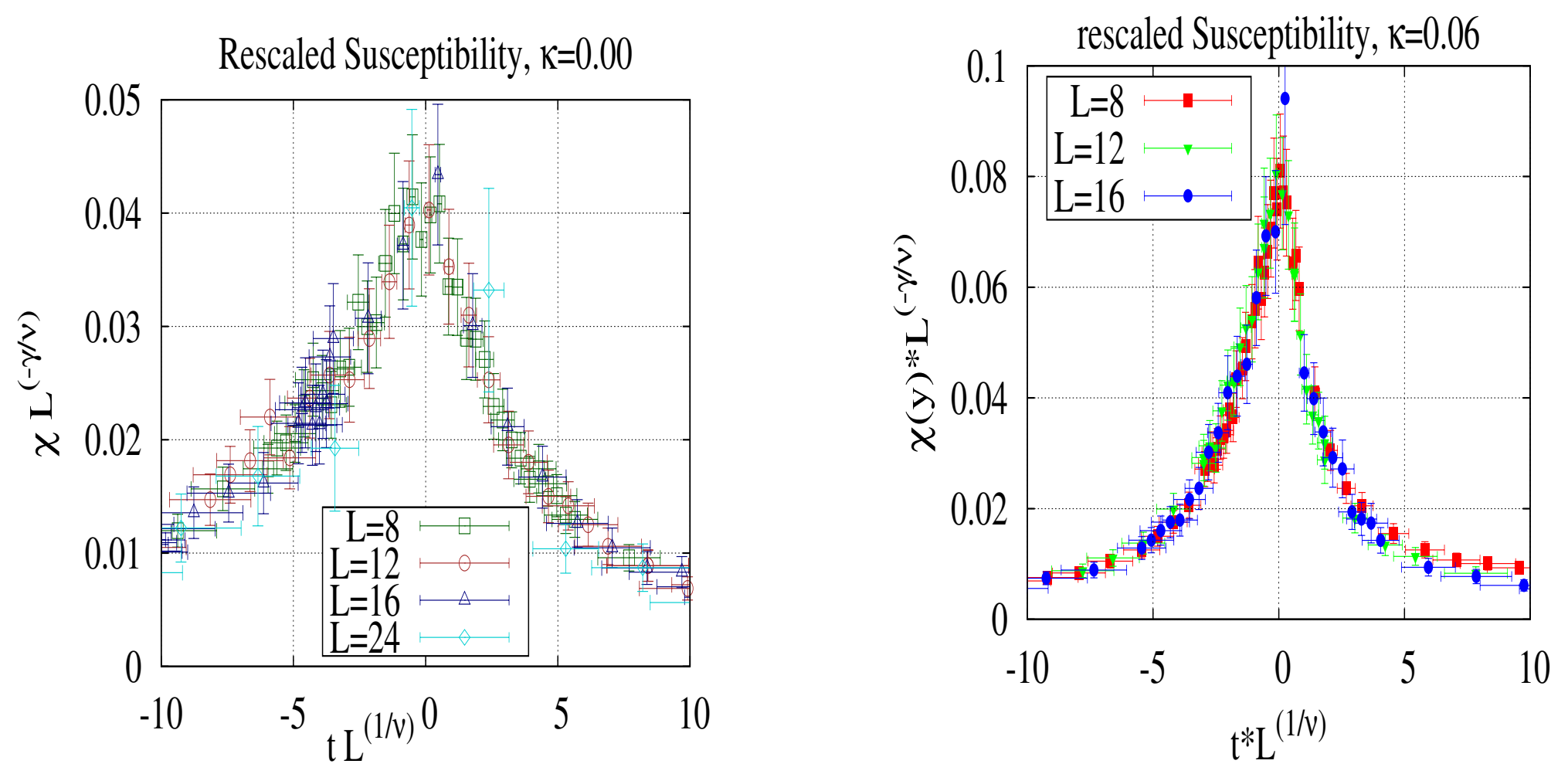


\section{Finite-size scaling of Binder's cumulant}
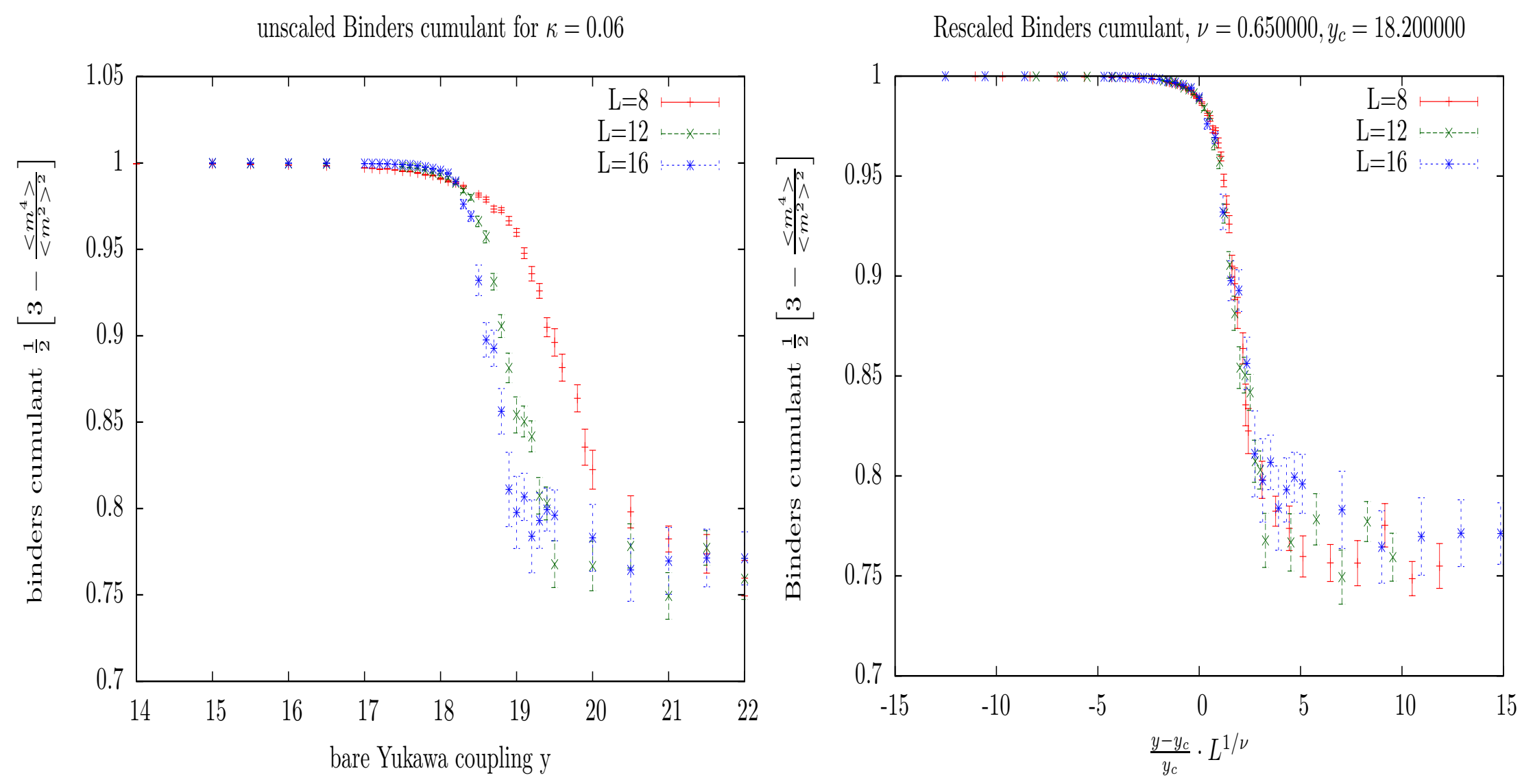


\section{Probing the phase structure using susceptibility}

\begin{tabular}{c|c|c|c} 
& $\kappa=0.00$ & $\kappa=0.06$ & $\mathrm{O}(4)$ scalar model \\
\hline$y_{\text {crit }}$ & $16.57 \pm 0.06$ & $18.11 \pm 0.06$ & $\mathrm{~N} / \mathrm{A}$ \\
\hline$\gamma$ & $0.97 \pm 0.02$ & $1.08 \pm 0.01$ & 1 \\
\hline$\nu$ & $0.52 \pm 0.01$ & $0.66 \pm 0.02$ & 0.5 \\
\hline$b$ & $2.18 \pm 0.09$ & $2.04 \pm 0.20$ & $?$ \\
\hline
\end{tabular}

- Quoted errors are statistical.

- Estimate systematics by changing the fit range in $y$.

- Systematic effects

- $y_{\text {crit }}$ is very stable.

$-\gamma$ can change by $\sim 2 \%$.

$-\nu$ can vary by $\sim 8 \%$. $\Rightarrow$ Different from $O(4)$ scalar model? 


\section{Outlook}

- Improving results by

- running more at large lattices, $24^{3} \times 48$. (finishing soon.)

- using more sophisticated procedure to investigate the susceptibility.

- studying the details of the scaling behaviour of Binder's cummulant.

- More information:

- Compute three renormalised couplings to "trade" with $\kappa, \hat{\lambda}$ and $y$.

- Study the spectrum in the strong Yukawa regime.

A lot more to do and to understand. 


\section{The Collaboration}

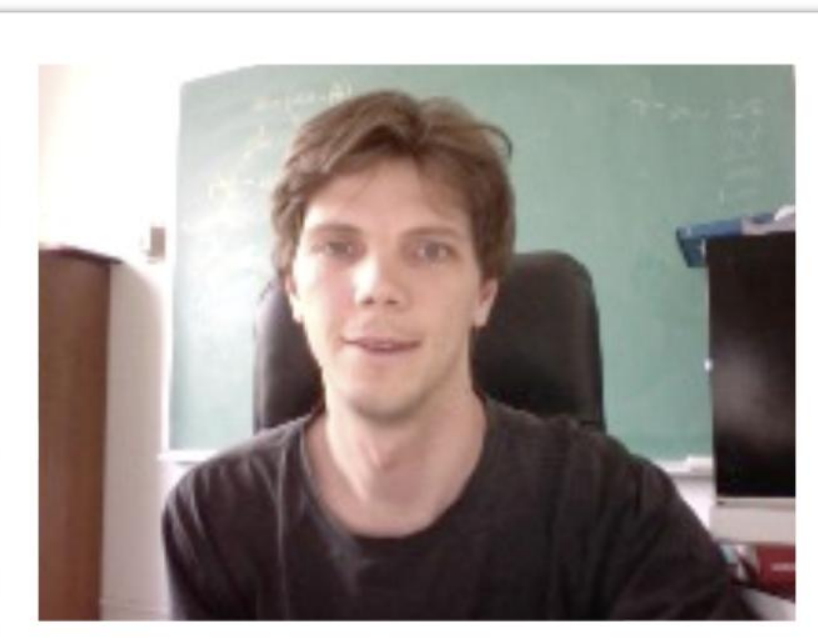

William Detmold

(William \& Mary /

JLab)

HWL

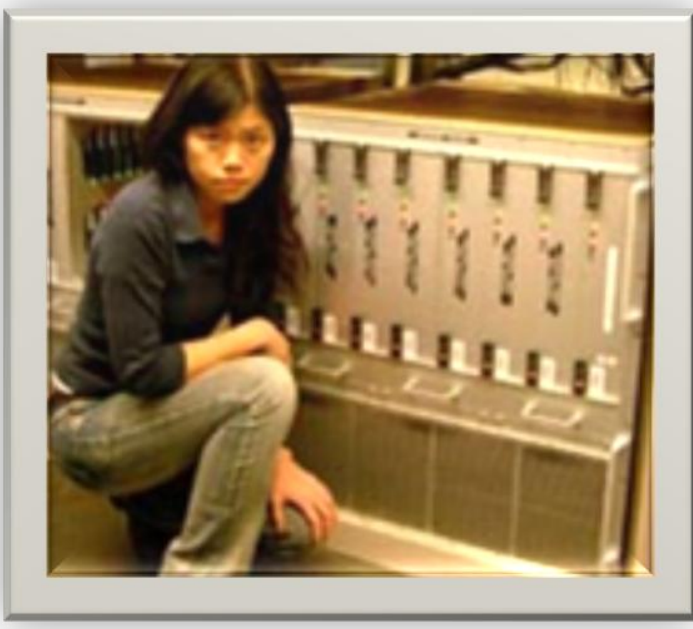

(U. of Washington)
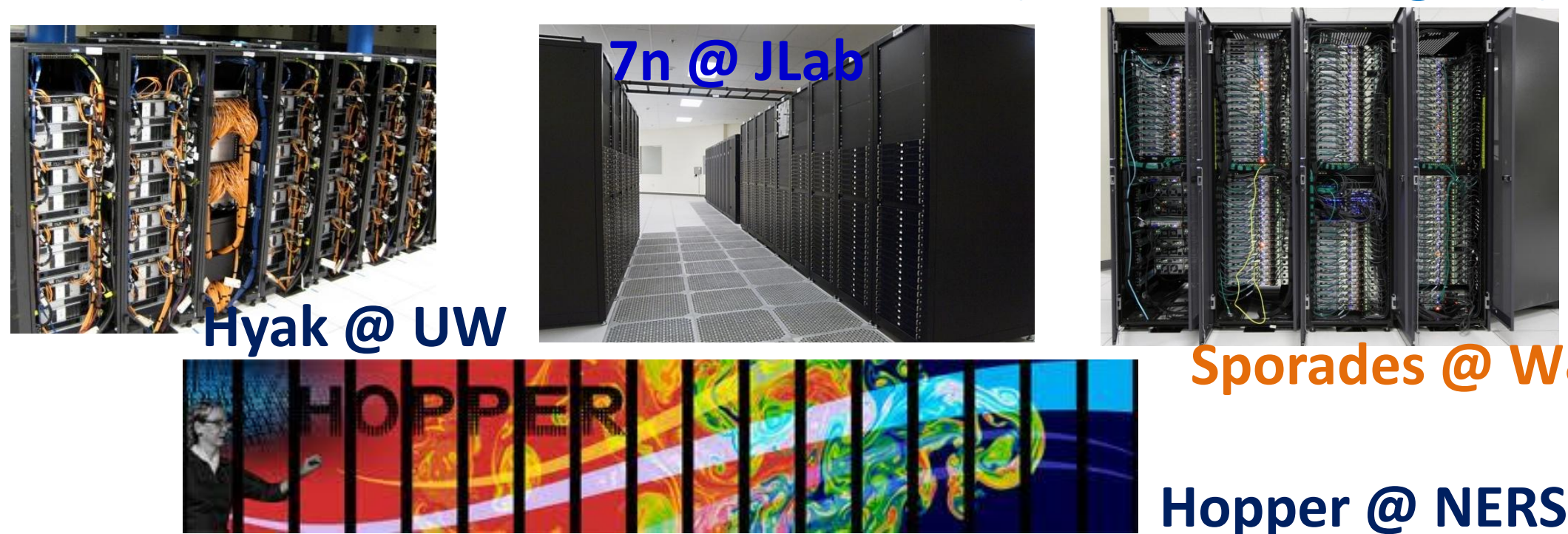

Sporades@W\&M

Hopper @ NERSC 


\section{Medium Modification}

\section{$\S$ Parton structure function in nuclear medium}

¿o The famous EMC effect

\& Significant deviations between heavy nuclei and deuterium

so Many models:

pion enhancement

nucleon expansion

multiquark clusters

rescaling

shadowing

local correlations $\ldots$

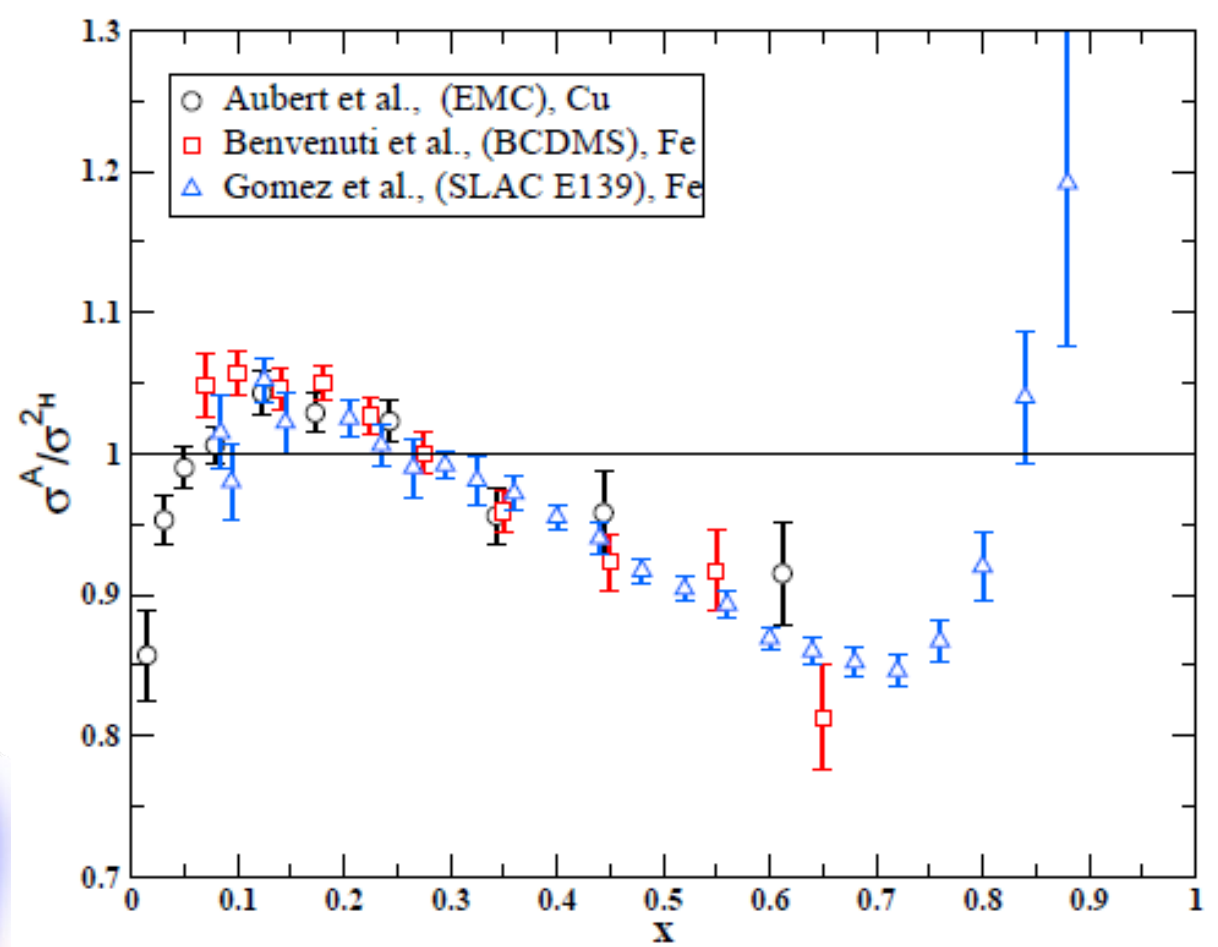

J. J. Aubert et al.

Phys. Lett. 123, 275 (1983)

$\S$ No universal understanding 


\section{Medium Modification}

$\S$ Not only significant for heavy nuclei, also important for light-nuclear systems

J. Seely et al., Phys. Rev. Lett. 103, 202301 (2009)

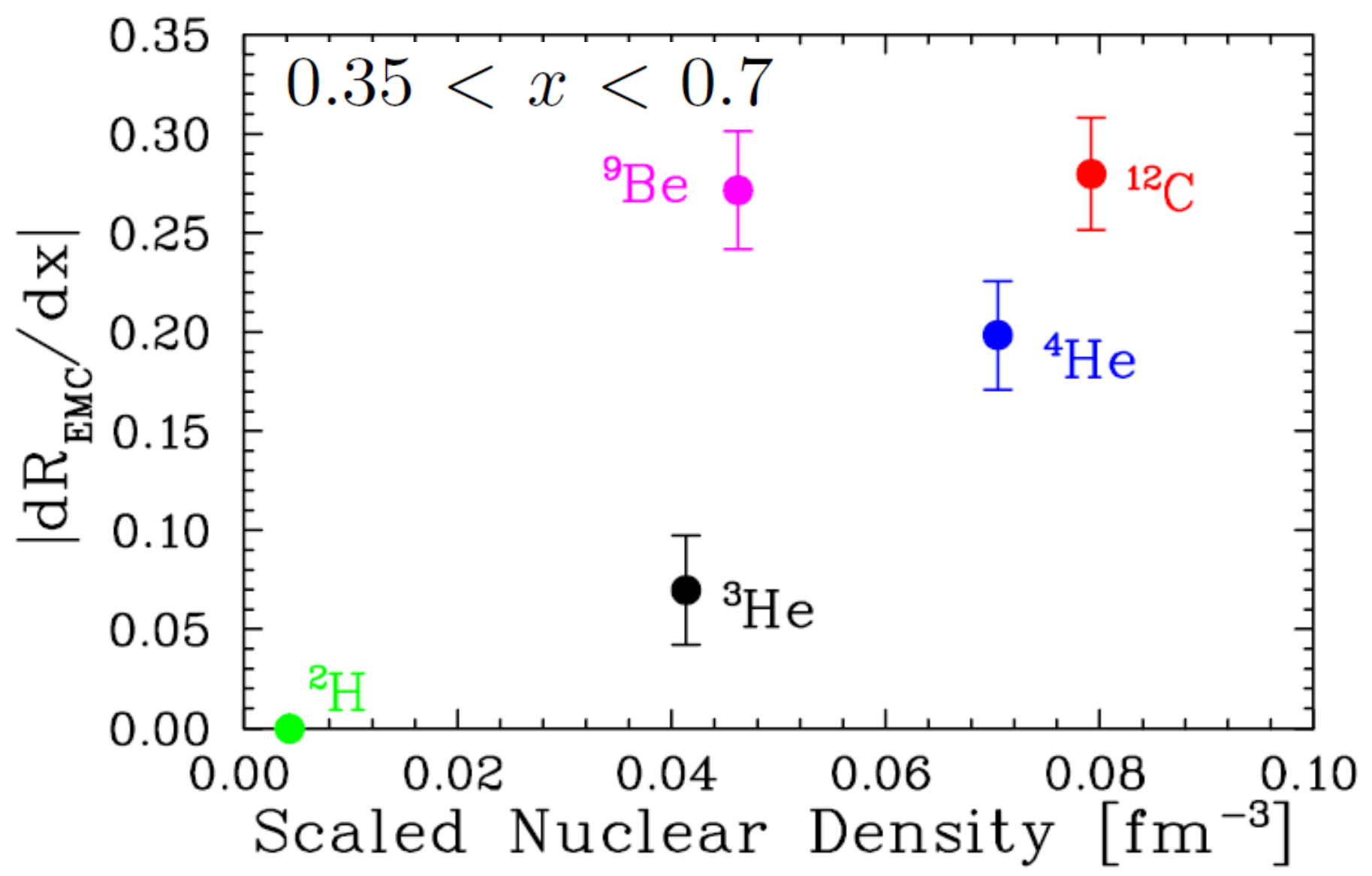




\section{Applications}

\section{$\S$ Important for tests of Standard Model}

$\S$ For example, NuTeV anomaly

๙ Weak mixing angle experiment

× 3 sigma away from $\mathrm{SM} \rightarrow$ "NuTeV anomaly"

G. P. Zeller et al.

Phys. Rev. Lett. 88, 091802 (2002)

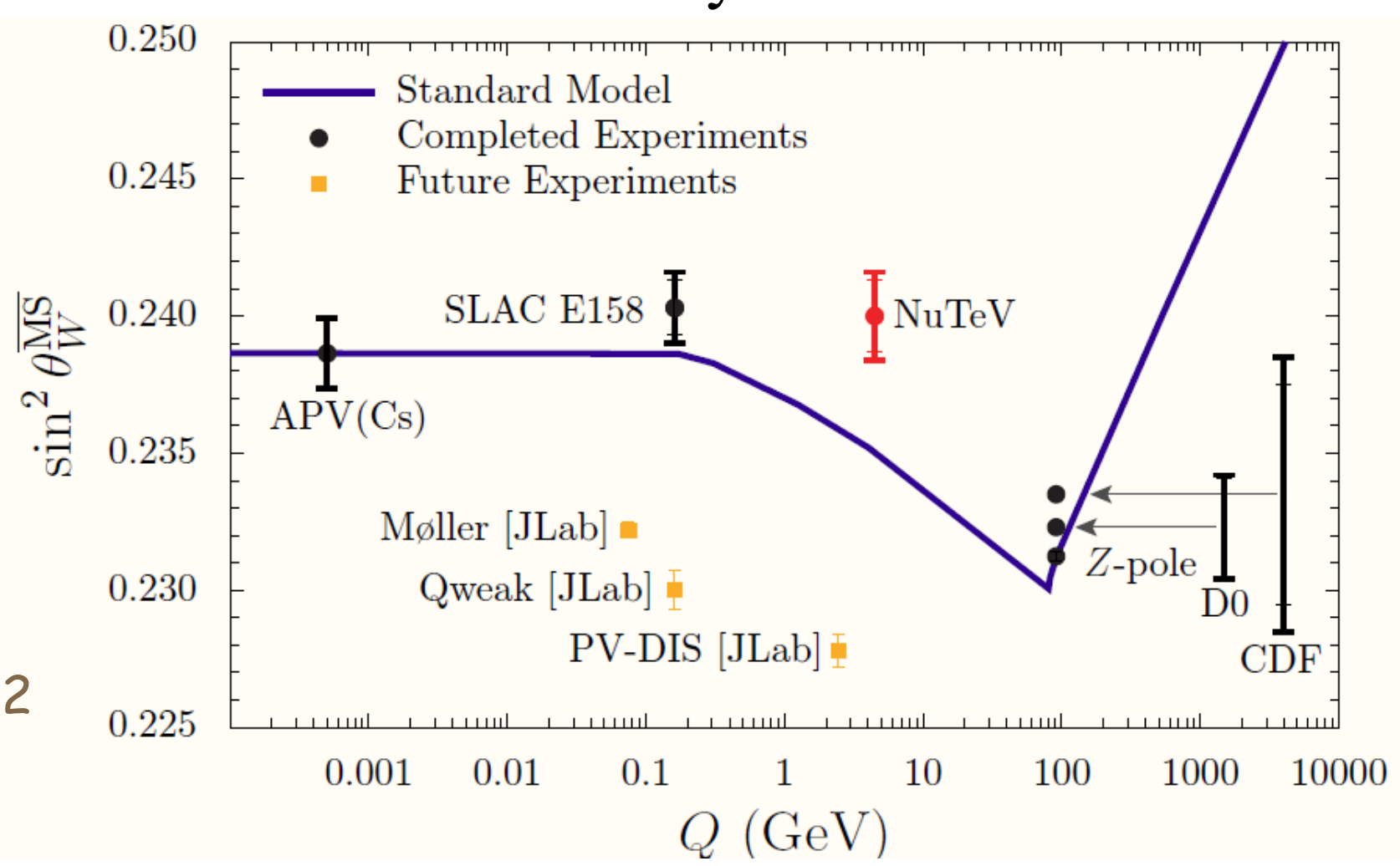




\section{Applications}

$\S$ Important for tests of Standard Model

$\S$ For example, NuTeV anomaly

s Weak mixing angle experiment

^ 3 sigma away from $\mathrm{SM} \rightarrow$ "NuTeV anomaly"

¿ Evidence for medium modification?

s Correction looks consistent with SM

I. Cloët, W. Bentz, A.
Phys. Lett. B693, 462 (2010)

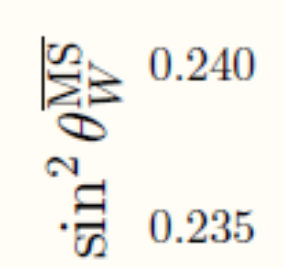

Thomas,

0.225

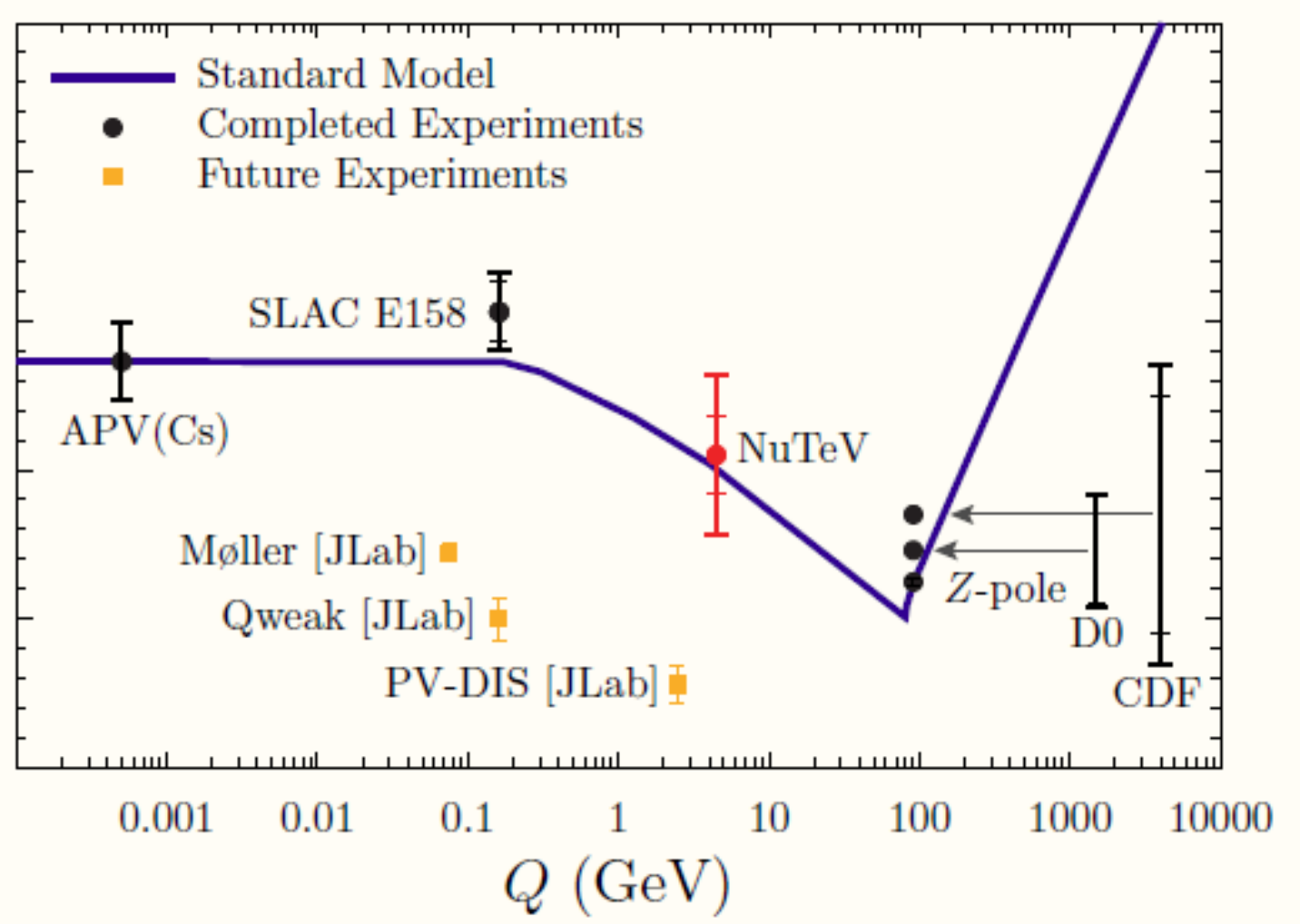




\section{Medium Modification}

$\S$ Interesting physics with multi-baryon systems

¿ Study with nonzero $\mu_{B}$ notorious "sign" problem in Monte Carlo calculation

2o Add nucleons to the system

complicated quark contraction and noise/signal issue

Number of contractions:

Signal Quality

$(A+Z) ! \times(2 A-Z)$ !

¿ Triton: $2880 \rightarrow 93$

${ }^{4} \mathrm{He}: 518400 \rightarrow 1107$

$\S$ Take a step back and look at \&o What you want:

What you get:
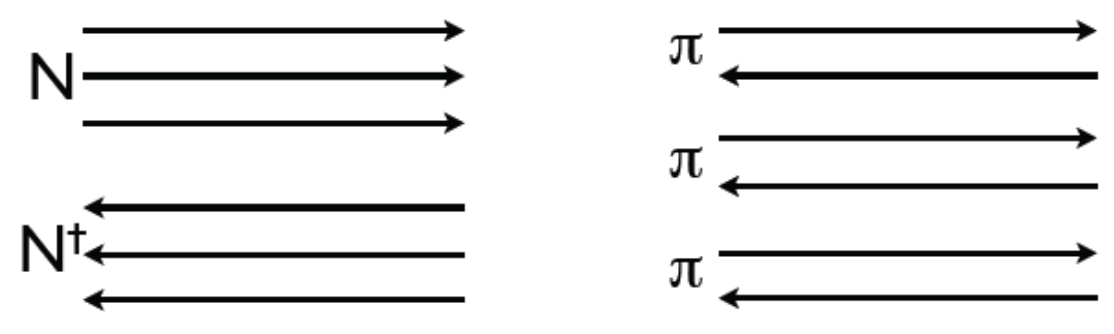

nonzero-isospin/multi-meson systems

\&o Many studies: "QCD at Finite Isospin Density", Son \& Stephanov

¿ Gain insight and experience for how to deal with nuclear systems 


\section{We do it all the time...}

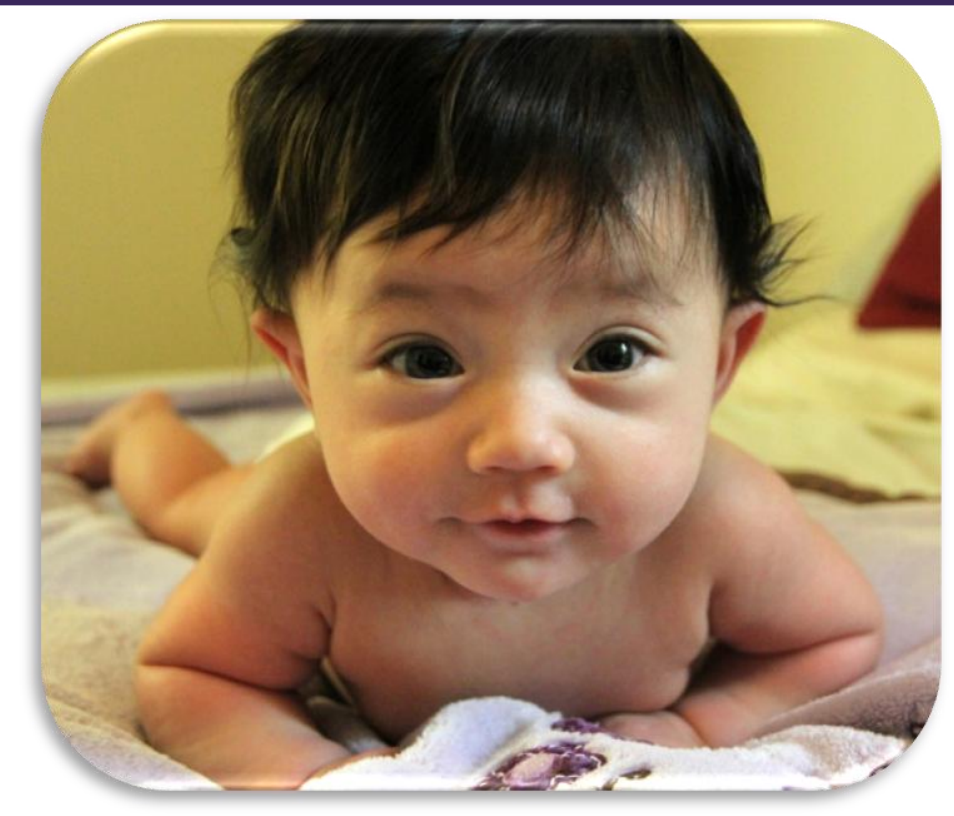




\section{We do it all the time...}

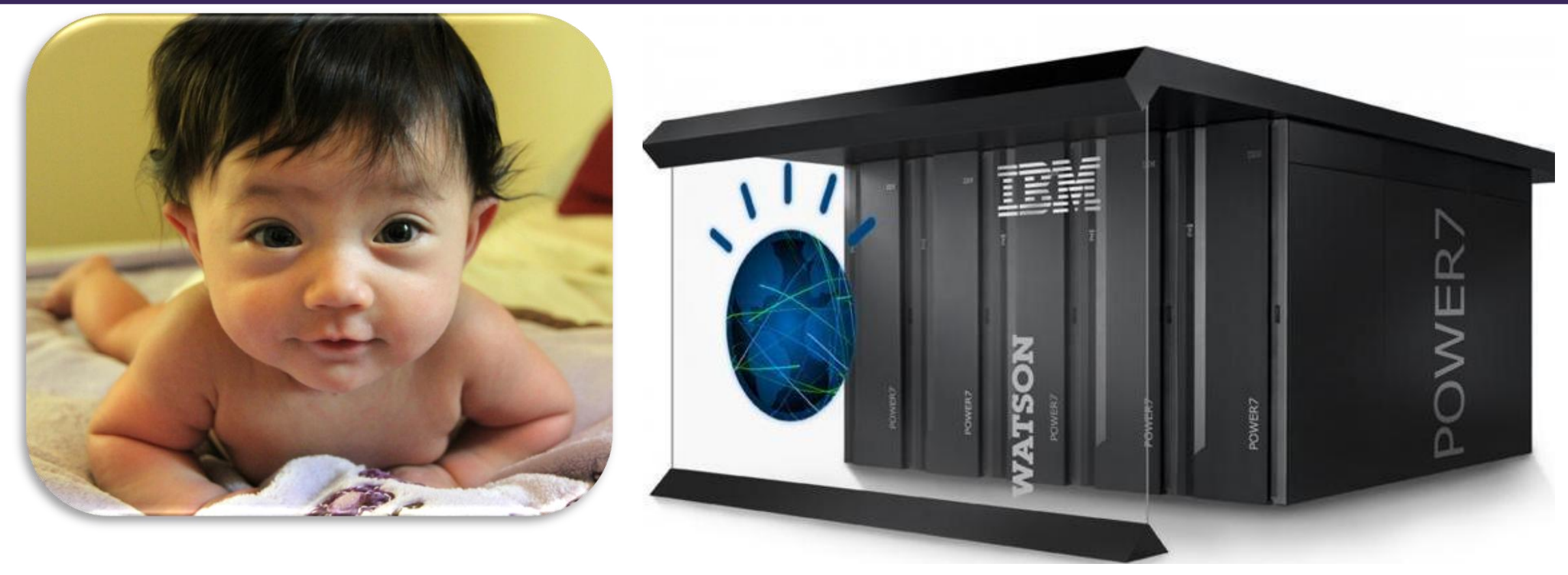




\section{We do it all the time...}

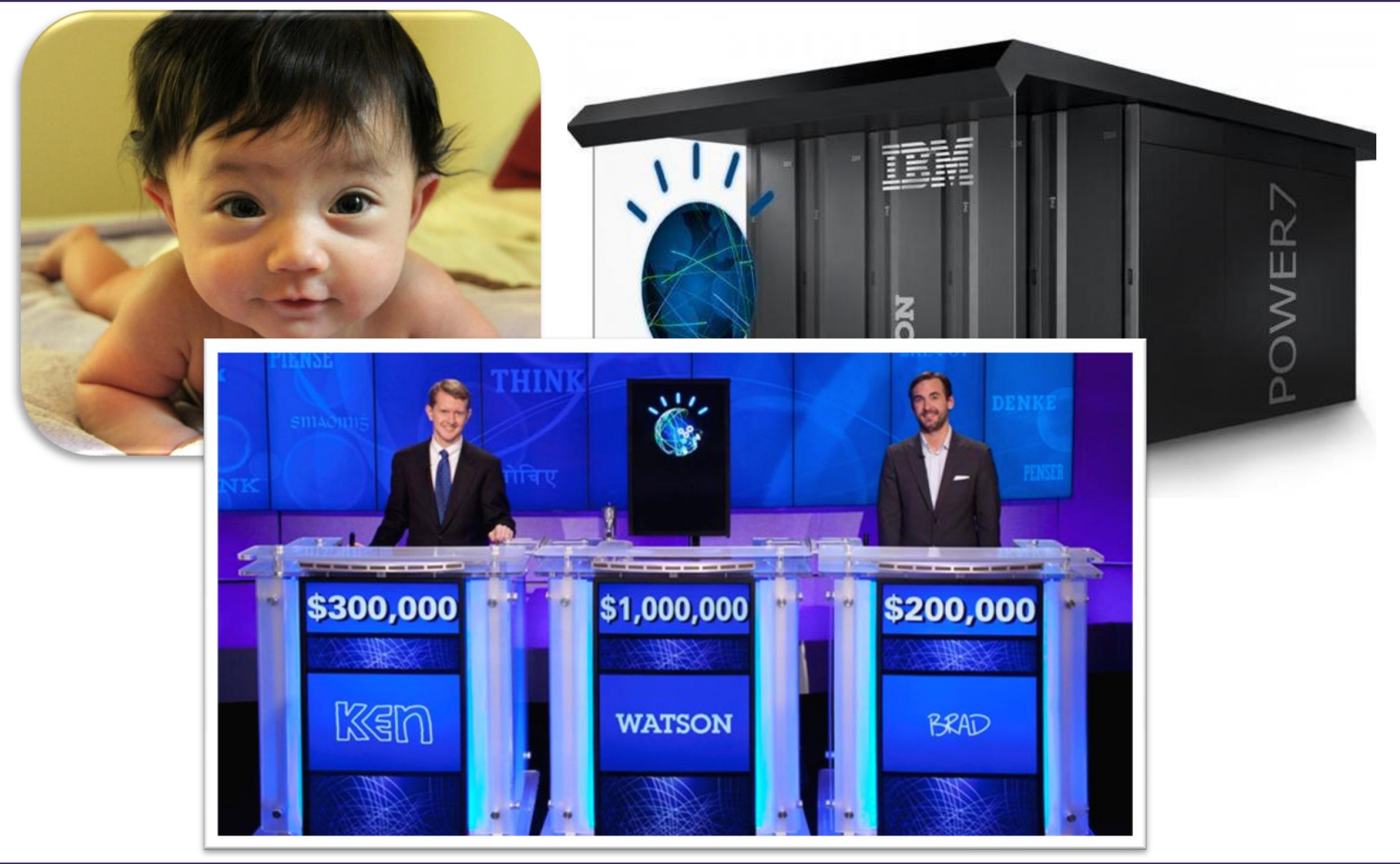




\section{$\mathcal{M} u l t i-\mathcal{M}$ eson System}

$\S$ First lattice $\pi-\pi$ calculation in '92

$\S$ Meson number $>2$ system is first studied on the lattice S. Beane et al., PRL 100: 082004 (2008)

$\S$ Followed by more complicated systems for

scattering length and 3-body interactions

W. Detmold et al., PRD 78: 014507 (2008); W. Detmold et al., PRD 78: 054514 (2008); W. Detmold et al., PRL 102: 032004 (2009); W. Detmold et al., 1103.4362 (2011)

$\S$ Effective theory

S. Beane, PRD 76: 074507 (2007); W. Detmold et al., PRD 77: 057502 (2008); Smigielski, PRD 79: 054506 (2009)

$\S$ Color-screening effects

W. Detmold et al., PRL 102: 032004 (2009)

$\S$ Study for $n \geq 13$ system

W. Detmold et al., PRD 82: 014511 (2010); Z. Shi and W. Detmold, PoS (2011) 


\section{Boring Details}

$\S$ The tool: Lattice gauge theory

$\S$ The actions

\section{a Domain-wall fermions on staggered sea}

OS Free forward propagators from LHPC and NPLQCD

os Chiral symmetry preserved at finite lattice spacing; good for spin physics and weak matrix elements

$\S$ The parameters

\begin{tabular}{ccccccc} 
Label & $a[\mathrm{fm}]$ & $L^{3} \times T$ & $m_{\pi}[\mathrm{MeV}]$ & $m_{\pi} L$ & $m_{\pi} T$ & Measurements \\
\hline $\mathrm{F}$ & 0.09 & $28^{3} \times 96$ & 320 & 4.1 & 14.0 & 432 \\
$\mathrm{C} 1$ & 0.12 & $20^{3} \times 64$ & 290 & 3.7 & 11.7 & 1450 \\
$\mathrm{C} 2$ & 0.12 & $20^{3} \times 64$ & 350 & 4.4 & 14.2 & 837 \\
$\mathrm{C} 3$ & 0.12 & $20^{3} \times 64$ & 490 & 6.2 & 19.9 & 1000 \\
\multicolumn{6}{l}{ C'S $^{3} \in\{16,20,24,28,32\}$} \\
F'
\end{tabular}




\section{$\pi^{+}$in Medium}

$\S$ We calculate

$$
C_{m}(t, \mathbf{p})=\left\langle 0\left|\left(\prod_{i=1}^{m} \sum_{\mathbf{x}} e^{i \mathbf{p}_{i} \cdot \mathbf{x}} \pi^{+}(\mathbf{x}, t)\right)\left(\pi^{-}\left(x_{0}\right)\right)^{m}\right| 0\right\rangle \quad \mathbf{p}=\sum_{i=1}^{m} \mathbf{p}_{i}
$$

$\S$ Contractions

a Examples from 3- $\pi^{+}$system

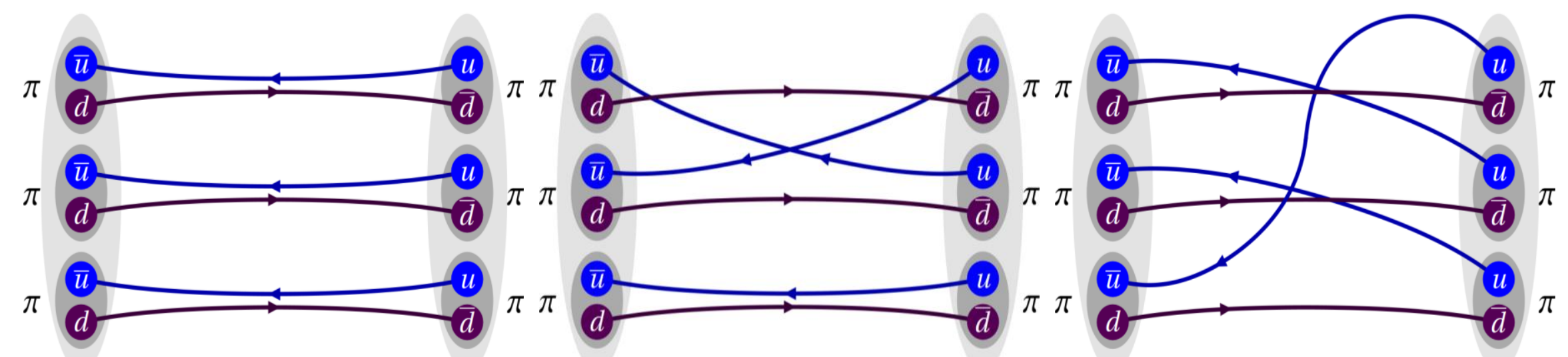




\section{$\pi^{+}$in Medium}

$\S$ We calculate

$$
C_{m}(t, \mathbf{p})=\left\langle 0\left|\left(\prod_{i=1}^{m} \sum_{\mathbf{x}} e^{i \mathbf{p}_{i} \cdot \mathbf{x}} \pi^{+}(\mathbf{x}, t)\right)\left(\pi^{-}\left(x_{0}\right)\right)^{m}\right| 0\right\rangle \quad \mathbf{p}=\sum_{i=1}^{m} \mathbf{p}_{i}
$$

$\S$ Contractions

$\curvearrowright$ Simplified with recursion relation

W. Detmold et al., Phys.Rev.D82:014511 (2010)

$$
\begin{aligned}
C_{3}(t) & =\operatorname{tr}[\Pi]^{3}-3 \operatorname{tr}[\Pi] \operatorname{tr}\left[\Pi^{2}\right]+2 \operatorname{tr}\left[\Pi^{3}\right] \\
\Pi & =\sum_{\mathbf{x}} \gamma_{5} S(\mathbf{x}, t ; 0) \gamma_{5} S^{\dagger}(\mathbf{x}, t ; 0)
\end{aligned}
$$

$\S$ Energy extraction from $n-\pi^{+}$system

$$
C_{m}(t, \mathbf{0}) \longrightarrow \sum_{\ell=0}^{m}\left(\begin{array}{c}
m \\
\ell
\end{array}\right) Z_{m}^{(\ell)} e^{-E_{m-\ell} t} e^{-E_{\ell}(T-t)}+\ldots
$$




\section{n- $\pi^{+}$Energy}

\section{$\S$ Fitted energies and effective-mass plot}

\section{a Examples}

$a=0.09 \mathrm{fm}$, $M_{\pi} \approx 320 \mathrm{MeV}$
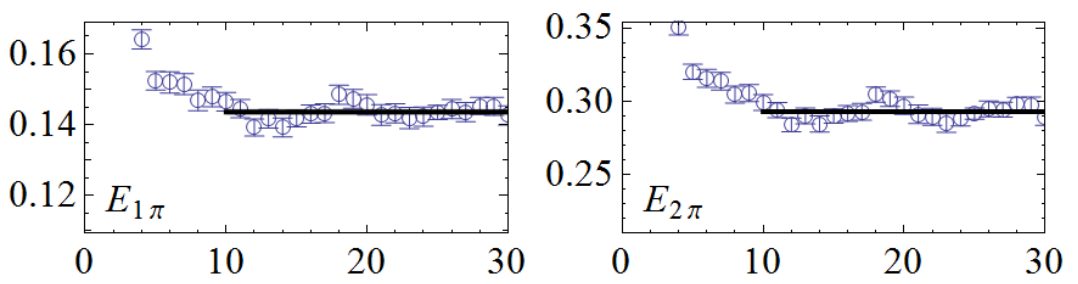

$\log (C(t) / C(t+1))$
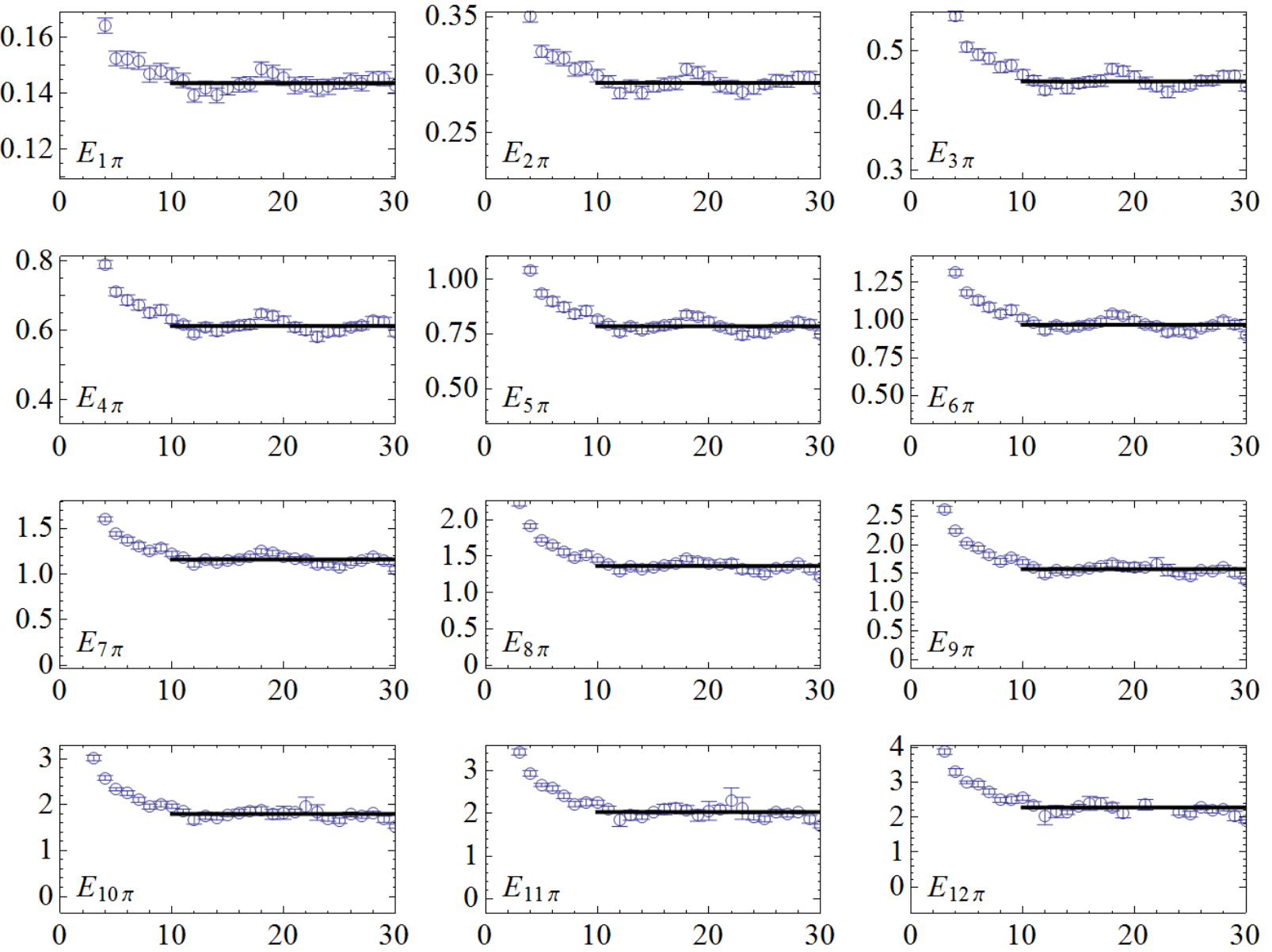


\section{$\mathcal{M}_{\pi}$ in Medium}

$\S$ How pion mass shifts in $K^{+}, \pi^{+}$medium

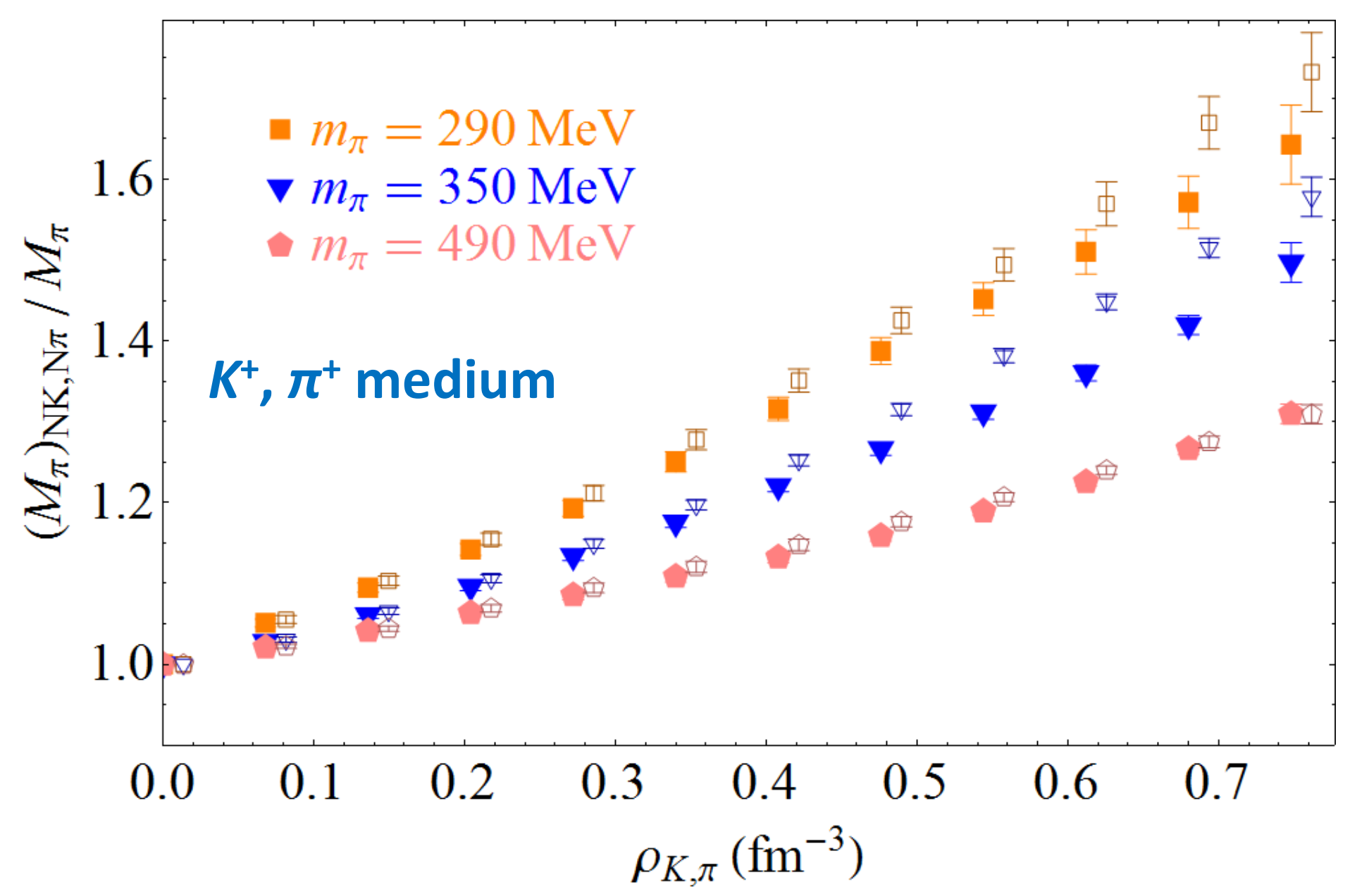




\section{Pion Structure Function}

$\S$ We calculate

$$
\begin{gathered}
C_{m}^{(n)}(\tau, t, \mathbf{p})=\left\langle 0\left|\left(\prod_{i=1}^{m} \sum_{\mathbf{x}} e^{i \mathbf{p}_{i}: \mathbf{x}} \pi^{+}(\mathbf{x}, t)\right)\left(\sum_{\mathbf{y}} O_{\left\{\mu_{0} \mu_{1} \ldots \mu_{n}\right\}}(\mathbf{y}, \tau)\right)\left(\pi^{-}\left(x_{0}\right)\right)^{m}\right| 0\right\rangle \\
\text { where } O_{\left\{\mu_{0} \mu_{1} \ldots \mu_{n}\right\}}=\left(\frac{i}{2}\right)^{n} \bar{\psi} \gamma_{\mu_{0}} \overleftrightarrow{D}_{\mu_{1}} \ldots \overleftrightarrow{D}_{\mu_{n}} \psi-\text { trace }
\end{gathered}
$$

\section{$\S$ Contractions}

¿ Examples from 3- $\pi^{+}$system
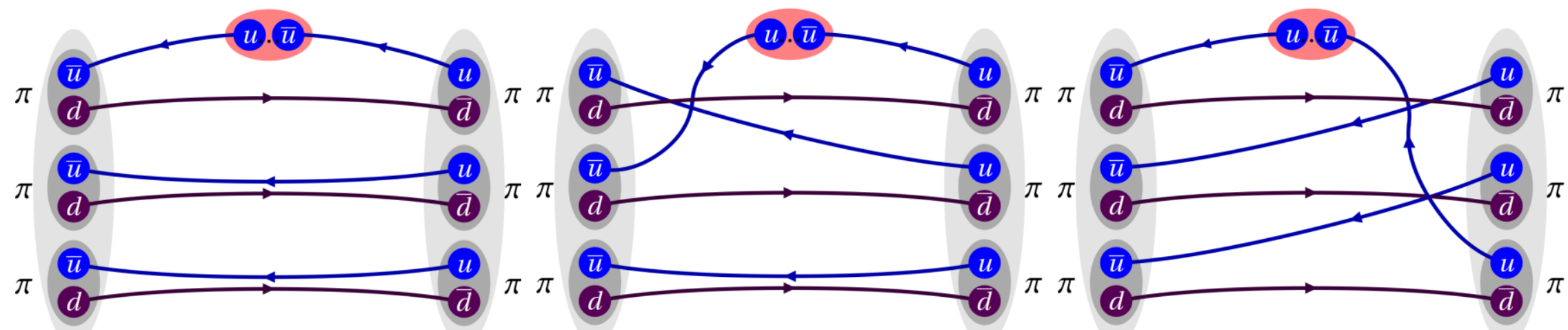


\section{Pion Structure Function}

$\S$ We calculate

$$
\begin{gathered}
C_{m}^{(n)}(\tau, t, \mathbf{p})=\left\langle 0\left|\left(\prod_{i=1}^{m} \sum_{\mathbf{x}} e^{i \mathbf{p}_{i} \cdot \mathbf{x}} \pi^{+}(\mathbf{x}, t)\right)\left(\sum_{\mathbf{y}} O_{\left\{\mu_{0} \mu_{1} \ldots \mu_{n}\right\}}(\mathbf{y}, \tau)\right)\left(\pi^{-}\left(x_{0}\right)\right)^{m}\right| 0\right\rangle \\
\text { where } O_{\left\{\mu_{0} \mu_{1} \ldots \mu_{n}\right\}}=\left(\frac{i}{2}\right)^{n} \bar{\psi} \gamma_{\mu_{0}} \overleftrightarrow{D}_{\mu_{1}} \ldots \overleftrightarrow{D}_{\mu_{n}} \psi-\text { trace }
\end{gathered}
$$

$\S$ Wick contractions

¿ Use mixed-meson recursion relations

W. Detmold, B. Smigielski, Phys.Rev.D84:014508 (2011)

$$
\begin{aligned}
& \Pi=\sum_{\mathbf{x}} \gamma_{5} S(\mathbf{x}, t ; 0) \gamma_{5} S^{\dagger}(\mathbf{x}, t ; 0) \\
& \tilde{\Pi}=\sum_{\mathbf{x}, \mathbf{y}} \gamma_{5} S(\mathbf{x}, t ; \mathbf{y}, \tau) O S(y, \tau ; 0) \gamma_{5} S^{\dagger}(\mathbf{x}, t ; 0) \Rightarrow \begin{array}{l}
\text { Treated as diff. } \\
\text { meson spices }
\end{array}
\end{aligned}
$$




\section{Pion Structure Function}

$\S$ We calculate

$$
\begin{gathered}
C_{m}^{(n)}(\tau, t, \mathbf{p})=\left\langle 0\left|\left(\prod_{i=1}^{m} \sum_{\mathbf{x}} e^{i \mathbf{p}_{i} \mathbf{x}} \pi^{+}(\mathbf{x}, t)\right)\left(\sum_{\mathbf{y}} O_{\left\{\mu_{0} \mu_{1} \ldots \mu_{n}\right\}}(\mathbf{y}, \tau)\right)\left(\pi^{-}\left(x_{0}\right)\right)^{m}\right| 0\right\rangle \\
\text { where } O_{\left\{\mu_{0} \mu_{1} \ldots \mu_{n}\right\}}=\left(\frac{i}{2}\right)^{n} \bar{\psi} \gamma_{\mu_{0}} \overleftrightarrow{D}_{\mu_{1}} \ldots \overleftrightarrow{D}_{\mu_{n}} \psi-\text { trace }
\end{gathered}
$$

$\S$ Wick contractions

¿ Examples from $n-\pi^{+}$system

$$
\begin{gathered}
C_{3}^{(n)}=-2 \operatorname{tr}[\Pi] \operatorname{tr}[\tilde{\Pi} \Pi]+2 \operatorname{tr}\left[\tilde{\Pi} \Pi^{2}\right]+\operatorname{tr}[\Pi]^{2} \operatorname{tr}[\tilde{\Pi}]-\operatorname{tr}\left[\Pi^{2}\right] \operatorname{tr}[\tilde{\Pi}] \\
\Pi=\sum_{\mathbf{x}} \gamma_{5} S(\mathbf{x}, t ; 0) \gamma_{5} S^{\dagger}(\mathbf{x}, t ; 0), \tilde{\Pi}=\sum_{\mathbf{x}, \mathbf{y}} \gamma_{5} S(\mathbf{x}, t ; \mathbf{y}, \tau) O S(y, \tau ; 0) \gamma_{5} S^{\dagger}(\mathbf{x}, t ; 0)
\end{gathered}
$$

$\S$ Matrix elements extraction (naively)

$$
C_{m}^{(n)}(\tau, t, \mathbf{p}) \stackrel{t \gg \tau \gg 0}{\longrightarrow} A e^{-E_{n} t}\langle n \pi|\mathcal{O}| n \pi\rangle+\ldots
$$




\section{Pion Momentum Fraction}

$\S\langle x\rangle^{\pi, N} /\langle x\rangle^{\pi, 0}$ without thermal-state degrees of freedom $t_{\text {sep }}=T / 2$

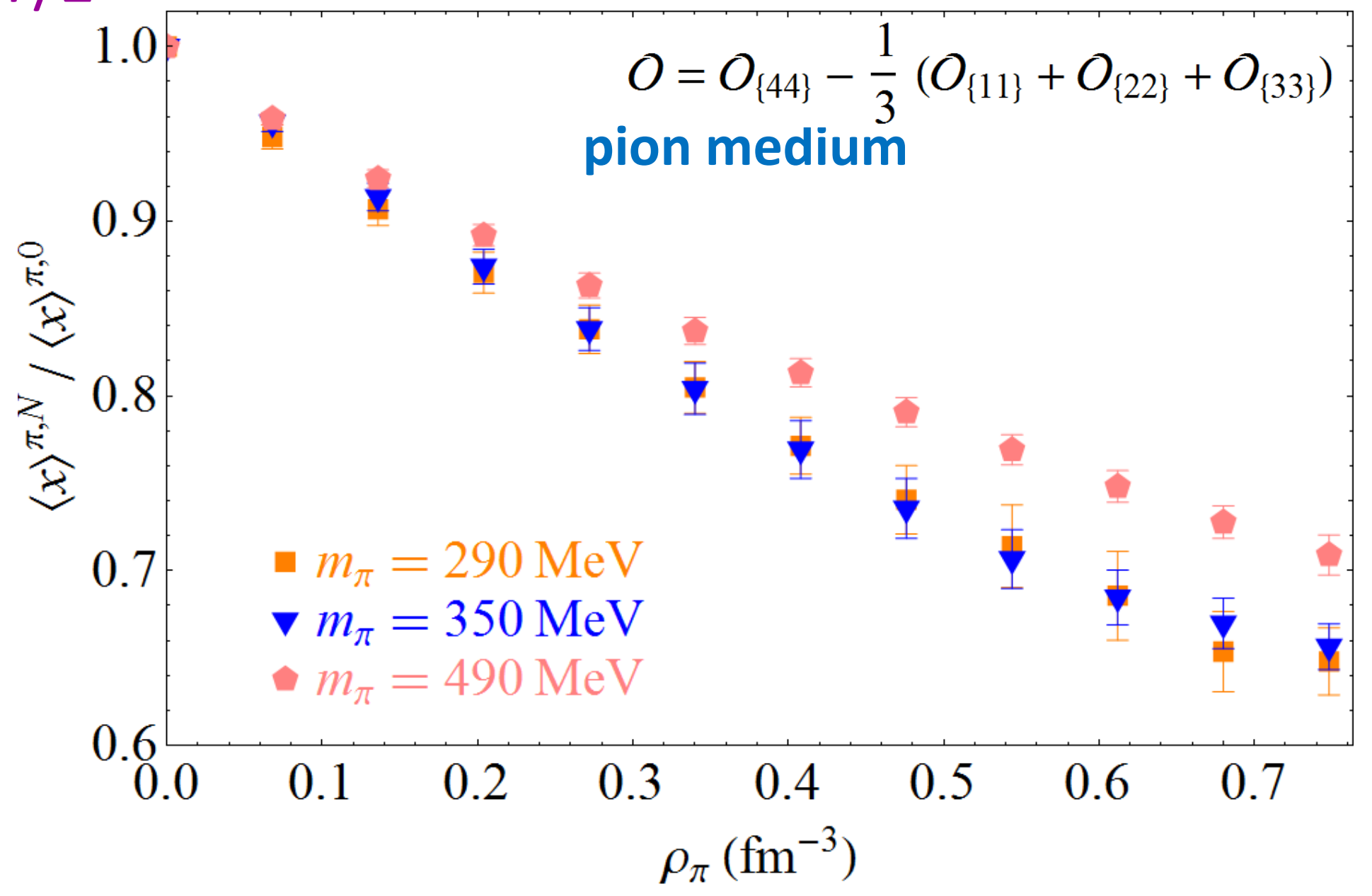




\section{Thermal Contamination}

$\S$ Significant for $n-\pi^{+}$system through amplitudes

$\S$ For example, $a=0.12 \mathrm{fm}$ ensemble, $T=64$

$$
a=0.12 \mathrm{fm}, M_{\pi}=290 \mathrm{MeV}, M_{\pi} T=11.6 \quad a=0.12 \mathrm{fm}, M_{\pi}=490 \mathrm{MeV}, M_{\pi} T=19.9
$$
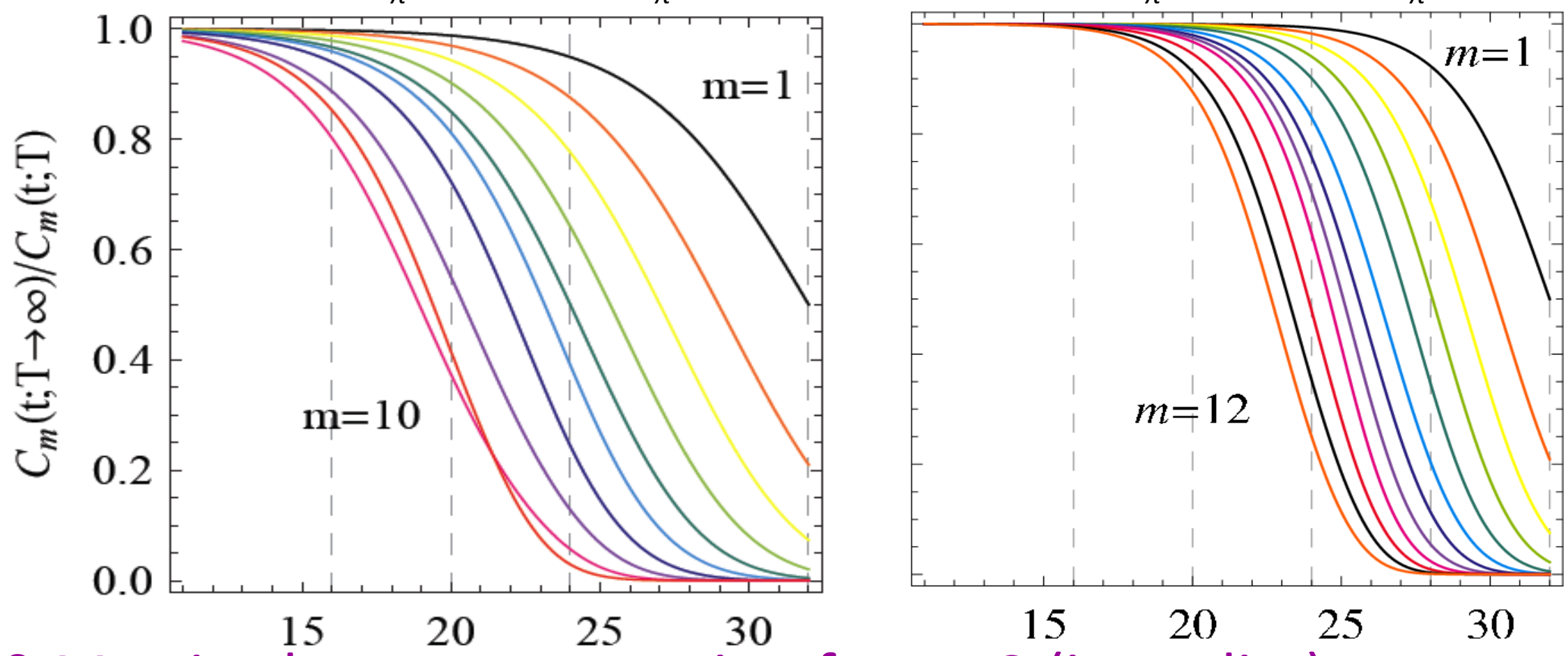

$\S$ Matrix elements extraction for $\boldsymbol{p}=\mathbf{0}$ (in reality)

$$
C_{m}^{(n)}(\tau, t, 0) \longrightarrow \sum_{\ell=0}^{m}\left(\begin{array}{c}
m \\
\ell
\end{array}\right) Z_{m}^{(\ell)}\left\langle O_{m-\ell}^{(n)}\right\rangle e^{-E_{m-\ell} t} e^{-E_{\ell}(T-t)}+\ldots
$$




\section{Pion Momentum Fraction}

$\S\langle x\rangle^{\pi, N} /\langle x\rangle^{\pi, 0}$ with thermal-state degrees of freedom multiple $t_{\text {sep }}$ used

$$
O=O_{\{44\}}-\frac{1}{3}\left(O_{\{11\}}+O_{\{22\}}+O_{\{33\}}\right)
$$

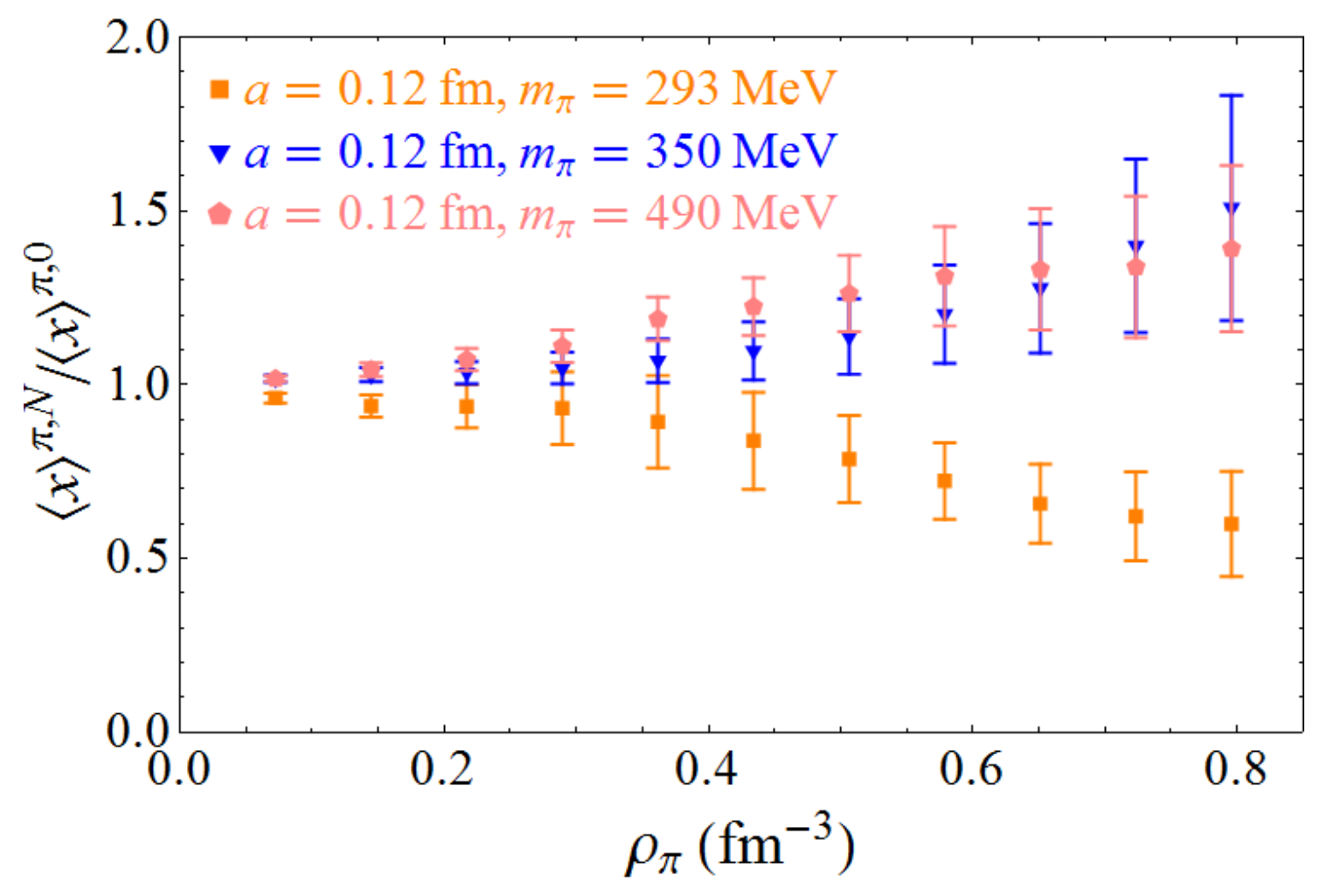




\section{Pion Momentum Fraction}

$\S\langle x\rangle^{\pi, N} /\langle x\rangle^{\pi, 0}$ with thermal-state degrees of freedom multiple $t_{\text {sep }}$ used

$$
O=O_{\{44\}}-\frac{1}{3}\left(O_{\{11\}}+O_{\{22\}}+O_{\{33\}}\right)
$$

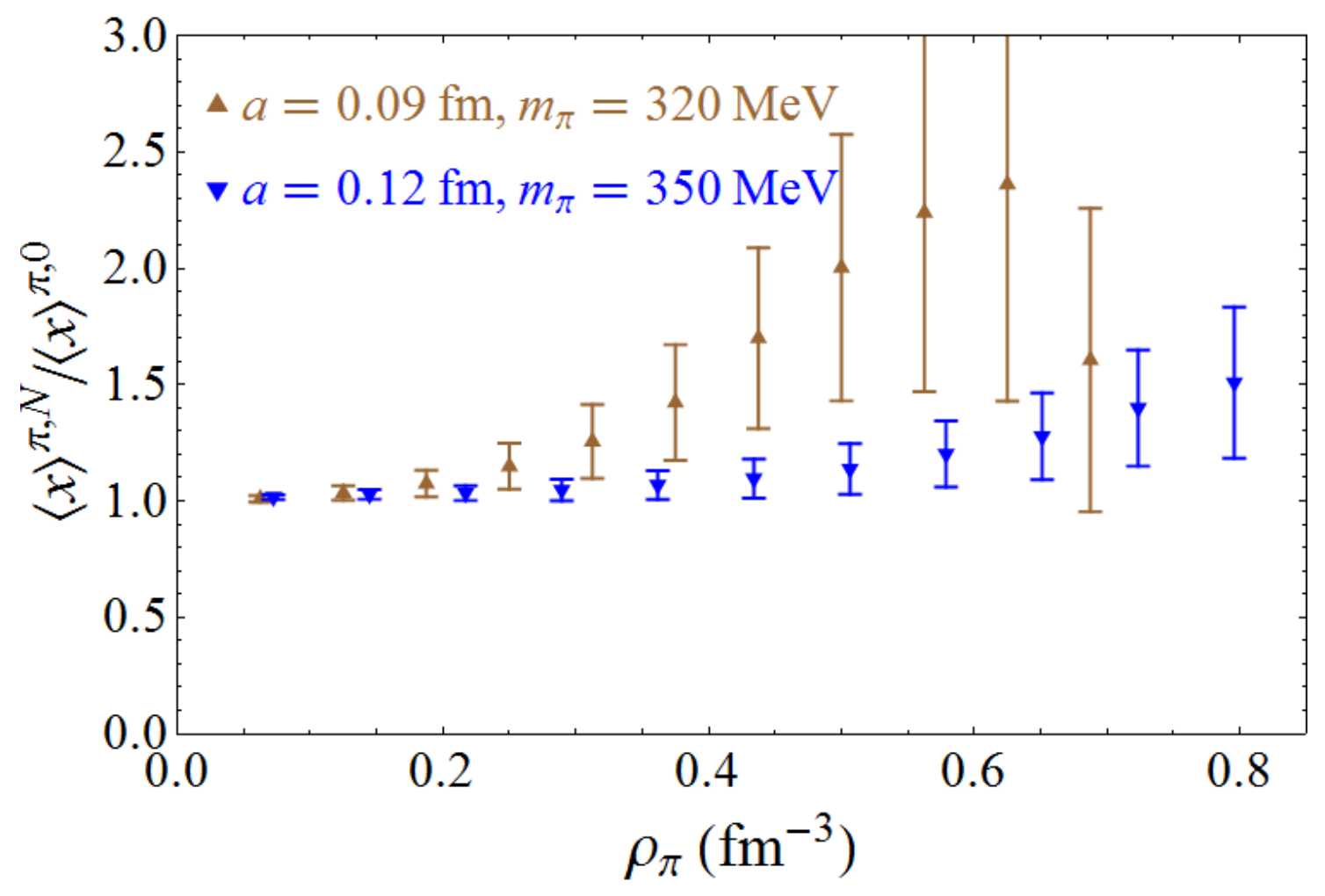




\section{Summary and Outlook}

$\S$ First lattice-QCD attempt to measure EMC effects $\curvearrowright$ Pion momentum fraction in pion medium $\curvearrowright_{0}$ With $m_{\pi} \approx 290-490 \mathrm{MeV}, 2$ lattice spacings

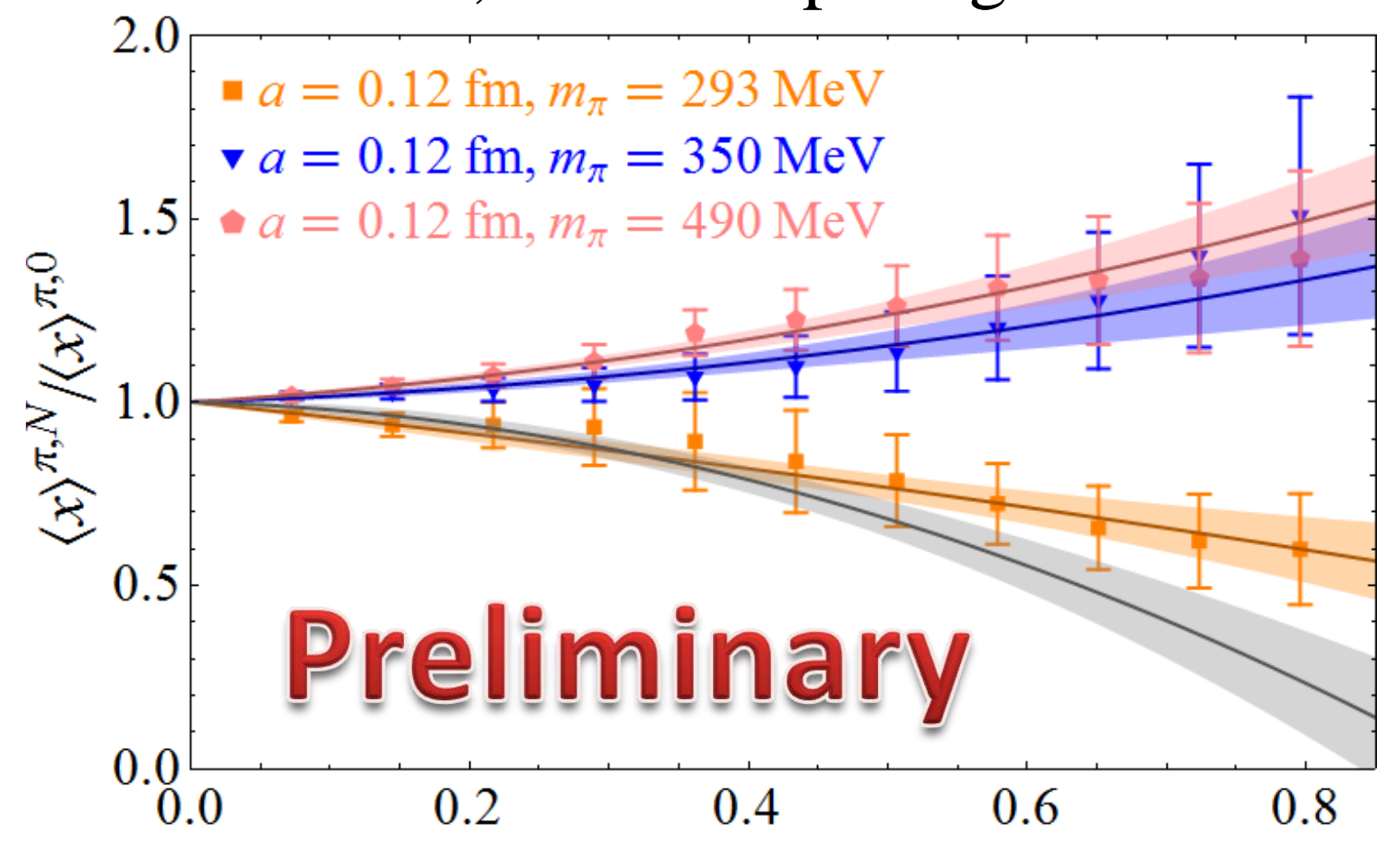

New Horizons $\rho_{\pi}\left(\mathrm{fm}^{-3}\right)$

$\S$ Nucleon structure in meson and light-nuclear media 


\title{
Many-Flavor Domain Wall Fermions and Fixed Topology
}

\author{
Meifeng Lin
}

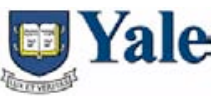

New Horizons for Lattice Computations with Chiral Fermions BNL, May 14 - 16, 2012 


\title{
Outline
}

\author{
Motivation
}

LSD Calculations with Domain Wall Fermions

Recap: Results for $N_{f}=2,6$

New: Results for $N_{f}=10$

\section{Summary}




\section{Motivation}

- $\mathrm{SU}(\mathrm{N})$ gauge theories with many flavors of fermions may exhibit behaviors that are fundamentally different from QCD.

- Increasing $N_{f}$ causes the gauge coupling to run slowly, and "walking" may occur, which could lead to some properties desirable for Technicolor model building.

- Specifically, we are interested in

- enhanced chiral condensate

- small $S$ parameter

- new pattern in hadron spectrum

- When $N_{f}>N_{f}^{c}$, the theory becomes conformal, and spectrum vanishes at the chiral limit. Also on our wishlist is to

- locate $N_{f}^{c}$ non-perturbatively 


\section{The Lattice Strong Dynamics Collaboration}

Tom Appelquist Yale U.

Ron Babich NVIDIA

Rich Brower Boston U.

Michael Buchoff LLNL

Michael Cheng Boston U.

Mike Clark NVIDIA

Saul Cohen U. Washington

George Fleming Yale U.

Joe Kiskis U. of Carlifornia, Davis

Meifeng Lin Yale U.

\author{
Heechang $\mathrm{Na}$ ANL \\ Ethan Neil Fermilab \\ James Osborn ANL \\ Claudio Rebbi Boston U. \\ David Schaich Boston U. \\ Chris Schroeder LLNL \\ Sergey Syritsyn LBL \\ Joe Wasem LLNL \\ Oliver Witzel Boston U. \\ Gennady Voronov Yale U. \\ Pavlos Vranas LLNL
}




\section{Gauge Ensembles}

- Fermion action: domain wall fermions.

- Gauge action: Iwasaki.

- Ensemble size: Typically 1200 MD trajectories.

[Cost grows as $N_{f}^{3 / 2}$ ]

\begin{tabular}{|c|c|c|c|c|c|}
\hline \hline$N_{f}$ & Volume & $L_{s}$ & $\beta$ & $a m_{f}$ & $a m_{\text {res }}$ \\
\hline 2 & $32^{3} \times 64$ & 16 & 2.70 & $0.005 \ldots 0.030$ & $2.5 \times 10^{-5}$ \\
\hline 6 & $32^{3} \times 64$ & 16 & 2.10 & $0.005 \ldots 0.030$ & $8.2 \times 10^{-4}$ \\
\hline 10 & $32^{3} \times 64$ & 16 & 1.95 & $0.005 \ldots 0.030$ & $1.7 \times 10^{-3}$ \\
\hline \hline
\end{tabular}

- For $N_{f}=2, a M_{V} \approx 0.21 \Rightarrow a^{-1} \approx 3.7 \mathrm{GeV}$.

- For $N_{f}=6$ and 10 , match $a M_{V}$ to within $10 \%$ of $N_{f}=2$. 


\section{Condensate Enhancement}

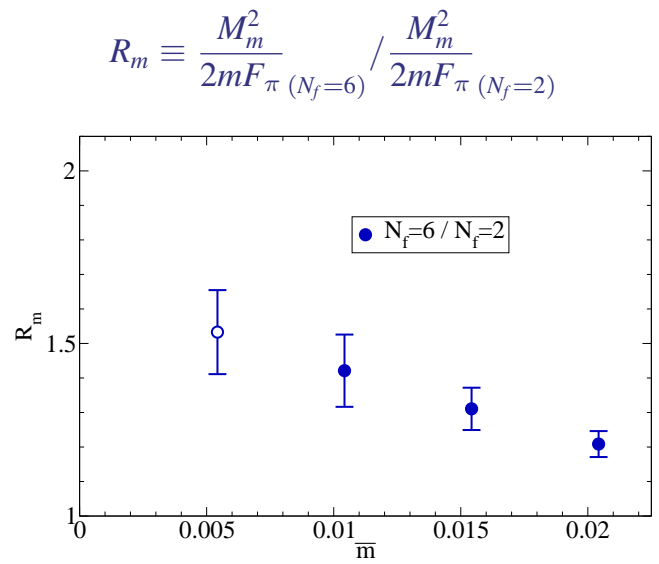

T. Appelquist et al. (LSD Collaboration), PRL104:071601,2010 


\section{The S Parameter}

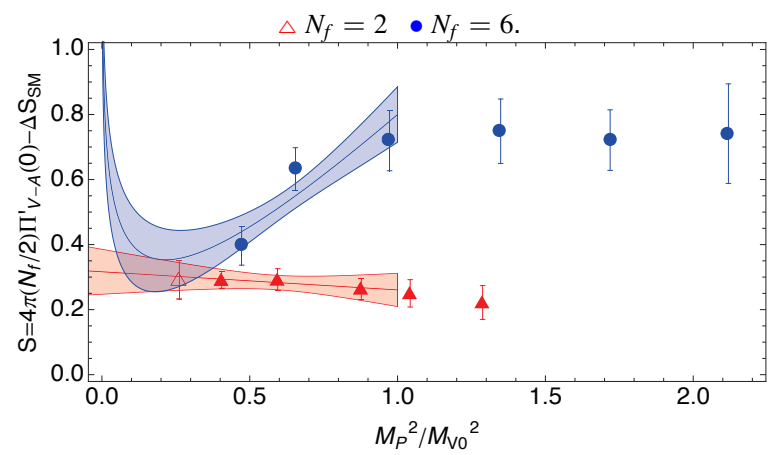

T. Appelquist et al. (LSD Collaboration), PRL 106 (2011) 231601 


\section{A New Observation for $N_{f}=10$}

- Two independent ensembles for each quark mass, with ordered and disordered start.

- The ensembles thermalize to different values

- NOT observed for $N_{f}=2$ or 6 .
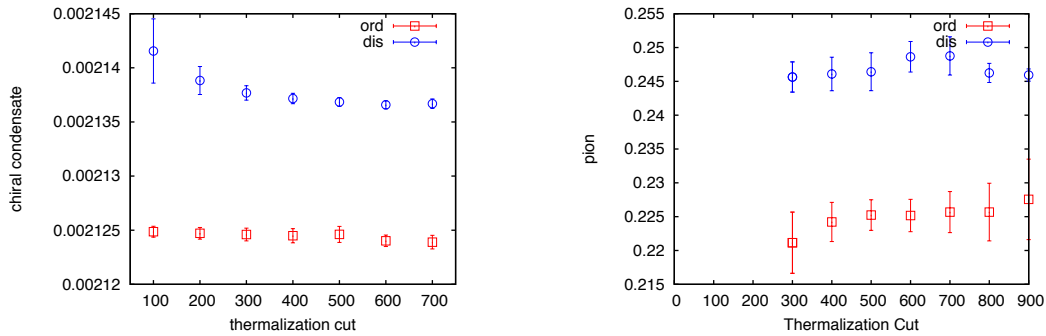


\section{Starting-Config. Dependence}

Statistically significant difference between the ordered-start and disordered-start ensembles are observed for almost all the ensembles.
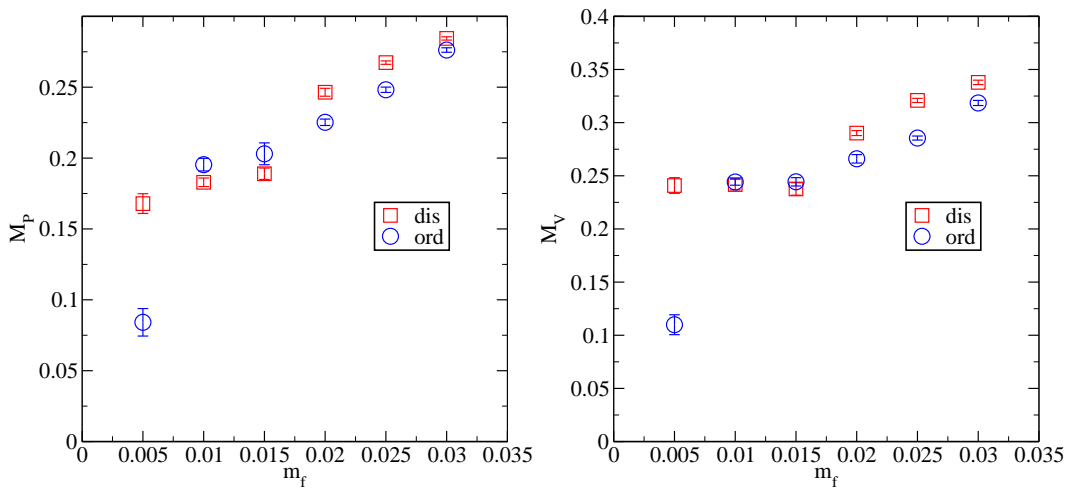


\section{Slowly-moving/Fixed Topology}

\begin{tabular}{c|c|c|c}
\hline \hline$m_{f}$ & start & trajectory range & $Q$ \\
\hline 0.005 & $\mathrm{O}$ & $500-1319$ & 0 \\
0.005 & $\mathrm{D}$ & $500-919$ & 3 \\
\hline 0.010 & $\mathrm{O}$ & $500-1373$ & 0 \\
0.010 & $\mathrm{D}$ & $500-1259$ & 1 \\
\hline 0.015 & $\mathrm{O}$ & $500-1204$ & 0 \\
0.015 & $\mathrm{D}$ & $500-1450$ & -1 \\
\hline 0.020 & $\mathrm{O}$ & $500-1239$ & 0 \\
0.020 & $\mathrm{D}$ & $500-1221$ & 12 \\
\hline 0.025 & $\mathrm{O}$ & $500-1256$ & 0 \\
0.025 & $\mathrm{D}$ & $500-609$ & -19 \\
& & $610-942$ & -18 \\
& & $943-1095$ & -17 \\
& & $1096-1097$ & -16 \\
& & $1098-1382$ & -17 \\
& & $1383-1484$ & -16 \\
\hline 0.030 & $\mathrm{O}$ & $500-1415$ & 0 \\
0.030 & $\mathrm{D}$ & $500-1227$ & 9 \\
\hline \hline
\end{tabular}




\section{Effects of Fixed Topology}

- The slight topological tunneling at $m_{f}=0.025$ (D) allows us to analyze the data for different topological sectors separately.

- Given the limited number of measurements for each topology, we only block every trajectory, hence the errors will be underestimated.

- Nevertheless, such analysis will give us a crude idea how much the fixed topology may affect our results.

R. Brower, S. Chandrasekharan, John W. Negele, and U.J. Wiese. 2003 has the following formula for the $Q$-dependence

$$
M_{Q}=M(0)+\frac{1}{2} M^{(2)}(0) \frac{1}{V \chi_{t}}\left(1-\frac{Q^{2}}{V \chi_{t}}\right)+O\left(\frac{1}{V^{3}}\right) .
$$

$\chi_{t}$ is the topological susceptibility, $M^{(2)}(0)$ is the second derivative with respect to $\theta$ at $\theta=0$, and $M(0)$ is the mass at $\theta=0$ (or equivalently, at non-fixed $Q$ ). 


\section{Observed $Q$-Dependence}
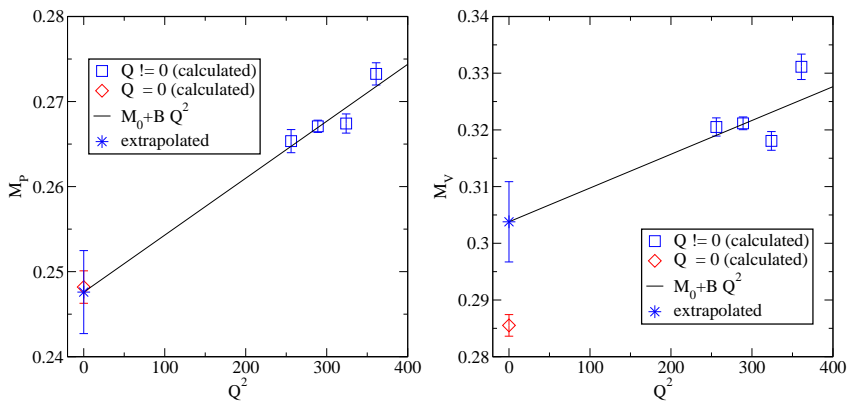

- The discrepancy between ordered and disordered ensembles can be described by the dependence on the fixed topology.

- To get results at $\theta=0$, we still need to know the topological susceptibility $\rightarrow$ work in progress.

- Large discrepancies $\Rightarrow$ small $\chi_{t} ? V$ too small? 


\section{Combining Datasets}

Lacking the results for the topological susceptibility, can we still combine the two ensembles and obtain some best estimates of the "true" value, $M(0)$ ?

- From our fits, $M^{(2)}(0)$ is negative, so $M(0)$ is between the results for $Q=0$ and $Q \neq 0$.

- Take $\bar{M}=\left(M_{0}+M_{Q}\right) / 2$ as the central value.

- Take the largest relative difference between $M_{Q}$ and $M_{0}$ as systematic error.

- Combine statistical and systematic errors as final error. 


\section{Testing Conformality - Qualitatively}

In a conformal theory with a small finite mass,

$$
M_{X}=C_{X} m^{1 /\left(1+\gamma^{*}\right)}
$$

so the vector-to-pseudoscalar meson mass ratio $M_{V} / M_{P}$ should look roughly constant.

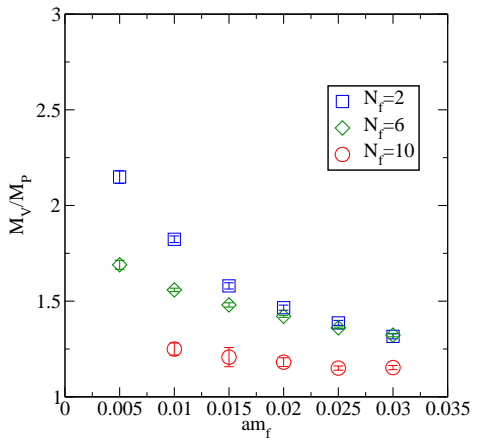

- The $N_{f}=2$ data are diverging as $m \rightarrow 0$, as expected since $M_{P} \rightarrow 0$.

- Similaryly for $N_{f}=6$, though not as fast as $N_{f}=2$.

- For $N_{f}=10$ the ratios seem to be roughly constant. 


\section{Testing Conformality - Quantitatively}
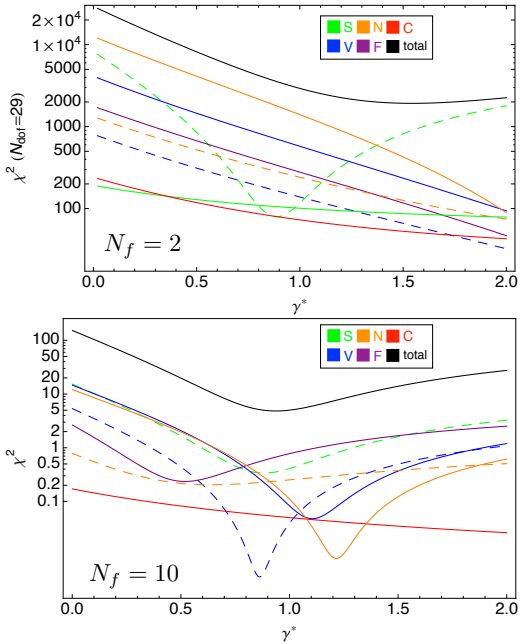

\begin{tabular}{|c|c|c|c|}
\hline Obs. & $m_{f} \geq 0.010$ & $m_{f} \geq 0.015$ & $m_{f} \geq 0.020$ \\
\hline \hline$\gamma^{\star}$ & $1.45(15)$ & $0.94(16)$ & $1.18(44)$ \\
{$[68 \% \mathrm{CI}]$} & {$[0.88,2.47]$} & {$[0.68,1.44]$} & {$[1.01,1.69]$} \\
\hline \hline$C_{P}$ & $0.978(9)$ & $1.44(21)$ & $1.21(42)$ \\
$C_{V}$ & $1.168(10)$ & $1.70(25)$ & $1.42(49)$ \\
$C_{A}$ & $1.429(13)$ & $2.14(32)$ & $1.79(63)$ \\
$C_{N}$ & $1.749(16)$ & $2.53(37)$ & $2.10(73)$ \\
$C_{N^{\star}}$ & $2.232(25)$ & $3.35(56)$ & $2.87(1.02)$ \\
$C_{F P}$ & $0.121(12)$ & $0.190(29)$ & $0.164(57)$ \\
\hline \hline$\chi^{2} /$ d.o.f. & $69 / 31$ & $14 / 23$ & $3 / 15$ \\
\hline
\end{tabular}

Finite volume effects may be large for $a m_{f} \leq 0.01$. 


\section{Summary}

- First SU(3) lattice simulations with $N_{f}=6$ and 10 domain wall fermions have been performed.

- Hints of condensate enhancement and reduced $S$ paramter have been seen with $N_{f}=6$.

- With increasing $N_{f}$, the topology becomes more difficult to evolve. Effects of fixed topology are big for $N_{f}=10$.

- Spectrum for $N_{f}=10$ is consistent with conformality. But cannot rule out the chirally-broken scenario, as the masses are too heavy to make use of ChPT.

Future Work

- Calculate topological susceptibility at fixed $Q$ for $N_{f}=10$.

S. Aoki et al., 2007, 2008

- Quantative studies of finite volume effects

- Smaller quark masses 


\section{Progress with Overlap Fermions}

- Overlap on $2+1$ flavor DWF configurations with deflation and low-mode sustituion $\longleftrightarrow$ quark loops with LMA

- Improvement of nucleon correlator

- Strangeness content in nucleon and other FF

- Glue momentum and angulalr momentum in nucleon

- Roper resonance with wall source

\section{XQCD Collaboration:}

A. Alexandru, Y. Chen, M. Deka, T. Doi, S.J. Dong, T. Draper, M. Gong,

I. Horvath, B. Joo, F. Lee, A. Li, H.W. Lin, K.F. Liu, N. Mathur, H. Thacker,

Y. Yang, J.B. Zhang

http://eagle.phys.gwu.edu/ fxlee/chiQCD.html

New Horizons for Lattice Calculations with Chiral Fermions, BNL, May 14-16, 2012 


\section{Overlap on 2+1 Flavor DWF configurations with HYP Smearing}

- Mixed action

- For chirally symmetric valence, it is a generalization of partial quenching with one extra low-energy constant in valence-sea mass

$$
\begin{aligned}
& m_{v_{1} v_{2}}^{2}=B_{o v}\left(m_{v_{1}}+m_{v_{2}}\right), \\
& m_{v s}^{2}=B_{o v} m_{v}+B_{d w}\left(m_{s}+m_{\text {res }}\right)+a^{2} \Delta_{\text {mix }}, \\
& m_{s_{1} s_{2}}^{2}=B_{d w}\left(m_{s_{1}}+m_{s_{2}}+2 m_{\text {res }}\right), \\
& \Delta_{\text {mix }}=0.030(6)(5) \mathrm{GeV}^{4} \quad \text { M. Lujan et al., } 1204.6256
\end{aligned}
$$

- Mixed action partially quenched chiral PT at NLO 


\section{2+1 flavor DWF configurations (RBC-UKQCD)}

La $2.8 \mathrm{fm}$

$\mathrm{m}_{\pi} \sim 330 \mathrm{MeV}$

\section{$24^{\wedge} 3 \times 32, a=0.115 \mathrm{fm}$}

$\left(\mathrm{O}\left(\mathrm{a}^{2}\right)\right.$ extrapolation)

$\mathrm{La} \sim 2.7 \mathrm{fm}$ $\mathrm{m}_{\pi} \sim 295 \mathrm{MeV}$

$32^{\wedge} 3 \times 64, a=0.085 \mathrm{fm}$

DSDR

$\mathrm{La} \sim 4.5 \mathrm{fm}$ $\mathrm{m}_{\pi} \sim 180 \mathrm{MeV}$ 


\section{Some Desirable Features of Overlap}

- Calculating eigenmodes is relatively easy

- Normality $\left(D^{+} D=D D+\right)$ and $G W$ relation

$\rightarrow$ Eigenvalues are on a unit circle and $\lambda=0,2$ are chiral modes.

The rest are complex pairs.

- Normality and $\left[\gamma_{5}, D^{\dagger} D\right]=0$

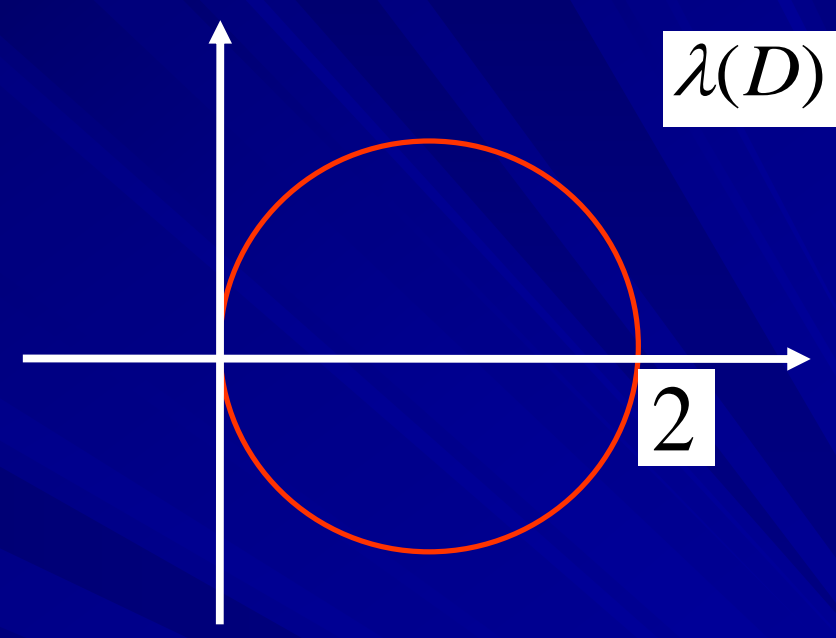

$\Rightarrow D^{\dagger} D \varphi_{L, R}=|\lambda|^{2} \varphi_{L, R} ;$

Diagonalize $\left\langle\varphi_{L, R}|D| \varphi_{L, R}\right\rangle \Rightarrow D \psi=\lambda \psi$ 


\section{Overlap with Deflation (multimass with same eigenvectors)}

$D(m, \rho) X_{L, R}^{H}=\eta_{L, R}-\sum_{i=1}^{n}\left(1 \mp \gamma_{5}\right)|i\rangle\left\langle i \mid \eta_{L, R}\right\rangle$

where,

$D(0, \rho)|i\rangle=\lambda_{i}|i\rangle ; \quad D(0, \rho) \gamma_{5}|i\rangle=\lambda_{i}^{*} \gamma_{5}|i\rangle$

Therefore,

$X_{L, R}^{H}=D^{-1}(m, \rho) \eta_{L, R}-X_{L, R}^{L}$

where,

$X_{L, R}^{L}=\sum_{i=1}^{n}\left[\frac{|i\rangle\left\langle i \mid \eta_{L, R}\right\rangle}{\rho \lambda_{i}+m\left(1-\lambda_{i} / 2\right)} \mp \frac{\gamma_{5}|i\rangle\left\langle i \mid \eta_{L, R}\right\rangle}{\rho \lambda_{i}^{*}+m\left(1-\lambda_{i}^{*} / 2\right)}\right]$

and

$\mathrm{X}=\left(\mathrm{X}_{L}^{H}+X_{R}^{H}\right)+\left(\mathrm{X}_{L}^{L}+X_{R}^{L}\right)$ except for the zero modes. 


\section{Speedup with deflation and HYP smearing}

\begin{tabular}{|l|c|c|c|c|c|c|c|c|c|c|}
\hline & & \multicolumn{3}{|c|}{$16^{\wedge} \mathbf{3} \times \mathbf{3 2}$} & \multicolumn{3}{|c|}{$24 \wedge \mathbf{3} \times 64$} & \multicolumn{3}{|c|}{$32^{\wedge} \mathbf{3} \times 64$} \\
\hline & res & w/o D & D & D+S & w/o D & D & D+S & w/o D & D & D+S \\
\hline Low mode & $10^{-8}$ & 0 & 200 & 200 & 0 & 200 & 200 & 0 & 400 & 400 \\
\hline Inner iter & $10^{-11}$ & 340 & 321 & 108 & 344 & 341 & 107 & 309 & 281 & 101 \\
\hline Outer iter & $10^{-8}$ & 627 & 72 & 85 & 2931 & 147 & 184 & 4028 & 132 & 156 \\
\hline $\begin{array}{l}\text { Speedup } \\
\text { Overhead }\end{array}$ & & & 23 & & & 51 & & & 79 \\
\hline
\end{tabular}

$$
D(0, \rho)=1+\gamma_{5} \varepsilon=1+\gamma_{5} \frac{H_{W}(\rho)}{\sqrt{H_{W}{ }^{2}(\rho)}} \approx 1+\gamma_{5} H_{W} \sum_{i=1}^{n} \frac{b_{i}}{H_{W}^{2}+c_{i}}
$$

- No critical slowing down

- Multii-mass inversion (30 masses)

A. Li, et al, PRD 82, 114501 (2010) 


\section{$Z_{3}$ grid (64) source with low-mode substitution}
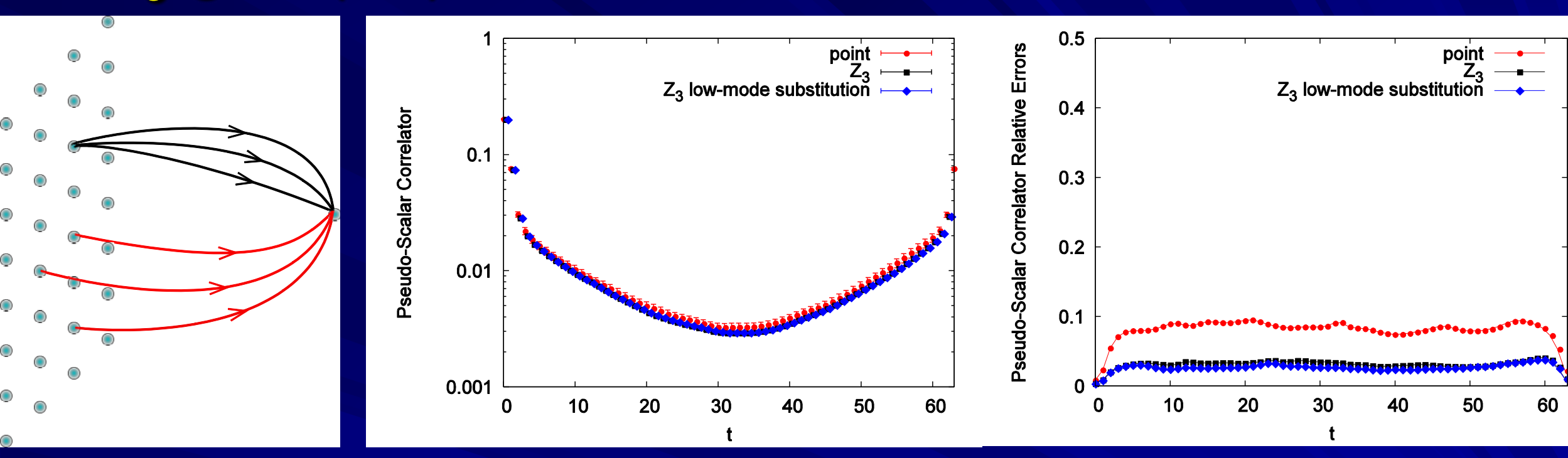

$32^{3} \times 64$ lattice, $m_{\mathrm{I}}(\mathrm{sea})=0.004$ at $\mathrm{m}_{\Pi} \sim 200 \mathrm{MeV}, 50$ conf.
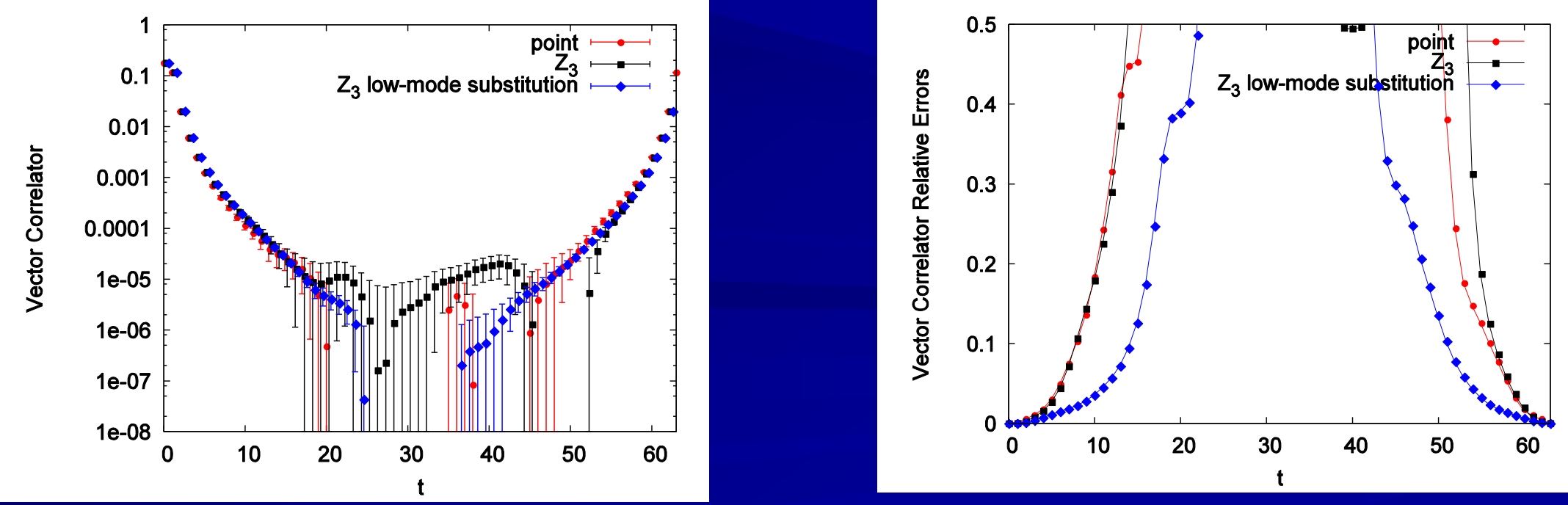


\section{Nucleon with LLL and HLL substitution}

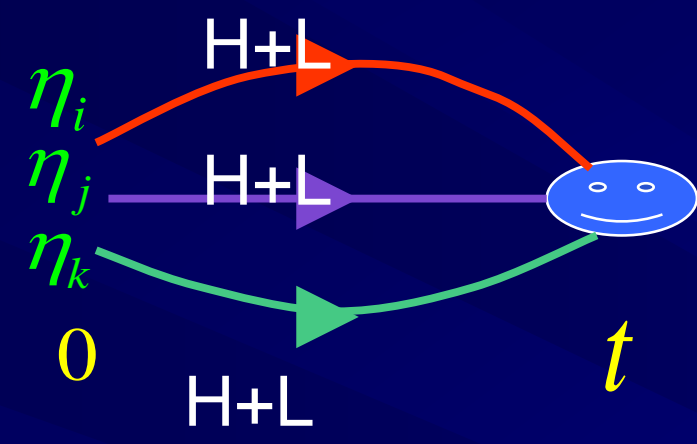

$\left\langle\eta_{i} \eta_{j} \eta_{k}\right\rangle=\delta_{i j} \delta_{j k}$

$\left\langle\eta_{i}^{\dagger} \eta_{j}\right\rangle=\delta_{i j}$

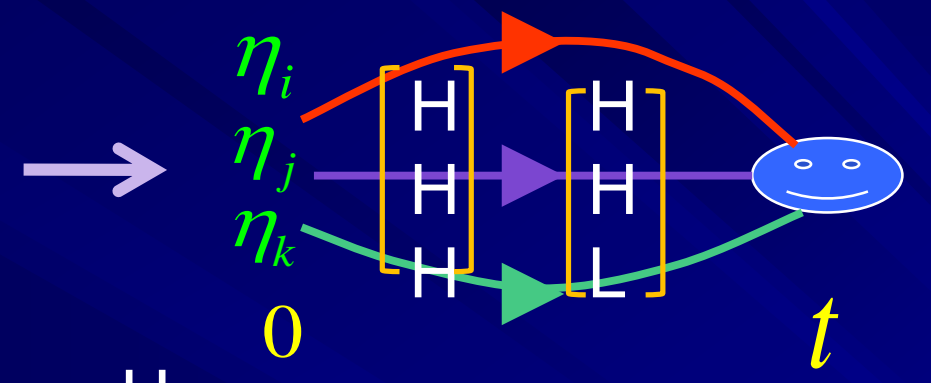
$\frac{C_{N}(t)}{\sigma_{N}(t)} \approx \sqrt{\frac{n}{V_{3}}} e^{-\left(m_{N}-32 m_{2}\right) x}$

$\mathrm{m}_{\pi} \sim 300 \mathrm{MeV}$
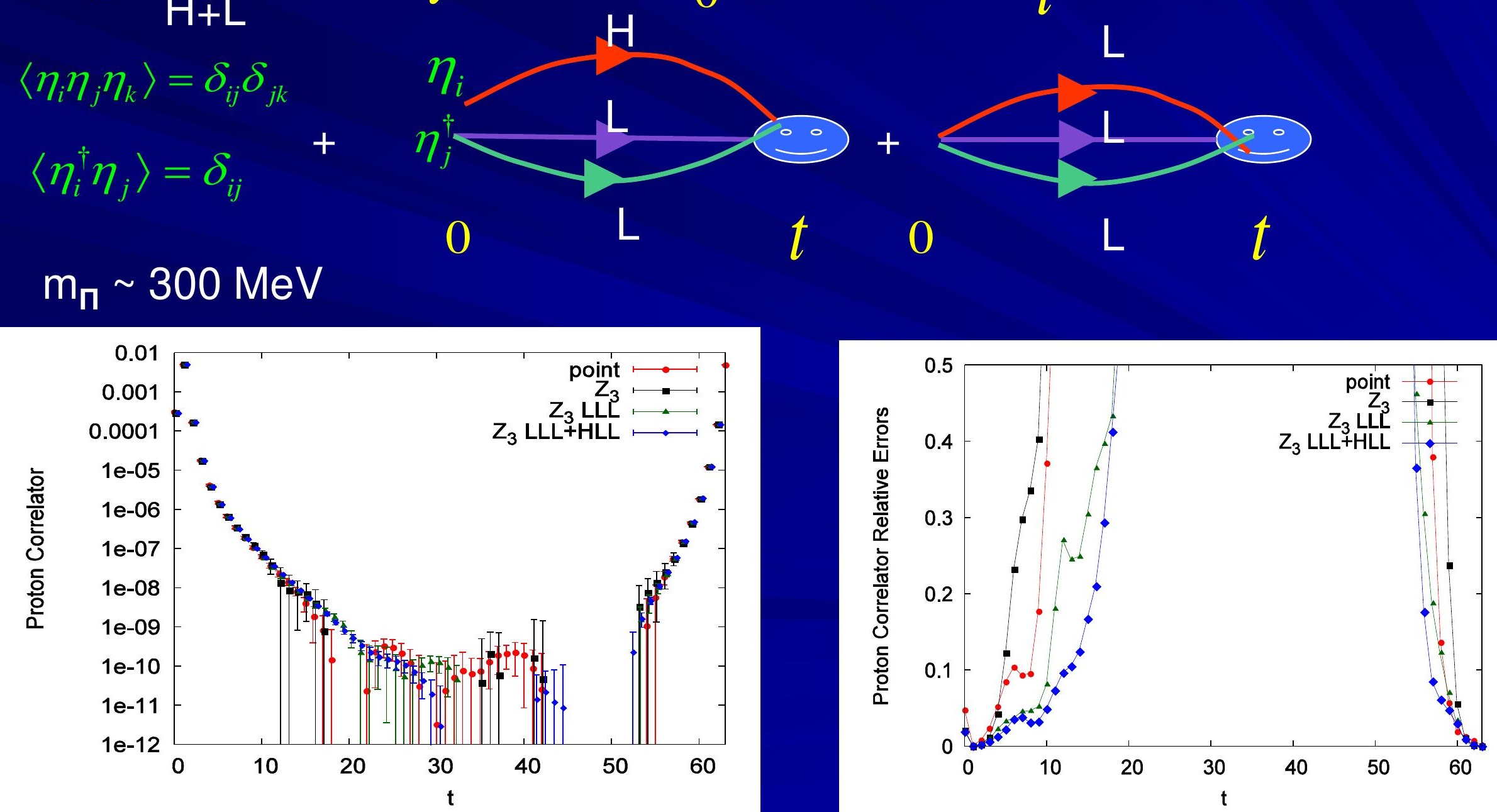


\section{Status of Projects in Progress}

Charmonium and $D_{S}$ spetra, $f_{D \prime}, f_{D s}$ are under global analysis with three sea masses and two lattice spacings.

Improvement of nucleon correlator with low-mode substitution

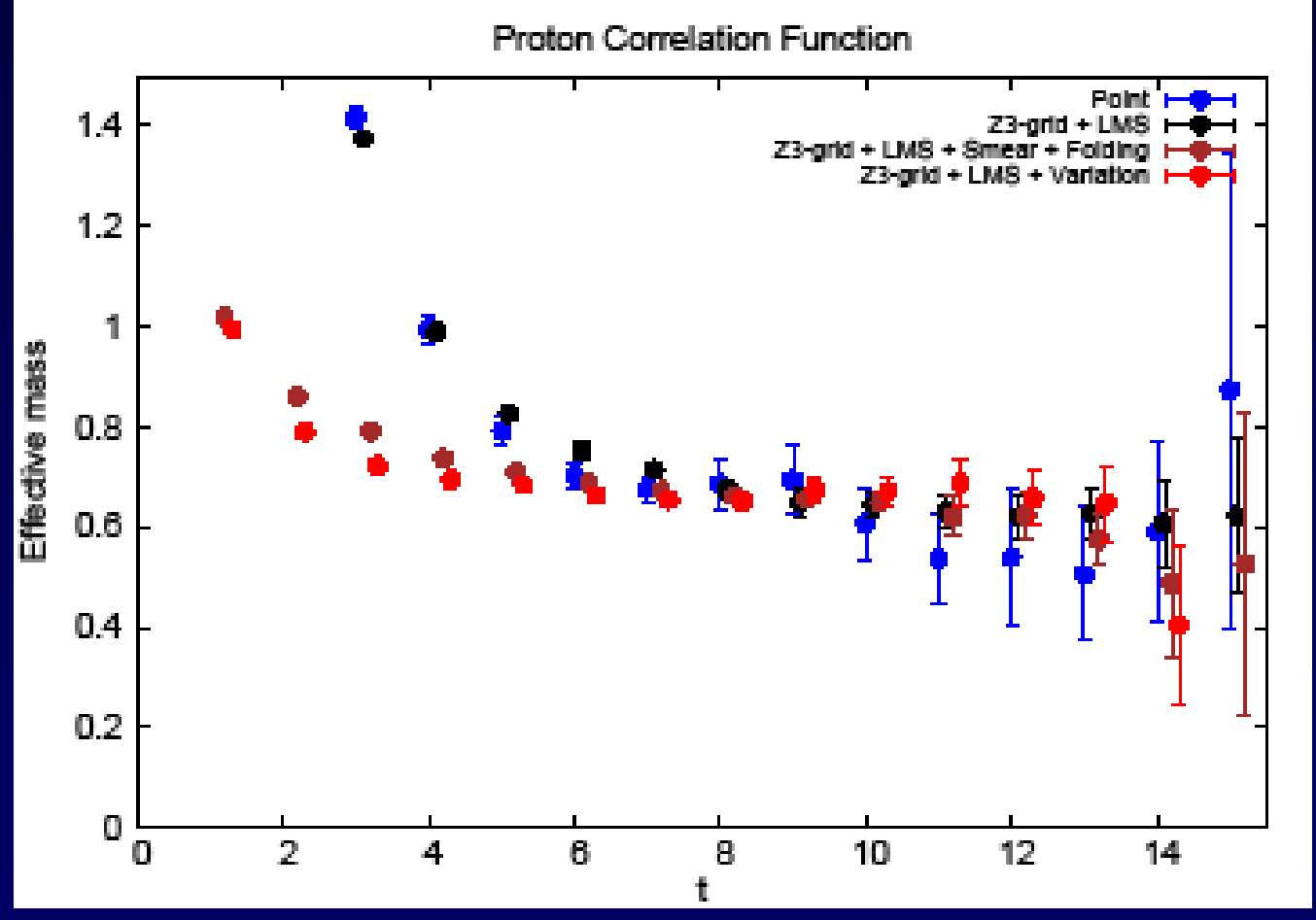

$24^{3} \times 64$ lattice with $m_{\pi}=331 \mathrm{MeV}, \mathrm{a}=1.73 \mathrm{GeV}^{-1}$

47 configurations

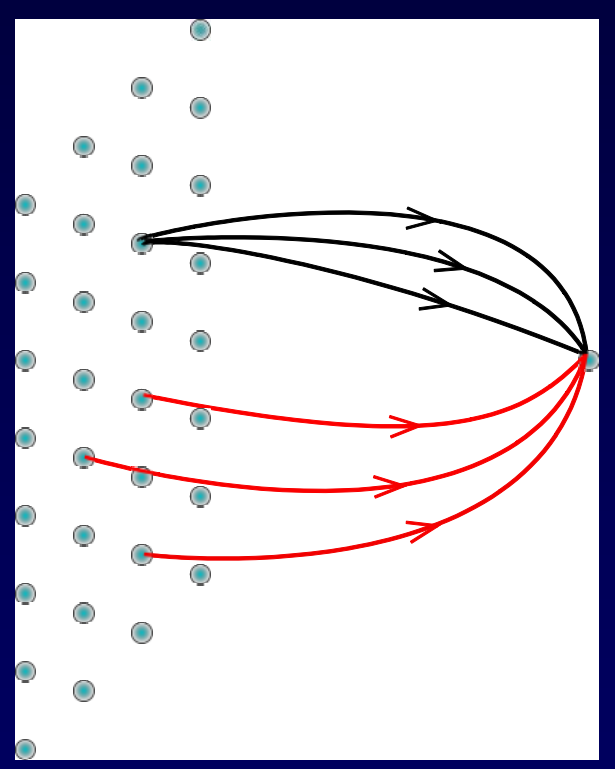

Point source: $\quad m_{N}=1.13(14) \mathrm{GeV}$;

$Z_{3}$ grid source: $m_{N}=1.08(5) \mathrm{GeV}$;

$\mathrm{Z}_{3}$ grid smeared source: $m_{N}=1.14(2) \mathrm{GeV}$;

Variation: $\quad m_{N}=1.16(1) \mathrm{GeV}$ 
- Quark loop with low-mode averaging and $Z_{4}$ noise estimate of high modes with grids and time dilution
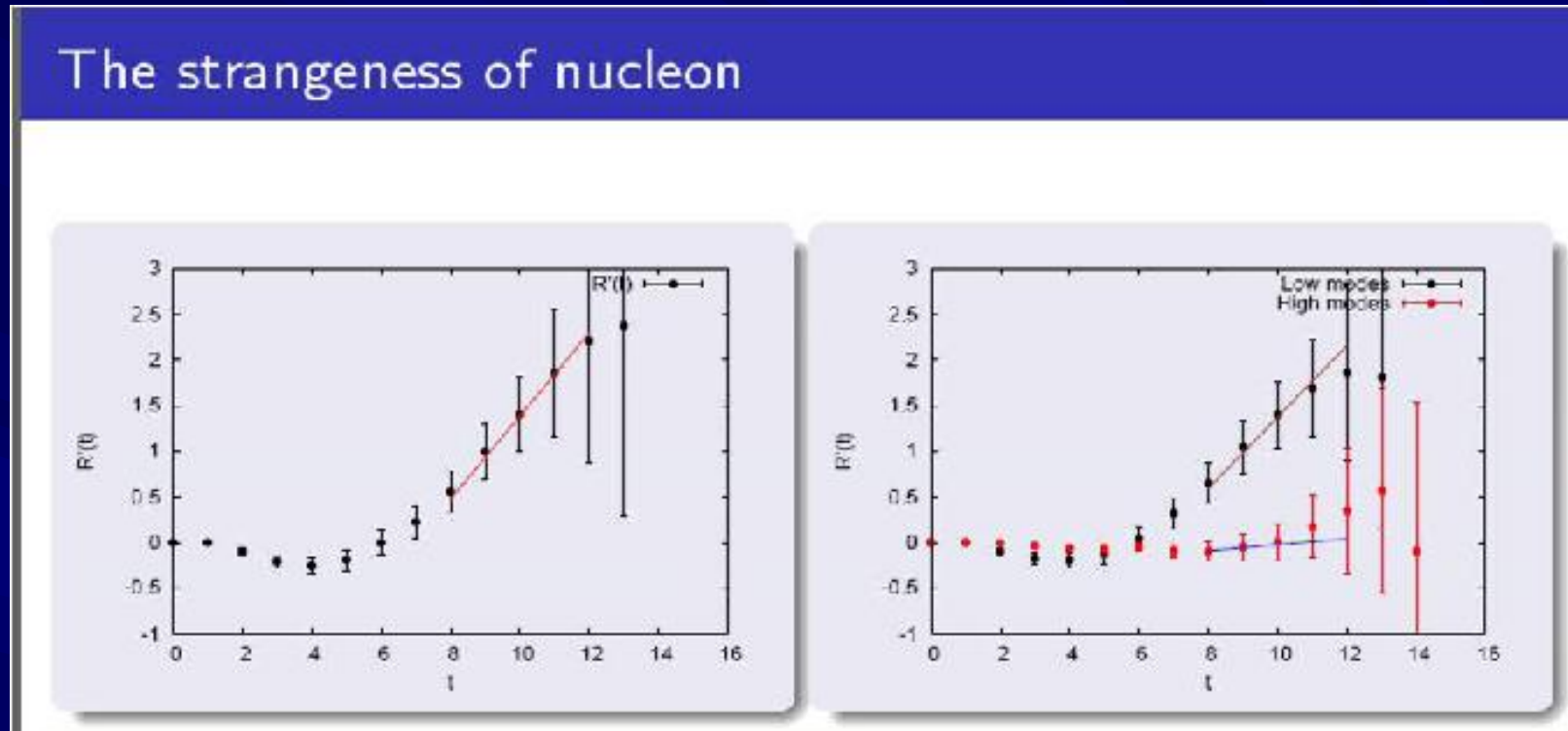

M. Gong

Lattice 2011

The fitting result

For $m_{s}=0.067, m_{a d}=0.016$ case :

$f_{T_{s}}=0.048(15), f_{T_{s}}{ }^{L}=0.041(12), f_{T_{s}}{ }^{H}=0.003(5)$

\section{constant $+\mathrm{m}_{s}<N|\bar{S} S| N>t$}

$24^{3} \times 64, m_{l}=0.005, m_{s}=0.04,50$ conf. $\Longrightarrow 25-30 \%$ error 


\section{$\langle\bar{\Psi} \Psi\rangle$ on different time slices}

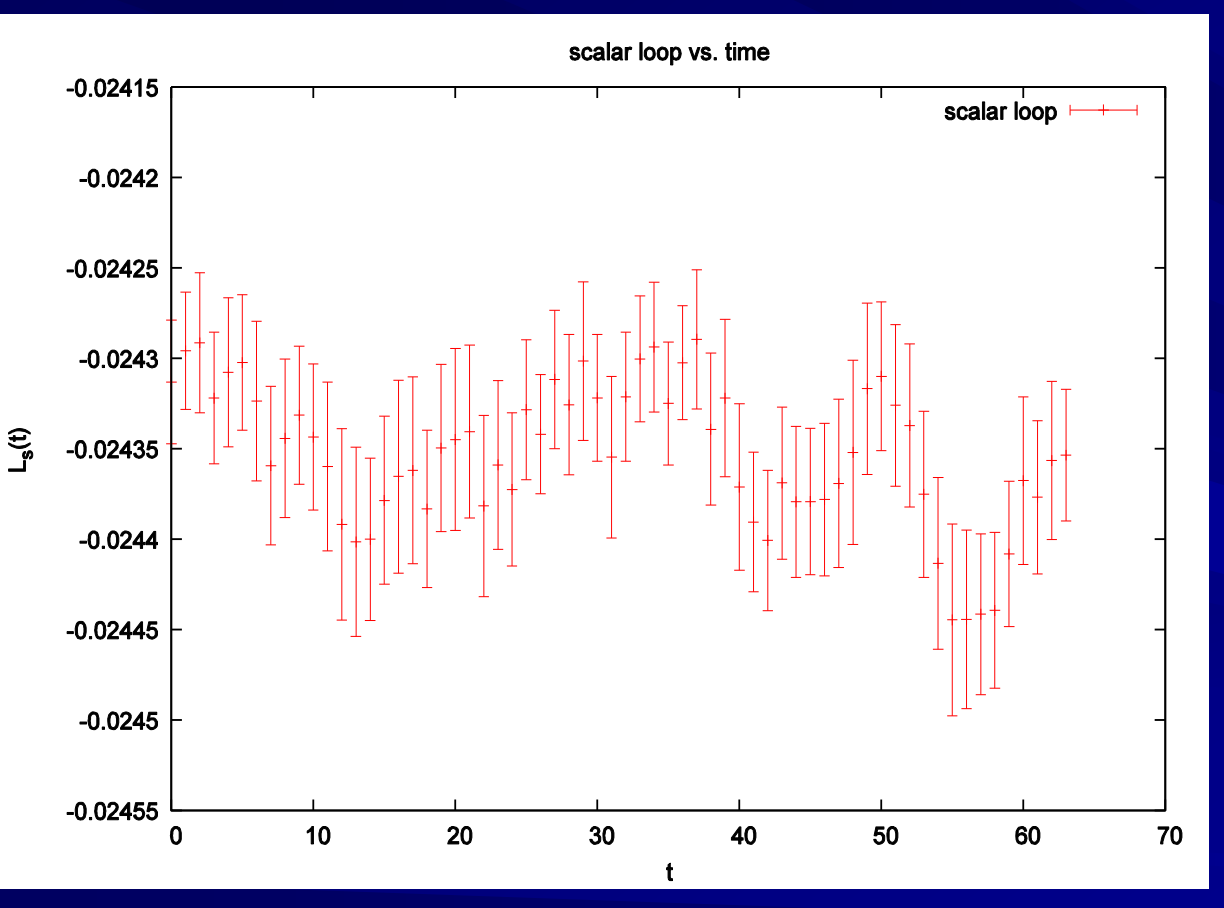

50 configurations: Low+High modes

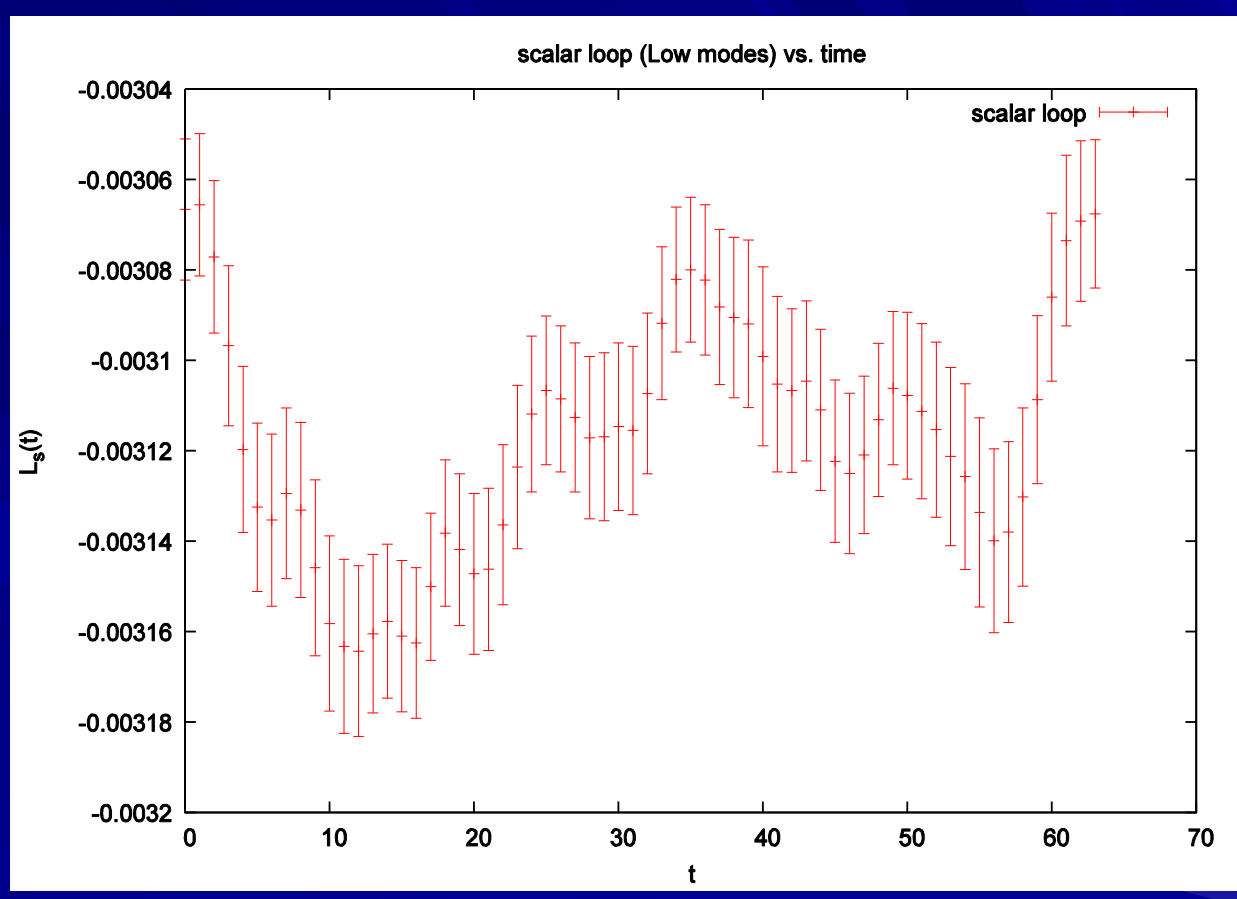

150 configurations: Low modes only 


\section{Momenta and Angular Momenta of Quarks and Glue}

Energy momentum tensor operators decomposed in quark and glue parts gauge invariantly --- Xiangdong Ji (1997)

$T_{\mu \nu}^{q}=\frac{i}{4}\left[\bar{\psi} \gamma_{\mu} \vec{D}_{\nu} \psi+(\mu \leftrightarrow v)\right] \rightarrow \overrightarrow{\mathrm{J}}_{q}=\int d^{3} x\left[\frac{1}{2} \bar{\psi} \vec{\gamma} \gamma_{5} \psi+\vec{x} \times \bar{\psi} \gamma_{4}(-i \vec{D}) \psi\right]$

$T_{\mu \nu}^{g}=F_{\mu \lambda} F_{\lambda v}-\frac{1}{4} \delta_{\mu \nu} F^{2} \quad \rightarrow \overrightarrow{\mathrm{J}}_{g}=\int d^{3} x[\vec{x} \times(\vec{E} \times \vec{B})]$

Nucleon form factors

$\left\langle p, s\left|T_{\mu \nu}\right| p^{\prime} s^{\prime}\right\rangle=\bar{u}(p, s)\left[T_{1}\left(q^{2}\right) \gamma_{\mu} \bar{p}_{v}-T_{2}\left(q^{2}\right) \bar{p}_{\mu} \sigma_{v \alpha} q_{\alpha} / 2 m\right.$

$\left.-\mathrm{iT}_{3}\left(q^{2}\right)\left(q_{\mu} q_{\nu}-\delta_{\mu \nu} q^{2}\right) / m+T_{4}\left(q^{2}\right) \delta_{\mu \nu} m / 2\right] u\left(p^{\prime} s^{\prime}\right)$

- Momentum and Angular Momentum

$$
Z_{q, g} T_{1}(0)_{q, g}[\mathrm{OPE}] \rightarrow\langle x\rangle_{q / g}(\mu, \overline{\mathrm{M}} \overline{\mathrm{S}}), Z_{q, g}\left[\frac{T_{1}(0)+T_{2}(0)}{2}\right]_{q, g} \rightarrow J_{q / g}(\mu, \overline{\mathrm{M}} \overline{\mathrm{S}})
$$




\section{$T_{1}\left(q^{2}\right)$ and $T_{2}\left(q^{2}\right)$}

3-pt to 2-pt function ratios

$\left.G_{\mu \nu}^{3 p t}\left(\vec{p}, t_{2} ; \vec{q}, t_{1}\right)=\sum_{\vec{x}_{1}, \vec{x}_{2}} e^{-i \vec{p} \cdot \vec{x}_{2}+i \vec{q} \cdot \vec{x}_{1}}\langle 0| T\left[\chi_{N}\left(\vec{x}_{2}, t_{2}\right) T_{\mu \nu} \bar{\chi}_{N}(0)\right]\right) ;$

$\operatorname{Tr}\left[\Gamma_{m} G_{\mu v}^{3 p t}\left(\vec{p}=0, t_{2} ; \vec{q}, t_{1}\right)\right]=W e^{-m\left(t_{2}-t_{1}\right)} e^{-E t_{1}}\left[T_{1}\left(q^{2}\right)+T_{2}\left(q^{2}\right)\right]$

- Need both polarized and unpolarized nucleon and different kinematics $\left(p_{i}, q_{j}, s\right)$ to separate out $T_{1}\left(q^{2}\right), T_{2}\left(q^{2}\right)$ and $T_{3}\left(q^{2}\right)$ 


\section{Status of Proton Spin}

- Quark spin $\Delta \Sigma \sim 20$ - 30\% of proton spin (DIS, Lattice)

- Quark orbital angular momentum? (lattice calculation (LHPC,QCDSF) $\rightarrow \sim 0$ )

- Glue spin $\triangle \mathrm{G} / \mathrm{G}$ small (COMPASS, STAR) ?

- Glue orbital angular momentum is small (Brodsky and Gardner)?

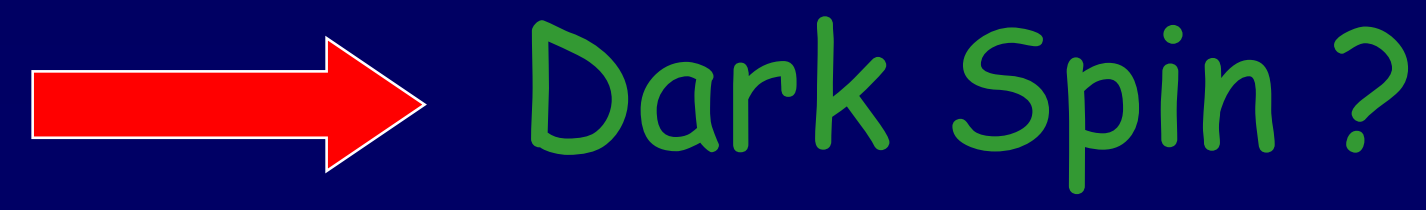


- Field strength tensor from overlap operator

$$
\begin{aligned}
& \operatorname{tr}_{s} \sigma_{\mu \nu} a D_{o v}(x, x)=c^{T} a^{2} F_{\mu \nu}+O\left(a^{3}\right), \\
& c^{T}=\rho \int_{-\pi}^{\pi} \frac{d^{4} k}{(2 \pi)^{4}} \frac{2\left[\left(\rho+r \sum_{\lambda}\left(c_{\lambda}-1\right)\right) c_{\mu} c_{\nu}+2 r c_{\mu} s_{\nu}^{2}\right]}{\left(\sum_{\mu}^{2} s_{\mu}^{2}+\left[\rho+\sum_{\nu}\left(c_{v}-1\right)\right]^{2}\right)^{3 / 2}} \\
& \text { where, } \quad r=1, \quad \rho=1.368, \quad c^{T}=0.11157
\end{aligned}
$$

where,

Liu, Alexandru, Horvath - PLB 659, 773 (2007)

- Noise estimation $D_{o v}(x, x) \rightarrow\left\langle\eta_{x}^{\dagger}\left(D_{o v} \eta\right)_{x}\right\rangle$ with $\mathrm{Z}_{\mathbf{4}}$ noise with color-spin dilution and some dilution in space-time as well.

- Quark and Glue momenta and angular momenta in the nucleon (quenched approximation) 


\section{Disconnected Insertions of $\mathrm{T}_{1}\left(\mathrm{q}^{2}\right)$ and $\mathrm{T}_{2}\left(\mathrm{q}^{2}\right)$ for $\mathrm{u} / \mathrm{d}$ Quarks}
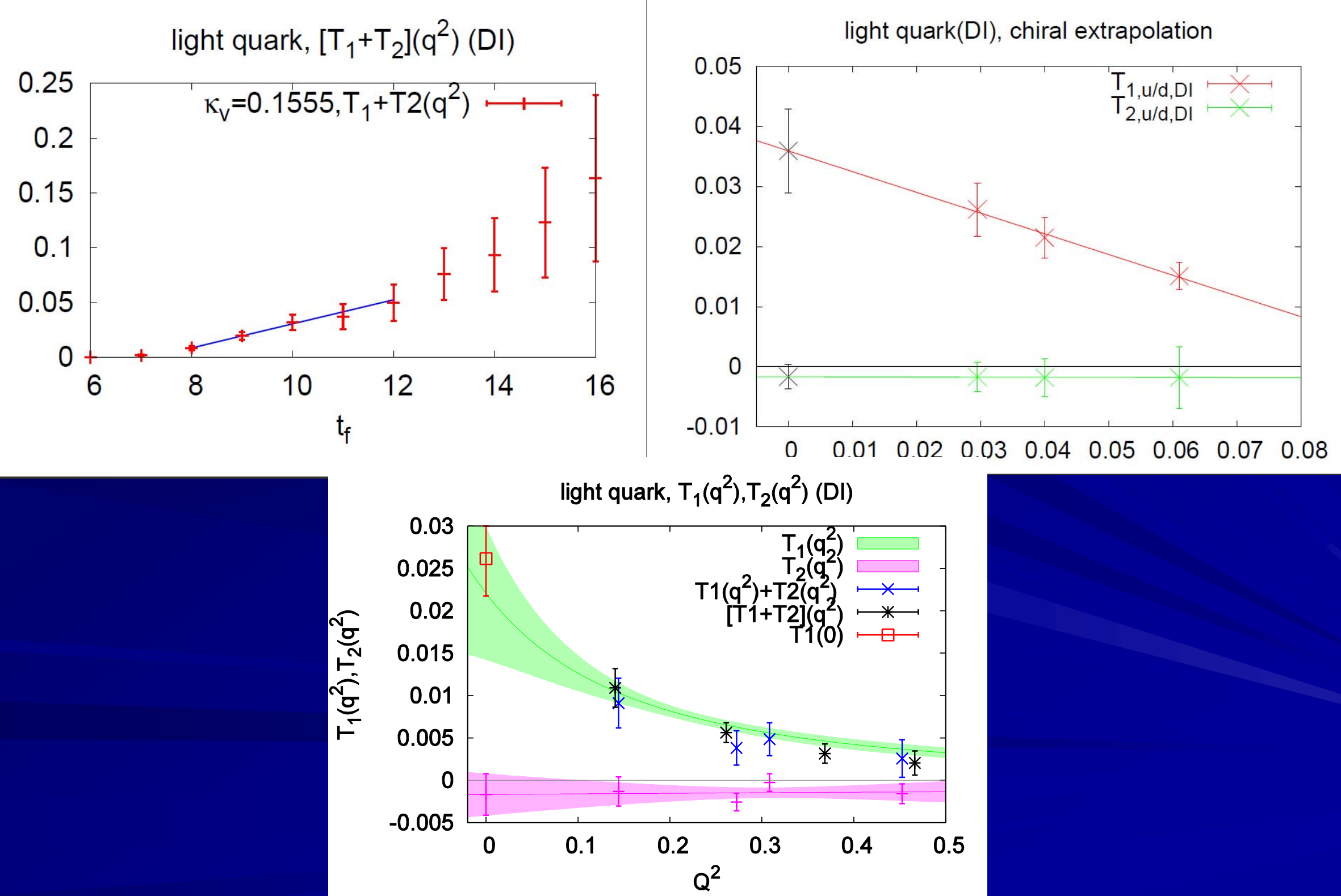


\section{Glue $T_{1}\left(q^{2}\right)$ and $T_{2}\left(q^{2}\right)$}
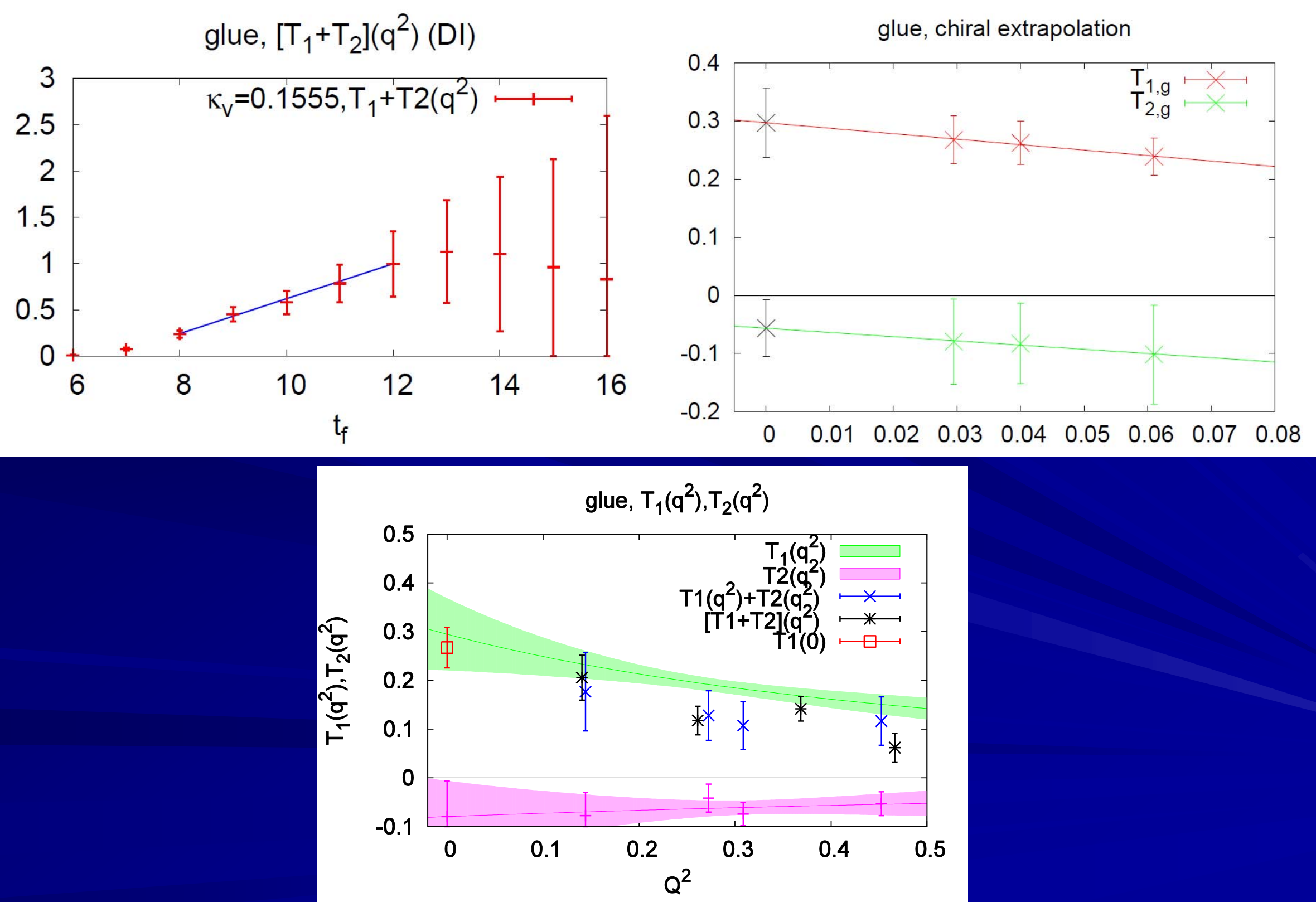


\section{Renormalization and Quark-Glue Mixing}

Momentum and Angular Momentum Sum Rules

$\langle x\rangle_{q}^{R}=Z_{q}\langle x\rangle_{q}^{L},\langle x\rangle_{g}^{R}=Z_{g}\langle x\rangle_{g}^{L}$,

$J_{q}^{R}=Z_{q} J_{q}^{L}, \quad J_{g}^{R}=Z_{g} J_{g}^{L}$,

$Z_{q}\langle x\rangle_{q}^{L}+Z_{g}\langle x\rangle_{g}^{L}=1, \quad\left\langle Z_{q} T_{1}^{q}(0)+Z_{g} T_{1}^{g}(0)=1\right.$,

$Z_{q} J_{q}^{L}+Z_{g} J_{g}^{L}=\frac{1}{2} \Rightarrow\left\{\begin{array}{l}Z_{q}\left(T_{1}^{q}+T_{2}^{q}\right)(0)+Z_{g}\left(T_{1}^{g}+T_{2}^{g}\right)(0)=1, \\ Z_{q} T_{2}^{q}(0)+Z_{g} T_{2}^{g}(0)=0\end{array}\right.$

Matching and Mixing

$$
\left[\begin{array}{c}
\langle x\rangle_{q}^{\bar{M} \bar{S}}(\mu) \\
\langle x\rangle_{g}^{\bar{M} \bar{S}}(\mu)
\end{array}\right]=\left[\begin{array}{ll}
C_{q q}(\mu) & C_{q g}(\mu) \\
C_{g q}(\mu) & C_{g g}(\mu)
\end{array}\right]\left[\begin{array}{l}
\langle x\rangle_{q}^{R} \\
\langle x\rangle_{g}^{R}
\end{array}\right]
$$

M. Glatzmaier 
Renormalized results: $\quad Z_{\mathbf{q}}=1.05, Z_{\mathbf{g}}=1.05$

\begin{tabular}{|c|c|c|c|c|c|c|}
\hline & $\mathrm{CI}(\mathrm{u})$ & $\mathrm{CI}(\mathrm{d})$ & $\mathrm{CI}(\mathrm{u}+\mathrm{d})$ & $\mathrm{DI}(\mathrm{u} / \mathrm{d})$ & $\mathrm{DI}(\mathrm{s})$ & Glue \\
\hline$<x>$ & $\begin{array}{c}0.428 \\
(40)\end{array}$ & $\begin{array}{c}0.156 \\
(20)\end{array}$ & $\begin{array}{c}0.586 \\
(45)\end{array}$ & $\begin{array}{c}0.038 \\
(6)\end{array}$ & $\begin{array}{c}0.024 \\
(6)\end{array}$ & $\begin{array}{l}0.313 \\
(56)\end{array}$ \\
\hline $\mathrm{T}_{2}(0)$ & $\begin{array}{l}0.297 \\
(112)\end{array}$ & $\begin{array}{c}-.218 \\
(80)\end{array}$ & $\begin{array}{c}0.064 \\
(22)\end{array}$ & $\begin{array}{c}-0.002 \\
(2)\end{array}$ & $\begin{array}{c}-.001 \\
(3)\end{array}$ & $\begin{array}{l}-.059 \\
(52)\end{array}$ \\
\hline $2 \mathrm{~J}$ & $\begin{array}{l}0.726 \\
(128)\end{array}$ & $\begin{array}{c}-.072 \\
(82)\end{array}$ & $\begin{array}{c}0.651 \\
(51)\end{array}$ & $\begin{array}{c}0.036 \\
(7)\end{array}$ & $\begin{array}{c}0.023 \\
(7)\end{array}$ & $\begin{array}{c}0.254 \\
(76)\end{array}$ \\
\hline
\end{tabular}

S. Brodsky et al. NPB 593, 311(2001) $\rightarrow$ no anomalous gravitomagnetic moment

E. Leader, arXiv:1109.1230 $\rightarrow$ transverse angular momentum 
Renormalized results:

\begin{tabular}{|c|c|c|c|c|c|c|}
\hline & $\mathrm{CI}(\mathrm{u})$ & $\mathrm{CI}(\mathrm{d})$ & $\mathrm{CI}(\mathrm{u}+\mathrm{d})$ & $\mathrm{DI}(\mathrm{u} / \mathrm{d})$ & $\mathrm{DI}(\mathrm{s})$ & Glue \\
\hline & 0.726 & -.072 & 0.651 & 0.036 & 0.023 & 0.254 \\
$2 \mathrm{~J}$ & $(128)$ & $(82)$ & $(51)$ & $(7)$ & $(7)$ & $(76)$ \\
\hline & 0.95 & -0.32 & 0.65 & -0.12 & -0.12 & \\
$\mathrm{~g}_{\mathrm{A}}$ & $(11)$ & $(12)$ & $(8)$ & $(1)$ & $(1)$ & \\
\hline & -0.25 & 0.26 & 0.00 & 0.17 & 0.15 & \\
$2 \mathrm{~L}$ & $(18)$ & $(14)$ & $(10)$ & $(2)$ & $(2)$ & \\
\hline
\end{tabular}




\title{
Quark Spin, Orbital Angular Momentum, and Gule Angular Momentum
}

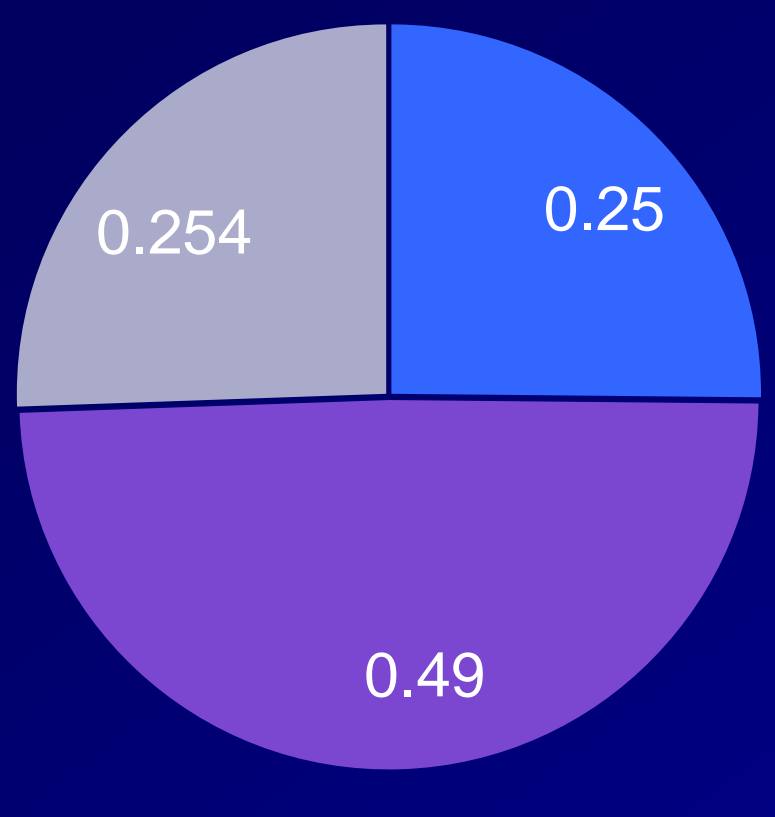

\author{
$2 \mathrm{~J}$ \\ - Quark Spin \\ - Qurak OAM \\ - Glue AM
}

$$
\begin{aligned}
& \Delta q \approx 0.25 \\
& 2 L_{q} \approx 0.49(0.0(\mathrm{CI})+0.49(\mathrm{DI})) \\
& 2 \mathrm{~J}_{g} \approx 0.25
\end{aligned}
$$




\section{Glozman and Riska Challenge Phys. Rep. 268, 263 (1996)}

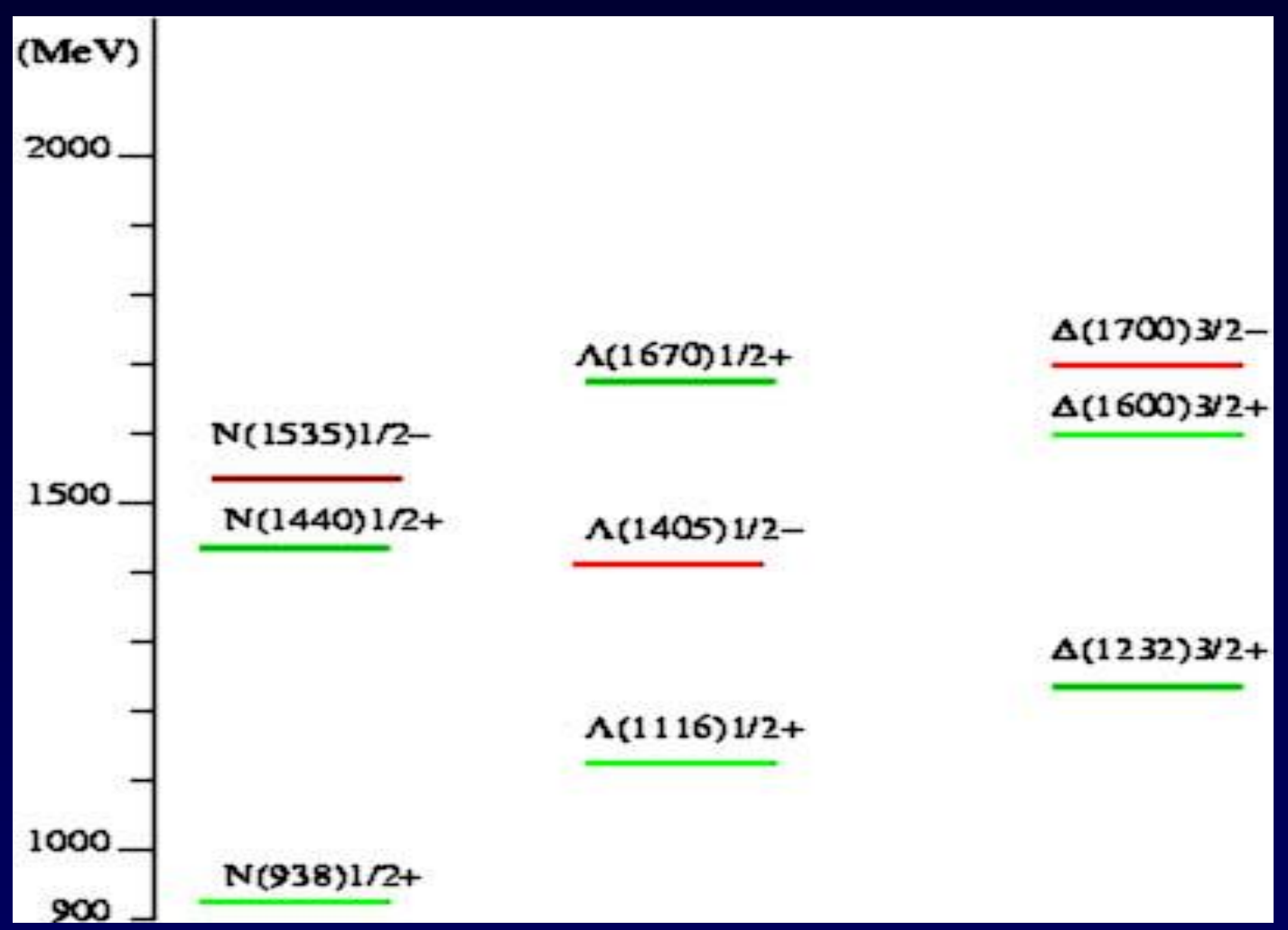

Hyperfine Interaction of Quarks in Baryons

- Color-spin

$\lambda_{1}^{c} \cdot \lambda_{2}^{c} \vec{\sigma}_{1} \cdot \vec{\sigma}_{2}$

- One-gluon exchange
- Flavor-spin

$$
\lambda_{1}^{F} \cdot \lambda_{2}^{F} \vec{\sigma}_{1} \cdot \vec{\sigma}_{2}
$$

- Goldstone boson exchange 


\section{Quenched Roper Calculation}

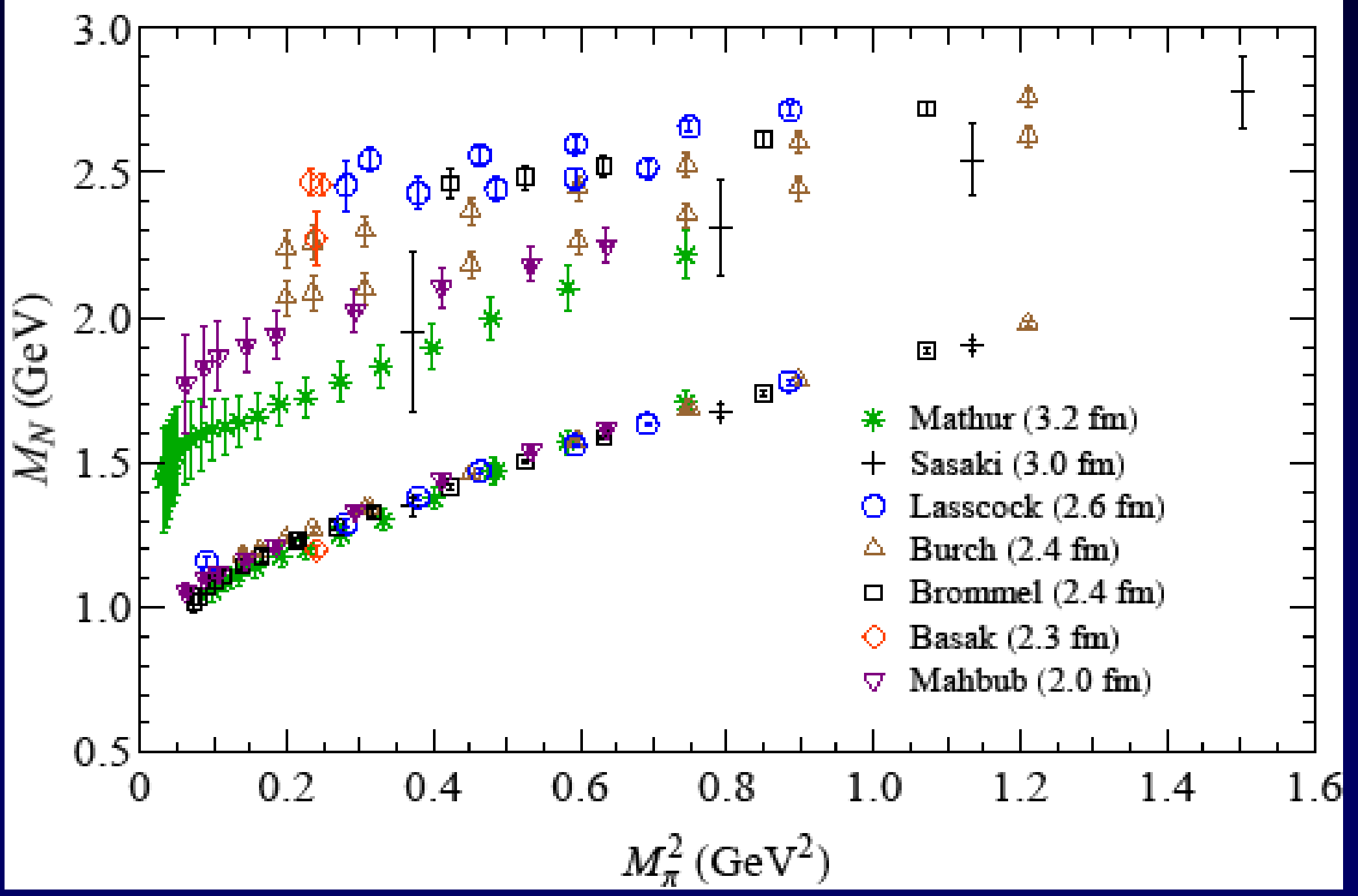




\section{Status of Projects in Progress}

Charmonium and $D_{S}$ spetra, $f_{D}, f_{D s}$, are under global analysis with three sea masses and two lattice spacings.

- Improvement of nucleon correlator with low-mode substitution

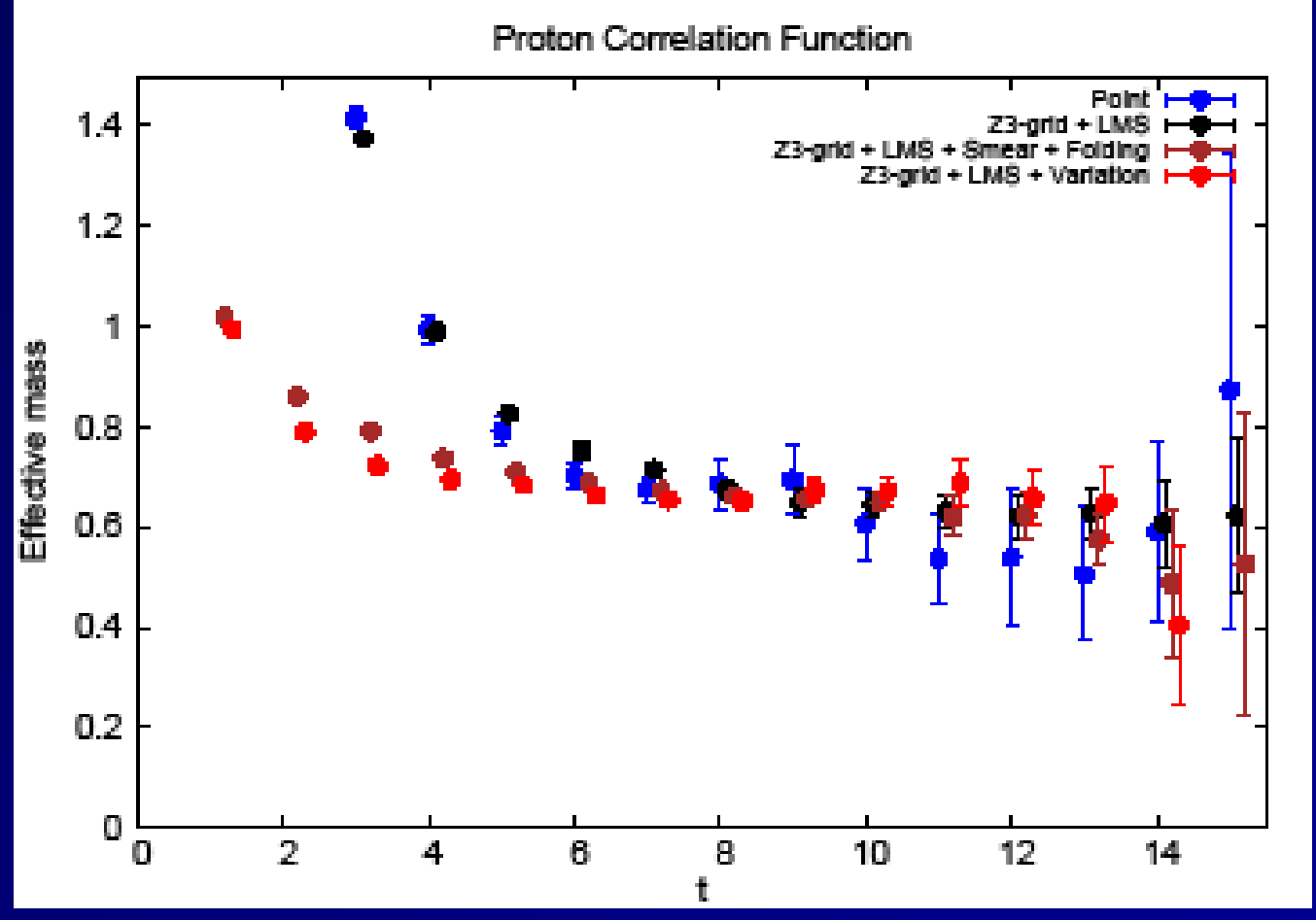

$24^{3} \times 64$ lattice with $m_{\pi}=331 \mathrm{MeV}, \mathrm{a}=1.73 \mathrm{GeV}^{-1}$

47 configurations

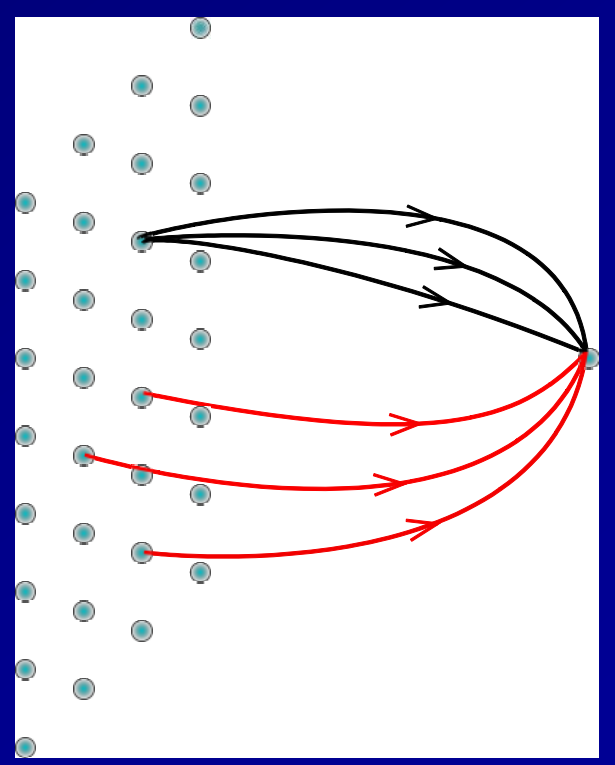

Point source: $\quad m_{N}=1.13(14) \mathrm{GeV}$;

$\mathrm{Z}_{3}$ grid source: $m_{N}=1.08(5) \mathrm{GeV}$;

$\mathrm{Z}_{3}$ grid smeared source: $m_{N}=1.14(2) \mathrm{GeV}$;

Variation: $\quad m_{N}=1.16(1) \mathrm{GeV}$ 


\section{- Roper resonance from Coulomb wall source}
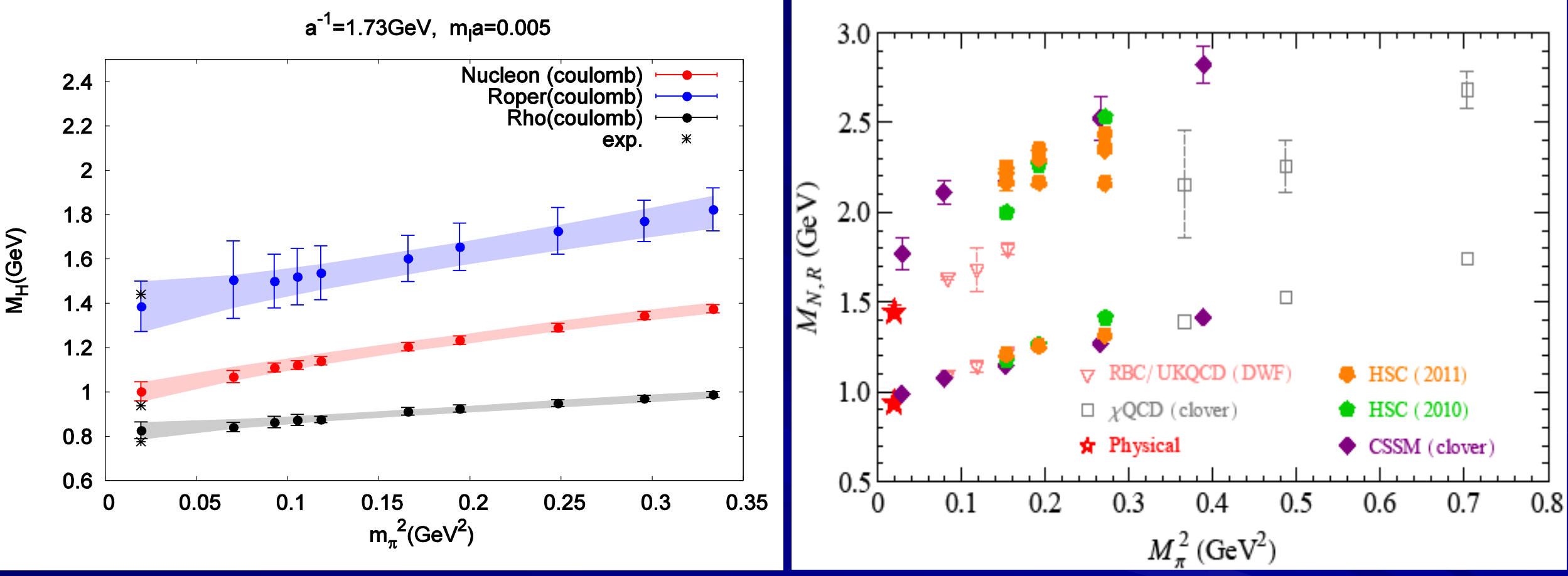

$m_{N}=m_{0}+c_{1} m_{\pi}^{2}+c_{2} m_{\pi}^{3}($ mixed $)+c_{3} m_{\pi}^{4} \ln \left(m_{\pi}^{2} / \mu^{2}\right)+\ldots$

$24^{3} \times 64$ lattice with $m_{\pi}=331 \mathrm{MeV}($ sea $), \mathrm{a}=1.73 \mathrm{GeV}^{-1}$ 


\section{Vector meson effective mass from Coulomb wall source}

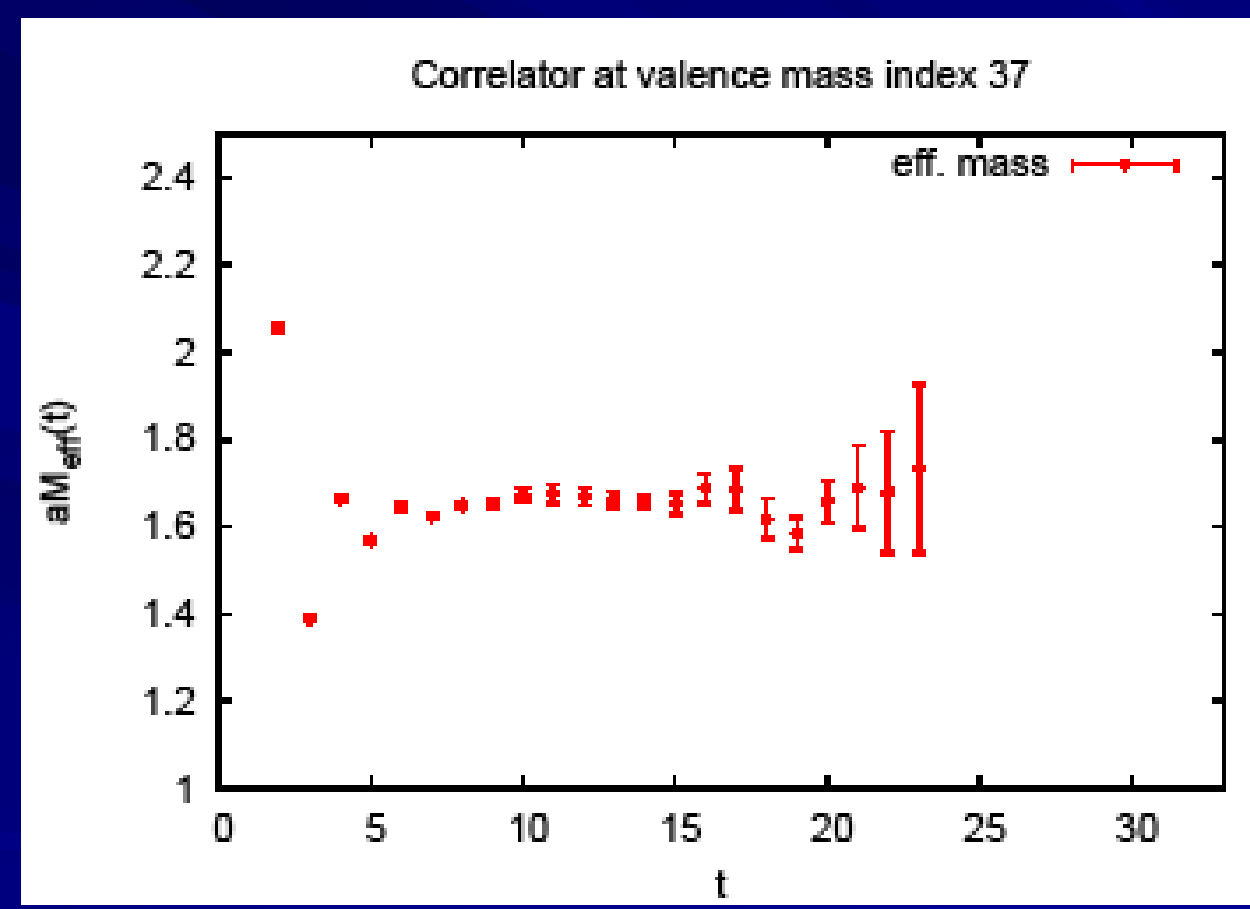

$$
C(t)=\sum_{n} W_{n} e^{-m_{i} t}+(-)^{t} W e^{-M t}
$$




\section{Summary of overlap on DWF config.}

- Good: same fermion for charm and light, eigenmodes, inversion with deflation and multimass, correlators with low-mode substitution, quark loop with low-mode averaging, glue field strength tensor.

$\star$ - Not so good: residual mass, oscillatory behavior at short time slices (DWF sea), not unitary (mixed action), auto-correlation.

- Can and should get better: precise approximation of the matrix sign function, reduced auto-correlation. 
page 29 


\section{Gauge Operators from the Overlap Dirac Operator}

- Overlap operator

$$
D_{o v}=1+\gamma_{5} \varepsilon(H) ; \quad H=\gamma_{5} D_{W}\left(m_{0}\right)
$$

- Index theorem on the lattice (Hasenfratz, Laliena, Niedermayer, Lüscher)

$$
\text { index } D_{o v}=-\operatorname{Tr} \gamma_{5}\left(1-\frac{a}{2} D_{o v}\right)
$$

- Local version (Kikukawa \& Yamada, Adams, Fujikawa, Suzuki)

$$
q_{L}(x)=-\operatorname{tr} \gamma_{5}\left(1-\frac{a}{2} D_{o v}(x, x)\right) \underset{a \rightarrow 0}{\longrightarrow} a^{4} q(x)+O\left(a^{6}\right)
$$

- Study of topological structure of the vacuum

- Sub-dimensional long range order of coherent charges (Horvàth et al; Thacker talk in Lattice 2006)

- Negativity of the local topological charge correlator (Horvàth et al) 


\section{Some Desirable Features of Overlap}

- Renormalization with chiral Ward identity

- Light mesons

$$
Z_{A} \partial_{\mu} A_{\mu}^{3}=2 \mathbb{Z}_{S}^{-1} m \mathbb{Z}_{P} P \Rightarrow f_{\pi}=\lim _{t \square a} 2 m \sqrt{\frac{G_{P P}(\vec{p}=0, t)}{m_{\pi}^{3}}} \mathrm{e}^{m_{\pi} t / 2}
$$

- Heavy-light meson K.F. Liu and S.J. Dong, Int.J.Mod.Phys. A20 (2005) 7241

$$
\tilde{Z}_{A}\left(m_{1}, m_{2}\right) \partial_{\mu} A_{\mu 12}=\left(\tilde{Z}_{S}^{-1}\left(m_{1}\right) m_{1}+\tilde{Z}_{S}^{-1}\left(m_{2}\right) m_{2}\right) \tilde{Z}_{P}\left(m_{1}, m_{2}\right) P_{12}
$$

- Gellmann-Oaks-Renner relation

$$
\begin{gathered}
\frac{1}{V} \int d^{4} x\left\langle\pi_{12}^{a \dagger}(x) \pi_{12}^{a}(0)\right\rangle=\frac{-2\left[\left\langle\bar{\Psi}_{1} \Psi_{1}\right\rangle+\left\langle\bar{\Psi}_{2} \Psi_{2}\right\rangle\right]}{m_{1}+m_{2}} \text { (renormalized and unrenormalized) } \\
\tilde{Z}_{P}\left(m_{1}, m_{2}\right)=\frac{\tilde{Z}_{S}\left(m_{1}\right)\left\langle\bar{\Psi}_{1} \Psi_{1}\right\rangle+\tilde{Z}_{S}\left(m_{2}\right)\left\langle\bar{\Psi}_{2} \Psi_{2}\right\rangle}{\left\langle\bar{\Psi}_{1} \Psi_{1}\right\rangle+\left\langle\bar{\Psi}_{2} \Psi_{2}\right\rangle} \frac{m_{1}+m_{2}}{\tilde{Z}_{S}^{-1}\left(m_{1}\right) m_{1}+\tilde{Z}_{S}^{-1}\left(m_{2}\right) m_{2}}
\end{gathered}
$$




\section{Decaying Pseudoscalars from DWF LQCD}

New Horizons for Lattice Computations with Chiral Fermions Brookhaven National Laboratory

May 15, 2012

Robert Mawhinney

Columbia University 


\begin{tabular}{|c|c|c|c|}
\hline Generic Process & Examples & Experiment & LQCD calculates \\
\hline Kl2 & $\begin{array}{l}K^{+} \rightarrow \mu^{+} \nu_{\mu} \\
K^{+} \rightarrow e^{+} \nu_{e}\end{array}$ & $f_{K}$ & $f_{K}$ \\
\hline Kl3 & $\begin{array}{l}K^{+} \rightarrow \pi^{0} l^{+} \nu_{l} \\
K^{0} \rightarrow \pi^{-} l^{+} \nu_{l}\end{array}$ & $\left|V_{u s} f^{+}(0)\right|^{2}$ & $f^{+}(0)$ \\
\hline Kl4 & $K \rightarrow \pi \pi l \bar{\nu}_{l}$ & & $? ?$ \\
\hline $\begin{array}{l}K \rightarrow \pi \pi \\
(\mathrm{CP} \text { conserving })\end{array}$ & $\begin{array}{l}K^{0} \rightarrow \pi^{+} \pi^{-} \\
K^{+} \rightarrow \pi^{+} \pi^{0}\end{array}$ & $\begin{array}{l}\left|A_{0}\right| \\
\left|A_{2}\right| \\
\end{array}$ & $\begin{array}{c}\left|A_{0}\right|\left|A_{2}\right| \\
\left(\mathrm{SM}_{\mathrm{cpc}} \text { inputs }\right)\end{array}$ \\
\hline$\Delta_{(\mathrm{CP} \text { conserving })}$ & $\begin{array}{c}K^{0} \leftrightarrow \pi \pi \leftrightarrow \bar{K}^{0} \\
\text { (long distance physics) } \\
K^{0} \leftrightarrow O_{\Delta S=2} \leftrightarrow \bar{K}^{0} \\
\text { (short distance physics) }\end{array}$ & $\Delta m_{K}$ & $\begin{array}{c}\Delta m_{K} \\
\left(\mathrm{SM}_{\mathrm{cpc}} \text { inputs }\right)\end{array}$ \\
\hline$\underset{\text { (indirect } \mathrm{CP} \text { violation) }}{K^{0}} \rightarrow \pi \pi$ & $\begin{array}{c}K_{L} \rightarrow \pi \pi \\
\qquad\left(K^{0} \leftrightarrow \bar{K}^{0}\right) \rightarrow \pi \pi \\
\text { independent of } \pi \pi \text { isospin }\end{array}$ & $\epsilon=\frac{\hat{B}_{K} F_{K}^{2} \mathrm{SM}}{\Delta m_{K}}$ & $\hat{B}_{K}$ \\
\hline$\underset{\text { (direct CP violation) }}{K^{0} \rightarrow \pi}$ & $\begin{array}{c}K_{L} \rightarrow \pi \pi \\
\text { depends on } \pi \pi \text { isospin }\end{array}$ & $\begin{array}{c}\operatorname{Re}\left(\epsilon^{\prime} / \epsilon\right) \\
=f\left(A_{0}, A_{2}, \mathrm{SM}\right)\end{array}$ & $\begin{array}{cc}A_{0} & A_{2} \\
\left(\mathrm{SM}_{\mathrm{cpc}}\right. & \text { inputs }) \\
\end{array}$ \\
\hline
\end{tabular}

$\mathrm{SM}_{\mathrm{cpc}}=$ Standard Model CP-conserving parameters 


\section{RBC/UKQCD 2+1 flavor DWF ensembles}

$m_{\pi}$ (unitary, degenerate quarks) and $a^{2}$ for DWF ensembles

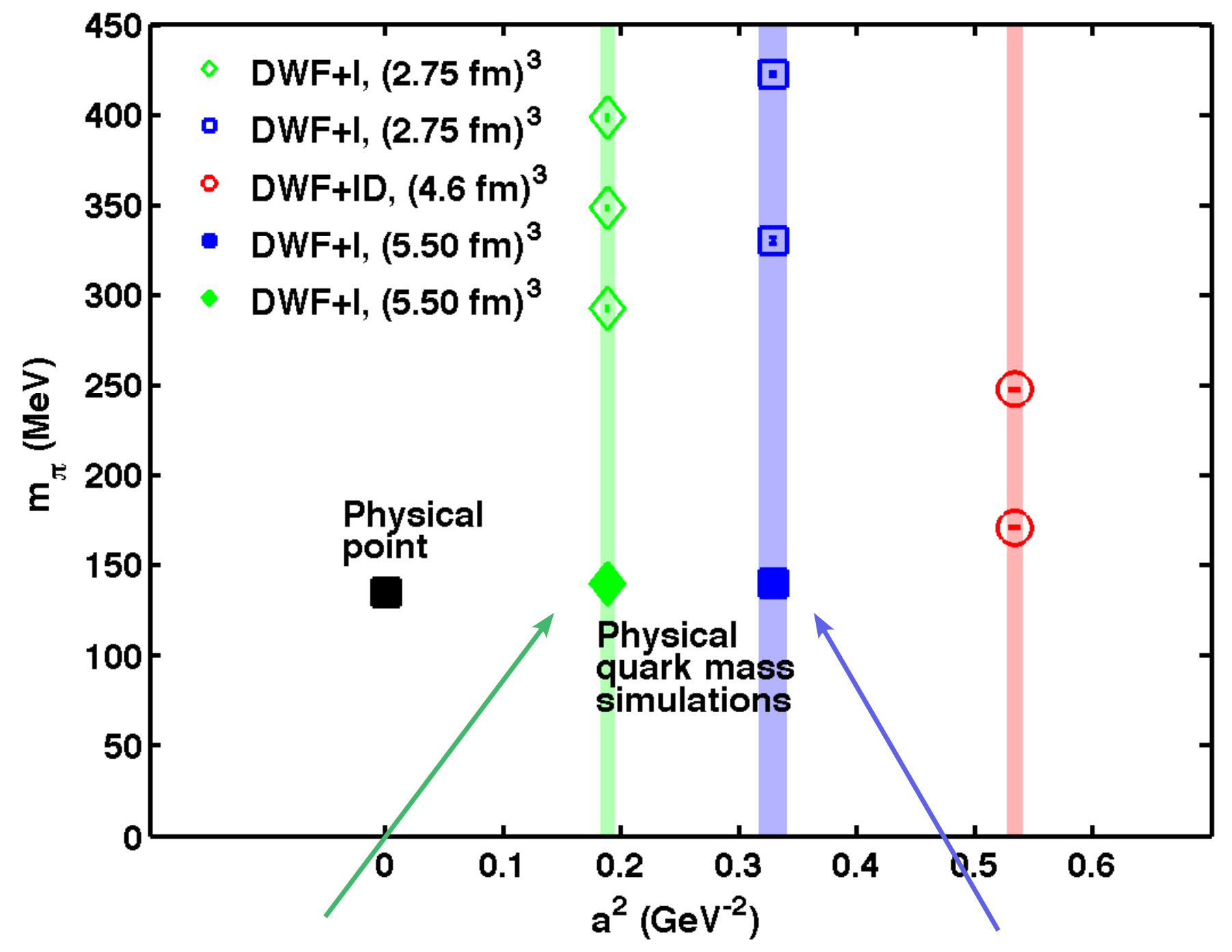

Proposed

Thermalizing on BNL BGQ 


\section{Improving Domain Wall Fermions}

- When underlying gauge field changes topology, the DWF modes can extend farther in the fifth dimension

- This gives a non-perturbative contribution to residual chiral symmetry breaking

- Becomes problematic at strong coupling

- Add ratio of determinants of twisted Wilson fermions to suppress these gauge field dislocations

- Tune to minimize residual mass while still preserving toplogical ergodicity

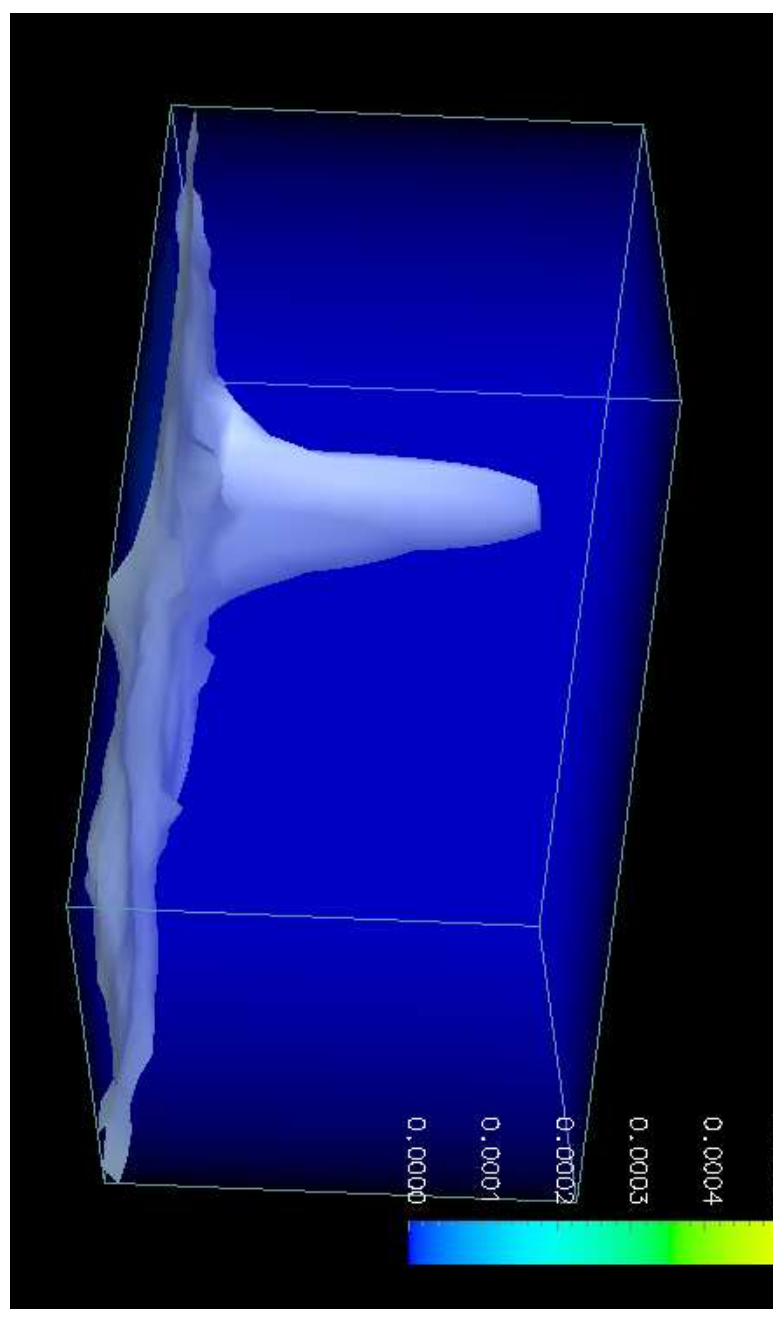

$\frac{\operatorname{det}\left[D_{W}\left(-M+i \varepsilon_{f} \gamma^{5}\right)^{\dagger} D_{W}\left(-M+i \varepsilon_{f} \gamma^{5}\right)\right]}{\operatorname{det}\left[D_{W}\left(-M+i \varepsilon_{b} \gamma^{5}\right)^{\dagger} D_{W}\left(-M+i \varepsilon_{b} \gamma^{5}\right)\right]}=\prod_{i} \frac{\lambda_{i}^{2}+\varepsilon_{f}^{2}}{\lambda_{i}^{2}+\varepsilon_{b}^{2}}$

$\lambda_{i}$ are eigenvalues of the Hermitian Wilson operator $\gamma^{5} D_{W}$ 


\section{Force Gradient Integrator}

- Proposed by Clark and Kennedy. Implemented (and simplified) in CPS by Hantao Yin

- For $16^{3} \times 32 \times 16$ volumes, no speed-up compared to $\mathrm{O}\left(\delta \tau^{2}\right)$ Omelyan

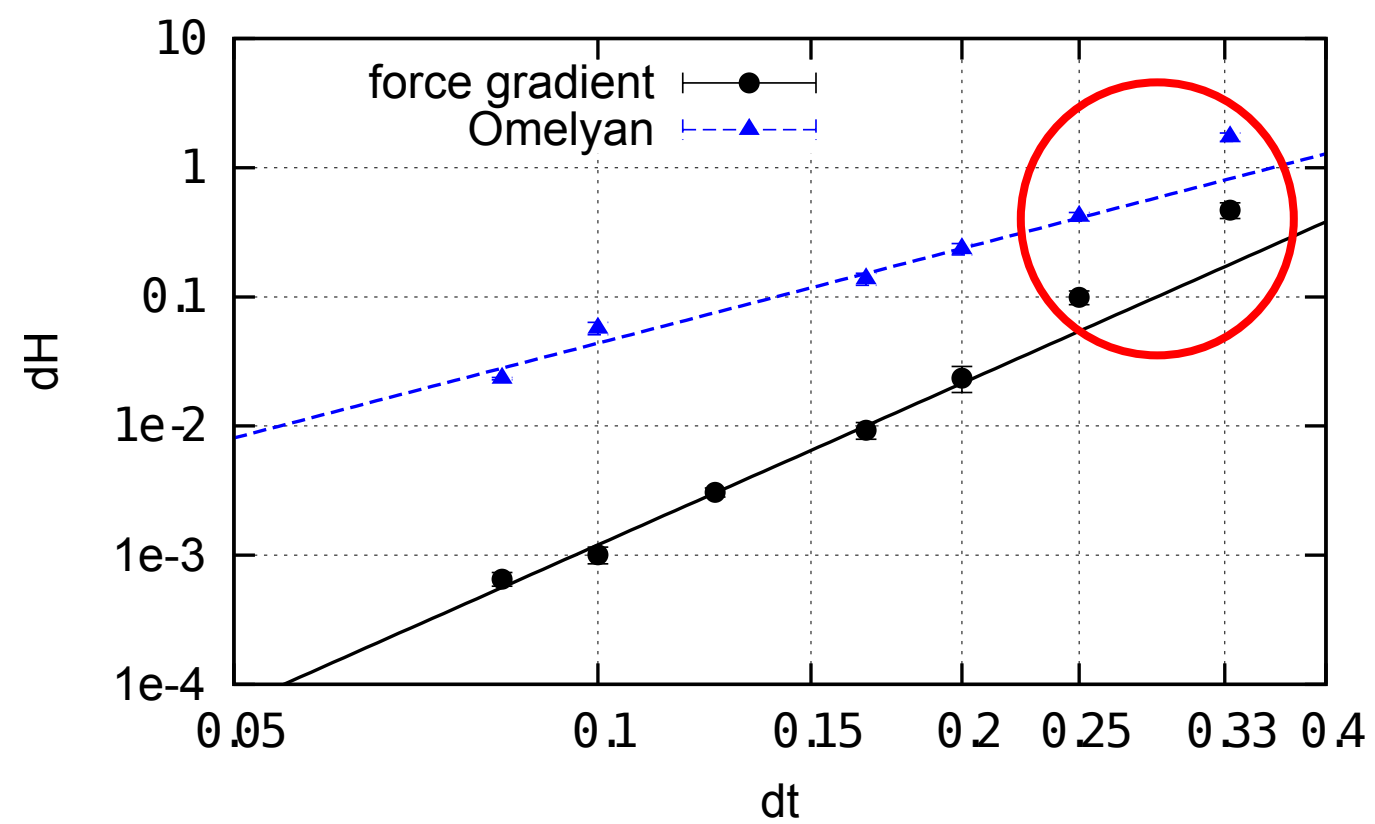

- For larger volumes, where $\delta H$ grows with volume, force gradient may be helpful

- Tests on $48^{3} \times 64 \times 16$ with $220 \mathrm{Mev}$ pions using force gradient and retuning Hasenbush masses, 184 minutes/accepted configuration went down to 108 minutes/accepted configuration. 


\section{MADWF Solver}

- Other chiral fermion formulations may achieve a smaller $\mathrm{m}_{\text {res }}$ for smaller $\mathrm{L}_{\mathrm{s}}$

- Mobius is one example: similar to DWF, but same $\mathrm{m}_{\text {res }}$ for $\sim \mathrm{L}_{\mathrm{s}} / 2$

- We have many simulations at different lattice spacings to put into our global fits, so not easy to change actions. May also change topological tunneling, ...

- Idea: use Mobius fermions to accelerate the linear solver for DWF

$$
\text { MADWF }=\text { Mobius Accelerated DWF }
$$

- Developed and implemented by Hantao Yin

- Gives $2 \times$ or more speed-up in quark propagator solves for current measurements.

\begin{tabular}{|c|c|c|c|c|c|}
\hline \multicolumn{3}{|c|}{ Direct CG solve } & \multicolumn{3}{|c|}{ Möbius Accelerated DWF } \\
\hline operation & Op. count & time $(\mathrm{s})$ & operation & Op. count & time $(\mathrm{s})$ \\
\hline \multirow{11}{*}{ CG solve(1e-10) } & \multirow{11}{*}{$11290 * 32$} & \multirow{11}{*}{2672} & initial DWF(1e-2) & $16^{*} 32$ & 3 \\
\hline & & & DWF $(1 \mathrm{e}-5)$ & $121^{*} 32$ & 28 \\
\hline & & & Möbius(1e-5) & $4447^{*} 12$ & 275 \\
\hline & & & DWF(1e-5) & $106 * 32$ & 25 \\
\hline & & & DWF $(1 e-5)$ & $101 * 32$ & 24 \\
\hline & & & Möbius(1e-5) & $4581^{*} 12$ & 284 \\
\hline & & & DWF $(1 \mathrm{e}-5)$ & $106^{*} 32$ & 25 \\
\hline & & & DWF(1e-5) & $102^{*} 32$ & 24 \\
\hline & & & Möbius(1e-5) & $4775^{*} 12$ & 296 \\
\hline & & & DWF(1e-5) & $106^{*} 32$ & 25 \\
\hline & & & final DWF $(1 \mathrm{e}-10)$ & $517^{*} 32$ & 121 \\
\hline total & $3.61 \mathrm{e} 5$ & 2672 & total & $2.03 \mathrm{e} 5$ & 1138 \\
\hline
\end{tabular}

Table 1: Comparison of MADWF CG solver with a regular (zero started) CG solver. $L=32, L^{\prime}=12$, with

$b=1.841556, c=0.841556$. Data obtained from a 512 node partition on BG/P, both solve to 1e-10. 


\section{Scaling at unphysical light quark mass}

Compare

- DWF+I: $1 / \mathrm{a}=2.28 \mathrm{GeV}$

- DWF+I: $1 / \mathrm{a}=1.73 \mathrm{GeV}$ (Phys. Rev. D83 (2011) 074508)

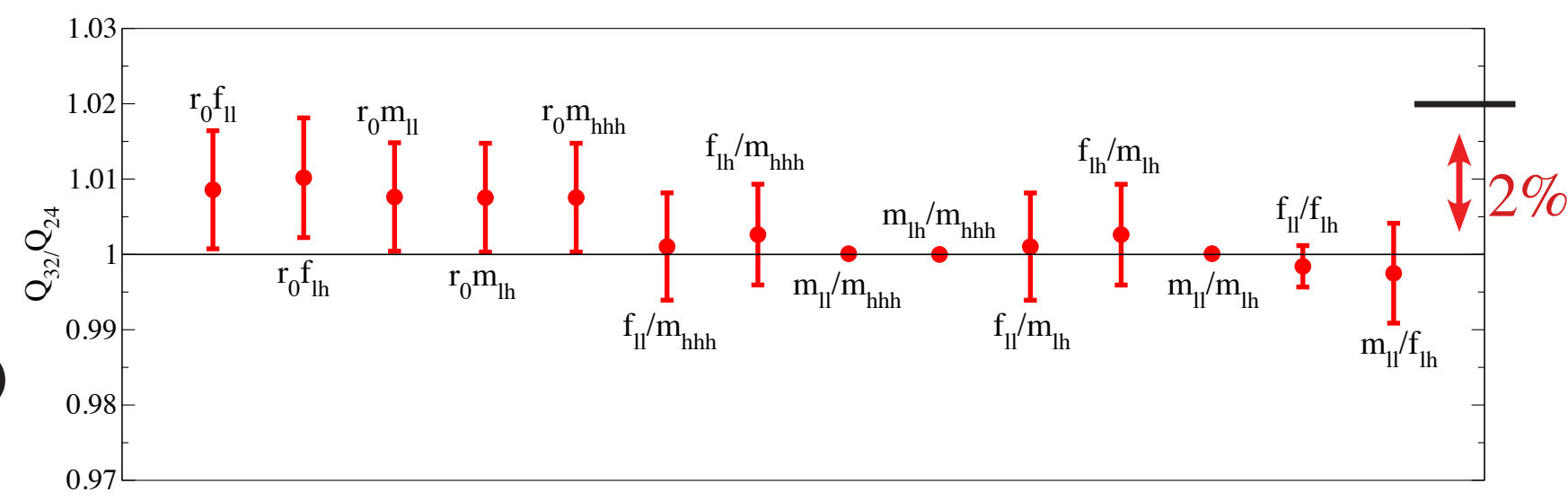

Compare

- DWF+I: $1 / \mathrm{a}=2.28 \mathrm{GeV}$

- DWF+ID: $1 / \mathrm{a}=1.37 \mathrm{GeV}$ (RBC/UKQCD to appear)

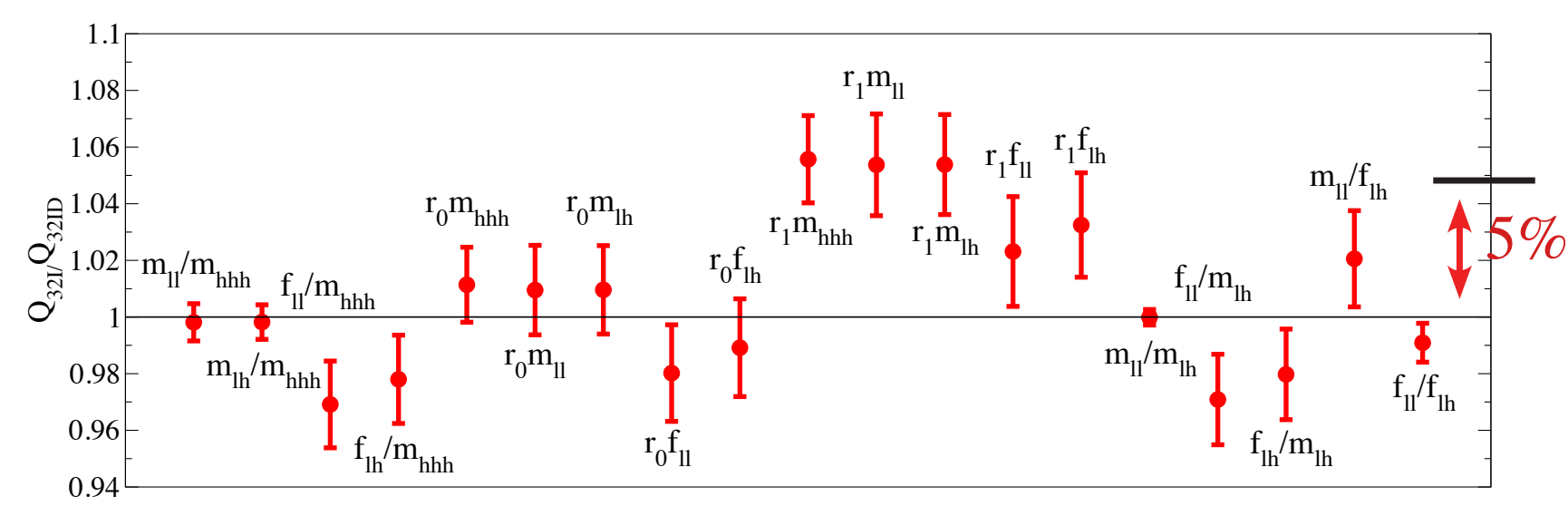

See few percent scaling errors from $1 / a=1.73 \mathrm{GeV} \rightarrow \infty$, with larger $O(5 \%)$ errors from $1 / \mathrm{a}$ $=1.37 \mathrm{GeV}$ 


\section{Parameters in DWF+I and DWF+ID Global Fits}

- Simultaneous fit to $\mathrm{m}_{\pi}^{2}, \mathrm{~m}_{\mathrm{K}}^{2}, \mathrm{f}_{\pi}, \mathrm{f}_{\mathrm{K}}$, and $\mathrm{m}_{\Omega}$

- $\mathrm{m}_{\pi}, \mathrm{m}_{\mathrm{K}}$ and $\mathrm{m}_{\Omega}$ chosen to be quantities without $\mathrm{O}\left(\mathrm{a}^{2}\right)$ corrections

- Parameters in $\mathrm{SU}(2)$ chiral expansion:

$$
\begin{array}{ll}
* & \mathrm{~m}_{\pi}^{2} \text { and } \mathrm{f}_{\pi}: 8 \text { parameters }-2 \mathrm{LO}, 4 \mathrm{NLO}, 2 \mathrm{O}\left(\mathrm{a}^{2}\right) \\
* & \mathrm{~m}_{\mathrm{K}}^{2} \text { and } \mathrm{f}_{\mathrm{K}}: 6 \text { parameters }-2 \mathrm{LO}, 4 \mathrm{NLO}, 2 \mathrm{O}\left(\mathrm{a}^{2}\right) \\
* & \mathrm{~m}_{\Omega}: 1 \mathrm{LO}, 1 \mathrm{NLO} \\
* & \text { Total: } 18 \text { parameters }
\end{array}
$$

- Fits also determine

* 3 lattice spacings

* 2 ratios of light quark mass renormalization factors

* 2 ratios of strange quark mass renormalization factors

* $\mathrm{m}_{\mathrm{s}}$ 


\section{Global Fits to Multiple Ensembles}

- Fit $\mathrm{m}_{\pi}^{2}, \mathrm{f}_{\pi}, \mathrm{m}_{\mathrm{K}}{ }^{2}, \mathrm{f}_{\mathrm{K}}$ and $\mathrm{m}_{\Omega}$ to an expansion in powers of $\mathrm{a}^{2}$ and $\mathrm{m}_{1}$, including SU(2) logs where appropriate. Examples are

$$
\begin{gathered}
m_{l l}^{2}=\chi_{l}\left[1+c_{B} a^{2}\right]+\chi_{l} \cdot\left\{\frac{16}{f^{2}}\left(\left(2 L_{8}^{(2)}-L_{5}^{(2)}\right)+2\left(2 L_{6}^{(2)}-L_{4}^{(2)}\right)\right) \chi_{l}+\frac{1}{16 \pi^{2} f^{2}} \chi_{l} \log \frac{\chi_{l}}{\Lambda_{\chi}^{2}}\right\} \\
f_{l l}=f\left[1+c_{f} a^{2}\right]+f \cdot\left\{\frac{8}{f^{2}}\left(2 L_{4}^{(2)}+L_{5}^{(2)}\right) \chi_{l}-\frac{\chi_{l}}{8 \pi^{2} f^{2}} \log \frac{\chi_{l}}{\Lambda_{\chi}^{2}}\right\} .
\end{gathered}
$$

- Note different $O\left(a^{2}\right)$ coefficients used for DWF+I and DWF+ID

- Fit all partially quenched data, including SU(2) ChPT finite volume corrections in fit

- Reweight data from simulation $\mathrm{m}_{\mathrm{h}}$ to self-consistently determined $\mathrm{m}_{\mathrm{s}}$ (Jung)

- Interpolate valence propagators to self-consistently determined $\mathrm{m}_{\mathrm{s}}$

- Use $\mathrm{m}_{\pi} \mathrm{m}_{\mathrm{K}}$ and $\mathrm{m}_{\Omega}$ set scale. 


\section{$\mathrm{m}_{\pi}^{2} / \mathrm{m}_{\mathrm{f}}$ versus $\mathrm{m}_{\mathrm{f}}$}

Degenerate $\mathrm{m}_{\pi}^{2} / \mathrm{m}_{\mathrm{x}}$ versus $\mathrm{m}_{\mathrm{x}}\left(=\mathrm{m}_{\mathrm{y}}\right)$

$\mathrm{NLO}+$ FVglobal fit, physical match point $\mathrm{NLO}+\mathrm{FV}$ curves plotted, original data

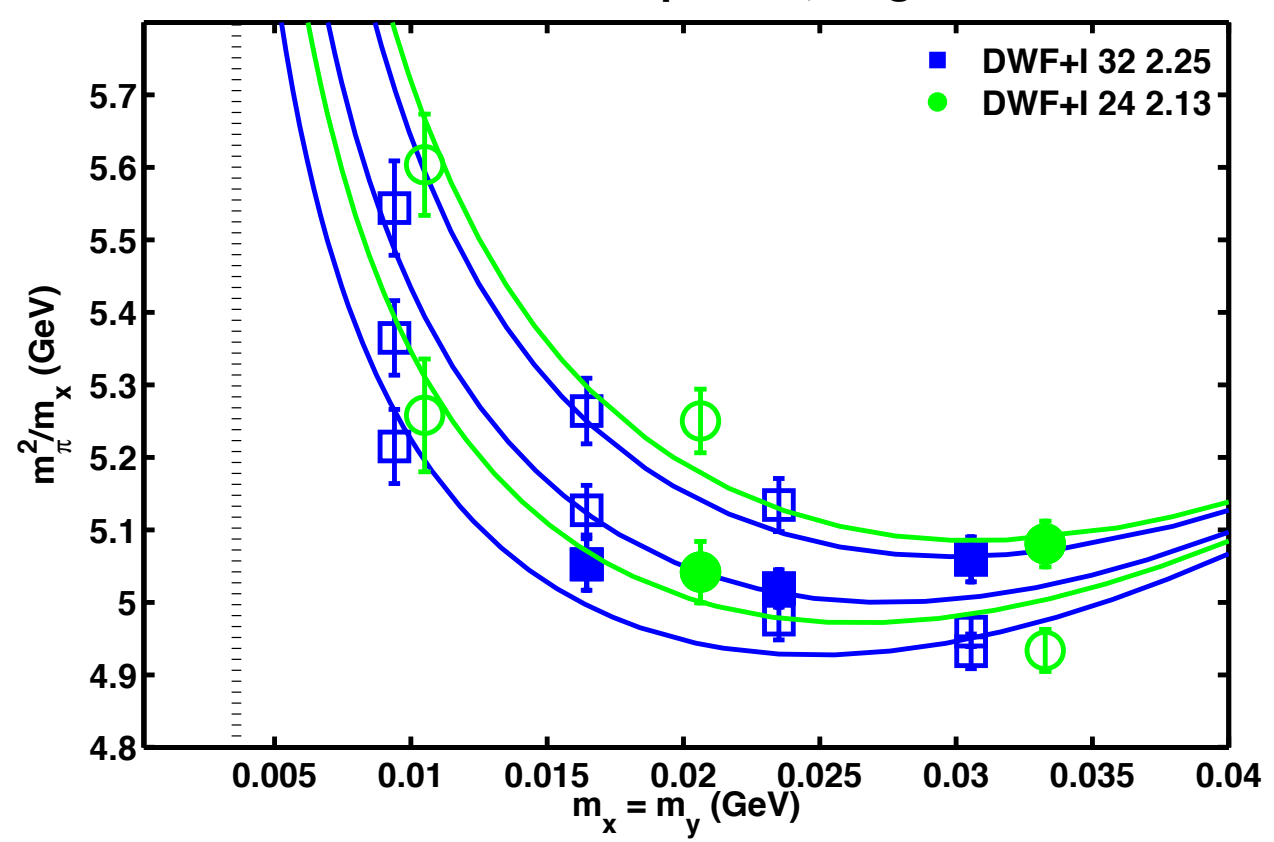

- Early fits from partial DWF+ID dataset

- Data consistent with chiral logarithms

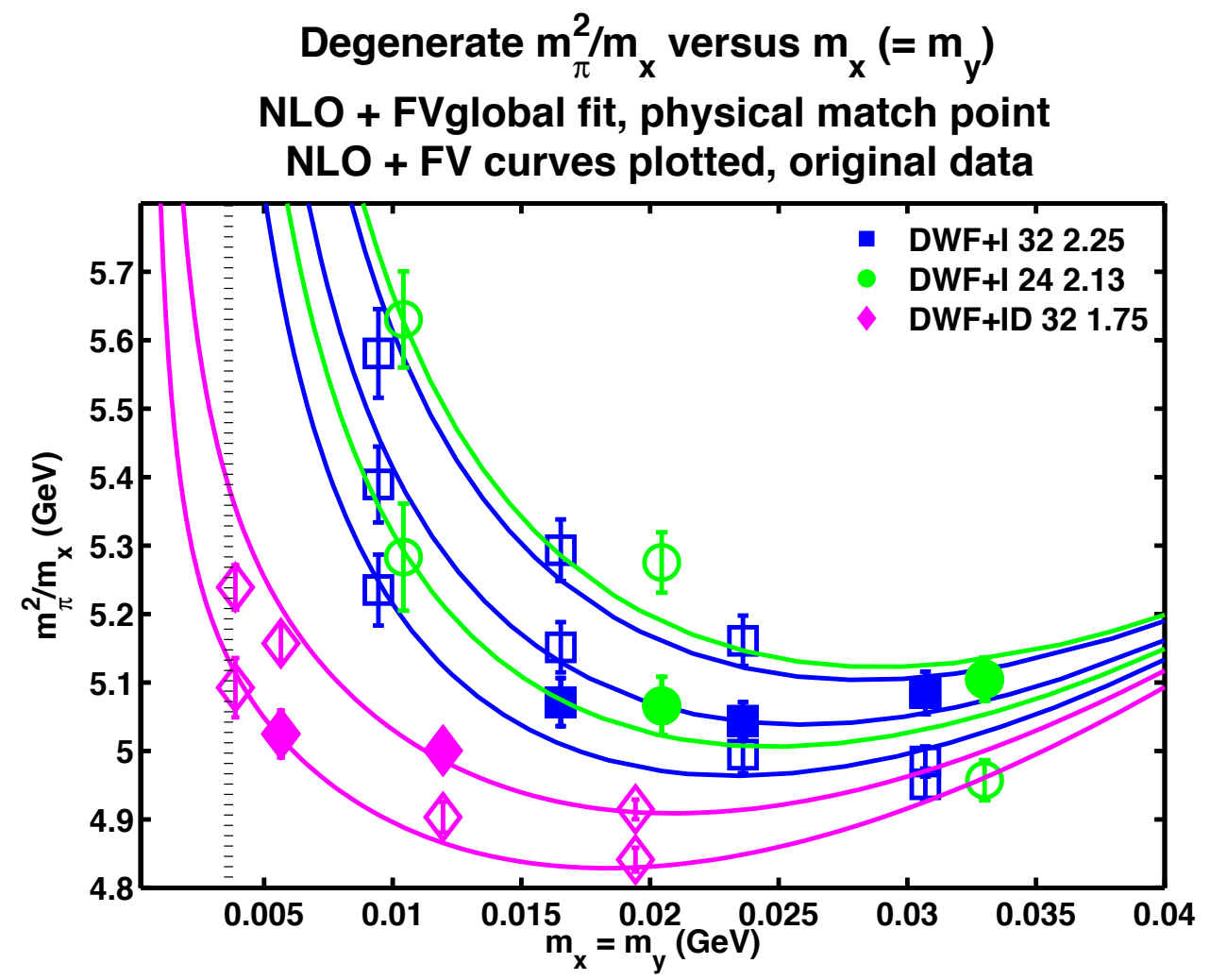

Degenerate $\mathrm{m}_{\pi}^{2} / \mathrm{m}_{x}$ versus $\mathrm{m}_{\mathrm{x}}\left(=\mathrm{m}_{\mathrm{y}}\right)$ $\mathrm{NLO}+$ FVglobal fit, physical match point NLO + FV curves plotted, original data 


\section{$\mathrm{m}_{\pi}^{2} / \mathrm{m}_{\mathrm{f}}$ versus $\mathrm{m}_{\mathrm{f}}$}
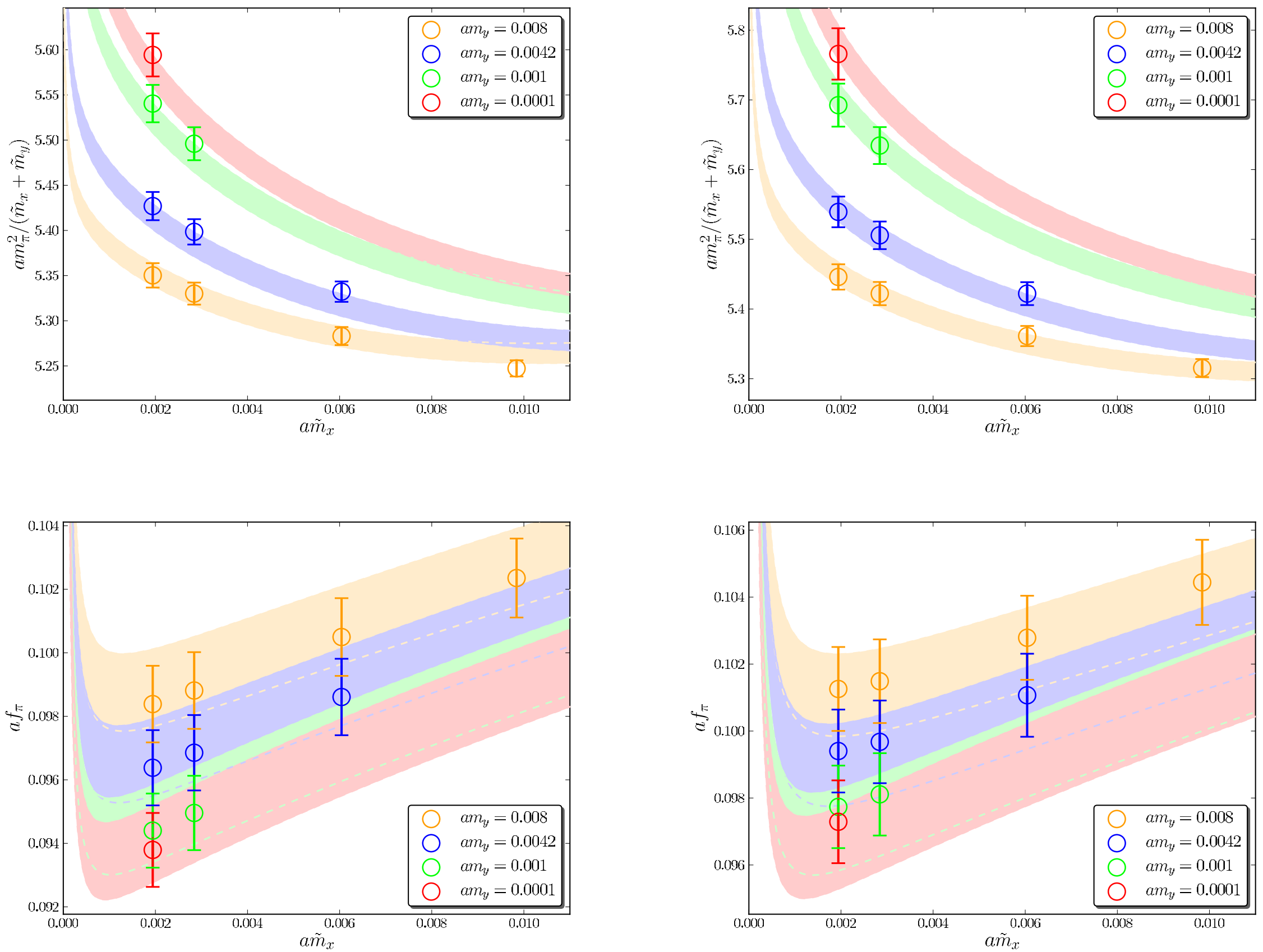


\section{Some physical results}

\begin{tabular}{|c|c|}
\hline DWF+I & DWF+I and DWF+ID \\
\hline$f_{\pi}^{\text {continuum }}=124(2)(5) \mathrm{MeV}$ & $f_{\pi}=127.1(2.7)(0.7)(2.5) \mathrm{MeV}$, \\
$f_{K}^{\text {continuum }}=149(2)(4) \mathrm{MeV}$ & $f_{K}=152.4(3.0)(0.1)(1.5) \mathrm{MeV}$, \\
$\left(f_{K} / f_{\pi}\right)^{\text {continuum }}=1.204(7)(25)$, & $f_{K} / f_{\pi}=1.1991(116)(69)(116)$. \\
\hline$m_{u d}^{\overline{\mathrm{MS}}}(2 \mathrm{GeV})=(3.59 \pm 0.21) \mathrm{MeV}$ & $m_{u / d}(\overline{\mathrm{MS}}, 3 \mathrm{GeV})=3.05(8)(6)(1)(4) \mathrm{MeV}$ \\
$m_{s}^{\overline{\mathrm{MS}}}(2 \mathrm{GeV})=(96.2 \pm 2.7) \mathrm{MeV}$ & $m_{s}(\overline{\mathrm{MS}}, 3 \mathrm{GeV})=83.6(1.7)(0.7)(0.4)(1.0) \mathrm{MeV}$ \\
$\frac{m_{s}}{m_{u d}}=26.8(0.8)_{\text {stat }}(1.1)_{\text {sys }}$. & $\frac{m_{s}}{m_{u / d}}=27.36(39)(30)(22)(0)$ \\
\hline$B_{K}(\overline{\mathrm{MS}}, 3 \mathrm{GeV})=0.529(5)(15)(2)(11)$ & $B_{K}(\overline{\mathrm{MS}}, 3 \mathrm{GeV})=0.535(8)(7)(3)(11)$ \\
& $(\mathrm{stat}, \mathrm{chiral}$, finite $\mathrm{V}$, pert. theory $)$ \\
\hline
\end{tabular}

Chiral extrapolation errors markedly reduced 


\section{Non-perturbative Renormalization}

- Many of the quantities discussed in this talk require renormalization

- Needed to match to continuum schemes where low energy effective Hamiltonians are determined to $\mathrm{N}^{\mathrm{n}} \mathrm{LO}$ and renormalized at some scale $\mu$

- Schrodinger functional and RI-MOM NPR schemes well understood

- $\quad$ RI-MOM is primarily used for kaons - simplicity?

- Recent improvements in RI-MOM

* Non-exceptional symmetric momenta - RI-SMOM

* Twisted b.c. to allow selection of continuous range of momenta

* Volume sources reduce statistical error dramatically

* Compute non-perturbative continuum running from fine lattices, use for coarse lattices (Rudy Arthur, Peter, Boyle, PRD 83 (2011) 114511).

* Implemented for $K \rightarrow \pi \pi$ (N. Garron) for RBC-UKQCD

simulations on coarse lattices $(1 / \mathrm{a}=1.37 \mathrm{GeV})$.

$$
\lim _{a_{1} \rightarrow 0} \underbrace{\left[Z\left(\mu_{1}, a_{1}\right) Z^{-1}\left(\mu_{0}, a_{1}\right)\right]}_{\text {fine lattice }} \times \underbrace{Z\left(\mu_{0}, a_{0}\right)}_{\text {coarse lattice }}=Z\left(\mu_{1}, a_{0}\right)
$$




\section{Some $K \rightarrow \pi \pi$ physics}

A neutral kaon beam will contain only long-lived $\mathrm{K}_{\mathrm{L}}$ far enough from source.

Dominant decay is $K_{L} \rightarrow \pi \pi \pi$, small phase space gives long lifetime.

Experiments measure decay amplitudes for $\mathrm{K}_{\mathrm{L}}$ compared to $\mathrm{K}_{\mathrm{S}}$ (2 complex numbers).

$$
\eta_{+-}=\frac{A\left(K_{L} \rightarrow \pi^{+} \pi^{-}\right)}{A\left(K_{S} \rightarrow \pi^{+} \pi^{-}\right)} \quad \eta_{00}=\frac{A\left(K_{L} \rightarrow \pi^{0} \pi^{0}\right)}{A\left(K_{S} \rightarrow \pi^{0} \pi^{0}\right)}
$$

If $\mathrm{K}_{\mathrm{L}}$ is CP eigenstate, $\eta_{+-}=\eta_{00}=0$.

Difference in $\eta_{+-}$and $\eta_{00}$ dominantly due to difference in isospin of final state.

Consider amplitudes to decay to states of definite isospin

$$
A\left(K^{0} \rightarrow \pi \pi(I)\right)=A_{I} \exp \left(i \delta_{I}\right) \quad\left(\text { I labels isospin, } \delta_{I} \text { is } \pi \pi \text { phase shift }\right)
$$

Parameters appearing in description of neutral kaon system

$$
\begin{aligned}
\eta_{+-}=\varepsilon+\frac{\varepsilon^{\prime}}{1+\omega e^{i \theta^{\prime}}} \approx \varepsilon+\varepsilon^{\prime} & \eta_{00}=\varepsilon-\frac{2 \varepsilon^{\prime}}{1-\sqrt{2} \omega e^{i \theta^{\prime}}} \approx \varepsilon-2 \varepsilon^{\prime} \\
\varepsilon=\tilde{\varepsilon}+i\left(\frac{\operatorname{Im}\left(A_{0}\right)}{\operatorname{Re}\left(A_{0}\right)}\right) & \mathcal{E}^{\prime}=\frac{\omega}{\sqrt{2}} e^{i \theta^{\prime}}\left(\frac{\operatorname{Im}\left(A_{2}\right)}{\operatorname{Re}\left(A_{2}\right)}-\frac{\operatorname{Im}\left(A_{0}\right)}{\operatorname{Re}\left(A_{0}\right)}\right) \\
\omega=\frac{\operatorname{Re}\left(A_{2}\right)}{\operatorname{Re}\left(A_{0}\right)}=0.045 & \theta=\tan ^{-1}\left[\frac{2 \Delta M}{\Gamma_{1}-\Gamma_{2}}\right]=43.67 \pm 0.14^{\circ} \\
\theta^{\prime}=\delta_{2}-\delta_{0}+\pi / 2=(43 \pm 6)^{\circ} &
\end{aligned}
$$




\section{$B_{K}$ and corrections to $\varepsilon$}

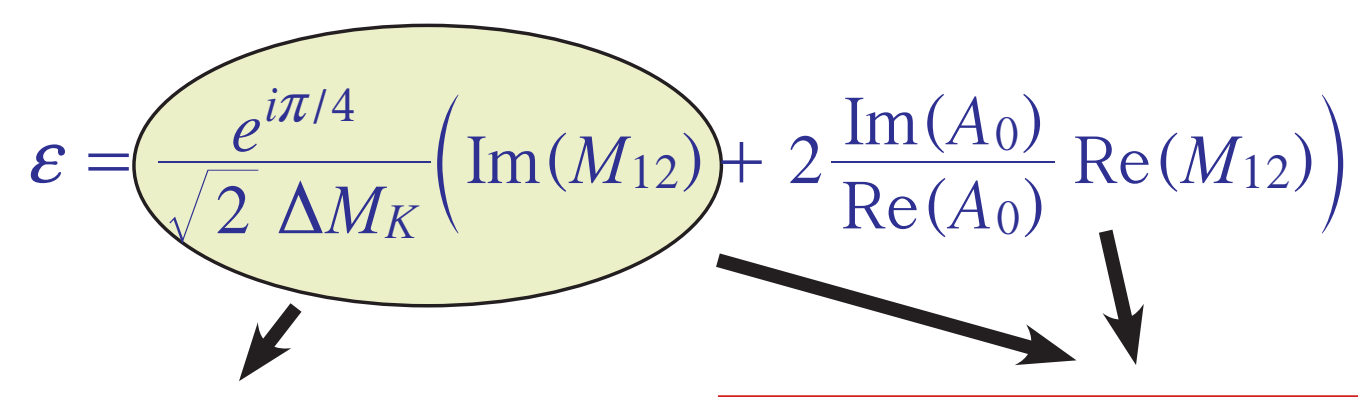

This has been focus, $O\left(G_{F}^{2}\right)$ contribution from $O^{\Delta S=2}$ operator

$$
B_{K}(\mu) \equiv \frac{\left\langle\bar{K}^{0}\left|(\bar{s} d)_{V-A}(\bar{s} d)_{V-A}\right| K^{0}\right\rangle}{\frac{8}{3} f_{K}^{2} m_{K}^{2}}
$$

Long distance physics hep-ph/0201071 (page 58, Nierste) Buras, Guadagnoli (PRD 78 (2008) 033005 Buras, Guadagnoli, Isidori

(PLB 688 (2010) 309

$$
\begin{aligned}
M_{12}= & \frac{1}{2 m_{K}}\left\langle K^{0}\left|H^{|\Delta S|=2}\right| \bar{K}^{0}\right\rangle-\operatorname{Disp} \frac{i}{4 m_{K}} \int \mathrm{d}^{4} x\left\langle K^{0}\left|H^{|\Delta S|=1}(x) H^{|\Delta S|=1}(0)\right| \bar{K}^{0}\right\rangle \\
\Gamma_{12} & =\operatorname{Abs} \frac{i}{2 m_{K}} \int \mathrm{d}^{4} x\left\langle K^{0}\left|H^{|\Delta S|=1}(x) H^{|\Delta S|=1}(0)\right| \bar{K}^{0}\right\rangle \\
& =\frac{1}{2 m_{K}} \sum_{f}(2 \pi)^{4} \delta^{4}\left(p_{K}-p_{f}\right)\left\langle K^{0}\left|H^{|\Delta S|=1}\right| f\right\rangle\left\langle f\left|H^{|\Delta S|=1}\right| \bar{K}^{0}\right\rangle \simeq \frac{1}{2 m_{K}} A_{0}^{*} \bar{A}_{0}
\end{aligned}
$$

- Norman Christ: measure these by extending Lellouch-Lüscher finite volume methods

- Jianglei Yu: numerical investigation of signal and renormalization for connected graphs 
$B_{K}$

$$
\varepsilon=\frac{e^{i \pi / 4}}{\sqrt{2} \Delta M_{K}}\left(\operatorname{Im}\left(M_{12}\right)+2 \frac{\operatorname{Im}\left(A_{0}\right)}{\operatorname{Re}\left(A_{0}\right)} \operatorname{Re}\left(M_{12}\right)\right)=\kappa_{\varepsilon} \frac{e^{i \phi_{\varepsilon}}}{\sqrt{2}}\left[\frac{\operatorname{Im}\left(M_{12}^{O^{\Delta s=2}}\right)}{\Delta m_{K}}\right]
$$
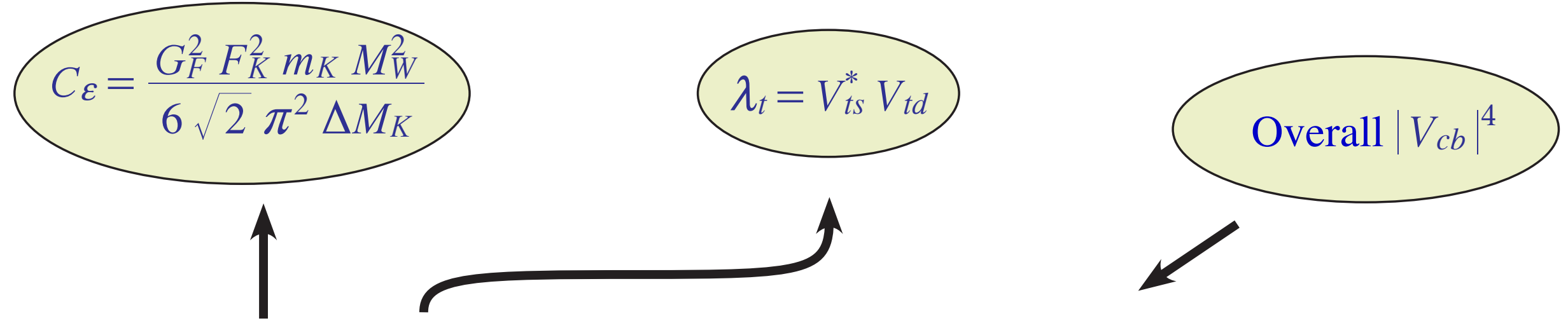

$\varepsilon_{K}=\kappa_{\varepsilon} C_{\varepsilon} \hat{B}_{K} \operatorname{Im}\left(\boldsymbol{\lambda}_{t}\right)\left\{\operatorname{Re}\left(\boldsymbol{\lambda}_{c}\right)\left[\eta_{1} S_{0}\left(x_{c}\right)-\eta_{3} S_{0}\left(x_{c}, x_{t}\right)\right]-\operatorname{Re}\left(\boldsymbol{\lambda}_{t}\right) \eta_{2} S_{0}\left(x_{t}\right)\right\} e^{i \pi / 4}$

$\downarrow$

$0.94 \pm 0.02$

Buras,Guadagnoli, Isidori

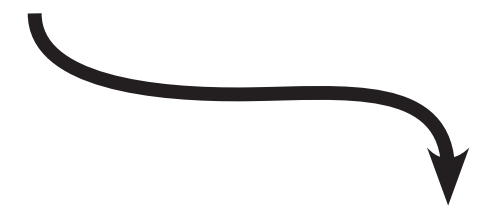

$$
\eta_{3}^{N L O} \equiv \eta_{c t}^{N L O} \equiv 0.457 \pm 0.073
$$$$
\eta_{3}^{N N L O} \equiv \eta_{c t}^{N N L O} \equiv 0.496 \pm 0.047
$$

Brod and Gorbahn, PRD 82094026 (2010) $3 \%$ overall change in $\varepsilon$ 


\section{$K \rightarrow \pi \pi$ Decays via Penguins}
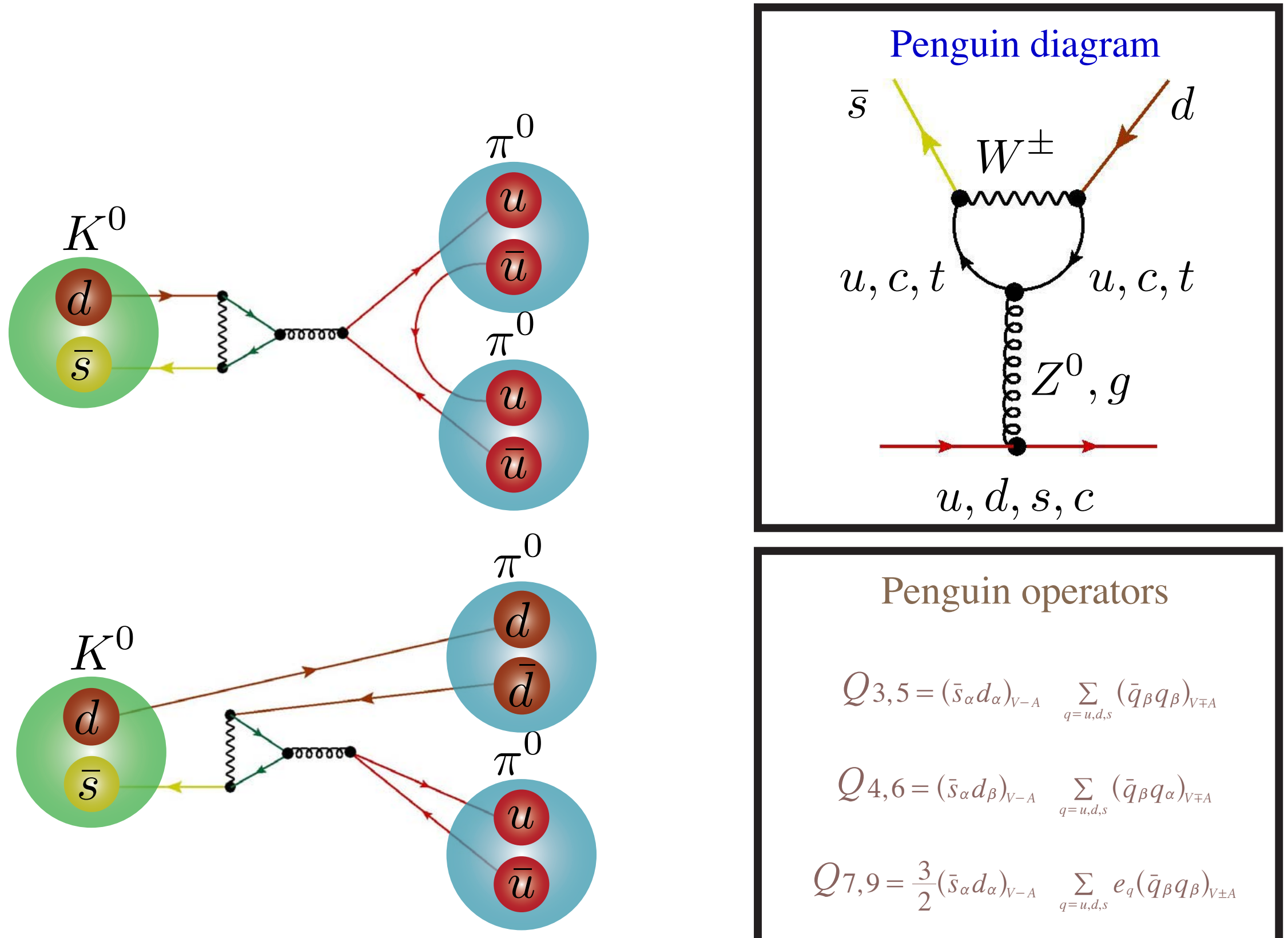

Penguin operators

$$
Q_{3,5}=\left(\bar{s}_{\alpha} d_{\alpha}\right)_{V-A} \sum_{q=u, d, s}\left(\bar{q}_{\beta} q_{\beta}\right)_{V \mp A}
$$$$
Q_{4,6}=\left(\bar{s}_{\alpha} d_{\beta}\right)_{V-A} \sum_{q=u, d, s}\left(\bar{q}_{\beta} q_{\alpha}\right)_{V \mp A}
$$

$Q_{7,9}=\frac{3}{2}\left(\bar{s}_{\alpha} d_{\alpha}\right)_{V-A} \sum_{q=u, d, s} e_{q}\left(\bar{q}_{\beta} q_{\beta}\right)_{V \pm A}$

$Q_{8,10}=\frac{3}{2}\left(\bar{s}_{\alpha} d_{\beta}\right)_{V-A} \sum_{q=u, d, s} e_{q}\left(\bar{q}_{\beta} q_{\alpha}\right)_{V \pm A}$ 


\section{Direct calculations of $K \rightarrow \pi \pi \Delta \mathrm{I}=3 / 2$ amplitudes}

- $\quad$ RBC-UKQCD DWF+ID (Iwasaki + DSDR gauge action) ensemble

$$
\mathcal{W}\left(M ; \epsilon_{f} ; \epsilon_{b}\right)=\frac{\operatorname{det}\left[D_{\mathcal{W}}\left(-M+i \epsilon_{b} \gamma^{5}\right)^{\dagger} D_{\mathcal{W}}\left(-M+i \epsilon_{b} \gamma^{5}\right)\right]}{\operatorname{det}\left[D_{\mathcal{W}}\left(-M+i \epsilon_{f} \gamma^{5}\right)^{\dagger} D_{\mathcal{W}}\left(-M+i \epsilon_{f} \gamma^{5}\right)\right]}=\prod_{i} \frac{\lambda_{i}^{2}+\epsilon_{f}^{2}}{\lambda_{i}^{2}+\epsilon_{b}^{2}}
$$

- $m_{\pi}^{\mathrm{dyn}}=170 \mathrm{MeV}, 32^{3} \times 64 \times 16$ lattice volume, $(4.60 \mathrm{fm})^{3}$ physical volume,

$1 / \mathrm{a}=1.37(2) \mathrm{GeV}(\mathrm{a}=0.146(2) \mathrm{fm}), m_{\mathrm{res}}^{M S}(\mu=2 \mathrm{GeV})=3.7 \mathrm{MeV}$

- $\quad m_{\pi}^{\mathrm{PQ}}=142(2) \mathrm{MeV}, m_{K}=508(9) \mathrm{MeV}, \vec{p}_{\pi}=199(4) \mathrm{MeV}$

- $\quad$ Physical decays have $m_{\pi}=140 \mathrm{MeV}, m_{K}=500 \mathrm{MeV}, \vec{p} \pi=200 \mathrm{MeV}$

Single wall source for $\pi$ 's on given lattice Multiple kaon locations, since inexpensive Results from 62 configurations

M. Lightman and E. Goode, Lattice 2010 M. Lightman, Columbia PhD thesis, 2011 E. Goode, talk Lattice 2011

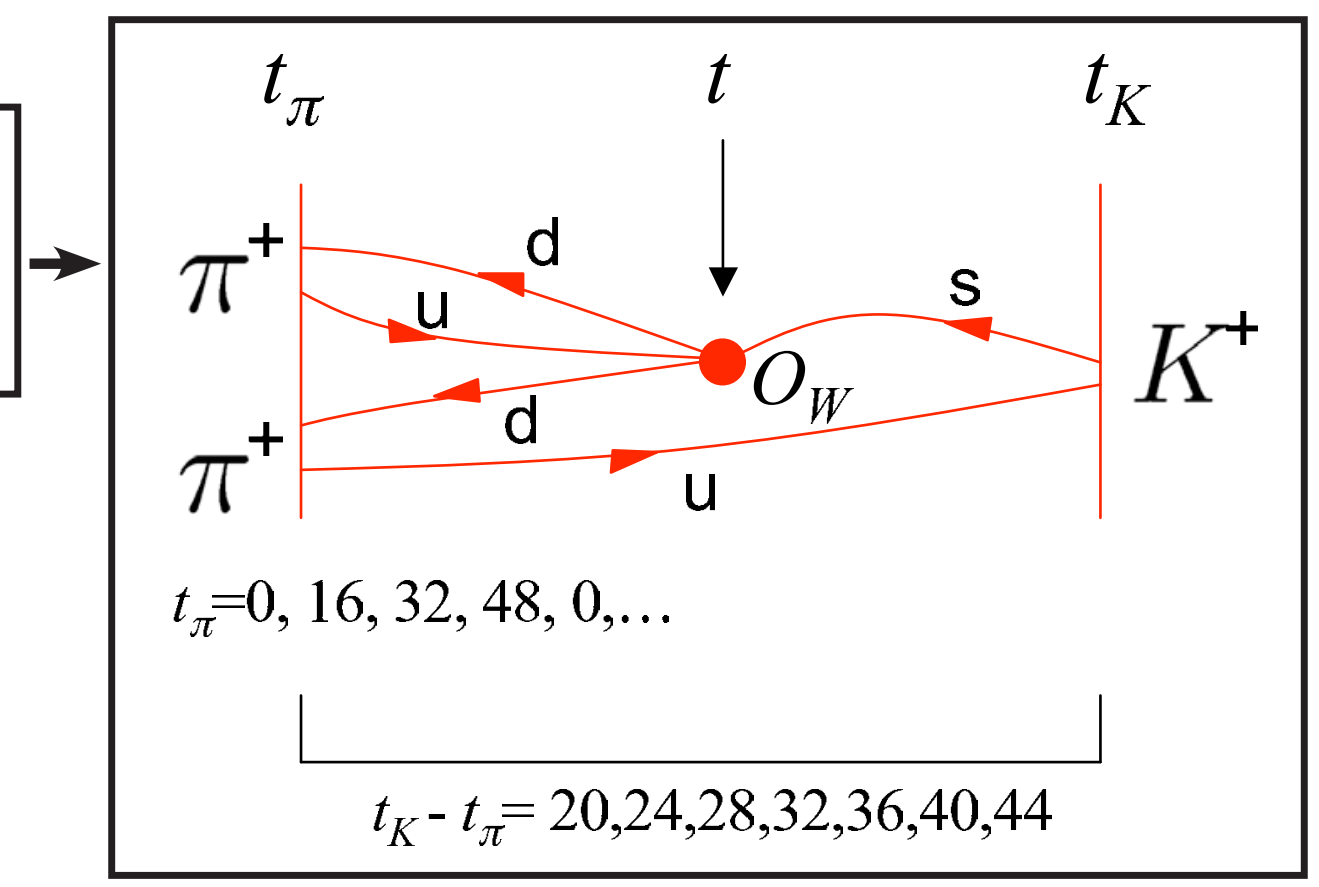




\section{Results for $K \rightarrow \pi \pi \Delta \mathrm{I}=3 / 2$ amplitudes}

- Simulations also done on quenched lattices, at many kinematic points, which help to estimate errors from extrapolations to physical kinematics on unquenched lattices

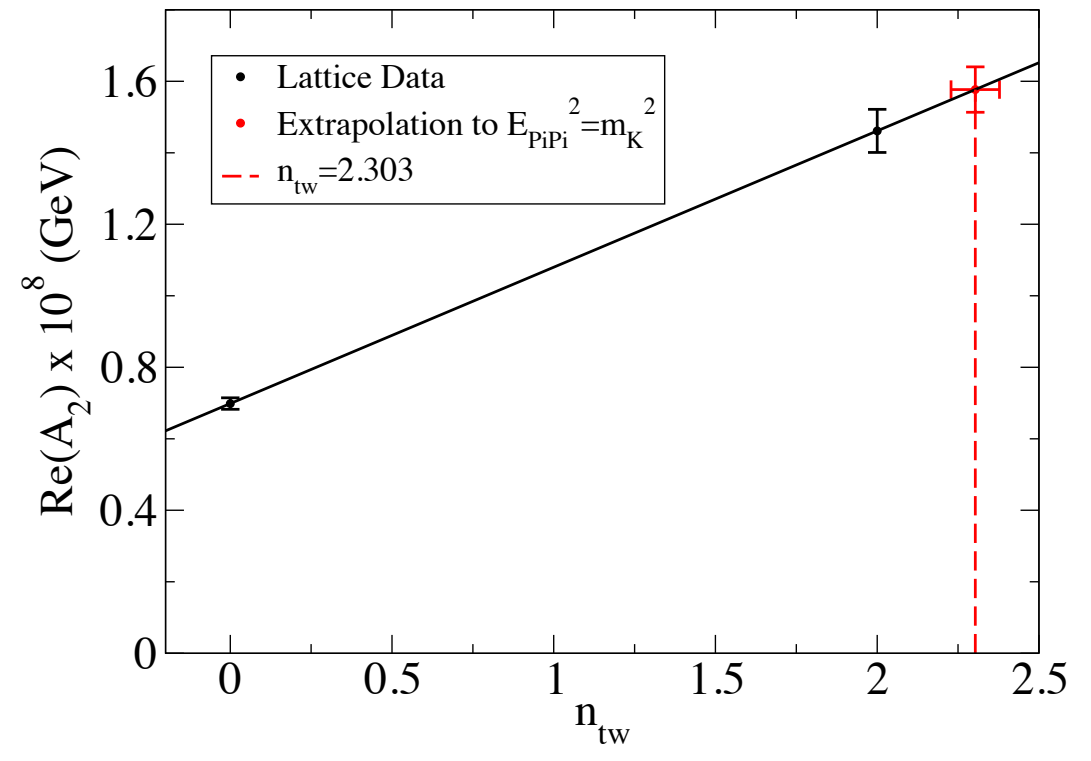

Extrapolation of $\operatorname{Re}\left(\mathrm{A}_{2}\right)$ to physical kinematics

\begin{tabular}{|c|c|c|}
\hline & $\operatorname{Re} A_{2}$ & $\operatorname{Im} A_{2}$ \\
\hline lattice artefacts & $15 \%$ & $15 \%$ \\
finite-volume corrections & $6.2 \%$ & $6.8 \%$ \\
partial quenching & $3.5 \%$ & $1.7 \%$ \\
renormalization & $1.7 \%$ & $4.7 \%$ \\
unphysical kinematics & $3.0 \%$ & $0.22 \%$ \\
derivative of the phase shift & $0.32 \%$ & $0.32 \%$ \\
Wilson coefficients & $7.1 \%$ & $8.1 \%$ \\
\hline Total & $18 \%$ & $19 \%$ \\
\hline
\end{tabular}

Error estimates (M. Lightman thesis)

- N. Garron and A. Lytle have NPR results now, using 4 RI-SMOM schemes.

- Reweighting to physical light dynamical mass

$$
\begin{gathered}
\operatorname{Re}\left(A_{2}\right)=1.397(81) \times 10^{-8} \mathrm{GeV} \stackrel{\text { reweighting }}{\longrightarrow} 1.46(15) \times 10^{-8} \mathrm{GeV} \\
\operatorname{Im}\left(A_{2}\right)=-5.65(31) \times 10^{-13} \mathrm{GeV} \stackrel{\text { reweighting }}{\longrightarrow}-5.79(39) \times 10^{-13} \mathrm{GeV}
\end{gathered}
$$




\section{Results for $K \rightarrow \pi \pi \Delta \mathrm{I}=3 / 2$ amplitudes}

- 63 configurations analyzed, in ongoing calculation.

- $\quad$ PRL 108 (2012) 141601

$$
\begin{gathered}
\operatorname{Re} A_{2}=\left(1.436 \pm 0.062_{\text {stat }} \pm 0.258_{\text {syst }}\right) 10^{-8} \mathrm{GeV} \\
\operatorname{Im} A_{2}=-\left(6.83 \pm 0.51_{\text {stat }} \pm 1.30_{\text {syst }}\right) 10^{-13} \mathrm{GeV} \\
\frac{\operatorname{Im} A_{2}}{\operatorname{Re} A_{2}}=-4.76(37)_{\text {stat }}(81)_{\text {syst }} \times 10^{-5} \\
\operatorname{Re}\left(A_{2}\right)=1.484 \times 10^{-8} \mathrm{GeV} \text { (experiment) }
\end{gathered}
$$




\section{Some observations and opinions}

- With DWF (or Mobius) plus BGQ, 2+1 flavor simulations with $m_{\pi}=140 \mathrm{MeV}$ are underway

* $48^{3} \times 96 \times 32 \mathrm{DWF}+\mathrm{I}$ with $1 / \mathrm{a}=1.74 \mathrm{GeV}$ gives $(5.5 \mathrm{fm})^{3}$ box 70 time units/BGQ-rack-month $->500$ time units/BGQ-rack-month

* $64^{3} \times 128 \times 16 \mathrm{DWF}+\mathrm{I}$ with $1 / \mathrm{a}=2.28 \mathrm{GeV}$ gives $(5.5 \mathrm{fm})^{3}$ box $2 \mathrm{x}$ to $4 \mathrm{x}$ harder than $1 / \mathrm{a}=1.74 \mathrm{GeV}$

* Many hundreds of configurations with a few BGQ rack-years

* Ideal for many physics measurements

- No chiral extrapolations!

* Still interesting in their own right, for better determination of LEC's

* Might need even lighter pions to know more about convergence of ChPT

* Not an issue for real-world QCD physics

- Adding DSDR term gives viable action for finite temperature studies

- We have reached the point where $2+1$ flavor QCD with full continuum symmetries, physical pions, physical kaons and large volumes can be done! 

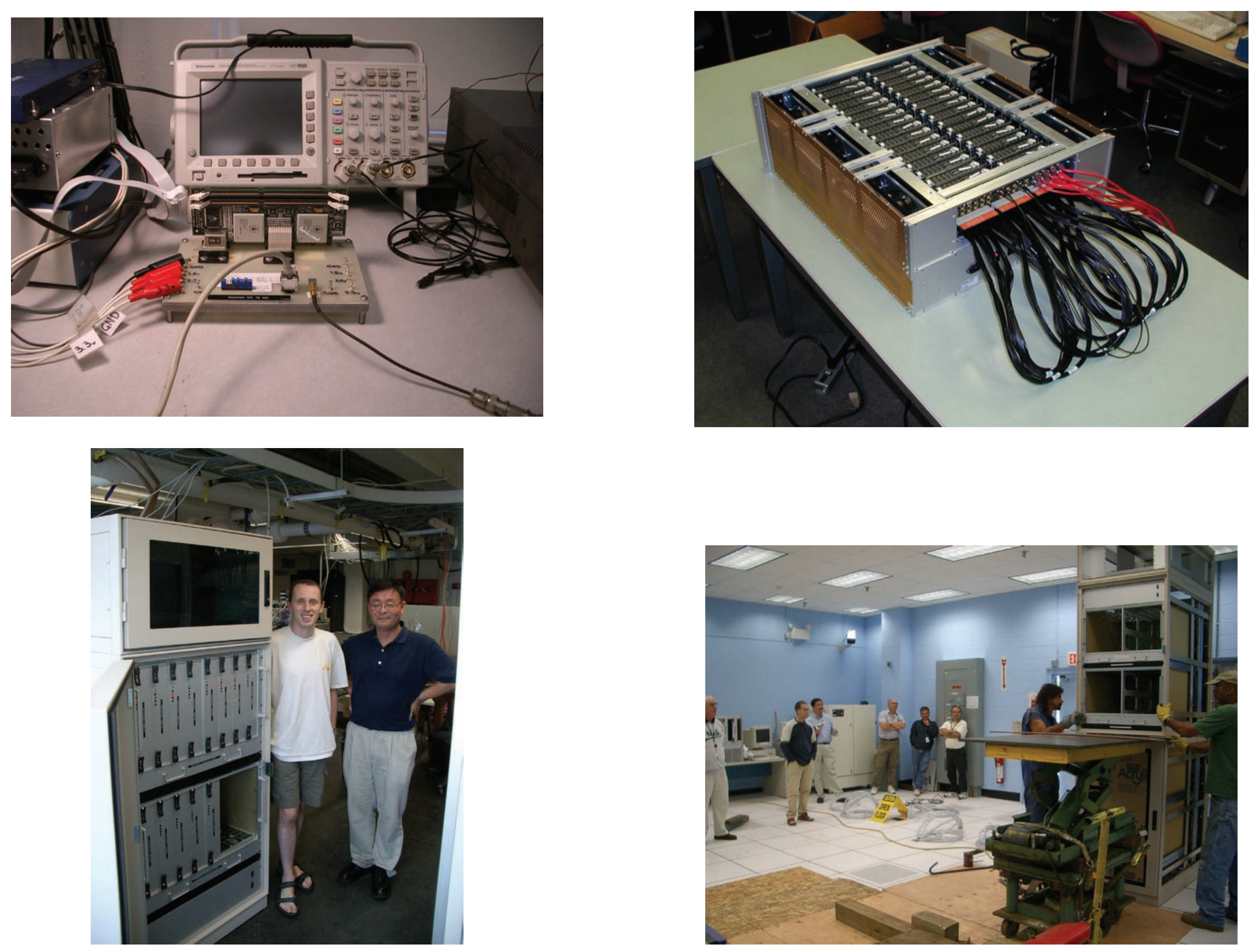

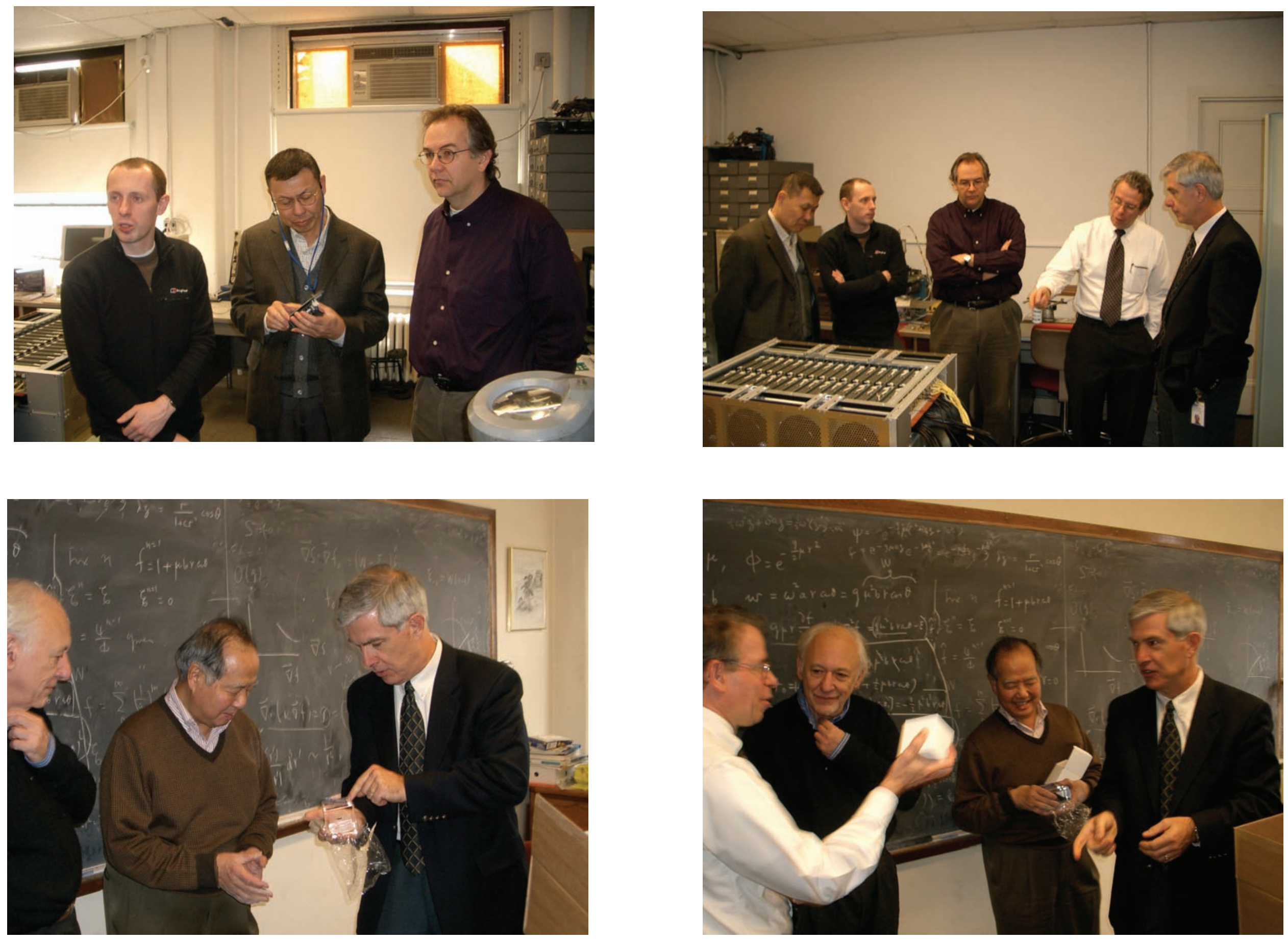

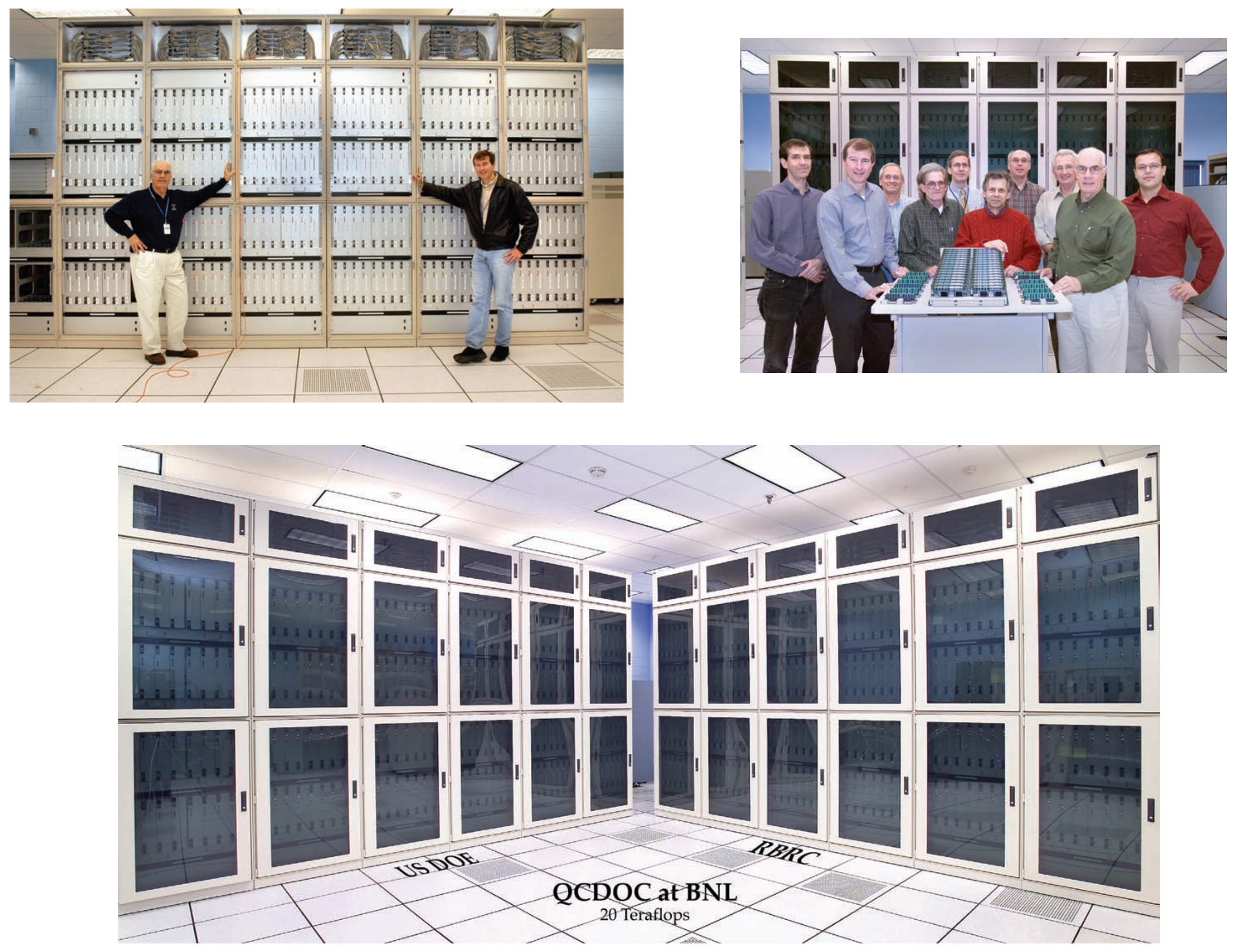
Shutdown of QCDOC, Sept. 18, 2011
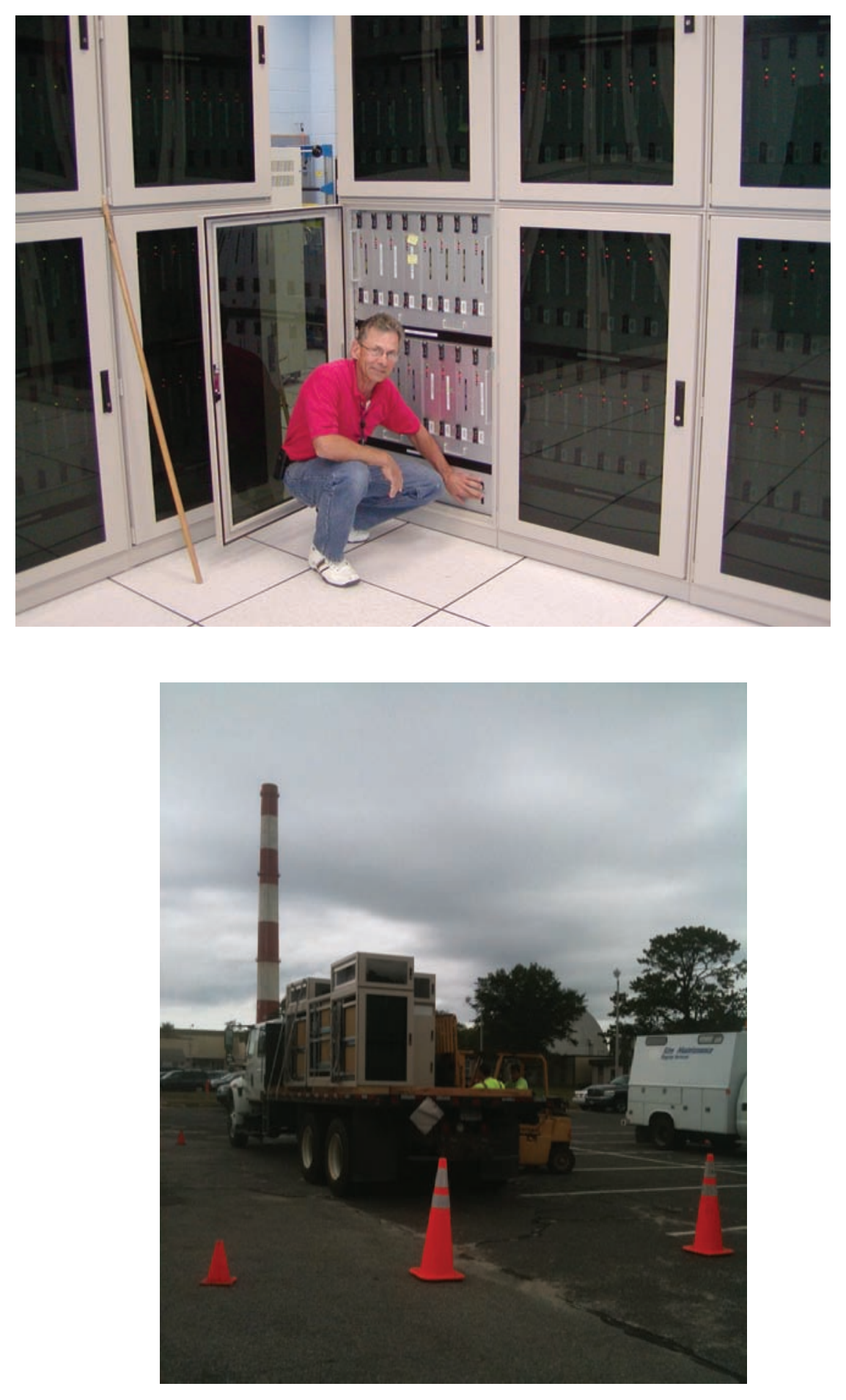

Last days of QCDSP, Nov. 2003
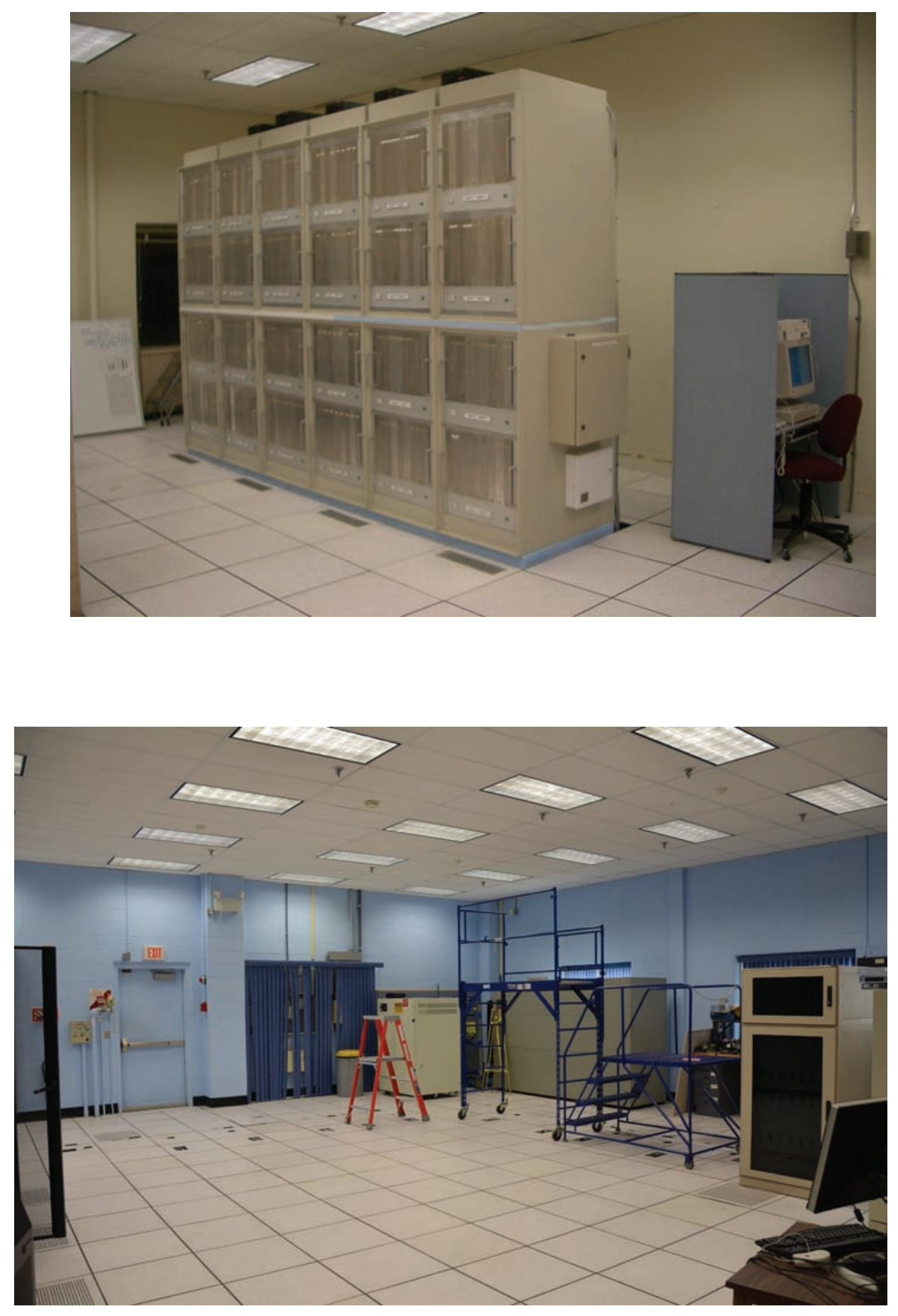


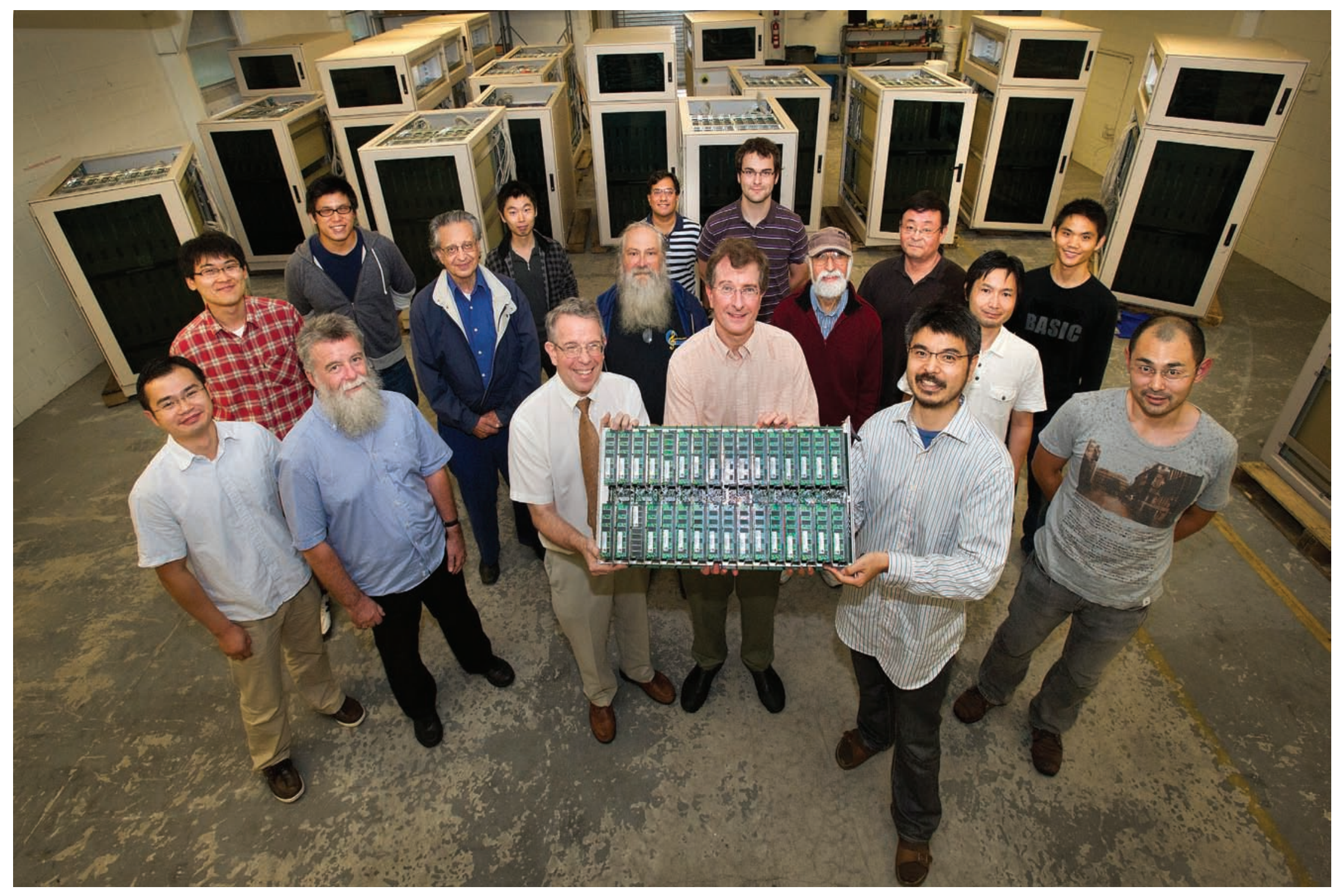




\section{BGQ at BNL}

- BNL currently has 3+ racks of preproduction BGQ hardware

* 1 rack is owned by BNL

* 2 complete racks are owned by the RIKEN-BNL Research Center (RBRC)

* A fourth partially populated RBRC rack will be used to hold a few small BGQ partitions for code development and testing.

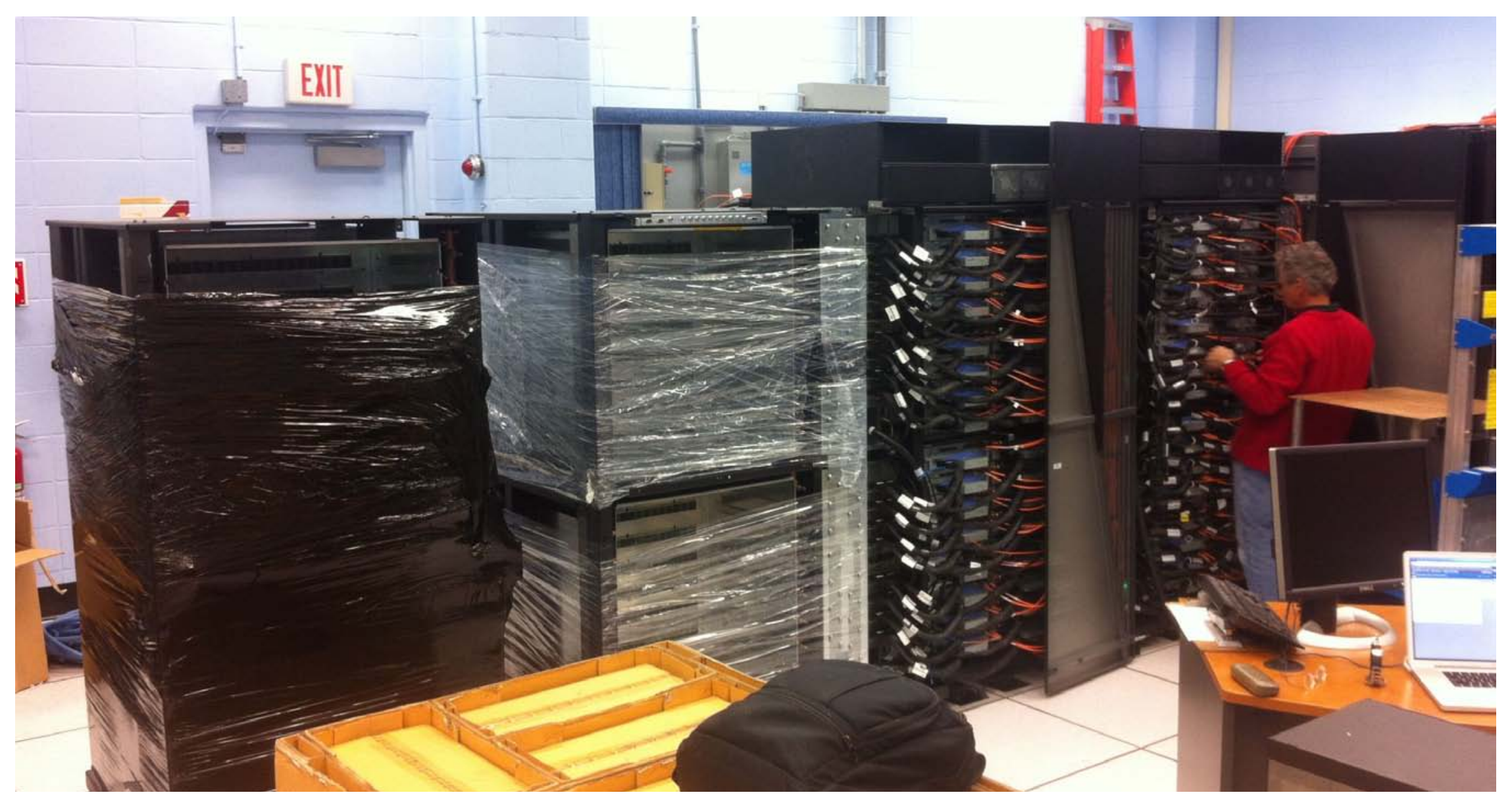




\section{Strong Scaling of BAGEL DWF CG Inverter on $64^{\wedge} 4$ volume}

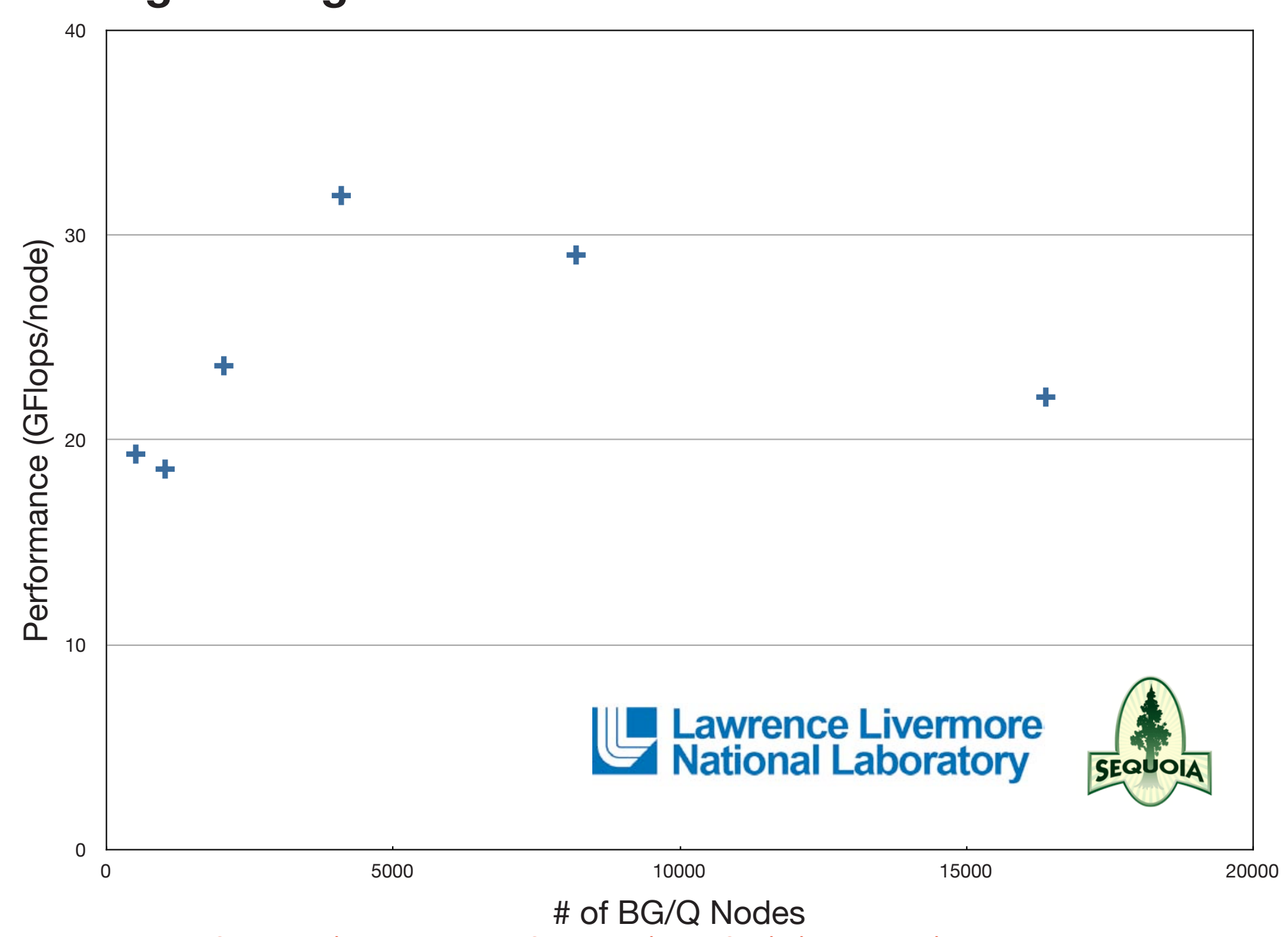

Tests were performed with the STFC funded DiRAC facility at Edinburgh 


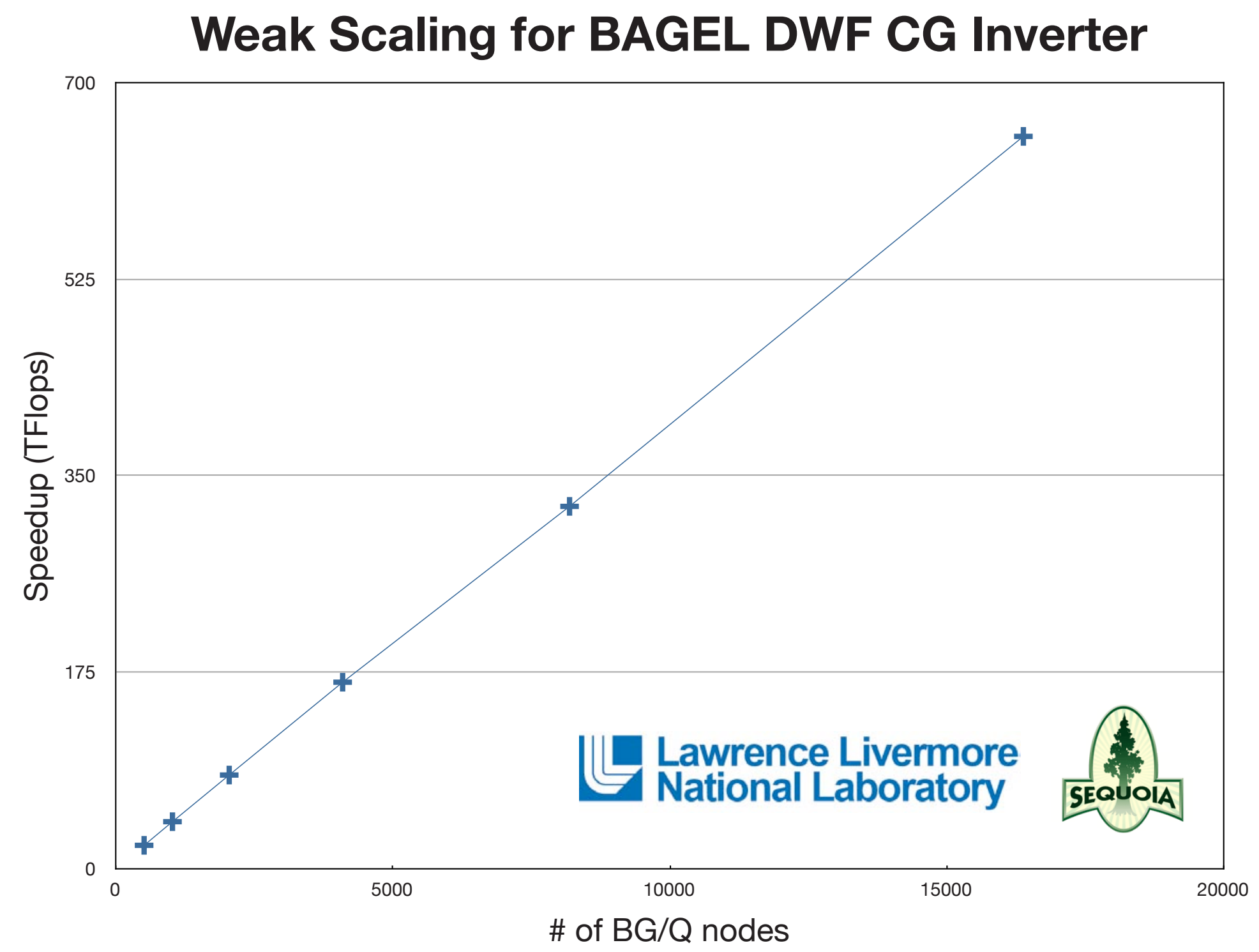

Tests were performed with the STFC funded DiRAC facility at Edinburgh 


\section{Axial couplings and strong decay widths of heavy hadrons}

\section{Stefan Meinel}

The College of William and Mary

In collaboration with William Detmold and C.-J. David Lin

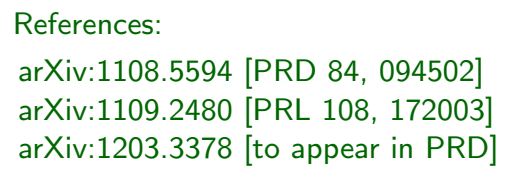

New Horizons for Lattice Computations with Chiral Fermions Brookhaven National Laboratory, May 2012 


\section{Heavy-hadron chiral perturbation theory}

Effective field theory combining heavy-quark symmetry and chiral symmetry [M. Wise 1992, T. M. Yan et al. 1992, G. Burdman and J. F. Donoghue 1992, P. Cho 1992]

- pions:

$$
\xi=\sqrt{\Sigma}=\exp (i \Phi / f)
$$

- heavy-light mesons with $s_{I}=1 / 2$ :

$$
\left(P^{i}\right)=\left(\begin{array}{c}
B^{+} \\
B^{0}
\end{array}\right),\left(P^{* i}\right)_{\mu}=\left(\begin{array}{c}
B^{*+} \\
B^{* 0}
\end{array}\right)_{\mu}
$$

combined into a single field

$$
H^{i}=\left[-P^{i} \gamma_{5}+P_{\mu}^{* i} \gamma^{\mu}\right] \frac{1-\psi}{2}
$$




\section{Heavy-hadron chiral perturbation theory}

- heavy-light baryons with $s_{I}=1$ :

$$
\left(B^{i j}\right)=\left(\begin{array}{cc}
\Sigma_{b}^{+} & \frac{1}{\sqrt{2}} \Sigma_{b}^{0} \\
\frac{1}{\sqrt{2}} \Sigma_{b}^{0} & \Sigma_{b}^{-}
\end{array}\right),\left(B^{* i j}\right)_{\mu}=\left(\begin{array}{cc}
\Sigma_{b}^{*+} & \frac{1}{\sqrt{2}} \Sigma_{b}^{* 0} \\
\frac{1}{\sqrt{2}} \Sigma_{b}^{* 0} & \Sigma_{b}^{*-}
\end{array}\right)_{\mu}
$$

combined into a single field

$$
S_{\mu}^{i j}=\sqrt{\frac{1}{3}}\left(\gamma_{\mu}+v_{\mu}\right) \gamma_{5} B^{i j}+B_{\mu}^{* i j}
$$

- heavy-light baryons with $s_{I}=0$ :

$$
\left(T^{i j}\right)=\frac{1}{\sqrt{2}}\left(\begin{array}{cc}
0 & \Lambda_{b} \\
-\Lambda_{b} & 0
\end{array}\right)
$$




\section{Heavy-hadron chiral perturbation theory}

At leading order in chiral- and heavy-quark expansion,

$$
\begin{aligned}
\mathcal{L}= & \frac{f^{2}}{8}\left(\partial^{\mu} \Sigma^{\dagger}\right)_{i j} \partial_{\mu} \Sigma^{j i}-i \operatorname{Tr}\left(\bar{H}_{i} v \cdot \mathscr{D} H^{i}\right)-i \bar{S}_{i j}^{\mu} v \cdot \mathscr{D} S_{\mu}^{i j}+\Delta \bar{S}_{i j}^{\mu} S_{\mu}^{i j}+i \bar{T}_{i j} v \cdot \mathscr{D} T^{i j} \\
& +g_{1} \operatorname{Tr}\left(\bar{H}_{i}\left(\mathscr{A}^{\mu}\right)_{j}^{i} \gamma_{\mu} \gamma_{5} H^{j}\right) \\
& -i g_{2} \epsilon_{\mu \nu \sigma \lambda} \bar{S}_{k i}^{\mu} v^{\nu}\left(\mathscr{A}^{\sigma}\right)_{j}^{i}\left(S^{\lambda}\right)^{j k} \\
& +\sqrt{2} g_{3}\left[\bar{S}_{k i}^{\mu}\left(\mathscr{A}_{\mu}\right)_{j}^{i} T^{j k}+\bar{T}_{k i}\left(\mathscr{A}^{\mu}\right)_{j}^{i} S_{\mu}^{j k}\right]
\end{aligned}
$$

where

$$
\mathscr{A}^{\mu}=\frac{i}{2}\left(\xi^{\dagger} \partial^{\mu} \xi-\xi \partial^{\mu} \xi^{\dagger}\right)=-\frac{1}{f} \partial^{\mu} \Phi+\ldots
$$

This gives the vertices
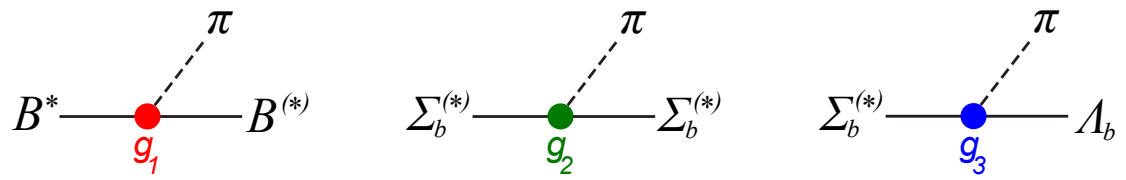

$\left[g_{1} \sim g \sim \hat{g} \sim g_{\pi} \sim g_{B^{*} B \pi}\right]$ 


\section{Heavy-hadron chiral perturbation theory}

Example application 1: strong decay $\Sigma_{b} \rightarrow \Lambda_{b} \pi$

$$
\begin{gathered}
\Sigma_{b}^{(*)}-\sigma_{g_{3}^{\prime}} \Lambda_{b} \\
\Gamma\left(\Sigma_{b}^{+} \rightarrow \Lambda_{b} \pi^{+}\right) \text {LO }=\frac{g_{3}^{2}}{6 \pi f^{2}}\left|\mathbf{p}_{\pi}\right|^{3}
\end{gathered}
$$

Example application 2: quark-mass dependence of $f_{B}$ in $S U(2) \chi \mathrm{PT}$ :

$$
\begin{aligned}
& B \otimes \\
& f_{B}=A\left[1+\frac{3}{4}\left(1+3 g_{1}^{2}\right) \frac{m_{\pi}^{2}}{(4 \pi f)^{2}} \log \left(\frac{m_{\pi}^{2}}{(4 \pi f)^{2}}\right)\right]+B m_{\pi}^{2}
\end{aligned}
$$




\section{Previous knowledge of $g_{1}, g_{2}, g_{3}$ :}

\begin{tabular}{|c|c|c|c|c|}
\hline Reference & Method & $g_{1}$ & $g_{2}$ & $g_{3}$ \\
\hline Yan et al., 1992 & Nonrelativistic quark model & 1 & 2 & $\sqrt{2}$ \\
\hline Colangelo et al., 1994 & Relativistic quark model & $1 / 3$ & $\ldots$ & $\ldots$ \\
\hline Bećirević, 1999 & Quark model with Dirac eq. & $0.6 \pm 0.1$ & $\ldots$ & $\ldots$ \\
\hline Guralnik et al., 1992 & Skyrme model & +0 & 1.6 & 1.3 \\
\hline Colangelo et al., 1994 & Sum rules & $0.15-0.55$ & $\ldots$ & $\ldots$ \\
\hline Belyaev et al., 1994 & Sum rules & $0.32 \pm 0.02$ & $\ldots$ & $\ldots$ \\
\hline Dosch and Narison, 1995 & Sum rules & $0.15 \pm 0.03$ & $\ldots$ & $\ldots$ \\
\hline Colangelo and Fazio, 1997 & Sum rules & $0.09-0.44$ & $\ldots$ & $\ldots$ \\
\hline Pirjol and Yan, 1997 & Sum rules & & $<\sqrt{6-g_{3}^{2}}$ & $<\sqrt{2}$ \\
\hline Zhu and Dai, 1998 & Sum rules & & $1.56 \pm 0.30 \pm 0.30$ & $0.94 \pm 0.06 \pm 0.20$ \\
\hline Cho and Georgi, 1992 & $\mathcal{B}\left[D^{*} \rightarrow D \pi, D \gamma\right]$ & $0.34 \pm 0.48$ & $\ldots$ & $\ldots$ \\
\hline Arnesen et al., 2005 & $\mathcal{B}\left[D_{(s)}^{*} \rightarrow D_{(s)} \pi, D_{(s)} \gamma\right]$ & 0.51 & $\ldots$ & . \\
\hline Li et al., 2010 & $\mathrm{~d} \Gamma[B \rightarrow \pi \ell \nu]$ & $<0.87$ & $\ldots$ & $\ldots$ \\
\hline Cheng, 1997 & $\Gamma\left[\Sigma_{c}^{*} \rightarrow \Lambda_{c} \pi\right], \mathrm{NRQM}$ & $0.70 \pm 0.12$ & $1.40 \pm 0.24$ & $0.99 \pm 0.17$ \\
\hline De Divitiis et al., 1998 & $n_{f}=0$ Lattice QCD & $0.42 \pm 0.09$ & . & $\cdots$ \\
\hline Abada et al., 2004 & $n_{f}=0$ Lattice QCD & $0.48 \pm 0.11$ & $\ldots$ & $\ldots$ \\
\hline Negishi et al., 2007 & $n_{f}=0$ Lattice QCD & $0.517 \pm 0.016$ & $\ldots$ & $\ldots$ \\
\hline Ohki et al., 2008 & $n_{f}=2$ Lattice QCD & $0.516 \pm 0.052$ & $\ldots$ & $\ldots$ \\
\hline Bećirević et al., 2009 & $n_{f}=2$ Lattice QCD & $0.44 \pm 0.03_{-0.00}^{+0.07}$ & $\cdots$ & $\cdots$ \\
\hline Bulava et al., 2010 & $n_{f}=2$ Lattice QCD & $0.51 \pm 0.02$ & $\ldots$ & $\ldots$ \\
\hline
\end{tabular}




\section{This work}

Complete calculation of $g_{1}, g_{2}, g_{3}$ from lattice QCD, controlling all systematic uncertainties

- RBC/UKQCD ensembles with 2+1 flavors of domain-wall fermions

- $227 \mathrm{MeV} \leq m_{\pi} \leq 352 \mathrm{MeV}$ (three unitary, three partially quenched)

- $L=2.7 \mathrm{fm}$

- NLO SU(4|2) chiral fits including finite-volume effects

- continuum extrapolation using two lattice spacings $a=0.112 \mathrm{fm}$, $a=0.085 \mathrm{fm}$

- chiral symmetry simplifies nonperturbative renormalization, provides automatic $\mathcal{O}(a)$ improvement 


\section{How can we calculate $g_{1}, g_{2}, g_{3}$ from QCD?}

- We compute suitable hadronic observables both in $\mathrm{HH} \chi \mathrm{PT}$ and in lattice QCD.

- The expressions derived from $\mathrm{HH} \chi \mathrm{PT}$ are then fitted to the lattice data, and in these fits the axial couplings are parameters.

- The simplest quantities that depend on the axial couplings already at leading order are the matrix elements of the axial current between single-hadron states.

$$
\begin{aligned}
\left\langle P^{* d}\left|A_{\mu}\right| P^{u}\right\rangle & =-2\left(g_{1}\right)_{\text {eff }} \varepsilon_{\mu}^{*}, \\
\left\langle S^{d d}\left|A_{\mu}\right| S^{d u}\right\rangle & =-(i / \sqrt{2})\left(g_{2}\right)_{\text {eff }} v^{\sigma} \epsilon_{\sigma \mu \nu \rho} \bar{U}^{\nu} U^{\rho}, \\
\left\langle S^{d d}\left|A_{\mu}\right| T^{d u}\right\rangle & =-\left(g_{3}\right)_{\text {eff }} \bar{U}_{\mu} \mathcal{U}
\end{aligned}
$$




\section{How can we calculate $g_{1}, g_{2}, g_{3}$ from QCD?}

In $S U(2)$ chiral perturbation theory and for $m_{u}=m_{d}$, one finds

$$
\begin{aligned}
&\left(g_{1}\right)_{\mathrm{eff}}=g_{1} {\left[1-\frac{2}{f^{2}} I\left(m_{\pi}\right)+\frac{4 g_{1}^{2}}{f^{2}} H\left(m_{\pi}, 0\right)+\text { analytic terms }\right], } \\
&\left(g_{2}\right)_{\mathrm{eff}}=g_{2} {\left[1-\frac{2}{f^{2}} I\left(m_{\pi}\right)+\frac{3 g_{2}^{2}}{2 f^{2}} H\left(m_{\pi}, 0\right)\right.} \\
&\left.+\frac{g_{3}^{2}}{f^{2}}\left\{H\left(m_{\pi},-\Delta\right)-2 K\left(m_{\pi},-\Delta, 0\right)\right\}+\text { analytic terms }\right], \\
&\left(g_{3}\right)_{\mathrm{eff}}=g_{3}\left[1-\frac{2}{f^{2}} I\left(m_{\pi}\right)+\frac{g_{2}^{2}}{f^{2}}\left\{-2 H\left(m_{\pi}, \Delta\right)+H\left(m_{\pi}, 0\right)\right\}\right. \\
&+ \frac{g_{3}^{2}}{2 f^{2}}\left\{H\left(m_{\pi},-\Delta\right)+9 H\left(m_{\pi}, \Delta\right)-2 K\left(m_{\pi}, \Delta, 0\right)\right\} \\
&+ \text { analytic terms }]
\end{aligned}
$$

Derived in our paper arXiv:1108.5594, also for $S U(4 \mid 2), S U(6 \mid 3)$, and in finite volume 


\section{Lattice calculation of axial-current matrix elements}

Use the interpolating fields

$$
\begin{aligned}
P^{i} & =\left(\gamma_{5}\right)_{\alpha \beta} \bar{Q}_{a \alpha} \tilde{q}_{a \beta}^{i}, \\
P_{\mu}^{* i} & =\left(\gamma_{\mu}\right)_{\alpha \beta} \bar{Q}_{a \alpha} \tilde{q}_{a \beta}^{i}, \\
S_{\mu \alpha}^{i j} & =\epsilon_{a b c}\left(C \gamma_{\mu}\right)_{\beta \gamma} \tilde{q}_{a \beta}^{i} \tilde{q}_{b \gamma}^{j} Q_{c \alpha}, \\
T_{\alpha}^{i j} & =\epsilon_{a b c}\left(C \gamma_{5}\right)_{\beta \gamma} \tilde{q}_{a \beta}^{i} \tilde{q}_{b \gamma}^{j} Q_{c \alpha}, \\
A_{\mu} & =Z_{A} \bar{d}_{a \alpha}\left(\gamma_{\mu} \gamma_{5}\right)_{\alpha \beta} u_{a \beta} .
\end{aligned}
$$

Static heavy quark $Q$ : Eichten-Hill action with HYP smearing

Light quarks $u, d$, s: domain wall action. Nonperturbative $Z_{A}$ (thanks to chiral symmetry) from RBC/UKQCD [Aoki et al., 2011]

$$
Z_{A}= \begin{cases}0.7019(26) & \text { for } a=0.112 \mathrm{fm} \\ 0.7396(17) & \text { for } a=0.085 \mathrm{fm}\end{cases}
$$




\section{Three-point correlators}

For example

$$
\left\langle S^{d d \mu}(\mathbf{x}, t) \sum_{\mathbf{x}^{\prime}} A^{\mu}\left(\mathbf{x}^{\prime}, t^{\prime}\right) \bar{T}_{d u}(\mathbf{x}, 0)\right\rangle
$$

Static heavy quark propagator requires hadron interpolating fields to be at same spatial point $\mathbf{x}$.

We generate pairs of light-quark propagators with sources at $(\mathbf{x}, 0)$ and $(\mathbf{x}, t)$ for multiple values of $t$.

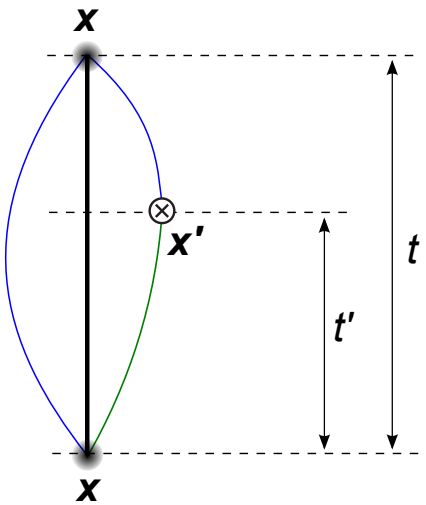




\section{Correlator ratios}

Simple ratios for mass-degenerate $P^{*} \rightarrow P$ and $S \rightarrow S$ matrix elements

$$
\begin{aligned}
& R_{1}\left(t, t^{\prime}\right)=-\frac{1}{3} \frac{\sum_{\mu=1}^{3}\left\langle P^{* d \mu}(t) A^{\mu}\left(t^{\prime}\right) P_{u}^{\dagger}(0)\right\rangle}{\left\langle P^{u}(t) P_{u}^{\dagger}(0)\right\rangle} \underset{t, t^{\prime},\left|t-t^{\prime}\right| \rightarrow \infty}{\longrightarrow}\left(g_{1}\right)_{\mathrm{eff}}, \\
& R_{2}\left(t, t^{\prime}\right)=i \frac{\sum_{\mu, \nu, \rho=1}^{3} \epsilon_{0 \mu \nu \rho}\left\langle S^{d d \mu}(t) A^{\nu}\left(t^{\prime}\right) \bar{S}_{d u}^{\rho}(0)\right\rangle}{\sum_{\mu=1}^{3}\left\langle S^{d d \mu}(t) \bar{S}_{d d}^{\mu}(0)\right\rangle} \underset{t, t^{\prime},\left|t-t^{\prime}\right| \rightarrow \infty}{\longrightarrow}\left(g_{2}\right)_{\mathrm{eff}},
\end{aligned}
$$

Double ratio for $S \rightarrow T$ transition matrix element

$$
\begin{aligned}
R_{3}\left(t, t^{\prime}\right) & =\left[\frac{1}{3} \frac{\sum_{\mu, \nu=1}^{3}\left\langle S^{d d \mu}(t) A^{\mu}\left(t^{\prime}\right) \bar{T}_{d u}(0)\right\rangle\left\langle T^{d u}(t) A^{\nu \dagger}\left(t^{\prime}\right) \bar{S}_{d d}^{\nu}(0)\right\rangle}{\sum_{\mu=1}^{3}\left\langle S^{d d \mu}(t) \bar{S}_{d d}^{\mu}(0)\right\rangle\left\langle T^{d u}(t) \bar{T}_{d u}(0)\right\rangle}\right]^{1 / 2} \\
t, t^{\prime},\left|t-t^{\prime}\right| \rightarrow \infty & \left(g_{3}\right)_{\mathrm{eff}}
\end{aligned}
$$




\section{Lattice parameters}

$\mathrm{RBC} / \mathrm{UKQCD}$ ensembles with $2+1$ flavors of domain wall fermions

\begin{tabular}{cccccl}
\hline \hline$L^{3} \times T$ & $m_{u / d}^{\text {(sea) }}$ & $m_{u / d}^{\text {(val) }}$ & $a(\mathrm{fm})$ & $m_{\pi}^{\text {(vs) }}(\mathrm{MeV})$ & $m_{\pi}^{(\mathrm{vv})}(\mathrm{MeV})$ \\
\hline $24^{3} \times 64$ & 0.005 & 0.005 & $0.1119(17)$ & $336(5)$ & $336(5)$ \\
$24^{3} \times 64$ & 0.005 & 0.002 & $0.1119(17)$ & $304(5)$ & $270(4)$ \\
$24^{3} \times 64$ & 0.005 & 0.001 & $0.1119(17)$ & $294(5)$ & $245(4)$ \\
& & & & & \\
$32^{3} \times 64$ & 0.006 & 0.006 & $0.0848(17)$ & $352(7)$ & $352(7)$ \\
$32^{3} \times 64$ & 0.004 & 0.004 & $0.0849(12)$ & $295(4)$ & $295(4)$ \\
$32^{3} \times 64$ & 0.004 & 0.002 & $0.0849(12)$ & $263(4)$ & $227(3)$ \\
\hline \hline
\end{tabular}




\section{Examples of ratios}

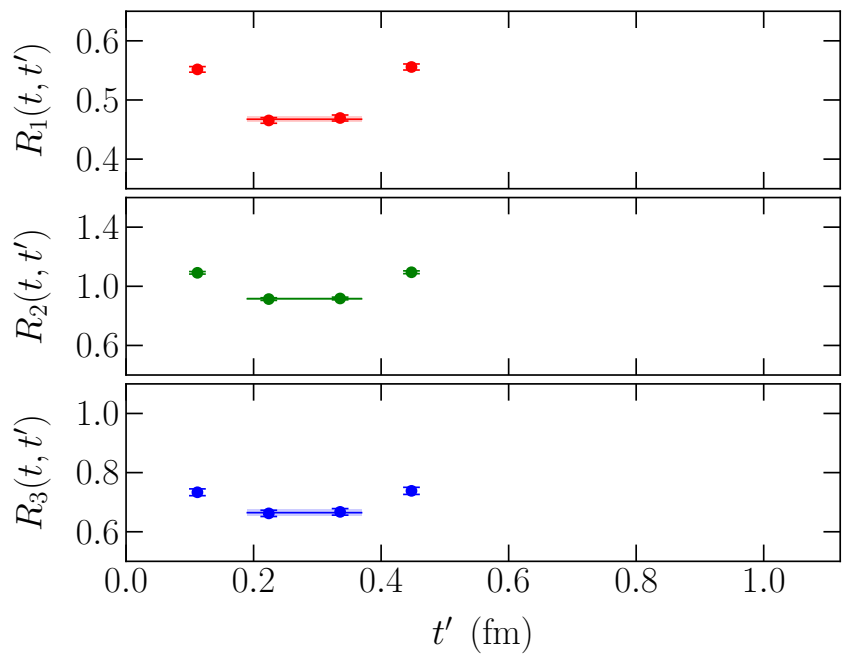

$$
\left[a=0.112 \mathrm{fm}, a m_{u / d}^{(\mathrm{val})}=0.002, t / a=5\right]
$$




\section{Examples of ratios}

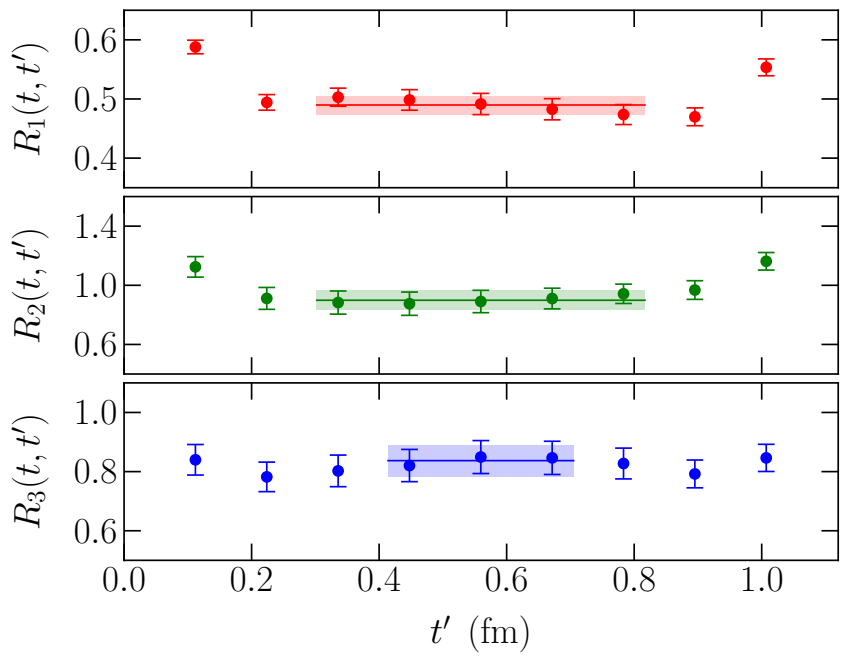

$\left[a=0.112 \mathrm{fm}, a m_{u / d}^{(\mathrm{val})}=0.002, t / a=10\right]$ 


\section{Extrapolation to infinite source-sink separation}

Fit $t$-dependence including first excited-state contamination using

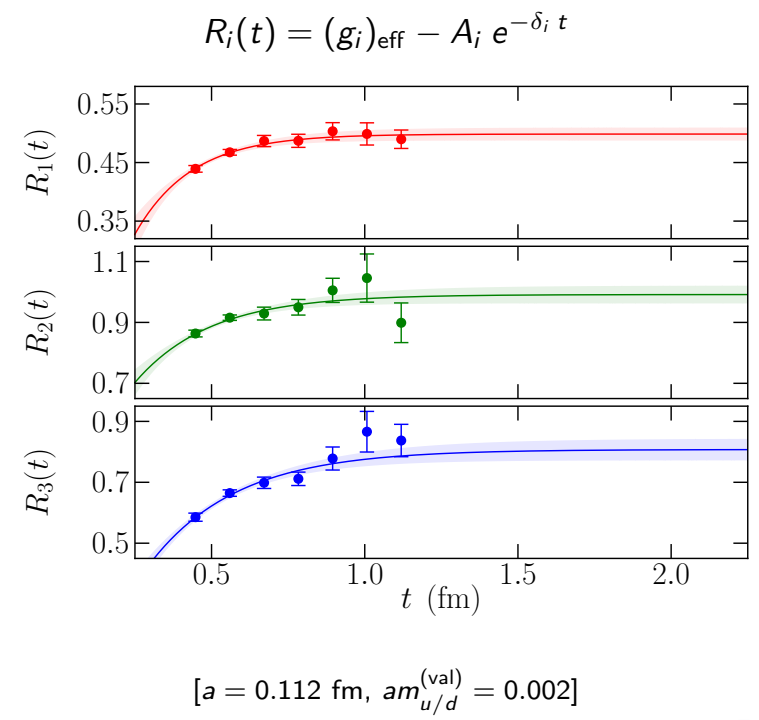




\section{Chiral-continuum fits using $S U(4 \mid 2) \mathrm{HH} \chi \mathrm{PT}$}

$$
\begin{aligned}
& \left(g_{1}\right)_{\mathrm{eff}}=g_{1}\left[1+f_{1}\left(g_{1}, m_{\pi}^{(\mathrm{vv})}, m_{\pi}^{(\mathrm{vs})}, L\right)+d_{1, n_{\mathrm{HYP}}} a^{2}\right. \\
& \left.+c_{1}^{(\mathrm{vv})}\left[m_{\pi}^{(\mathrm{vv})}\right]^{2}+c_{1}^{(\mathrm{vs})}\left[m_{\pi}^{(\mathrm{vs})}\right]^{2}\right], \\
& \left(g_{2}\right)_{\mathrm{eff}}=g_{2}\left[1+f_{2}\left(g_{2}, g_{3}, m_{\pi}^{(\mathrm{vv})}, m_{\pi}^{(\mathrm{vs})}, \Delta, L\right)+d_{2, n_{\mathrm{HYP}}} a^{2}\right. \\
& \left.+c_{2}^{(\mathrm{vv})}\left[m_{\pi}^{(\mathrm{vv})}\right]^{2}+c_{2}^{(\mathrm{vs})}\left[m_{\pi}^{(\mathrm{vs})}\right]^{2}\right], \\
& \left(g_{3}\right)_{\mathrm{eff}}=g_{3}\left[1+f_{3}\left(g_{2}, g_{3}, m_{\pi}^{(\mathrm{vv})}, m_{\pi}^{(\mathrm{vs})}, \Delta, L\right)+d_{3, n_{\mathrm{HYP}}} a^{2}\right. \\
& \left.+c_{3}^{(\mathrm{vv})}\left[m_{\pi}^{(\mathrm{vv})}\right]^{2}+c_{3}^{(\mathrm{vs})}\left[m_{\pi}^{(\mathrm{vs})}\right]^{2}\right]
\end{aligned}
$$

$(\triangle=200 \mathrm{MeV})$ 


\section{Fits using $S U(4 \mid 2) \mathrm{HH} \chi \mathrm{PT}$}

Plotted at $L=\infty, m_{\pi}^{(\mathrm{vs})}=m_{\pi}^{(\mathrm{vv})}$ :

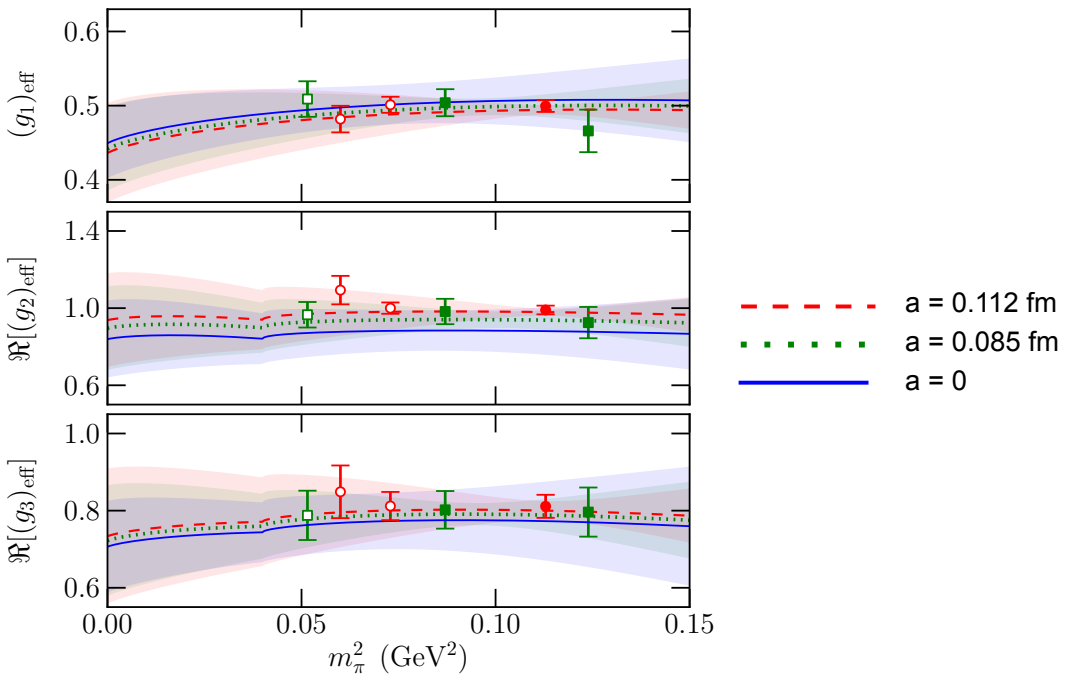




\section{Final results for axial couplings}

Final results:

$$
\begin{aligned}
& g_{1}=0.449 \pm 0.047_{\text {stat }} \pm 0.019_{\text {syst }}=0.449 \pm 0.051 \\
& g_{2}=0.84 \pm 0.20_{\text {stat }} \pm 0.04_{\text {syst }}=0.84 \pm 0.20 \\
& g_{3}=0.71 \pm 0.12_{\text {stat }} \pm 0.04_{\text {syst }}=0.71 \pm 0.13
\end{aligned}
$$

Systematic uncertainties:

\begin{tabular}{lccc}
\hline \hline Source & $g_{1}$ & $g_{2}$ & $g_{3}$ \\
\hline NNLO terms in fits of $m_{\pi^{-}}$and a-dep & $3.6 \%$ & $2.8 \%$ & $3.7 \%$ \\
Higher excited states in fits to $R_{i}(t)$ & $1.7 \%$ & $2.8 \%$ & $4.9 \%$ \\
Unphysical value of $m_{s}^{\text {(sea) }}$ & $1.5 \%$ & $1.5 \%$ & $1.5 \%$ \\
Total & $4.2 \%$ & $4.3 \%$ & $6.3 \%$ \\
\hline \hline
\end{tabular}




\section{$g_{1}$ : comparison with previous lattice results}

\begin{tabular}{lccl}
\hline \hline Reference & $n_{f}$, action & {$\left[m_{\pi}^{(\mathrm{vv})}\right]^{2}\left(\mathrm{GeV}^{2}\right)$} & $g_{1}$ \\
\hline De Divitiis et al., 1998 & 0, clover & $0.58-0.81$ & $0.42 \pm 0.04 \pm 0.08$ \\
Abada et al., 2004 & 0, clover & $0.30-0.71$ & $0.48 \pm 0.03 \pm 0.11$ \\
Negishi et al., 2007 & 0, clover & $0.43-0.72$ & $0.517 \pm 0.016$ \\
Ohki et al., 2008 & 2, clover & $0.24-1.2$ & $0.516 \pm 0.005 \pm 0.033 \pm 0.028$ \\
Bećirević et al., 2009 & 2, clover & $0.16-1.2$ & $0.44 \pm 0.03_{-0.00}^{+0.07}$ \\
Bulava et al., 2010 & 2, clover & $0.063-0.49$ & $0.51 \pm 0.02$ \\
This work & 2+1, DW & $0.052-0.12$ & $0.449 \pm 0.047_{\text {stat }} \pm 0.019_{\text {syst }}$ \\
\hline \hline
\end{tabular}

Note: none of the previous works use correct NLO $\chi \mathrm{PT}$ (linear extrapolation or tadpole missing) 


\section{Impact of NLO $\chi \mathrm{PT}$ corrections}

In $S U(2)$ :

Strong decay: $\mathcal{M}\left(P^{*} \rightarrow P \pi\right) \propto g_{1}\left[1-4 g_{1}^{2} \frac{m_{\pi}^{2}}{\left(4 \pi f_{\pi}\right)^{2}} \log \frac{m_{\pi}^{2}}{\mu^{2}}+\tilde{c} m_{\pi}^{2}\right]$.

Axial current m. elt.: $\left(g_{1}\right)_{\text {eff }}=g_{1}\left[1-\left(2+4 g_{1}^{2}\right) \frac{m_{\pi}^{2}}{\left(4 \pi f_{\pi}\right)^{2}} \log \frac{m_{\pi}^{2}}{\mu^{2}}+c m_{\pi}^{2}\right]$.

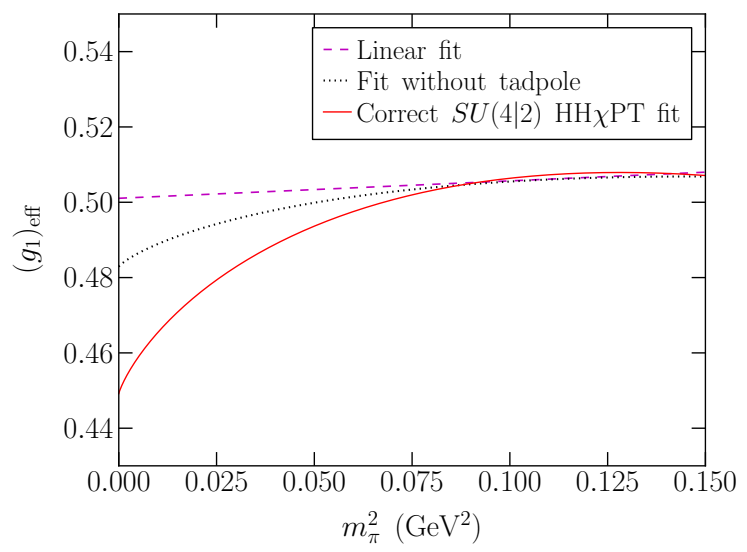




\section{Comparison with other results}

\begin{tabular}{|c|c|c|c|c|}
\hline Reference & Method & $g_{1}$ & $g_{2}$ & $g_{3}$ \\
\hline Yan et al., 1992 & Nonrelativistic quark model & 1 & 2 & $\sqrt{2}$ \\
\hline Colangelo et al., 1994 & Relativistic quark model & $1 / 3$ & $\ldots$ & $\ldots$ \\
\hline Bećirević, 1999 & Quark model with Dirac eq. & $0.6 \pm 0.1$ & & $\ldots$ \\
\hline Guralnik et al., 1992 & Skyrme model & & 1.6 & 1.3 \\
\hline Colangelo et al., 1994 & Sum rules & $0.15-0.55$ & $\ldots$ & $\ldots$ \\
\hline Belyaev et al., 1994 & Sum rules & $0.32 \pm 0.02$ & $\ldots$ & $\ldots$ \\
\hline Dosch and Narison, 1995 & Sum rules & $0.15 \pm 0.03$ & $\ldots$ & $\ldots$ \\
\hline Colangelo and Fazio, 1997 & Sum rules & $0.09-0.44$ & $\ldots$ & $\ldots$ \\
\hline Pirjol and Yan, 1997 & Sum rules & $\ldots$ & $<\sqrt{6-g_{3}^{2}}$ & $<\sqrt{2}$ \\
\hline Zhu and Dai, 1998 & Sum rules & & $1.56 \pm 0.30 \pm 0.30$ & $0.94 \pm 0.06 \pm 0.20$ \\
\hline Cho and Georgi, 1992 & $\mathcal{B}\left[D^{*} \rightarrow D \pi, D \gamma\right]$ & $0.34 \pm 0.48$ & $\ldots$ & $\ldots$ \\
\hline Stewart, 1998 & $\mathcal{B}\left[D_{(s)}^{*} \rightarrow D_{(s)} \pi, D_{(s)} \gamma\right]$ & $0.27_{-0.02-0.02}^{+0.04+0.05}$ & $\cdots$ & $\cdots$ \\
\hline Li et al., 2010 & $\mathrm{~d} \Gamma[B \rightarrow \pi \ell \nu]$ & $<0.87$ & $\ldots$ & \\
\hline Cheng, 1997 & $\Gamma\left[\Sigma_{c}^{*} \rightarrow \Lambda_{c} \pi\right], \mathrm{NRQM}$ & $0.70 \pm 0.12$ & $1.40 \pm 0.24$ & $0.99 \pm 0.17$ \\
\hline This work & Lattice QCD & $0.449 \pm 0.051$ & $0.84 \pm 0.20$ & $0.71 \pm 0.13$ \\
\hline
\end{tabular}

Our QCD results are MUCH smaller than the quark-model predictions (even for $g_{A}^{u d}=0.75$, as needed to get correct nucleon $g_{A}$ ) 
Heavy baryon decays 


\section{$S \rightarrow T$ strong decays: leading-order width}

At LO in chiral and heavy quark expansion, $\mathrm{HH} \chi \mathrm{PT}$ predicts

$$
\Gamma[S \rightarrow T \pi]=c_{\mathrm{f}}^{2} \frac{1}{6 \pi f_{\pi}^{2}} g_{3}^{2} \frac{M_{T}}{M_{S}}\left|\mathbf{p}_{\pi}\right|^{3}
$$

where

$$
c_{\mathrm{f}}= \begin{cases}1 & \text { for } \quad \Sigma_{Q}^{(*)} \rightarrow \Lambda_{Q} \pi^{ \pm} \\ 1 & \text { for } \quad \Sigma_{Q}^{(*)} \rightarrow \Lambda_{Q} \pi^{0} \\ 1 / \sqrt{2} & \text { for } \quad \Xi_{Q}^{\prime(*)} \rightarrow \Xi_{Q} \pi^{ \pm} \\ 1 / 2 & \text { for } \quad \Xi_{Q}^{\prime(*)} \rightarrow \Xi_{Q} \pi^{0}\end{cases}
$$

and

$$
\left|\mathbf{p}_{\pi}\right|=\frac{\sqrt{\left[\left(M_{S}-M_{T}\right)^{2}-m_{\pi}^{2}\right]\left[\left(M_{S}+M_{T}\right)^{2}-m_{\pi}^{2}\right]}}{2 M_{S}}
$$

(kinematic factors not expanded in $1 / m_{Q}$ here) 


\section{$S \rightarrow T$ strong decays: including $1 / m_{Q}$ correction}

LO in chiral expansion is precice enough, because physical $m_{\pi}$ is so small.

$\mathrm{LO}$ in $\mathrm{HQ}$ expansion is not good enough. Include generic $1 / m_{Q}$ correction

$$
\Gamma[S \rightarrow T \pi]=c_{\mathrm{f}}^{2} \frac{1}{6 \pi f_{\pi}^{2}}\left(g_{3}+\frac{\kappa_{J}}{m_{Q}}\right)^{2} \frac{M_{T}}{M_{S}}\left|\mathbf{p}_{\pi}\right|^{3}
$$

Determine $\kappa_{1 / 2}$ and $\kappa_{3 / 2}$ by fitting experimental data for $\Sigma_{c}^{++}, \Sigma_{c}^{0}$ and $\Sigma_{c}^{*++}, \Sigma_{c}^{* 0}$ with $g_{3}$ constrained to $L Q C D$ value, and $m_{Q}=\frac{1}{2} M_{J / \psi}$. 


\section{$S \rightarrow T$ strong decays: including $1 / m_{Q}$ correction}

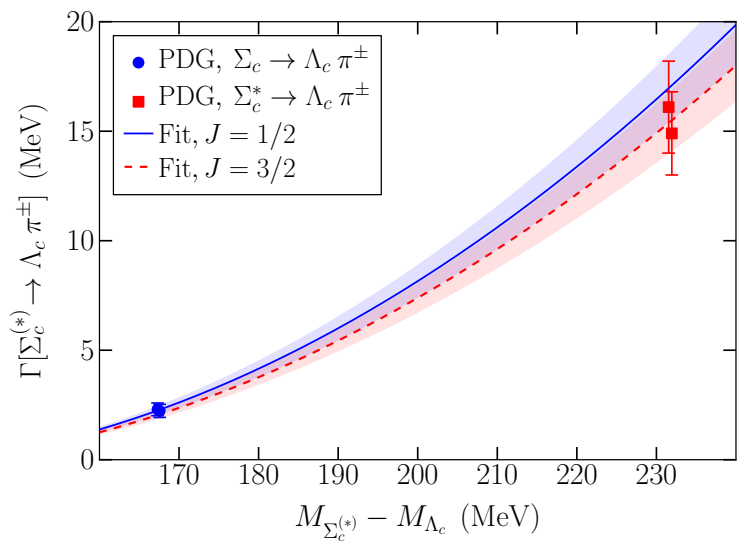

Result:

$$
\begin{aligned}
& \kappa_{1 / 2}=0.55(21) \mathrm{GeV}, \quad \operatorname{Cov}\left(\kappa_{1 / 2}, g_{3}\right)=-0.025 \mathrm{GeV} \\
& \kappa_{3 / 2}=0.47(21) \mathrm{GeV}, \quad \operatorname{Cov}\left(\kappa_{3 / 2}, g_{3}\right)=-0.025 \mathrm{GeV}
\end{aligned}
$$




\section{$S \rightarrow T$ strong decays: including $1 / m_{Q}$ correction}

Effective coupling vs $1 / m_{Q}$ :

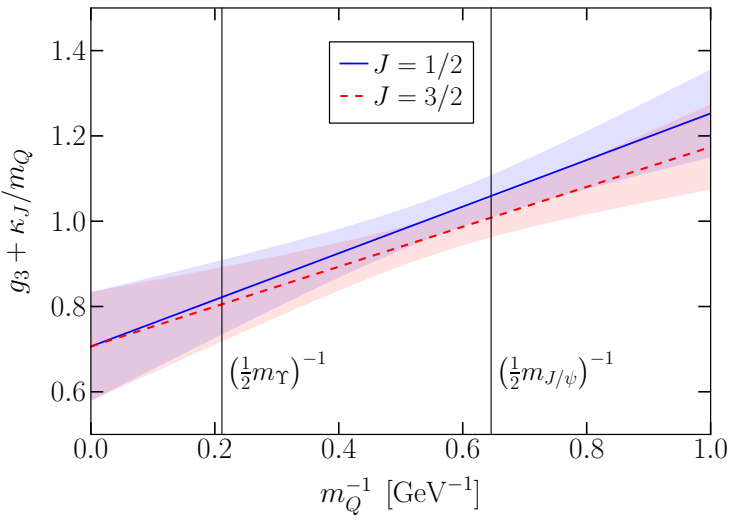

$\rightarrow$ Evaluate for $m_{Q}=\frac{1}{2} M_{\Upsilon}$ to make predictions for bottom baryons 


\section{$S \rightarrow T$ strong decays: predictions for bottom baryons}

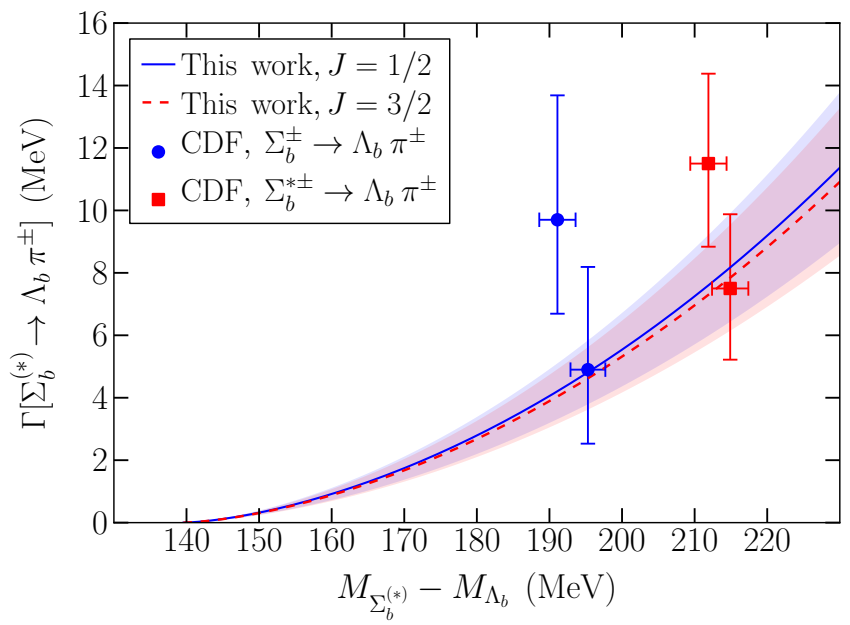

Also: $\Xi_{b}^{* 0}$, recently discovered by CMS

$\Gamma\left[\Xi_{b}^{* 0}\right]=2.1 \pm 1.7 \mathrm{MeV}(\mathrm{CMS}), \quad \Gamma\left[\Xi_{b}^{* 0}\right]=0.51 \pm 0.26 \mathrm{MeV}$ (this work) 


\section{Conclusions}

- First complete lattice QCD determination of heavy-hadron axial couplings, Results are $g_{1}=0.449 \pm 0.051, g_{2}=0.84 \pm 0.20$, $g_{3}=0.71 \pm 0.13$

- Systematic uncertainties very small, statistical uncertainties by far dominant

- Chiral expansion of axial-current matrix elements well-behaved here, better than for light baryons

- $g_{1}, g_{2}, g_{3}$ are much smaller than NRQM prediction, but ratios close to NRQM

- Compare to $g_{A} \approx 1.26,\left|g_{N \Delta}\right| \sim 1.6$ and $g_{\Delta \Delta} \sim-1.9$

- $1 / m_{Q}$ corrections in $\Gamma\left[\Sigma_{Q}^{(*)} \rightarrow \Lambda_{Q} \pi\right]$ are significant even for $Q=b$

- Calculated $\Gamma\left[\Sigma_{b}^{(*)} \rightarrow \Lambda_{b} \pi\right]$ widths agree with experiment

- Many future applications (e.g. chiral extrapolations for spectroscopy) 


\section{Extra slides}




\section{Systematic uncertainties from higher excited states}

$$
R_{i}(t)=\left(g_{i}\right)_{\mathrm{eff}}-A_{i} e^{-\delta_{i} t}
$$

\begin{tabular}{lcccccc}
\hline \hline Fit & $\left(g_{1}\right)_{\text {eff }}$ & $\delta\left(g_{1}\right)_{\text {eff }}$ & $\left(g_{2}\right)_{\text {eff }}$ & $\delta\left(g_{2}\right)_{\text {eff }}$ & $\left(g_{3}\right)_{\text {eff }}$ & $\delta\left(g_{3}\right)_{\text {eff }}$ \\
\hline Original & $0.499(11)$ & 0 & $0.993(29)$ & 0 & $0.810(36)$ & 0 \\
$t / a=4$ removed & $0.496(13)$ & $0.0030(76)$ & $0.975(35)$ & $0.016(19)$ & $0.783(43)$ & $0.026(15)$ \\
$t / a=4,5$ removed & $0.494(12)$ & $0.0041(76)$ & $0.984(41)$ & $0.009(26)$ & $0.807(54)$ & $0.003(30)$ \\
Second exp. added & $0.498(11)$ & $0.0009(77)$ & $0.988(30)$ & $0.005(21)$ & $0.796(40)$ & $0.014(36)$ \\
\hline \hline
\end{tabular}

Conservative estimate of uncertainty:

$$
\sqrt{\left[\delta\left(g_{i}\right)_{\mathrm{eff}}\right]^{2}+\left[\sigma \delta\left(g_{i}\right)_{\mathrm{eff}}\right]^{2}}
$$

$g_{1}: 1.7 \%$

$g_{2}: 2.8 \%$

$g_{3}: 4.9 \%$ 


\section{Summation Method}

Summed ratio:

$$
S_{i}(t)=a \sum_{t^{\prime}=0}^{t} R_{i}\left(t, t^{\prime}\right)
$$

For large $t$ :

$$
S_{i}(t) \rightarrow c_{i}+\left(g_{i}\right)_{\mathrm{eff}} t
$$

Use slope to extract $\left(g_{i}\right)_{\text {eff }}$ :

$$
R_{i}^{\text {sum }}(t)=\frac{\mathrm{d}}{\mathrm{d} t} S_{i}(t)
$$

Expect (if off-diagonal excited state contrib $\neq 0$ ):

$$
\begin{aligned}
R_{i}(t)-\left(g_{i}\right)_{\text {eff }} & =O\left(e^{-\frac{1}{2} \delta_{i} t}\right), \\
R_{i}^{\text {sum }}(t)-\left(g_{i}\right)_{\text {eff }} & =O\left(t e^{-\delta_{i} t}\right)
\end{aligned}
$$

[Maiani et al 1987, Gusken et al. 1989, Bulava et al. 2011] 


\section{Summation Method}

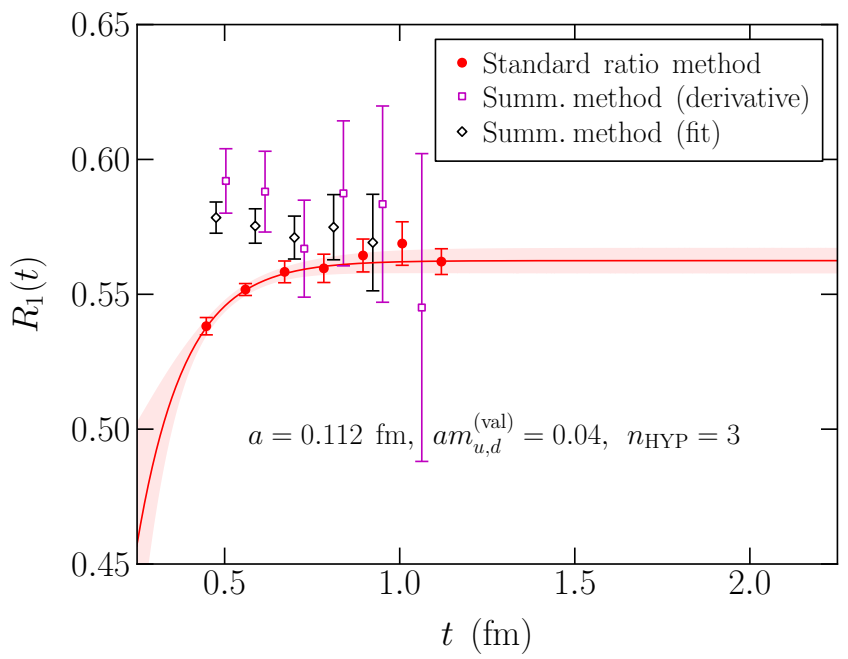




\section{Summation Method}

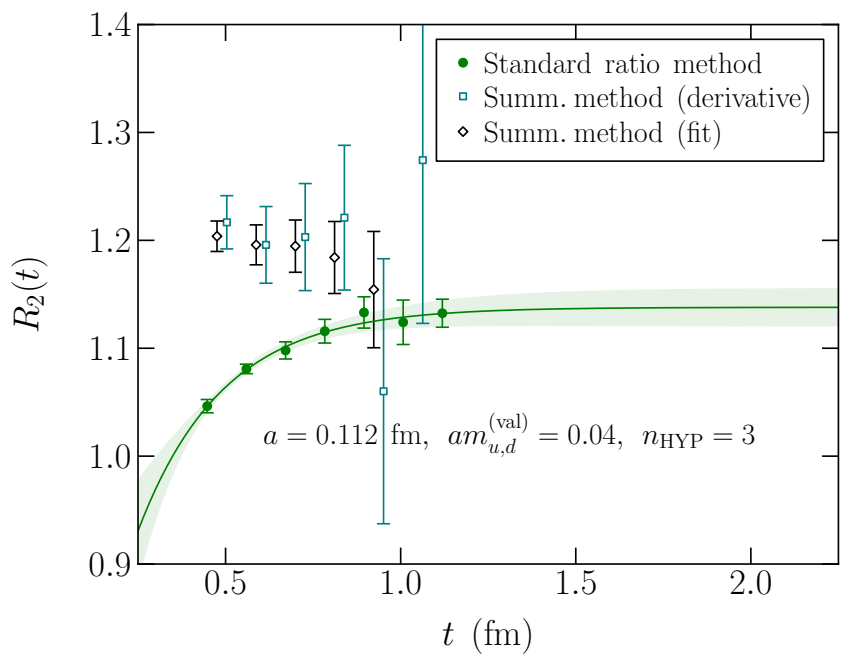




\section{Fits using $\mathrm{SU}(4 \mid 2) \mathrm{HH} \chi \mathrm{PT}$ : estimates of systematic}

\section{uncertainties}

Add higher-order analytic terms.

$$
\begin{aligned}
\left(g_{i}\right)_{\mathrm{eff}}^{(\mathrm{NLO}+\mathrm{HO})}\left(a, m, n_{\mathrm{HYP}}\right) & \\
= & \left(g_{i}\right)_{\mathrm{eff}}^{(\mathrm{NLO})}\left(a, m, n_{\mathrm{HYP}}\right) \\
+ & g_{i}\left[c_{i}^{(\mathrm{vv}, \mathrm{vv})}\left[m_{\pi}^{(\mathrm{vv})}\right]^{4}+c_{i}^{(\mathrm{vs}, \mathrm{vs})}\left[m_{\pi}^{(\mathrm{vs})}\right]^{4}+c_{i}^{(\mathrm{vv}, \mathrm{vs})}\left[m_{\pi}^{(\mathrm{vv})}\right]^{2}\left[m_{\pi}^{(\mathrm{vs})}\right]^{2}\right. \\
& \left.+d_{i, n_{\mathrm{HYP}}}^{(\mathrm{vv})} a^{2}\left[m_{\pi}^{(\mathrm{vv})}\right]^{2}+d_{i, n_{\mathrm{HYP}}}^{(\mathrm{vs})} a^{2}\left[m_{\pi}^{(\mathrm{vs})}\right]^{2}+h_{i, n_{\mathrm{HYP}}} a^{4}\right]
\end{aligned}
$$




\section{Fits using $\mathrm{SU}(4 \mid 2) \mathrm{HH} \chi \mathrm{PT}$ : estimates of systematic}

\section{uncertainties}

Constrain with Gaussian priors. In terms of the natural scales,

$$
\begin{aligned}
& c_{i}^{(\mathrm{vv}, \mathrm{vv})}=0 \pm w / \Lambda_{\chi}^{4}, \\
& c_{i}^{(\mathrm{vs}, \mathrm{vs})}=0 \pm w / \Lambda_{\chi}^{4}, \\
& c_{i}^{(\mathrm{vv}, \mathrm{vs})}=0 \pm w / \Lambda_{\chi}^{4}, \\
& d_{i, n_{\mathrm{HYP}}}^{(\mathrm{vv})}=0 \pm w \Lambda_{\mathrm{QCD}}^{2} / \Lambda_{\chi}^{2}, \\
& d_{i, n_{\mathrm{HYP}}}^{(\mathrm{vs})}=0 \pm w \Lambda_{\mathrm{QCD}}^{2} / \Lambda_{\chi}^{2}, \\
& h_{i, n_{\mathrm{HYP}}}=0 \pm w \Lambda_{\mathrm{QCD}}^{4} .
\end{aligned}
$$

$w$ is the width 


\section{Fits using $\mathrm{SU}(4 \mid 2) \mathrm{HH} \chi \mathrm{PT}$ : estimates of systematic}

\section{uncertainties}

\begin{tabular}{ccccccc}
\hline \hline$w$ & \multicolumn{1}{c}{$g_{1}$} & $\delta \sigma\left(g_{1}\right)$ & $g_{2}$ & $\delta \sigma\left(g_{2}\right)$ & $g_{3}$ & $\delta \sigma\left(g_{3}\right)$ \\
\hline 0 & $0.449(47)$ & 0 & $0.84(20)$ & 0 & $0.71(12)$ & 0 \\
1 & $0.449(47)$ & 0.0020 & $0.84(20)$ & 0.0023 & $0.71(12)$ & 0.0045 \\
5 & $0.452(48)$ & 0.0089 & $0.84(20)$ & 0.014 & $0.70(12)$ & 0.017 \\
10 & $0.455(50)$ & 0.016 & $0.84(20)$ & 0.024 & $0.70(12)$ & 0.026 \\
50 & $0.464(72)$ & 0.054 & $0.82(22)$ & 0.099 & $0.68(15)$ & 0.094 \\
100 & $0.452(94)$ & 0.082 & $0.78(26)$ & 0.17 & $0.63(21)$ & 0.17 \\
\hline
\end{tabular}

Conservative estimate: with $w=10$, use

$$
\delta \sigma\left(g_{i}\right)=\sqrt{\sigma^{2}\left(g_{i}\right)^{(\mathrm{NLO}+\mathrm{HO})}-\sigma^{2}\left(g_{i}\right)^{(\mathrm{NLO})}}
$$

$g_{1}: 3.6 \%$
$g_{2}: 2.8 \%$
$g_{3}: 3.7 \%$ 


\section{Fits using $\mathrm{SU}(4 \mid 2) \mathrm{HH} \chi \mathrm{PT}$ : finite-volume corrections}

\begin{tabular}{ccccc}
\hline \hline$m_{\pi}^{(\mathrm{vs})}(\mathrm{MeV})$ & $m_{\pi}^{(\mathrm{vv})}(\mathrm{MeV})$ & $\frac{\left(g_{1}\right)_{\text {eff }}^{(\infty)}-\left(g_{1}\right)_{\text {eff }}^{(L)}}{\left(g_{1}\right)_{\text {eff }}^{(\infty)}}$ & $\frac{\left(g_{2}\right)_{\text {eff }}^{(\infty)}-\left(g_{2}\right)_{\text {eff }}^{(L)}}{\left(g_{2}\right)_{\text {eff }}^{(\infty)}}$ & $\frac{\left(g_{3}\right)_{\text {eff }}^{(\infty)}-\left(g_{3}\right)_{\text {eff }}^{(L)}}{\left(g_{3}\right)_{\text {eff }}^{(\infty)}}$ \\
\hline 294 & 245 & 0.0057 & 0.015 & 0.0074 \\
304 & 270 & 0.0040 & 0.0070 & 0.0027 \\
336 & 336 & 0.0016 & 0.00037 & -0.00079 \\
263 & 227 & 0.0072 & 0.028 & 0.013 \\
295 & 295 & 0.0031 & 0.00027 & -0.0012 \\
352 & 352 & 0.0013 & 0.00033 & -0.00071 \\
\hline \hline
\end{tabular}




\section{New fermion discretizations and their applications}

\section{Tatsuhiro MISUMI (BNL)}

In collaborations with M.Creutz(BNL), T.Kimura(RIKEN), A.Ohnishi(YITP),

S. Sharpe (Washington), S. Aoki(Tsukuba) 


\section{Table of contents}

1. Flavored mass 2-flavor QCD

2. Central branch Many-flavor QCD

3. Flavored chemical potential Finite (T, $\mu) \mathrm{QCD}$ 
Naive

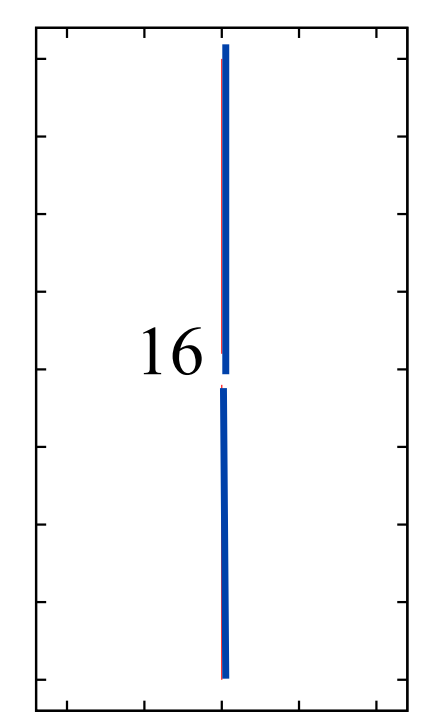

$\mathrm{U}(4) \times \mathrm{U}(4)$

\section{Wilson}
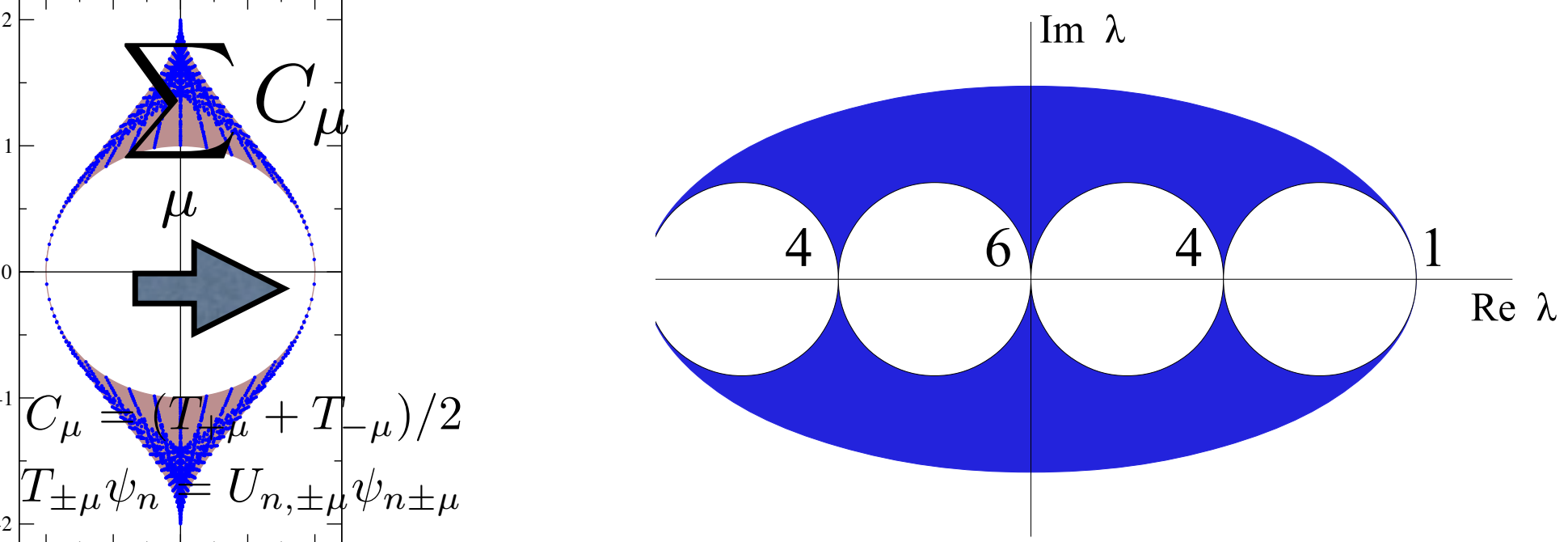


\section{Naive}

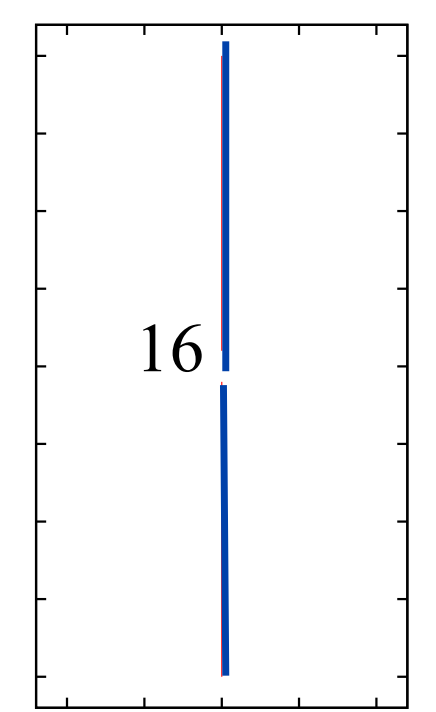

$\mathrm{U}(4) \times \mathrm{U}(4)$

\section{Wilson}
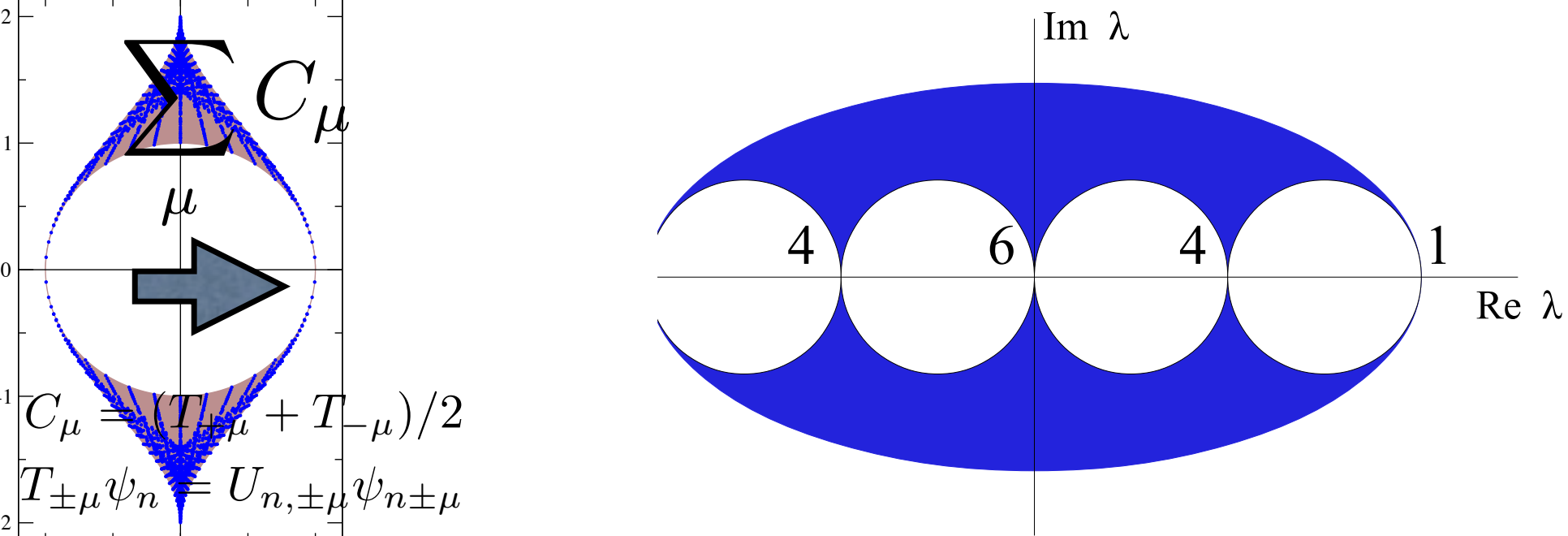

$$
\begin{aligned}
& \Gamma_{X}^{(+)} \in\left\{\mathbf{1}_{4},(-1)^{n_{1}+\ldots+n_{4}} \gamma_{5},(-1)^{\check{n}_{\mu}} \gamma_{\mu},(-1)^{n_{\mu}} i \gamma_{\mu} \gamma_{5},(-1)^{n_{\mu, \nu}} \frac{\left[\gamma_{\mu}, \gamma_{\nu}\right]}{2}\right\} \\
& \Gamma_{X}^{(-)} \in\left\{(-1)^{n_{1}+\ldots+n_{4}} \mathbf{1}_{4}, \gamma_{5},(-1)^{n_{\mu}} \gamma_{\mu},(-1)^{\check{n}_{\mu}} \gamma_{\mu} \gamma_{5},(-1)^{\check{n}_{\mu, \nu}} \frac{\left[\gamma_{\mu}, \gamma_{\nu}\right]}{2}\right\} \\
& \psi_{n} \rightarrow \psi_{n}^{\prime}=\exp \left[\mathrm{i} \sum_{X}\left(\theta_{X}^{(+)} \Gamma_{X}^{(+)}+\theta_{X}^{(-)} \Gamma_{X}^{(-)}\right)\right] \psi_{n}, \quad \bar{\psi}_{n} \rightarrow \bar{\psi}_{n}^{\prime}=\bar{\psi}_{n} \exp \left[\mathrm{i} \sum_{X}\left(-\theta_{X}^{(+)} \Gamma_{X}^{(+)}+\theta_{X}^{(-)} \Gamma_{X}^{(-)}\right)\right]
\end{aligned}
$$




\section{Naive}

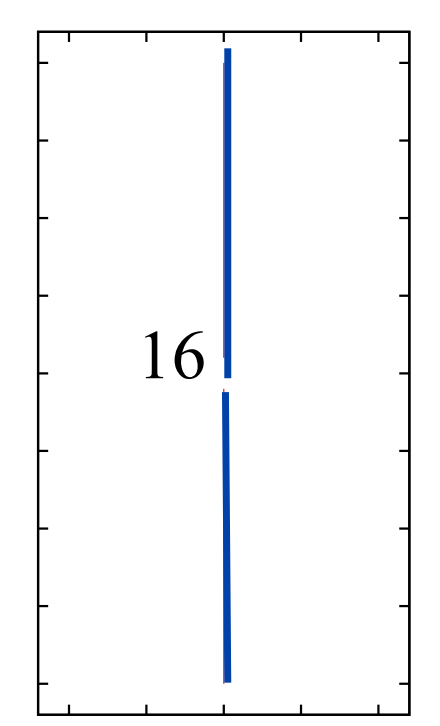

$\mathrm{U}(4) \times \mathrm{U}(4)$

$$
\begin{aligned}
& \Gamma_{X}^{(+)} \in\left\{\mathbf{1}_{4},\right. \\
& \Gamma_{X}^{(-)} \in\{
\end{aligned}
$$

\section{Wilson}
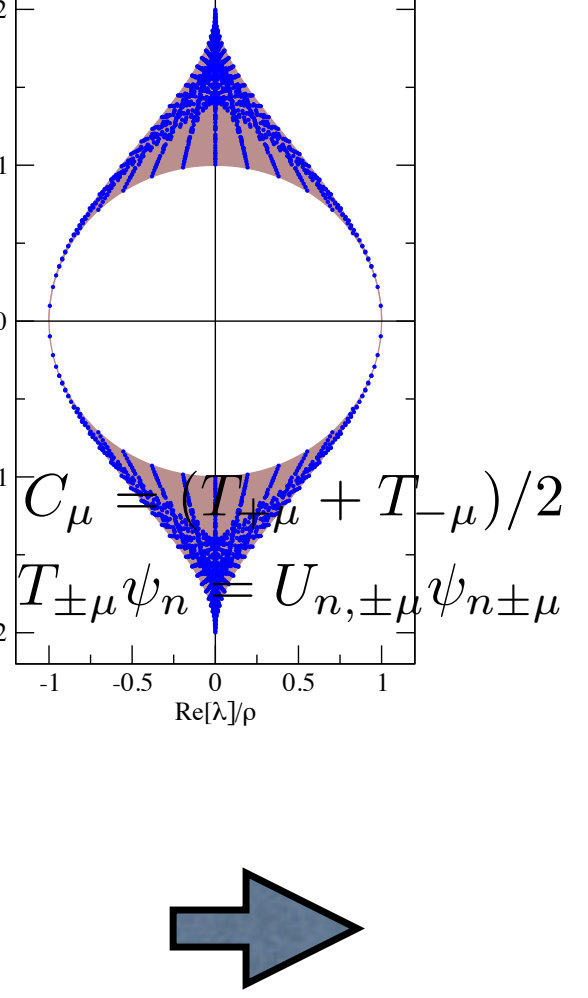

$\mathrm{U}(1)$
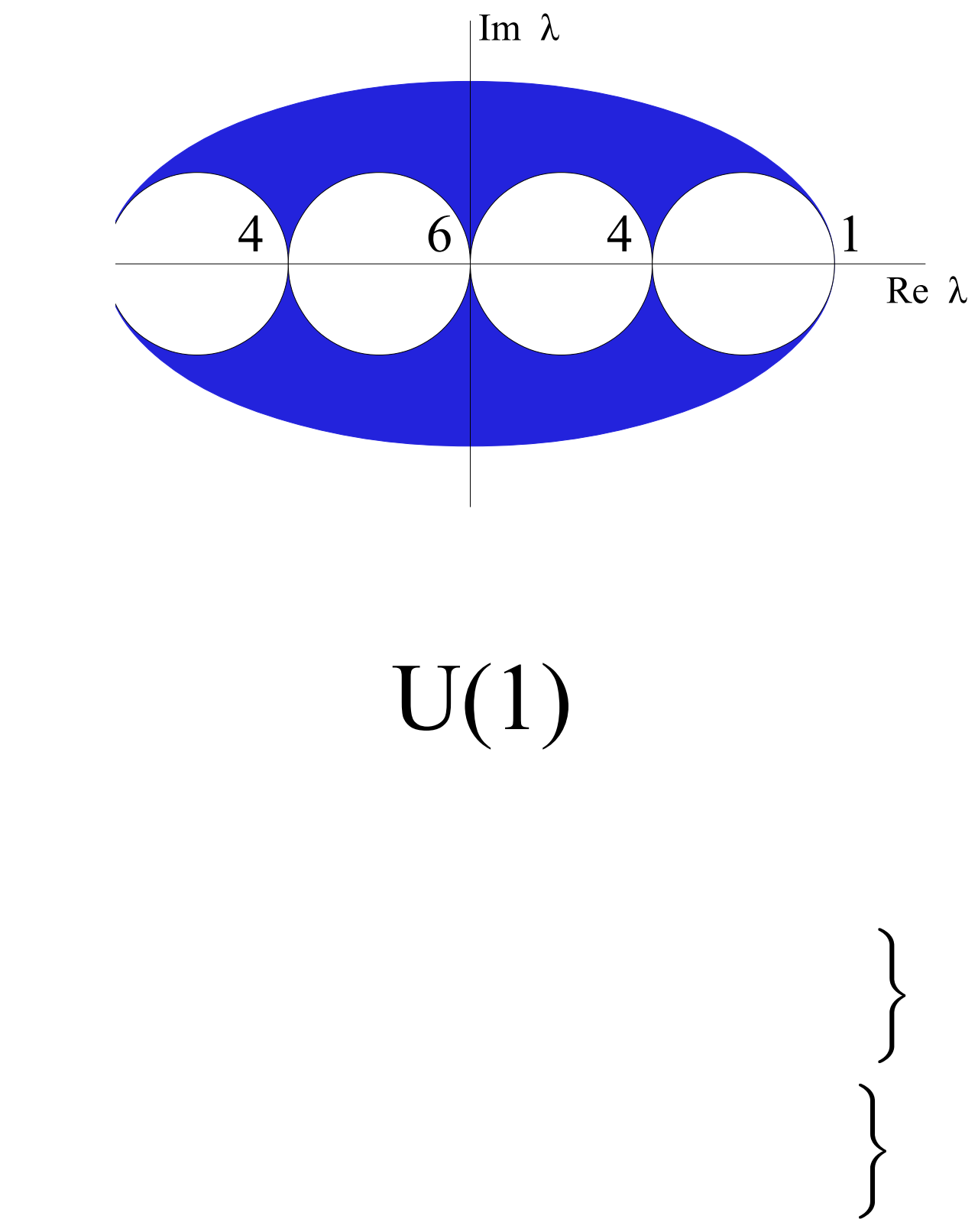


\section{Naive}

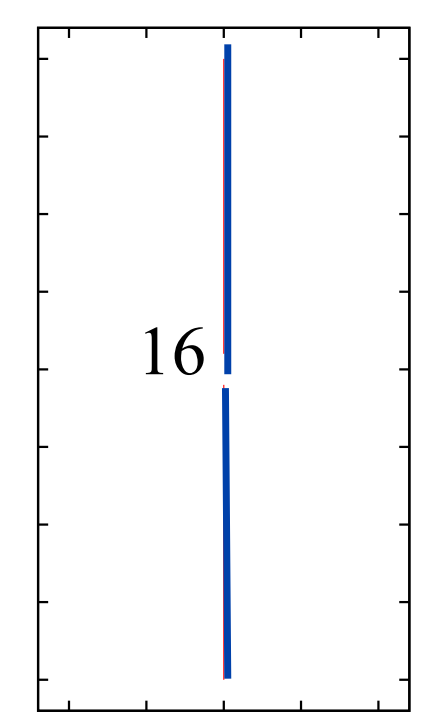

$\mathrm{U}(4) \times \mathrm{U}(4)$

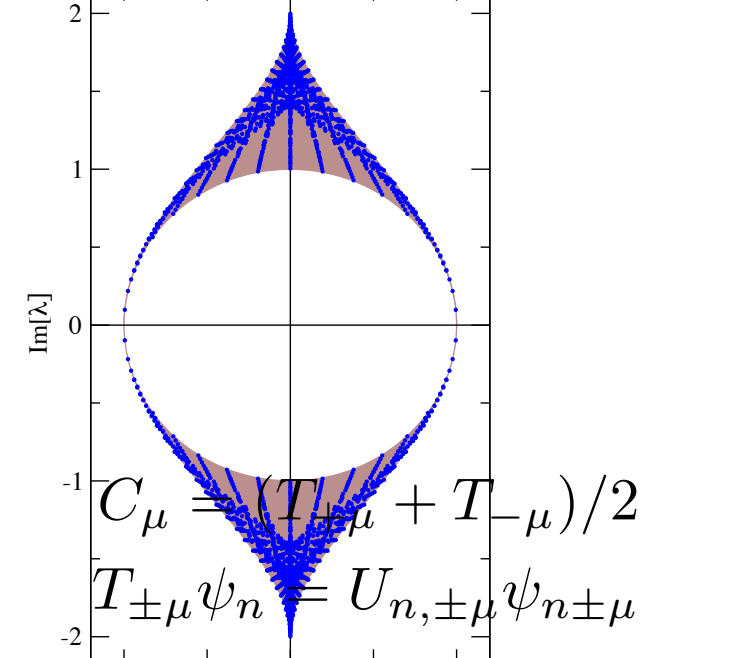

$C_{\mu}=\left(T_{+\mu}+T_{\mu}\right) / 2$
$T_{ \pm}=U_{n, \pm \mu} \psi_{n \pm \mu}$

$$
\begin{aligned}
& \Gamma_{X}^{(+)} \in\left\{\mathbf{1}_{4},(-1)^{n_{1}+\ldots+n_{4}} \gamma_{5},\right. \\
& \Gamma_{X}^{(-)} \in\{
\end{aligned}
$$

\section{Wilson'}

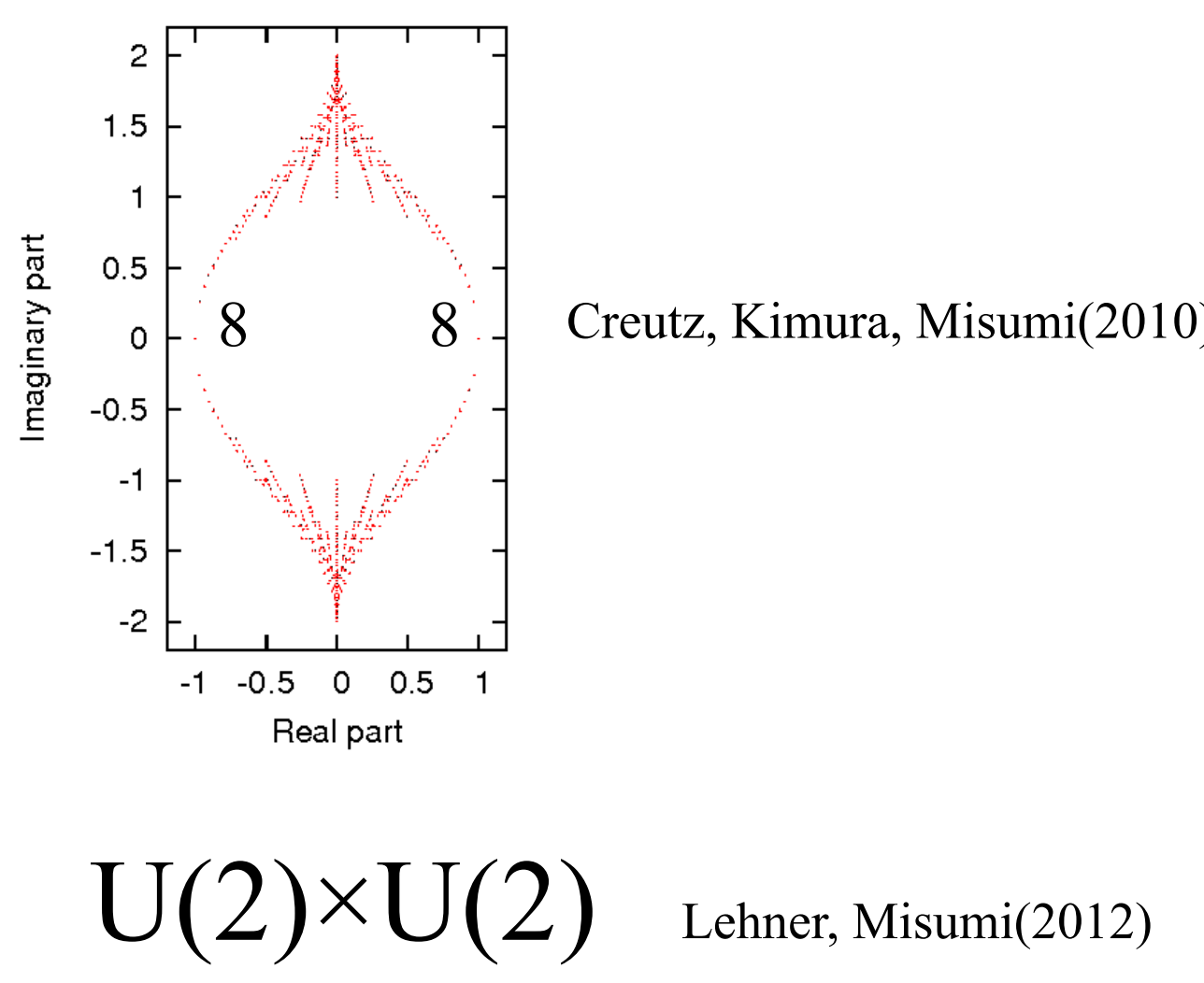




\section{Flavored mass Crut, Kimura, Misumizic010)}

$$
\begin{array}{cc}
M_{\mathrm{V}}=\sum_{\mu} C_{\mu}, & \text { Vector (1-link) } \\
M_{\mathrm{T}}=\sum_{\text {perm. }} \sum_{\text {sym. }} C_{\mu} C_{\nu}, & \text { Tensor (2-link) } \\
M_{\mathrm{A}}=\sum_{\text {perm. }} \sum_{\text {sym. }} \prod_{\nu} C_{\nu}, & \text { Axial-V (3-link) } \\
M_{\mathrm{P}}=\sum_{\text {sym. }} \prod_{\mu=1}^{4} C_{\mu}, & \text { Pseudo-S (4-link) }
\end{array}
$$

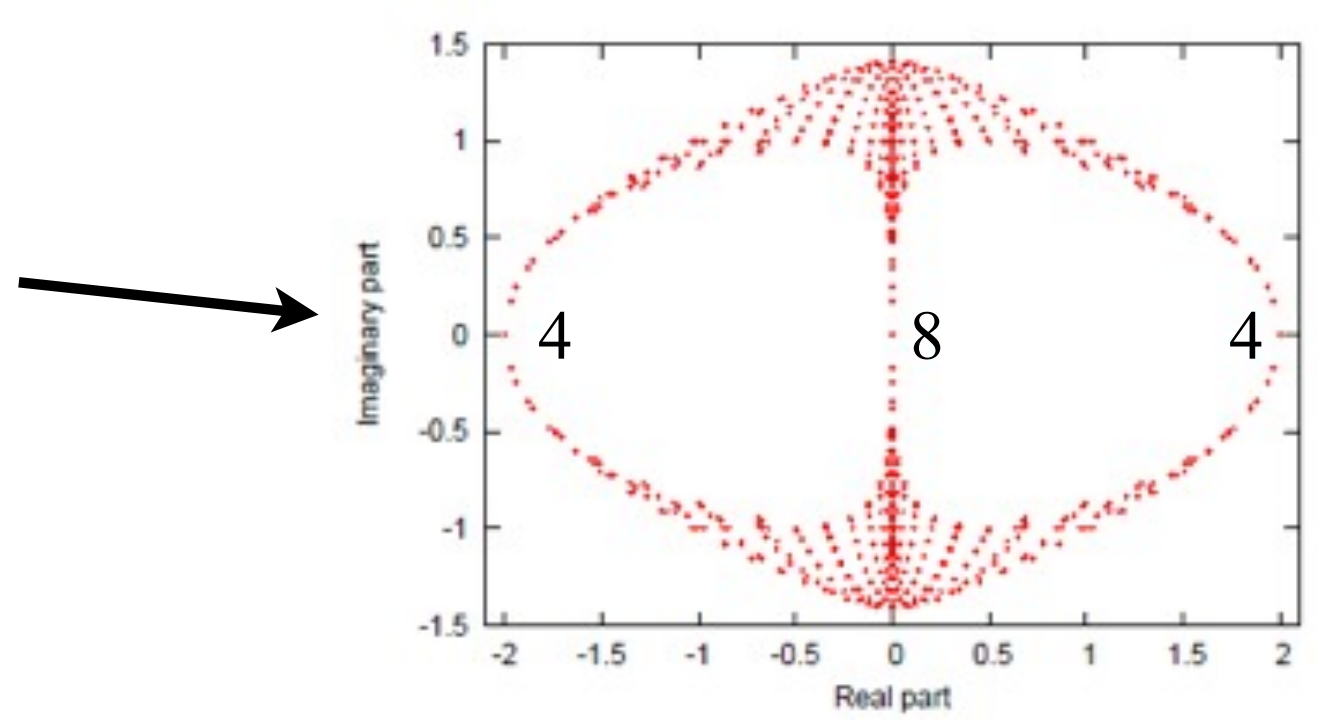

- gamma-5 hermiticity

- 2 nd derivative terms $\quad \sum_{n} \bar{\psi}_{n}\left(M_{P}-1\right) \psi_{n} \rightarrow-a \int d^{4} x \bar{\psi}(x) D_{\mu}^{2} \psi(x)+O\left(a^{2}\right)$

- Cousins of Wilson fermion 
Naive

\section{Flavored-mass}

\section{Wilson \\ Domain-wall $^{(\text {) }}$ \\ Overlap ${ }^{(2)}$}

Spin diag.

Staggered 
Naive

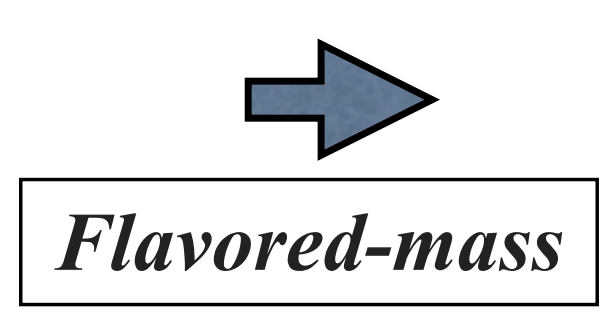

Wilson ${ }^{()}$

St.Wilson

Spin diag.

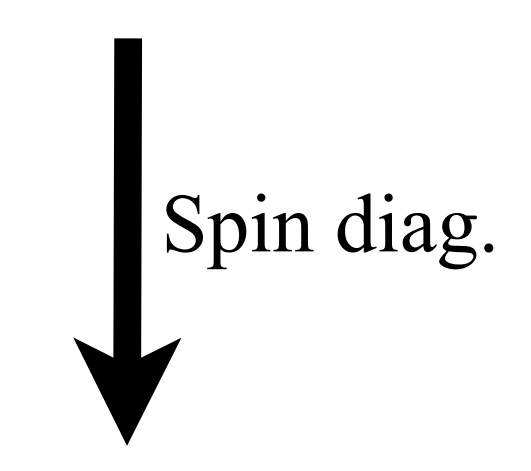

$\underset{\text { Stavored-mass }}{\text { Staggered }} \Rightarrow$

Staggered
Domain-wall $^{(\text {) }}$

Overlap ${ }^{(2)}$

Spin diag.

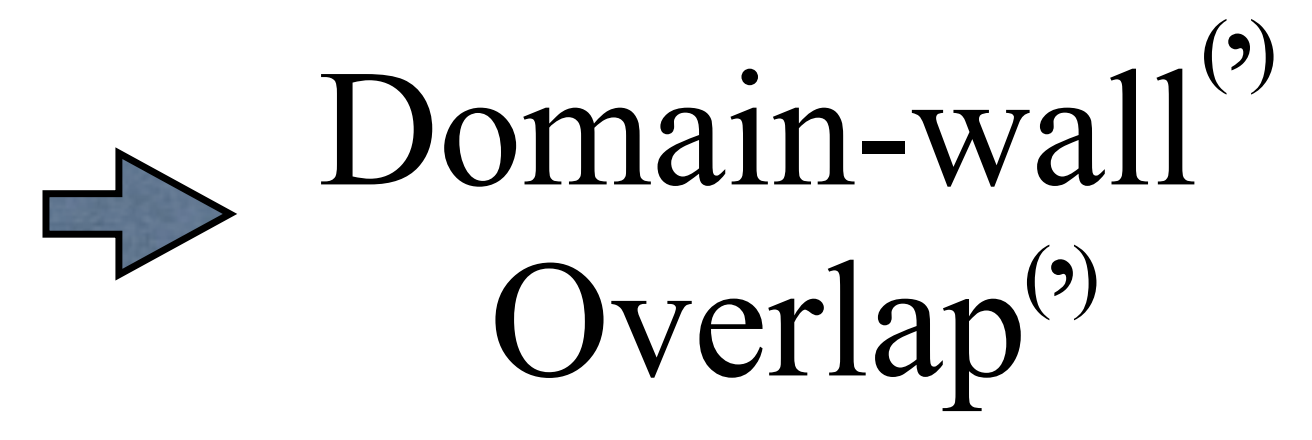

St.Dm-wall

St.Overlap 


\section{- Staggered-Wilson}

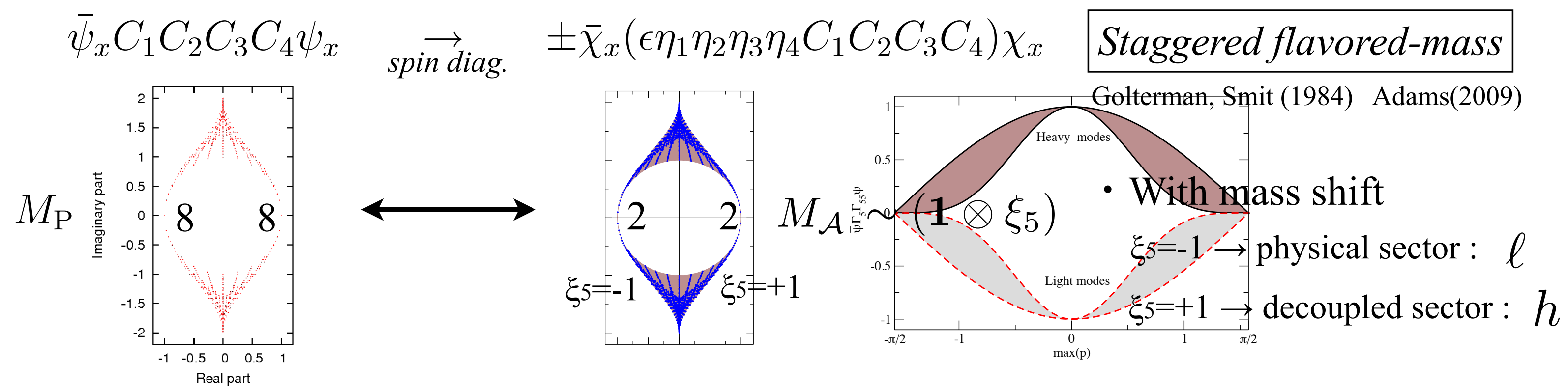

- Index theorem Adams (09), Creutz,Kimura,Misumi(10), Follana, et.al.(11)

- Aoki phase Creutz, Kimura Misumi (11)

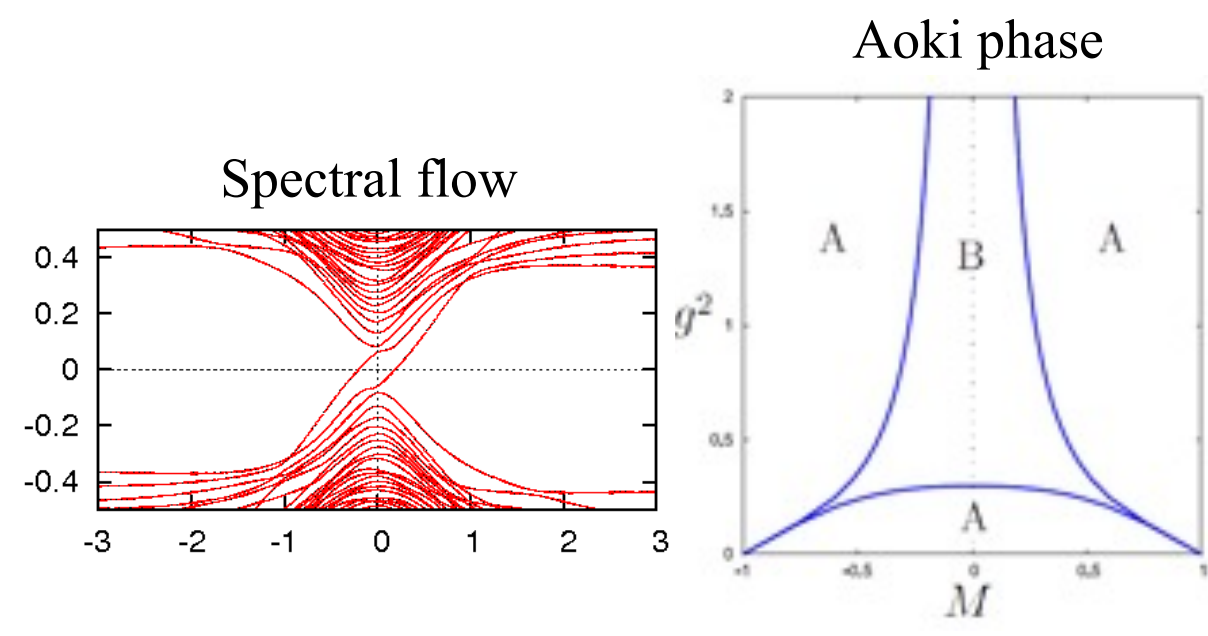

Aoki phase

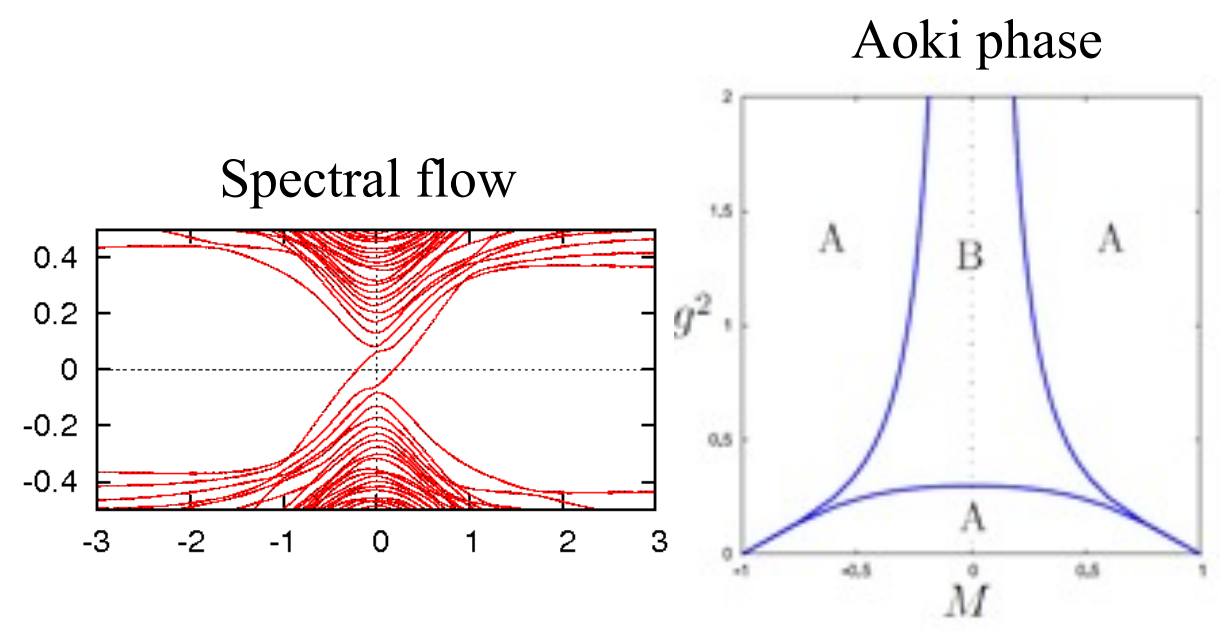

$\S$ Potential advantages of $\eta_{\mu} D_{\mu}+r\left(1+M_{\mathcal{A}}\right)$

- could reduce numerical costs in 2-flavor overlap de Forcrand, Kurkela, Panero(2012)

- could reduce influence of taste-breaking for 2-flavor Sharpe (2012) 
$\S$ Staggered sym. $\left\{C_{0}, \Xi_{\mu}, I_{s}, R_{\mu \nu}\right\} \times\left\{U^{\epsilon}(1)\right\}_{m=0} \Rightarrow\left\{C_{0}, \Xi_{j}, I_{s}, R_{i j}\right\}$ Transfer-matrix sym.

$\checkmark$ classify 15 pseudoscalar operators Golterman (1986)

$1: \xi_{4}, \xi_{45}, \xi_{5}$

$\mathbf{3}: \xi_{i}, \xi_{i 5}, \xi_{i j} \xi_{i 4}$ cf.) ChPT by Lee, Sharpe (1999) $1: \xi_{5}$, 4 irreps of $\mathrm{SO}(4)$ upto $4: \xi_{\mu}, \xi_{\mu 5}$, $O\left(a^{4}\right), O\left(a^{2} m\right) O\left(a^{2} p^{2}\right) \quad 6: \xi_{\mu \nu}$.

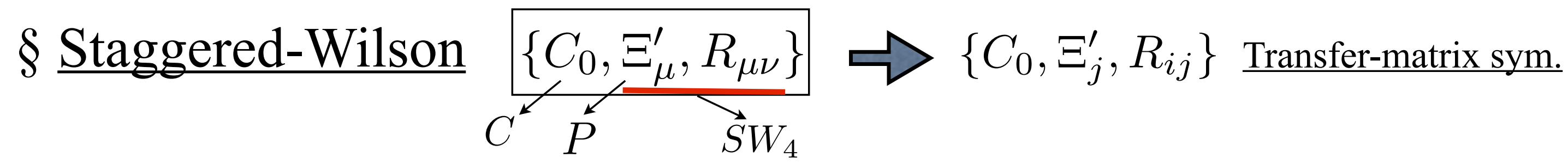

Irreps mix in $\xi_{5}$ pairs

Physical sector

$1 \quad \& \quad \xi_{5} \quad \longrightarrow \quad \bar{\ell} \ell, \quad \bar{h} h \quad \longrightarrow \bar{\ell}\left(\gamma_{5} \otimes \mathbf{1}\right) \ell \quad \eta^{\prime}$

$\Rightarrow \xi_{4} \quad \& \quad \xi_{45} \rightarrow \quad \bar{\ell} h, \bar{h} \ell$

$\xi_{i 4} \quad \& \quad \xi_{i 45} \longrightarrow \bar{\ell} \sigma_{j} \ell, \bar{h} \sigma_{j} h \longrightarrow \bar{\ell}\left(\gamma_{5} \otimes \sigma_{i}\right) \ell \pi_{0}, \pi_{ \pm} \quad$ States in 3d irrep

$\xi_{i} \quad \& \quad \xi_{i 5} \rightarrow \bar{\ell} \sigma_{j} h, \bar{h} \sigma_{j} \ell$

Sharpe (2012)

Discrete symmetries seem sufficient for degenerate pion triplet!

cf.) $\mathrm{ChPT} \rightarrow \mathrm{SU}(2)$ restoration upto $O\left(a^{4}\right), O\left(a^{2} m\right) O\left(a^{2} p^{2}\right)$ 
Naive

\section{Flavored-mass}

Wilson ${ }^{(9)}$

St.Wilson
Domain-wall $^{(\text {) }}$

Overlap (')

Spin diag.

Staggered

Flavored-mass
St.Dm-wall St.Overlap 
Naive

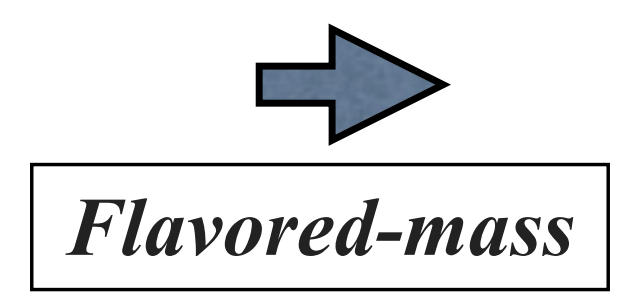

Spin diag.
Wilson ${ }^{(1)}$

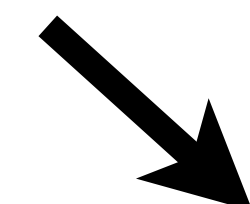

\section{Domain-wall $^{(\text {) }}$ \\ Overlap ${ }^{(2)}$}

\section{Central-branch}

St.Wilson

\section{St.Dm-wall \\ St.Overlap}




\section{Central-branch}

Creutz, Kimura, Misumi (2011)

Kimura, Komatsu, Misumi, Noumi, Torii, Aoki (2012)

- Wilson w/o onsite term $M_{W} \equiv m+4 r=0$

$$
\begin{aligned}
S= & \frac{1}{2} \sum_{x, \mu} \bar{\psi}_{x}\left[\gamma_{\mu}\left(\psi_{x+\mu}-\psi_{x-\mu}\right)-\left(\psi_{x+\mu}+\psi_{x-\mu}\right)\right] \\
&
\end{aligned}
$$

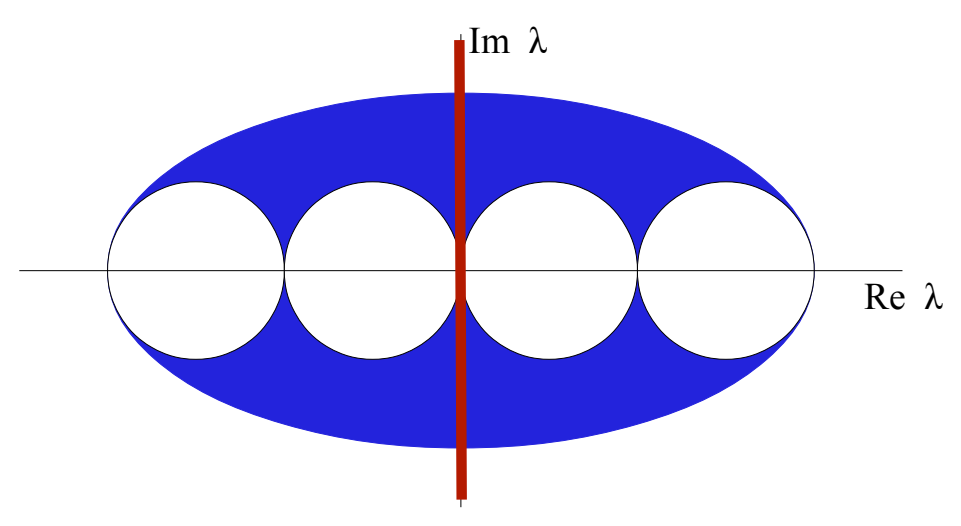

$$
\psi_{x} \rightarrow e^{i \theta(-1)^{x_{1}+x_{2}+x_{3}+x_{4}}}, \quad \bar{\psi}_{x} \rightarrow \bar{\psi}_{x} e^{i \theta(-1)^{x_{1}+x_{2}+x_{3}+x_{4}}}
$$

$$
\begin{aligned}
& \Gamma_{X}^{(+)} \in\left\{\mathbf{1}_{4},(-1)^{n_{1}+\ldots+n_{4}} \gamma_{5},(-1)^{\check{n}_{\mu}} \gamma_{\mu},(-1)^{n_{\mu}} i \gamma_{\mu} \gamma_{5},(-1)^{n_{\mu, \nu}} \frac{\left[\gamma_{\mu}, \gamma_{\nu}\right]}{2}\right\} \\
& \Gamma_{X}^{(-)} \in\left\{(-1)^{n_{1}+\ldots+n_{4}} \mathbf{1}_{4}, \gamma_{5},(-1)^{n_{\mu}} \gamma_{\mu},(-1)^{\check{n}_{\mu}} \gamma_{\mu} \gamma_{5},(-1)^{\check{n}_{\mu, \nu}} \frac{\left[\gamma_{\mu}, \gamma_{\nu}\right]}{2}\right\}
\end{aligned}
$$




\section{Central-branch}

Creutz, Kimura, Misumi (2011)

Kimura, Komatsu, Misumi, Noumi, Torii, Aoki (2012)

- Wilson w/o onsite term $M_{W} \equiv m+4 r=0$

$$
\begin{aligned}
S= & \frac{1}{2} \sum_{x, \mu} \bar{\psi}_{x}\left[\gamma_{\mu}\left(\psi_{x+\mu}-\psi_{x-\mu}\right)-\left(\psi_{x+\mu}+\psi_{x-\mu}\right)\right] \\
&
\end{aligned}
$$

$$
\psi_{x} \rightarrow e^{i \theta(-1)^{x_{1}+x_{2}+x_{3}+x_{4}}}, \quad \bar{\psi}_{x} \rightarrow \bar{\psi}_{x} e^{i \theta(-1)^{x_{1}+x_{2}+x_{3}+x_{4}}}
$$

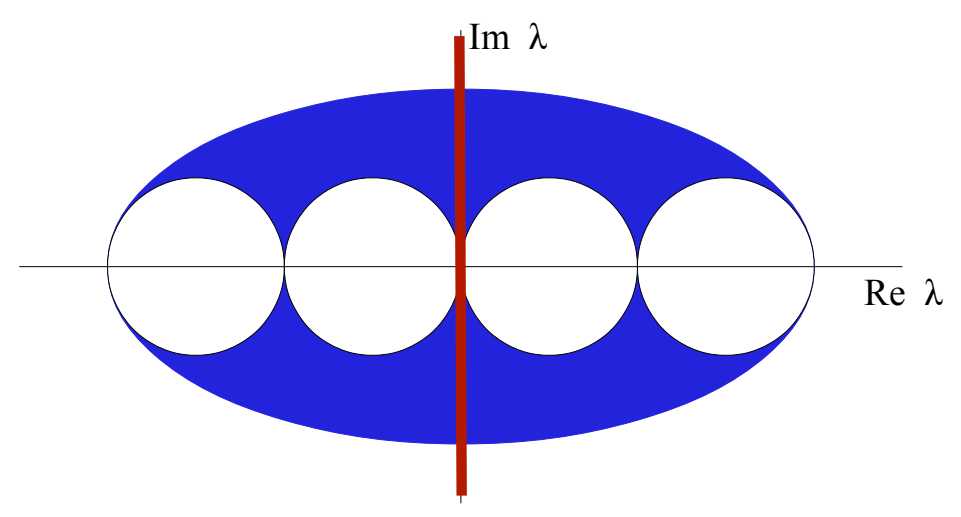

$$
\begin{aligned}
& \Gamma_{X}^{(+)} \in\left\{\mathbf{1}_{4},\right. \\
& \Gamma_{X}^{(-)} \in\left\{\frac{(-1)^{n_{1}+\ldots+n_{4}} \mathbf{1}_{4}}{}\right. \\
& \searrow \text { Prohibits additive mass renormalization! } \\
& \text { SSB gives NG boson! }
\end{aligned}
$$


- Strong-coupling QCD

$$
\cosh \left(m_{S P A}\right)=1+\frac{2 M_{W}^{2}\left(16+M_{W}^{2}\right)}{16-15 M_{W}^{2}}
$$

- Pion (eta) condensate $\left\langle\bar{\psi} \gamma_{5} \psi\right\rangle \neq 0$

- No chiral condensate $\langle\bar{\psi} \psi\rangle=0$
NG boson associated with SSB of $U(1)$

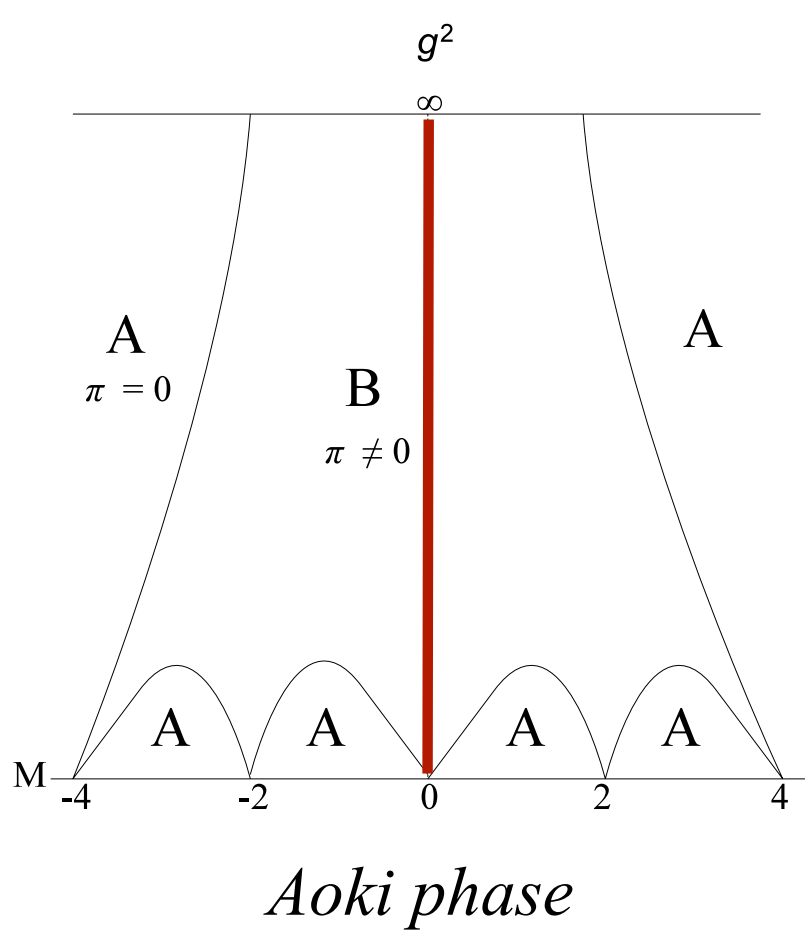

$\S$ Advantages

- No additive mass renormalization (no fine-tuning)

$\bar{\psi} \psi \leftrightarrow \bar{\psi} \gamma_{5} \psi$ change of mass base

- SSB of U(1) and massless NG boson

6-flavor massless $Q C D$

$\S$ Potential drawbacks

- sign problem

- U(1) problem

Twisted-mass works?

$\rightarrow$ 12-flavor massless QCD

- Quark mass 
Flavoredchem. pot.

Naive

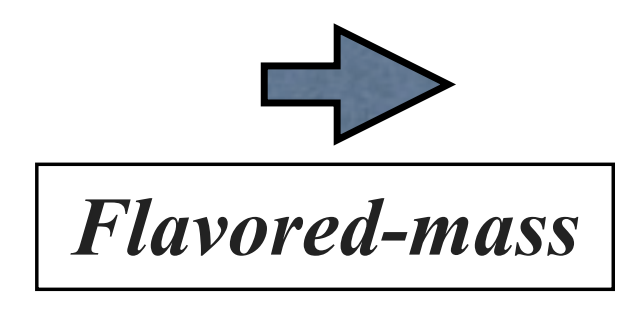

Spin diag.
Wilson ${ }^{(0)}$

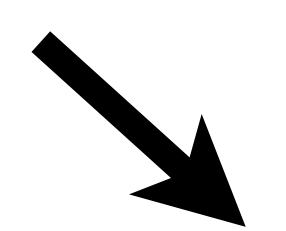

Domain-wall" Overlap ${ }^{(2)}$

\section{Central-branch}

St.Dm-wall St.Overlap 
FCP

\section{$\Rightarrow$ FCP-Wilson}

Flavored-mass

\section{FCP Dm-wall FCP Overlap \\ 5}

Flavored-

chem. pot.
Naive

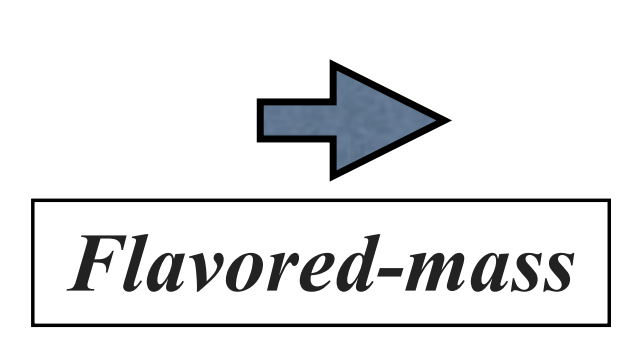

Wilson')

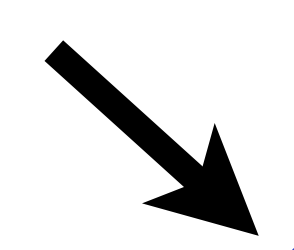

Domain-wall Overlap ${ }^{(2)}$

\section{Central-branch}

Spin diag.

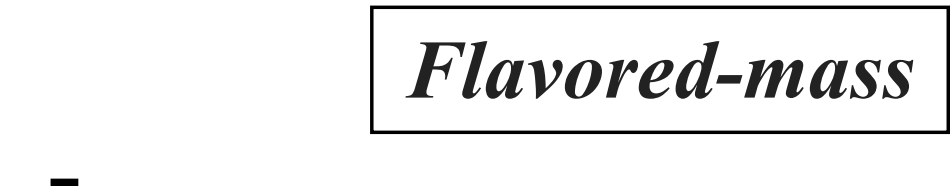

r

\section{Staggered}

St.Dm-wall

St.Wilson 


\section{Flavored chemical potential Misum (2021)}

Wilson

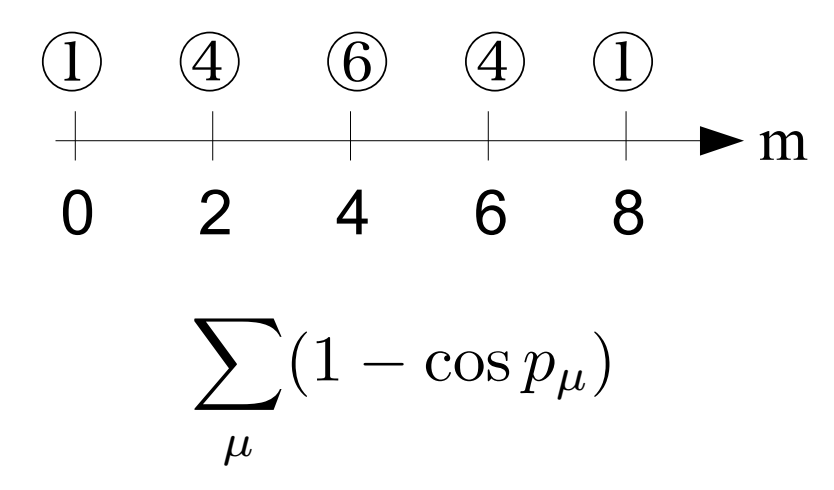

Flavored chemical-pot.

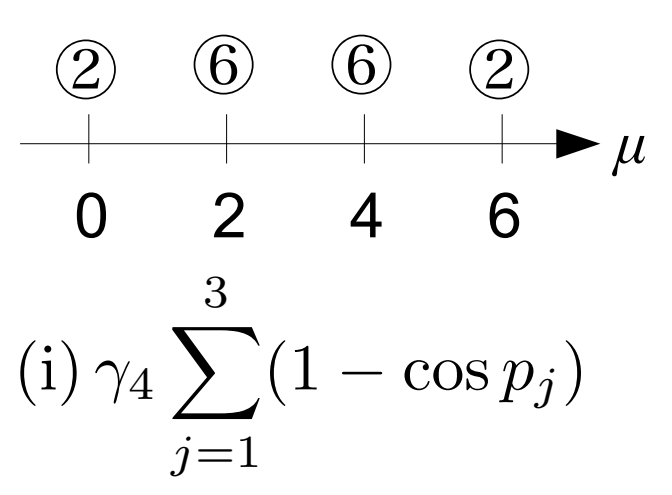

- Real type $\rightarrow$ Sign problem

- Imaginary type $\rightarrow$ No sign problem “Minimal-doubling" $\begin{aligned} & \text { Karsten(81)Wilczek(87)Creutz, } \\ & \text { Borici(07)Creutz\&Misumi(10) }\end{aligned}$

cf.)Bedaque, Buchoff, Tiburzi, Walker-Loud(08)

\section{$\sqrt{7 \text { Finite-mass system(Wil) } \leftrightarrows \text { Finite-density system(FCP) }}$}

- Advantage

- U(1) chiral symmetry

- 2 flavor possible
(1) $U(1)_{V} \times \underline{U(1)_{A}}$

(2) $\mathrm{P}$

(3) $\mathrm{CT}$

(4) Cubic symmetry

- Drawbacks

- $\mathrm{O}(1 / a)$ chemical potential renormalization

- Tuning a parameter even for finite- $\mu$ QCD

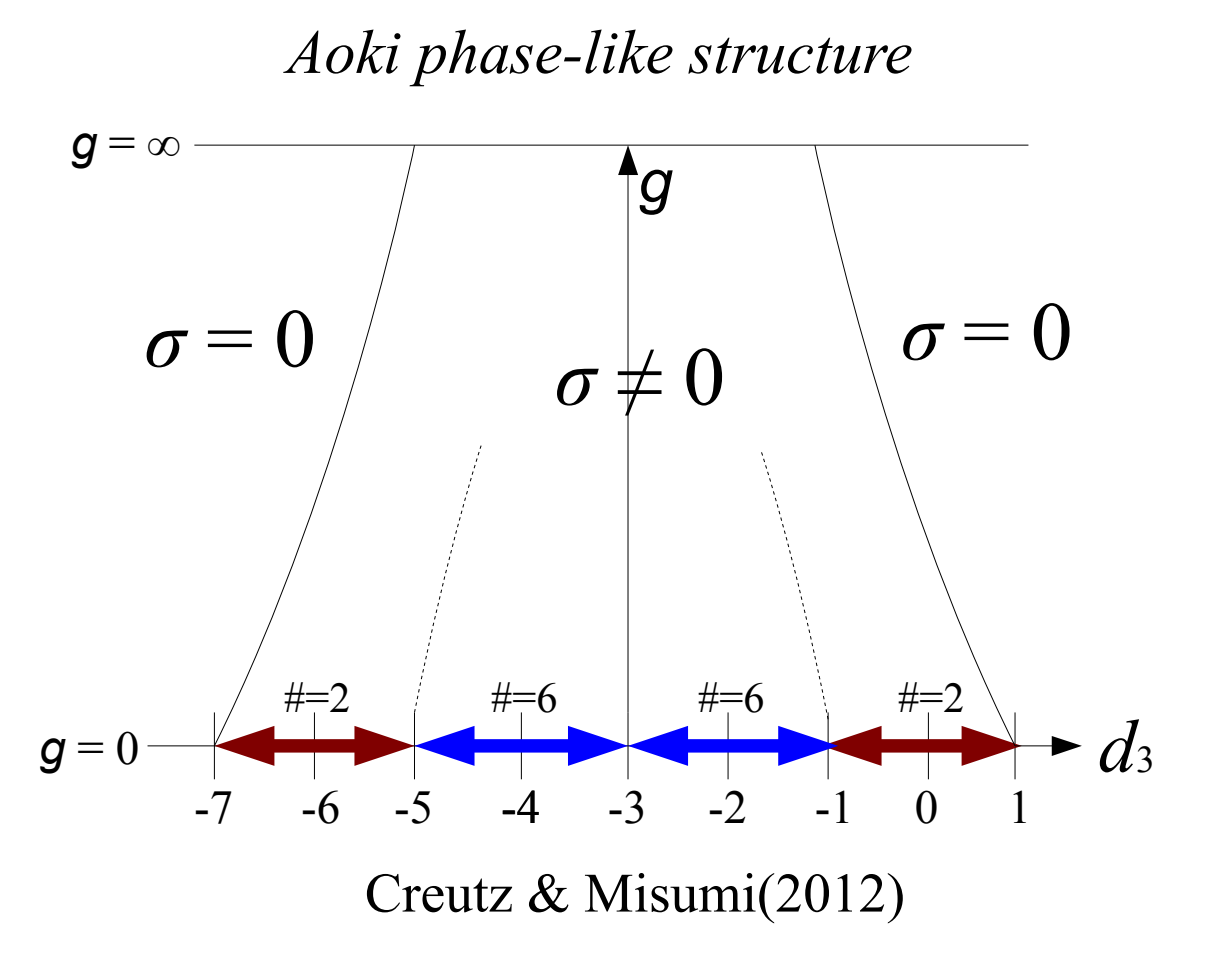


- Finite $(T, \mu)$ QCD with FCP Misumi, Kimura, Ohnishi (2012)

$$
\begin{aligned}
S_{\mathrm{md}}=\sum_{x} & {\left[\frac{1}{2} \sum_{j=1}^{3} \bar{\psi}_{x} \gamma_{j}\left(U_{x, x+j} \psi_{x+j}-U_{x, x-j} \psi_{x-j}\right)+\frac{1}{2} \bar{\psi}_{x} \gamma_{4}\left(e^{\mu} U_{x, x+4} \psi_{x+4}\right.\right.} \\
+ & \left.\frac{i}{2} \sum_{j=1}^{3} \bar{\psi}_{x} \gamma_{4}\left(2 \psi_{x}-U_{x, x+j} \psi_{x+j}-U_{x, x-j} \psi_{x-j}\right)+i d_{3} \bar{\psi}_{x} \gamma_{4} \psi_{x}\right]
\end{aligned}
$$

$\S$ Strong-coupling study

Effective potential of $\sigma$ as a function of $T, \mu$ and $d 3$

$$
\begin{aligned}
\mathcal{F}_{\text {eff }}\left(\sigma ; m, T, \mu, d_{3}\right)=\frac{9}{2} \sigma^{2} & -\frac{3}{2} \log \left(1+\left(d_{3}+3\right)^{2}\right) \\
& -\max \left\{3 \operatorname{arcsinh}\left(\frac{3 \sqrt{2} \sigma}{2 \sqrt{1+\left(d_{3}+3\right)^{2}}}\right), \mu_{B}\right\} .
\end{aligned}
$$
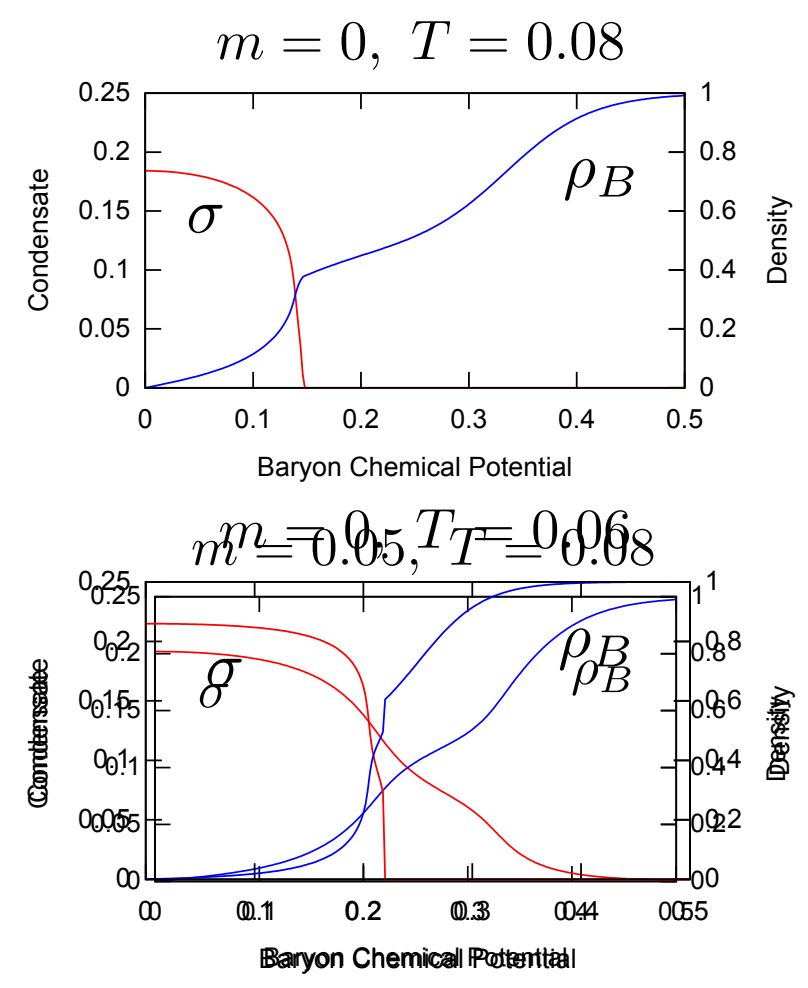

3 Chiral phase structure

- 1 st and 2 nd phase transition $(\mathrm{m}=0)$

- 1 st, critical point and crossover $(\mathrm{m} \neq 0)$

New possibility of $(T, \mu)$ lattice $Q C D$ !

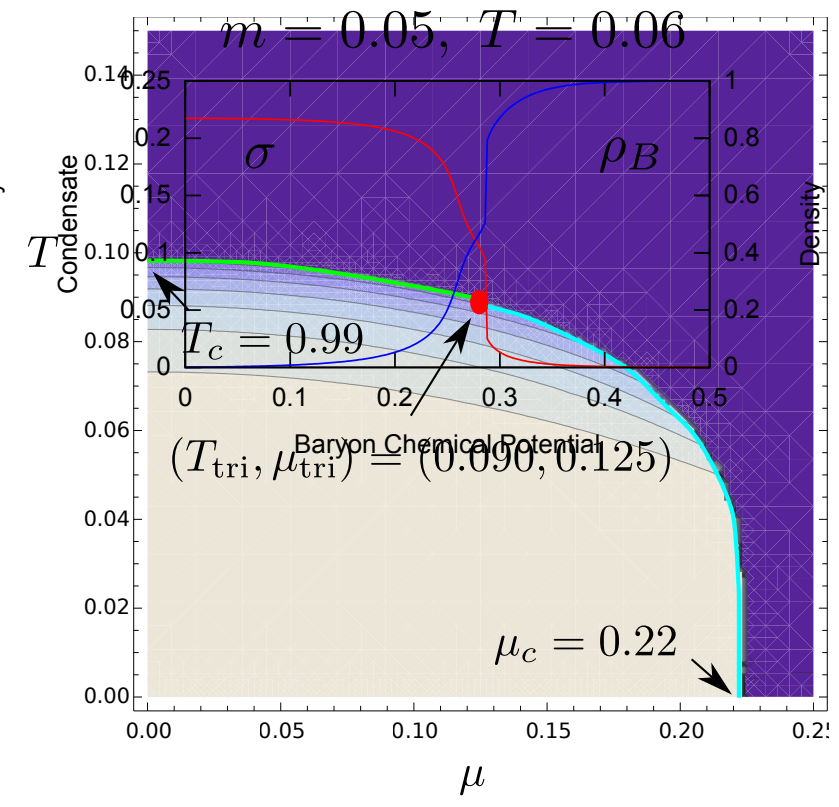




\section{Summary}

1. Flavored-mass terms give us new types of Wilson and overlap fermions.

2. Staggered-Wilson can be an alternative Wilson and overlap for 2-flavor QCD (3 degenerate pion spectrum)

3. Central-branch fermion is a new possibility of use of Wilson for many-flavor QCD without fine-tuning of parameters.

4. Flavored-chemical-potential fermion would be useful for finite-temperature \& density lattice QCD. 


\section{Back-up slides}




\section{Adams-type flavored mass}

$$
\begin{aligned}
\bar{\psi}_{x} \psi_{x+\hat{1}+\hat{2}+\hat{3}+\hat{4}} & =\bar{\chi}_{x} \gamma_{4}^{x_{4}} \gamma_{3}^{x_{3}} \gamma_{2}^{x_{2}} \gamma_{1}^{x_{1}} \gamma_{1}^{x_{1}+1} \gamma_{2}^{x_{2}+1} \gamma_{3}^{x_{3}+1} \gamma_{4}^{x_{4}+1} \chi_{x+\hat{1}+\hat{2}+\hat{3}+\hat{4}} \\
& =(-1)^{x_{2}+x_{4}} \bar{\chi}_{x} \gamma_{5} \chi_{x+\hat{1}+\hat{2}+\hat{3}+\hat{4}} \quad\left(\gamma_{5} \text { diagonalized }\right) \\
& \rightarrow \pm \bar{\chi}_{x} \epsilon \eta_{1} \eta_{2} \eta_{3} \eta_{4} \chi_{x+\hat{1}+\hat{2}+\hat{3}+\hat{4}}
\end{aligned}
$$

\section{Hoelbling-type flavored mass Hoelbing (2010), de Forrand (2010)}

- spin diagonalization

$$
\begin{aligned}
\bar{\psi}_{x} \psi_{x+\hat{1}+\hat{2}}+\bar{\psi}_{x} \psi_{x+\hat{3}+\hat{4}} & =(-1)^{x_{2}} \bar{\chi}_{x} \gamma_{1} \gamma_{2} \chi_{x+\hat{1}+\hat{2}}+(-1)^{x_{4}} \bar{\chi}_{x} \gamma_{3} \gamma_{4} \chi_{x+\hat{3}+\hat{4}} \\
& \rightarrow \pm \bar{\chi}_{x} i \epsilon_{12} \eta_{1} \eta_{2} \chi_{x+\hat{1}+\hat{2}} \pm \bar{\chi}_{x} i \epsilon_{34} \eta_{3} \eta_{4} \chi_{x+\hat{3}+\hat{4}}
\end{aligned}
$$

※ two terms simultaneously diagonalizable : $\left[\sigma_{12}, \sigma_{34}\right]=0$
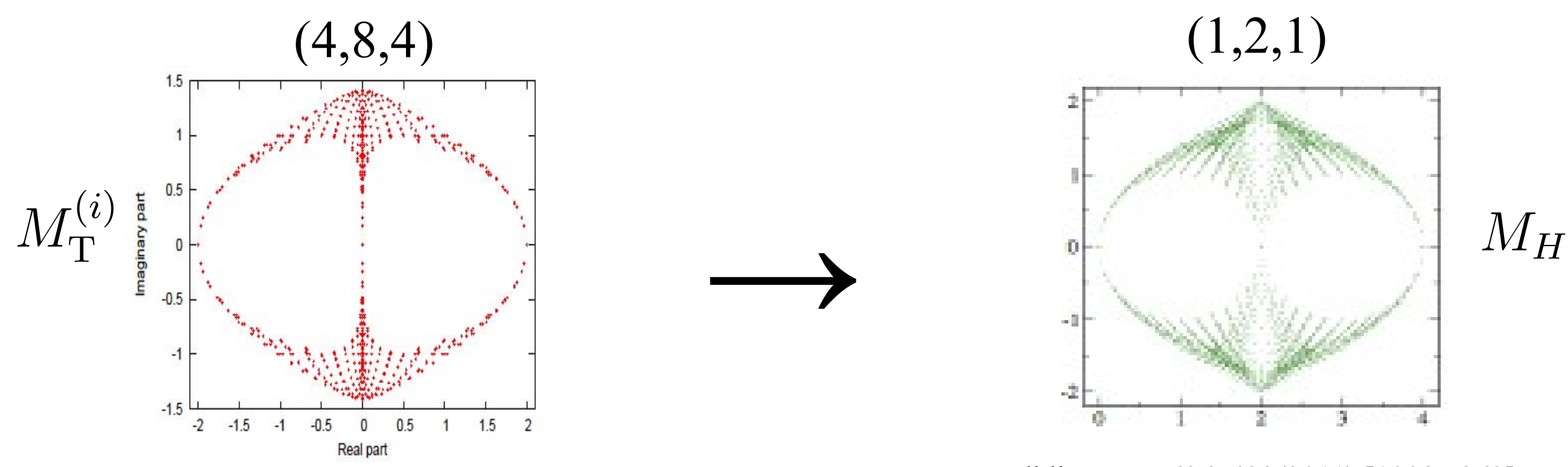

Hoelbling, PLB696, 422(2011) [1009.5362]. 


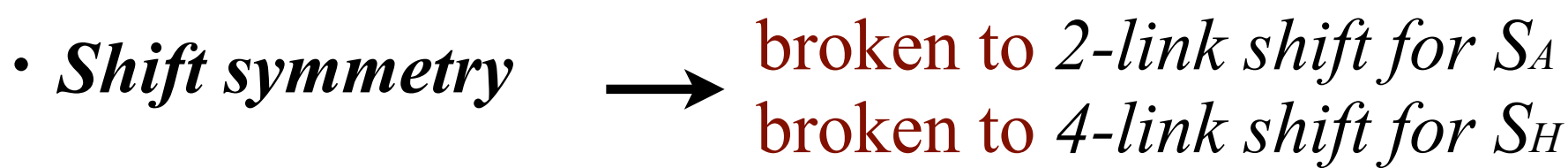

$$
\mathcal{S}_{\rho}: \chi_{x} \rightarrow \zeta_{\rho}(x) \chi_{x+\hat{\rho}}, \quad \bar{\chi}_{x} \rightarrow \zeta_{\rho}(x) \bar{\chi}_{x+\hat{\rho}}, \quad U_{\mu, x} \rightarrow U_{\mu, x+\hat{\rho}} \quad \mathcal{S}_{\mu}: \phi(p) \rightarrow \exp \left(i p_{\mu}\right) \Xi_{\mu} \phi(p)
$$

- Axis reversal $\rightarrow$ broken to shifted axis reversal

$$
\mathcal{I}_{\rho}: \chi_{x} \rightarrow(-1)^{x_{\rho}} \chi_{I x}, \quad \bar{\chi}_{x} \rightarrow(-1)^{x_{\rho}} \bar{\chi}_{I x}, \quad U_{\mu, x} \rightarrow U_{\mu, I x} \quad \mathcal{I}_{\rho}: \phi(p) \rightarrow \Gamma_{\rho} \Gamma_{5} \Xi_{\rho} \Xi_{5} \phi(I p)
$$

- Rotation $\rightarrow$ remain in $S_{A}$

$$
\text { broken to subgroup in } S_{H}
$$

$$
\mathcal{R}_{\rho \sigma}: \chi_{x} \rightarrow S_{R}\left(R^{-1} x\right) \chi_{R^{-1} x}, \quad \bar{\chi}_{x} \rightarrow S_{R}\left(R^{-1} x\right) \bar{\chi}_{R^{-1} x}, \quad U_{\mu, x} \rightarrow U_{\mu, R x} \quad \mathcal{R}_{\rho \sigma}: \phi(p) \rightarrow \exp \left(\frac{\pi}{4} \Gamma_{\rho} \Gamma_{\sigma}\right) \exp \left(\frac{\pi}{4} \Xi_{\rho} \Xi_{\sigma}\right) \phi\left(R^{-1} p\right)
$$

- Conjugation

$$
\longrightarrow \quad \begin{array}{r}
\text { remain in } S_{A} \\
\text { broken in } S_{H}
\end{array}
$$

$$
\mathcal{C}: \chi_{x} \rightarrow \epsilon_{x} \bar{\chi}_{x}^{T}, \bar{\chi}_{x} \rightarrow-\epsilon_{x} \bar{\chi}_{x}^{T}, U_{\mu, x} \rightarrow U_{\mu, x}^{*}
$$

$$
\mathcal{C}: \phi(p) \rightarrow \bar{\phi}(-p)^{T}
$$


Details of StWil symmetries

$$
\begin{aligned}
& \left\{\Xi_{\mu}, I_{s}, R_{\mu \nu}\right\} \rightarrow \Gamma_{4} \rtimes S W_{4} \\
& \left\{\Xi_{\mu}^{\prime}, R_{\mu \nu}\right\} \rightarrow \Gamma_{3} \rtimes S W_{4}
\end{aligned}
$$

Physical-sector symmetry

$$
\begin{aligned}
\Xi_{j}^{\prime} \Xi_{4}^{\prime} R_{j 4}^{2} & =\Xi_{j} \Xi_{4} \sim\left(1 \otimes \sigma_{j}\right) \\
\Xi_{4}^{\prime} R_{34}^{2} R_{12}^{2} & =\Xi_{4} I_{s} \sim\left(\gamma_{4} \otimes \mathbf{1}\right) \\
C_{0} \Xi_{2}^{\prime} \Xi_{4}^{\prime} R_{24}^{2} & \sim C
\end{aligned}
$$




\section{Details of timeslice symmetries}

Enlarged staggered sym : $\left\{C_{0}, \Xi_{\mu}, I_{s}, R_{\mu \nu}, T_{\mu}^{1 / 2}\right\} \quad \Xi_{\mu}^{2}=1$

$$
\longrightarrow T_{\mu}^{1 / 2} \rtimes\left[\left\{C_{0}, \Xi_{\mu}\right\} \rtimes\left\{R_{\mu \nu}, I_{s}\right\}\right]=\left(\otimes_{j} Z_{N_{\mu}}\right) \rtimes\left[\Gamma_{4,1} \rtimes W_{4}\right]
$$

Timeslice sym : $\quad T_{\mu}^{1 / 2} \rtimes\left[\left\{C_{0}, \Xi_{\mu}\right\} \rtimes\left\{R_{i j}, I_{s}\right\}\right]=\left(\otimes_{j} Z_{N_{j}}\right) \rtimes\left[\Gamma_{4,1} \rtimes W_{3}\right]$

Relevant group at rest

$$
\begin{aligned}
\Gamma_{4,1} \rtimes W_{3} & \left.\sim \underline{\left[R_{i j}, \Xi_{i j}\right\}} \times\left\{C_{0}, \Xi_{4}, \Xi_{123}, I_{s}\right\}\right] / Z_{2} \\
& \left.=\underline{\left[R_{i j}, \tilde{R}_{4 i} \equiv \epsilon_{i j k} R_{j k} \Xi_{k j}\right\}} \times\left\{C_{0}, \Xi_{4}, \Xi_{123}, C_{0} \Xi_{4} I_{s}\right\}\right] / Z_{2} \\
& \left.=\underline{\left[W_{4}\right.} \times \Gamma_{2,2}\right] / Z_{2}
\end{aligned}
$$

Staggered-Wilson

$$
\begin{aligned}
\left\{C_{0}, \Xi_{\mu}^{\prime}, R_{\mu \nu}, T_{\mu}^{\prime 1 / 2}\right\} & \sim\left[\left\{R_{i j}, \Xi_{i j}^{\prime}\right\} \times\left\{C_{0}, \Xi_{4}^{\prime}, \Xi_{123}^{\prime}, I_{s}\right\}\right] / Z_{2} \\
& =\left[\left\{R_{i j}, \tilde{R}_{4 i} \equiv \epsilon_{i j k} R_{j k} \Xi_{k j}^{\prime}\right\} \times\left\{C_{0}, \Xi_{4}, \Xi_{123}\right] / Z_{2}\right. \\
& =\underline{\left[S W_{4} \times \Gamma_{1,2}\right] / Z_{2}}
\end{aligned}
$$


Dim3, 4: $\bar{Q}\left(1 \otimes \xi_{F}\right) Q \quad \bar{Q}\left(\gamma_{\mu} \otimes \xi_{F}\right) D_{\mu} Q \quad$ for $\xi_{F}=1$ or $\xi_{5} \quad \leadsto \bar{\ell} \gamma_{\mu} D_{\mu} \ell, \quad \bar{\ell} \ell$ $\operatorname{Dim} 5 \mathrm{O}(a): \quad \bar{Q}\left(i \sigma_{\mu \nu} F_{\mu \nu} \otimes \xi_{F}\right) Q$ for $\xi_{F}=1$ or $\xi_{5} \leftrightharpoons \bar{\ell} i \sigma_{\mu \nu} F_{\mu \nu} \ell$

No unphysical term nor taste-breaking term up to $O(a)$

Dim6 $\mathrm{O}\left(\mathfrak{a}^{2}\right): 2$ types of four-fermi operators $\quad \mathcal{L}_{6}^{F F(A)}$ and $\mathcal{L}_{6}^{F F(B)}$

In $\mathcal{L}_{6}^{F F(A)}$ the spin and flavor independently forms scalar

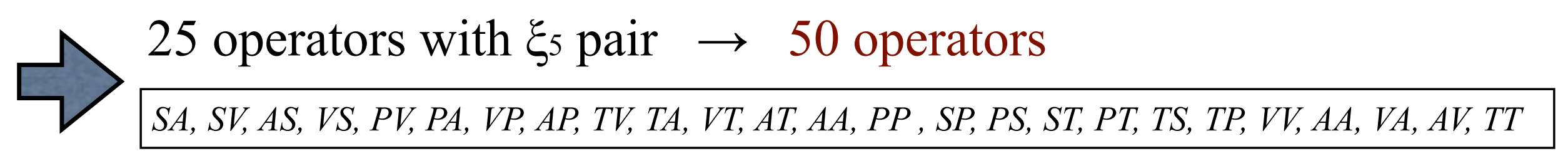

$\rightarrow$ No taste-breaking. No derivative terms. Contributes to potential $\mathcal{V}_{6}^{F F(A)}$

In $\mathcal{L}_{6}^{F F(B)}$ the spin and flavor are not independent

510 operators with $\xi_{5}$ pair $\rightarrow 20$ operators

$\rightarrow$ Taste-breaking. Derivative terms. No contribution to potential $\quad \mathcal{V}_{6}^{F F(B)}$

No taste-breaking in ChPT potential upto $O\left(a^{2}\right)$ : $S U(2)$ 
- Central cusps for other flavored masses

- For other naive flavored mass terms

$$
\begin{aligned}
& M_{\mathrm{A}}: \mathrm{U}(1) \text { restored } \\
& M_{\mathrm{T}}^{(i)}: \mathrm{U}(2) \text { restored } \\
& M_{\mathrm{P}}: \mathrm{U}(2) \times \mathrm{U}(2) \text { restored }
\end{aligned}
$$

- For staggered flavored mass terms

$M_{\mathcal{A}}: \mathrm{C}_{\mathrm{T}} \Xi, \mathrm{C}_{\mathrm{T}} \mathrm{I}$ restoed

$M \mathfrak{H}: \mathrm{C}_{\mathrm{T}}$ restored

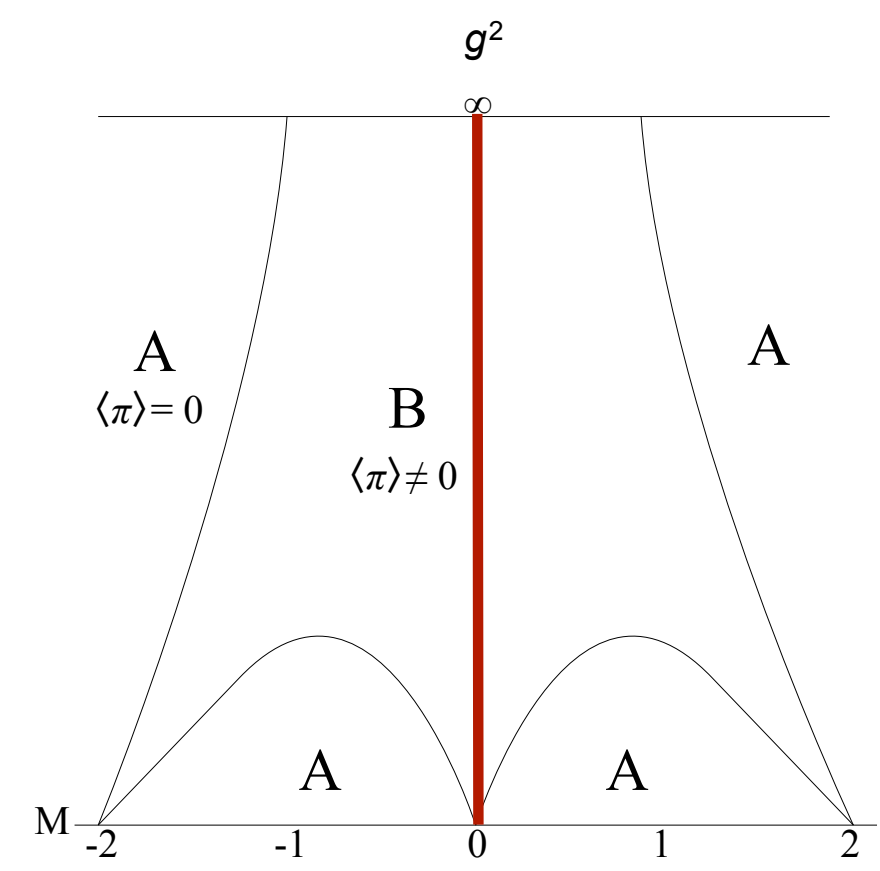

$$
\mathcal{C}: \chi_{x} \rightarrow \bar{\chi}_{x}^{T}, \quad \bar{\chi}_{x} \rightarrow \chi_{x}^{T}, \quad U_{\mu, x} \rightarrow U_{\mu, x}^{*}
$$

\begin{tabular}{ccccccc}
\hline \hline & $C_{T}^{\prime}$ & $\Xi_{\mu}$ & $I_{\mu}$ & $C_{T}^{\prime} \Xi_{\mu}$ & $C_{T}^{\prime} I_{\mu}$ & $\Xi_{\mu} I_{\mu}$ \\
\hline$S_{s t}$ & $\circ$ & $\circ$ & $\circ$ & $\circ$ & $\circ$ & $\circ$ \\
$S_{\mathcal{A}}$ & $\times$ & $\times$ & $\times$ & $\circ$ & $\circ$ & $\circ$ \\
$S_{\mathcal{H}}$ & $\circ$ & $\times$ & $\times$ & $\times$ & $\times$ & $\circ$ \\
$S_{m}$ & $\times$ & $\circ$ & $\circ$ & $\times$ & $\times$ & $\circ$ \\
\hline \hline
\end{tabular}




\subsection{Strong coupling analysis}

Now we employ the strong coupling analysis to show that there appears an NG boson associated with the $\mathrm{U}(1)_{V}^{-}$symmetry breaking in the presence of the pion condensate. An effective action for mesons in the strong coupling limit $[42,9,10]$ can be written in general as

$$
\begin{aligned}
S_{\mathrm{eff}}(M) & =N_{c} \sum_{n}\left[\sum_{\mu} \operatorname{Tr} f\left(\Lambda_{n, \mu}\right)+\operatorname{tr} \hat{M} M(n)-\operatorname{tr} \log M(n)\right] \\
\Lambda_{n, \mu} & =\frac{V_{n, \mu} \bar{V}_{n, \mu}}{N_{c}^{2}}, \quad M(n)^{\alpha \beta}=\frac{\sum_{a} \bar{\psi}_{n}^{a, \alpha} \psi_{n}^{a, \beta}}{N_{c}}
\end{aligned}
$$

where $N_{c}$ is the number of colors, $\operatorname{Tr}$ ( $\operatorname{tr}$ ) means a trace over color(spinor) index, and $M(n)$ is a meson field. The explicit form of the function $f$ is determined by performing a one-link integral of the gauge field. More explicitly we can write

$$
\begin{aligned}
V_{n, \mu}^{a b} & =\bar{\psi}_{n}^{b} P_{\mu}^{-} \psi_{n+\hat{\mu}}^{a}, \quad \bar{V}_{n, \mu}^{a b}=-\bar{\psi}_{n+\hat{\mu}}^{b} P_{\mu}^{+} \psi_{n}^{a}, \\
\operatorname{Tr} f\left(\Lambda_{n, \mu}\right) & =-\operatorname{tr} f\left(-M(n)\left(P_{\mu}^{+}\right)^{\mathrm{T}} M(n+\hat{\mu})\left(P_{\mu}^{-}\right)^{\mathrm{T}}\right),
\end{aligned}
$$

where $4 \times 4$ matrices $P_{\mu}^{ \pm}$are specified later. In the large $N_{c}$ limit, it is known that $f(x)$ can be analytically evaluated as

$$
f(x)=\sqrt{1+4 x}-1-\ln \frac{1+\sqrt{1+4 x}}{2}=x+O\left(x^{2}\right) .
$$

However, in the following part of this paper, we will approximate $f(x)$ as $f(x)=x$ unless otherwise stated because qualitative features such as an appearance of NG bosons remain unchanged by this approximation.

To calculate meson masses we expand the meson field $\mathrm{as}^{5}$

$$
M(n)=M_{0}^{\mathrm{T}}+\sum_{X} \pi^{X}(n) \Gamma_{X}^{\mathrm{T}}, \quad X \in\left\{S, P, V_{\alpha}, A_{\alpha}, T_{\alpha \beta}\right\}
$$

where $M_{0}$ is the vacuum expectation value (VEV) of $M(n)$, and

$$
\Gamma_{S}=\frac{\mathbf{1}_{4}}{2}, \Gamma_{P}=\frac{\gamma_{5}}{2}, \Gamma_{V_{\alpha}}=\frac{\gamma_{\alpha}}{2}, \Gamma_{A_{\alpha}}=\frac{\mathrm{i} \gamma_{5} \gamma_{\alpha}}{2}, \Gamma_{T_{\alpha \beta}}=\frac{\gamma_{\alpha} \gamma_{\beta}}{2 \mathrm{i}}(\alpha<\beta)
$$

Then the effective action at the second order of $\pi^{X}$ is given by

$$
\begin{aligned}
S_{\mathrm{eff}}^{(2)} & =N_{c} \sum_{n}\left[\frac{1}{2} \operatorname{tr}\left(M_{0}^{-1} \Gamma_{X} M_{0}^{-1} \Gamma_{Y}\right) \pi^{X}(n) \pi^{Y}(n)+\sum_{\mu} \operatorname{tr}\left(\Gamma_{X} P_{\mu}^{-} \Gamma_{Y} P_{\mu}^{+}\right) \pi^{X}(n) \pi^{Y}(n+\hat{\mu})\right] \\
& =N_{c} \int \frac{d^{4} p}{(2 \pi)^{4}} \pi^{X}(-p) D_{X Y}(p) \pi^{Y}(p)
\end{aligned}
$$

where

$$
\begin{aligned}
& D_{X Y}(p)=\frac{1}{2}\left(\widetilde{D}_{X Y}(p)+\widetilde{D}_{Y X}(-p)\right) \\
& \widetilde{D}_{X Y}(p)=\frac{1}{2} \operatorname{tr}\left(M_{0}^{-1} \Gamma_{X} M_{0}^{-1} \Gamma_{Y}\right)+\sum_{\mu} \operatorname{tr}\left(\Gamma_{X} P_{\mu}^{-} \Gamma_{Y} P_{\mu}^{+}\right) e^{\mathrm{i} p_{\mu}} .
\end{aligned}
$$

In the case of the Wilson fermion, $\hat{M}=(m+4 r) \mathbf{1}_{4} \equiv M_{W} \mathbf{1}_{4}$ and $P_{\mu}^{ \pm}=\frac{\gamma_{\mu} \pm r}{2}$. By taking $M_{0}=\sigma \mathbf{1}_{4}+\mathrm{i} \pi \gamma_{5}$, we have

$$
\left\{\begin{array}{lll}
\sigma=\frac{-M_{W} \pm \sqrt{M_{W}^{2}+8\left(1-r^{2}\right)}}{4\left(1-r^{2}\right)}, & \pi=0, & M_{W}^{2} \geq M_{c}^{2} \\
\sigma=\frac{M_{W}}{4 r^{2}}, & \pi^{2}=\frac{1}{16 r^{4}\left(1+r^{2}\right)}\left(8 r^{4}-M_{W}^{2}\left(1+r^{2}\right)\right), & M_{W}^{2}<M_{c}^{2}
\end{array}\right.
$$

where $M_{c}^{2}=\frac{8 r^{4}}{1+r^{2}}$.

As discussed in the previous subsection, at $M_{W}=0$ we have an additional U(1) symmetry, $\mathrm{U}(1)_{V}^{-}$. Since this parameter regime resides in the parity broken phase, in which $\pi^{2} \neq 0$ and $M_{W}^{2}<M_{c}^{2}, \mathrm{U}(1)_{V}^{-}$is spontaneously broken by the VEV of $\pi$ in this case.

To compute the meson mass, we hereafter take $r^{2}=1$ for simplicity. Because $D(p)$ is blockdiagonal, we concentrate on its submatrix $D_{X Y}(p)$ with $X, Y \in\left\{S, P, A_{\alpha}\right\}$. Then, by setting $p=\left(\pi, \pi, \pi, \pi+\mathrm{i} m_{S P A}\right)$, we find that the $S-P-A_{\alpha}$ sector mass $m_{S P A}$ is given by

$$
\cosh \left(m_{S P A}\right)=1+\frac{20 M_{W}^{2}}{6-7 M_{W}^{2}} .
$$

Note that since the transformation (22) involves the site-dependent quantity $(-1)^{n_{1}+\cdots+n_{4}}$, it is natural to expand the momentum $p$ around $(\pi, \pi, \pi, \pi)$. Eq. (33) tells us that the meson becomes a massless NG boson at $M_{W}=0$ as expected. If we use the exact form of $f(x)$ in the large $N_{c}$ limit, we then obtain

$$
\cosh \left(m_{S P A}\right)=1+\frac{2 M_{W}^{2}\left(16+M_{W}^{2}\right)}{16-15 M_{W}^{2}},
$$

which again shows that a massless NG boson appears at $M_{W}=0$. 


\section{A. Effective potential}

We first need to derive effective potential of meson fields including scalar one $\sigma$ from (4) in the strong-coupling limit $\left(g^{2} \rightarrow \infty\right)$. We here consider general color number as $N_{c}$ for $S U\left(N_{c}\right)$ gauge group and general space-time dimensions as $D+1$. For the purpose we perform the 1-link integral for the gauge field in the $D$-dimensional spatial part, and introduce auxiliary fields to eliminate the 4-point interaction as

$$
\begin{aligned}
& \int \mathcal{D} U_{1} \cdots \mathcal{D} U_{D} \exp [\left.-\sum_{x} \sum_{j=1}^{D}\left(\bar{\psi}_{x} P_{j}^{+} U_{j}(x) \psi_{x+\hat{j}}-\bar{\psi}_{x+\hat{j}} P_{j}^{-} U_{j}^{\dagger}(x) \psi_{x}\right)\right] \\
&=\exp \left[N_{c} \sum_{x}\left(\sum_{j=1}^{D} \operatorname{tr} \mathcal{M}(\mathrm{x})\left(P_{\mathrm{j}}^{+}\right)^{\mathrm{T}} \mathcal{M}(\mathrm{x}+\hat{\mathrm{j}})\left(P_{\mathrm{j}}^{-}\right)^{\mathrm{T}}\right)+\mathcal{O}(1 / \sqrt{D})\right] \\
&=\int \mathcal{D} \sigma \mathcal{D} \pi_{4} \exp \left[-N_{c} \sum_{x}\left(D\left(\left(1+r^{2}\right) \sigma^{2}+\left(1-r^{2}\right) \pi_{4}^{2}\right)\right.\right. \\
&\left.\left.-\frac{D}{2} \operatorname{tr}\left(\sqrt{1+\mathrm{r}^{2}} \sigma-\mathrm{i} \sqrt{1-\mathrm{r}^{2}} \pi_{4} \gamma_{4}\right) \mathcal{M}(\mathrm{x})\right)\right]
\end{aligned}
$$

with

$$
P_{\mu}^{ \pm}= \begin{cases}\left(\gamma_{\mu} \pm i r \gamma_{4}\right) / 2 & (\mu \neq 4) \\ \gamma_{4} / 2 & (\mu=4)\end{cases}
$$

where we introduce the mesonic field as

$$
\mathcal{M}^{\alpha \beta}(x)=\frac{1}{N_{c}} \delta_{a b} \bar{\psi}_{x}^{a, \alpha} \psi_{x}^{b, \beta} .
$$

We note that two auxiliary fields $\sigma$ and $\pi_{4}$ are required to get rid of four-fermi interactions in this case because of the Karsten-Wilczek term. $\pi_{4}$ condensate can be a signal of nonzero effective imaginary chemical potential, and we will discuss on this later. We also note that 
we dropped next-leading order of $O(1 / \sqrt{D})$ expansions in (5), which corresponds to large $D$ limit. We now have an intermediate form of the effective action from (4),

$$
\begin{aligned}
S_{\mathrm{eff}}= & \sum_{x}\left[\frac{1}{2}\left(\bar{\psi}_{x} e^{\mu} U_{4}(x) \gamma_{4} \psi_{x+\hat{4}}-\bar{\psi}_{x+\hat{4}} e^{-\mu} U_{4}^{\dagger}(x) \gamma_{4} \psi_{x}\right)+\bar{\psi}_{x}\left(m \mathbf{1}+i\left(d_{3}+D r\right) \gamma_{4}\right) \psi_{x}\right. \\
& \left.+N_{c} D\left(\left(1+r^{2}\right) \sigma^{2}+\left(1-r^{2}\right) \pi_{4}^{2}\right)+\frac{N_{c}}{2} D \operatorname{tr}\left(\sqrt{1+\mathrm{r}^{2}} \sigma-\mathrm{i} \sqrt{1-\mathrm{r}^{2}} \pi_{4} \gamma_{4}\right) \mathcal{M}(\mathrm{x})\right] .
\end{aligned}
$$

We here defined complex chemical potential as $\mu \equiv \mu_{\mathrm{Re}}+i \mu_{\mathrm{Im}}$. We make fourier transformation of the temporal direction $(\mu=4)$ by introducing Matsubara modes as,

$$
\psi_{\tau, \vec{x}}=\frac{1}{\sqrt{N_{\tau}}} \sum_{n=1}^{N_{\tau}} e^{i k_{n} \tau} \tilde{\psi}_{n, \vec{x}}, \quad \bar{\psi}_{\tau, \vec{x}}=\frac{1}{\sqrt{N_{\tau}}} \sum_{n=1}^{N_{\tau}} e^{-i k_{n} \tau} \tilde{\bar{\psi}}_{n, \vec{x}}, \quad k_{n}=\frac{2 \pi}{N_{\tau}}\left(n-\frac{1}{2}\right) .
$$

We here take Polyakov gauge and the link variable in the temporal direction is given by,

$$
U_{4}(\vec{x})=\left(\begin{array}{cccc}
e^{i \phi_{1}(\vec{x}) / N_{\tau}} & & & \\
& e^{i \phi_{2}(\vec{x}) / N_{\tau}} & & \\
& & \ddots & \\
& & & e^{i \phi_{N_{c}}(\vec{x}) / N_{\tau}}
\end{array}\right), \quad \sum_{a=1}^{N_{c}} \phi_{a}(\vec{x})=0 .
$$

with $\phi_{a}$ defined as components of gauge fields. It enables us to calculate fermionic determinant analytically as

$$
\begin{aligned}
\operatorname{det} D & =\prod_{\vec{x}} \prod_{a=1}^{N_{c}} \prod_{n=1}^{N_{\tau}} \operatorname{det}\left[\left(m+\frac{D}{2} \sqrt{1+r^{2}} \sigma\right) 1+i \gamma_{4}\left(\sin \bar{k}_{n}^{(a)}+d_{3}+D r-\frac{D}{2} \sqrt{1-r^{2}} \pi_{4}\right)\right] \\
& \equiv \prod_{\vec{x}} \prod_{a=1}^{N_{c}} \prod_{n=1}^{N_{\tau}} \operatorname{det}\left[B+i \gamma_{4} A \sin \tilde{k}_{n}^{(a)}\right] \\
& =\prod_{\vec{x}} \prod_{a=1}^{N_{c}} \prod_{n=1}^{N_{\tau}}\left(A^{2} \sin ^{2} \tilde{k}_{n}^{(a)}+B^{2}\right)^{2} \\
& =\prod_{\vec{x}} A^{4 N_{c} N_{\tau}} \prod_{a=1}^{N_{c}}\left(2 \cosh N_{\tau} E+2 \cos \left(\phi_{a}-i N_{\tau} \mu\right)\right)^{4}
\end{aligned}
$$

where we define

$$
\begin{gathered}
A^{2}=1+\left(d_{3}+D r-\frac{D}{2} \sqrt{1-r^{2}} \pi_{4}\right)^{2}, \quad B=m+\frac{D}{2} \sqrt{1+r^{2}} \sigma \\
E=\operatorname{arcsinh}\left(\frac{B}{A}\right)=\log \left[\frac{B}{A}+\sqrt{1+\left(\frac{B}{A}\right)^{2}}\right]
\end{gathered}
$$

with $\bar{k}_{n}^{(a)}=k_{n}+\phi_{a} / N_{\tau}-i \mu$. By integrating the temporal gauge field $\phi_{a}$ we derive

$$
\begin{gathered}
\int \mathcal{D} U_{4} \prod_{\vec{x}} A^{4 N_{c} N_{\tau}} \prod_{a=1}^{N_{c}}\left(2 \cosh N_{\tau} E+2 \cos \left(\phi_{a}-i N_{\tau} \mu\right)\right)^{4}=\prod_{\vec{x}}\left[\sum_{n \in Z} \operatorname{det}\left(Q_{n+i-j}\right)_{1 \leq i, j \leq N_{c}}\right] \\
Q_{n}=\int_{-\pi}^{\pi} \frac{d \phi}{2 \pi}\left(2 \cosh N_{\tau} E+2 \cos \theta\right)^{4} e^{-i n \phi}, \quad \theta=\phi-i N_{\tau} \mu
\end{gathered}
$$

For $N_{c}=3$ these $Q_{n}$ are explicitly given as

$$
\begin{aligned}
Q_{0} & =2\left(8 \cosh ^{4} N_{\tau} E+24 \cosh ^{2} N_{\tau} E+3\right) \\
Q_{ \pm 1} & =8 \cosh N_{\tau} E\left(4 \cosh ^{2} N_{\tau} E+3\right) e^{ \pm N_{\tau} \mu} \\
Q_{ \pm 2} & =4\left(6 \cosh ^{2} N_{\tau} E+1\right) e^{ \pm 2 N_{\tau} \mu} \\
Q_{ \pm 3} & =8 \cosh N_{\tau} E e^{ \pm 3 N_{\tau} \mu} \\
Q_{ \pm 4} & =e^{ \pm 4 N_{\tau} \mu} \\
Q_{|n| \geq 5} & =0
\end{aligned}
$$

As a result, the effective free energy is given by

$$
\begin{aligned}
\mathcal{F}_{\text {eff }}\left(\sigma, \pi_{4} ; m, T, \mu, d_{3}\right)= & \frac{N_{c} D}{4}\left(\left(1+r^{2}\right) \sigma^{2}+\left(1-r^{2}\right) \pi_{4}^{2}\right)-N_{c} \log A \\
& -\frac{T}{4} \log \left(\sum_{n \in Z} \operatorname{det}\left(Q_{n+i-j}\right)_{1 \leq i, j \leq N_{c}}\right) .
\end{aligned}
$$

Here we redefine the free energy $4 \mathcal{F}_{\text {eff }} \rightarrow \mathcal{F}_{\text {eff. }}$ The calculation of the determinant part for $N_{c}=3$ is moved to Appendix A 2. We here show only the result as

$$
\begin{aligned}
& \sum_{n \in Z} \operatorname{det}\left(Q_{n+i-j}\right)_{1 \leq i, j \leq N_{c}} \\
= & 8\left(1+12 \cosh ^{2} \frac{E}{T}+8 \cosh ^{4} \frac{E}{T}\right)\left(15-60 \cosh ^{2} \frac{E}{T}+160 \cosh ^{4} \frac{E}{T}-32 \cosh ^{6} \frac{E}{T}+64 \cosh ^{8} \frac{E}{T}\right) \\
& +64 \cosh \frac{\mu_{B}}{T} \cosh \frac{E}{T}\left(-15+40 \cosh ^{2} \frac{E}{T}+96 \cosh ^{4} \frac{E}{T}+320 \cosh ^{8} \frac{E}{T}\right) \\
& +80 \cosh \frac{2 \mu_{B}}{T}\left(1+6 \cosh ^{2} \frac{E}{T}+24 \cosh ^{4} \frac{E}{T}+80 \cosh ^{6} \frac{E}{T}\right) \\
& +80 \cosh \frac{3 \mu_{B}}{T} \cosh \frac{E}{T}\left(-1+\cosh ^{2} \frac{E}{T}\right)+2 \cosh \frac{4 \mu_{B}}{T} .
\end{aligned}
$$

with

$$
\mu_{B}=3 \mu
$$




\title{
Thermodynamics with Domain Wall Fermions and anomaly in hot QCD
}

\author{
Swagato Mukherjee \\ BROOKHBVEN \\ NATIONAL LABORATORY
}




\section{$S U_{L}(2) \otimes S U_{R}(2) \otimes U_{A}(1) \otimes U_{B}(1)$}

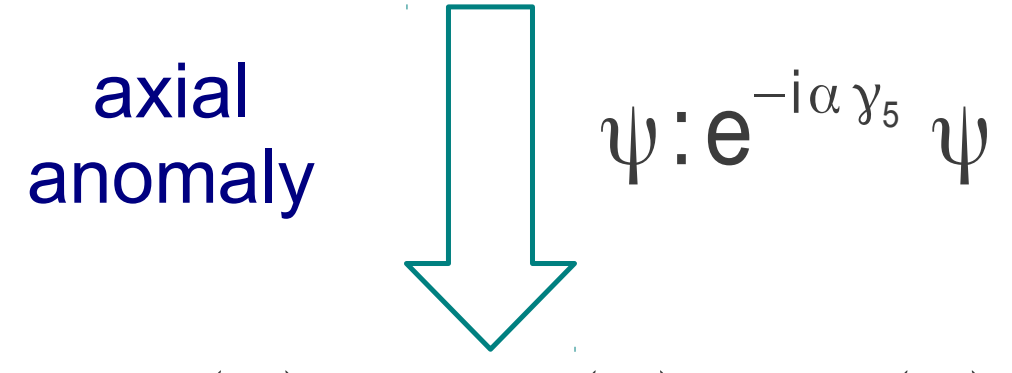

$S U_{L}(2) \otimes S U_{R}(2) \otimes U_{B}(1)$

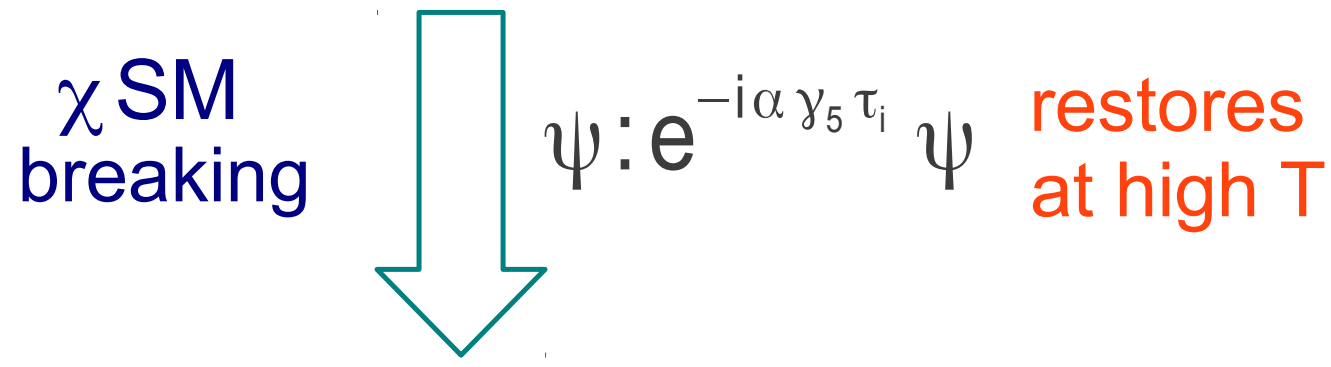

$S U_{v}(2) \otimes U_{B}(1)$
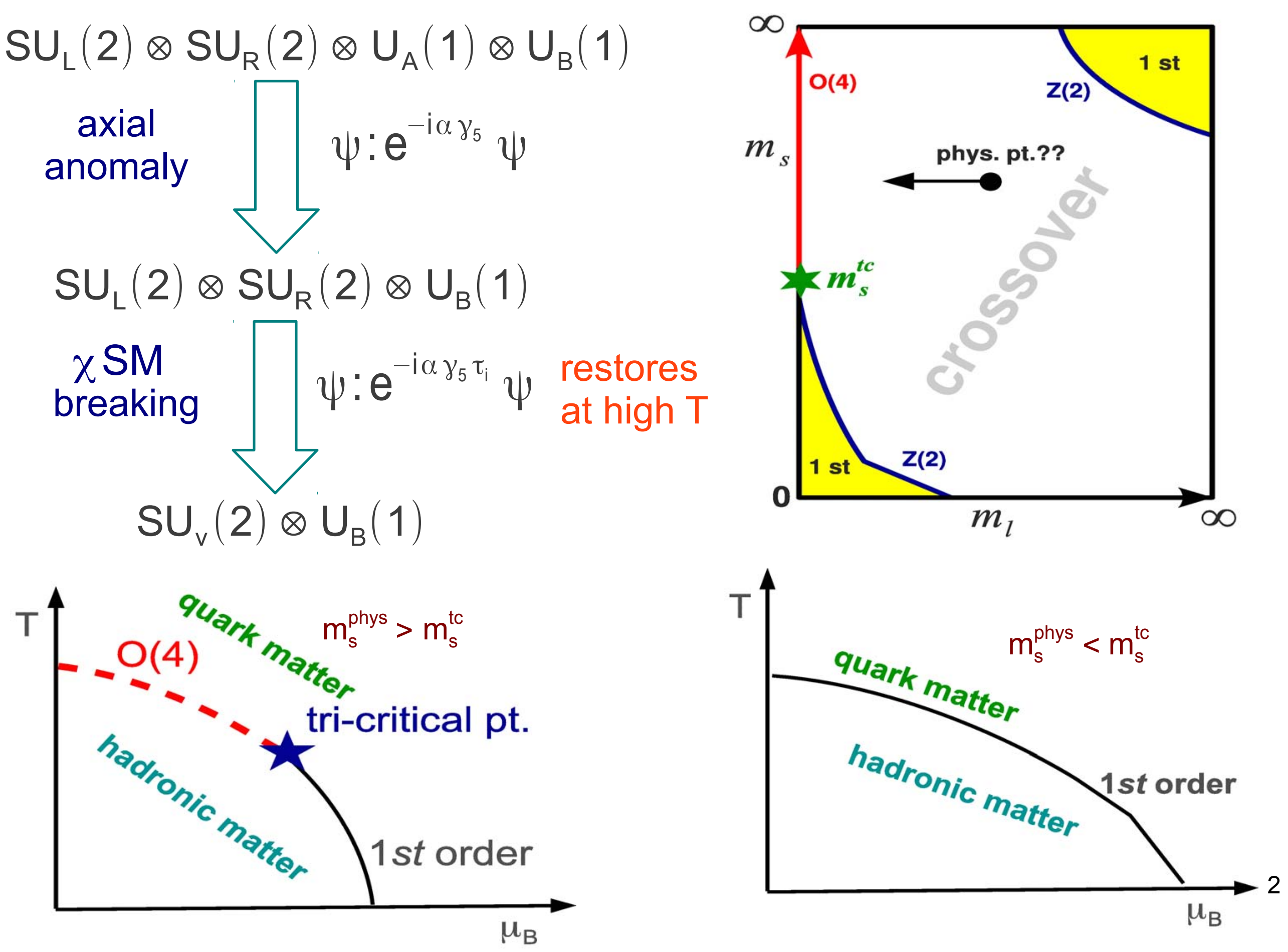

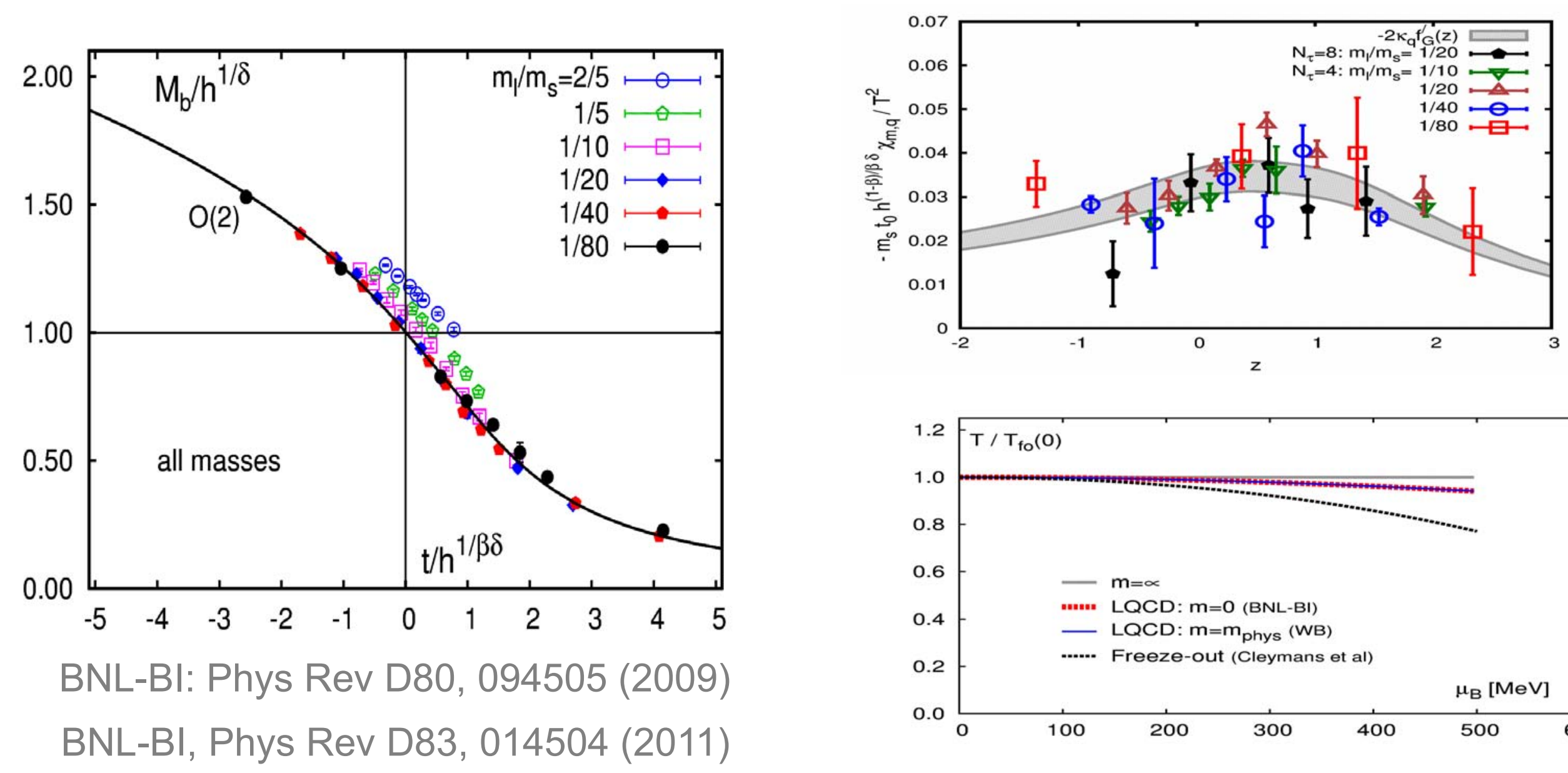

staggered fermions: intertwined chiral \& continuum limit

$a \neq 0, m \rightarrow 0: O(2)$, instead of continuum $O(4)$

need exact chiral symmetry for $a \neq 0$

chiral fermions: Domain Wall Fermions 


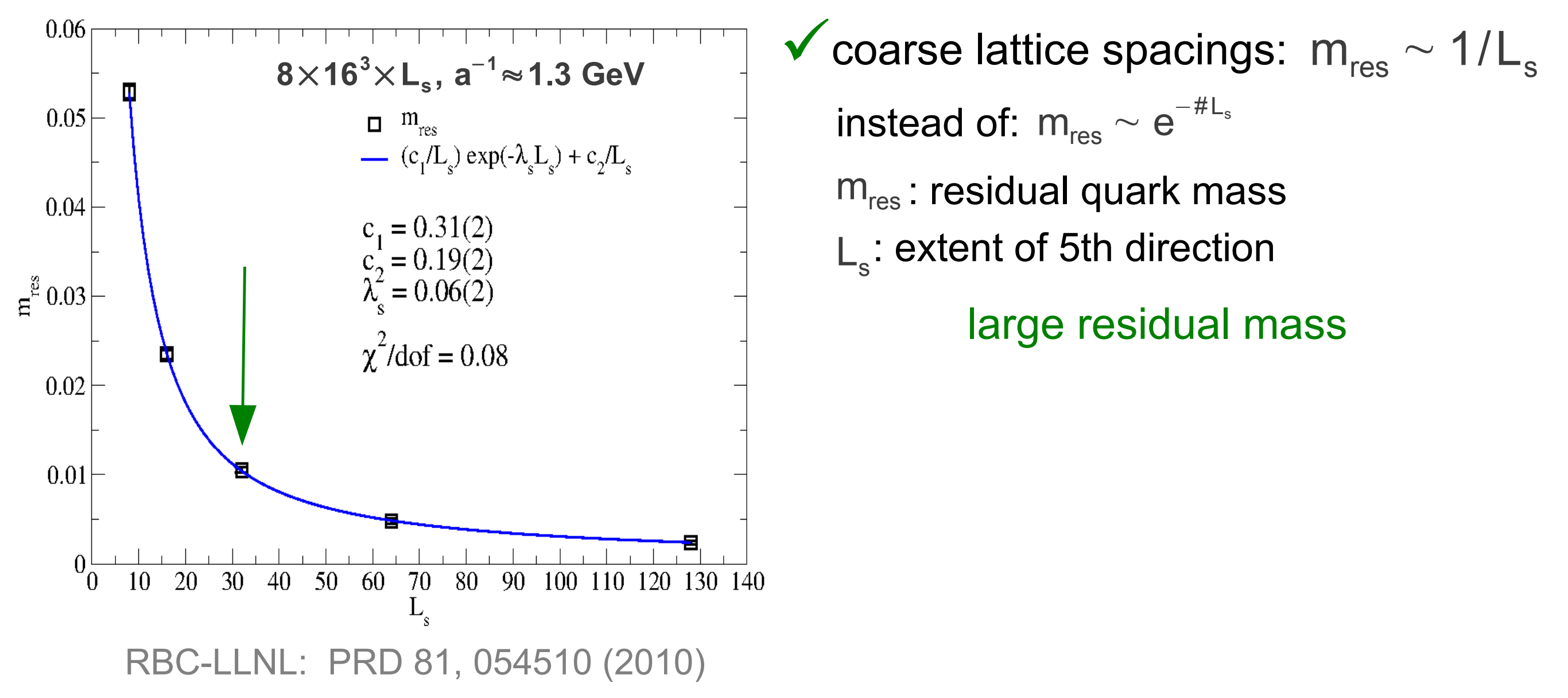




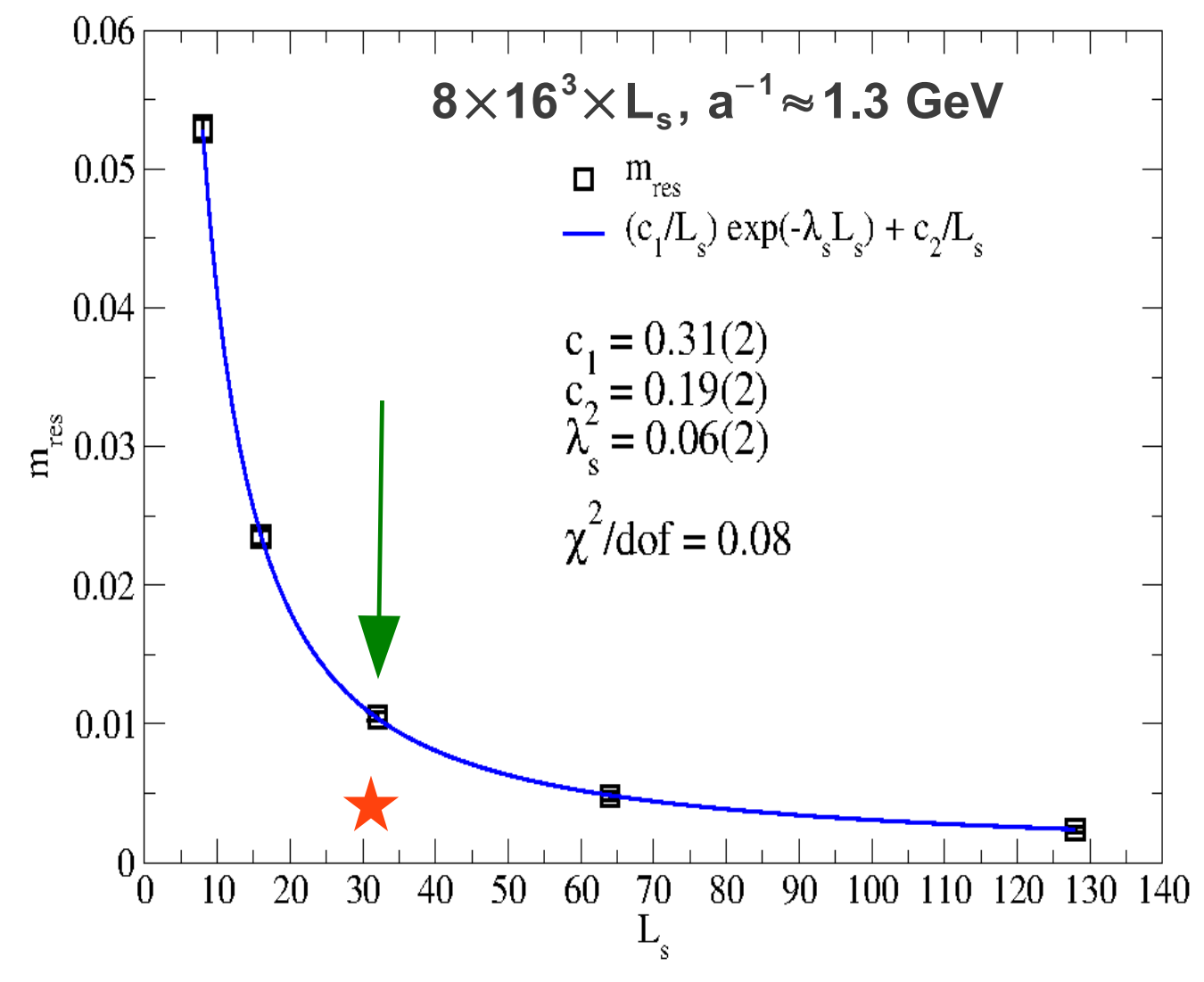

RBC-LLNL: PRD 81, 054510 (2010)

small residual mass for coarser lattices and moderate extent of the 5th direction $\checkmark$ coarse lattice spacings: $m_{\text {res }} \sim 1 / L_{s}$ instead of: $\mathrm{m}_{\text {res }} \sim \mathrm{e}^{-\# L_{\mathrm{s}}}$

$\mathrm{m}_{\text {res }}$ : residual quark mass

$\mathrm{L}_{\mathrm{s}}$ : extent of 5 th direction

large residual mass

\section{$\star$ DSDR}

Dislocation Suppressing Determinant Ratio

$$
\frac{\operatorname{det}\left[D_{W}^{+}\left(-M_{5}+i \epsilon_{f} \gamma_{5}\right) D_{W}\left(-M_{5}+i \epsilon_{f} \gamma_{5}\right)\right]}{\operatorname{det}\left[D_{W}^{+}\left(-M_{5}+i \epsilon_{b} \gamma_{5}\right) D_{W}\left(-M_{5}+i \epsilon_{b} \gamma_{5}\right)\right]}
$$

suppresses gauge field dislocations, but ensures topological tunneling, minimally affects the UV modes

(1) HotQCD: $8 \times 16^{3} \times 32(48), \mathrm{m}_{\pi}=200 \mathrm{MeV}, \mathrm{m}_{\mathrm{K}}=$ phys. $\mathrm{T}=135-200 \mathrm{MeV}$

(2) RBC-LLNL: $8 \times 32^{3} \times 32(48)$ 


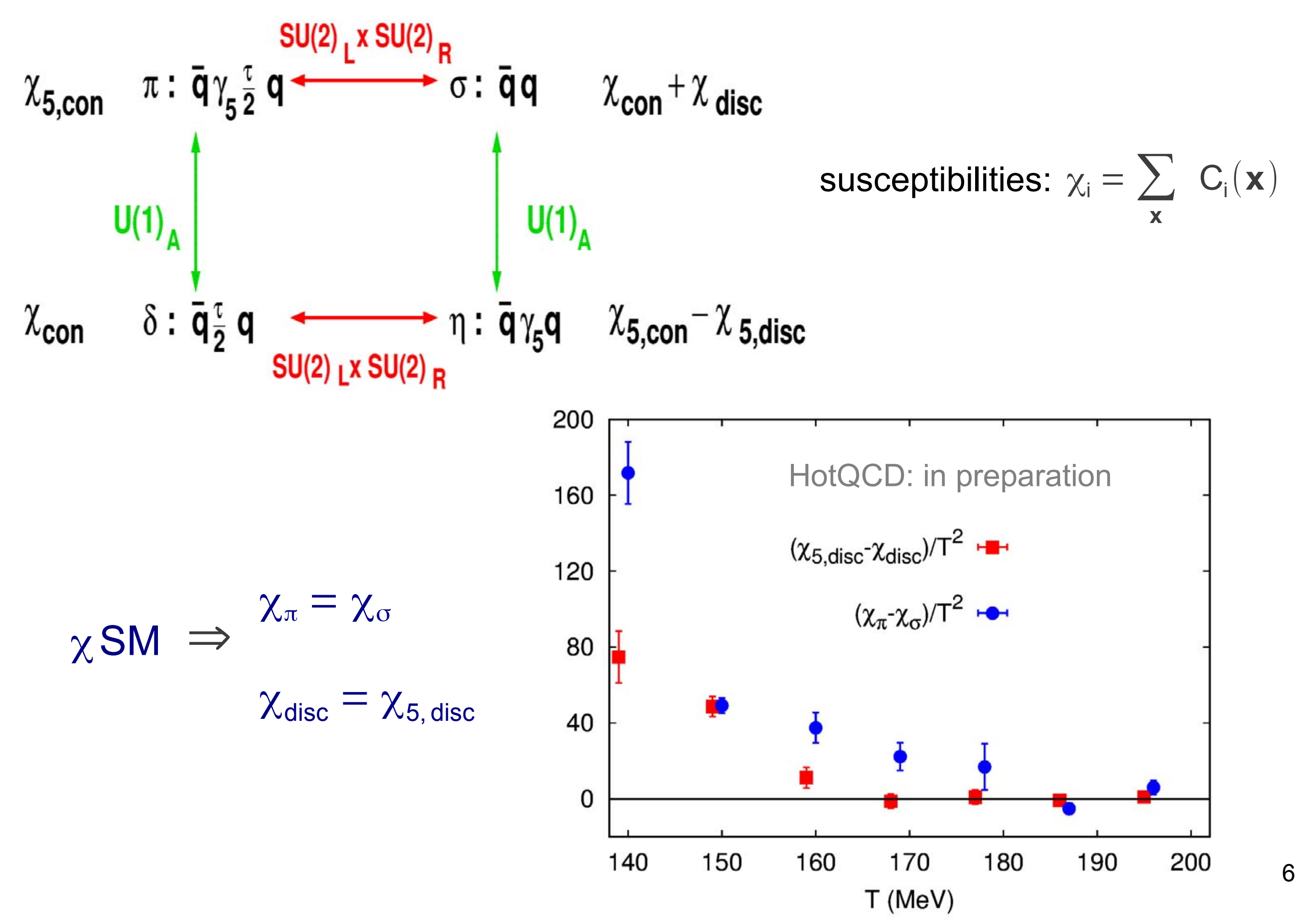




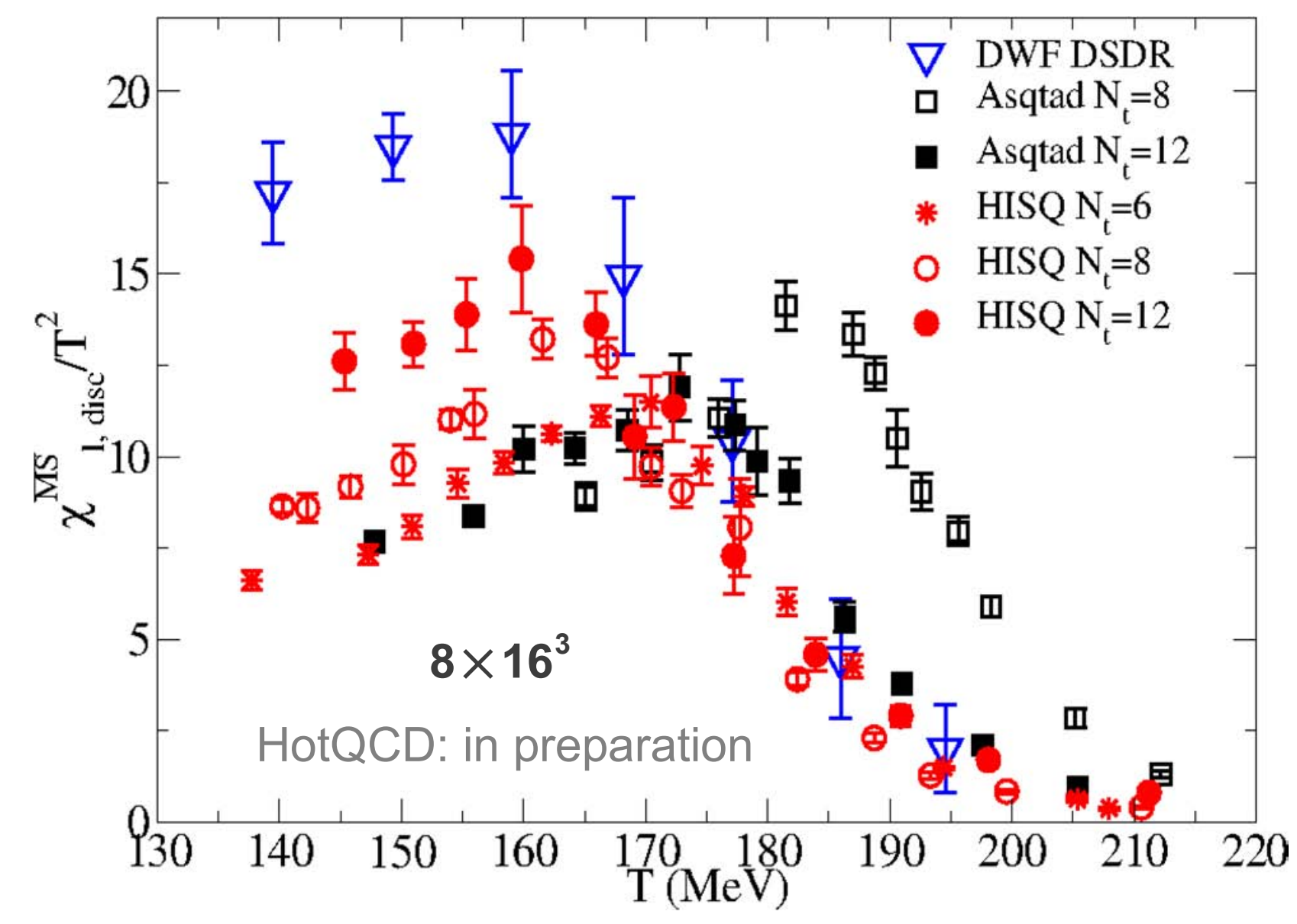

\section{disconnected chiral susceptibility}

re-normalized at scale $2 \mathrm{GeV}$ in MS scheme 


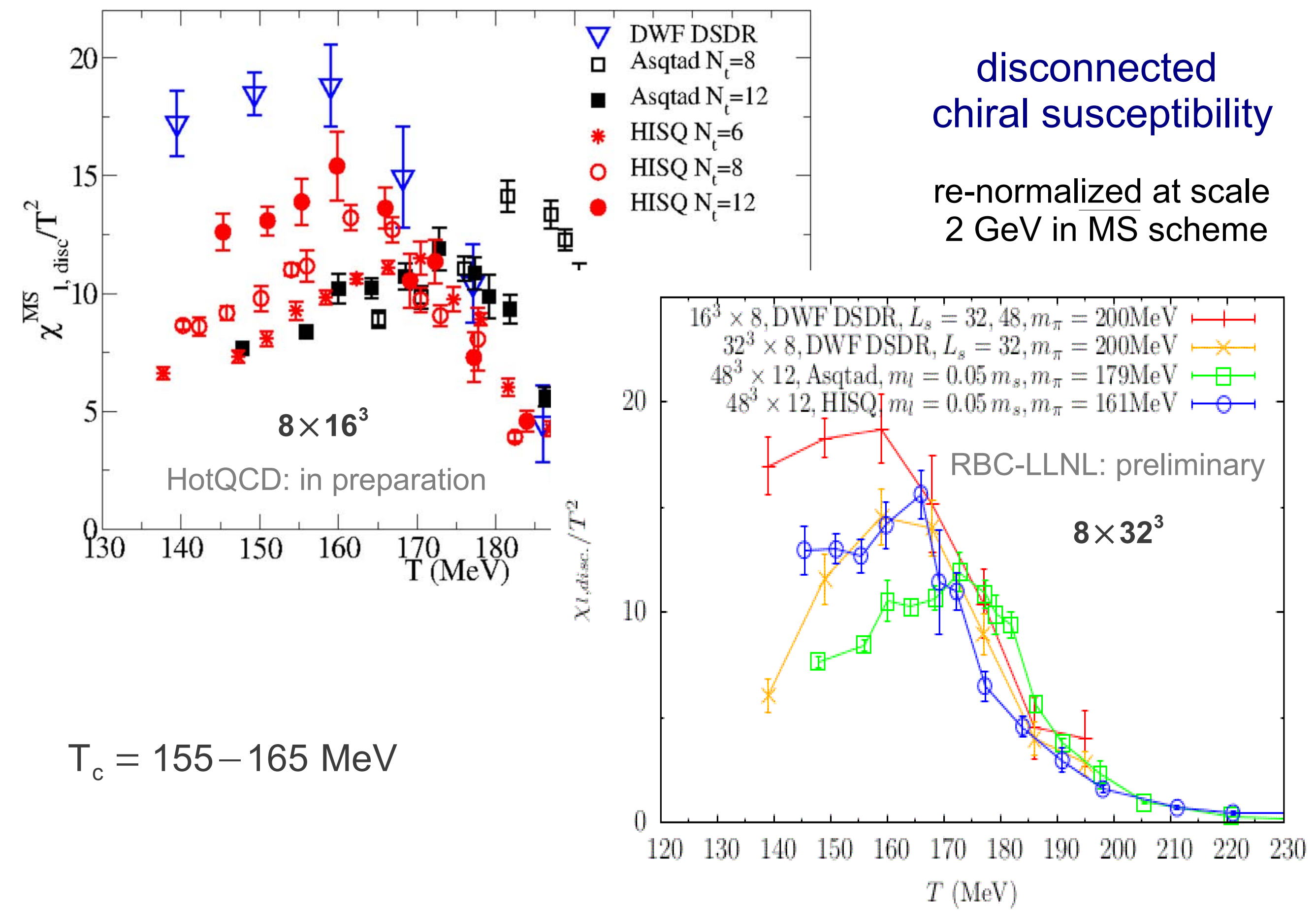


$\checkmark$ vacuum: non-zero $\langle\bar{\psi} \psi\rangle$ breaks $U_{\mathrm{A}}(1)$

$\checkmark T>T_{\mathrm{c}}^{\chi}: U_{\mathrm{A}}(1)$ is broken due to presence of topologically non-trivial configurations (e.g. instantons)

complex non-perturbative mechanism for axial symmetry breaking?

$\checkmark$ high T: exponential suppression of instanton density due to screening chromo-electric fields leads to exponentially small $U_{A}(1)$ breaking, dilute instanton gas approximation 


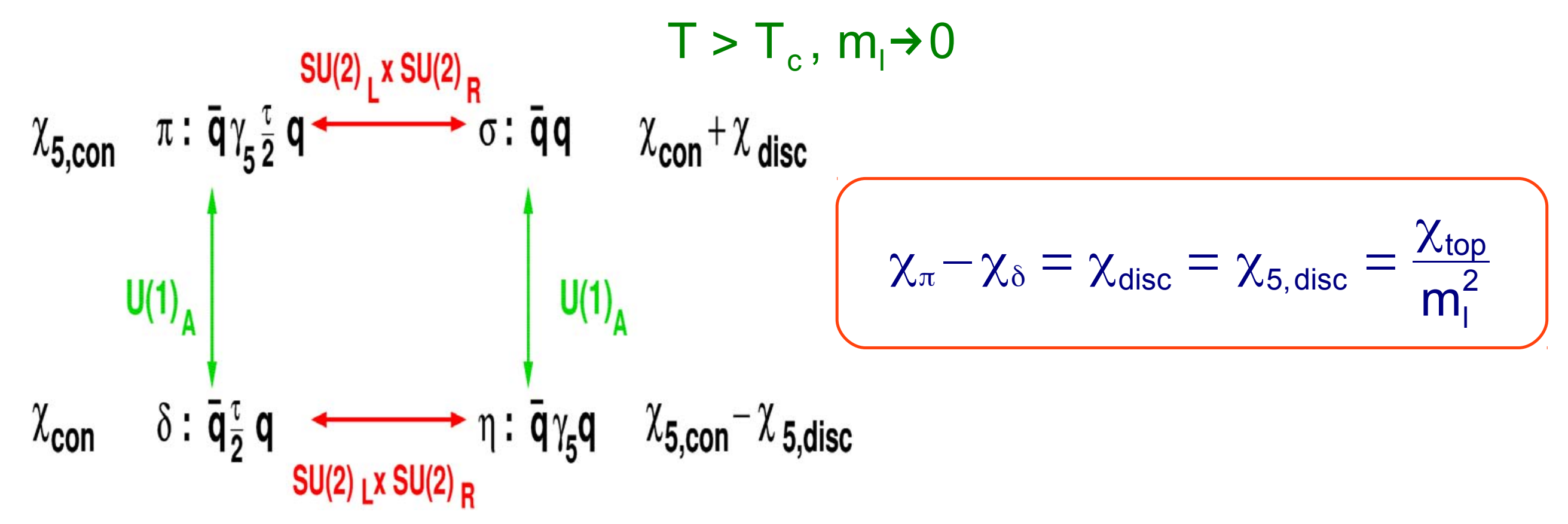




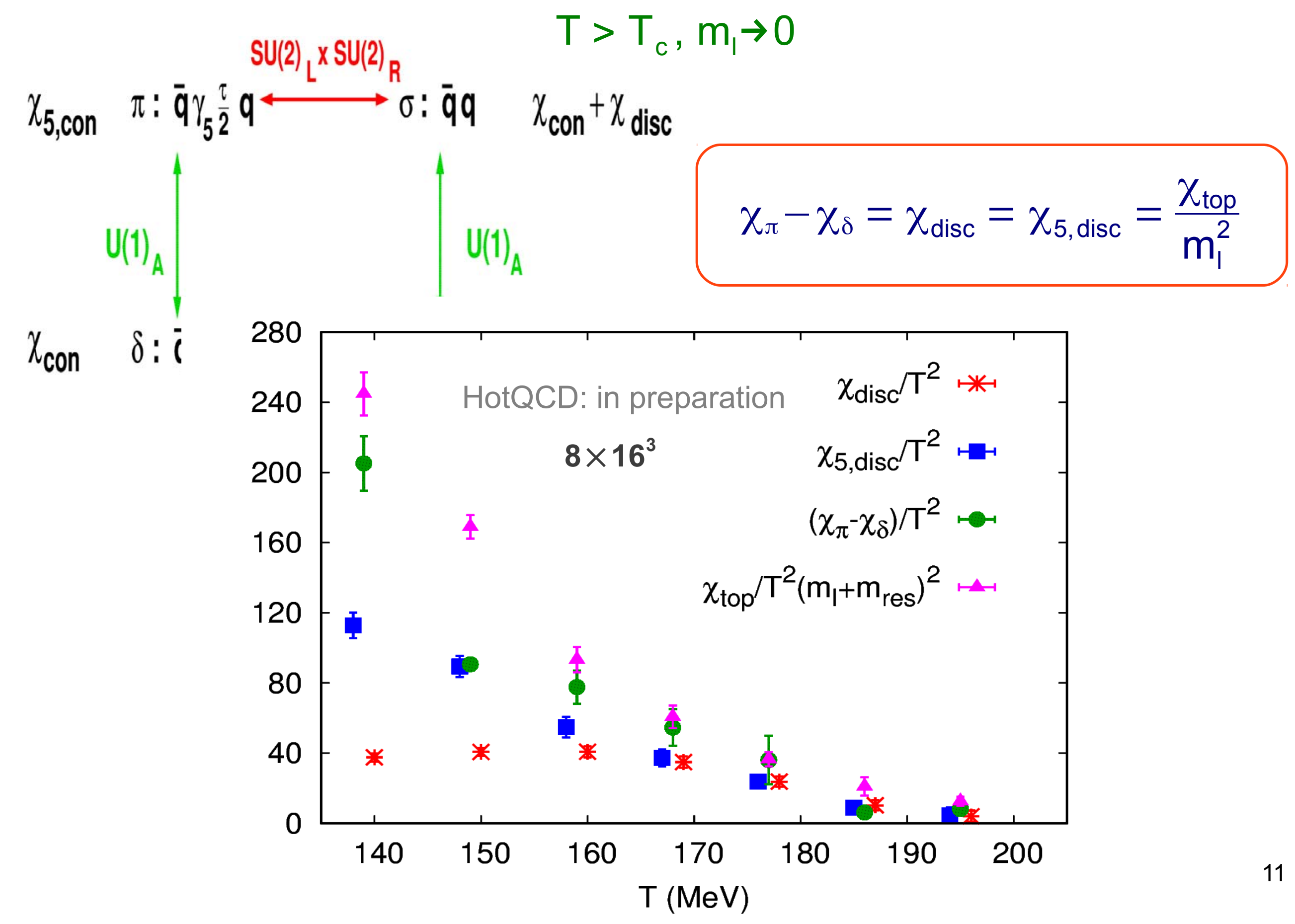


$\chi S M:\langle\bar{\psi} \psi\rangle=\int_{0}^{\infty} \mathrm{d} \lambda \frac{2 \mathrm{~m}_{1} \rho(\lambda)}{\mathrm{m}_{1}^{2}+\lambda^{2}} \quad \mathrm{U}_{\mathrm{A}}(1): \chi_{\pi}-\chi_{\delta}=\int_{0}^{\infty} \mathrm{d} \lambda \frac{4 \mathrm{~m}_{1}^{2} \rho(\lambda)}{\left(\mathrm{m}_{1}^{2}+\lambda^{2}\right)^{2}}$

Banks-Casher: $\lim _{\mathrm{m} \rightarrow 0}\langle\bar{\psi} \psi\rangle=\pi \rho(0)$

a gap in the Dirac spectrum for $T>T_{c}$

$\chi \mathrm{SM}:\langle\bar{\psi} \psi\rangle=0$

$$
\cup_{A}(1): \chi_{\pi}-\chi_{\delta}=0 ? !
$$

one obvious resolution: dilute instanton gas approximation

$$
\rho(\lambda) \sim \mathrm{m}_{1}^{2} \delta(\lambda)
$$




\section{Axial anomaly in hot QCD \& the Dirac spectrum}
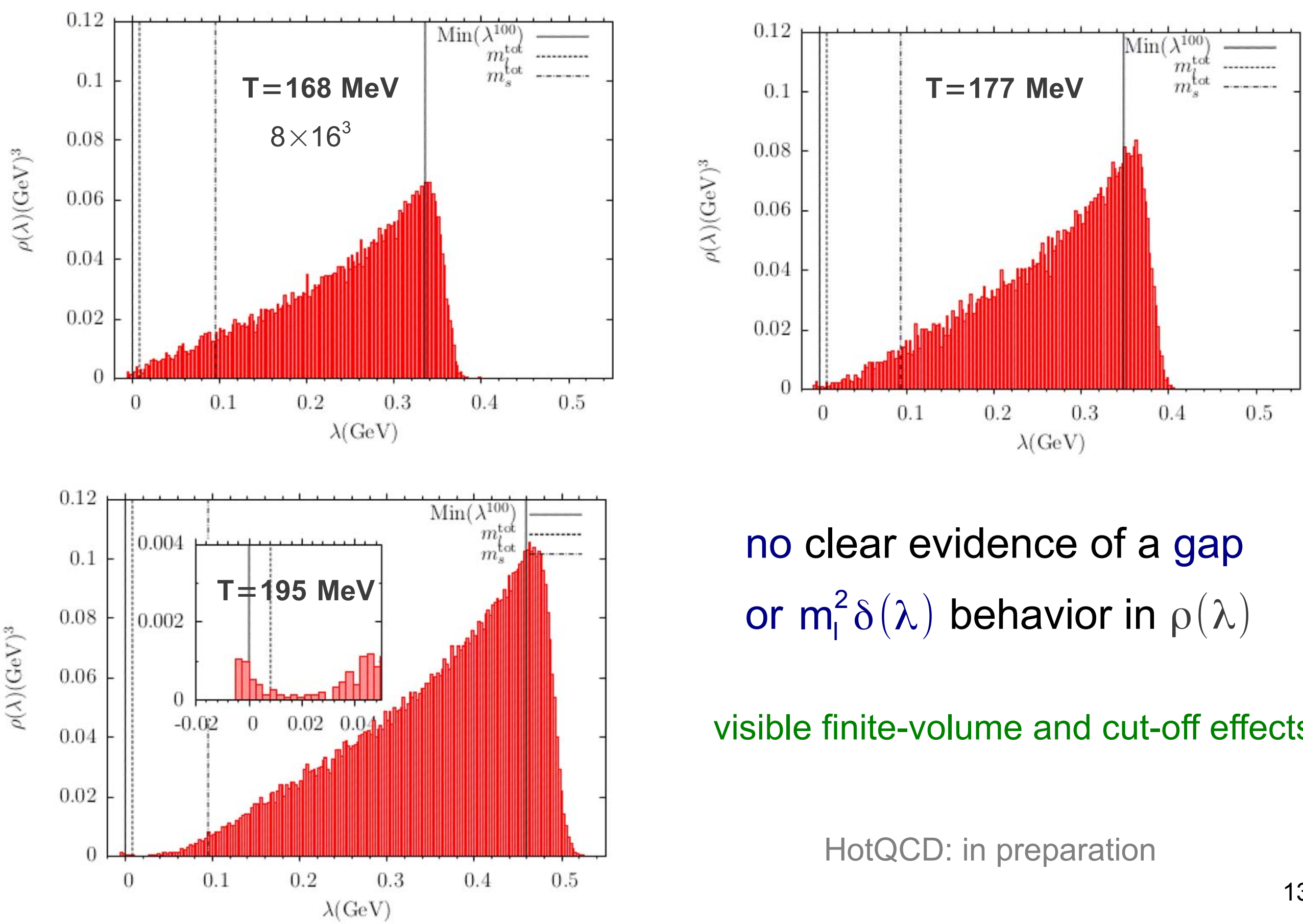

no clear evidence of a gap or $m_{1}^{2} \delta(\lambda)$ behavior in $\rho(\lambda)$

visible finite-volume and cut-off effects HotQCD: in preparation 


\section{(simple) mechanisms for axial anomaly} in the chirally symmetric phase ??

$$
\begin{aligned}
& \mathrm{m}_{1} \rightarrow 0: \\
& \langle\bar{\psi} \psi\rangle=\int_{0}^{\infty} \mathrm{d} \lambda \frac{2 \mathrm{~m}_{1} \rho(\lambda)}{\mathrm{m}_{1}^{2}+\lambda^{2}}=0 \\
& \chi_{\pi}-\chi_{\delta}=\int_{0}^{\infty} \mathrm{d} \lambda \frac{4 \mathrm{~m}_{1}^{2} \rho(\lambda)}{\left(\mathrm{m}_{1}^{2}+\lambda^{2}\right)^{2}} \neq 0 \\
& \chi_{\pi}=\langle\bar{\psi} \psi\rangle / \mathrm{m}_{1}=\text { finite } \\
& \chi_{\text {disc }}=\int_{0}^{\infty} \mathrm{d} \lambda \frac{2 \mathrm{~m}_{1}(\mathrm{~d} \rho(\lambda) / \mathrm{dm})}{\mathrm{m}_{1}^{2}+\lambda^{2}}=\chi_{\pi}-\chi_{\delta} \\
& \chi_{\delta}=\int_{0}^{\infty} \mathrm{d} \lambda 2 \rho(\lambda) \frac{\mathrm{d}}{\mathrm{dm}}\left[\frac{2 \mathrm{~m}_{1}}{\mathrm{~m}_{1}^{2}+\lambda^{2}}\right]=\text { finite }
\end{aligned}
$$

$$
\lambda \rightarrow 0 \text { : }
$$

$$
\begin{aligned}
& \checkmark_{\rho}(\lambda) \sim \mathrm{m}_{1}^{2} \delta(\lambda) \\
& x_{\rho}(\lambda) \sim \lambda \\
& \checkmark_{\rho(\lambda)} \sim \mathrm{m}_{1}
\end{aligned}
$$

need to confirm using chiral / domain wall fermions

requires many quark masses and larger volumes 
$\checkmark$ chiral fermions are essential for deeper insight into the nature of the QCD chiral transition

$\checkmark$ chiral fermions are required to understand the mechanism of the axial anomaly in QCD

$\checkmark$ RBC-LLNL + HotQCD have started serious efforts to address these questions using Domain Wall Fermions

$\checkmark$ immense technical progress for DWF thermo

$\checkmark$ more physics results coming soon $\ldots$ 


\title{
Large $\mathrm{N}$ gauge theories with adjoint fermions
}

\author{
Rajamani Narayanan \\ Florida International University
}

work done in collaboration with Ari Hietanen

arXiv:09I I.2449, arXiv:I0II.2I50, arXiv:I204.033 I

New Horizons for Lattice Computations with Chiral Fermions

14-16 May 2012, Brookhaven National Laboratory 


\section{The single site model}

Fermion

action:

$$
\begin{gathered}
H_{o}(\mu)=\frac{1}{2}\left[(1+\mu) \gamma_{5}+(1-\mu) \epsilon(H)\right] \\
b=\frac{1}{g^{2} N} \quad \text { is the inverse lattice ' } \mathrm{t} \text { Hooft coupling }
\end{gathered}
$$

$\mu$ is the fermion mass $\quad f$ is the number of Dirac flavors

We can ignore gauge field topology in the large $\mathbf{N}$ limit and assume all gauge fields are in the zero topological sector

Due to the chiral symmetry obeyed by the overlap-Dirac operator, the eigenvalues of $\mathrm{H}_{\circ}(0)$ come in doubly degenerate positive-negative pairs.

The determinant is positive definite and we can set $f$ to any real value 


\section{Weak Coupling Perturbation Theory}

$$
\begin{aligned}
& D_{\mu}^{i j}=e^{i \theta_{\mu}^{i}} \delta_{i j} \begin{array}{c}
\text { Polyakov loop } \\
\text { eigenvalues }
\end{array} \\
& U_{\mu}=e^{i a_{\mu}} D_{\mu} e^{-i a_{\mu}} \\
& \text { Leading order result } \\
& S_{g}=\sum_{i \neq j} \ln \left[\sum_{\mu} \sin ^{2} \frac{1}{2}\left(\theta_{\mu}^{i}-\theta_{\mu}^{j}\right)\right] \\
& S_{f}=-2 f \sum_{i, j} \ln \left[\frac{1+\mu^{2}}{2}+\frac{1-\mu^{2}}{2} \frac{2 \sum_{\mu} \sin ^{2} \frac{\theta_{\mu}^{i}-\theta_{\mu}^{j}}{2}-m}{\sqrt{\left(2 \sum_{\mu} \sin ^{2} \frac{\theta_{\mu}^{i}-\theta_{\mu}^{j}}{2}-m\right)^{2}+\sum_{\mu} \sin ^{2}\left(\theta_{\mu}^{i}-\theta_{\mu}^{j}\right)}}\right] \\
& \text { If all } \theta_{\mu}^{i}=0 \text { then } S_{g} \rightarrow-\infty \quad \text { But, if } \mu=0 \text { then } S_{f} \rightarrow \infty
\end{aligned}
$$




\section{Single site Polyakov loop eigenvalues and momenta on the infinite lattice}

At lowest order in weak coupling perturbation theory adjoint fermions on a single site lattice see momentum modes

$$
e^{i\left(\theta_{\mu}^{i}-\theta_{\mu}^{j}\right)} \quad 1 \leq i, j \leq N
$$

The $N^{2}-1$ angles, $\theta_{\mu}^{i}-\theta_{\mu}^{j}$, approach a continuum of momenta, $p_{\mu}$, as $\mathrm{N}$ approaches infinity We want the measure to be $\int \prod_{\mu} d p_{\mu}$ in order to reproduce correct infinite volume perturbation theory

Why do we need overlap fermions on a single site lattice?

\section{$\underline{\text { Naive fermions }}$}

$$
S_{g}=\sum_{i \neq j} \ln \left[\sum_{\mu} \sin ^{2} \frac{1}{2}\left(\theta_{\mu}^{i}-\theta_{\mu}^{j}\right)\right] \quad S_{f}=-2 f \sum_{i, j} \ln \left[\mu^{2}+\sum_{\mu} \sin ^{2}\left(\theta_{\mu}^{i}-\theta_{\mu}^{j}\right)\right]
$$

Momenta, $p_{\mu} \in\left[\frac{\pi}{2}, \pi\right]$, will spoil the uniform measure in the large $\mathrm{N}$ limit. 


\section{Massless overlap fermions}

$$
S_{f}=-2 f \sum_{i \neq j} \ln \left[\frac{1}{2}+\frac{1}{2} \frac{2 \sum_{\mu} \sin ^{2} \frac{\theta_{\mu}^{i}-\theta_{\mu}^{j}}{2}-m}{\sqrt{\left(2 \sum_{\mu} \sin ^{2} \frac{\theta_{\mu}^{i}-\theta_{\mu}^{j}}{2}-m\right)^{2}+\sum_{\mu} \sin ^{2}\left(\theta_{\mu}^{i}-\theta_{\mu}^{j}\right)}}\right]
$$

Unlike naive fermions, momenta $p_{\mu} \in\left[0, \frac{\pi}{2}\right]$ and $p_{\mu} \in\left[\frac{\pi}{2}, \pi\right]$ are not identified We need $m>2 \sum_{\mu} \sin ^{2} \frac{\theta_{\mu}^{i}-\theta_{\mu}^{j}}{2}$ for the mode corresponding to that momentum to be massless $m \rightarrow \infty$ is the naive fermion limit and so we cannot make it too large Making it too small will restrict the region inside the Brillouin zone, $p_{\mu} \in[-\pi, \pi]$ where we have massless fermions and therefore the correct momentum measure 


\section{Correlated versus uncorrelated momenta}

Correlated momenta $\theta_{\mu}^{j^{-}}=\frac{2 \pi j}{N} j=1 \cdots, N$ in all four directions $S_{c}$ : :action

Uncorrelated momenta $\theta_{\mu}^{j}=\frac{2 \pi \pi_{j}^{\mu}}{N} \pi^{\mu}$ is a permutation of $j=1 \cdots, N S_{u}$ :action

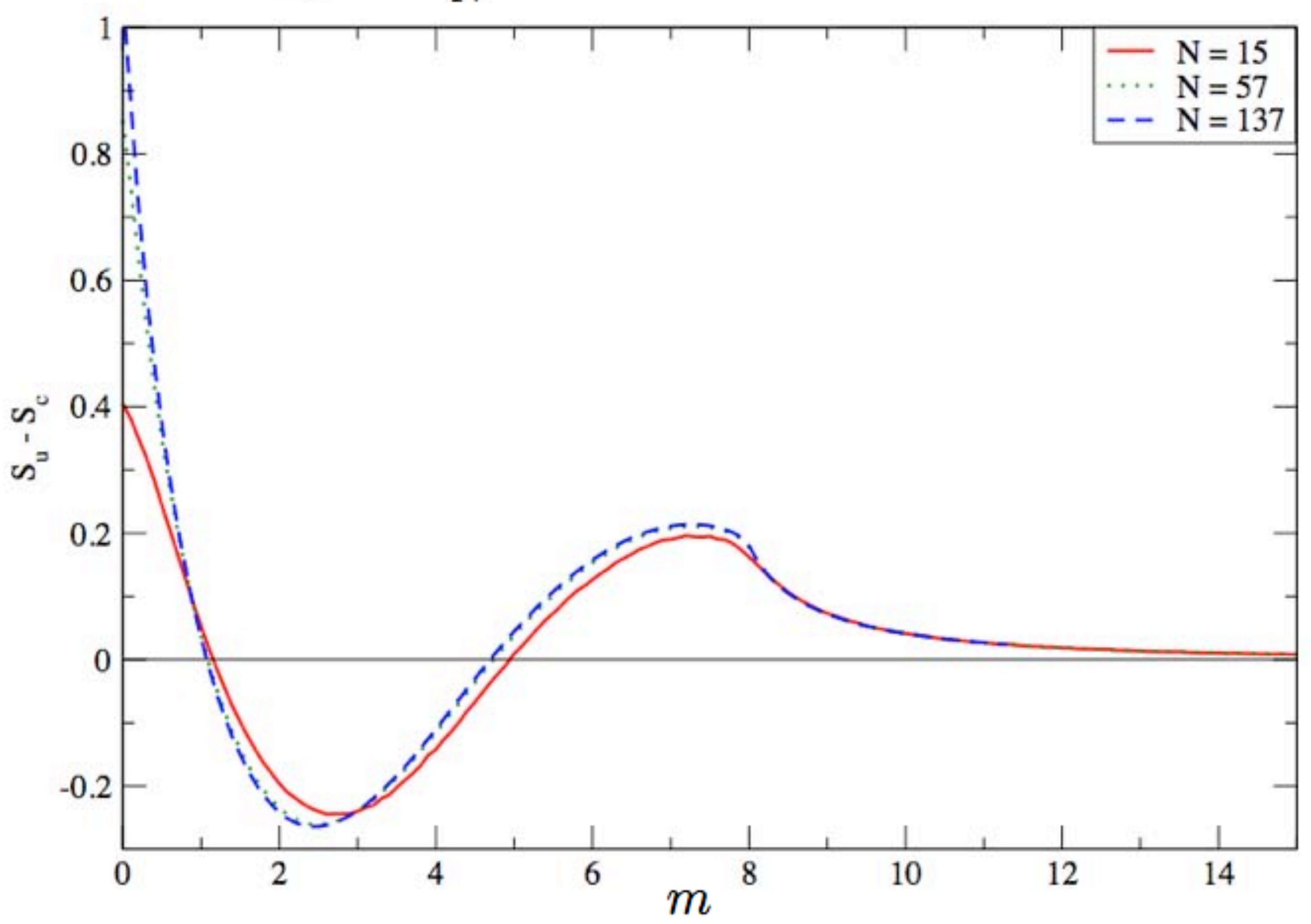

$m \in[3.5,4.5]$

is a good choice 
Distribution of Polyakov loop eigenvalues

$$
P_{\mu}=\frac{1}{2}\left(1-\frac{1}{N^{2}}\left|\operatorname{Tr} U_{\mu}\right|^{2}\right)=\frac{1}{N^{2}} \sum_{i, j} \sin ^{2} \frac{1}{2}\left(\theta_{\mu}^{i}-\theta_{\mu}^{j}\right)
$$

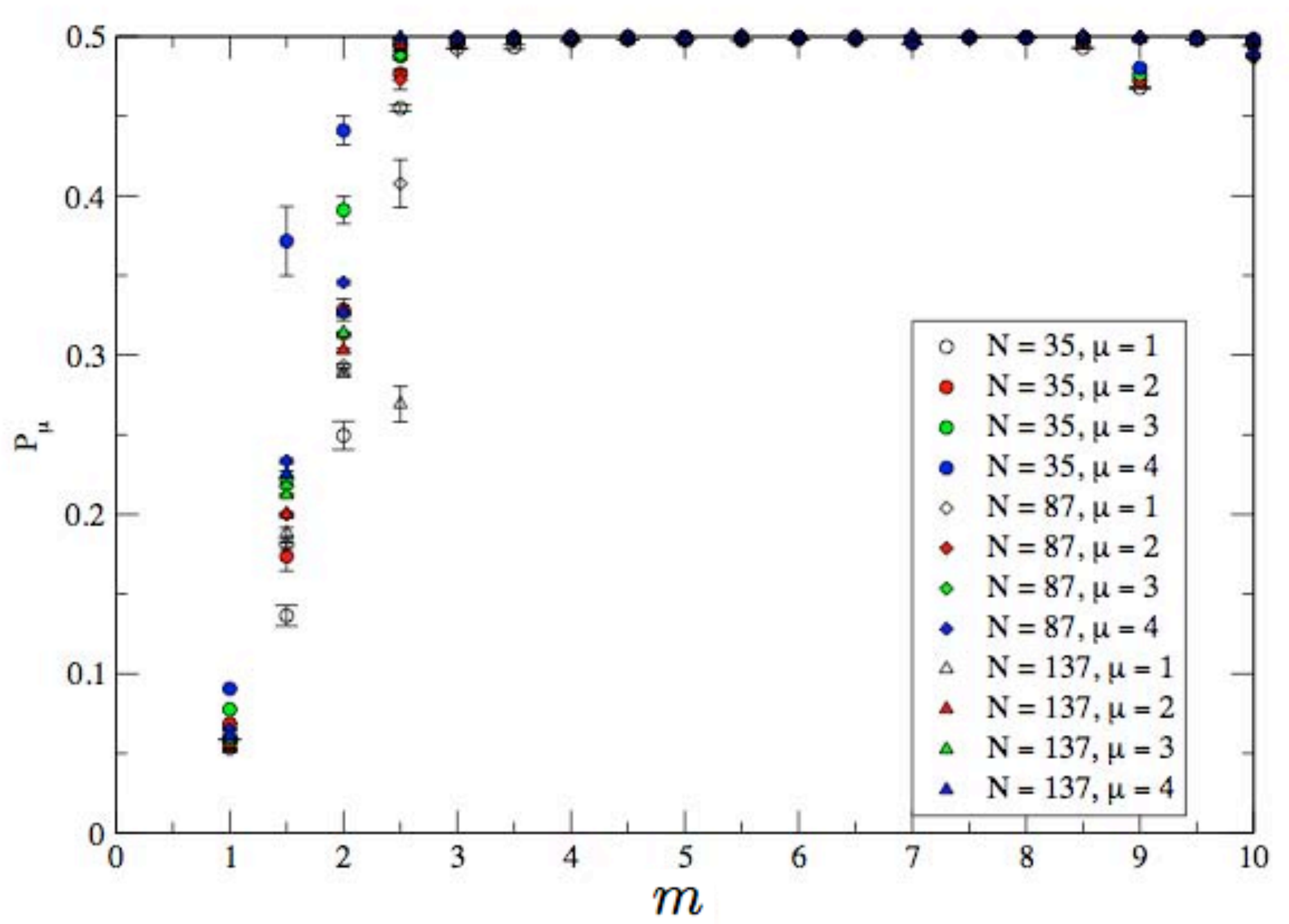




\section{A numerical proposal}

- Use Hybrid Monte Carlo Algorithm with Pseudo-fermions.

- Works for integer number of Dirac flavors.

- A direct fermion HMC algorithm for non-integer Dirac flavors.

- Pick $N$ and $b$ such that we are in the large $N$ limit for that $b$.

-We expect $N$ to increase as $b$ increases.

- Pick a quantity to set the scale.

- Lowest positive eigenvalue of the overlap Dirac operator.

- Strong to weak coupling transition.

- Find the region of $b$ where we observe scaling.

- Measure physically interesting quantities.

We will report on progress toward this goal by showing some results with

$$
\begin{aligned}
& \text { single Dirac flavor } \\
& b=\frac{1}{\lambda}=\frac{1}{g^{2} N}=\frac{\beta}{2 N^{2}} \\
& \text { massless fermions } \\
& \mathrm{N}=18 \\
& b=0.35 \\
& \beta=2.8, N=2 \\
& \text { b from } 0.32 \text { to } 0.70 \\
& \beta=6.3, N=3
\end{aligned}
$$




\section{beta function}

Let $t=\ln a(b)$ be a logarithmic scale where $a(b)$ could be the square root of the string tension at the lattice coupling, $b$.

beta function: $\quad \beta(b)=\frac{d b(t)}{d t} \quad$ Perturbation theory: $\quad \beta(b)=-b_{0}-\frac{b_{1}}{b}+\cdots$

$$
b_{0}=\frac{11-4 f}{24 \pi^{2}} ; \quad b_{1}=\frac{17-16 f}{192 \pi^{4}} \quad \begin{array}{r}
\text { Higher order coefficients depend on the choice } \\
\text { of } \mathrm{a}(\mathrm{b}) \text { and the form of the lattice action }
\end{array}
$$

The perturbative beta function $b_{*}(f)=-\frac{b_{1}}{b_{0}}=\frac{1}{8 \pi^{2}} \frac{16 f-17}{11-4 f} . \quad$ if $\quad \frac{11}{4}>f>\frac{17}{16}$
has a zero at

If $f$ is close to $\mathrm{II} / 4$, the zero occurs at very weak coupling and we can expect the zero in perturbation theory to remain essentially unaltered.

If $f$ is close to $17 / 16$, the zero occurs at very strong coupling and it is quite possible that it depends on the choice of $\mathrm{a}(\mathrm{b})$ and the form of the lattice action

Since a zero of the beta function could be an infra-red or ultra-violet fixed point, we need to study the behavior of the coupling close to this zero in order to see if we can define a continuum theory at the location of this zero. 

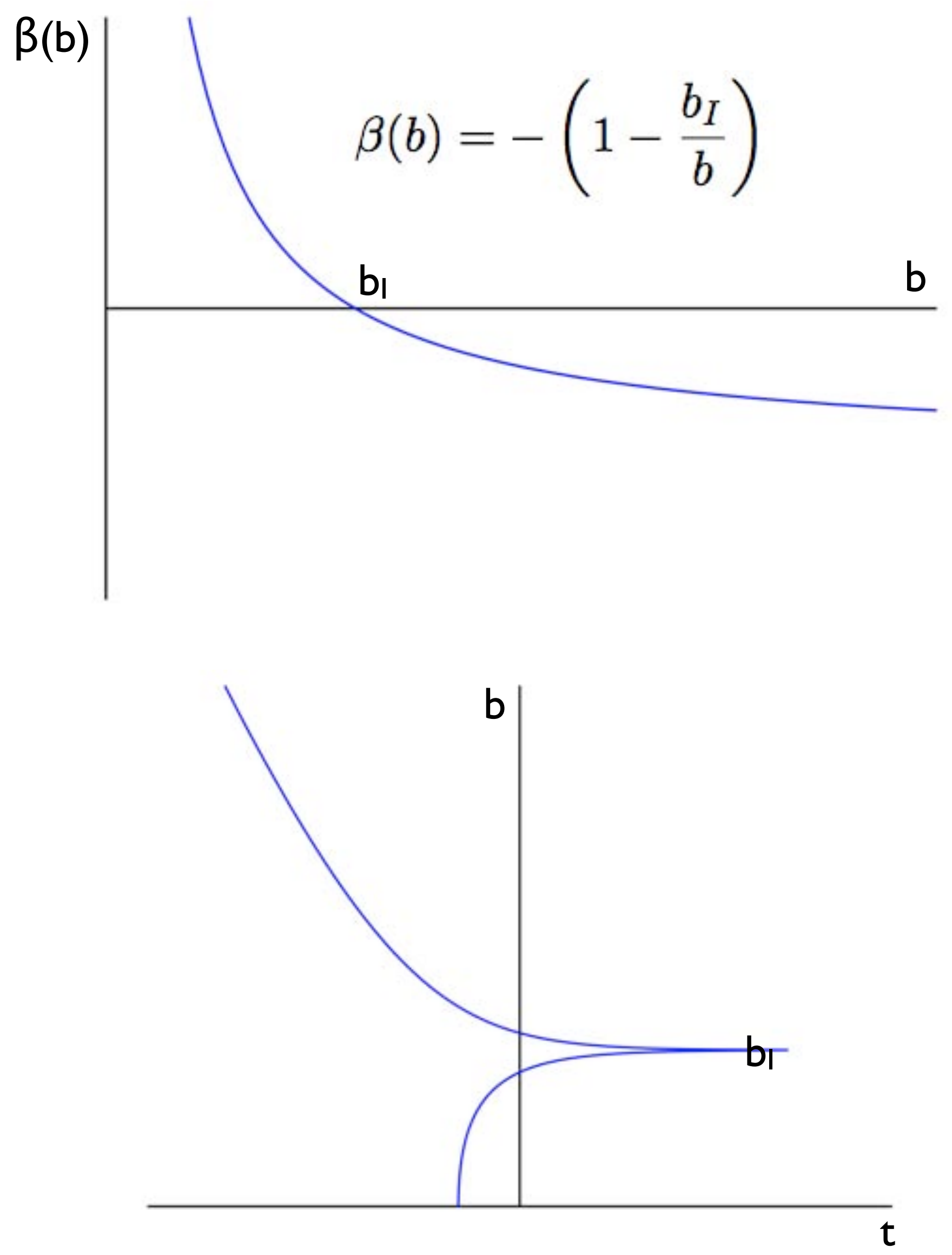
$\beta$ (b)

$$
\beta(b)=-\left(1-\frac{b_{I}}{b}\right)\left(\left(1-\frac{b_{U}}{b}\right)\right.
$$
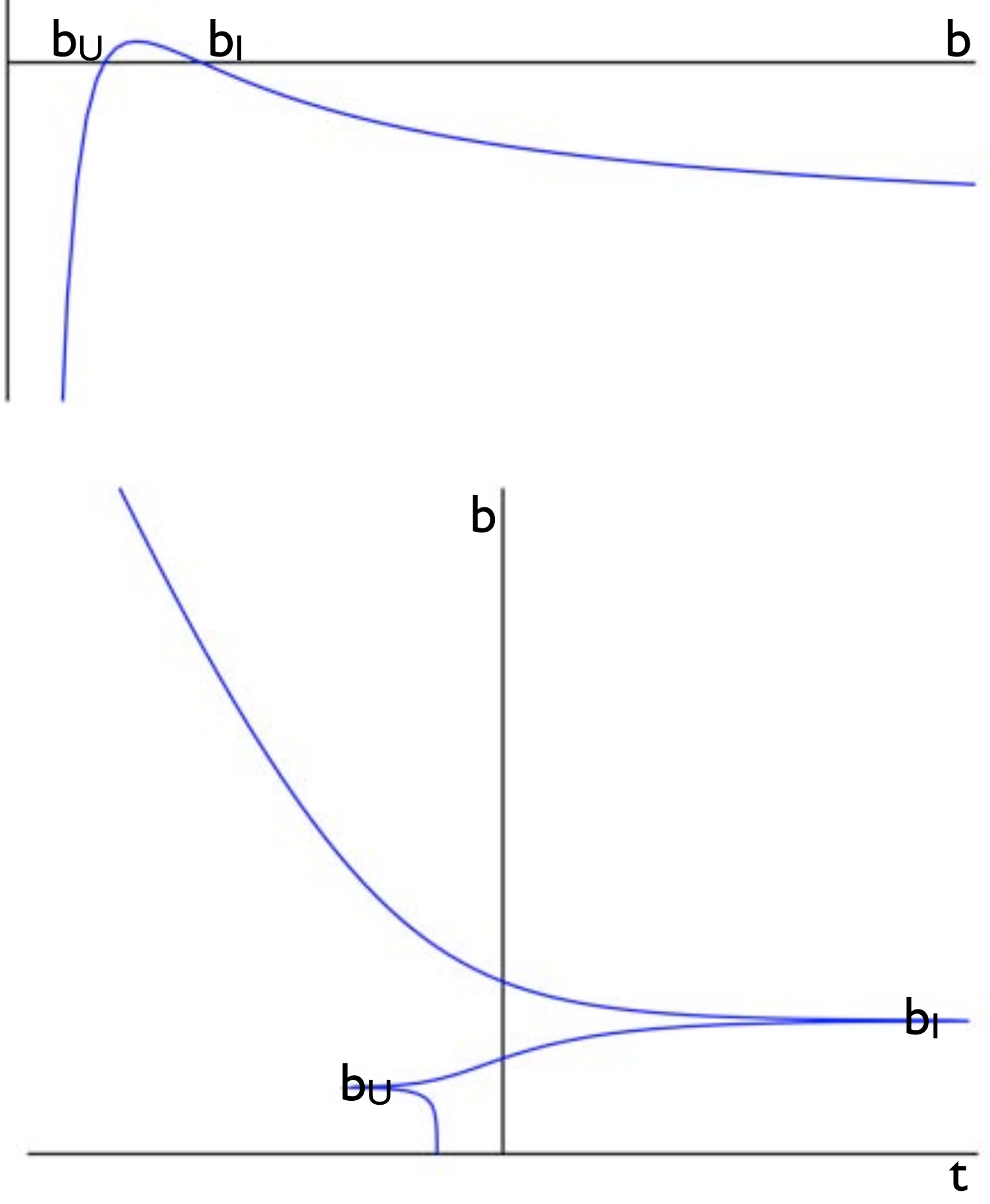

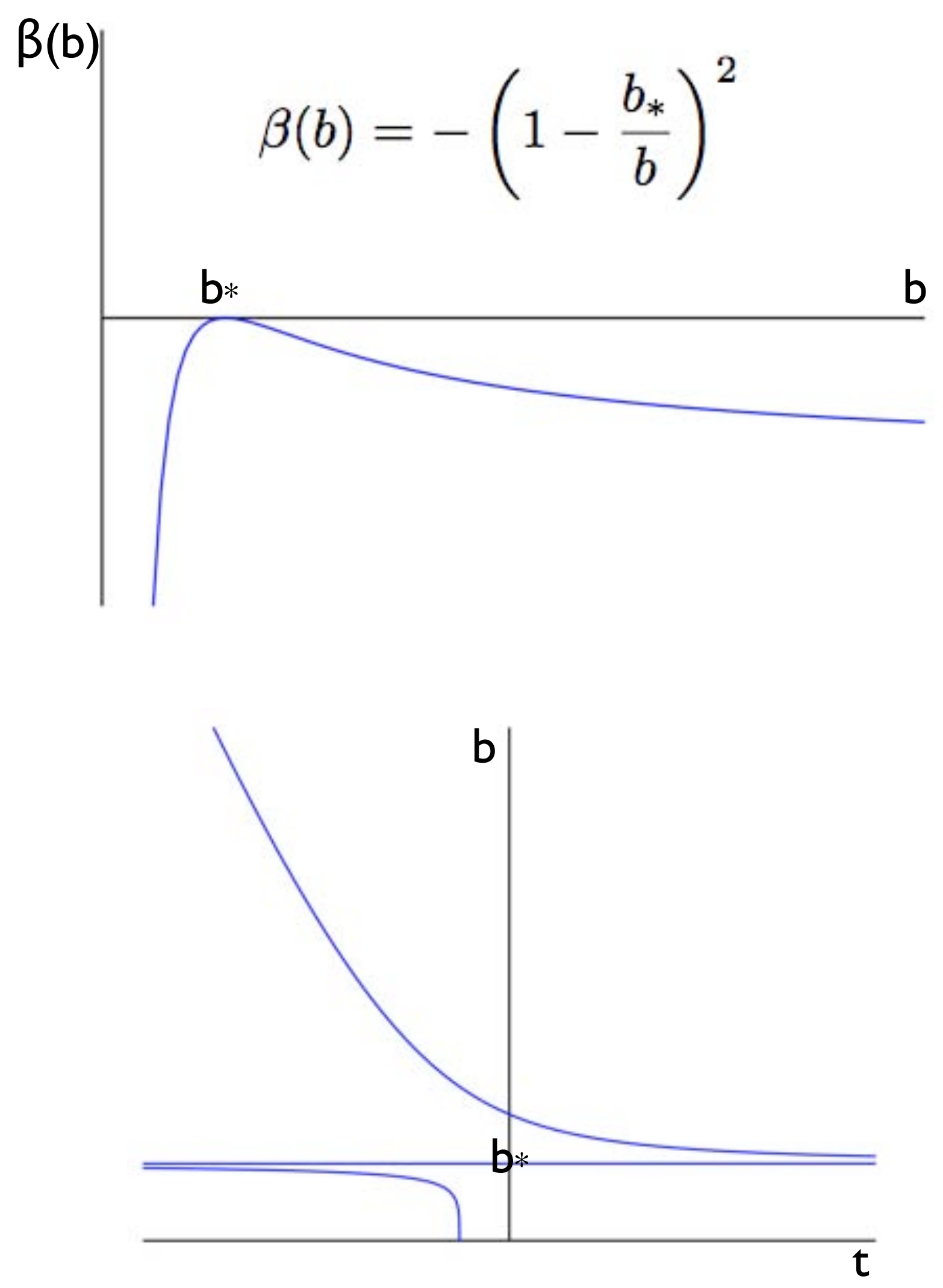
$\beta(b)$

$$
\beta(b)=-\left(1-\frac{b_{*}}{b}\right)^{2}-\frac{\alpha^{2}}{b^{2}}
$$

b*

b

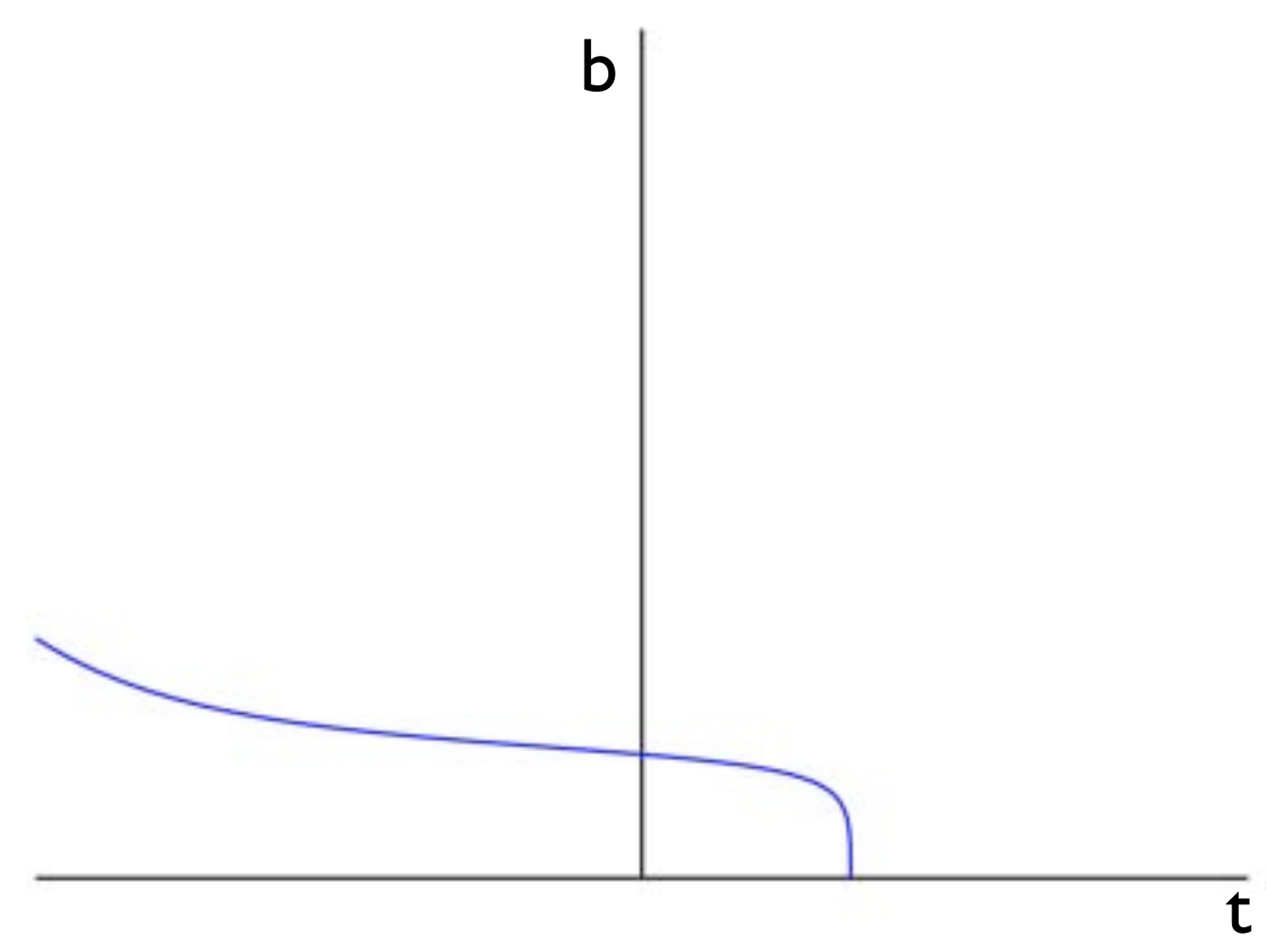




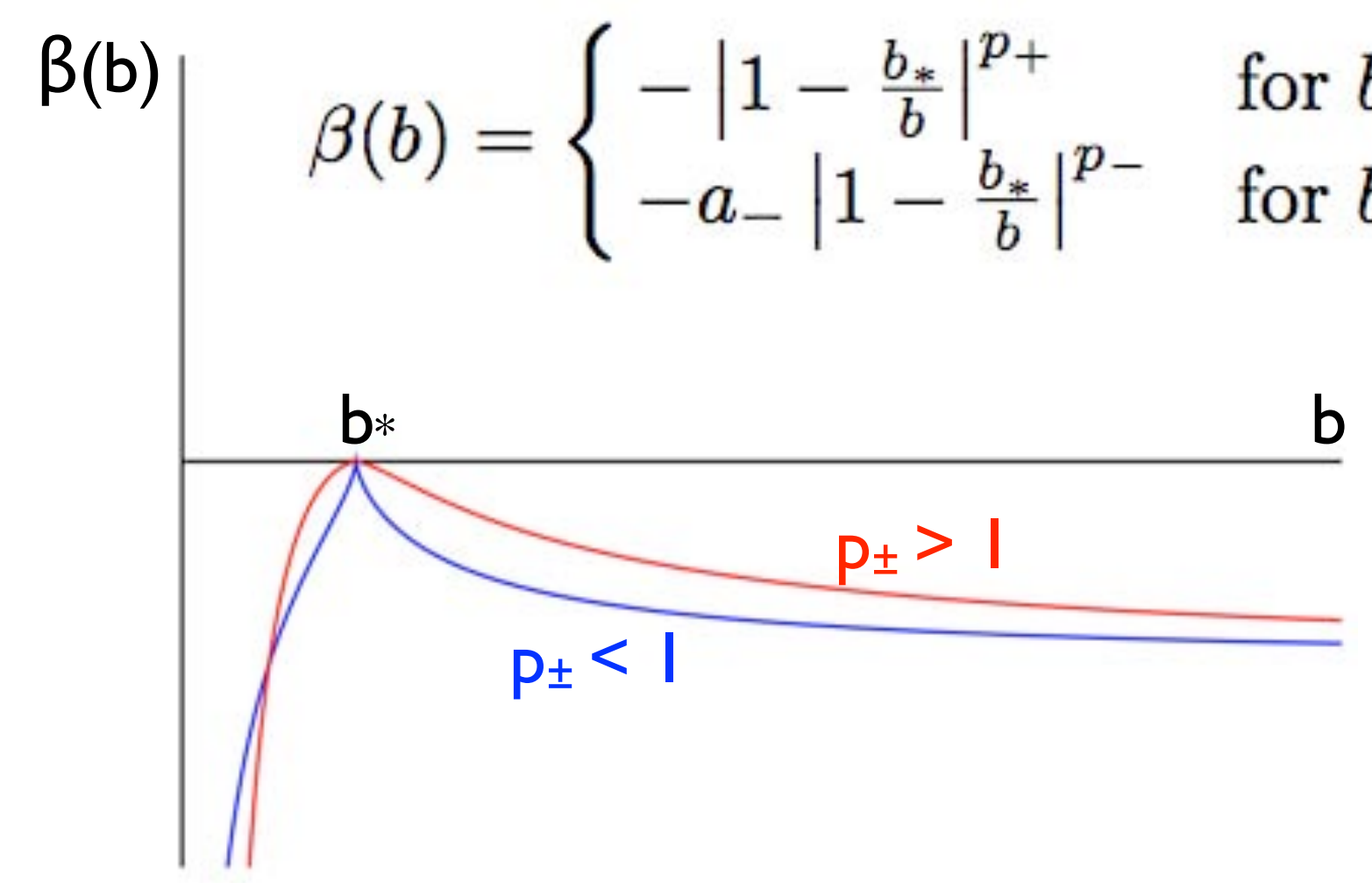

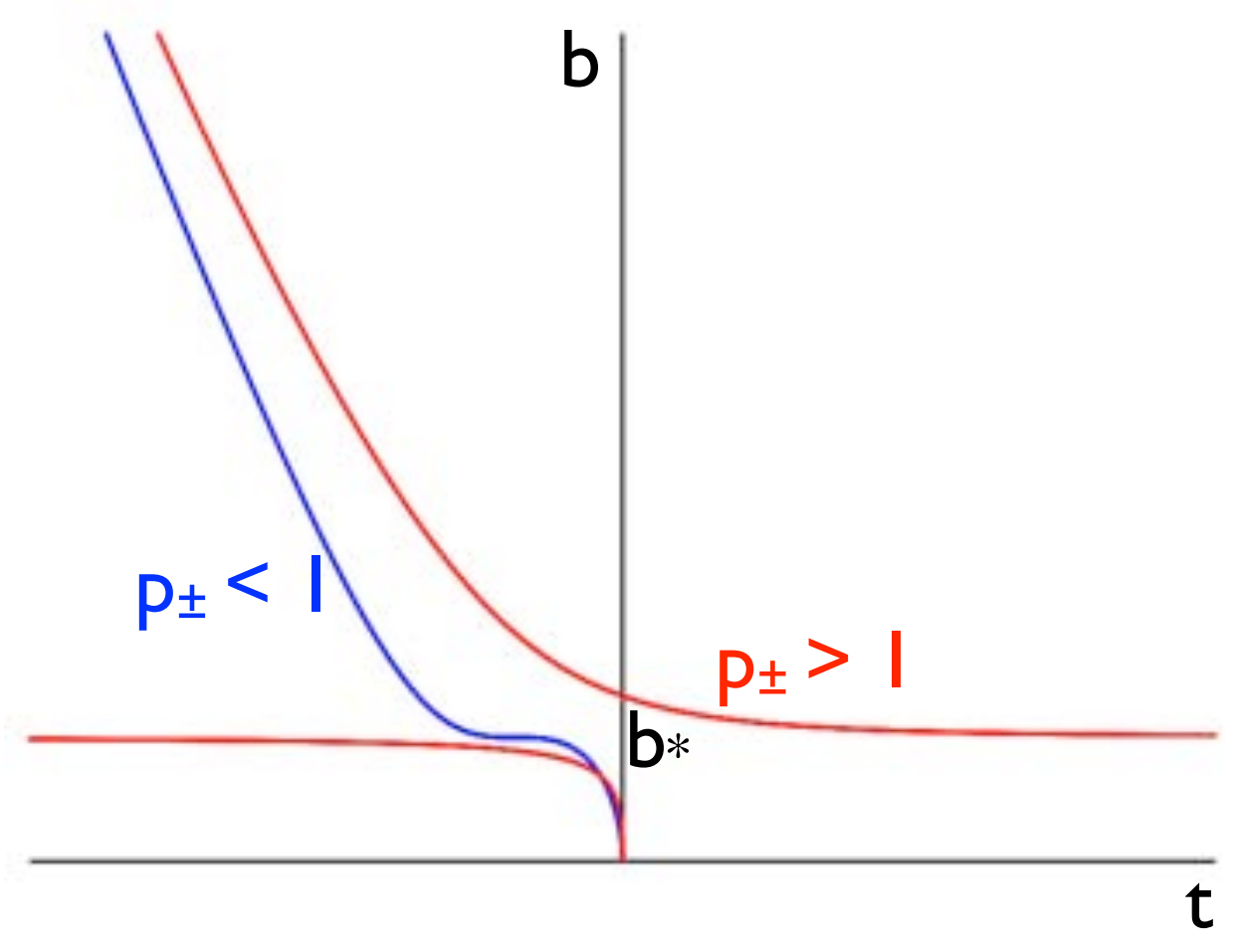




\section{Folded Wilson loop operators}

An LXLWilson loop operator is given by $\quad W(L)=U_{\mu}^{L} U_{\nu}^{L} U_{\mu}^{\dagger^{L}} U_{\nu}^{\dagger L}$

Eigenvalues of this operator are gauge invariant and lie on a unit circle in the complex plane All eigenvalues will be close to $+I$ if the loop is small and there will be gap around $-I$

Eigenvalues will be distributed over the full unit circle for large loops

There is a critical size, $L_{c}(b, N)$, where the gap will close

$$
\text { We can define } a(b)=\frac{1}{L_{c}(b, N)} \quad \text { as a scale }
$$

\section{Eigenvalues of the Hermitian massless overlap Dirac operator}

$$
\pm \lambda_{\mathrm{k}} \quad 0<\lambda_{k}<1, k=1, \cdots, N^{2}-1 \text { with } \lambda_{k}<\lambda_{k+1}
$$

$$
\text { We can define } \lambda(b)=\left\langle\lambda_{1}\right\rangle \text { as another scale }
$$

If chiral symmetry is broken, we expect the ratios

$$
r_{k}=\left\langle\frac{\lambda_{1}}{\lambda_{k}}\right\rangle
$$

to be independent of coupling for small $\mathrm{k}$ at finite $\mathrm{N}$ 


\section{Average value of the plaquette}

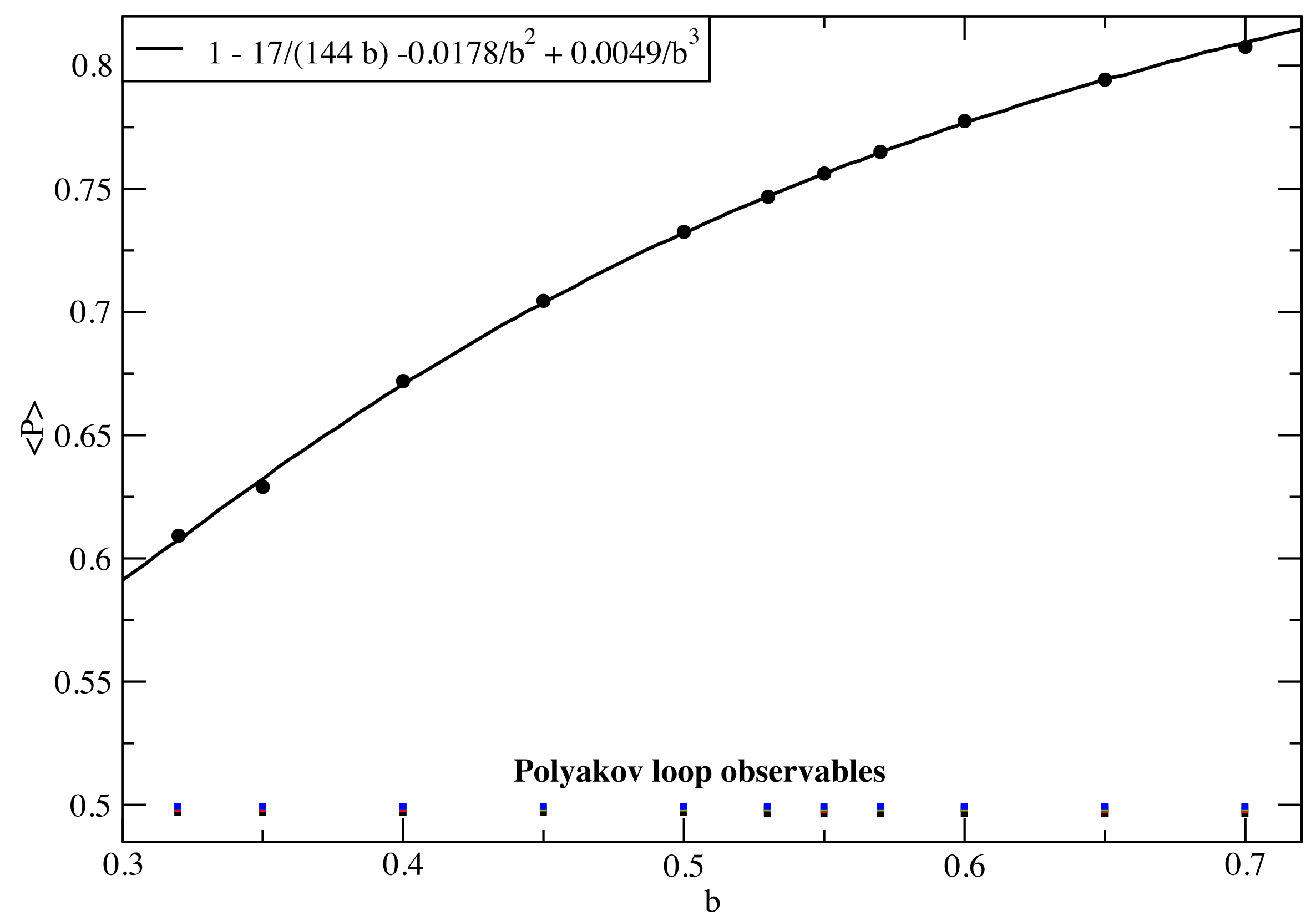

Polyakov loop observables show that we are in the continuum phase 


\section{Running of the scale obtained from the folded Wilson loop operator}

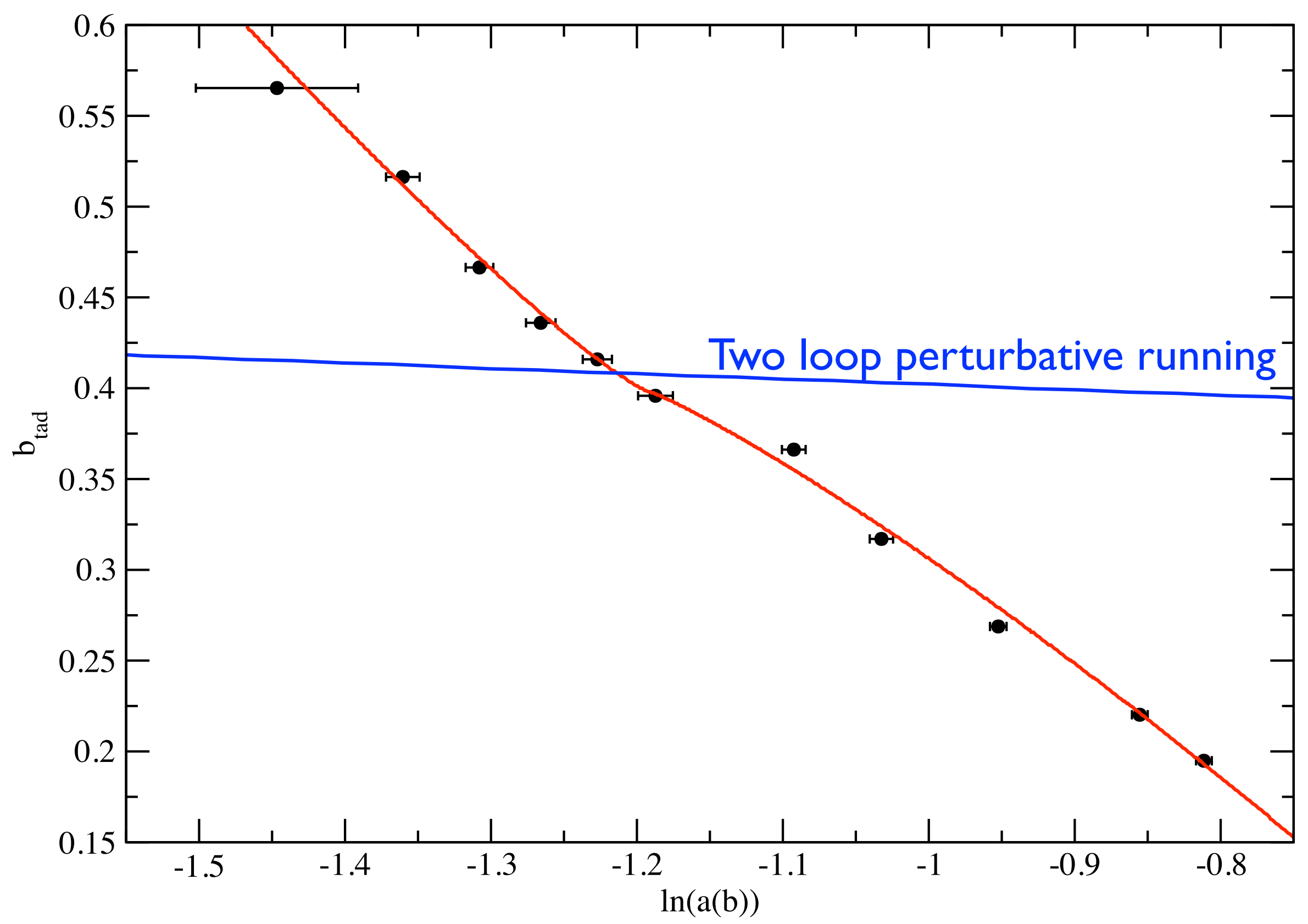


Distribution of the Wilson loop operator eigenvalues at, above and below criticality

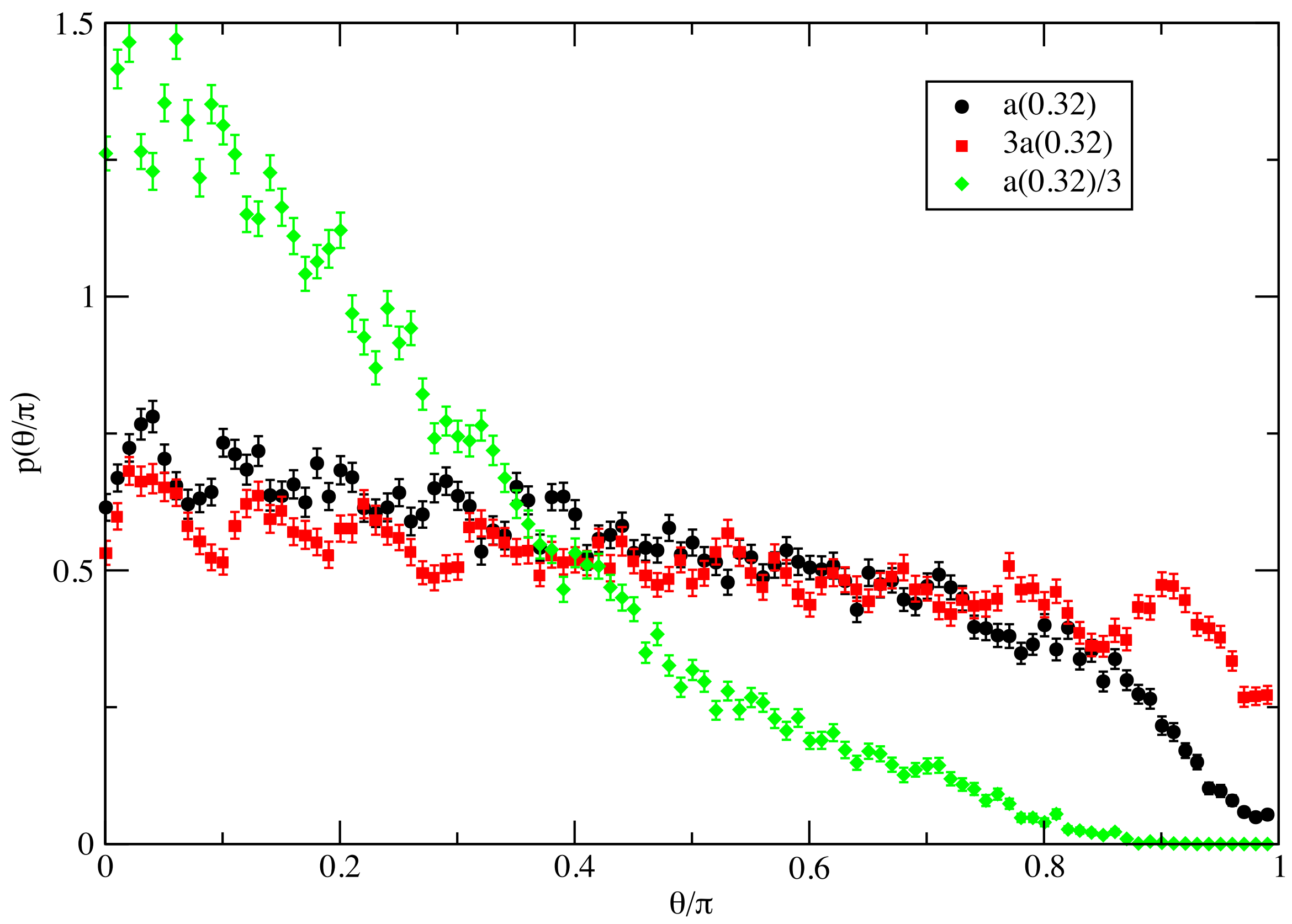


Running of the scale obtained from the lowest positive eigenvalue of the Dirac operator

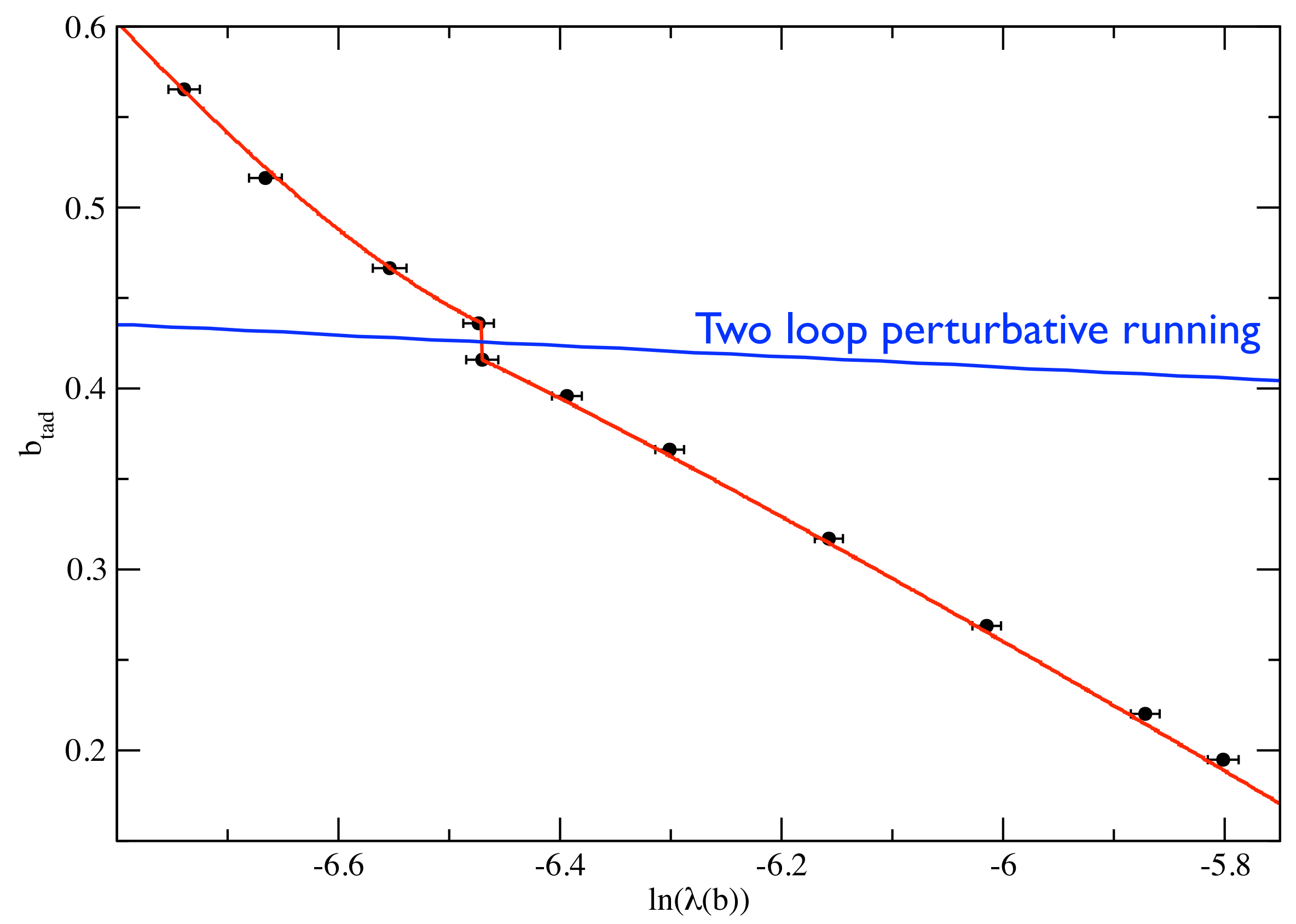


Full distribution of the eigenvalues of the overlap Dirac operator at three different couplings

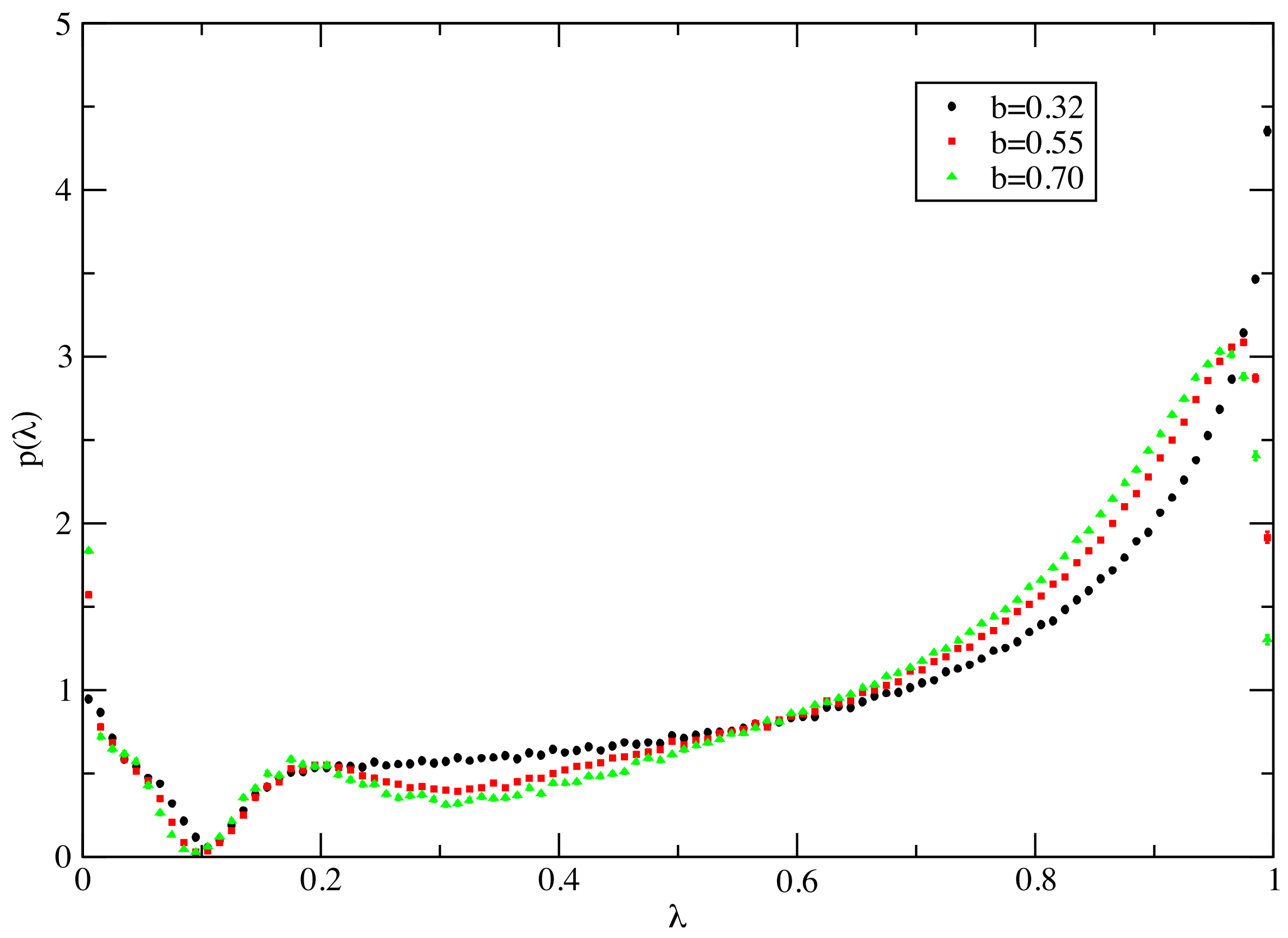


Ratios of the eigenvalues of the overlap Dirac operator

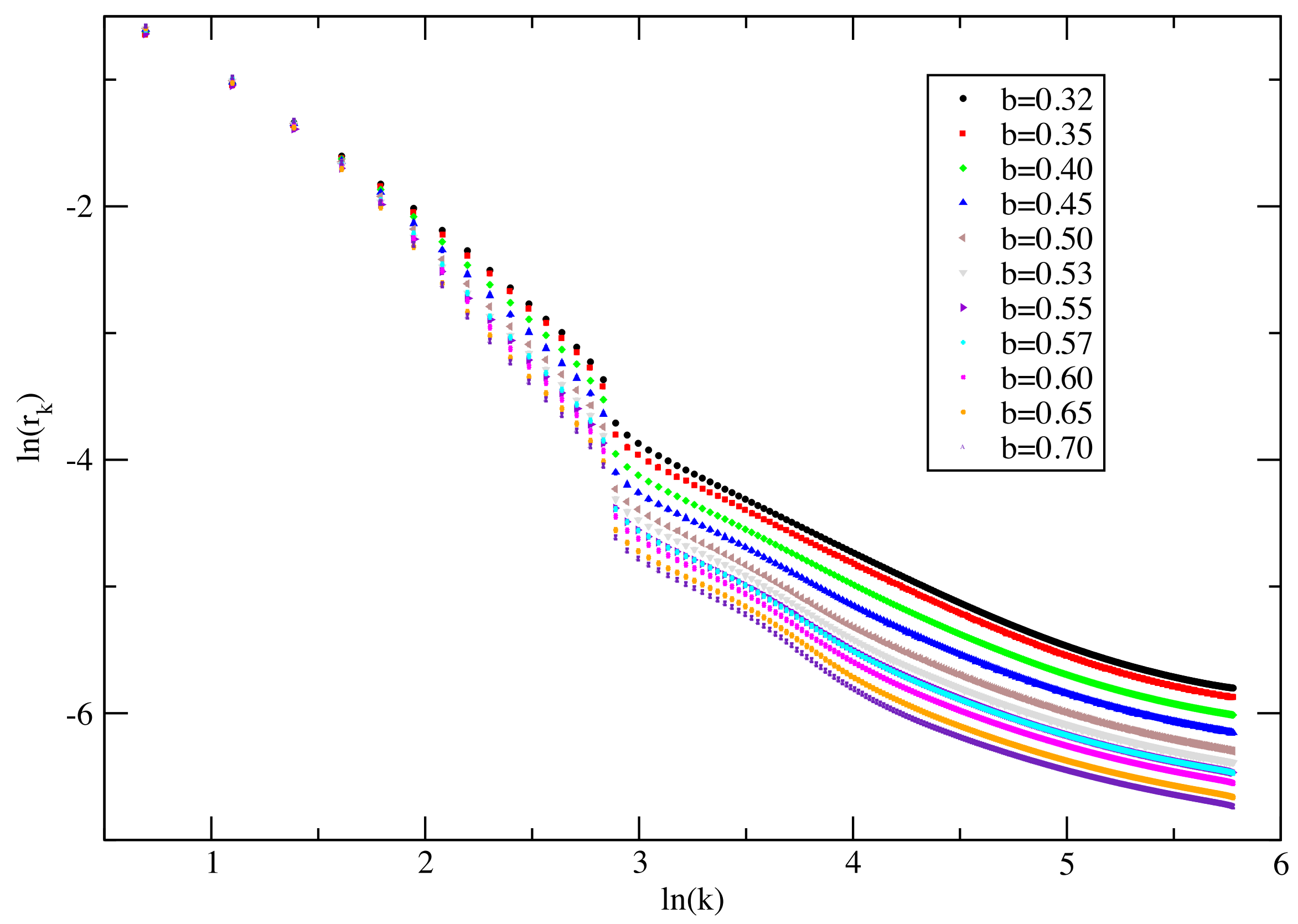




\section{Results for the $f=I$ massless theory}

$$
\begin{gathered}
\text { using } \mathrm{a}(\mathrm{b}) \text { as the scale } \\
\beta\left(b_{\mathrm{tad}}\right)= \begin{cases}-\left|1-\frac{0.398}{b_{\mathrm{tad}}}\right|^{0.16} & \text { for } b_{\mathrm{tad}}>0.398 \\
-0.64\left|1-\frac{0.398}{b_{\mathrm{tad}}}\right|^{0.12} & \text { for } b_{\mathrm{tad}}<0.398\end{cases}
\end{gathered}
$$

using $\lambda(b)$ as the scale

$$
\beta\left(b_{\mathrm{tad}}\right)= \begin{cases}-\left|1-\frac{0.419}{b_{\mathrm{tad}}}\right|^{0.37} & \text { for } b_{\mathrm{tad}}>0.419 \\ -0.36\left|1-\frac{0.419}{b_{\mathrm{tad}}}\right|^{0.05} & \text { for } b_{\mathrm{tad}}<0.419\end{cases}
$$

Combining the two results, we say that $\quad b_{*}=0.408(11) \quad$ is a location of non-analyticity

All powers are less than unity and so we cannot define a continuum theory at $b *$ for the $f=I$ theory 


\section{Conclusions}

- We can use matrix model in the large $\mathrm{N}$ limit to numerically study gauge theories coupled to adjoint fermions.

- We can look at conformal and near-conformal theories by extending $f$, the number of fermion flavors, to take on any real value.

- One can use exact HMC algorithm (compute the fermion force exactly) or use pseudofermion HMC algorithm.

- The beta function is expected to show non-analytical behavior at the infra-red/ultra-violet fixed point.

- It is possible to add a mass term and study the theory. 


\title{
Lattice Radial Quantization.
}

\section{Richard Brower, George Fleming and Herbert Neuberger (speaker)}

\author{
Department of Physics \\ Rutgers University \\ Piscataway, NJ08854
}

New Horizons for Lattice Gauge Theory Computations, Brookhaven National Laboratory, May 14-16, 2012, NY 


\section{Abstract}

We regularize conformal field theories in radial quantization using lattice techniques. Only angular separations matter. Scale is logarithmically discretized in equal intervals. As an application, we set out to compute the critical exponent $\eta$ for the $3 \mathrm{D}$ Ising model and present some preliminary results. 


\section{Outline}

Beautiful theories

Conformal theories

Foliations

The icosahedral Transfer matrix

Spectrum

Regularization

Velocity of light renormalization

Preliminary numerical results 


\title{
Outline
}

\author{
Beautiful theories
}

Conformal theories

Foliations

The icosahedral Transfer matrix

Spectrum

Regularization

Velocity of light renormalization

Preliminary numerical results

Lattice Radial Quantization. 


\section{Being a snob}

"Beauty" $=\frac{\text { nr. of nontrivial results }}{\text { nr. of free continuos parameters }}$. To be worthwhile of our time, a beautiful theory ought to be relevant to Nature. Such beautiful theories never are exactly soluble, nor are they completely perturbative. The top examples for particle physicists are

- The most relevant beautiful particle theory is QCD with a moderate number of massless quarks.

- The most beautiful unparticle theory is $S U(N)$ gauge theory with massless fermionic matter in the conformal window. Maybe it is relevant to Nature.

QCD is essentially a single scale theory, and lattice techniques work and are in principle exact. Walking technicolor type of theories are harder because they have too wide a range of scales for an ordinary lattice approach. One can make progress however by considering their conformal cousins. Of primary importance are the anomalous dimensions of some composite scaling fields in the IR. 


\section{Outline}

\section{Beautiful theories}

Conformal theories

\section{Foliations}

The icosahedral Transfer matrix

Spectrum

Regularization

Velocity of light renormalization

Preliminary numerical results 


\section{Main point and application}

In a conformal theory there is no scale and only angles matter. Rather than uniformly discretizing scales one should uniformly discretize their logarithm as per AMR logic. Simulating a conformal theory by uniformly discretizing scales might be impractical. What to do in the "almost conformal" case is another topic.

There is no point in further generalities. I'll focus on the critical $3 \mathrm{D}$ Ising model because one can simulate it very efficiently using cluster algorithms. The objective would be to exploit it being a CFT in order to extract the anomalous dimension of the magnetization operator. Our numerical results are preliminary. 


\section{Outline}

\section{Beautiful theories}

Conformal theories

Foliations

\section{The icosahedral Transfer matrix}

Spectrum

Regularization

Velocity of light renormalization

Preliminary numerical results 


\section{Radial quantization and its cousins}

In radial quantization one views $R^{3}$ as a sequence of 2D concentric spherical shells whose radial density is uniform in the logarithm of the radius measured from the common center.

The coordinates on the shells are angles. By a conformal transformation flat $R^{3}$ gets mapped into an infinite cylinder raised on top of a $2 \mathrm{D}$ sphere. One quantizes by defining a transfer matrix along the cylinder. The logarithm of the eigenvalues of this transfer matrix provide the spectrum of dimensions of all operators.

We could foliate $R^{3}$ in other ways. We choose to foliate it into concentric $2 \mathrm{D}$ icosahedral shells, again at uniform density in the logarithm of the distance from the common center. The shells have 20 flat equilateral triangular faces and 12 corners. One can define now an icosahedral transfer matrix. 


\section{Outline}

\section{Beautiful theories}

Conformal theories

Foliations

The icosahedral Transfer matrix

\section{Spectrum}

Regularization

Velocity of light renormalization

Preliminary numerical results 


\section{Spectrum and states}

The icosahedral transfer matrix $T$ has the same spectrum as the radial one: it consists of the exponent of all the eigenvalues of the dilatation operator in the Wilson-Fisher CFT.

The eigenstates of $T$ are different from the radial case. Most

spectral regularities would seem accidental because the $S O(3)$ invariance is hidden. Regularities reflecting the discrete symmetry group I of the icosahedron remain evident. A multiplet of angular momentum / under $\mathrm{SO}(3)$ would transform irreducibly under $I$ if $I=0,1,2$ because the icosahedron has 3 -fold and 5 -fold symmetry axes. The remaining 2 irreps of I appear in the decomposition of $I=3$; their degeneracy would seem accidental if we did not know about the hidden $S O(3)$. Let $M(x)$ be the exact scaling field corresponding to the highest eigenvalue of $T$ in the $Z_{2}$-odd sector. One can construct out of it 5 orthogonal multiplets transforming irreducibly under $\mathbf{I}$ in representations subduced from the $I=1,2,3$ irreps of $S O(3)$. 


\section{Outline}

\section{Beautiful theories}

Conformal theories

Foliations

The icosahedral Transfer matrix

Spectrum

Regularization

Velocity of light renormalization

Preliminary numerical results 


\section{Descendants}

Let $m_{0}$ be the dimension of $M(x)$ and $m_{l}$ the dimensions of the descendants constructed from $M(x)$ for $I=0,1,2$. One has

$$
m_{l}=m_{0}+l
$$

At this point we went beyond just using scaling, which reflects only dilatation invariance. The integer spacing is a consequences of full conformal invariance. Our regularization preserves (if present in the continuum) the crucial discrete inversion transformation which extends the symmetry in the flat case to the full conformal group. 


\section{Outline}

\section{Beautiful theories}

Conformal theories

Foliations

The icosahedral Transfer matrix

Spectrum

Regularization

Velocity of light renormalization

Preliminary numerical results 


\section{The lattice}

Each equilateral triangle is replaced by a fine triangular mesh defined by adding $s-1$ extra points at equal distance on each icosahedral edge. The total number of sites per shell is $10 s^{2}+2$. Our UV cutoff $\Lambda$ will be $\propto s$ for large $s$ because the size of a shell is some fixed number in terms of angular extent. The UV cutoff is dimensionless in radial quantization. On each site we place an Ising spin. To all intra- and inter- links connecting sites $i$ and $j$ we attach the standard weight

$$
e^{b \sigma_{i} \sigma_{j}}
$$

$b>0$ is the Ising coupling and needs to be tuned to $b_{c}$ to get into the domain of attraction of the Wilson Fisher fixed point. The SW cluster update algorithm only needs the abstract graph of the structure in order to proceed. 


\section{Outline}

\section{Beautiful theories}

Conformal theories

Foliations

The icosahedral Transfer matrix

Spectrum

Regularization

Velocity of light renormalization

Preliminary numerical results 


\section{Fixing homogeneity}

Except at the corners, the shells are locally a flat triangular lattice. Neighboring shells are connected chain like. There is local regularity, but the approximate local discrete rotational invariance around a vertex does no mix in-shell with intra-shell directions.

For a given $s$, the eigenvalues corresponding to $m_{l}$ are $\mu_{l}$ and the near shell separation is $\Delta \tau=1$. The continuum shell separation is $\Delta t$. We define $\kappa$ so that $m_{l}=\kappa \mu_{l} \wedge$. Then, $t=\frac{\tau}{\Lambda}$ to ensure $m_{l} t=\kappa \mu_{l} \tau$.

We can extract $\kappa \Lambda$ from

$$
\mu_{l}^{\prime}-\mu_{I}=\frac{l^{\prime}-I}{\kappa \Lambda}
$$

Then we can extract $m_{0}$ from $m_{0}=\kappa \Lambda \mu_{0}$. The anomalous dimension of $M(x), \eta$ is defined by

$$
m_{0}=\frac{1+\eta}{2}
$$




\section{Outline}

\section{Beautiful theories}

Conformal theories

Foliations

The icosahedral Transfer matrix

Spectrum

Regularization

Velocity of light renormalization

Preliminary numerical results

Lattice Radial Quantization. 
The refinement level is $s$ and the number of shells is $16 s$; at $b=b_{c}, \kappa \Lambda \propto s$ must hold to leading order in $s . b=0.16107$.

\begin{tabular}{|l|l|l|l|l|}
\hline$b$ & $s$ & $\frac{\mu_{2}-\mu_{1}}{\mu_{1}-\mu_{0}}$ & $\frac{s}{\kappa \Lambda}$ & $\eta$ \\
\hline 0.16107 & 6 & $0.966(5)$ & $0.724(2)$ & $0.084(2)$ \\
\hline 0.16107 & 7 & $0.966(5)$ & $0.728(1)$ & $0.066(2)$ \\
\hline 0.16107 & 8 & $0.958(2)$ & $0.7288(2)$ & $0.052(1)$ \\
\hline 0.16107 & 9 & $0.954(2)$ & $0.7311(2)$ & $0.036(1)$ \\
\hline 0.16107 & 10 & $0.957(2)$ & $0.7298(2)$ & $0.044(1)$ \\
\hline 0.16107 & 11 & $0.961(2)$ & $0.7267(2)$ & $0.064(1)$ \\
\hline 0.16107 & 12 & $0.973(2)$ & $0.7181(2)$ & $0.123(1)$ \\
\hline
\end{tabular}

Previous simulations, using cubic shells and a different approach, gave, after extrapolation to infinite cutoff, $\eta=0.002 \pm 0.010$; the expected value is $\eta \approx 0.036$. Maybe we found the correct value of $\eta$ at $s=9$ not by accident. 


\section{"Masses" or more correctly dimensions}

We probably are not at $b=b_{c}(\infty)$. Something is happening at $s=9$. Maybe we are in the would-be $Z_{2}$ broken side of $b_{c}(\infty)$. Maybe periodic boundary conditions are better. So far, our analysis has been rough.

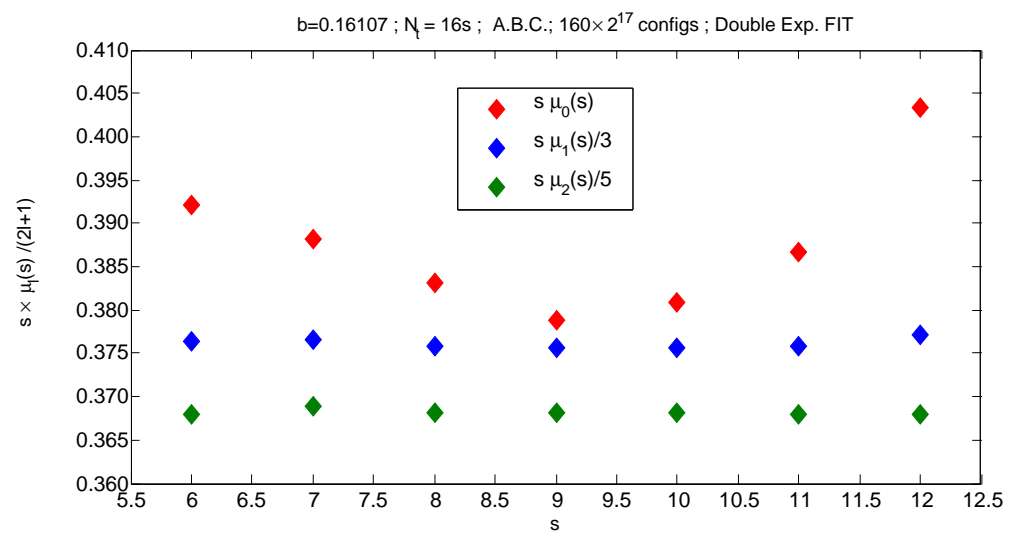

Lattice Radial Quantization. 


\section{He \\ LATTICE QGD FOR \\ NUGLEAR PHYSICS \\ Kostas Orginos}




\section{NPLQCD Collaboration}

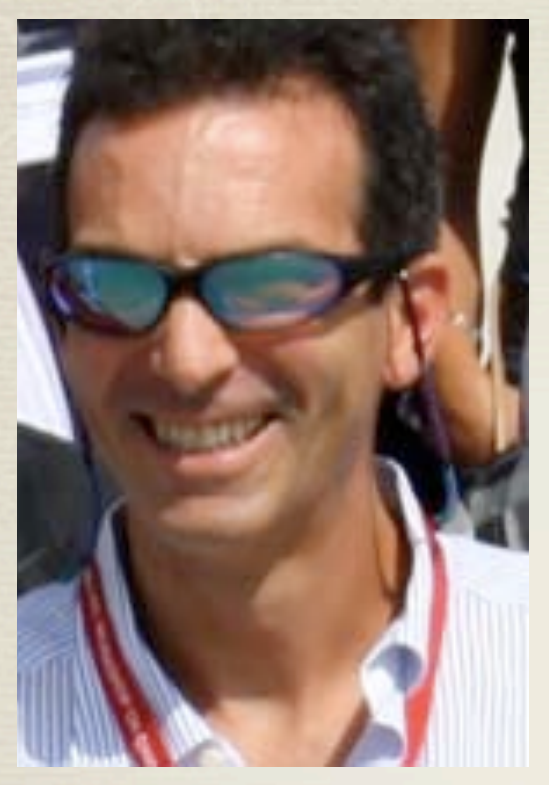

Silas R. Beane

New Hampshire

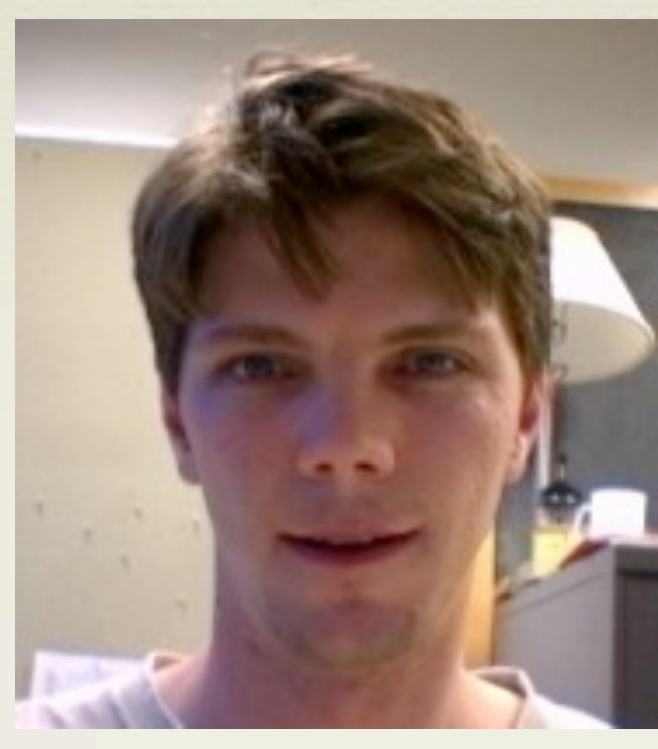

William Detmold William \& Mary

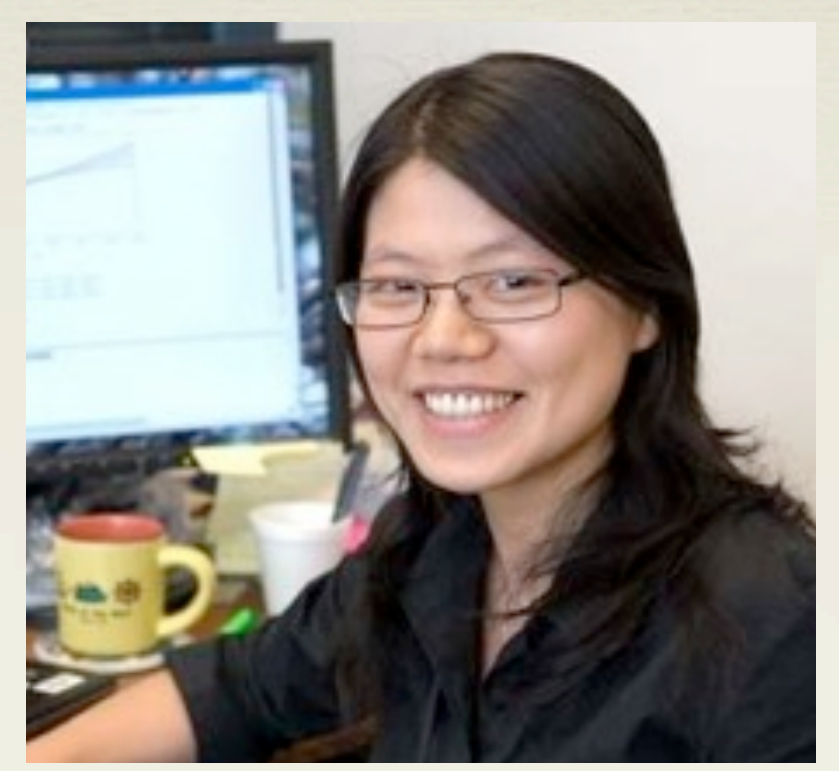

Huey-Wen Lin

$\mathrm{U}$ of Washington

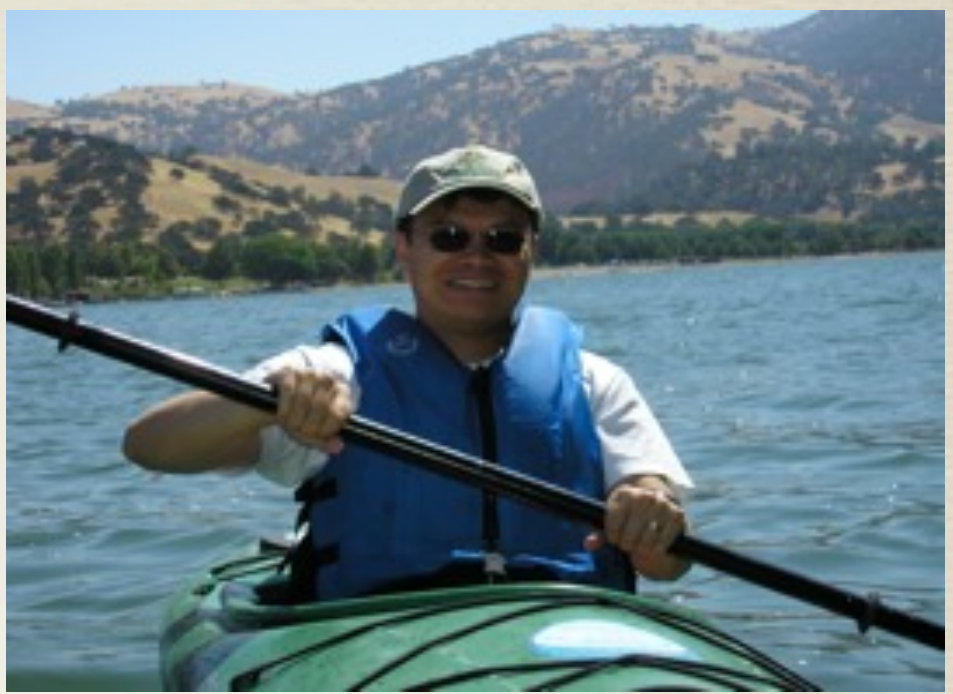

Tom Luu

Livermore

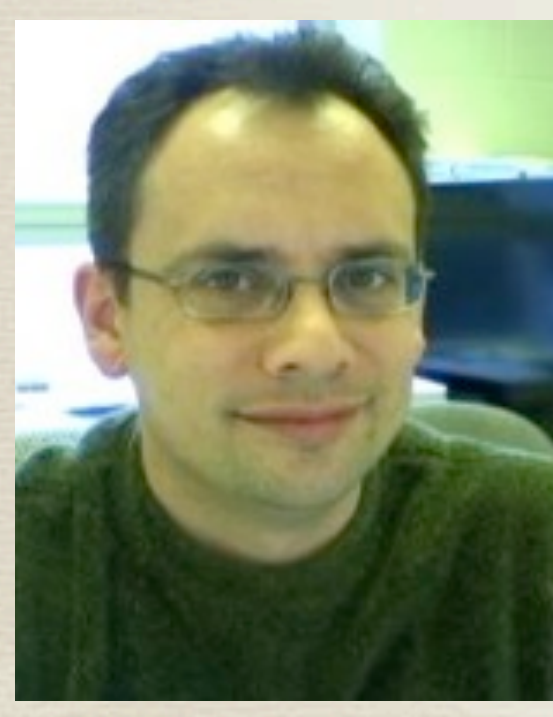

Kostas Orginos William \& Mary

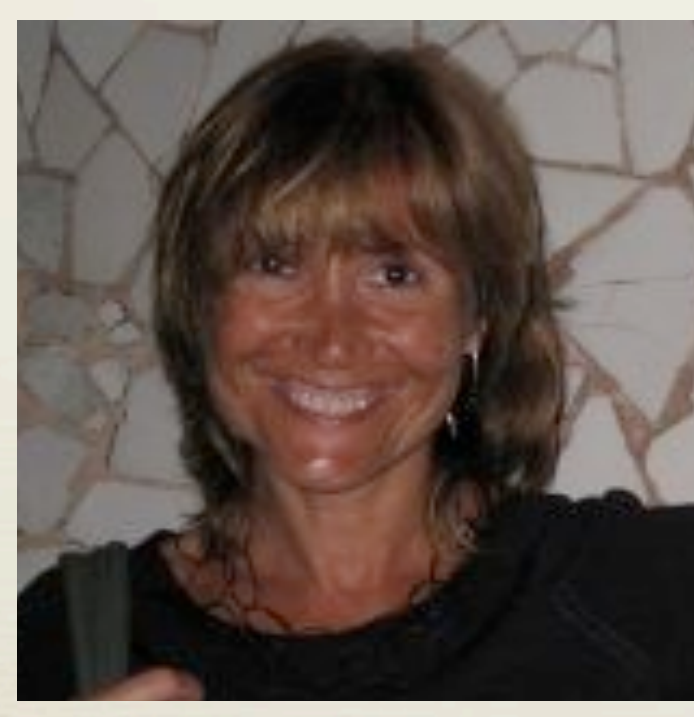

Assumpta Parreño Barcelona

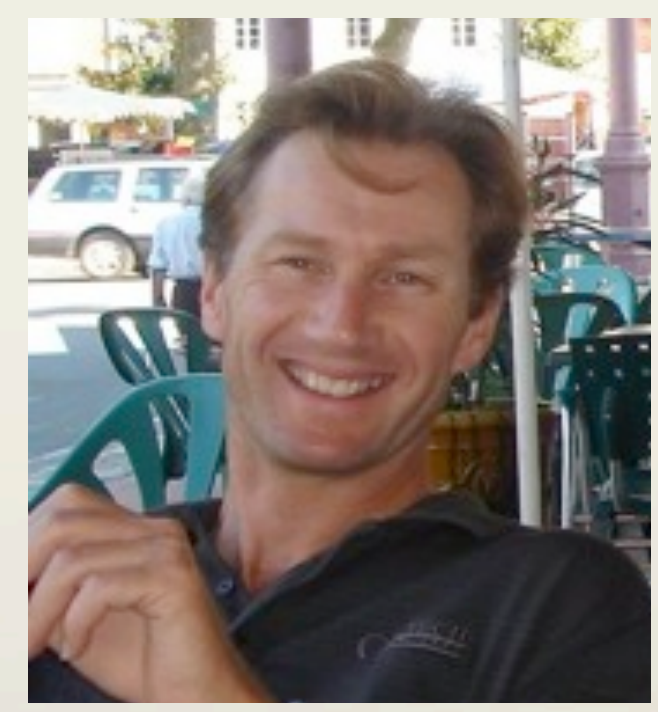

Martin J. Savage U of Washington

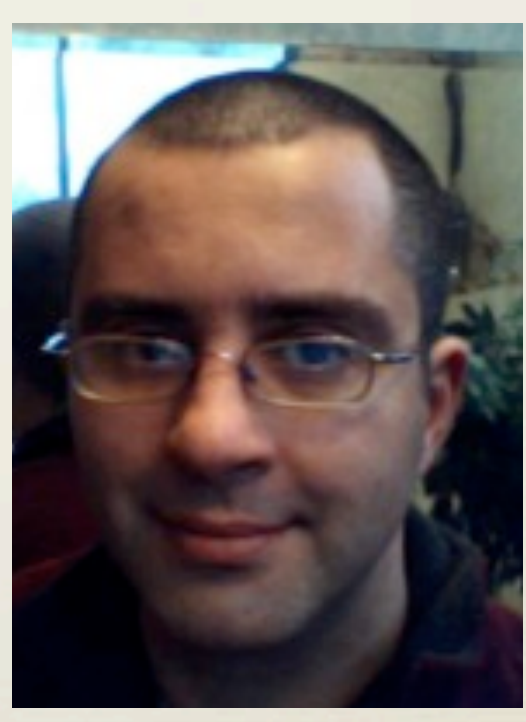

Aaron Torok Indiana

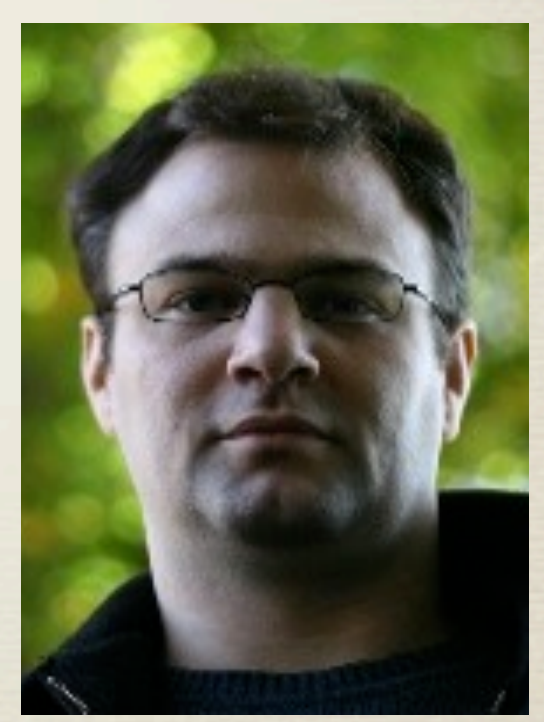

André Walker-Loud William \& Mary

Emmanuel Chang 


\section{Hadron Interactions}

\section{Goals:}

* Challenge: Compute properties of nuclei from QCD

* Spectrum and structure

* Confirm well know experimental observation for two nucleon systems

* Explore the largely unknown territory of hyper-nuclear physics

* Provide input for the equation of state for nuclear matter in neutron stars

* Provide input for understanding the properties of multi-baryon systems

\section{Jefferson Lab}

FAIR

4.

(E) KEK

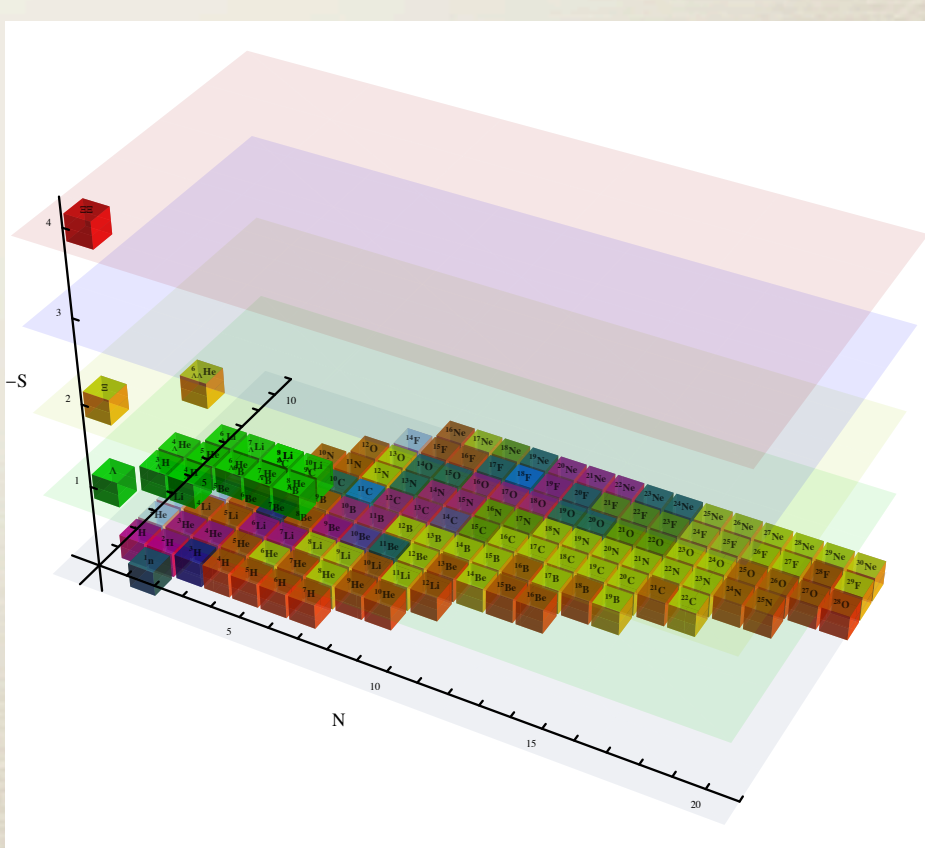




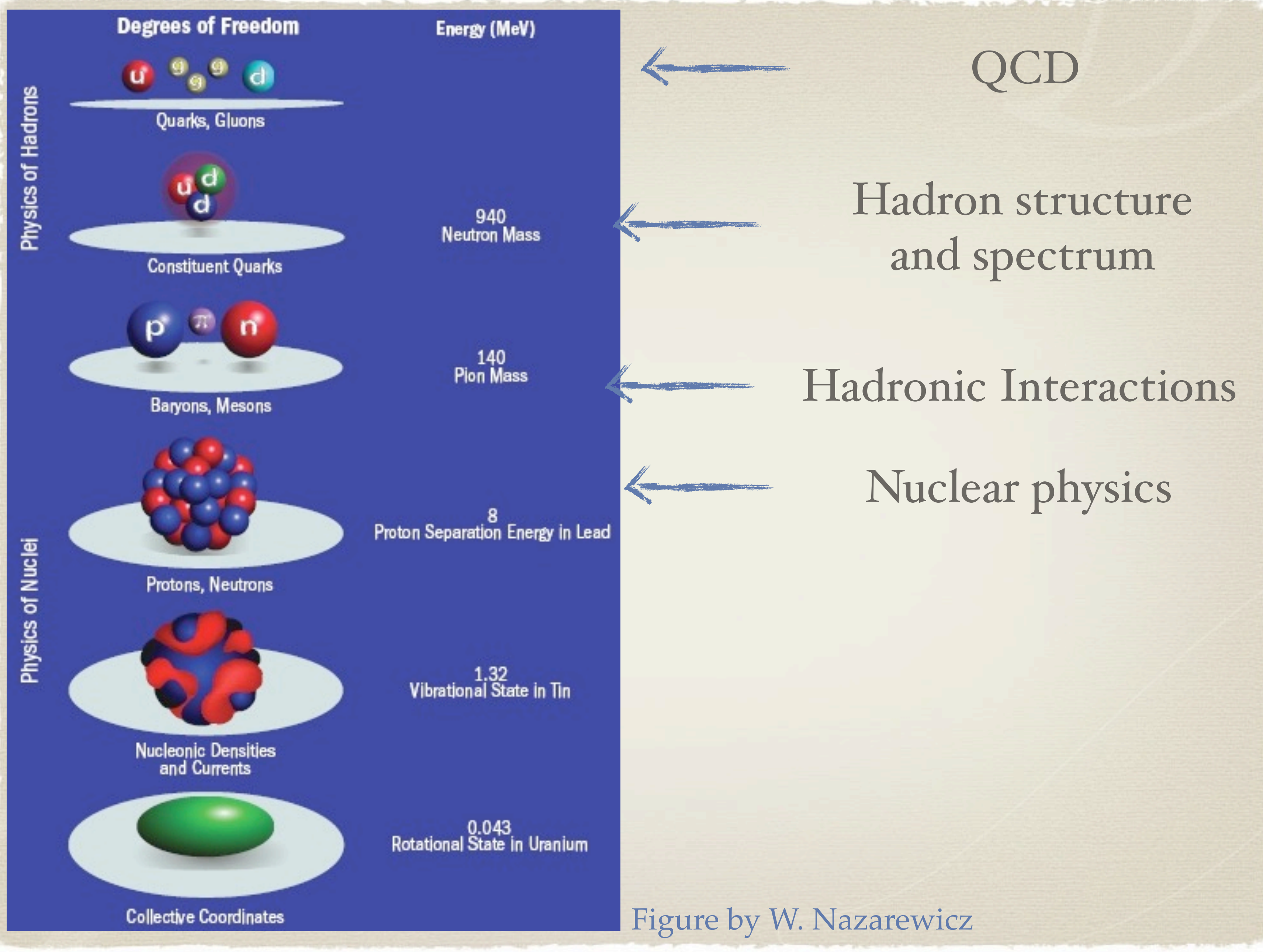




\section{What does it take?}

- Hadronic Scale: $1 \mathrm{fm} \sim 1 \times 10^{-13} \mathrm{~cm}$

- Lattice spacing $<<1 \mathrm{fm}$

- take $\mathrm{a}=0.1 \mathrm{fm}$

- Lattice size La $>>1 \mathrm{fm}$

- take $\mathrm{La}=3 \mathrm{fm}$

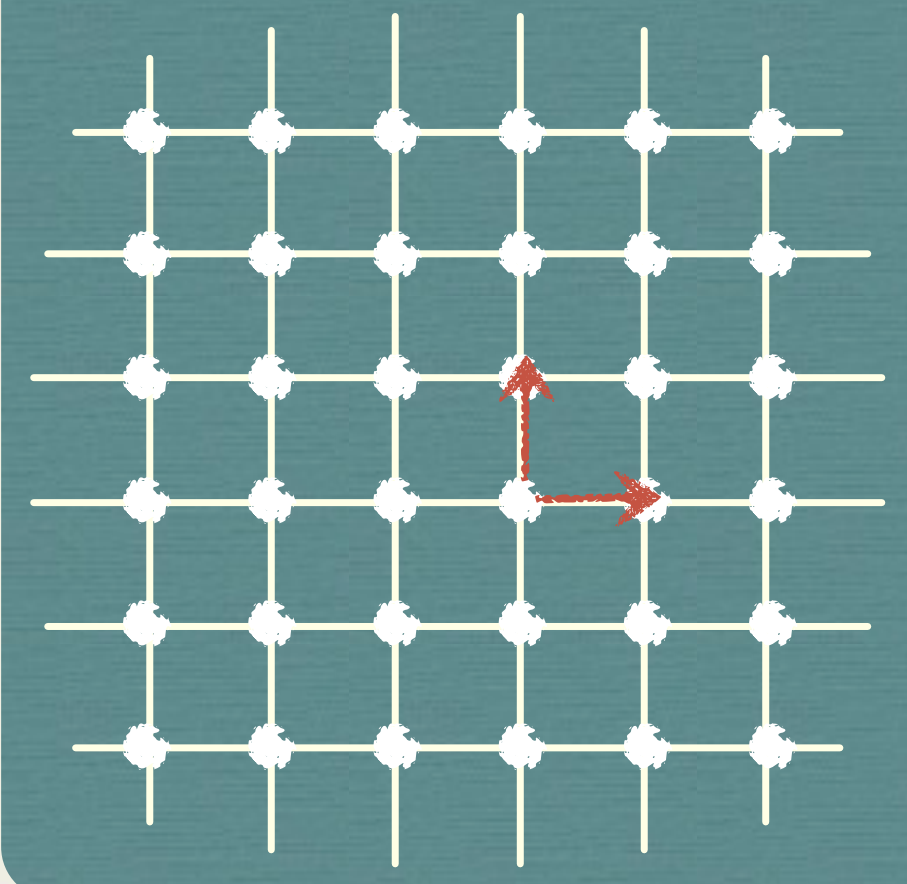

- Lattice $32^{4}$

- Gauge degrees of freedom: $8 \times 4 \times 32^{4}=3.4 \times 10^{7}$

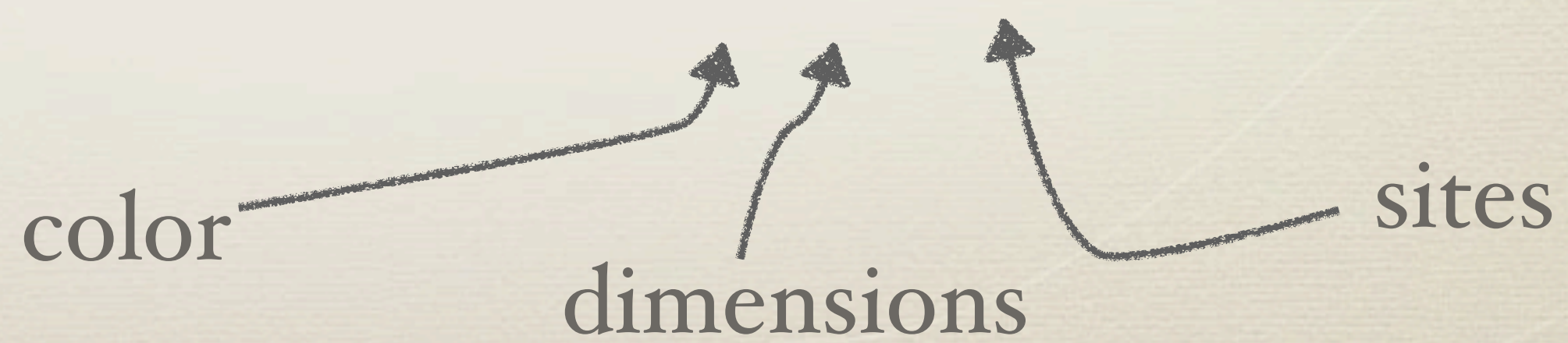


The pion mass is an additional small scale

Volume corrections $\sim e^{-m_{\pi} L}$

6-7 fm boxes might be needed at the physical point $\sim 1 / \mathrm{m}_{\pi} \sim 1.4 \mathrm{fm}$

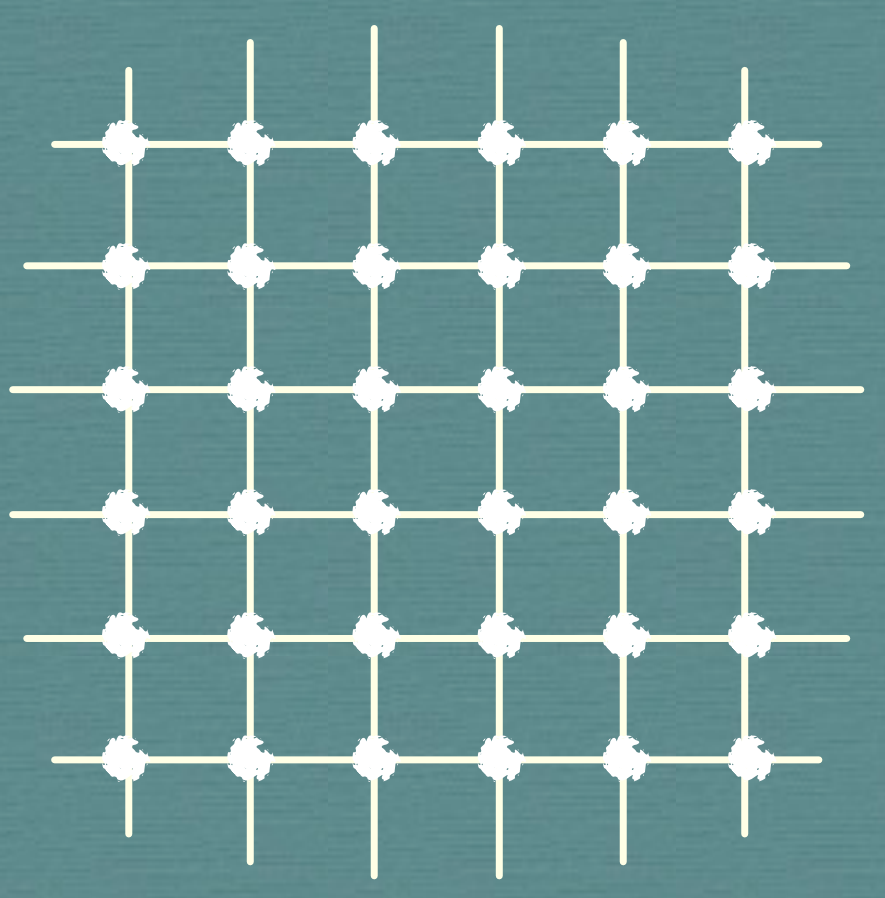

Smaller scale: Binding momentum of the deuteron $\sim 4 \mathrm{OMeV}$

Nuclear energy level splittings are a few $\mathrm{MeV}$ 


\section{Scattering on the Lattice}

Luscher Comm. Math. Phys 105, 153 '86

Elastic scattering amplitude (s-wave):

$$
\begin{array}{r}
A(p)=0+\cdots \\
A(p)=\frac{4 \pi}{m} \frac{1}{p \cot \delta-i p}
\end{array}
$$

At finite volume one can show:

$$
E_{n}=2 \sqrt{p_{n}^{2}+m^{2}}
$$

$p \cot \delta(p)=\frac{1}{\pi L} \mathbf{S}\left(\frac{p^{2} L^{2}}{4 \pi^{2}}\right)$

$\mathbf{S}(\eta) \equiv \sum_{\mathbf{j}}^{|\mathbf{j}|<\Lambda} \frac{1}{|\mathbf{j}|^{2}-\eta}-4 \pi \Lambda$

Small p:

$$
p \cot \delta(p)=\frac{1}{a}+\frac{1}{2} r p^{2}+\ldots
$$

$a$ is the scattering length 


\section{Bound States}

Luscher Comm. Math. Phys 104, 177 '86

$$
\begin{array}{ll}
E_{b}=\sqrt{p^{2}+m_{1}^{2}}+\sqrt{p^{2}+m_{2}^{2}}-m_{1}-m_{2} & p^{2}<0 \\
E_{b} \approx \frac{p^{2}}{2 \mu}=-\frac{\kappa^{2}}{2 \mu} & \kappa=|p|
\end{array}
$$

$\kappa$ is the "binding momentum" and $\mu$ the reduced mass

Finite volume corrections:

$$
\Delta E_{b}=-3|A|^{2} \frac{e^{-\kappa L}}{\mu L}+\mathcal{O}\left(e^{-\sqrt{2} \kappa L}\right) \quad \text { cubic group irrep: } A_{1}^{+}
$$




\section{Two Nucleon spectrum}

free nucleons

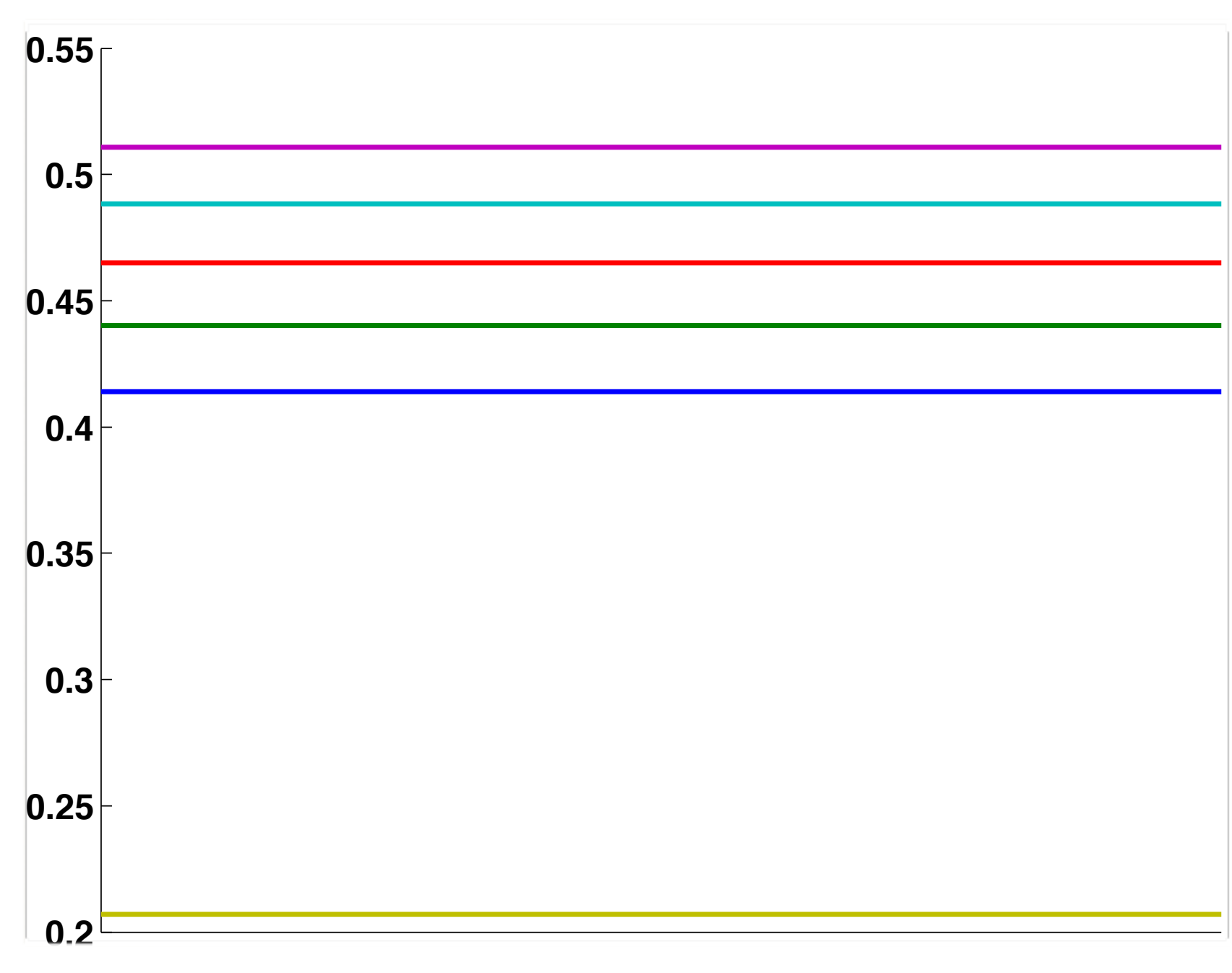

$3 f m$ box

$24^{3}$ Lallice

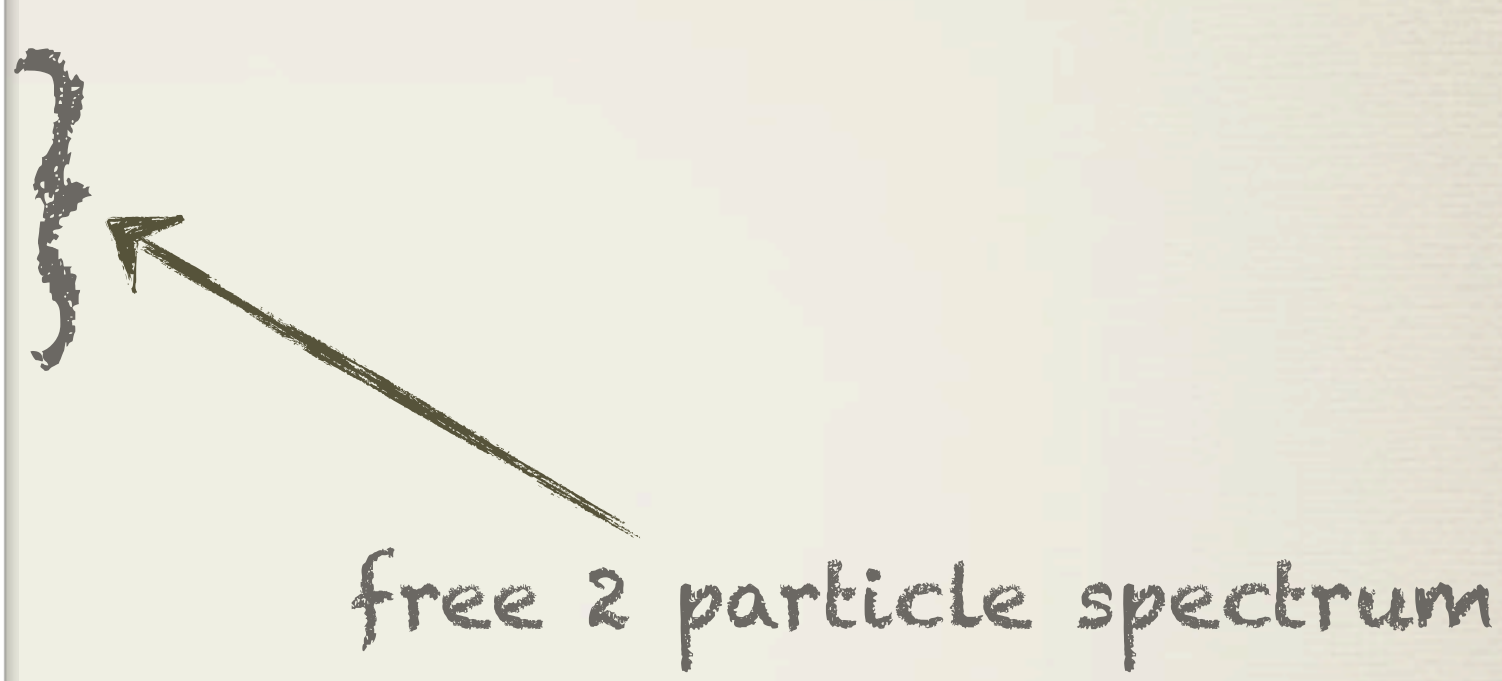

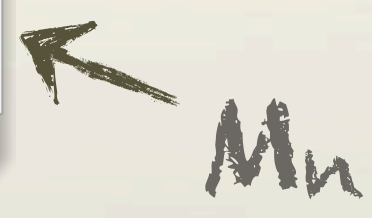

anisotropy factor 3.5
$M_{\pi}=390 \mathrm{MeV}$ 


\section{Signal to Noise ratio for correlation functions}

$$
\begin{gathered}
C(t)=\langle N(t) \bar{N}(0)\rangle \sim E e^{-M_{N} t} \\
\operatorname{var}(C(t))=\langle N \bar{N}(t) N \bar{N}(0)\rangle \sim A e^{-2 M_{N} t}+B e^{-3 m_{\pi} t} \\
S t o N=\frac{C(t)}{\sqrt{\operatorname{var}(C(t))}}=\sim A e^{-\left(M_{N}-3 / 2 m_{\pi}\right) t}
\end{gathered}
$$

* The signal to noise ratio drops exponentially with time

* The signal to noise ratio drops exponentially with decreasing pion mass

* For two nucleons: $\operatorname{StoN}(2 \mathrm{~N})=\operatorname{StoN}(1 \mathrm{~N})^{2}$ 


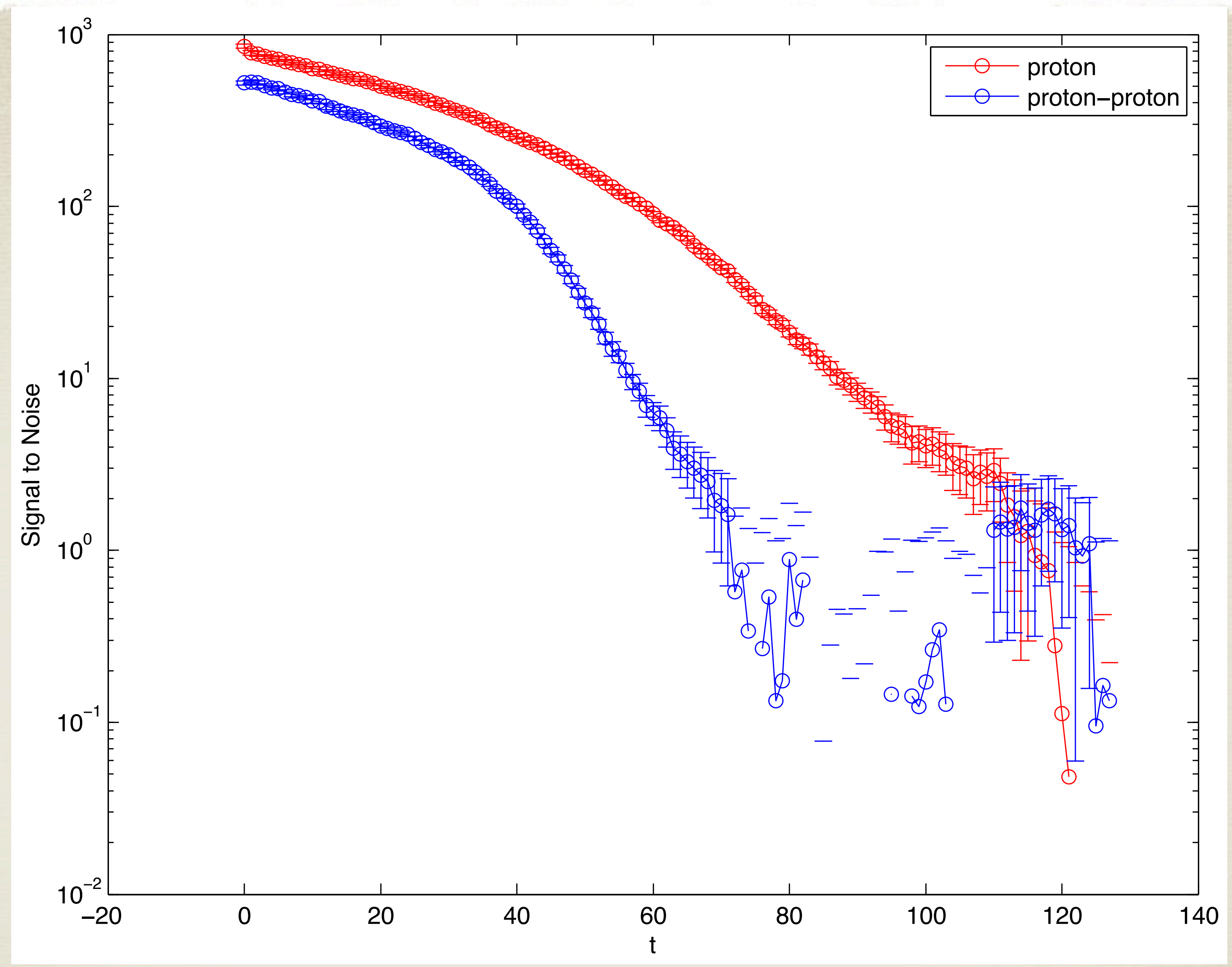

\section{Signal to Noise}

$323 \times 256$ $\mathrm{M}_{\pi}=390 \mathrm{MeV}$ 
We need to fit for several low lying states for reliable estimation of the ground state of the two particle system in a finite box.

\section{Use "variational methods"}

We need very high statistics to be able to resolve excited state contamination 
We need to fit for several low lying states for reliable estimation of the ground state of the two particle system in a finite box.

\section{Use "variational methods"}

We need very high statistics to be able to resolve excited state contamination

We really need better algorithms to deal with an exponentially hard problem 
I. Hyperon-Hyperon Interactions

2. Hyperon-Nucleon Interactions 


\section{H-dibaryon}

R. L. Jaffe, Phys. Rev. Lett. 38, 195 (1977)

* Proposed by R. Jaffe I977

* Perturbative color-spin interactions are attractive for

$S=-2$, (uuddss)$$
B=2 \text {, }
$$

* Diquark picture of scalar diquarks (ud)(ds)(su)

* Experimental searches of the $\mathrm{H}$ have not found it

* BNL RHIC (+model): Excludes the region $[-95$, o] $\mathrm{MeV}$

A. L. Trattner, PhD Thesis, LBL, UMI-32-54109 (2006),

* KEK: Resonance near threshold

C. J. Yoon et al., Phys. Rev. C 75, 022201 (2007).

* Several Lattice QCD calculations have been addressing the existence of a bound $\mathrm{H}$ 


\section{Lattice}

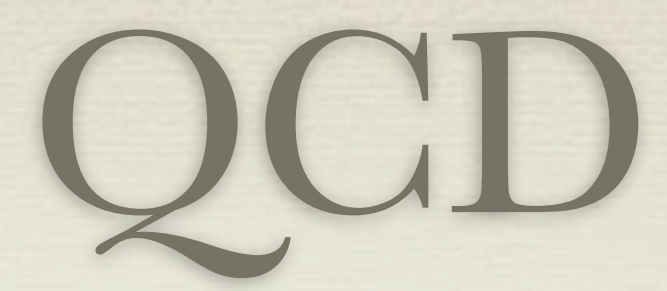

* P. B. Mackenzie and H. B. Thacker, Phys. Rev. Lett. 55,2539 (I985)

* Did not find it

* Y. Iwasaki, T. Yoshie and Y. Tsuboi, Phys. Rev. Lett. 6o, I37I (I988)

* Found it

* A. Pochinsky, J. W. Negele and B. Scarlet, Nucl. Phys. Proc. Suppl. 73, 255 (I999)

* Concluded $\mathrm{H}$ is not bound in the infinite volume limit

* I. Wetzorke and F. Karsch Nucl. Phys. Proc. Suppl. II9, 287 (2003)

* Concluded the $\mathrm{H}$ is not bound 


\section{Lattice}

\section{QGD}

* NLPQCD Phys.Rev.Lett.ro6.I6200r 2011

* HALQCD Phys.Rev.Lett.Io6.I62002 20II

* Luo, Loan and Liu arXiv:IIo6.I945

* Anisotropic Clover (quenched)

* Multiple lattice spacings and volumes

* Result: $\mathrm{B}_{\mathrm{H}}=7 \mathrm{O}(\mathrm{II})(\mathrm{I} 5) \mathrm{MeV}$ 


\section{NPLQCD: lattice set up}

* Anisotropic 2+1 clover fermion lattices

* $\mathrm{a} \sim 0.125 \mathrm{fm}$ (anisotropy of $\sim 3.5$ )

Hadron Spectrum/JLAB

* pion mass $\sim 390 \mathrm{MeV}$

* Volumes $16^{3}$ x $128,20^{3} \times 128,24^{3}$ x $128,32^{3} \times 256$

* Smeared source - 3 sink interpolating fields

* Interpolating fields have the structure of s-wave $\Lambda$ - $\Lambda$ system

* $\mathrm{I}=0, \mathrm{~S}=-2, \mathrm{~A}_{1}$, positive parity 


\section{NPLQCD methods}

* Compute the ground state energy using Euclidian correlators

* Use a single source operator with three sinks operator (varied smearing)

* "Diagonalize" the correlators to isolate the ground state

* Matrix Prony and Multi-exponential fits

* Extract the binding momentum

* Extrapolate to infinite volume using multiple volumes

* Use very high statistics $O(500 \mathrm{~K})$ correlation functions 


\section{NPLQCD}
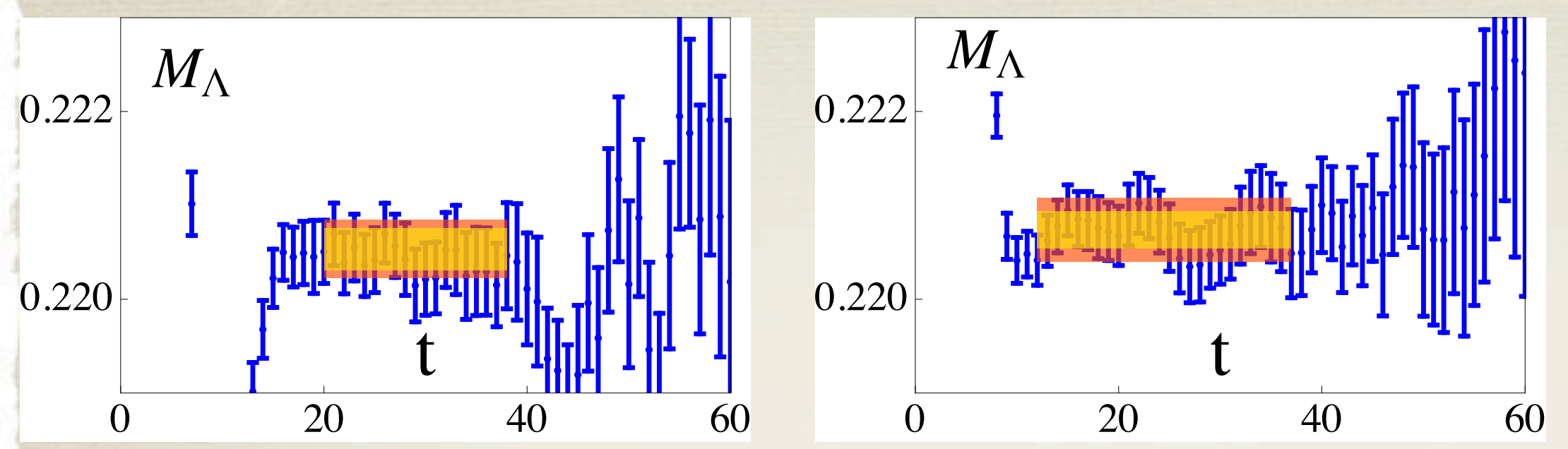

$\Lambda \Lambda$ Energy

$E_{\Lambda \Lambda}=2 \sqrt{p^{2}+m_{\Lambda}^{2}}$
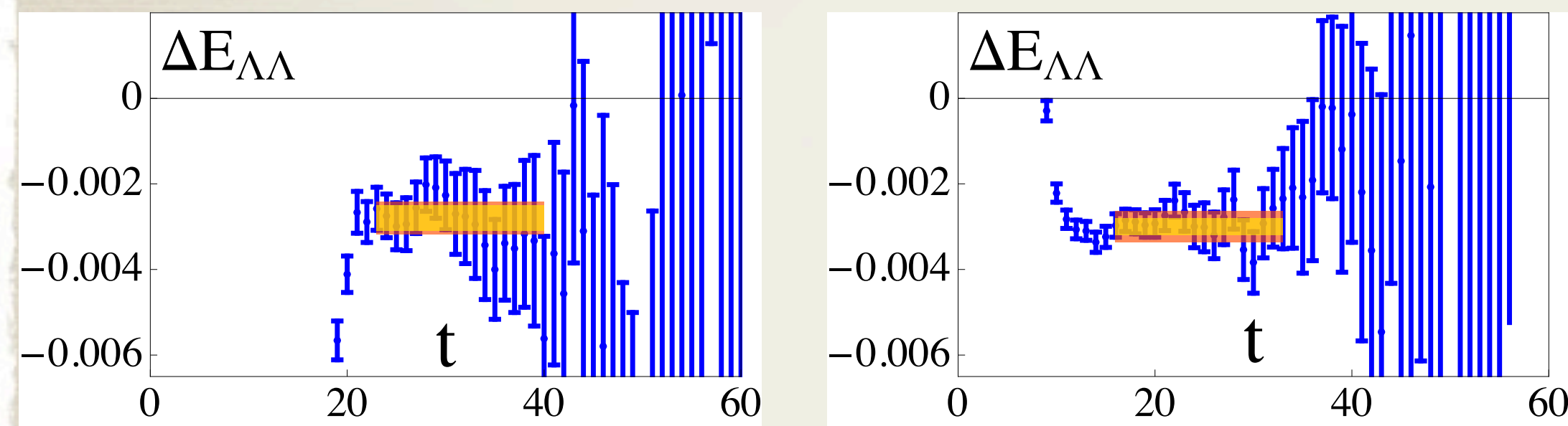

binding momentum

$\kappa=\gamma+\frac{g_{1}}{L}\left(e^{-\gamma L}+\sqrt{2} e^{-\sqrt{2} \gamma L}\right)$

$$
\kappa=|p|
$$

$B_{H}=\frac{\gamma^{2}}{m_{\Lambda}}$ 


\section{NPLQCD: H-dibaryon}

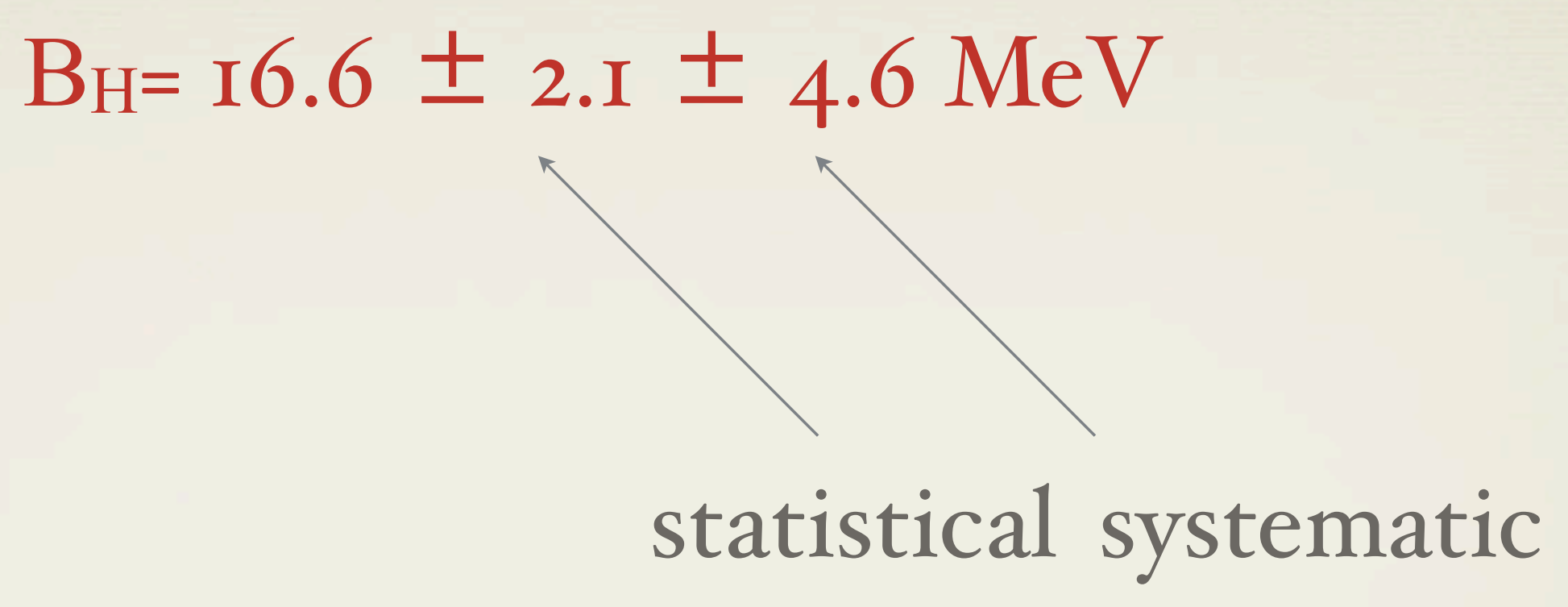

$\mathrm{M}_{\pi}=390 \mathrm{MeV}$

2+I Clover anisotropic fermions
NPLQCD: arXiv:1012.3812

Phys. Rev. Lett. 106, 162001

(Published April 20, 2011) 


\section{NPLQCD: H-dibaryon}

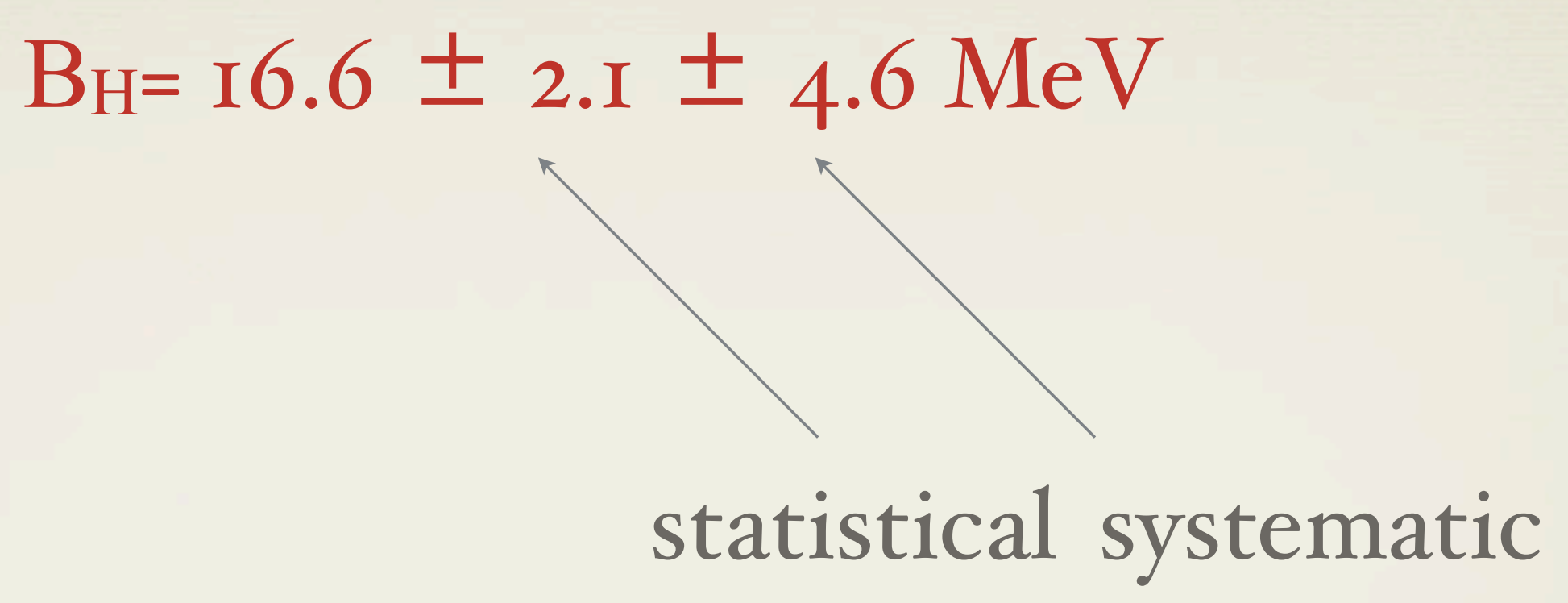

$\mathrm{M}_{\pi}=390 \mathrm{MeV}$

2+I Clover anisotropic fermions
NPLQCD: arXiv:1012.3812

Phys. Rev. Lett. 106, 162001

(Published April 20, 2011)
Continuum limit?

Physical pion mass?
Isospin breaking?

Electromagnetism? 


\section{NPLQCD: H-dibaryon}

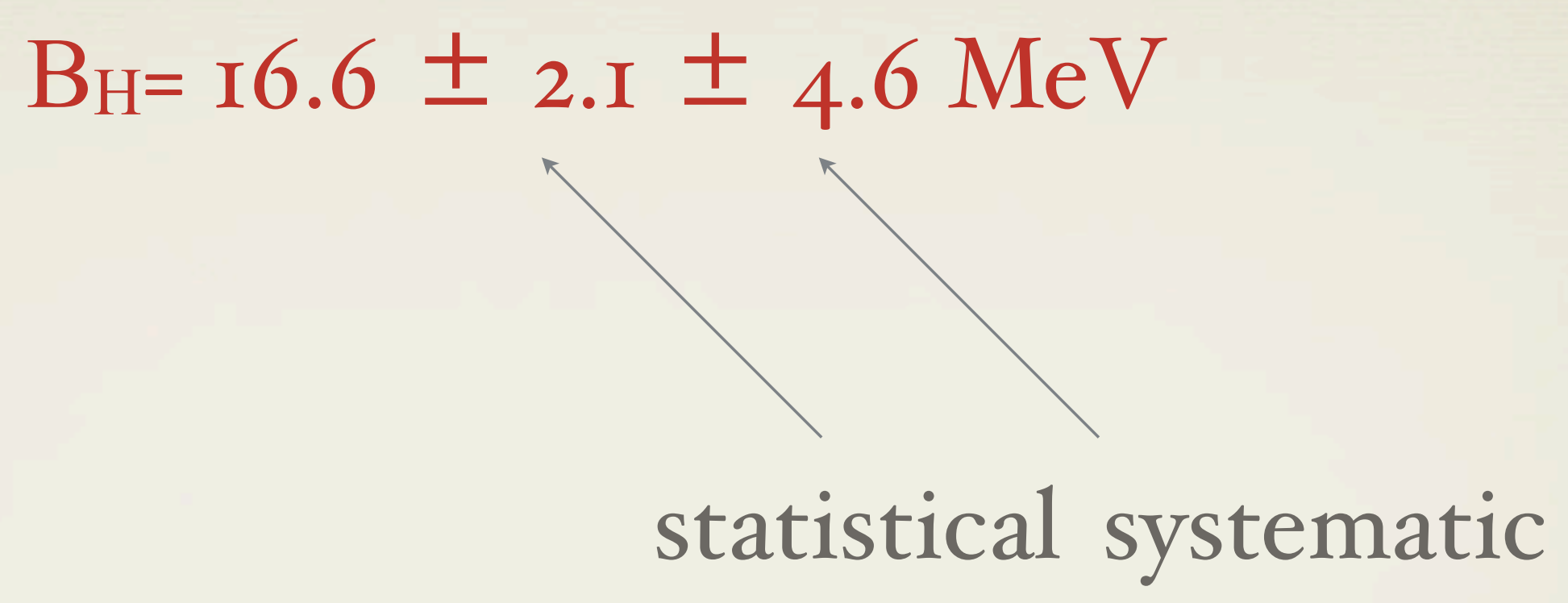

$\mathrm{M}_{\pi}=390 \mathrm{MeV}$

2+I Clover anisotropic fermions
NPLQCD: arXiv:1012.3812

Phys. Rev. Lett. 106, 162001

(Published April 20, 2011)
Continuum limit?

Physical pion mass?
Isospin breaking?

Electromagnetism? 


\section{Chiral extrapolation}

P. Shanahan et.al. arXiv:IIO6.285I

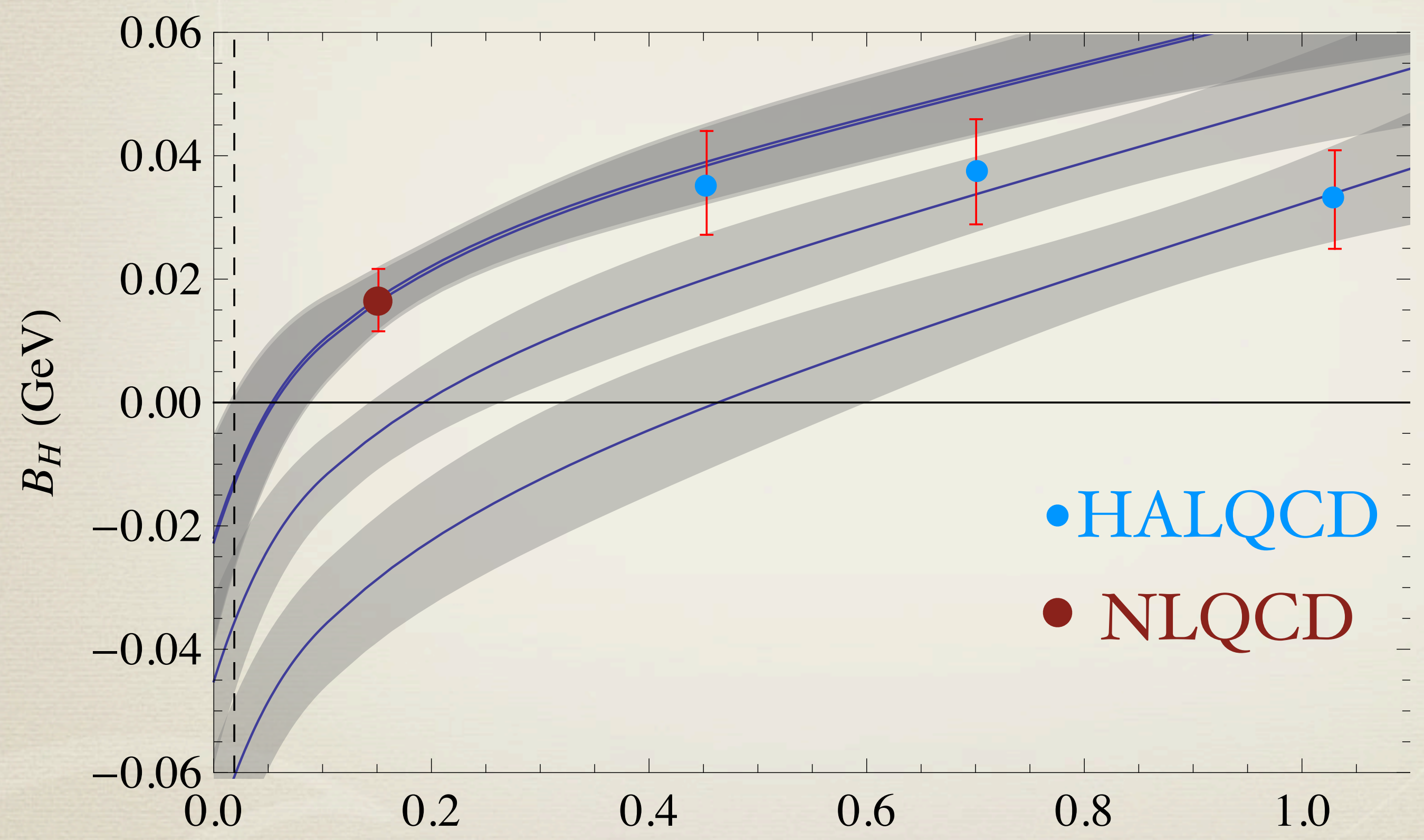

$m_{\pi}^{2}\left(\mathrm{GeV}^{2}\right)$

The H may not be bound 


\section{Chiral extrapolation}

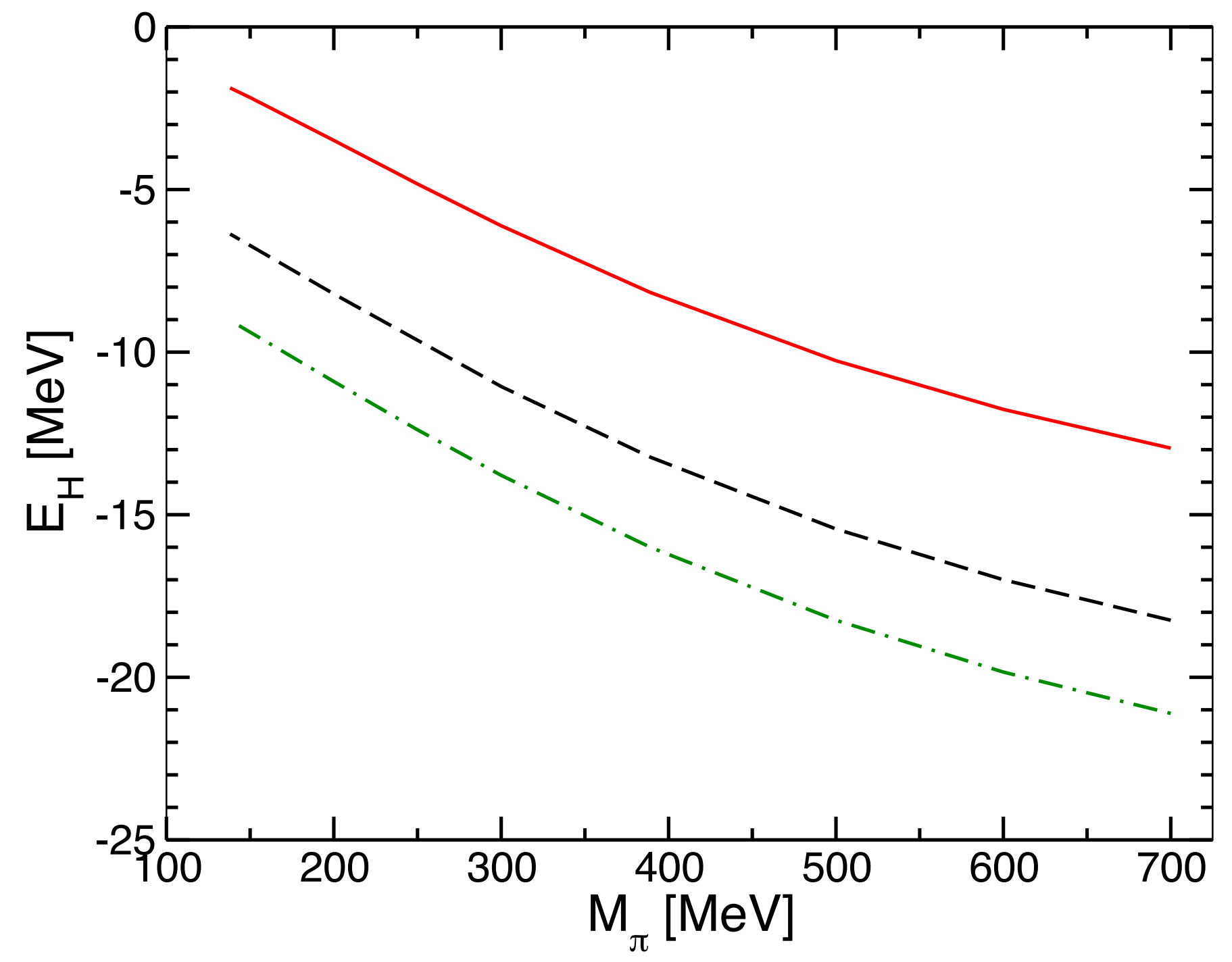

J. Haidenbauer, Ulf-G. Meisner arXiv:IIO9.3590 


\section{H-dibaryon: Towards the physical point}

[ S. Beane et.al. arXiv:1103.2821 Mod. Phys. Lett. A26: 2587, 2011]

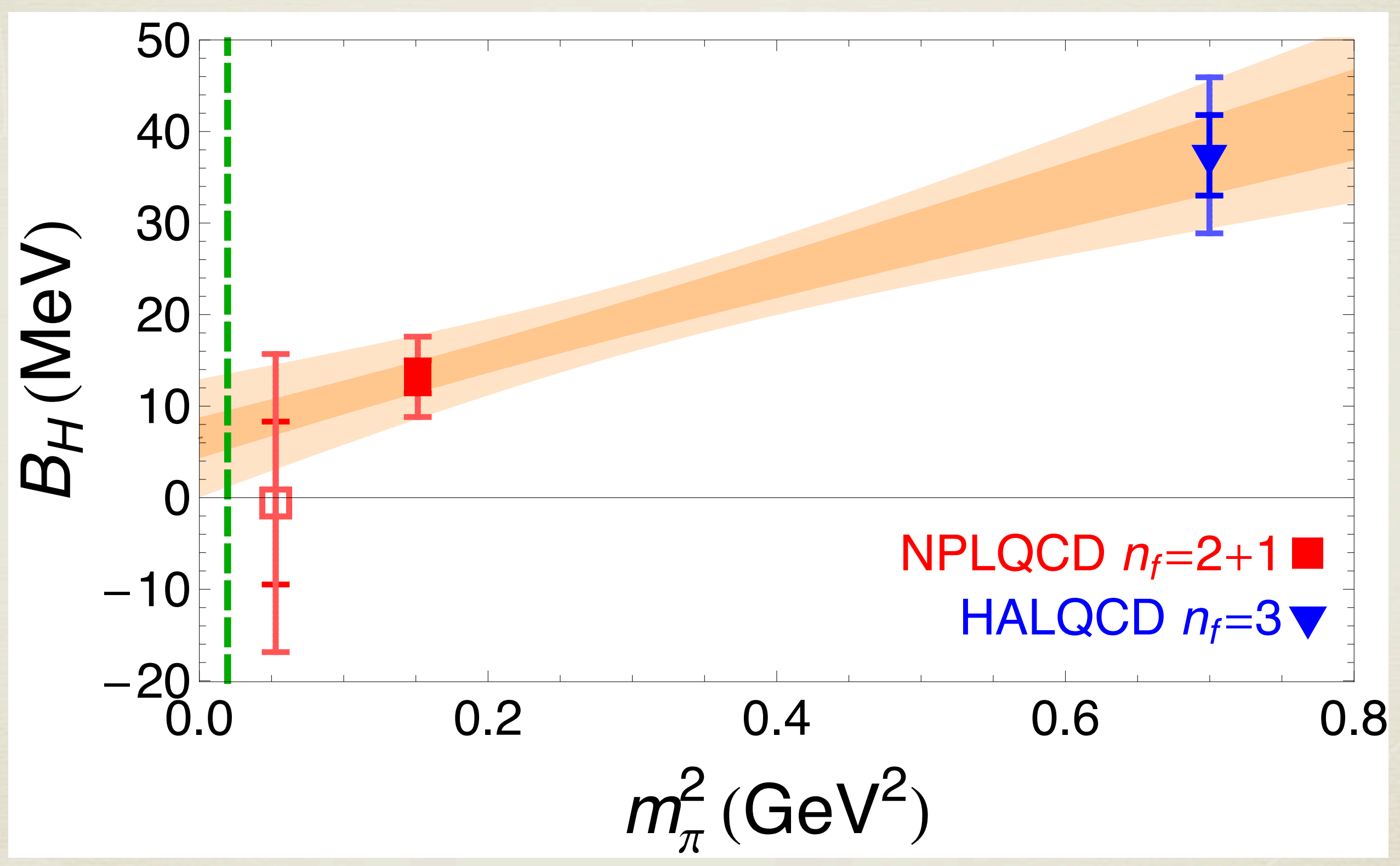

HALQCD:Phys.Rev.Lett. 106:162002,2011

H-dibaryon:

Is bound at heavy quark masses. May be unbound at the physical point 


\section{H-dibaryon: Towards the physical point}

[ S. Beane et.al. arXiv:1103.2821 Mod. Phys. Lett. A26: 2587, 2011]

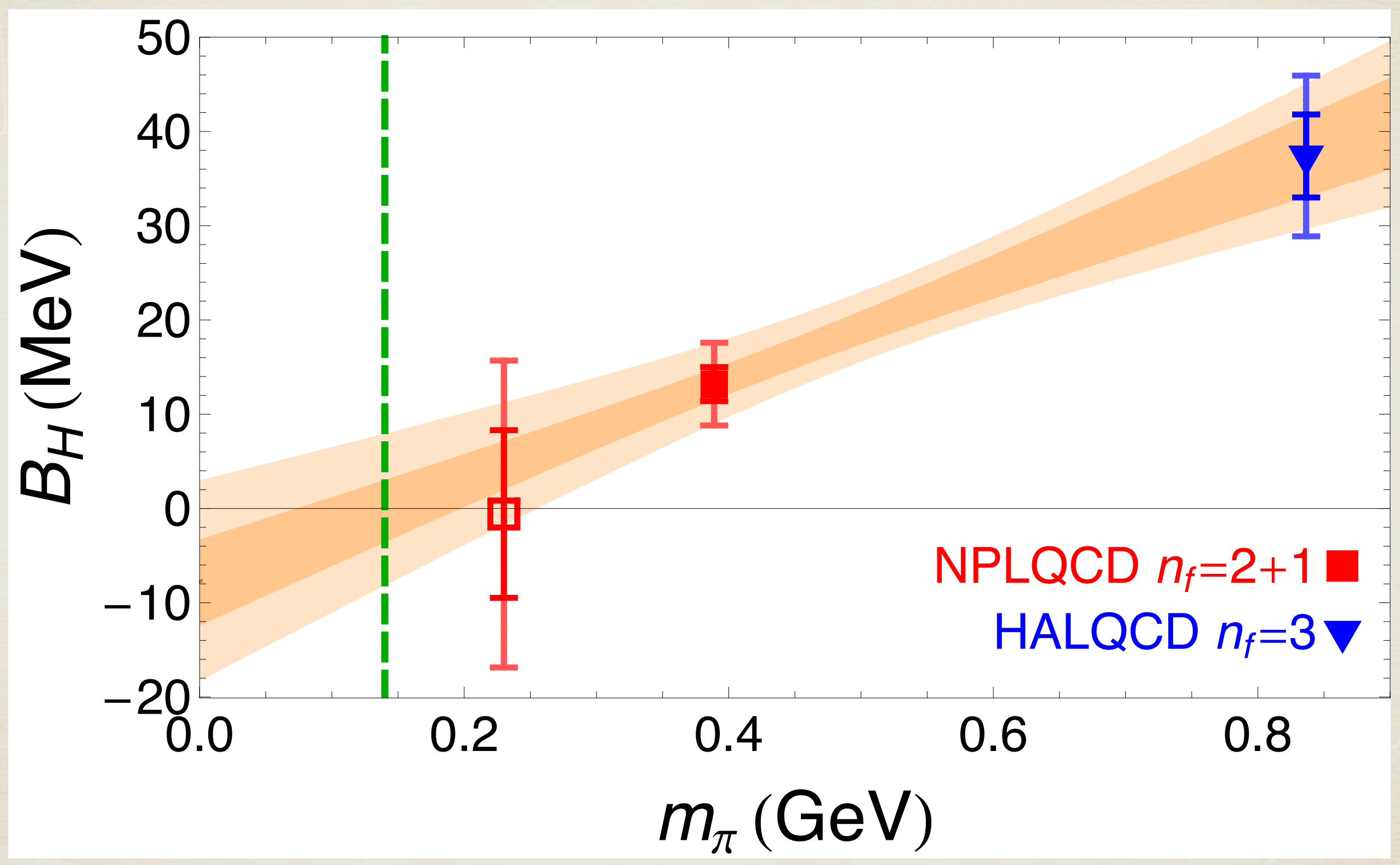

HALQCD:Phys.Rev.Lett.106:162002,2011

H-dibaryon:

Is bound at heavy quark masses. May be unbound at the physical point 


\section{Two baryon bound states}

[ S. Beane et.al. arXiv:IIo8.2889 submitted to Phys.Rev.D]
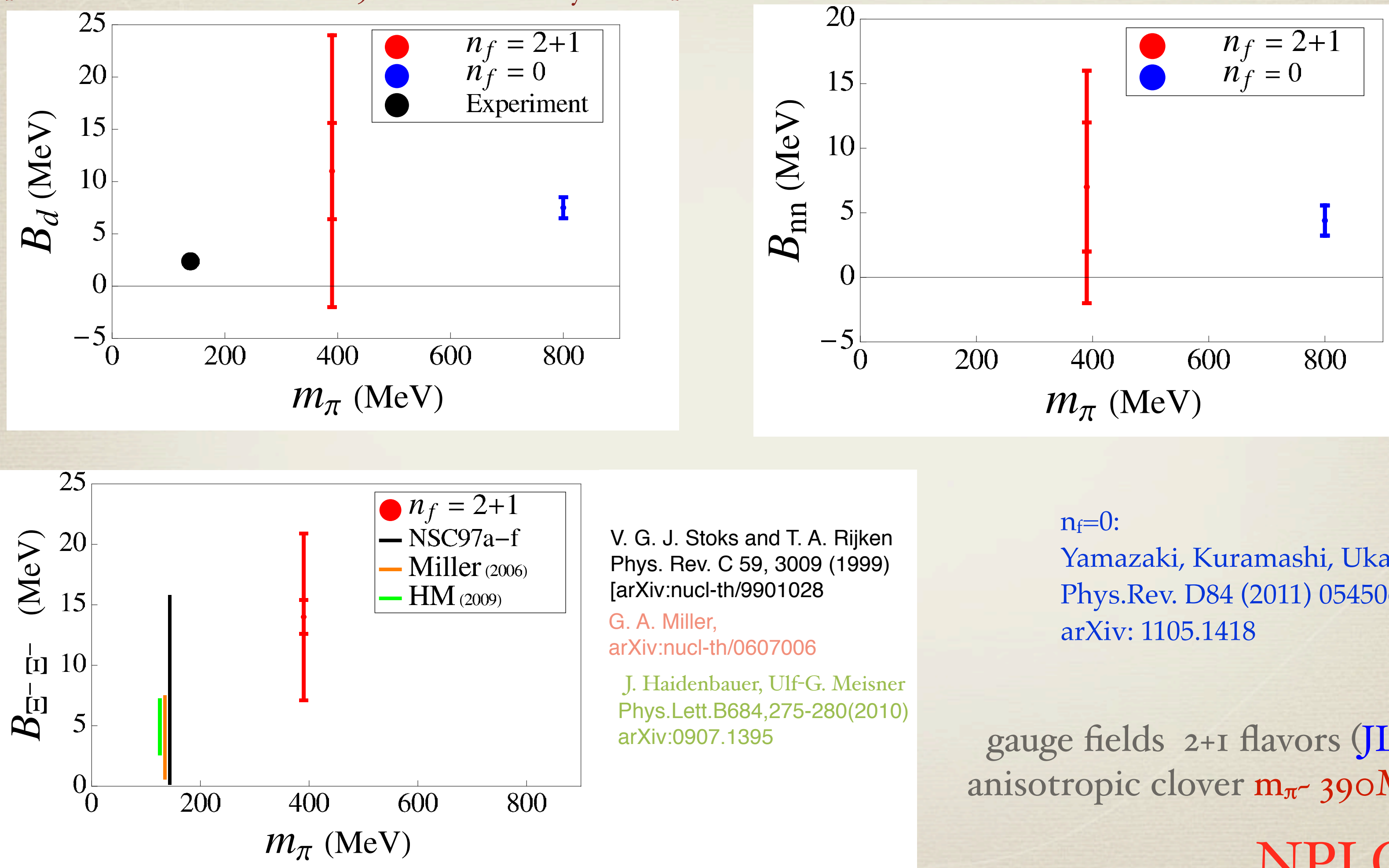

V. G. J. Stoks and T. A. Rijken Phys. Rev. C 59, 3009 (1999) [arXiv:nucl-th/9901028

G. A. Miller,

arXiv:nucl-th/0607006

J. Haidenbauer, Ulf-G. Meisner Phys.Lett.B684,275-280(2010) arXiv:0907.1395 $\mathrm{n}_{\mathrm{f}}=0$ :

Yamazaki, Kuramashi, Ukawa Phys.Rev. D84 (2011) 054506 arXiv: 1105.1418

gauge fields 2+I flavors (JLab) anisotropic clover $m_{\pi} 390 \mathrm{MeV}$ 


\section{Hyperon-Nucleon interactions}
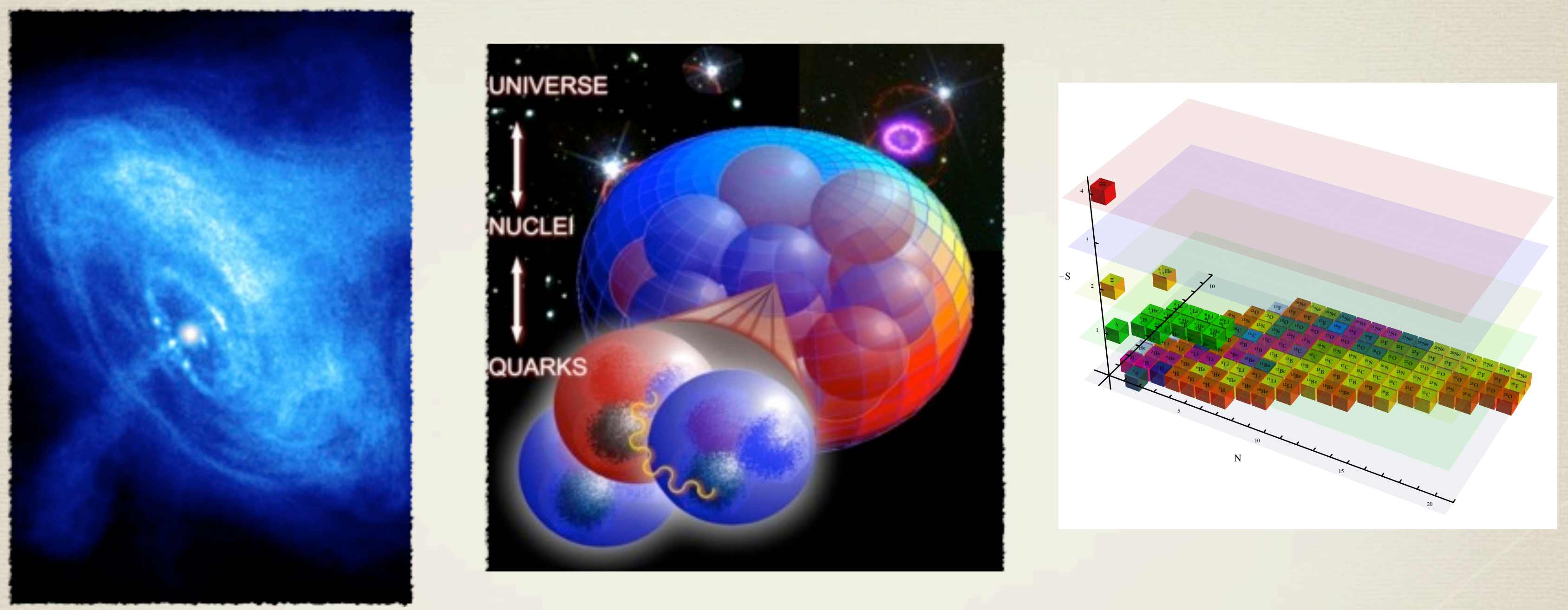

equation of state for nuclear matter in neutron stars

hyper-nuclear physics 


\section{$\mathrm{n} \Sigma^{-}$interactions}
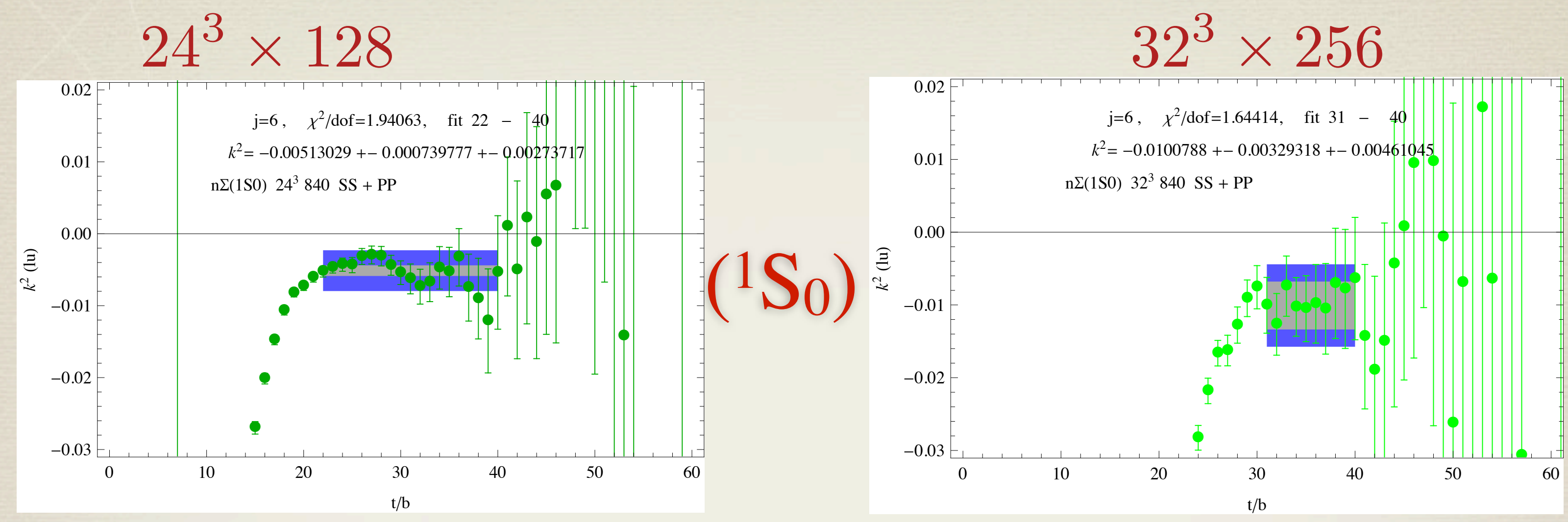

Finite volume scaling: $\kappa=\gamma+\frac{g_{1}}{L}\left(e^{-\gamma L}+\sqrt{2} e^{-\sqrt{2} \gamma L}\right)$

$$
B_{n \Sigma}=\frac{\gamma^{2}}{2 \mu_{n \Sigma}}=25 \pm 9.3 \pm 11 \mathrm{MeV}
$$




\title{
Hyperon-Nucleon
}

[ S. Beane et.al. arXiv:I204.3606 submitted to Phys. Rev. Lett.]

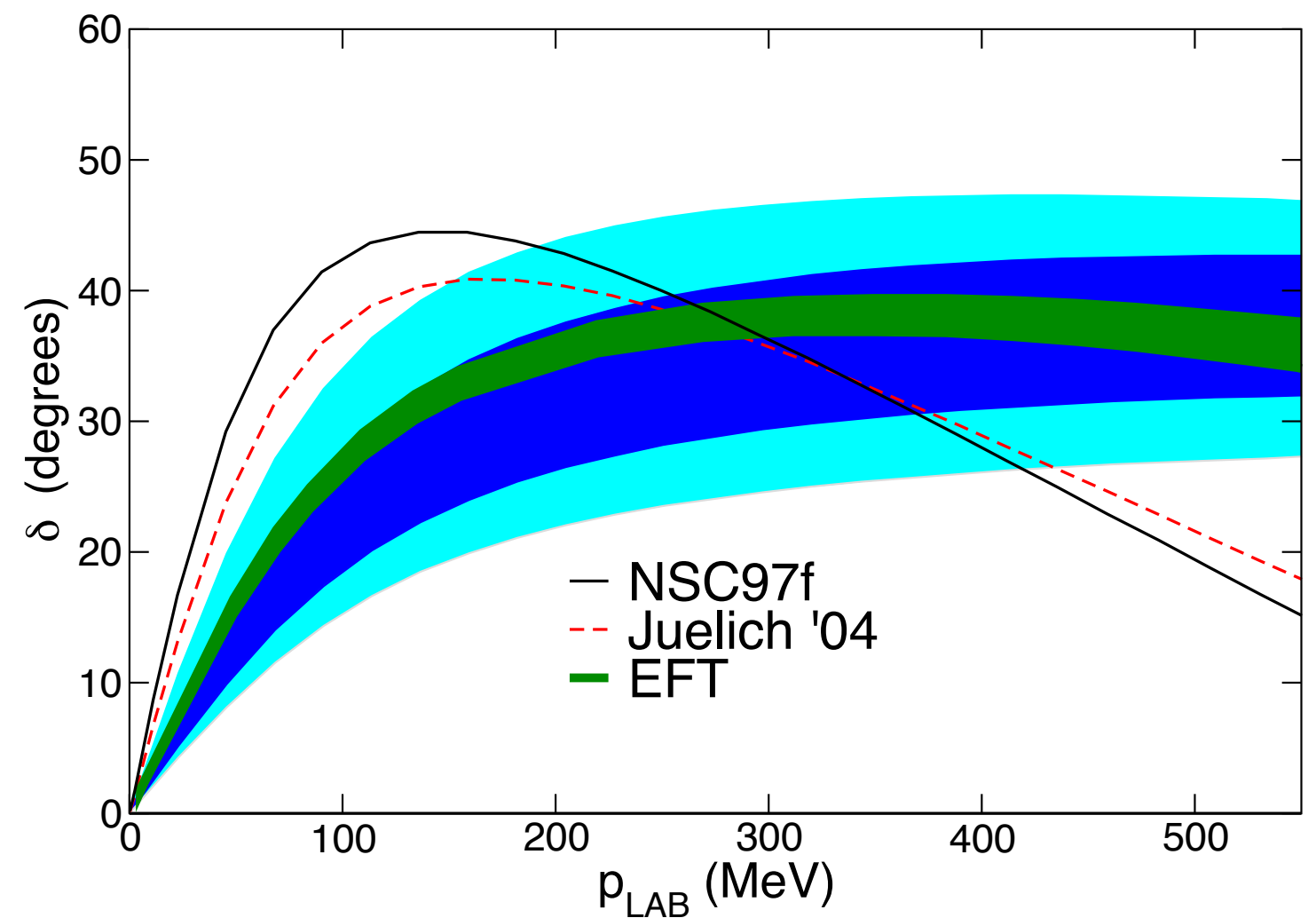

$\mathrm{n}-\sum$ spin singlet

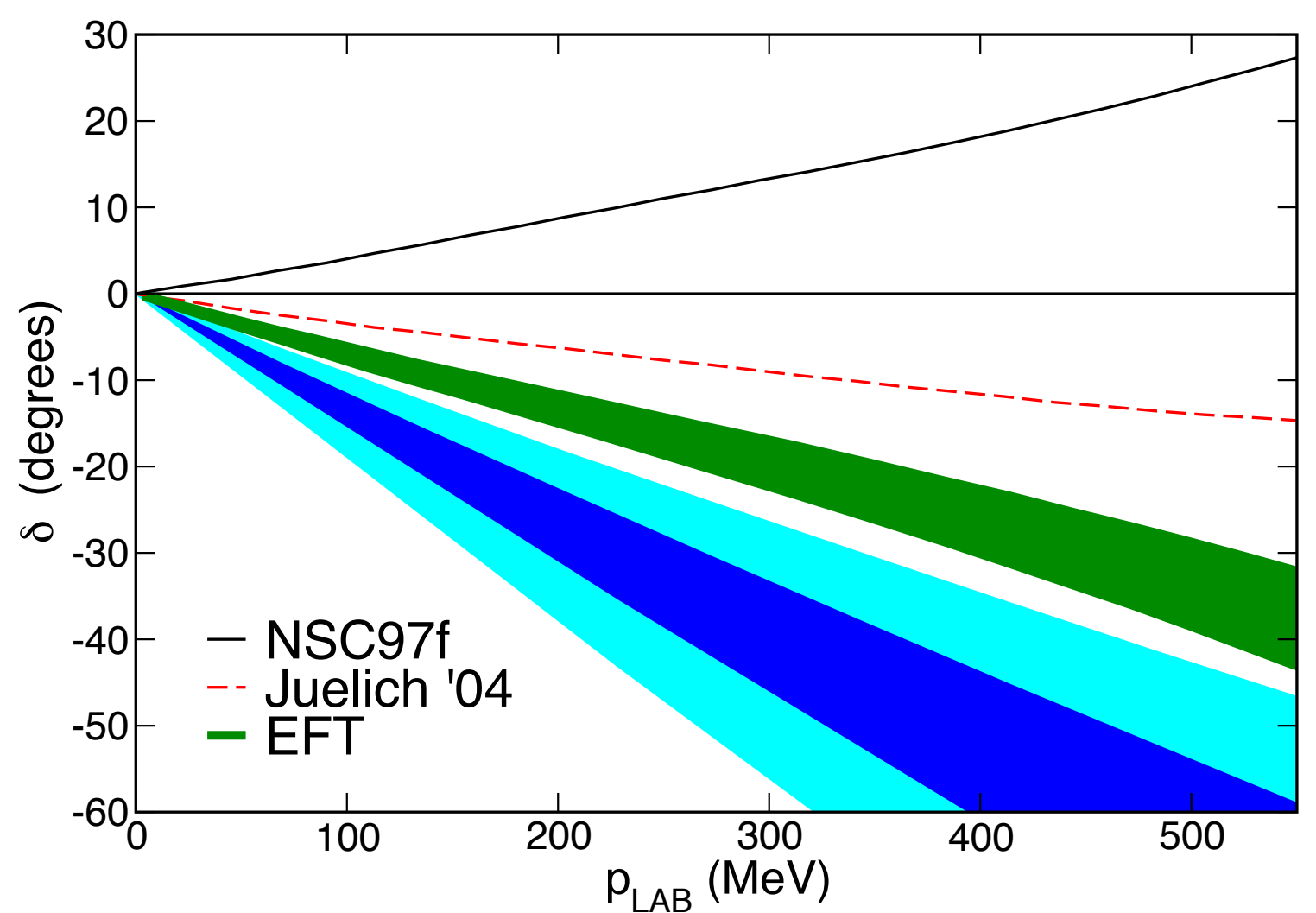

$\mathrm{n}-\sum$ spin triplet

\author{
gauge fields 2+I flavors (JLab) \\ anisotropic clover \\ $\mathrm{m}_{\pi \sim} 390 \mathrm{MeV}$
}




\section{Conclusions}

* Observables related to Hadronic interactions are now being computed

* Meson-Meson sector: Precision results already exist

* Progress in the Baryon sector

* Calculation of deuteron and He binding energy on the way

Yamazaki, Kuramashi, Ukawa (PACS-CS) Phys.Rev.D81:111504 (2010)

* Petaflop computing is here

Phys.Rev.D84:054506 (2011)

* Significant aid in achieving precision in the baryon sector

* Will take us a long way in understanding how the nuclear force emerges from QCD

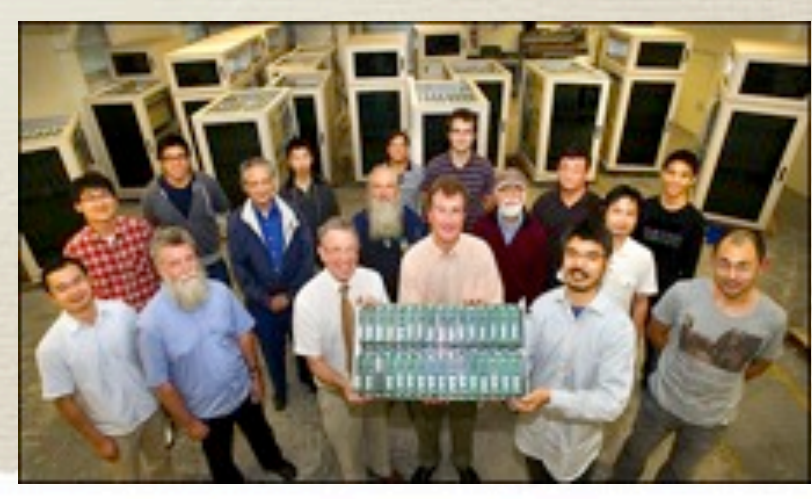




\section{Conclusions}

* Observables related to Hadronic interactions are now being computed

* Meson-Meson sector. Precision results alreadv exist

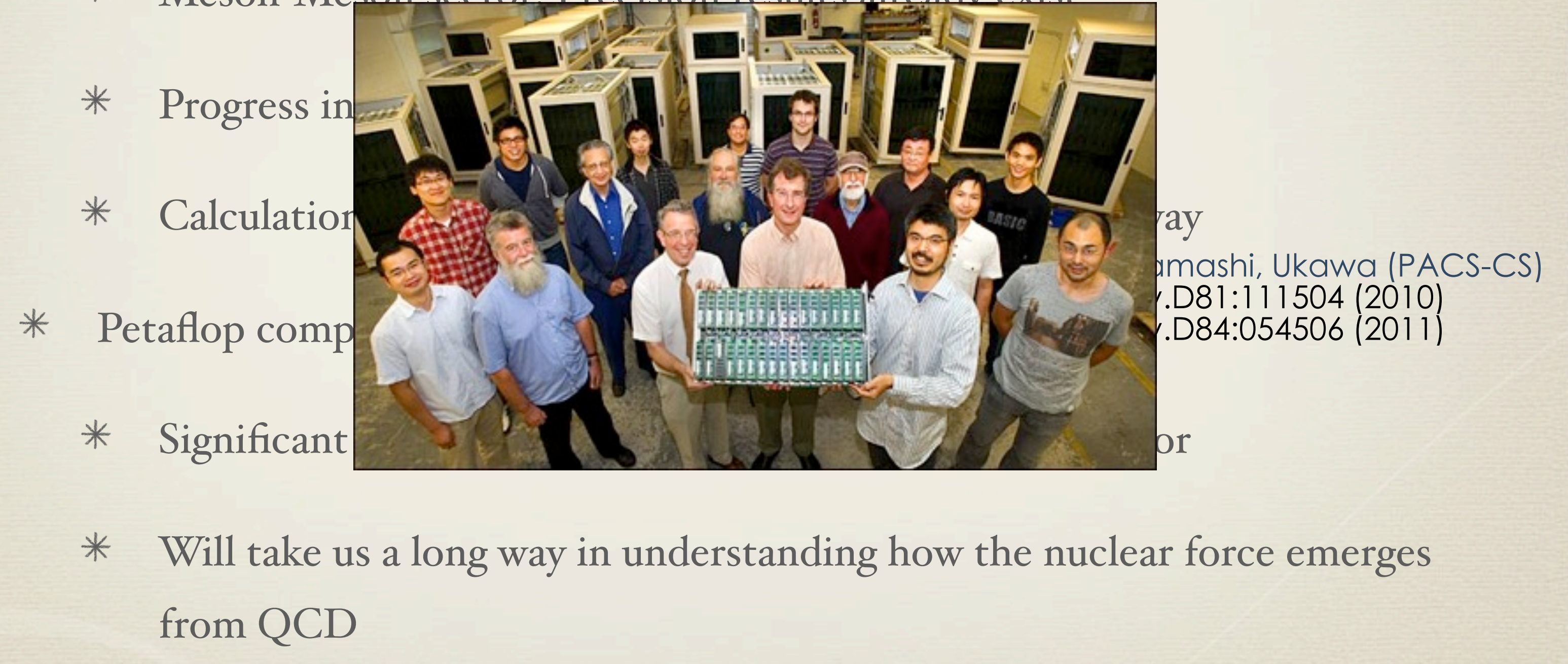




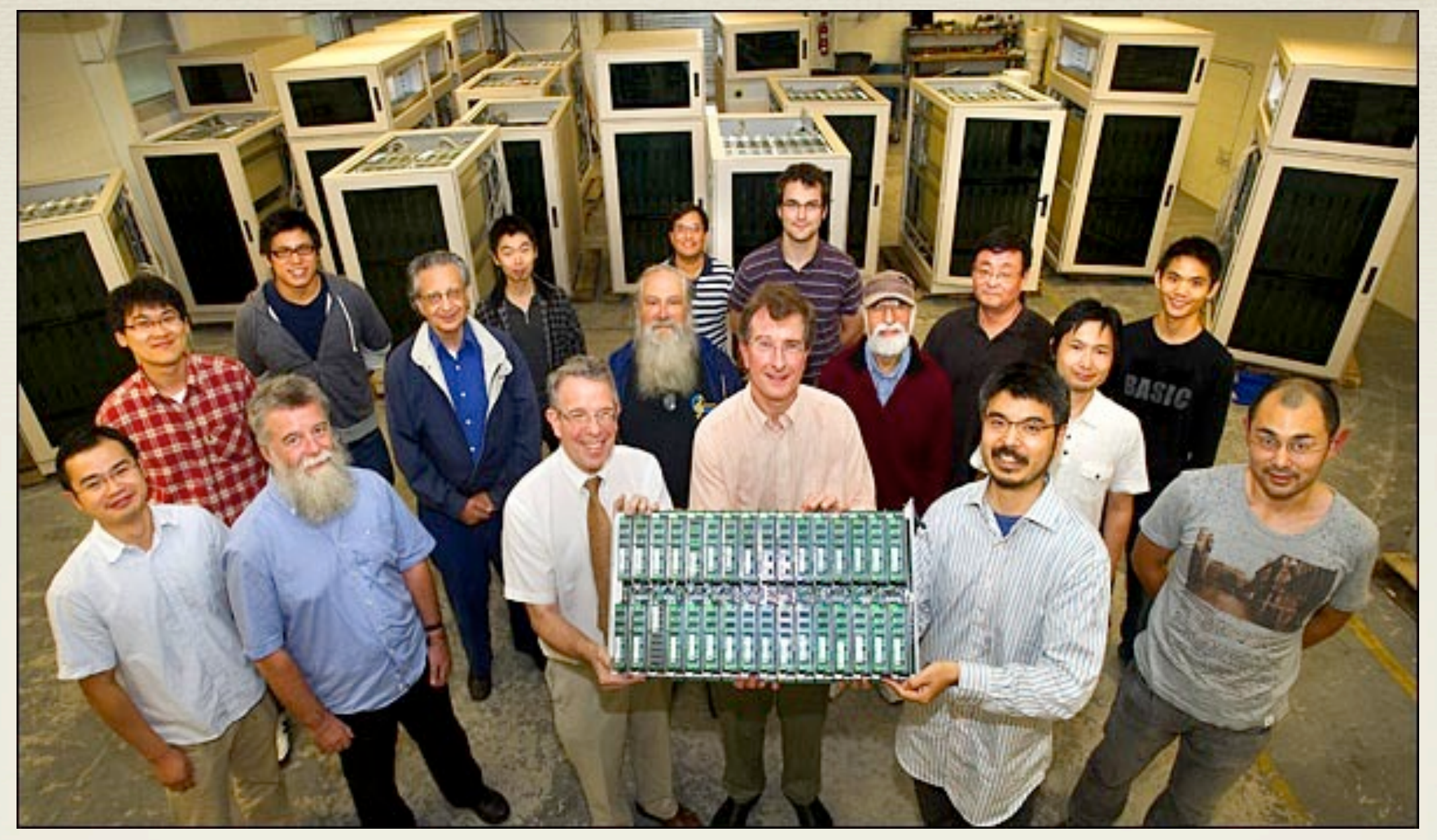




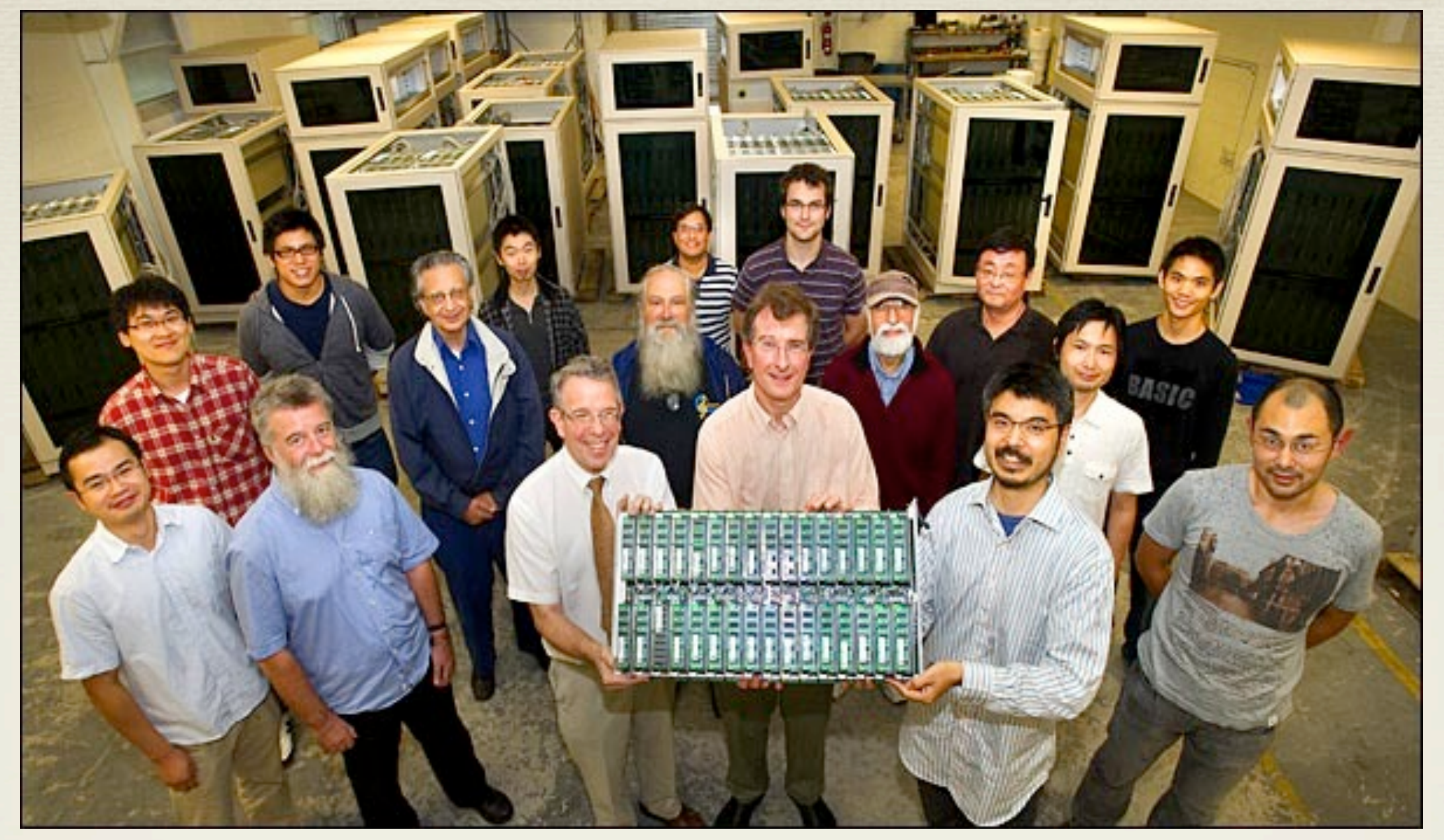

Оวளวठ

QCDCQ

9DADด 


\section{The anomaly triangle and muon $g-2$}

SANTI PERIS (SFSU and UAB) 


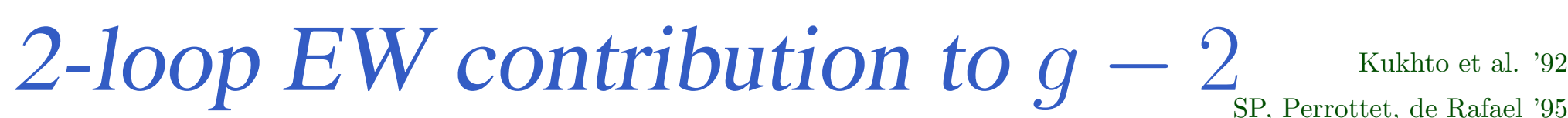

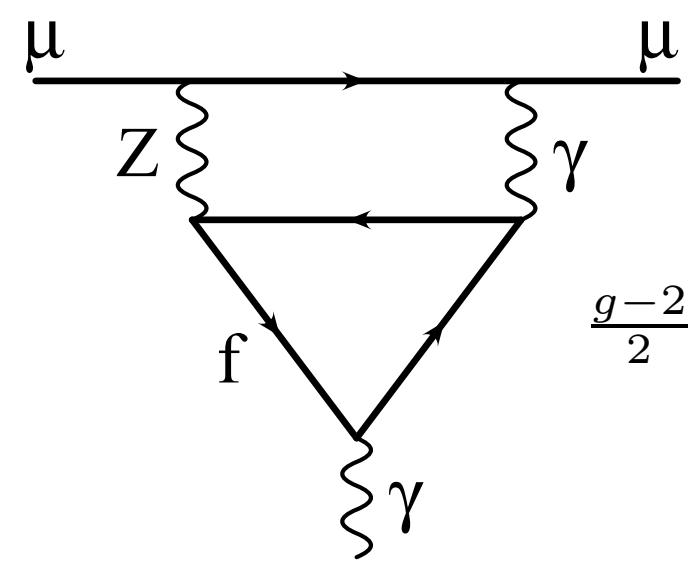

Czarnecki, Krause, Marciano '95, '96

Knecht, SP, Perrottet, de Rafael '02

Czarnecki, Marciano,Vainshtein '03

$\underbrace{\left[w_{L}\left(Q^{2}\right)+\frac{M_{Z}^{2}}{M_{Z}^{2}+Q^{2}} w_{T}\left(Q^{2}\right)\right]}_{w_{L}=2 w_{T}=2 \frac{N_{C}}{Q^{2}} \quad\left(\text { one-loop, } m_{f}=0\right)}$

$$
\left.\frac{g-2}{2}\right|_{e, u, d} \sim 2 \times 10^{-11}
$$

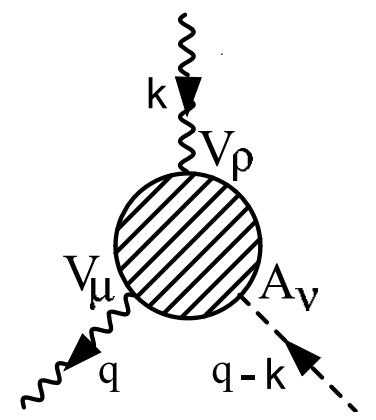

$$
Q^{2}=-q^{2}, \quad \text { "Gluon-irreducible" triangle }
$$

$$
\begin{aligned}
W_{\mu \nu \rho}(q, k)= & T_{f}^{(3)} Q_{f}^{2}\left[w_{L}\left(Q^{2}\right) q_{\nu} \epsilon_{\mu \rho \alpha \beta} q^{\alpha} k^{\beta}+\right. \\
& \left.w_{T}\left(Q^{2}\right) k^{\sigma}\left(q^{2} \epsilon_{\mu \nu \rho \sigma}+q_{\nu} \epsilon_{\mu \rho \lambda \sigma} q^{\lambda}+q_{\mu} \epsilon_{\rho \nu \lambda \sigma} q^{\lambda}\right)\right]+\mathcal{O}\left(k^{2}\right)
\end{aligned}
$$

- $w_{L}\left(Q^{2}\right)$ related to the chiral anomaly $\Longrightarrow \sum_{\nu, e, u, d} w_{L}=0$.

- $w_{T}\left(Q^{2}\right)$ not but... 


\section{Theorem}

In the massless limit, to all orders in $\alpha_{s}$ :

$$
w_{L}\left(Q^{2}\right)=2 w_{T}\left(Q^{2}\right)
$$

and, since anomaly does not get renormalized: $\quad w_{L}=2 \frac{N_{c}}{Q^{2}} \quad$ (Adler,Bardeen '69; Witten '83)

$\Rightarrow$ neither does $2 w_{T}=2 \frac{N_{c}}{Q^{2}}$, to all orders in $\alpha_{s}$.

Using $L_{\mu}^{(3)}=\sum_{\ell=\nu, e} \overline{\ell_{L}} \gamma_{\mu} T^{(3)} \ell_{L}+\sum_{q=u, d} \overline{q_{L}} \gamma_{\mu} T^{(3)} q_{L}$, etc...in $S U(2)_{L} \times U(1)_{Y}$ :

$Q^{2}\left[w_{L}\left(Q^{2}\right)-2 w_{T}\left(Q^{2}\right)\right] \propto \int d^{4} x d^{4} y e^{i q x}(y-x)_{\lambda} \epsilon^{\mu \nu \rho \lambda} \underbrace{\left\langle T\left\{L_{\mu}^{(3)}(x) V_{\nu}^{(Y)}(y) R_{\rho}^{(Y)}(0)\right\}\right\rangle}_{\nexists \mathbb{1}_{L} \otimes \mathbb{1}_{R}}$

- i.e., $w_{L}-2 w_{T}$ has no pert. contributions in $\alpha_{s}$, it is like, e.g., $\langle L R\rangle=\langle V V-A A\rangle$. 


\section{Non-perturbative effects}

1) Adler-Bardeen-Witten : $w_{L}\left(Q^{2}\right)=2 \frac{N_{c}}{Q^{2}} \quad$ (exact for all $Q$ !)

2) However, for $w_{T}\left(Q^{2}\right)$ :

- Large $Q^{2}$ :

$$
2 w_{T}\left(Q^{2}\right) \approx 2 \frac{N_{c}}{Q^{2}}\left(1+\underline{N O \alpha_{s}}\right)+(\text { const. }) \alpha_{s} \chi \frac{\langle\bar{\psi} \psi\rangle^{2}}{Q^{6}}+\mathcal{O}\left(1 / Q^{8}\right)
$$

Magnetic susceptibility, $\quad \chi=\frac{\Pi_{V T}(0)}{\langle\bar{\psi} \psi\rangle}$, very poorly known.

- Small $Q^{2}$ :

$$
2 w_{T}\left(Q^{2}\right) \approx(\text { const. }) C_{22}^{\left(p^{6}\right)}+\mathcal{O}\left(Q^{2}\right) \quad, \quad C_{22}^{\left(p^{6}\right)} \sim 1 / M_{\text {Hadron }}^{2} \quad \text { (unknown) }
$$

Chiral Pert. Theory, $\mathcal{L}_{\text {eff }}$ (parity-odd):

$$
\mathcal{L}_{\mathcal{O}\left(p^{6}\right)}=C_{22}^{\left(p^{6}\right)} \epsilon_{\mu \nu \alpha \beta} \underbrace{\operatorname{Tr}\left(u^{\mu}\left\{\nabla_{\gamma} f_{+}^{\gamma \nu}, f_{+}^{\alpha \beta}\right\}\right)}_{\pi, \eta, \ldots}+\ldots \quad\left(S U_{N_{F}} \times S U_{N_{F}} \rightarrow S U_{N_{F}}\right)
$$




\section{Conjectures}

- $\underline{\text { Conjecture 1: }} \quad w_{L}\left(Q^{2}\right)-2 w_{T}\left(Q^{2}\right)=-2 \frac{N c}{f_{\pi}^{2}} \Pi_{L R}\left(Q^{2}\right) \quad$ (Son-Yamamoto '10)

in wide class of "AdS/QCD" models (chiral limit, $N_{c} \rightarrow \infty$ )

(not without caveats, e.g. OPE is exponential; mismatch in pert. theory if treated as a WI)

( Knecht, SP, de Rafael '11)

Chiral log's respect this relation. (Gorksy, Kopnin,Krikun, Vainshtein '12)

Test: $\quad C_{22}^{\left(p^{6}\right)}(\mu)=-\frac{N_{c}}{32 \pi^{2} f_{\pi}^{2}} L_{10}^{\left(p^{4}\right)}(\mu) \quad$ ??

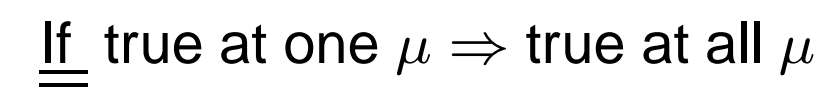

- Conjecture 2: $\quad \chi=-\frac{N_{c}}{4 \pi^{2} f_{\pi}^{2}} \sim-9 \mathrm{GeV}^{-2} \quad$ (Magnetic susceptibility) ??

(Vainshtein '02):

Other results: $\chi \sim-3 \mathrm{GeV}^{-2}$, sum rules, VMD, (Ioffe, Fadin, Lipatov '10; Balitsky et al. '85; Belyaev et al. '84; Ball et al. '02) 


\section{Another Perturbative Surprise}

Up to now, special kinematic configuration in $\langle V V A\rangle$.

However, it has been found at two loops for all momenta that :

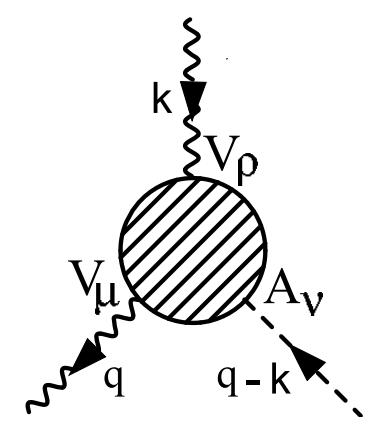

$$
W_{\mu \nu \rho}(q, k)=\left.W_{\mu \nu \rho}(q, k)\right|_{\text {one-loop }}(1+\underbrace{\mathcal{O}\left(\alpha_{s}\right)}_{=0 ! !})
$$

i.e., no renormalization, not just for the anomaly, but for the whole triangle !

Given the non-trivial momentum dependence,

can this be just a coincidence ?

could this be true to all orders in $\alpha_{s}$ ? 


\section{Summary}

- VVA is a very interesting theoretical laboratory for QCD

- Most results obtained in chiral limit: can lattice help/check ?

- The LbL $\longleftrightarrow\langle V V A\rangle$ connection: $k_{1} \approx k_{2} \gg k_{3}$

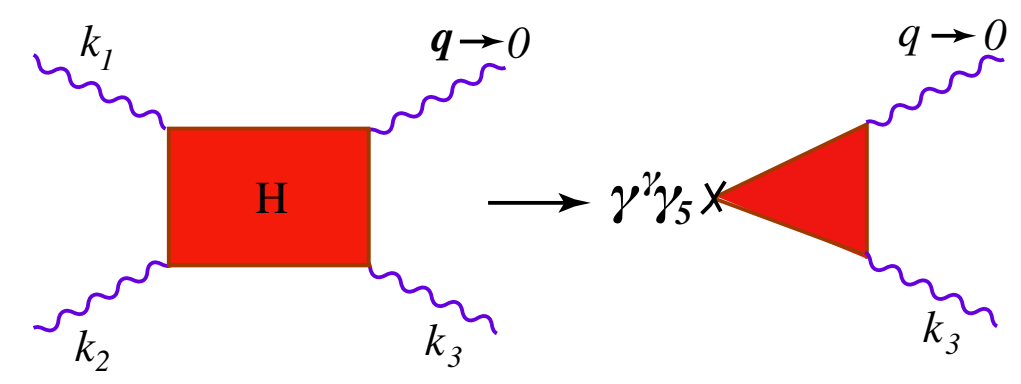




\title{
Last year's good news: the lattice can do it!
}

\section{Two-Flavor QCD Correction to Lepton Magnetic Moments at Leading Order in the Electromagnetic Coupling}

\author{
Xu Feng, ${ }^{1,2, *}$ Karl Jansen, ${ }^{1}$ Marcus Petschlies, ${ }^{3}$ and Dru B. Renner ${ }^{1, \dagger}$ \\ ${ }^{1}$ NIC, DESY, Platanenallee 6, D-15738 Zeuthen, Germany \\ ${ }^{2}$ Universität Münster, Institut für Theoretische Physik, Wilhelm-Klemm-Strasse 9, D-48149, Germany \\ ${ }^{3}$ Institut für Physik, Humboldt-Universität zu Berlin, D-12489, Berlin, Germany \\ (Received 28 March 2011; published 17 August 2011)
}

We present a reliable nonperturbative calculation of the QCD correction, at leading order in the electromagnetic coupling, to the anomalous magnetic moment of the electron, muon, and tau leptons using two-flavor lattice QCD. We use multiple lattice spacings, multiple volumes, and a broad range of quark masses to control the continuum, infinite-volume, and chiral limits. We examine the impact of the commonly ignored disconnected diagrams and introduce a modification to the previously used method that results in a well-controlled lattice calculation. We obtain $1.513(43) \times 10^{-12}, 5.72(16) \times 10^{-8}$, and $2.650(54) \times 10^{-6}$ for the leading-order two-flavor QCD correction to the anomalous magnetic moment of the electron, muon, and tau, respectively, each accurate to better than $3 \%$.

DOI: 10.1103/PhysRevLett.107.081802

PACS numbers: 13.40.Em, 12.38.Gc, 14.60.Ef 


\section{In the beginning there was Dirac}

\section{$i\left(\partial_{\mu}-i e A_{\mu}(x)\right) \gamma^{\mu} \psi(x)=m \psi(x)$}

predicted electron magnetic moment

$$
\begin{aligned}
\vec{\mu} & =g\left(\frac{Q e}{2 m}\right) \vec{s}, \quad e>0 \\
g & \equiv 2
\end{aligned}
$$

However, experimentally $\mathbf{g}>2$; need to add a Pauli term Qe $4 m$

$$
a \bar{\psi}(x) F_{\mu \nu}(x) \sigma^{\mu \nu} \psi(x)
$$

dimension 5 operator where $a$ is the anomaly,

$$
g=2(1+a)
$$

(only from loops) 


\section{In the QED, a becomes an expansion in $(\alpha / \pi)$ from loops}

New Physics contribution to a at some scale $\Lambda$

$$
a(\text { NewPhysics })=C\left(\frac{m}{\wedge}\right)^{2}
$$

where $\mathbf{C}$ could be $\mathcal{O}(1)$, or 


\section{Magnetic and Electric Dipole Interactions}

$$
\begin{gathered}
\Gamma_{\beta}=e F_{1} \bar{\psi}_{R} \gamma_{\beta} \psi_{R}+\frac{i e}{2 m} F_{2} \bar{\psi}_{R} \sigma_{\beta \delta} q^{\delta} \psi_{L} \\
+H C
\end{gathered}
$$

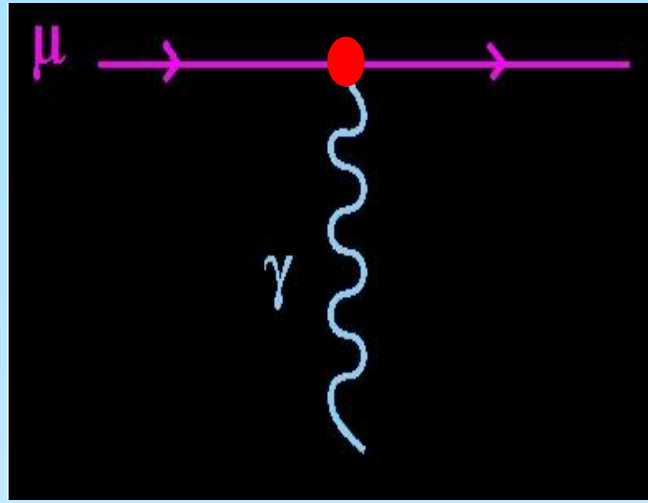

- Muon Magnetic Dipole Momoment $\mathrm{a}_{\mu}$ chiral changing

$$
\begin{gathered}
\bar{u}_{\mu}\left[e F_{1}\left(q^{2}\right) \gamma_{\beta}+\frac{i e}{2 m_{\mu}} F_{2}\left(q^{2}\right) \sigma_{\beta \delta} q^{\delta}\right] u_{\mu} \\
F_{1}(0)=1 \quad F_{2}(0)=a_{\mu}
\end{gathered}
$$

- Muon EDM

$$
\begin{gathered}
\bar{u}_{\mu}\left[\frac{i e}{2 m_{\mu}} F_{2}\left(q^{2}\right)-F_{3}\left(q^{2}\right) \gamma_{5}\right] \sigma_{\beta \delta} q^{\delta} u_{\mu} \\
F_{2}(0)=a_{\mu} \quad F_{3}(0)=d_{\mu} ; \text { EDM }
\end{gathered}
$$


$\mathrm{a}_{\mu}{ }^{\text {had }}($ LO) Analyticity + Optical Theorem $a_{\mu}(\mathrm{had})=\left(\frac{\alpha m_{\mu}}{3 \pi}\right)^{2} \int_{4 m_{\pi}^{2}}^{\infty} \frac{d s}{s^{2}} K(s) \quad\left(\frac{\sigma\left(e^{+} e^{-} \rightarrow \text { hadrons }\right)}{\sigma\left(e^{+} e^{-} \rightarrow \mu^{+} \mu^{-}\right)}\right)$
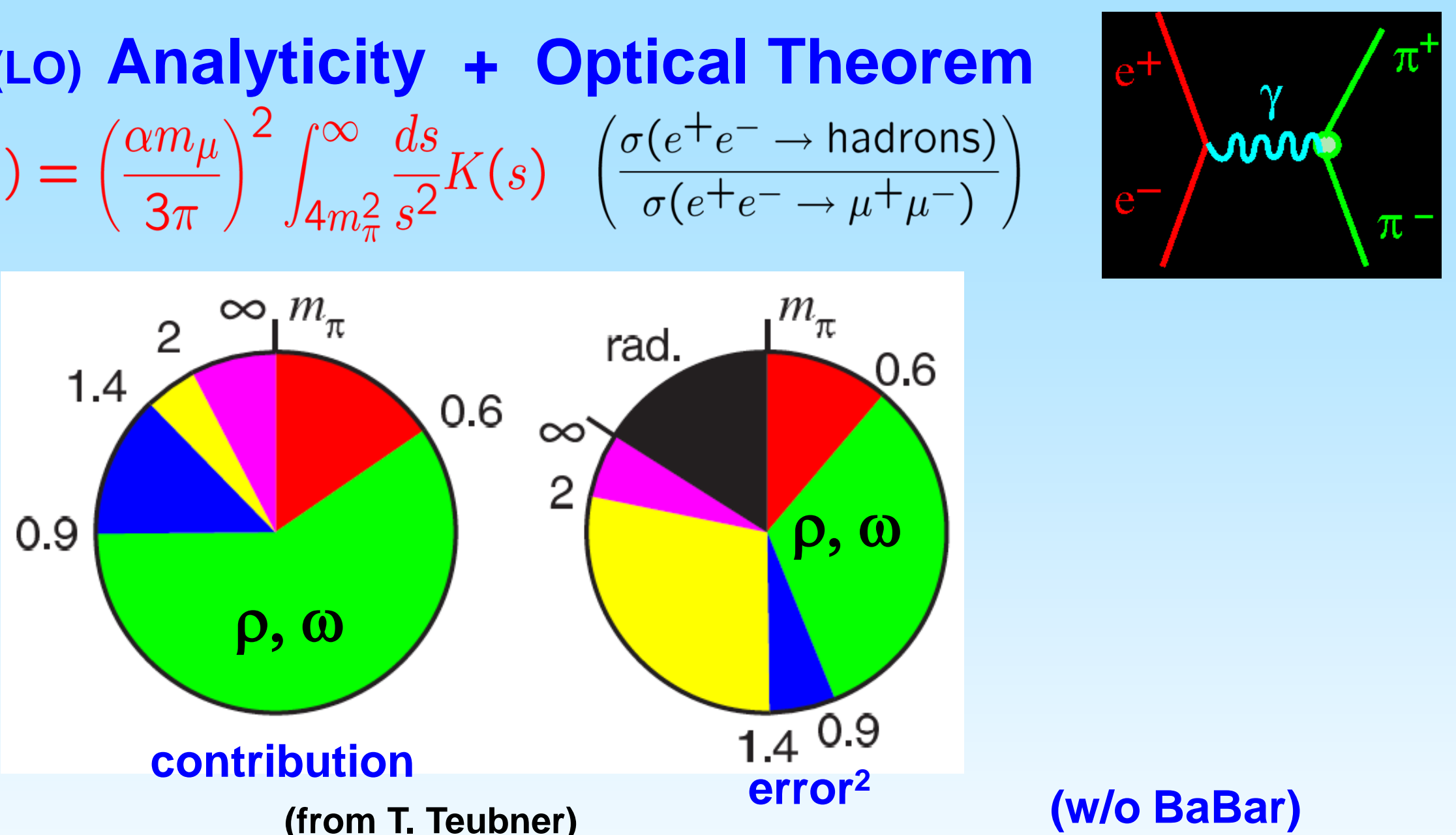

- Future efforts will reduce errors

- CMD3 at VEPP2000, up to $2.0 \mathrm{GeV}$ (next 5 years)

- perhaps Belle 


\section{Measured Cross section for $\mathrm{e}^{+} \mathrm{e}^{-} \rightarrow \pi^{+} \pi^{-}$}
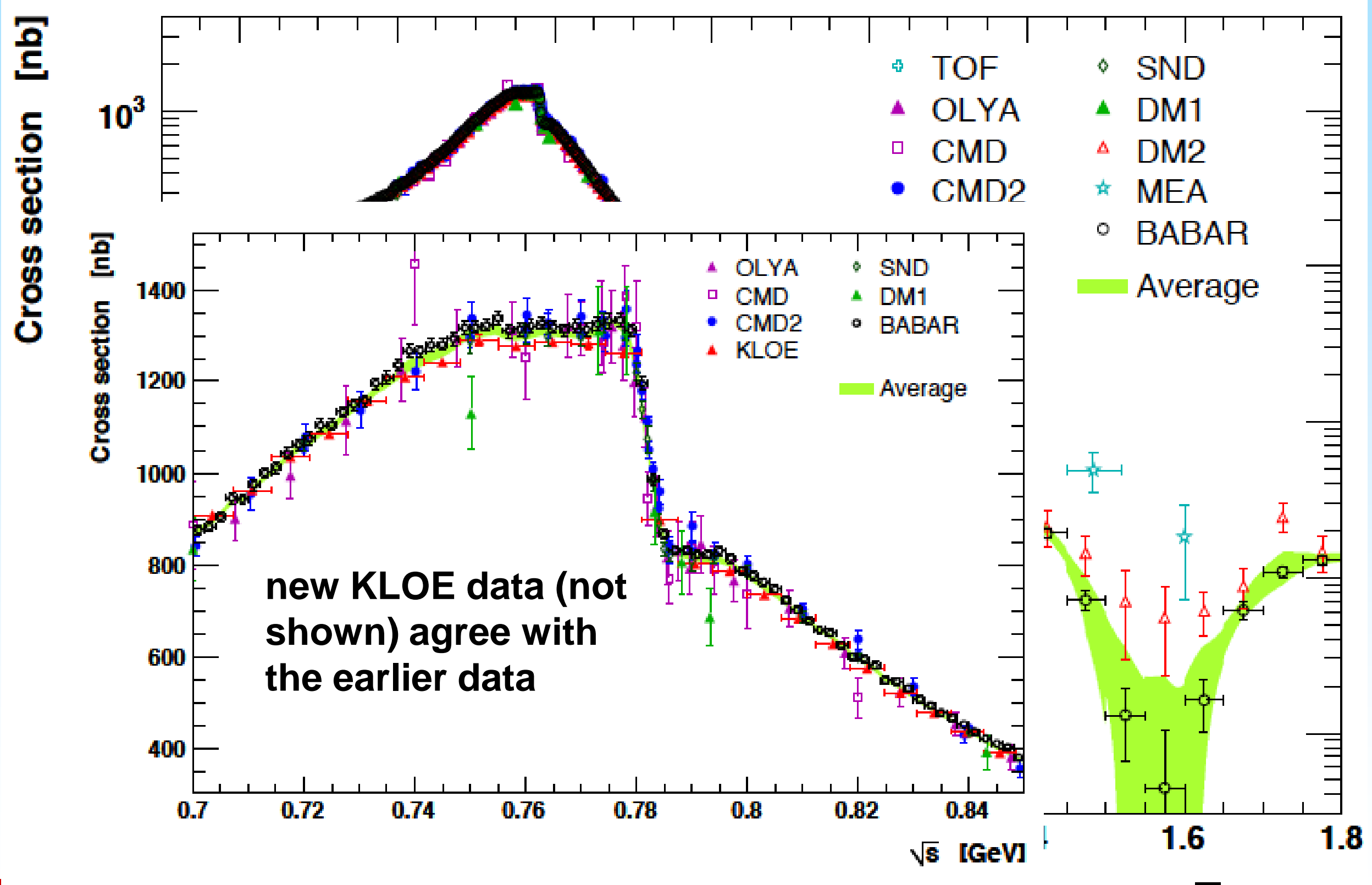

$\sqrt{5}[G e V]$ 


\section{The SM Value for $a_{\mu}$ from $\mathrm{e}^{+} \mathrm{e}^{-} \rightarrow$ hadrons (Updated 9/09)}

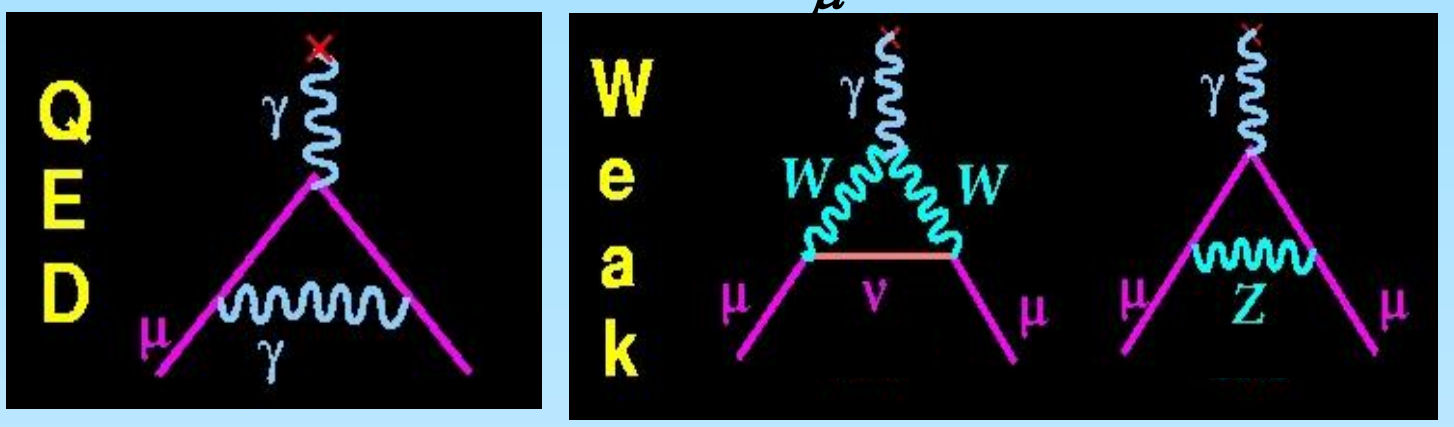

well known

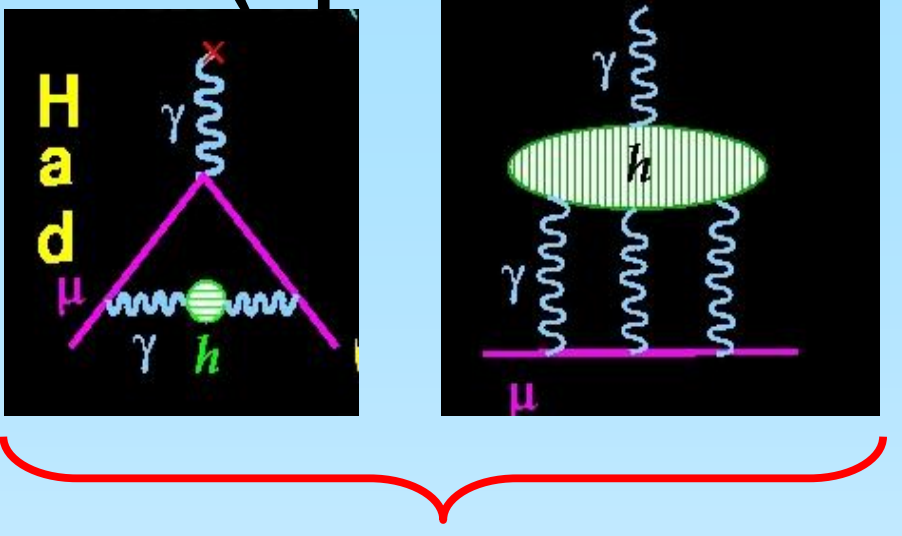

significant work ongoing

\begin{tabular}{|c|c|}
\hline & VALUE $\left(\times 10^{-11}\right)$ UNITS \\
\hline $\mathrm{QED}(\gamma+\ell)$ & $116584718.09 \pm 0.14 \pm 0.04_{\alpha}$ \\
\hline $\mathrm{HVP}(\mathrm{lo})$ & w. BaBar, no KLOE LA $\quad 6923 \pm 42$ \\
\hline $\mathrm{HVP}(\mathrm{ho})$ & $-97.9 \pm 0.9$ \\
\hline $\mathrm{EW}$ & \\
\hline Total SM & $116591802 \pm 42_{\mathrm{H}-\mathrm{LO}} \pm 26_{\mathrm{H}-\mathrm{HO}} \pm 2_{\mathrm{other}}\left( \pm 49_{\text {tot }}\right)$ \\
\hline
\end{tabular}




\section{I will return the the theory issue soon, but first a word about the experimental value}

- I'll give the cartoon version of the experiment for theorists 
We measure the difference frequency between the spin and momentum precession

$\omega_{a}=\omega_{S}-\omega_{C}=\left(\frac{g-2}{2}\right) \frac{Q e B}{m} B \Rightarrow\langle B\rangle_{\mu-\text { dist }}$

With an electric quadrupole field for vertical focusing without vertical focussing

$$
\vec{\omega}_{a}=-\frac{Q e}{m}\left[a_{\mu} \vec{B}-\left(\frac{\overrightarrow{\omega_{\mu}}-1}{c}\right]\right.
$$
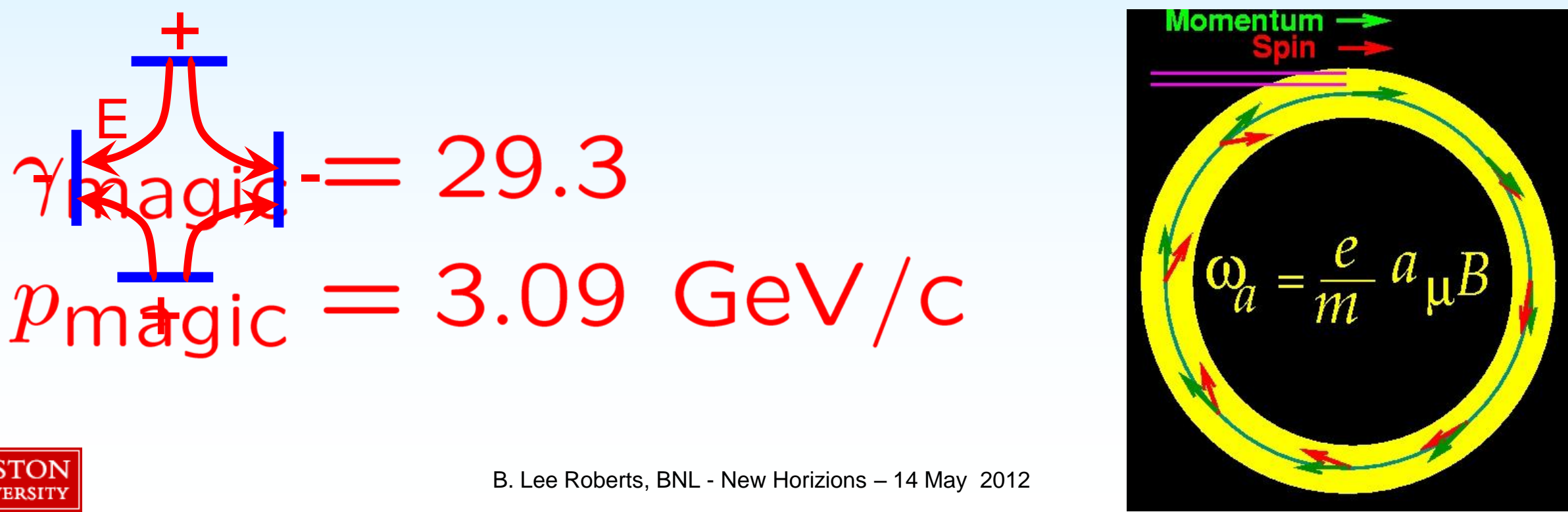


\section{Experimental Technique}

25ns bunch of $5 \times 10^{12}$ protons from AGS

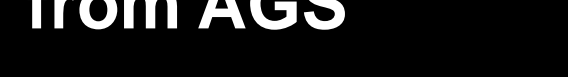

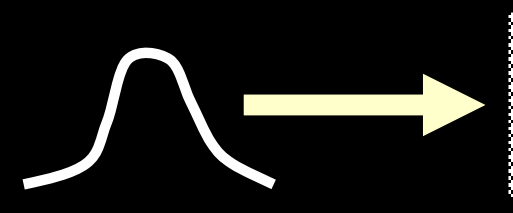

Target

- Muon polarization

- Muon storage ring

- injection \& kicking

- focus with Electric Quadrupoles

- 24 electron calorimeters

$\vec{\omega}_{a}=-\frac{e}{m} a_{\mu} \vec{B}$

Electric Quadrupoles

$$
\begin{aligned}
& x_{c} \approx 77 \mathrm{~mm} \\
& \beta \approx 10 \mathrm{mrad}
\end{aligned}
$$

B. dl $\approx 0.1 \mathrm{Tm}$ 
e PRL 100, 120801 (2008 $\quad \boldsymbol{\mu}$ PRL, 92, 161802 (2004)
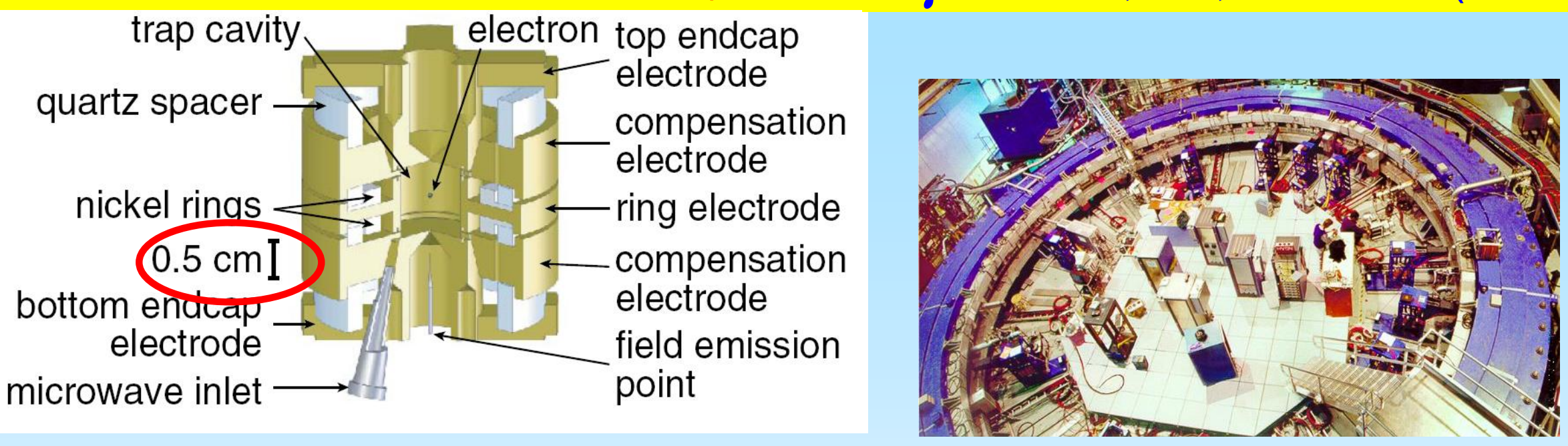

$$
\begin{aligned}
& a_{\mu}=(116592089 \pm 63) \times 10^{-11}(0.54 \mathrm{ppm}) \\
& a_{e}=(115965218073 \pm 28) \times 10^{-14}(0.24 \mathrm{ppb}) \\
& \begin{array}{l}
\text { muon more sensitive to } \\
\text { heavier physics by }
\end{array} \sim\left(\frac{m_{\mu}}{m_{e}}\right)^{2} \simeq 42,000
\end{aligned}
$$

and interpretation of the electron anomaly limited by precision of independent measurements of $\alpha, \sim 4.5 \mathrm{ppb}$. 


\section{To determine $\mathrm{a}_{\mu}$ we measure two numbers:}

The muon spin frequency: $\omega_{a}$

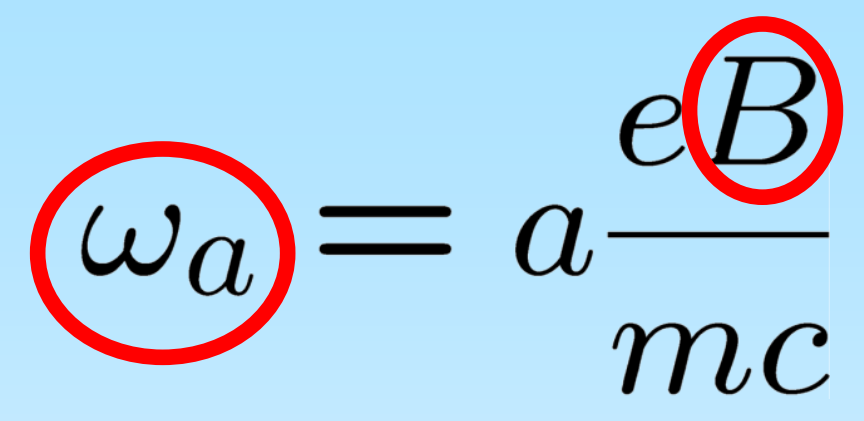

The magnetic field normalized to the

Larmor frequency of the free proton: $\omega_{p}$

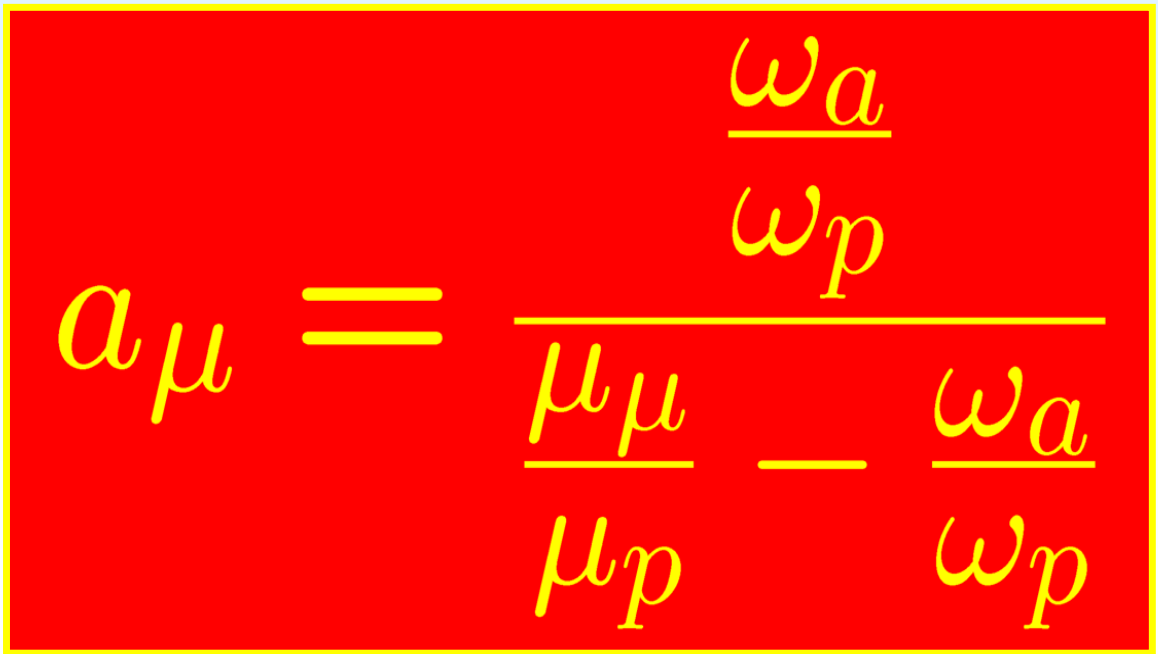


To measure $\omega_{a}$ we used $\mathrm{Pb}$-scintillating fiber calorimeters.
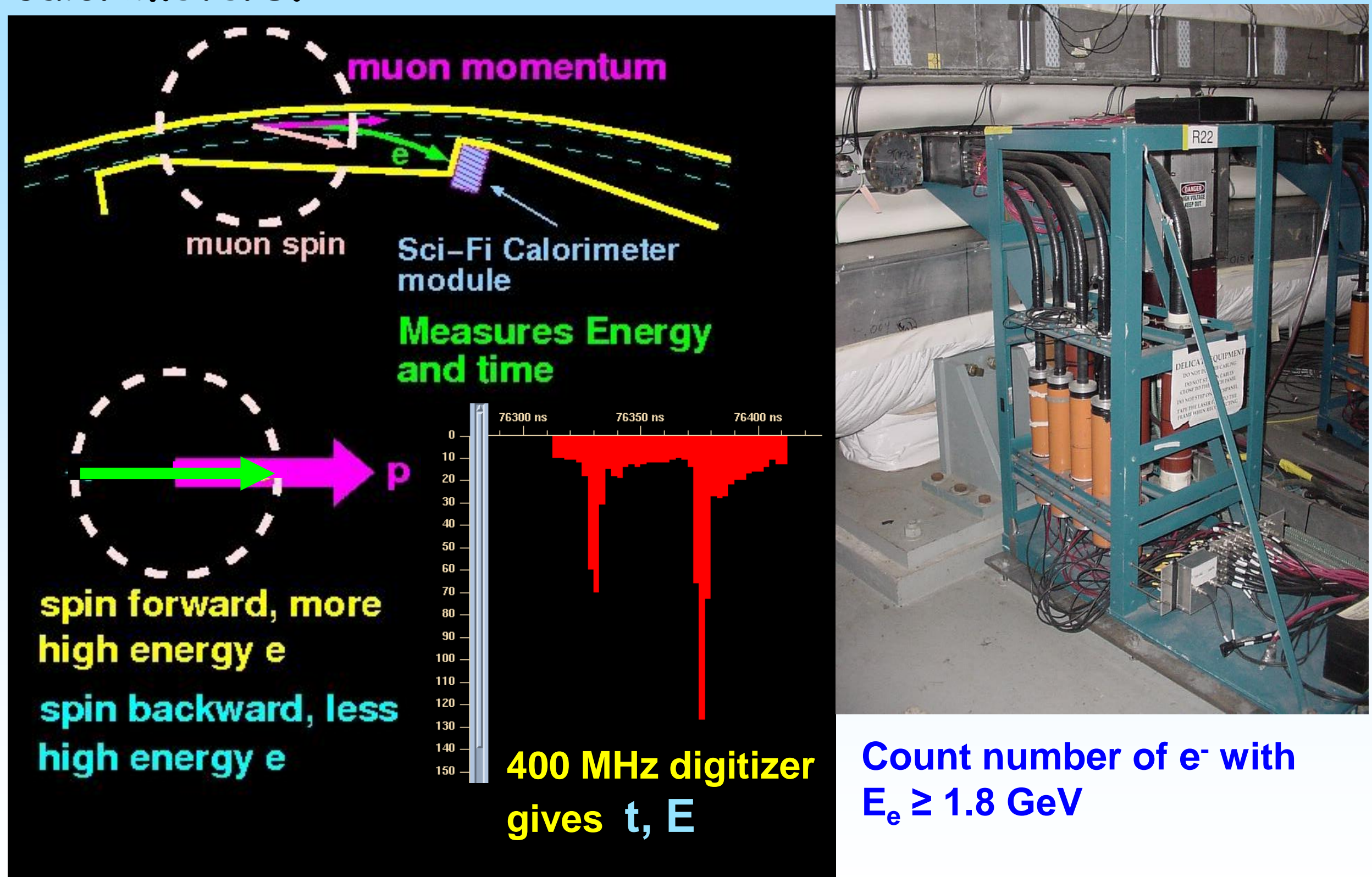


\section{Arrival time spectrum}

$$
\begin{gathered}
\left.f(t) \simeq N_{0} e^{-\lambda t}\left[1+A \cos \omega_{a} t+\phi\right)\right] \\
\quad 4 \times 10^{9} e^{-}, E_{e^{-}} \geq 1.8 \mathrm{GeV} \\
\text { electron time spectrum (2001) }
\end{gathered}
$$

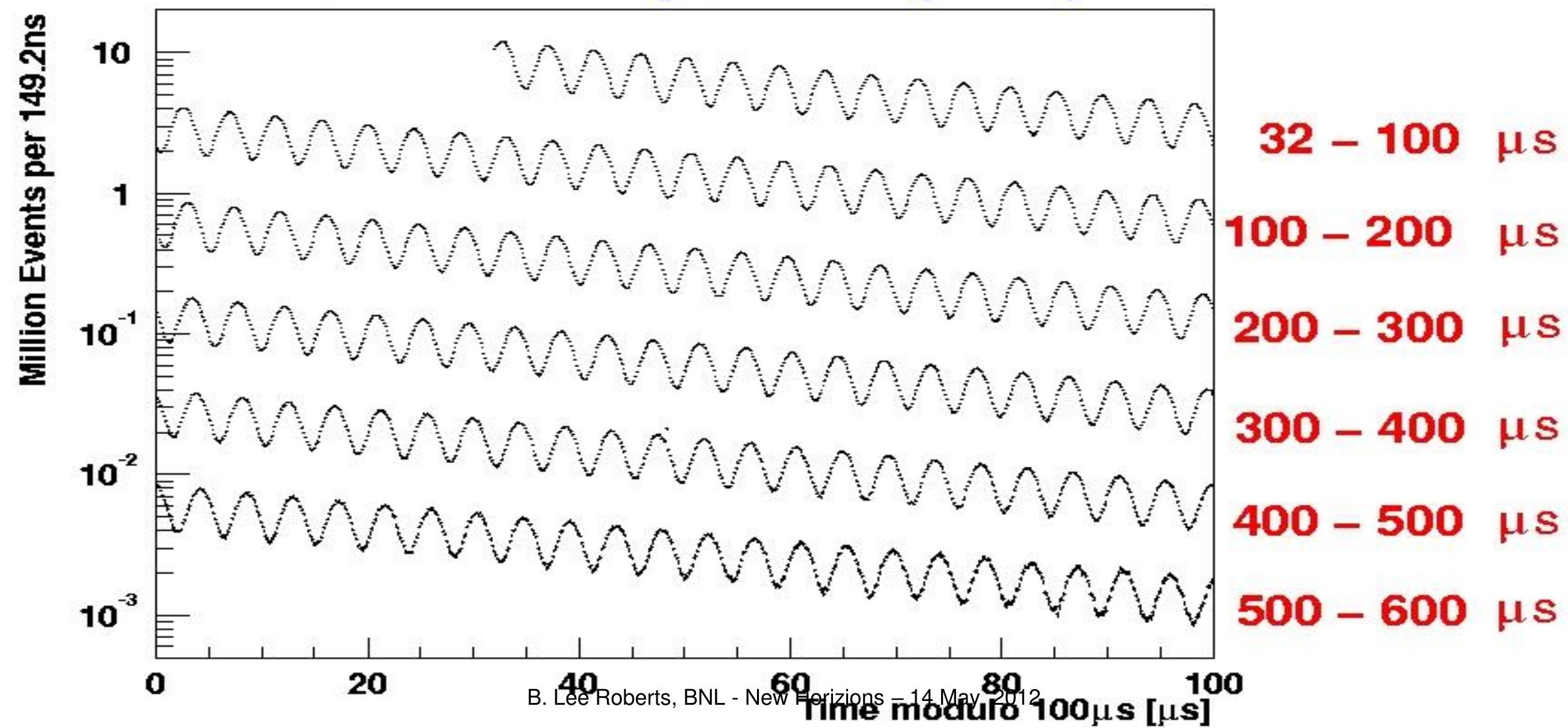


The magnetic field is measured and controlled using pulsed NMR and the free-induction decay.

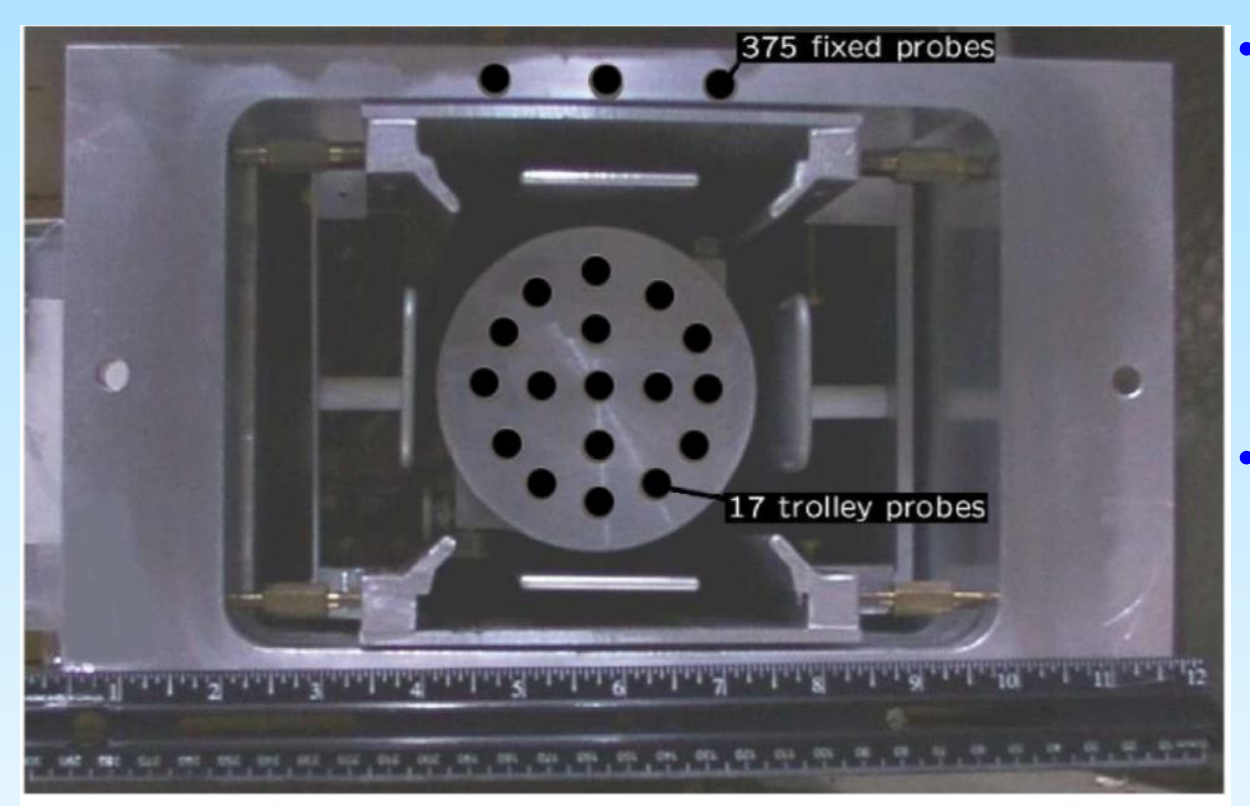

Free induction decay signals:
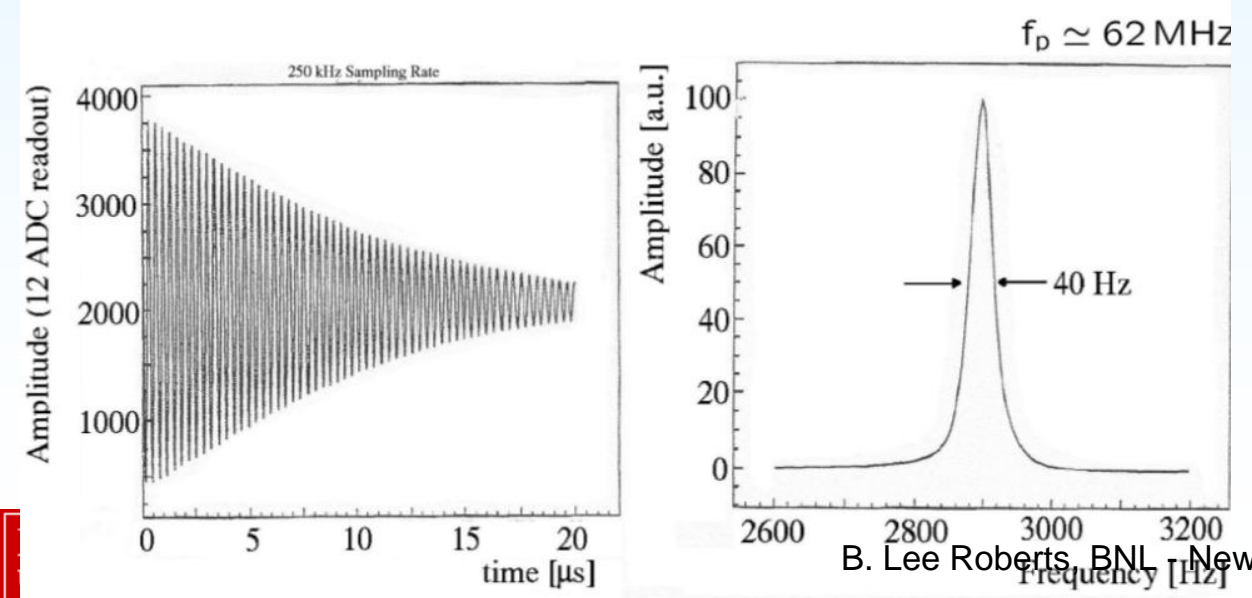

Calibration to a spherical water sample that ties the field to the Larmor frequency of the free proton $\omega_{p}$.

We measure $\omega_{a}$ and $\omega_{p}$

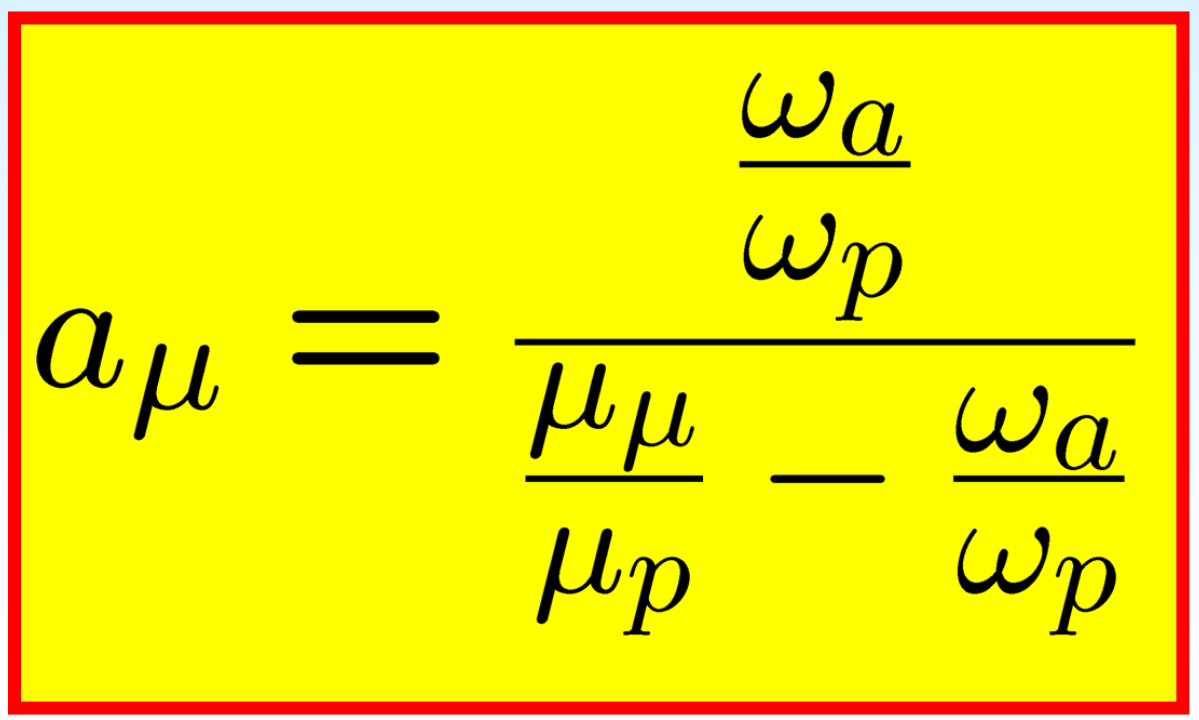

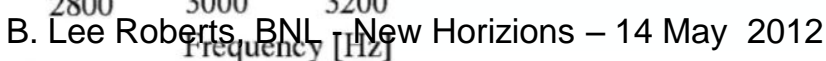


E821 achieved $\pm 0.54 \mathrm{ppm}$. The $\mathrm{e}^{+} \mathrm{e}^{-}$based theory is at the $\sim 0.4 \mathrm{ppm}$ level. Difference is $>3 \sigma$

SM: Davier et al, , Eur.

Phys. J. C (2011) 71:1515

Hagiwara, et al., J.Phys. G 38 (2011) 085003

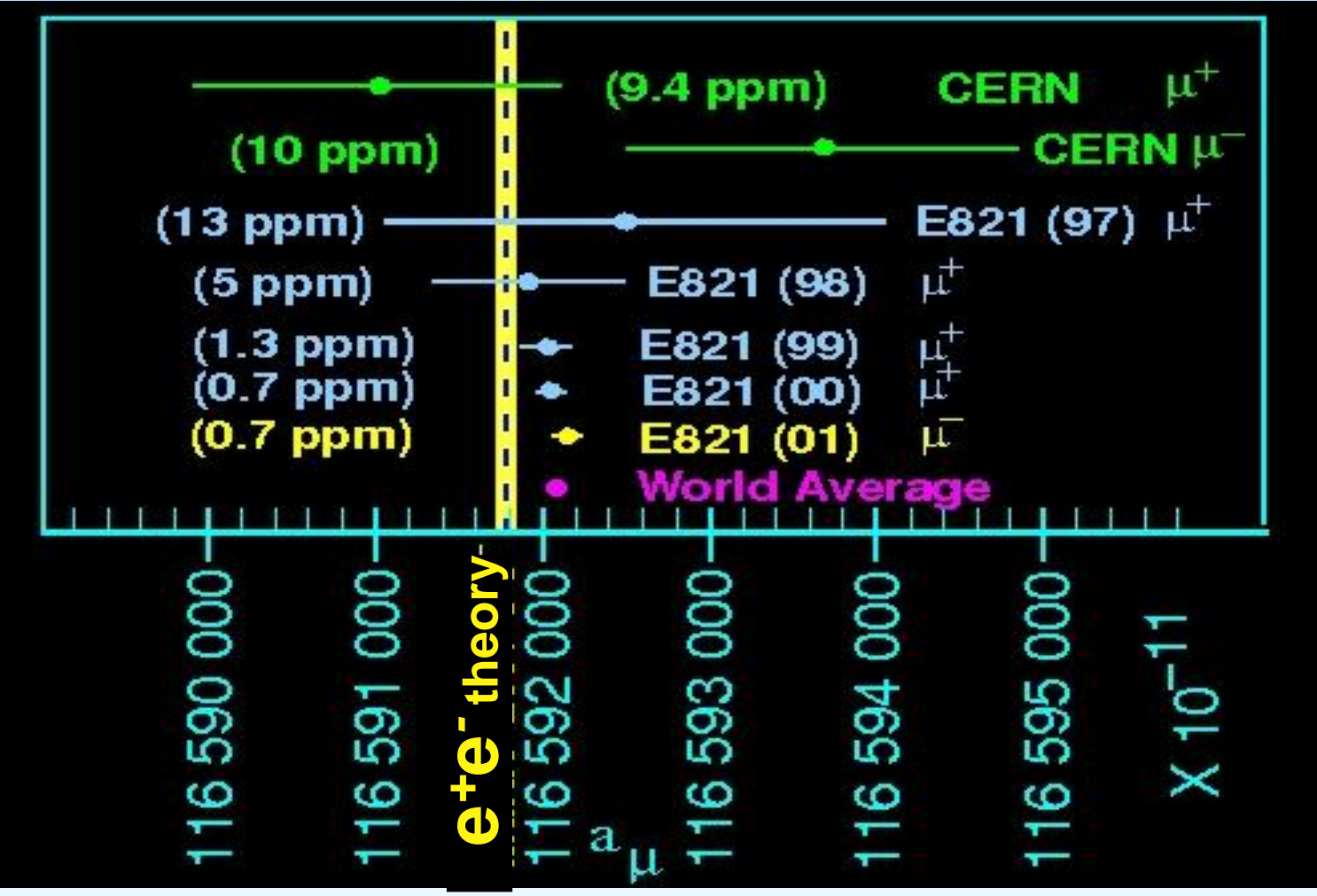

$$
\begin{gathered}
a_{\mu}^{S M}=116591802(49) \times 10^{-11}(0.42 \mathrm{ppm}) \\
a_{\mu}^{e x p}=116592089(63) \times 10^{-11}(0.54 \mathrm{ppm}) \\
\triangle a_{\mu} \equiv a_{\mu}^{e x p}-a_{\mu}^{S M}=(287 \pm 80) \times 10^{-11}
\end{gathered}
$$

$$
a_{\mu}^{E W}=154(1)(2) \times 10^{-11}
$$


Muon $\mathrm{g}-2$ is a powerful discriminator between models; chiral-changing, flavor and CP conserving interaction.

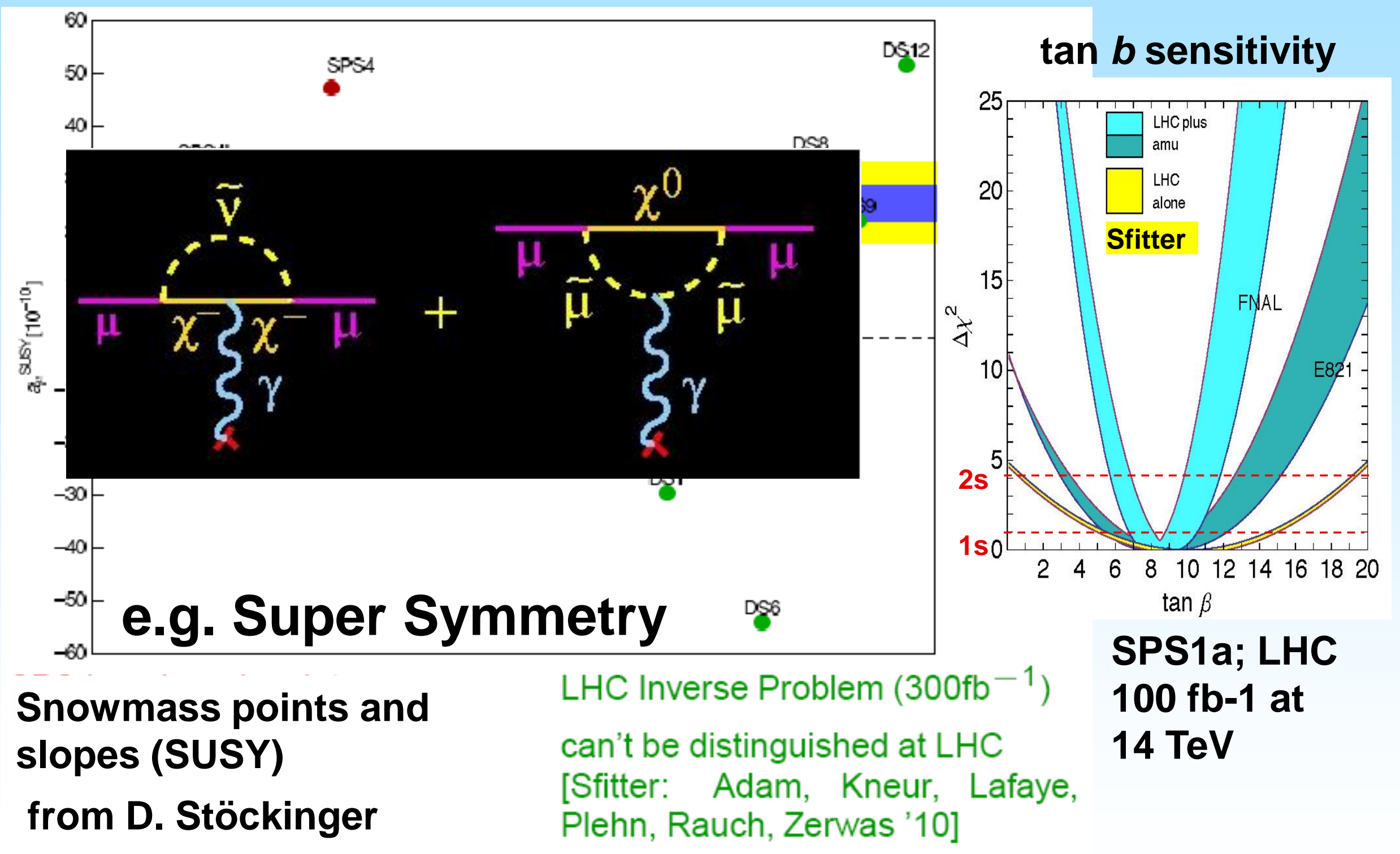




\section{Other Models}

- Technicolor

- small $\Delta a_{\mu}$

- Littlest Higgs with T-parity

- small $\Delta \mathrm{a}_{\mu}$

- Universal Extra Dimensions

- small $\Delta \mathrm{a}_{\mu}$

- Randall Sundrum

- could accommodate large $\Delta \mathrm{a}_{\mu}$

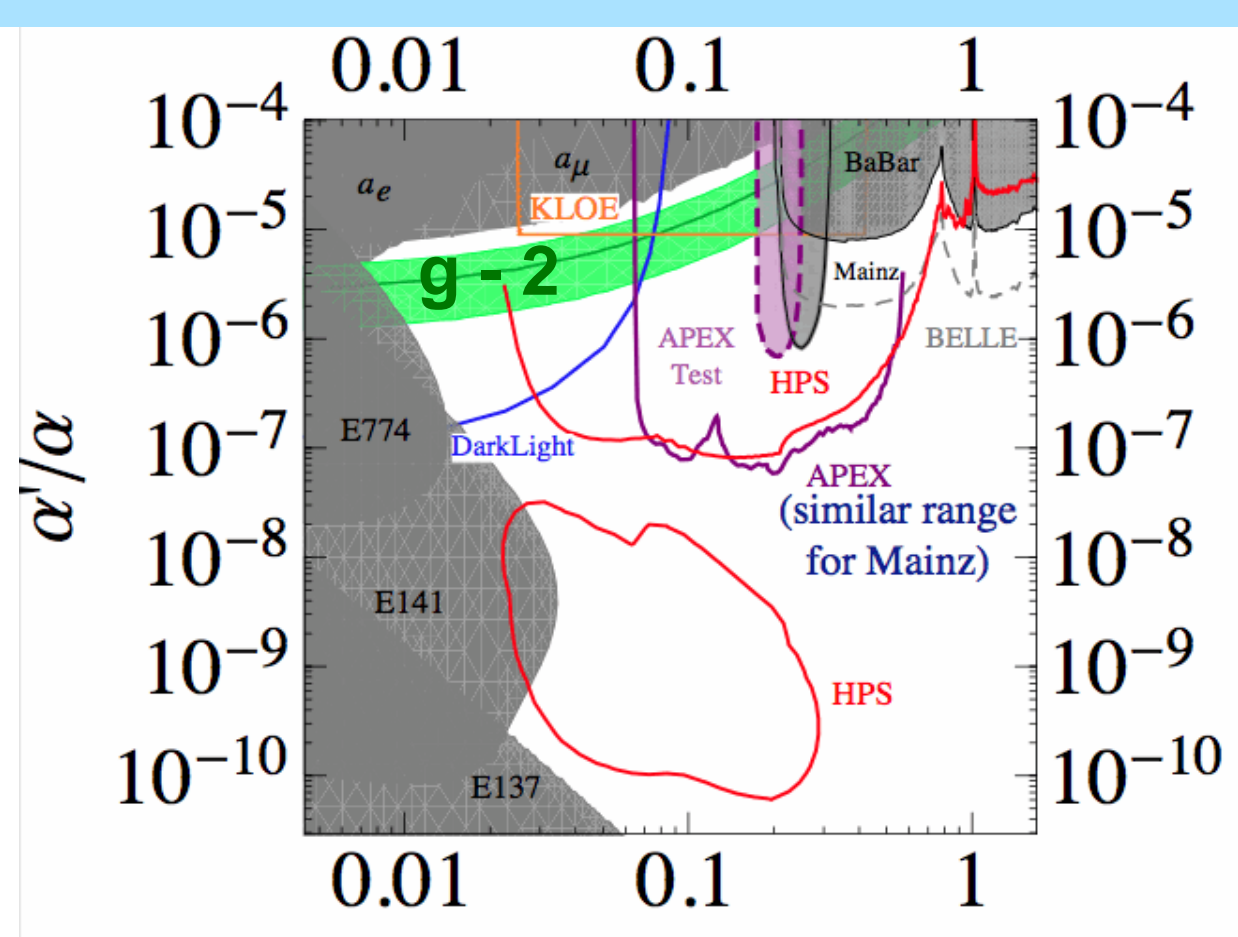

Natalia Toro, Aspen $2011 m_{A^{\prime}}($ GeV)

- Two Higgs doublets, shadow Higgs

- small $\Delta \mathrm{a}_{\mu}$

- Additional light bosons that can affect EM interactions (difficult to study at LHC)

- secluded U(1), etc., could have significant $\Delta \mathrm{a}_{\mu}$ 
How to clarify this $3.3-3.6 \sigma$ situation?

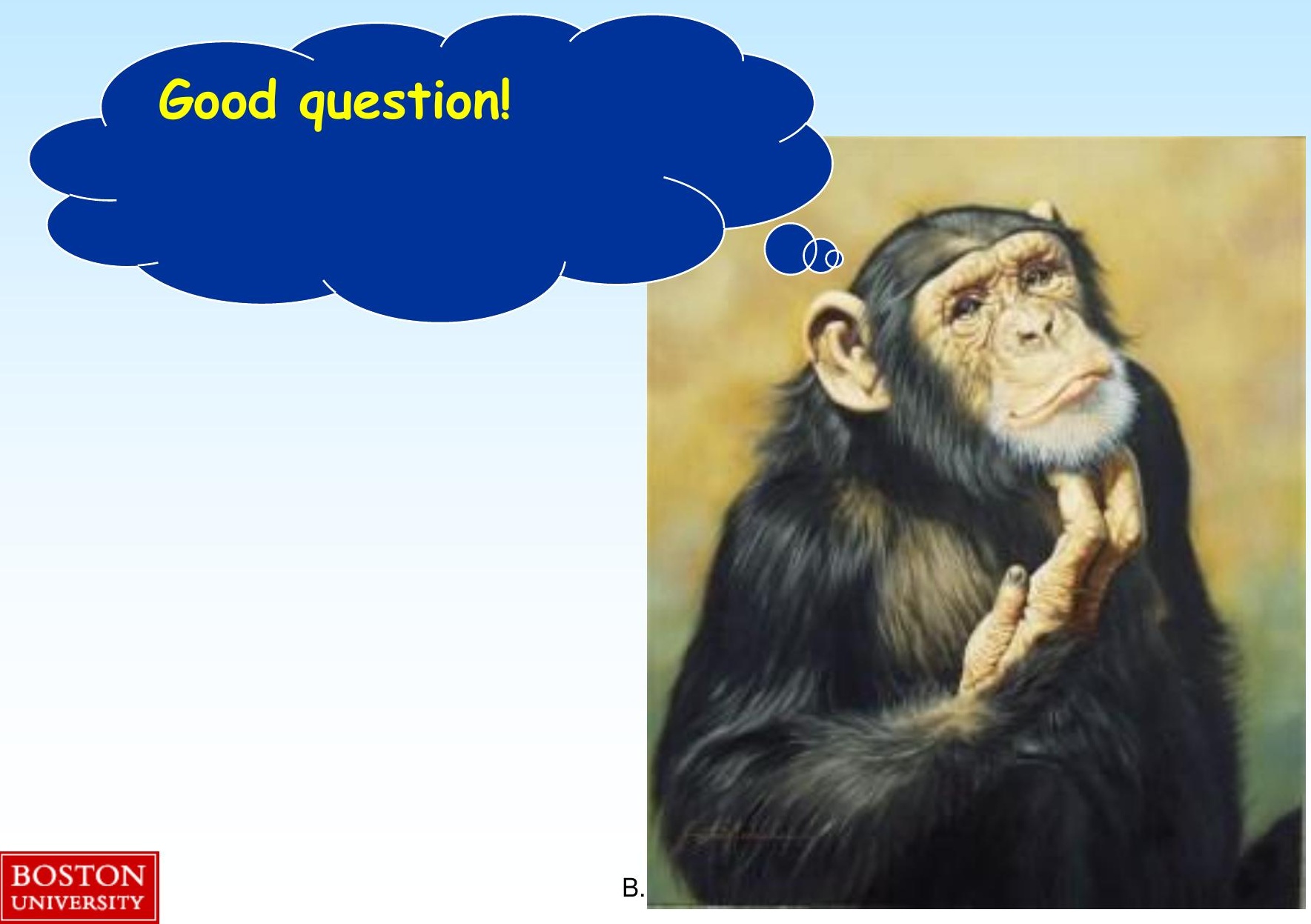




\section{$3.3-3.6 \sigma:$ Theory \& Experiment must do better}

- Experiment: E989 at Fermilab $\geq$ X4 better

- move the storage ring to Fermilab

- use the p-bar debuncher (now called the delivery ring) as a long decay line. 


\section{Fermilab Muon Beam}

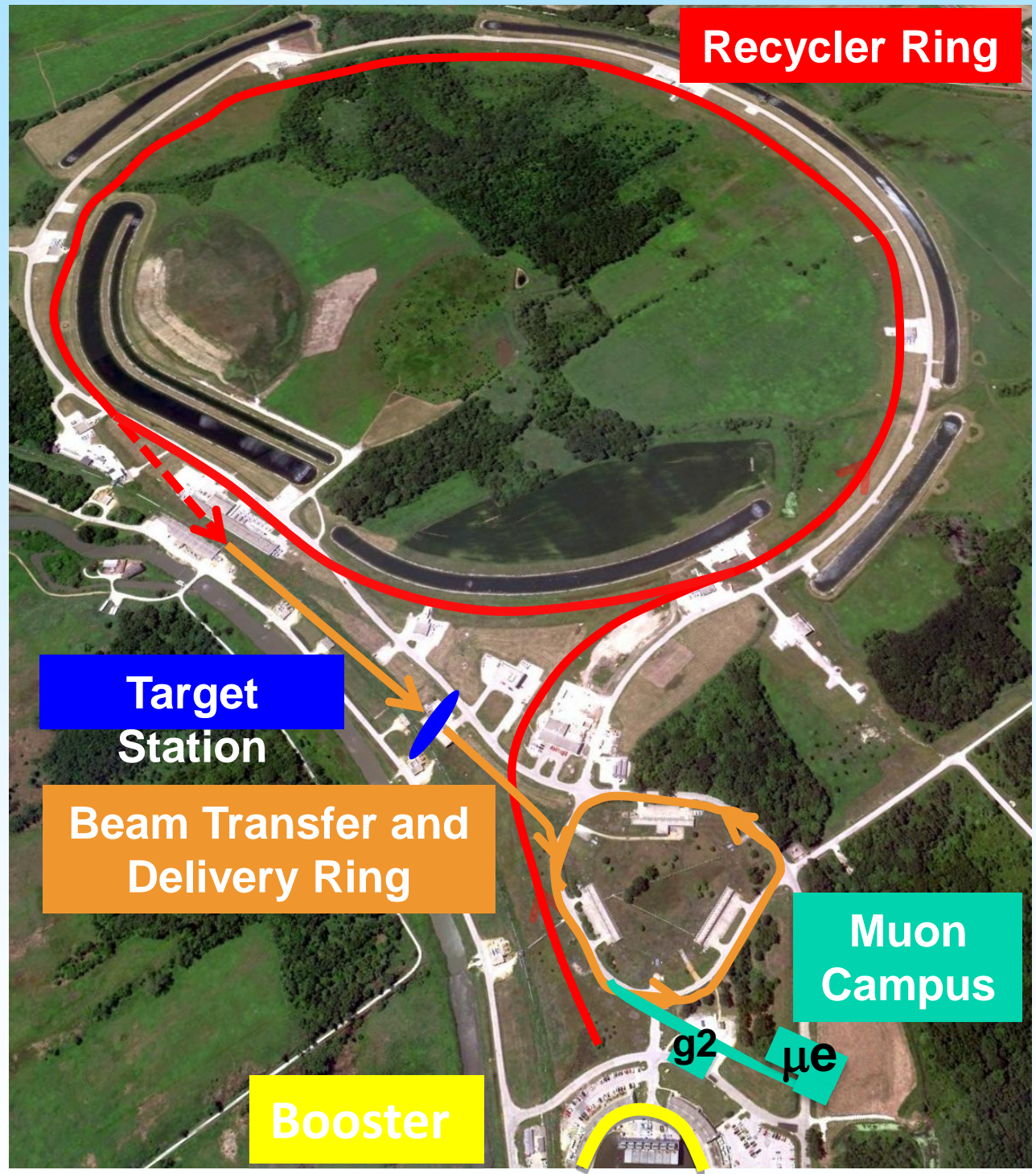




\section{Polarized muons delivered and stored in the ring at the magic momentum, 3.094 GeV/c}

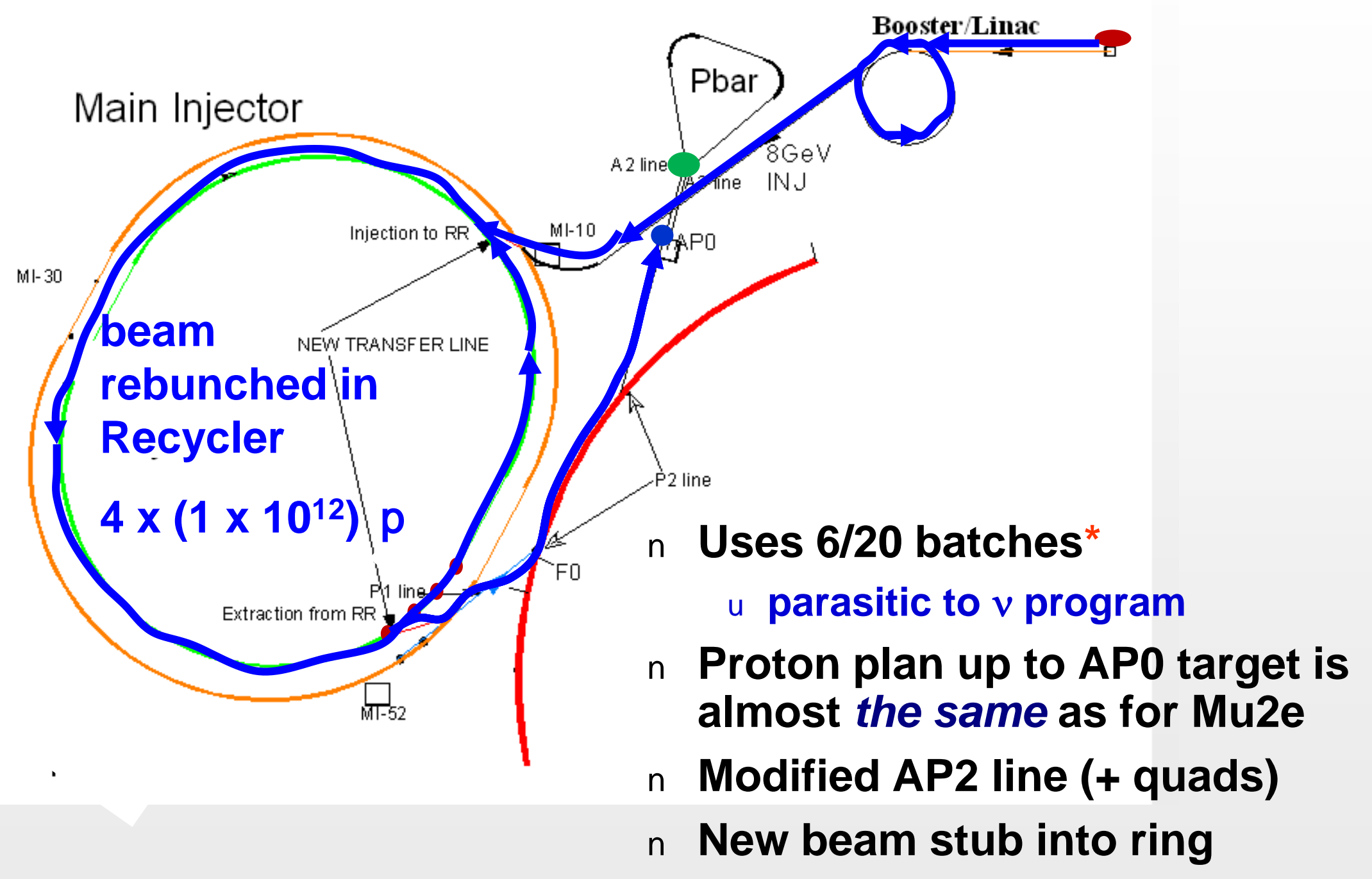


Theory: It's the hadronic contribution

$$
\sigma_{\mathrm{H}-\mathrm{LO}}=42 \quad \sigma_{\mathrm{H}-\mathrm{LBL}}=26 \quad \sigma_{\mathrm{Tot}}=49
$$

Dispersion relation and data?
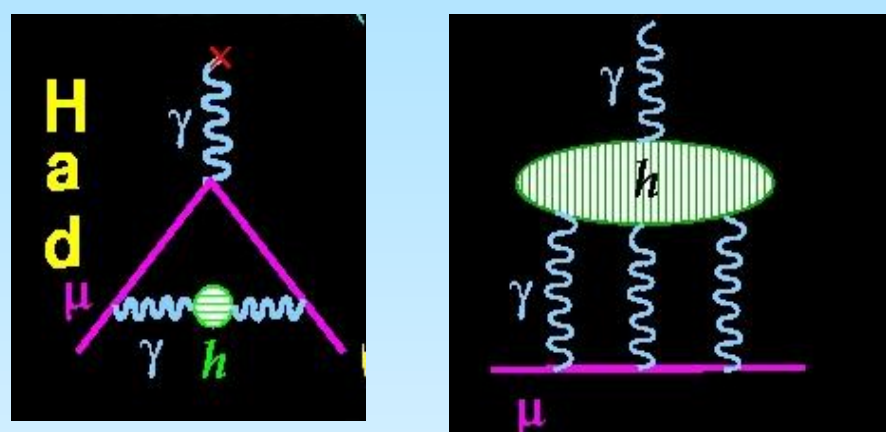

Models

Are data relevant?

- The lowest-order hadronic comes from $\mathrm{e}^{+} \mathrm{e}^{-}$data and a dispersion relation

- can't they just take more data?

- It's not so simple because of the $\tau$-decay data 


\section{So what's the problem}

- It looks like data can determine the lowest-order hadronic just fine
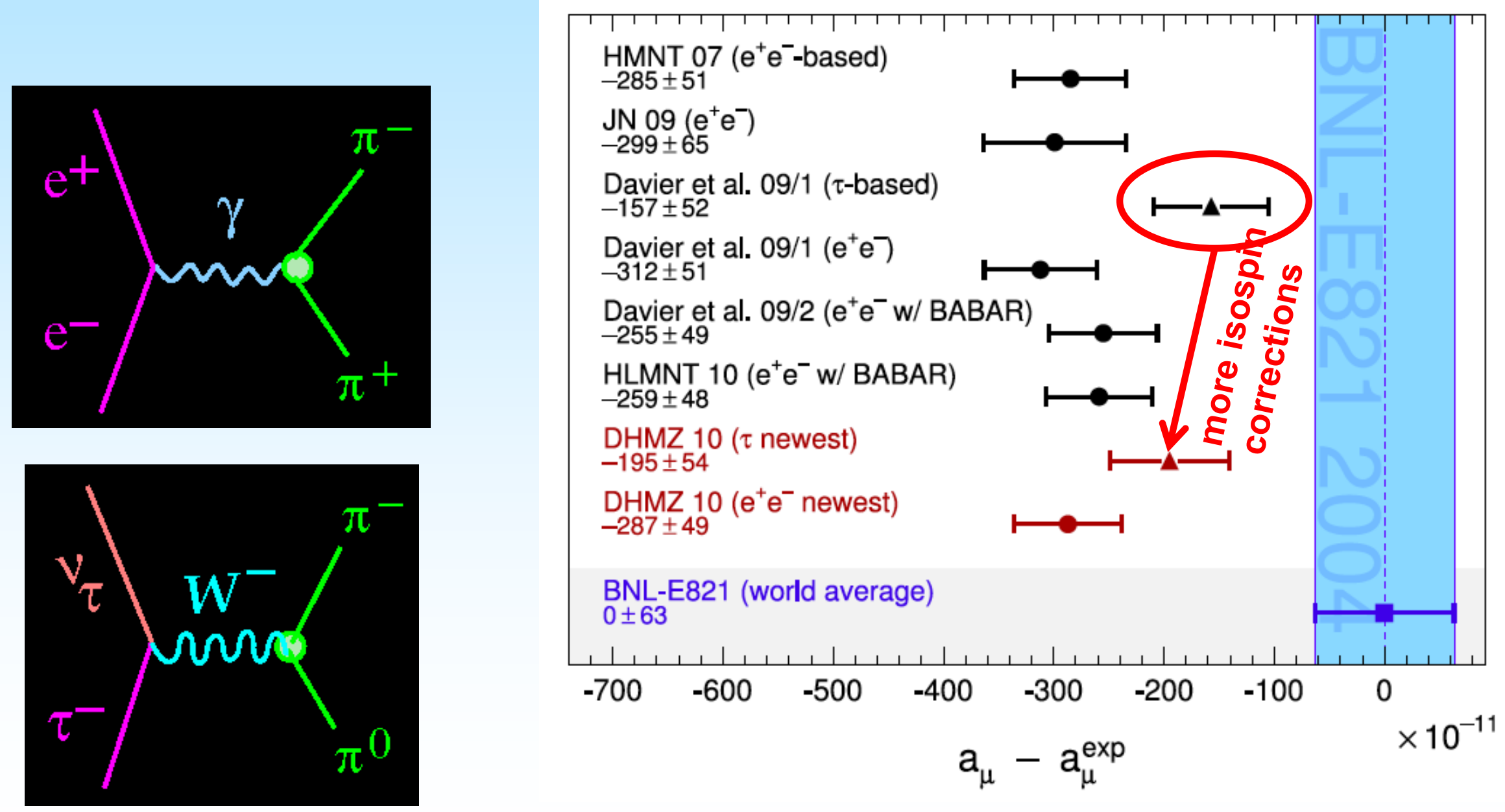


\section{Clarifying the $\tau$ problem for H-LO}

- It is claimed that if $\rho-\gamma$ mixing is correctly included the $\tau-\mathrm{e}^{+} \mathrm{e}^{-}$difference goes away.

- What's the path forward

- More $\mathrm{e}^{+} \mathrm{e}^{-}$data?

-The Lattice!

- The lattice can make an important contribution in the determination of the lowestorder hadronic contribution

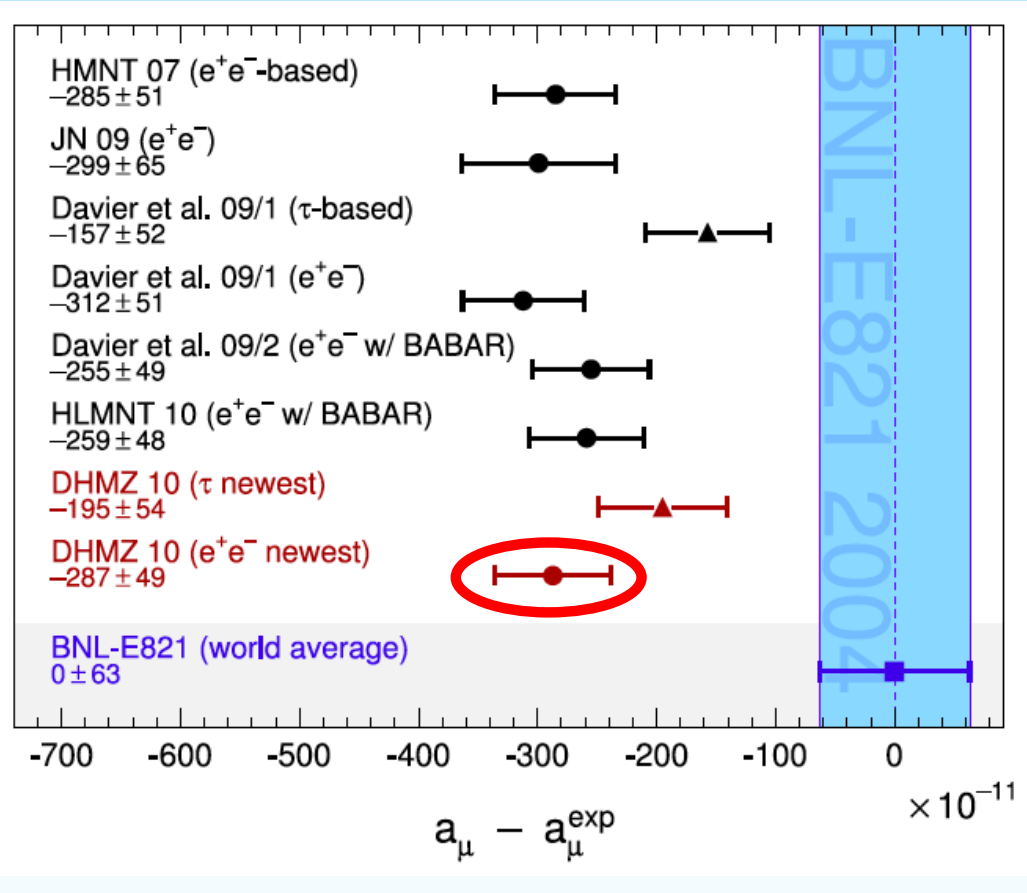

- The proof of principle was published last year 


\section{Even more urgent: Hadronic light-by-light}

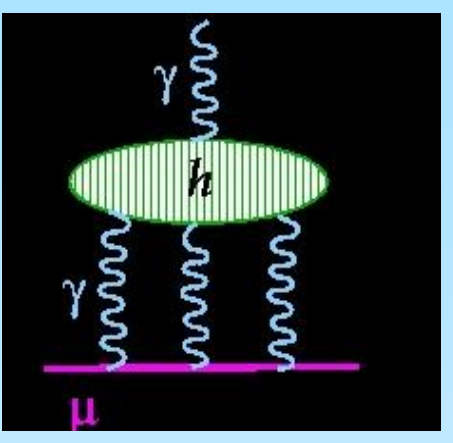

$$
a_{\mu}^{(\mathrm{HLbyL})}=(105 \pm 26) \times 10^{-11}
$$

- Model calculation

- (Prades, de Rafael, Vainshtein compilation)

- Here the lattice can make an extremely important calculation.

- even 10 to $20 \%$ would be fabulous 


\section{Summary and Conclusions}

- Fermilab E989 will improve g-2 by at least X4

- Additional data for the dispersion relation will be taken at CMD3 (Novosibirsk), BESS3, and Super Belle.

- The Lattice has the opportunity to play an important role in the confrontation between the Standard Model and experiment

- lowest-order hadronic

- hadronic light-by-light

- It's an opportunity to make a huge impact in the search for physics beyond the standard model. 


\title{
Prospects for the computation of rare kaon-decay amplitudes
}

\author{
Chris Sachrajda \\ (based on discussions with P.Boyle, E.Goode, P.Kassel and A.Lytle)
}

\author{
School of Physics and Astronomy \\ University of Southampton \\ Southampton SO17 1BJ \\ UK \\ (RBC-UKQCD)
}

New Horizons for Lattice Computations with Chiral Fermions Brookhaven National Laboratory, 14-16 May 2012

\section{Southampinton \\ School of Physics \\ and Astronomy}




\section{Motivation and Introduction}

- Lattice simulations are contributing to a wide variety of fundamental issues in particle physics and increasingly to nuclear physics.

- For me personally, a major motivation is the important complementarity of high-energy experiments (most notably the LHC) and precision flavour physics in discovering and unraveling the next layer of fundamental physics.

- If, as expected/hoped the LHC experiments discover new elementary particles, then precision flavour physics will be necessary to understand the underlying framework.

- The discovery potential of precision flavour physics should also not be underestimated. (In principle, the reach is about two-orders of magnitude deeper than the LHC!)

- Precision flavour physics requires control of hadronic effects for which lattice QCD simulations are essential.

- For many years we have been calculating matrix elements of the forms:

$$
\begin{aligned}
\langle 0|O(0)| h\rangle & \rightarrow f_{P}, \text { for example } \\
\left\langle h_{2}|O(0)| h_{1}\right\rangle & \rightarrow B_{P}, \text { semileptonic form factors, } \cdots .
\end{aligned}
$$

$\Rightarrow$ unitarity triangle tests of the SM, determination of CKM matrix elements etc. 


\section{Extending the reach of Lattice Simulations}

- Recent RBC-UKQCD extensions to the above include the evaluation of $K \rightarrow \pi \pi$ decay amplitudes.

- More recently we have began to consider long-distance contributions to physical quantities. These are not given in terms of matrix elements of local operators, but require the evaluation for example of:

$$
\int d^{4} x \int d^{4} y\left\langle h_{2}\left|T\left\{O_{1}(x) O_{2}(y)\right\}\right| h_{1}\right\rangle .
$$

- The most advanced of our projects is on the evaluation of long-distance contributions to the $K_{L}-K_{S}$ mass difference.

Jianglei Yu, arXiv:1111.6953; paper in preparation.

$$
\int d^{4} x \int d^{4} y\left\langle\bar{K}^{0}\left|T\left\{H_{W}(x) H_{W}(y)\right\}\right| K^{0}\right\rangle .
$$

- Here instead, I will present some preliminary thoughts about the rare kaon decays $K \rightarrow \pi v \bar{v}$ and $K \rightarrow \pi \ell^{+} \ell^{-}$:

$$
\int d^{4} x e^{-i q \cdot x} \int d^{4} y\left\langle\pi\left|T\left\{J^{\mu}(x) H_{W}(y)\right\}\right| K^{0}\right\rangle .
$$

- Up to now, the main theoretical tool for these processes has been Chiral Perturbation Theory with its many limitations and uncertainties. 
- Rare kaon decays which are dominated by short-distance FCNC processes, $K \rightarrow v \bar{v}$ in particular, provide a potentially valuable window on new physics at high-energy scales.

- The decays $K_{L} \rightarrow \pi^{0} e^{+} e^{-}$and $K_{L} \rightarrow \pi^{0} \mu^{+} \mu^{-}$are also considered promising because the long-distance effects are reasonably under control using ChPT.

- They are sensitive to different combinations of short-distance FCNC effects and hence in principle provide additional discrimination to the neutrino modes.

A challenge for the lattice community is therefore either to calculate the long-distance effects reliably or at least to determine the Low Energy Constants of ChPT. 
There are three main contributions to the amplitude:

1 Short distance contributions:

$$
H_{\mathrm{eff}}=-\frac{G_{F} \alpha}{\sqrt{2}} V_{t s}^{*} V_{t d}\left\{y_{7 V}\left(\bar{s} \gamma_{\mu} d\right)\left(\bar{\ell} \gamma^{\mu} \ell\right)+y_{7 A}\left(\bar{s} \gamma_{\mu} d\right)\left(\bar{\ell} \gamma^{\mu} \gamma_{5} \ell\right)\right\}+\text { h.c. }
$$

- Direct CP-violating contribution.

- In BSM theories other effective interactions are possible.

2 Long-distance indirect CP-violating contribution

$$
A_{I C P V}\left(K_{L} \rightarrow \pi^{0} \ell^{+} \ell^{-}\right)=\varepsilon A\left(K_{1} \rightarrow \pi^{0} \ell^{+} \ell^{-}\right) .
$$

The two-photon CP-conserving contribution $K_{L} \rightarrow \pi^{0}\left(\gamma^{*} \gamma^{*} \rightarrow \ell^{+} \ell^{-}\right)$.

(a)

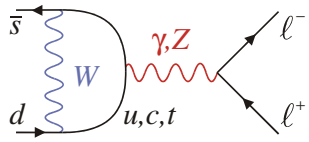

(b)

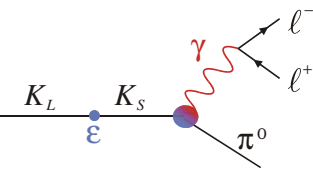

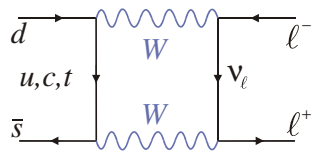

(c)

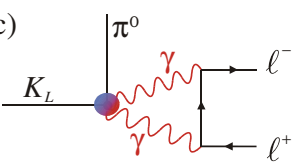


- The current phenomenological status for the SM predictions is nicely summarised by:

V.Cirigliano et al., arXiv1107.6001

$$
\begin{aligned}
\operatorname{Br}\left(K_{L} \rightarrow \pi^{0} e^{+} e^{-}\right)_{\mathrm{CPV}} & =10^{-12} \times\left\{15.7\left|a_{S}\right|^{2} \pm 6.2\left|a_{S}\right|\left(\frac{\operatorname{Im} \lambda_{t}}{10^{-4}}\right)+2.4\left(\frac{\operatorname{Im} \lambda_{t}}{10^{-4}}\right)^{2}\right\} \\
\operatorname{Br}\left(K_{L} \rightarrow \pi^{0} \mu^{+} \mu^{-}\right)_{\mathrm{CPV}} & =10^{-12} \times\left\{3.7\left|a_{S}\right|^{2} \pm 1.6\left|a_{S}\right|\left(\frac{\operatorname{Im} \lambda_{t}}{10^{-4}}\right)+1.0\left(\frac{\operatorname{Im} \lambda_{t}}{10^{-4}}\right)^{2}\right\}
\end{aligned}
$$

$\lambda_{t}=V_{t d} V_{t s}^{*}$ and $\operatorname{Im} \lambda_{t} \simeq 1.35 \times 10^{-4}$.

- $\left|a_{S}\right|$, the amplitude for $K_{S} \rightarrow \pi^{0} \ell^{+} \ell^{-}$at $q^{2}=0$ as defined below, is expected to be $O(1)$ but the sign of $a_{S}$ is unknown. $\left|a_{S}\right|=1.06_{-0.21}^{+0.26}$.

a For $\ell=e$ the two-photon contribution is negligible.

- Taking the positive sign (?) the prediction is

$$
\begin{aligned}
\operatorname{Br}\left(K_{L} \rightarrow \pi^{0} e^{+} e^{-}\right)_{\mathrm{CPV}} & =(3.1 \pm 0.9) \times 10^{-11} \\
\operatorname{Br}\left(K_{L} \rightarrow \pi^{0} \mu^{+} \mu^{-}\right)_{\mathrm{CPV}} & =(1.4 \pm 0.5) \times 10^{-11} \\
\operatorname{Br}\left(K_{L} \rightarrow \pi^{0} \mu^{+} \mu^{-}\right)_{\mathrm{CPC}} & =(5.2 \pm 1.6) \times 10^{-12} .
\end{aligned}
$$

- The current experimental limits $(\mathrm{KTeV})$ are:

$$
\operatorname{Br}\left(K_{L} \rightarrow \pi^{0} e^{+} e^{-}\right)<2.8 \times 10^{-10} \text { and } \operatorname{Br}\left(K_{L} \rightarrow \pi^{0} \mu^{+} \mu^{-}\right)<3.8 \times 10^{-10} .
$$


CPC Decays: $K_{S} \rightarrow \pi^{0} \ell^{+} \ell^{-}$and $K^{+} \rightarrow \pi^{+} \ell^{+} \ell^{-}$

G.Isidori, G.Martinelli and P.Turchetti, hep-lat/0506026

- We now turn to the CPC decays $K_{S} \rightarrow \pi^{0} \ell^{+} \ell^{-}$and $K^{+} \rightarrow \pi^{+} \ell^{+} \ell^{-}$and consider

$$
T_{i}^{\mu}=\int d^{4} x e^{-i q \cdot x}\left\langle\pi(p)\left|\mathrm{T}\left\{J_{\mathrm{em}}^{\mu}(x) Q_{i}(0)\right\}\right| K(k)\right\rangle,
$$

where $Q_{i}$ is an operator from the effective Hamiltonian.

- Gauge invariance implies that

$$
T_{i}^{\mu}=\frac{\omega_{i}\left(q^{2}\right)}{(4 \pi)^{2}}\left\{q^{2}(p+k)^{\mu}-\left(m_{K}^{2}-m_{\pi}^{2}\right) q^{\mu}\right\} .
$$

- Within ChPT the Low energy constants $a_{+}$and $a_{S}$ are defined by

$$
a=\frac{1}{\sqrt{2}} V_{u s}^{*} V_{u d}\left\{C_{1} \omega_{1}(0)+C_{2} \omega_{2}(0)+\frac{2 N}{\sin ^{2} \theta_{W}} f_{+}(0) C_{7 V}\right\}
$$

where $Q_{1,2}$ are the two current-current GIM subtracted operators and the $C_{i}$ are the Wilson coefficients. ( $C_{7 V}$ is proportional to $y_{7 V}$ above).

G.Ambosio, G.Ecker, G.Isidori and J.Portoles, hep-ph/9808289

- Phenomenological values: $a_{+}=-0.578 \pm 0.016$ and $\left|a_{S}\right|=1.06_{-0.21}^{+0.26}$.

Can we do better in lattice simulations? 


\section{The fiducial volume}

- Ideas developed (or being developed) from our $\Delta M_{K}$ project.

- How do you prepare the states $h_{1,2}$ in

$$
\int d^{4} x \int d^{4} y\left\langle h_{2}\left|T\left\{O_{1}(x) O_{2}(y)\right\}\right| h_{1}\right\rangle,
$$

when the time of the operators is integrated.

- The practical solution is to integrate over a large subinterval in time $t_{A} \leq t_{x, y} \leq t_{B}$, but to create $h_{1}$ and to annihilate $h_{2}$ well outside of this region:

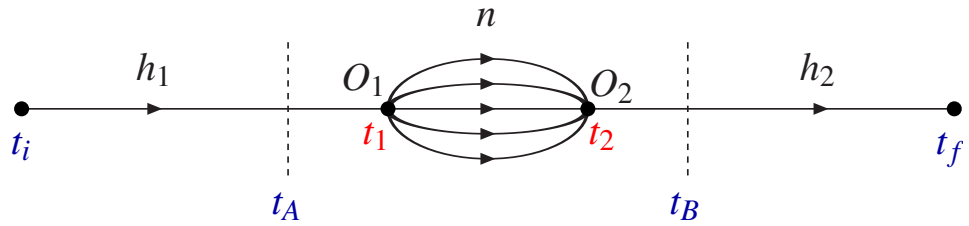

- This is the natural modification of standard field theory for which the asymptotic states are prepared at $t \rightarrow \pm \infty$ and then the operators are integrated over all time.

- This approach has been successfully implemented in the $\Delta M_{K}$ project.

N.Christ arXiv:1012.6034; Jianglei Yu arXiv:1111.6953; paper in preparation 
- The generic non-local matrix elements which we wish to evaluate is

$$
\begin{aligned}
X & \equiv \int_{-\infty}^{\infty} d t_{x} d^{3} x\langle\pi(p)|\mathrm{T}[J(0) H(x)]| K\rangle \\
& =i \sum_{n} \frac{\langle\pi(p)|J(0)| n\rangle\langle n|H(0)| K\rangle}{m_{K}-E_{n}+i \varepsilon}-i \sum_{n_{s}} \frac{\left\langle\pi(p)|H(0)| n_{s}\right\rangle\left\langle n_{s}|J(0)| K\right\rangle}{E_{n_{s}}-E_{\pi}+i \varepsilon},
\end{aligned}
$$

- $\{|n\rangle\}$ and $\left\{\left|n_{s}\right\rangle\right\}$ represent complete sets of non-strange and strange sets.

- In Euclidean space we envisage calculating correlation functions of the form

$$
C \equiv \int_{-T_{a}}^{T_{b}} d t_{x}\left\langle\phi_{\pi}\left(\vec{p}, t_{\pi}\right) \mathrm{T}\left[J(0) H\left(t_{x}\right)\right] \phi_{K}^{\dagger}\left(t_{K}\right)\right\rangle \equiv \sqrt{Z_{K}} \frac{e^{-m_{K}\left|t_{K}\right|}}{2 m_{K}} X_{E} \sqrt{Z_{\pi}} \frac{e^{-E_{\pi} t_{\pi}}}{2 E_{\pi}},
$$

where

$$
\begin{aligned}
& X_{E_{-}}=-\sum_{n} \frac{\langle\pi(p)|J(0)| n\rangle\langle n|H(0)| K\rangle}{m_{K}-E_{n}}\left(1-e^{\left(m_{K}-E_{n}\right) T_{a}}\right) \text { and } \\
& X_{E_{+}}=\sum_{n_{s}} \frac{\left\langle\pi(p)|H(0)| n_{s}\right\rangle\left\langle n_{s}|J(0)| K\right\rangle}{E_{n_{s}}-E_{\pi}}\left(1-e^{-\left(E_{n_{s}}-E_{\pi}\right) T_{b}}\right) .
\end{aligned}
$$


- Chiral ward identities imply that we can add a term proportional to the scalar density $\bar{s} d$ to the Hamiltonian without changing physical results. We can therefore subtract the single pion intermediate state by imposing $\left\langle\pi\left|H+c_{S} \bar{s} d\right| K\right\rangle=0$.

- It is instructive to see how this works in the present case at lowest order in chiral perturbation theory. The scalar density in the effective theory can be written as

$$
S^{s d}=\operatorname{Tr}\left[\lambda^{s d}\left(\Sigma+\Sigma^{\dagger}\right)\right] \quad \text { where } \quad \lambda^{s d}=\left(\begin{array}{lll}
0 & 0 & 0 \\
0 & 0 & 0 \\
0 & 1 & 0
\end{array}\right) .
$$

- The em current is of the form

$$
J^{\mu}=i \frac{f^{2}}{4} \operatorname{Tr}\left[Q\left(\Sigma \partial^{\mu} \Sigma^{\dagger}+\Sigma \partial^{\mu} \Sigma\right)\right]
$$

- The $c_{S}$ term leads to additional diagrams:
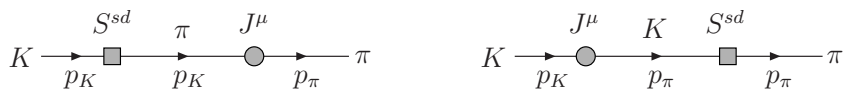

which are proportional to

$$
\frac{\left(p_{\pi}+p_{K}\right)^{\mu}}{p_{K}^{2}-m_{\pi}^{2}}+\frac{\left(p_{\pi}+p_{K}\right)^{\mu}}{p_{\pi}^{2}-m_{K}^{2}} .
$$

On shell, when $p_{K}^{2}=m_{K}^{2}$ and $p_{\pi}^{2}=m_{\pi}^{2}$, the sum of the two terms indeed gives zero. 


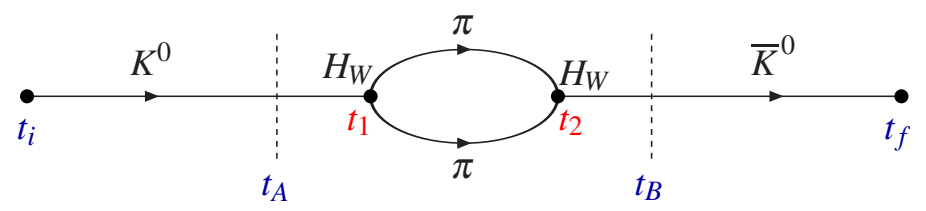

- In the $\Delta M_{K}$ computation, there is, of course, a two-pion intermediate state and we have had to control the corresponding finite-volume effects.

- This has been done on the assumption that the dominant intermediate states below $m_{K}$ are the two-pion states. 
- We have seen that we can remove the single pion intermediate state.

- Which intermediate states contribute?

Are there any states below $M_{K}$ ?

We can control $q^{2}$ and stay below the two-pion threshold.
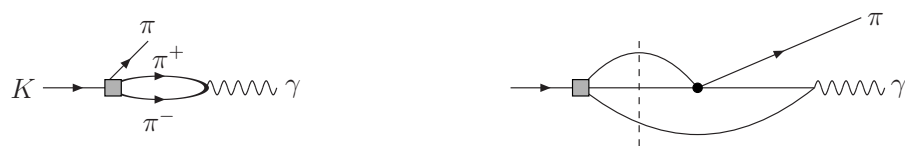

Are there two-pion intermediate states as a result of the Wess-Zumino term?

- Do we need to consider three-pion intermediate states?

Answers to the above questions will affect what the finite-volume corrections are?

- The ChPT-based phemomenology community neglect such possibilities.

\section{All to be investigated further!}

- It looks as though the FV corrections are much simpler than for $\Delta M_{K}$ and may be exponentially small? 


\section{Short Distance Effects}

$$
T_{i}^{\mu}=\int d^{4} x e^{-i q \cdot x}\left\langle\pi(p)\left|\mathrm{T}\left\{J^{\mu}(x) Q_{i}(0)\right\}\right| K(k)\right\rangle,
$$

- Each of the two local $Q_{i}$ operators can be normalized in the standard way and $J$ can be normalized.

- Calculation of long-distance effects $\Rightarrow$ must treat additional divergences as $x \rightarrow 0$.

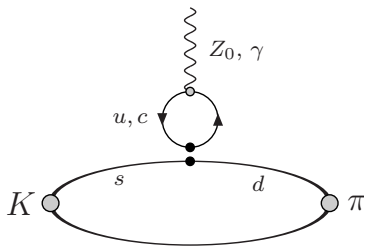

- Quadratic divergence is absent by gauge invariance $\Rightarrow$ Logarithmic divergence.

- Checked explicitly for Wilson and Clover at one-loop order.

G.Isidori, G.Martinelli and P.Turchetti, hep-lat/0506026

- Absence of power divergences does not require GIM.

- Logarithmic divergence cancelled by GIM.

11. For DWF the same applies for the axial current.

- Control of short-distance effects also appears to be much simpler than for $\Delta M_{K}$.

To be investigated further! 


\section{Lots of diagrams to evaluate!}

- Sample diagrams:
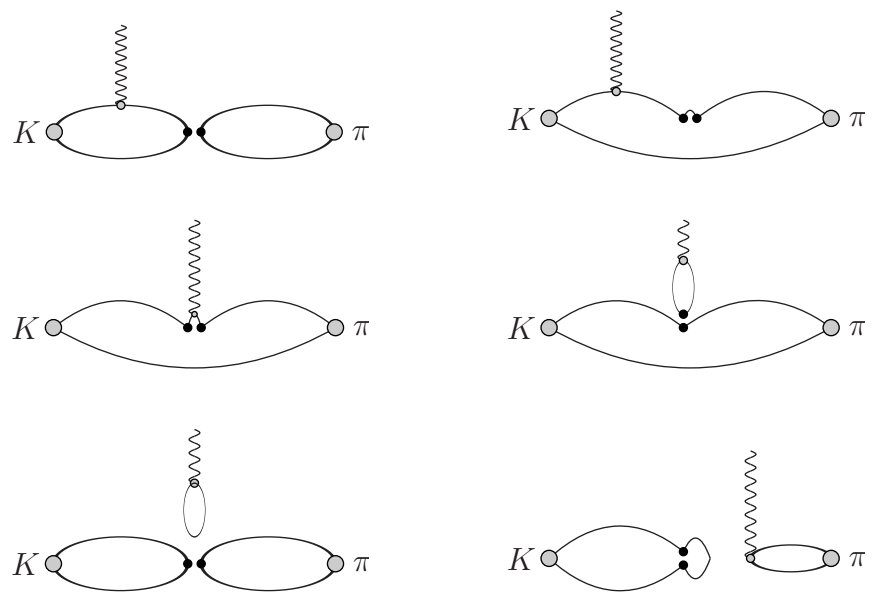

+ lots more

- The last two diagrams are examples of disconnected diagrams. 


\section{Summary and Conclusions}

- Our community must continue to strive to

1 improve the precision of computations of quantities we know well how to compute;

extend the range of quantities which can be computed.

This is necessary of precision flavour physics is to play a complementary rôle to large $p_{\perp}$ experiments in exploring the limits of the standard model and unravelling the basic framework of new physics.

- I have reviewed one such possible future extension, that of the calculation of long-distance physics in rare kaon decays.

- There are many similarities to our calculation of the long-distance contributions to $\Delta M_{K}$ which is further advanced.

Although much still remains to be done, the theoretical background appears to be simpler than for $\Delta M_{K}$ (both finite-volume effects and short distance subtractions).

- The calculations will rely on progress in the computation of disconnected diagrams.

- There are many important rare $B$-decays which should be studied but

- I still don't know how to tackle nonleptonic B decays, even in principle. 


\section{SuperKEKB project}

\section{Upgrade of KEKB/Belle}

KEKB

SuperK

Luminosity : $2.1 \times 10^{34} \rightarrow 8 \times 10^{35}(\times 40)$

Total Data: $\quad 1 \mathrm{ab}^{-1} \rightarrow>50 \mathrm{ab}^{-1}$ (x50)

Detector: Belle $\rightarrow$ Belle II

KEK

High Energy Accelerator Reseach Organization

(E) 


\section{High Energy Physics}

\section{PARTICLE PHYSICS}

Mainly using particle Accelerators

\section{Goal :}

\section{Ultimate nature of Matter}

Fundamental law

Elementary particle Interactions, Symmetries

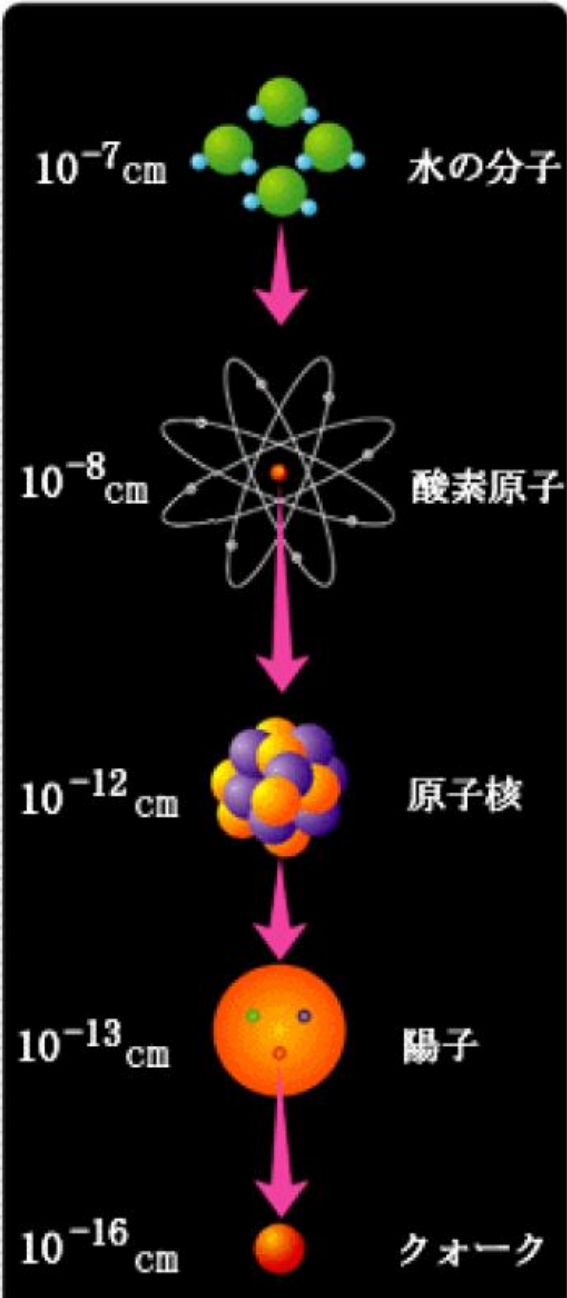




\section{Particle Physics \& Universe}

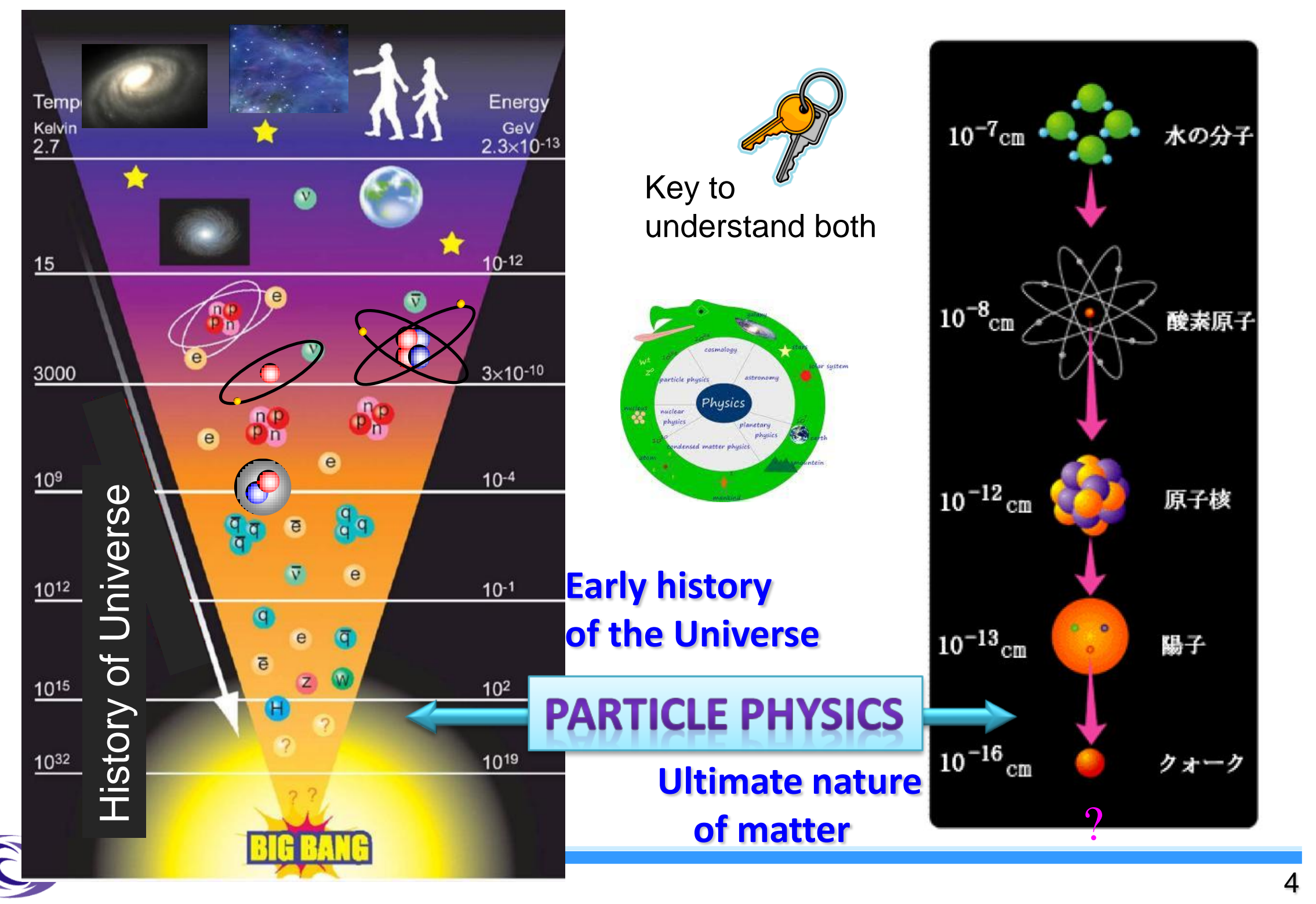




\section{"Standard Model"}

Elementary Particles in SM

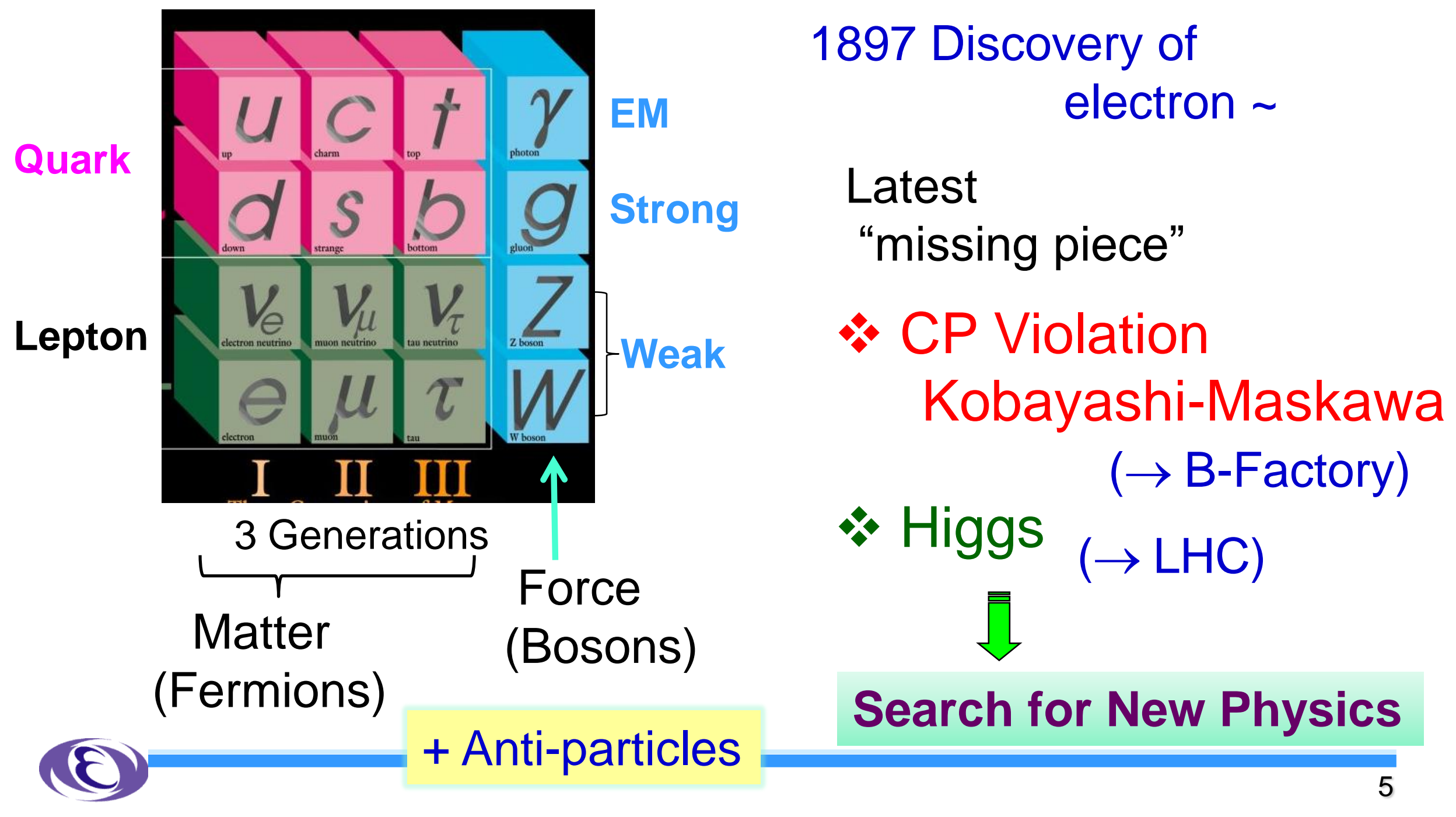




\section{Goal/Milestones of B-factory}

Step1

Discovery of CPV in B decays 2001 summer!

Step2 Precise test of KM and SM

Step3

Search for NP Hints of NP

(SUSY, Extra-dim...) Establish procedures

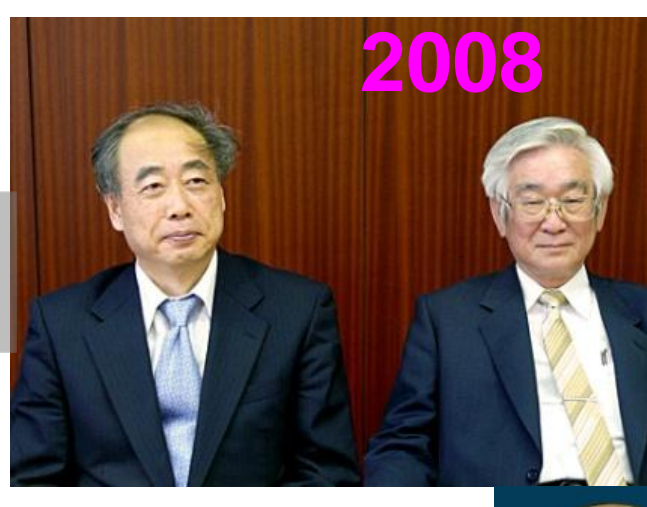

SuperB-factory

50 times more data

(higher luminosity) 


\section{CP Violation}

\section{Difference between particle \& anti-particle (matter \& anti-matter)}

Universe: almost "matter" only (no anti-matter)

Big-Bang $\rightarrow \mathrm{N}($ particles $)=\mathrm{N}($ anti-particles $)$

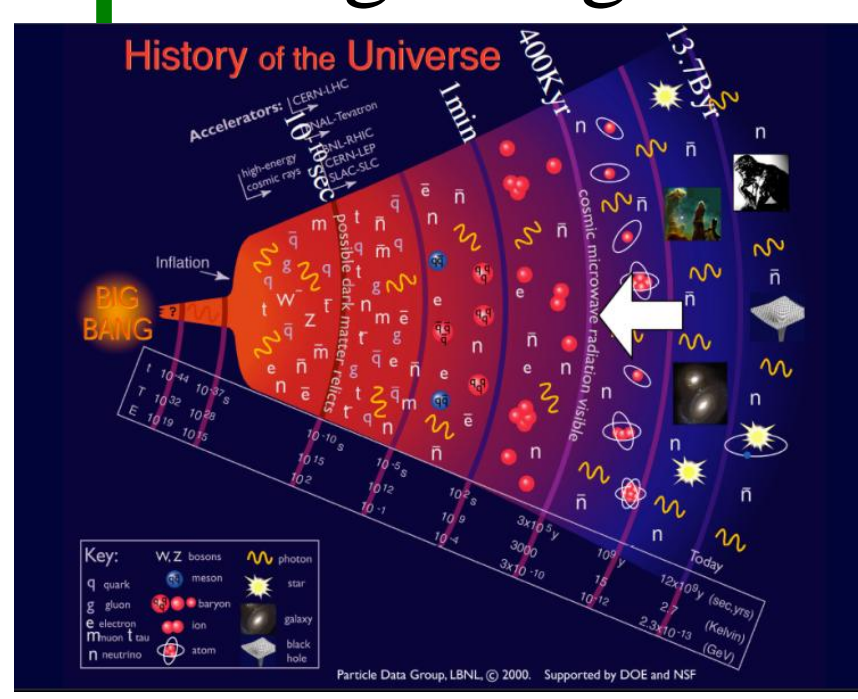

Sakhalov's 3 conditions (1967):

1. baryon number violation

2. CP violation

3. existence of non-equiblium

CPV is a key for Existence of Universe \& us ! Andrei Sakharov (1921-1989) 


\section{Kobayashi-Maskawa}

( 1973

Mechanism of CPV

Weak interaction

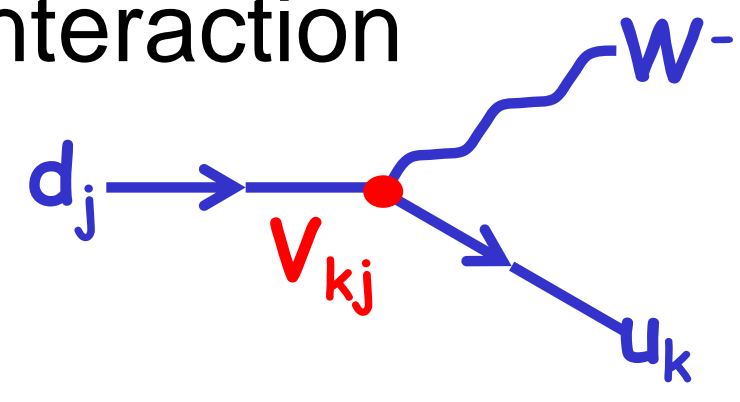

CKM Matrix

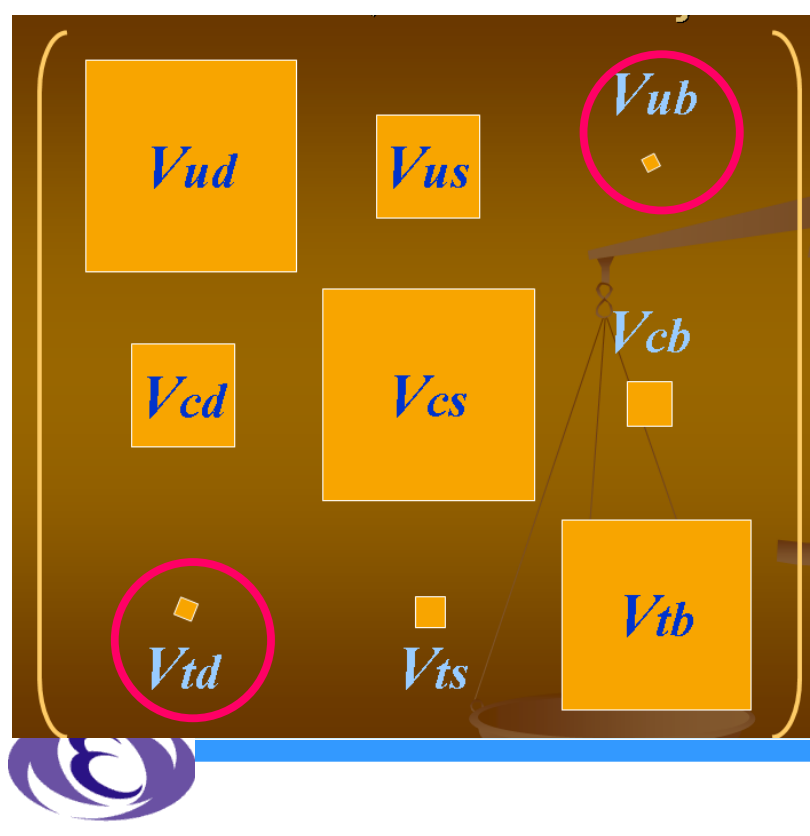

Irreducible Complex Phase

( 3 Generation )

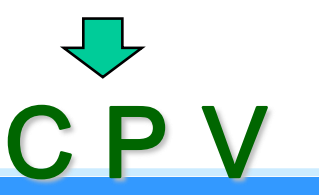

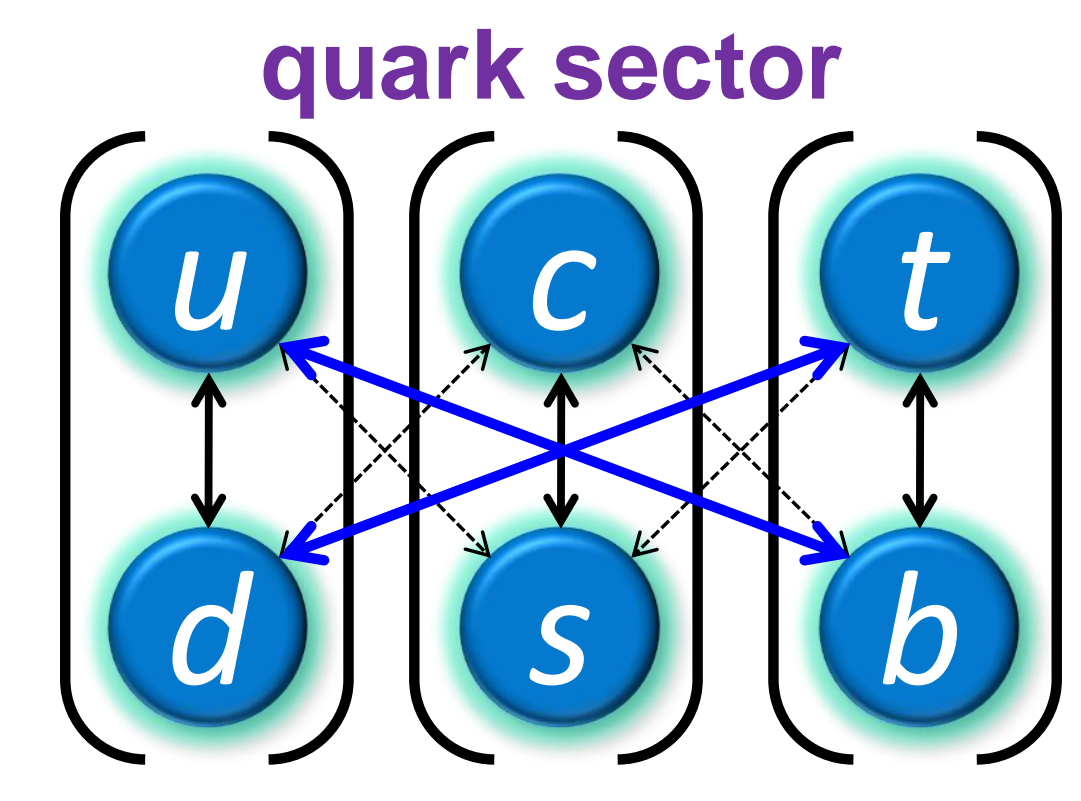

( Unitarity Triangle )
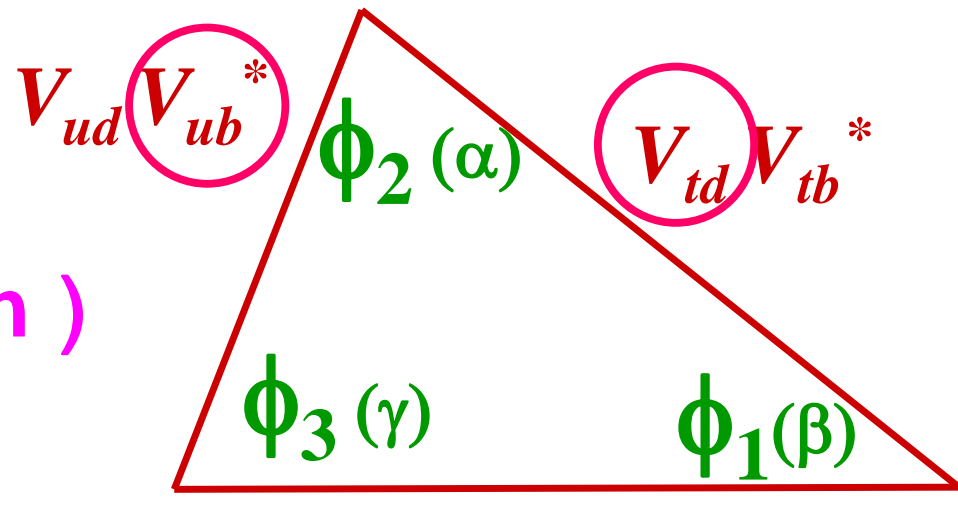

$$
V_{c d} V_{c b}^{*} \quad 8
$$




\section{KEKB accelerator}

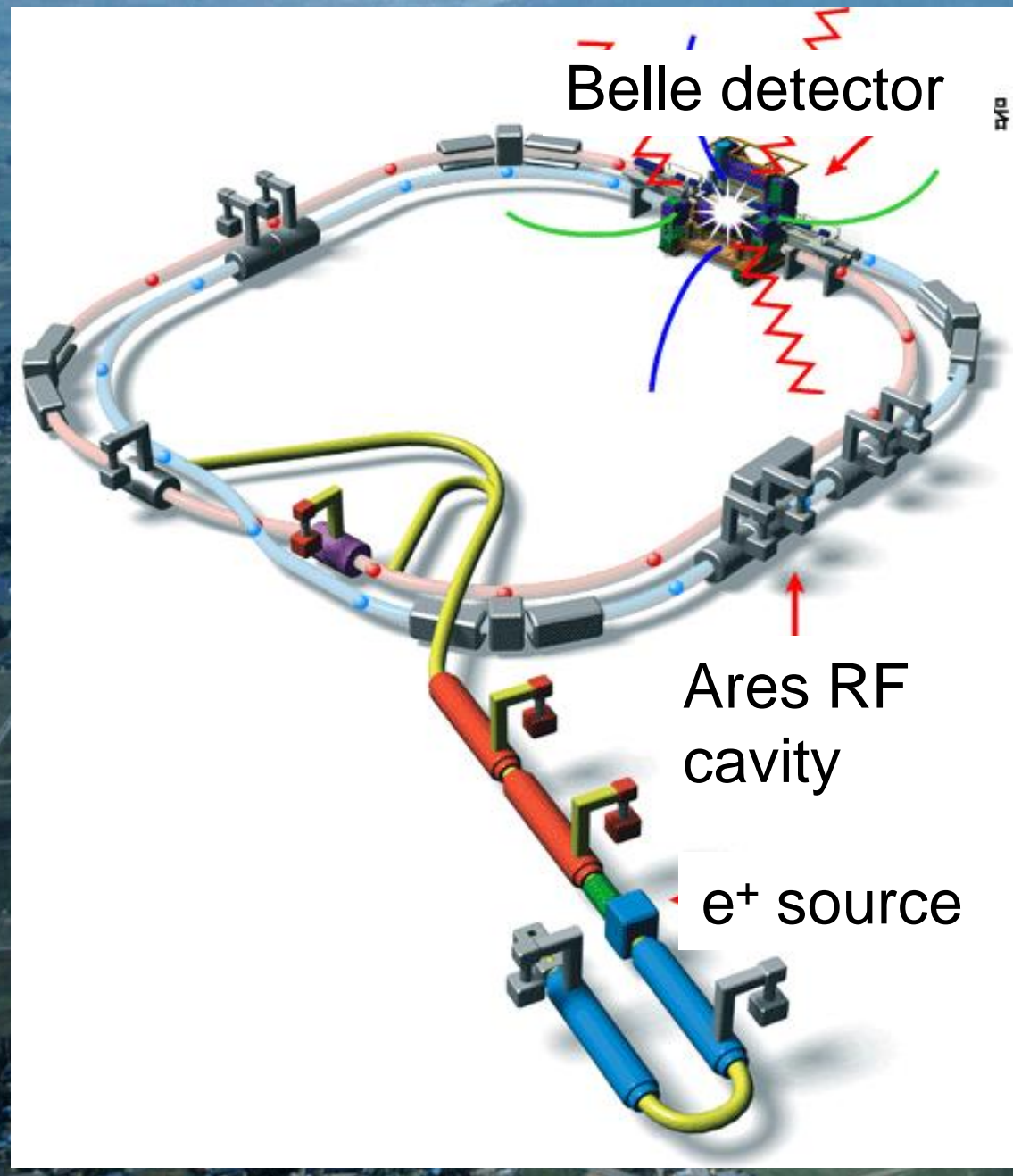

- Asymmetric energy collider ( $8 \mathrm{GeV}$ e- x $3.5 \mathrm{GeV}$ e+)

- Finite angle beam crossing (22mrad)

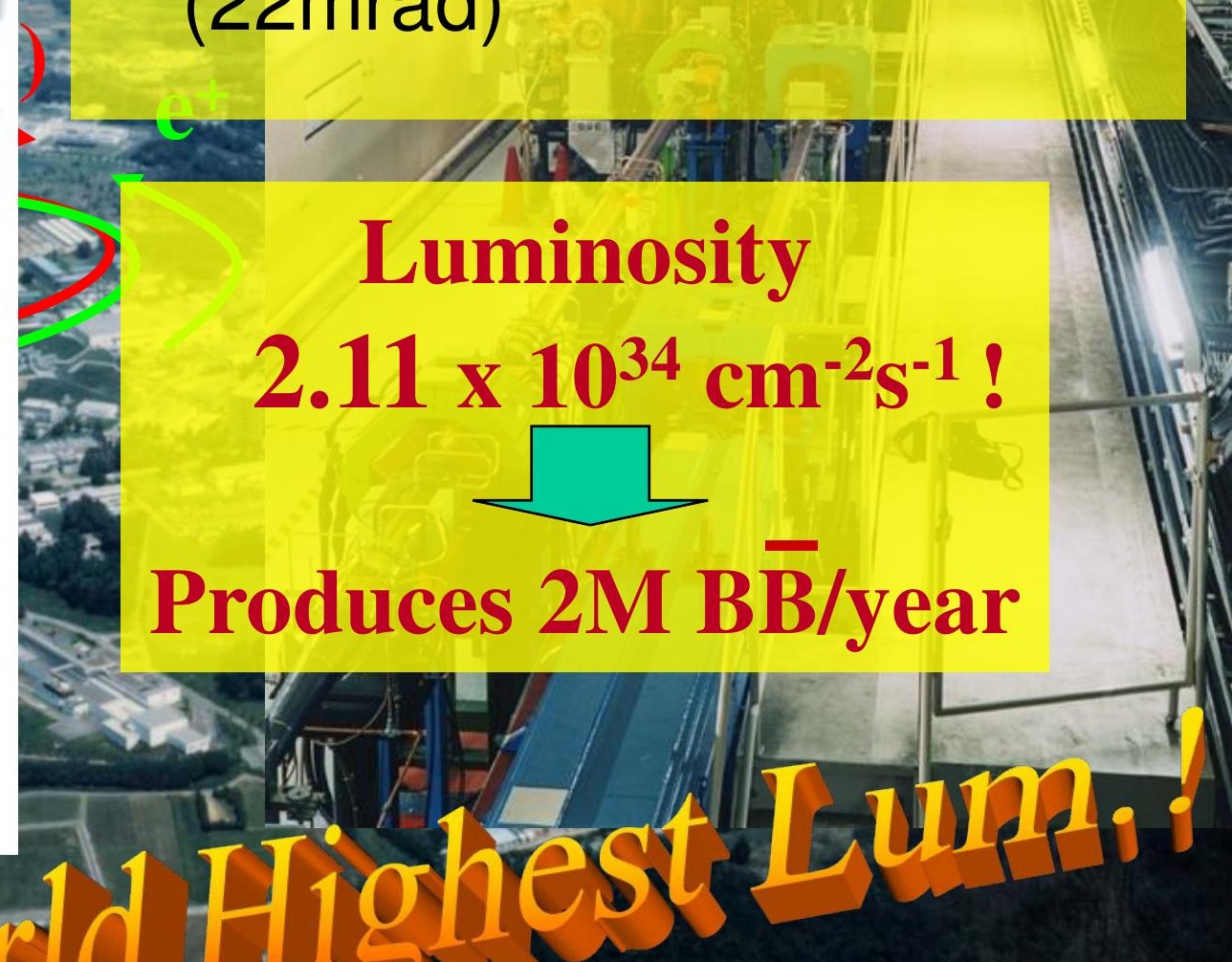




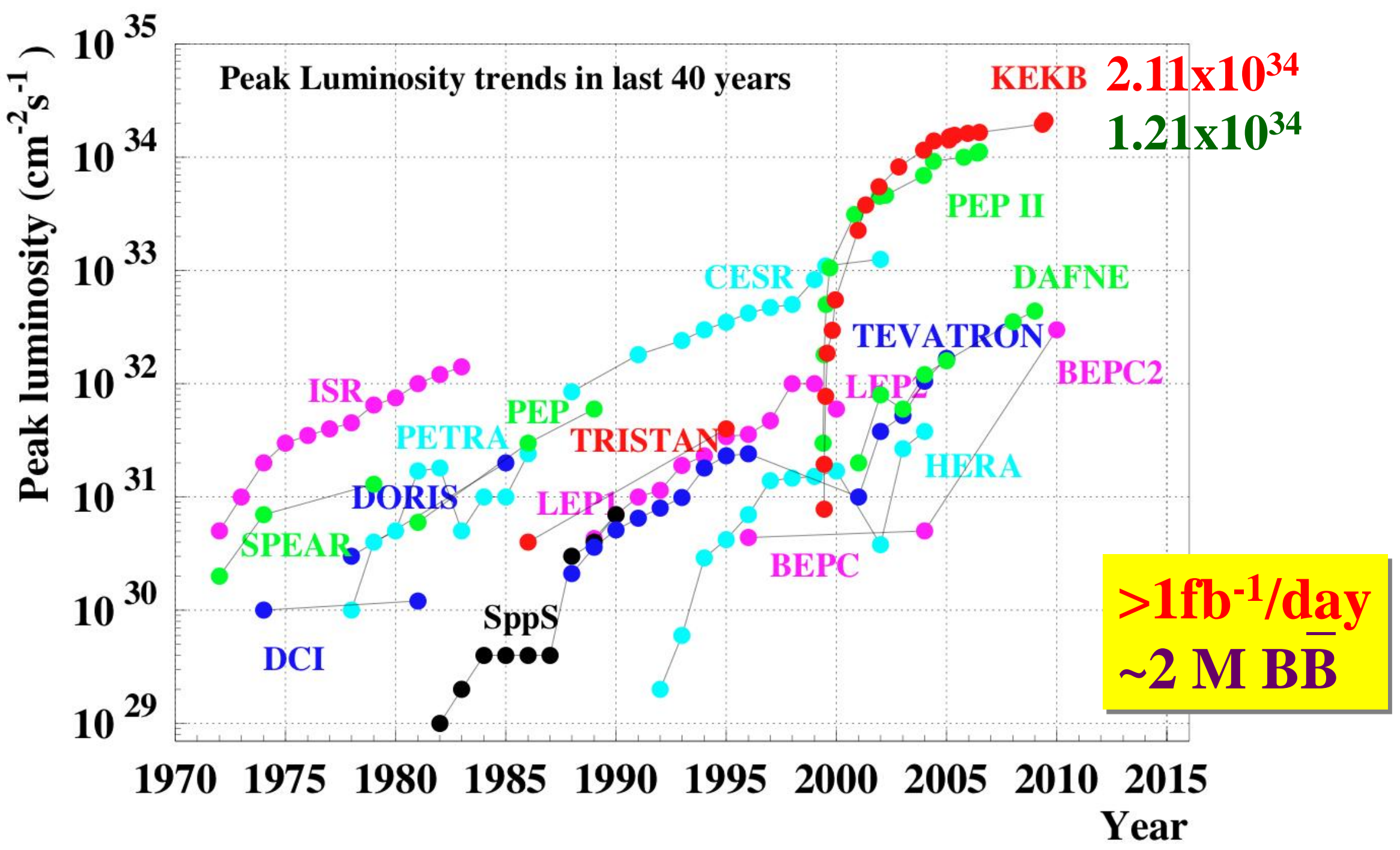




\section{Data at KEKB/Belle}

$\left(\mathrm{fb}^{-1}\right)$

1200

1000

800
600

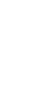

400
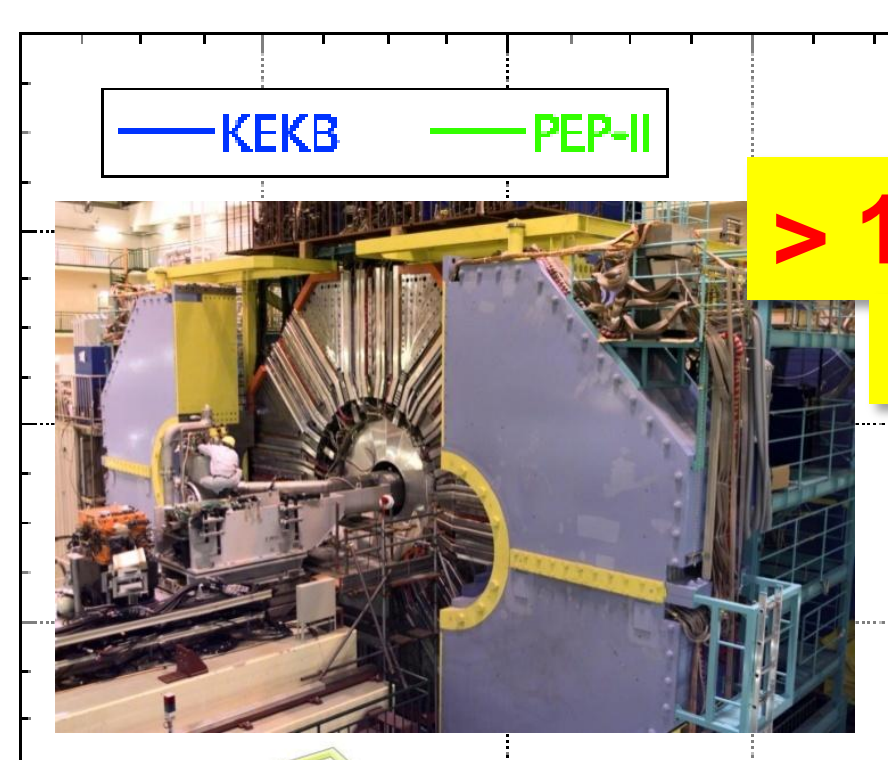

200

0

1998/1 2000/1 2002/1 2004/1 2006/1 2008/1 2010/1 2012/1
On resonance: $Y(5 \mathrm{~S}) \cdot 121 \mathrm{fb}^{-1}$ $Y(4 \mathrm{~S}): 711 \mathrm{fb}^{-1}$ $Y(3 \mathrm{~S}): 3 \mathrm{fb}^{-1}$ $Y(2 \mathrm{~S}): 24 \mathrm{fb}^{-1}$ $Y(1 \mathrm{~S}): 6 \mathrm{fb}^{-1}$ Off reson./scan $\sim 100 \mathrm{fb}^{-1}$

$$
\sim 550 \mathrm{fb}^{-1}
$$

On resonance: $Y(4 \mathrm{~S}): 433 \mathrm{fb}^{-1}$ $Y(3 \mathrm{~S}): 30 \mathrm{fb}^{-1}$ $Y(2 \mathrm{~S}): 14 \mathrm{fb}^{-1}$ Off resonance: $\sim 54 \mathrm{fb}^{-1}$ 



\section{CPV in $\mathrm{B}^{0}$ decays}
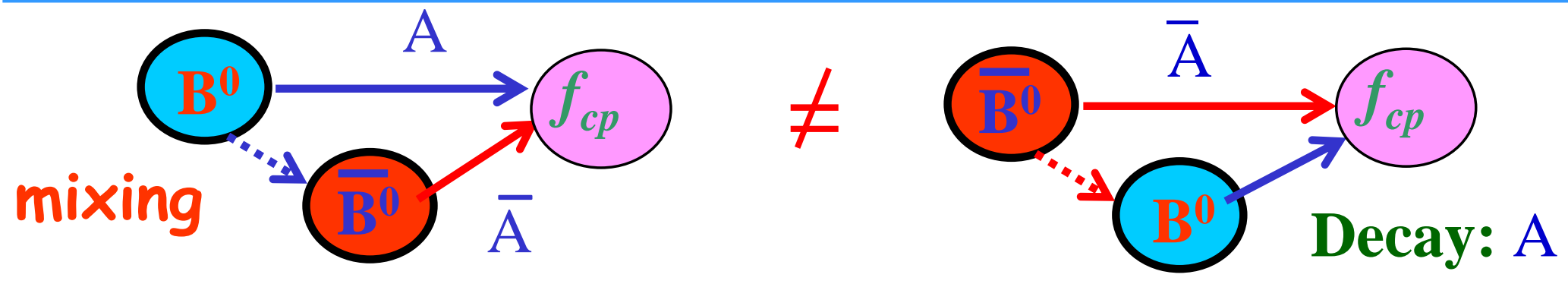

Sanda

Bigi

Carter

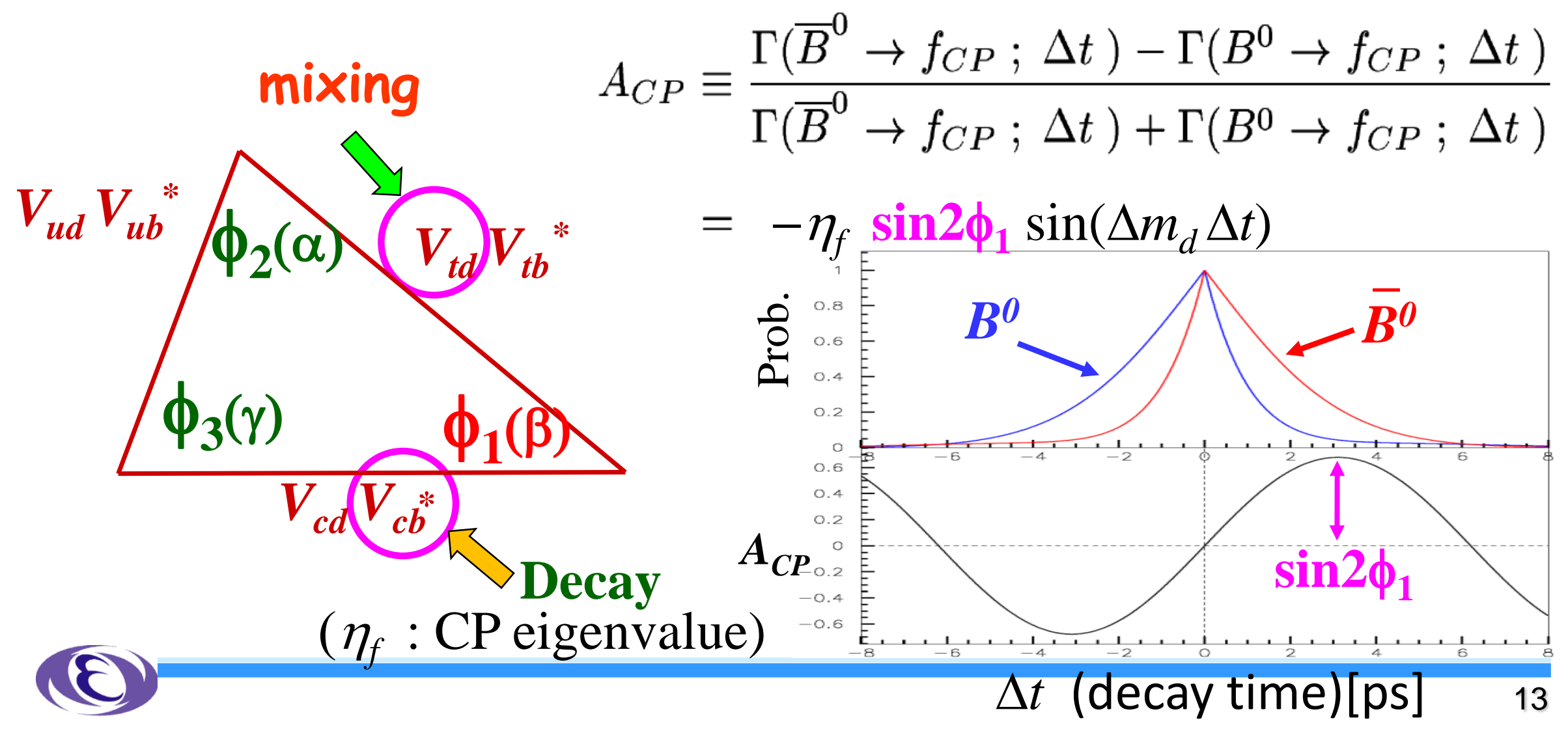




\section{Discovery of CPV in B decays}

$$
B^{0} \rightarrow J / \psi K^{0}
$$

\section{1(31M BB') Discovery!}

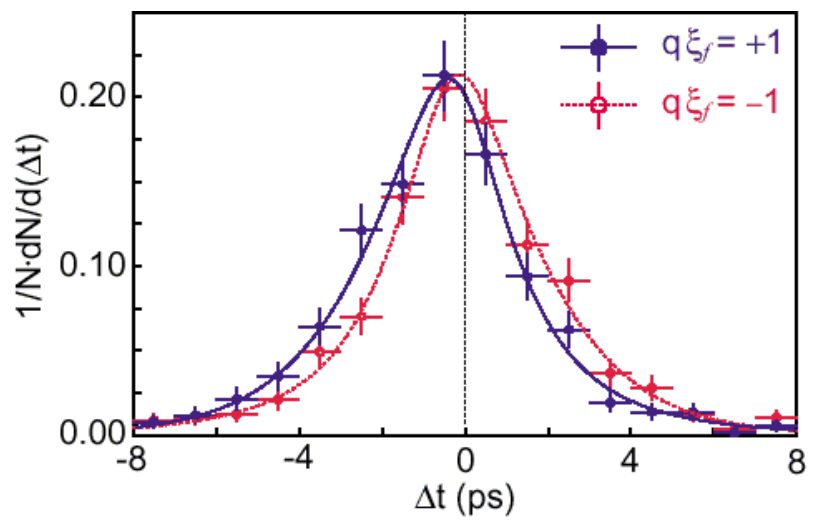

$$
\begin{aligned}
A_{C P}(\Delta t) & =\frac{\Gamma\left(\bar{B}^{0} \rightarrow f_{C P}\right)-\Gamma\left(B^{0} \rightarrow f_{C P}\right)}{\Gamma\left(\bar{B}^{0} \rightarrow f_{C P}\right)+\Gamma\left(B^{0} \rightarrow f_{C P}\right)} \\
& =-\xi_{f} \sin 2 \phi_{1} \sin \Delta m \Delta t
\end{aligned}
$$

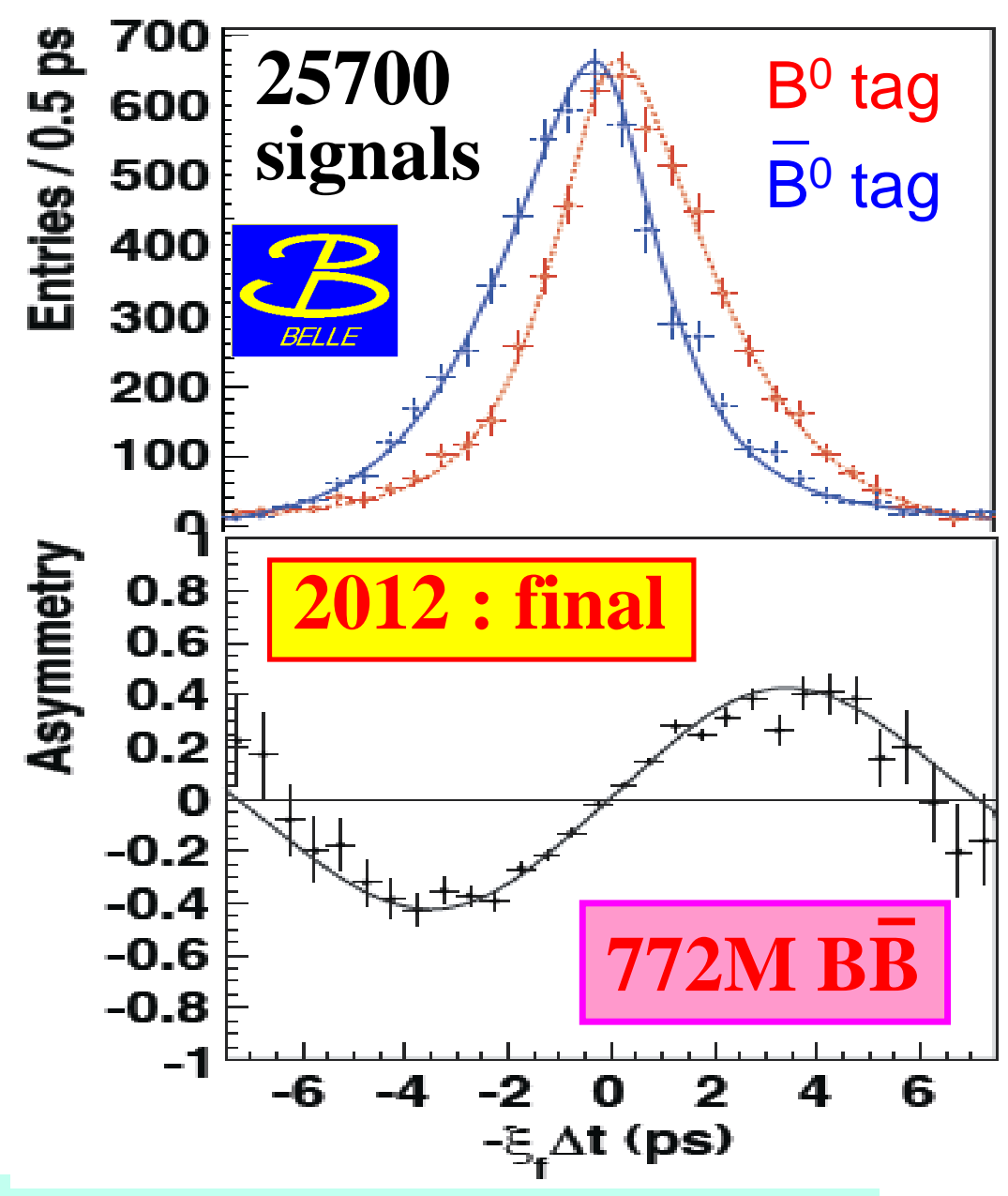

Precise Measurement [PRL 108, 171802] $\sin 2 \phi_{1}=0.667 \pm 0.023$ (stat) \pm 0.012 (syst) 


\section{Complete Test of KM \& SM}

\section{Measurements of CKM}

$$
\mathrm{B} \rightarrow \pi \pi, \rho \pi, \rho \rho
$$

$b \rightarrow u l v$

$\mathrm{B} \rightarrow \pi / \rho l v$

$$
\begin{gathered}
V_{u d} \\
{ }_{m} K^{-}
\end{gathered}
$$

$$
\begin{aligned}
& B^{-} \rightarrow D_{c o m} K^{-} \\
& B^{0} \rightarrow D^{(*)+} \pi
\end{aligned}
$$

\section{Determination of UT}

LQCD: important roles

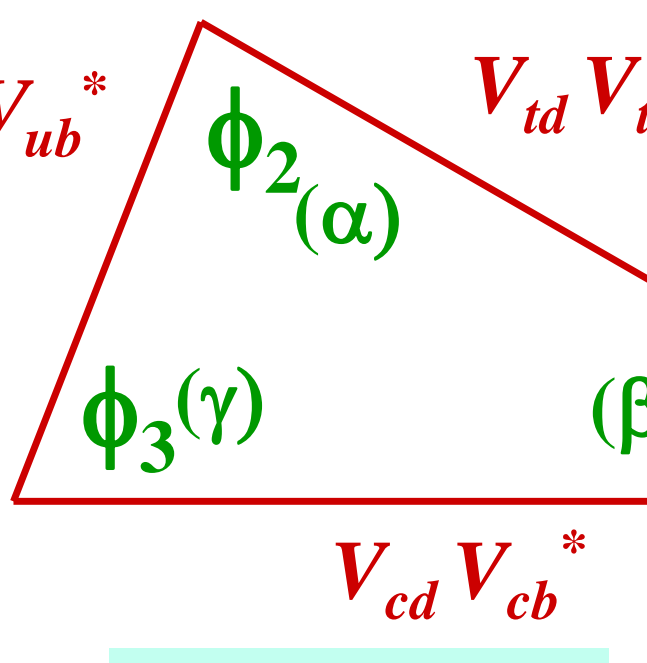

$$
\begin{aligned}
& b \rightarrow c l v \\
& \mathbf{B} \rightarrow \mathrm{D}^{(*)} l v
\end{aligned}
$$

$B^{0}$-mixing $\left(\Delta m_{d}\right)$

$B \rightarrow \rho \gamma$

(ß) $\phi_{1}$

$$
\begin{aligned}
& \mathbf{B}^{\mathbf{0}} \rightarrow(\mathbf{c c}) \mathbf{K}^{(*) 0} \\
& \left.\mathbf{B}^{\mathbf{0}} \rightarrow \mathbf{D}^{*+} \mathbf{D}^{(*)-(} \mathbf{K}\right)
\end{aligned}
$$

Over constraint !

B experiments can provide all measurements ! 


\section{Verification of KM for CPV}

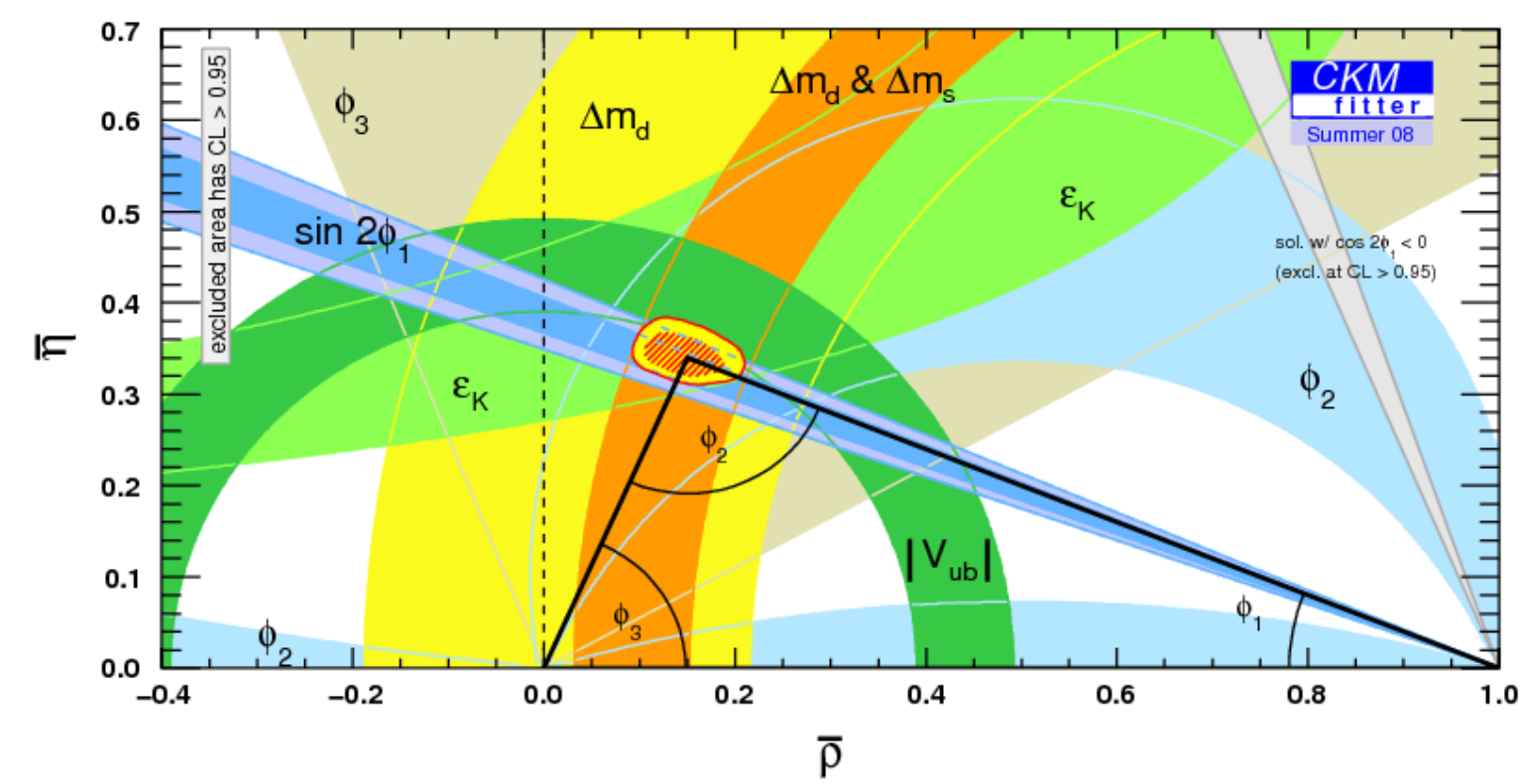

All consistent

CPV:

caused by a single phase of CKM matrix
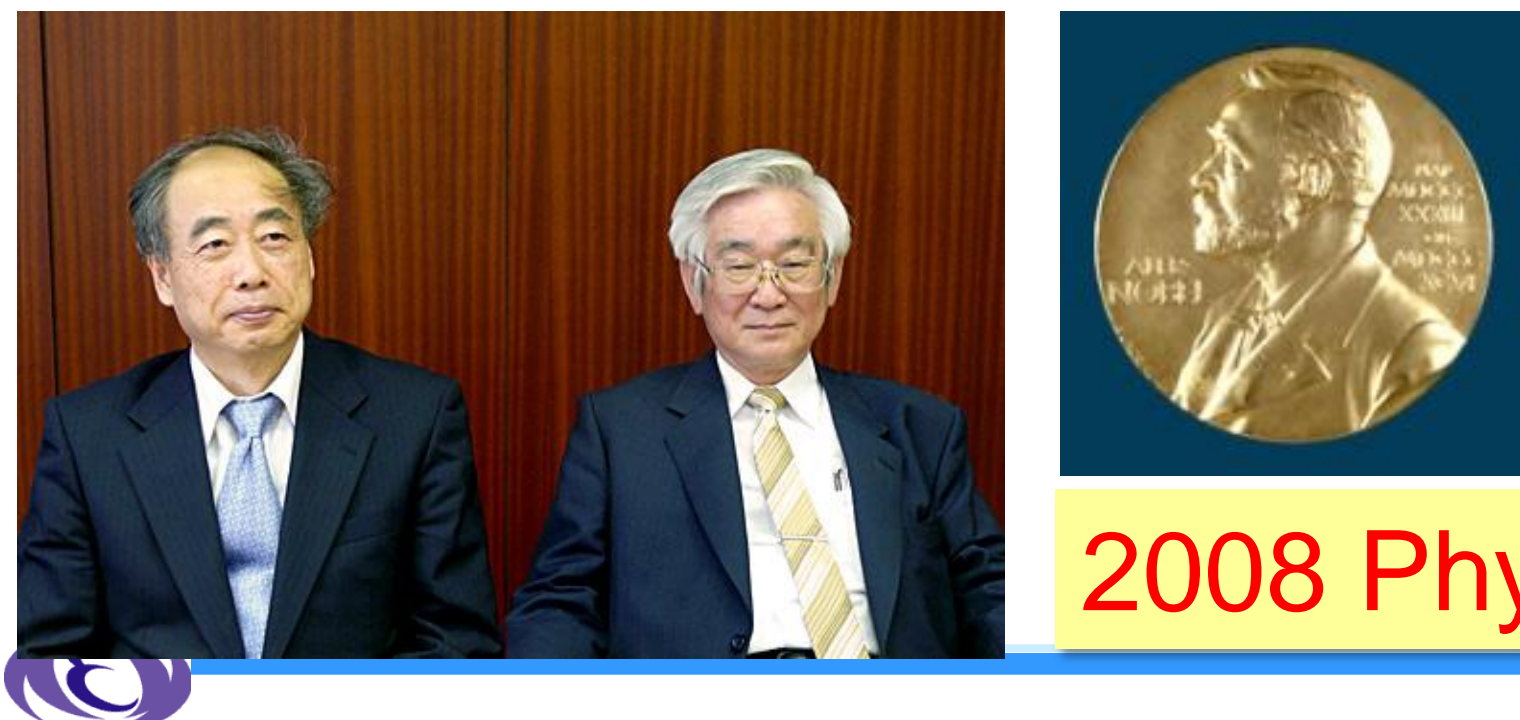

Verified by B-factory experiments

2008 Physics Nobel Prize 


\section{Next Challenge}

In spite of Great Success of SM, there must be

New Physics beyond SM at High Energy scale

(SM is valid effective theory at current $\mathrm{E}$-scale)

Observed CPV in SM is not enough to explain matter dominance of Universe $\left[>0\left(10^{10}\right)\right]$ !

New Source of CPV should exists (beyond SM)

One of Next important goals of Flavor Physics

$$
\Leftrightarrow \text { Energy Frontier }
$$

Note) NP effects appear in Flavor Physics in various way! 


\section{Search for New Physics}

CPV in B provide Powerful tool for Search NP ( New Phase ) [ $b \rightarrow s q q \mathrm{tCPV}]$

Rare B decays excellent opportunities for NP search Loop diagram Penguins $\left[\mathrm{b} \rightarrow \mathrm{s}(\mathrm{d}) \gamma, \mathrm{b} \rightarrow \mathrm{s}(\mathrm{d}) l^{+} l^{-}\right]$ Decays involving $\tau\left(\leftrightarrow \mathbf{H}^{ \pm}\right)$

$$
A_{\mathrm{NP}} \sim A_{\mathrm{SM}} \text { (small/forbidden) }
$$

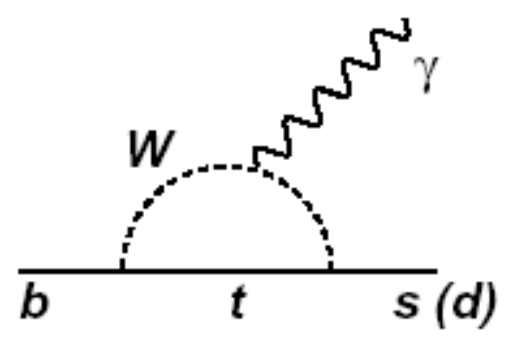

$\tau$ Decays $($ Lepton Flavor Violation $=\mathrm{NP}): \mathrm{B}$-factory $=\tau$-factory

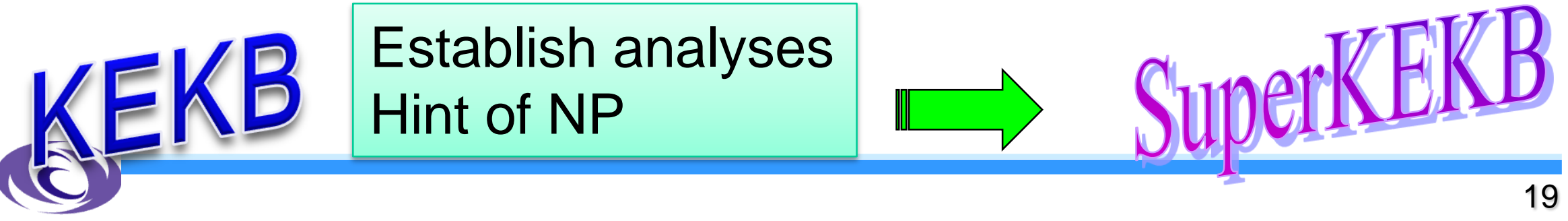




\section{Energy Frontier vs Flavor Physics}

Direct Production by High Energy Coll.

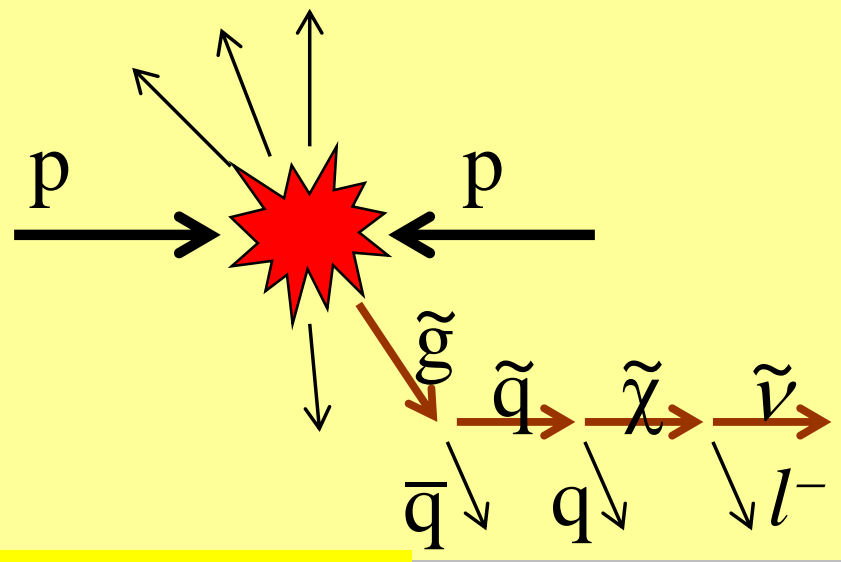

Energy Frontier
Virtual Production via Quantum Eff.

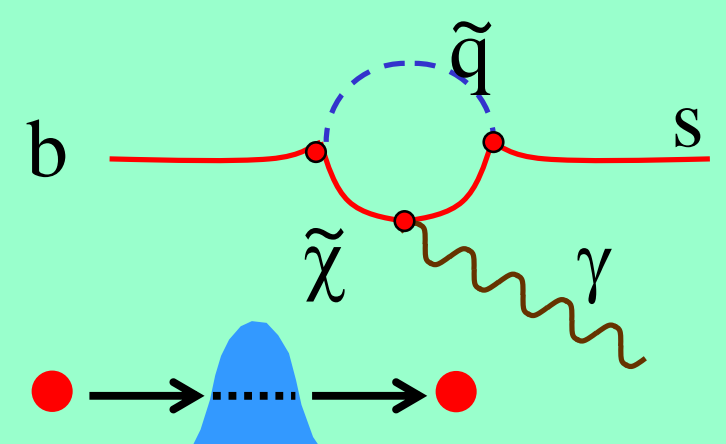

Tunnel effect

Luminosity Frontier

Diagonal terms $\rightarrow m_{11}^{2} \quad m_{12}^{2} \quad m_{13}^{2}$

$$
\left(m_{\tilde{q}}^{2}\right)_{i j}=\left(\begin{array}{lll}
m_{21}^{2} & m_{22}^{2} & m_{23}^{2} \\
m_{31}^{2} & m_{32}^{2} & m_{33}^{2}
\end{array}\right.
$$

Off-diagonal terms

Higher Energy Scale Can be searched (even if LHC finds no New Physics) 


\section{LHC vs SuperKEKB}

Illustrative reach of NP searches

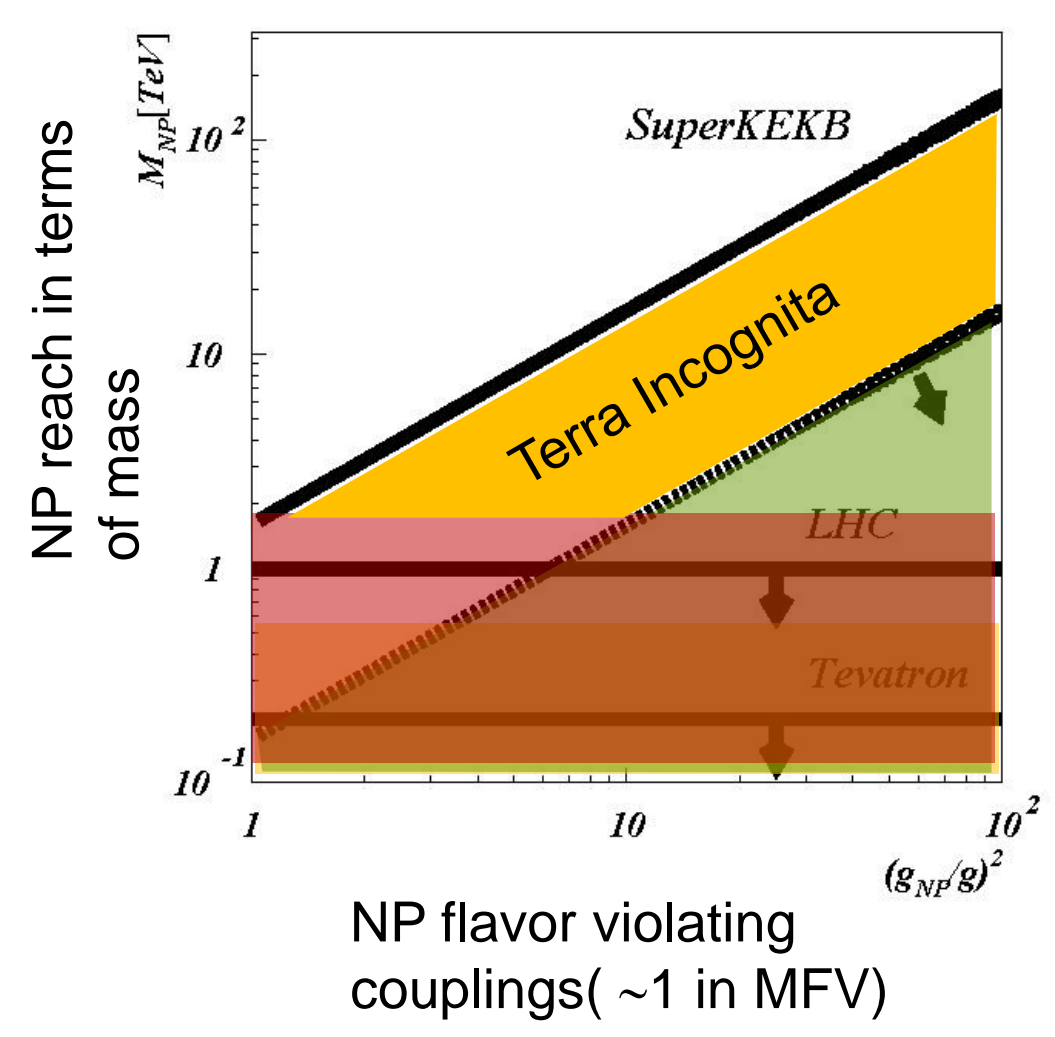

LHCb vs SuperKEKB

$\mathrm{LHCb}$ is producing nice Flavor Physics results

$>$ SuperKEKB: can do Missing-E, Inclusive Neutrals

$>$ Single measurement only Confirmation is important

[ similar in LQCD ] 


\section{Hints/Sensitive to NP}

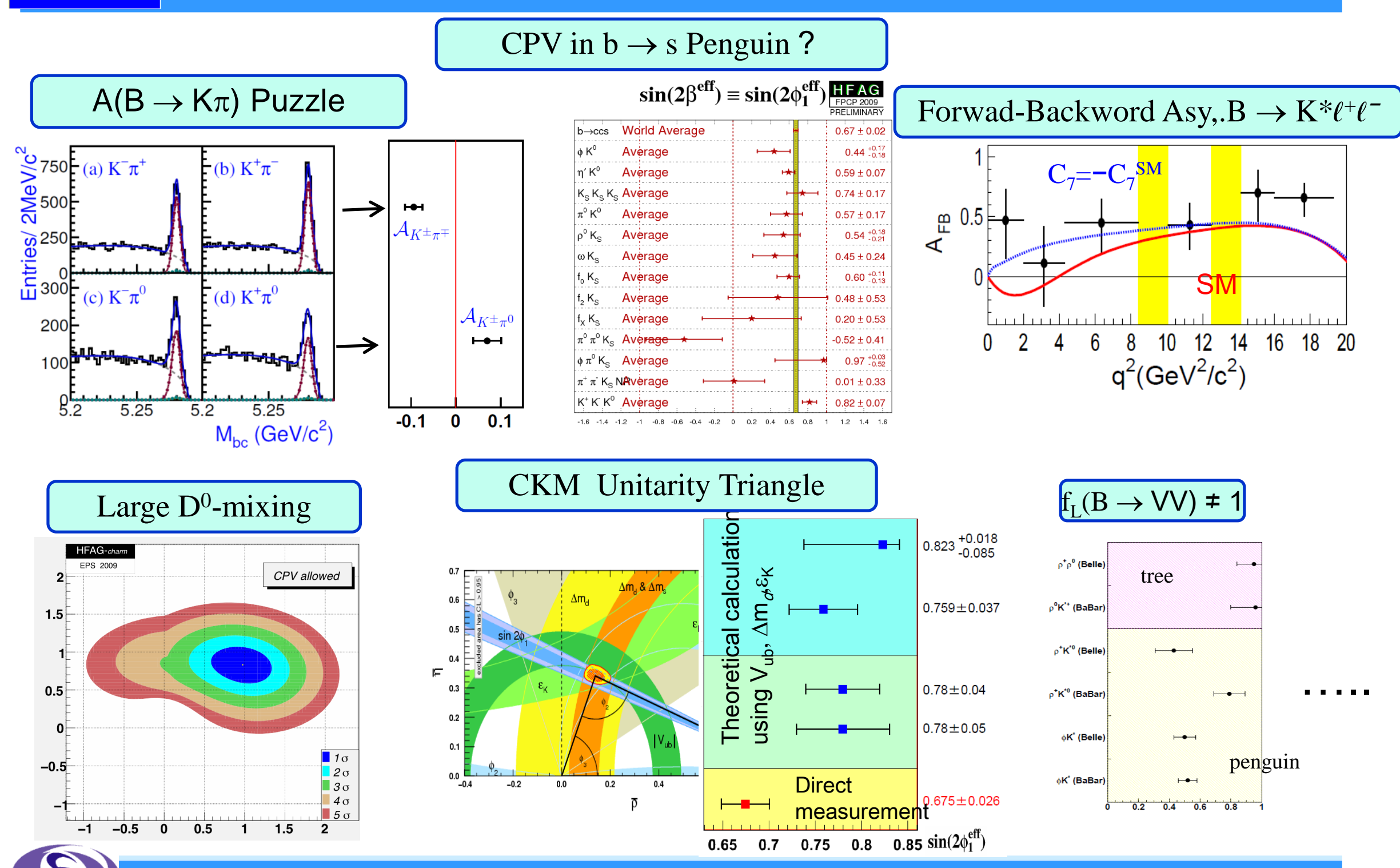




\section{NP search : Precise CKM}

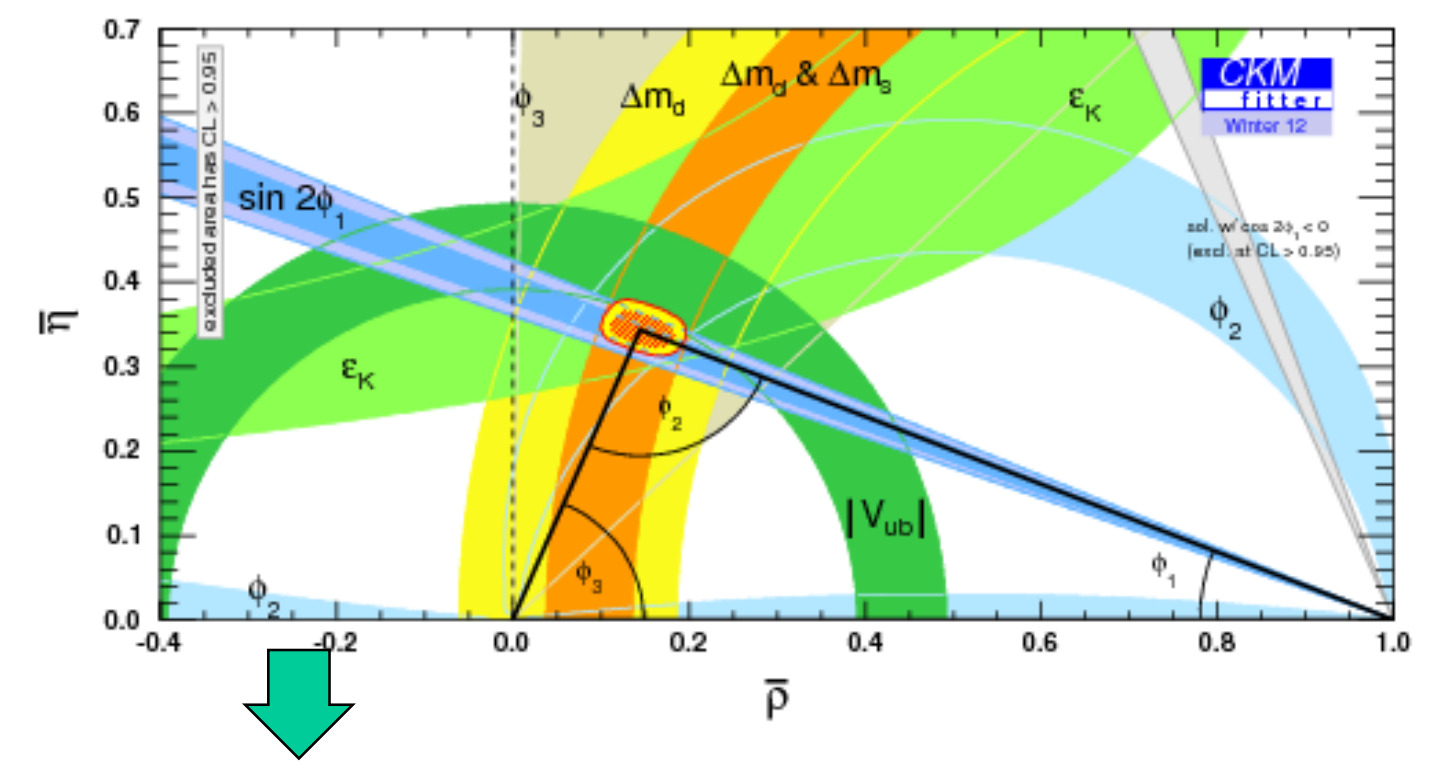

\section{Still $10 \%$ room for NP}
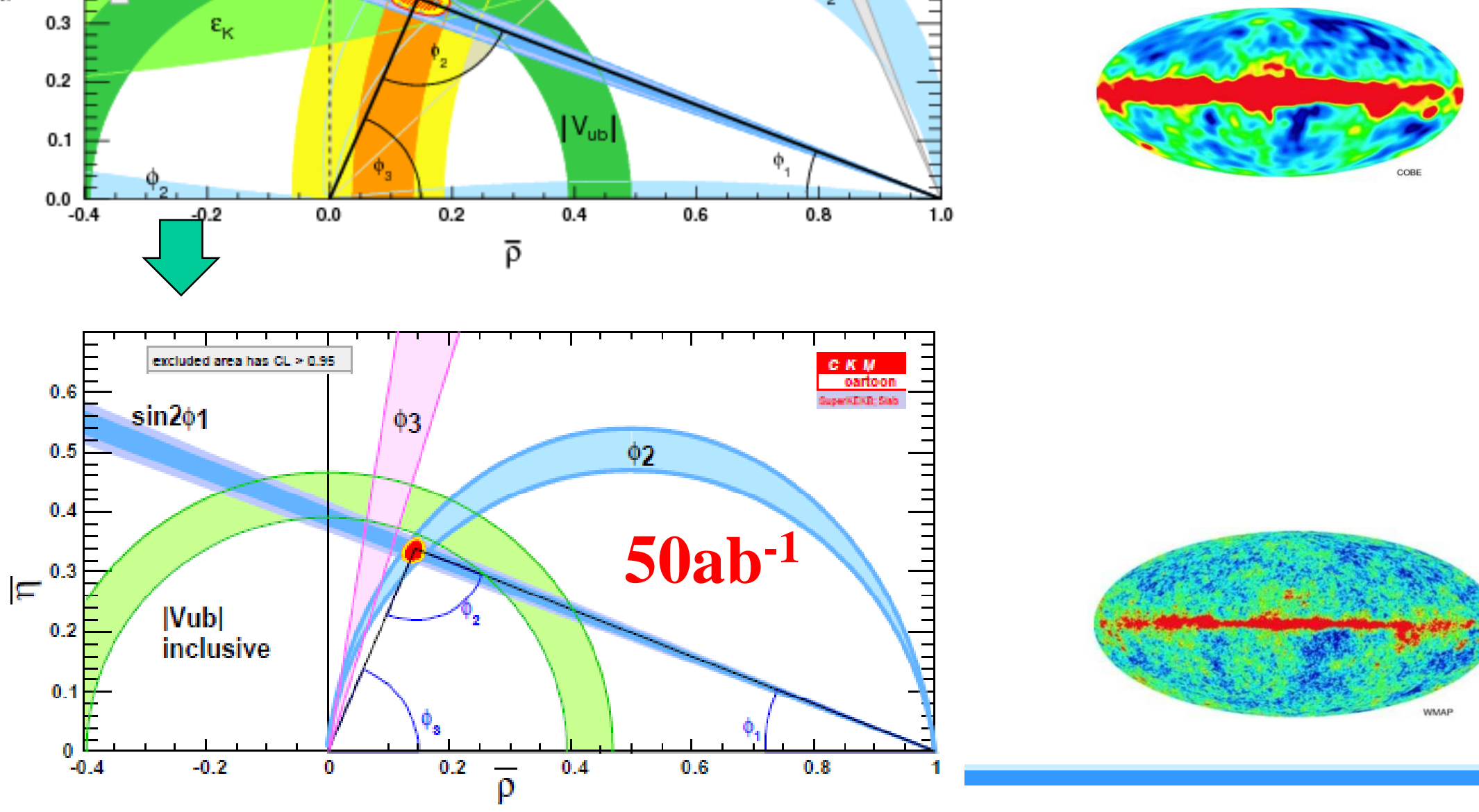


\section{New Source of CPV: $b \rightarrow s q \bar{q}$}

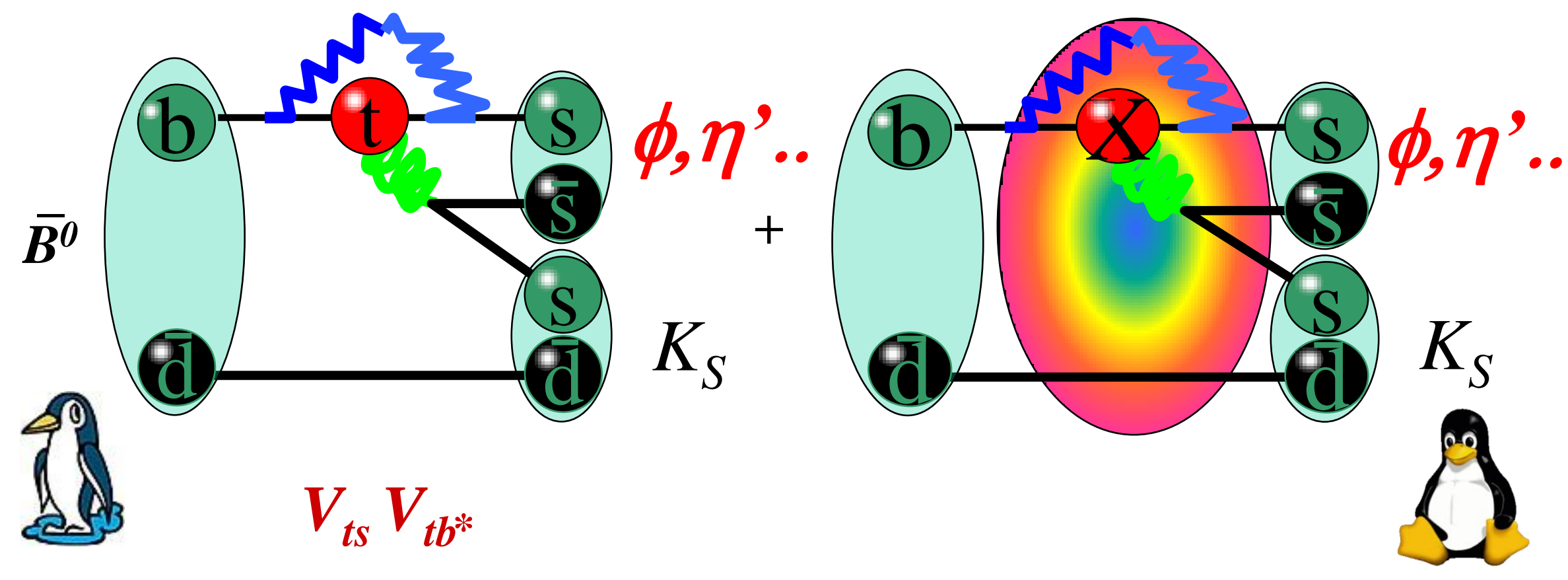

SM: $b \rightarrow$ s Penguin phase $=(\mathbf{c} \overline{\mathbf{c}}) \mathbf{K}^{0}$

\section{+ New Physics with New Phase $\mathrm{S}_{\mathrm{bs}} \neq \mathrm{S}_{\mathrm{bc}}, \mathrm{A}_{\mathrm{DCP}}$ can $\neq 0$}

"b $\rightarrow$ ccs: $\sin 2 \phi_{1} "$ (SM reference) $\square$ deviation 


\section{Summary of New CPV search}
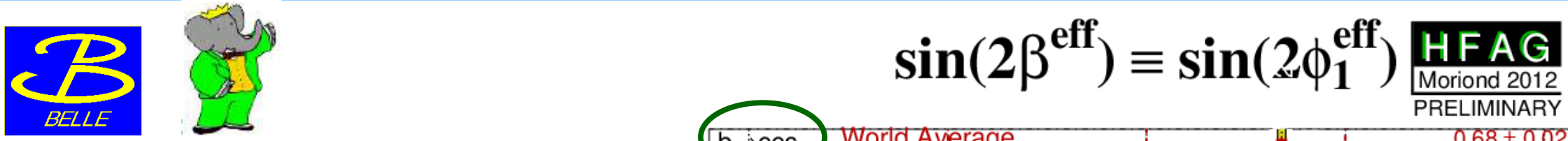

$B^{0} \rightarrow J / \psi K^{0}$

Reference point of SM

\section{No clear deviation seen in all modes $(1 \sim 2 \sigma)$}

New CPV effect can be seen with much larger data

\section{$\Rightarrow$ Super B-factory}

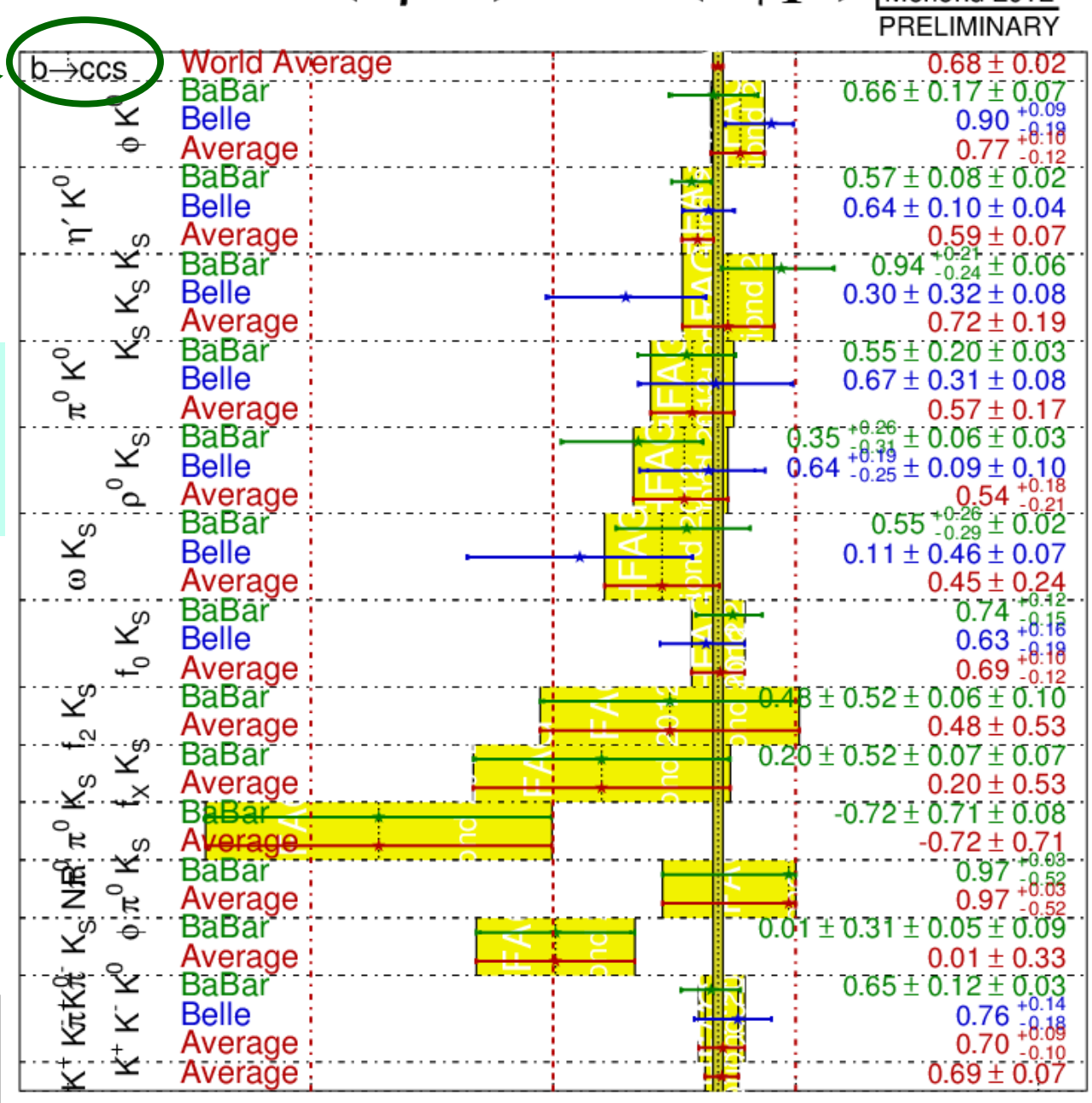

$-2$ 


\section{SuperKEKB prospect}

$B \rightarrow \phi K^{0}$ at 50/ab with $\sim 2010$ WA values
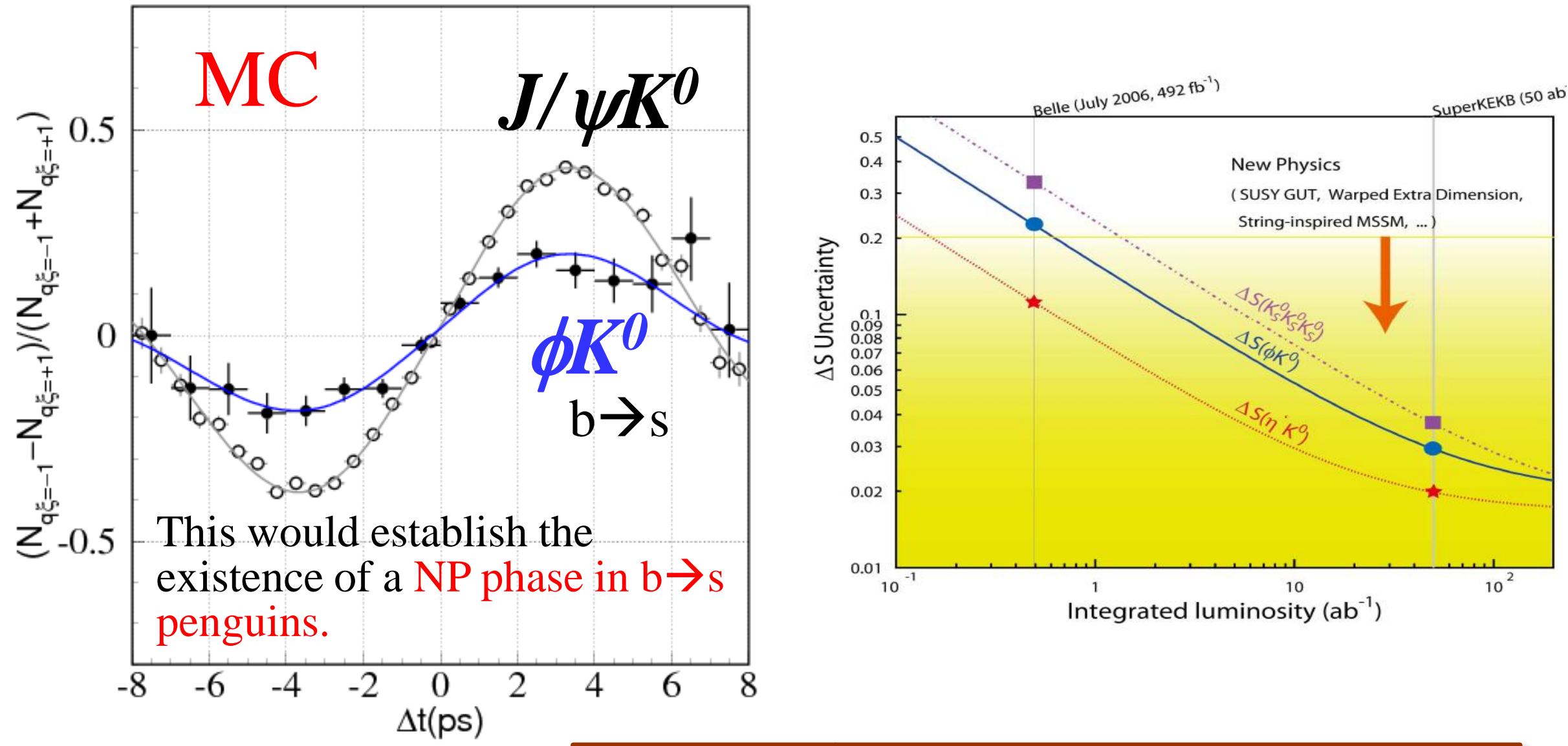

\section{Prospect $\delta\left(S_{b \rightarrow s}\right) \sim 0.012 @ 50 a^{-1}$}




\section{Charged Higgs Hunting}

B-Factory:

Variety of Modes sensitive to Charged Higgs

Some are only possible at B-Factory 


\section{Inclusive $\mathbf{b} \rightarrow \mathbf{s} \gamma$}
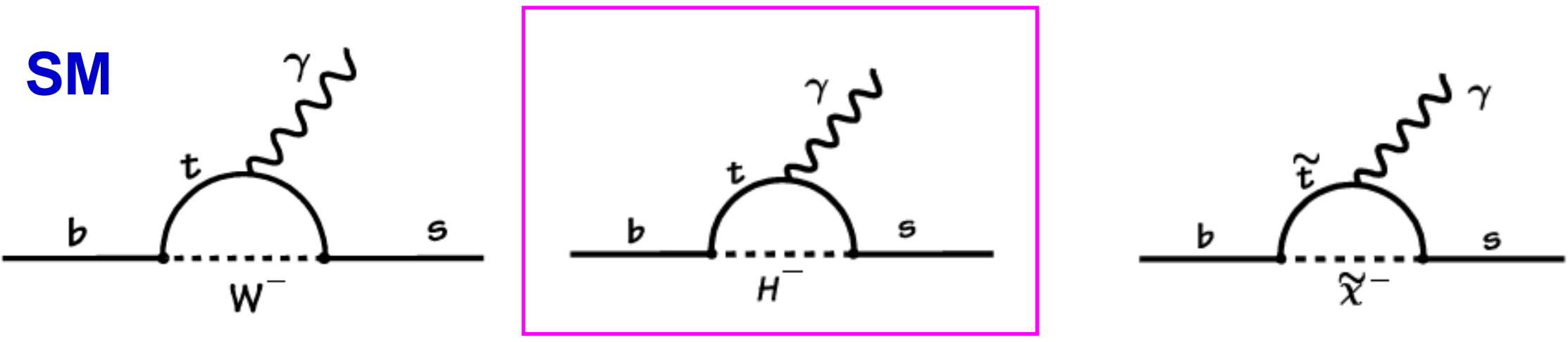

Fully Inclusive measurement
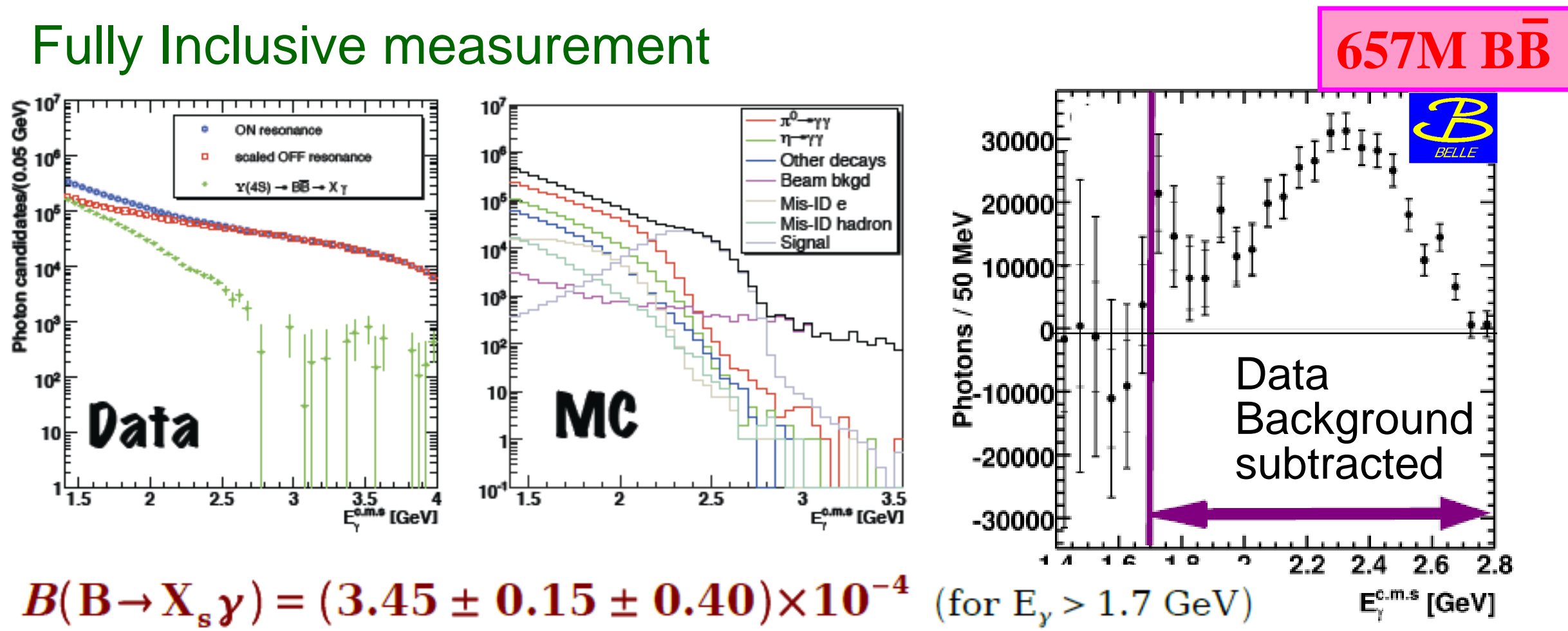

$B\left(B \rightarrow X_{\mathrm{s}} \gamma\right)=(3.45$

Most precise measurement of $B\left(\mathrm{~B} \rightarrow \mathrm{X}_{\mathrm{s}} \gamma\right)$ (lowest $\mathrm{E}_{\gamma}$ threshold) 


\section{b $\rightarrow$ s $\gamma$ Summary}

HFAG 2010: $B\left(\mathrm{~B} \rightarrow \mathrm{X}_{\mathrm{s}} \gamma\right)=(3.55 \pm 0.26) \times 10^{-4}\left(\right.$ for $\left.\mathrm{E}_{\gamma}>1.6 \mathrm{GeV}\right)$ VS

$\mathrm{SM}: \quad B\left(\mathrm{~B} \rightarrow \mathrm{X}_{\mathrm{s}} \gamma\right)=(3.15 \pm 0.23) \times 10^{-4}\left(\right.$ for $\left.\mathrm{E}_{\gamma}>1.6 \mathrm{GeV}\right)$

CLEO [9.1 $\left.\mathrm{fb}^{-1}\right]$ (2001) untag

BaBar $\left[82 \mathrm{fb}^{-1}\right]$ (2005) sum-of-excl BaBar $\left[82 \mathrm{fb}^{-1}\right]$ (2007) lep-tag

BaBar $\left[210 \mathrm{fb}^{-1}\right]$ (2008) breco-tag

Belle $\left[5.8 \mathrm{fb}^{-1}\right]$ (2001) sum-of-excl Belle $\left[605 \mathrm{fb}^{-1}\right]$ (2009) untag+lep-tag HFAG 2010

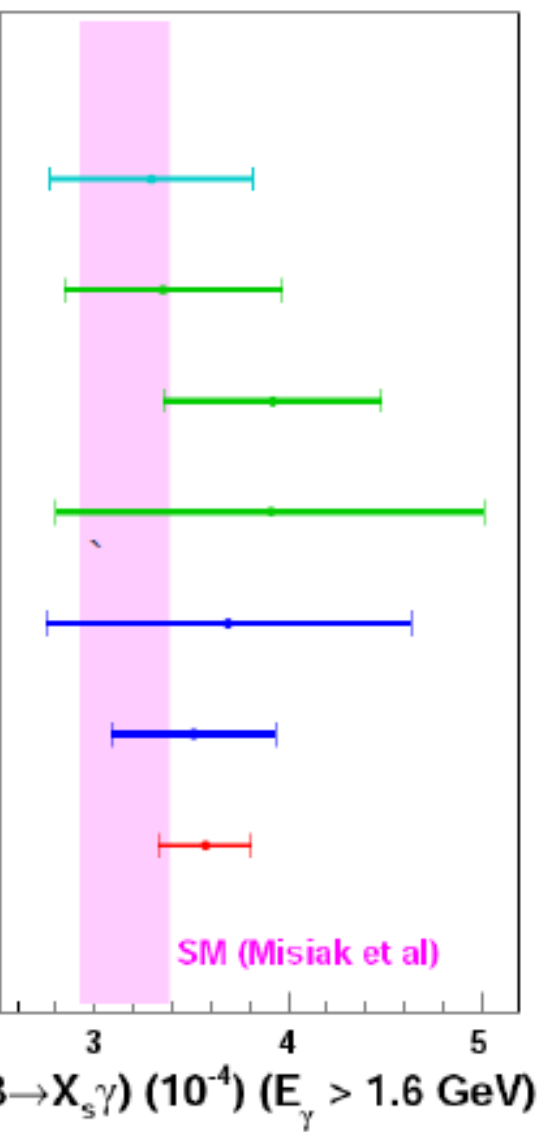

Charged Higss bound (2HDM TypeII) $\mathrm{M}_{\mathrm{H}^{+}}>300 \mathrm{GeV}$

M. Misiak et al., PRL98, 022002 (2007)

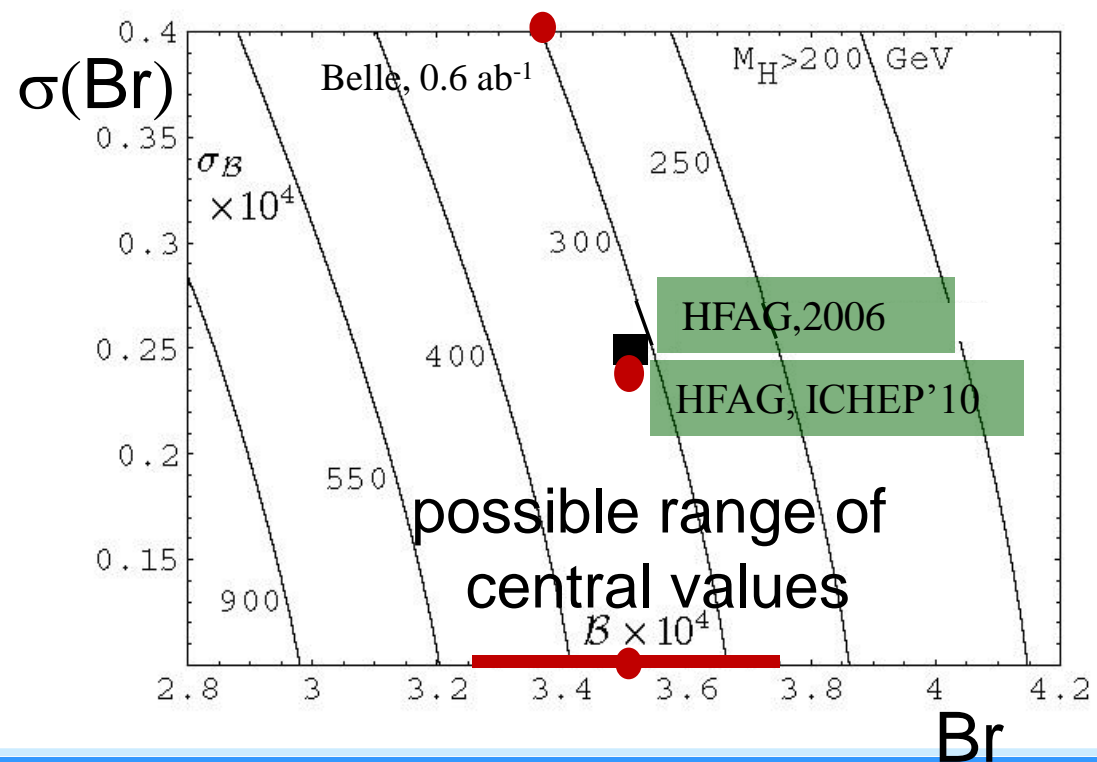




\section{$H^{+}$Search: $B^{+} \rightarrow \tau^{+} v_{\tau}$}

(Decays with Large Missing Energy)

Sensitivity to new physics from charged Higgs

$\mathrm{b}$ $1^{+}$

SM:

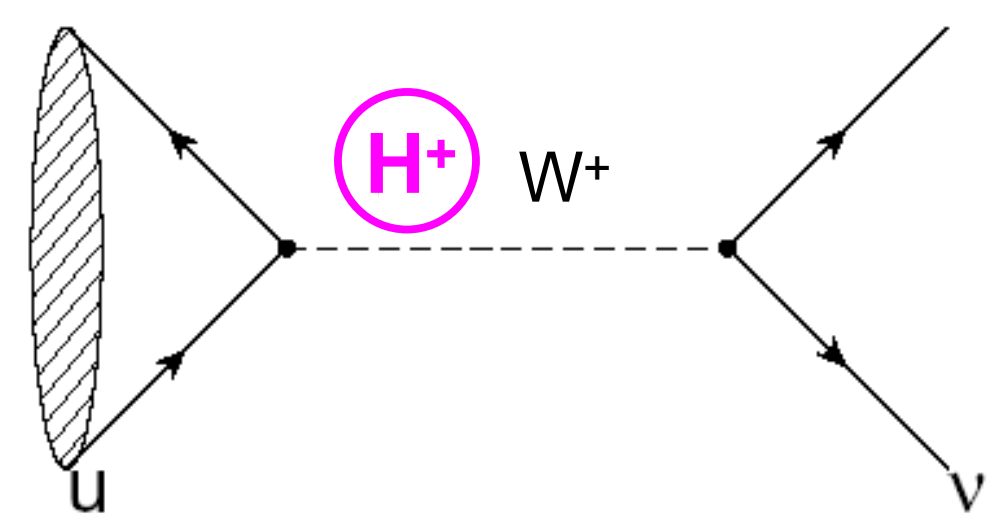

$$
\begin{array}{r}
\mathcal{B}\left(B^{+} \rightarrow \tau^{+} \nu_{\tau}\right)= \\
\text { The B meson decay constant } \\
\text { LQCD }
\end{array}
$$

\section{LQCD}

$\left|V_{u b}\right|$ : from indep. measurements. 


\section{$B->\tau v$ : Experimental Challenge}
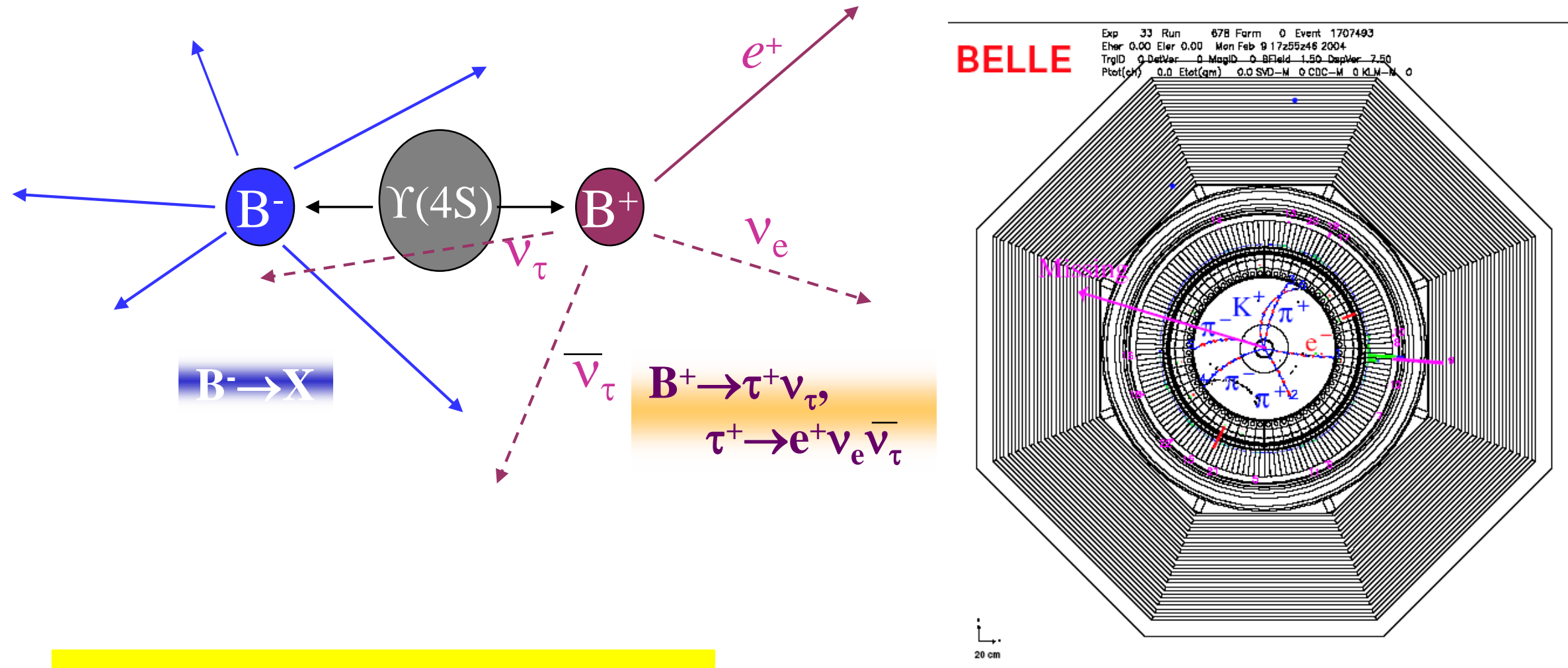

Always $\geq 2$ neutrinos appear in $B \rightarrow \tau v$ decay

Signature : 1 track +invisible

Experimental Challenge! 


\section{$B->\tau v$ : Experimental Challenge}
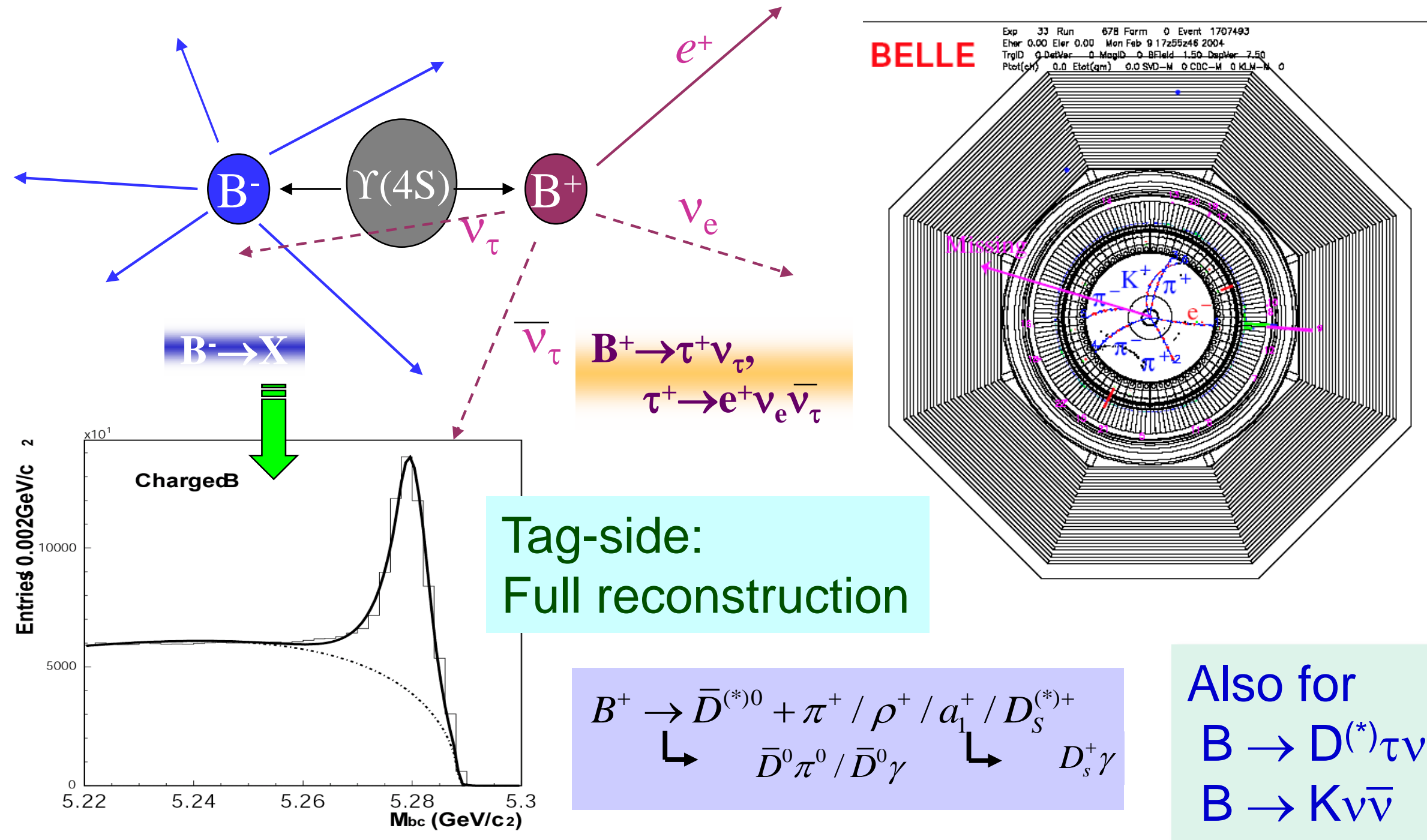

Full reconstruction

$$
\begin{aligned}
& B^{+} \rightarrow \bar{D}^{(*) 0}+\pi^{+} / \rho^{+} / a_{1}^{+} / D_{S}^{(*)+} \\
& \stackrel{\longrightarrow}{\longrightarrow} \bar{D}^{0} \pi^{0} / \bar{D}^{0} \gamma \quad \longrightarrow \quad D_{s}^{+} \gamma
\end{aligned}
$$

Also for

$\mathrm{B} \rightarrow \mathrm{D}^{(*)} \tau \nu$

$\mathrm{B} \rightarrow \mathrm{K} v \bar{v}$

Can be measured only by B-Factory! 


\section{$B->\tau v$ Results}

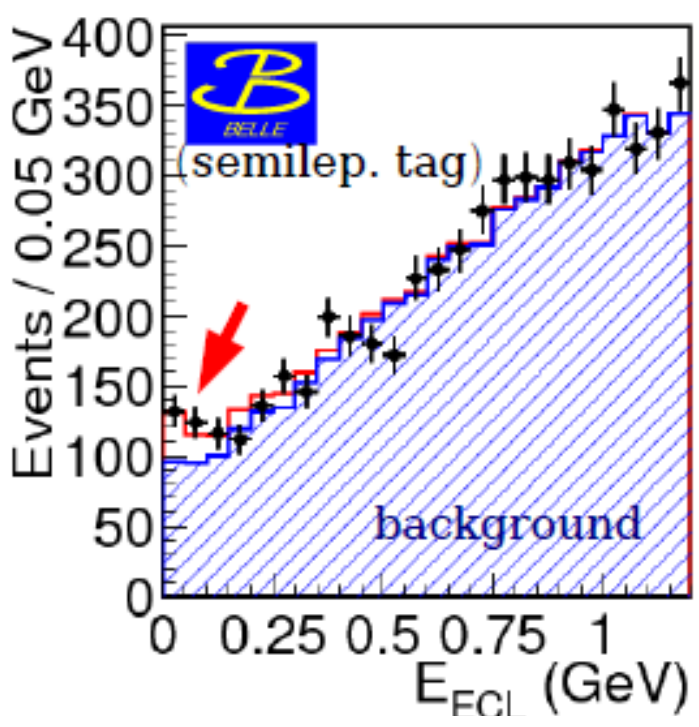

$\sigma(\mathcal{B}) \cdot 10^{-4}$

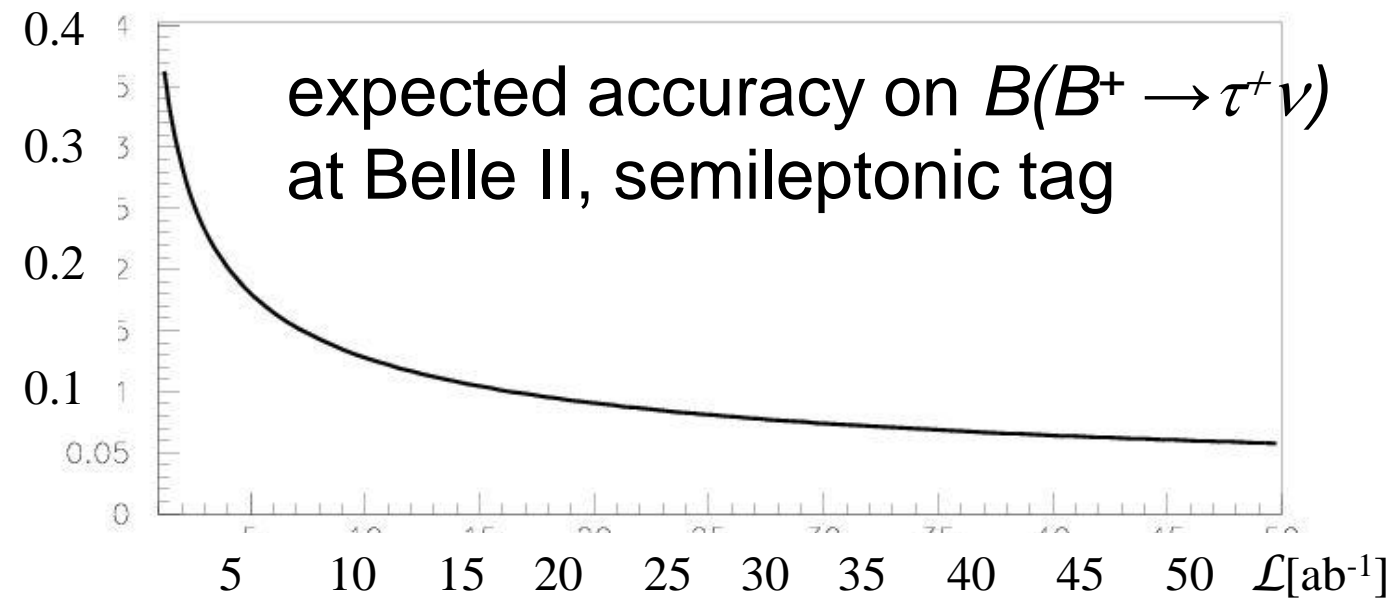

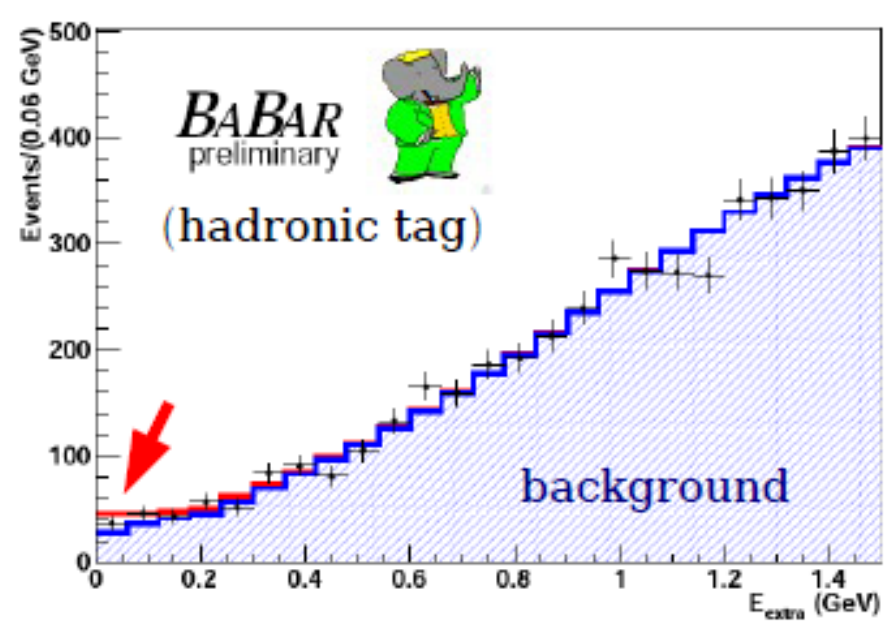

Extra calorimeter energy : $\mathrm{E}_{\mathrm{ECL} / \text { extra }}(\mathrm{GeV})$ 


\section{$\boldsymbol{B} \rightarrow \boldsymbol{D}^{(*)} \tau \boldsymbol{v}$}
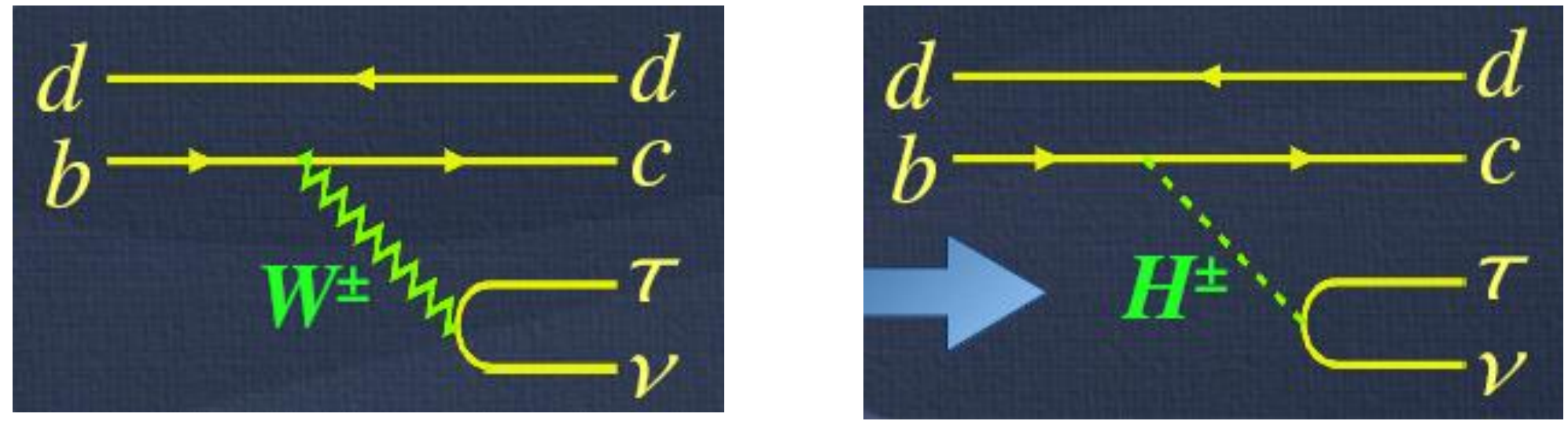

- $B \rightarrow D^{*} \tau v$ : Lepton $(\tau)$ polarization info.

Expected $\mathscr{B} \sim 1.4 \%$ in SM (large) [e.g. D.S.Hwang EPJ C14,271(2000)] But, large background $\left(D^{* * *} l \nu, D^{*} X\right)$

$>$ Always involve $\geq 2 v($ Missing E): 


\section{$B \rightarrow D^{(*)} \tau v$ Results}

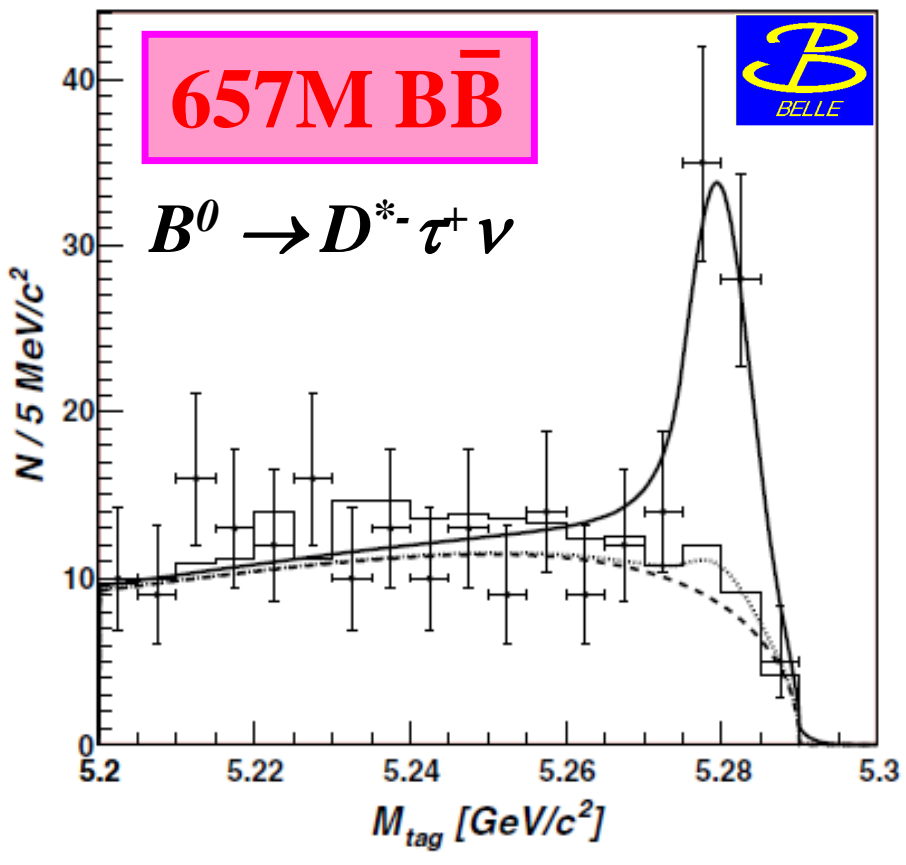

[PRL 99, 191807(2007)]

\section{First Observation !}
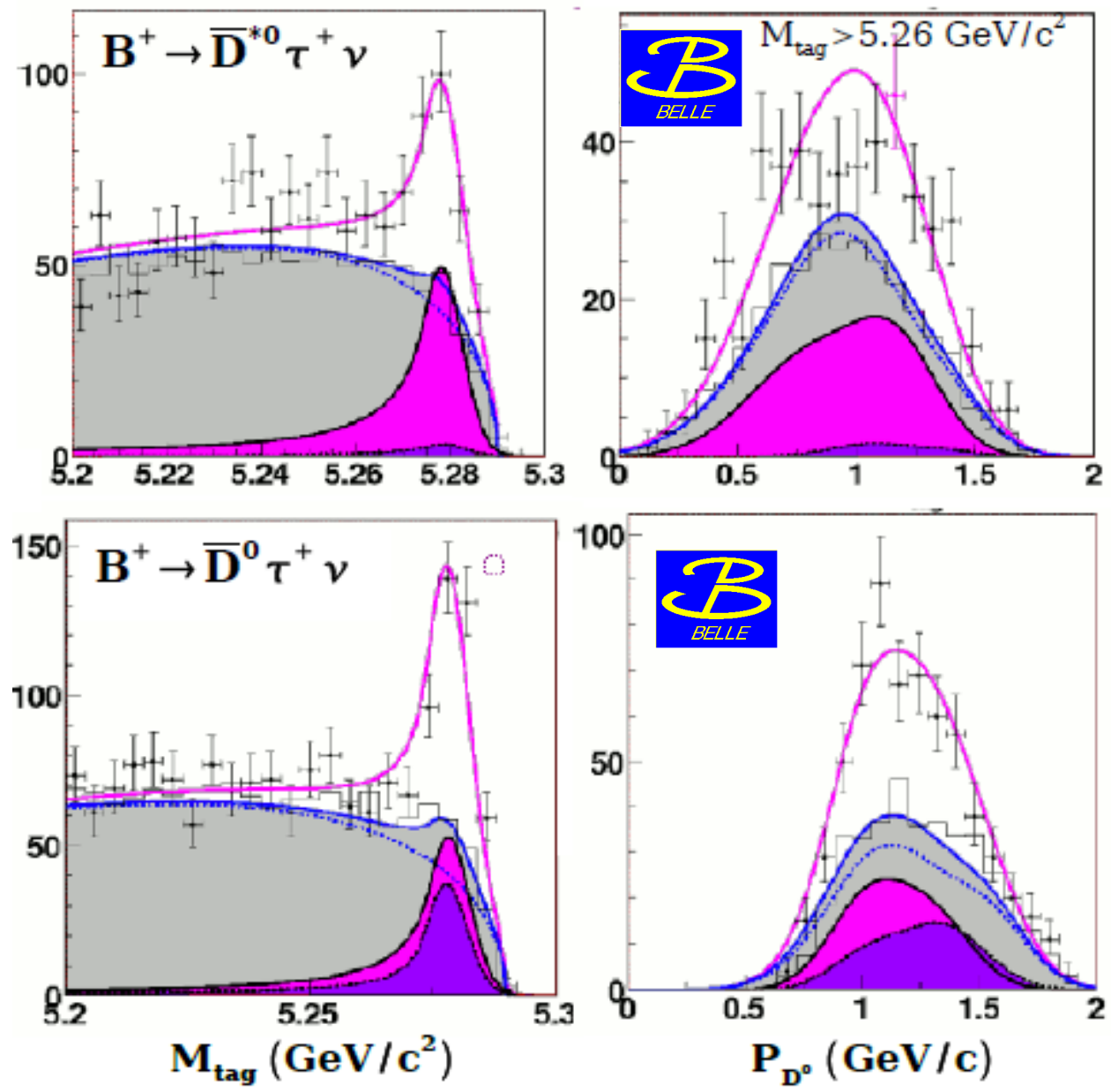

[PRD 82, 0720005(2010)] 


\section{$\boldsymbol{B} \rightarrow \boldsymbol{D}^{(*)} \tau v$ Summary}
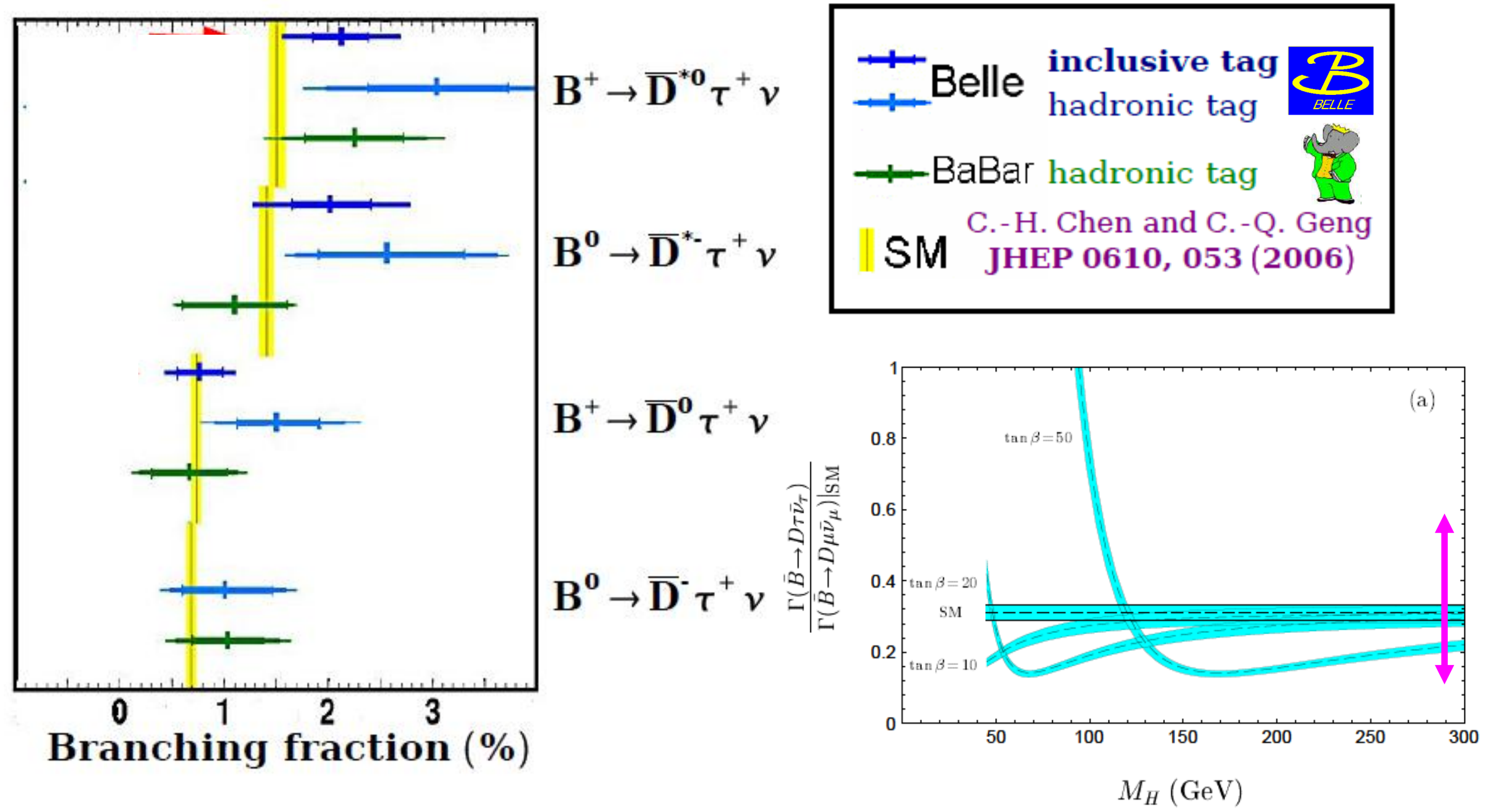

[M.Tanaka Z.Phys. C67,321(1995)] 


\section{Constraints on charged Higgs}

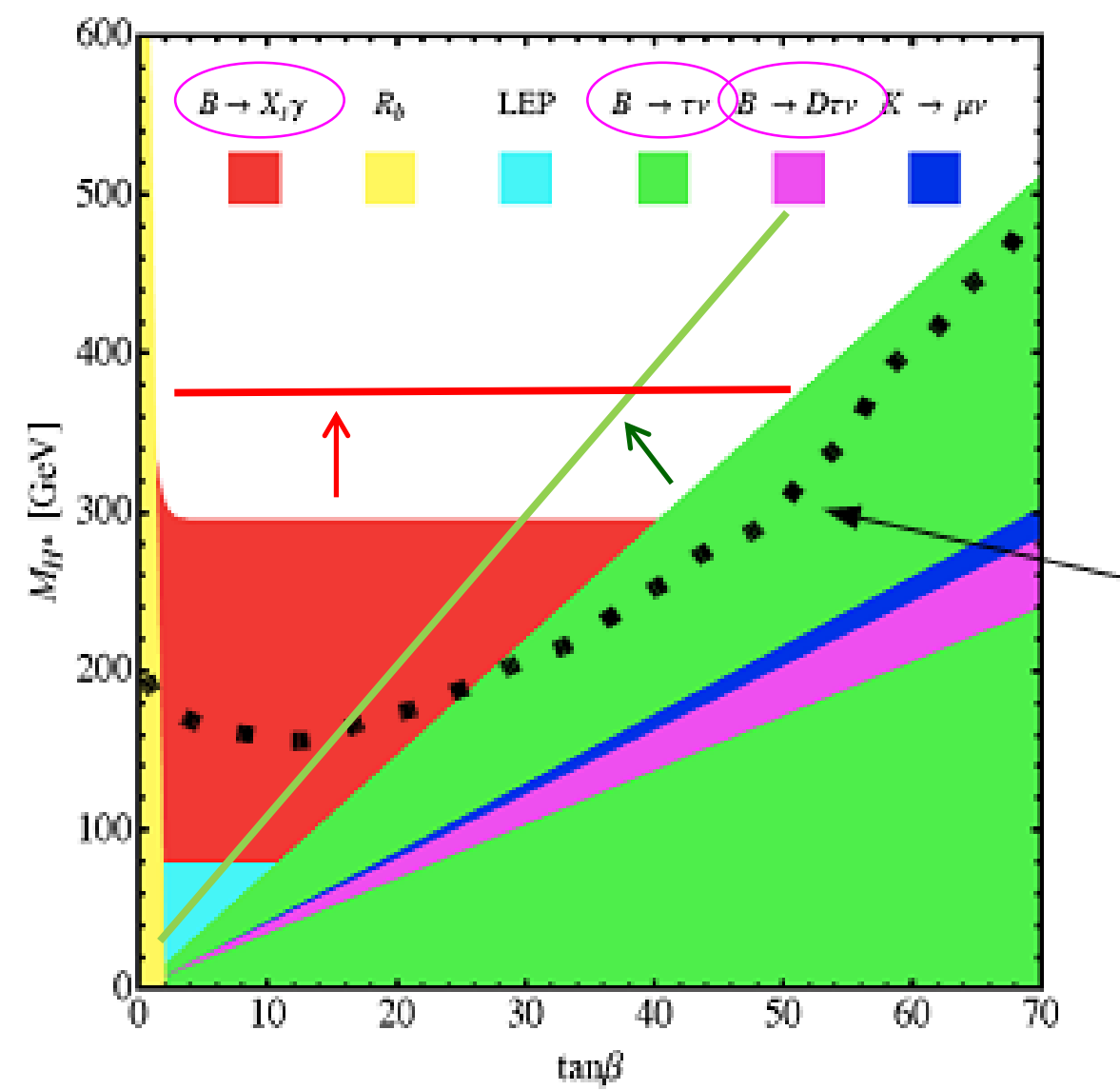

U. Haisch, hep-ph/0805.2141; ATLAS curve added by Steve Robertson
Current flavour constraints are already very competitive with LHC expected direct search sensitivity for charged Higgs

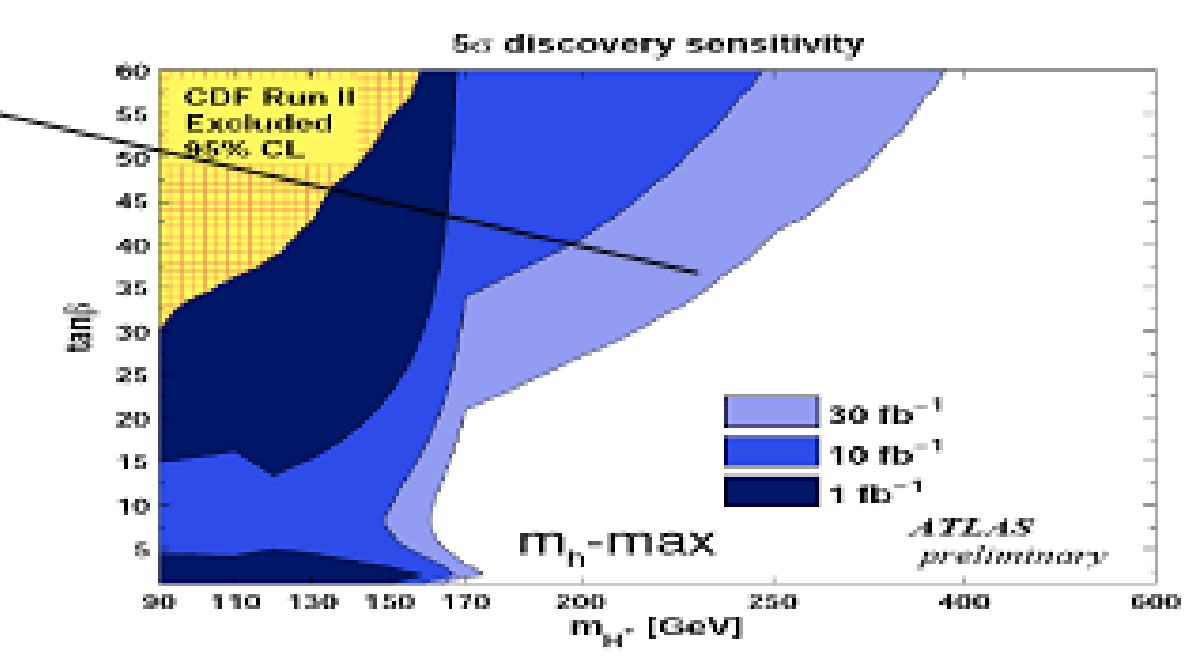

Also see (MSSM),D.

Eriksson,F.Mahmoudi and 0.Stal 


\section{New Physics Prospects}

\section{Complemantarity}

Super B factory

\section{$\mathrm{LHCb}$}

K experiments

$\longrightarrow$ theory uncertainty matches the expected exp. precision

$\longrightarrow$ theory uncertainty will match the expected exp. precision with expected progress in LQCD

G. Isidori et al.,

Ann.Rev.Nucl.Part.Sci. 60, 355 (2010)

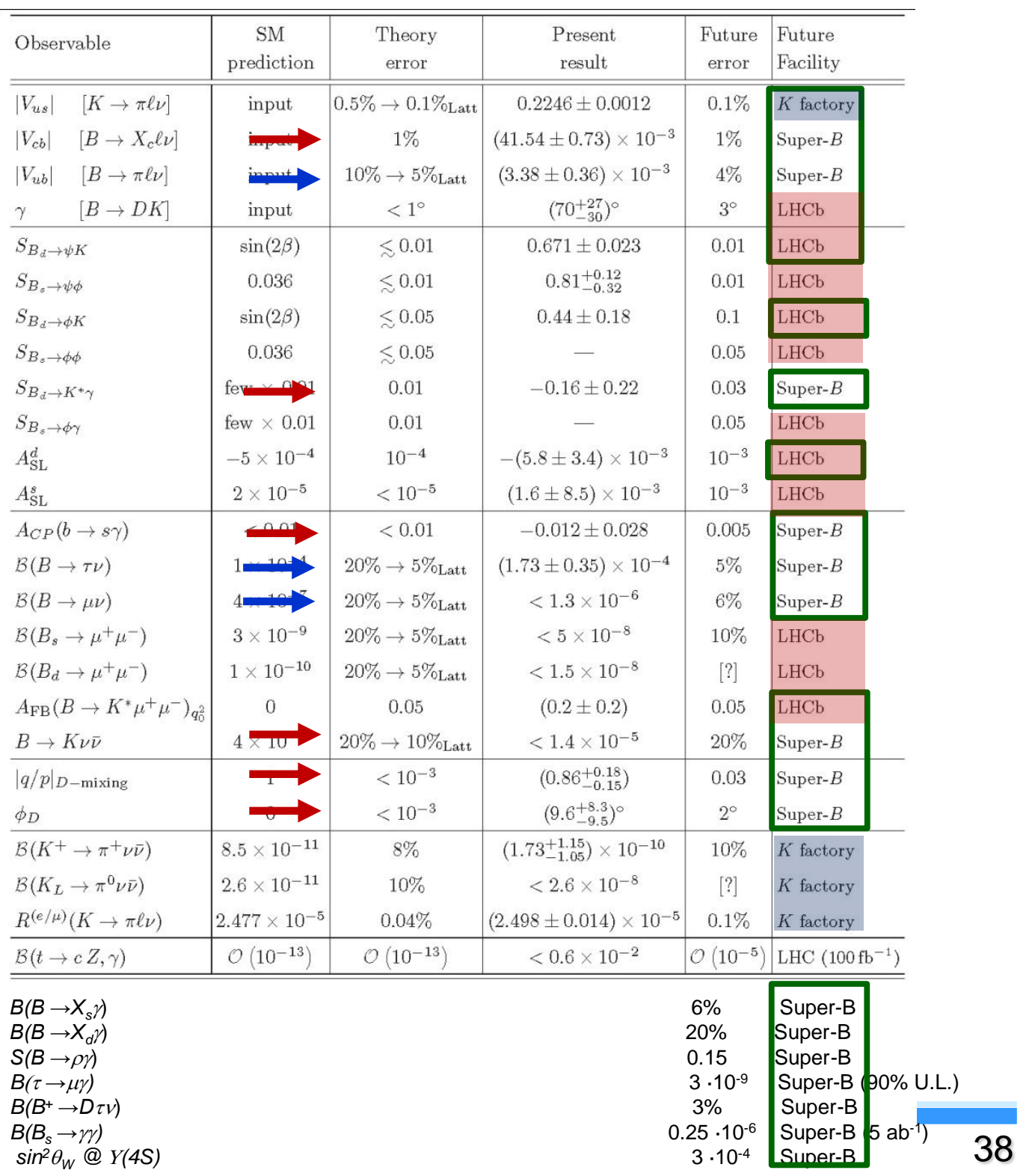




\section{Identification of NP type}

$\Rightarrow$ Identify by the pattern of deviations from SM

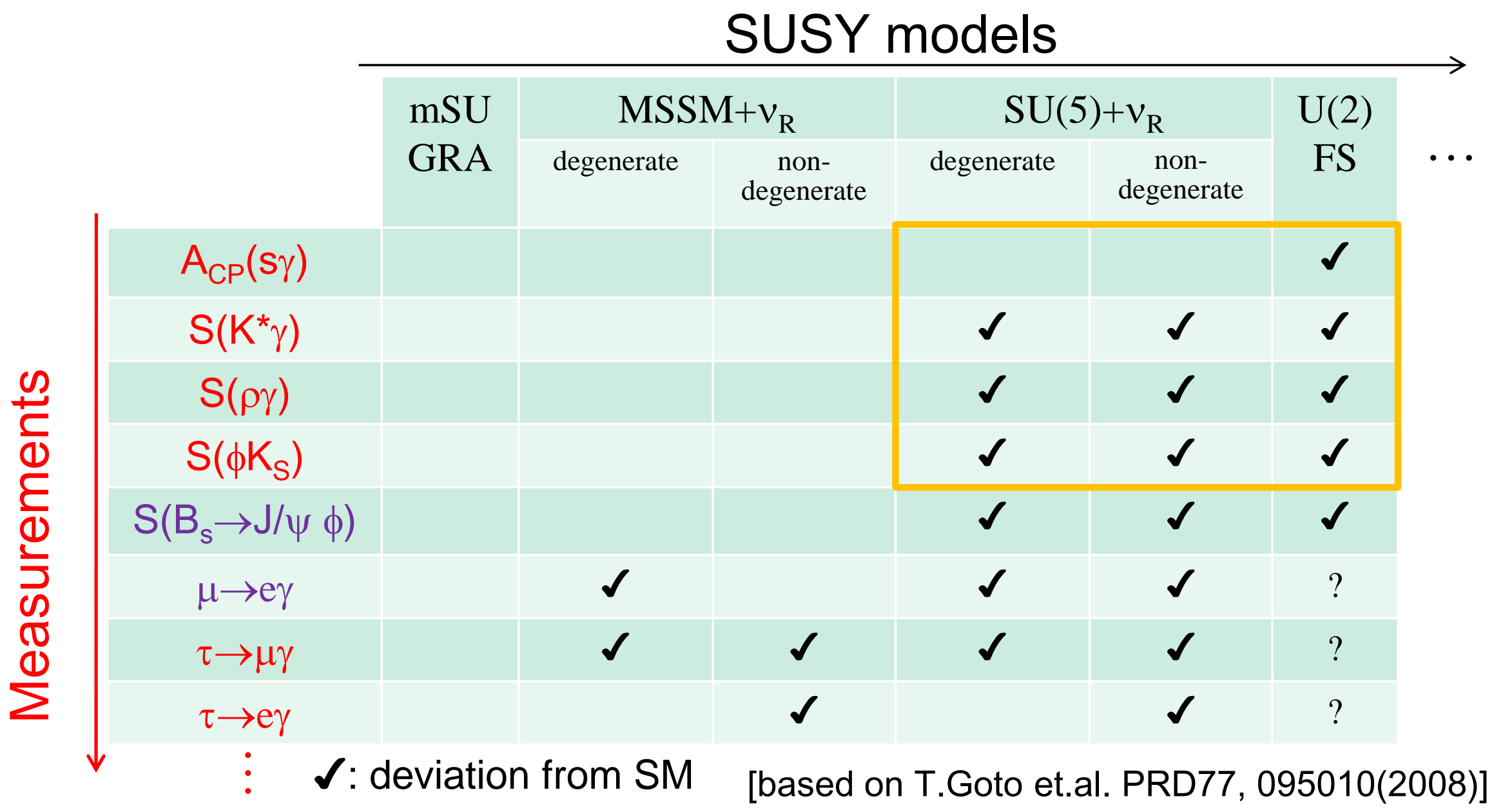




\section{Physics at Super B-factory}

\section{is "DNA chip of}

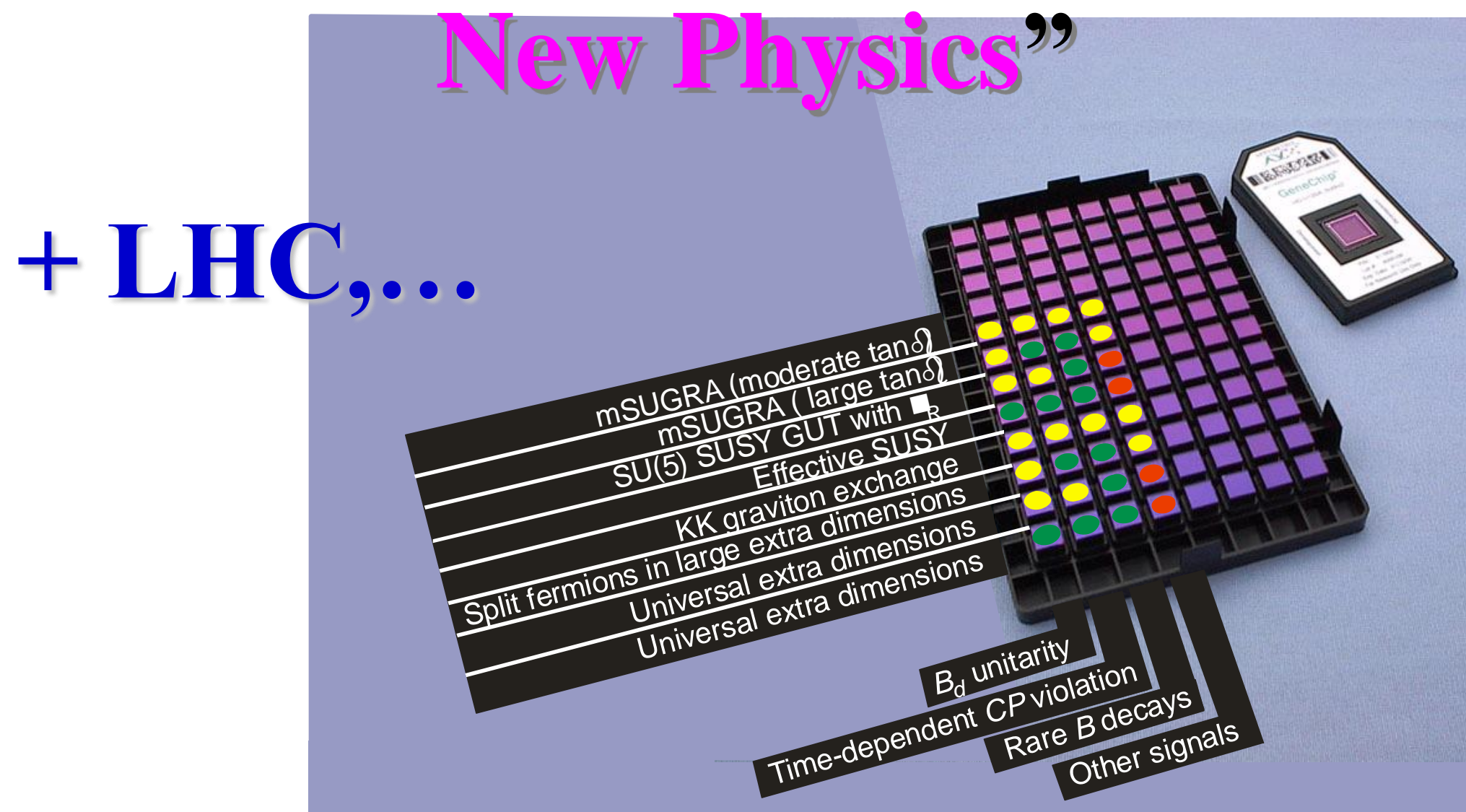

(c) D. Hitlin 


\section{New Hadron Structures: QCD}

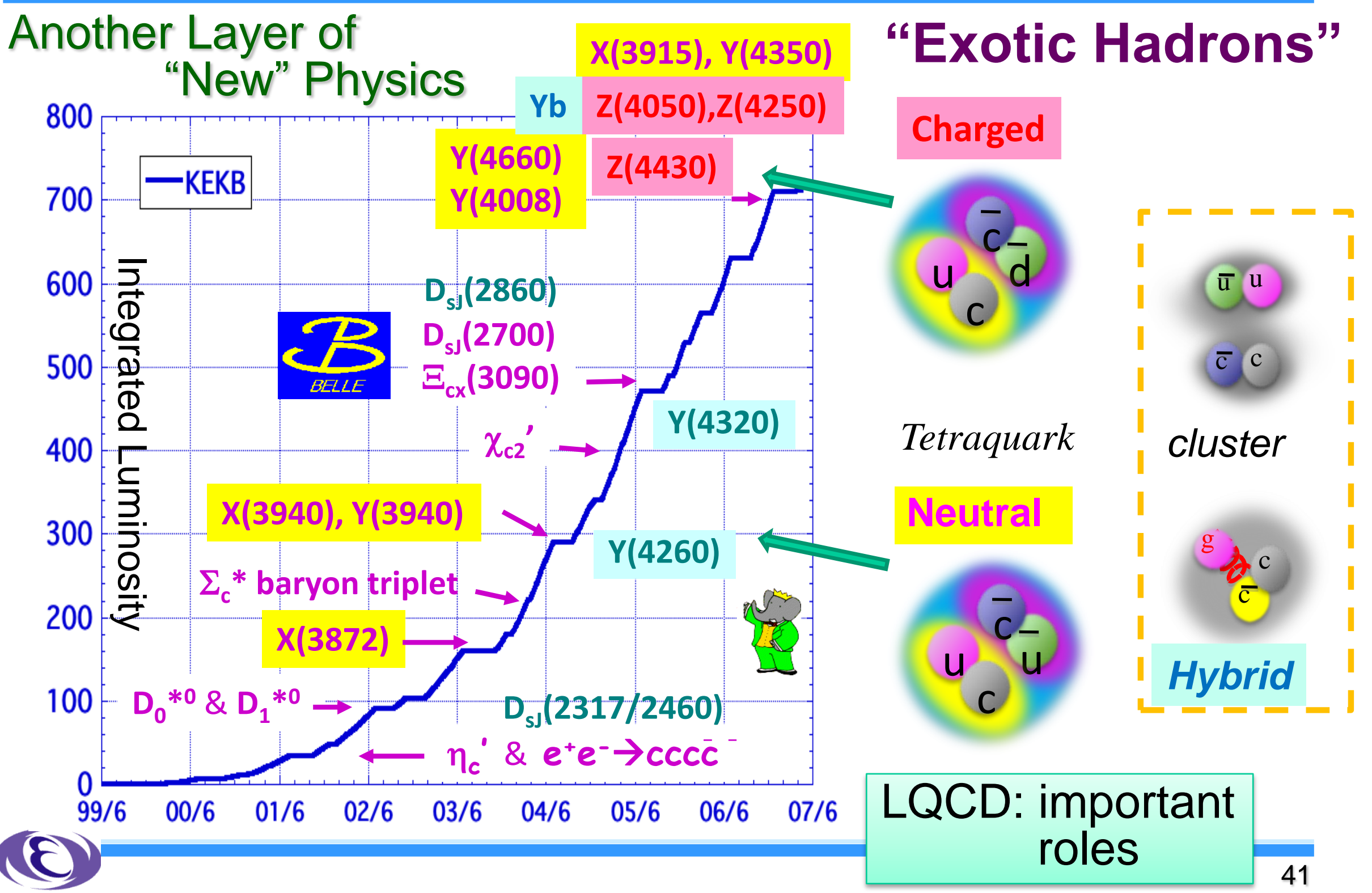


$\overbrace{\sim}^{10} 10^{35}$ Peak Luminosity trends in last 40 years

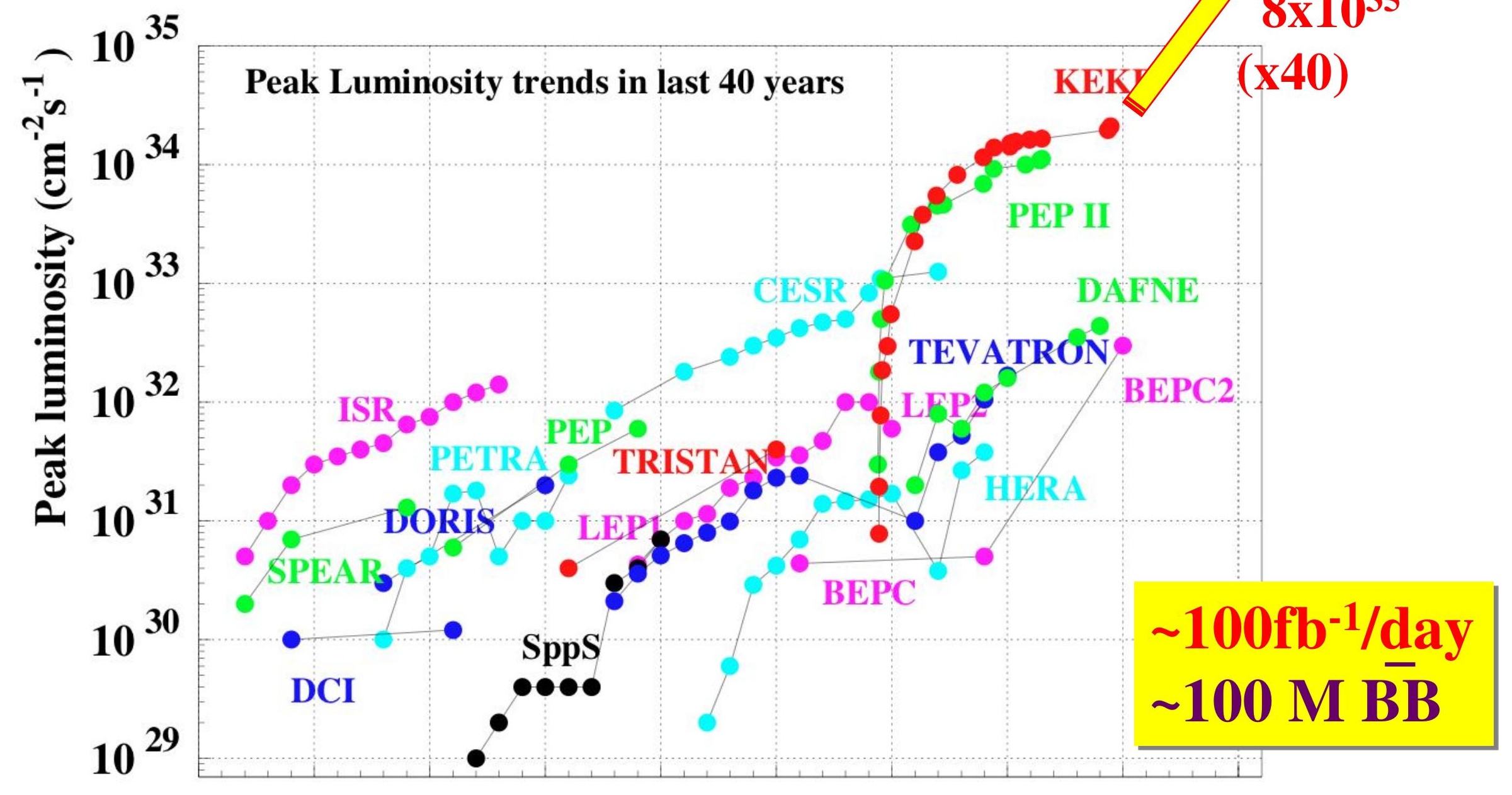

$\begin{array}{llllllllll}1970 & 1975 & 1980 & 1985 & 1990 & 1995 & 2000 & 2005 & 2010 & 2015\end{array}$

Year 


\section{To High Luminosity}

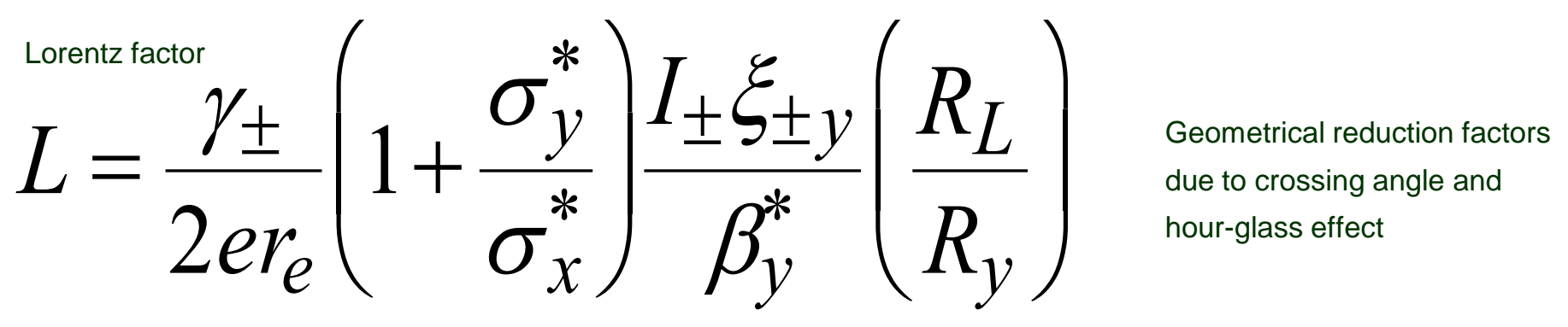

Classical electron radius Beam size ratio

- Increase beam current, I x 2

- Larger beam-beam par, $\xi_{y}$ same

- Smaller $\beta^{*}$

$<1 / 20$ +low emmittance

(long bunch)

\section{Nano-beam approach}




\section{Nano-Beam Scheme}

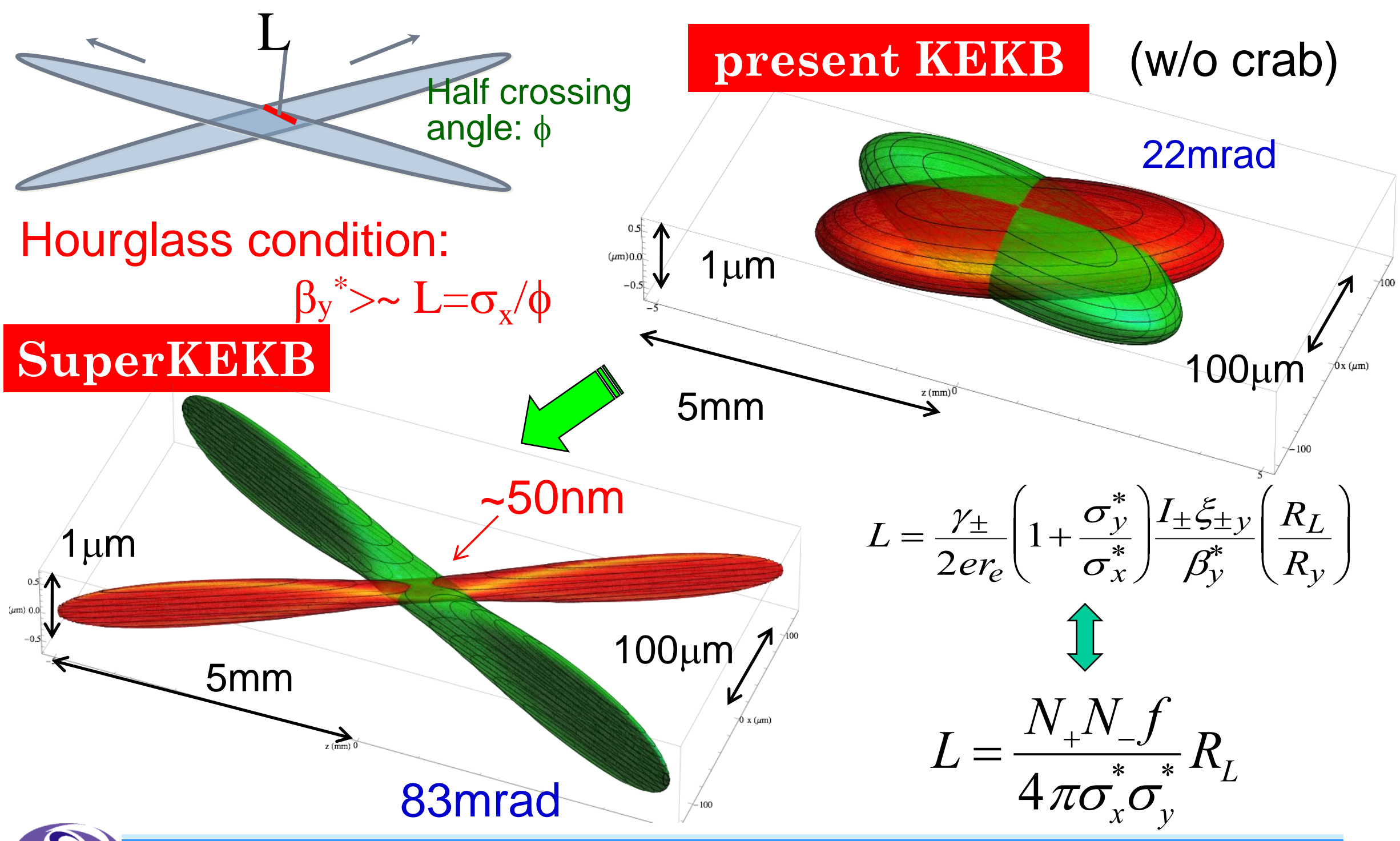




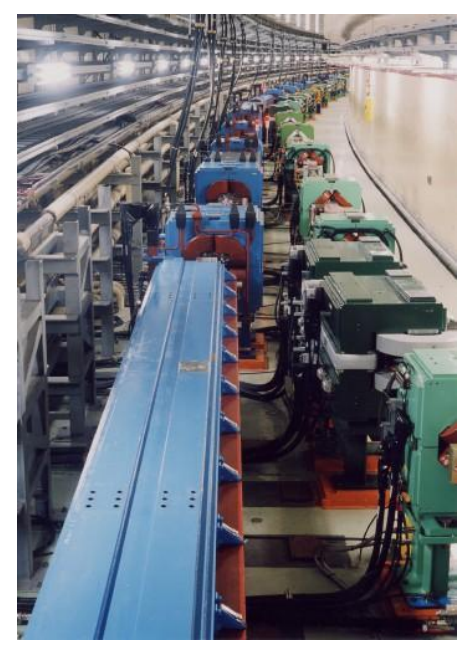

Replace short dipoles with longer ones (LER)

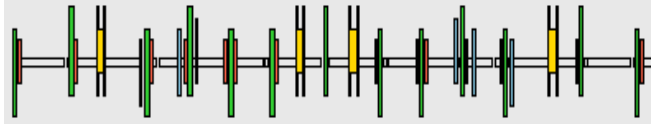
$\square$

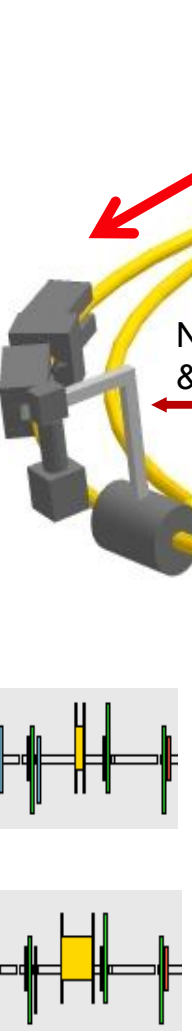

Redesign the lattices of HER \& LER to squeeze the emittance

TiN-coated beam pipe with antechambers

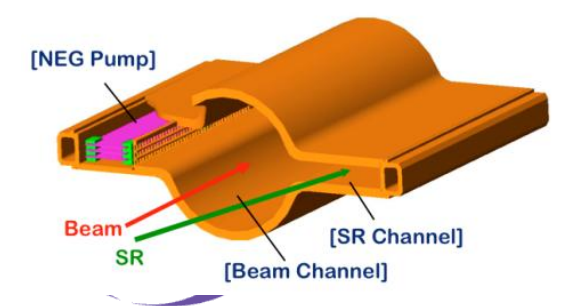

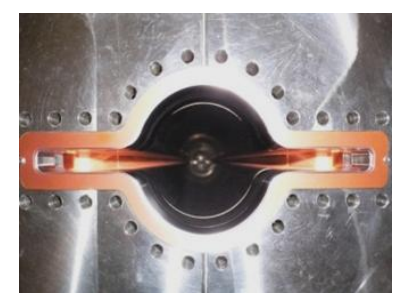

Colliding bunches

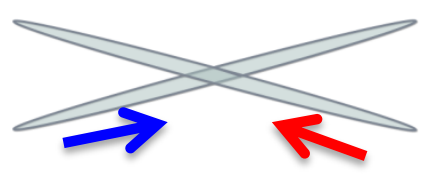

New superconducting /permanent final focusing quads near the IP
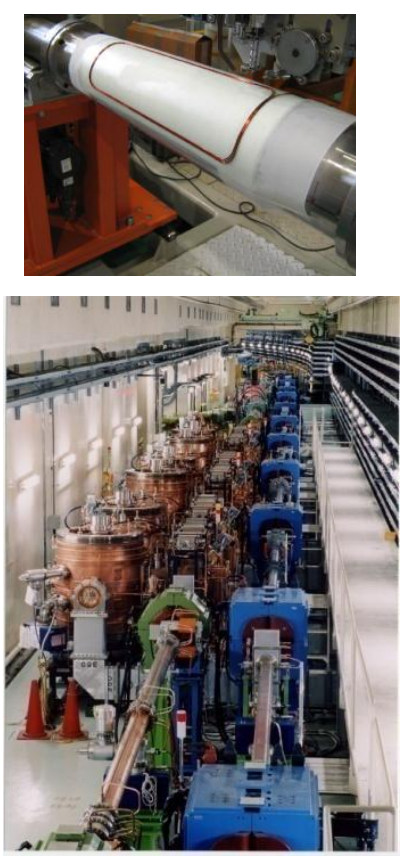

Add / modify RF systems for higher beam current

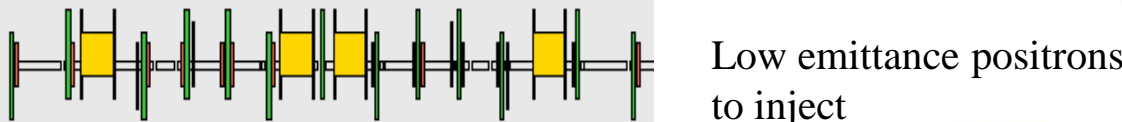

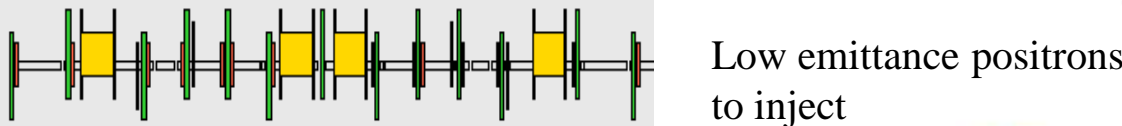

Damping ring

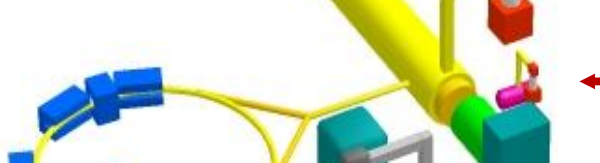

Positron source

New positron target / capture section

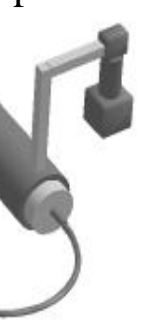

$L=8 \cdot 10^{35} \mathrm{~s}^{-1} \mathrm{~cm}^{-2}$

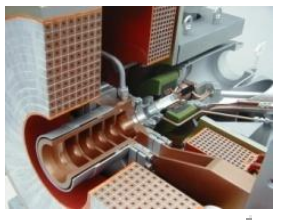

Low emittance electrons to inject

$$
L=\frac{\gamma_{ \pm}}{2 e r_{e}}\left(1+\frac{\sigma_{y}^{*}}{\sigma_{x}^{*}}\right) \frac{I_{ \pm} \xi_{ \pm y}}{\beta_{y}^{*}}\left(\frac{R_{L}}{R_{y}}\right)
$$

\section{x 40 Gain in Luminosity}




\section{IR Design}

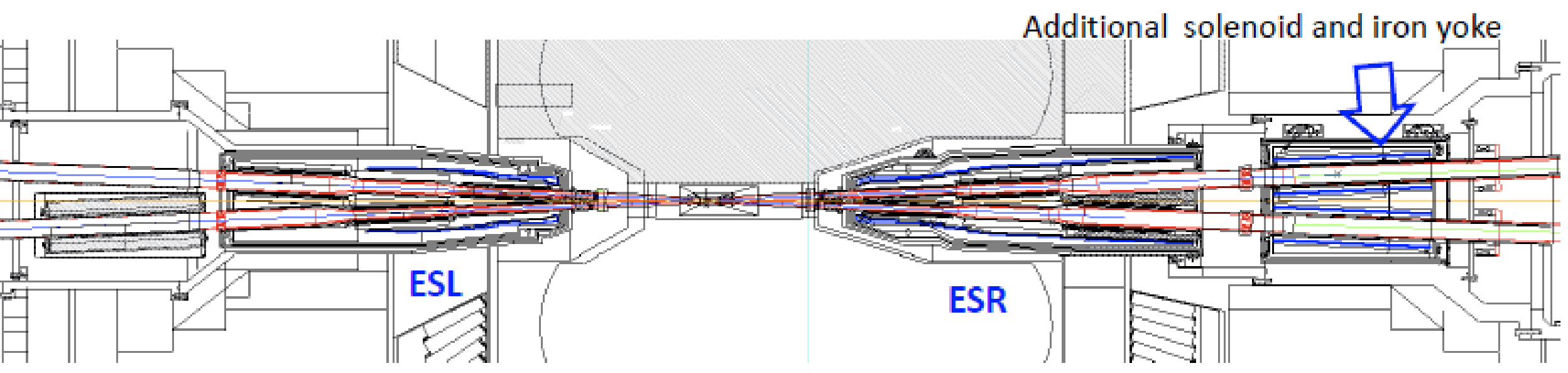

IR is one of crucial items of SuperKEKB

QCS and Compensation Solenoids

Beam Focusing, Orbit Stability

Background to detector 


\section{Luminosity Prospects}

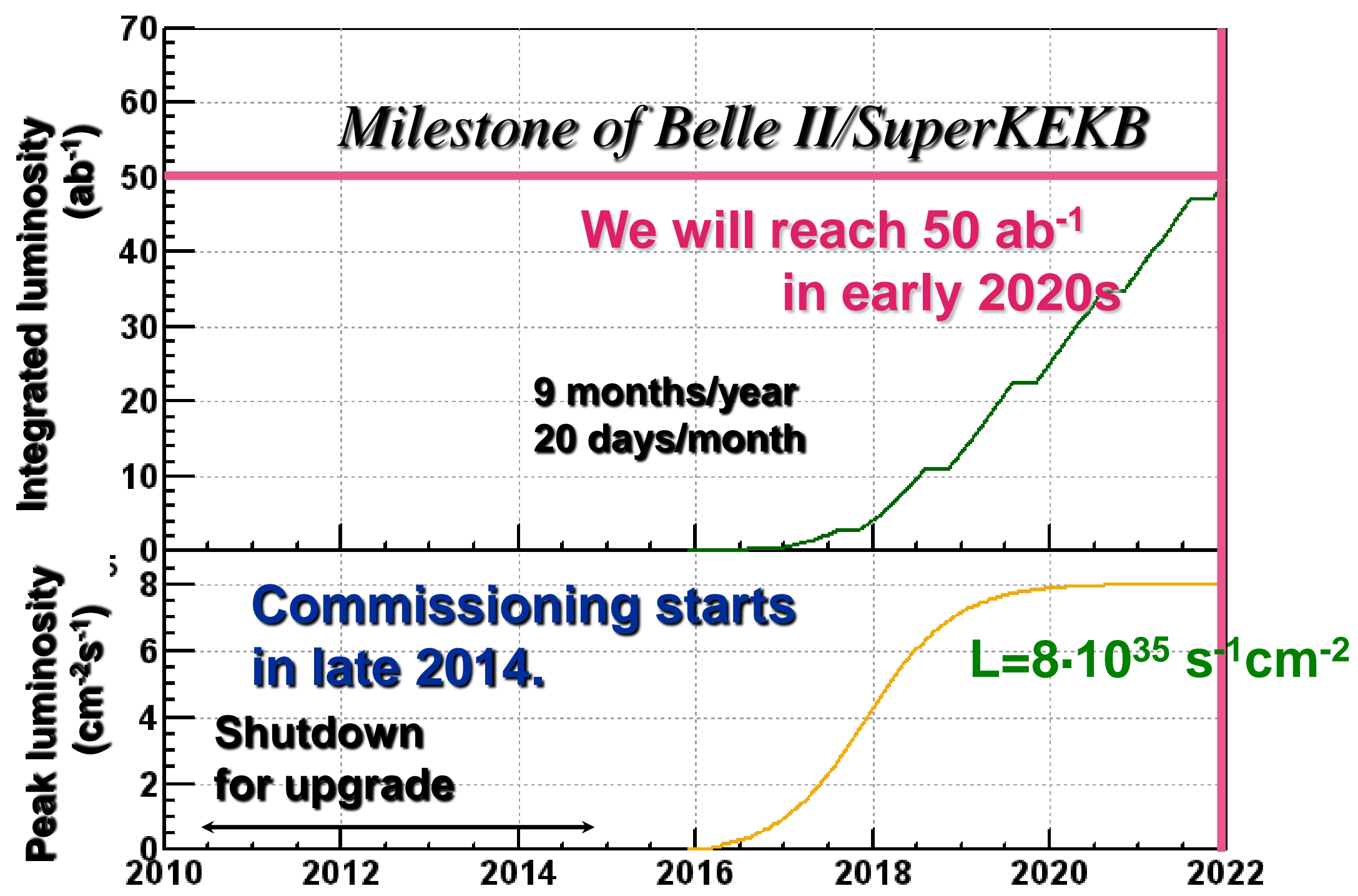




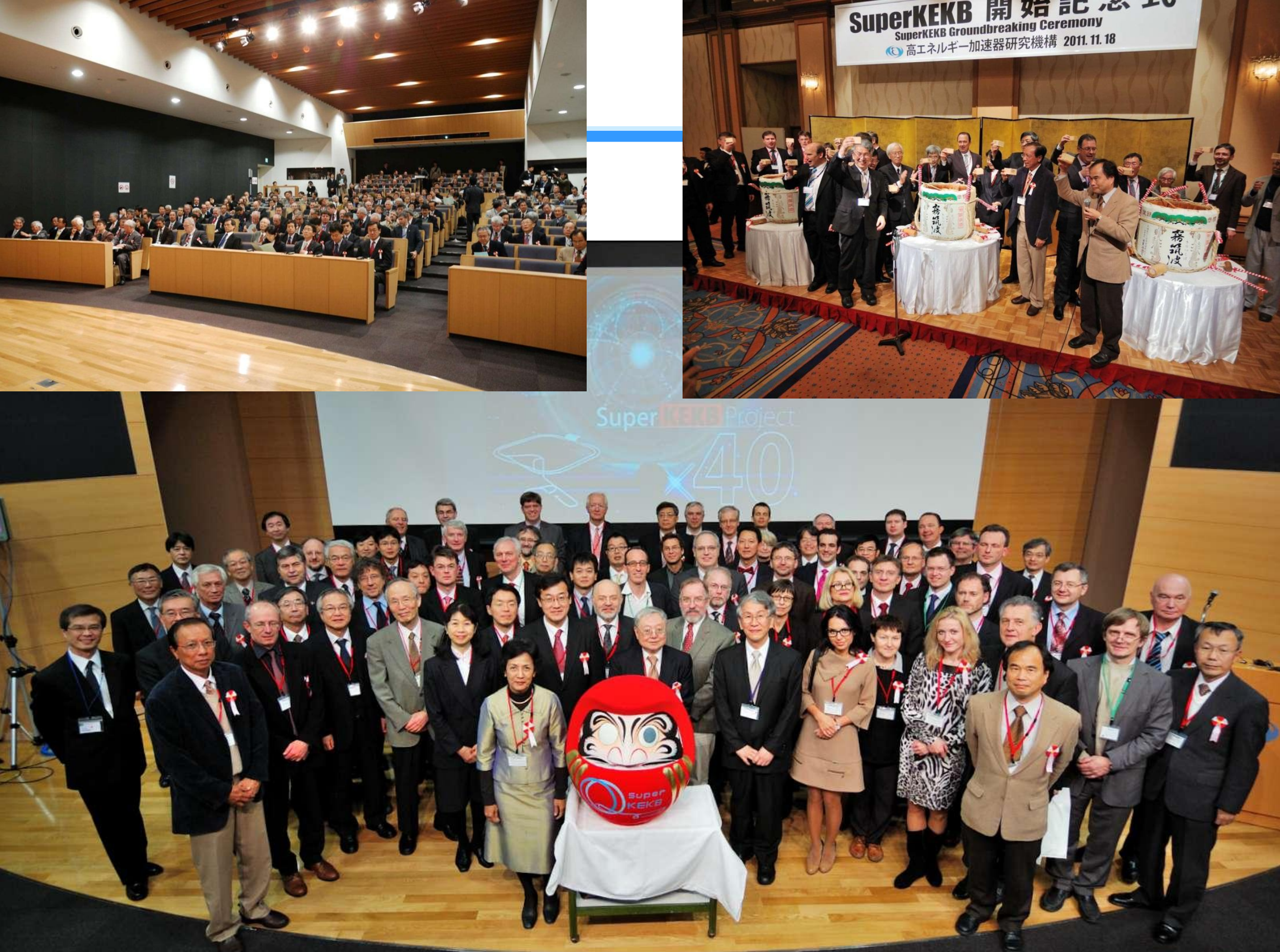




\section{(Q)}
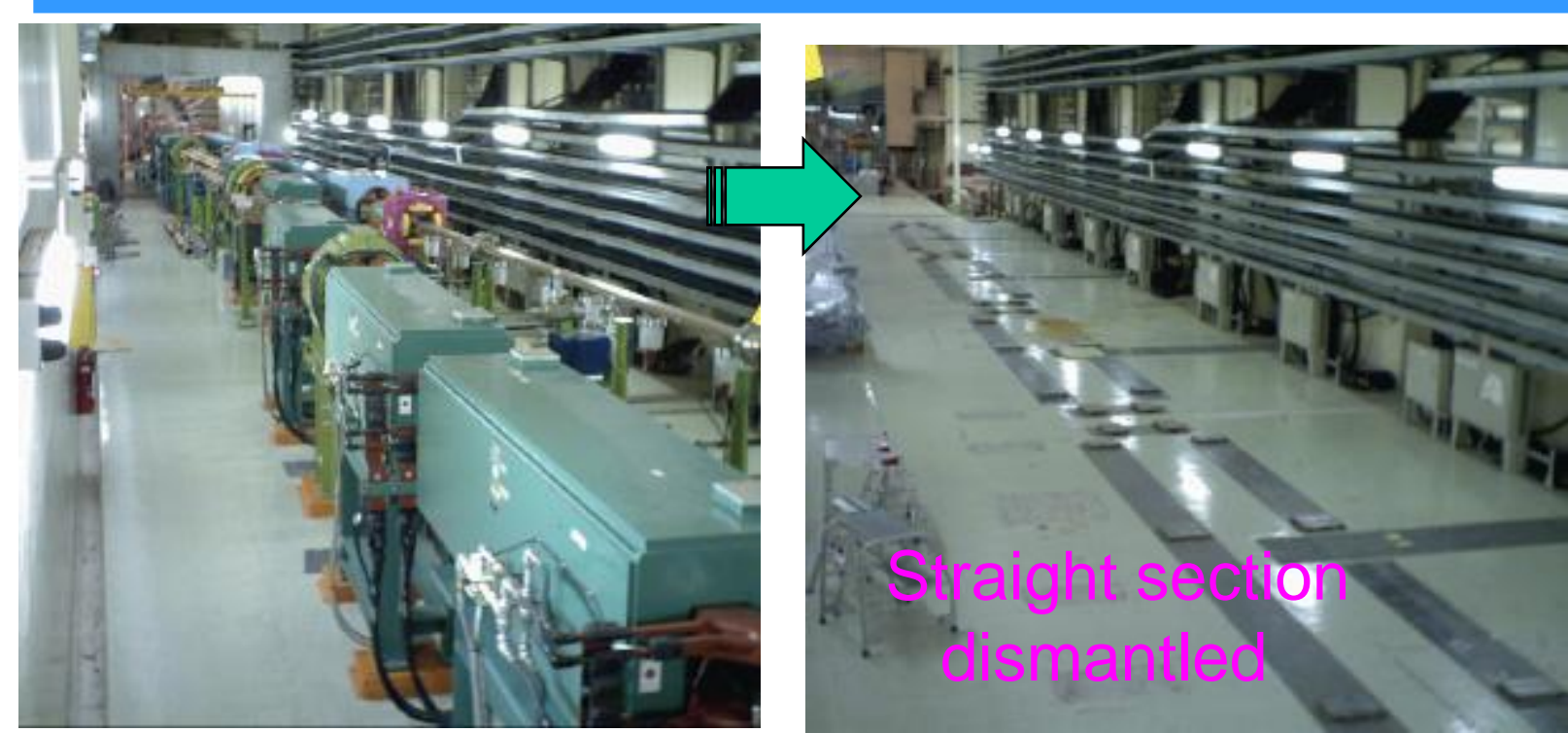

New LER Dipole

Field measurement set up
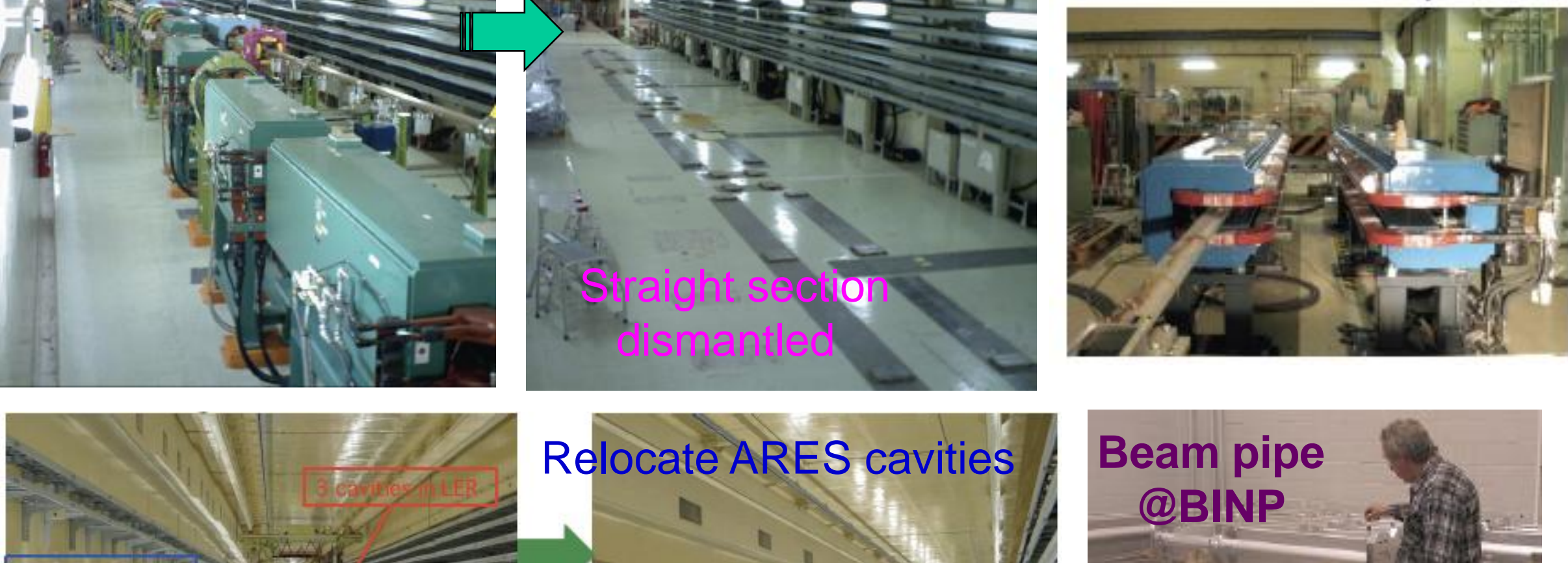

Relocate ARES cavities
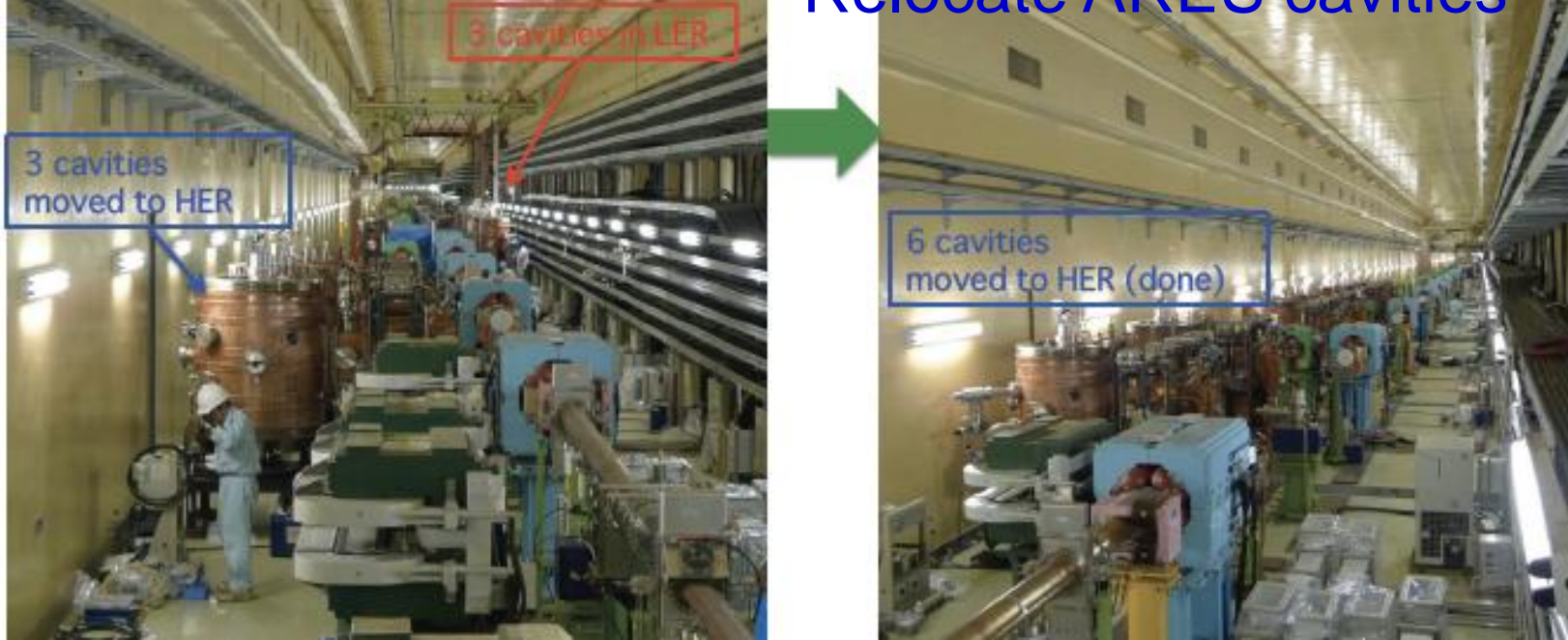

Beam pipe @BINP

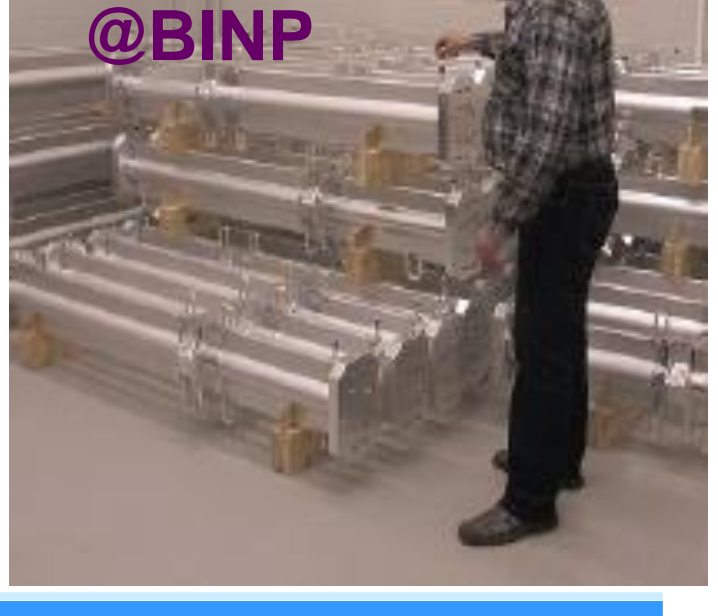




\section{Belle II Collaboration}

2004.06 SuperKEKB Lol

2008.01 KEK Roadmap

$2008.03 \quad 1^{\text {st }}$ Proto collaboration meeting

2008.10 Detector study report

2008.12 New collaboration, Belle-II, started

$\sim 400$ collaborators from 57 institutions in $\mathbf{1 3}$ countries

Peter Krizan (Ljubljana) elected as the first spokesperson 2012.03 Series of open collaboration meetings

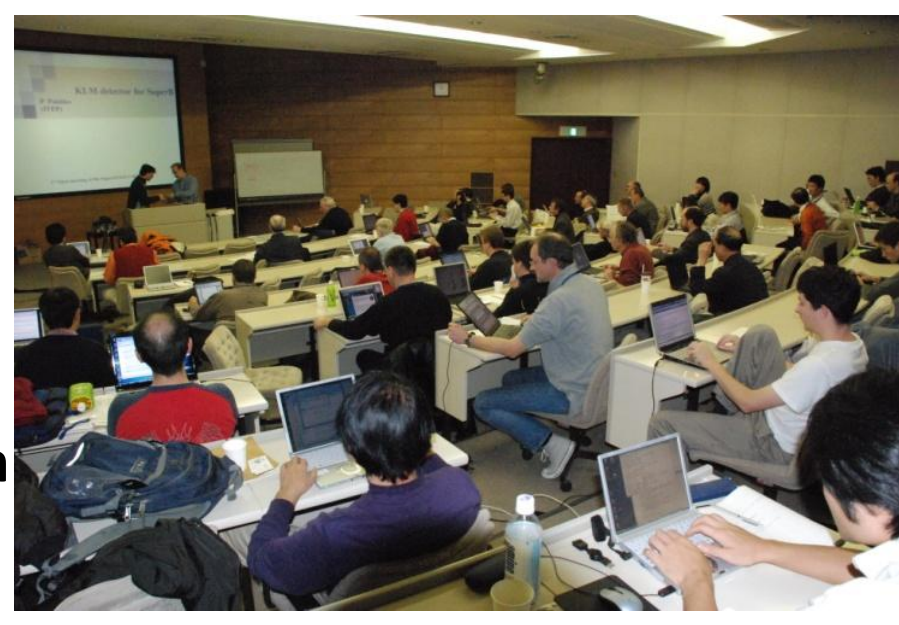

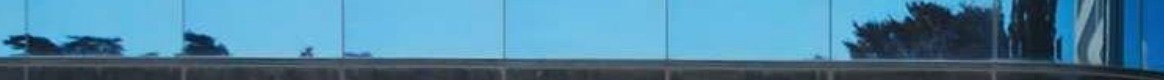

$a_{1}$

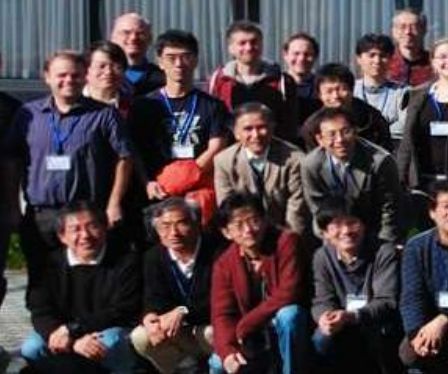

1

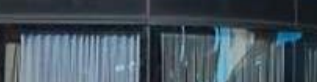

I
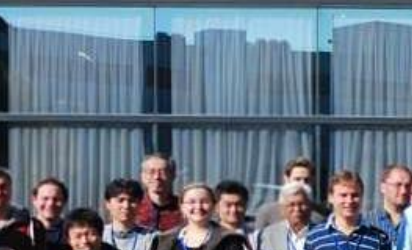

(1)

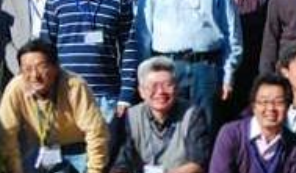

II

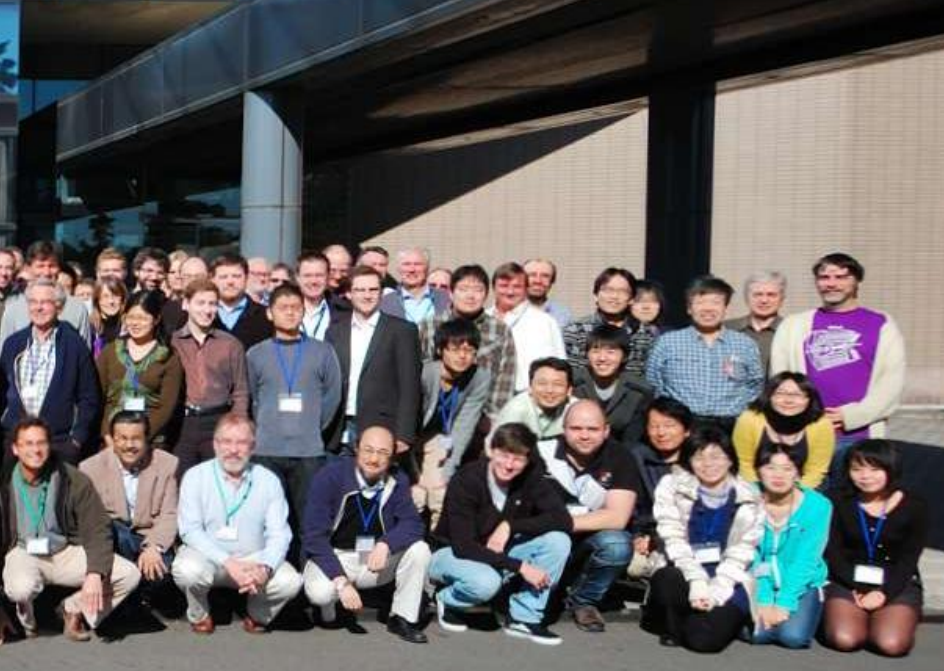




\section{$\mathcal{B}$ Belle II Detector}

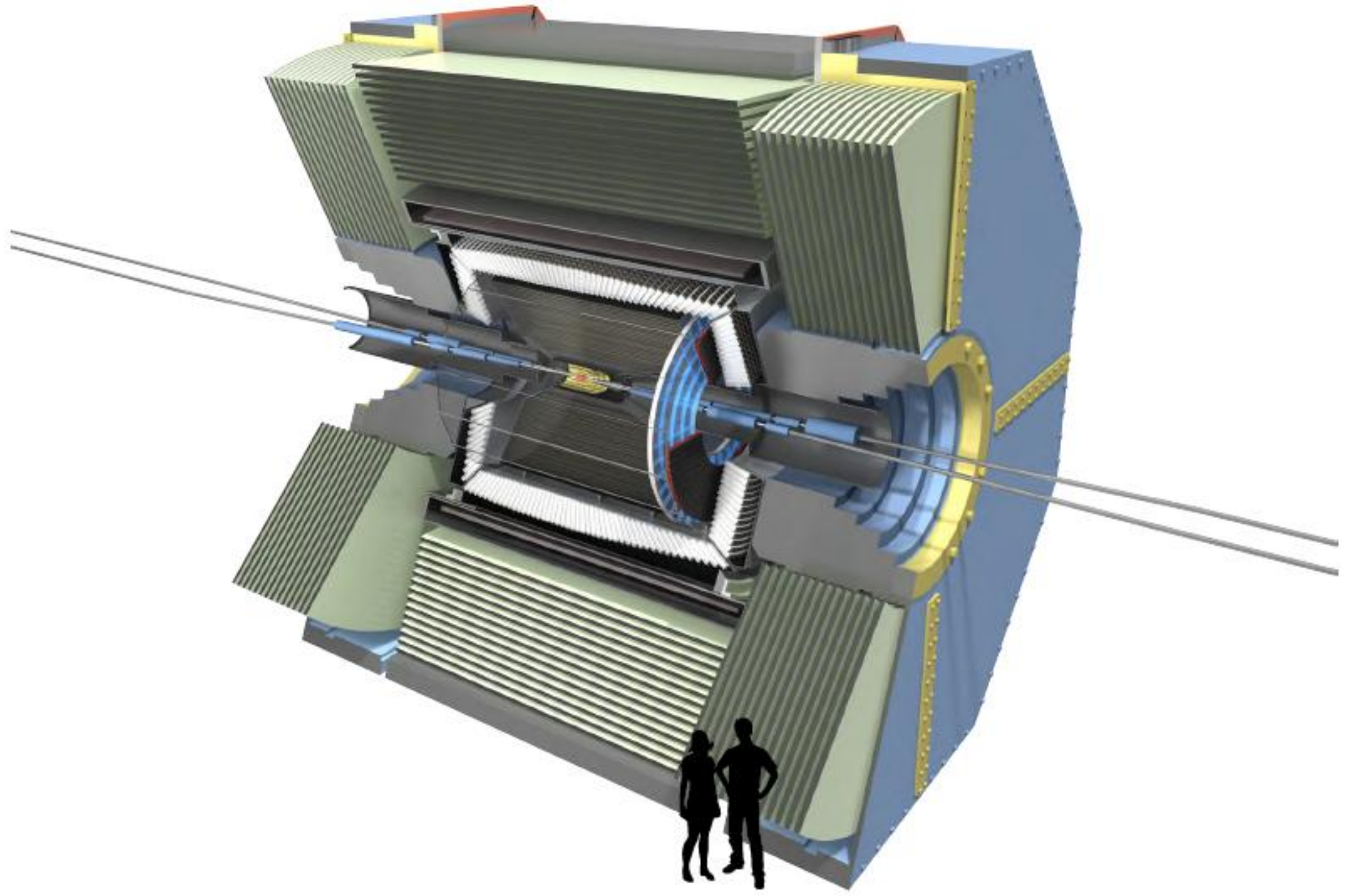

(E) 


\section{$\mathcal{B} \quad$ Belle $\rightarrow$ Belle II}

Belle II Ku

mascerat

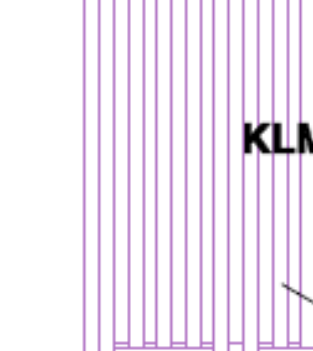

KLM
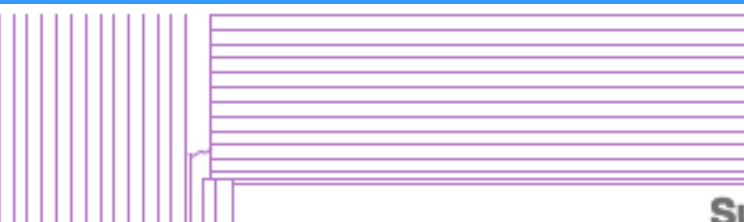

uper conducting coil

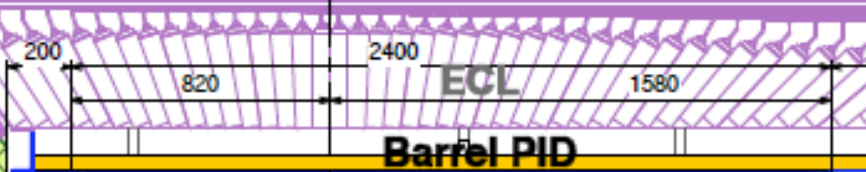

KLM
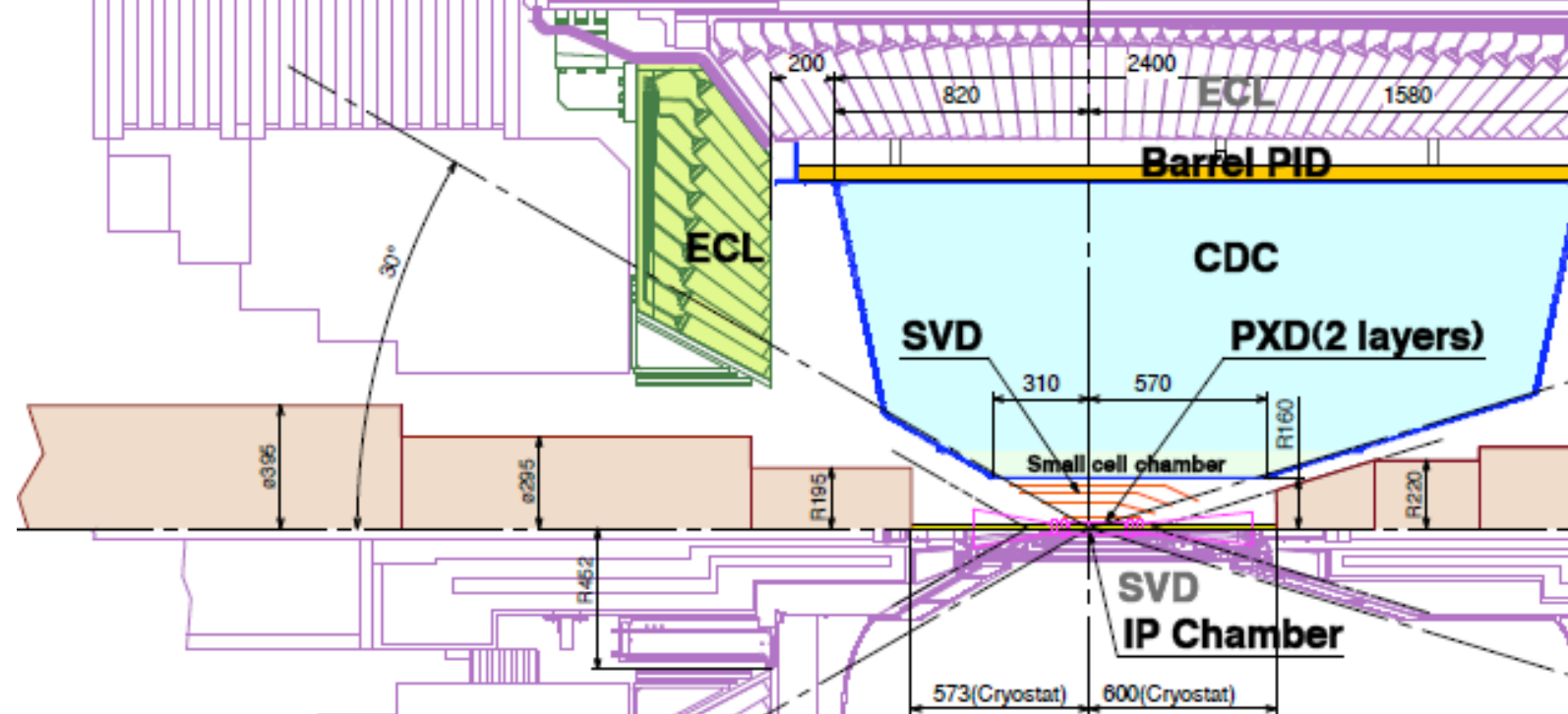

IP Chamber

\begin{tabular}{|l|l|}
573 (Cryostat) & 600 (Cryostat) \\
\hline
\end{tabular}

CDC

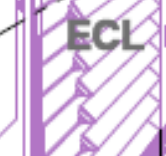

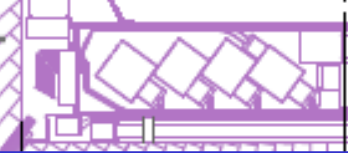

SVD: 4 DSSD lyrs $\rightarrow 2$ DEPFET + 4 DSSD lyrs

$C D C$ : small cell, long lever arm

$\mathrm{ACC}+\mathrm{TOF} \rightarrow \mathrm{TOP}+\mathrm{A}-\mathrm{RICH}$

ECL: waveform sampling (+pure Csl end-caps)

KLM: RPC $\rightarrow$ Scintillator + MPPC(end-caps)

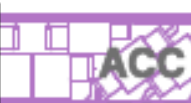

ce
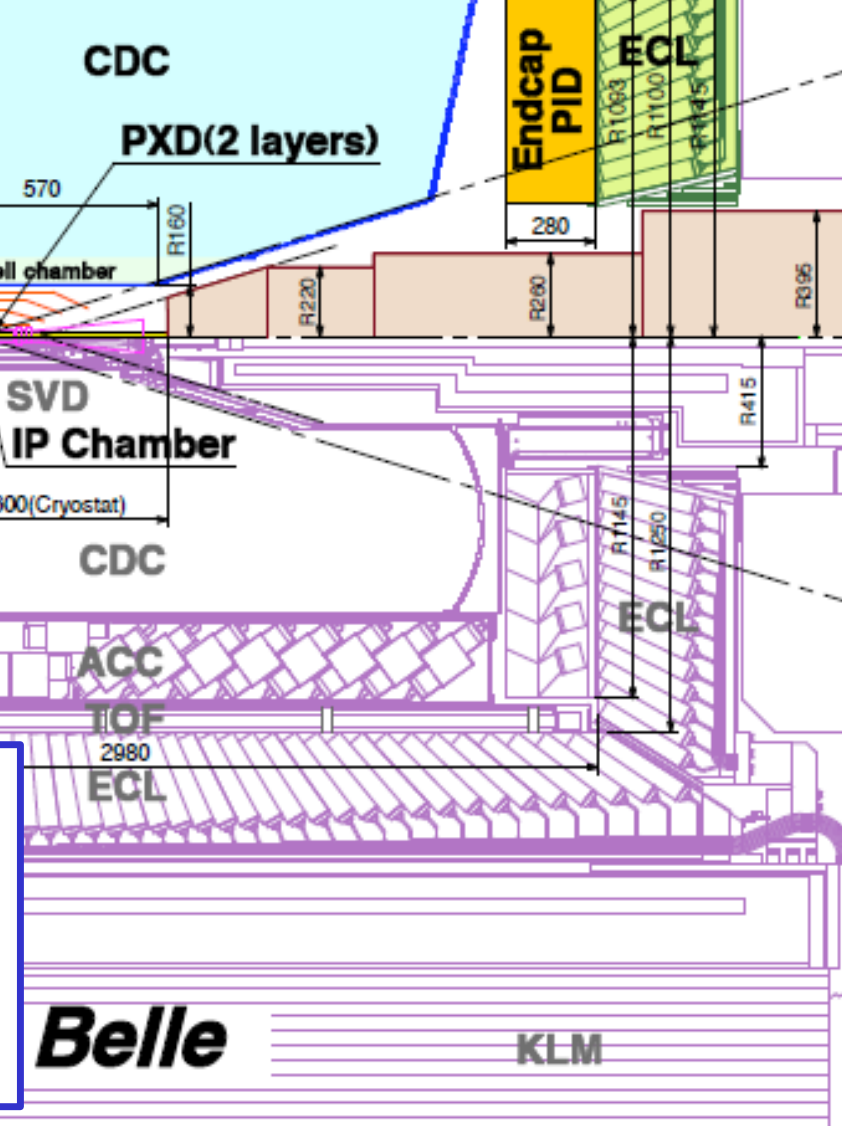

을 छ욜

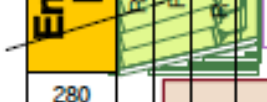

$\int$
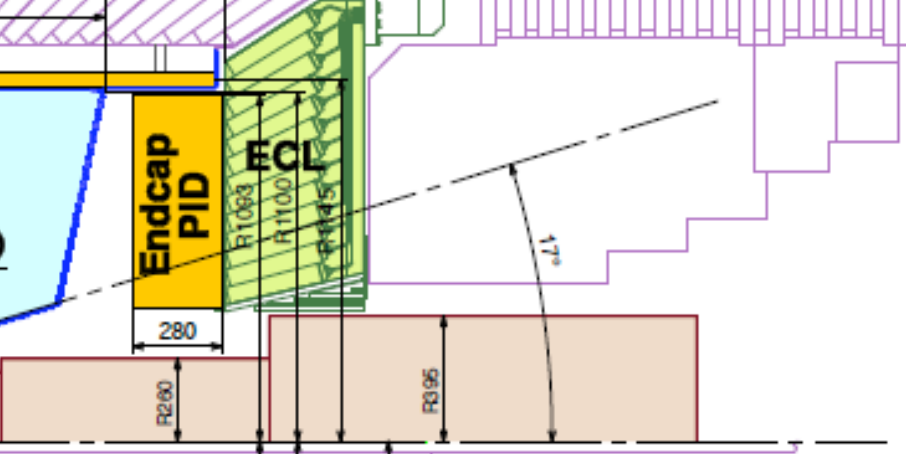


\section{$\mathcal{B}$ Vertex detectors PXD/SVD}

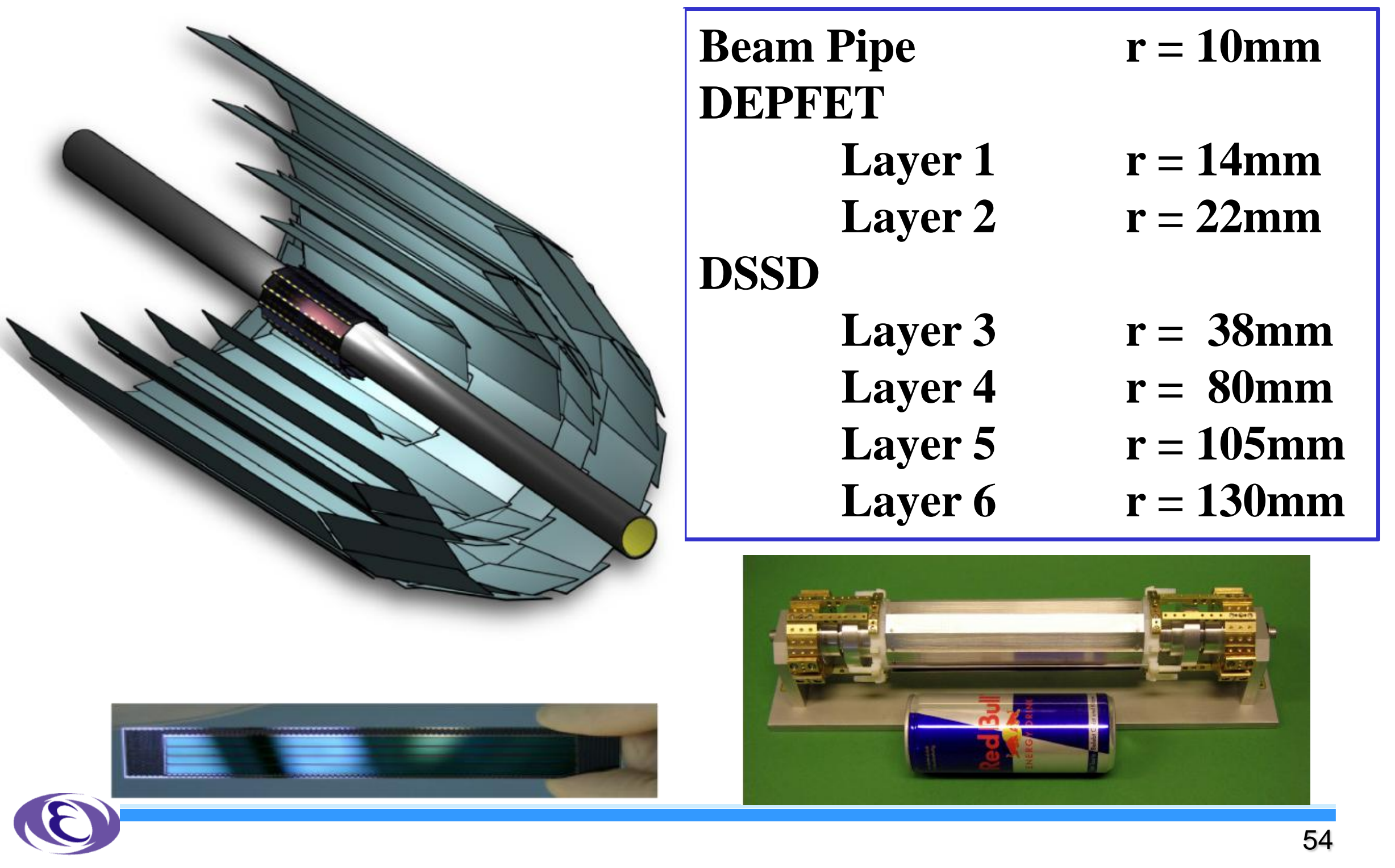




\section{$\mathcal{B}$ Expected performance $\sigma=a+\frac{b}{p \beta \sin ^{\prime} \theta}$}

Significant improvement in IP resolution!

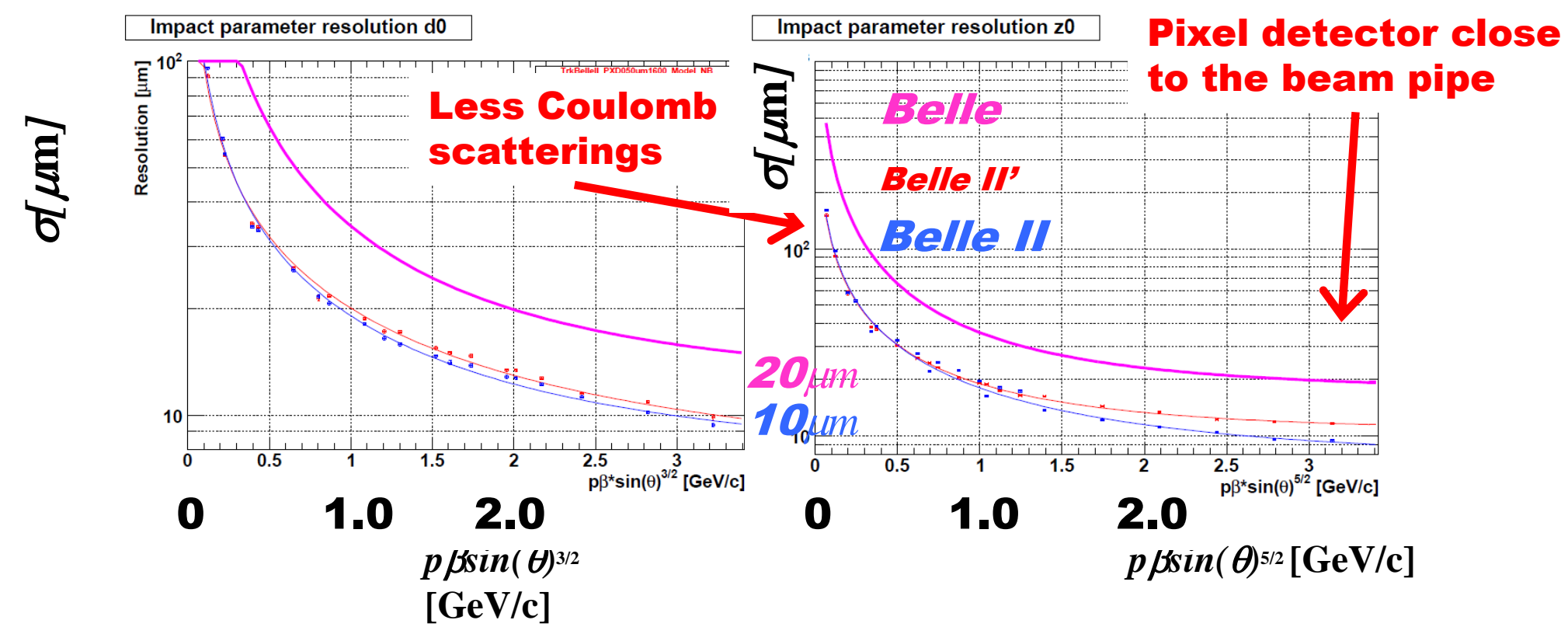

Significant improvement in $\delta S\left(K_{\mathrm{S}} \pi^{0} \gamma\right)^{\omega^{n I}}$

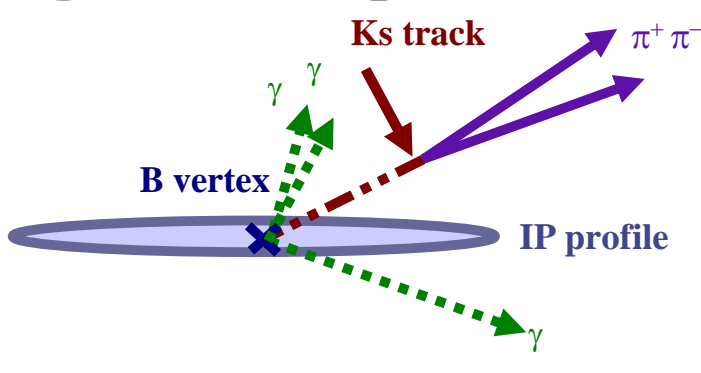

B decay point reconstruction with $\mathrm{K}_{\mathrm{S}}$ trajectory

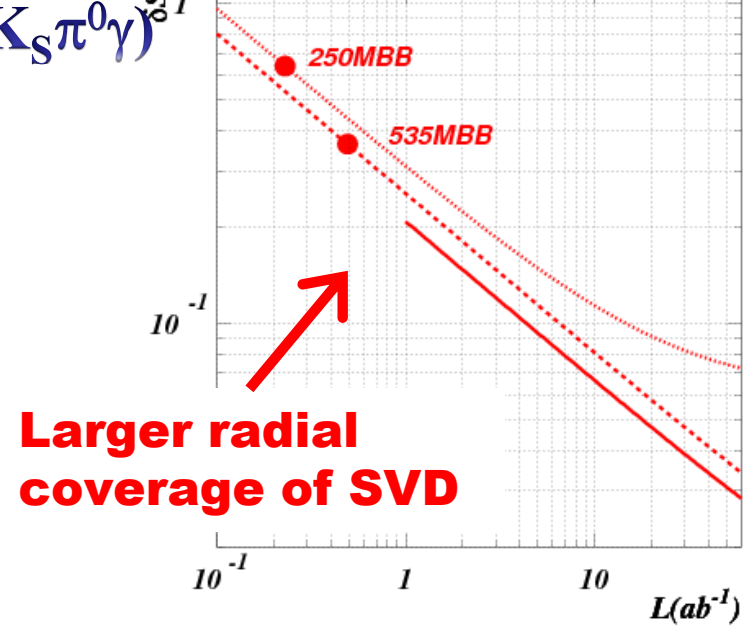




\section{CDC}

\section{Belle \\ )}

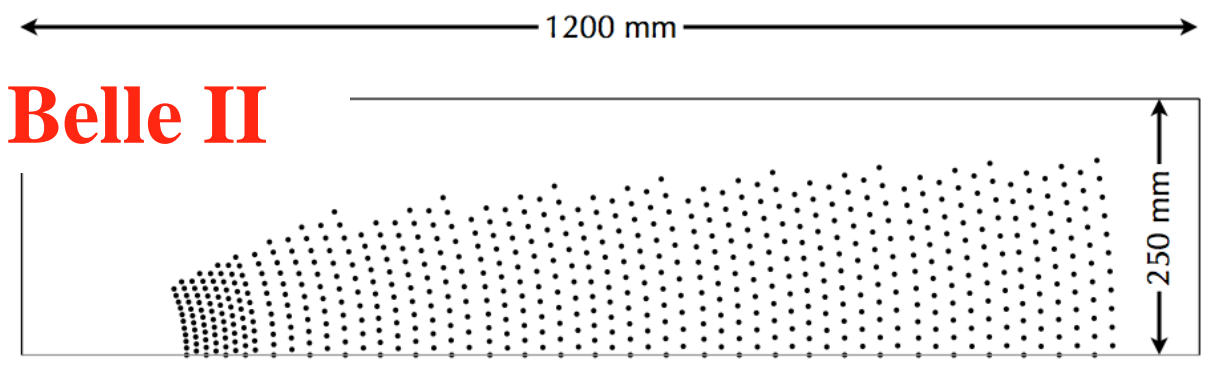

longer lever arm improve resolution of momentum and $\mathrm{dE} / \mathrm{dx}$

$$
\sigma_{P_{t}} / P_{t}=0.19 P_{t} \oplus 0.30 / \beta
$$$$
\sigma_{P_{t}} / P_{t}=0.11 P_{t} \oplus 0.30 / \beta
$$

new readout system dead time $1-2 \mu \mathrm{s} \rightarrow 200 \mathrm{~ns}$

\section{small cell}

smaller hit rate for each wire shorter maximum drift time

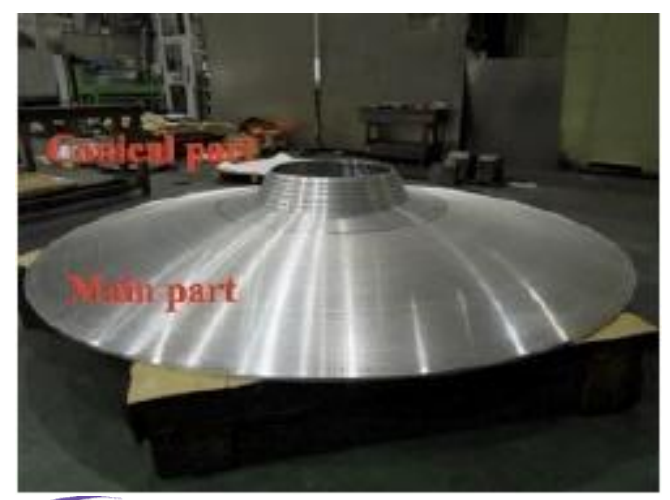

\begin{tabular}{|c|c|c|}
\hline & Belle & Belle II \\
\hline radius of inner most sense wire & 88 & 168 \\
\hline radius of outer most sense wire & 863 & 1111.4 \\
\hline Number of layers & 50 & 56 \\
\hline Number of total sense wire & 8400 & 14336 \\
\hline Gas & $\mathrm{He}_{2} \mathrm{C}_{2} \mathrm{H}_{6}$ & $\mathrm{He}: \mathrm{C}_{2} \mathrm{H}_{6}$ \\
\hline sense/field wire & $\mathrm{W}(\Phi 30 \mu \mathrm{m}) / \mathrm{Al}(\Phi 120 \mu \mathrm{m})$ & $\mathrm{W}(\Phi 30 \mu \mathrm{m}) / \mathrm{Al}(\Phi 120 \mu \mathrm{m})$ \\
\hline
\end{tabular}




\section{$\underset{B}{B} \quad$ Particle ID}

\section{Barrel PID: Time of Propagation Counter (TOP)}

Quartz radiator Forward

Small expansion block

Focusing mirror Hamamatsu MCP-PMT (measure $t, x$ and $y$ )

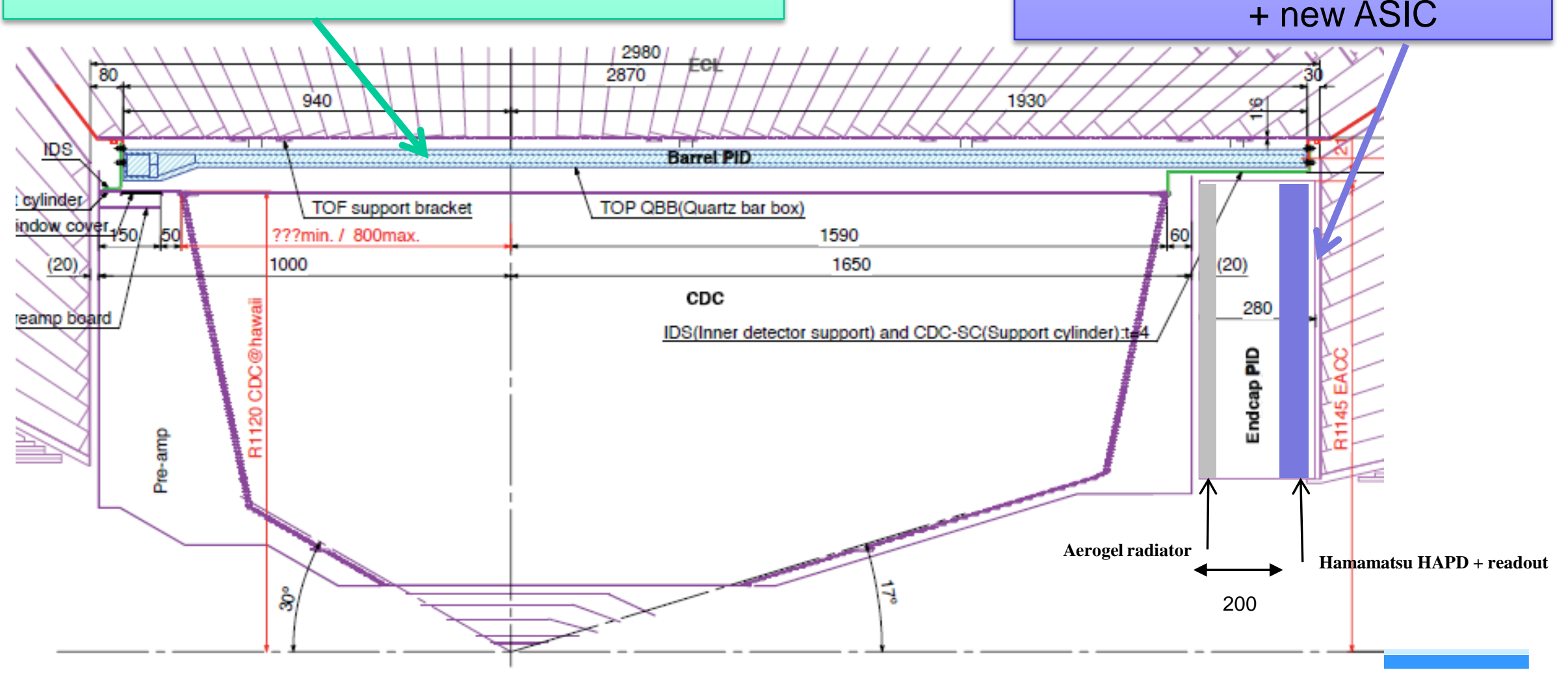




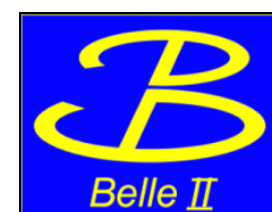

\section{TOP counter}

- Quartz radiator

$-2.6 \mathrm{~m}^{\mathrm{L}} \times 45 \mathrm{~cm}^{\mathrm{W}} \times 2 \mathrm{~cm}^{\top}$

- Excellent surface accuracy

- MCP-PMT

- Hamamatsu 16ch MCP-PMT

- Good TTS (<35Ps) \& enough lifetime

- Multialkali photo-cathode $\rightarrow$ SBA

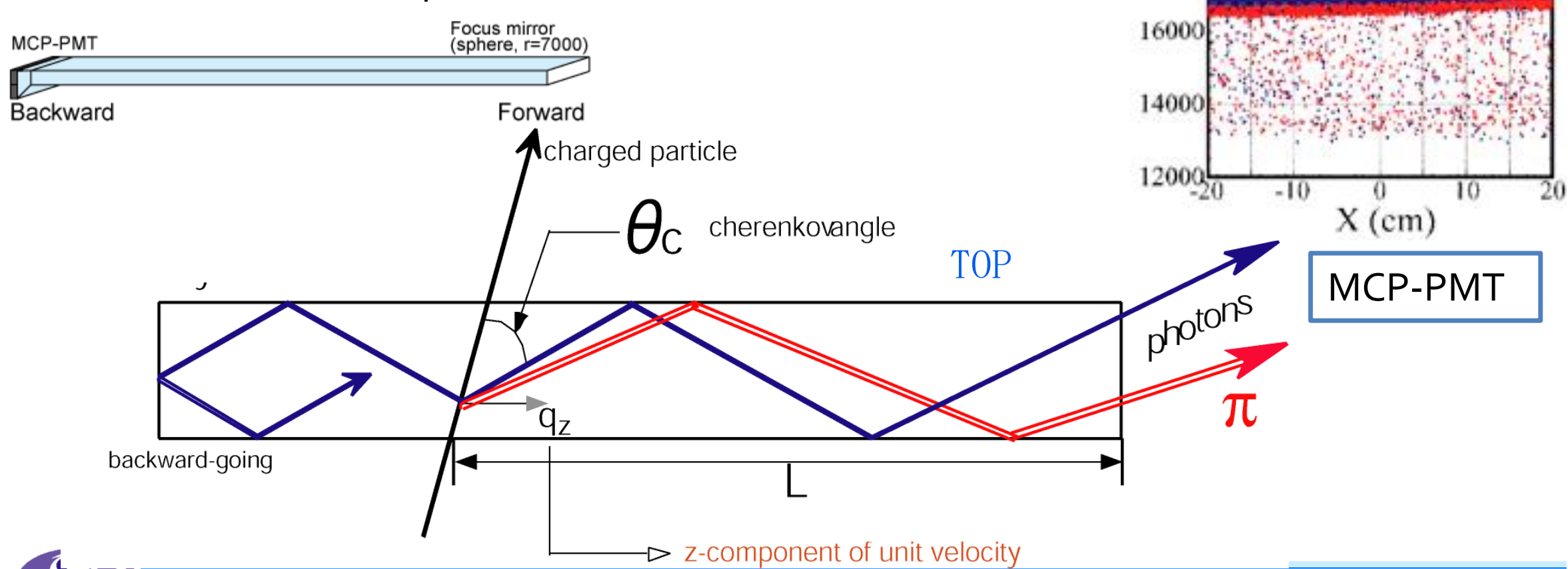




\section{KLM}

- Endcap \& Barrel 2 lyrs

$-\mathrm{RPC} \rightarrow$ Scintillator+MPPC

- (7-10)x40 $\mathrm{mm}^{2}$ scintillator strip+WLS

- 14 layers Superlayer $(\mathrm{X}, \mathrm{Y})$

- MPPC from HPK

- same design as T2K

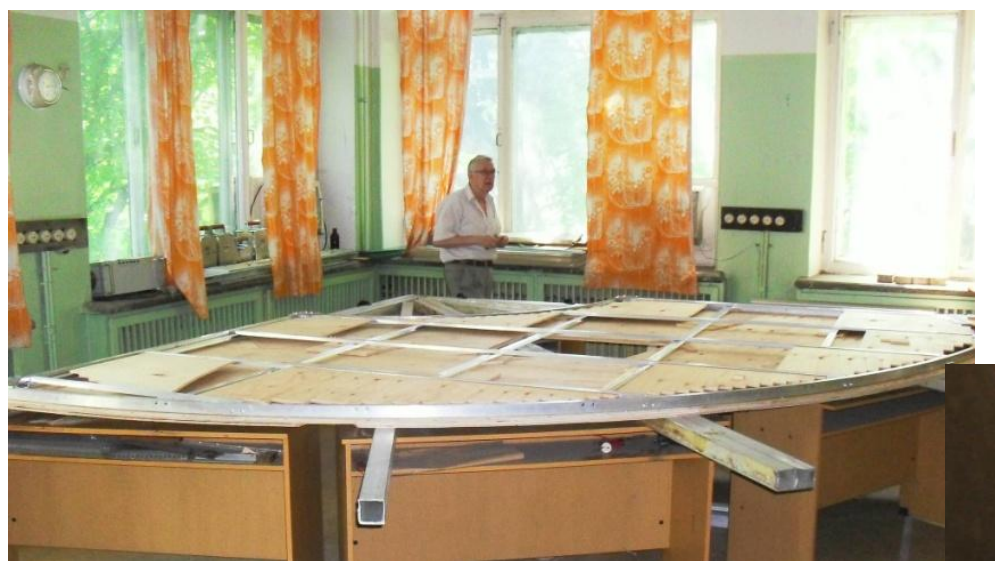

Mechanical Mockup

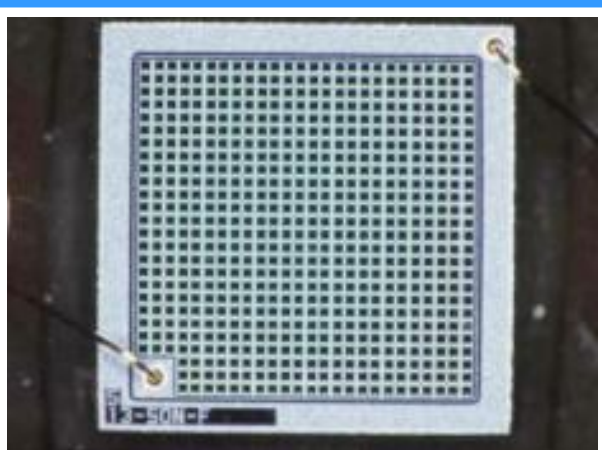

MPPC: Hamamatsu

$1.3 \times 1.3 \mathrm{~mm} 667$ pixels (used in T2K ND)

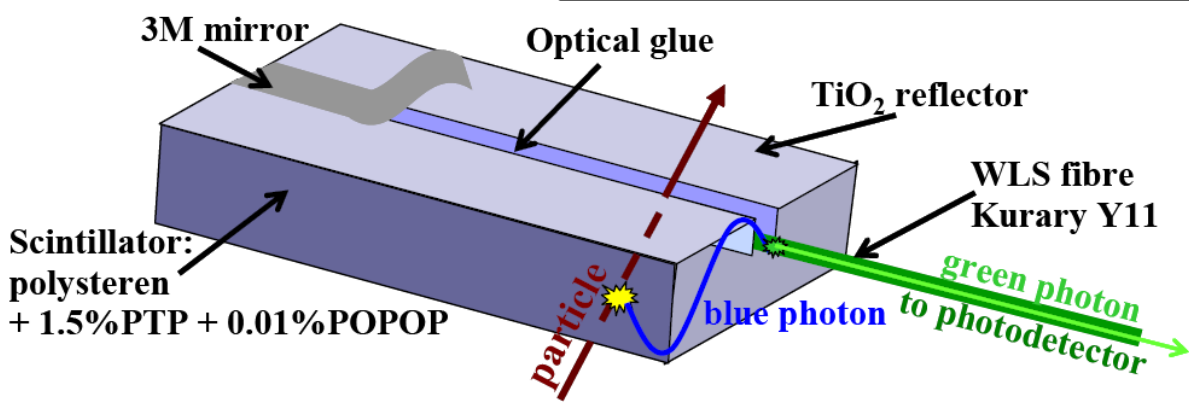

Scintillator bar: Vladimir (Russia) (used in T2K ND) 


\section{Summary}

Step1

Discovery of CPV in B decays

\section{1 summer!}

Step2

Precise test of $\mathrm{KM}$ and $\mathrm{SM}$

Step3

Search for NP Hints of NP

(SUSY, Extra-dim...) Establish procedures

2008

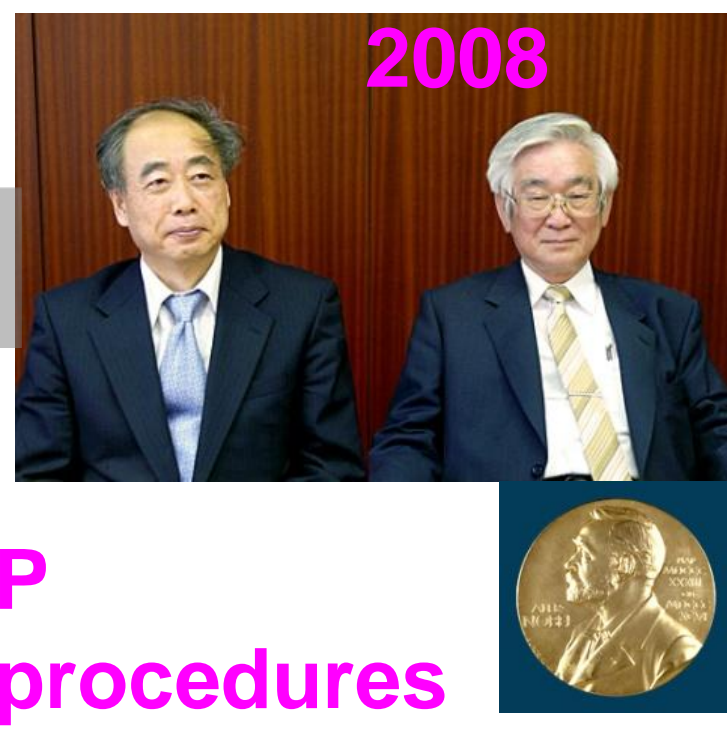

LHC: New particle, masses

SuperB-factory SuperKEKB: couplings Understand NP $\rightarrow$ need Both $\sim 50 \mathrm{ab}^{-1}$ data $\left(\mathrm{L} \sim 10^{36} \mathrm{~cm}^{-2} \mathrm{~s}^{-1}\right)$ 


\section{Summary}
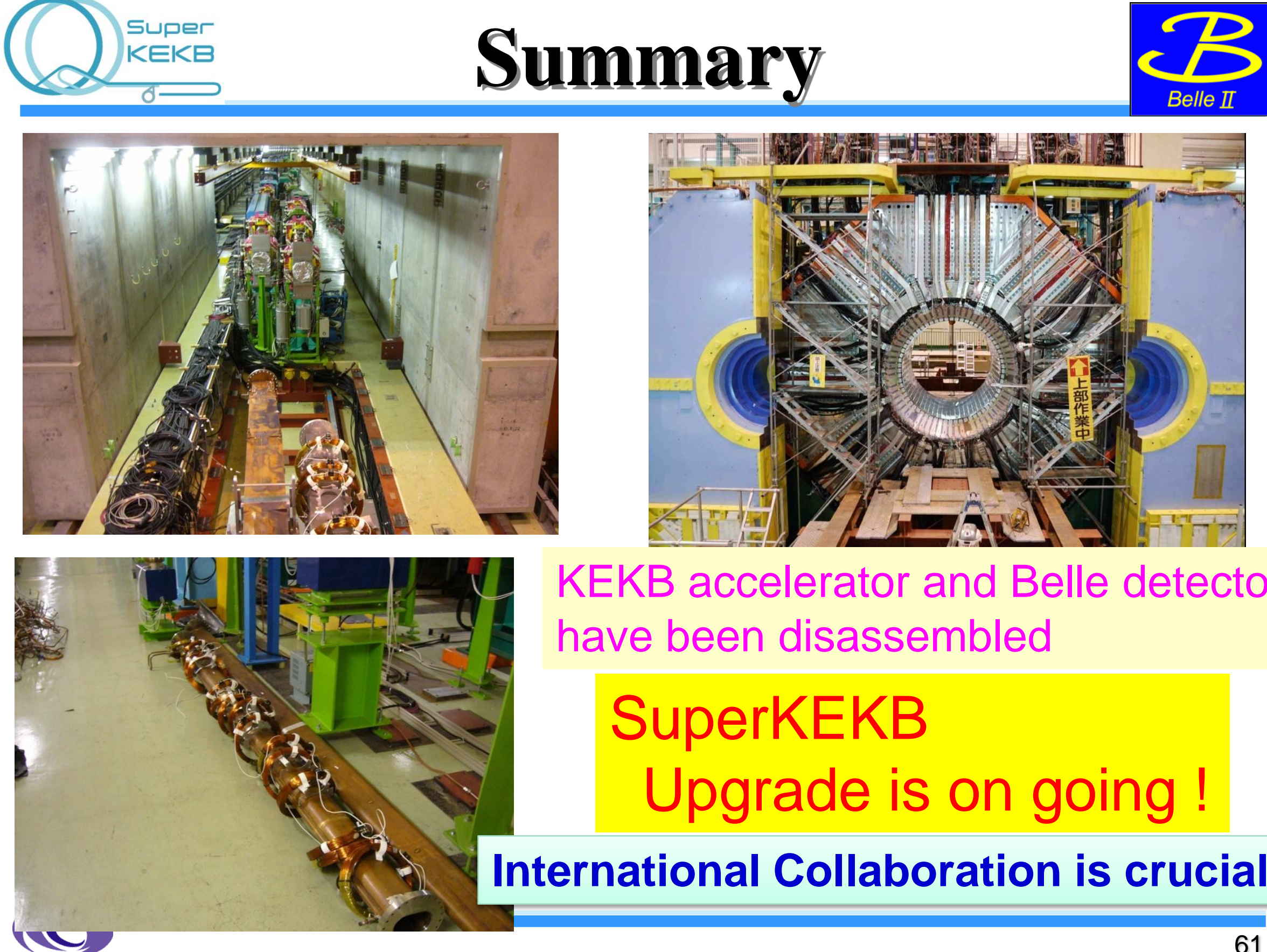

KEKB accelerator and Belle detector have been disassembled

\section{SuperKEKB \\ Upgrade is on going!}

International Collaboration is crucial ! 
(E) 62 


\section{Machine Parameters}

\begin{tabular}{|c|c|c|c|c|}
\hline 2011/July/20 & LER & HER & unit & \\
\hline $\mathrm{E}$ & 4.000 & 7.007 & $\mathrm{GeV}$ & \\
\hline I & 3.6 & 2.6 & A & \\
\hline Number of bunches & \multicolumn{2}{|c|}{2,500} & & \\
\hline Bunch Current & 1.44 & 1.04 & $\mathrm{~mA}$ & \\
\hline Circumference & \multicolumn{2}{|c|}{$3,016.315$} & m & \\
\hline$\varepsilon_{\mathrm{x}} / \varepsilon_{\mathrm{y}}$ & $3.2(1.9) / 8.64(2.8)$ & $4.6(4.4) / 11.5(1.5)$ & $\mathrm{nm} / \mathrm{pm}$ & 0:zero current \\
\hline Coupling & 0.27 & 0.28 & & Includes beam-bean \\
\hline$\beta_{x^{*}} / \beta_{y}^{*}$ & $32 / 0.27$ & $25 / 0.30$ & $\mathrm{~mm}$ & \\
\hline Crossing angle & \multicolumn{2}{|c|}{83} & mrad & \\
\hline$\alpha_{p}$ & $3.25 \times 10^{-4}$ & $4.55 \times 10^{-4}$ & & \\
\hline$\sigma_{5}$ & $8.08(7.73) \times 10^{-4}$ & $6.37(6.31) \times 10^{-4}$ & & 0:zero current \\
\hline$V_{c}$ & 9.4 & 15.0 & MV & \\
\hline$\sigma_{z}$ & $6.0(5.0)$ & $5(4.9)$ & $\mathrm{mm}$ & 0:zero current \\
\hline$V_{5}$ & -0.0247 & -0.0280 & & \\
\hline$v_{x} / v_{y}$ & $44.53 / 44.57$ & $45.53 / 43.57$ & & \\
\hline $\mathrm{U}_{0}$ & 1.87 & 2.43 & $\mathrm{MeV}$ & \\
\hline $\mathrm{Tx}, y / \mathrm{Ts}_{\mathrm{s}}$ & $43.1 / 21.6$ & $58.0 / 29.0$ & msec & \\
\hline$\xi_{x} / \xi_{y}$ & $0.0028 / 0.0881$ & $0.0012 / 0.0807$ & & \\
\hline Luminosity & \multicolumn{2}{|c|}{$8 \times 10^{35}$} & $\mathrm{~cm}^{-2} \mathrm{~s}^{-1}$ & \\
\hline
\end{tabular}




\section{Construction Schedule}

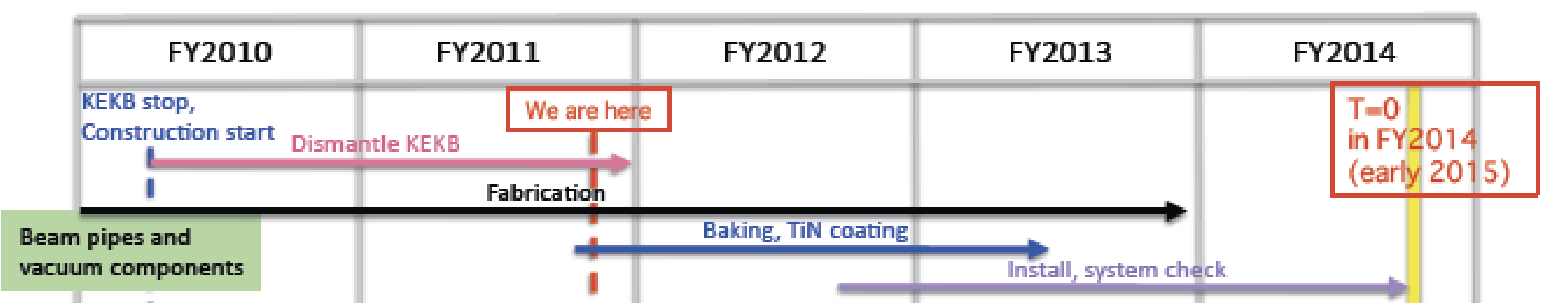

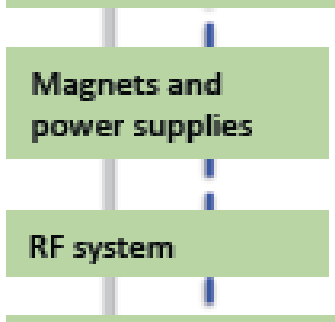

Monitor \& control,

Beam transport
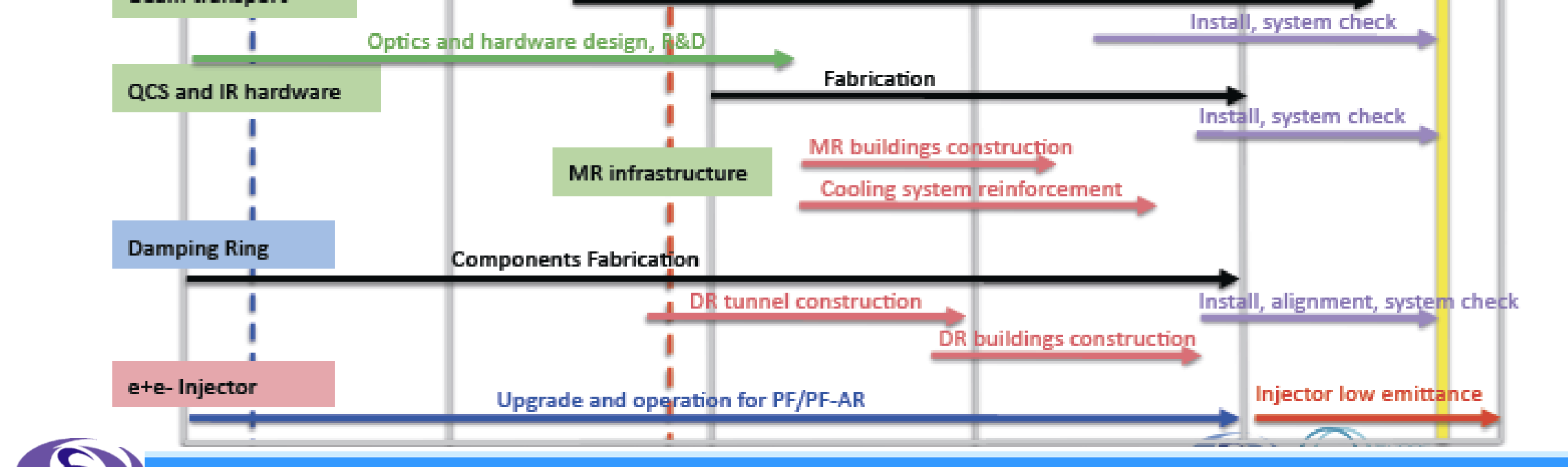

Optics and hardware design, P\&D

Fabrication
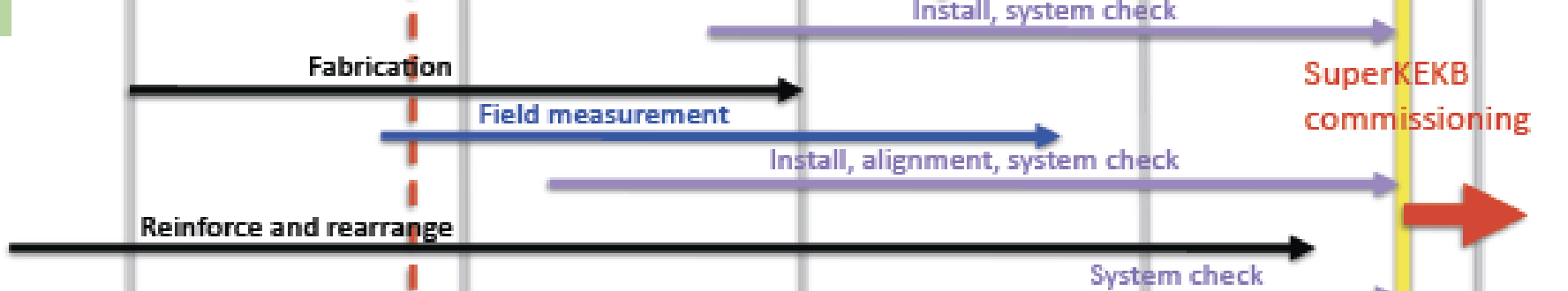


\section{Kobayashi-Maskawa: CPV}

CPV: due to a complex phase in the quark mixing matrix

CKM matrix

$$
\left(\begin{array}{c}
d^{\prime} \\
s^{\prime} \\
b^{\prime}
\end{array}\right)=\left(\begin{array}{c}
V_{u d} V_{u s} V_{u b} \\
V_{c d} V_{c s} V_{c b} \\
V_{t d} V_{t s} V_{t b}
\end{array}\right)\left(\begin{array}{c}
d \\
s \\
b
\end{array}\right)
$$

Wolfenstein representation
$1-\lambda^{2} / 2$
$\lambda$
$-\lambda$
$1-\lambda^{2} / 2$
$A \lambda^{3}(\propto-i \eta)$
$A \lambda^{3}(1-\rho-i \eta)-A \lambda^{2}$

$A \lambda^{2}$
1

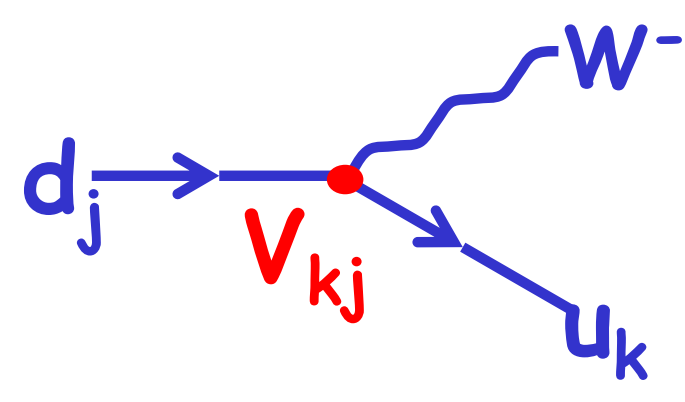

Unitarity triangle

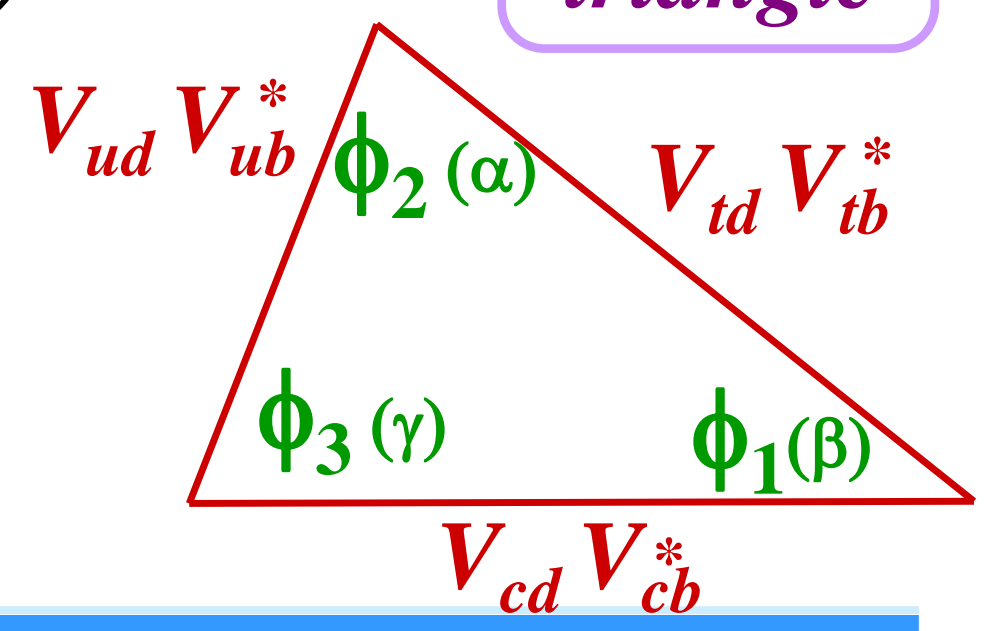




\section{DAQ \& Trigger}

KEK, IHEP, Korea

\section{$\sim 1 \mathrm{MB} / \mathrm{ev}$ $\sim 30 \mathrm{kHz}$ \\ Size reduction $=1 / 10$ voise reduction by track ass}

PXD
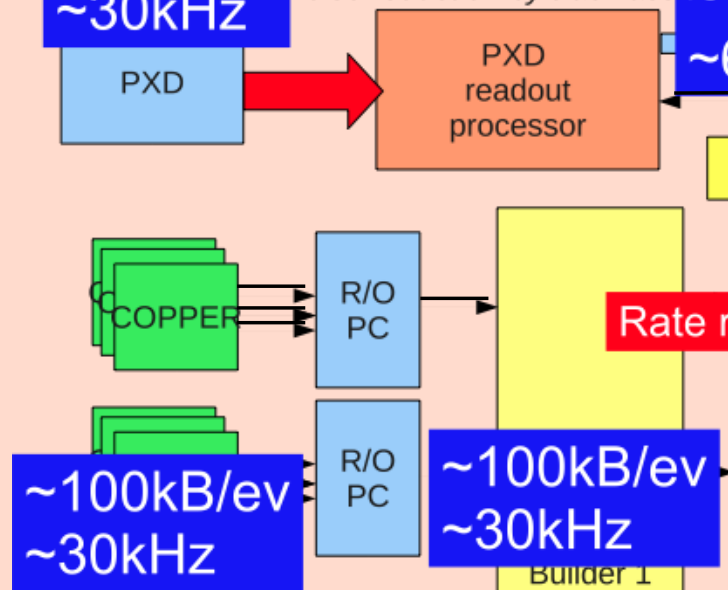

detector No size reduction except Pixel

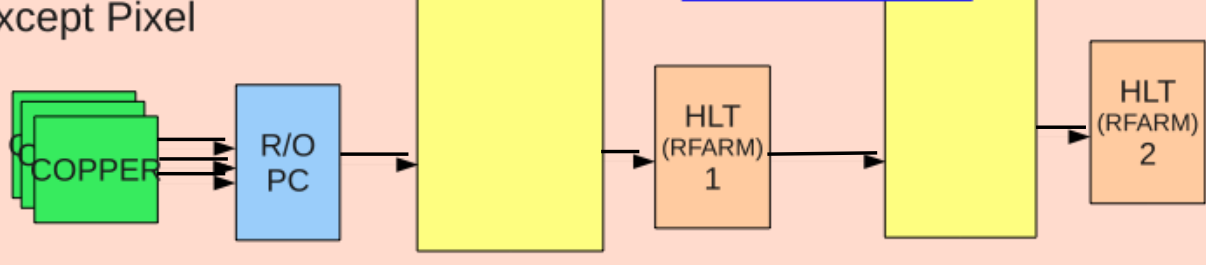
just data nodes)

\begin{tabular}{|c|c|c|}
\hline & Belle & Belle II \\
\hline Trigger rate & $500 \mathrm{~Hz}$ & $30 \mathrm{kHz}$ \\
\hline Event size & $40 \mathrm{kB}$ & $300 \mathrm{kB}(\max )$ \\
\hline
\end{tabular}

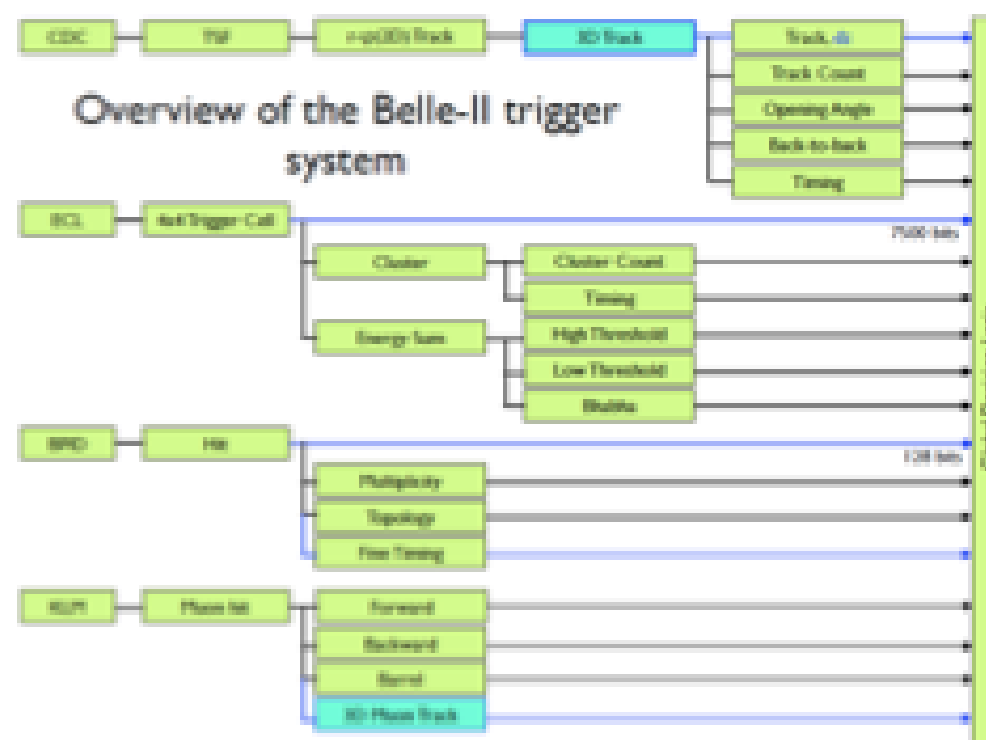

(could be recording

3D trigger study with CDC test chamber
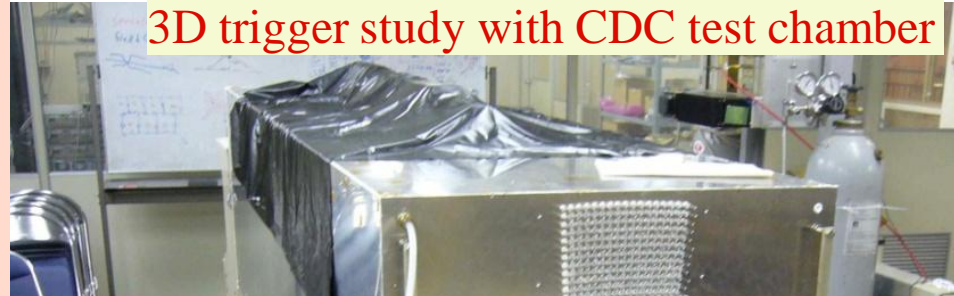

New trigger board

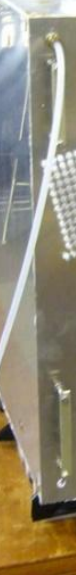




\section{Computing}

\begin{tabular}{|c|c|c|}
\hline & HLT rate & event size \\
\hline Belle II & $3.6 \mathrm{kHz}$ & $300 \mathrm{kB}$ \\
\hline ATLAS & $0.2 \mathrm{kHz}$ & $1.6 \mathrm{MB}$ \\
\hline
\end{tabular}

same amount of Accumulated data

Belle:Centralised computing $\rightarrow$ Belle II:Distributed computing

$50 a b^{-1}$ data

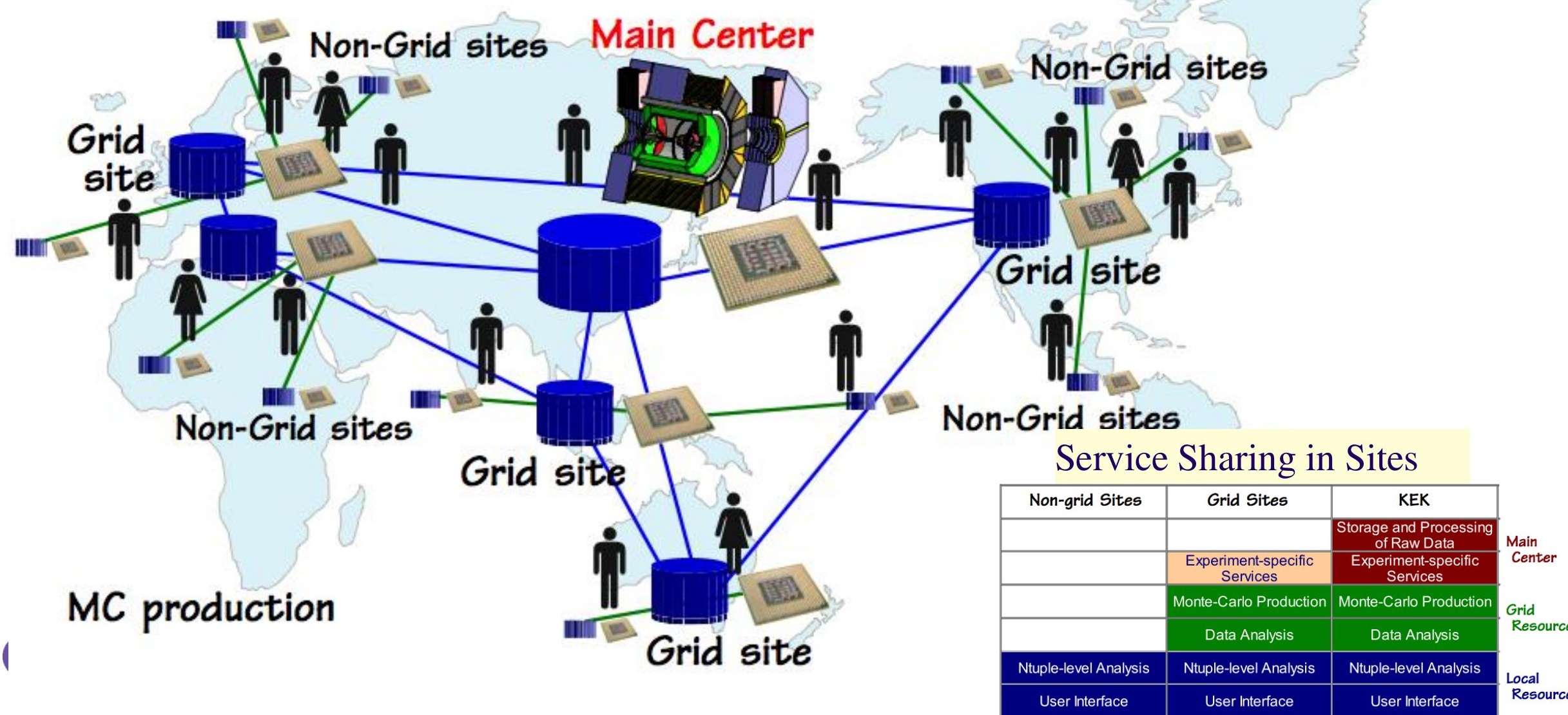




\section{Physics at B factory}

- B physics ( 1.1nb)

- CP violation \& CKM

- Rare decays

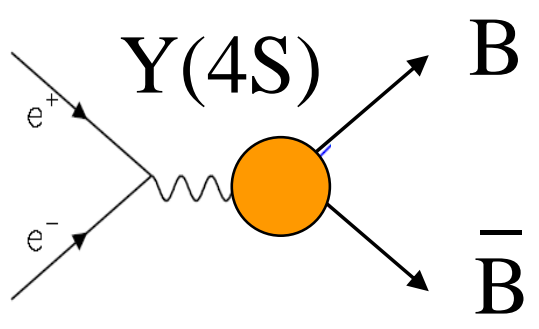

- Charm physics ( 1.3 nb)

- $\tau$ physics $(\sim 0.9 \mathrm{nb})$

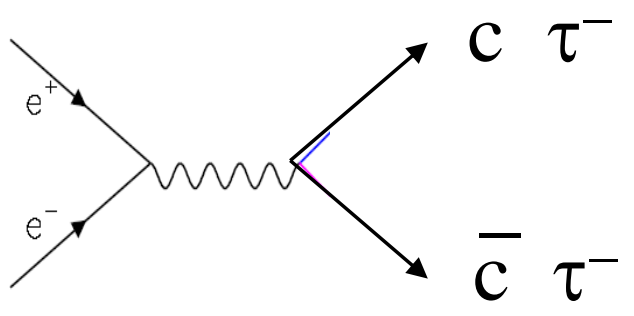

- two-photon processes

- New Resonance

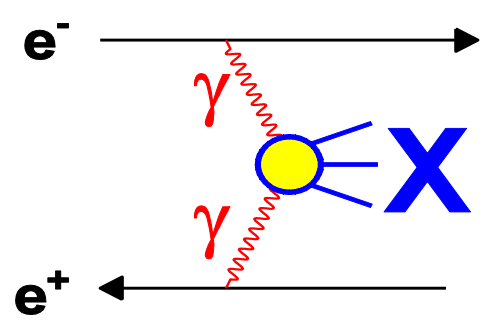

- ordinary \& exotics

\section{Variety of Physics !}

\section{Complement/Cooperative with $\tau /$ Charm factory !}




\section{Physics Prospects: $\tau /$ charm}

\section{Also, Super-Factory for $\tau /$ charm !}

$\tau$ LFV decays

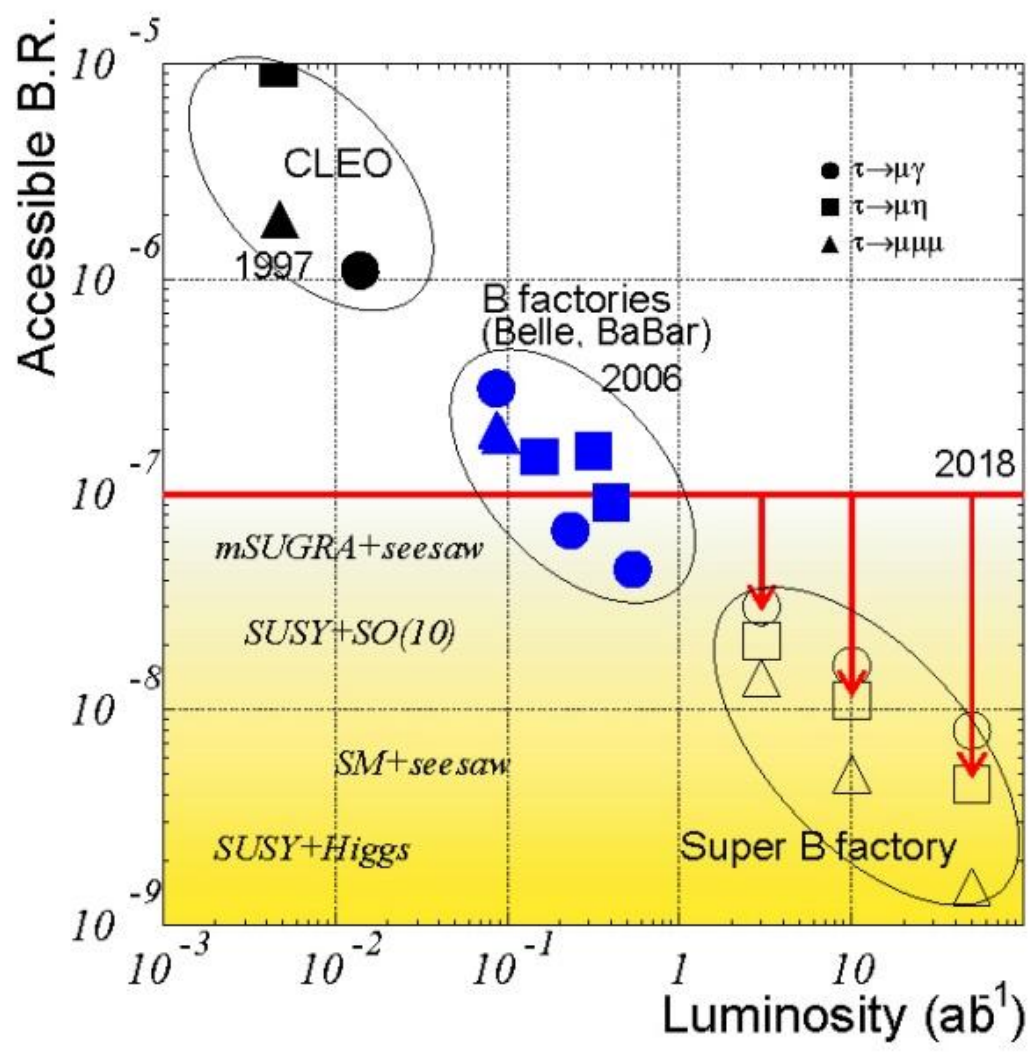

$D^{0}$-mixing

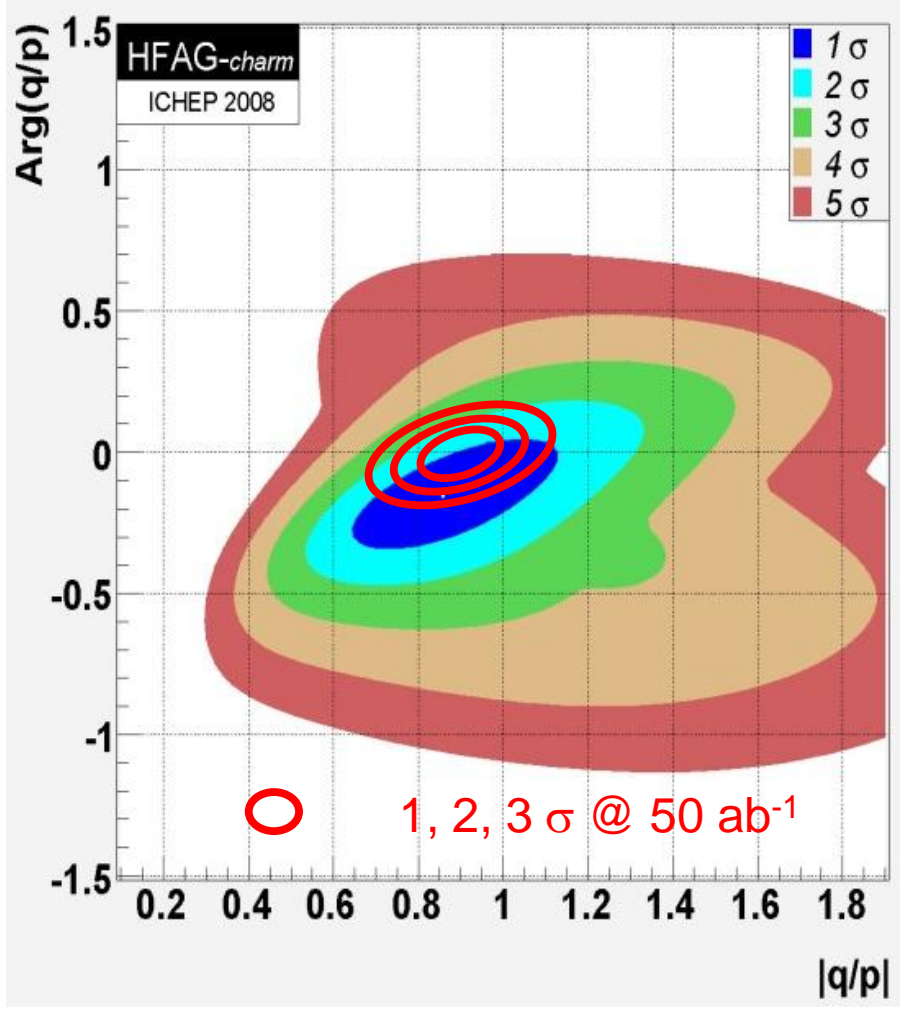

\section{LFV, CPV in $\mathrm{D} / \tau$ : Clear New Physics!}




\title{
Lattice QCD with open boundary conditions
}

\author{
Stefan Schaefer
}

CERN

May 14, 2012

Based on work done in collaboration with Martin Lüscher 
Rising cost as $a \rightarrow 0$

- Need more points for fixed volume $L=$ const $\rightarrow N=L^{4} a^{-4}$.

- Monte Carlo time scales as $a^{-2}$.

n Topological sectors emerge $\rightarrow$ simulation gets stuck

\section{Solutions}

- Fix topological sector.

- Loss of unitarity.

- Deal with $1 / V$ corrections.

- Open the lattice. 


\section{Scaling in pure gauge theory}

- Topological charge shows dramatic slow down: periodic b.c.

n Pure gauge theory

SOMMER, VIROTTA, ST.S' 10

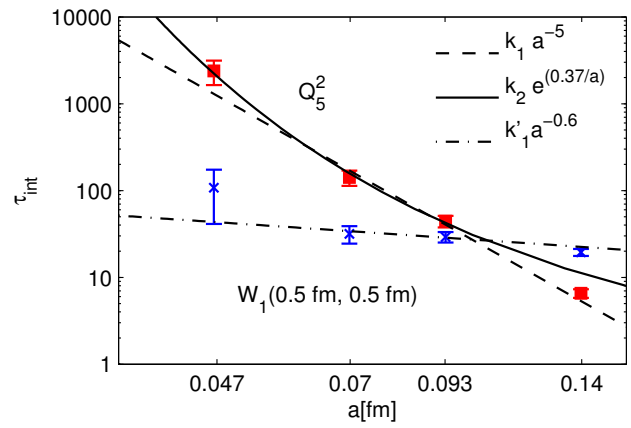




\section{Topological Charge}

Slowing down

- Topological sectors emerge in continuum limit.

- Simulation gets stuck.

\section{Fermions}

n Some folklore that fermions solve the problem.

- Distribution of $Q$ gets narrower at light quark mass.

- Different effective gluonic action

$\rightarrow$ influences coefficient.

- Slow topology observed, e.g., by MILC, ALPHA. 


\section{Open boundary conditions}

Proposed solution

- open boundary condition in time direction

$\rightarrow$ same transfer matrix, same particle spectrum

- periodic boundary condition in spatial directions

$\rightarrow$ momentum projection possible

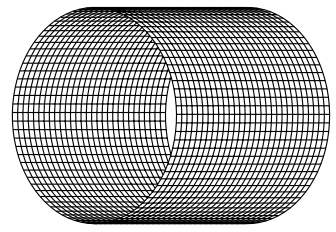




\section{Open boundary conditions}

- Periodic boundary conditions in space.

- Neumann boundary conditions in time.

- Gauge fields

$$
\left.F_{0 k}\right|_{x_{0}=0}=\left.F_{0 k}\right|_{x_{0}=T}=0, \quad k=1,2,3
$$

- Fermion fields

$$
\begin{aligned}
& \left.P_{+} \psi(x)\right|_{x_{0}=0}=\left.P_{-} \psi(x)\right|_{x_{0}=T}=0 \quad P_{ \pm}=\frac{1}{2}\left(1 \pm \gamma_{0}\right) \\
& \left.\bar{\psi}(x) P_{-}\right|_{x_{0}=0}=\left.\bar{\psi}(x) P_{+}\right|_{x_{0}=T}=0
\end{aligned}
$$




\section{On shell improvement}

Boundary terms

Gauge action

$$
\delta S_{G, b}=\frac{1}{2 g_{0}^{2}}\left(c_{G}-1\right) \sum_{p_{s}} \operatorname{tr}\left(1-U\left(p_{s}\right)\right)
$$

Fermion action

$$
\delta S_{F, b}=a^{3}\left(c_{F}-1\right) \sum_{\vec{x}}\left(\left.\bar{\psi}(x) \psi(x)\right|_{x_{0}=a}+\left.\bar{\psi}(x) \psi(x)\right|_{x_{0}=T-a}\right)
$$

- Very similar to Schrödinger functional.

- If one stays clear of boundaries, might not be needed. 


\section{Pure gauge theory: $\tau_{\text {int }}$ vs $a^{-2}$}

\section{LÜSCHER, ST.S, JHEP 1107 (2011) 036}

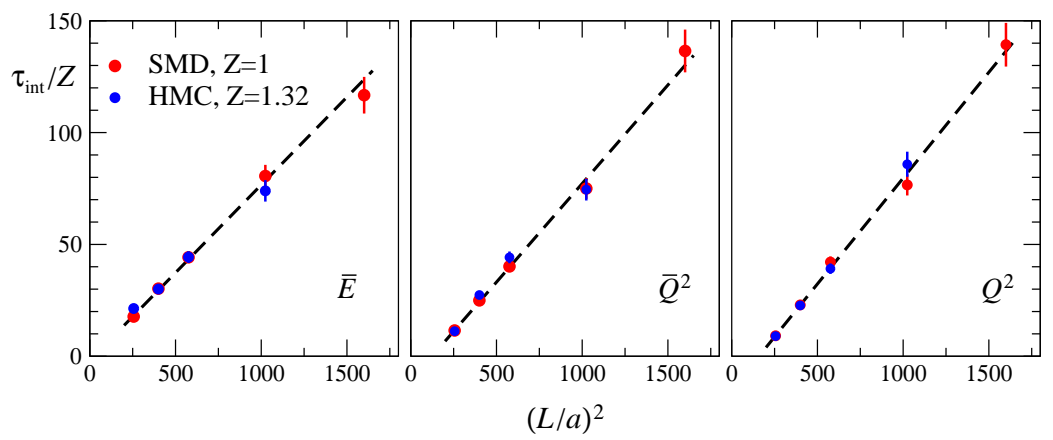

- $L=$ const

- scaling linear in $a^{-2}$.

- no effect of sector forming visible. 


\section{Pure gauge theory: Periodic vs Open boundaries}

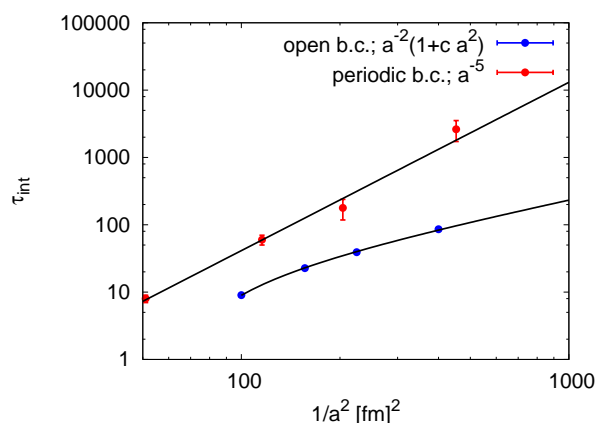

- Open boundary conditions solve problem.

- Scaling of the topological charge same as other observables.

- Already at typical $a$ sizable improvement. 
Finite volume

- For $T \rightarrow \infty$ the effect of the b.c. vanishes.

- But also the effect on observables vanishes as $V^{-1}$.

\section{Dependence on $T$}

- Width of distribution of $Q$ is $\propto \sqrt{T L^{3}}$.

- Change of charge through boundary $\propto \sqrt{L^{3}}$.

$\rightarrow$ expect $\tau_{\text {int }} \propto T$, for random walk

- For each $T$, there is an $a$ from which the boundary tunneling dominates over the bulk tunneling. 


\section{Analysis}

- Physics in the center as with period. bound. cond.

- Boundary effects decay with lightest state of vaccuum quantum numbers. $\rightarrow 2 \pi$

- How is the effect in actual simulations? 


\section{Action}

- $N_{\mathrm{f}}=2+1 \mathrm{NP}$ improved Wilson fermions

- Iwasaki gauge action

- $64 \times 32^{3}$ lattice with $a=0.09 \mathrm{fm}$

- studied extensively by PACS-CS

- $m_{\pi}=200 \mathrm{MeV} ; m_{\pi} L=3$

\section{Reweighting}

- Simulate fermion action with spectral gap.

- Include reweighting factor in measurement.

- Stabil simulation, no ergodicity problems. 


\section{Yang-Mills action density}

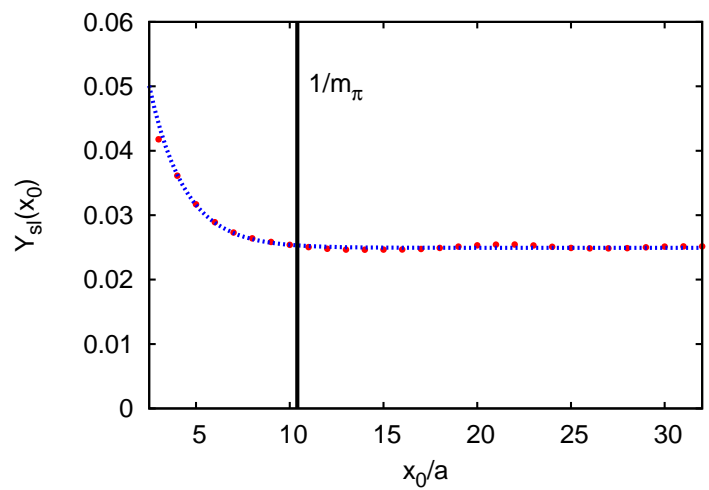

- Gauge action density from smoothed links.

- Boundary effects decay with mass $\approx 1 \mathrm{GeV}$.

- $m_{\pi} \approx 200 \mathrm{MeV}$. 


\section{Boundary conditions}

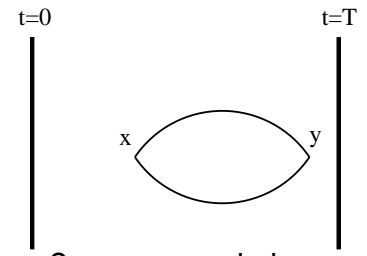

$$
C\left(x_{0}, y_{0}\right)=\sum_{\mathbf{x}, \mathbf{y}}\left\langle P\left(x_{0}, \mathbf{x}\right) P\left(y_{0}, \mathbf{y}\right)\right\rangle
$$

- Source point $y$, zero momentum projection

- With periodic bc get $\cosh \left(m\left(x_{0}-y_{0}\right)\right)$ behavior

Open boundary conditions

Dirichlet boundary condtions for hadron propagator

$$
C(x, y) \propto \sinh \left(m\left(T-x_{0}\right)\right) \quad \text { for } \quad x_{0}>y_{0}
$$




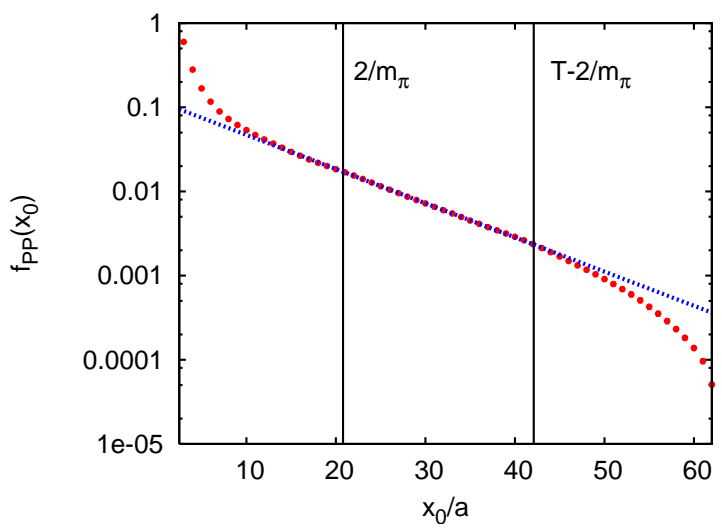

- source at $y_{0} / a=1$

- exponential fall-off $2 / m_{\pi}$ away from source/boundary 


\section{Pseudoscalar Correlator: effective mass}

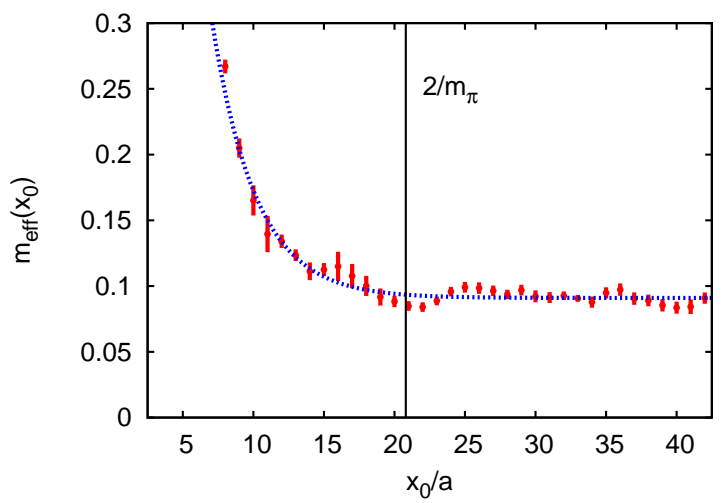

- Mass agrees with PACS-CS (interpolated) value 


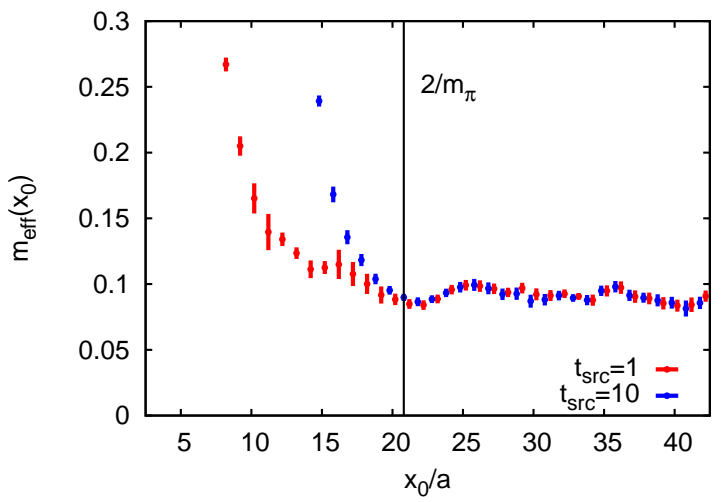

- Source on boundary couples strongy to excited states

- Plateau starts about at same time slice. 


\section{Conclusions}

- Simulations with reduced rate of tunneling cannot produce accurate results.

- Open boundary conditions in time solve the problem of frozen topology.

- Fermion simulations without particular problems.

- Measurements $2 / m_{\pi}$ from boundary.

- Reweighting makes Wilson simulations safe. 


\section{Storage ring Electric Dipole Moment} experiment for the proton Yannis K. Semertzidis, BNL

$\checkmark$ Goal: $10^{-29} \mathrm{e}_{\sqsubset} \mathrm{cm}$; Probe New Physics $\sim 10^{3} \mathrm{TeV}$

$\checkmark$ Systematics best in an all-electric ring and counter-rotating (CR) beams. 
Why is there so much matter after

\section{the Big Bang;}

Mistory of the Universe

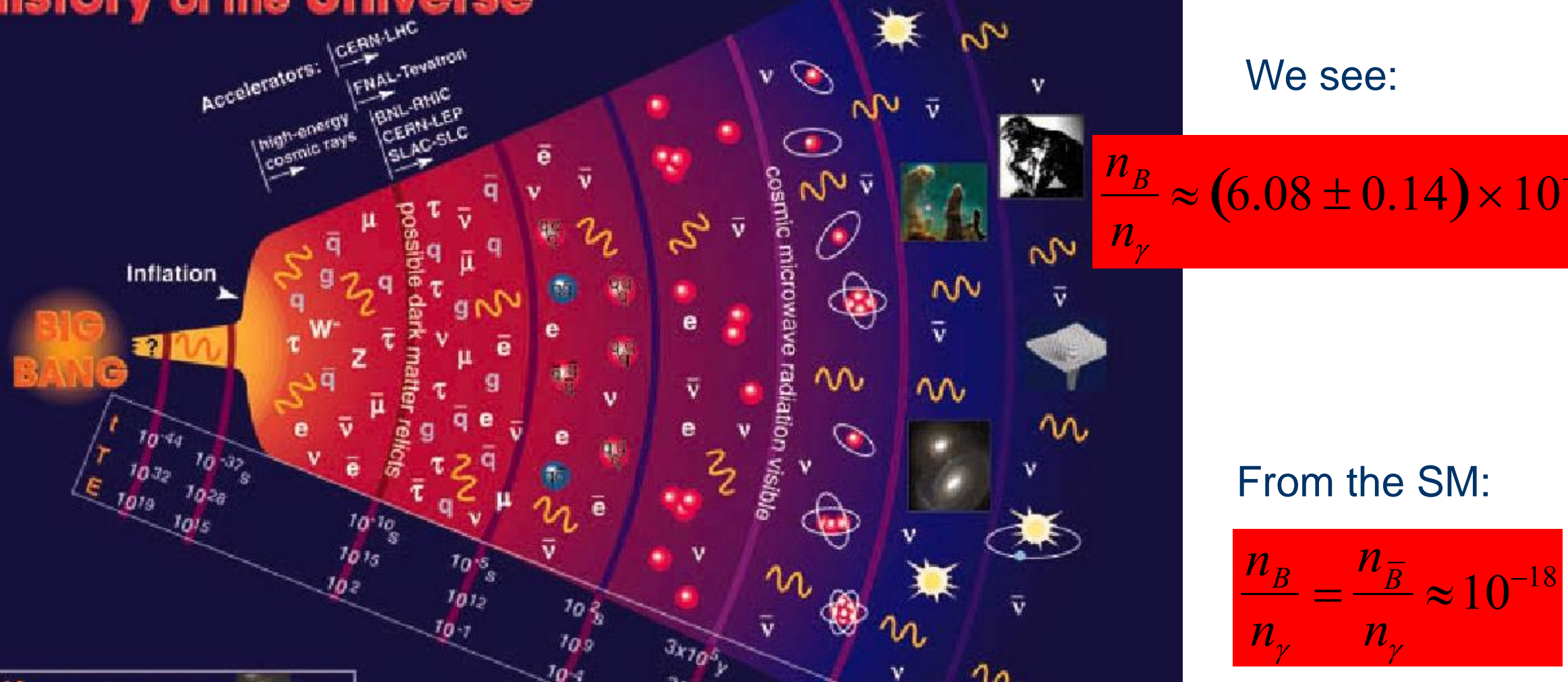

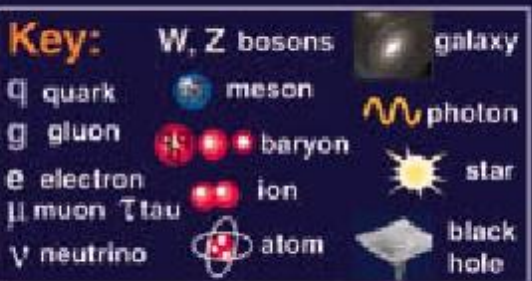

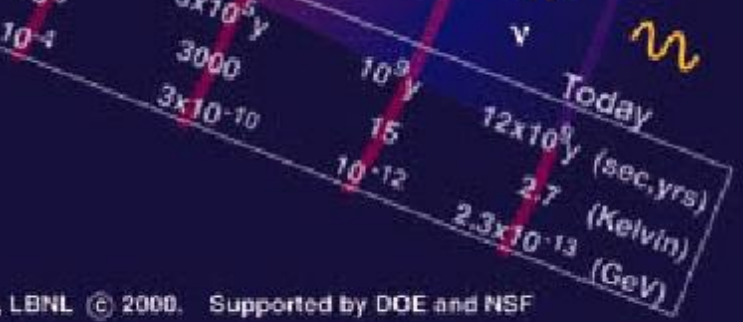


Spin is the only vector defining a direction of a "fundamental" particle with spin
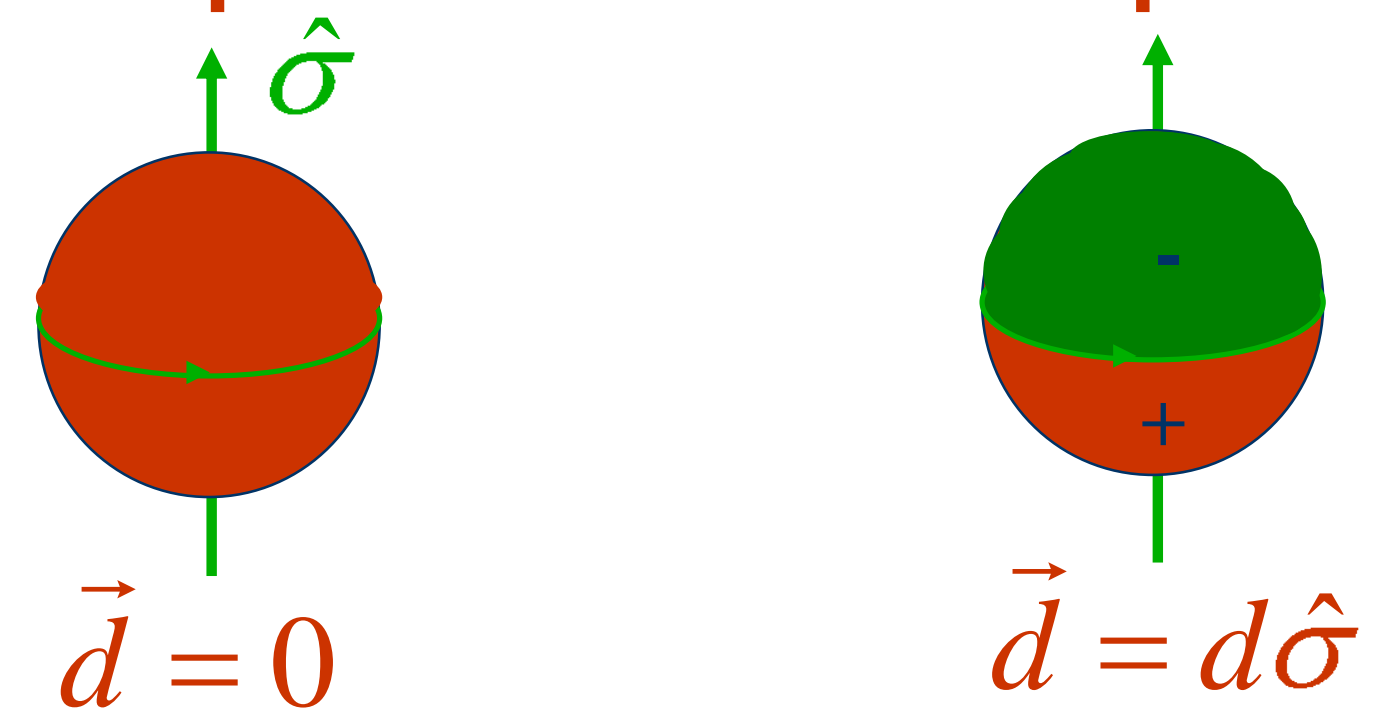
Electric Dipole Moment: two possibilities
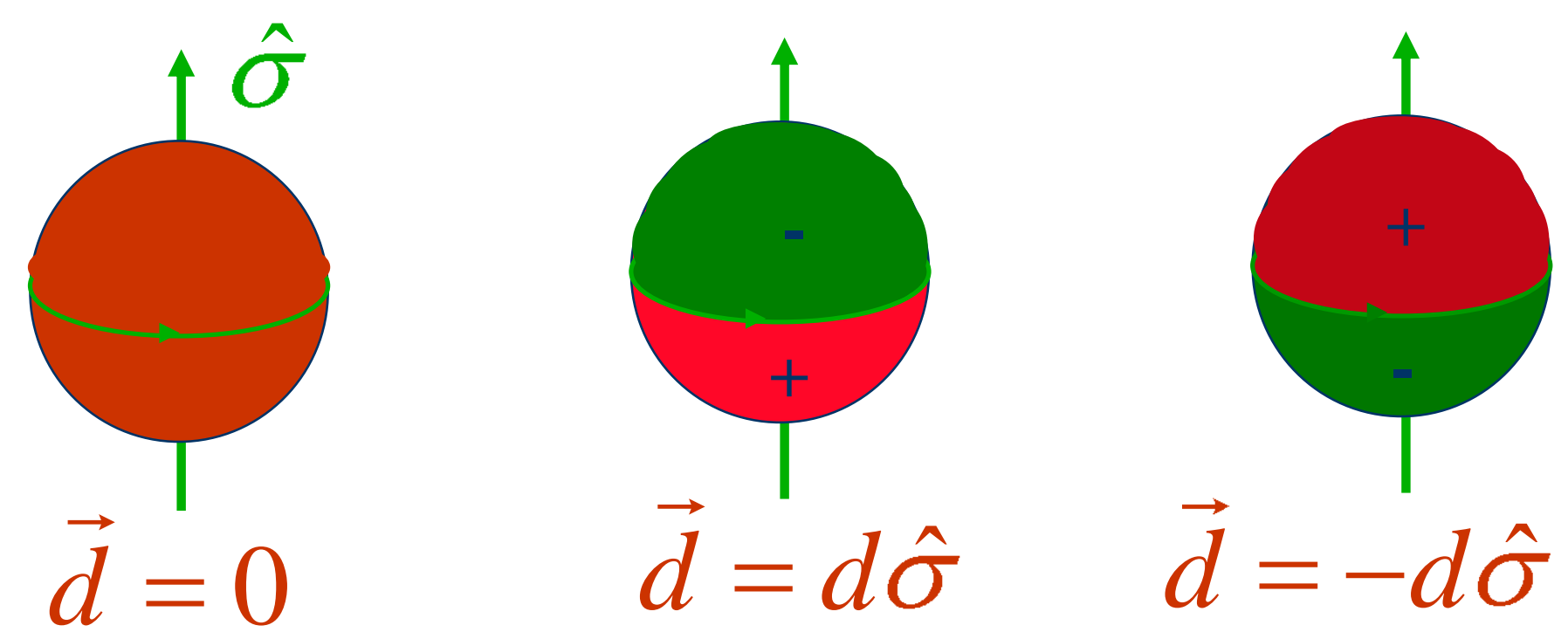


\section{If we discover that the proton}

- Has a non-zero EDM value, i.e. prefers only one of the two possible states:
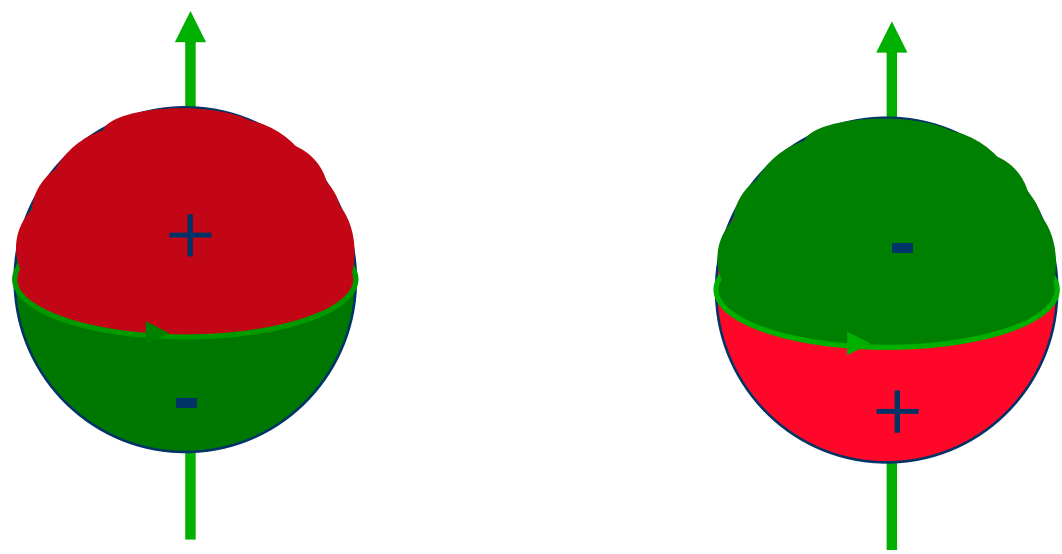

- Then $P$ and $T$ symmetries are violated and through CPT, CP-symmetry is also violated.

- CP-violation is one of three necessary conditions to obtain a matter dominated universe starting from a symmetric one... 


\section{Purcell and Ramsey:}

"The question of the possible existence of an electric dipole moment of a nucleus or of an elementary particle... becomes a purely experimental matter"

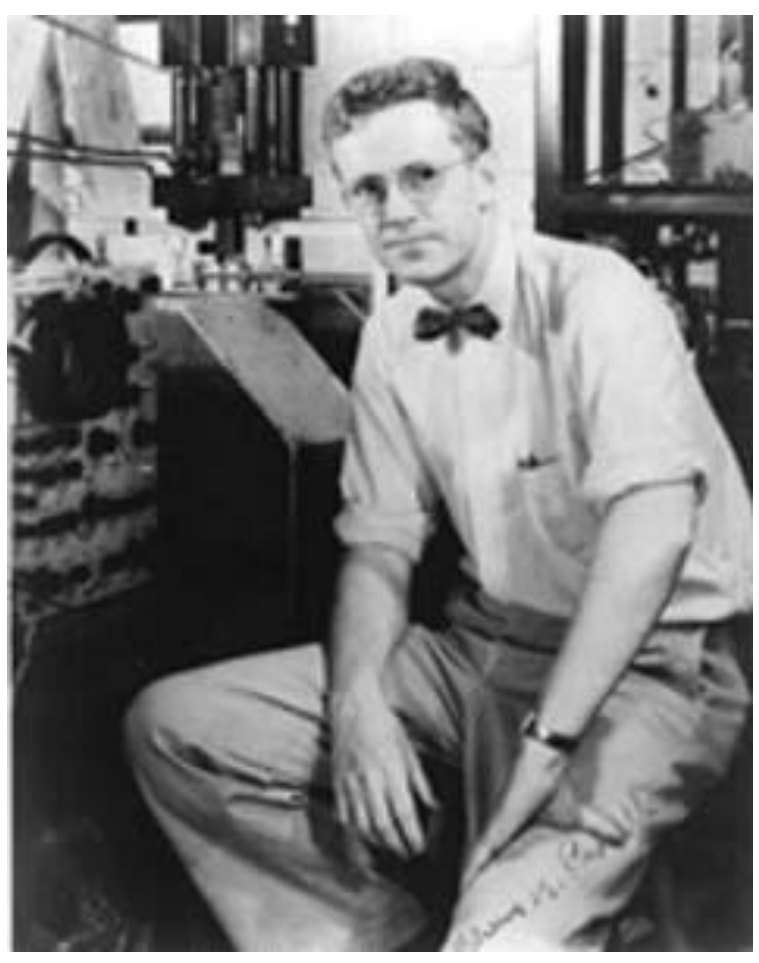

Phys. Rev. 78 (1950)

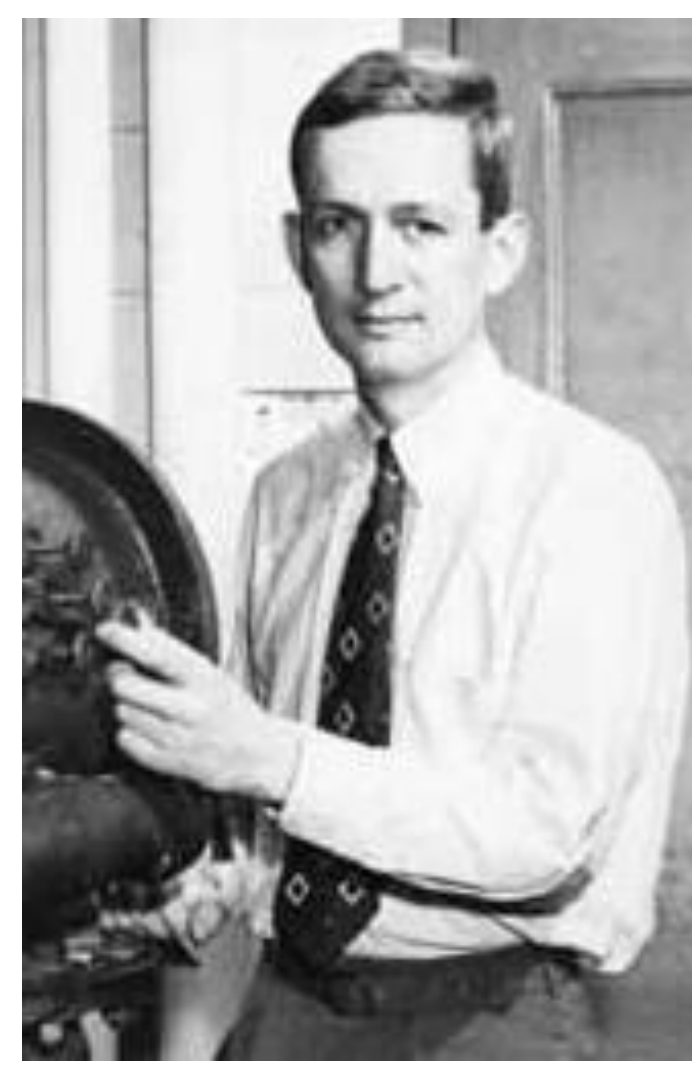




\section{Short History of EDM}

- 1950's neutron EDM experiment started to search for parity violation (Ramsey and Purcell).

- After P-violation $\rightarrow$ EDMs require both P,T-Violation

- 1960's EDM searches in atomic systems

- 1970's Indirect Storage Ring EDM method from the CERN muon g-2 exp.

- 1980's Theory studies on systems (molecules) w/ large enhancement factors

- 1990's First exp. attempts w/ molecules. Dedicated Storage Ring EDM method developed

- 2000's Proposal for sensitive dEDM exp. developed.

- 2010's Proposal for sensitive pEDM exp. developed. 


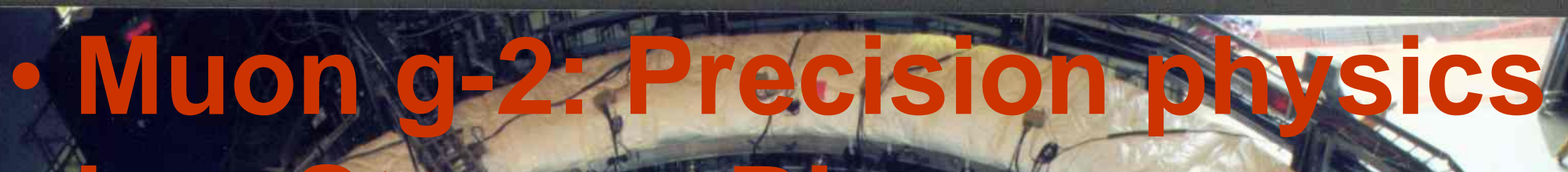
in $5 t$ iat

$y=y^{2}+1$ $3=-0^{2} a^{2}$ stale s limited... to line ove sensh ratagl 4 at Fermation
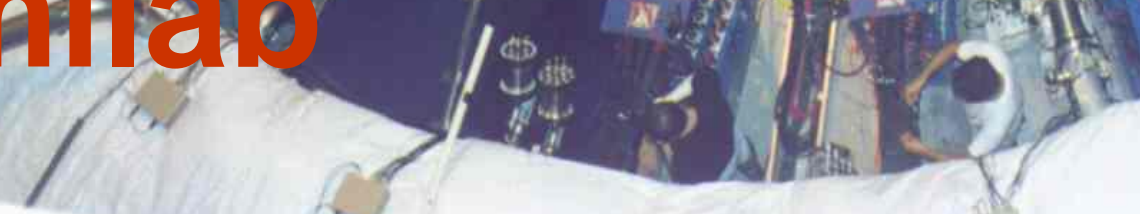


\section{Muon g-2: 4 Billion $\mathrm{e}^{+}$with $\mathrm{E}>2 \mathrm{GeV}$}

$d N / d t=N_{0} e^{-\frac{t}{\tau}}\left[+A \cos \phi_{a} t+\phi_{a}\right]$

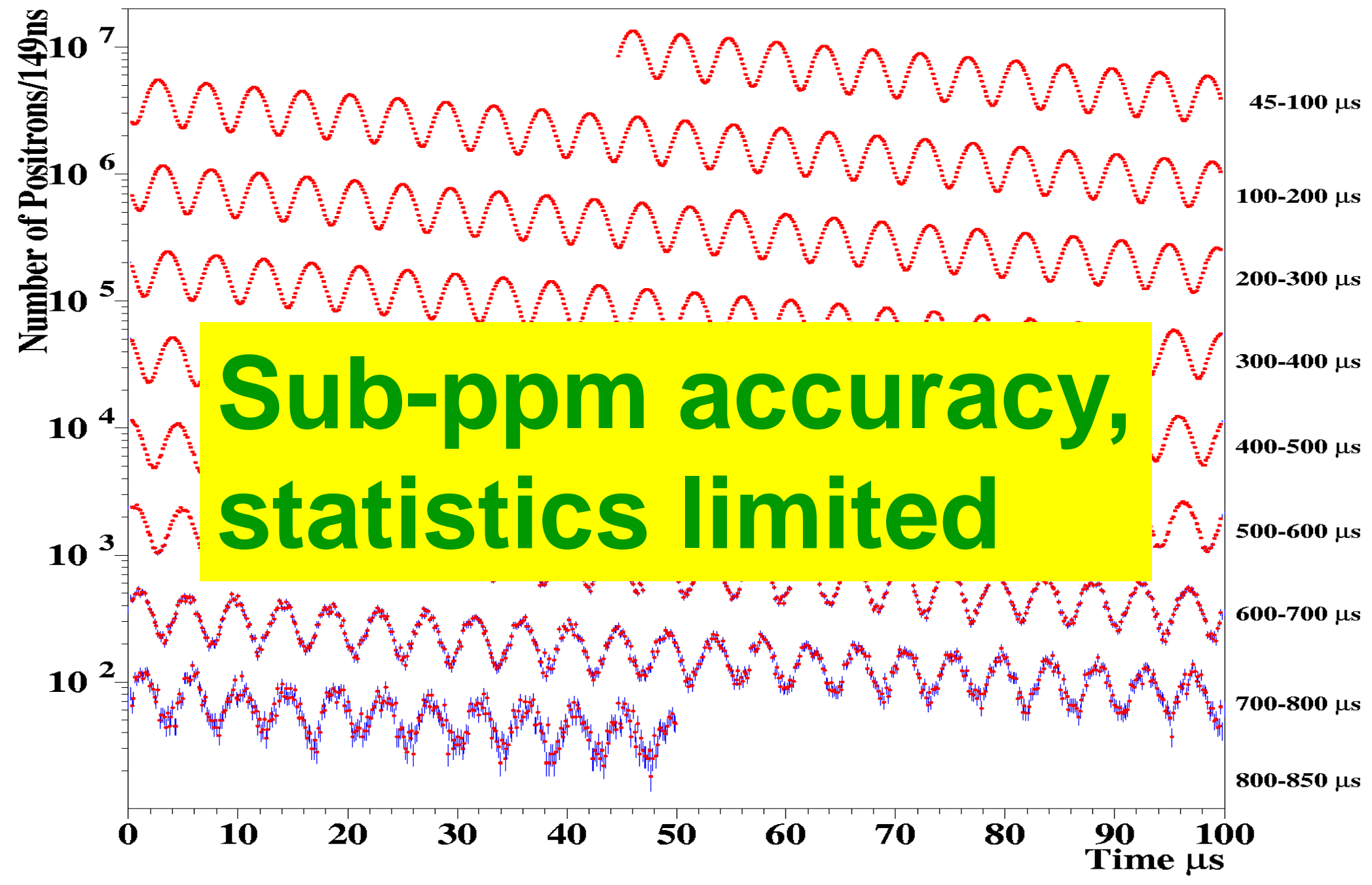


Breakthrough concept: Freezing the horizontal spin precession due to E-field

$$
\vec{\omega}_{a}=\frac{e}{m}\left\{a \vec{B}+\left[a-\left(\frac{m}{p}\right)^{2}\right] \frac{\vec{\beta} \times \vec{E}}{c}\right\}
$$

Muon g-2 focusing is electric: The spin precession due to E-field is zero at "magic" momentum (3.1GeV/c for muons, $0.7 \mathrm{GeV} / \mathrm{c}$ for protons, ...)

$$
p=\frac{m}{\sqrt{a}}, \text { with } a=\frac{g-2}{2}
$$

The "magic" momentum concept was used in the muon g-2 experiments at CERN, BNL, and ...next at FNAL. 
The proton EDM uses an ALL-ELECTRIC ring: spin is aligned with the momentum vector

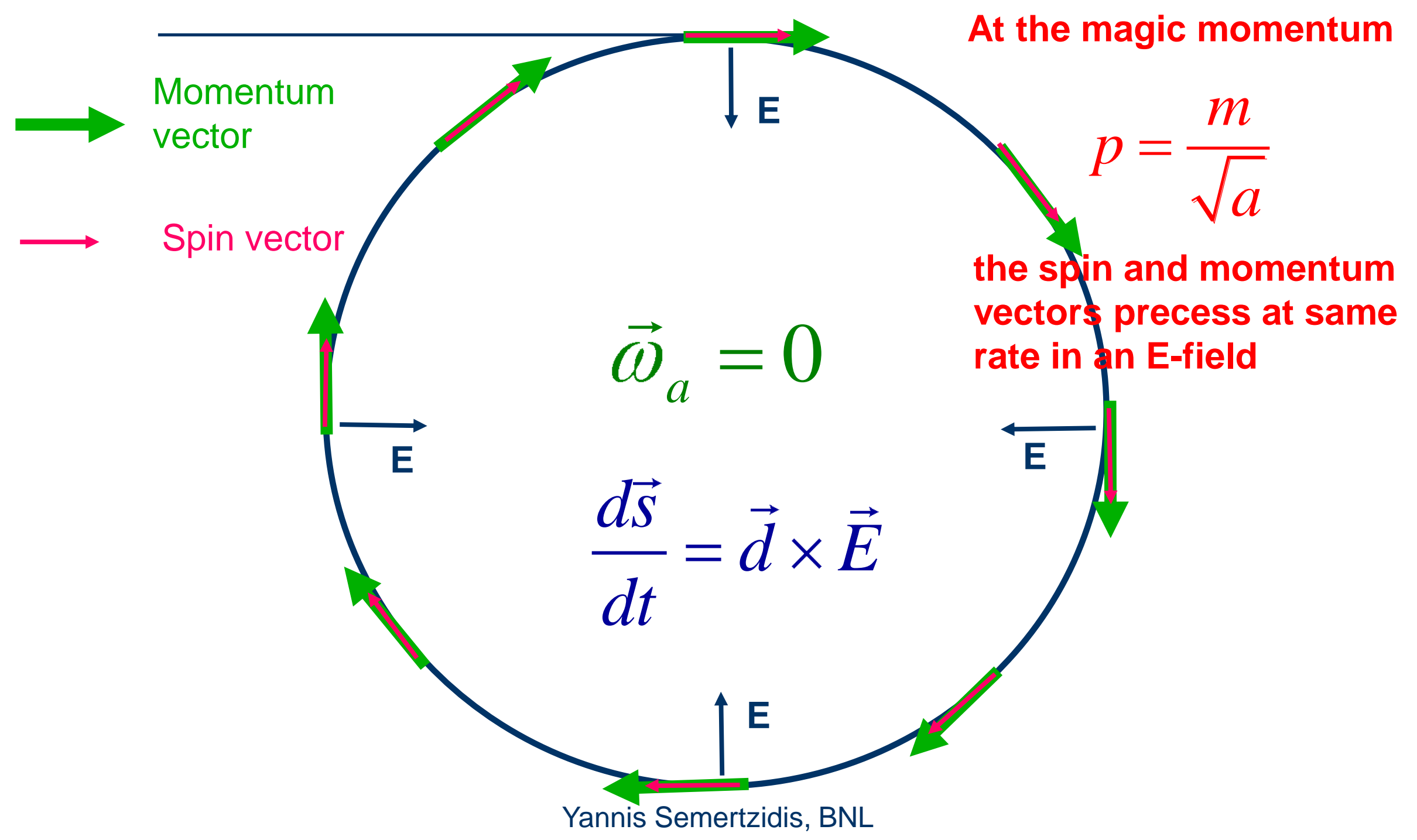




\section{Is the polarimeter analyzing power good at $\mathbf{P}_{\text {magic }}$ ? YES!}

Analyzing power can be further optimized

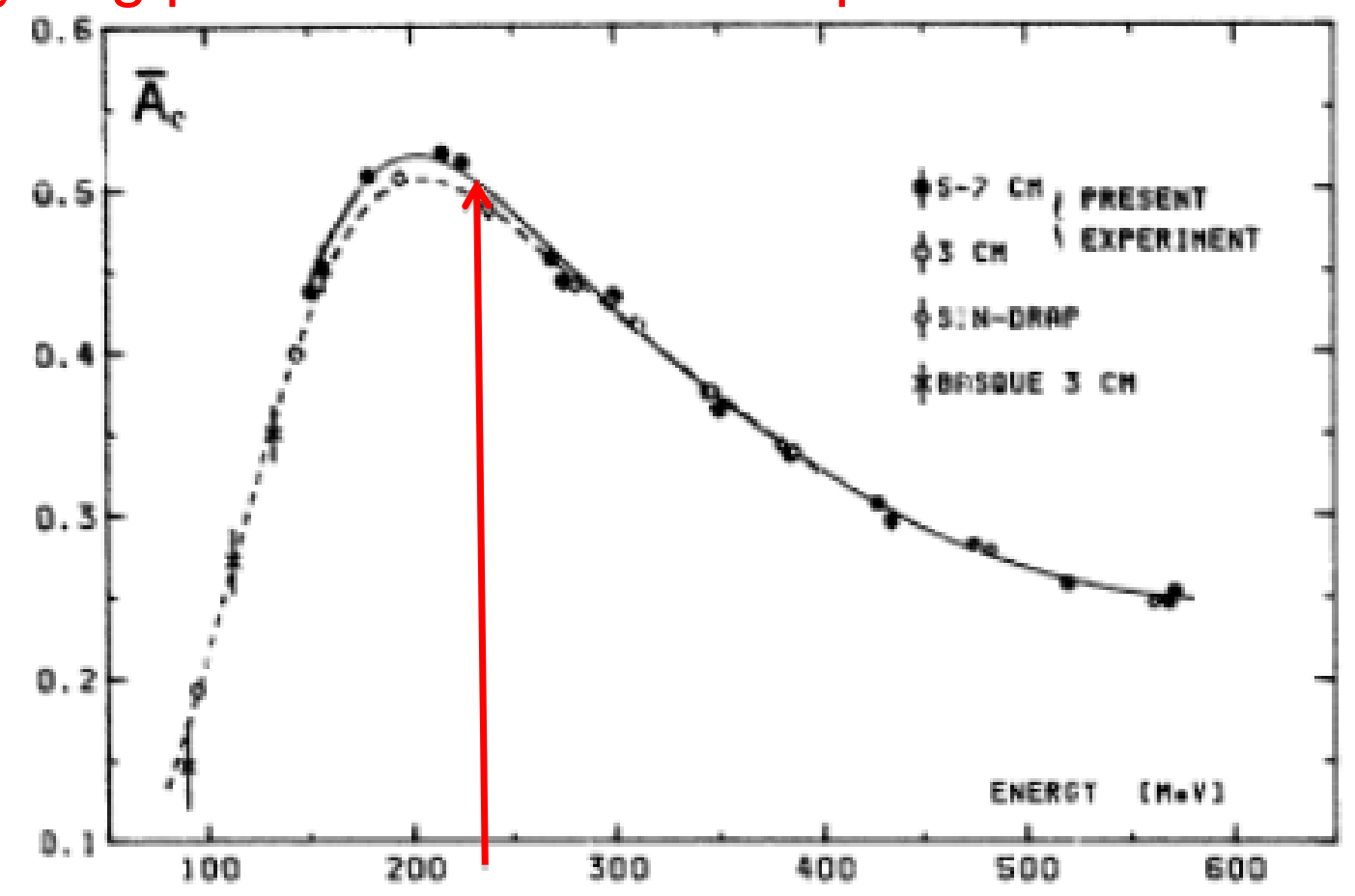

Fig 4. Angle-averaged effective analyzing power. Curves show our fits. Points are the data included in the fits. Errors are statistical eniy

Fig.4. The angle averaged effective analyzing power as a function of the proton kinetic energy. The magic momentum of $0.7 \mathrm{GeV} / \mathrm{c}$ corresponds to $232 \mathrm{MeV}$. 


\section{The proton}

As shown on the March 2011 review EDM ring

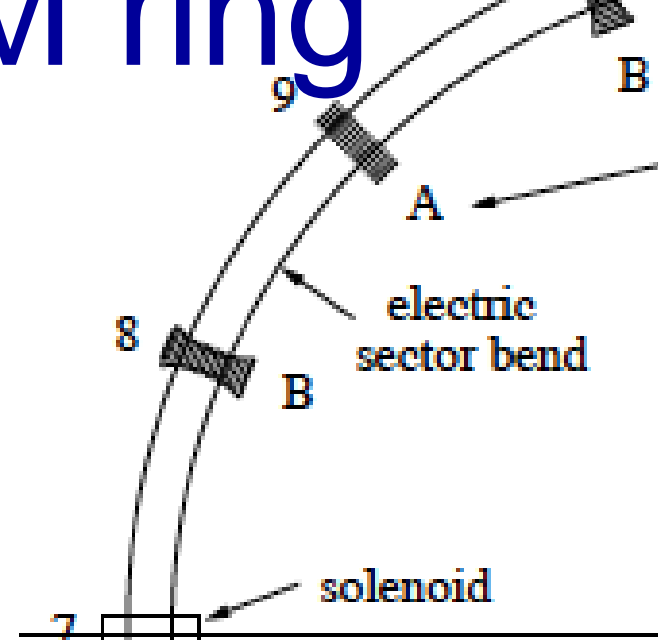
$\rightarrow 2 \mathrm{~m} \mid-$ with limited straight-section length

10
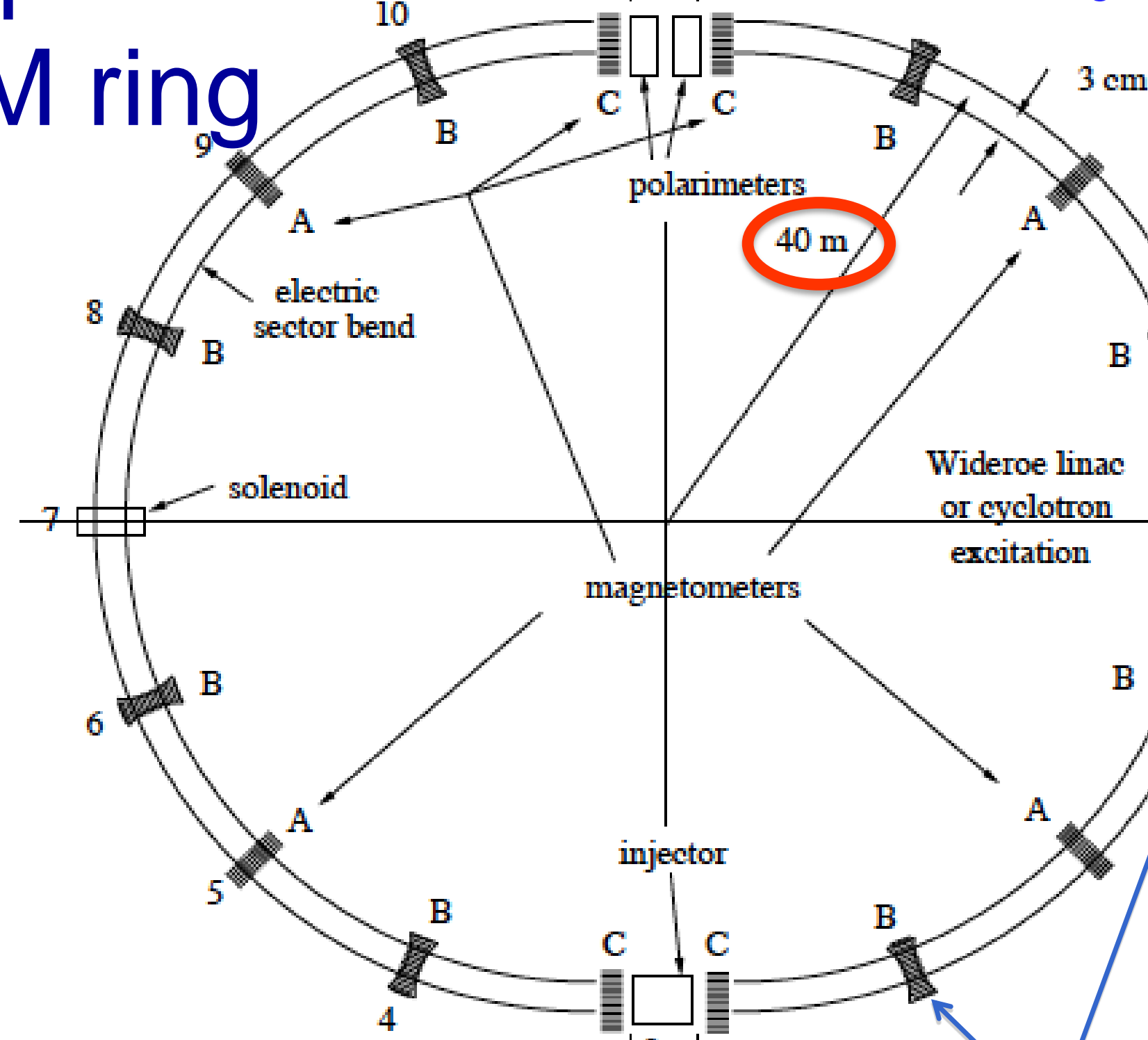

Weak vertical focusing to optimiz⿸丆⿰丨丶刀 SCT and BPM operation 


\section{Important Stages in an EDM Experiment}

1. Polarize: state preparation, intensity of beams

2. Interact with an E-field: the higher the better

3. Analyze: high efficiency analyzer

4. Scientific Interpretation of Result! Easier for the simpler systems (theory; lattice?) 
The grand issues in the proton EDM experiment

1. BPM magnetometers (need to demonstrate in a storage ring environment)

2. Polarimeter development: high efficiency, small systematic errors

3. Spin Coherence Time (SCT): study at COSY/simulations; Simulations for an allelectric ring: SCT and systematic error studies

4. Electric field development for large surface area plates 
Clock-wise (CW) \& Counter-Clock-wise Storage

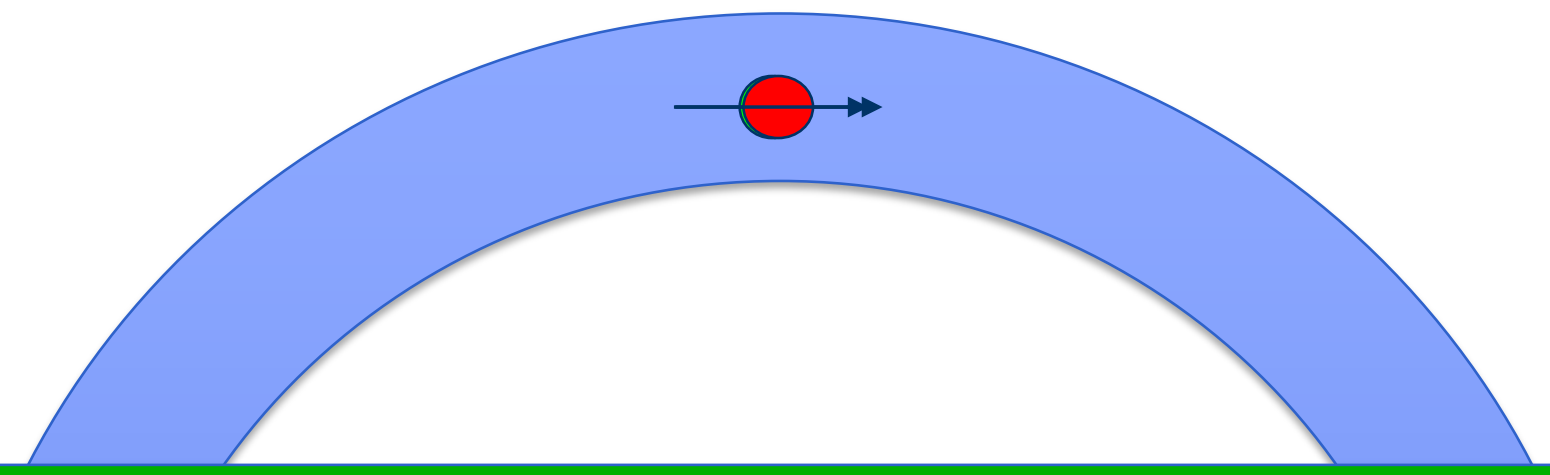

Any radial magnetic field sensed by the stored particles will also cause their vertical splitting. Unique feature among EDM experiments...

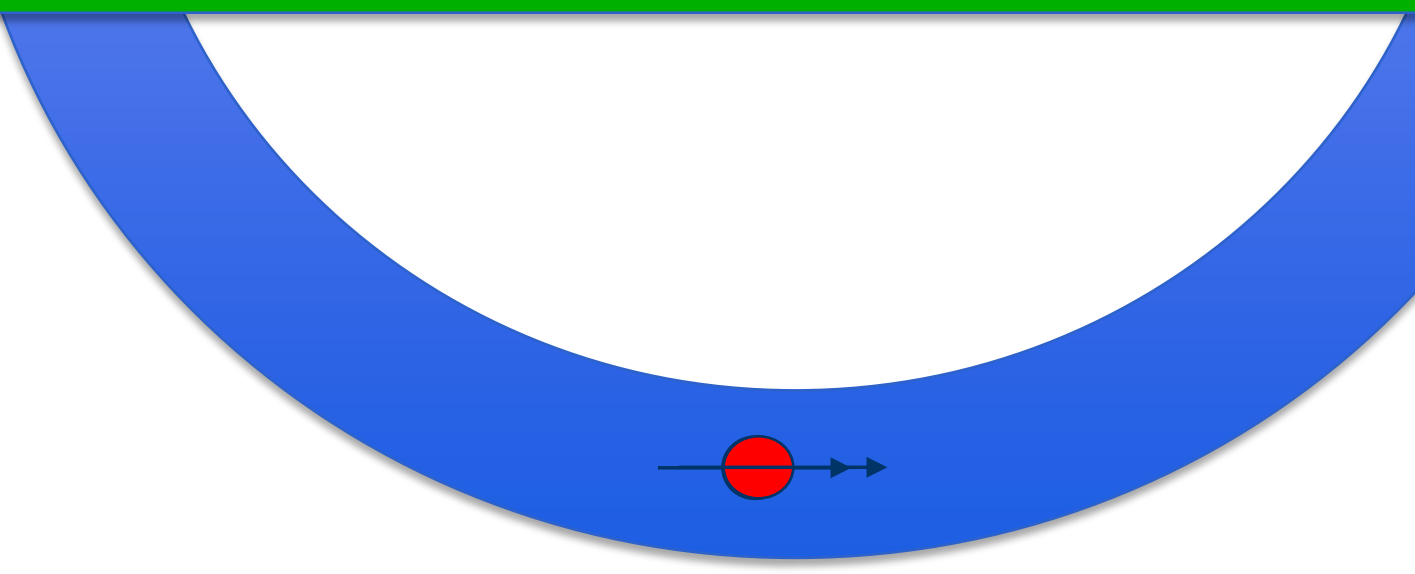




\section{Beam Position Monitors}

- Technology of choice: Low $T_{\mathrm{C}}$ SQUIDS, signal at $10^{2}-10^{4} \mathrm{~Hz}$ (10\% vertical tune modulation)

- R\&D sequence: (First funding from US-Japan)

1. Operate SQUIDS in a magnetically shielded area-reproduce current state of art

2. Operate in RHIC at an IP (evaluate noise in an accelerator environment);

3. Operate in E-field string test 


\section{Polarimeter Development}

- Polarimeter tests with runs at COSY

(Germany) demonstrated < 1ppm level

systematic errors: N. Brantjes et al., NIM A

664, 49, (2012)

- Technologies under investigation:

1. Micro-Megas/Greece: high rate, pointing capabilities, part of R\&D for ATLAS upgrade

2. MRPC/Italy: high energy resolution, high rate capability, part of ALICE development 


\section{Spin Coherence Time: need $>10^{2} \mathrm{~s}$}

- Not all particles have same deviation from magic momentum, or same horizontal and vertical divergence (all second order effects)

- They cause a spread in the g-2 frequencies:

$$
d \omega_{a}=a \vartheta_{x}^{2}+b \vartheta_{y}^{2}+c\left(\frac{d P}{P}\right)^{2}
$$

- Present design parameters allow for $10^{3} \mathrm{~s}$. Cooling/mixing during storage could prolong SCT (upgrade option?). 


\section{SCT Development}

We have a SCT working solution (precision tracking and analytically-work in progress).

Tests with polarized deuterons and protons at COSY to benchmark software

Test runs at COSY are very encouraging.

Bonus: Electric ring with weak vertical focusing $\rightarrow$ SCT is long enough for $10^{3}$ s storage 


\section{Electric Field Development}

$\checkmark$ Reproduce Cornell/JLAB results of stainless steel plates treated with high pressure water rinsing (part of ILC/ERL development work)

Recent Progress from ILC/ERL R
( $~ 5 \mathrm{~mm}$ gap tests) Cornell/JLab

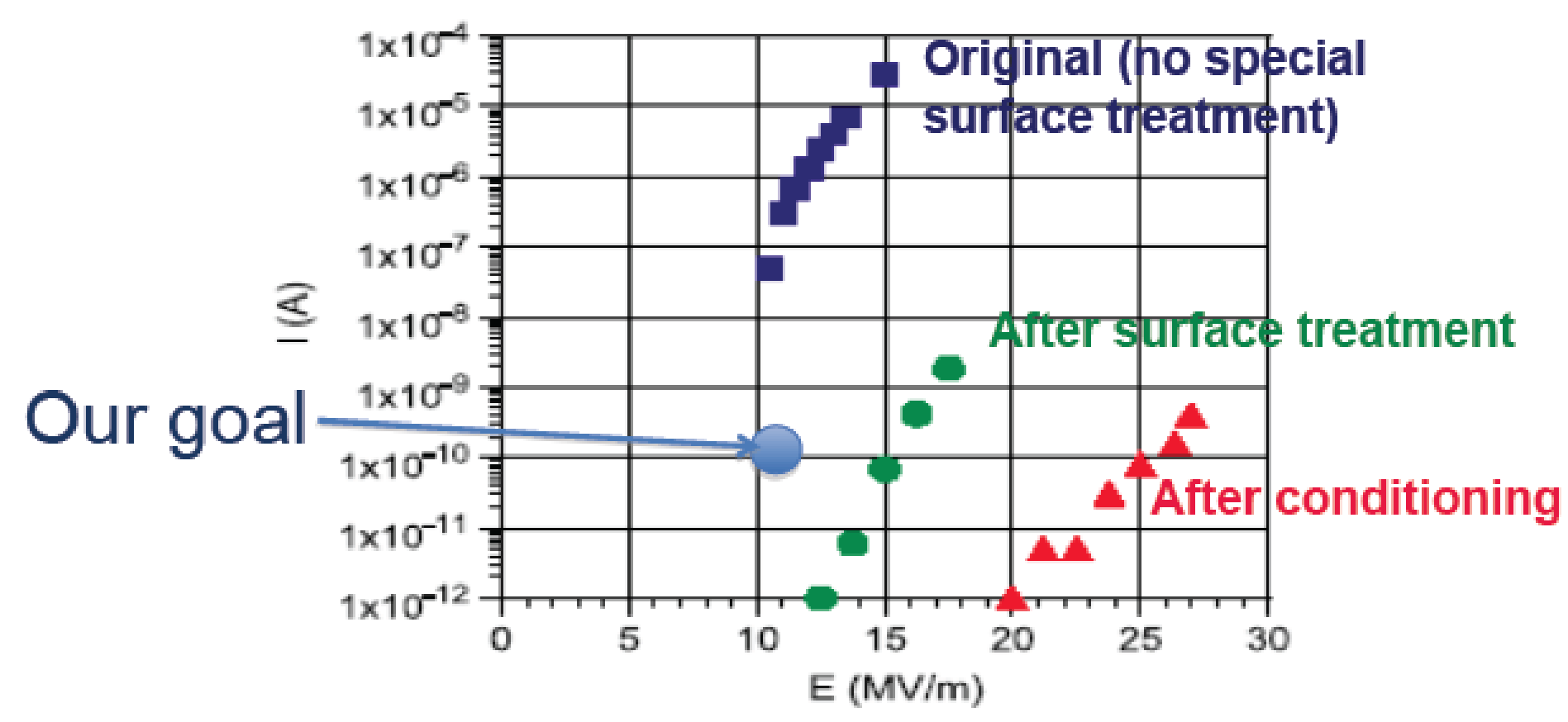

face treatment

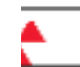

fter conditioning 


\section{Large Scale Electrodes, New: pEDM electrodes with HPWR}

\begin{tabular}{|c|c|c|c|}
\hline Parameter & $\begin{array}{c}\text { Tevatron pbar-p } \\
\text { Separators }\end{array}$ & $\begin{array}{c}\text { BNL K-pi } \\
\text { Separators }\end{array}$ & pEDM \\
\hline Length & $2.6 \mathrm{~m}$ & $4.5 \mathrm{~m}$ & $3 \mathrm{~m}$ \\
\hline Gap & $5 \mathrm{~cm}$ & $10 \mathrm{~cm}$ & $3 \mathrm{~cm}$ \\
\hline Height & $0.2 \mathrm{~m}$ & $0.4 \mathrm{~m}$ & $0.2 \mathrm{~m}$ \\
\hline Number & 24 & 2 & $10^{2}$ \\
\hline Max. HV & $\pm 180 \mathrm{KV}$ & $\pm 200 \mathrm{KV}$ & $\pm 150 \mathrm{KV}$ \\
\hline
\end{tabular}




\section{E-field plate module: Similar to the}

(26) FNAL Tevatron ES-separators Beamposition

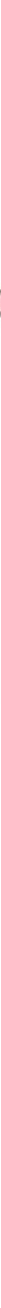




\section{E-field plate module: Similar to the}

\section{(26) FNAL Tevatron ES-separators}

bosition

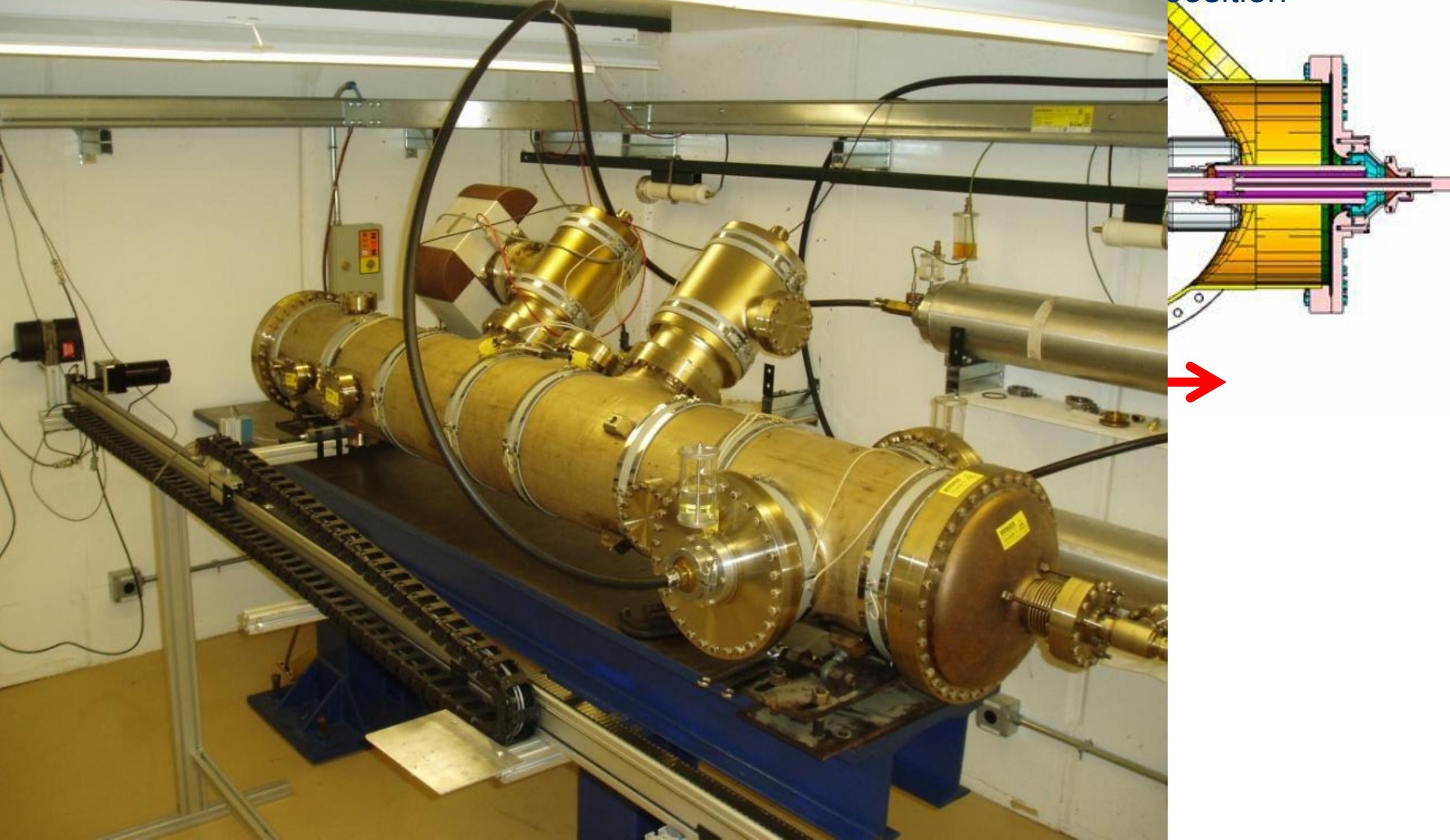




\section{The miracles that make the pEDM}

1. Magic momentum (MM): high intensity

charged beam in an all-electric storage ring

2. High analyzing power: $A>50 \%$ at the $M M$

3. Weak vertical focusing in an all-electric ring:

SCT allows for $10^{3}$ s beneficial storage; prospects for much longer SCT with mixing (cooling and heating)

4. The beam vertical position tells the average radial B-field; the main systematic error source 


\section{Technically driven pEDM timeline}

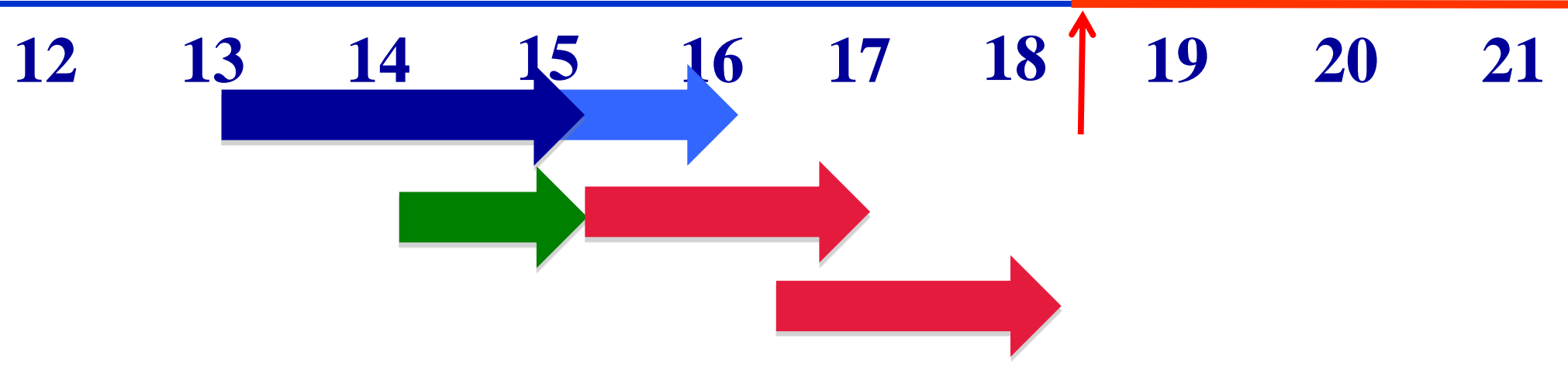

- Two years R\&D

- One year final ring design

- Two years ring/beam-line construction

- Two years installation

- One year "string test" 


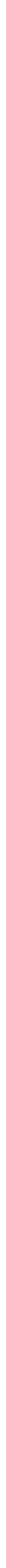




\section{Total cost: exp + ring + beamline for two different ring locations @ BNL}

\begin{tabular}{l|l|l|l}
\hline System & $\begin{array}{l}\text { Experiment } \mathrm{w} / \\
\text { indirects }\end{array}$ & $\begin{array}{l}\text { Conventional plus } \\
\text { beamline w/ indirects }\end{array}$ & Total \\
\hline pEDM at ATR & $\$ 25.6 \mathrm{M}$ & $\$ 20 \mathrm{M}$ & $\$ 45.6 \mathrm{M}$ \\
\hline pEDM at SEB & $\$ 25.6 \mathrm{M}$ & $\$ 14 \mathrm{M}$ & $\$ 39.6 \mathrm{M}$
\end{tabular}

\begin{tabular}{|c|c|c|c|}
\hline System & $\begin{array}{l}\text { Experiment } \mathrm{w} / \\
55 \% \text { contingency }\end{array}$ & $\begin{array}{l}\text { Conv. \& Beamline w/ } \\
\text { contingency }\end{array}$ & Total \\
\hline $\mathrm{pEDM}$ at ATR & $\$ 39.5 \mathrm{M}$ & $\$ 29.2 M$ & \$68.7M \\
\hline pEDM at SEB & $\$ 39.5 \mathrm{M}$ & $\$ 22.6 \mathrm{M}$ & $\$ 62.1 \mathrm{~N}$ \\
\hline
\end{tabular}




\section{Storage Ring EDM Collaboration}

Aristotle University of Thessaloniki, Thessaloniki/Greece

- Research Inst. for Nuclear Problems, Belarusian State University, Minsk/Belarus

- Brookhaven National Laboratory, Upton, NY/USA

- Budker Institute for Nuclear Physics, Novosibirsk/Russia

- Royal Holloway, University of London, Egham, Surrey, UK

$>20$ Institutions • Cornell University, Ithaca, NY/USA

$>80$ Collaborators • Institut für Kernphysik and Jülich Centre for Hadron Physics Forschungszentrum Jülich, Jülich/Germany

- Institute of Nuclear Physics Demokritos, Athens/Greece

- Mpiversity and INFN Ferhara, Ferrar/Italy

- Joint Institute for Nuclear Research, Dub no

- Indiana University, Indiana/USA

- Istanbul Technical University, Istanbul/Turkey

- University of Massachusetts, Amherst, Massachusetts/USA

- Michigan State University, East Lansing, Minnesota/USA

- Dipartimento do Fisica, Universita' "Tor Vergata" and Sezione INFN, Rome/Italy

- University of Patras, Patras/Greece

- CEA, Saclay, Paris/France

- KEK, High Energy Accel. Res. Organization, Tsukuba, Ibaraki 305-0801, Japan

- University of Virginia, Virginia/USA 


\section{Sensitivity to Rule on Several New Models}

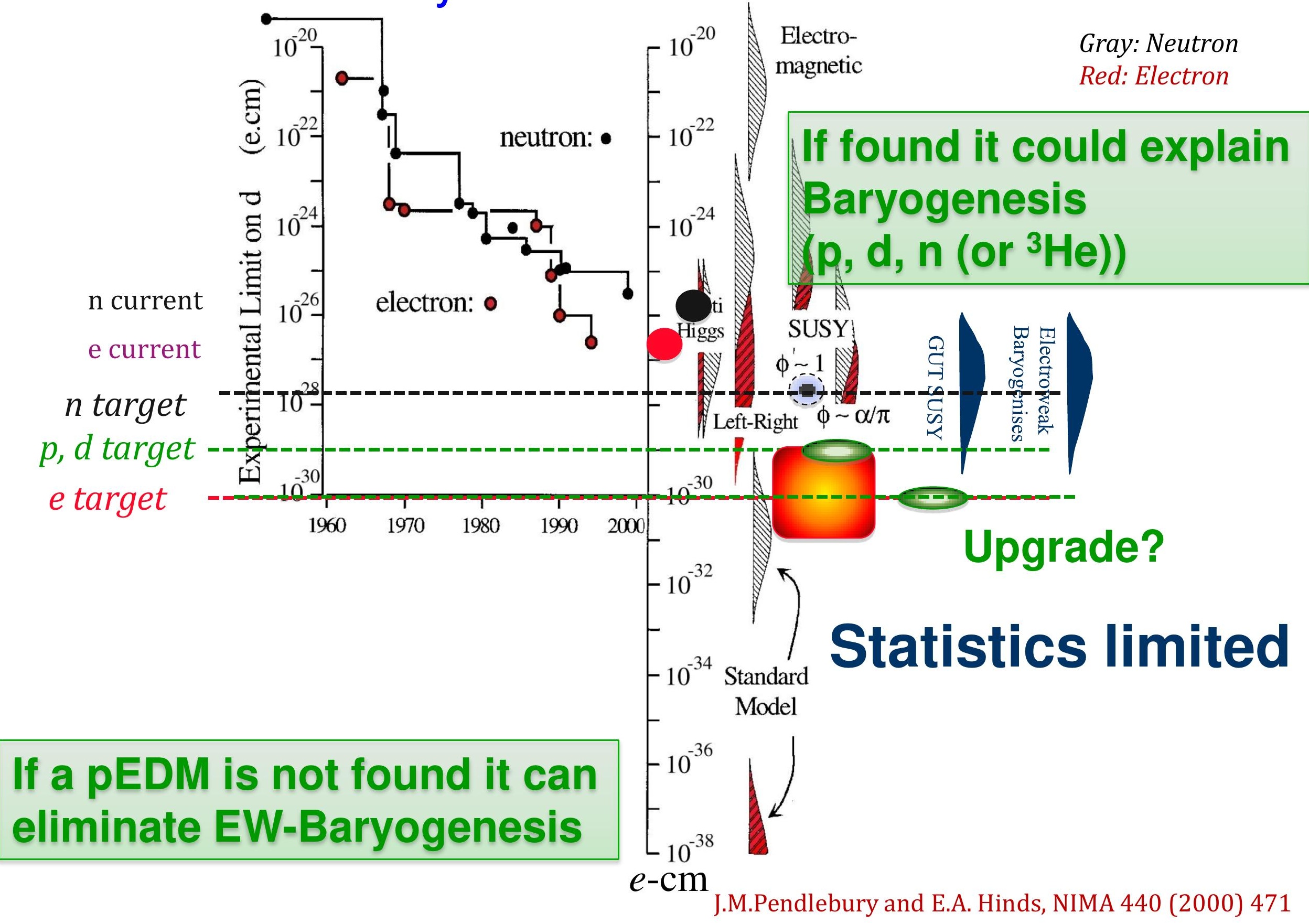




\section{EDMs of hadronic systems are mainly sensitive to}

- Theta-QCD (part of the SM)

- CP-violating sources beyond the SM

Alternative simple systems are needed to be able to differentiate the CP-violating source (e.g. neutron, proton, deuteron,...).

pEDM at $10^{-29} \mathrm{e} \cdot \mathrm{cm}$ is $>$ an order of magnitude more sens. than the best current nEDM plans 


\section{Measure all three: proton, ideuteron and neutron EDMs to determine CPV source}

Super-Symmetry (SUSY) model predictions:

$$
d_{s} \simeq 1.4\left(d_{s}-0.25 d_{N}\right)+0.83 e\left(d^{c}+d_{s}^{c}\right)-0.27 e\left(d^{c}-d^{c}\right)
$$

Theoretical estimation on the

lattice?

$d_{N}^{I-1} \simeq 0.87\left(d_{u}-d_{d}\right)+0.27 e\left(d_{u}^{c}-d_{d}^{c}\right)$

$d_{N}^{I-1}=\left(d_{p}-d_{n}\right) / 2$

$d_{N}^{I-0} \simeq 0.5\left(d_{u}+d_{d}\right)+0.83 e\left(d_{u}^{c}+d_{d}^{c}\right)$

$d_{N}^{I-0}=\left(d_{p}+d_{n}\right) / 2$ 


\section{Physics reach of magic pEDM ${ }_{\text {(Marciano) }}$}

- Currently: $\bar{\theta} \leq 10^{-10}$, Sensitivity with pEDM: $\bar{\theta}<0.3 \times 10^{-13}$

- Sensitivity to new contact interaction: $3000 \mathrm{TeV}$

- Sensitivity to SUSY-type new Physics:

$p E D M \approx 10^{-24} \mathrm{e} \cdot \mathrm{cm} \times \sin \delta \times\left(\frac{1 \mathrm{TeV}}{M_{\mathrm{SUSY}}}\right)^{2}$

The proton EDM at $10^{-29} \mathrm{e} \cdot \mathrm{cm}$ has a reach of $>300 \mathrm{TeV}$ or, if new physics exists at the LHC scale, $\delta<10^{-7}-10^{-6}$ rad CP-violating phase; an unprecedented sensitivity level.

The deuteron EDM sensitivity is similar. 


\section{The current status}

- Have developed R\&D plans (need \$1M/year for two years) for

1) BPM magnetometers, 2) SCT tests at COSY,

3) E-field development, and 4) Polarimeter

prototype

- We had two successful technical reviews: Dec 2009, and March 2011.

- Sent a proposal to DOE NP for a proton EDM experiment at BNL: November 2011 


\section{Common R\&D with COSY}

\section{EDM at Storage Rings}

\section{International srEDM Network}

Institutional (MoU) and Personal (Spokespersons ...) Cooperation

srEDM Collaboration (BNL)

srEDM Collaboration (FZJ)

RHIC

Beam Position Monitors

(...)

Spin Tracking

\section{Common R \& D}

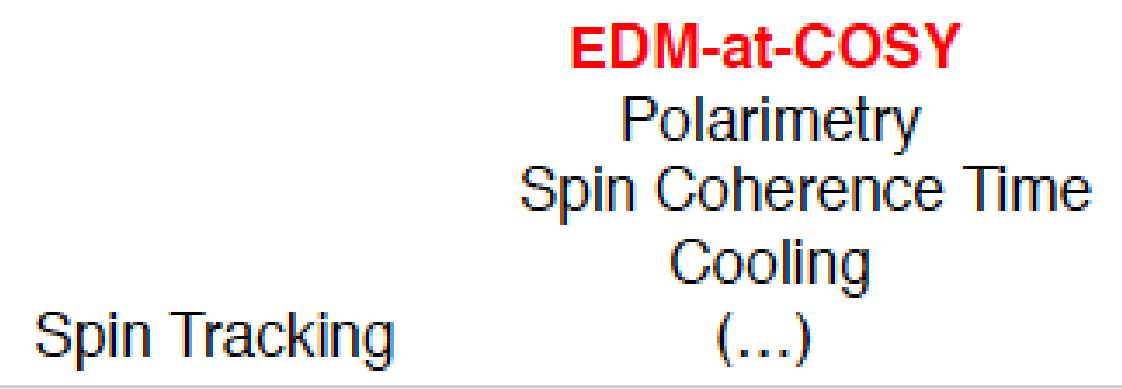

Slide by H. Stroeher, Director of IKP II

\section{DOE-Proposal}

$\mathrm{CDO}, 1, \ldots$

\section{Study Group}

Precursor; Ring Design

HGF Application(s) 


\section{Summary}

$\checkmark$ Proton EDM physics is a must do, $>$ order of magnitude improvement over the neutron EDM

$\checkmark$ E-field issues well understood

$\checkmark$ Working EDM lattice with long SCT and large enough acceptance $\left(\sim 10^{-29} \mathrm{e} \cdot \mathrm{cm} /\right.$ year $)$

$\checkmark$ Polarimeter work

- Planning BPM-prototype demonstration including tests at RHIC

- Old accumulator ring could house the proton EDM ring at Fermilab; BNL: new tunnel needed

$\checkmark$ At COSY a pre-cursor proposal to PAC 


\section{Extra slides}




\section{Physics strength comparison (Marciano)}

\begin{tabular}{|l|l|l|l|}
\hline System & $\begin{array}{l}\text { Current limit } \\
{[\mathrm{e} \cdot \mathrm{cm}]}\end{array}$ & Future goal & $\begin{array}{l}\text { Neutron } \\
\text { equivalent }\end{array}$ \\
\hline Neutron & $<1.6 \times 10^{-26}$ & $\sim 10^{-28}$ & $10^{-28}$ \\
\hline${ }^{199} \mathrm{Hg}$ atom & $<3 \times 10^{-29}$ & $<10^{-29}$ & $10^{-25}-10^{-26}$ \\
\hline $\begin{array}{l}129 \\
\text { Xe atom }\end{array}$ & $<6 \times 10^{-27}$ & $\sim 10^{-29}-10^{-31}$ & $10^{-25}-10^{-27}$ \\
\hline $\begin{array}{l}\text { Deuteron } \\
\text { nucleus }\end{array}$ & & $\sim 10^{-29}$ & $\begin{array}{l}3 \times 10^{-29} \\
5 \times 10^{-31}\end{array}$ \\
\hline $\begin{array}{l}\text { Proton } \\
\text { nucleus }\end{array}$ & $<7 \times 10^{-25}$ & $\sim 10^{-29}$ & $10^{-29}$ \\
\hline
\end{tabular}




$$
\gamma_{\mathrm{m}}=\sqrt{1+\frac{1}{a}}=1.248
$$

where $a=1.793$ is the proton anomaly. Consequently we have:

$$
\begin{aligned}
\beta_{\mathrm{m}} & =0.598 \\
p_{\mathrm{m}} & =\frac{m c}{\sqrt{a}}=0.701 \mathrm{GeV} / \mathrm{c} \\
U_{\mathrm{m}} & =\gamma_{\mathrm{m}} m c^{2}=1.171 \mathrm{GeV} \\
W_{\mathrm{m}} & =U_{\mathrm{m}}-m c^{2}=0.233 \mathrm{GeV} \\
m & =\text { proton mass }=0.938 \mathrm{GeV} / \mathrm{c}^{2}
\end{aligned}
$$

and

$$
B_{\text {eq }}=\frac{E_{\text {rad }}}{\beta c}, \quad \text { for } \quad E_{\mathrm{rad}}=1.5 \times 10^{7} \mathrm{Vm}^{-1}=150 \mathrm{kV} / \mathrm{cm}
$$

or

$$
B_{\mathrm{eq}}=8.37 \times 10^{-2} \text { Tesla, which yields a bending radius } \rho=\frac{p}{e B_{\mathrm{eq}}}=28 \mathrm{~m}
$$

$$
\nu_{\mathrm{H}}^{2}=2-\beta_{0}^{2}=1+\frac{1}{\gamma_{0}^{2}}
$$




\section{Why does the world need a Storage Ring EDM experiment at the $10^{-29}$ e-cm level ?}

1. The proton, deuteron and neutron combined can pin-down the CP-violating source should a non-zero EDM value is discovered. Critical: they can differentiate between a theta-QCD source and beyond the SM.

2. The proton and deuteron provide a path to the next order of sensitivity. 


\section{Why Storage Ring EDMs?}

- Storage rings offer a unique setting for a sensitive electric dipole moment (EDM) probe of charged particles. A number of simple systems can be probed with high accuracy: $p, d,{ }^{3} \mathrm{He}, \ldots$

- The mechanical (centrifugal) force balances the strong radial E-fields.

- Pencil-like, high intensity/high polarization beams of protons and deuterons have been around for decades.

- Ready for prime time. 


\section{Beam parameters}

C.R. proton beams

$\sim 10^{2} \mathrm{~m}$ base length
$0.7 \mathrm{GeV} / \mathrm{c} \quad \geq 80 \%$ polariz.; $\sim 4 \times 10^{10}$ protons/store
Repetition Beam energy: period: 20 minutes

Horizontal: Vertical:

$2 \mathrm{~mm}$ $\mathrm{mrad}$
Average beam power: $\sim 1 \mathrm{~mW}$

$(\mathrm{dp} / \mathrm{p})_{\mathrm{rms}}{ }^{\sim}$ $2 \times 10^{-4}$

- CW \& CCW injections: Average emittance parameters: same to $\sim 10 \%$ at injection.

Fermi would need to get into polarized beams physics 


\section{Proton EDM R\&D cost: \$2M}

- BPM development \& testing over two years: $\$ 0.6 \mathrm{M}$

- E-field prototype development \& testing: 1.8

years: $\$ 0.4 \mathrm{M}$

- SCT tests at COSY, 2 years: $\$ 0.4 \mathrm{M}$

- Polarimeter prototype, 2 years: $\$ 0.6 \mathrm{M}$ 


\section{Polarimeter rates:}

-Beam intensity with $2 \times 10^{10}$ pol. protons/ $\sim 10^{3} \mathrm{~s}$ and a detection efficiency of $1 \% \rightarrow$ $200 \mathrm{KHz}$ for $\sim 3000 \mathrm{~cm}^{2}$ area, or $\sim 100 \mathrm{~Hz} / \mathrm{cm}^{2}$ on average but much higher at small radius. Design: $\sim 1 \mathrm{KHz} /$ pad.
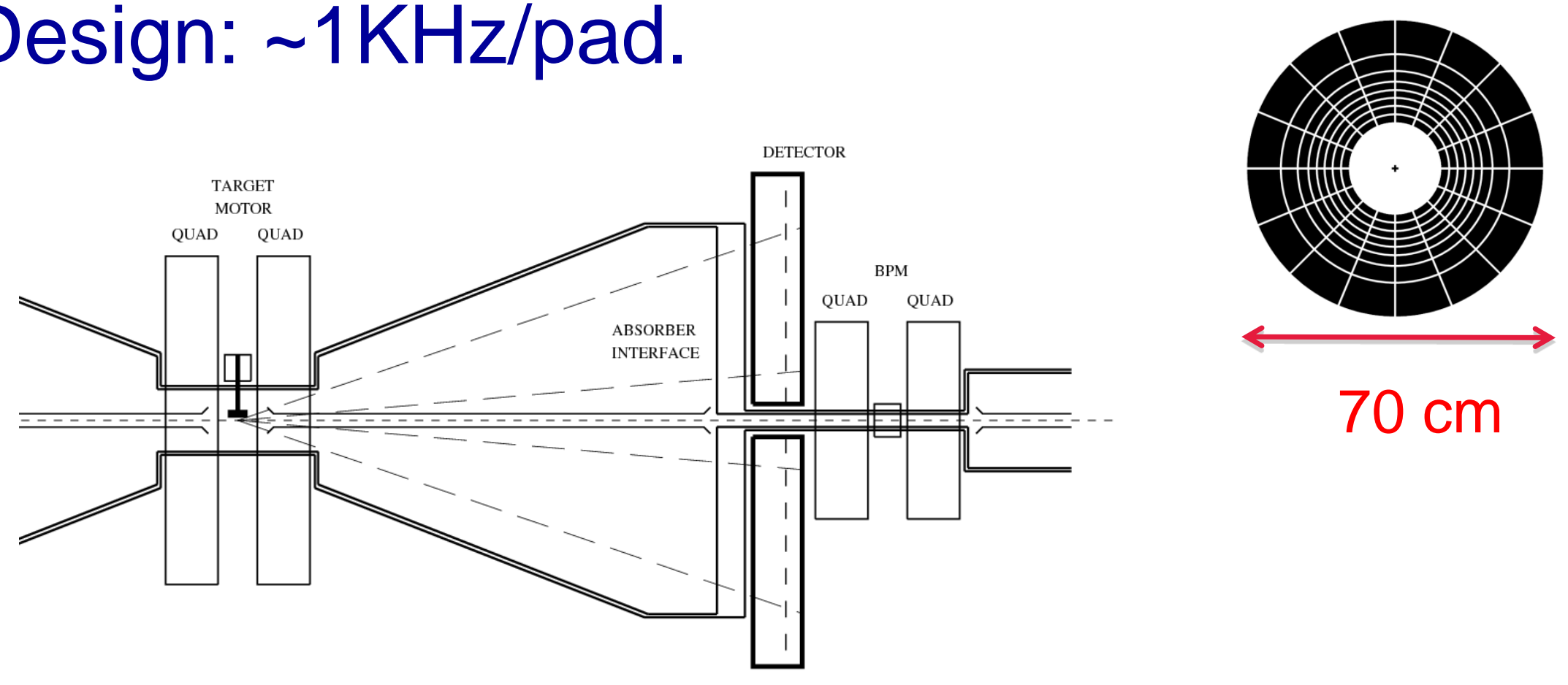


\section{The Electric Dipole Moment precesses in an Electric field}

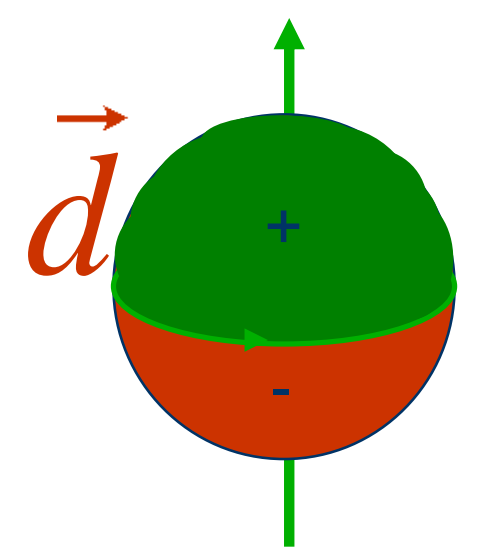

The EDM vector $d$ is along the particle spin direction

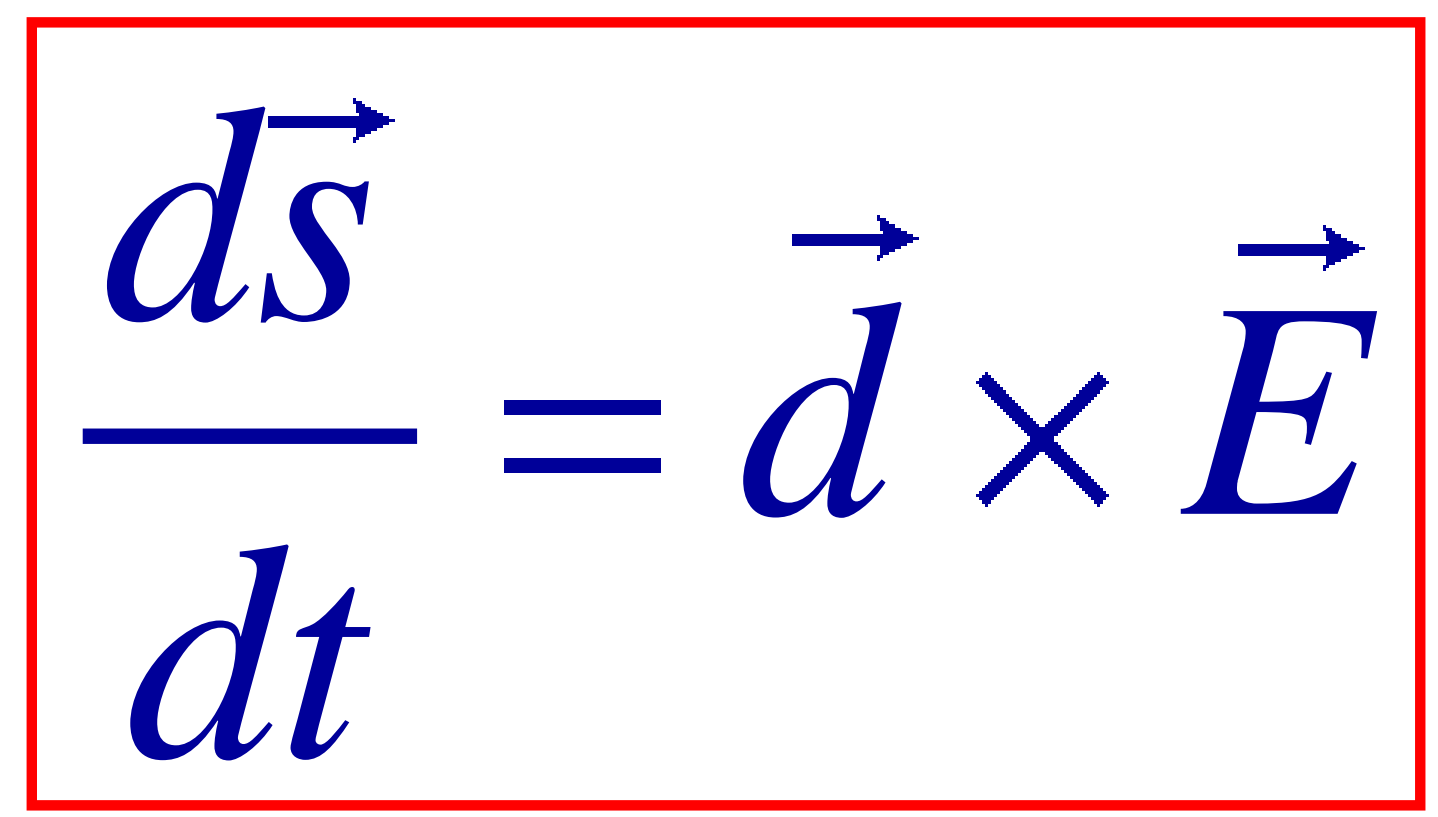

Yannis Semertzidis, BNL 
Table 2. The table of parameters for the proton EDM ring is shown here. The lattice has been estimated using the exact electric field and not an effective dipole magnetic field.

\begin{tabular}{|c|c|c|c|}
\hline \multirow{15}{*}{$\begin{array}{l}\text { Parameters of } \\
\text { current lattice }\end{array}$} & Parameter & Value & Comment \\
\hline & Proton Momentum & $0.7007405 \mathrm{GeV} / \mathrm{c}$ & $\begin{array}{l}\text { Kinetic energy: } 232.8 \mathrm{MeV} \text {, } \\
\beta=0.59838, \gamma=1.2481\end{array}$ \\
\hline & Ring bending radius & $40 \mathrm{~m}$ & \\
\hline & $\begin{array}{l}\text { Total length of straight } \\
\text { sections }\end{array}$ & $11.6 \mathrm{~m}$ & $\begin{array}{l}\text { If more straight section } \\
\text { length is needed the ring } \\
\text { bending radius has to } \\
\text { increase proportionally. }\end{array}$ \\
\hline & Radial E-field strength & $10.5 \mathrm{MV} / \mathrm{m}$ & $\begin{array}{l}\text { For plate separation of } 3 \mathrm{~cm} \\
\text { the voltage on the plates is } \\
\text { about } \pm 160 \mathrm{KV} \text {. }\end{array}$ \\
\hline & Number of sections & 16 & $\begin{array}{l}\text { The E-field plates within a } \\
\text { section are } \sim 16 \mathrm{~m} \text { long each. } \\
\text { They can be segmented into } \\
5 \text { pieces, } 3.14 \mathrm{~m} \text { long each. }\end{array}$ \\
\hline & $\begin{array}{l}\text { Radial E-field dependence } \\
\text { at } \mathrm{y}=0\end{array}$ & $R^{0.2}$ & $\begin{array}{l}\text { The E-field is slightly } \\
\text { increased at larger radius. }\end{array}$ \\
\hline & Total length of orbit & $263 \mathrm{~m}$ & \\
\hline & Horizontal tune & 1.3 & \\
\hline & Vertical tune & $0.2-0.1$ & $\begin{array}{l}\text { To be modulated by } \sim 10 \% \\
\text { around } 0.1\end{array}$ \\
\hline & $\beta_{\mathrm{x}, \max }$ & $28 \mathrm{~m}$ & Horizontal aperture: $3 \mathrm{~cm}$ \\
\hline & $\beta_{y, \max }$ & $240 \mathrm{~m}$ & Vertical aperture: $8 \mathrm{~cm}$ \\
\hline & Cyclotron frequency & $0.6839 \mathrm{MHz}$ & \\
\hline & $f_{r f}=135 \times 0.6839 \mathrm{MHz}$ & $90 \mathrm{MHz}$ & $\begin{array}{l}\text { Total RF voltage: } 5 \mathrm{KV} \text { for } \\
\text { synchrotron tune of } 0.01\end{array}$ \\
\hline & Slip factor & 0.45 & Sign is $-(\mathrm{TBC})$ \\
\hline
\end{tabular}


The EDM signal: early to late change - Comparing the (left-right)/(left+right) counts vs. time we monitor the vertical component of spin

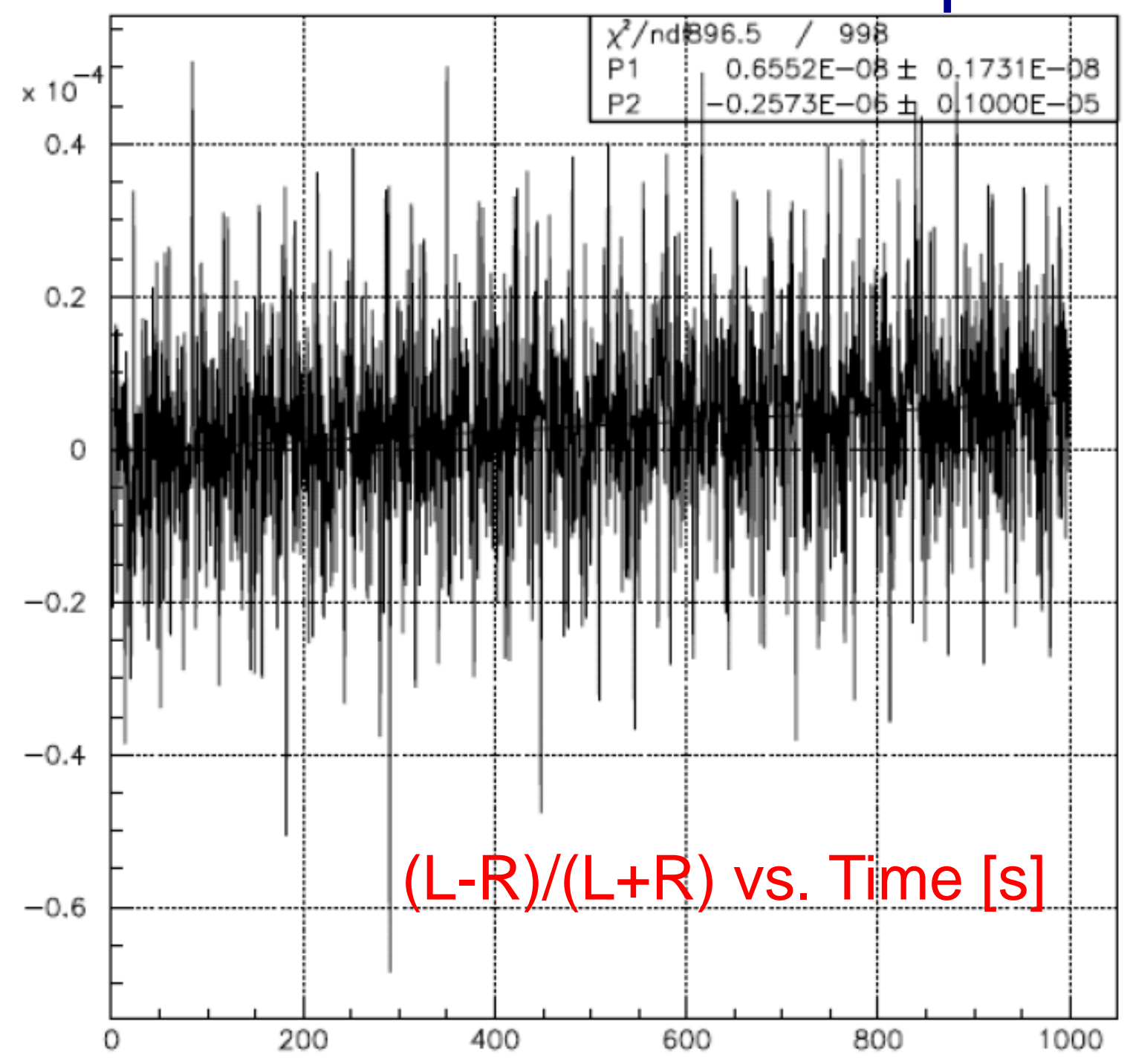

M.C. data 


\section{MINOS Tidal Data}

Difference in two sensors 90 meters apart

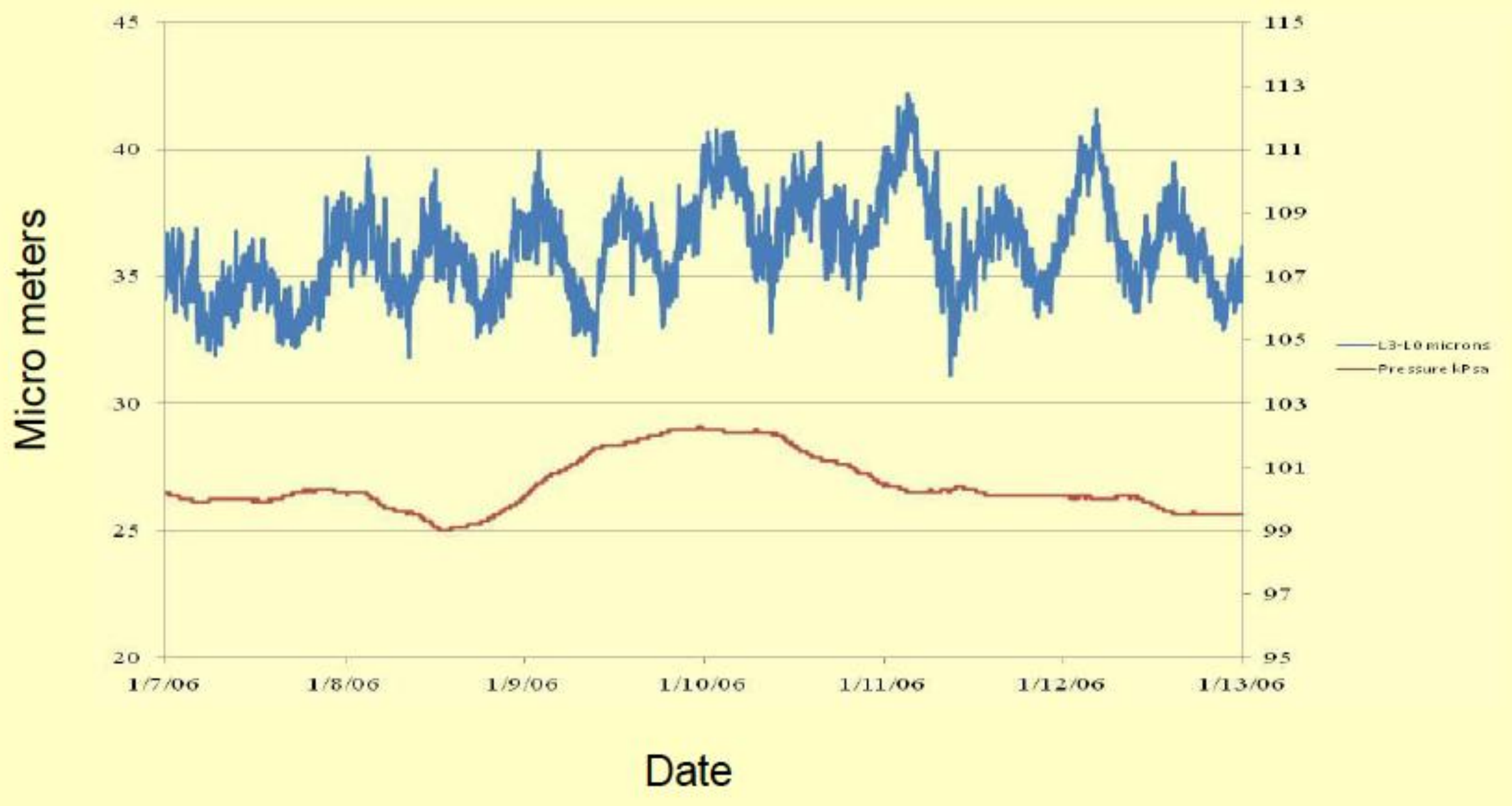

J T Volk Fermilab Dec 2008 


\section{Why COSY?}

Scale like EDM ring Polarized P/D beams Electron cooling Outside user program Available equipment

\section{History}

Proposal in 2007 Visit SPIN@COSY run Three polarimeter runs:

June 2008 - initial tests September 2008 - trial data June 2009 - final long run (paper in preparation) Polarization lifetime runs:

January 2011 - initial tests

Prior work at KVI, Groningen

d=C data, $2004+2005$

Systematic errors, 2007

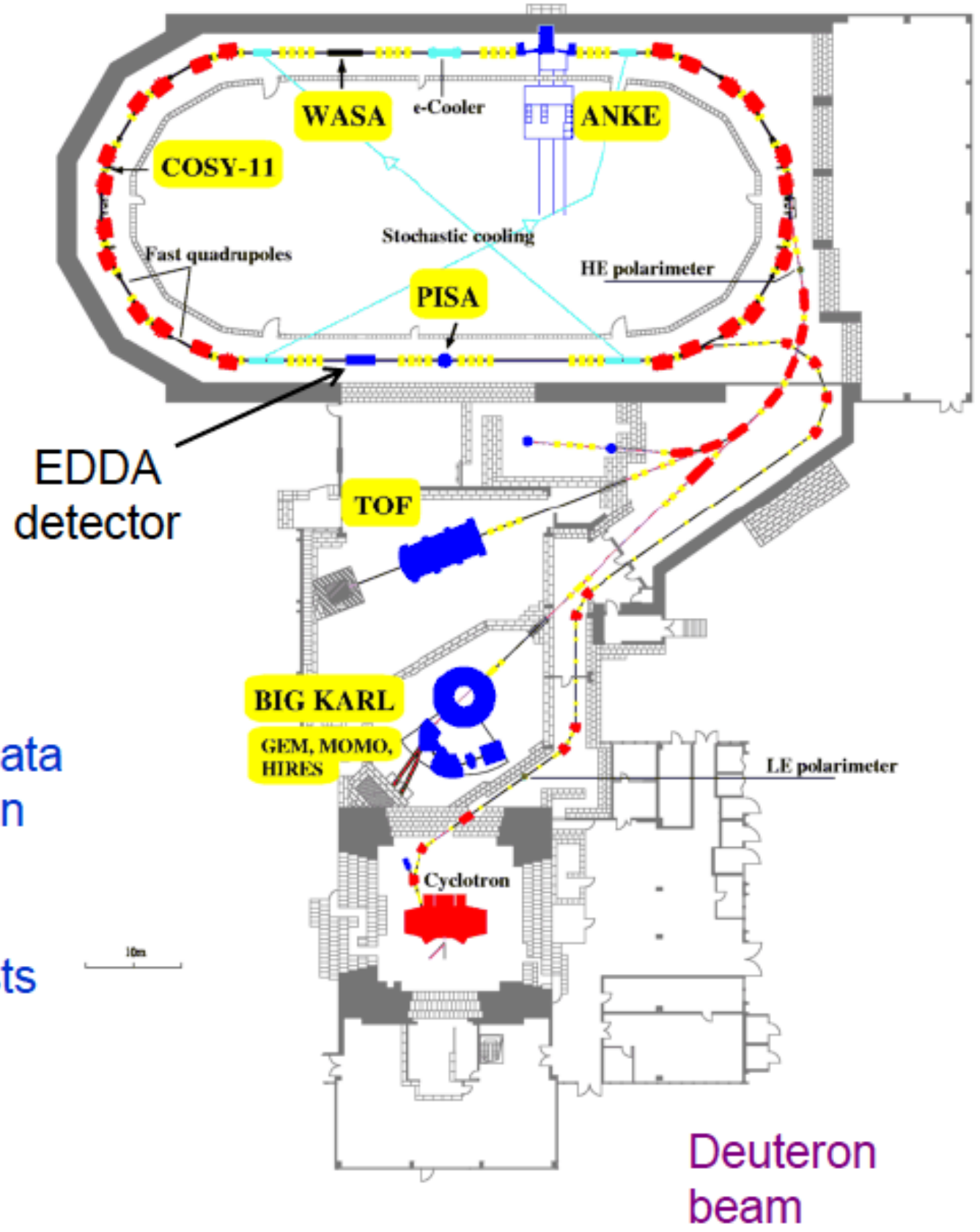




\section{How to Scale HPWR to 3cm gap?}

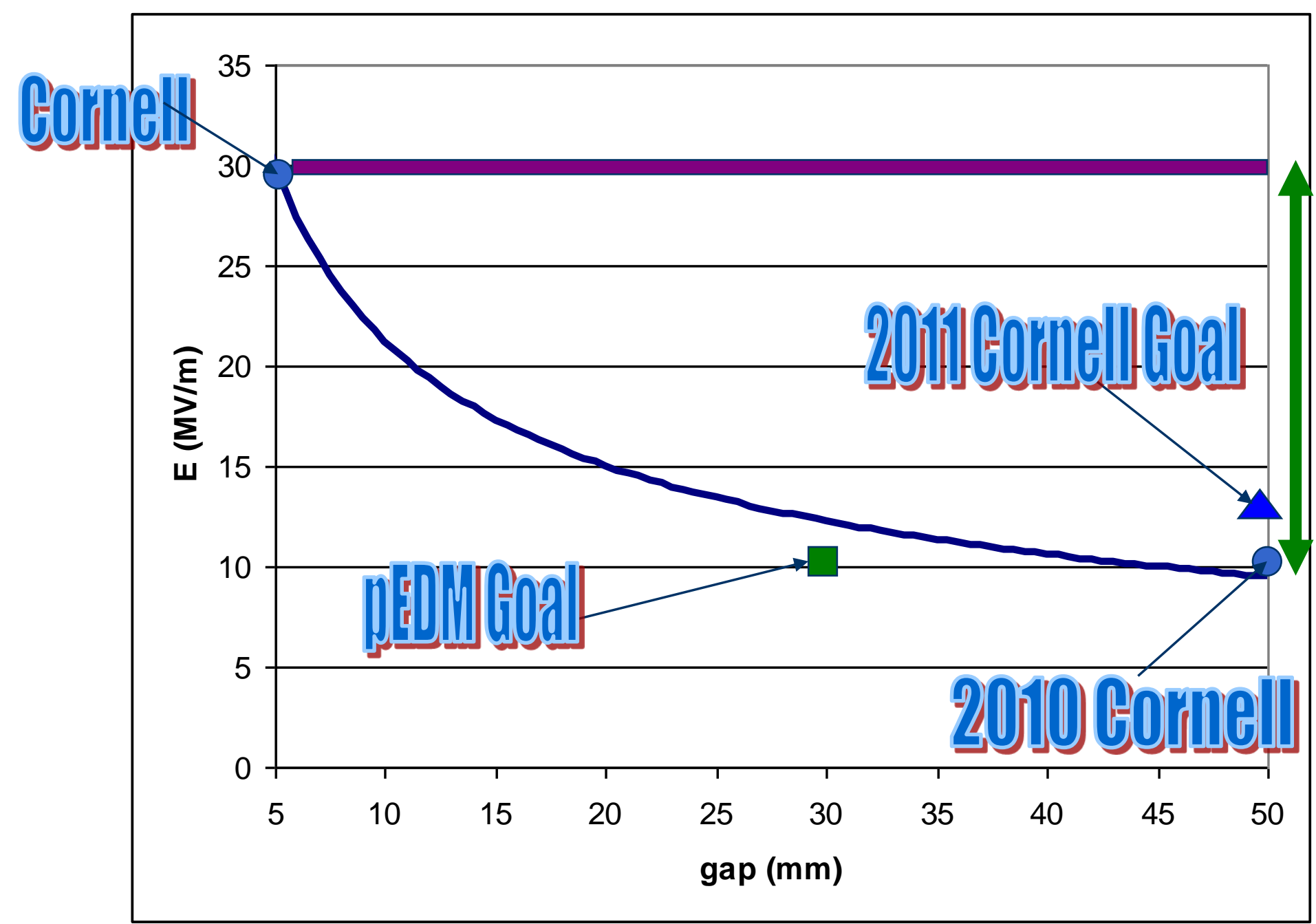

W. Morse PEDM Review 


\section{Detector systems: alternatives to scintillators}

Multi-resistive plate

A chambers(Italy)

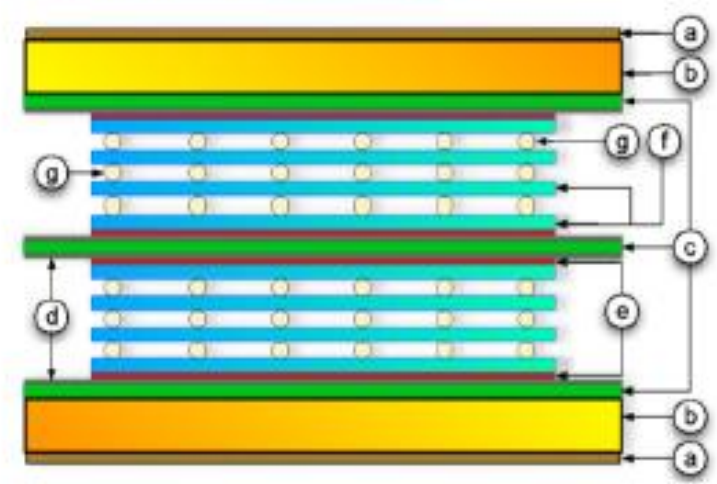

pickup electrodes (green) also shown in photograph

The $20 \mathrm{~cm} \times 50 \mathrm{~cm}$ prototype

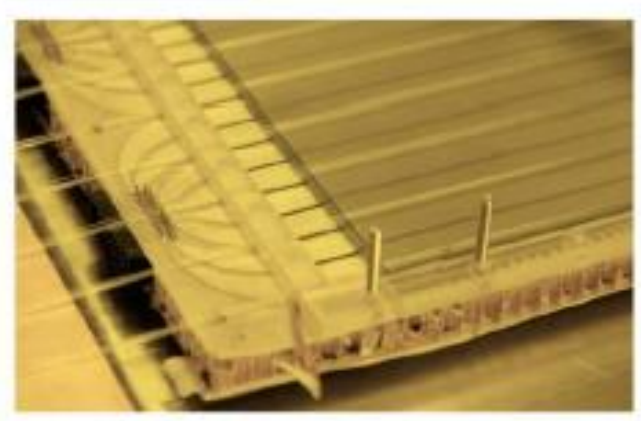

Micro-megas avalanche

B detection system Greece)

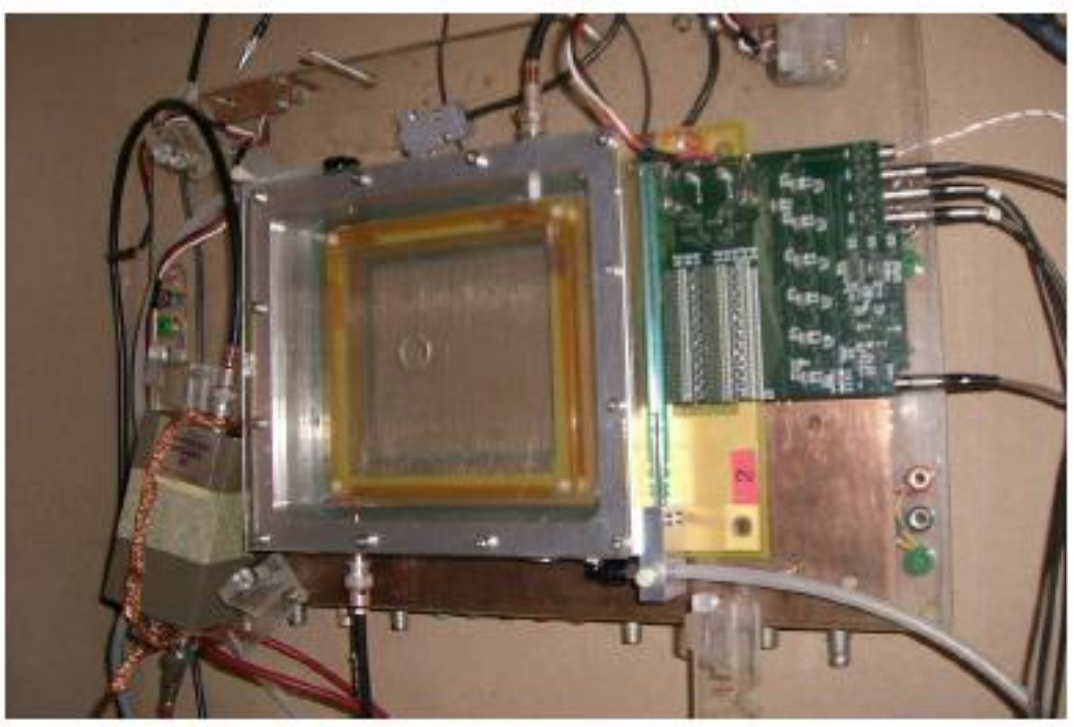

C Gas electron multiplier (GEM) system

In-beam tests are needed (COSY) to provide sample data sets. 


\section{Gas cluster ion beam surface treatment: getting rid of $\sim \mu \mathrm{m}$ level asperities}

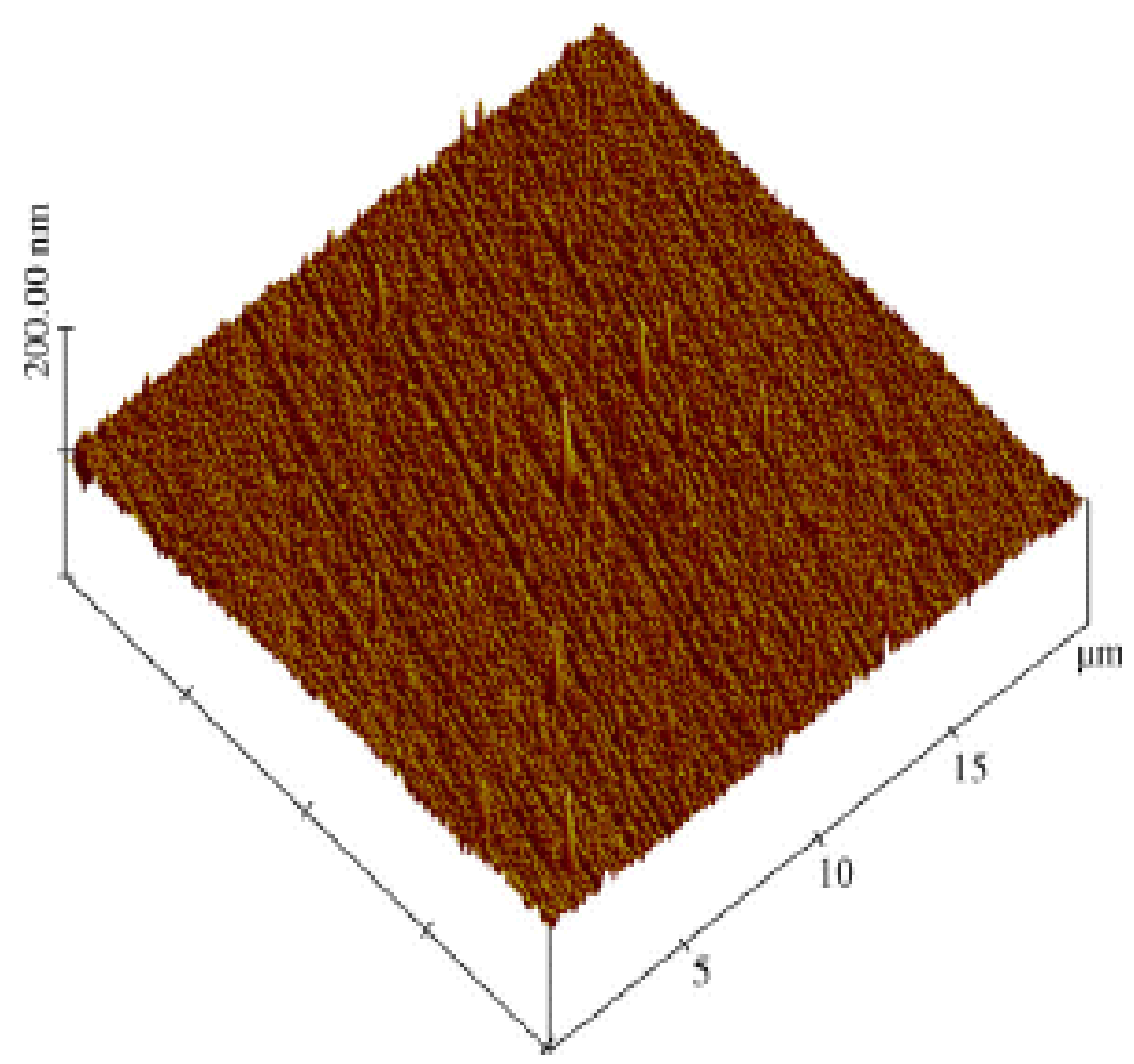

Fig. 1, AFM image $(20 \times 20 \mu \mathrm{m})$ of highly polisbed stainles sted eloctrode material before GCIB treatment showing asperities and scratch marks from polishing. The vertical scale is 120 m/division,

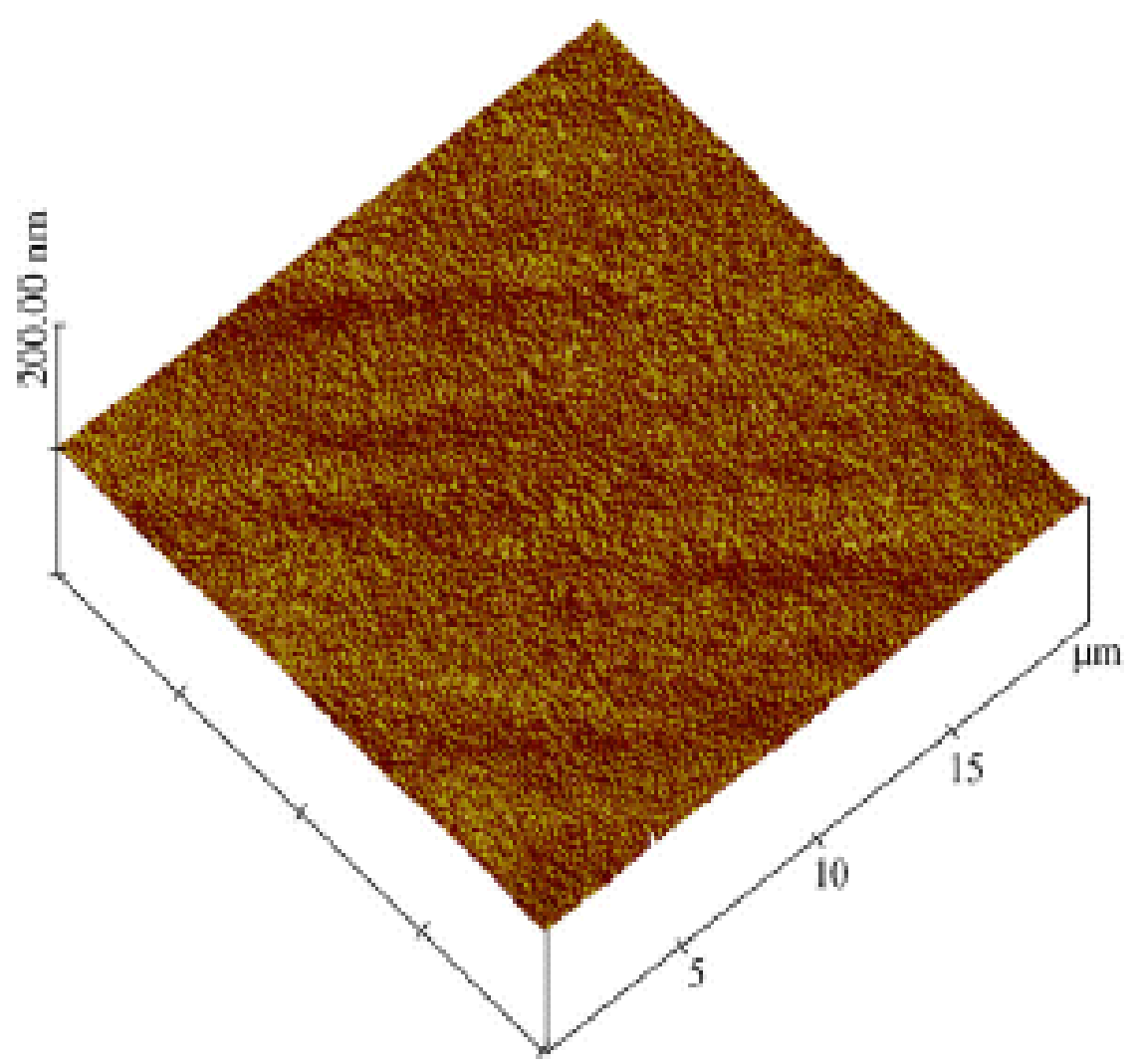

Fig. 2, AFM image $(20 \times 20 \mu \mathrm{m})$ of highly polished stainles stol eloctrode material after GCIB treatment. The vertical scale is $120 \mathrm{~mm} /$ division. 


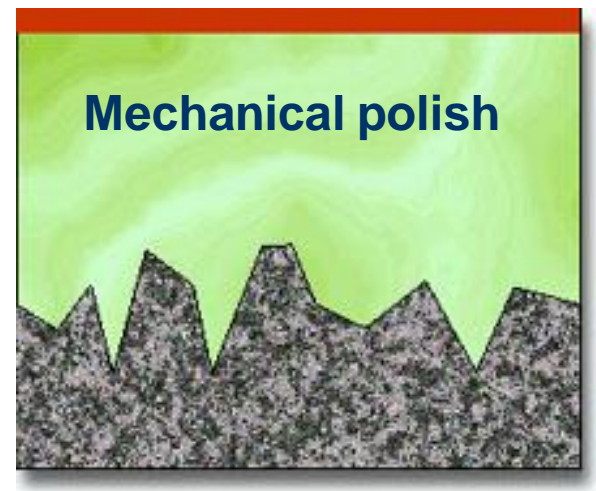

Roughness: 4 - 40 microinches (depends from abrasive grit number)

Mechanical polishing is an operation designed to prepare a metal surface for electropolishing or to satisfy non-critical surface roughness requirements.

Mechanical polishing reduces all surface ridges, microprotrusions, pits and discrepancies to provide a homogeneous appearance and roughness.

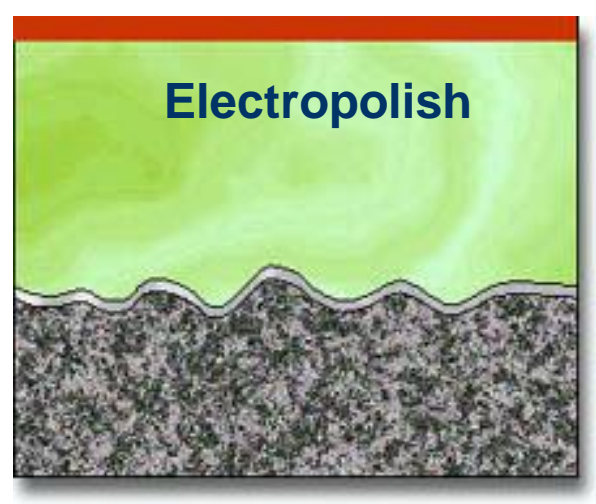

Roughness: 2 - 5 microinches

Electropolishing (used since early 1950's) is the electrochemical removal of microscopic irregularities or diminution scratches, burns and unwanted harp edges from metal surfaces. Typical material removal is .0001"- .0004" per surface.

Smoothness of the metal surface is one of the primary and most advantageous effects of electropolishing.

Electropolishing should improve separator performance. 


\section{High Voltage Electrical Breakdown in Vacuum}

It is generally agreed that a vacuum breakdown is a vapor arc, taking place in material evaporated from the electrodes.

Evidence is the observation of localized light during breakdown and electrode material transferred across the gap.

* Electron field emission mechanism for initiating the breakdown According with this model, electrons are assumed to be field emitted from the tip of microprotrusion at an isolated site on the surface of broad-area cathode. Question: where is the metal vapor produced at the anode or cathode?

Is it enough power to vaporize anode material by field emitted electrons bombarded anode or positive ions produced at the anode lead to rupture of the cathode or that resistive heating on the cathode causes them to melt and ultimately to vaporize. This mechanism dominates at gaps less than $2 \mathrm{~mm}$.

* Microparticle or "clump" model

Clump of loosely adhesive material is drawn across the gap by the electric field so as to strike the opposite electrode with enough energy to produce high local temperature in the electrode or clump material with melting and vaporizing.

Pre-operational electrode surface will be characterized by having a finite number of microscopic particles. These will originate from various stages of mechanical polishing, and may be in the form of either impurity particle of polishing material or dust particles. Another source of microparticles are those originated from thermal instabilities at either the cathode or anode "hot" spot. For uniform gaps the breakdown voltage should vary as the square root of the gap spacing. The model is dominating at large gaps.

\section{* Ion exchange mechanism}

This mechanism is assumed to be initiated by say random positive ion created in the gap that is then accelerated by the field to generate further negative ions on impact with cathode, which subsequently generate more positive ions on impact with the anode etc. Thus, if the ion multiplication factor $>1$, the process will develop in the breakdown mode. It is very sensitive to chemicals contaminations.

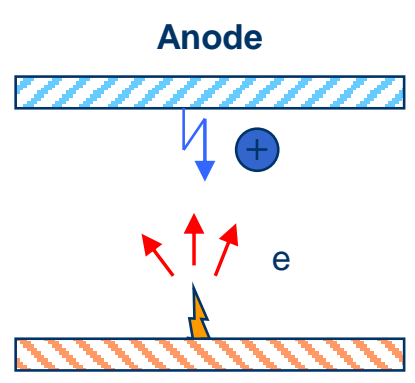

Cathode
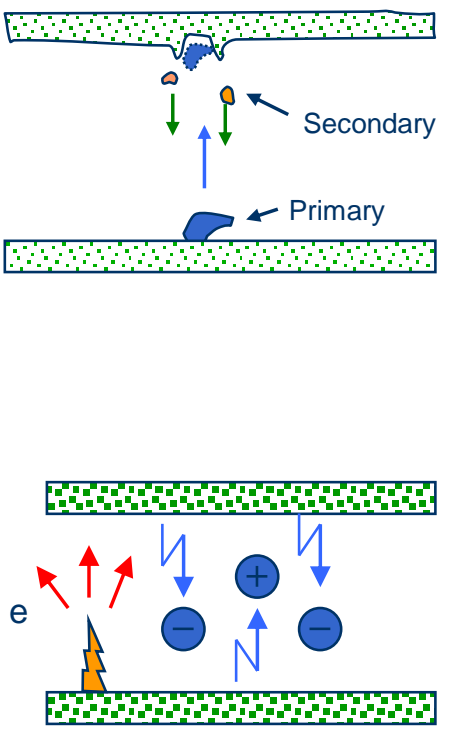

The breakdown consists of many complicated and complex phenomena with no single process involved. 
When $\mathrm{P}=\mathrm{P}_{\text {magic }}$ the spin follows the momentum

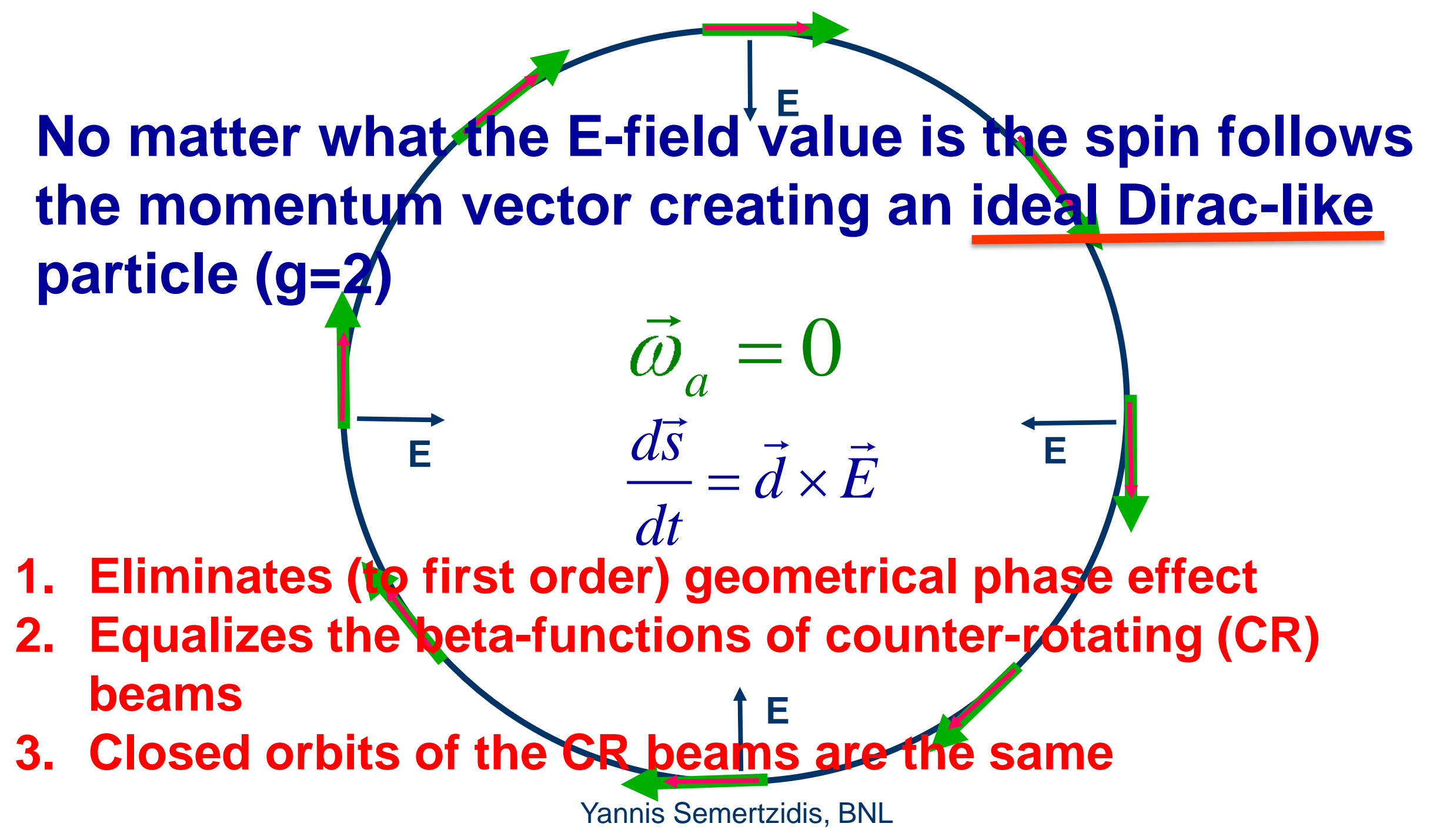




\section{Proton Statistical Error (230MeV):}

$$
\sigma_{d}=\frac{2 \hbar}{E_{R} P A \sqrt{N_{c} f \tau_{p} T_{t o t}}}
$$

$\tau_{p}: 10^{3} \mathrm{~s}$ Polarization Lifetime (Spin Coherence Time)

$A: 0.6 \quad$ Left/right asymmetry observed by the polarimeter

$P: 0.8 \quad$ Beam polarization

$N_{c}: 4 \times 10^{10} \mathrm{p} /$ cycle Total number of stored particles per cycle $T_{\text {Tot }}: 10^{7} \mathrm{~s}$

Total running time per year

$f: 0.5 \%$

Useful event rate fraction (efficiency for EDM)

$E_{R}: 10.5 \mathrm{MV} / \mathrm{m} \quad$ Radial electric field strength (95\% azim. cov.)

$\sigma_{d}=1.6 \times 10^{-29} \mathrm{e} \cdot \mathrm{cm} /$ year for uniform counting rate and

$\sigma_{d}=1.1 \times 10^{-29} \mathrm{e} \cdot \mathrm{cm} /$ year for variable counting rate 


\section{Physics/effort comparison}

- Physics reach $\sim 10^{3} \mathrm{TeV}$, similar to mu2e

(MECO) experiment at FNAL; moreover, it can explain BAU (EW-Baryogenesis)

- SUSY-like new physics at LHC scale, it probes CP-violating phases to sub micro-radian level, complementary to LHC (plus fine-tuned SUSY)

- At $10^{-29} \mathrm{e} \cdot \mathrm{cm}$ it's > an order of magnitude better than the best neutron EDM plans anywhere. Statistically superior to neutron EDM exps.

- Method can be applied to proton, deuteron, and ${ }^{3} \mathrm{He}$ to unravel the underlying physics. More than other methods can do. 


\title{
SU(N) gauge theories with symmetric-rep fermions
}

\author{
Yigal Shamir * \\ with \\ Tom DeGrand ${ }^{\diamond}$ and Ben Svetitsky*
}

Part I. $S U(N), \quad N=2,3,4$, with $N_{f}=2$ two-index sym rep Dirac fermions:

"continuum" issues; physics results

Part II: Fat (nHYP) links and what they do for us

$\Rightarrow$ It's a good idea to try DWF with fat links

* Tel Aviv University

$\diamond$ Boulder, CO 


\section{Walking [extended] Technicolor}

- Confinement \& Chiral symmetry breaking are lost for $N_{f}>N_{\text {critical }}$

- "Walking" theories are found just below the conformal window

- Condensate enhancement needed $\Leftrightarrow$ mass anomalous dimension $\gamma \approx 1$

- Natural candidates: $N_{f}=2$ higher-irrep fermions

\section{Schrödinger functional}

- Induce background field thru boundary conditions in $L^{4}$ box

- Measure $1 / g^{2}(L)$ from response to small change in boundary conditions

- Extract mass anomalous dimension $\gamma$ from scaling of pseudoscalar renormalization constant $Z_{P}$, on the same lattices 


\section{Life inside (or near) the conformal window}

Two-loop beta function, $\mathrm{SU}(2)$ with $N_{f}=2$ of:
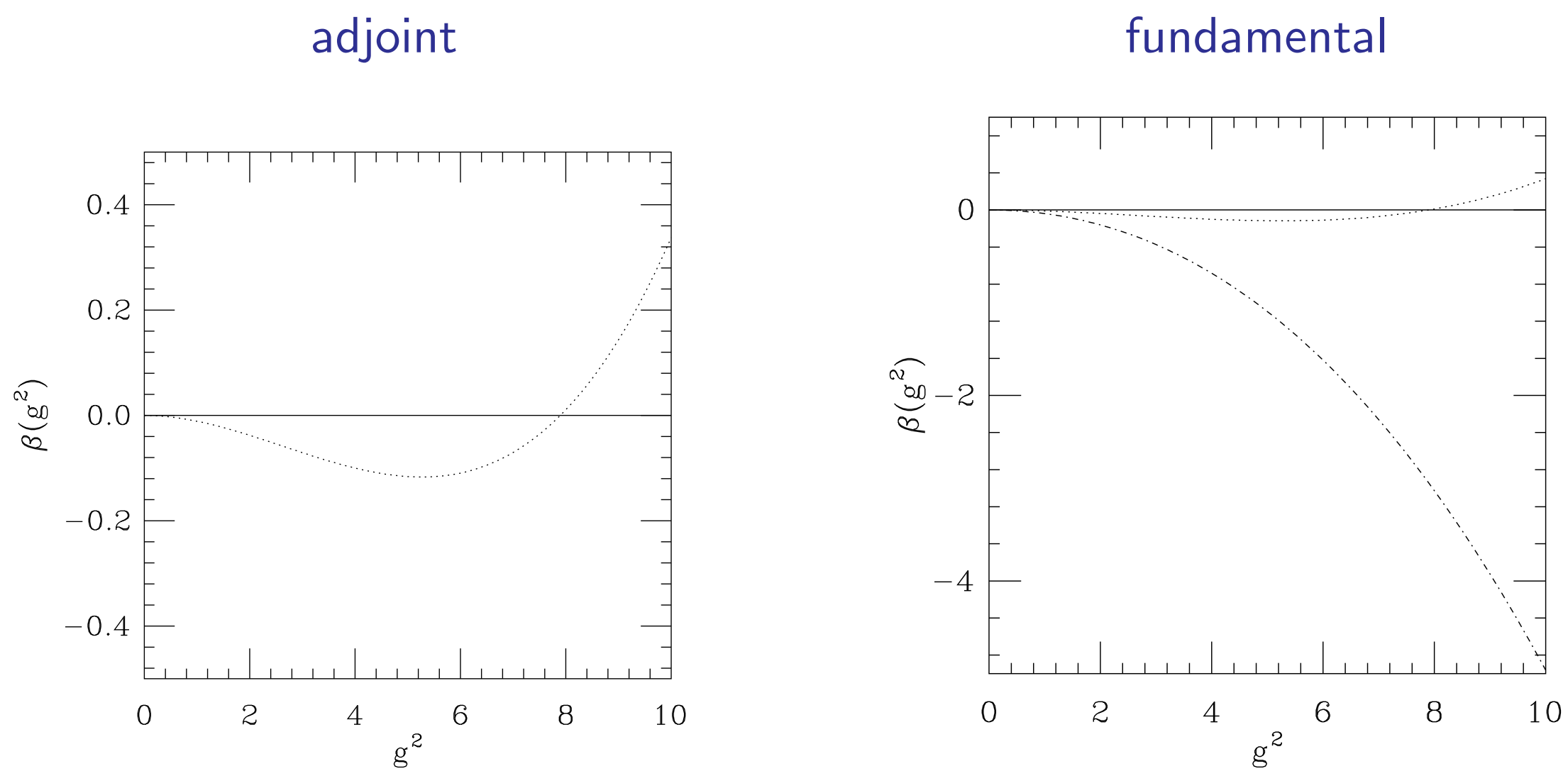

$\Rightarrow$ Strategy of ALPHA developed for QCD; performs poorly here 


\section{Nearly constant beta function}

define: $\quad \tilde{\beta}\left(1 / g^{2}\right) \equiv \frac{d\left(1 / g^{2}\right)}{d \log (L)}=2 \beta\left(g^{2}\right) / g^{4}$

- Solution: $1 / g^{2} \simeq a+b \log (L)$

- Exact at one loop

- Treat dataset at each bare $\beta$ as separate fitting problem

- slope $=$ beta fn.

- Systematics: add $\log ^{2}$ term, or remove smallest volume

- Slope changes sign $\Longrightarrow$ IRFP!

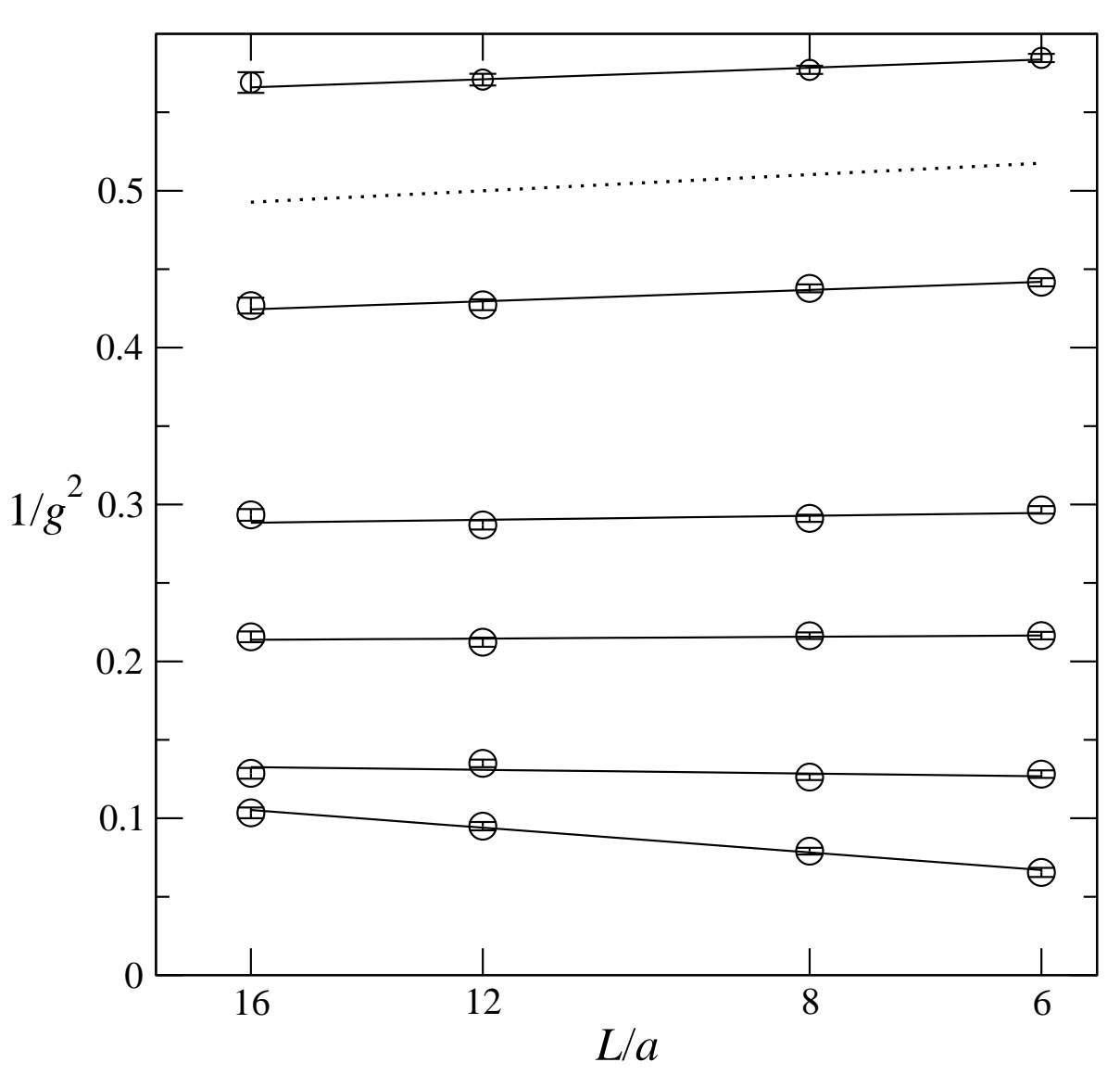




\section{$\mathrm{SU}(2) /$ adjoint}

Fitting $1 / g^{2}: \quad x=L / 8, \quad$ Black: $L=6,8,12,16, \quad$ Red: $L=8,12,16$
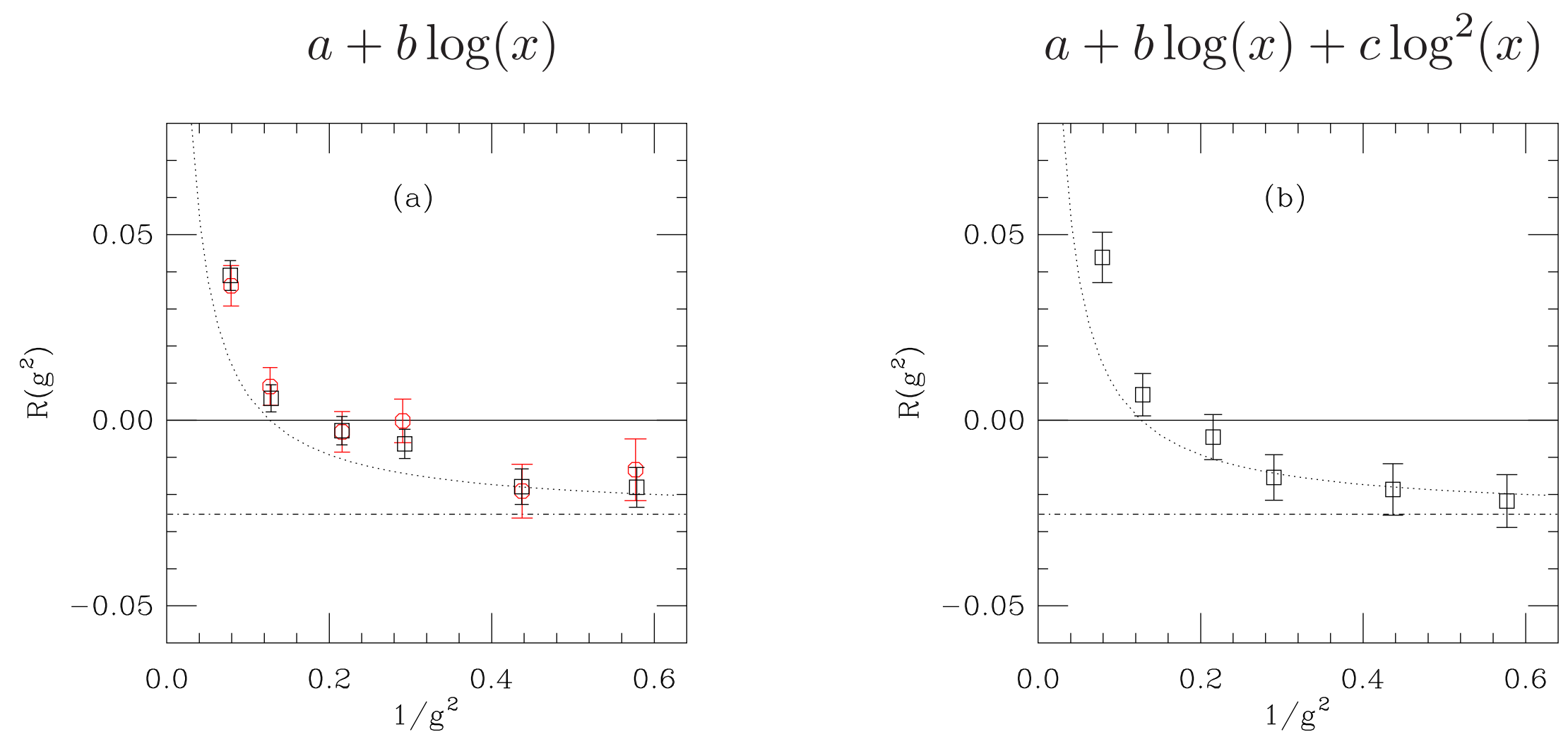

$$
1 / g_{*}^{2}=0.20(4)(3)
$$




\section{SU(2)/adjoint, SU(3)/sextet, SU(4)/decuplet}

- Qualitatively agrees with $\beta$ [2-loop]

- Actual $\beta>\beta^{\text {[2-loop] }}$

- With increasing $N_{c}$, IRFP moves to stronger coupling, or disappears

- Consistent with no IRFP of $\beta^{\text {[2-loop] }}$ for $N_{c} \rightarrow \infty$ limit

- Can one use SU(4)/decuplet for walking technicolor?

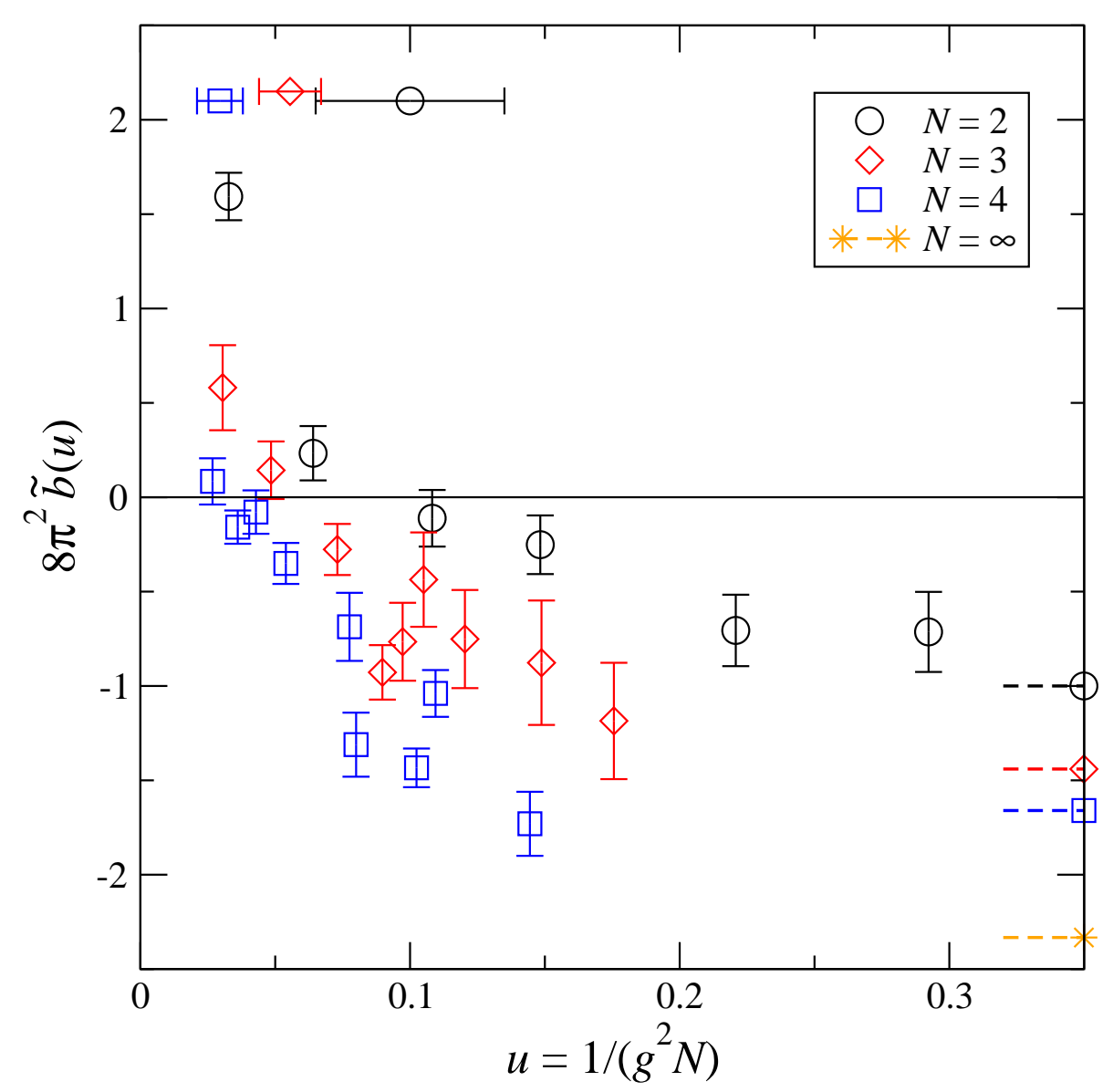




\section{Mass anomalous dimension}

- Dashed line:

one-loop for $N_{c} \rightarrow \infty$

- Remarkable universality

- Saturation: $\gamma \lesssim 0.45$

- Missed by analytic calculations!

- No good for walking technicolor

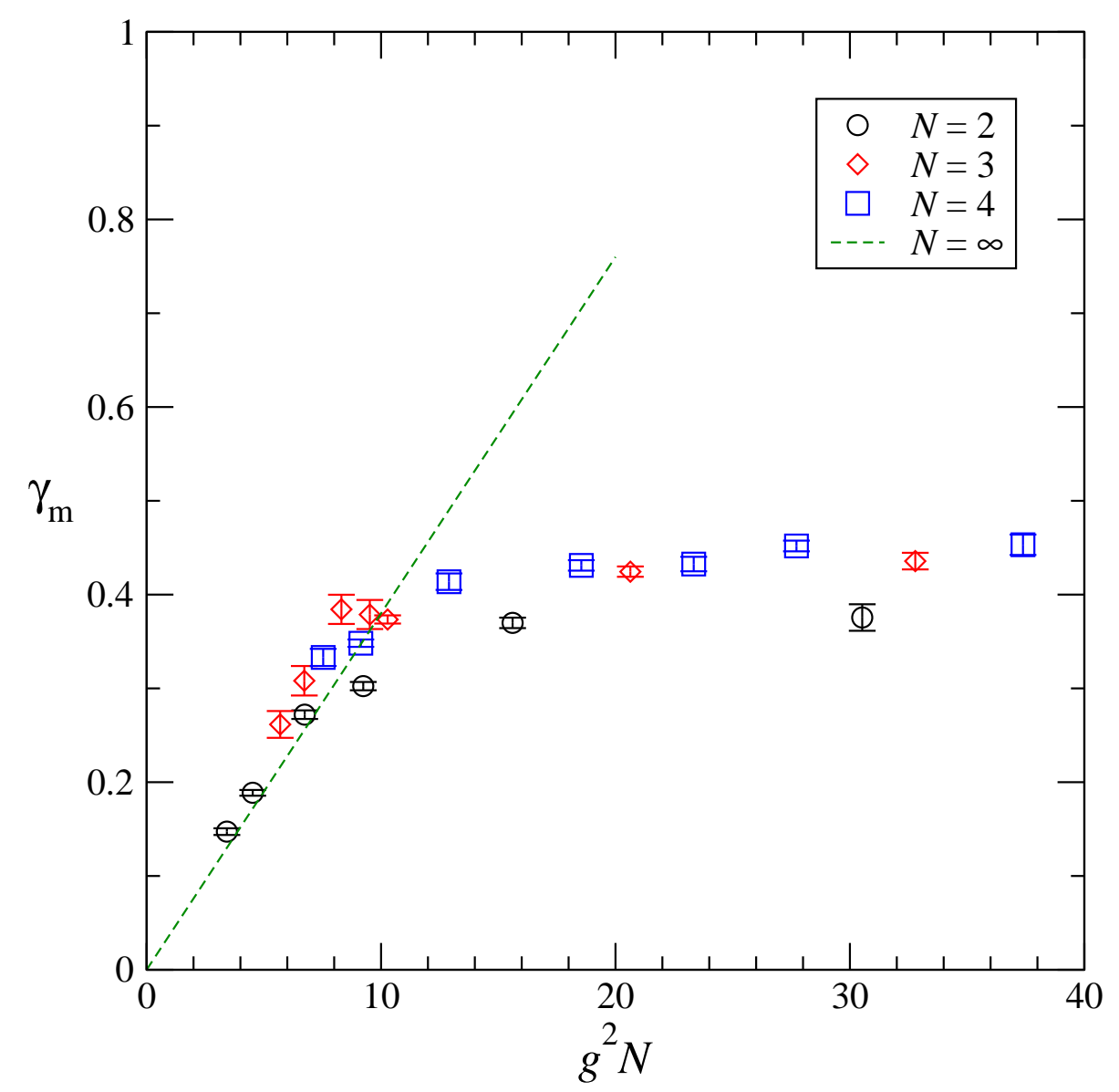




\section{Part II. What nHYP links do for us}

- Results from SU(3)/sextet

\begin{tabular}{c|ccccc}
\hline \hline & $\beta$ & $g_{S F}^{2}(L=6)$ & $\kappa_{c}$ & $m_{c}$ & $M$ \\
\hline \hline thin & 6.0 & $\sim 2$ & 0.1610 & -0.89 & 1.9 \\
\hline fat & 5.8 & 1.9 & 0.1283 & -0.10 & 1.1 \\
\hline \hline
\end{tabular}

Critical hopping parameter $\kappa_{c}=1 /\left(8+2 m_{c}\right)$

Optimal Domain-Wall height $\quad M=1+\left|m_{c}\right| \quad$ (to be explained later on)

- Similar improvement for optimal clover coefficient (big enough that we've decided to set $c_{S W}=1$ )

- Fat links allow us to probe much larger $g^{2}(L)$ 


\section{DWF primer}

- 5-d Wilson fermions, supercritical (negative bare mass), with chiral 4-d fields located near the boundaries $s=0$ and $s=L$

- Effective 4-dim operator: $\quad D_{\text {eff }}(L)=1+\gamma_{5} \tanh \left((L / 2) a_{5} H\right)$

- For $L \rightarrow \infty$ it becomes a GW operator: $\quad D_{\text {eff }}(\infty)=1+\gamma_{5} H / \sqrt{H^{2}}$

- DWF transfer matrix: $T=\exp \left(-a_{5} H\right)=1-a_{5} H_{W}+\cdots$ where $H_{W}=\gamma_{5} D_{W}$ is the 4-d hermitian Wilson operator

- Penetration into the 5-d bulk from near-unity eigenvalues of $T$, hence from near-zero eigenvalues of $H_{W}$

- PCAC: $\partial_{\mu} A_{\mu}=2 m_{q} J_{5}+2 m_{\text {res }}\left(J_{5}+\right.$ [more] lattice artifacts $)$

- Need $m_{\text {res }} \sim 10^{-3}$ (or smaller). Want $L$ to be small. 


\section{spectrum of $D_{W}$ in complex plane}

- Optimal DW height = center of leftmost "void"

- Fat links tame the Wilson spectrum: $m_{c}($ fat $) \ll m_{c}($ thin $)$

- Expect many more ev's inside the thin-links "void": $m_{\text {res }}($ fat $) \ll m_{\text {res }}($ thin $)$
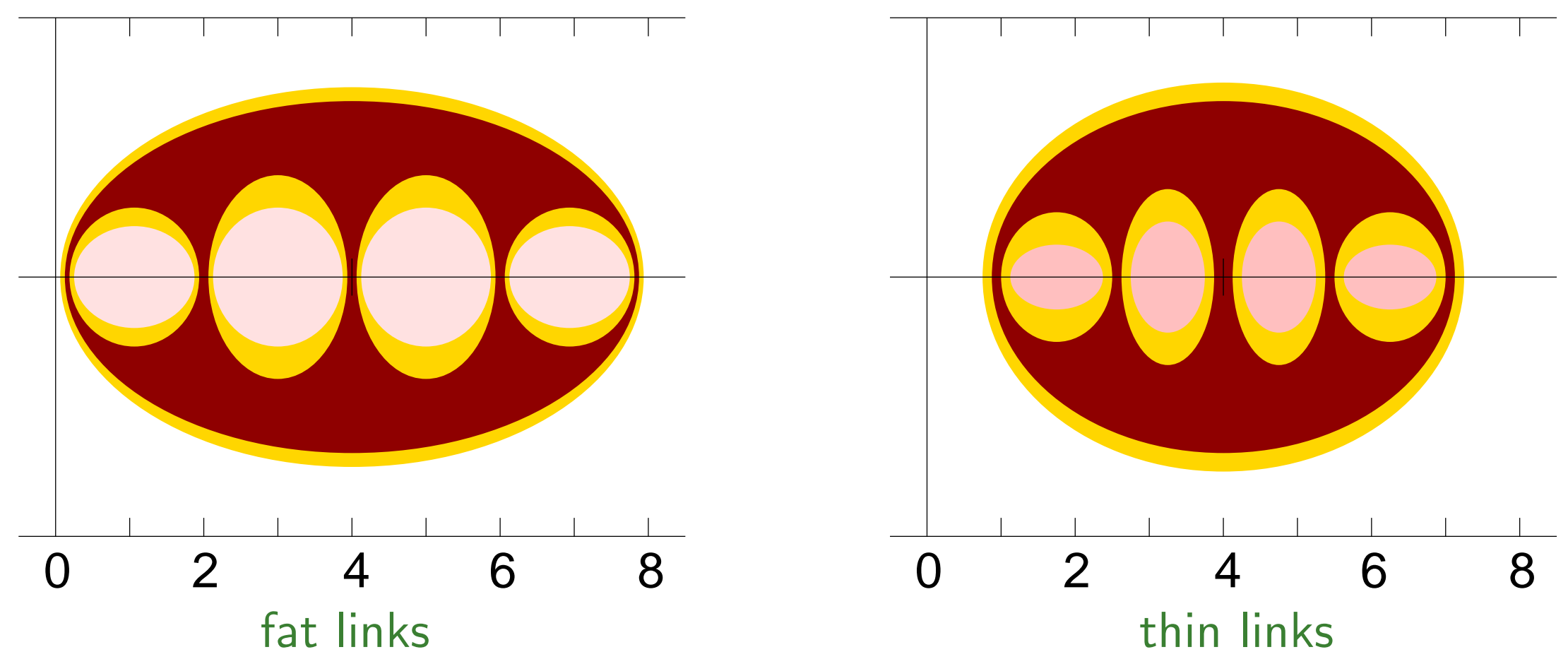


\section{Fat links and DWF}

- DWF on staggered MILC lattices:

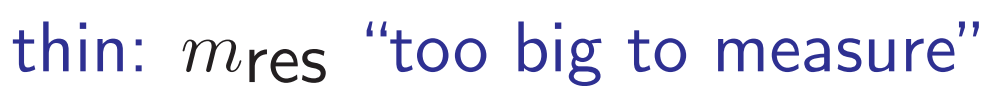
fat: $m_{\text {res }} \sim 10^{-3}$

- Locality

- It's true that $D_{W}$ is less local with fat links

- nHYP links only mildly nonlocal:

[Hasenfratz, Hoffmann \& Schaefer] Smeared link depends only on thin links that share a hypercube with it

- GW operators are never ultra-local

- Relevant notion: exponential localization of $D_{\text {eff }}(L)$.

- Depending on suppression of near-zero eigenmodes, in principle $D_{\text {eff }}(L)$ might be more local with fat links! 


\section{Summary}

- $S U(N), \quad N=2,3,4$, with $N_{f}=2$ two-index sym rep Dirac fermions are no good for walking technicolor

because mass anomalous dimension saturates at $\gamma \lesssim 0.45$

- While several nice tricks are already being used to bring down $m_{\text {res }}$ [improved gauge action, Dislocations Suppressing Determinant Ratio], and to speed up the inversion [Möbius], it's a good idea to try DWF with fat links 


\section{Walking (extended) Technicolor}

For acceptable flavor physics, candidate theories should have:

- Chiral symmetry breaking

- Small $S$-parameter

- Avoid massless GBs (except those eaten by $W^{ \pm}$and $Z$ )

- Condensate enhancement

$\Rightarrow$ need mass anomalous dimension $\gamma \approx 1$

$\Rightarrow$ this is expected for nearly conformal ("walking") theories

$\Rightarrow$ Natural candidates: $N_{f}=2$ higher-irrep fermions 


\section{Schrödinger functional}

- Prescribe gauge field $A_{k}(L, \eta)$ on time boundaries: $t=0$ and $t=L$

$\Rightarrow$ induce background color-electric field in the bulk

$$
\begin{aligned}
\Gamma(L, \eta) \equiv-\log (Z) & =\text { tree-level }+ \text { one-loop }+\cdots \\
& =\left(\frac{1}{g_{0}^{2}(a)}+\frac{b_{1}}{32 \pi^{2}} \log (L / a)+\cdots\right) S_{c l}(\eta) \\
& =\frac{1}{g^{2}(L)} S_{c l}(\eta)
\end{aligned}
$$

- Obtain $1 / g^{2}(L)$ from variation w.r.t. $\eta$, which is an observable

- Extract mass anomalous dimension $\gamma$ from scaling of pseudoscalar renormalization constant $Z_{P}$, on the same lattices 


\section{Soft gauge action}

- Soft action: $S_{\text {gauge }}=\beta \sum U_{\text {plaq }}($ thin links $)+\tilde{\beta} \sum U_{\text {plaq }}($ fat links $)$

- Pushes 1st-order bulk transition back into stronger bare coupling

- SU(3)/sextet:

- thin links: transition at $g_{S F}^{2} \approx 2.5$, lattice artifacts for $g_{S F}^{2} \gtrsim 2.0$

- fat links, $\tilde{\beta}=0$ : transition at $g_{S F}^{2} \approx 5$.0, lattice artifacts for $g_{S F}^{2} \gtrsim 3.5$

- fat links, $\tilde{\beta}=0.5$ : up to $g_{S F}^{2} \approx 11$ before running out of steam

- Stabilize nHYP reunitarization step:

$$
V_{\mu}=\Omega_{\mu}\left(\Omega_{\mu}^{\dagger} \Omega_{\mu}\right)^{-1 / 2} \Rightarrow V_{\mu}=\Omega_{\mu}\left(\Omega_{\mu}^{\dagger} \Omega_{\mu}+\epsilon\right)^{-1 / 2}
$$

We use $\epsilon=10^{-6}$ 


\section{Soft gauge action: weak-coupling universality}

bare coupling: $\quad \frac{1}{g_{0}^{2}}=\frac{\beta}{N} T(f)+\frac{\tilde{\beta}}{d_{R}} T(R)$,
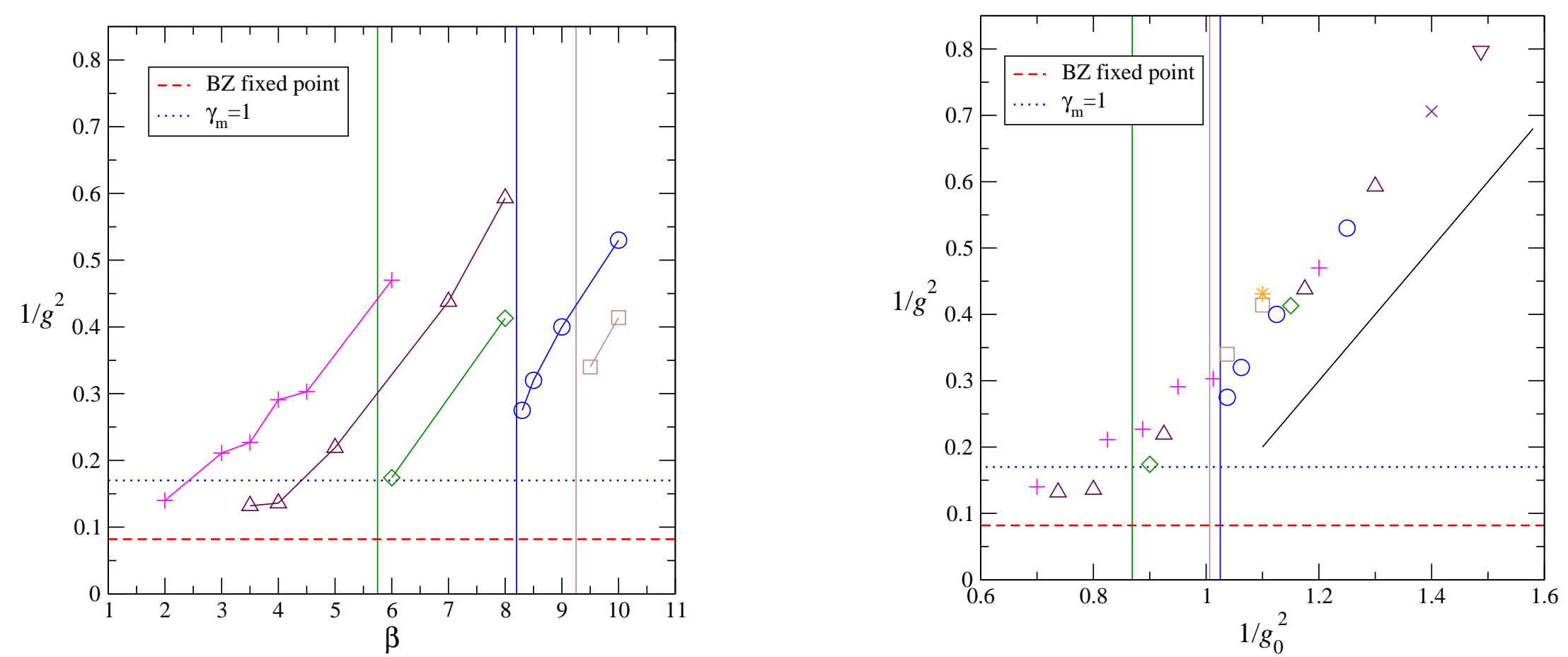

SU(4), Right to left: $\tilde{\beta}=-0.5,0.0,0.5,1.0,1.5$ 


\section{Soft gauge action: $\kappa_{c}$}

- Perturbation theory:

$\kappa_{c}$ increases with $\widetilde{\beta}$ at fixed $1 / g_{0}^{2}$

- Beyond $8 / g_{0}^{2} \approx 9$ trend reverses

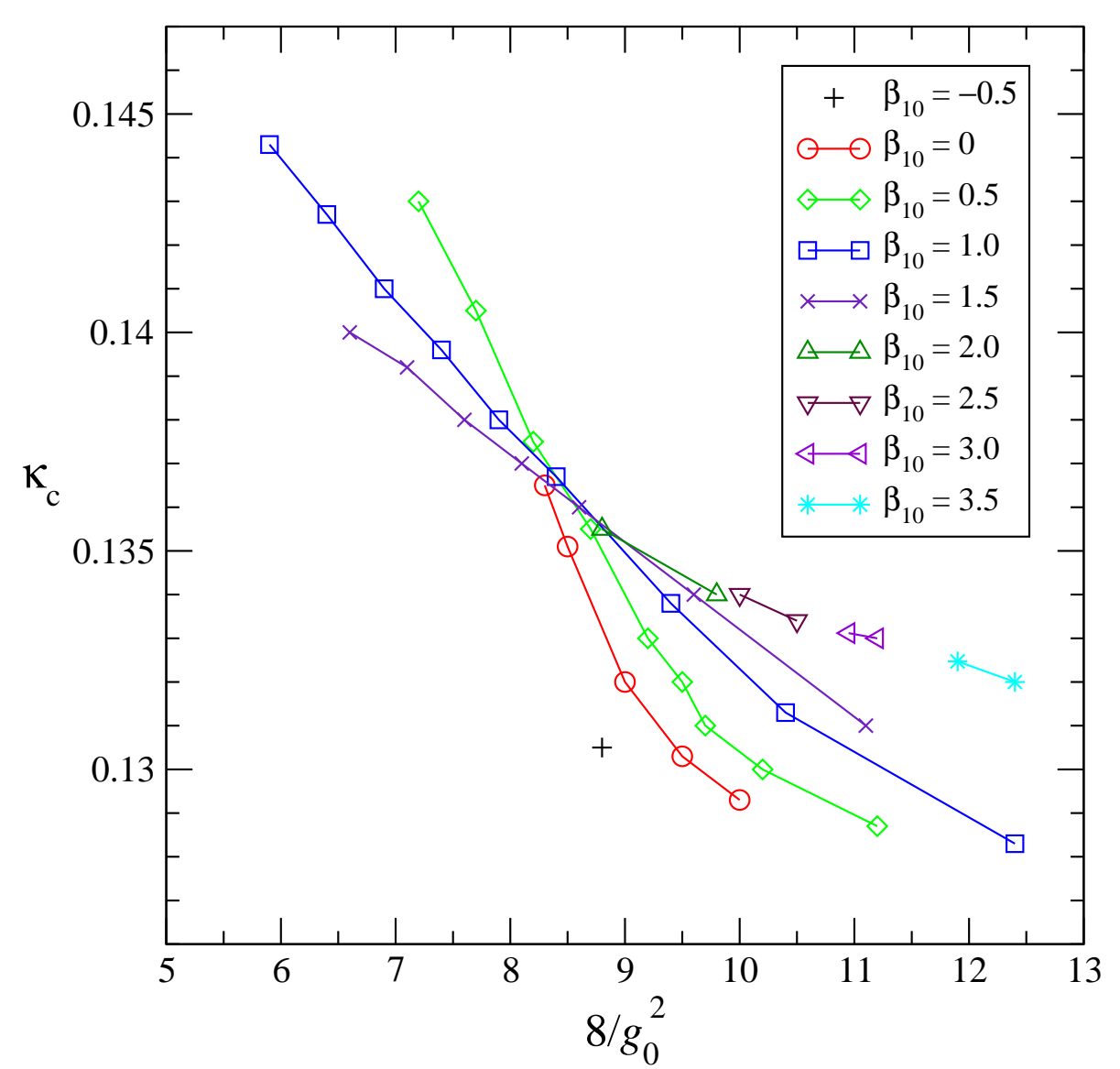




\section{Effects of the bulk transition, SU(3)}

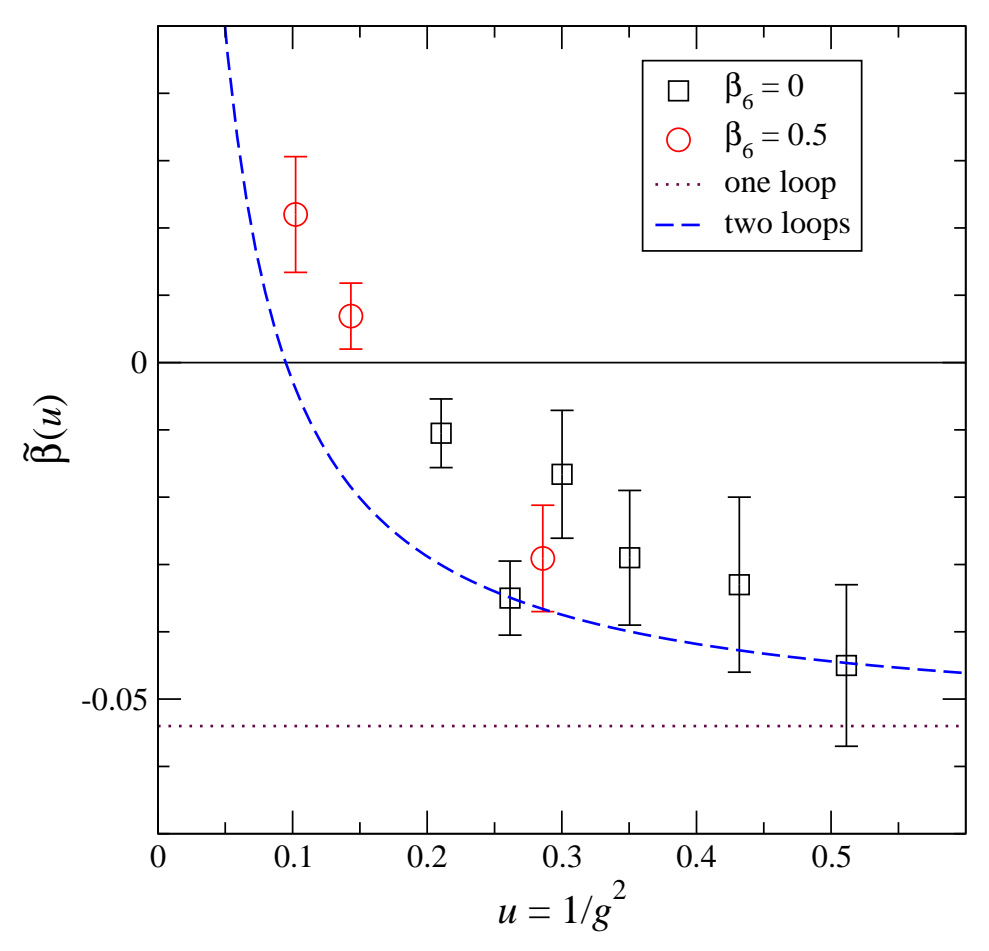

SF coupling: agreement

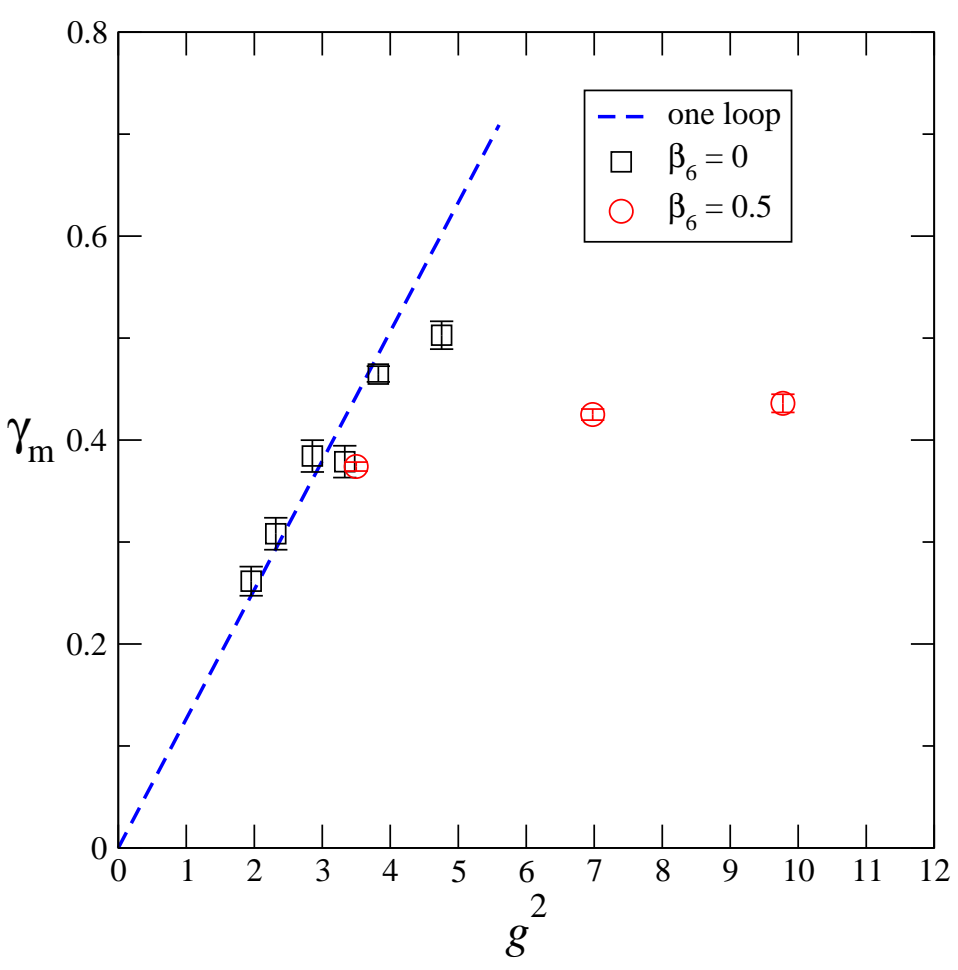

Mass anomalous dimension: discrepancy - due to proximity of bulk transition for $\tilde{\beta}=0$ 


\section{Nucleon EDM from Lattice QCD}

\section{Eigo Shintani (RIKEN-BNL) for $\mathrm{RBC} / \mathrm{UKQCD}$ collaboration}

I New Horizons for Lattice Computations with Chiral Fermions, May 162012 


\section{CP symmetry breaking in the SM}

- EW

D It has been known the CP violation occurs by the phase of CKM matrix

- $\mathrm{K}, \mathrm{D}, \mathrm{B}$ meson decay via direct and indirect $\mathrm{CP}$ violation

- Contribution to EDM is very tiny, $\Rightarrow d_{N}^{\mathrm{KM}} \simeq 10^{-30 \sim-33} \mathrm{e} \cdot \mathrm{cm}$ 6-orders magnitude below the exp. upper limit: $\left|d_{N}^{\exp }\right|<2.9 \times 10^{-26} \mathrm{e} \cdot \mathrm{cm}$

1 QCD

> $\theta$ term in the QCD Lagrangian:

$$
\mathcal{L}_{\theta}=\bar{\theta} \frac{1}{64 \pi^{2}} \int d^{4} x G \widetilde{G}, \quad \bar{\theta}=\theta+\arg \operatorname{det} M
$$

renormalizable and CP-violation comes due to topological charge density.

- EDM experiment provides very strong constraint on $\bar{\theta}<10^{-9}$

$\Rightarrow \theta$ and $\arg \operatorname{det} M$ need to be unnaturally canceled ! (strong CP problem) 


\section{CP symmetry breaking beyond the SM}

- Possible higher dimension operators

- Effective Hamiltonian with higher dimension than 4

$$
\begin{aligned}
& \mathcal{O}_{\mathrm{qEDM}}=\bar{q}(\sigma \cdot F) \gamma_{5} q \quad: \text { Quark-photon }
\end{aligned}
$$

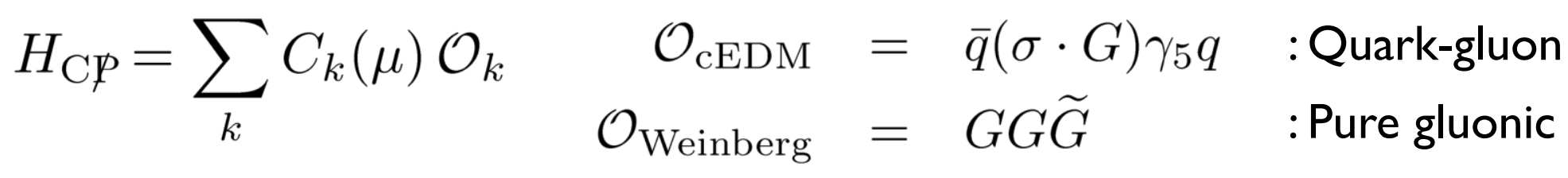

SUSY model
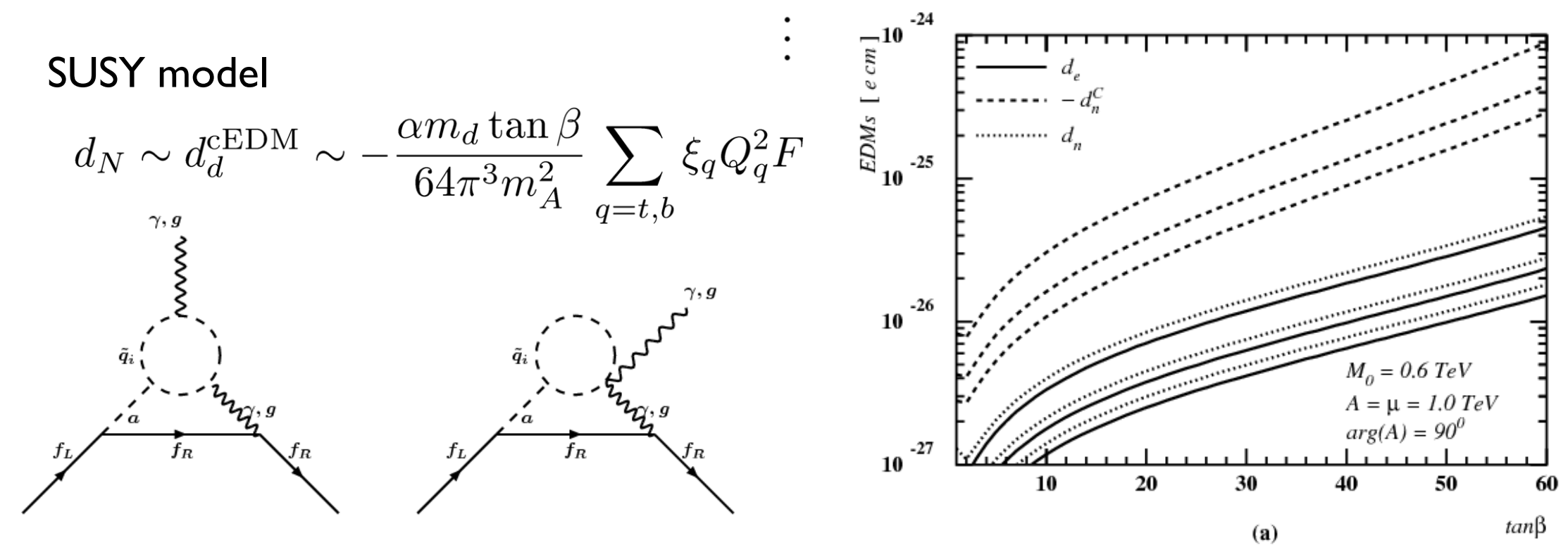

Chang, et al. (99), Ibrahim and Nath (08) 


\section{Constraint on nEDM}

- The present and future experiment is close to "exclude" of MSSM pEDM experiment @ BNL, nEDM experiment @ J-PARC,... $\Rightarrow$ reaching a sensitivity of $10^{-29} \mathrm{e} \cdot \mathrm{cm}$ !

- Current theoretical bound is based on quark model.

- Non-perturbative computation is necessary to draw more reliable conclusion.

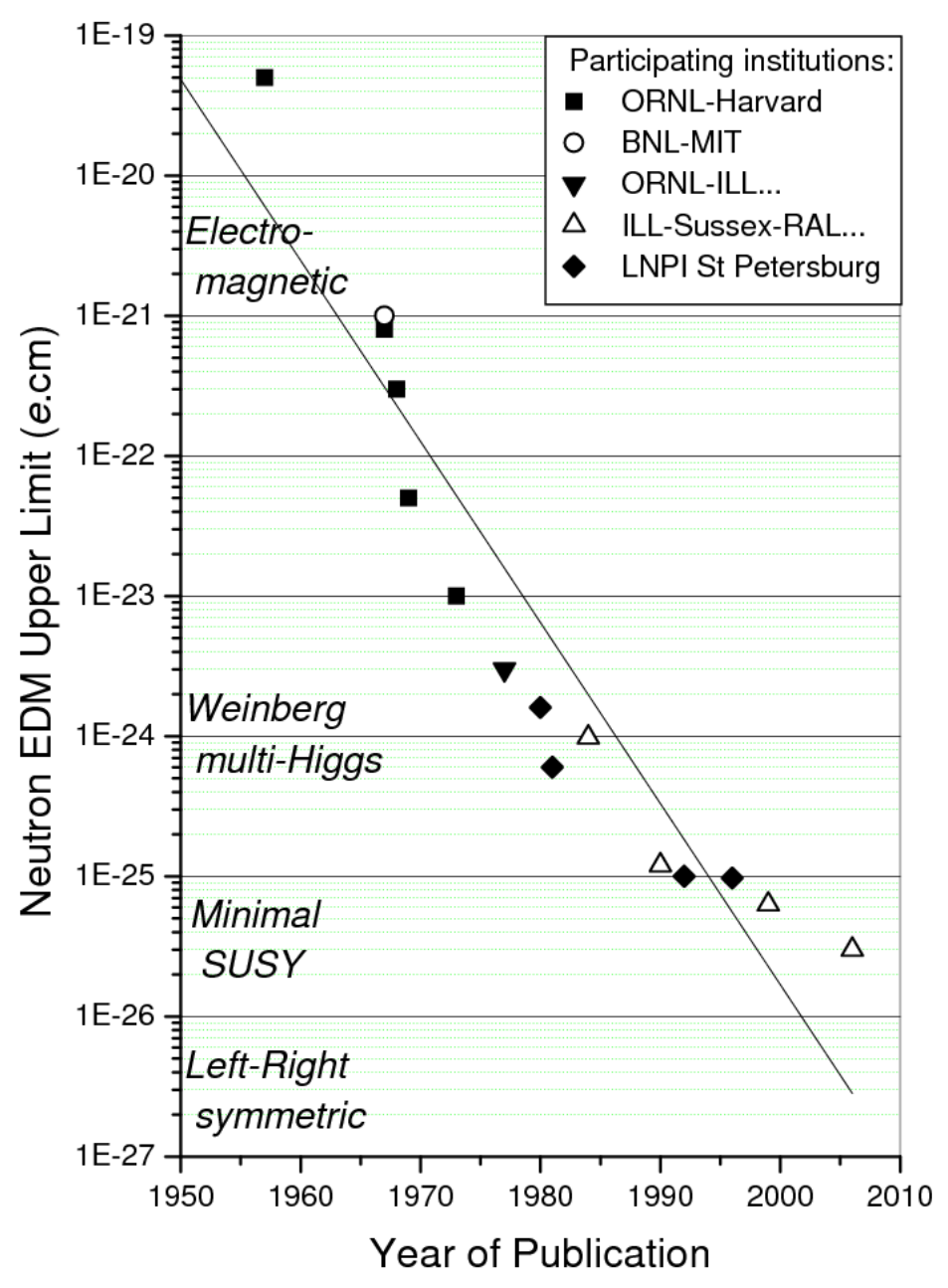

Harris, 0709.3100 


\section{What lattice QCD can do for $\mathrm{nEDM}$}

- In principle

D Direct estimate of neutron and proton EDM from $\theta$ term, higher dim. EP operators

- Matrix elements of higher dimension operators

$\left\langle N\left|\bar{q} \gamma_{5} \sigma \cdot F q\right| N\right\rangle,\left\langle N\left|\bar{q} \gamma_{5} \sigma \cdot G q\right| N\right\rangle,\langle N|G G \tilde{G}| N\rangle, \cdots$

- In practice there are some difficulties

- Statistical error

Source of CP violation comes from gauge background (topological charge, sea quark) which is intrinsically noisy.

Disconnected diagram is necessary because of flavor singlet contraction.

- Systematic error

Volume effect may be significant.

Chiral behavior is important, $\mathrm{d}_{\mathrm{N}} \sim \mathrm{O}(\mathrm{m})$ ? 


\section{Possible lattice methods}

- Spectrum method

Aoki-Gocksch(89), ES et al. CP-PACS $(06,07)$

- Spin splitting of nucleon energy in external electric field and $\theta$ term, which is given by 2 -pt function: $m_{\uparrow}-m_{\downarrow}=2 \mathrm{~d}_{N} \theta \mathrm{E}$

- Computational cost is cheap, and directly obtain EDM.

- Form factor

ES et al. CP-PACS(05), RBC(06)

$$
\left\langle n\left(P_{1}\right)\left|J_{\mu}^{\mathrm{EM}}\right| n\left(P_{2}\right)\right\rangle_{\theta}=\bar{u}_{N}^{\theta}[\underbrace{\frac{F_{3}^{\theta}\left(Q^{2}\right)}{2 m_{N}} \gamma_{5} \sigma_{\mu \nu} Q_{\nu}}_{\mathrm{P}, \mathrm{T} \text {-odd }}+\underbrace{F_{1} \gamma_{\mu}+\frac{F_{2}}{2 m_{N}} \sigma_{\mu \nu} Q_{\nu}}_{\mathrm{P}, \mathrm{T} \text {-even }}+\cdots] u_{N}^{\theta}
$$

1 $\mathrm{F}_{3}$ in $\mathrm{Q}^{2} \rightarrow 0$ provides $\mathrm{d}_{\mathrm{N}}$

- Subtraction to contribution of CP-odd phase in $\mathrm{n}$ propagator.

- Imaginary $\theta$

- Generate new configurations with imaginary $\theta$ term, which may enhance signal. Izubuchi (07), Horsley et al. (08) 


\section{Spectrum method}

ES et al. $(06,07)$

- Ratio of spin up and down

$R_{3}=\frac{\langle N(t) \bar{N}(0)\rangle_{\theta, E}^{\text {up }}}{\langle N(t) \bar{N}(0)\rangle_{\theta, E}^{\text {down }}} \simeq 1+d_{N} E \theta t$

Linear response, gradient is a signal of EDM.

- Remarks

> Reweighting works well for small real $\theta$

- Temporal periodicity is broken by electric field.
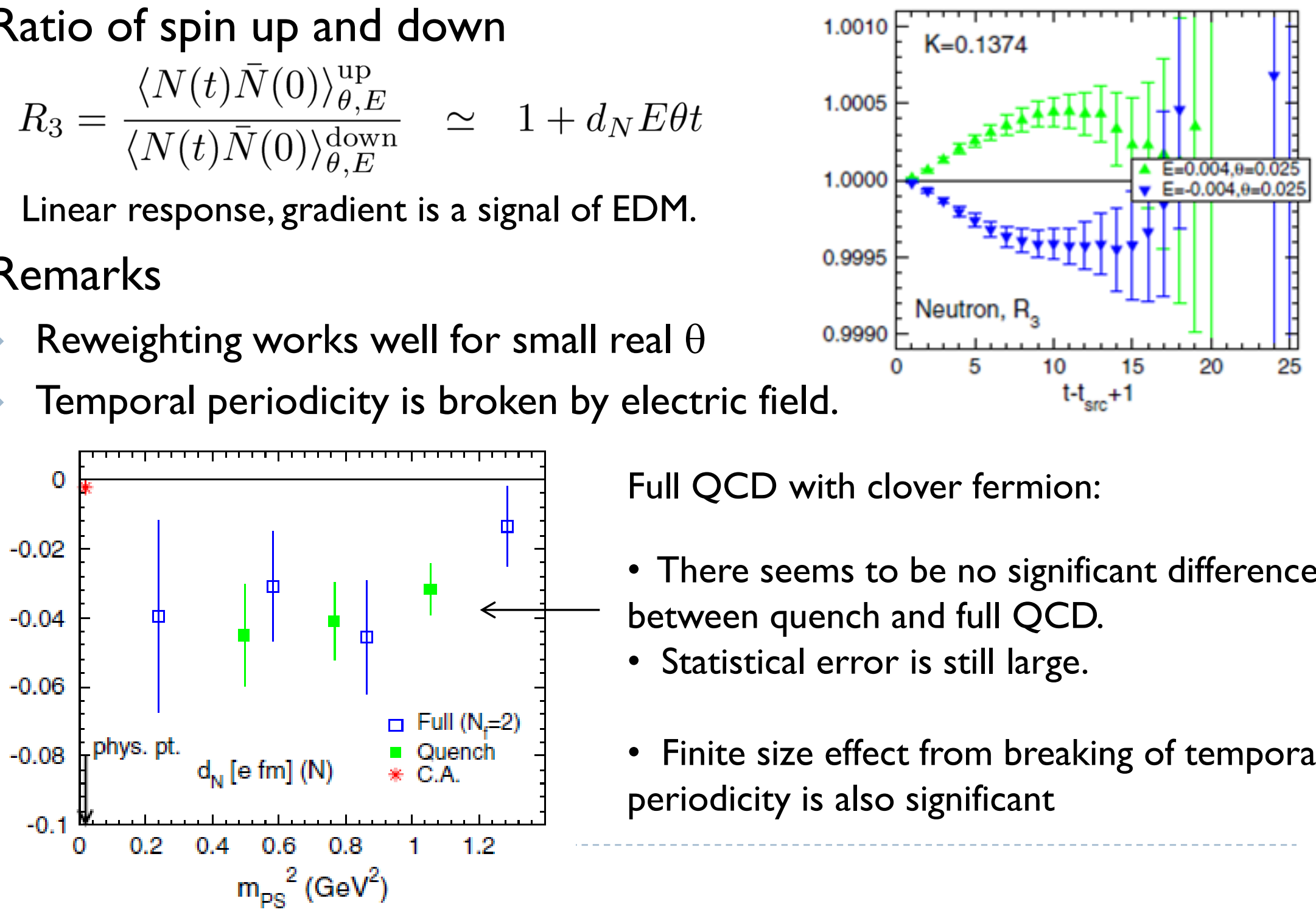

Full QCD with clover fermion:

- There seems to be no significant difference between quench and full QCD.

- Statistical error is still large.

- Finite size effect from breaking of temporal periodicity is also significant

$$
\mathrm{m}_{\mathrm{PS}}{ }^{2}\left(\mathrm{GeV}^{2}\right)
$$




\section{Form factor method}

- $\mathrm{F}_{3}$ signal
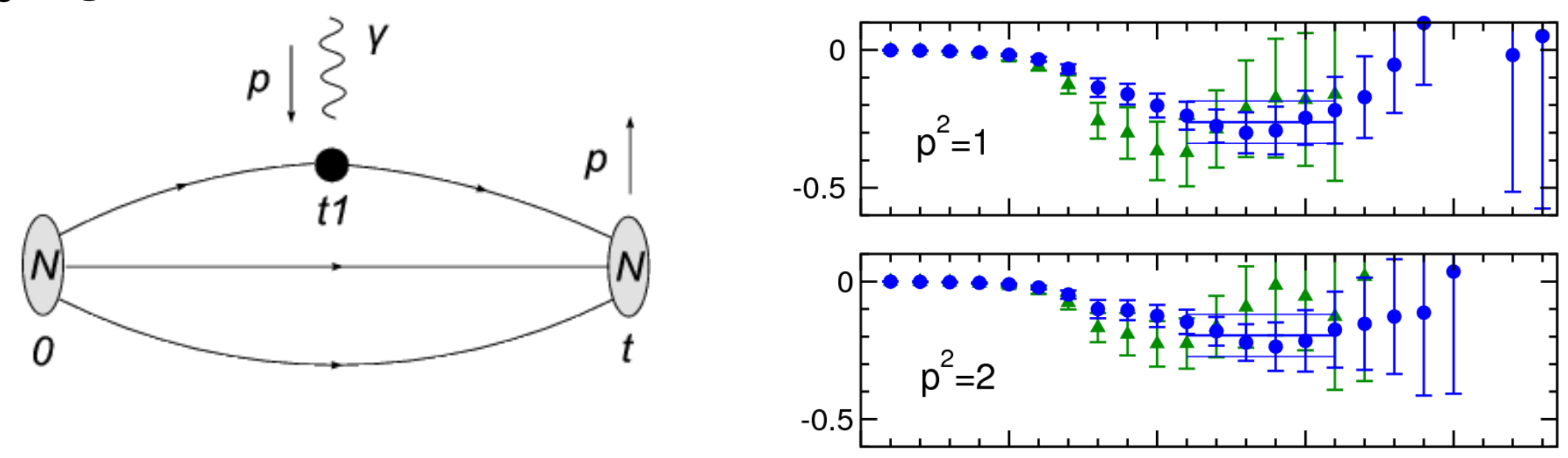

v $\mathrm{Nf}=2$ clover fermion

- Sequential source for $V$ current

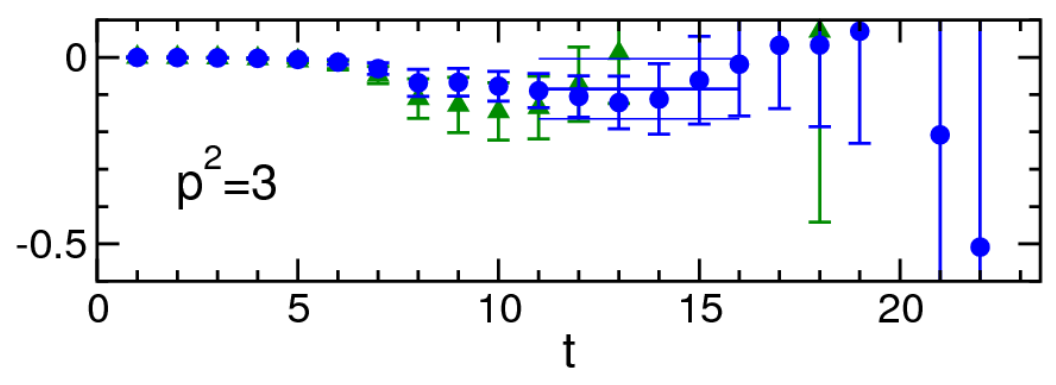




\section{Imaginary $\theta$}

- Analytically continued to pure imaginary, $\theta \rightarrow \mathrm{i} \theta^{\mathrm{I}}$

$$
\left\langle O e^{i \theta Q}\right\rangle \rightarrow\left\langle O e^{-\theta^{I} Q}\right\rangle
$$

1 There is no sign problem, expect better signal.

- Generate the QCD ensemble with $\theta^{\mathrm{I}}$. distribution of topological charge is shifted by $\theta^{\mathrm{I}}$
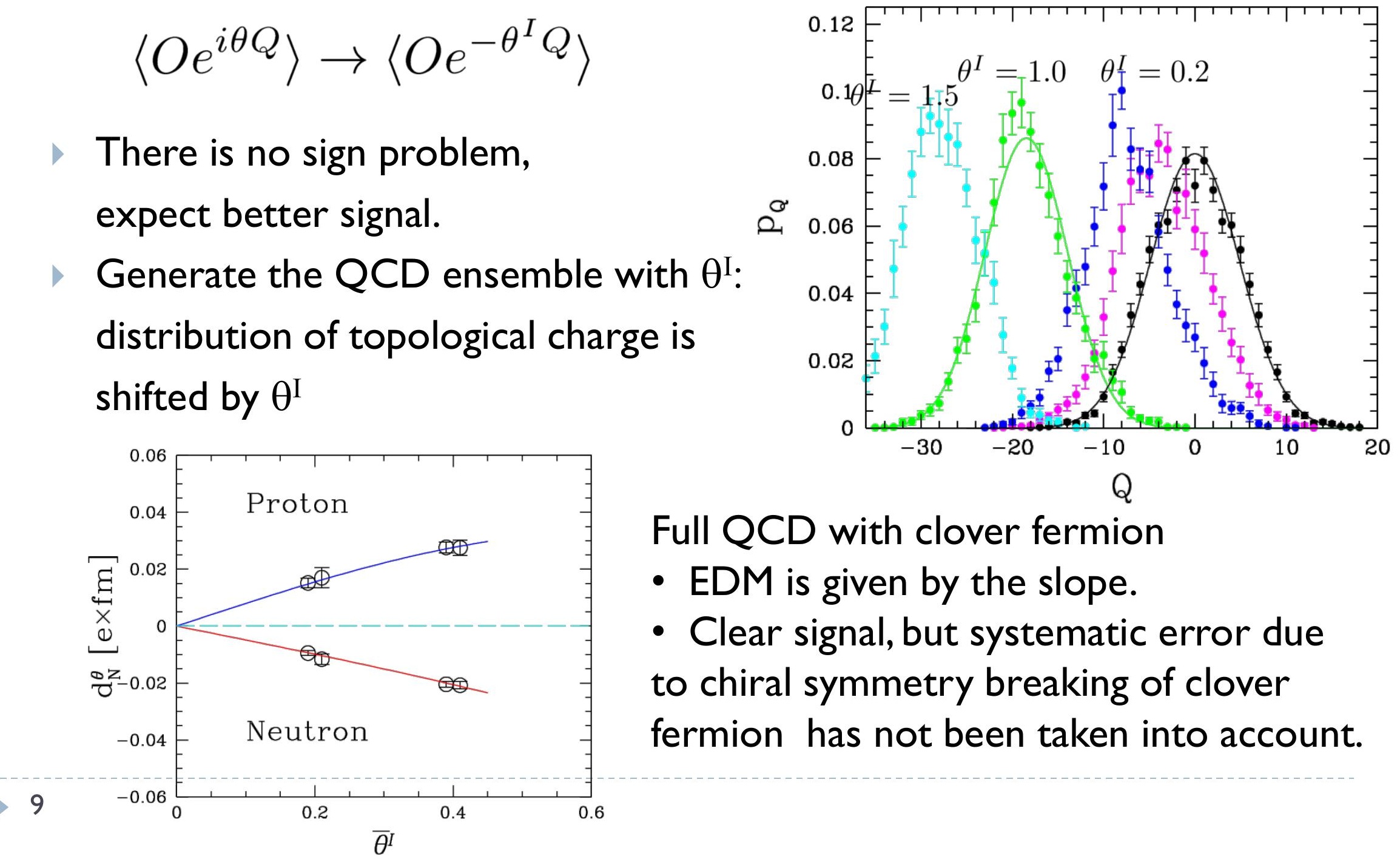

Full QCD with clover fermion

- EDM is given by the slope.

- Clear signal, but systematic error due to chiral symmetry breaking of clover fermion has not been taken into account. 


\section{Comparison of results}

- Full QCD

- Lattice results are consistent within I $\sigma$.

- An order of magnitude larger than the results of current algebra.

- $\mathrm{N}_{\mathrm{f}}=2+$ I DWF configs. (RBC/UKQCD) are available for near physical pion mass.

- Large statistical error is still problem.

$(\mathrm{O}(100)$ measurements is not

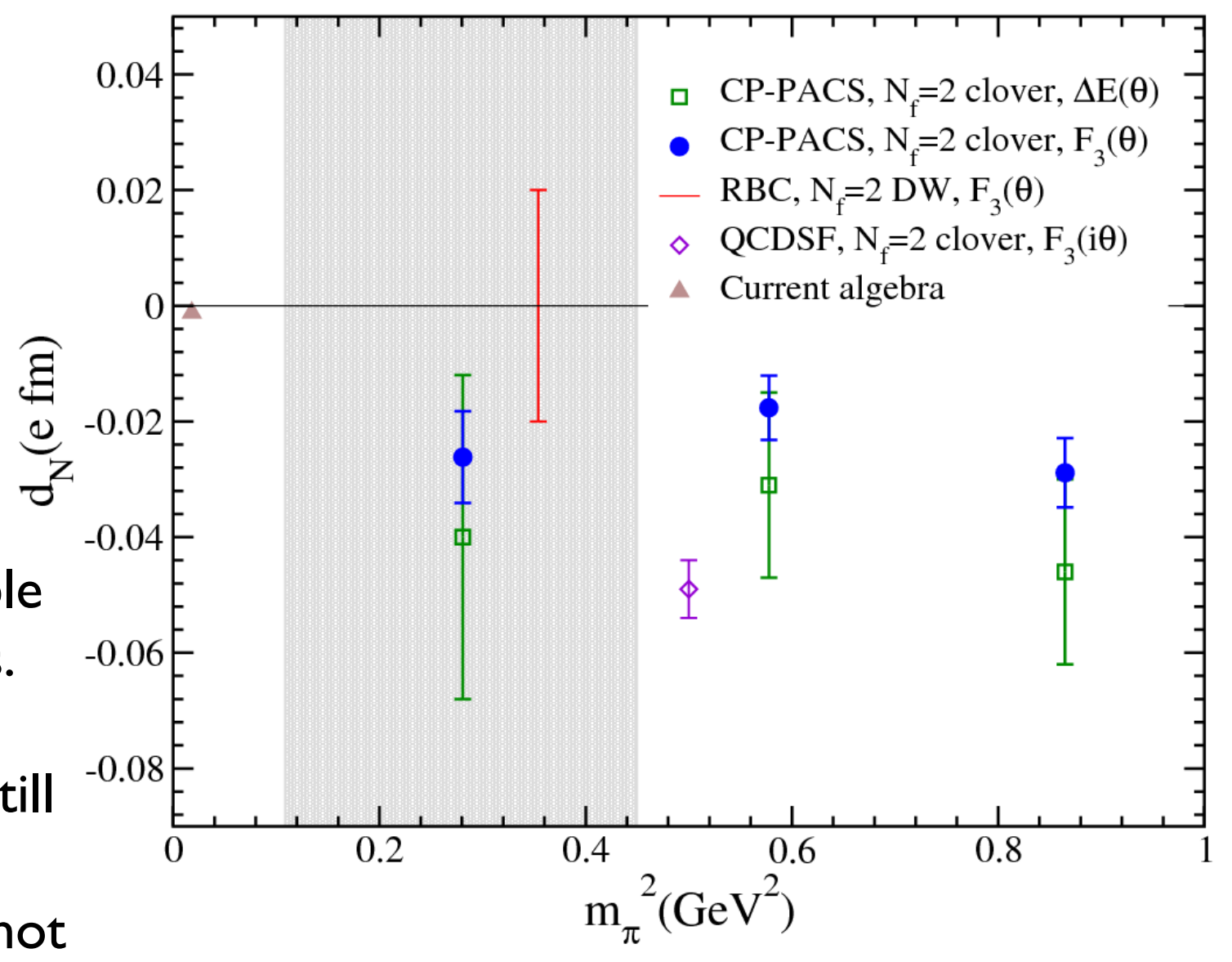
enough) 


\section{Error reduction techniques}

RBC in prep.

- Covariant approximation averaging (CAA)

- For original observables $O$, (unbiased) improved estimator

$$
\mathcal{O}^{(\mathrm{imp})}=\mathcal{O}^{(\text {rest })}+\frac{1}{N_{G}} \sum_{g \in G} \mathcal{O}^{(\text {appx }), g}, \quad \mathcal{O}^{(\text {rest })}=\mathcal{O}-\mathcal{O}^{(\text {appx })}
$$

which satisfies $\langle\mathrm{O}\rangle=\left\langle\mathrm{O}^{\mathrm{imp}}\right\rangle$ if approximation is covariant under lattice symmetry g, and error becomes err ${ }^{\mathrm{imp}} \simeq \mathrm{err} / \sqrt{N_{G}}$

- Ideal approximation
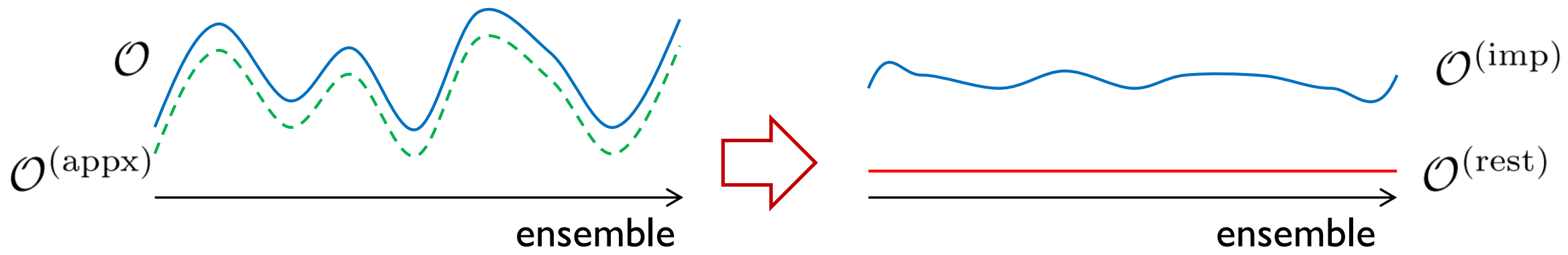

- Ignoring the error from $\mathrm{O}^{\text {(rest) }}$

- There may be many candidates of $O^{(\text {appx) }}$ e.g. LMA, heavy mass, ...

- The cost of approximated observable need to be smaller than the original. 


\section{Examples of CAA}

- Lowmode averaging (LMA)

Guisti et al.(04), Neff et al.(0I), DeGrand et al. (04)

> Using lowlying eigenmode of Dirac operator to approximate propagator:

$$
\mathcal{O}^{(\text {appx })}=\sum_{\lambda}^{N_{\lambda}} \mathcal{O}_{\lambda}^{\text {low }}
$$

where $\mathrm{N}_{\lambda}$ is number of lowmode computed by Lanczos.

Except for computational cost of eigenmode, $\operatorname{Cost}($ LMA $) \simeq 0$, but approximation is only lowmode part (long distance contribution).

- All-mode averaging (AMA)

- Using sloppy CG (loose stopping condition),

$$
\mathcal{O}^{(\text {appx })}=O^{\text {sloppy }}
$$

If stopping cond. is $0.003, \operatorname{Cost}(A M A) \simeq \operatorname{Cost}(C G) / 50$ (without deflation).

Approximation becomes better than LMA for other than lowmode dominanted observables (nucleon, finite momentum hadron, ...). 


\section{Examples of Covariant Approximations}

- All Mode Averaging AMA

Sloppy CG or Polynomial approximations

$$
\begin{aligned}
& \mathcal{O}^{\text {(appx) }}=\mathcal{O}\left[S_{l}\right], \\
& S_{l}=\sum_{\lambda} v_{\lambda} f(\lambda) v_{\lambda}^{\dagger}, \\
& f(\lambda)= \begin{cases}\frac{1}{\lambda}, & |\lambda|<\lambda_{\text {cut }} \\
P_{n}(\lambda) & |\lambda|>\lambda_{\text {cut }}\end{cases}
\end{aligned}
$$$$
P_{n}(\lambda) \approx \frac{1}{\lambda}
$$

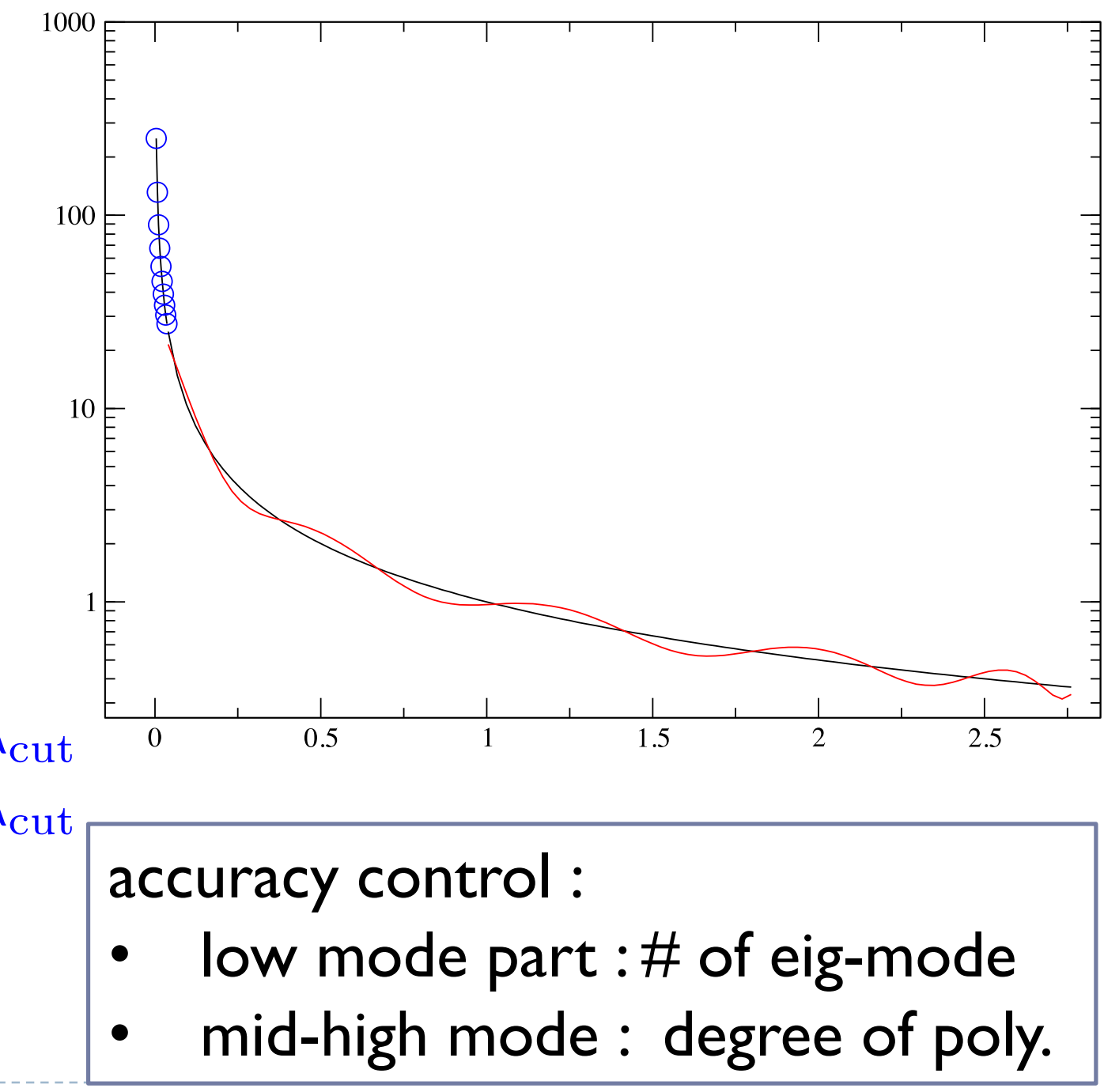




\section{Comparison between LMA/AMA}

$\mathrm{RBC}$ in prep.

- Preliminary result

1 8 configs, Gaussian smearing, $\mathrm{N}_{\mathrm{G}}=2^{3} \times 4=32$ sources, $24^{3} 64 \times 16$ DWF
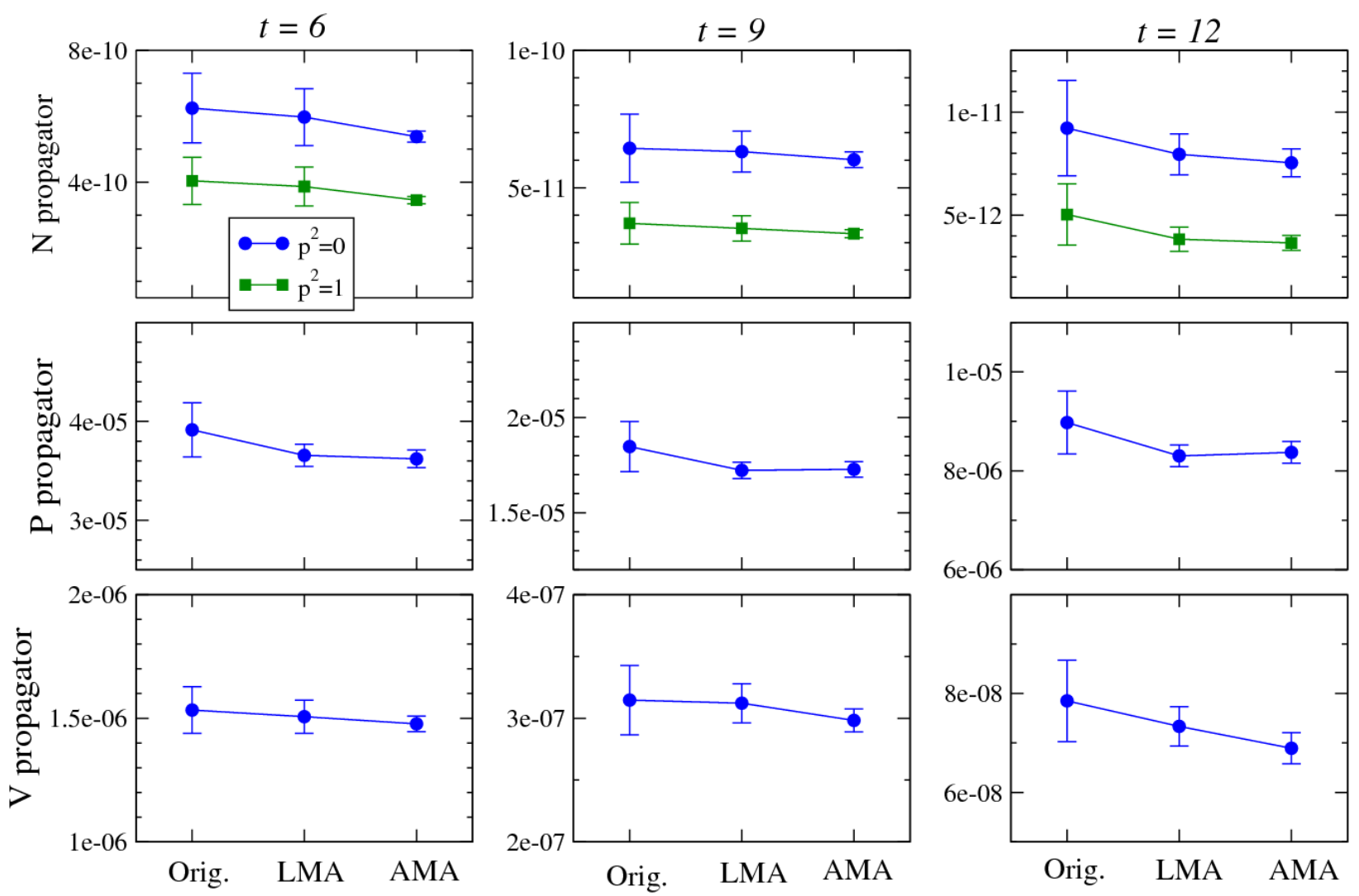

- $\mathrm{t}=6$ :

Error in AMA is actually reduced by factor 5 compared with orig. and LMA.

- $\mathrm{t}=12$ Error in AMA/LMA is reduced by factor 3--4 compared with original. 


\section{Comparison between LMA/AMA}

- Very preliminary

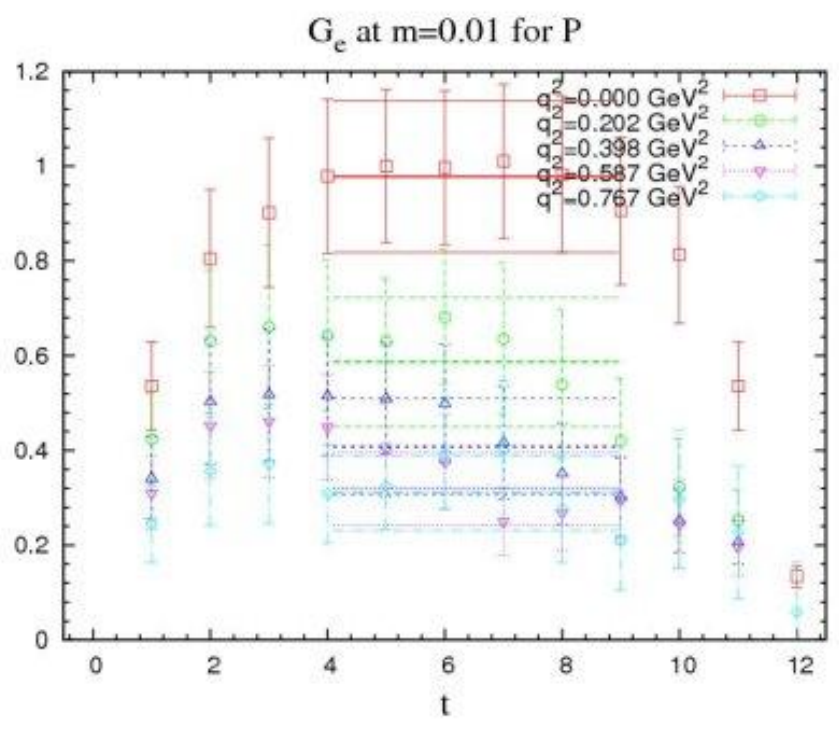

Proton Ge (Original)
Proton Ge (LMA)
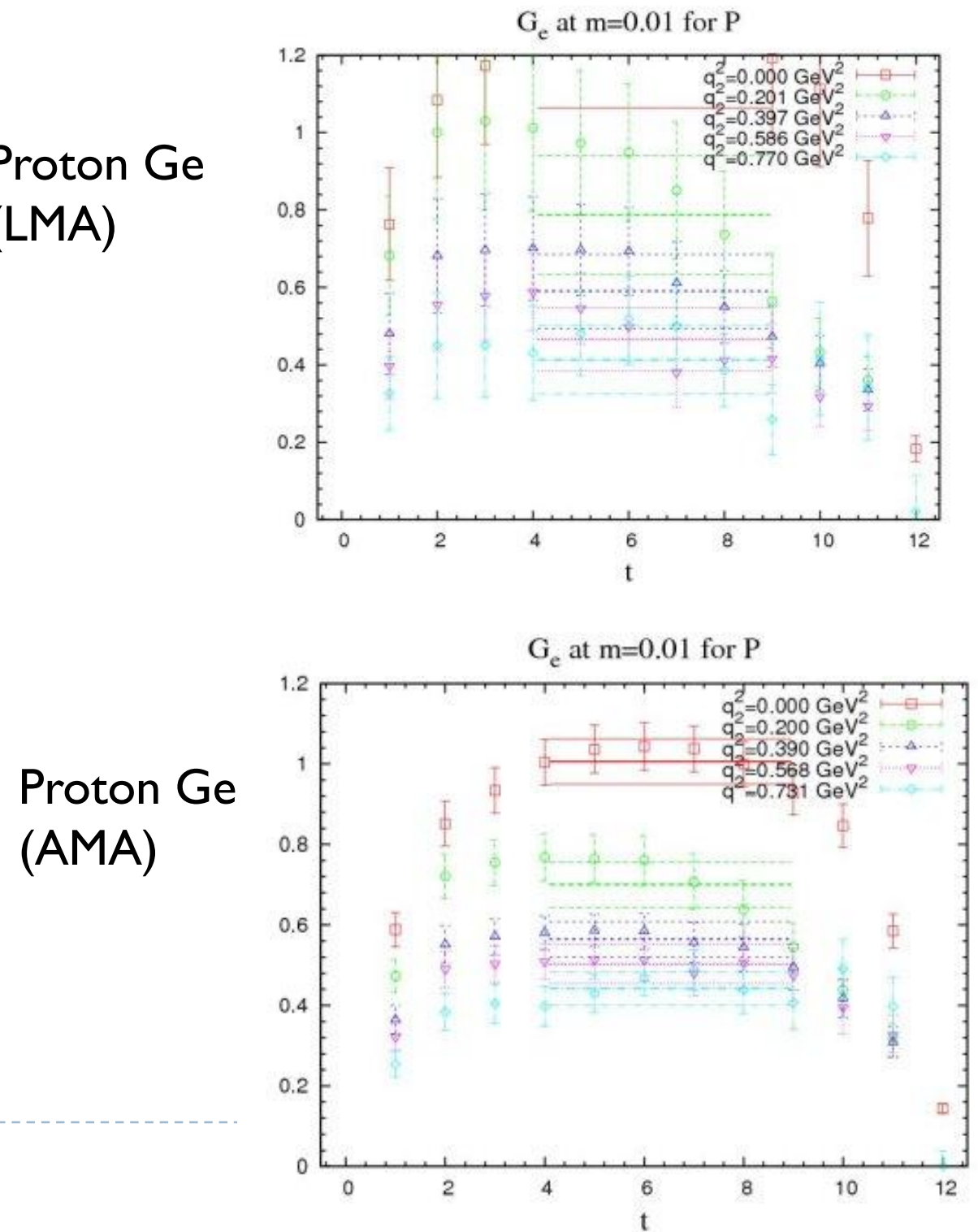


\section{Conclusion and future work}

- Nucleon EDM in lattice QCD

- Large statistical error is problem.

- LMA/AMA may work well.

- Aim for less than 10\% statistical error.

- Systematic study of finite size effect, chiral behavior, ...

> Other source EP effect

\section{Thank you.}




\section{Backup}




\section{Electric dipole moment (EDM)}

- $\mathrm{P}($ arity), $\mathrm{T}$ (ime reversal) $[=\mathrm{CP}]$ symmetry breaking EDM:

$$
\vec{D}=d \vec{S}, \quad \Delta H=\vec{D} \cdot \vec{E}
$$

under discrete symmetries, spin and $\mathrm{E}$ have different behavior

$$
\begin{array}{lll}
\mathrm{P}: & \vec{E} \rightarrow-\vec{E} & \vec{S} \rightarrow \vec{S} \\
\mathrm{~T}: & \vec{E} \rightarrow \vec{E} & \vec{S} \rightarrow-\vec{S}
\end{array}
$$

If $d \neq 0$,

$$
\mathrm{P}, \mathrm{T}: \Delta H \rightarrow-\Delta H
$$

Non-vanishing EDM is a signal of the $\mathrm{P}, \mathrm{CP}$ violation.

- In EW P, CP violation following Kobayashi-Maskawa mechanism.

- In QCD, it is natural to exist but there has been no signal the breaking would be also. 


\section{Strong CP problem ?}

- Possible solution

- Massless quark

One of the quark flavor is massless $\left(\mathrm{m}_{\mathrm{u}}=0\right.$ or $\left.\mathrm{m}_{\mathrm{d}}=0\right)$, i.e. $\arg \operatorname{det} M \propto m_{u} m_{d} m_{s} /\left(m_{u}+m_{d}+m_{s}\right)=0$

This has been refused by spectrum study in lattice QCD+QED.

- Axion model

- Pecci-Quinn (additional chiral) symmetry is spontaneously broken. Axion of (in-)visible model has been almost excluded by cosmology.

- Spontaneous breaking 


\section{CP symmetry breaking in the SM}

- Contribution to EDM from weak interaction is very small

- Vanishing I-loop (no Im part), 2-loop diagram

- Three-loop order(short) and pion loop correction (long):

Short distance

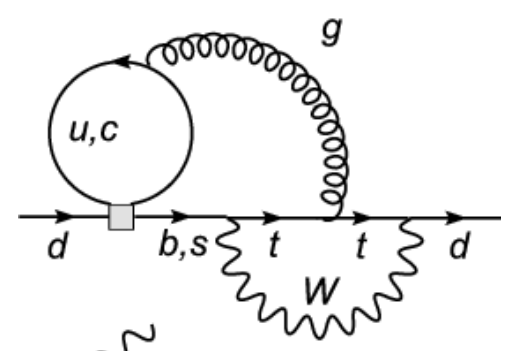

$\kappa_{V} \quad$ Czmechi, Krause (1997)

$d_{N}^{\mathrm{KM} \text { short }} \sim \mathcal{O}\left(\alpha_{s} G^{2}\right) \sim-10^{-34} \mathrm{e} \cdot \mathrm{cm}$
Long distance
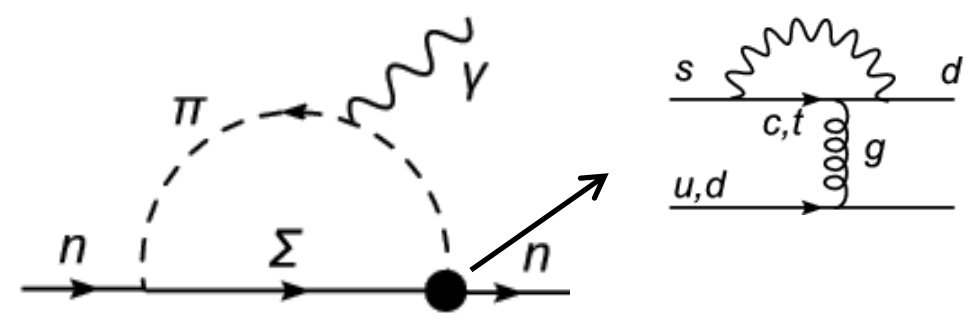

Khriplovich, Zhitnitsky ( 1982)

$$
d_{N}^{\mathrm{KM} \text { long }} \sim 10^{-30 \sim-33} \mathrm{e} \cdot \mathrm{cm}
$$

$\Rightarrow d_{N}^{\mathrm{KM}}=d_{N}^{\mathrm{KM} \text { short }}+d_{N}^{\mathrm{KM} \text { long }} \simeq 10^{-30 \sim-33} \mathrm{e} \cdot \mathrm{cm}$

which is the 6-order magnitude below the exp.

upper limit: $\left|d_{N}^{\exp }\right|<2.9 \times 10^{-26} \mathrm{e} \cdot \mathrm{cm}$ 


\section{Spectrum method}

Aoki-Gocksch(89), ES(06,07),

- Energy difference between nucleon spin Horsley(07), QCD-SF(08)

> Energy eigenvalue if $\theta$ exist in the background

$$
\mathcal{E}_{+S_{3}}(\vec{E})-\mathcal{E}_{-S_{3}}(\vec{E})=2 d_{N} \bar{\theta} S_{3} E_{3}+\mathcal{O}\left(E^{2}, \bar{\theta}^{2}, E \bar{\theta}\right)
$$

in the case of $\vec{E}(t)=\left(0,0, E_{3}\right)$

> 2-pt function provides EDM as exponents

$$
\begin{aligned}
& \langle N(t) \bar{N}(0)\rangle_{\theta, E}^{\text {upper }} \simeq Z\left(1+A_{N}\left(E^{2}\right) \theta E_{3}\right) \exp \left(-m_{N} t-d_{N} \theta E_{3} t / 2\right) \\
& \langle N(t) \bar{N}(0)\rangle_{\theta, E}^{\text {lower }} \simeq Z\left(1-A_{N}\left(E^{2}\right) \theta E_{3}\right) \exp \left(-m_{N} t+d_{N} \theta E_{3} t / 2\right)
\end{aligned}
$$

$d_{N}$ is given by fitting with the above asymptotic function.

> Two reweighting method

$$
\begin{aligned}
& \langle N \bar{N}\rangle_{\theta, E}=\left\langle N \bar{N}\left(U e^{q_{e} E}\right) e^{i \theta Q}\right\rangle \quad \text { Eucleadian } \mathrm{E} \text {, real } \theta \\
& =\left\langle N \bar{N}\left(U e^{i q_{e} E}\right) e^{\theta m_{q} \bar{q} \gamma_{5} q}\right\rangle \text { Minkowski E, imaginay } \theta
\end{aligned}
$$




\section{Form factor}

- Matrix element

$$
\left\langle n\left(P_{1}\right)\left|J_{\mu}^{\mathrm{EM}}\right| n\left(P_{2}\right)\right\rangle_{\theta}=\bar{u}_{N}^{\theta}[\underbrace{\frac{F_{3}^{\theta}\left(Q^{2}\right)}{2 m_{N}} \gamma_{5} \sigma_{\mu \nu} Q_{\nu}}_{\mathrm{P}, \mathrm{T} \text {-odd }}+\underbrace{F_{1} \gamma_{\mu}+\frac{F_{2}}{2 m_{N}} \sigma_{\mu \nu} Q_{\nu}}_{\mathrm{P}, \mathrm{T} \text {-even }}+\cdots] u_{N}^{\theta}
$$

- $\mathrm{F}_{3}$ in $\mathrm{Q}^{2} \rightarrow 0$ is equivalent to $\mathrm{d}_{\mathrm{N}}$

- Expansion of 3pt func. at $O(\theta)$ into different CP-odd sources:

$$
\begin{aligned}
& \left\langle N(t) J_{\mu}^{\mathrm{EM}}\left(t_{1}\right) \bar{N}(0) Q\right\rangle \\
& =\left[u_{N} \otimes \bar{u}_{N} \frac{F_{3}}{2 m_{N}} Q_{\nu} \sigma_{\mu \nu} \gamma_{5} u_{N} \otimes \bar{u}_{N}\right. \\
& +u^{\theta^{1}} \otimes \bar{u}_{N}^{\theta^{1}}\left(F_{1} \gamma_{\mu}+\frac{F_{2}}{2 m_{N}} Q_{\nu} \sigma_{\mu \nu}\right) u_{N} \otimes \bar{u}_{N} \\
& \left.+u_{N} \otimes \bar{u}_{N}\left(F_{1} \gamma_{\mu}+\frac{F_{2}}{2 m_{N}} Q_{\nu} \sigma_{\mu \nu}\right) u^{\theta^{1}} \otimes \bar{u}_{N}^{\theta^{1}}\right] e^{-E_{N}\left(P_{1}\right) t_{1}-E_{N}\left(P_{2}\right)\left(t-t_{1}\right)}
\end{aligned}
$$

Subtraction of CP-odd phase in $n$ propagator ( $2^{\text {nd }}$ and $3^{\text {rd }}$ terms) is essential. 


\section{Error reduction technique}

- Statistical error

$$
\text { err } \simeq C / \sqrt{N_{\mathrm{mes}}}
$$

- In order to reduce error, do more $\mathrm{N}_{\text {mes }}$ independent measurements. change to $C$ of observables with small fluctuation.

- Due to limited gauge ensembles, usually covariant observables under lattice symmetry $\mathrm{O}^{\mathrm{g}}$ are regarded as independent measurements:

$$
\langle O\rangle=\left\langle O^{g}\right\rangle
$$

e.g. $g$ : lattice rotation, translation, ...

- Problem is computational cost. 


\section{Lattice QCD's works}

- One of the most successful non-perturbative calculation in the particle physics.

D Reproduce the hadron spectrum using a few input parameters.

BMW, PACS-CS, ...

- Monte-Carlo simulation is powerful tool.

1 Precision of lattice computations are getting better year by year thanks to development of algorithm (improved HMC, CAA) and machine (GPGPU, Blue Gene, Kei, ...).

- Flexible methodology to apply other physics concerned with strong interaction (e.g. many flavor, Graphene, ...) 


\title{
$B$-physics with domain-wall light quarks and relativistic heavy quarks
}

\author{
Oliver Witzel \\ Center for Computational Science
}

\section{BOSTON UNIVERSITY}

BNL, May 15, 2012 


\section{Phenomenological Importance}

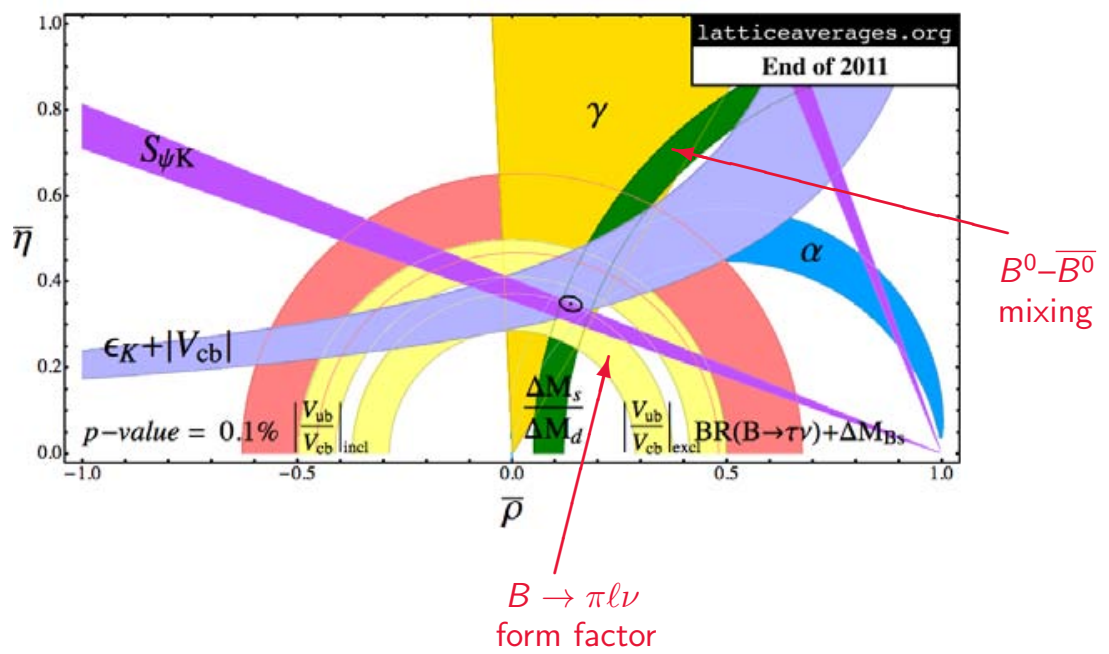




\section{$B^{0}-\overline{B^{0}}$ Mixing}

- Allows us to determine the CKM matrix elements

- Dominant contribution in SM: box diagram with top quarks

$$
\left.\begin{array}{c}
\left|V_{t d}^{*} V_{t b}\right| \text { for } B_{d} \text {-mixing } \\
\left|V_{t s}^{*} V_{t b}\right| \text { for } B_{s}-\text { mixing }
\end{array}\right\} \Delta M_{q}=\frac{G_{F}^{2} m_{W}^{2}}{6 \pi^{2}} \eta_{B} S_{0} M_{B_{q}} f_{B_{q}}^{2} B_{B_{q}}\left|V_{t q}^{*} V_{t b}\right|^{2}
$$

- Nonperturbative contribution: $f_{q}^{2} B_{B q}$

- Define the $S U(3)$ breaking ratio $\xi^{2}=f_{B_{s}}^{2} B_{B_{s}} / f_{B_{d}}^{2} B_{B_{d}}$

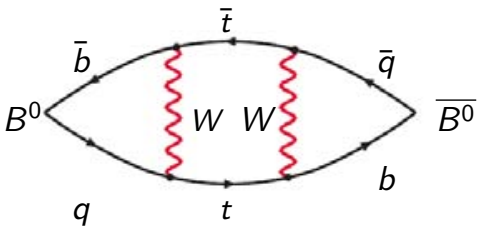

- CKM matrix elements are extracted by

$$
\frac{\Delta M_{s}}{\Delta M_{d}}=\frac{M_{B_{s}}}{M_{B_{d}}} \xi^{2} \frac{\left|V_{t s}\right|^{2}}{\left|V_{t d}\right|^{2}}
$$

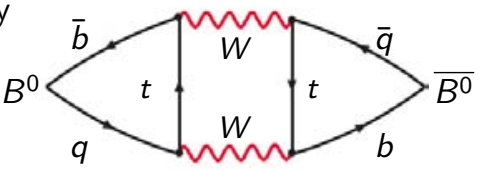

- Experimental error of $\Delta M_{q}$ is better than a percent; lattice uncertainty for $\xi$ is about $3 \%$ 


\section{$B \rightarrow \pi l \nu$ form factor}

- Allows to determine the CKM matrix element $V_{u b}$ from the experimental branching ratio $\frac{d \Gamma(B \rightarrow \pi l \nu)}{d q^{2}}=\frac{G_{F}^{2}\left|V_{u b}\right|^{2}}{192 \pi^{3} M_{B}^{3}}\left[\left(M_{B}^{2}+M_{\pi}^{2}-q^{2}\right)^{2}-4 M_{B}^{2} M_{\pi}^{2}\right]^{3 / 2}\left|f_{+}\left(q^{2}\right)\right|^{2}$

- Tension between exclusive determination and inclusive determinations of $V_{u b}$ is greater than $3 \sigma$ 


\section{Possible Deviations from the Standard Model}

\section{[Lunghi and Soni 2010/11]}

- Experimental value for $\sin (2 \beta)$ is $3.3 \sigma$ lower than $\mathrm{SM}$ expectation

- Measured value for $\operatorname{BR}(B \rightarrow \pi / \nu)$ is $2.8 \sigma$ lower than predicted

- Most likely source of deviation in $B_{d(s)}$ mixing and $\sin (2 \beta)$; less likely in $B \rightarrow \tau \nu$

\section{[Laiho, Lunghi and Van de Water 2012, http: //www. latticeaverages.org]}

- Scenario in which new physics is in $B \rightarrow \tau \nu$ decay and/or in $B_{d}$-mixing preferred

- If tension is taken at face value, points to physics at a few-GeV mass scale

See also: http://ckmfitter.in2p3.fr, http://utfit.roma1.infn.it 


\section{$2+1$ Flavor Lattice Calculations of $f_{B_{s}}, f_{B}, f_{B_{s}} / f_{B}, \xi, V_{u b}$}
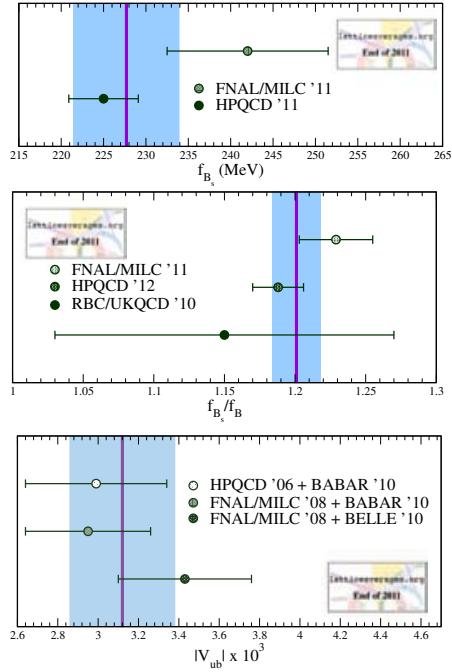
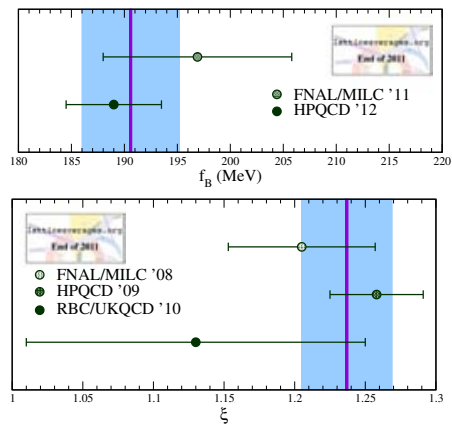

- HPQCD and FNAL-MILC result both based on the asqtad-improved staggered ensembles generated by MILC 


\section{Our Project}

- Use domain-wall light quarks and nonperturbatively tuned relativistic $b$-quarks to compute at few-percent precision

- $B^{0}-\overline{B^{0}}$ mixing

- Decay constants $f_{B}$ and $f_{B_{s}}$

- $B \rightarrow \pi \ell \nu$ form factor

- Tune RHQ parameters using bottom-strange states and high statistics

- Improve upon exploratory studies and verify made assumptions

- Validate tuning procedure by computing $b \bar{b}$ masses and splittings

- Derive lattice perturbation theory for matching lattice results to continuum 1-loop in tadpole-improve lattice perturbation

- Improve matching using a mostly-nonperturbative scheme for $f_{B}, f_{B_{s}}$ and $B \rightarrow \pi \ell \nu$ 


\section{2+1 Flavor Domain-Wall Gauge Field Configurations}

- Domain-wall fermions for the light quarks (u, d, s) [Kaplan 1992, Shamir 1993]

- Iwasaki gauge action [Iwasaki 1983]

- Configurations generated by RBC and UKQCD collaborations [C. Allton et al. 2008],

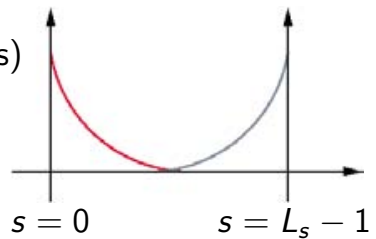

[Y. Aoki et al. 2010]

\begin{tabular}{ccccccc}
\hline $\mathrm{L}$ & $a(\mathrm{fm})$ & $m_{l}$ & $m_{s}$ & $m_{\pi}(\mathrm{MeV})$ & $\begin{array}{c}\text { approx. } \\
\text { \# configs. }\end{array}$ & $\begin{array}{c}\text { \# time } \\
\text { sources }\end{array}$ \\
\hline 24 & $\approx 0.11$ & 0.005 & 0.040 & 331 & 1636 & 1 \\
24 & $\approx 0.11$ & 0.010 & 0.040 & 419 & 1419 & 1 \\
\hline 32 & $\approx 0.08$ & 0.004 & 0.030 & 307 & 628 & 2 \\
32 & $\approx 0.08$ & 0.006 & 0.030 & 366 & 889 & 2 \\
32 & $\approx 0.08$ & 0.008 & 0.030 & 418 & 544 & 2 \\
\hline
\end{tabular}




\section{Relativistic Heavy Quark Action for the b-Quarks}

- Relativistic Heavy Quark action developed by Christ, Li, and Lin for the $b$-quarks in 2-point and 3-point correlation functions

\section{[Christ, Li, Lin 2007; Lin and Christ 2007]}

- Builds upon Fermilab approach [El Khadra, Kronfeld, Mackenzie 1997] by tuning all parameters of the clover action non-perturbatively; close relation to the Tsukuba formulation [Aoki, Kuramashi, Tominaga 2003]

- Heavy quark mass is treated to all orders in $\left(m_{b} a\right)^{n}$

- Expand in powers of the spatial momentum through $O(\vec{p} a)$

- Resulting errors will be of $O\left(\vec{p}^{2} a^{2}\right)$

- Allows computation of heavy-light quantities with discretization errors of the same size as in light-light quantities

- Applies for all values of the quark mass

- Has a smooth continuum limit 


\section{Tuning the Parameters of the RHQ Action}

$$
S=\sum_{n, n^{\prime}} \bar{\Psi}_{n}\left\{m_{0}+\gamma_{0} D_{0}-\frac{a D_{0}^{2}}{2}+\zeta\left[\vec{\gamma} \cdot \vec{D}-\frac{a(\vec{D})^{2}}{2}\right]-a \sum_{\mu \nu} \frac{i c_{P}}{4} \sigma_{\mu \nu} F_{\mu \nu}\right\}_{n, n^{\prime}} \Psi_{n^{\prime}}
$$

- Start from an educated guess for $m_{0} a, c_{P}$, and $\zeta$

$\left[\begin{array}{c}m_{0} a \\ c_{P} \\ \zeta\end{array}\right] \pm\left[\begin{array}{c}\sigma_{m_{0} a} \\ 0 \\ 0\end{array}\right],\left[\begin{array}{c}0 \\ \sigma_{c_{P}} \\ 0\end{array}\right],\left[\begin{array}{c}0 \\ 0 \\ \sigma_{\zeta}\end{array}\right]$

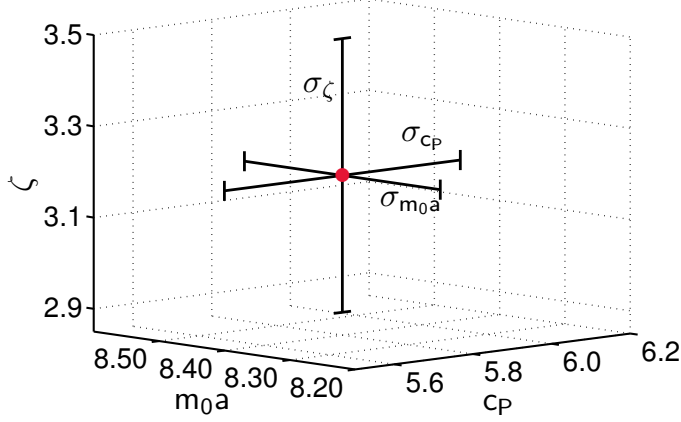


- Compute for all seven parameter sets

$\begin{array}{lllc}\text { spin-averaged mass } & \bar{M}=\left(M_{B_{s}}+3 M_{B_{s}^{*}}\right) / 4 & \rightarrow & 5403.1(1.1) \mathrm{MeV} \\ \text { hyperfine-splitting } & \Delta_{M}=\left(M_{B_{s}^{*}}-M_{B_{s}}\right) & \rightarrow & 49.0(1.5) \mathrm{MeV} \\ \text { ratio } & \frac{M_{1}}{M_{2}}=M_{\text {rest }} / M_{\text {kinetic }} & \rightarrow & 1\end{array}$

- Assuming linearity

$$
Y_{r}=\left[\begin{array}{c}
\bar{M} \\
\Delta_{M} \\
\frac{M_{1}}{M_{2}}
\end{array}\right]_{r}=J^{(3 \times 3)}\left[\begin{array}{c}
m_{0} a \\
c_{P} \\
\zeta
\end{array}\right]_{r}+A^{(3 \times 1)} \quad(r=1, \ldots, 7)
$$

and defining

$$
J=\left[\frac{Y_{3}-Y_{2}}{2 \sigma_{m_{0} a}}, \frac{Y_{5}-Y_{4}}{2 \sigma_{c_{P}}}, \frac{Y_{7}-Y_{6}}{2 \sigma_{\zeta}}\right] \quad A=\left[\begin{array}{c}
\bar{M} \\
\Delta_{M} \\
\frac{M_{1}}{M_{2}}
\end{array}\right]_{1}-J \times\left[\begin{array}{c}
m_{0} a \\
c_{P} \\
\zeta
\end{array}\right]_{1}
$$

- We extract the RHQ parameters and iterate until result is inside uncertainties

$$
\left[\begin{array}{c}
m_{0} a \\
c_{P} \\
\zeta
\end{array}\right]^{\mathrm{RHQ}}=J^{-1} \times\left(\left[\begin{array}{c}
\bar{M} \\
\Delta_{M} \\
\frac{M_{1}}{M_{2}}
\end{array}\right]^{\mathrm{PDG}}-A\right)
$$




\section{Nonperturbatively Tuned Parameters of the RHQ Action (preliminary)}

\begin{tabular}{llll}
\hline$m_{\text {sea }}^{\prime}$ & $m_{0} a$ & $c_{P}$ & $\zeta$ \\
\hline 0.005 & $8.43(7)$ & $5.7(2)$ & $3.11(9)$ \\
0.010 & $8.47(9)$ & $5.8(2)$ & $3.1(2)$ \\
\hline average & $8.45(6)$ & $5.8(1)$ & $3.10(7)$ \\
\hline
\end{tabular}

\begin{tabular}{llll}
\hline$m_{\text {sea }}^{\prime}$ & $m_{0} a$ & $c_{P}$ & $\zeta$ \\
\hline 0.004 & $4.07(6)$ & $3.7(1)$ & $1.86(8)$ \\
0.006 & $3.97(5)$ & $3.5(1)$ & $1.94(6)$ \\
0.008 & $3.95(6)$ & $3.6(1)$ & $1.99(8)$ \\
\hline average & $3.99(3)$ & $3.57(7)$ & $1.93(4)$ \\
\hline
\end{tabular}




\section{Preliminary Predictions for the Heavy-Heavy States}

$\checkmark$ RHQ action describes heavy-light as well as heavy-heavy mesons

- Tuning the parameters in the $B_{s}$ system we can predict bottomonium states and mass splittings
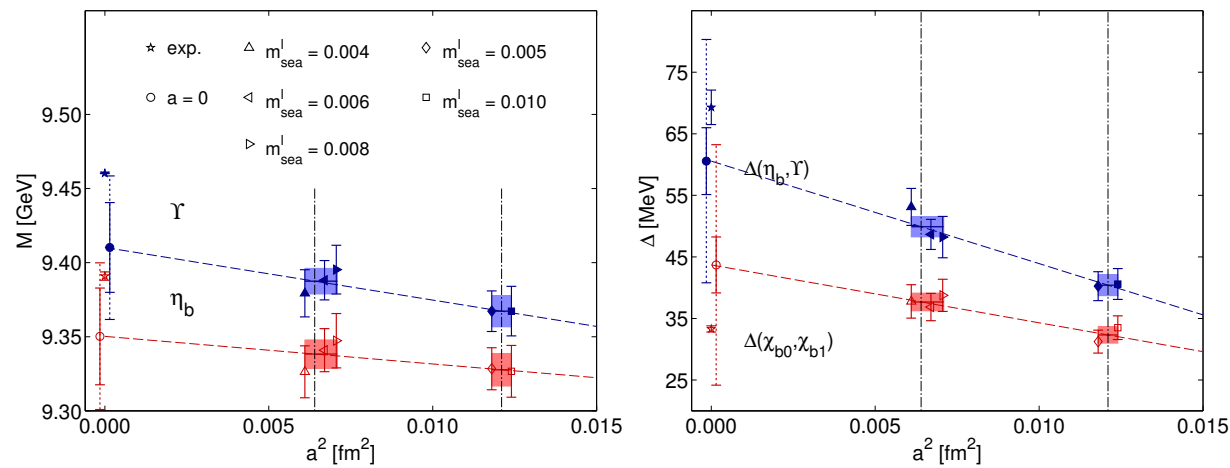

$$
\begin{aligned}
& \eta_{b}=9350(33)(37) \mathrm{MeV} \\
& \Upsilon=9410(30)(38) \mathrm{MeV}
\end{aligned}
$$

$$
\begin{aligned}
& \Delta\left(\eta_{b}, \Upsilon\right)=60(05)(20) \mathrm{MeV} \\
& \Delta\left(\chi_{b 0}, \chi_{b 1}\right)=44(05)(19) \mathrm{MeV}
\end{aligned}
$$



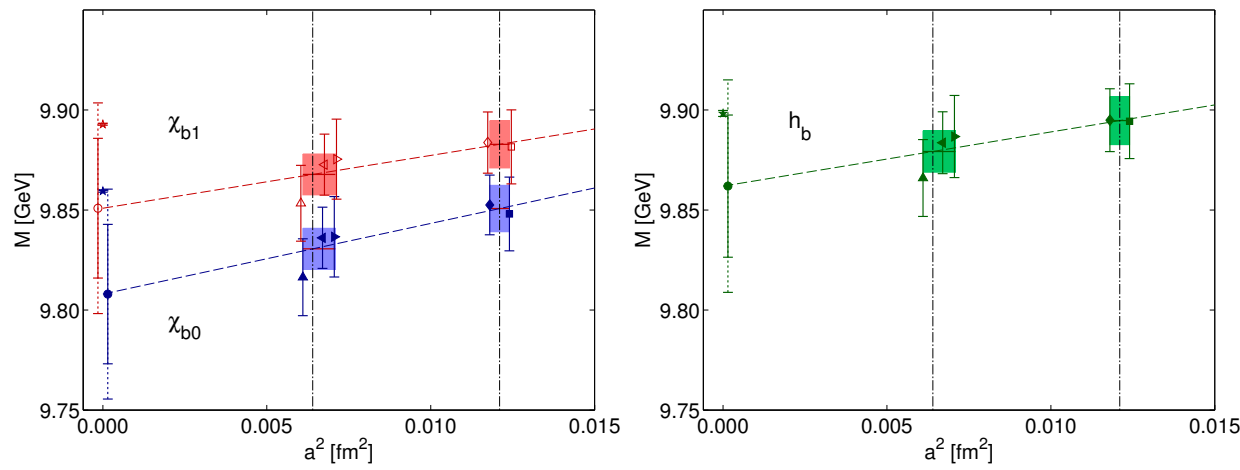

$$
\begin{aligned}
& \chi_{b 0}=9808(35)(39) \mathrm{MeV} \\
& \chi_{b 1}=9851(35)(39) \mathrm{MeV}
\end{aligned}
$$$$
h_{b}=9862(36)(39) \mathrm{MeV}
$$

- Publication on tuning and bottomonium spectroscopy to appear soon 


\section{RHQ Lattice Perturbation Theory [C. Lehner]}

Motivation - Knowing the RHQ parameters nonperturbatively we can compare the outcome with lattice perturbation theory

- Helps to build confidence that lattice perturbation theory is working also in cases where we do not have fully non-perturbative matching (e.g. decay constants, form factors)

- Computation at 1-loop order

- Mean field improved

- Use nonperturbative inputs for $\langle P\rangle,\langle R\rangle,\langle L\rangle$ and $m_{0} a$

- Predict: $c_{P}$ and $\zeta$

- Naive $\alpha_{S}^{2} \sim 5 \%$ power-counting estimate 

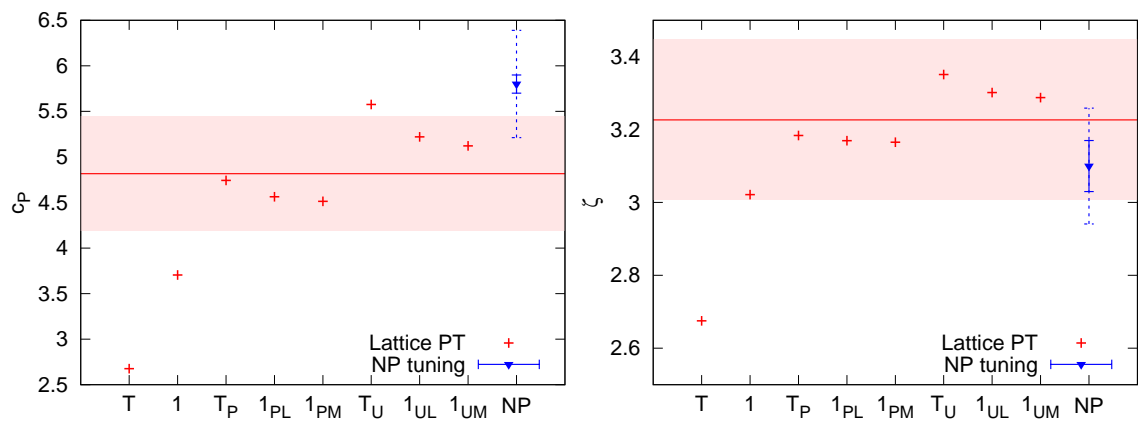

- Central values: average of one-loop mean-field improved values computed with $u_{0}$ obtained from the plaquette and from the spatial Landau link

- Error on perturbative $c_{P}$ : difference between mean field methods dominates

- Error on perturbative $\zeta$ : naive power-counting dominates

- Nonperturbative values include systematic errors from discretization errors in quantities used for tuning

- Agreement within errors $\Rightarrow$ MF-improved LPT can be trusted in situations for which NP matching factors are not available 


\section{$B^{0}-\overline{B^{0}}$ Mixing Matrix Element Calculation}

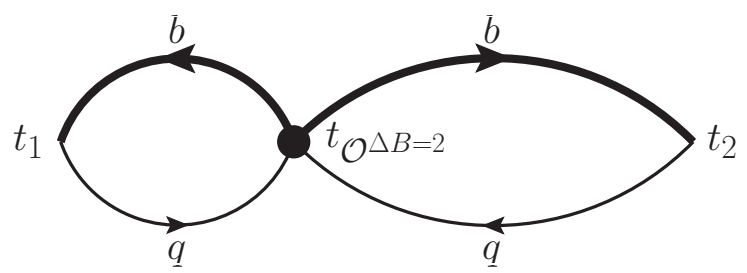

- Location of four-quark operator is fixed

- Location of $B$-mesons is varied over all possible time slices

- Need: one point-source light quark and one point-source heavy quark originating from operator location

- Propagators can be used for $B$ - and $\bar{B}$-meson

- Project out zero-momentum component using a Gaussian sink

- Optimize Gaussian wavefunction to minimize excited-state contamination in $B$-meson 2-point correlation function 


\section{Preliminary $B$ - and $B^{*}$-meson mass}

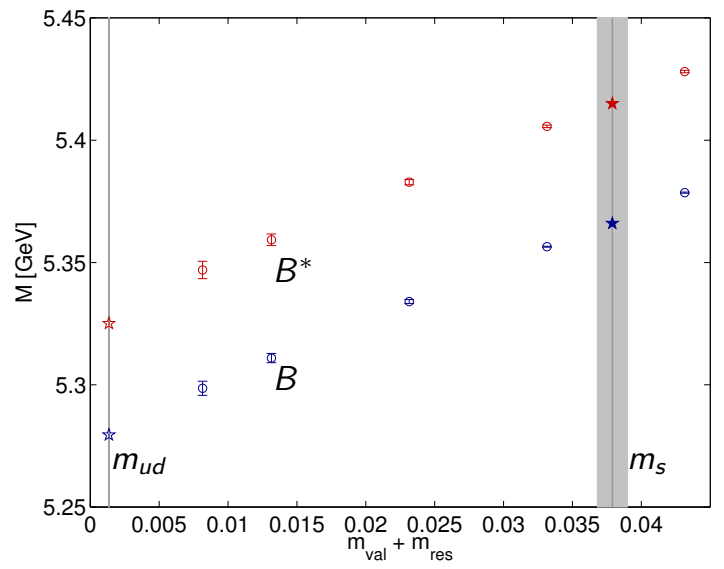

- $L=24, m_{\text {sea }}^{\prime}=0.005, N=1636$, only statistical uncertainty 


\section{Mostly Nonperturbative Renormalization}

For $f_{B}, f_{B_{s}}$ and $B \rightarrow \pi$ we plan to compute mostly non-perturbative renormalization factors á la [El Khadra et al. 2001]

$$
\varrho^{b l}=\frac{Z_{V}^{b l}}{\sqrt{Z_{V}^{b b} Z_{V}^{\prime \prime}}}
$$

- Compute $Z_{V}^{\prime \prime}$ and $Z_{V}^{b b}$ non-perturbatively and only $\varrho^{b /}$ perturbatively

- Enhanced convergence of perturbative serious of $\varrho^{b l}$ w.r.t. $Z_{V}^{b l}$ because tadpole diagrams cancel in the ratio

- Bulk of the renormalization is due to flavor conserving factor $\sqrt{Z_{v}^{\prime \prime} Z_{v}^{b b}} \sim 3$

- $\varrho^{b l}$ is expected to be of $\mathcal{O}(1)$; receiving only small corrections

- For domain-wall fermions $Z_{A}=Z_{V}+\mathcal{O}\left(m_{\text {res }}\right)$ i.e. we know $Z_{V}^{\prime \prime}$ [Y. Aoki et al. 2011]

- Mostly nonperturbative renormalization not yet computed for $B^{0}-\overline{B^{0}}$ mixing 


\section{$B$-meson Decay Constant Calculation}

- Re-use: point-source light quark and generate Gaussian smeared-source heavy quark

- Final result will use mostly nonperturbative renormalization
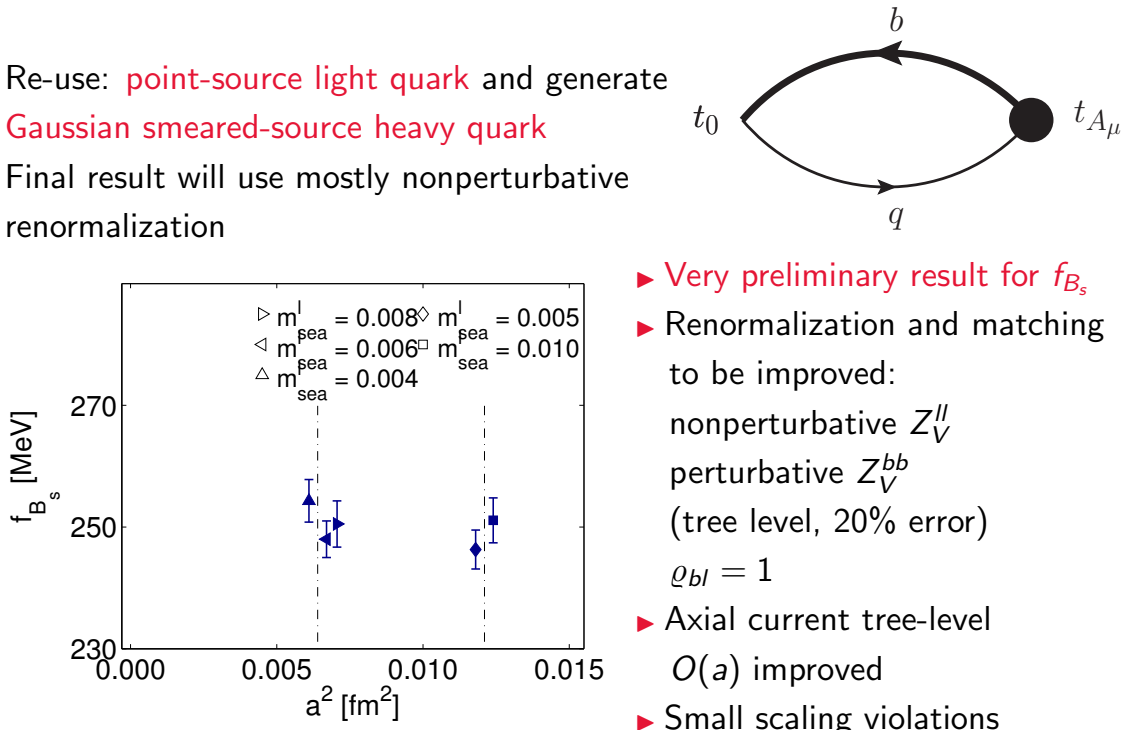

- Very preliminary result for $f_{B_{s}}$

- Renormalization and matching to be improved: nonperturbative $Z_{V}^{\prime \prime}$ perturbative $Z_{V}^{b b}$ (tree level, 20\% error) $\varrho_{b l}=1$

- Axial current tree-level $O(a)$ improved

- Small scaling violations 


\section{$B \rightarrow \pi l \nu$ form factor [T. Kawanai]}

- Compute matrix element of the $b \rightarrow u$ vector current between $B$-meson and pion

- Fix location of pion at $t_{0}$ and $B$ meson at $T-t_{\text {sink }}-t_{0}$

- Vary operator location $t_{V_{\mu}}$ in that range

- $B$-meson is at rest, inject momentum on pion side

- Using partially quenched daughter quark-masses should help to better resolve quark-mass dependence and pion-energy dependence

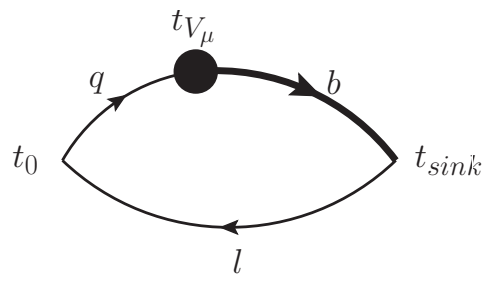




\section{Conclusion}

- We have completed tuning the parameters of the RHQ action for $b$-quarks, and find good agreement between our predictions for bottomonium masses and fine splittings with experiment.

- Given this success, we are now using this method for B-meson quantities such as decay constants and form factors, and expect to obtain errors competitive with other groups.

- The RHQ action can also be used for charm quarks, and Hao Peng is currently performing the necessary parameter tuning.

- We should have results for decay constants, mixing parameters, and form factors within the next year, and maybe sooner! 


\section{Exploring Many Flavor QCD with Wilson Fermion}

\section{N. Yamada (KEK/GUAS) \\ based on collaboration with}

M. Hayakawa (Nagoya), K.-I. Ishikawa (Hiroshima),

Y. Iwasaki (KEK/Tsukuba), Y. Osaki(Hiroshima),

S. Takeda (Kanazawa), S. Uno(Nagoya), T. Yoshie (Tsukuba) 


\section{Conformal Window}

Phase of a theory with $\mathrm{N}_{\mathrm{F}}$ degenerate massless flavors at $\mathrm{T}=\mathbf{0}$

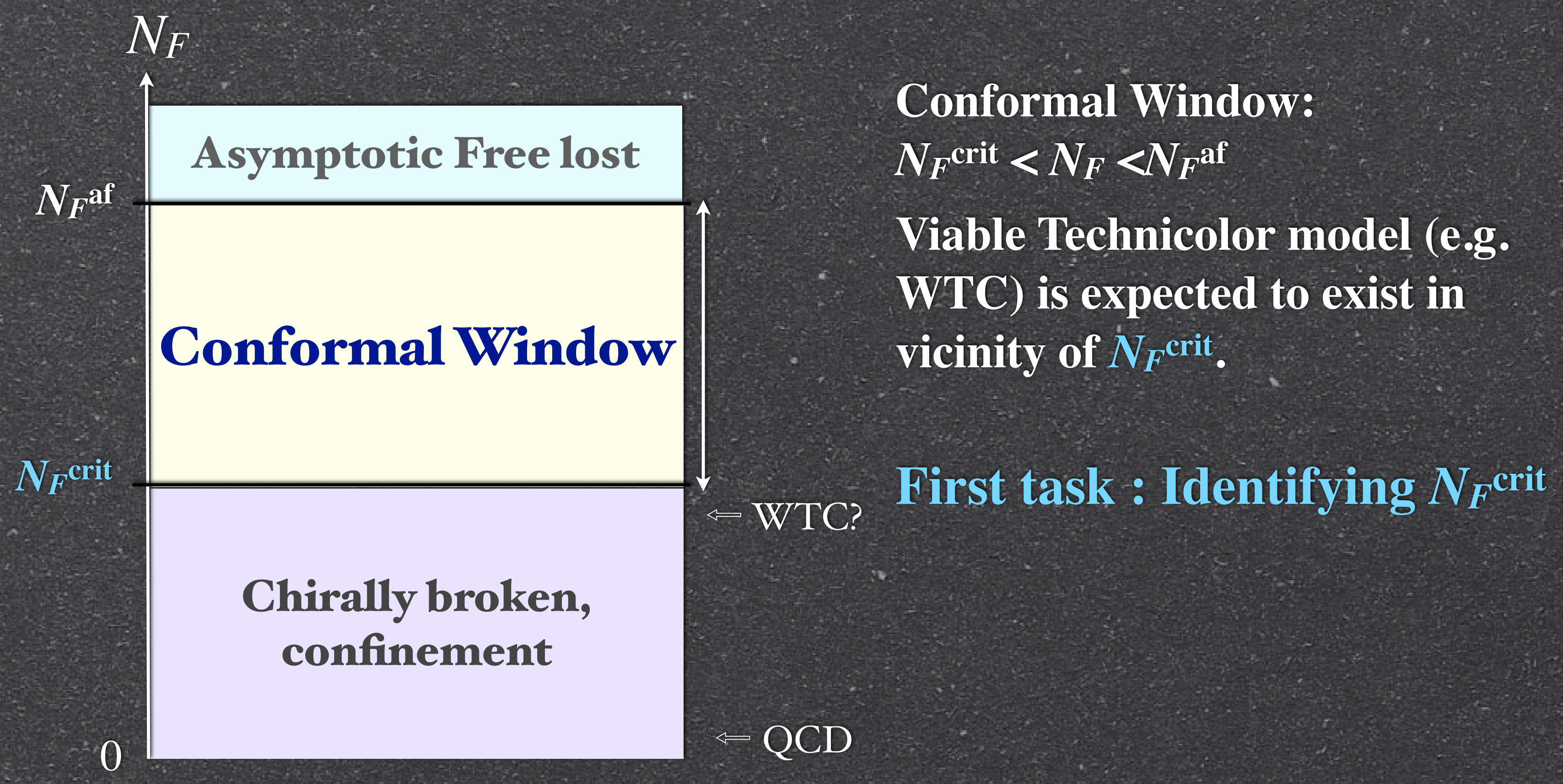

Phase diagram@T=0 
Disadvantages:

$\checkmark \mathrm{O}(\mathrm{a})$ scaling violation

$\checkmark$ Fine-tuning,

$\checkmark \ldots$
Advantages:

$\checkmark$ Simple, tractable and well understood

$\checkmark$ Able to study arbitrary $N_{F}$ without any subtlety

$\checkmark$ Independent check to KS (or other) results 


\section{Contents}

There are several approaches to identify $\mathrm{N}_{\mathrm{F}}{ }^{\text {crit }}$.

Focusing on SU(3) gauge theory, we are performing the following studies:

1. Running coupling and anomalous dimension in 10-flavor QCD. IRFP?

2. Finite temperature study of Many Flavor QCD $\left(\mathrm{N}_{\mathrm{F}}=6-10\right)$

- Strategy

- Future prospect 


\section{$\alpha(\mu)$ and $\gamma_{m}$ in \\ 10-flavor QCD \\ M. Hayakawa, K.-I. Ishikawa, Y. Osaki, S. Takeda, S. Uno, NY}




\section{$g_{\mathrm{SF}^{2}}(\mu)$ in 10 -flavor QCD}

Hayakawa, Ishikawa, Osaki, Takeda, Uno, NY, PRD(20II) and work in progress

\section{Preliminary}

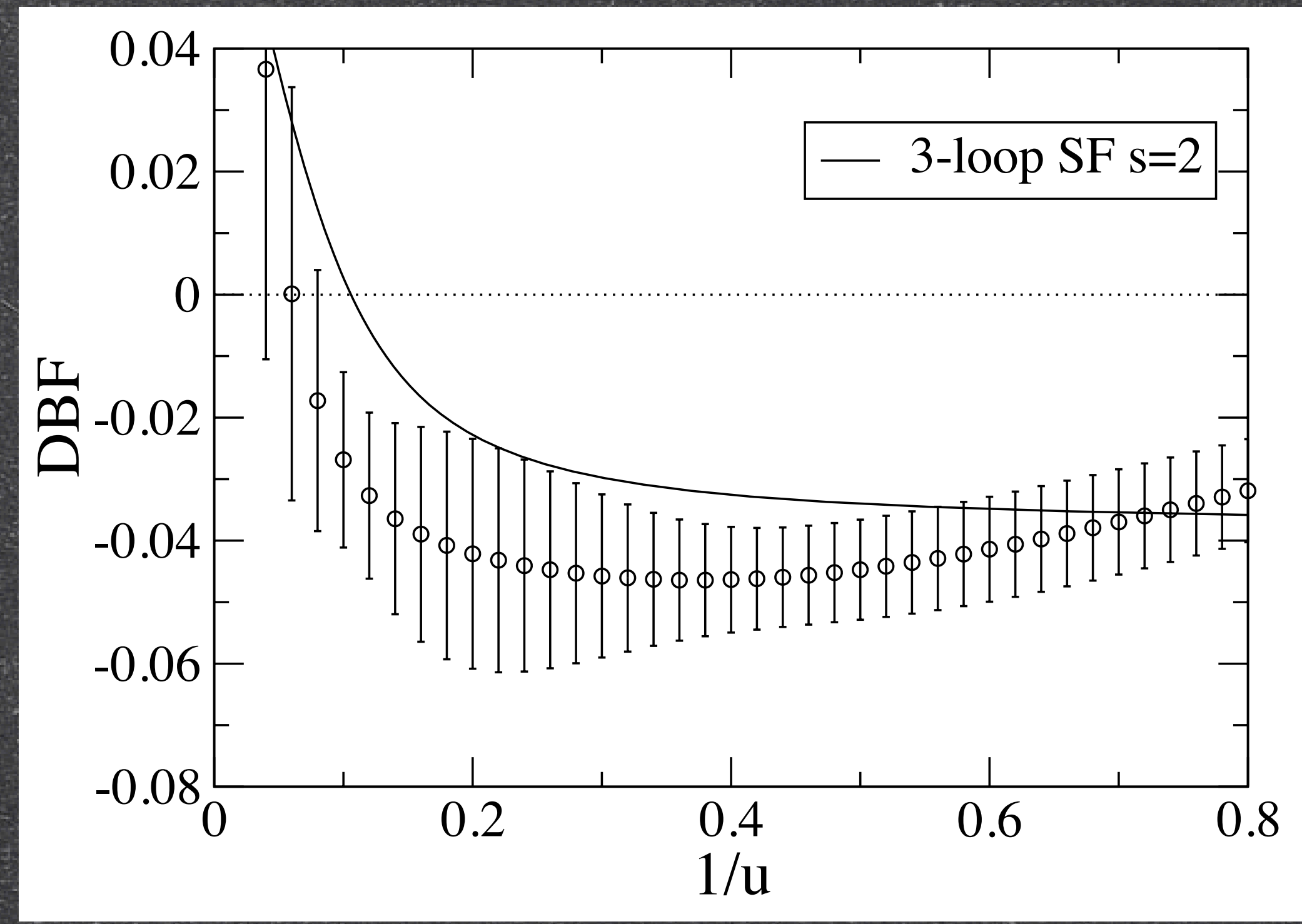

$$
B^{\mathrm{SF}}(u, s)=\frac{1}{g_{\mathrm{SF}}^{2}(u, s)}-\frac{1}{u}
$$

$\mathrm{DBF}=0 \Rightarrow \mathrm{IRFP}$

$\mathrm{g}^{2} \mathrm{FP} \geq 12$

Continuum extrapolation with two data points.

In order to have more confidence, large V calculation is on-going. 


\section{$g_{\mathrm{SF}^{2}}(\mu)$ in 10-flavor QCD}

Hayakawa, Ishikawa, Osaki, Takeda, Uno, NY, PRD(20II) and work in progress

\section{Preliminary}

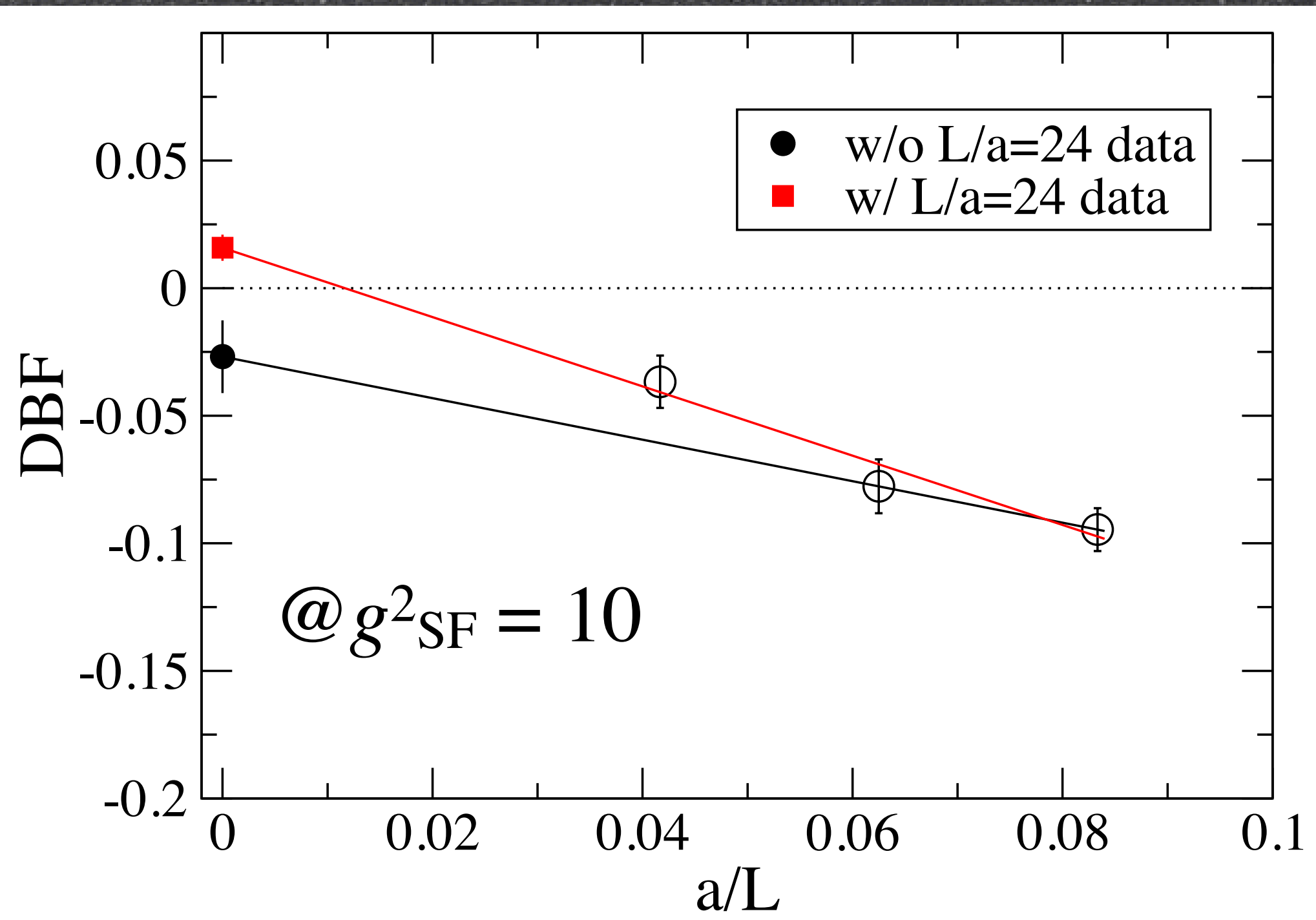

Adding large $\mathrm{V}$ data, the continuum limit shits upward.

$\mathrm{g}^{2} \mathrm{FP} \geq 12 \Rightarrow \mathrm{g}^{2} \mathrm{FP} \sim 10$ 7

More confident on IRFP 


\section{$\gamma_{m}$ of 10 -flavor QCD}

Hayakawa, Ishikawa, Osaki, Takeda, Uno, N.Y., work in progress

\section{Preliminary}

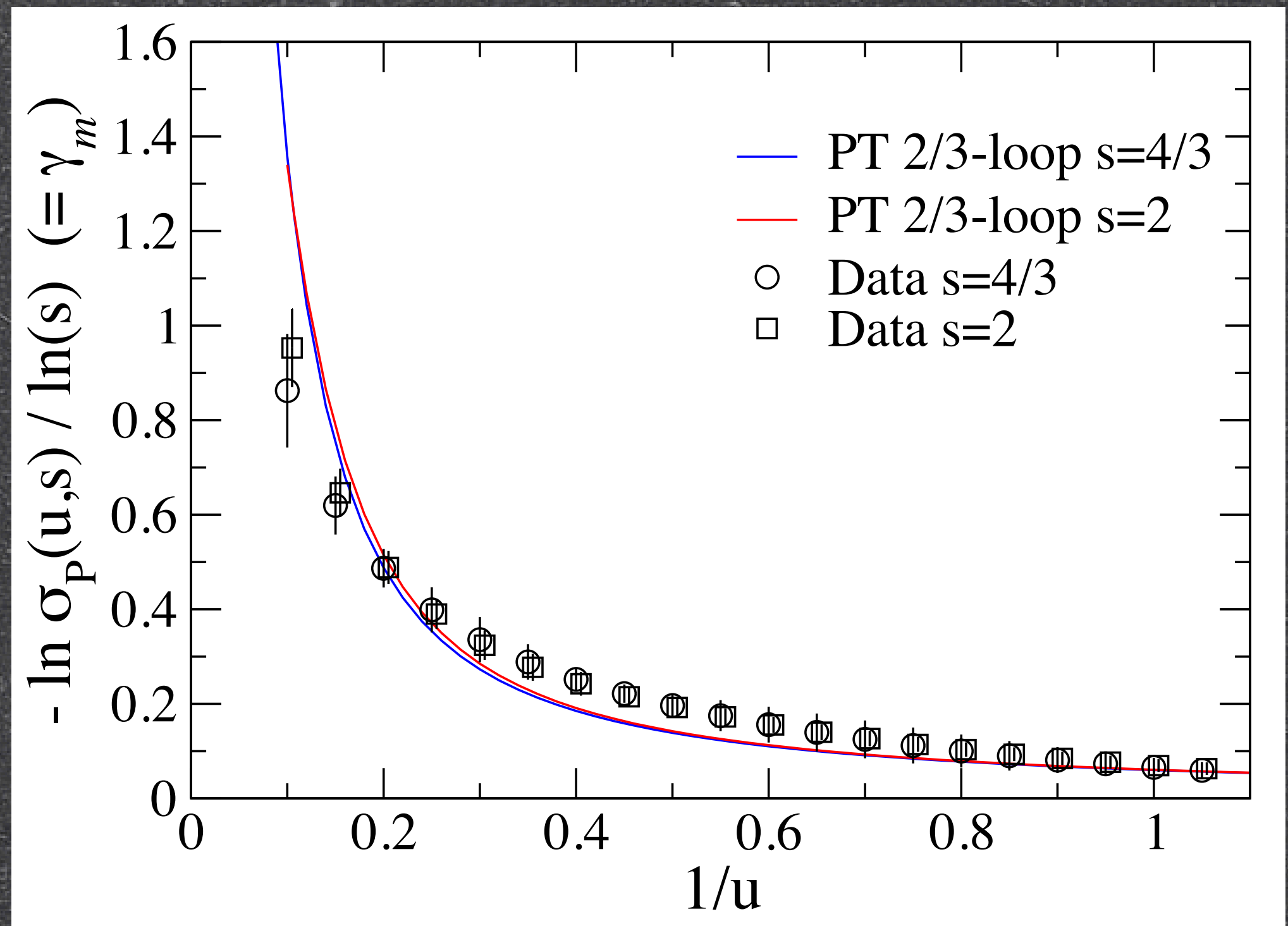

Two different step scaling factors give consistent result.

Assuming $g_{\mathrm{FP}^{2}} \sim 10, \gamma_{m} \sim 1$ !

10-flavor QCD appears to be in CW $\left(\mathrm{N}_{F}\right.$ crit $\left.<10\right)$ and have $\gamma_{m} \sim O(1)$. 
Finite temperature study of Many Flavor QCD WMF Collaboration: M. Hayakawa, K.-I. Ishikawa, Y. Iwasaki, S. Takeda, T. Yoshie, NY 


\section{Phase diagram of Wilson fermion for $\mathbf{N}_{\mathbf{F}}<\mathbf{N}_{\mathbf{F}}{ }^{\text {crit }}$ Iwasaki et al. (9I,04)}

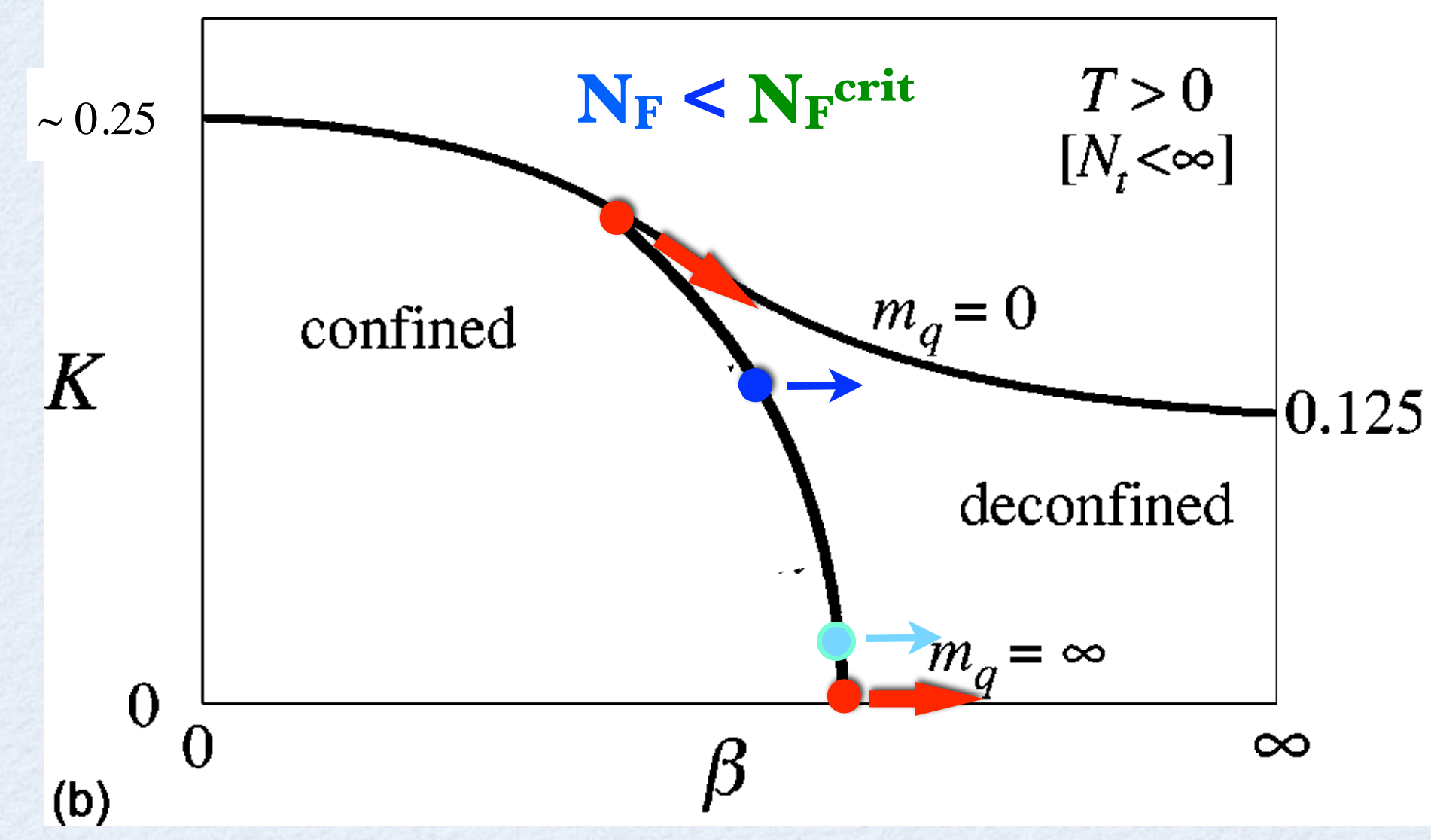

If the theory is confining, the transition line move to the right as $T$ decreases (or $V$ increases). 


\section{Phase diagram of Wilson fermion for $\mathbf{N}_{\mathbf{F}}<\mathbf{N}_{\mathbf{F}}{ }^{\text {crit }}$ Iwasaki et al. (9I,04)}

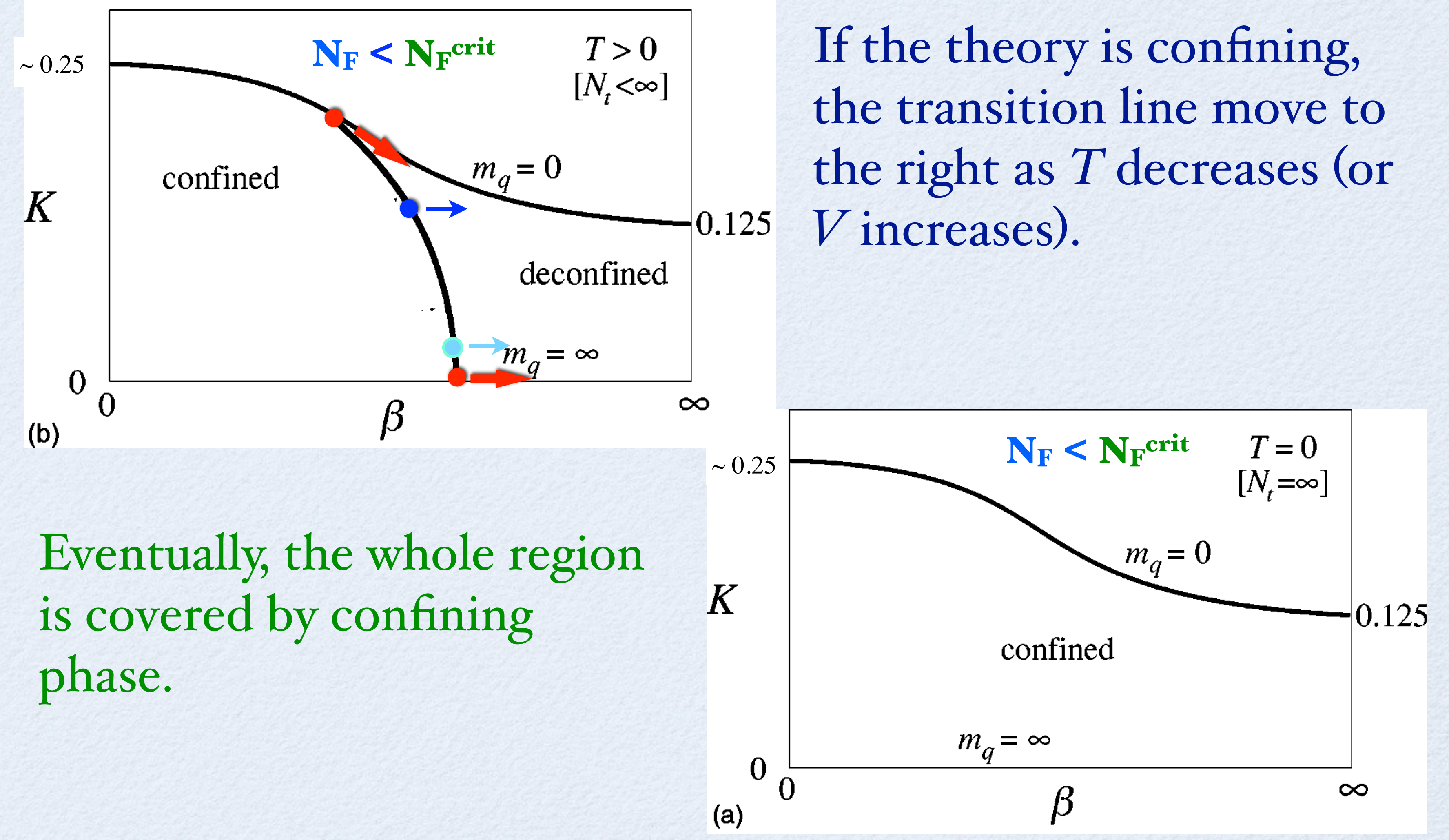




\section{Phase diagram of Wilson fermion with $\mathbf{N F}_{\mathrm{F}}^{\text {crit }}<\mathbf{N}_{\mathrm{F}}<\mathbf{N F}_{\mathrm{F}}^{\text {af }}$ (speculation)}

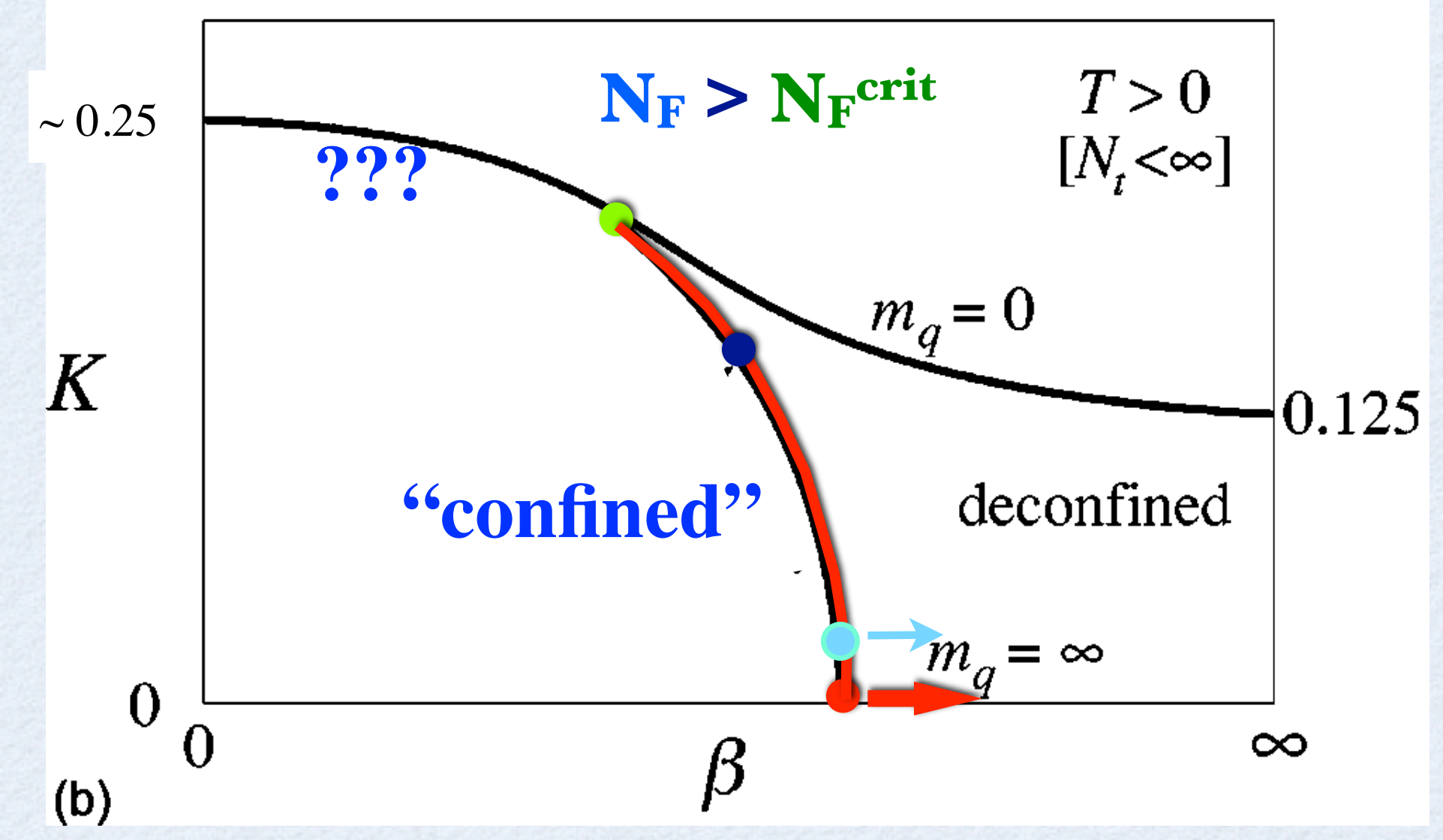

The one end of the transition line at $m_{q}=\infty$ moves to the right with $\mathrm{V}$ as before, while the other end at $m_{q}=0$ won't. 


\section{Phase diagram of Wilson fermion with $\mathbf{N F}_{\mathrm{F}}$ crit $<\mathbf{N}_{\mathrm{F}}<\mathbf{N F}_{\mathrm{F}}^{\text {af }}$ (speculation)}

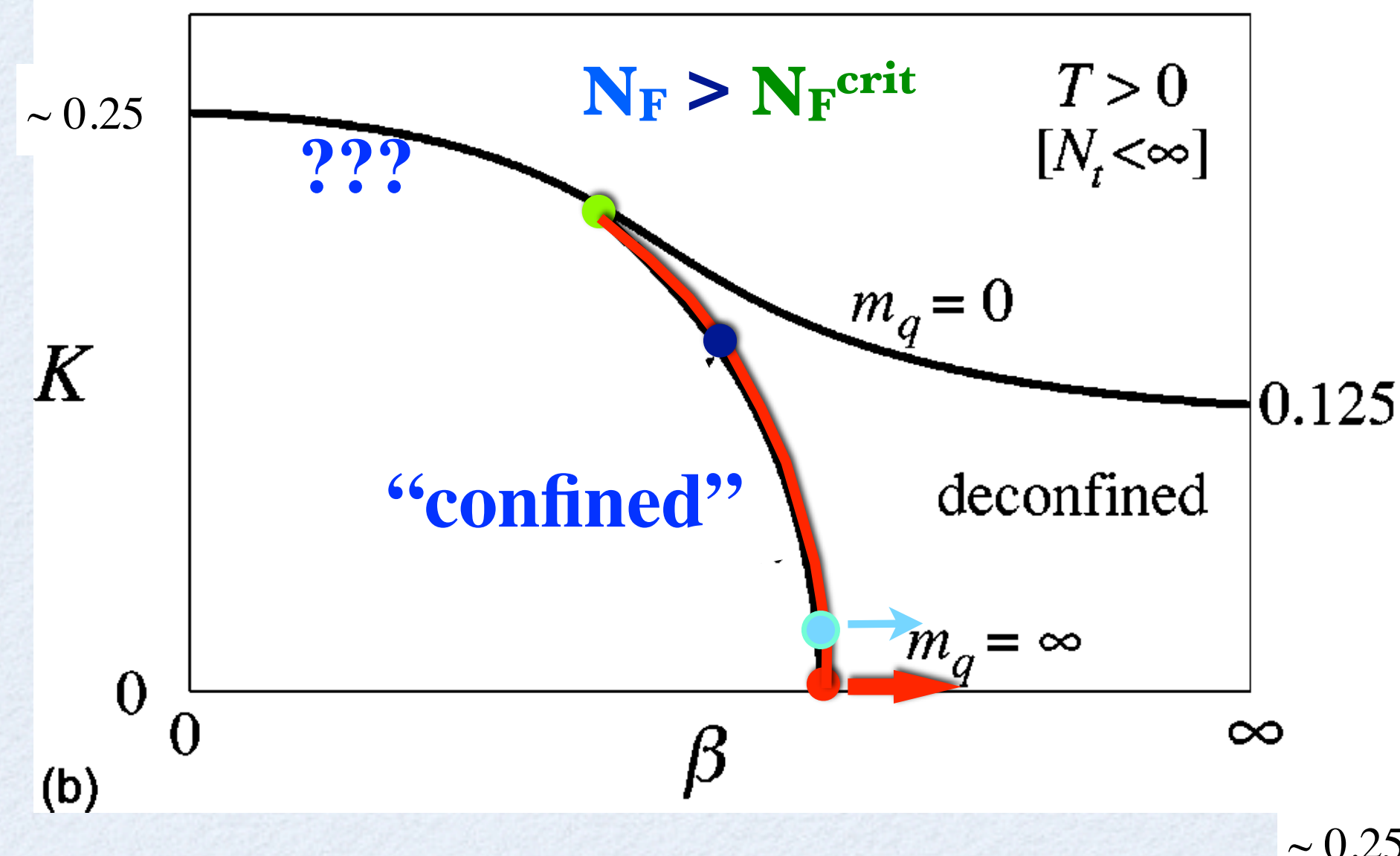

In the large $V$ limit, the relevant region is covered by "confined" $K$ phase except for the chiral limit In "confined", $\Lambda_{\mathrm{TC}}$ depends or $m_{q}$ and vanishes in the chiral limi1
The one end of the transition line at $m_{q}=\infty$ moves to the right with $\mathrm{V}$ as before, while the other end at $m_{q}=0$ won't.

$$
m_{q}=\infty
$$

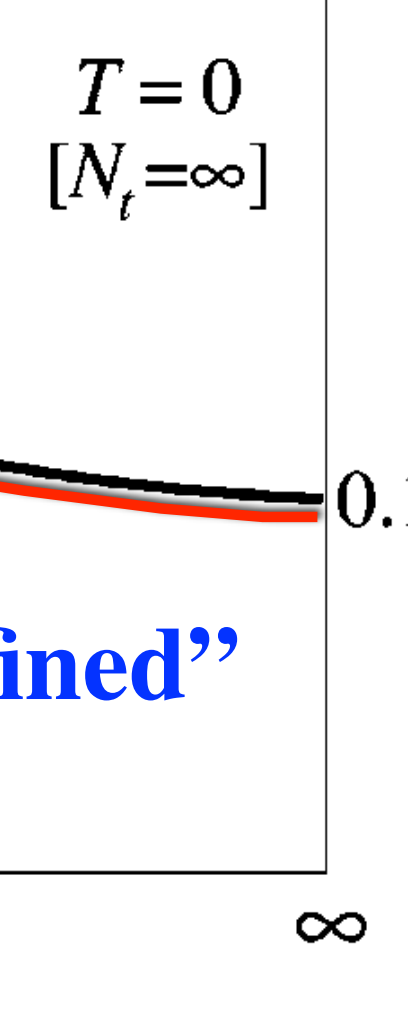




\section{General Strategy}

$\checkmark$ Find the critical endpoint around $\boldsymbol{m}_{q}=\mathbf{0}$.

$\checkmark$ Keep monitoring it while changing $\mathrm{V}$ to see whether it moves to the right with $\mathrm{N}_{\mathrm{T}}$ or $\mathrm{V}$.

$\checkmark$ If the endpoint moves to the right as in QCD, the theory is outside of Conformal Window. 


\section{This work $\left(\mathbf{N}_{\mathbf{F}}=\mathbf{1 0}\right)$}

$\mathrm{m}_{\mathrm{Q}}$ and $\beta$ dependence of Polyakov loop@ $\mathrm{N}_{\mathrm{F}}=10$

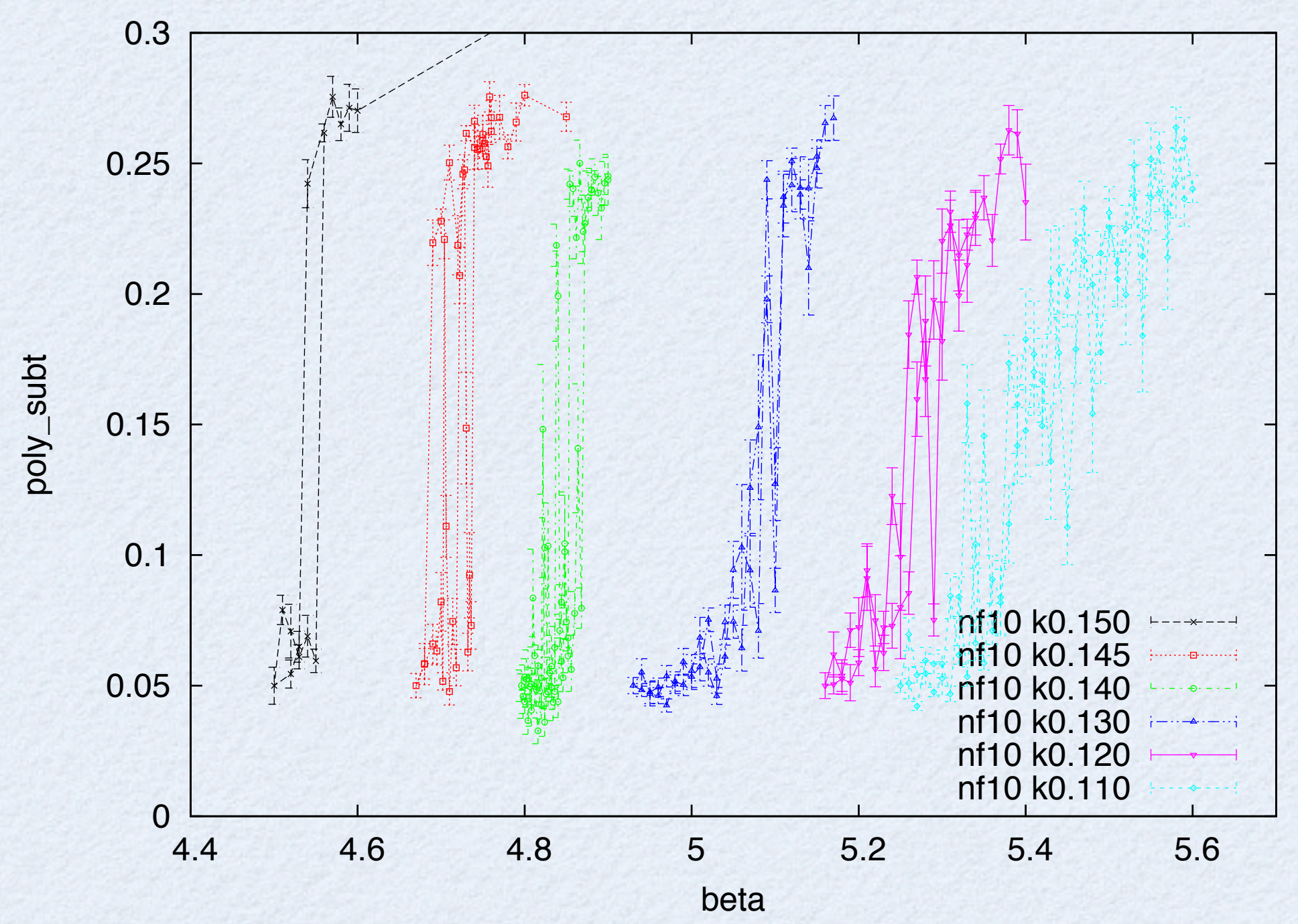

This work using Wilson Fermion on $V=4^{3} \times 4$.

1st oder weakens as going to small kappa. 


\section{Previous Results $\left(\mathbf{N}_{\mathrm{F}}=\mathbf{1 0}\right)$}

Fukugita, Ohta, Ukawa (88)

mQ and $\beta$ dependence of Polyakov loop@ $\mathrm{N}_{\mathrm{F}}=10$

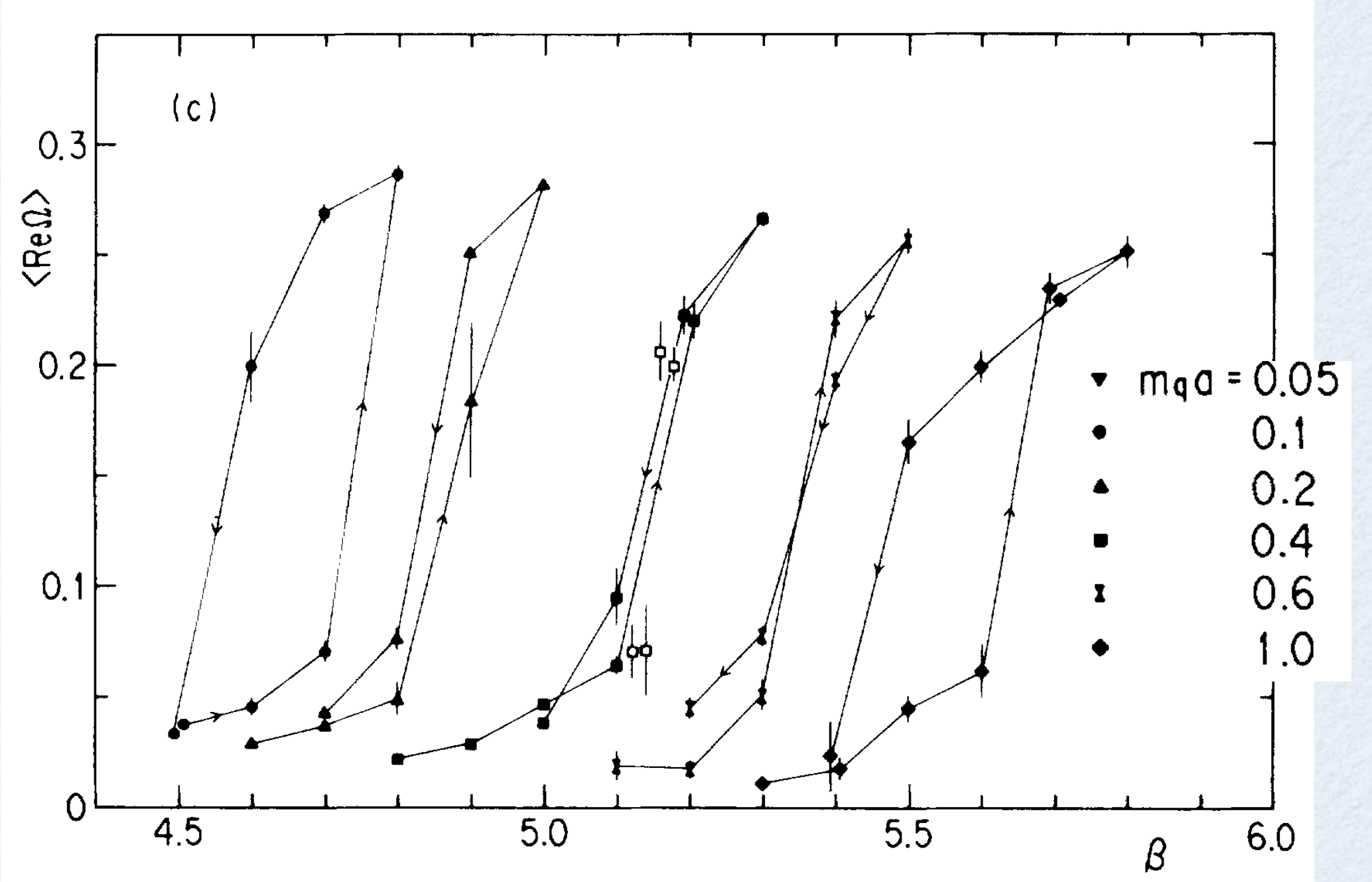

$\mathrm{KS}, \mathrm{V}=8^{3} \times 4$

Observation:

Transitions are 1st order over the entire range of fermion mass at $\mathrm{N}_{\mathrm{F}}=\mathbf{1 0}$. No end point. 


\section{$V=8^{3} \times 8, N_{F}=6-10$}

Preliminary Results for $\mathrm{N}_{\mathrm{F}}=6,7,8,10$
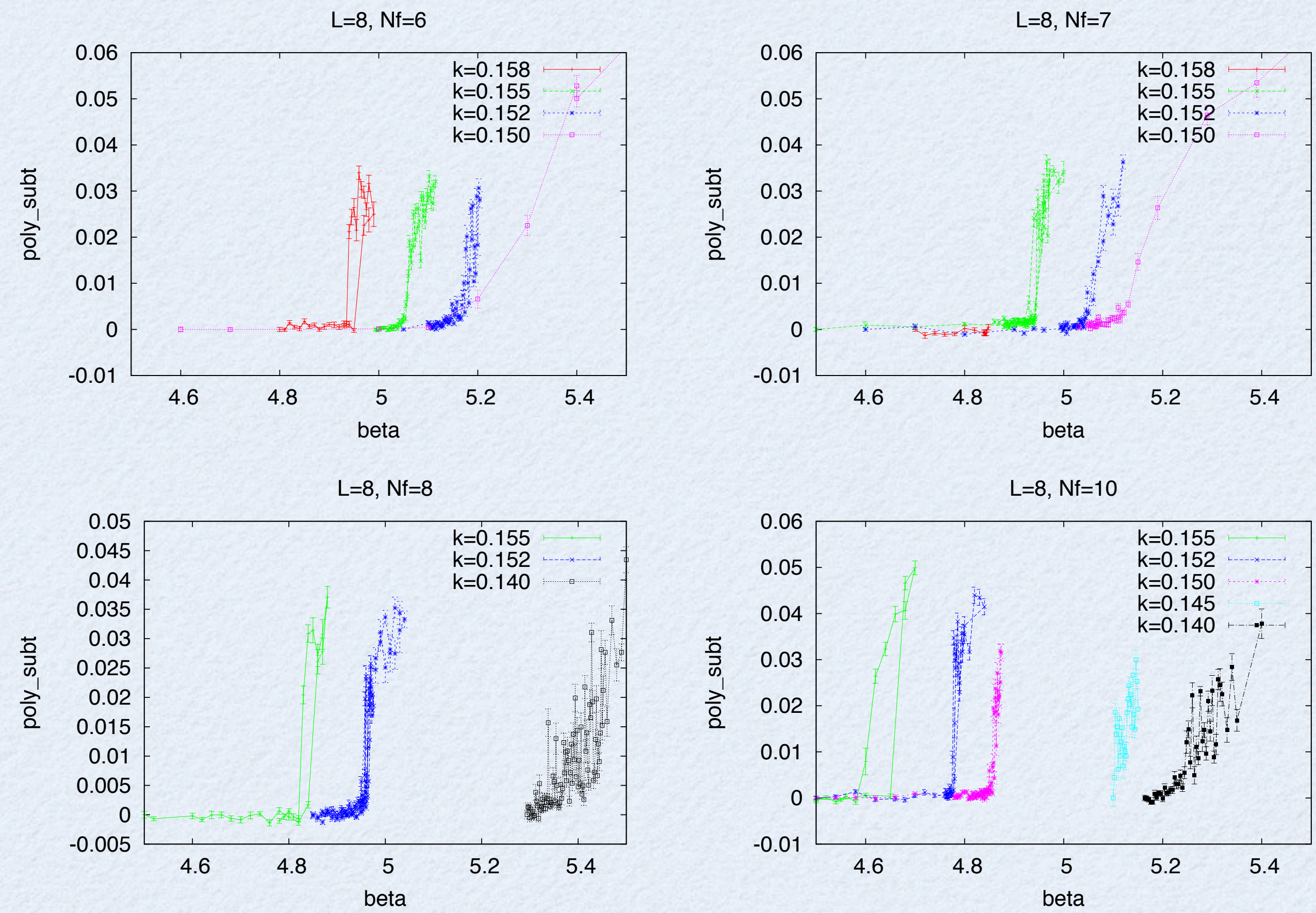

1st order at large kappa weakens as going to small kappa.

Calc. on $V=16^{4}$ is on going. 


\section{(Far) Future plan}

(strongly depends on what LHC@CERN observes.)

$\checkmark$ In realistic TC model, only two of $\mathrm{N}_{\mathrm{F}}$ flavors must be exact massless while the other $\mathrm{N}_{\mathrm{F}}-2$ flavors shouldn't be so.

$\checkmark$ Go to $2+\left(\mathrm{N}_{\mathrm{F}}-2\right) \mathrm{QCD}$

$\checkmark$ Nice to discuss such a theory on the basis of Columbia plot 


\section{Columbia plot}

Brown, Butler, Chen, Christ, Dong, Schaffer, Unger, and Vaccarino (90), N.H. Christ, Z. Dong (92) and N.H. Christ(92)

\section{Kanaya, Lattice 2010}
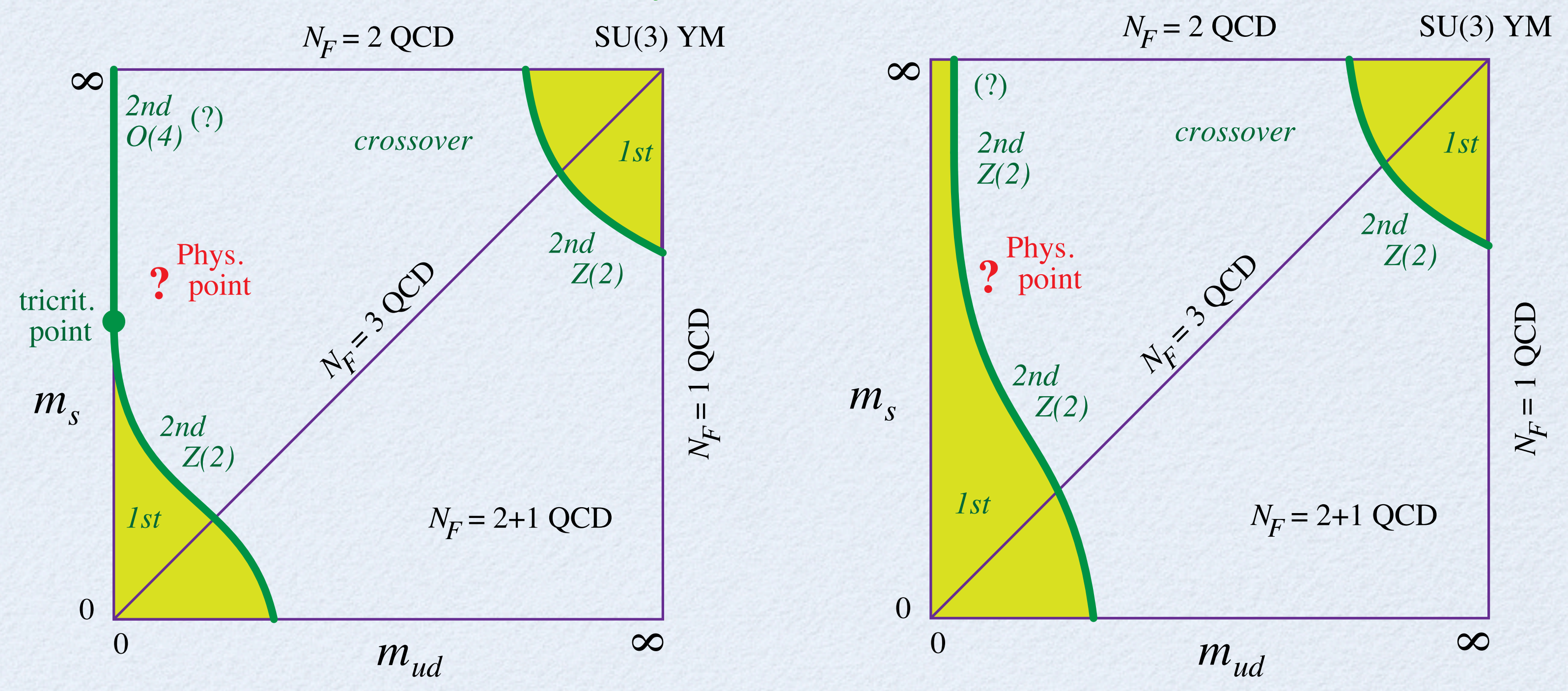

Recent lattice results seems to favor the left.

How does this plot for many flavor QCD look like? 


\section{$\mathbf{N}_{\mathrm{F}}=4\left(<\mathrm{N}_{\mathrm{F}}^{\text {crit }}\right)$}

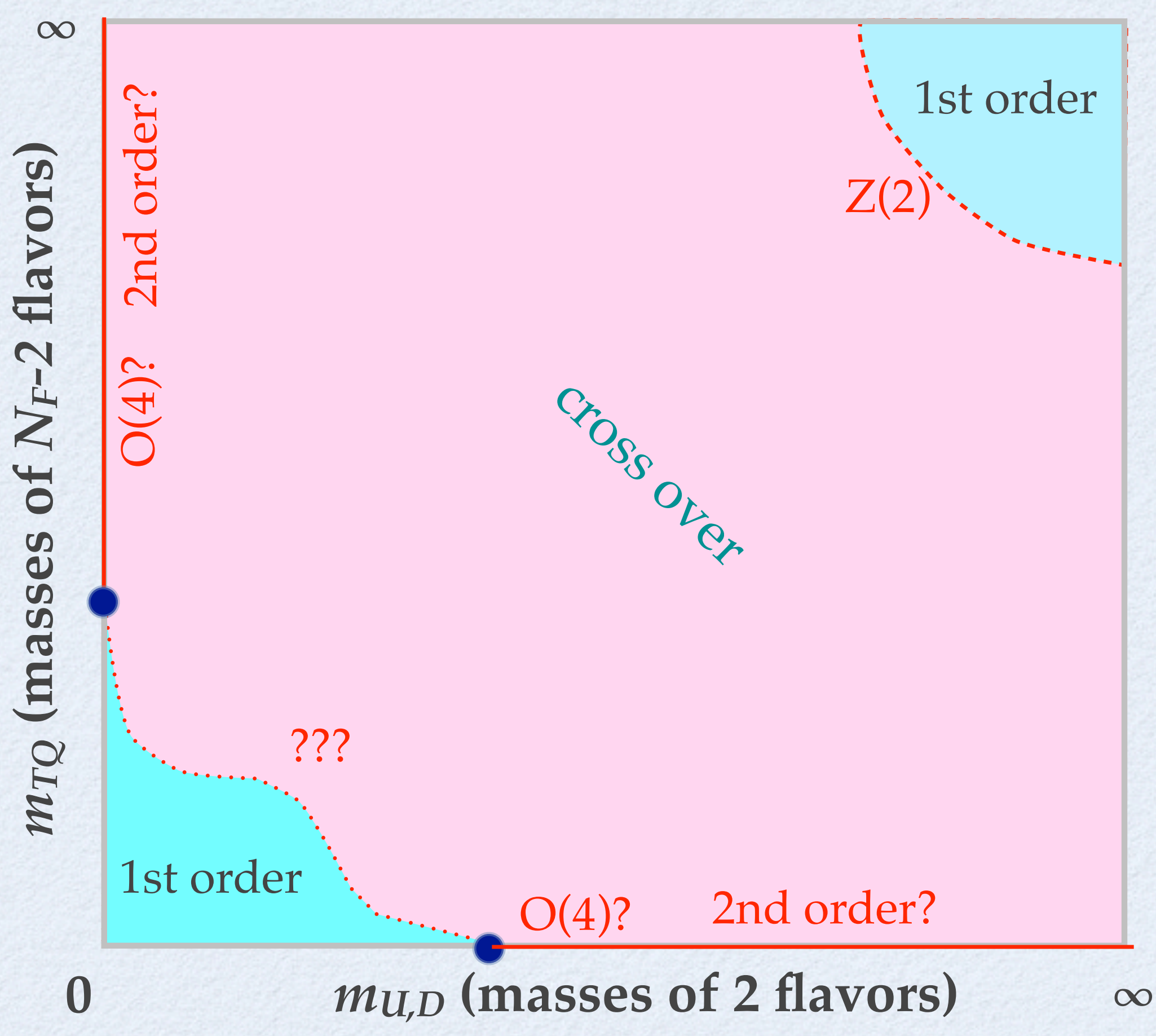

$\mathrm{N}_{\mathrm{F}}{ }^{\text {crit }}>4$ is assumed.

Symmetric phase diagram

Probably running is not slow enough.

Less interesting. 


\section{$5 \leqq N_{F}<N_{F}{ }^{c r i t}$}

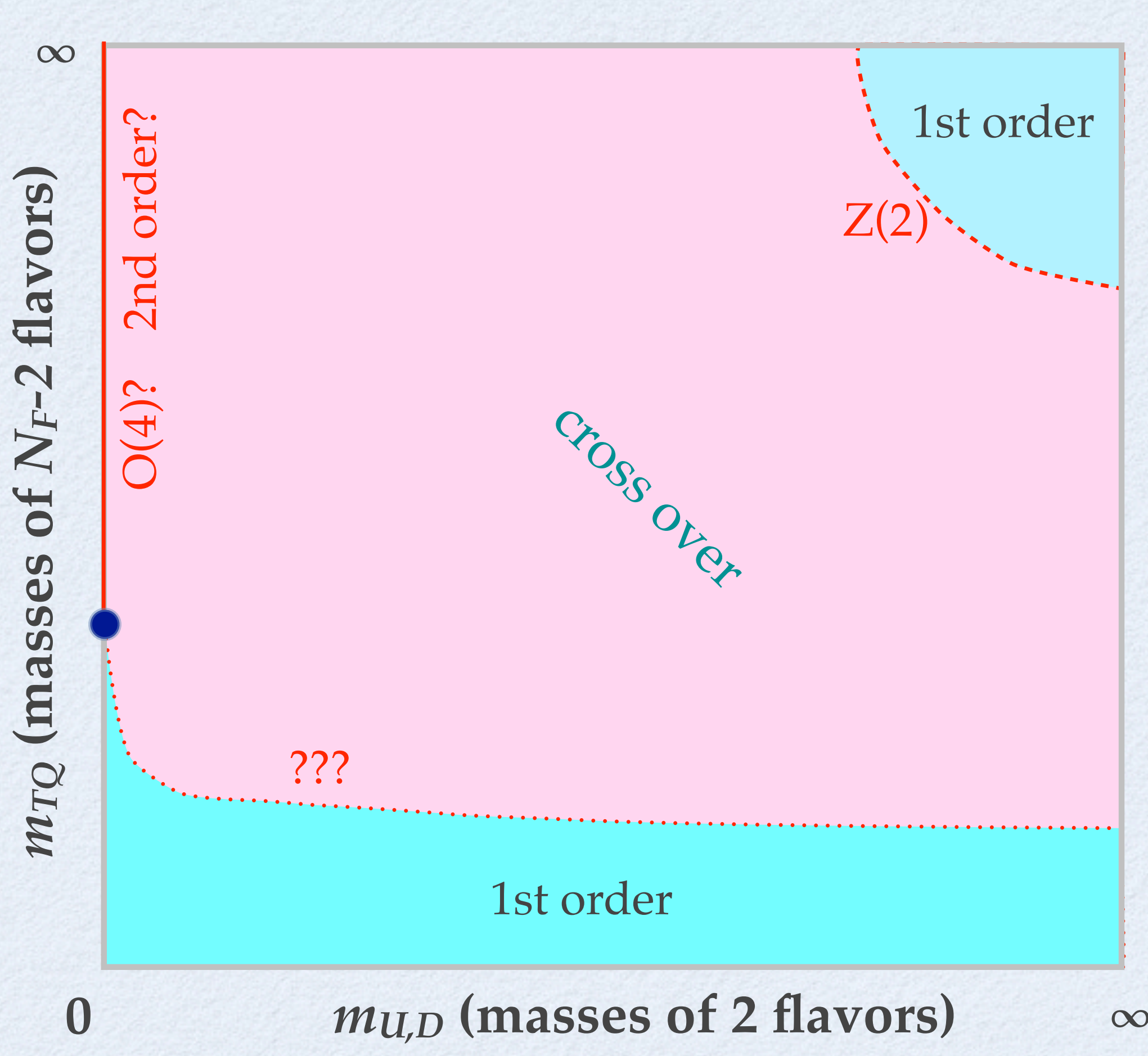

$N_{F}{ }^{\text {crit }}>5$ is assumed.

1 st order persists to $\mathrm{m}_{U, D}=\infty$ for small $\mathrm{m}_{T Q}$.

Slow running and large $\gamma_{m}$ may be expected at some $N_{F}$. 


\section{$5 \leqq N_{F}<N_{F}{ }^{c r i t}$}




\section{$\mathbf{N}_{\mathbf{F}}-2<\mathbf{N}_{\mathbf{F}}^{\text {crit }}<\mathbf{N}_{\mathbf{F}}($ Speculation)}

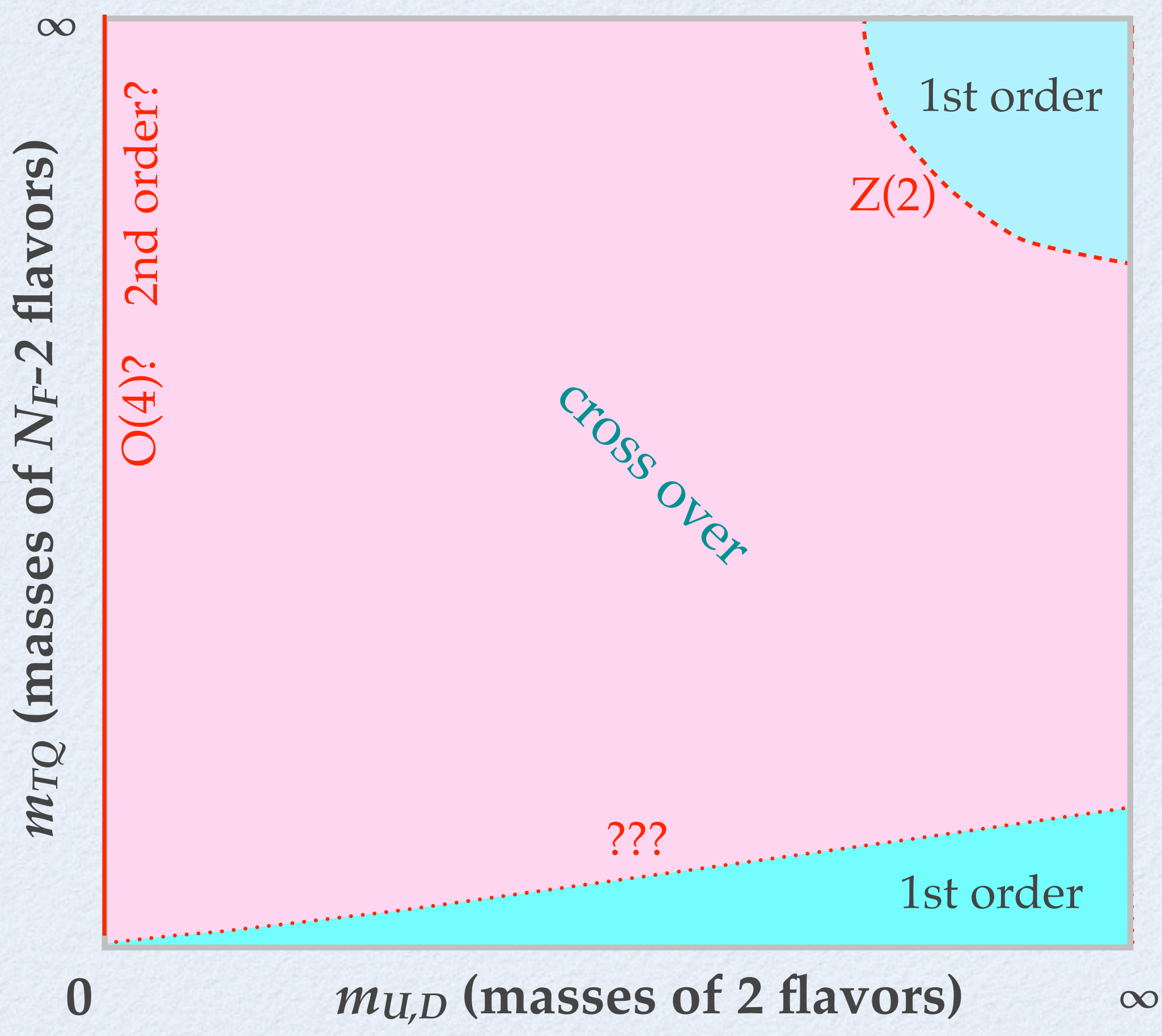

If this is the case,

EW Baryongenesis within TC seems to difficult. 


\section{$\mathbf{N}_{\mathbf{F}}{ }^{\text {crit }}<\mathbf{N}_{\mathrm{F}}-2<\mathbf{N}_{\mathrm{F}}($ Speculation $)$}

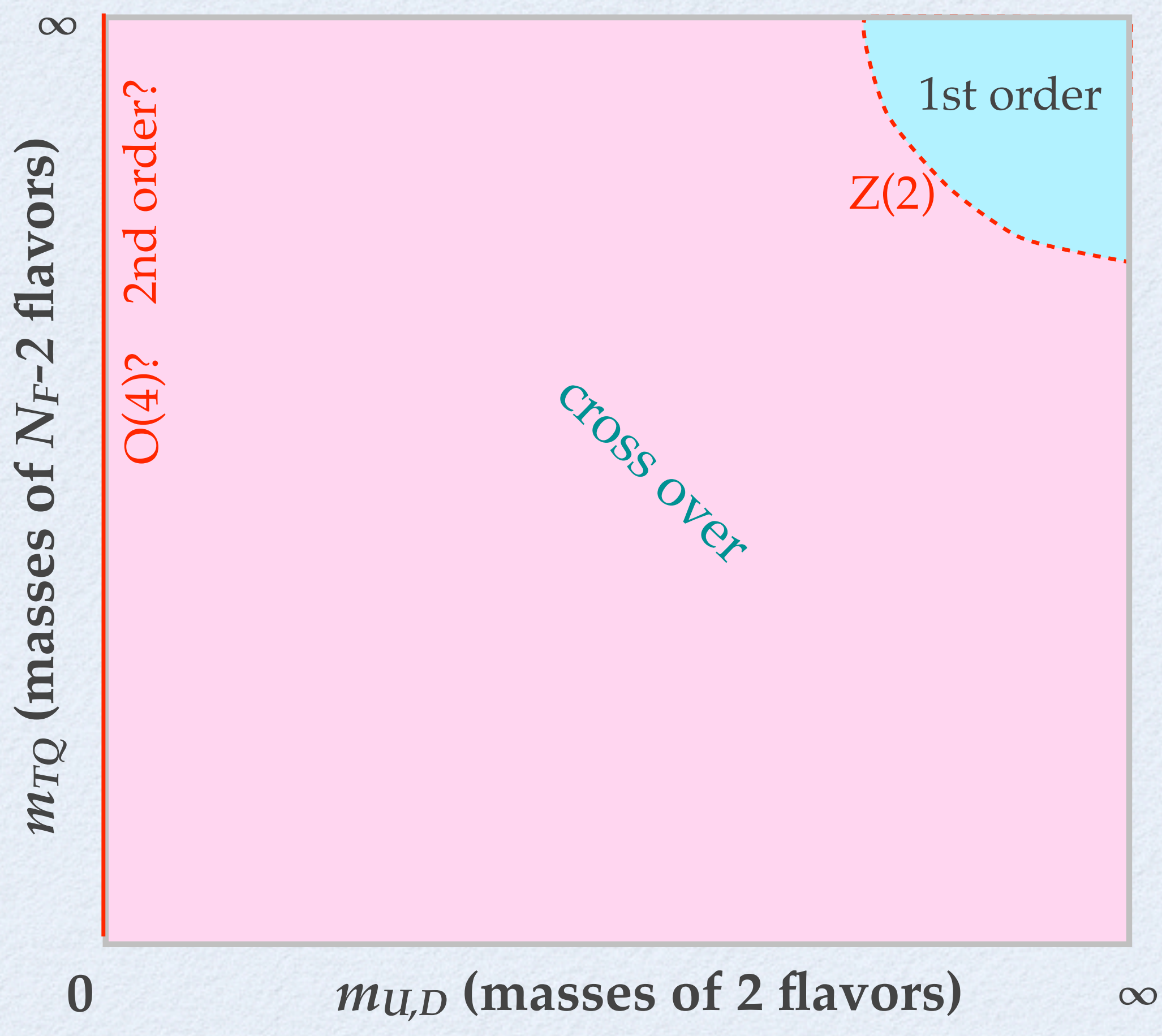

If this is the case, not interesting. 


\section{Summary}

$\checkmark$ Important to have several complementary approaches in the search for Conformal Window.

$\checkmark$ We employ Wilson fermion to study the properties of Many Flavor QCD.

$\checkmark$ Important to know Wilson Phase diagram when interpreting spectroscopy results.

$\checkmark$ Establishing Columbia plot for Many Flavor QCD clarifies phenomenologically interesting region. 


\section{Finite Volume effect}

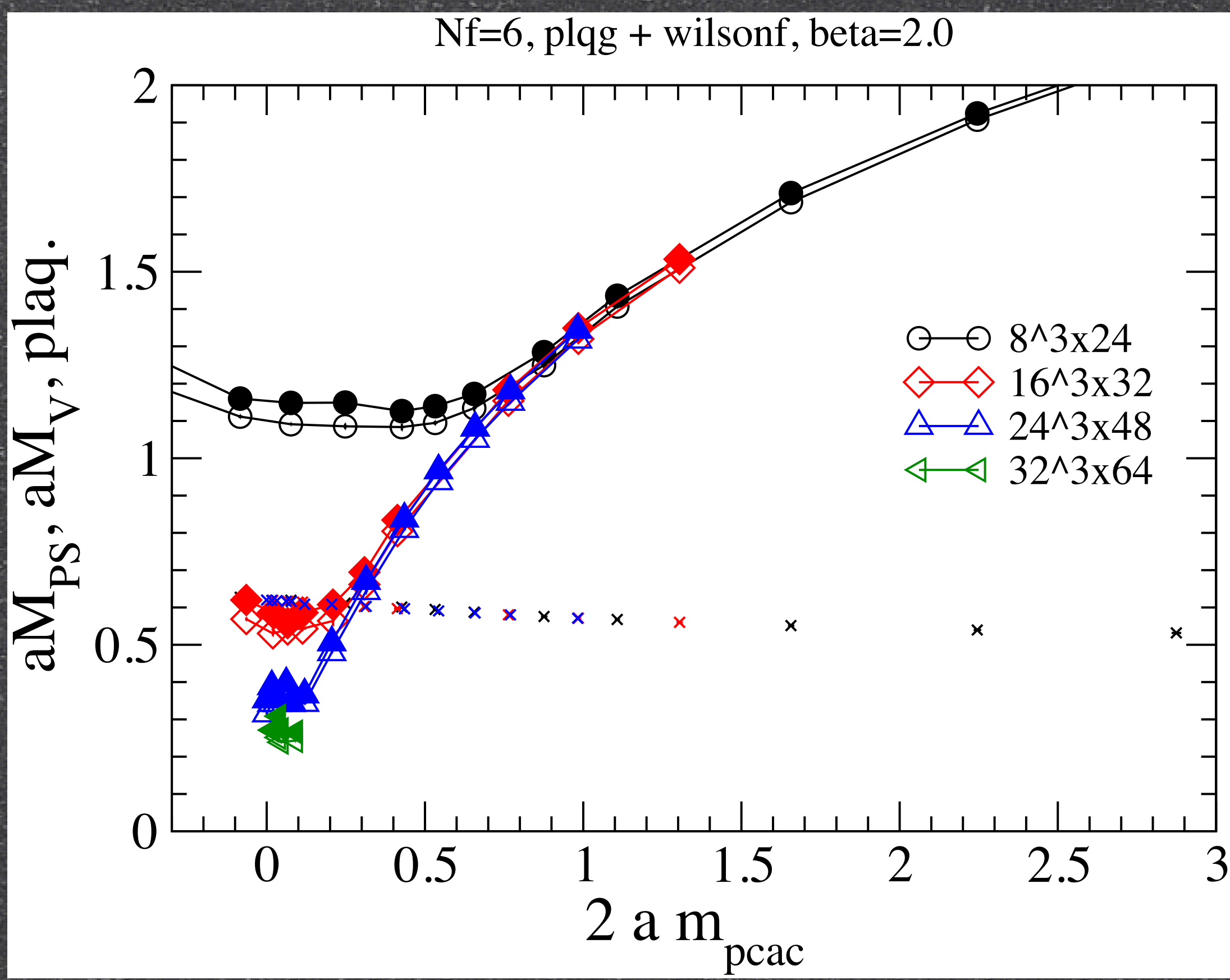

- Finite volume effect is significant.

- Masses are bounded from below.

- Minimum decreases as volume $\rightarrow$ large. 


\section{Expected behavior in Conformal Window}

\section{Del Debbio et al.(20IO)}

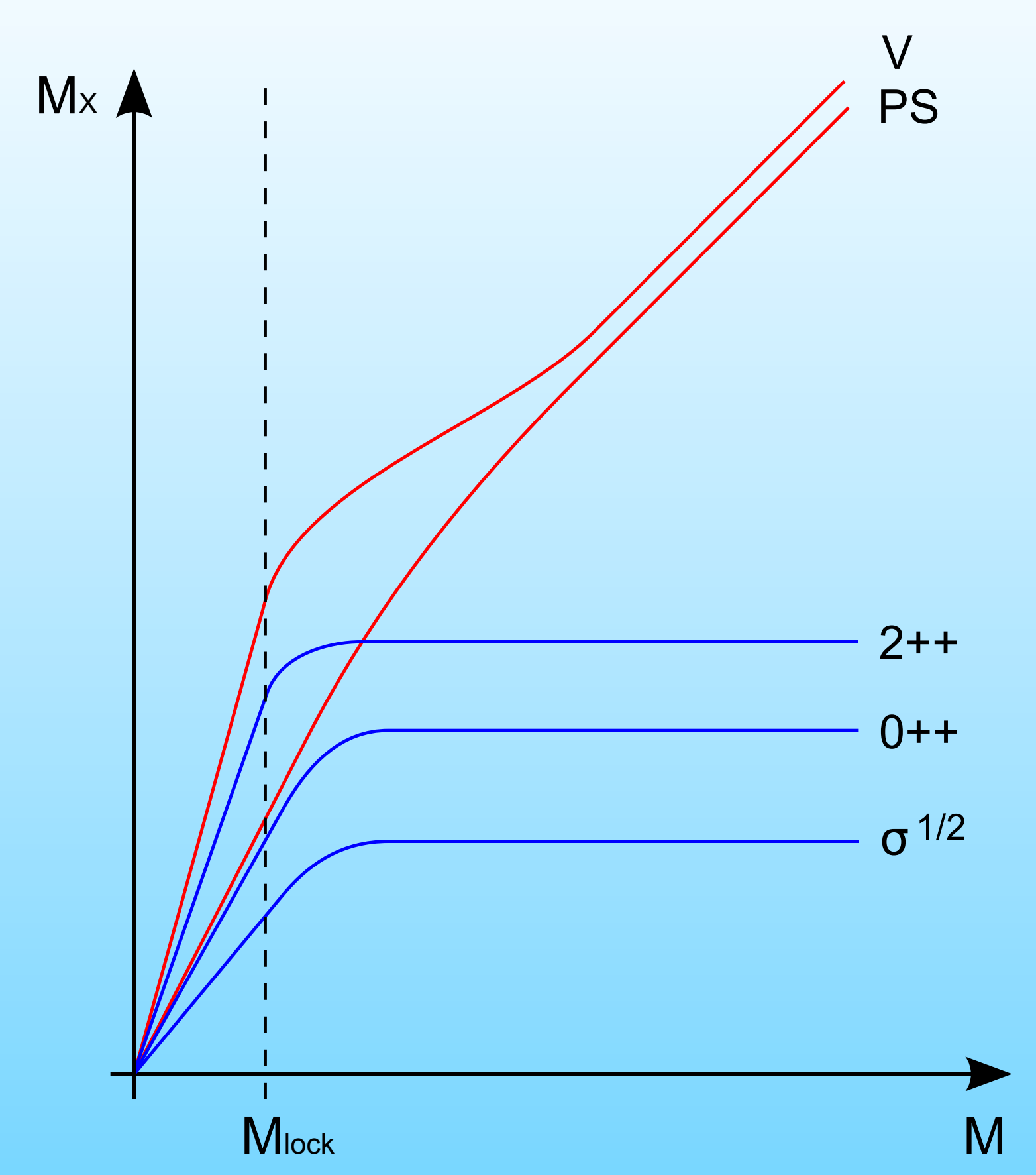

- Static limit = Quench

- In the massless limit, everything becomes massless.

- Dynamical scale (e.g. $\Lambda_{Q C D}$ in QCD) also vanishes there in contrast to QCD.

-Therefore, mass dependence of gluonic quantities is the key. 


\section{$M_{H}$ and $\sigma$}

- $M_{P} \approx M_{V}$ and $M_{S} \approx M_{A V}$

$\mathrm{Nf}=6$, plqg + wilsonf, beta $=2.0, \mathrm{~V}=24^{3} \times 48$

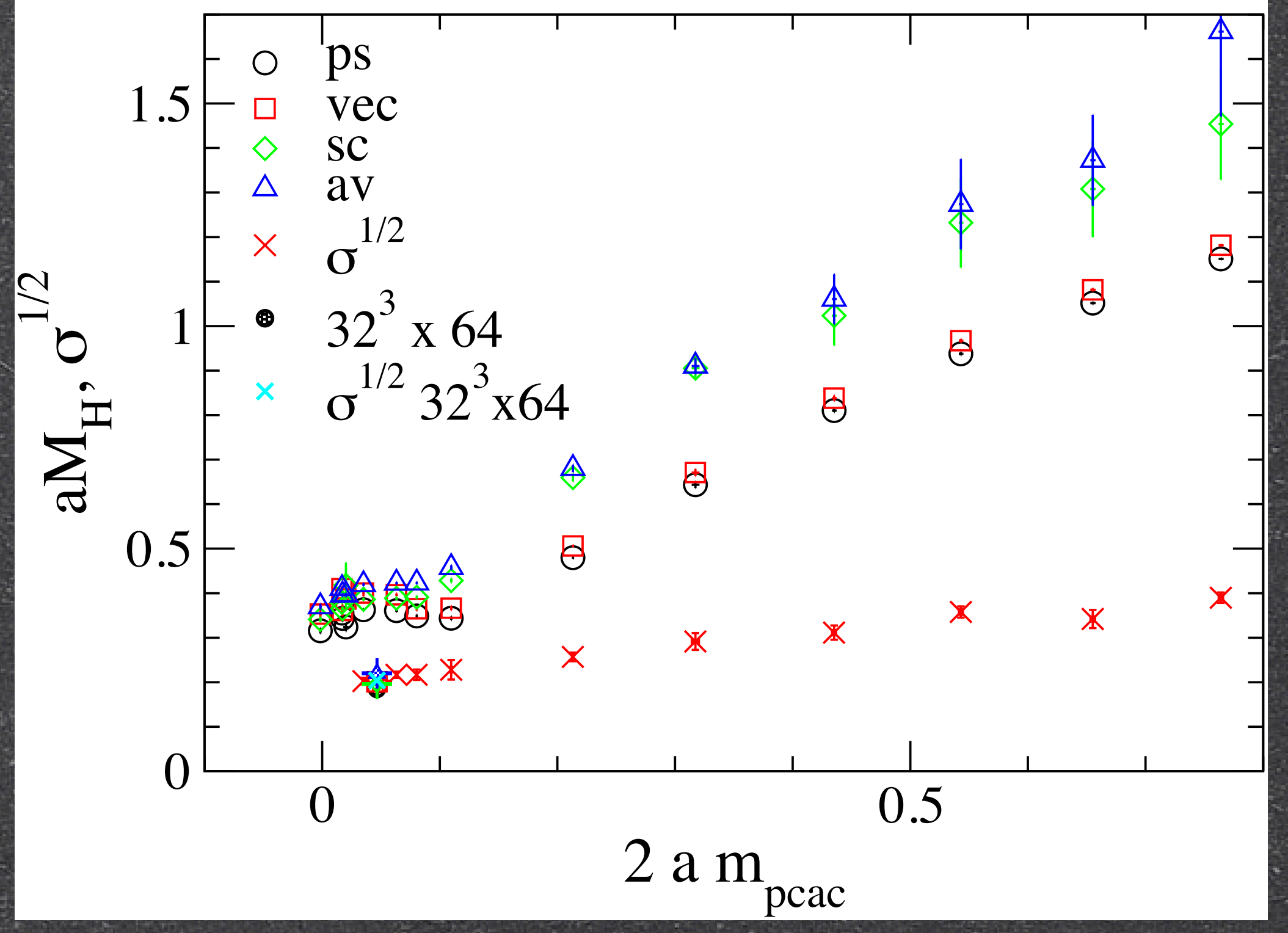
are typical pattern in the presence of heavy quark symmetry.

is smaller than $M_{H}$ in most region.

- At $V=323 \times 640 \% 2 \approx M$

-FVE is small for $\sigma^{1 / 2}$.

seems to remain finite in the chiral limit. -Confinement? 


\section{Light nuclei from quenched lattice QCD}

\section{Takeshi Yamazaki}

I M X Kobayashi-Maskawa Institute for the Origin of Particles and the Universe $\mathrm{K}] \mathrm{M}$ I

Nagoya University

Y. Kuramashi and A. Ukawa for PACS-CS Collaboration PRD81:111504(R)(2010); PRD84:054506(2011)

New Horizons for Lattice Computations with Chiral Fermions

@ BNL May 14-16 2012 
Outline

1. Introduction

2. Simulation parameters

3. Results

1. 3- and 4-nucleon channels

2. NN channels

4. Preliminary results of $N_{f}=2+1$

5. Summary and future work 


\section{Introduction}

Strong interaction

Bind $\left\{\begin{aligned} \text { protons and neutrons } & \rightarrow \text { nuclei } \\ \text { quarks and gluons } & \rightarrow \text { protons and neutrons }\end{aligned}\right.$

Spectrum of nuclei

success of Shell model since 1949: Jensen and Mayer degrees of freedom of protons and neutrons

Spectrum of proton and neutron (nucleons)

success of non-perturbative calculation of QCD

such as lattice QCD

degrees of freedom of quarks and gluons

Shell model

quarks and gluons $\rightarrow$ protons and neutrons $\rightarrow$ nuclei

(lattice) QCD 


\section{Introduction}

Strong interaction

Bind $\left\{\begin{aligned} \text { protons and neutrons } & \rightarrow \text { nuclei } \\ \text { quarks and gluons } & \rightarrow \text { protons and neutrons }\end{aligned}\right.$

Spectrum of nuclei

success of Shell model since 1949: Jensen and Mayer degrees of freedom of protons and neutrons

Spectrum of proton and neutron (nucleons)

success of non-perturbative calculation of QCD

such as lattice QCD

degrees of freedom of quarks and gluons

Motivation: Understand property of nuclei from (lattice) QCD

$\underbrace{\text { quarks and gluons } \rightarrow \overbrace{\text { protons and neutrons } \rightarrow \text { nuclei }}^{\text {model }}}_{\text {(lattice) QCD }}$ 
1. Introduction

Motivation :

Understand property and structure of nuclei from QCD

If we can study nuclei from QCD, we may be able to

1. reproduce spectrum of nuclei

2. predict property of nuclei hard to calculate or observe such as neutron rich nuclei

So far only few works for multi-baryon bound states Before studying such difficult problems, we should check

$\rightarrow$ Can we calculate known binding energy in a-few-nucleon systems? 


\section{Multi-baryon bound state from lattice QCD}

Not observed before '09 (except H-dibaryon '88 Iwasaki et al.)

Recent studies of lattice QCD for bound state of multi-baryon systems

1. 3- and 4-nucleon systems

'10 PACS-CS $N_{f}=0 m_{\pi}=0.8 \mathrm{GeV}$ PRD81:111504(R)(2010)

2. $\mathrm{H}$ dibaryon in $\wedge \wedge$ system $(\mathrm{S}=-2, \mathrm{I}=0)$

11 NPLQCD $N_{f}=2+1 m_{\pi}=0.39 \mathrm{GeV}$

' 11 HALQCD $N_{f}=3 m_{\pi}=0.67-1.02 \mathrm{GeV}$

'11 Luo et al. $N_{f}=0 m_{\pi}=0.5-1.3 \mathrm{GeV}$

3. NN systems

11 PACS-CS $N_{f}=0 m_{\pi}=0.8 \mathrm{GeV}$ PRD84:054506(2011)

'12 NPLQCD $N_{f}=2+1 m_{\pi}=0.39 \mathrm{GeV}$

4. 三三 system

'12 NPLQCD $N_{f}=2+1 m_{\pi}=0.39 \mathrm{GeV}$

presented in this talk

Other studies: 2- and 3-nucleon forces HALQCD, $\Omega \Omega$ channel Buchoff et al. 


\section{Problems of multi-nucleon bound state}

Traditional calculation method of Oth state energy

Example: ${ }^{4} \mathrm{He}$ channel $\left(J^{P}=0^{+}, I=0\right)$

$$
\begin{aligned}
C_{4}(t) & =\left\langle 0\left|O_{{ }^{4} \mathrm{He}}(t) \bar{O}_{4}{ }_{\mathrm{He}}(0)\right| 0\right\rangle \\
& =\sum_{n}\left\langle 0\left|O_{{ } \mathrm{He}}\right| n\right\rangle\left\langle n\left|\bar{O}_{{ }^{H} \mathrm{He}}\right| 0\right\rangle e^{-E_{n} t} \underset{t \gg 1}{\longrightarrow} A_{0} e^{-E_{0} t}
\end{aligned}
$$

1. Statistical error

$\rightarrow$ Unphysically heavy quark mass + large \# of measurements

2. Calculation cost

$\rightarrow$ Reduce calculation costs with several methods

Multi-meson systems: '10 Detmold and Savage

Multi-baryon systems: '12 Doi and Endres

3. Identification of bound state in finite volume

$\rightarrow$ Volume dependence of energy shift of Oth state 


\section{Simulation parameters}

Quenched Iwasaki gauge action at $\beta=2.416$

$$
a^{-1}=1.54 \mathrm{GeV} \text { with } r_{0}=0.49 \mathrm{fm}
$$

Tad-pole improved Wilson fermion action

$$
\begin{aligned}
& m_{\pi}=0.8 \mathrm{GeV} \text { and } m_{N}=1.62 \mathrm{GeV} \\
& \text { reduce large statistical fluctuation }
\end{aligned}
$$

Finite volume dependence of Oth state

$$
\left({ }^{4} \mathrm{He},{ }^{3} \mathrm{He},{ }^{3} \mathrm{~S}_{1} \text { and }{ }^{1} \mathrm{~S}_{0} \text { channels }\right)
$$

- Three volumes: $L=3.1,6.1,12.3 \mathrm{fm}$

\begin{tabular}{|c|c|c|c|}
\hline$L$ & $L[\mathrm{fm}]$ & $N_{\text {conf }}$ & $N_{\text {meas }}$ \\
\hline 24 & 3.1 & 2500 & 2 \\
\hline 48 & 6.1 & 400 & 12 \\
\hline 96 & 12.3 & 200 & 12 \\
\hline
\end{tabular}

- Two smearing sources: for consistency check

Simulations:

PACS-CS, T2K-Tsukuba at Univ. of Tsukuba, HA8000 at Univ. of Tokyo 


\section{Results}

Effective energy of ${ }^{4} \mathrm{He}$ channel at $L=48 E_{0}=\log \left(\frac{C_{\mathrm{He}}(t)}{C_{\mathrm{He}}(t+1)}\right)$

$$
\text { and } \Delta E_{L}=\log \left(\frac{R_{4} \mathrm{He}}{R_{4} \mathrm{He}_{\mathrm{e}}(t+1)}\right) \text { with } R_{4 \mathrm{He}}(t)=\frac{C_{4} \mathrm{He}_{\mathrm{e}}(t)}{\left(C_{N}(t)\right)^{4}}
$$
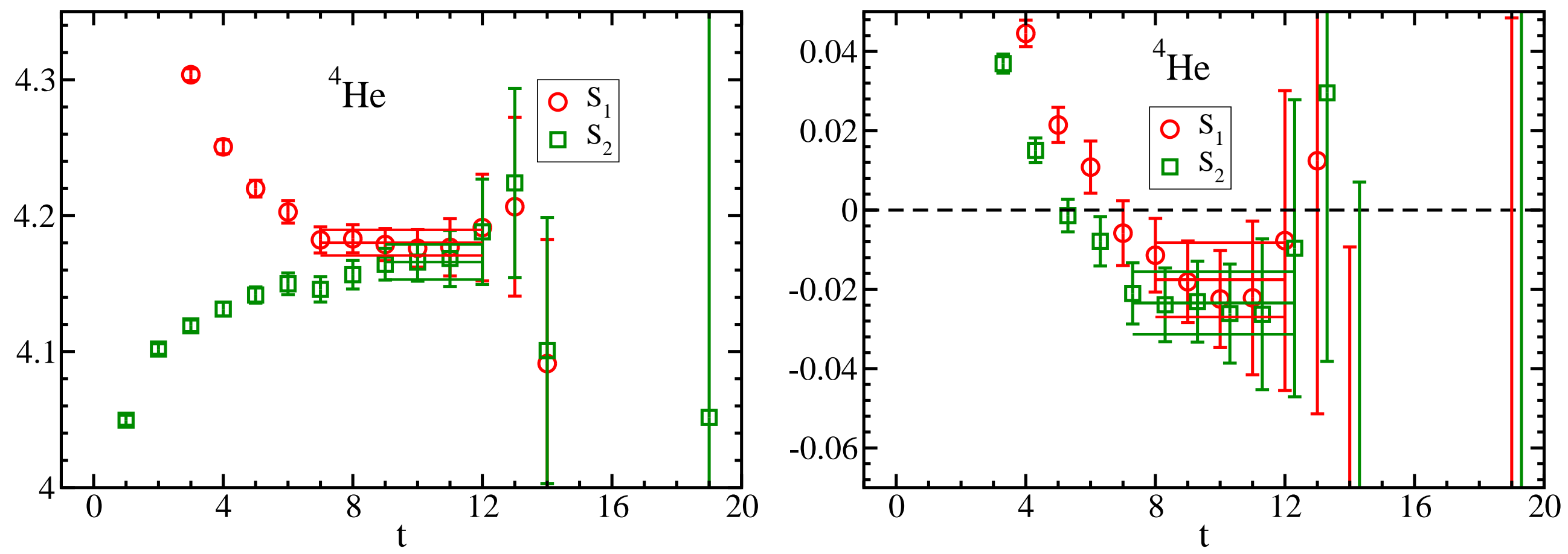

- Clear signal in $t<12$, but larger error in $t \geq 12$

- consistent plateaus in $8 \lesssim t \leq 12$ 


\section{Results}

3- and 4-nucleon channels $\Delta E_{L}=E_{0}-N_{N} m_{N}$

PACS-CS Collaboration, PRD81:111504(R)(2010)

Identification of bound state from volume dependence of $\Delta E$
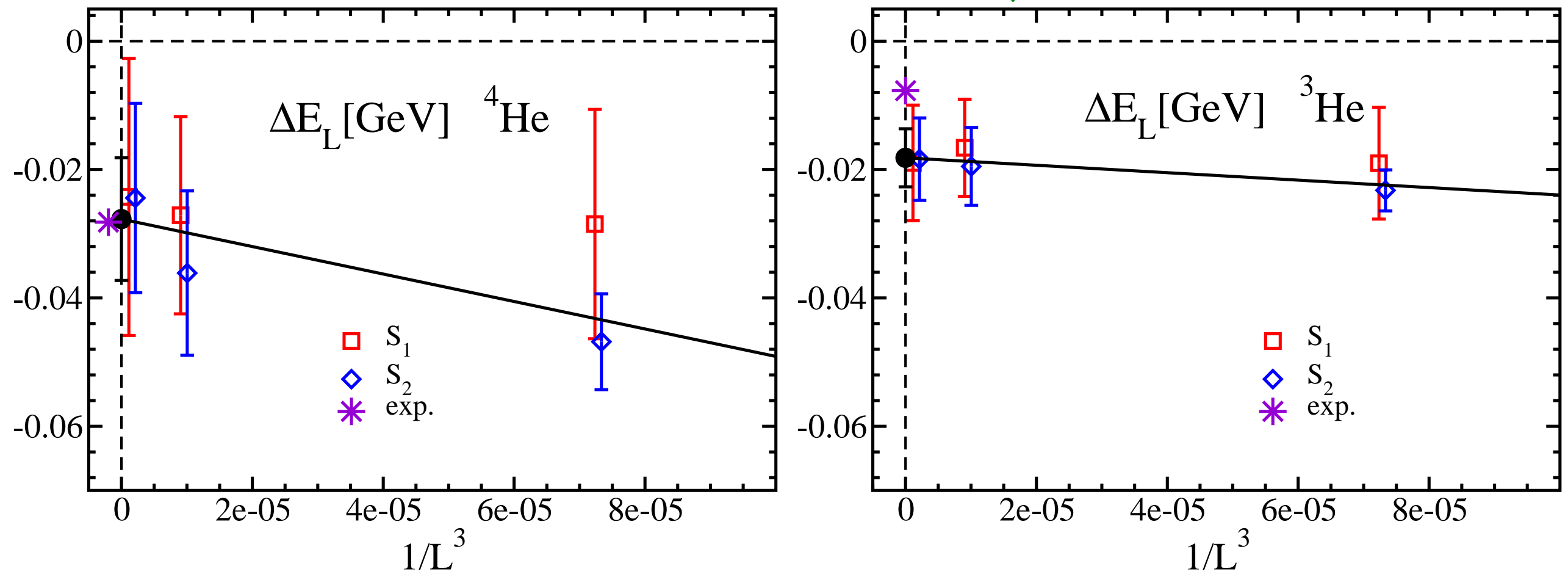

- $\Delta E_{L}<0$ in three volumes $\Leftarrow$ statistically independent ensembles

- Small volume dependence

- Infinite volume limit with $\Delta E_{L}=-\Delta E_{\text {bind }}+C / L^{3}$ 


\section{Results}

3- and 4-nucleon channels $\Delta E_{L}=E_{0}-N_{N} m_{N}$

PACS-CS Collaboration, PRD81:111504(R)(2010)

Identification of bound state from volume dependence of $\Delta E$
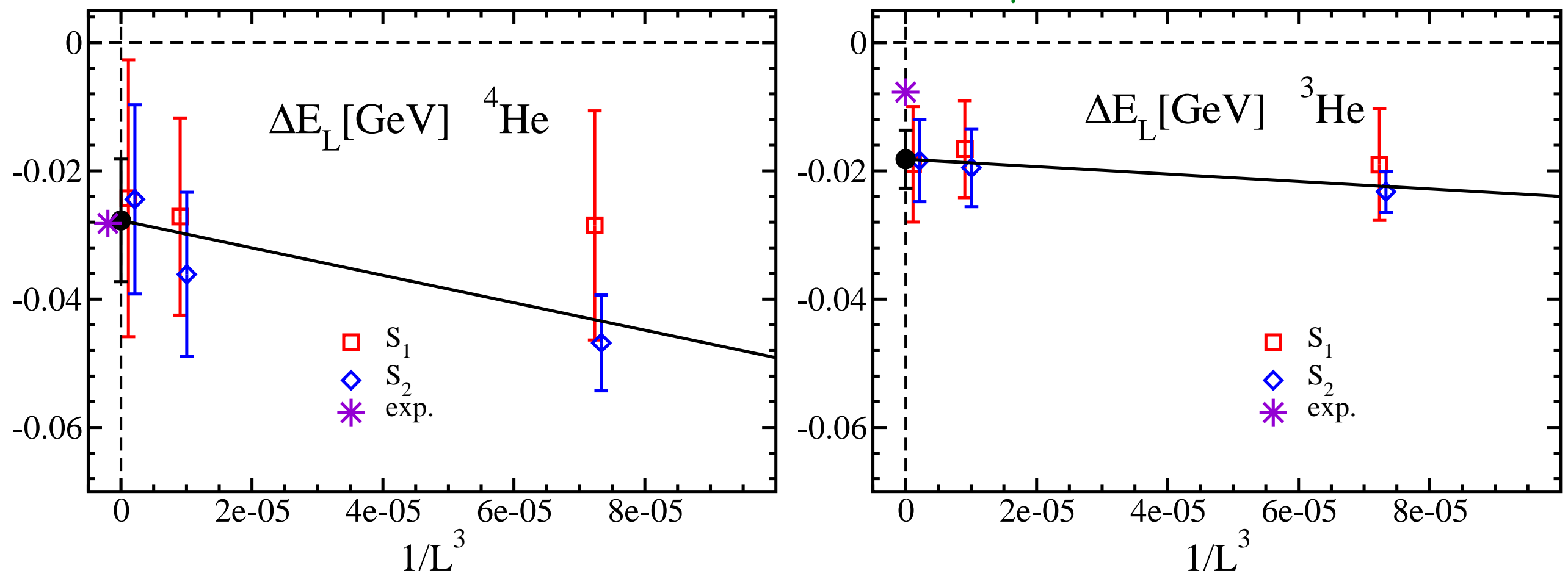

$\Delta E_{4 \mathrm{He}}=27.7(7.8)(5.5) \mathrm{MeV}$

$\Delta E_{3 \mathrm{He}}=18.2(3.5)(2.9) \mathrm{MeV}$

1. Observe bound state in both channels

2. Same order of $\Delta E$ to experiment

However, large systematic errors, e.g., $m_{\pi}=0.8 \mathrm{GeV}$ 


\section{Results}

NN $\left({ }^{3} \mathrm{~S}_{1}\right.$ and $\left.{ }^{1} \mathrm{~S}_{0}\right)$ channels $\Delta E_{L}=E_{0}-2 m_{N}$

PACS-CS Collaboration, PRD84:054506(2011)
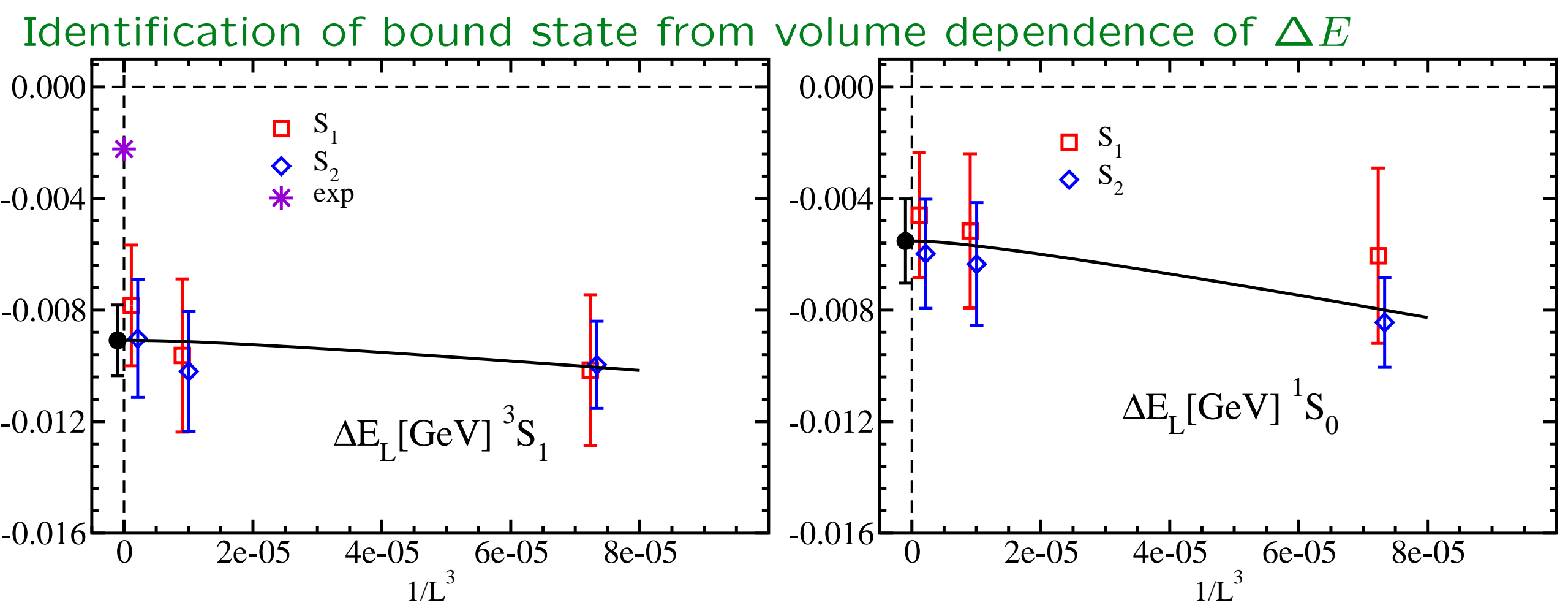

$\Delta E_{L}<0$ in three volumes Infinite volume extrapolation

'04 Beane et al., '06 Sasaki \& TY

$$
\Delta E_{L}=-\frac{\gamma^{2}}{m_{N}}\left\{1+\frac{C_{\gamma}}{\gamma L} \sum_{\vec{n}}^{\prime} \frac{\exp \left(-\gamma L \sqrt{\vec{n}^{2}}\right)}{\sqrt{\vec{n}^{2}}}\right\}, \Delta E_{\mathrm{bind}}=\frac{\gamma^{2}}{m_{N}}
$$

based on Lüscher's finite volume formula 


\section{Results}

NN $\left({ }^{3} \mathrm{~S}_{1}\right.$ and $\left.{ }^{1} \mathrm{~S}_{0}\right)$ channels $\Delta E_{L}=E_{0}-2 m_{N}$

PACS-CS Collaboration, PRD84:054506(2011)

Identification of bound state from volume dependence of $\Delta E$
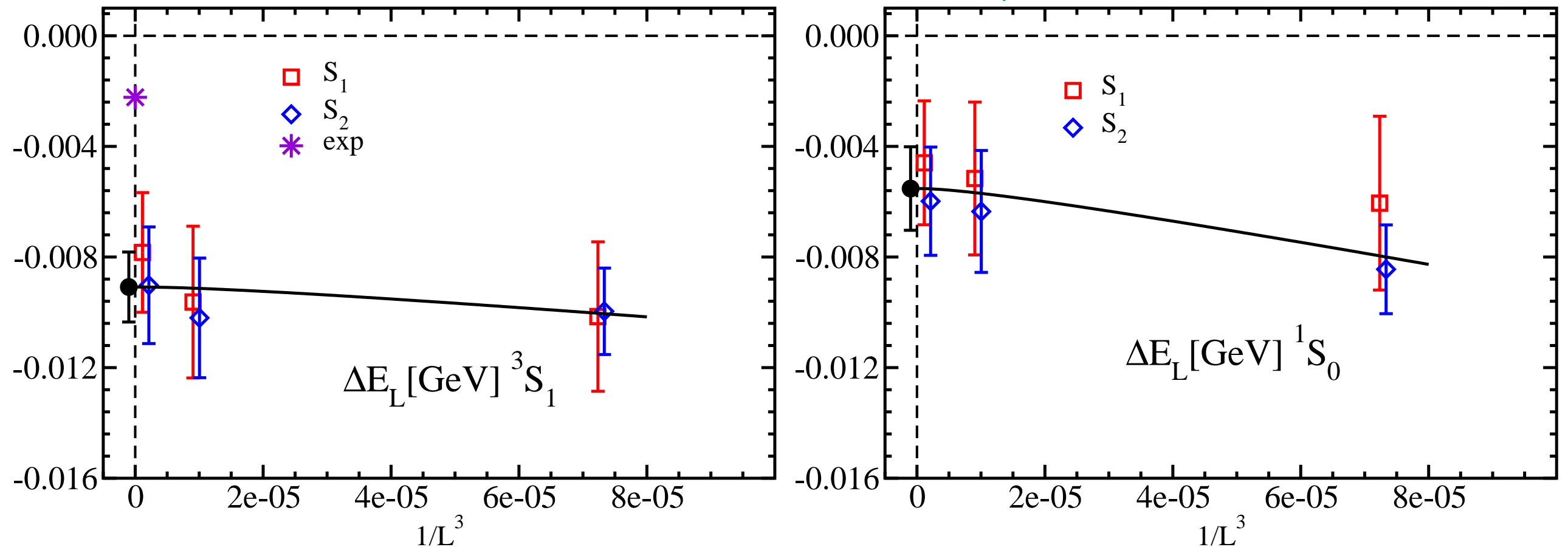

Bound state in both channels $\leftarrow$ different from experiment

$$
\Delta E_{3 \mathrm{~S}_{1}}=9.1(1.1)(0.5) \mathrm{MeV} \quad \Delta E_{1 \mathrm{~S}_{0}}=5.5(1.1)(1.0) \mathrm{MeV}
$$

Probably bound states in $N_{f}=2+1 m_{\pi}=0.39 \mathrm{GeV}$ ('12 NPLQCD) might be caused by heavy quark mass in calculation 
4. Preliminary results of $N_{f}=2+1$

Reduce systematic errors

Quark mass dependence of $\Delta E$ and $a_{0}$

Bound state in ${ }^{1} S_{0}$ vanishes as quark mass decreases?

Heavy quark mass

$$
m_{\pi}=0.8 \mathrm{GeV}
$$

Quenched effect

Finite lattice spacing

$$
a^{-1}=1.54 \mathrm{GeV}
$$


4. Preliminary results of $N_{f}=2+1$

Reduce systematic errors

Quark mass dependence of $\Delta E$ and $a_{0}$

Bound state in ${ }^{1} S_{0}$ vanishes as quark mass decreases?

Heavy quark mass

$$
\left.m_{\pi}=0.8 \mathrm{GeV} \rightarrow 0.5 \text { (and } 0.7\right) \mathrm{GeV}
$$

Quenched effect

$$
N_{f}=2+1 \text { QCD }
$$

Finite lattice spacing

$$
a^{-1}=1.54 \mathrm{GeV} \rightarrow a^{-1} \sim 2 \mathrm{GeV}
$$

Calculation of $\mathrm{NN},{ }^{3} \mathrm{He},{ }^{4} \mathrm{He}$ channels 


\section{Preliminary results of $N_{f}=2+1$}

\section{Simulation parameters}

- Iwasaki gauge action at $\beta=1.90$

$$
a^{-1}=2.194 \mathrm{GeV} \text { with } m_{\Omega}
$$

- Non-perturbative improved Wilson fermion action

$$
\begin{gathered}
m_{\pi}=0.5 \mathrm{GeV} \text { and } m_{N}=1.3 \mathrm{GeV} \\
m_{s} \sim \text { physical strange mass }
\end{gathered}
$$

Finite volume dependence of Oth state

Four volumes: $L=2.9,3.6,4.3,5.8 \mathrm{fm}$

Measurements: $\sim 200$ confs $\times O(100) /$ per conf

Simulations:

PACS-CS, T2K-Tsukuba, HA-PACS at Univ. of Tsukuba, HA8000 at Univ. of Tokyo and $\mathrm{K}$ at AICS 


\section{Preliminary results of $N_{f}=2+1$}

3- and 4-nucleon channels

Identification of bound state from volume dependence of $\Delta E$
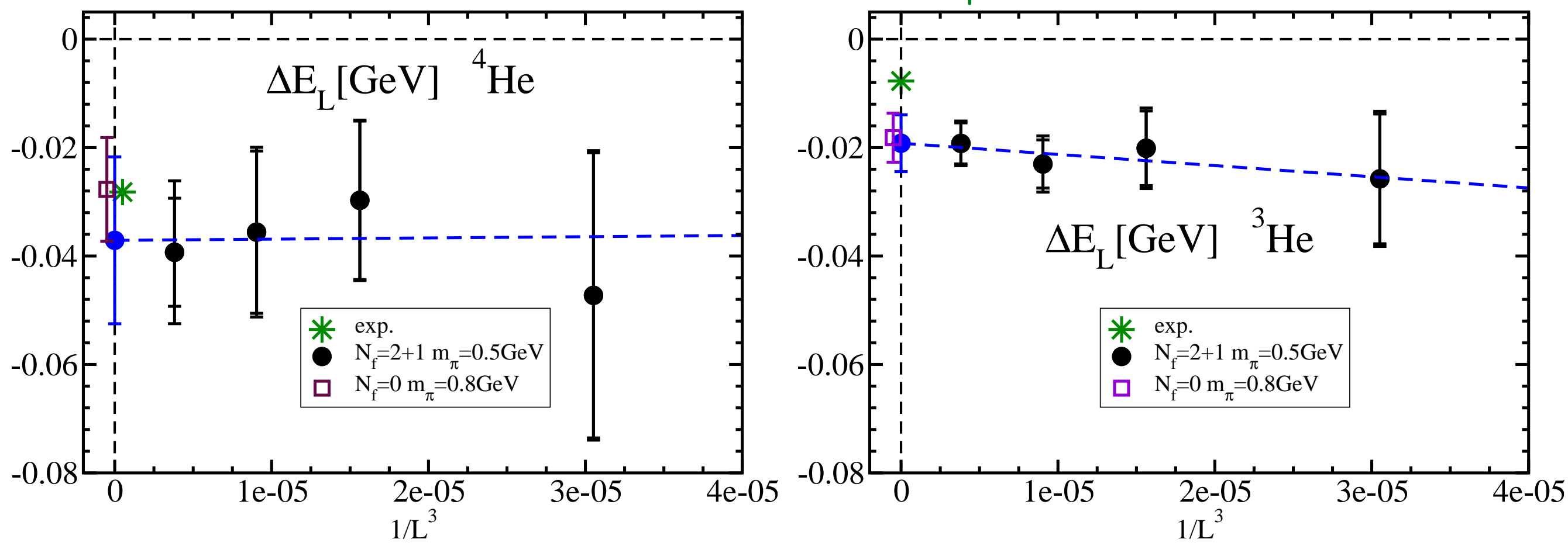

Similar results to $N_{f}=0 m_{\pi}=0.8 \mathrm{GeV}$ 


\section{Preliminary results of $N_{f}=2+1$}

\section{NN $\left({ }^{3} \mathrm{~S}_{1}\right.$ and $\left.{ }^{1} \mathrm{~S}_{0}\right)$ channels}
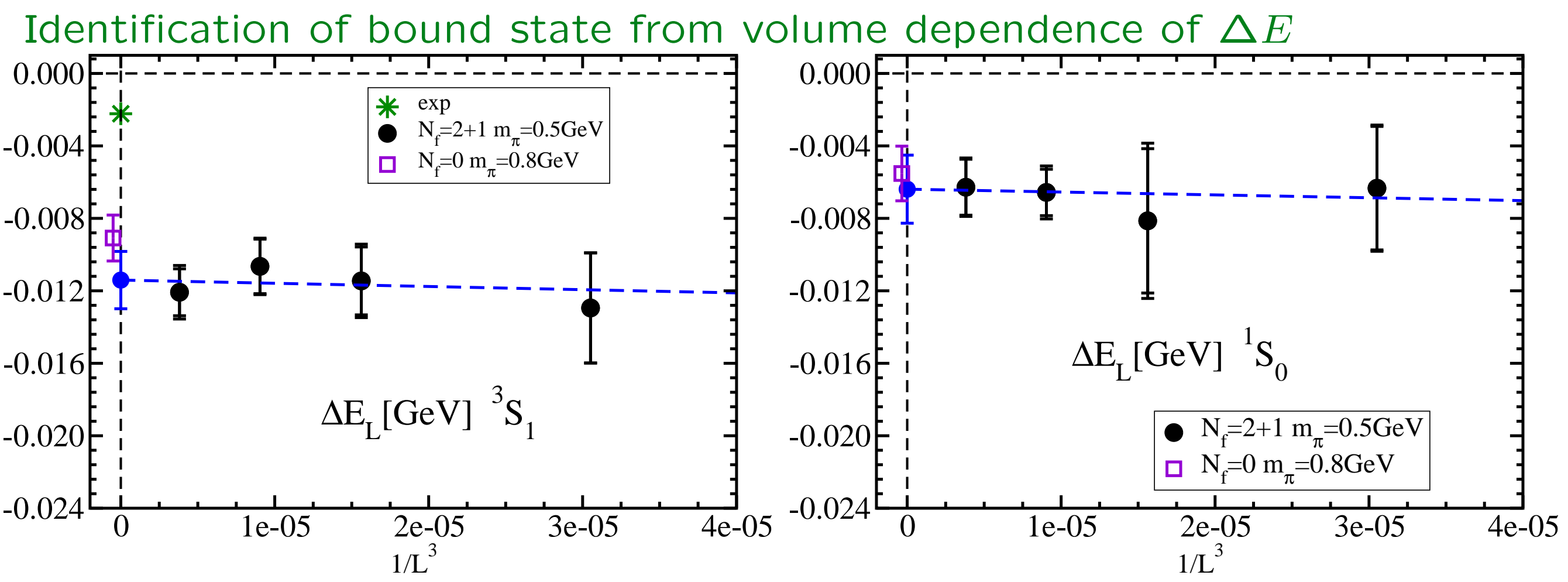

Similar results to $N_{f}=0 m_{\pi}=0.8 \mathrm{GeV}$ 


\section{Preliminary results of $N_{f}=2+1$}

\section{NN $\left({ }^{3} \mathrm{~S}_{1}\right.$ and $\left.{ }^{1} \mathrm{~S}_{0}\right)$ channels}

\section{Current status of $\Delta E$}
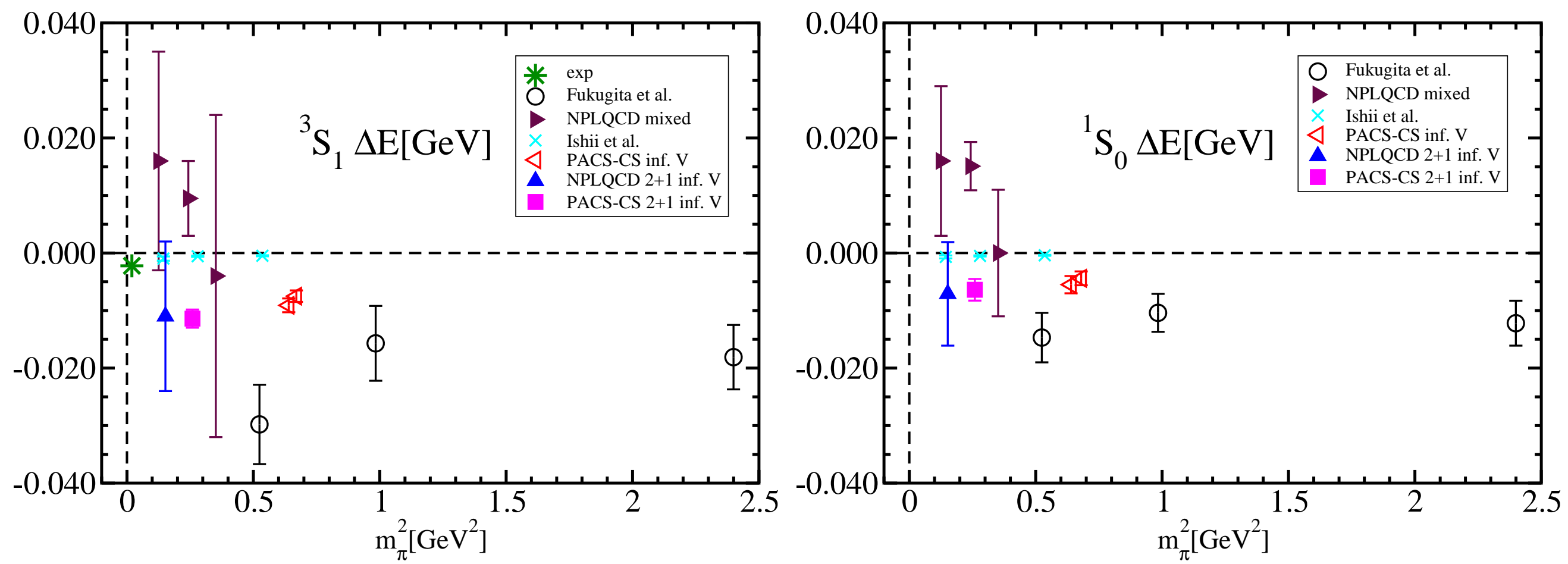

filled(open) symbols: $N_{f}=2+1$ (quenched) results

Roughly consistent with other results Still quark mass might be heavy Need further lighter quark mass for clear quark dependence 


\section{Summary and future work}

Exploratory study of light nuclei in quenched lattice QCD

- Unphysically heavy quark mass

- Volume dependence of energy shift of ground state

1. $\Delta E \neq 0$ of Oth state in infinite volume limit

(2. Expected properties of 1st excited state in NN)

$\rightarrow$ bound state in ${ }^{4} \mathrm{He},{ }^{3} \mathrm{He},{ }^{3} \mathrm{~S}_{1}$ and ${ }^{1} \mathrm{~S}_{0}$ at $m_{\pi}=0.8 \mathrm{GeV}$

Larger $\Delta E$ than experiment

Bound state in ${ }^{1} S_{0}$ not observed in experiment

probably observed $N_{f}=2+1$ at $m_{\pi}=0.39 \mathrm{GeV}$ ('12 NPLQCD)

Need to reduce systematic errors 
5. Summary and future work

$N_{f}=2+1$ calculation is on-going at $m_{\pi}=0.5 \mathrm{GeV}$

Similar result to quenched case

$\rightarrow$ bound state in ${ }^{4} \mathrm{He},{ }^{3} \mathrm{He},{ }^{3} \mathrm{~S}_{1}$ and ${ }^{1} \mathrm{~S}_{0}$ at $m_{\pi}=0.5 \mathrm{GeV}$

Need further lighter quark mass calculation 
Back up 
Very preliminary $N_{f}=2+1$ results at $m_{\pi}=0.7 \mathrm{GeV}$
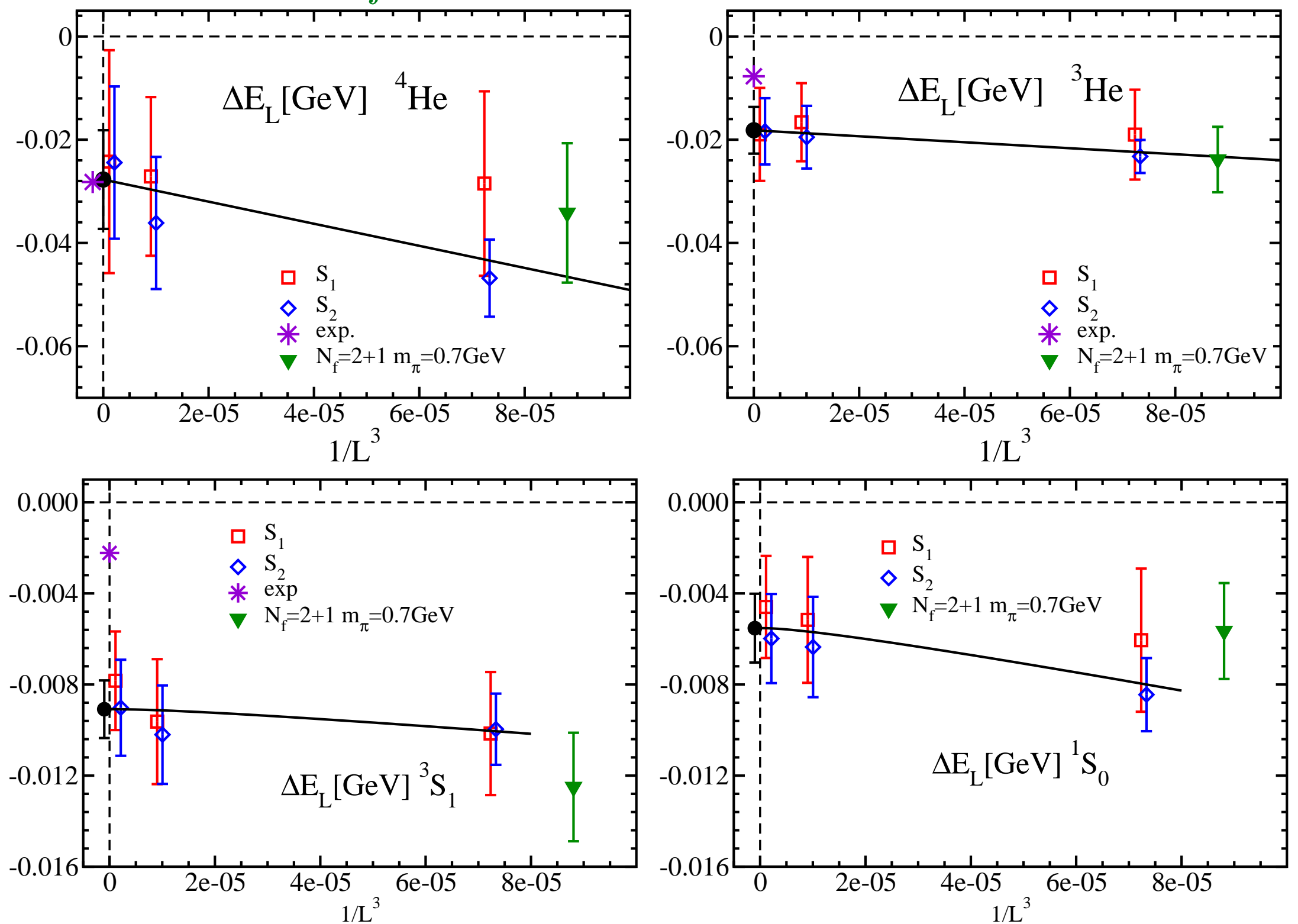

Larger volumes at $m_{\pi}=0.5$ and $0.7 \mathrm{GeV}$ underway 


\section{Example of large quark mass dependence}

rms radii from form factors $F_{1}$ and $F_{2}$

'09 RBC + UKQCD
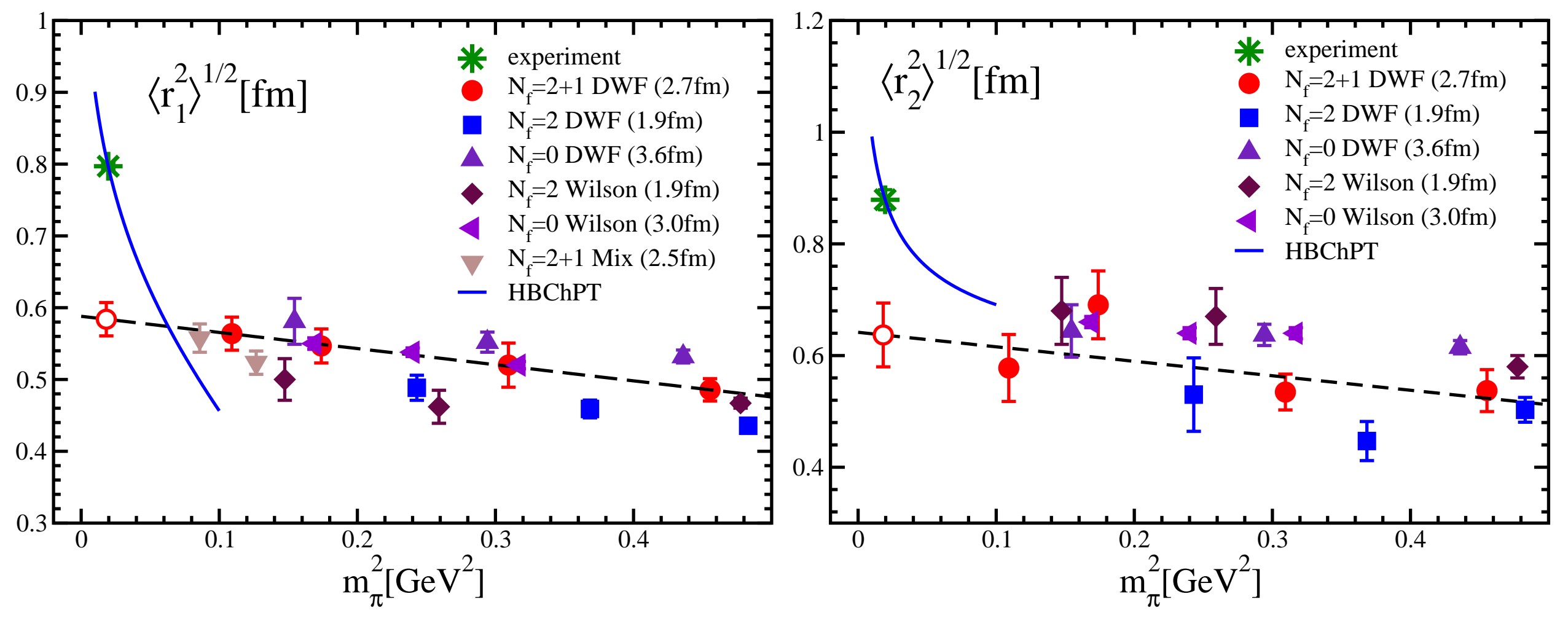
Effective energy shift $\Delta E_{L}=E_{\mathrm{NN}}-2 m_{N}$ of ${ }^{3} \mathrm{~S}_{1}$ at $L=24$ and $L=96$ $\Delta E_{L}(t)=\log \left(\frac{R(t)}{R(t+1)}\right), \quad R(t)=\frac{C_{\mathrm{NN}}(t)}{\left(C_{N}(t)\right)^{2}}$
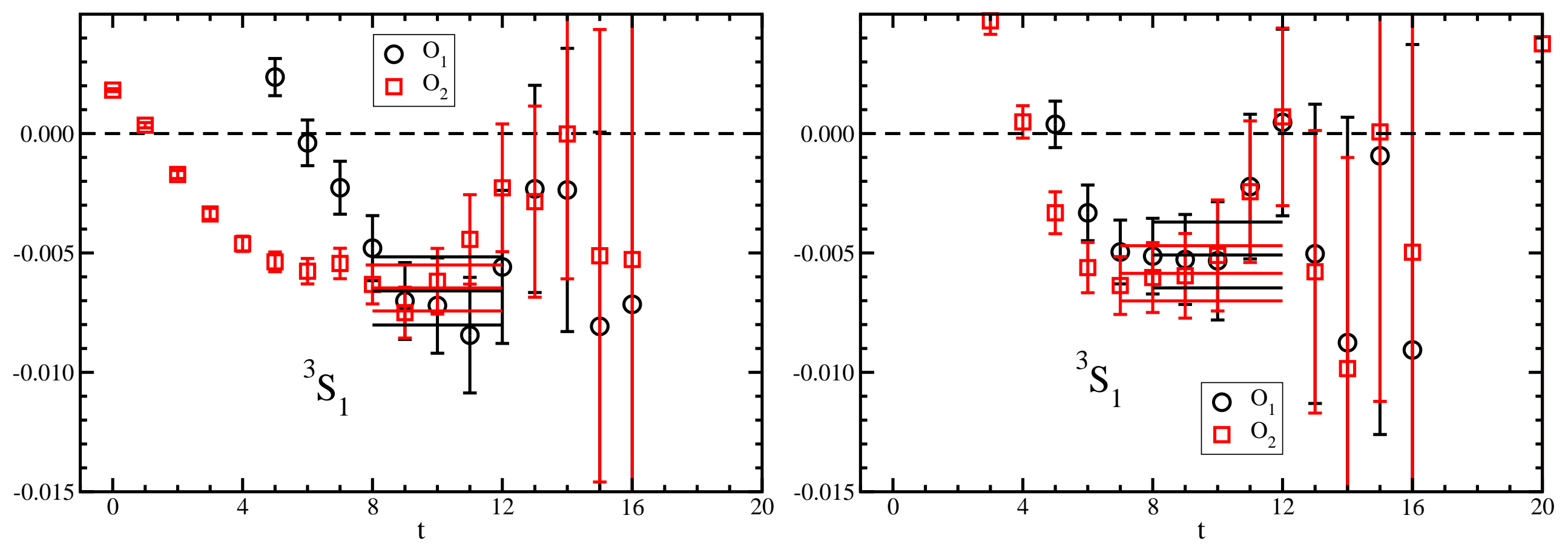
Smearing functions of sink operator at $L=32$ and $L=48$

$$
N(\mathbf{x}) W_{q^{2}}(|\mathbf{x}-\mathbf{y}|) N(\mathbf{y})
$$
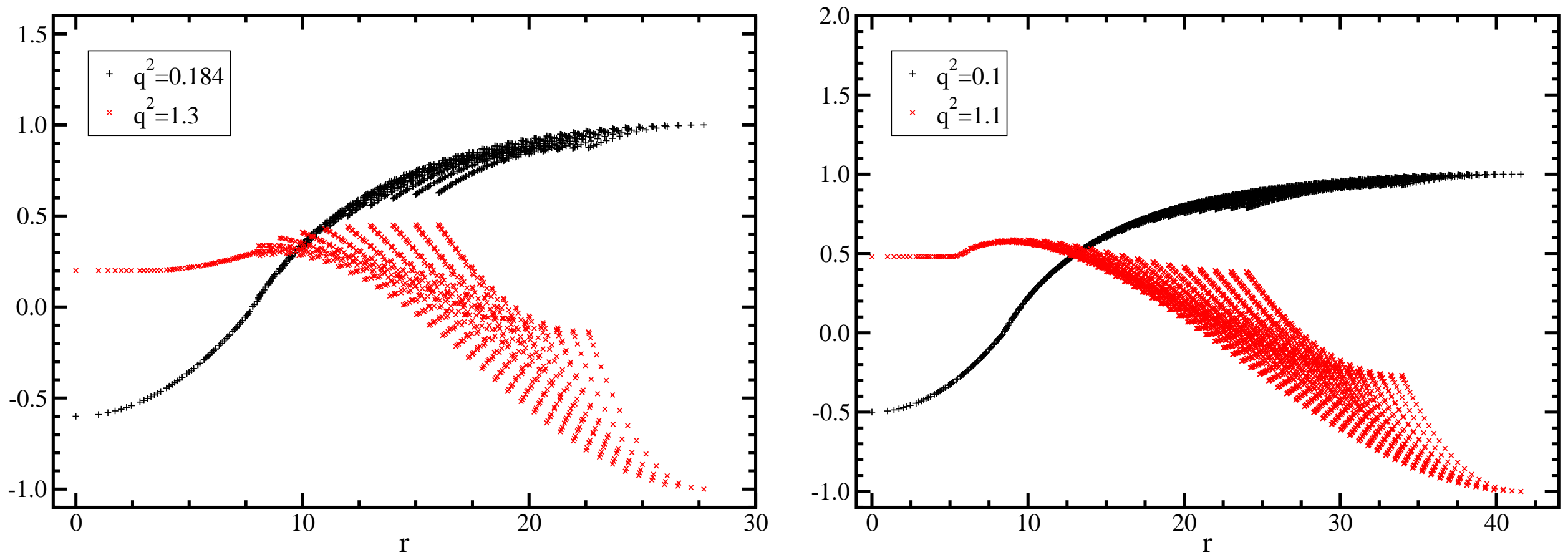
Effective nucleon mass at $L=48$

$$
M_{N}(t)=\log \left(\frac{C_{N}(t)}{C_{N}(t+1)}\right)
$$
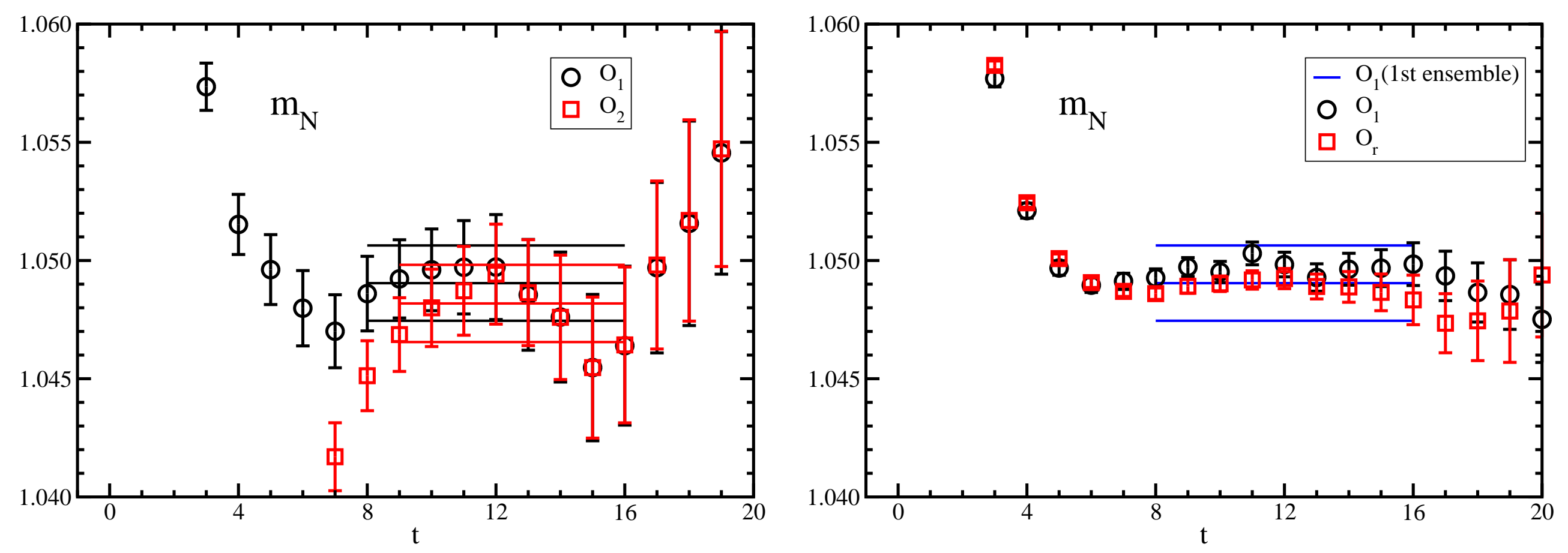
Effective energy before and after diagonalization $L=48$

$$
E_{i j}(t)=\log \left(\frac{G_{i j}(t)}{G_{i j}(t+1)}\right)
$$

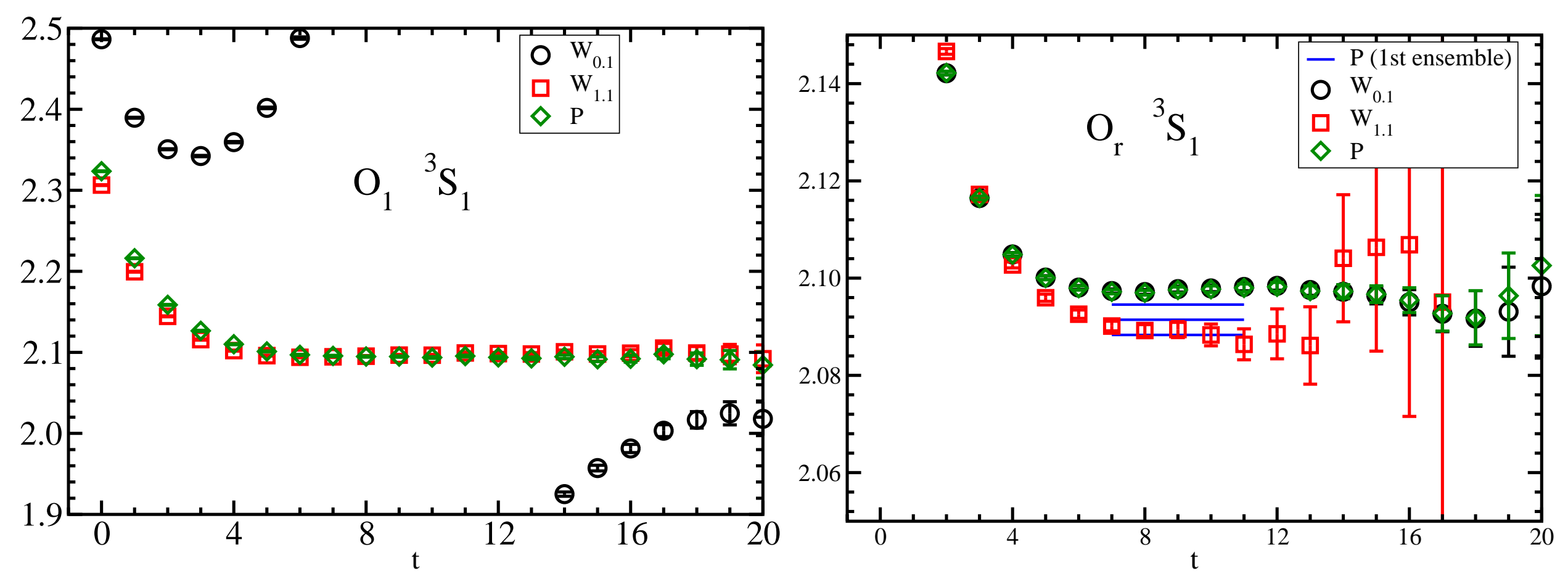


Effective energy before and after diagonalization $L=32$

$$
E_{i j}(t)=\log \left(\frac{G_{i j}(t)}{G_{i j}(t+1)}\right)
$$
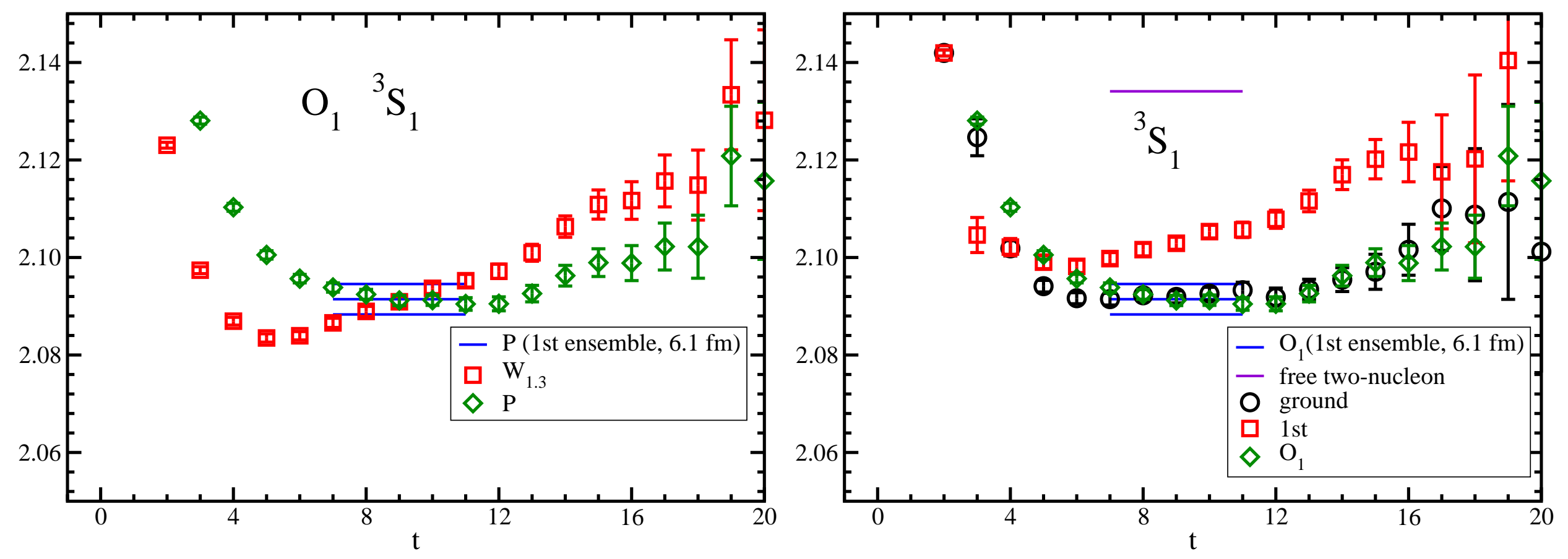
Effective energy shift $\Delta \bar{E}_{L, \alpha}=\bar{E}_{\alpha}-2 m_{N}$

for ground and 1st excited states of ${ }^{3} S_{1}$ at $L=48$ $\Delta \bar{E}_{L, \alpha}(t)=\log \left(\frac{\bar{R}_{\alpha}(t)}{\bar{R}_{\alpha}(t+1)}\right), \quad \bar{R}_{\alpha}(t)=\frac{\lambda_{\alpha}(t)}{\left(C_{N}(t)\right)^{2}}$
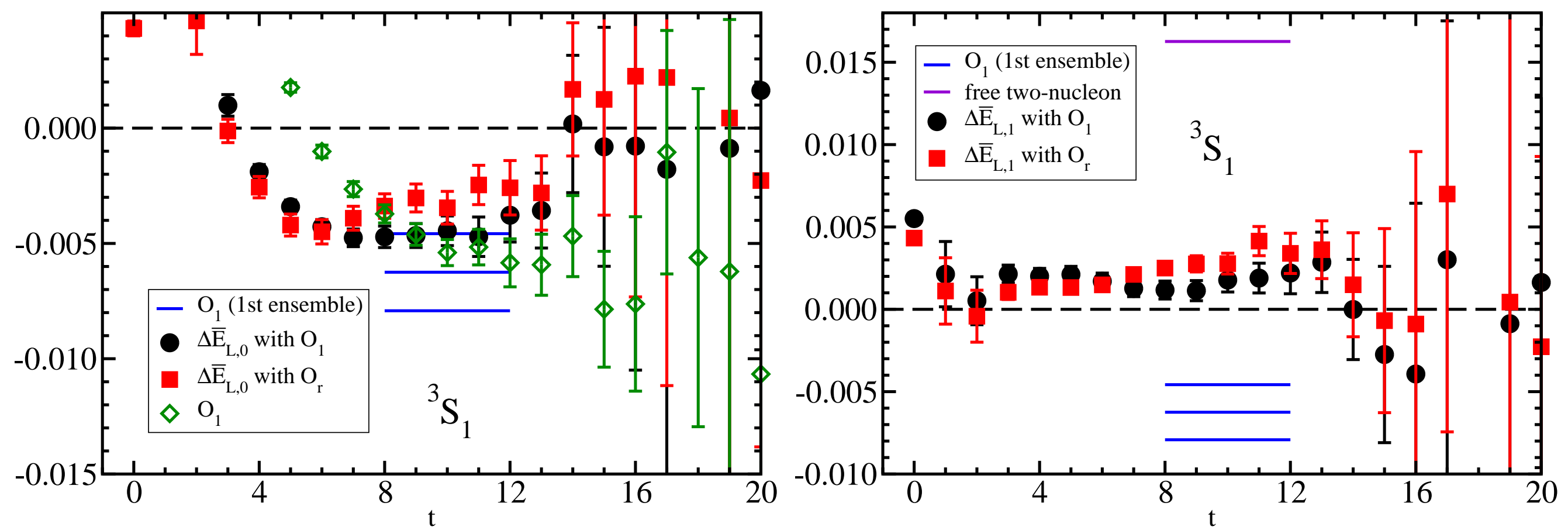
Effective energy shift $\Delta \bar{E}_{L, \alpha}=\bar{E}_{\alpha}-2 m_{N}$

for ground and 1st excited states of ${ }^{1} \mathrm{~S}_{0}$ at $L=48$ $\Delta \bar{E}_{L, \alpha}(t)=\log \left(\frac{\bar{R}_{\alpha}(t)}{\bar{R}_{\alpha}(t+1)}\right), \quad \bar{R}_{\alpha}(t)=\frac{\lambda_{\alpha}(t)}{\left(C_{N}(t)\right)^{2}}$
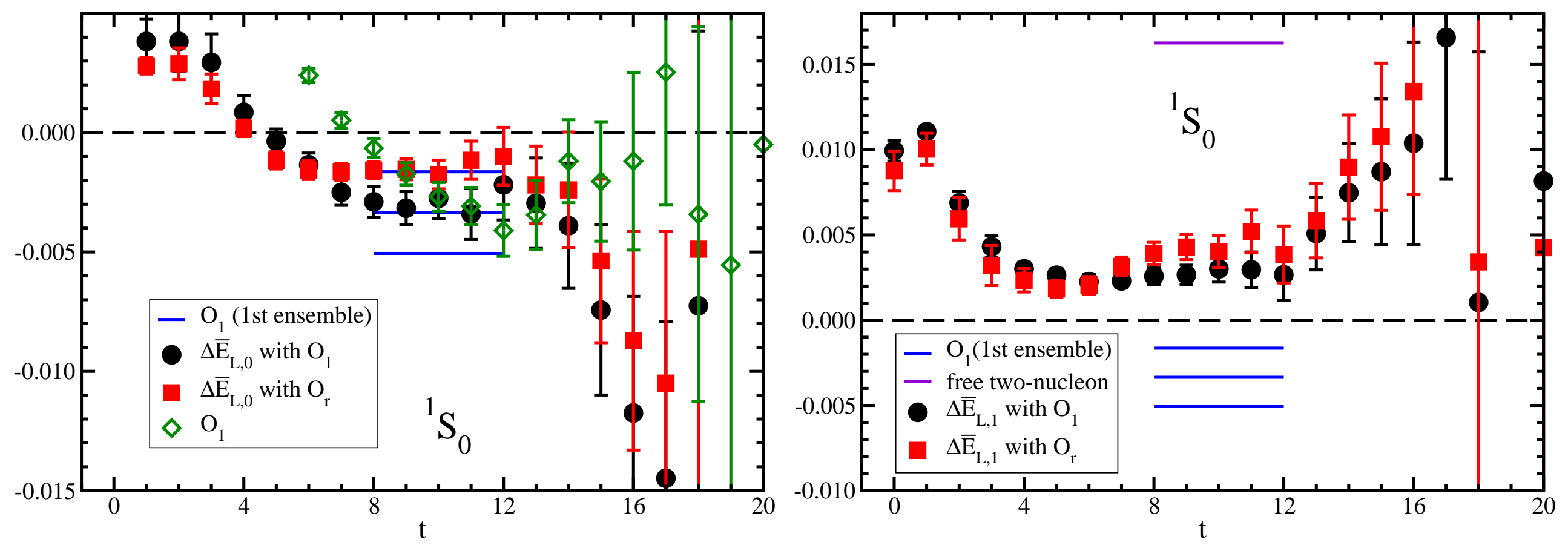


\section{Results (cont'd) \\ 2. Two-state analysis}

Volume dependence of $\Delta E_{L}$ and $\Delta \bar{E}_{L, 0}$
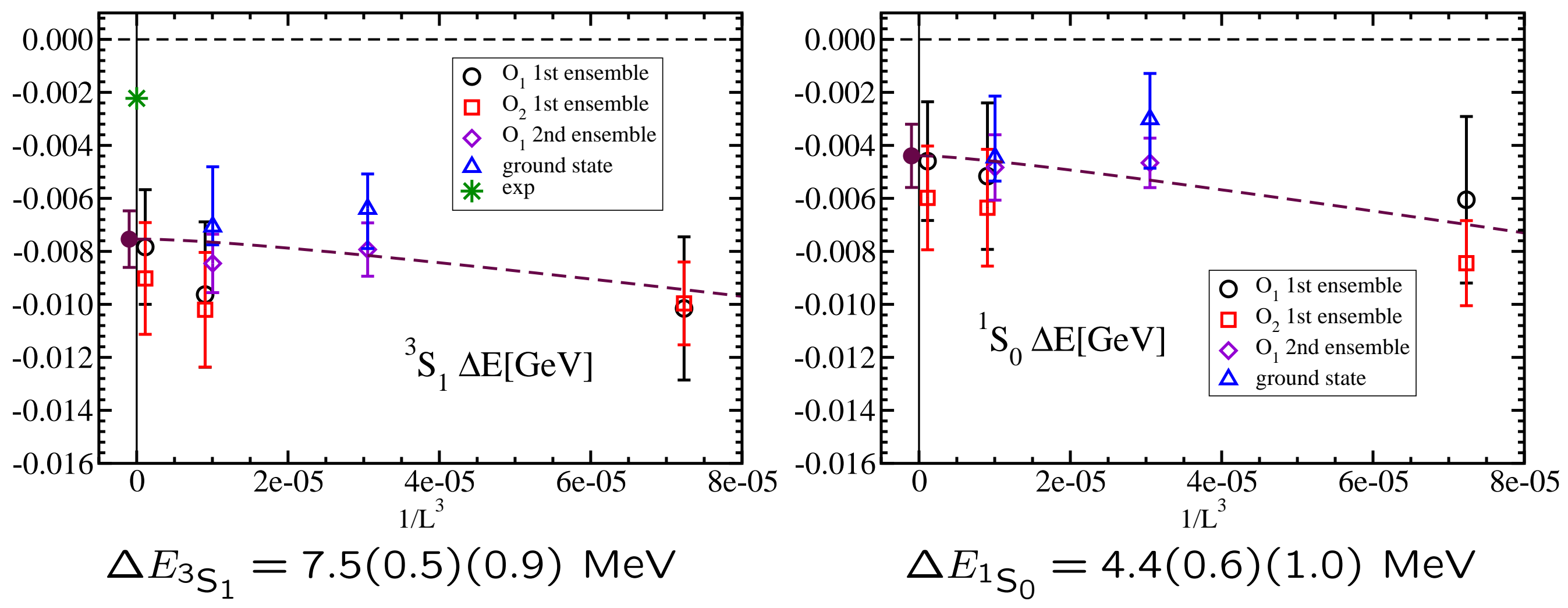

${ }^{3} S_{1}$ : almost four times larger than experimental values

${ }^{1} \mathrm{~S}_{0}$ : bound state exists different from experiment

mainly caused by heavy quark mass in calculation, probably 


\section{Future work quark mass dependence}

Assume: our results smoothly continue to physical one

\section{Quark mass \}

${ }^{3} \mathrm{~S}_{1}: \Delta E \searrow$ and $\left|a_{0}\right| \nearrow$

${ }^{1} \mathrm{~S}_{0}: \Delta E \searrow$ and $\left|a_{0}\right| \nearrow$

$\rightarrow$ bound state vanishes, $\left|a_{0}\right|=\infty$ and $\operatorname{sign}\left(a_{0}\right)=+$
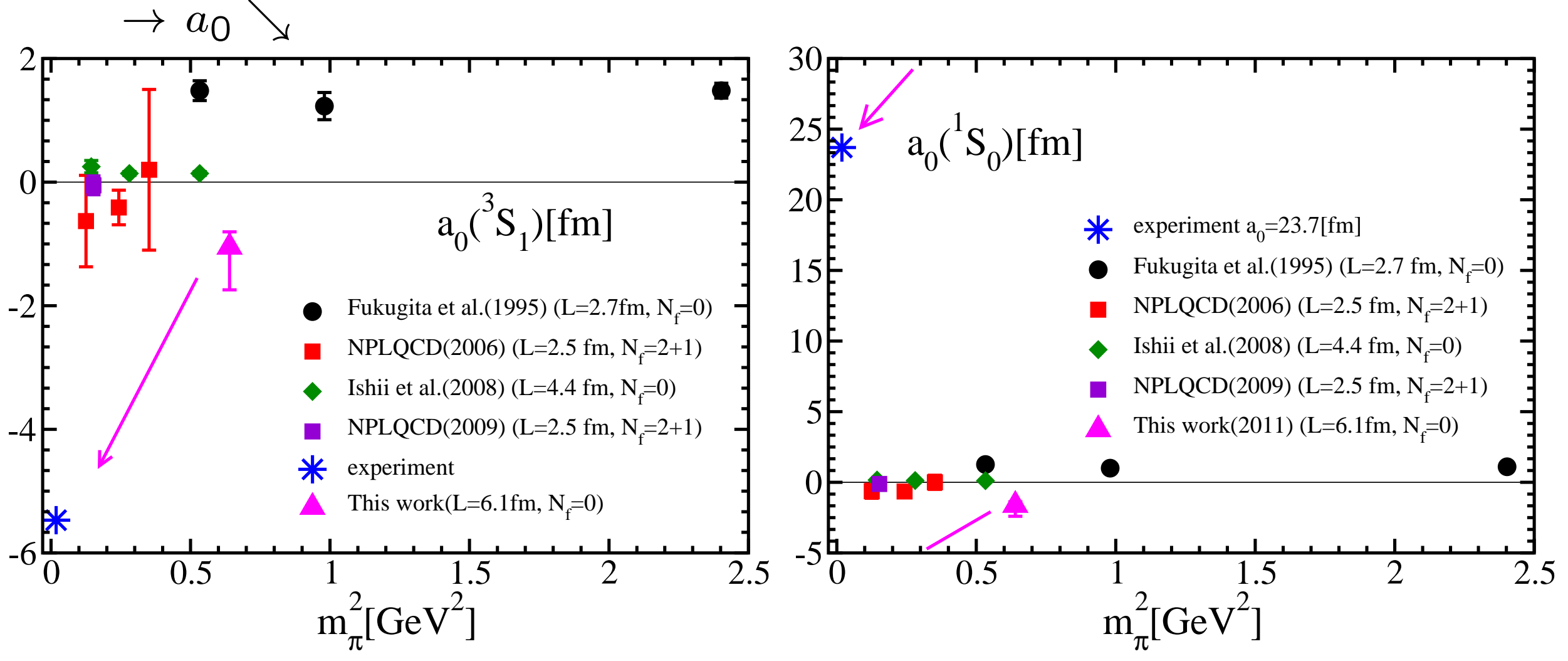

Certainly need to study quark mass dependence 


\section{Simulation parameters}

- Quenched Iwasaki gauge action at $\beta=2.416$

$$
a^{-1}=1.54 \mathrm{GeV} \text { with } r_{0}=0.49 \mathrm{fm}
$$

- Tad-pole improved Wilson fermion action

$$
m_{\pi}=0.8 \mathrm{GeV} \text { and } m_{N}=1.62 \mathrm{GeV}
$$

reduce large statistical fluctuation

1. Single state analysis

- Three volumes

\begin{tabular}{|c|c|c|c|}
\hline$L$ & $L[\mathrm{fm}]$ & $N_{\text {conf }}$ & $N_{\text {meas }}$ \\
\hline 24 & 3.1 & 2500 & 2 \\
\hline 48 & 6.1 & 400 & 12 \\
\hline 96 & 12.3 & 200 & 12 \\
\hline
\end{tabular}

- Exponential smearing sources $q(\vec{x})=A \exp (-B|\vec{x}|)$

$$
\mathrm{O}_{1} \quad \mathrm{O}_{2}
$$

$$
\begin{aligned}
& (A, B)=(0.5,0.5),(0.5,0.1) \text { for } L=24 \\
& (A, B)=(0.5,0.5),(1.0,0.4) \text { for } L=48,96
\end{aligned}
$$

- quark operator with non-relativistic projection in nucleon operator 


\section{Simulation parameters}

2. Two-state analysis with $2 \times 2$ diagonalization

- Two volumes

- Exponential smearing sources $q(\vec{x})=A \exp (-B|\vec{x}|)$

\begin{tabular}{|c|c|c|c|c|c|c|}
\hline$L$ & $L[\mathrm{fm}]$ & $N_{\text {conf }}$ & $N_{\text {meas }}$ & $N_{r}$ & $q_{1}^{2}$ & $q_{2}^{2}$ \\
\hline 32 & 4.1 & 300 & 192 & 40 & 0.184 & 1.3 \\
\hline 48 & 6.1 & 300 & 144 & 32 & 0.1 & 1.1 \\
\hline
\end{tabular}

$$
O_{1}:(A, B)=(0.5,0.5)
$$

$O_{r}$ : spread random source with $(A, B)=(0.5,0.5)$

- Two Wavefunction smearing sinks $N(\mathbf{x}) W_{q^{2}}(|\mathbf{x}-\mathbf{y}|) N(\mathbf{y})$

$W_{q^{2}}(|\mathbf{r}|)$ : Based on solution of 3-dimension Helmholtz equation parameters: $q^{2}$ and overall normalization

- quark operator with non-relativistic projection in nucleon operator

Simulations:

PACS-CS, T2K-Tsukuba at Univ. of Tsukuba HA8000 at Univ. of Tokyo 


\section{Results}

1. Single state analysis

Effective two-nucleon energy at $L=6.1 \mathrm{fm}$

$$
E_{\mathrm{NN}}(t)=\log \left(\frac{C_{\mathrm{NN}}(t)}{C_{\mathrm{NN}}(t+1)}\right)
$$
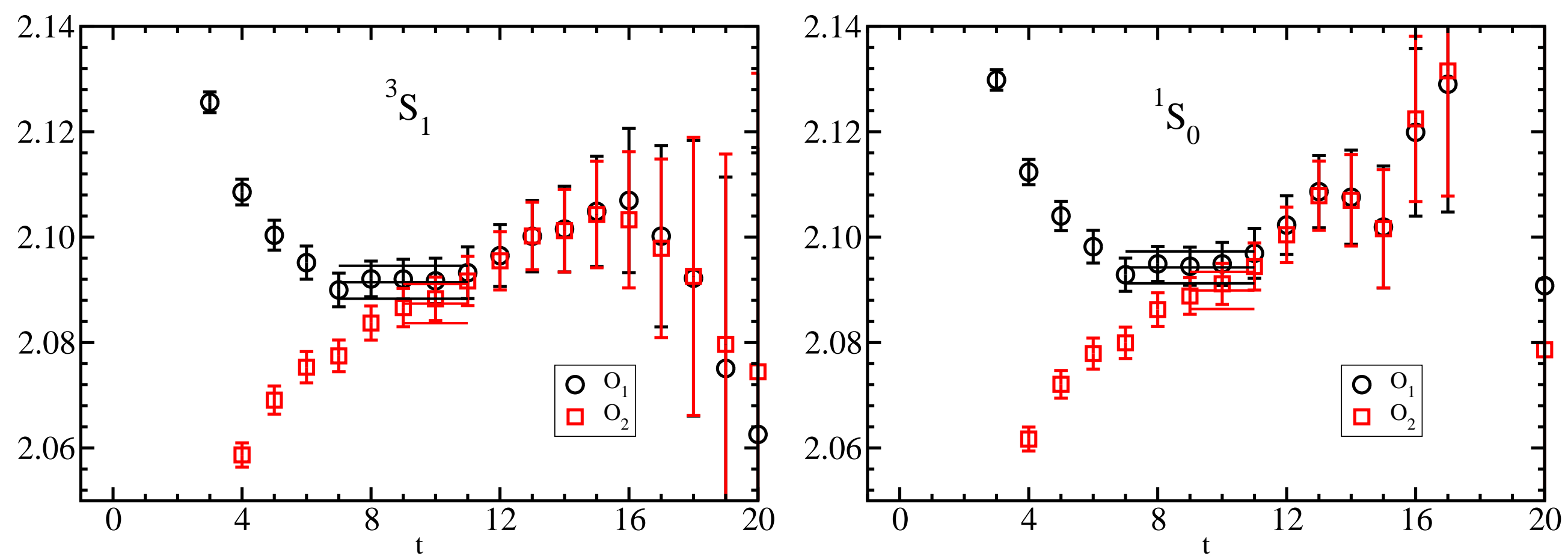

- Clear signal in $t<12$, but larger error in $t \geq 11$

- consistent plateaus in $8 \lesssim t \leq 11$ 
2. Two-state analysis

Effective two-nucleon energy after diagonalization at $L=6.1 \mathrm{fm}$

$$
\begin{gathered}
\bar{E}_{\alpha}(t)=\log \left(\frac{\lambda_{\alpha}(t)}{\lambda_{\alpha}(t+1)}\right), \operatorname{Diag}\left[G^{-1}\left(t_{0}\right) G(t)\right]=\lambda(t) \text { with } t_{0}=6 \\
G_{i j}(t)=\left\langle 0\left|O_{i}(t) O_{j}(0)\right| 0\right\rangle
\end{gathered}
$$
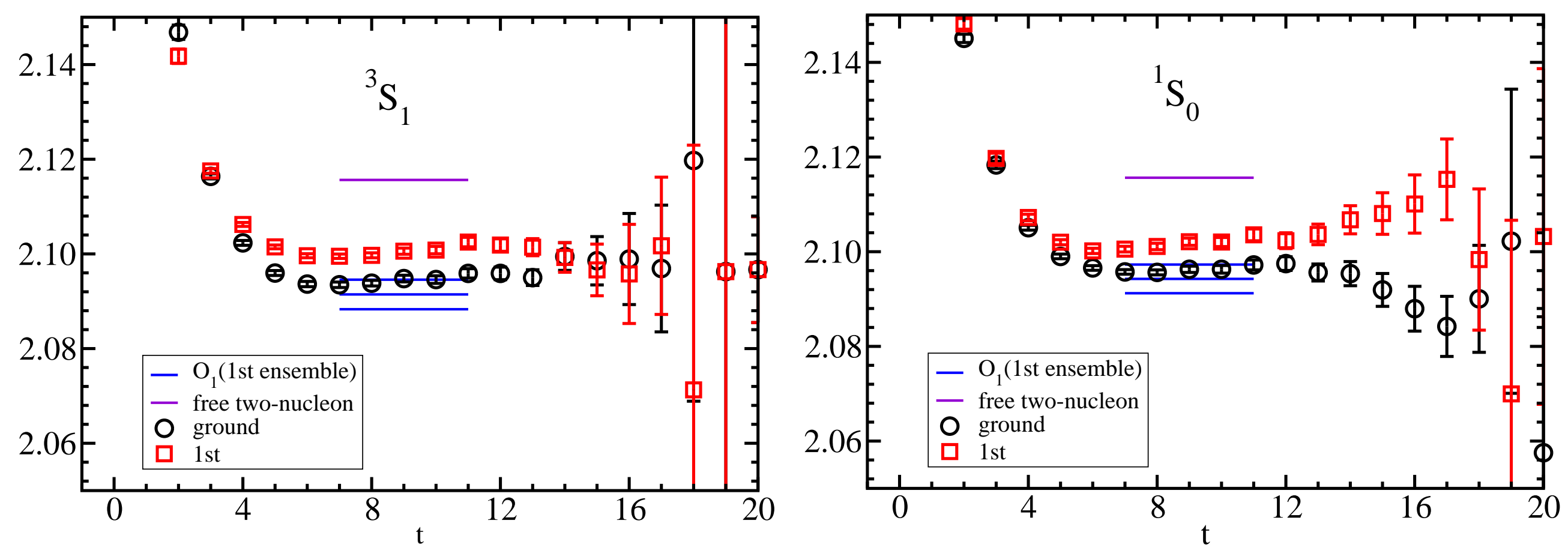

- Close two states, but clearly split

- ground state agree with one in single state analysis

- 1 st excited state below free state $2 \sqrt{M_{N}^{2}+(2 \pi / L)^{2}}$ 


\section{Results}

1. Single state analysis

Effective energy shift $\Delta E_{L}=E_{\mathrm{NN}}-2 m_{N}$ at $L=6.1 \mathrm{fm}$

$$
\Delta E_{L}=\log \left(\frac{R(t)}{R(t+1)}\right), \quad R(t)=\frac{C_{\mathrm{NN}}(t)}{\left(C_{N}(t)\right)^{2}}
$$
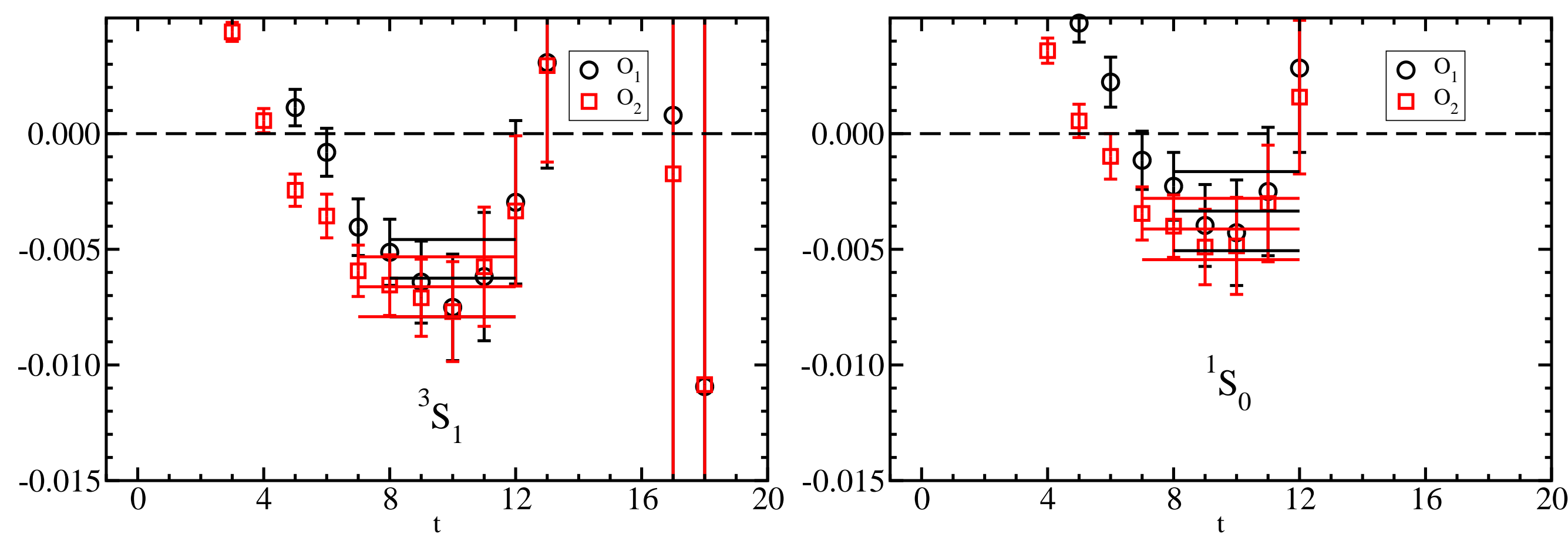

- $\Delta E_{L}<0$ in $8 \lesssim t \leq 11$

- consistent plateaus in $8 \lesssim t \leq 11$ 
Existence of bound state for $a_{0}$

\begin{tabular}{|c|c|c|}
\hline System & w/ bound state & w/o bound state \\
\hline Oth & bound state & scattering state \\
\hline 1 st & scattering state & scattering state \\
\hline$a_{0}$ & $<0$ from 1st & $>0$ from 0 th \\
\hline
\end{tabular}

Bound state exists $\rightarrow a_{0}$ never obtained from Oth state

$$
\Delta E_{L}=E_{N N}^{0}-2 m_{N}=-\frac{4 \pi a_{0}}{m_{N} L^{3}}+\cdots \quad \text { ('86, '91 Lüsher) }
$$

Need to check existence of bound state to calculate $a_{0}$

Two properties in w/ bound state system

1. Oth state energy

2. $a_{0}$ (1st state energy) 
2. 1st excited state energy

Effective energy shift for ground and 1st excited states at $L=6.1 \mathrm{fm}$

$\operatorname{Diag}\left[G^{-1}\left(t_{0}\right) G(t)\right]=\lambda(t)$ with $G_{i j}(t)=\left\langle 0\left|O_{i}(t) O_{j}(0)\right| 0\right\rangle$

$$
\Delta \bar{E}_{L, \alpha}=E_{\alpha}-2 m_{N}=\log \left(\frac{\bar{R}_{\alpha}(t)}{\bar{R}_{\alpha}(t+1)}\right), \quad \bar{R}_{\alpha}(t)=\frac{\lambda_{\alpha}(t)}{\left(C_{N}(t)\right)^{2}}
$$
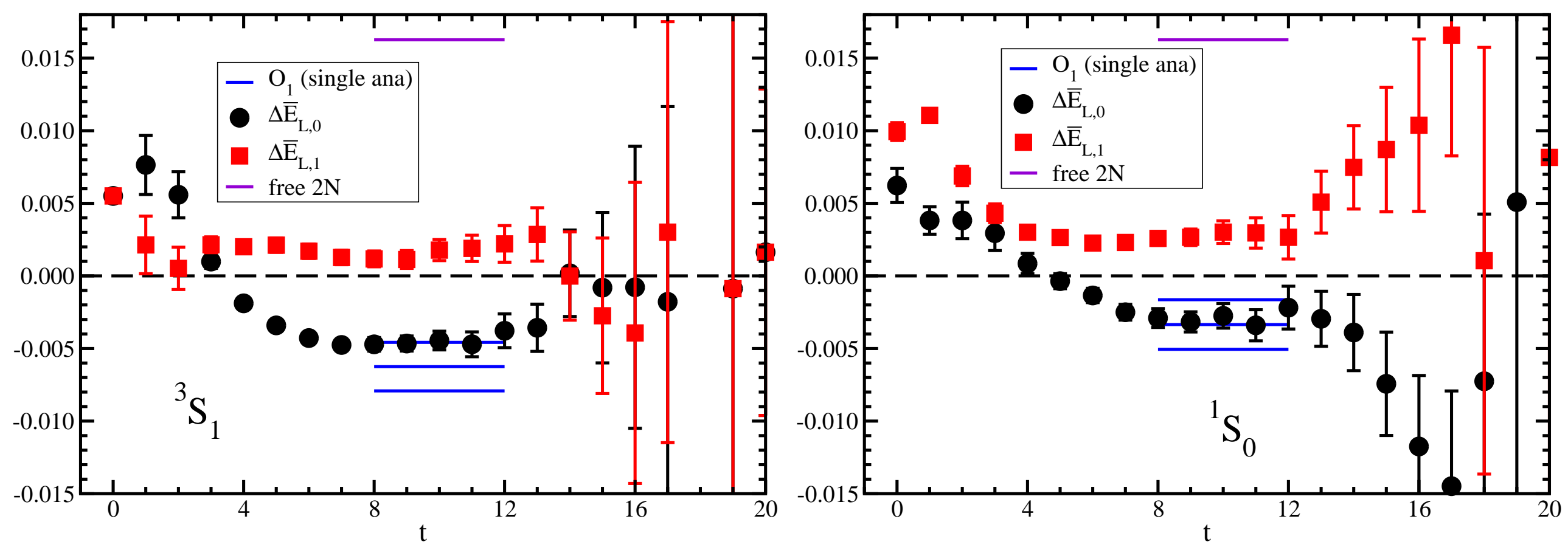

- $\Delta \bar{E}_{L, 0}<0$ and consistent with $\Delta E_{L}$

- small, but $\Delta \bar{E}_{L, 1}>0$ as expected 


\section{Exploratory study of three- and four-nucleon systems}

PACS-CS Collaboration, PRD81:111504(R)(2010)

Traditional method for example ${ }^{4} \mathrm{He}$ channel

$$
\left\langle 0\left|O_{{ }_{4} \mathrm{He}}(t) O_{{ }^{H} \mathrm{He}}^{\dagger}(0)\right| 0\right\rangle=\sum_{n}\left\langle 0\left|O_{{ }_{H} \mathrm{He}}\right| n\right\rangle\left\langle n\left|O_{{ }_{4} \mathrm{He}^{\dagger}}\right| 0\right\rangle e^{-E_{n} t} \underset{t \gg 1}{\longrightarrow} A_{0} e^{-E_{0} t}
$$

Difficulties for multi-nucleon calculation

1. Statistical error

Statistical error $\propto \exp \left(N_{N}\left[m_{N}-\frac{3}{2} m_{\pi}\right] t\right)$

2. Calculation cost

Wick contraction for ${ }^{4} \mathrm{He}=p^{2} n^{2}=(u d u)^{2}(d u d)^{2}: 518400$

3. Identification of bound state on finite volume

Finite volume effect in energy $E_{0}$ 


\section{Exploratory study of three- and four-nucleon systems}

PACS-CS Collaboration, PRD81:111504(R)(2010)

Traditional method for example ${ }^{4} \mathrm{He}$ channel

$$
\left\langle 0\left|O_{4 \mathrm{He}}(t) O_{{ }_{\mathrm{He}}}^{\dagger}(0)\right| 0\right\rangle=\sum_{n}\left\langle 0\left|O_{{ }_{4} \mathrm{He}}\right| n\right\rangle\left\langle n\left|O_{{ }_{\mathrm{He}}}^{\dagger}\right| 0\right\rangle e^{-E_{n} t} \underset{t \gg 1}{\longrightarrow} A_{0} e^{-E_{0} t}
$$

Difficulties for multi-nucleon calculation

1. Statistical error

Statistical error $\propto \exp \left(N_{N}\left[m_{N}-\frac{3}{2} m_{\pi}\right] t\right)$

$\rightarrow$ heavy quark mass corresponding to $m_{\pi}=0.8 \mathrm{GeV}$

c.f. physical pion mass $m_{\pi}=0.135 \mathrm{GeV}$

2. Calculation cost

Wick contraction for ${ }^{4} \mathrm{He}=p^{2} n^{2}=(u d u)^{2}(d u d)^{2}: 518400$

$\rightarrow 1107$ using symmetries

$$
p \leftrightarrow p, n \leftrightarrow n, p \leftrightarrow n, u(d) \leftrightarrow u(d) \text { in } p(n)
$$

3. Identification of bound state on finite volume

Finite volume effect in energy $E_{0}$

$\rightarrow$ Finite volume dependence of $\Delta E=E_{0}-N_{N} m_{N}$ 


\section{Introduction}

Hadron spectrum from $N_{f}=2+1$ QCD

'09 PACS-CS Collaboration

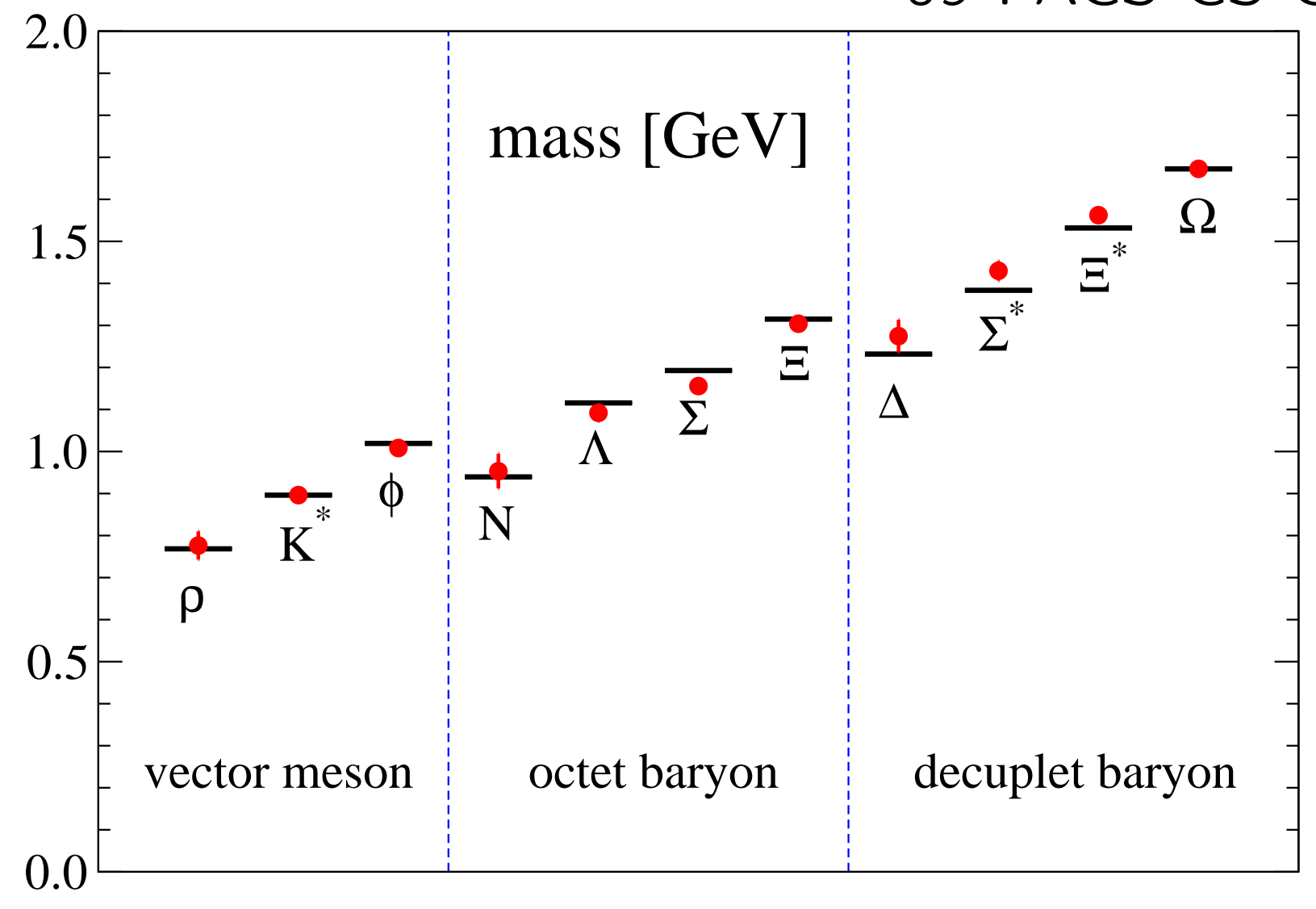

Input: $m_{\pi}, m_{K}$ and $m_{\Omega}$ 


\section{Multi-baryon system from lattice QCD at '09}

1. $\wedge$ M system (Quenched QCD)

'85 Mackenzie \& Thacker '00 Wetzorke et al.

'88 Iwasaki et al.

'02 Wetzorke \& Karsch

'99 Pochinsky et al.

'09 NPLQCD $\left(N_{f}=2+1\right)$

$\mathrm{H}$ dibaryon: unbound

2. NN system ${ }^{3} \mathrm{~S}_{1}$ and ${ }^{1} \mathrm{~S}_{0}$

'95 Fukugita et al. : Quenched QCD

'06 NPLQCD: $N_{f}=2+1$ QCD

'08 Ishii et al. : Quenched and $N_{f}=2+1 \mathrm{QCD}$

'09 NPLQCD : $N_{f}=2+1$ QCD

Deuteron: unbound due to $m_{\pi} \gtrsim 0.3 \mathrm{GeV}$

3. NNN system

'09 NPLQCD: $N_{f}=2+1$ QCD

Triton: likely unbound 


\section{Statistical error}

$C_{4}(t)=\left\langle 0\left|O_{4} \mathrm{He}(t) \bar{O}_{4_{\mathrm{He}}}\right| 0\right\rangle \underset{t \gg 1}{\longrightarrow} A_{0} e^{-E_{0} t}$

Rough estimate of noise-to-signal ratio for $N_{N^{-}}$nucleon channel

$$
\frac{\delta C_{N_{N}}(t)}{C_{N_{N}}(t)} \propto \frac{1}{\sqrt{N_{\text {meas }}}} \exp \left(N_{N}\left[m_{N}-\frac{3}{2} m_{\pi}\right] t\right)
$$

$m_{\pi}$ : proportional to (quark mass) ${ }^{1 / 2}$

$\begin{array}{cl}\text { quark mass } & \rightarrow \text { small } \\ N_{N} & \rightarrow \text { large } \\ N_{\text {meas }} & \rightarrow \text { small }\end{array} \Rightarrow \frac{\delta C_{N_{N}}(t)}{C_{N_{N}}(t)} \rightarrow$ large $\Rightarrow \frac{\delta E_{0}}{E_{0}} \rightarrow$ large

Avoid large statistical fluctuation

- unphysically heavy quark mass $m_{\pi}=0.8 \mathrm{GeV}$ and $m_{N}=1.62 \mathrm{GeV}$

- larger number of measurements than typical calculation

Lighter quark mass

$\rightarrow$ new method or much larger number of measurements 


\section{Calculation cost}

$$
C_{4}(t)=\left\langle 0\left|O_{4 \mathrm{He}}(t) \bar{O}_{4_{\mathrm{He}}}(0)\right| 0\right\rangle \text { with } O_{4_{\mathrm{He}}}=p^{2} n^{2}=[u d u]^{2}[d u d]^{2}
$$

Number of Wick contraction $N_{u} ! \times N_{d} !=\left(2 N_{p}+N_{n}\right) ! \times\left(2 N_{n}+N_{p}\right)$ !

$$
\begin{aligned}
& { }^{4} \mathrm{He}: \quad 6 ! \times 6 !=518400 \\
& { }^{3} \mathrm{He}: \quad 5 ! \times 4 !=2880
\end{aligned}
$$$$
\mathrm{NN}: \quad 4 ! \times 2 !=48
$$

but contain identical contractions

Summation of $\sim(\text { color and dirac })^{2 N_{N}}$ in each contraction

Reduction of contractions

Symmetries

$$
p \leftrightarrow p, n \leftrightarrow n \text { in }{ }^{4} \text { He operator }
$$

Isospin all $p \leftrightarrow$ all $n$

Calculate two contractions simultaneously

$u \leftrightarrow u$ in $p$ or $d \leftrightarrow d$ in $n$ 
2. Calculation cost (cont'd)

$C_{4}(t)=\left\langle 0\left|O_{4 \mathrm{He}}(t) \bar{O}_{4_{\mathrm{He}}}(0)\right| 0\right\rangle$ with $O_{4 \mathrm{He}}=p^{2} n^{2}=[u d u]^{2}[d u d]^{2}$

Number of Wick contraction $N_{u} ! \times N_{d} !=\left(2 N_{p}+N_{n}\right) ! \times\left(2 N_{n}+N_{p}\right)$ !

${ }^{4} \mathrm{He}: 6 ! \times 6 !=518400 \longrightarrow 1107$

${ }^{3} \mathrm{He}: 5 ! \times 4 !=2880 \quad \longrightarrow \quad 93$

Summation of $\sim(\text { color and dirac })^{2 N_{N}}$ in each contraction

Further reduction: avoid same calculations of dirac and color indices

Block of three quark propagators $B_{3}$

zero momentum nucleon operator in sink time slice

Blocks of two $B_{3}$

$1,2,3$ dirac contractions carried out

Multi-meson systems : '10 Detmold and Savage

Recursion relations: $R_{n+1}=\left\langle R_{n}\right\rangle \cdot R_{1}-n R_{n} \cdot R_{1}$

Multi-baryon systems : '12 Doi and Endres

Unified contraction algorithm 
3. Identification of bound state in finite volume $C_{4}(t)=\left\langle 0\left|O_{4}{ }_{\mathrm{He}}(t) \bar{O}_{4 \mathrm{He}}\right| 0\right\rangle \underset{t \gg 1}{\longrightarrow} A_{0} e^{-E_{0} t}$

Observed state is whether bound state or not?

Example) Two-particle system

observe small $\Delta E=E-2 m<0$ at single $L$

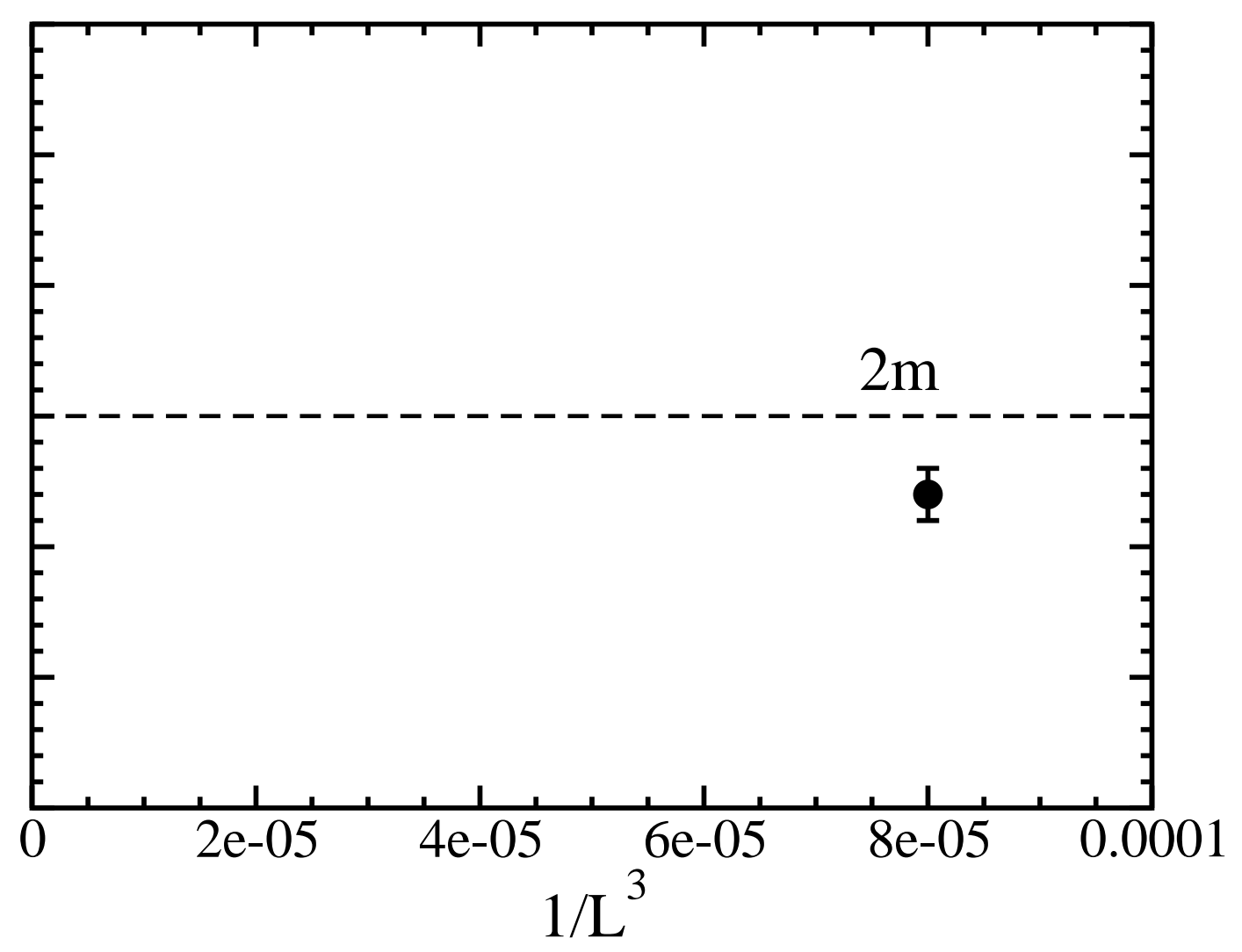


3. Identification of bound state in finite volume (cont'd) Example) Two-particle system

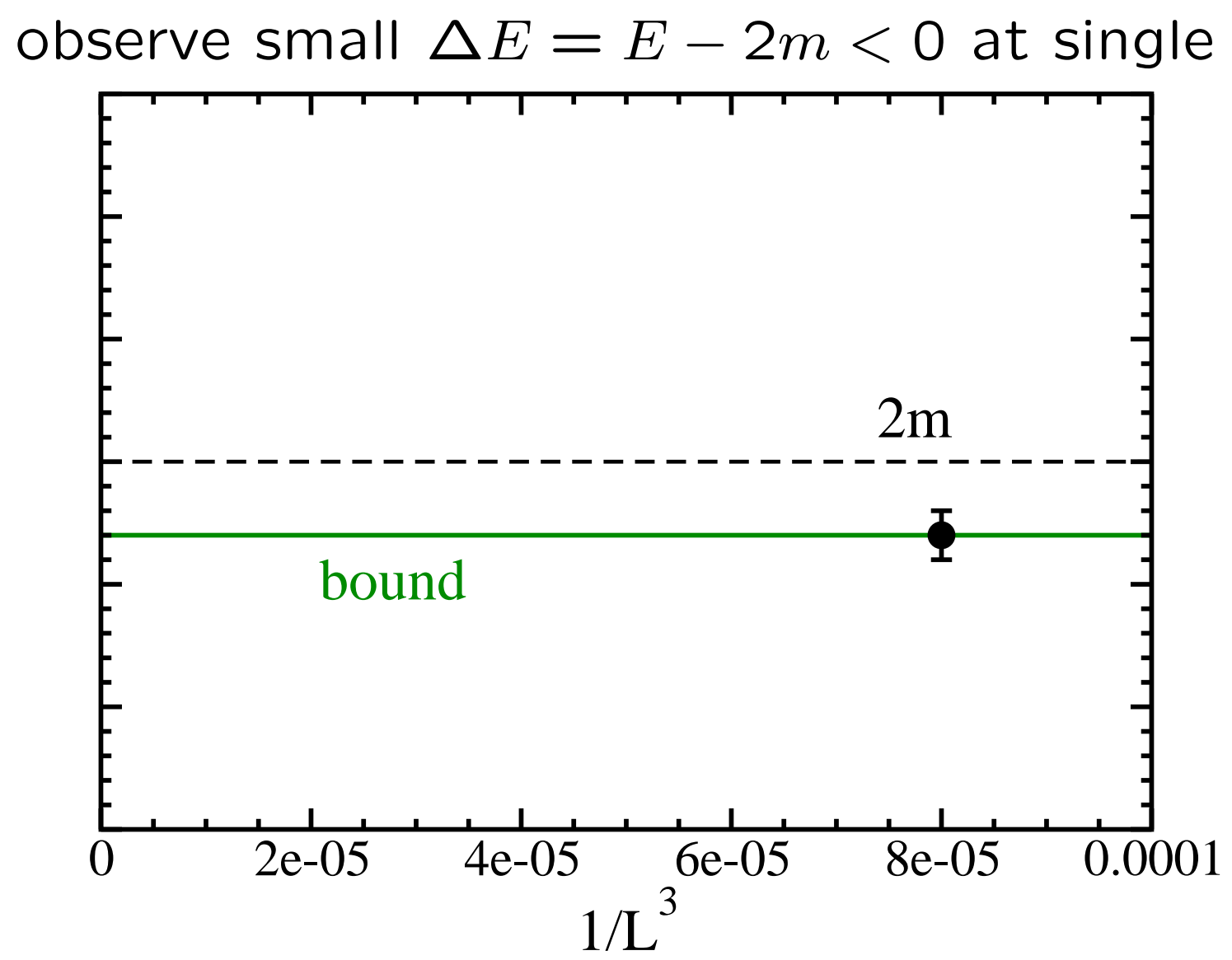

Bound state: $\Delta E=-\Delta E_{\text {bind }}+O\left(\mathrm{e}^{-\gamma L}\right)<0$

Beane et al., PLB585:106(2004), Sasaki \& TY, PRD74:114507(2006) 
3. Identification of bound state in finite volume (cont'd) Example) Two-particle system

$$
\text { observe small } \triangle E=E-2 m<0 \text { at single } L
$$

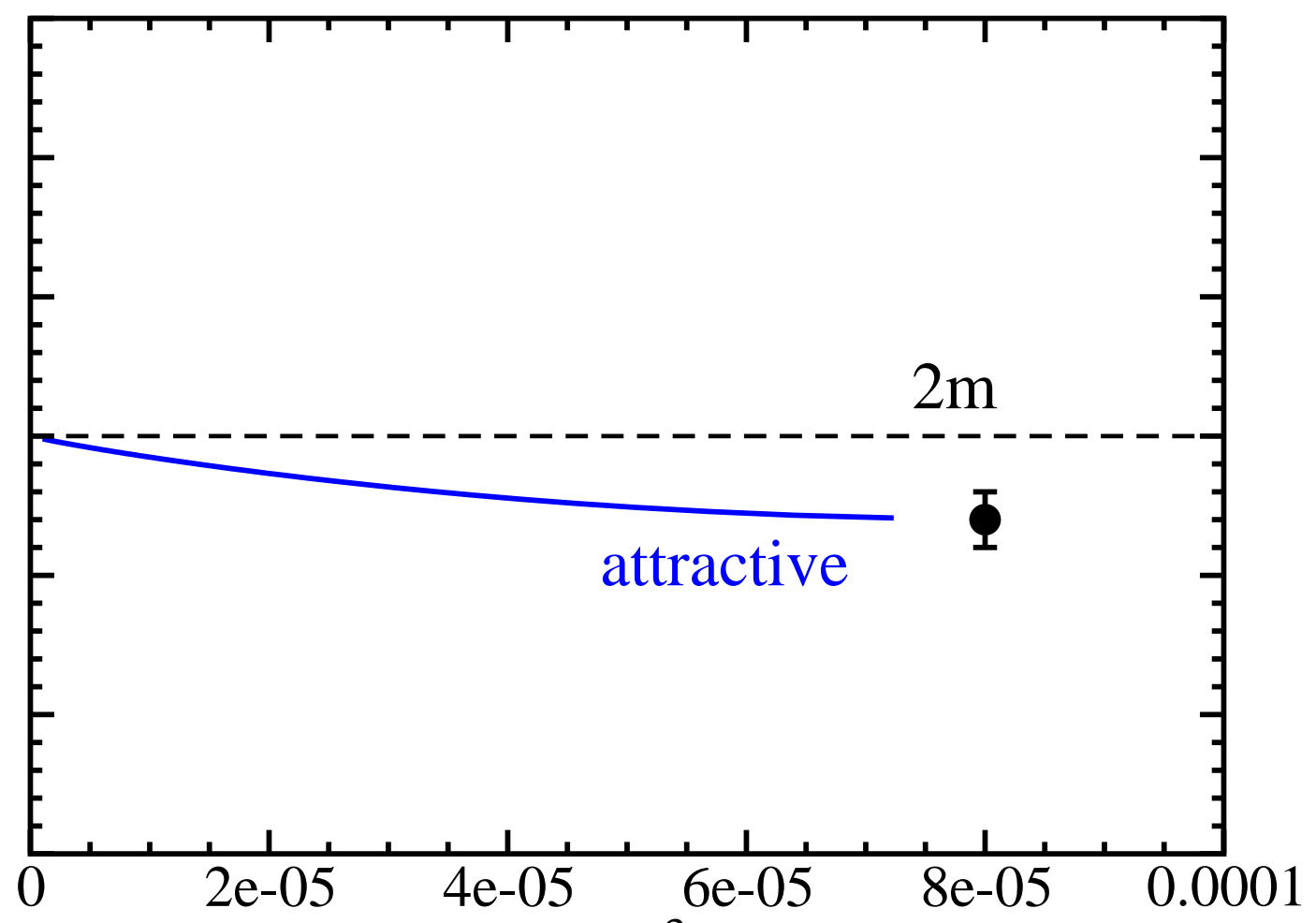

$$
1 / \mathrm{L}^{3}
$$

Attractive scattering state : $\triangle E=O\left(-\frac{a_{0}}{M L^{3}}\right)<0 \quad\left(a_{0}>0\right)$

Lüscher, CMP105:153(1986), NPB354:531(1991)

c.f.) $N$-particle scattering state : $\triangle E=E_{\text {scat }}-N m=O\left(-\frac{{ }_{N} C_{2} a_{0}}{M L^{3}}\right)$

Beane et al., PRD76:074507(2007) 
3. Identification of bound state in finite volume (cont'd) Example) Two-particle system

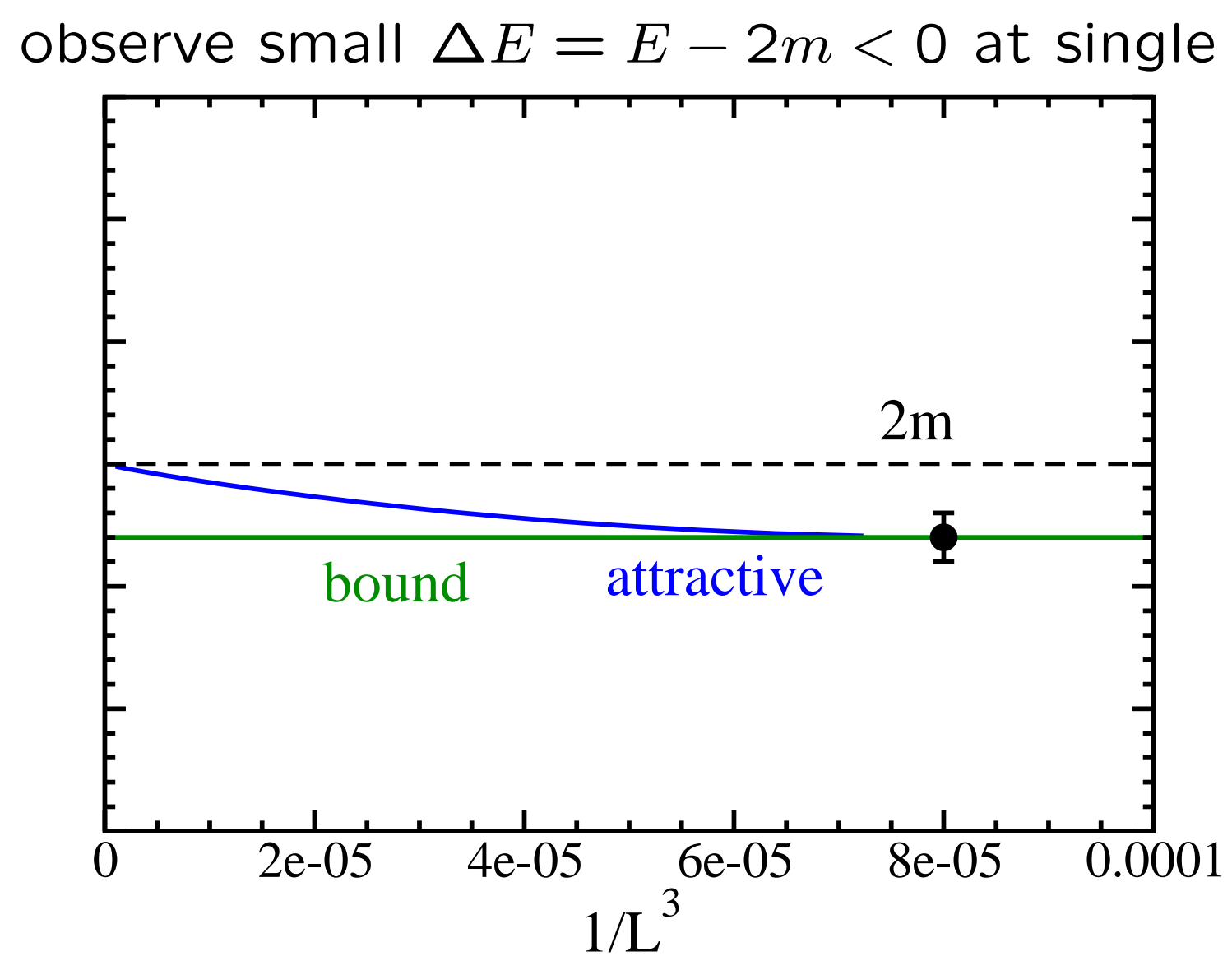

Hard to distinguish at single $L$

Bound state and Attractive scattering state 
3. Identification of bound state in finite volume (cont'd) Example) Two-particle system

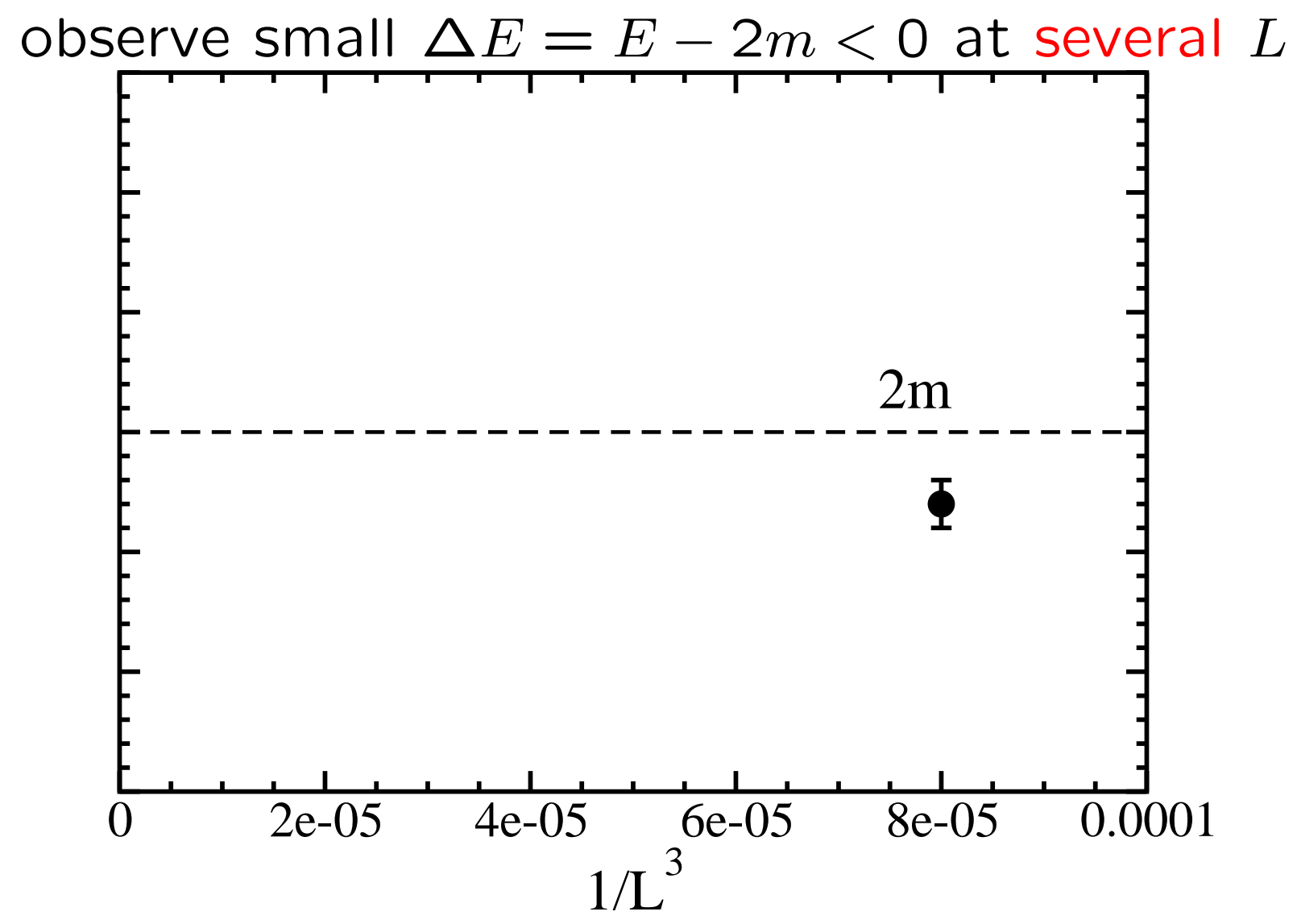


3. Identification of bound state in finite volume (cont'd) Example) Two-particle system

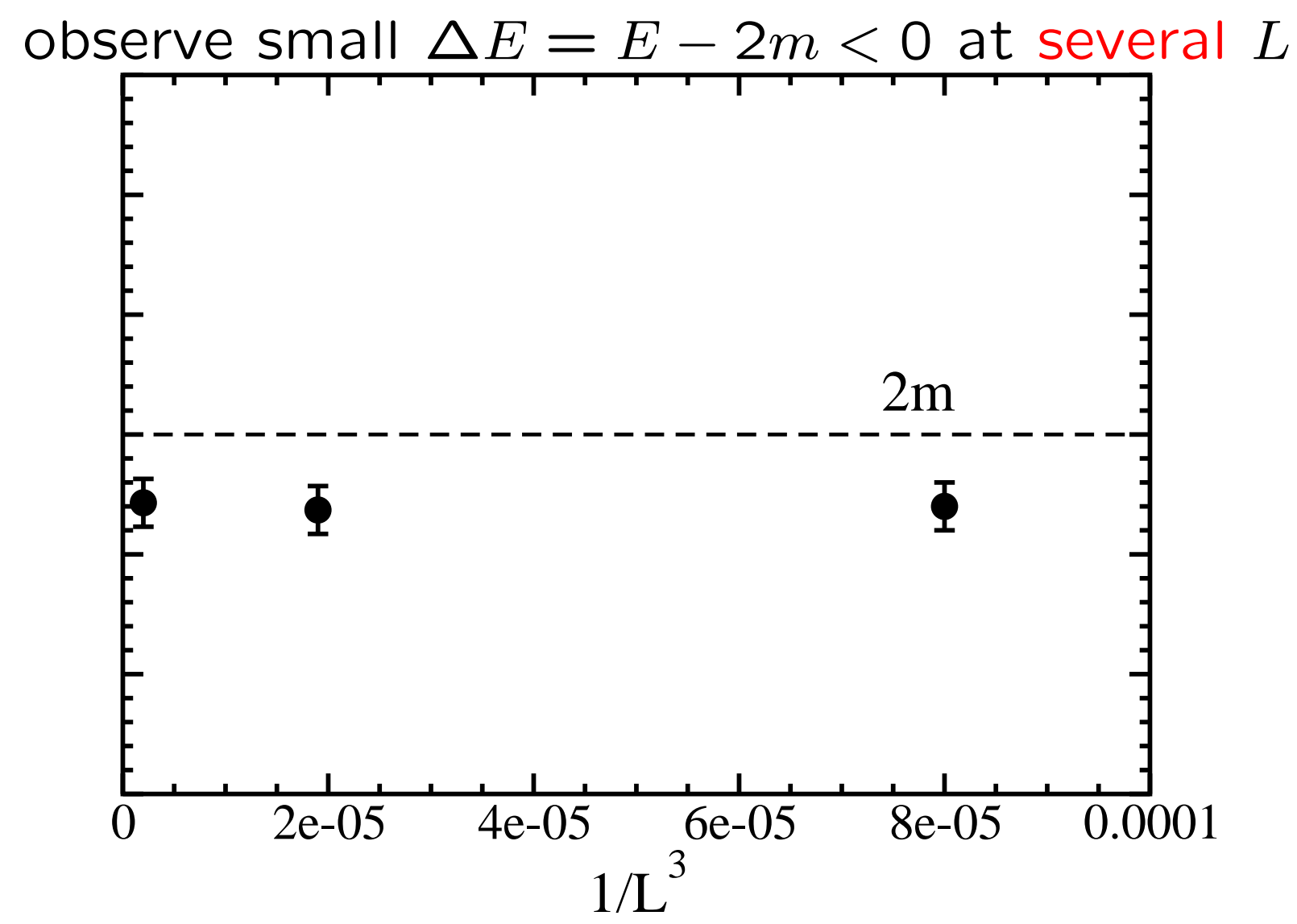


3. Identification of bound state in finite volume (cont'd) Example) Two-particle system

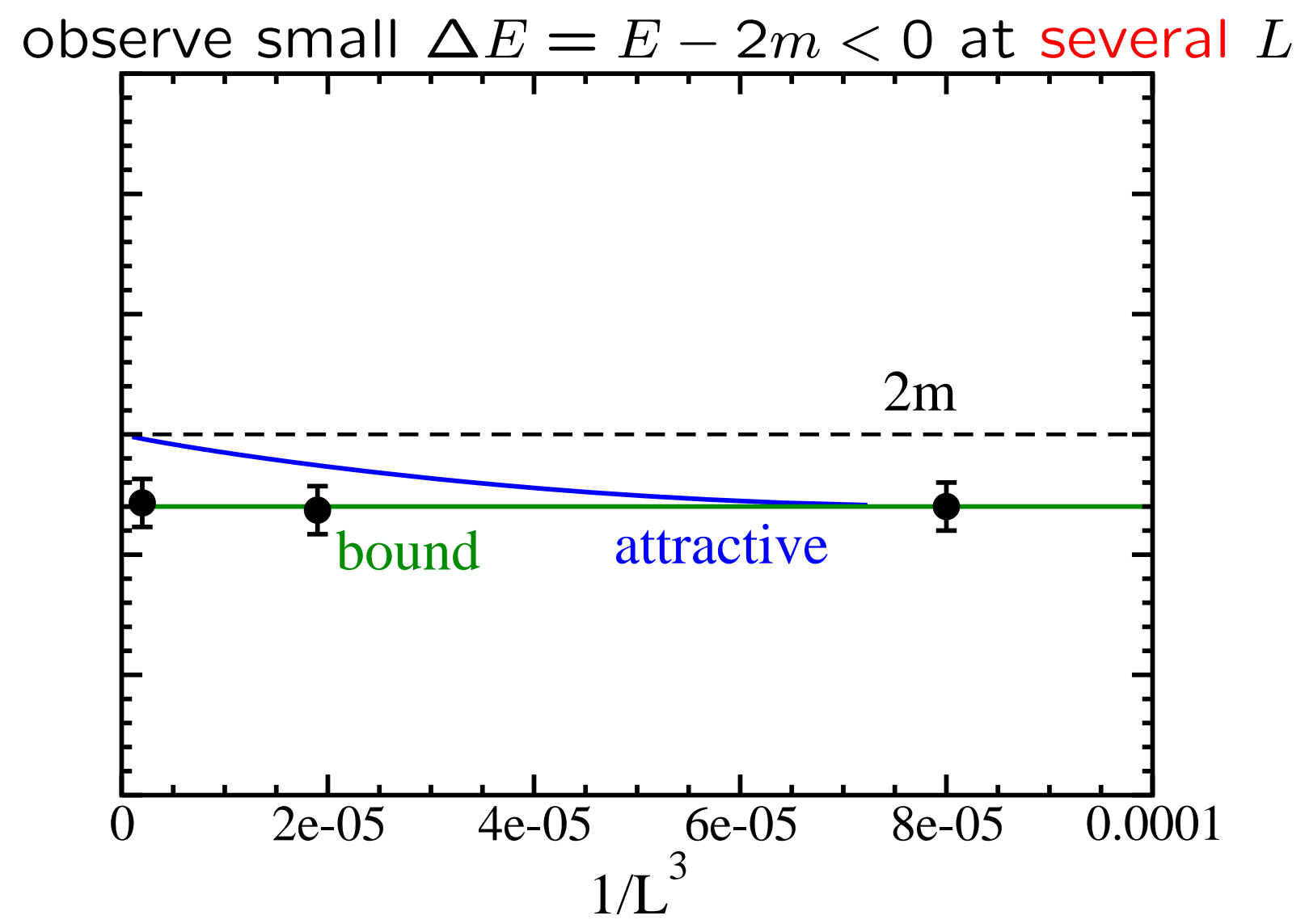

Identify bound state from volume dependence of $\Delta E$

observe constant in infinite volume limit with $L=3.1,6.1,12.3 \mathrm{fm}$

Other methods: spectral weight: Mathur et al., PRD70:074508(2004) anti-periodic boundary.: Ishii et al., PRD71:034001(2005) 
2. Problems of multi-nucleon bound state NN channels

1. Statistical error

Not so severer than 3- and 4-nucleon channels

$$
\frac{\delta C_{N_{N}}(t)}{C_{N_{N}}(t)} \propto \frac{1}{\sqrt{N_{\text {meas }}}} \exp \left(N_{N}\left[m_{N}-\frac{3}{2} m_{\pi}\right] t\right)
$$

2. Calculation cost

Not so severer than 3- and 4-nucleon channels \# of contraction $<10$ after reduction

3. Identification of bound state in finite volume Similar to 3- and 4-nucleon channels

Bound state in simplest multi-nucleon system, NN system? 
Scattering length $a_{0}$ in NN system at '09
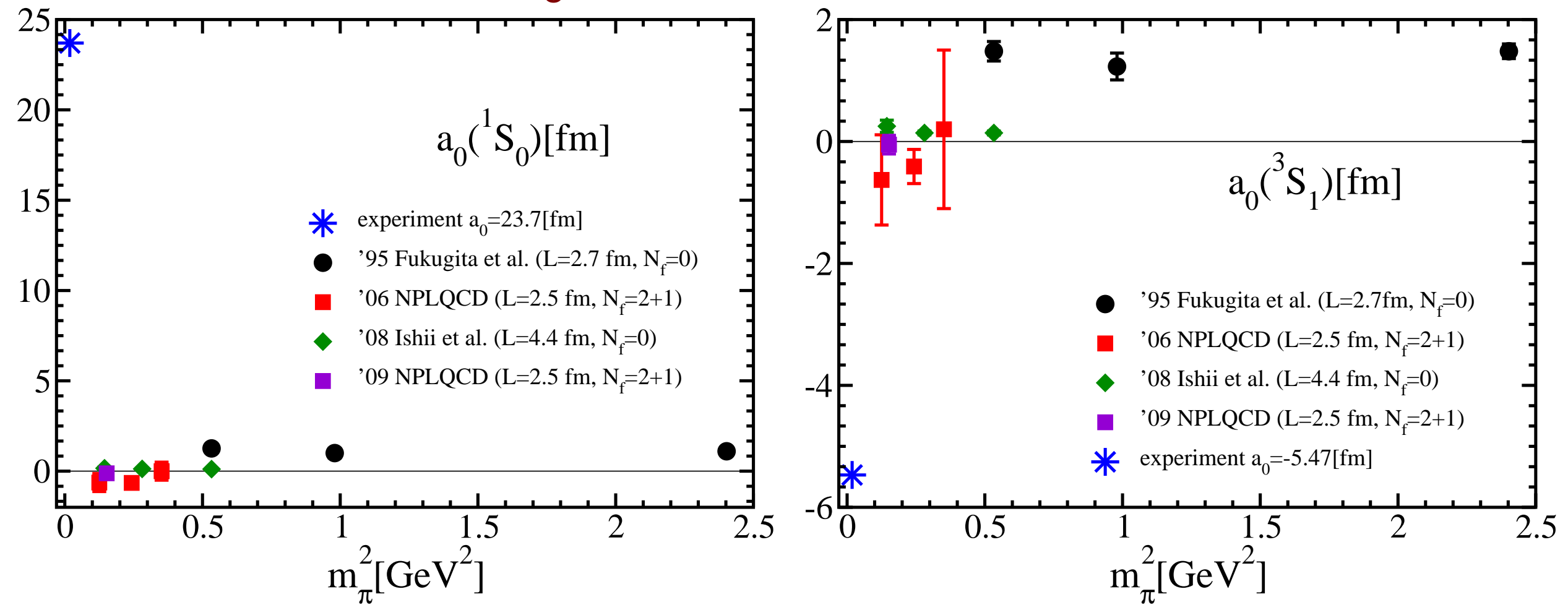

$a_{0}$ : Far from experiments due to $m_{\pi} \gtrsim 0.3 \mathrm{GeV}$

Assumption: Deuteron in ${ }^{3} S_{1}$ channel unbound due to $m_{\pi} \gtrsim 0.3 \mathrm{GeV}$

Aim of this work: check assumption by simpler method c.f. using nuclear force '09 HALQCD 
Existence of bound state for $a_{0}$

\begin{tabular}{|c|c|c|}
\hline System & w/ bound state & w/o bound state \\
\hline Oth & bound state & scattering state \\
\hline 1 st & scattering state & scattering state \\
\hline$a_{0}$ & $<0$ from 1st & $>0$ from 0 th \\
\hline
\end{tabular}

Bound state exists $\rightarrow a_{0}$ never obtained from Oth state

by Lüscher's finite volume method

$$
\Delta E_{L}=E_{N N}^{0}-2 M=-\frac{4 \pi a_{0}}{M L^{3}}+\cdots \quad(' 86, \text { '91 Lüsher })
$$

Need to check existence of bound state to calculate $a_{0}$

Two methods

1. Volume dependence of Oth state

2. Properties of 1st state energy 
Properties of 1st excited state energy in finite volume '06 Sasaki \& TY bound state exists $\rightarrow 1$ st excited state $=$ scattering state with $a_{0}<0$ $\rightarrow \Delta E_{L, 1}>0$ at finite $L$

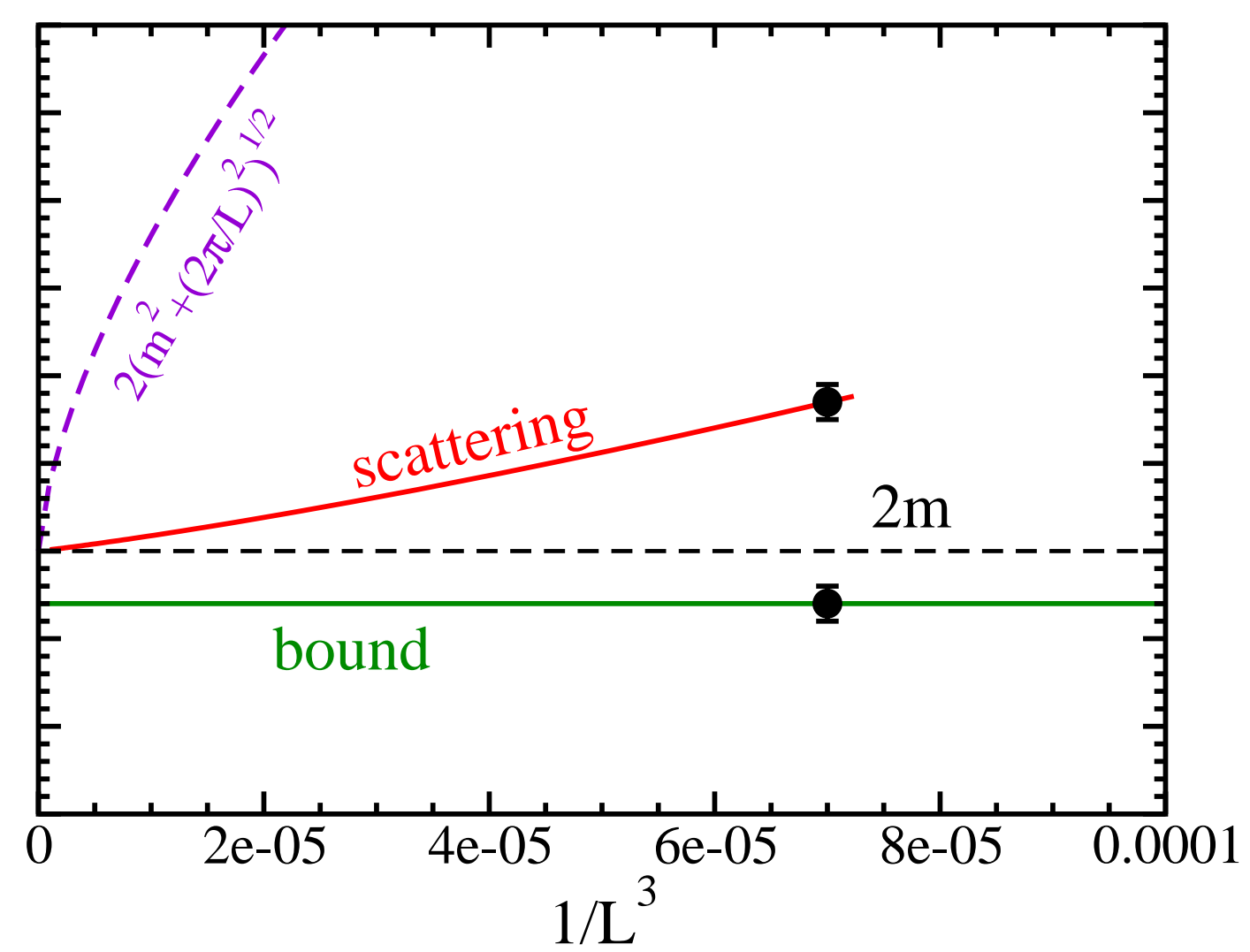

Scattering state : $\Delta E_{L}=O\left(-\frac{a_{0}}{m L^{3}}\right)>0 \quad\left(a_{0}<0\right) \quad$ '86, '91 Lüscher 1st excited state $\leftarrow$ diagonalization method '90 Lüscher \& Wolff 


\section{Results}

NN $\left({ }^{3} \mathrm{~S}_{1}\right.$ and $\left.{ }^{1} \mathrm{~S}_{0}\right)$ channels $\Delta E_{L, 1}=E_{1}-2 m_{N}$

PACS-CS Collaboration, PRD84:054506(2011)

Volume dependence of $\Delta E_{L, 1}$

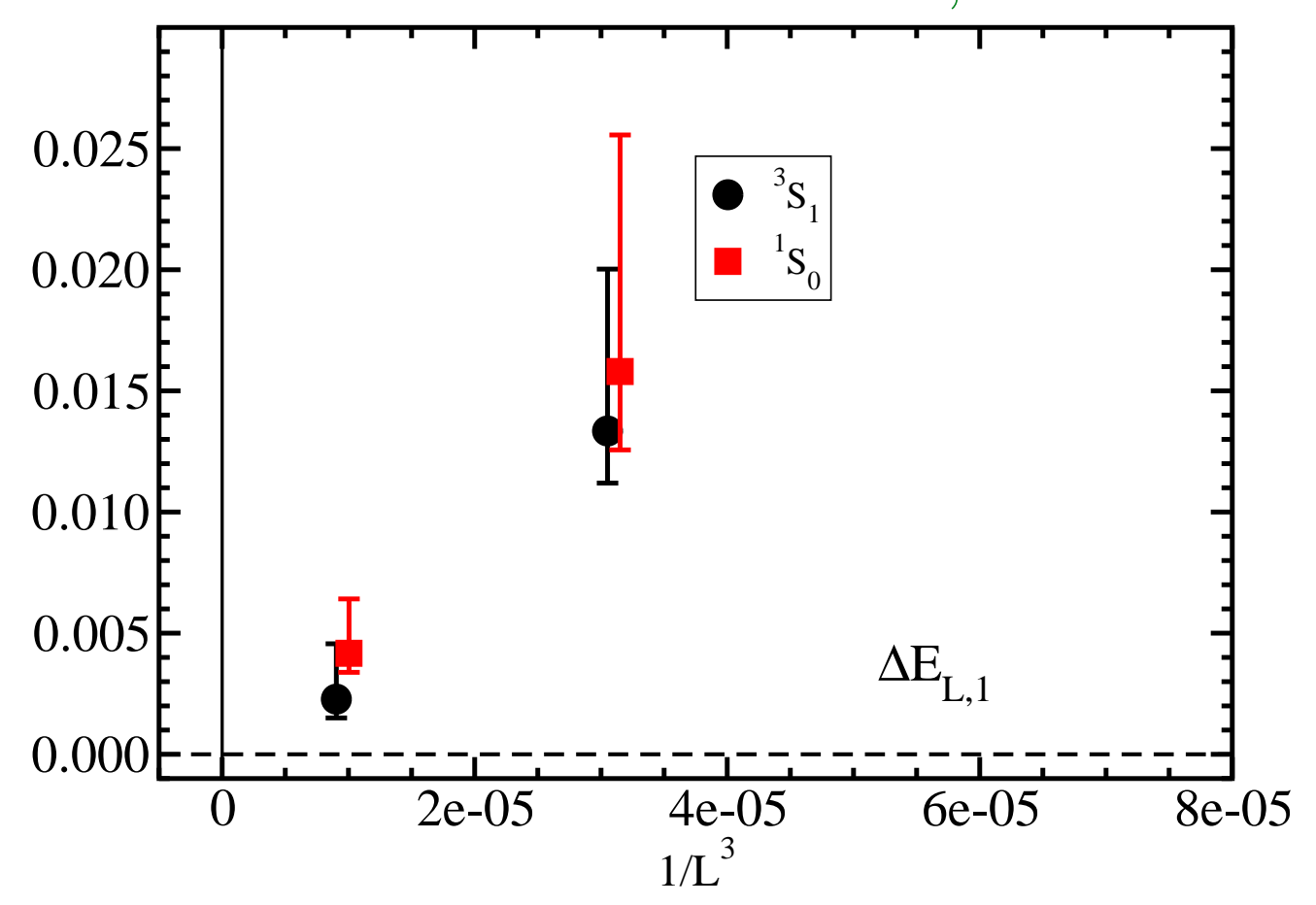

$\triangle E_{L, 1}>0$ and $1 / L^{3}$ tendency

Scattering length $a_{0} \mathrm{fm}$

\begin{tabular}{|c|c|}
\hline $\mathrm{L}[\mathrm{fm}]$ & ${ }^{3} \mathrm{~S}_{1}$ \\
\hline 3.1 & $-1.5(0.2)\left(\begin{array}{l}+0.2 \\
-1.4\end{array}\right)$ \\
\hline 6.1 & $-1.05(24)\left(\begin{array}{l}+0.05 \\
-0.65\end{array}\right)$ \\
\hline $\mathrm{L}[\mathrm{fm}]$ & ${ }^{1} \mathrm{~S}_{0}$ \\
\hline 3.1 & $-1.8(0.3)\left(\begin{array}{l}+0.4 \\
-12.9\end{array}\right)$ \\
\hline 6.1 & $-1.62(24)\left(\begin{array}{l}+0.01 \\
-0.75\end{array}\right)$ \\
\hline
\end{tabular}

Observe expected properties of 1st excited state 


\title{
Computing the $\mathrm{K}_{\mathrm{L}}$ - $\mathrm{K}_{s}$ mass difference in Lattice QCD
}

\author{
Jianglei Yu \\ RBC and UKQCD Collaborations
}

May 15 20I2, BNL 


\section{Outline}

- Introduction

- Summary of the method

- Setup of the calculation

- Short distance effect

- Long distance effect

- Mass difference

- Conclusion and future plans 


\section{Introduction}

- $K^{0}-\bar{K}^{0}$ mixing :

$\Delta M_{K}=3.4583(6) \times 10^{-12} \mathrm{Mev}$

- Perturbative calculation can explain $70 \%$ of the mass difference

- Long distance effect

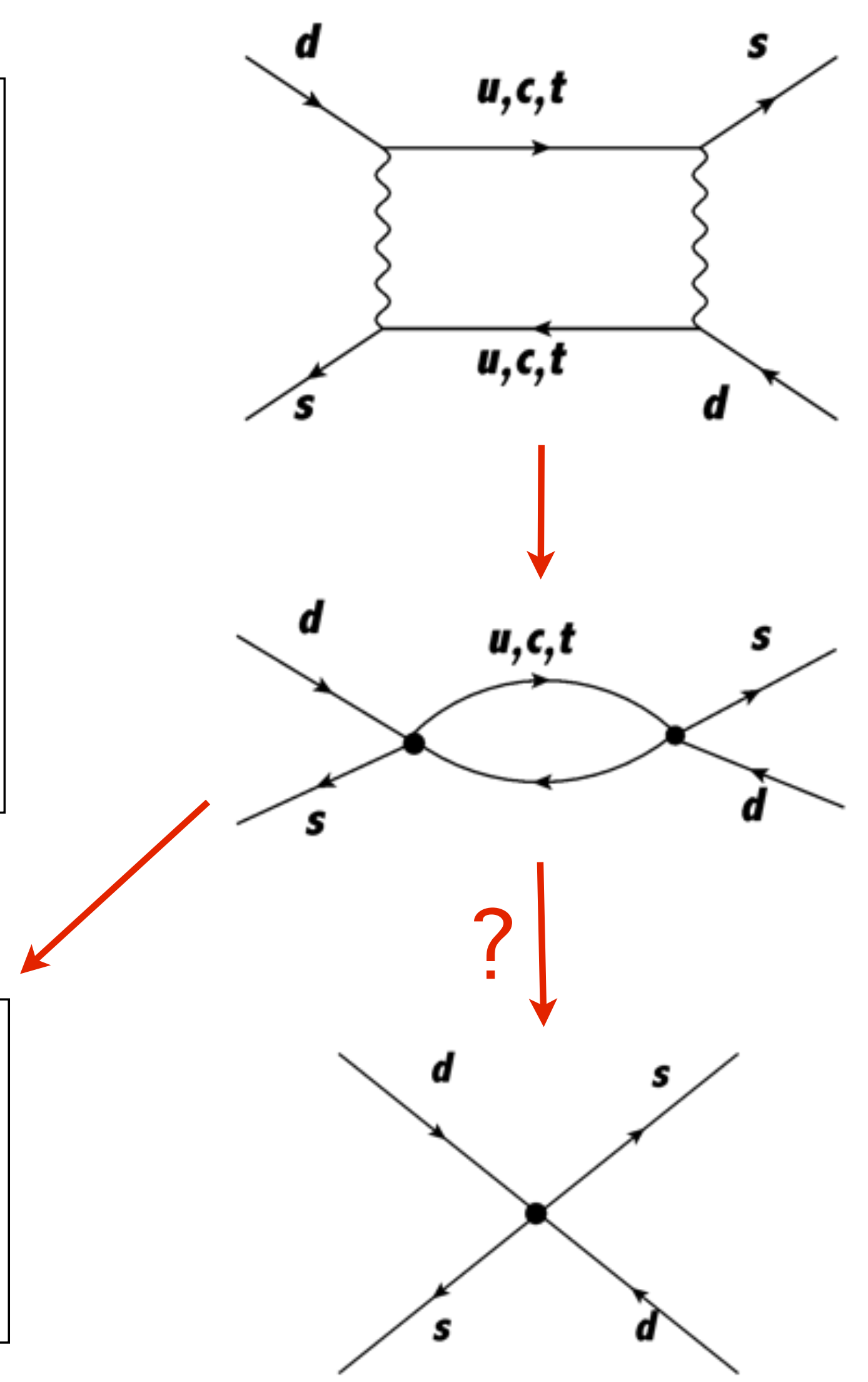

Directly evaluate second order weak process on a Lattice 


\section{Summary of the method}

Neglect $C P$ violation, $K_{L}-K_{S}$ mass difference is given by :

$$
\Delta M_{K}=2 \mathscr{P} \int d E_{n} \frac{\left\langle\bar{K}^{0}\left|H_{W}\right| n\right\rangle\left\langle n\left|H_{W}\right| K^{0}\right\rangle}{M_{K}-E_{n}}
$$

Two parts to calculate $\Delta M_{K}$ :

$\checkmark$ Evaluate lattice four point function

- Correct finite volume effect
Principal part should be taken when dealing with $M_{K}=E_{n}$ singularity

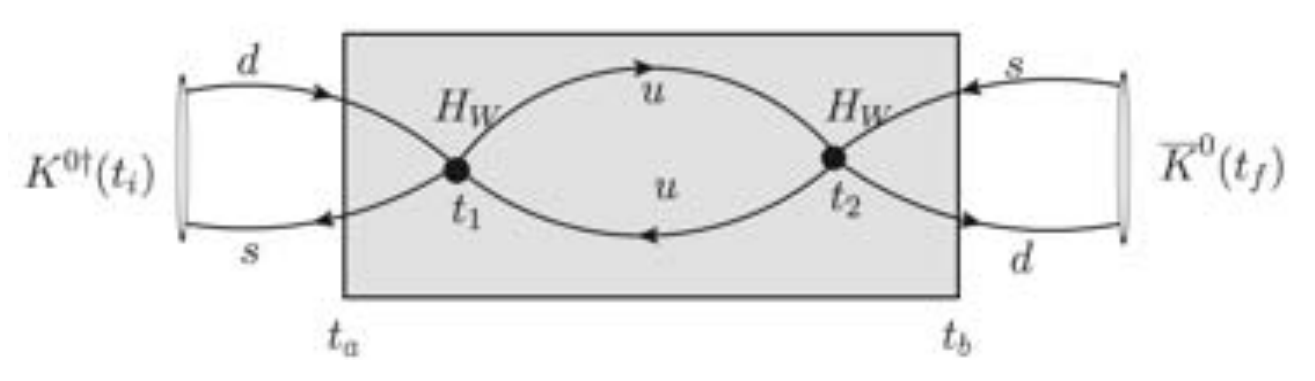




\section{Lattice four point function}

Four point correlator :

$$
G\left(t_{f}, t_{1}, t_{2}, t_{i}\right)=\left\langle\overline{K^{0}}\left(t_{f}\right) H_{W}\left(t_{2}\right) H_{W}\left(t_{1}\right) K^{0 \dagger}\left(t_{i}\right)\right\rangle
$$

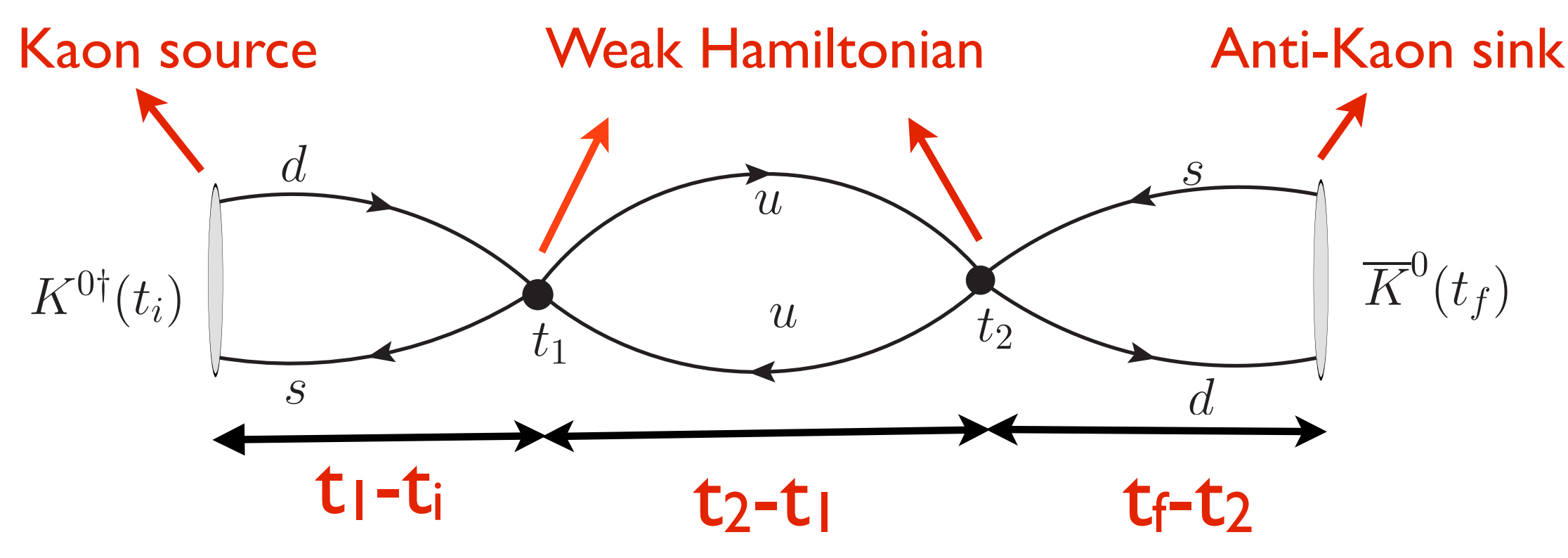

- $t_{1}-t_{i}$ and $t_{f}-t_{2}$ should be sufficiently large to get a kaon

- Fix $t_{i}$ and $t_{f}$, correlator depends only on $t_{2}-t_{1}$

- Refer to this quantity as unintegrated correlator 


\section{Integrated Correlator}

Integrate the unintegrated correlator over a time interval :

$$
\mathscr{A}=\frac{1}{2} \sum_{t_{1}=t_{a}}^{t_{b}} \sum_{t_{2}=t_{a}}^{t_{b}}\left\langle\overline{K^{0}}\left(t_{f}\right) H_{W}\left(t_{2}\right) H_{W}\left(t_{1}\right) K^{0 \dagger}\left(t_{i}\right)\right\rangle
$$

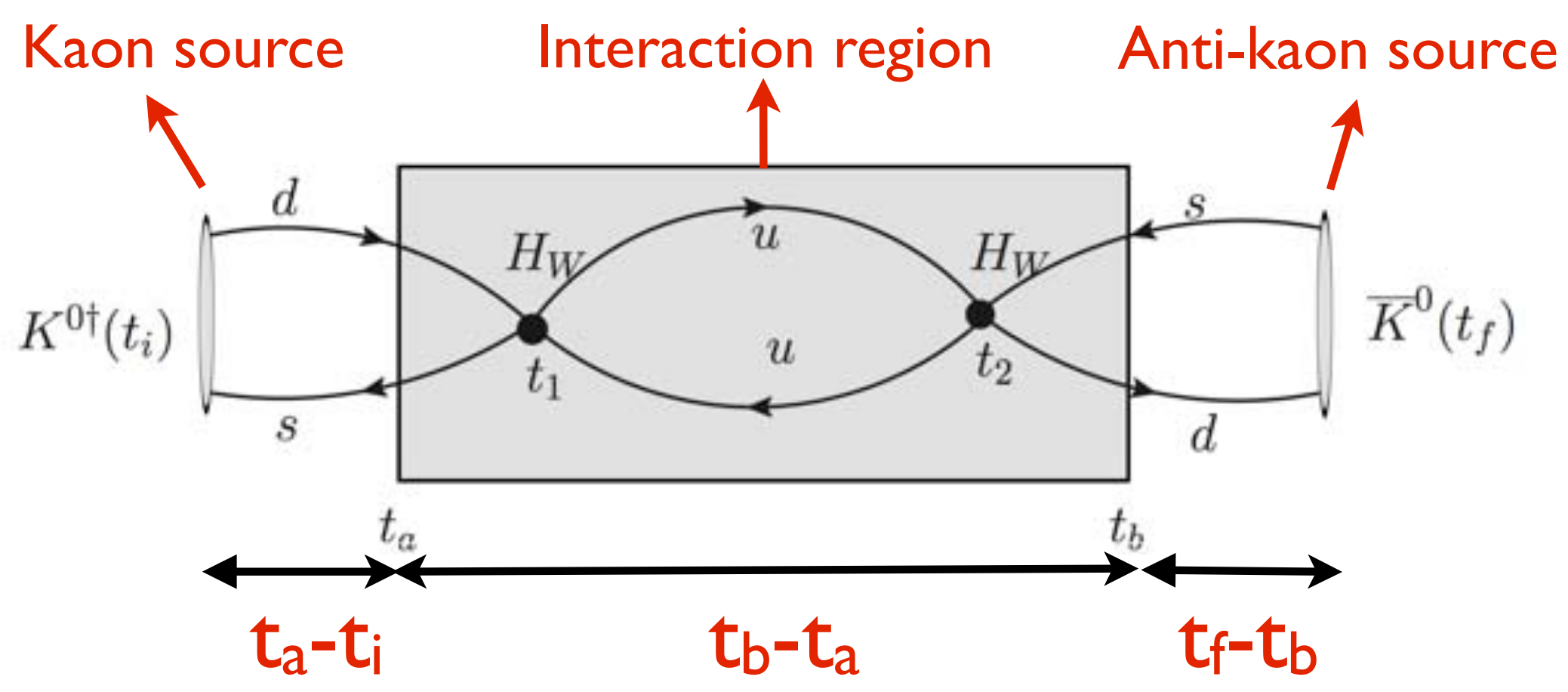

- $t_{f}-t_{b}$ and $t_{a}-t_{j}$ should be sufficiently large to get a kaon

- Fix $t_{i}$ and $t_{f}$, correlator depends only on $t_{b}-t_{a}$

- Refer to this quantity as integrated correlator 
After inserting a sum over intermediate states one obtains :

$$
\begin{aligned}
\mathscr{A} & =N_{K}^{2} e^{-M_{K}\left(t_{f}-t_{i}\right)}\left\{\sum_{n \neq n_{0}} \frac{\left\langle\bar{K}^{0}\left|H_{W}\right| n\right\rangle\left\langle n\left|H_{W}\right| K^{0}\right\rangle}{M_{K}-E_{n}}\left(-T-\frac{1}{M_{K}-E_{n}}+\frac{e^{\left(M_{K}-E_{n}\right) T}}{M_{K}-E_{n}}\right)\right. \\
& \left.+\frac{1}{2}\left\langle\bar{K}^{0}\left|H_{W}\right| n_{0}\right\rangle\left\langle n_{0}\left|H_{W}\right| K^{0}\right\rangle T^{2}\right\} \backslash
\end{aligned}
$$

5

$T=t_{b}-t_{a}+I$ is the integration range, the terms in correlator fall into five categories:

I. Linear term, the coefficient gives finite volume approximation to $\Delta M_{K}$

2. Constant term, which is trival

3. Exponential decreasing term, come from states $E_{n}>M_{K}$

4. Exponential increasing term, come from states $E_{n}<M_{K}$ Subtract from

15. Quadratic term, come from state $E_{n}=M_{K}$ 


\section{Correct finite volume effect}

Finite Volume :

- $\Delta M_{K}$ is given by finite volume sum

- Tune lattice so

$E_{\pi \pi}=M_{K}$

- Use degenerate perturbation theory, relate $\mathrm{E}_{\pi \pi}$ with $\Delta M_{K}$
Infinite volume :

- $\Delta M_{K}$ is given by infinite volume integral

- $\pi-\pi$ phase shift relate to $\Delta M_{K}$ through kaon pole

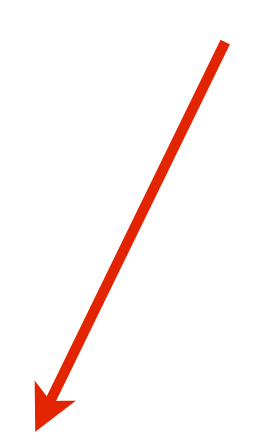

Luscher condition:

$$
\phi(E)+\delta_{0}(E)+\delta_{W}(E)=n \pi
$$




\section{Result for $\Delta M_{K}$}

Leading order term

- Tune volume so $M_{K}=E_{\pi \pi}$

- Remove $\pi$ Tा state

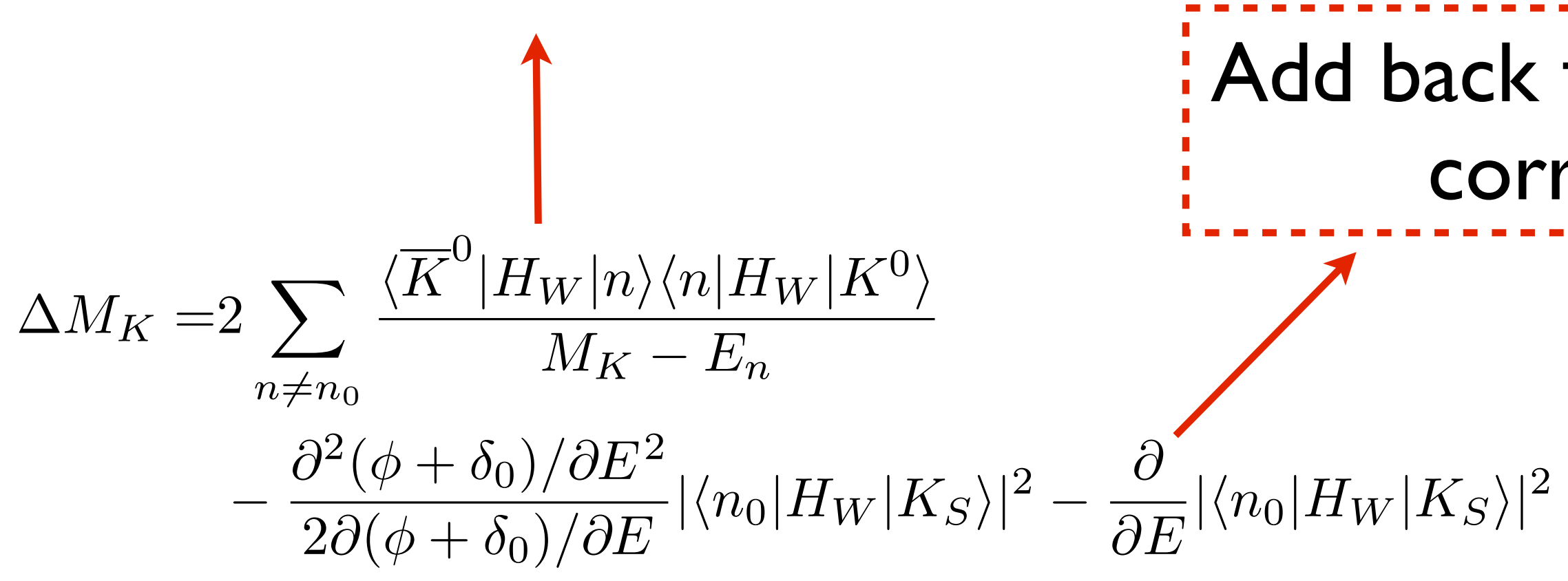

The finite volume correction
is not done in this calculation 


\section{Setup of the calculation}

Lattice ensemble :

- $16^{3} \times 32 \times 16,2+1$ flavor DWF

- Inverse lattice spacing I.73(3) Gev

- $M_{\pi}=42 \mathrm{I} \mathrm{Mev}, M_{\mathrm{K}}=559 \mathrm{Mev}$

- 800 configurations, each separated by 10 time units

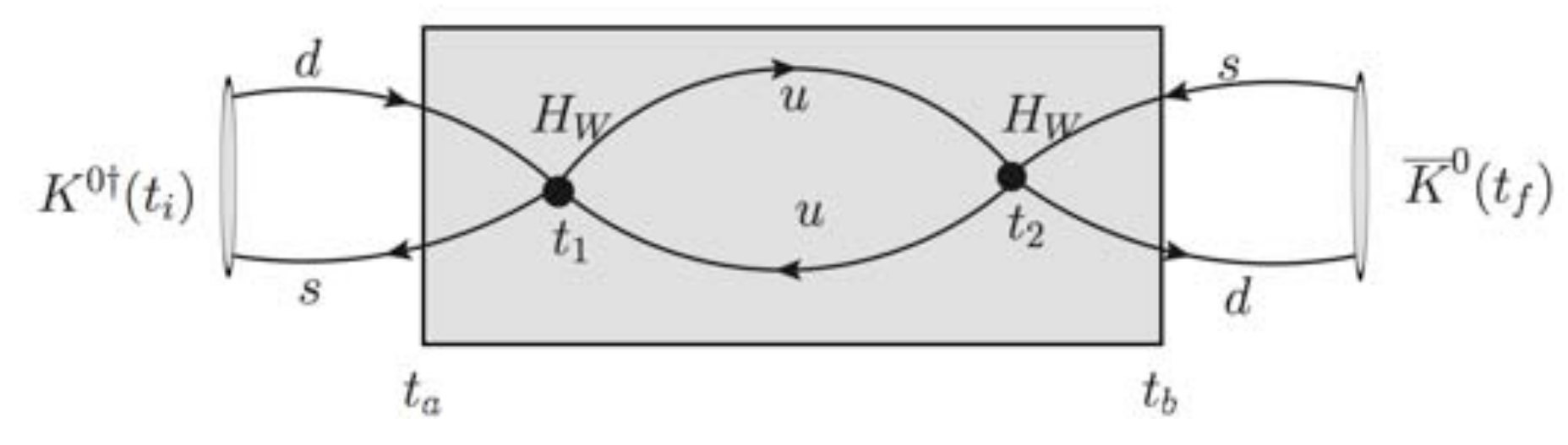

- Kaon wall sources at $\mathrm{t}_{\mathrm{i}}=0$ at $\mathrm{t}_{\mathrm{f}}=27$

- Weak Hamiltonian act between $t_{a}=4$ and $t_{b}=23$ 


\section{Effective weak Hamiltonian}

The $\Delta S=$ I effective weak Hamiltonian in a 4 flavor theory:

$$
H_{W}=\frac{G_{F}}{\sqrt{2}} \sum_{q, q^{\prime}=u, c} V_{q d} V_{q^{\prime} s}^{*}\left(C_{1} Q_{1}^{q q^{\prime}}+C_{2} Q_{2}^{q q^{\prime}}\right)
$$

Here we only include current-current operators :

$$
\begin{aligned}
& Q_{1}^{q q \prime}=\left(\bar{s}_{i} d_{i}\right)_{V-A}\left(\bar{q}_{j} q_{j}^{\prime}\right)_{V-A} \\
& Q_{2}^{q q \prime}=\left(\bar{s}_{i} d_{j}\right)_{V-A}\left(\bar{q}_{j} q_{i}^{\prime}\right)_{V-A}
\end{aligned}
$$

All the penguin operators are neglected, since they are highly suppressed because of GIM cancellation 


\section{Type I diagrams}

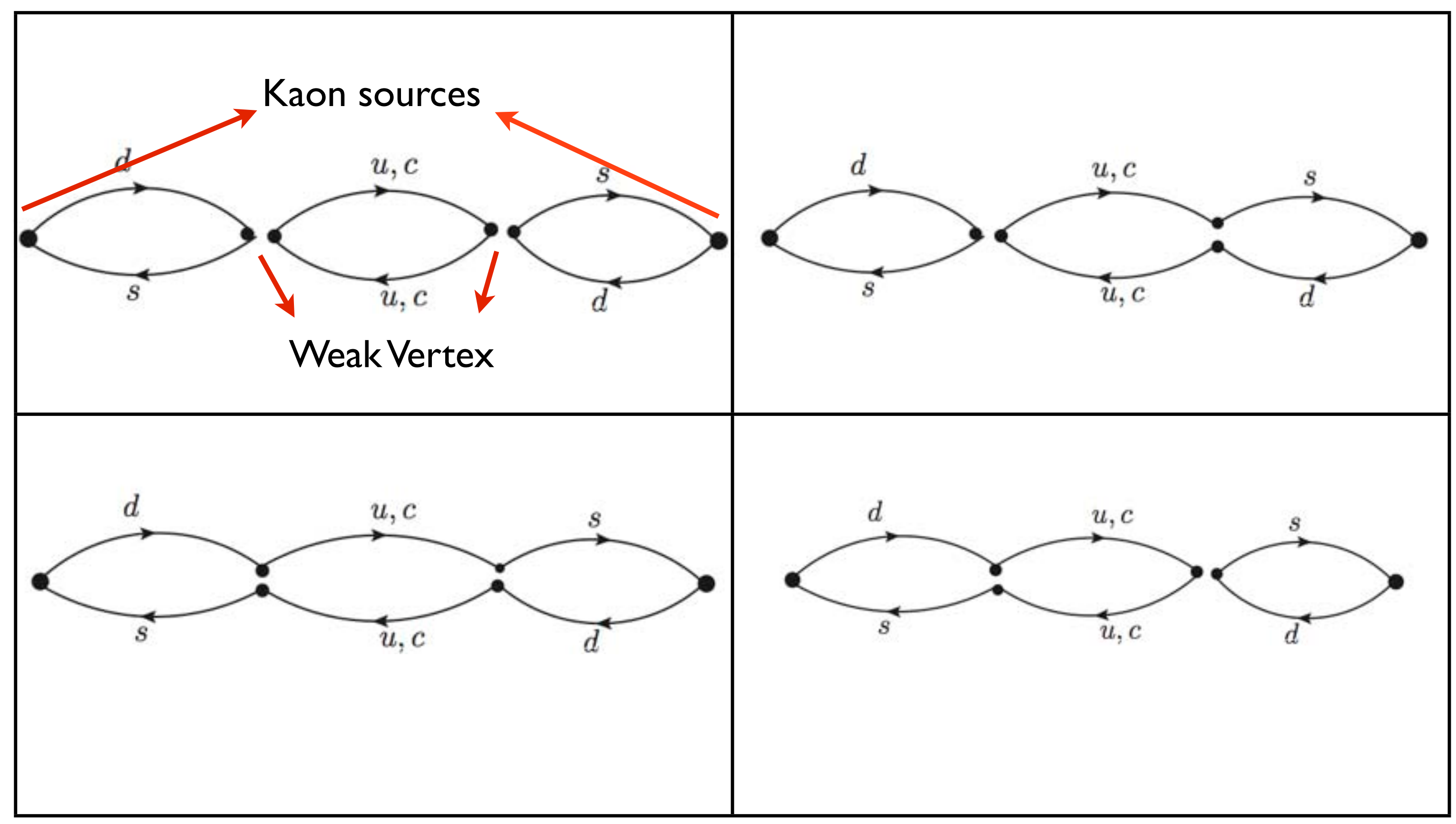




\section{Type 2 diagrams}

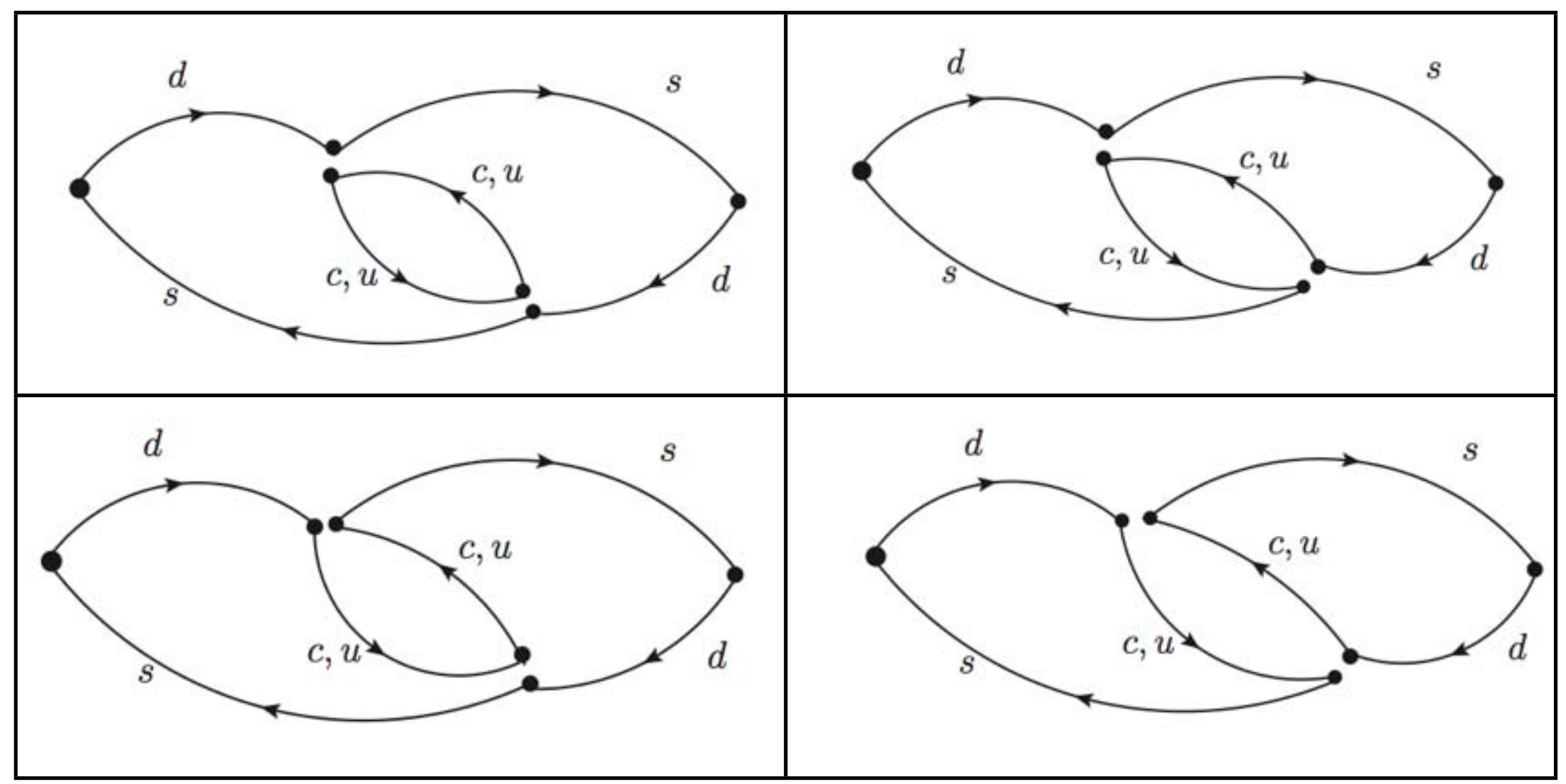




\section{Type 3 digrams, not calculated}

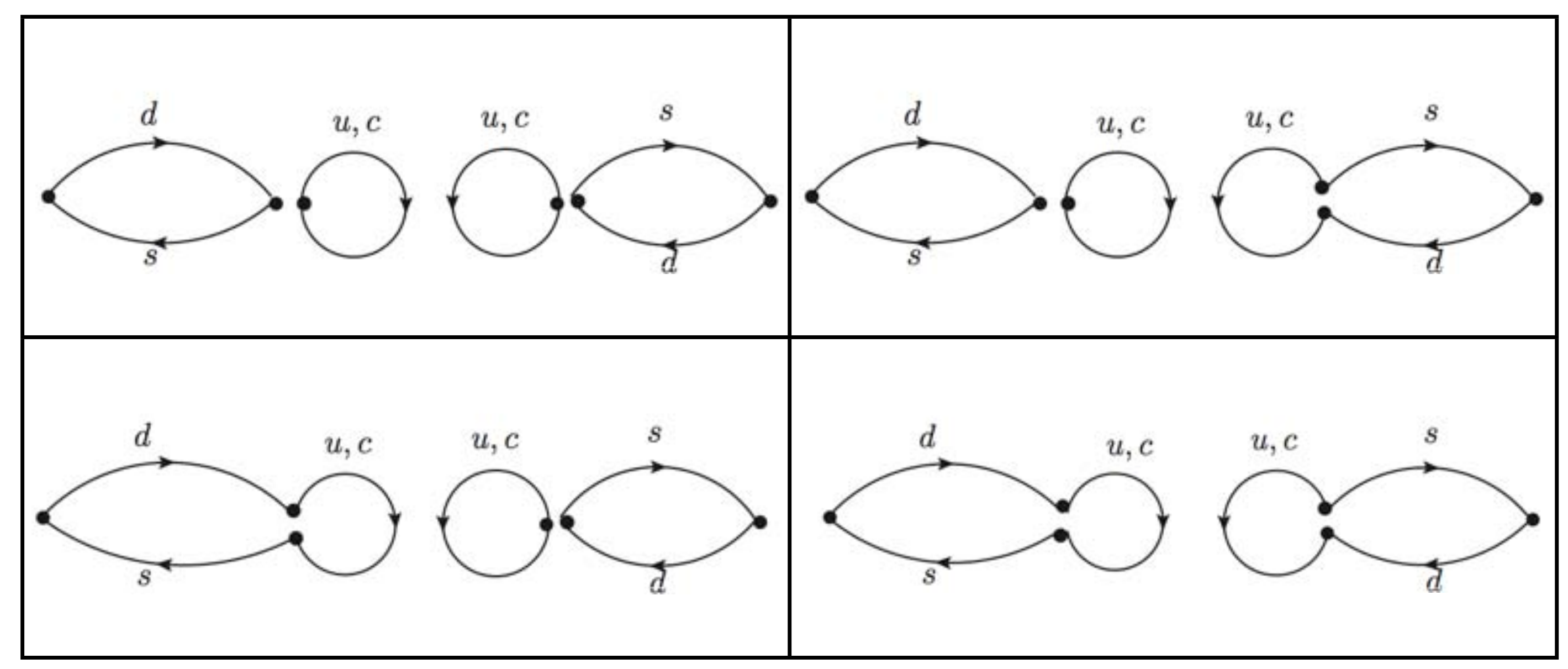




\section{Type 4 diagrams, not calculated}

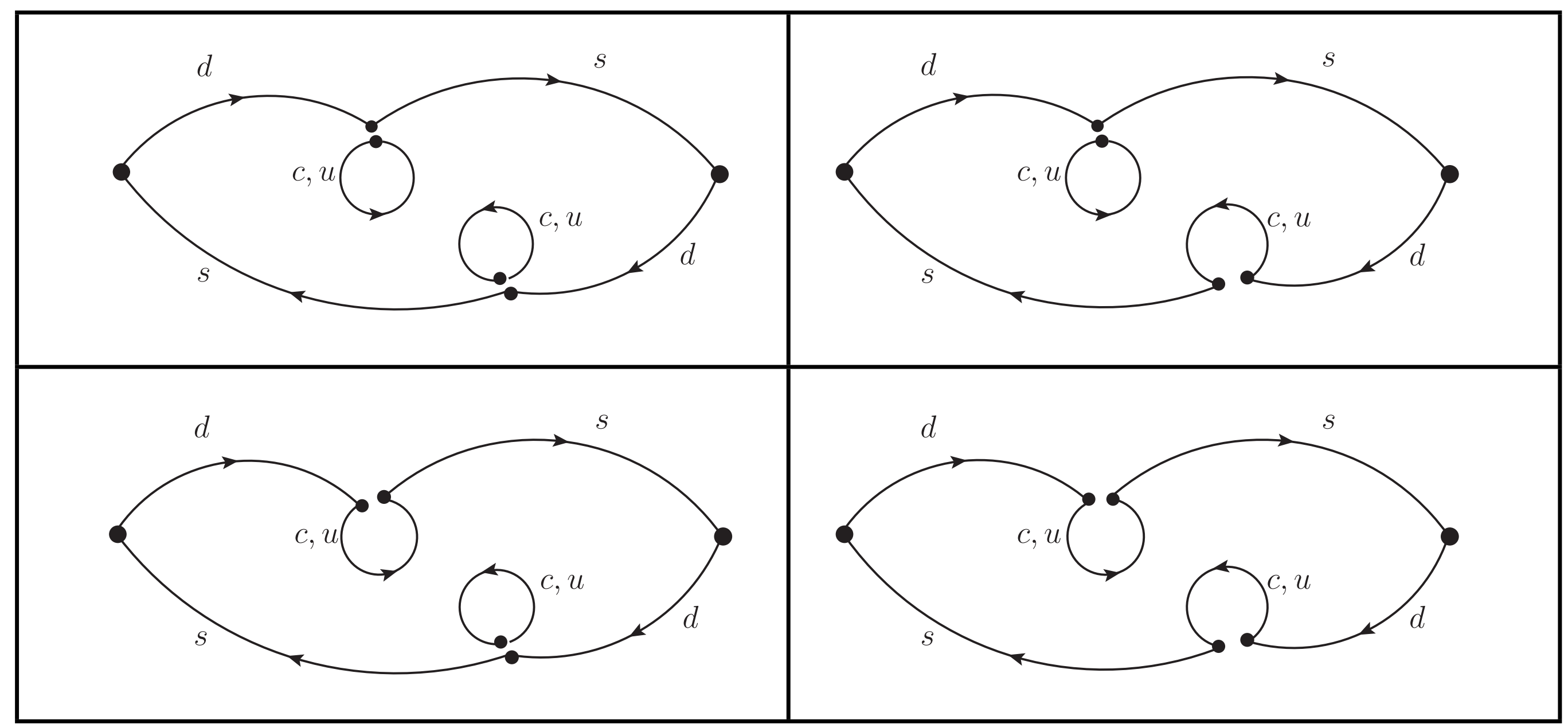




\section{$\Pi^{0}$ intermediate state}

$\pi^{0}$ intermediate state contributes an exponentially increasing term in the integrated correlator, which must be identified and removed. In this non unitary calculation, $\pi^{0}$ and $\eta$ have same mass, since only up quark can appear in our intermediate state, we define $\pi^{0}=i \bar{u} \gamma_{5} u$, calculate following diagrams to compute kaon to pion decay amplitude :
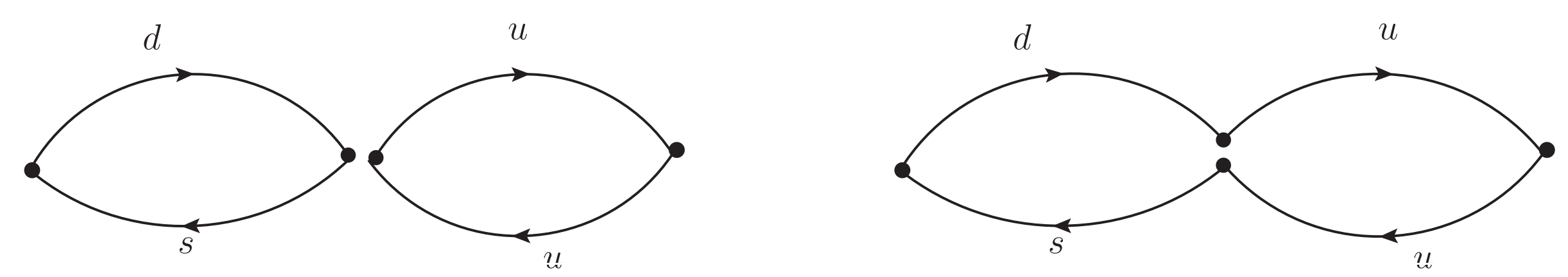


\section{Result without GIM cancellation}

- Both operators are $Q_{1}$

- Without GIM, there will be divergent short distance effect

- The dependence of correlator on time is almost linear imply that largest contribution comes from short distance
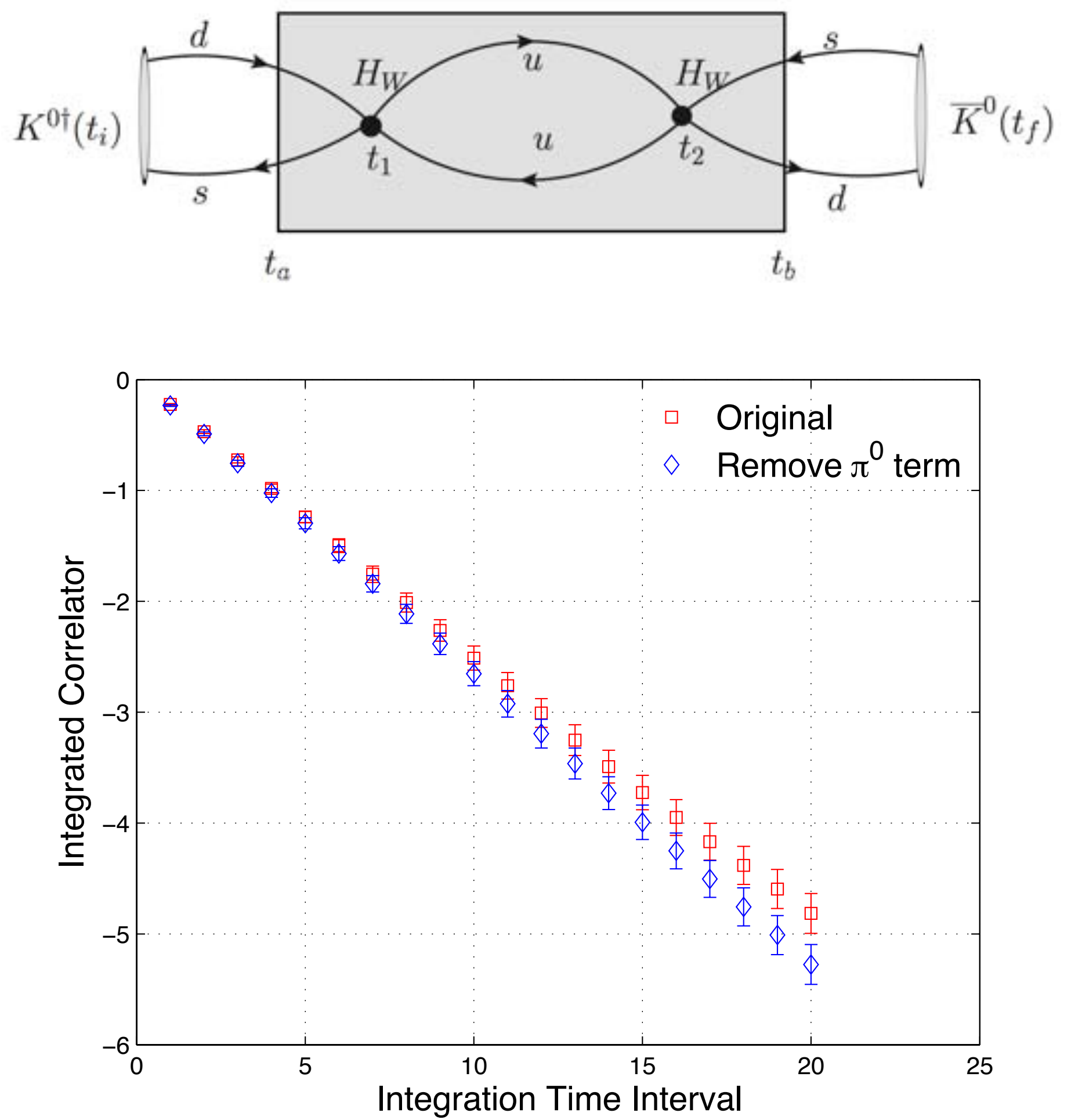


\section{Artificial cutoff}

We can impose an artificial cutoff, require $\left|x_{2}-x_{1}\right| \geq r$ while doing integral :
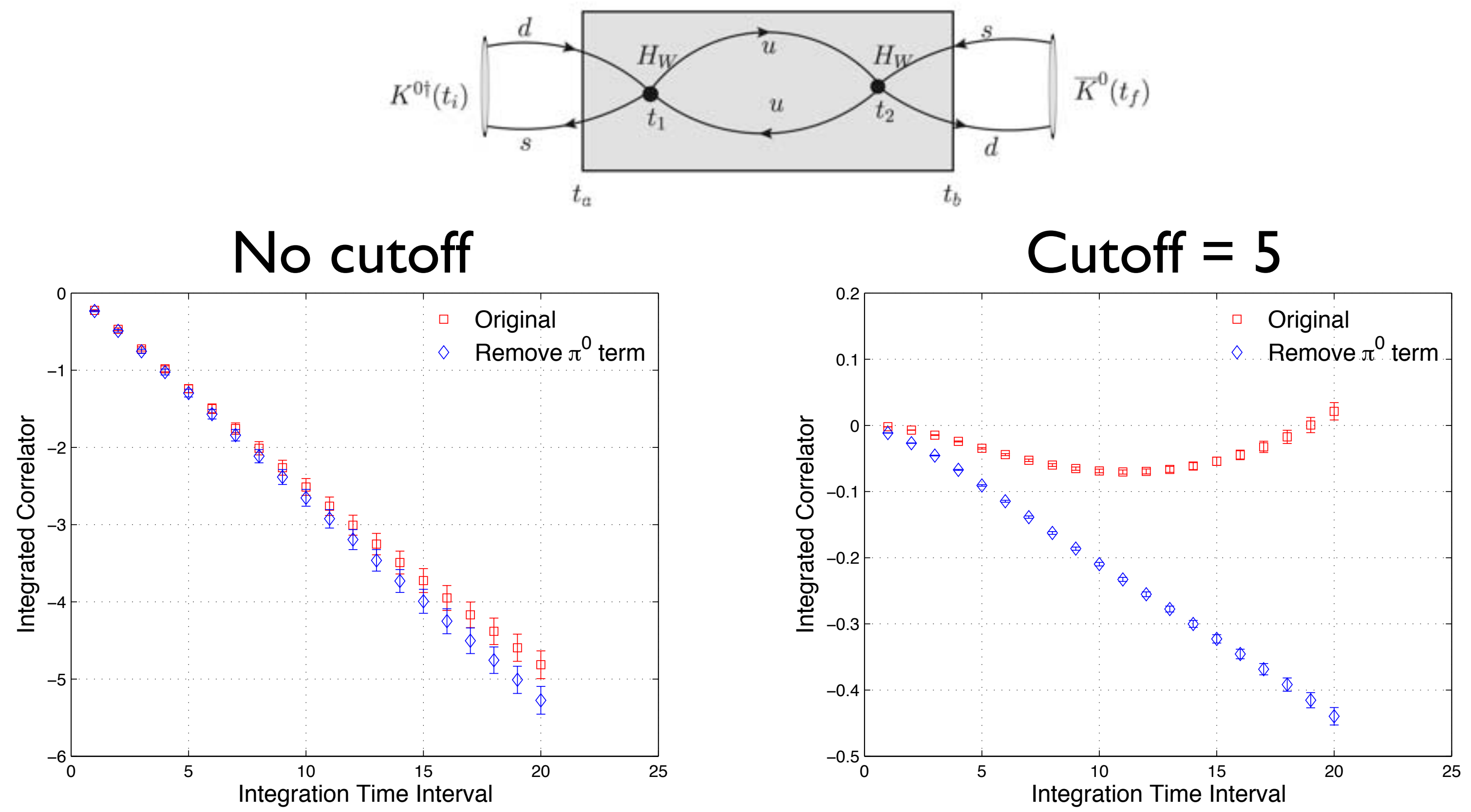


\section{Quadratic divergence}

Mass difference is given by the slope of integrated correlator plot while the integration range is large enough.

$$
\Delta M_{K}(R)=\frac{a}{R^{2}}+b
$$

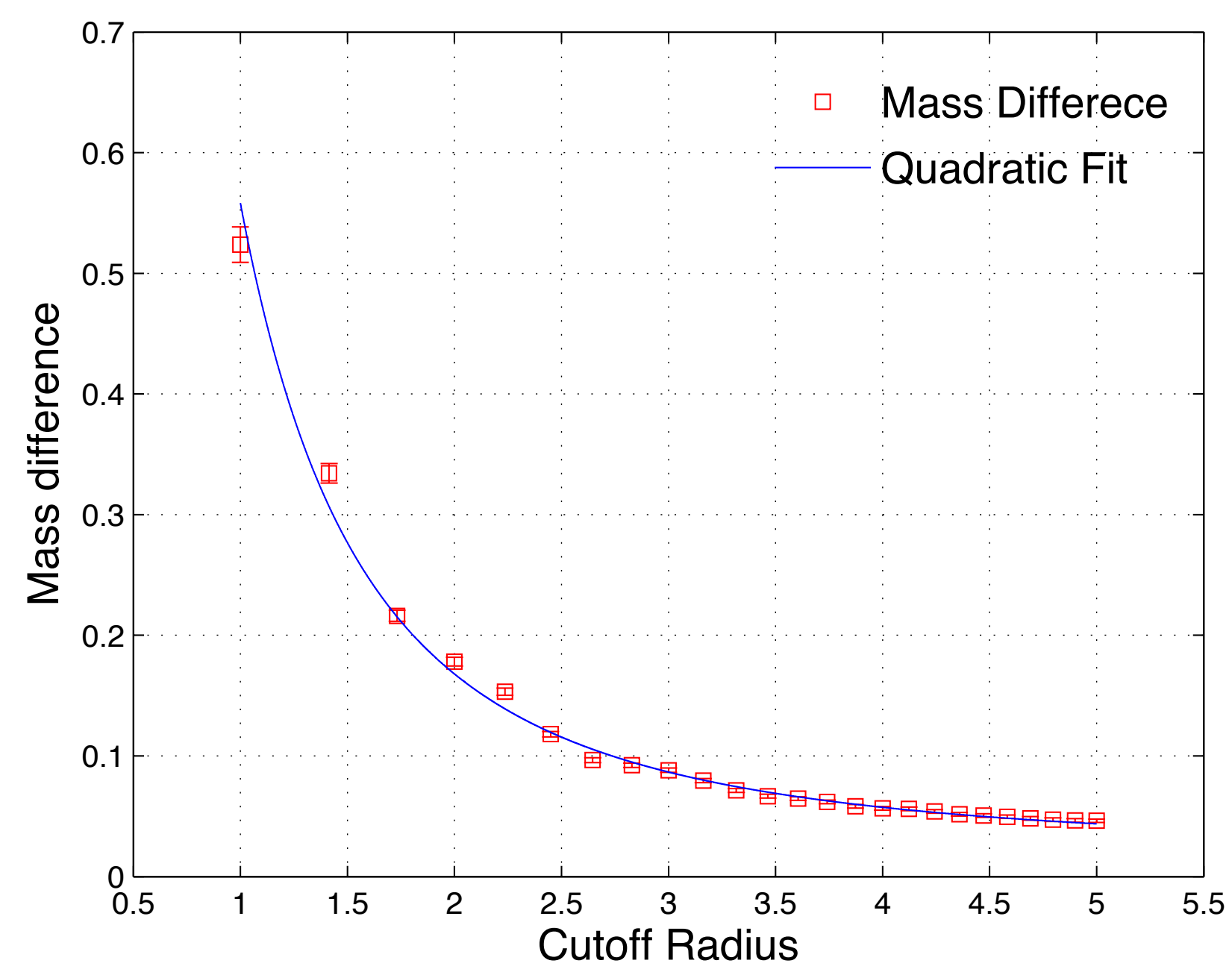




\section{GIM remove the divergence in short distance :}
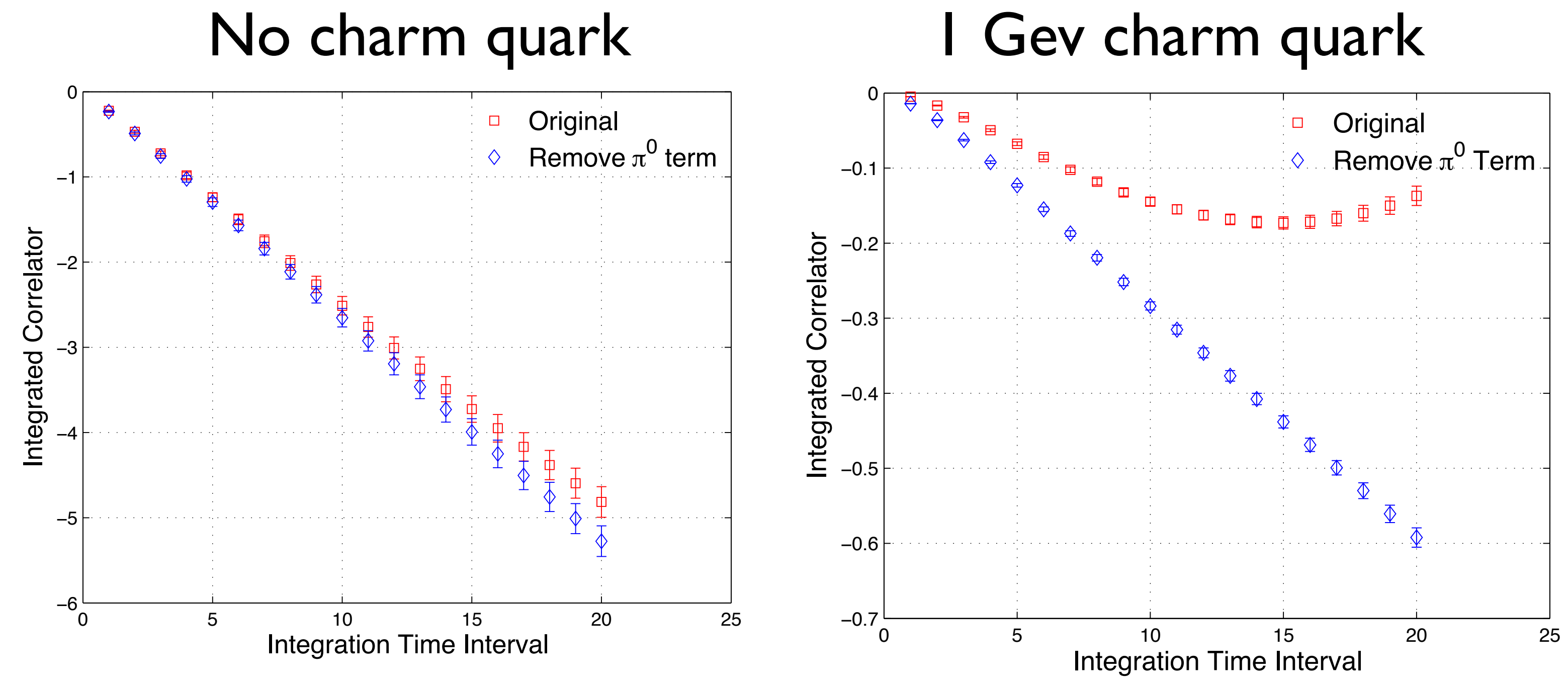
Quadratic Fit :

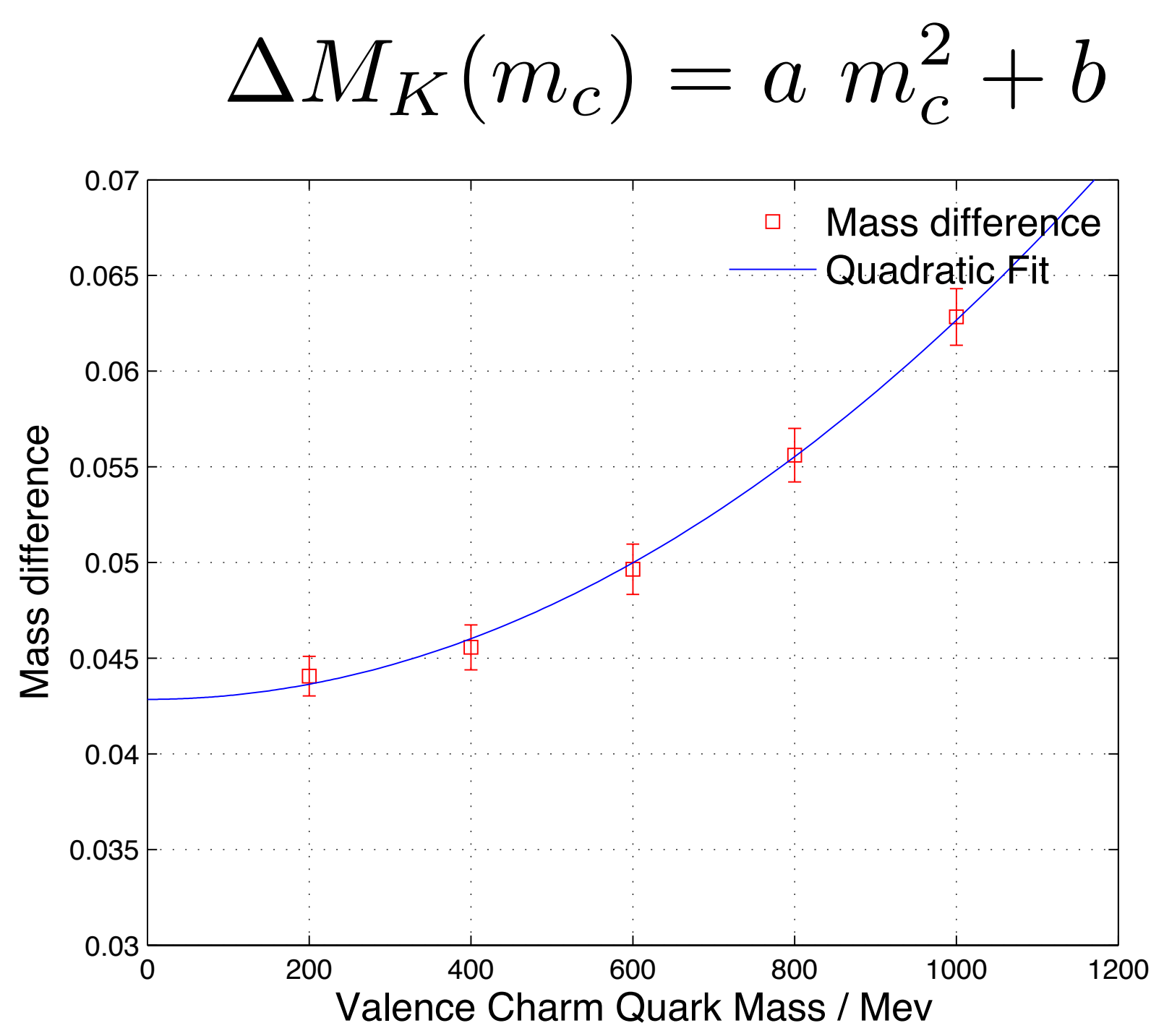

Is I Gev charm too heavy?

Quadratic dependence on $\mathrm{m}_{\mathrm{c}}$ will be cutoff buy lattice spacing if charm is too heavy. The fitting result suggest we haven't reach that region 


\section{Long distance effect}

Investigate unintegrated correlator :

$$
G\left(T ; t_{i}, t_{f}\right)=N_{K}^{2} e^{-M_{K}\left(t_{f}-t_{i}\right)} \sum_{n}\left\langle\overline{K^{0}}\left|H_{W}\right| n\right\rangle\left\langle n\left|H_{W}\right| K^{0}\right\rangle e^{-\left(E_{n}-M_{K}\right) T}
$$

Here $T$ is the time separation between to weak Hamiltonian

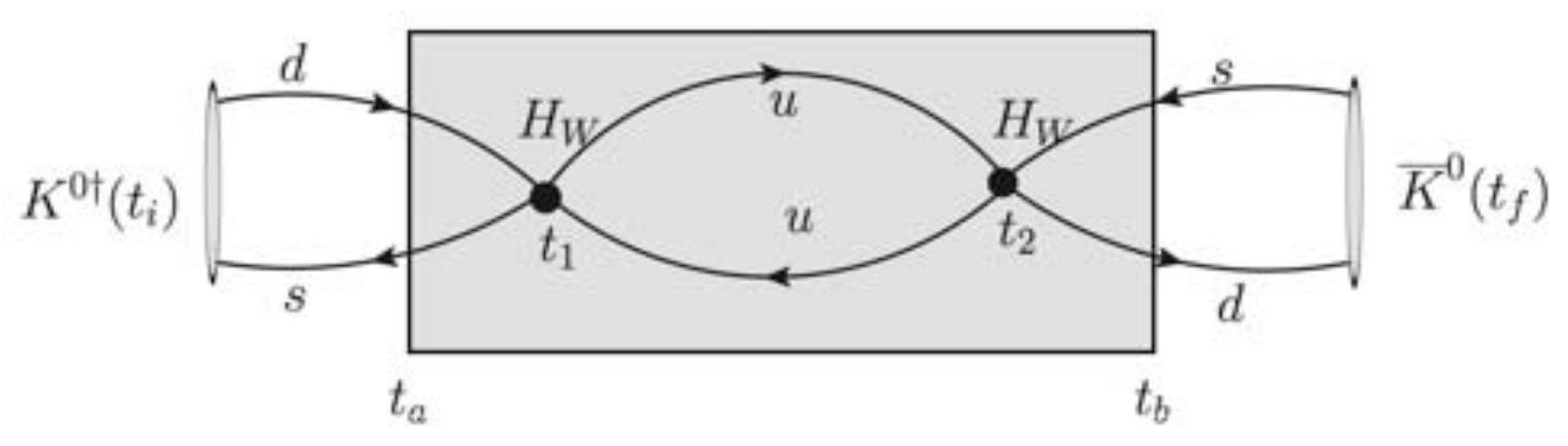

- Separate the Hamiltonian into two parity channel :

- Parity conserving channel, long distance effect dominate by $\pi^{0}$ intermediate state

- Parity violating channel, long distance effect dominate by $\pi \pi$ intermediate state

- Use various kaon masses 


\section{Parity conserving channel}

In long distance, correlator dominate by $\pi^{0}$ term :

$$
G\left(T ; t_{i}, t_{f}\right)=N_{K}^{2} e^{-M_{K}\left(t_{f}-t_{i}\right)}\left\langle\overline{K^{0}}\left|H_{W}\right| \pi^{0}\right\rangle\left\langle\pi^{0}\left|H_{W}\right| K^{0}\right\rangle e^{-\left(E_{\pi}-M_{K}\right) T}
$$
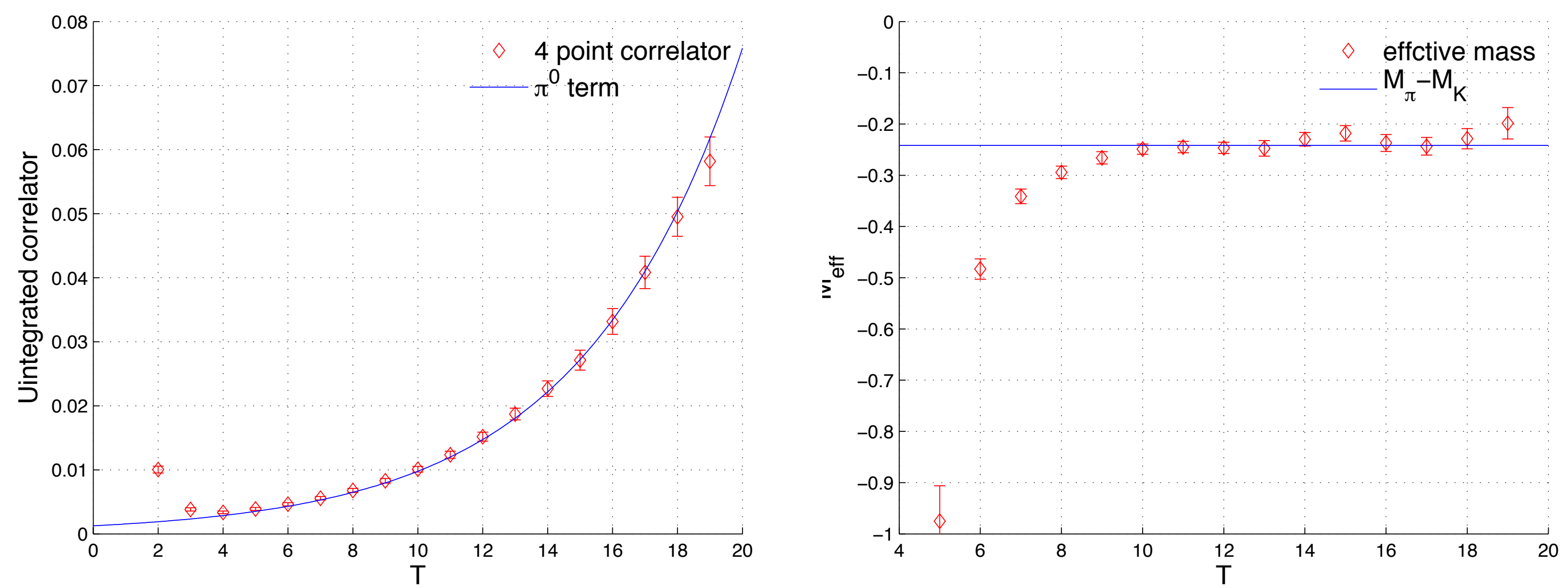


\section{Parity conserving channel}

- Points are the fitting results from unintegrated correlators at various kaon masses

- Horizontal line is the "exact" pion mass given by two point correlator calculation

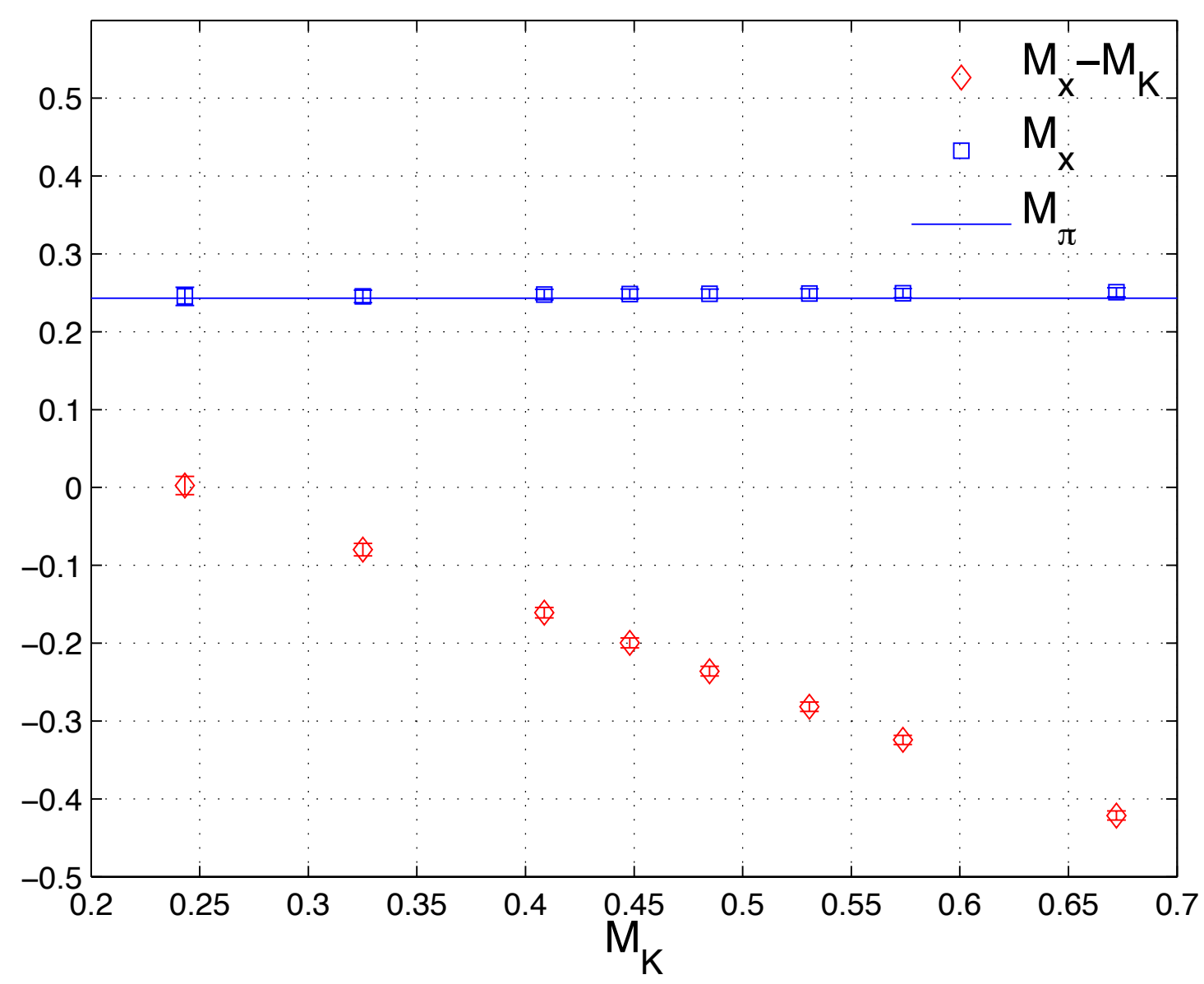




\section{Parity violating channel}

In long distance, correlator dominate by $\pi \pi$ term :

$$
G\left(T ; t_{i}, t_{f}\right)=N_{K}^{2} e^{-M_{K}\left(t_{f}-t_{i}\right)}\left\langle\overline{K^{0}}\left|H_{W}\right| \pi \pi\right\rangle\left\langle\pi \pi\left|H_{W}\right| K^{0}\right\rangle e^{-\left(E_{\pi \pi}-M_{K}\right) T}
$$

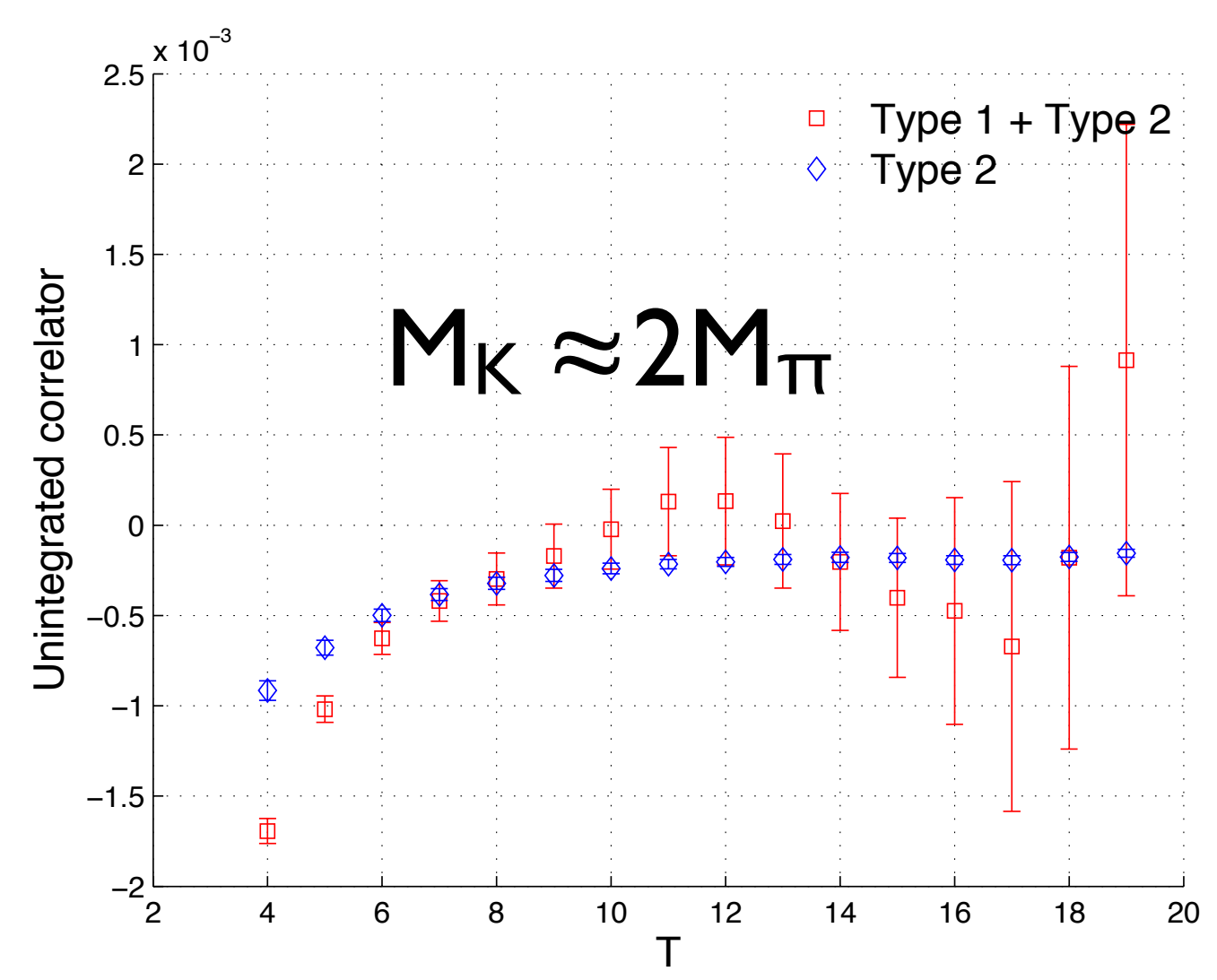

We expect to see plateau at long distance :

- No signal at long distance

- Good signal from type 2 diagrams only 


\section{Parity violating channel}

Type I

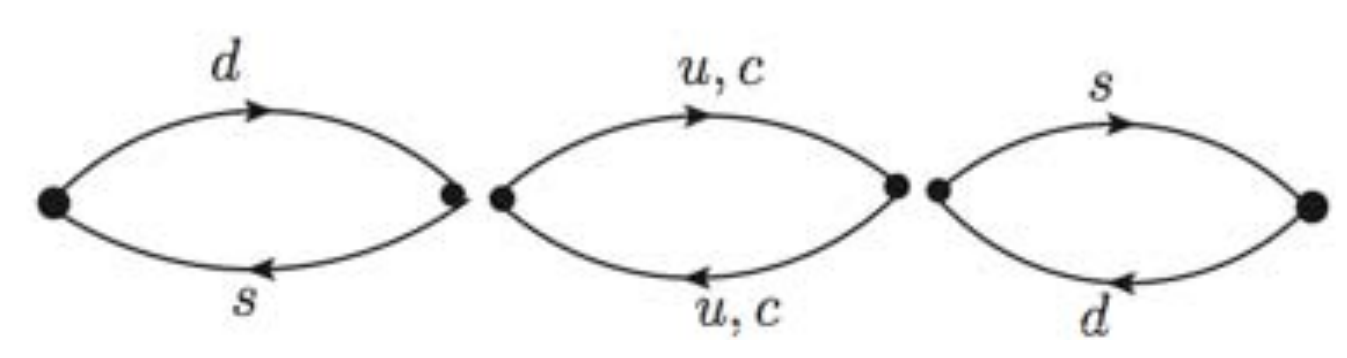

Noise behave like $\pi$,exponentially increasing noise to signal ratio
Type 2

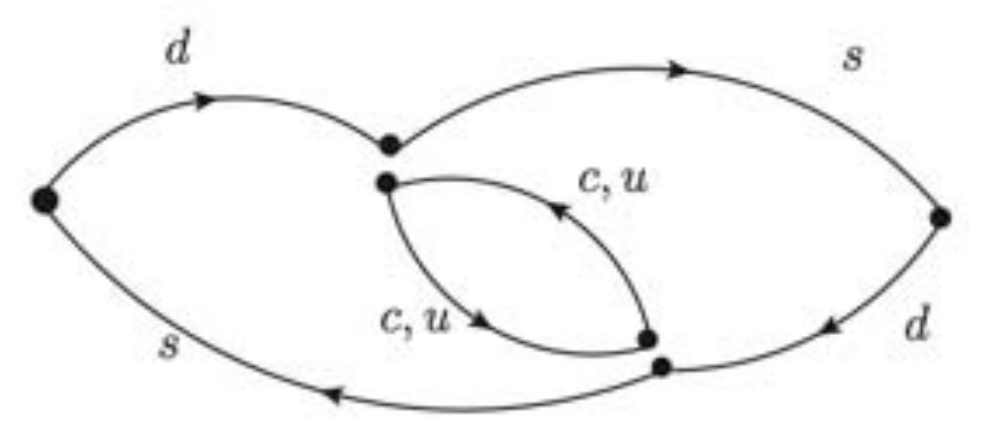

The signal from type

2 contractions don't have such noise 


\section{Parity violating channel}

- Points are the fitting results from unintegrated correlators at various kaon masses, type 2 contractions only

- Horizontal line is the "exact" 2 pion mass given by two point correlator

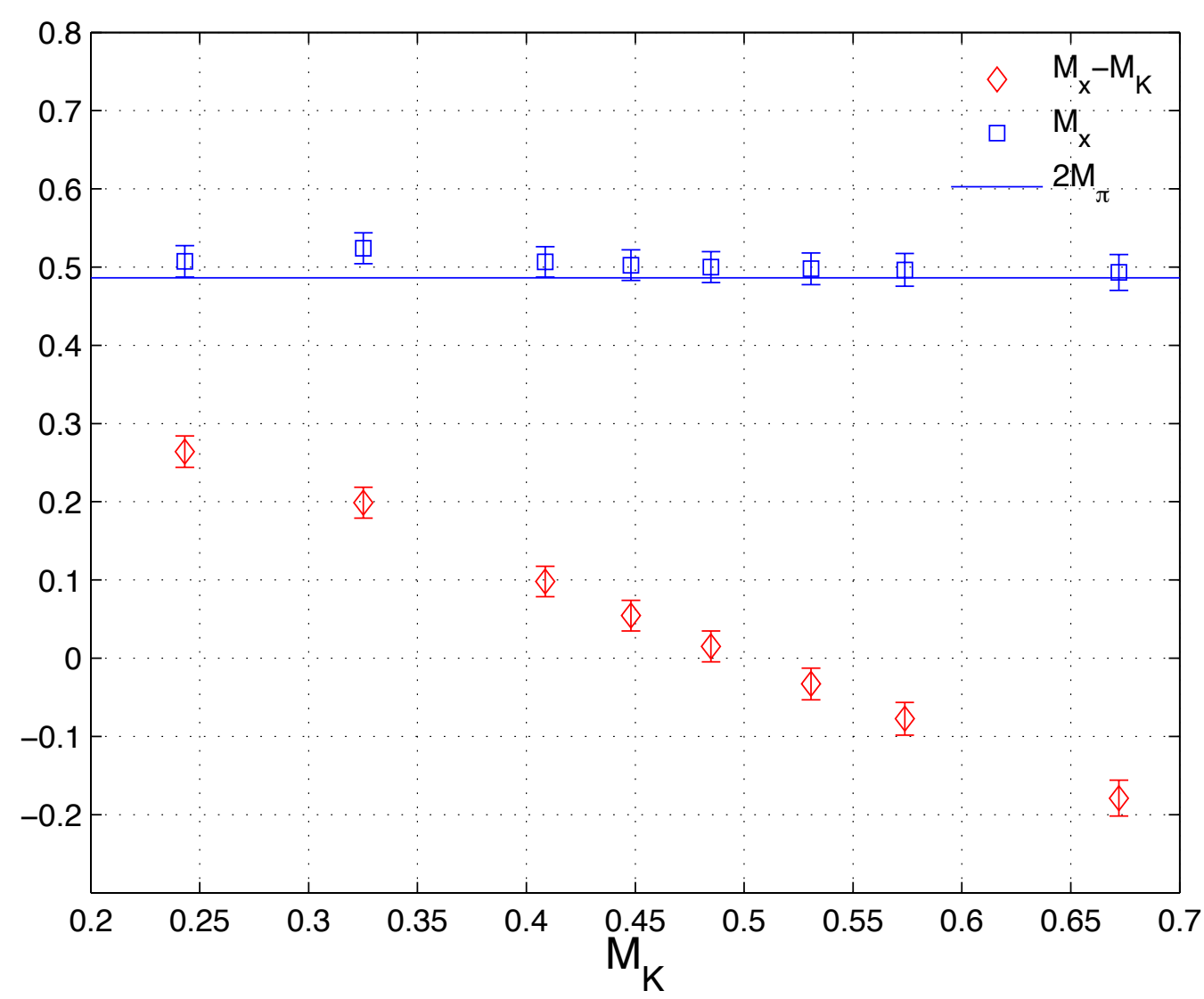
calculation 


\section{Mass difference}

$M_{\pi}=42 \mathrm{I} \mathrm{Mev} \quad$ Only included statistical error $M_{c}=1 \mathrm{Gev}$

- Finite volume effect not corrected

$\Delta M_{K}^{\mathrm{exp}}=3.483(6) \times 10^{-12} \mathrm{Mev}$

\begin{tabular}{c|ccc|c}
\hline$M_{K}(\mathrm{Mev})$ & $\Delta M_{K}^{11}$ & $\Delta M_{K}^{12}$ & $\Delta M_{K}^{22}$ & $\Delta M_{K}\left(\times 10^{-12} \mathrm{Mev}\right)$ \\
\hline 563 & $6.38(14)$ & $-2.64(14)$ & $1.47(8)$ & $5.52(24)$ \\
707 & $8.90(21)$ & $-2.96(23)$ & $2.10(12)$ & $7.38(37)$ \\
775 & $10.63(27)$ & $-3.18(30)$ & $2.48(15)$ & $8.61(49)$ \\
839 & $12.56(34)$ & $-3.62(40)$ & $2.89(20)$ & $9.93(65)$
\end{tabular}




\section{Conclusions and future plans}

- Lattice calculation of $\Delta M_{\mathrm{K}}$ is possible :

$\checkmark$ Use GIM to remove divergence in short distance

$\checkmark$ Remove $\pi$ exponentially term

- Use on-shell K $\rightarrow \pi \pi$ kinematics, remove quadratic term from integrated correlator

- Add finite volume correction term

- Include type 3 and type 4 diagrams in future

- Use Low mode averaging or A2A to collect statistics more efficiently 


\section{Operator mixing and renormalization}

Three group of operators :

Equivalent basis :

$$
\begin{aligned}
\tilde{Q}_{1} & =\left(\bar{s}_{i} d_{i}\right)_{V-A}\left(\bar{u}_{j} u_{j}\right)_{V-A} \\
& -\left(\bar{s}_{i} d_{i}\right)_{V-A}\left(\bar{c}_{j} c_{j}\right)_{V-A} \\
\tilde{Q}_{2} & =\left(\bar{s}_{i} d_{j}\right)_{V-A}\left(\bar{u}_{j} u_{i}\right)_{V-A} \\
& -\left(\bar{s}_{i} d_{j}\right)_{V-A}\left(\bar{c}_{j} c_{i}\right)_{V-A} \\
Q_{1}^{c u} & =\left(\bar{s}_{i} d_{i}\right)_{V-A}\left(\bar{c}_{j} u_{j}\right)_{V-A} \\
Q_{2}^{c u} & =\left(\bar{s}_{i} d_{j}\right)_{V-A}\left(\bar{c}_{j} u_{i}\right)_{V-A} \\
Q_{1}^{u c} & =\left(\bar{s}_{i} d_{i}\right)_{V-A}\left(\bar{u}_{j} c_{j}\right)_{V-A} \\
Q_{2}^{u c} & =\left(\bar{s}_{i} d_{j}\right)_{V-A}\left(\bar{u}_{j} c_{i}\right)_{V-A}
\end{aligned}
$$

$$
\begin{aligned}
& Q_{+}^{X}=Q_{1}^{X}+Q_{2}^{X} \\
& Q_{-}^{X}=Q_{1}^{X}-Q_{2}^{X}
\end{aligned}
$$

$\mathrm{X}=\sim, \mathrm{cu}, \mathrm{uc} \quad \mathrm{SU}(4) \times \mathrm{SU}(4)$

- Operators will not mix with penguin

- Renormalization for three groups of operators should be identical 


\section{Correct finite volume effect}

- Singular energy denominator $I /\left(M_{K}-E_{n}\right)$ will introduce uncontrolled errors

- Use generalized Lellouch-Lucsher method :

- Tune lattice so $E_{\pi \pi}=M_{K}$

- Finite volume $E_{\pi \pi}$ depend on finite volume sum

- Infinite volume $\pi-\pi$ resonant phase shift $\delta_{w}$ depend on infinite volume integral

- Luscher condition relate them :

$$
\phi(E)+\delta_{0}(E)+\delta_{W}(E)=n \pi
$$




\section{Finite volume energy}

- Let $\left|n_{0}\right\rangle$ be the $\pi-\pi$ state degenerate with kaon

- Second order perturbation theory

$$
\left(\begin{array}{cc}
M_{K}+\sum_{n \neq n_{0}} \frac{\left|\left\langle n\left|H_{W}\right| K\right\rangle\right|^{2}}{M_{K}-E_{n}} & \left\langle K\left|H_{W}\right| n_{0}\right\rangle \\
\left\langle n_{0}\left|H_{W}\right| K\right\rangle & E_{n_{0}}+\sum_{n \neq K} \frac{\left|\left\langle n\left|H_{W}\right| n_{0}\right\rangle\right|^{2}}{E_{n_{0}}-E_{n}}
\end{array}\right)
$$

$-\pi-\pi$ state energy is given by:

$$
\begin{aligned}
E_{ \pm} & =M_{K} \pm\left\langle K\left|H_{W}\right| n_{0}\right\rangle \\
& +\frac{1}{2}\left\{\sum_{n \neq n_{0}} \frac{\left|\left\langle n\left|H_{W}\right| K\right\rangle\right|^{2}}{M_{K}-E_{n}}+\sum_{n \neq K} \frac{\left|\left\langle n\left|H_{W}\right| n_{0}\right\rangle\right|^{2}}{E_{n_{0}}-E_{n}}\right\}
\end{aligned}
$$




\section{Infinite volume scattering}

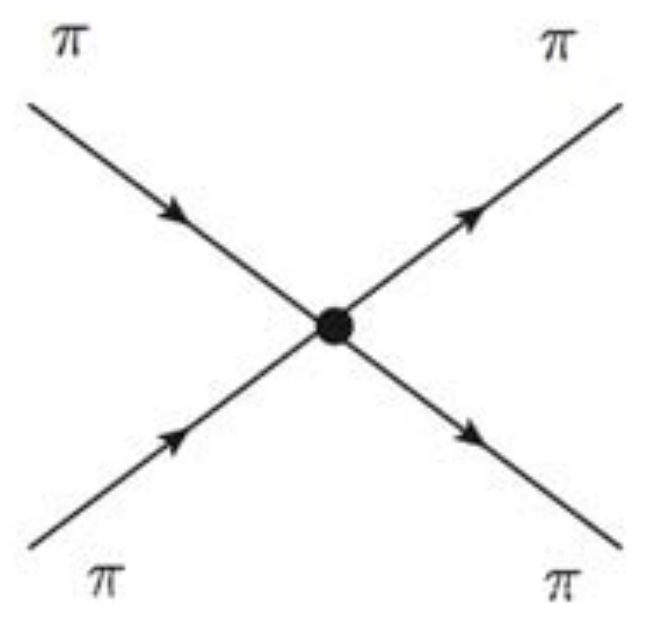

Strong interaction

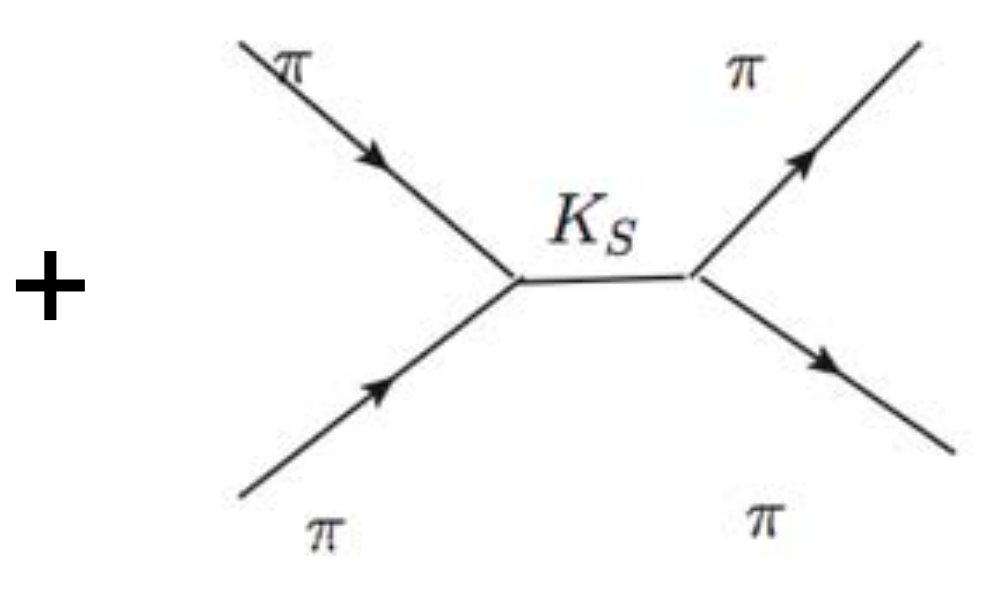

Kaon pole

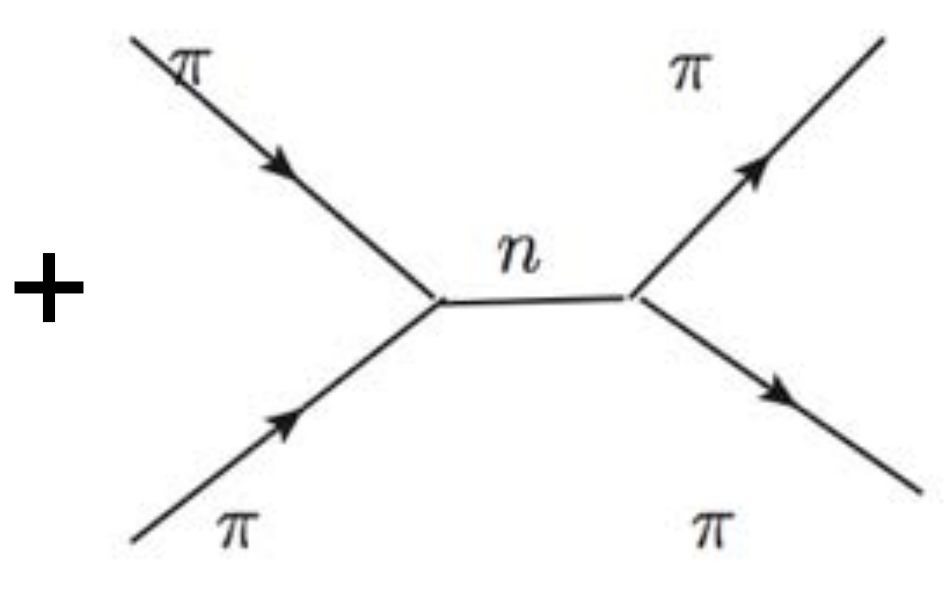

Weak interaction

Total phase shift is given by :

$$
\delta(E)=\delta_{0}(E)+\arctan \left(\frac{\Gamma(E) / 2}{M_{K}+\Delta M_{K}-E}\right)-\pi \sum_{n \neq K} \frac{\left.\left\langle n\left|H_{W}\right| \pi \pi\right\rangle\right|^{2}}{E-E_{n}}
$$

Require that :

$$
\delta\left(E_{ \pm}\right)+\phi\left(E_{ \pm}\right)=n \pi
$$




\title{
Form factors for $B \rightarrow \mathrm{KI}^{+} \mathrm{I}^{-}$semileptonic decay from three-flavor lattice QCD
}

\author{
Ran Zhou \\ Indiana University \\ (In collaboration with FNAL/MILC) \\ New Horizons for Lattice Computations with Chiral Fermions, \\ BNL, NY
}

$05 / 15 / 2012$ 


\section{Motivations and theoretical background}

$B \rightarrow K / l$ semileptonic decay occurs through Penguin diagram $(b \rightarrow s / l)$.

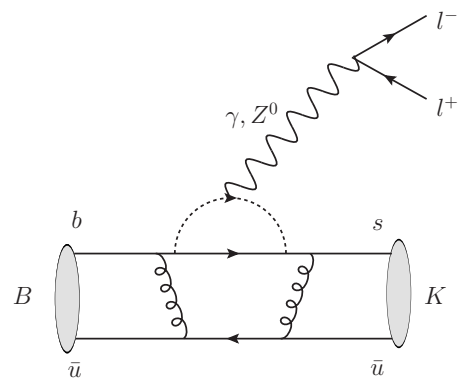

- Standard Model (SM) contributes via FCNC (suppressed)

- Suitable process to detect physics BSM

- Studied by many experiment groups (BABAR, Belle, CDF, LHCb etc.) 


\section{Example of observable in $B \rightarrow K I I$ process}

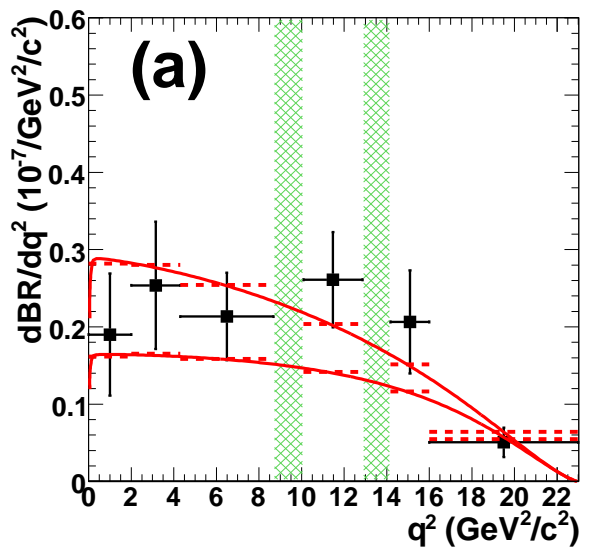

- $B^{+} \rightarrow K^{+} \mu^{+} \mu^{-}$differential branching ratio from CDF 2011

- Uncertainties in form factors are crucial to theoretical predictions(Red lines).

- High intensity front experiment( $\mathrm{LHCb}, \mathrm{SuperB})$ will come to with more accurate result. 


\section{Studies of $B \rightarrow K / l$ form factors from lattice QCD}

Quenched lattice QCD:

- A. Al-Haydari et al. (QCDSF) Eur. Phys. J. A 43, 107120 (2010)

- D. Becirevic et al. Nucl. Phys. B 769, 31 (2007)

- L. Del Debbio et al. Phys. Lett. B 416, 392 (1998)

- A. Abada et al. Phys. Lett. B 365, 275 (1996)

Recent studies on dynamical $\mathbf{N f}=\mathbf{2}+\mathbf{1}$ flavors ensembles:

- FNAL/MILC. $(B \rightarrow K I I)$ hep-lat/1111.0981

- Cambridge group. $\left(B \rightarrow K / K^{*} / I\right)$ hep-ph/1101.2726 
Lattice ensembles used in $B \rightarrow K / l$ work

\begin{tabular}{cccc}
\hline$a^{-1}(\mathrm{fm})$ & size & $a m_{l} / a m_{s}$ & $N_{\text {meas }}$ \\
\hline 0.12 & $20^{3} \times 64$ & $0.02 / 0.05$ & 2052 \\
0.12 & $20^{3} \times 64$ & $0.01 / 0.05$ & 2259 \\
0.12 & $20^{3} \times 64$ & $0.007 / 0.05$ & 2110 \\
0.12 & $20^{3} \times 64$ & $0.005 / 0.05$ & 2099 \\
\hline 0.09 & $28^{3} \times 96$ & $0.0124 / 0.031$ & 1996 \\
0.09 & $28^{3} \times 96$ & $0.0062 / 0.031$ & 1931 \\
0.09 & $32^{3} \times 96$ & $0.00465 / 0.031$ & 984 \\
0.09 & $40^{3} \times 96$ & $0.0031 / 0.031$ & 1015 \\
0.09 & $64^{3} \times 96$ & $0.00155 / 0.031$ & 791 \\
\hline 0.06 & $48^{3} \times 144$ & $0.0036 / 0.018$ & 673 \\
0.06 & $64^{3} \times 144$ & $0.0018 / 0.018$ & 827 \\
\hline
\end{tabular}

Table: Ensembles of QCD gauge field configurations used in the current B2K analysis. Four sources $\left(0, \frac{N_{t}}{4}, \frac{N_{t}}{2}, \frac{3 N_{t}}{4}\right)$ are used for all measurements 
Form factors in $B \rightarrow K / l$ semileptonic decays

- Two matrix elements are needed in $B \rightarrow K l l$ work:

$\left\langle B(p)\left|\bar{b} \gamma^{\mu} s\right| K(k)\right\rangle,\left\langle B(p)\left|\bar{s} \sigma^{\mu \nu} b\right| K(k)\right\rangle$

$$
\begin{gathered}
\left\langle B(p)\left|\bar{b} \gamma^{\mu} s\right| K(k)\right\rangle=f_{+}\left(p^{\mu}+k^{\mu}-\frac{m_{B}^{2}-m_{K}^{2}}{q^{2}} q^{\mu}\right)+f_{0} \frac{m_{B}^{2}-m_{K}^{2}}{q^{2}} q^{\mu} \\
=\sqrt{2 m_{B}}\left[f_{\|} \frac{p^{\mu}}{m_{B}}+f_{\perp} p_{\perp}^{\mu}\right] \\
\left\{\begin{array}{l}
f_{\|}\left(E_{K}\right)=\frac{\left\langle B(p)\left|\bar{b} \gamma^{0} s\right| K(k)\right\rangle}{\sqrt{2 m_{B}}} \\
f_{\perp}\left(E_{K}\right)=\frac{\left\langle B(p)\left|\bar{b} \gamma^{i} s\right| K(k)\right\rangle}{2 \sqrt{m_{B}}} \frac{1}{p_{i}}
\end{array}\right. \\
\left\{\begin{array}{l}
f_{0}\left(E_{K}\right)=\frac{2 m_{B}}{m_{B}^{2}-m_{K}^{2}}\left[\left(m_{B}-E_{K}\right) f_{\|}\left(E_{K}\right)+\left(E_{K}^{2}-m_{K}^{2}\right) f_{\perp}\left(E_{K}\right)\right] \\
f_{+}\left(E_{K}\right)=\frac{1}{\sqrt{2 m_{B}}}\left[f_{\|}\left(E_{K}\right)+\left(m_{B}-E_{K}\right) f_{\perp}\left(E_{K}\right)\right]
\end{array}\right.
\end{gathered}
$$




\section{Form factors in $B \rightarrow K / l$ semileptonic decays}

Semileptonic $B \rightarrow K$ transition from tensor current:

$$
q_{\nu}\left\langle K(k)\left|\bar{s} \sigma^{\mu \nu} b\right| B(p)\right\rangle=\frac{i f_{T}}{m_{B}+m_{K}}\left[q^{2}\left(p^{\mu}+k^{\mu}\right)-\left(m_{B}^{2}-m_{K}^{2}\right) q^{\mu}\right]
$$

Solve for $f_{T}$ :

$$
f_{T}=\frac{m_{B}+m_{K}}{\sqrt{2 m_{B}}} \frac{\left\langle K(k)\left|i b \sigma^{0 i} s\right| B(p)\right\rangle}{\sqrt{2 m_{B}} k^{i}}
$$




\section{$B_{x} \rightarrow P_{x y} / /$ semileptonic decays in NLO SChPT}

$$
\begin{aligned}
f_{\|} & =\frac{C_{0}}{f}\left(1+\operatorname{logs}+C_{1} m_{x}+C_{2} m_{y}+C_{3} E+C_{4} E^{2}+C_{4} a^{2}\right) \\
f_{\perp} & =\frac{C_{0}}{f}\left[\frac{g}{E+\Delta_{B}^{*}+D}\right] \\
& +\frac{\left(C_{0} / f\right) g}{E+\Delta_{B}^{*}}\left(\operatorname{logs}+C_{1} m_{x}+C_{2} m_{y}+C_{3} E+C_{4} E^{2}+C_{5} a^{2}\right)
\end{aligned}
$$

where $\Delta_{B}^{*}=m_{B_{s}^{*}}-m_{B}, D$ and logs are chiral log terms.

- We use SU(2) chiral logs in the chiral fit.

- The same formula are used for $f_{T}$ and $f_{\perp}$. 


\section{$f_{\|}, f_{\perp}$ and $f_{T}$ chiral-continuum extrapolations}

Fpara NLO SU(2) ChPT fit. p-val=0.41
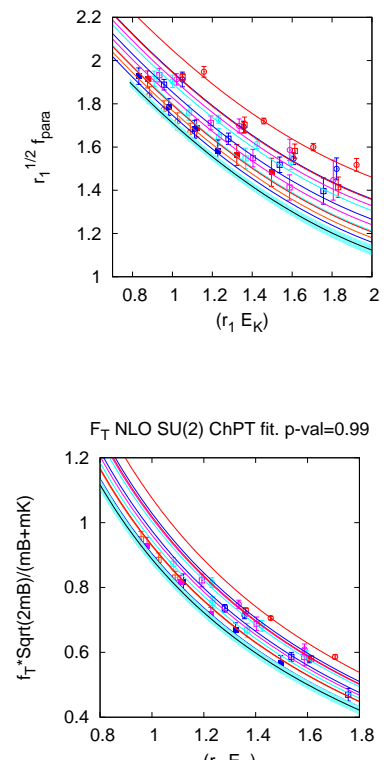

Ran Zhou (Indiana University)
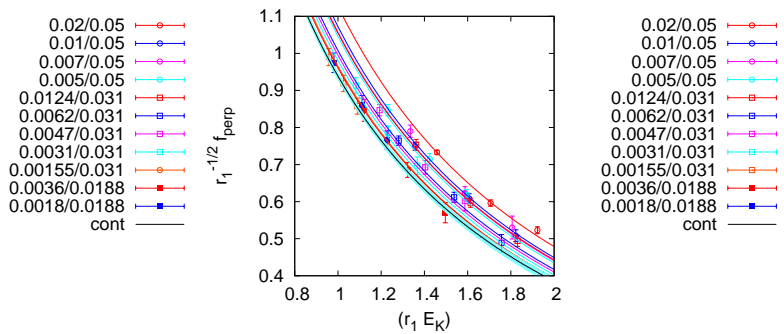

- Chiral-Continuum extrapolations give FFs at small $E_{K}$. (large $\left.q^{2}\right)$

- $q^{2}=\left(p_{B}-p_{K}\right)^{2}=$ $m_{B}^{2}+m_{K}^{2}-2 m_{B} E_{K}$

- z-expansion is a model independent extrapolation method to small $q^{2}$.
$0.02 / 0.05$

$0.007 / 0.05$

$0.005 / 0.05$

$0.0124 / 0.031$

$0.0062 / 0.031 \longmapsto$

$0.0047 / 0.031$

$0.0031 / 0.031$

$0.00155 / 0.031$

$0.0036 / 0.0188$

$0.0018 / 0.0188$

cont 


\section{$z$-expansion on $B \rightarrow K / l$ form factors}

- $z$-expansion maps $q^{2}$ to $z$ by:

$$
z\left(q^{2}, t_{0}\right)=\frac{\sqrt{t_{+}-q^{2}}-\sqrt{t_{-}-t_{0}}}{\sqrt{t_{+}-q^{2}}+\sqrt{t_{-}-t_{0}}}, \quad t_{ \pm}=\left(m_{B} \pm m_{K}\right)^{2}
$$

- Choose $t_{0}=t_{+}\left(1-\sqrt{\left.1-\frac{t_{-}}{t_{+}}\right)} \rightarrow z \in(-0.15,0.15)\right.$ for $B \rightarrow K I I$, corresponding $q^{2} \in(0,23)$.

- Expand form factors as a function of $z$.

$$
f\left(q^{2}\right)=\frac{1}{B(z) \phi(z)} \sum_{k=0}^{\infty} a_{k} z^{k}
$$

where $B(z)=z\left(q^{2}, m_{R}^{2}\right)$ and $\phi(z)$ is selected such that $\sum_{k=0}^{\infty} a_{k}^{2} \leq 1$ 


\section{$z$-expansion on $B \rightarrow K / l$ form factors}

$$
f\left(q^{2}\right)=\frac{1}{B(z) \phi(z)} \sum_{k=0}^{\infty} a_{k} z^{k},
$$

Numerical recipe for $z$-expansion:

- Fit $f\left(q^{2}\right) B(z) \phi(z)$ as a polynomial of $z$ in the range of $z \in(-0.15,0)$

- Extrapolate $z$-expansion fit to $z=0.15$

- Convert variable $z$ back to $q^{2}$

Now, we extend lattice measured form factors from $q^{2} \in(15,23) \mathrm{GeV}^{2}$ to whole $q^{2}$ range. 


\section{$z$-expansion on $B \rightarrow K / l$ form factors}

$z$-expansion on $B \rightarrow K I /$ form factors. (Statistical error only.)
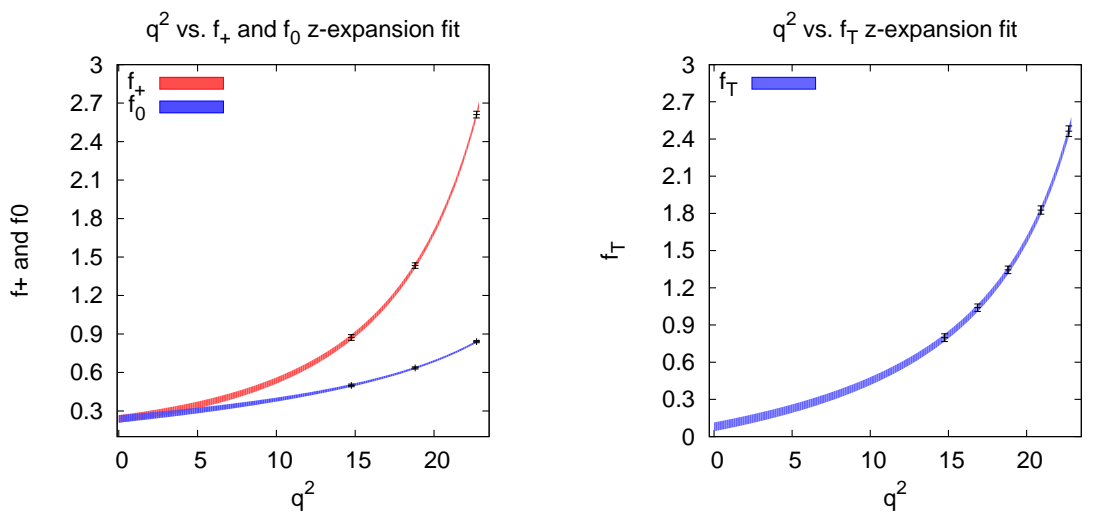

kinematic constraint, $f_{+}\left(q^{2}=0\right)=f_{0}\left(q^{2}=0\right)$, is applied in $z$-expansion fit. 


\section{$z$-expansion on $B \rightarrow K / l$ form factors}

$z$-expansion on $B \rightarrow K I /$ form factors. (Statistical and systematic error.)
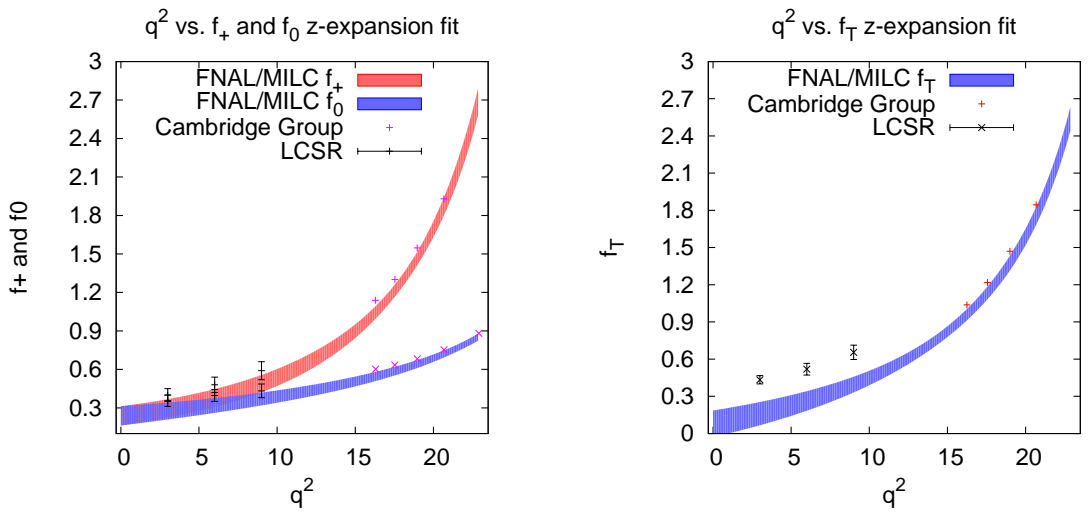


\section{Systematic error budget}

System Error Analysis on $f_{+}$
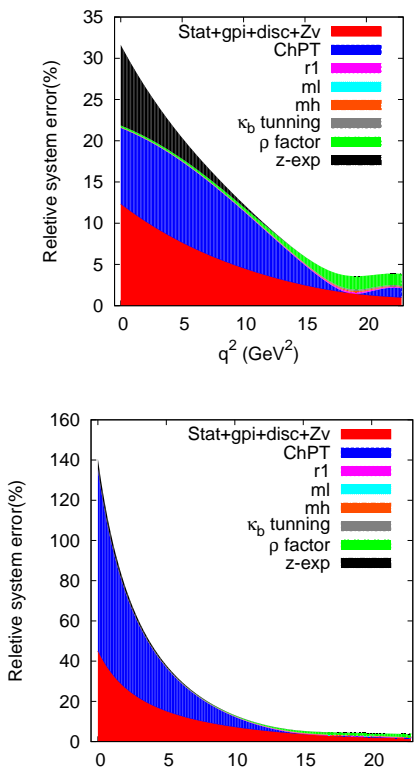

System Error Analysis on $\mathrm{f}_{0}$

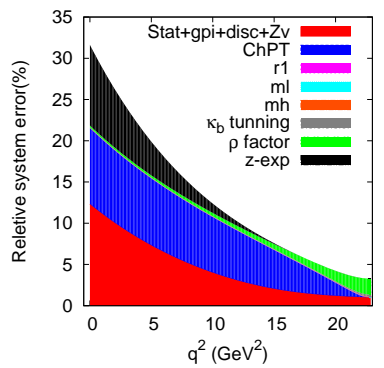

- Renormalization error could be smaller in the final result.

- ChPT error is important.

- Direct measurement of form factors at small $q^{2}$ is valuable. 


\section{Future work}

- Study possible impact from the accurate form factors

- Consider to measure form factors at smaller quark masses and lower $q^{2}$.

- Consider form factors in $B \rightarrow K^{*} / /$ semileptonic decay 


\section{Backup slides}

$f_{\|} \mathrm{SU}(3)$ chiral log:

$$
\begin{aligned}
& \frac{1}{(4 \pi f)^{2}}\left\{\frac { 1 } { 1 6 } \sum _ { \equiv } \left[\frac{2-3 g_{\pi}^{2}}{2} I_{1}\left(m_{K, \equiv}\right)-3 g_{\pi}^{2} I_{1}\left(m_{\pi, \Xi}\right)+\frac{1}{2} I_{1}\left(m_{S, \Xi}\right)\right.\right. \\
& \left.+2 I_{2}\left(m_{K, \equiv}, E\right)+I_{2}\left(m_{S, \Xi}, E\right)\right] \\
& -\frac{1}{2} I_{1}\left(m_{S, I}\right)+\frac{3 g_{\pi}^{2}}{4} I_{1}\left(m_{\pi, I}\right)+\frac{8-3 g_{\pi}^{2}}{12} I_{1}\left(m_{\eta, I}\right)+I_{2}\left(m_{\eta, I}, E\right)-I_{2}\left(m_{S, I}, E\right) \\
& +a^{2} \delta_{V}^{\prime}\left[\frac{I_{1}\left(m_{\eta^{\prime}, V}\right)-I_{1}\left(m_{\eta, V}\right)+I_{2}\left(m_{\eta^{\prime}, V}, E\right)-I_{2}\left(m_{\eta, V}, E\right)}{m_{\eta^{\prime}, V}^{2}-m_{\eta, V}^{2}}\right. \\
& -\sum_{j \in\left\{S, \eta, \eta^{\prime}\right\}} R_{j}^{[3,1]}\left(\left\{m_{S, V}, m_{\eta, V}, m_{\eta^{\prime}}, V\right\} ;\left\{m_{\pi, V}\right\}\right)\left(\frac{1}{2} I_{1}\left(m_{j, V}\right)+I_{2}\left(m_{j, V}, E\right)\right) \\
& \left.+\frac{3 g_{\pi}^{2}}{2} \sum_{j \in\left\{\pi, \eta, \eta^{\prime}\right\}} R_{j}^{[3,1]}\left(\left\{m_{\pi, V}, m_{\eta, V}, m_{\eta^{\prime}, V}\right\} ;\left\{m_{S, V}\right\}\right) I_{1}\left(m_{j, V}\right)\right] \\
& +[V \rightarrow A]\}
\end{aligned}
$$




\section{Backup slides}

$f_{\|} \mathrm{SU}(2)$ chiral log:

$$
\begin{aligned}
\operatorname{logs}^{B \rightarrow K}= & \frac{1}{(4 \pi f)^{2}}\left\{\frac{1}{16} \sum_{\equiv}\left[-3 g_{\pi}^{2} I_{1}\left(m_{\pi, \Xi}\right)\right]+\frac{3 g_{\pi}^{2}}{4} I_{1}\left(m_{\pi, l}\right)\right. \\
& \left.+\frac{3 g_{\pi}^{2}}{2}\left[2 I_{1}\left(m_{\pi, V}\right)-2 I_{1}\left(m_{\eta, V}\right)\right]+[V \rightarrow A]\right\}
\end{aligned}
$$




\section{New Horizons for Lattice Computations with Chiral Fermions}

Hamilton Seminar Room

Chemistry Department, Bldg. 555

May 14 - 16, 2012

\section{Monday, May 14, 2012}

\section{Chair: Taku Izubuchi}

08:00 - 08:25

$08: 25-08: 40$

$08: 40-09: 20$

$09: 20-09: 40$

09:40 - 10:00

10:00 - 10:20

10:20 - 10:50

\section{Chair: Norman Christ}

10:50 - 11:20 AOKI, Sinya

11:20 - 11:40 COSSU, Guido

11:40 - 12:00 MUKHERJEE, Swagato

12:00 - 12:30 GIUSTI, Leonardo

$12: 30-14: 00$

\section{Chair: Yasumichi Aoki}

$\begin{array}{ll}\text { 14:00 - 14:30 } & \text { FODOR, Zoltan } \\ \text { 14:30 - 15:00 } & \text { SHAMIR, Yigal } \\ \text { 15:00 - 15:30 } & \text { LIN, C.-J. David } \\ \text { 15:30 - 16:00 } & \text { KUTI, Julius }\end{array}$

$16: 00-16: 30$

\section{Chair: Artan Borici}

16:30 - 17:00

$17: 00-17: 20$

$17: 20-17: 40$

$17: 40-18: 00$

NARAYANAN, Rajamani

SCHAEFER, Stefan

MISUMI, Tatsuhiro

CREUTZ, Michael

\section{Registration / Coffee / Light Snacks}

Welcome

The New Muon g-2 Experiment at Fermilab: A Challenge for Theorists and Experimentalists

On the Hadronic Vacuum Polarization Contribution to g-2

The Anomaly Triangle and $\mathrm{g}-2$

The Two-Photon Decay of Neutral Pion from Lattice QCD

.Coffee Break

Chiral Symmetry Restoration and Eigenvalue Density of Dirac Operator at Finite Temperature The Axial Symmetry at Finite Temperature in Lattice QCD with Overlap Fermions

Thermodynamics with Domain Wall Fermions and Anomaly in Hot QCD

Thermodynamic Potentials from Shifted Boundary Conditions Lunch Break

\section{High-precision Scale Setting in Lattice QCD}

SU(N) Gauge Theories with Symmetric-representation Fermions

Lattice Study of the Phase Structure of the Strong-Yukawa Model

The Composite Higgs Mechanism and the Conformal Window

Coffee Break

Large N Gauge Theories with Adjoint Fermions

Lattice QCD with Open Boundary Conditions

New Fermion Discretizations and their Application

On Quark Masses

...Reception / Light Meal (Berkner Hall Lobby) 


\section{Tuesday, May 15, 2012}

\section{Chair: Stefan Schaefer}

08:00 - 08:30

08:30 - 09:00

09:00 - 09:20

09:20 - 09:40

09:40 - 10:10

10:10 - 10:40

BROWER, Richard

COHEN, Saul

KIM, Hyung-Jin

ORGINOS, Kostas

\section{Chair: Shoji Hashimoto}

10:40 - 11:10 BERNARD, Claude

11:10 - 11:40 LEE, Weonjong

11:40 - 12:10 MAWHINNEY, Robert

12:10 - 12:40 YU, Jianglei

$12: 40-14: 20$

\section{Chair: Ruth Van de Water}

$14: 50-15: 10$

SACHRAJDA, Christopher

$15: 10-15: 30$
14:20 - 14:50

\section{..Coffee / Light Snacks}

Prospects of Multi-grid and Domain Decomposition Dirac Solvers on GPU

Multigrid Acceleration for Domain-Wall Fermion Inversion

Recent Progress in Multi GPU Conjugate Gradient Convertor with Staggered Fermion

Lattice Gauge Theory and Applications to Nuclear Physics

...Coffee Break / Group Photo

Status and Prospects for Some MILC and Fermilab/MILC Projects

Recent Progress in Calculating B_K using Staggered Fermions

Decaying Pseudoscalars from DWF LQCD

Computing the K_L - K_S Mass Difference in Lattice QCD

.Lunch Break

Prospects for the Computation of Rare Kaon Decay Amplitudes

Kaon Semileptonic Decays in QCD with Exact Chiral Symmetry

..Coffee Break

\section{Chair: Taku Izubuchi}

15:30 - 16:30

$16: 30-16: 40$

SAKAI, Yoshihide

Physics Prospects and Status of SuperKEKB/Belle II Break

\section{Chair: Jonathan Flynn}

16:40 - 17:00

$17: 00-17: 20$

$17: 20-17: 40$

$17: 40-18: 00$

KELLY, Christopher

WITZEL, Oliver

AUBIN, Christopher

ZHOU, Ran

Progress Towards Delta I=1/2 K to pi pi Decays with G-parity Boundary Conditions B-physics with Domain-wall Light and Relativistic Heavy Quarks

An Approach to Non-leptonic B-decays on the Lattice

B to Kll and B K\gamma Decay Form Factors from Three-flavor Lattice QCD

$19: 00-20: 30$

.Workshop Dinner (Brookhaven Center, South Room) 


\section{Wednesday, May 16, 2012}

\section{Chair: Tom Blum}

08:00 - 08:30....

08:30 - 09:10

09:10 - 09:30

09:30 - 09:50

09:50 - 10:10

10:10 - 10:40

\section{Chair: Michael Creutz}

10:40 - 11:00

$11: 00-11: 20$

$11: 20-11: 50$

$11: 50-12: 20$

$12: 20-13: 50$

\section{Chair: Hideo Matsufuru}

13:50 - 14:20

$14: 20-14: 50$

$14: 50-15: 20$

$15: 20-15: 40$

$15: 40-16: 10$

JANSEN, Karl

KURAMASHI, Yoshinobu

CHIU, Ting-Wai

BORICI, Artan

\section{Chair: Maarten Golterman}

16:10 - 16:40

$16: 40-17: 00$

$17: 00-17: 20$

$17: 20-17: 40$

$17: 40-18: 00$

18:00 - 18:02
HASENFRATZ, Anna

AOKI, Yasumichi

LIN, Meifeng

YAMADA, Norikazu

JIN, Xiao-Yong

ISHIKAWA, Tomomi
...Coffee / Light Snacks

Storage Ring Electric Dipole Moment Experiment for the Proton

Nucleon EDM from Lattice QCD

Medium-Modification Effects on Pion Structure

Axial Couplings and Strong Decay Widths of Heavy Hadrons

..Coffee Break

Exploring Three-Nucleon Forces in Lattice QCD

Light Nuclei from Quenched Lattice QCD

Progress with Overlap Fermions

Lattice Radial Quantization

..Lunch Break

Status of Four Flavor Simulations using Maximally Twisted Mass Fermions

1+1+1 Flavor QCD+QED Simulation at the Physical Point

Optimal Domain-Wall Fermions

Chiral Fermions via Energy Level Splitting

Coffee Break

A Novel Phase with Many Flavors of Staggered Fermions

Exploring for Technicolor from QCD

Many-flavor Domain Wall Fermions and Fixed Topology

Exploring Many Flavor QCD with Wilson Fermion

Exploring the Phases of QCD with Many Flavors

Closing 


\section{New Horizons for Lattice Computations with Chiral Fermions}

\begin{tabular}{|c|c|c|}
\hline Name & Affiliation & E-Mail Address \\
\hline Sinya Aoki & University of Tskuba & saoki@het.ph.tsukuba.ac.jp \\
\hline Yasumichi Aoki & KMI, Nagoya University & yaoki@kmi.nagoya-u.ac.jp \\
\hline Rudy Arthur & University of Edinburgh & R.Arthur@sms.ed.ac.uk \\
\hline Christopher Aubin & Fordham University & caubin@fordham.edu \\
\hline Claude Bernard & Washington University, St. Louis & cb@wustl.edu \\
\hline Tom Blum & University of CT & tblum@phys.uconn.edu \\
\hline Artan Borici & University of Tirana & borici@proteus.al \\
\hline Peter Boyle & University of Edinburgh & paboyle@ph.ed.ac.uk \\
\hline Richard Brower & Boston University & brower@bu.edu \\
\hline Ting-Wai Chiu & National Taiwan University & twchiu@phys.ntu.edu.tw \\
\hline Norman Christ & Columbia University & nhc@phys.columbia.edu \\
\hline Saul Cohen & University of Washington & sdcohen@uw.edu \\
\hline Guido Cossu & KEK High Energy Phy Res Center & neo.cossu@gmail.com \\
\hline Michael Creutz & $\mathrm{BNL}$ & mike@latticeguy.net \\
\hline Takumi Doi & RIKEN & doi@ribf.riken.jp \\
\hline Robert Edwards & JLab & edwards@jlab.org \\
\hline Xu Feng & KEK & pkufengxu@gmail.com \\
\hline Zoltan Fodor & University of Wuppertal & fodor@bodri.elte.hu \\
\hline Jonathan Flynn & University of Southampton & j.m.flynn@soton.ac.uk \\
\hline Leonardo Giusti & University of Milano-Bicocca & Leonardo.Giusti@cern.ch \\
\hline Maarten Golterman & San Francisco State University & maarten@stars.sfsu.edu \\
\hline Anna Hasenfratz & University of Colorado & anna@eotvos.colorado.edu \\
\hline Shoji Hashimoto & KEK & shoji.hashimoto@kek.jp \\
\hline Urs Heller & American Physical Society & heller@aps.org \\
\hline Tomomi Ishikawa & RBRC & tomomi@quark.phy.bnl.gov \\
\hline Taku Izubuchi & RBRC \& BNL & izubuchi@bnl.gov \\
\hline Karl Jansen & NIC, DESY & karl.jansen@desy.de \\
\hline Xiao-Yong Jin & RIKEN AICS & xjin@riken.jp \\
\hline Chulwoo Jung & BNL & chulwoo@bnl.gov \\
\hline Takashi Kaneko & KEK & takashi.kaneko@kek.jp \\
\hline Christopher Kelly & Columbia University & giltirn@gmail.com \\
\hline Hyung-Jin Kim & BNL & windy510@gmail.com \\
\hline Yoshinobu Kuramashi & University of Tsukuba & kuramasi@het.ph.tsukuba.ac.jp \\
\hline Julius Kuti & U.C. San Diego & jkuti@ucsd.edu \\
\hline Weonjong Lee & Seoul National University & wlee@snu.ac.kr \\
\hline Christoph Lehner & RBRC & clehner@bnl.gov \\
\hline Matthew Lightman & Washington University & mlightman@physics.wustl.edu \\
\hline C.-J. David Lin & National Chiao-Tung University & dlin1971@gmail.com \\
\hline Huey-Wen Lin & University of Washington & hwlin@phys.washington.edu \\
\hline Meifeng Lin & Yale University & meifeng.lin@yale.edu \\
\hline Zhongjie Lin & Columbia University & jasper@phys.columbia.edu \\
\hline Keh-Fei Liu & University of Kentucky & liu@pa.uky.edu \\
\hline Andrew Lytle & University of Southampton & atlytle@gmail.com \\
\hline Paul Mackenzie & FermiLab & mackenzie@fnal.gov \\
\hline Hideo Matsufuru & KEK & hideo.matsufuru@kek.jp \\
\hline Robert Mawhinney & Columbia University & rdm@physics.columbia.edu \\
\hline
\end{tabular}




\begin{tabular}{|c|c|c|}
\hline \multicolumn{3}{|c|}{ New Horizons for Lattice Computations with Chiral Fermions } \\
\hline \multicolumn{3}{|c|}{ Registered Participants } \\
\hline Name & Affiliation & E-Mail Address \\
\hline Stefan Meinel & College of William and Mary & smeinel@wm.edu \\
\hline Tatsuhiro Misumi & BNL & tatsuhiromisumi@gmail.com \\
\hline Swagato Mukherjee & BNL & swagato@bnl.gov \\
\hline Rajamani Narayanan & Florida International University & rajamani.narayanan@fiu.edu \\
\hline Herbert Neuberger & Rutgers University & NEUBERG@PHYSICS.RUTGERS.EDU \\
\hline Junichi Noaki & KEK & noaki@post.kek.jp \\
\hline Shigemi Ohta & KEK \& RBRC & shigemi.ohta@kek.jp \\
\hline Kostas Orginos & College of William and Mary & kostas@wm.edu \\
\hline Santiago Peris & SFSU/UAB & peris@ifae.es \\
\hline Frank Quarant & BNL & quarant@bnl.gov \\
\hline David Richards & JLab & $\operatorname{dgr} @$ jlab.org \\
\hline Bradley Roberts & Boston University & roberts@bu.edu \\
\hline Christopher Sachrajda & University of Southampton & cts@soton.ac.uk \\
\hline Yoshihide Sakai & KEK & Yoshihide.Sakai@kek.jp \\
\hline Stefan Schaefer & CERN & stefan.schaefer@cern.ch \\
\hline Yannis Semertzidis & BNL & yannis@bnl.gov \\
\hline Yigal Shamir & Tel Aviv University & shamir@post.tau.ac.il \\
\hline Eigo Shintani & RBRC & shintani@riken.jp \\
\hline Amarjit Soni & BNL & adlersoni@gmail.com \\
\hline Ruth Van de Water & BNL & ruthv@bnl.gov \\
\hline Chip Watson & Thomas Jefferson Nat'l Accelerator Facility & watson@jlab.org \\
\hline Oliver Witzel & Boston University & owitzel@bu.edu \\
\hline Norikazu Yamada & KEK & norikazu.yamada@kek.jp \\
\hline Takeshi Yamazaki & KMI, Nagoya Univ & yamazaki@kmi.nagoya-u.ac.jp \\
\hline Hantao Yin & Columbia University & yinnht@phys.columbia.edu \\
\hline Jianglei Yu & Columbia University & physhark@gmail.com \\
\hline Daiqian Zhang & Columbia University & dz2203@columbia.edu \\
\hline Ran Zhou & Indiana University & ZHOURAN123@GMAIL.COM \\
\hline
\end{tabular}




\section{RBRC Workshop Proceedings}

Volume 108 - Hyperon-Hyperon Interactions and Searches for Exotic Di-Hyperons in Nuclear Collisions, February 29 - March 2, 2012 - BNL-97035-2012

Volume 107 - Future Directions in High Energy QCD, October 20-22, 2011 - BNL-98119-2011

Volume 106 - Fluctuations, Correlations and RHIC Low Energy Runs, October 3-5, 2011 - BNL-96514-2011

Volume 105 - Opportunities for Polarized He-3 in RHIC and EIC, September 28-30, 2011 - BNL-96418-2011

Volume 104 - Brookhaven Summer Program on Quarkonium Production in Elementary and Heavy Ion Collisions, June 6-18, 2011 - BNL-96171-2011

Volume 103 - Opportunities for Drell-Yan Physics at RHIC, May 11-13, 2011 - BNL-95236-2011-2011

Volume 102 - Initial State Fluctuations and Final-State Particle Correlations, February 2-4, 2011 - BNL-94704-2011

Volume 101 - RBRC Scientific Review Committee Meeting, October 27-29, 2010 - BNL-94589-2011

Volume 100 - Summer Program on Nucleon Spin Physics, July 14-27, 2010 - BNL-96163-2011

Volume 99 - The Physics of W and Z Bosons, June 24-25, 2010 - BNL-94287-2010

Volume 98 - Saturation, the Color Glass Condensate and the Glasma: What Have we Learned from RHIC? - May 10-12, 2010 - BNL-94271-2010

Volume 97 - RBRC Scientific Review Committee Meeting, October 21-22, 2009 - BNL-90674-2009

Volume 96 - P- and CP-Odd Effects in Hot and Dense Matter, April 26-30, 2010 - BNL-94237-2010

Volume 95 - Progress in High-pT Physics at RHIC, March 17-19, 2010 - BNL-94214-2010

Volume 94 - Summer Program on Nucleon Spin Physics at LBL, June 1-12, 2009

Volume 93 - PHENIX Spinfest School 2009 at BNL - July 1-31, 2009. BNL-90343-2009 Link: PHENIXSpinfestSchool2009@,BNL

Volume 92 - PKU-RBRC Workshop on Transverse Spin Physics, June 30 - July 4, 2008, Beijing, China - BNL-81685-2008

Volume 91 - RBRC Scientific Review Committee Meeting, November 17-18, 2008 - BNL-81556-2008

Volume 90 - PHENIX Spinfest School 2008 at BNL, August 4-8, 2008 - BNL-81478-2008

Volume 89 - Understanding QGP through Spectral Functions and Euclidean Correlators, April 23-25, 2008 - BNL-813182008

Volume 88 - Hydrodynamics in Heavy Ion Collisions and QCD Equation of State, April 21-22, 2008 - BNL-81307-2008

Volume 87 - RBRC Scientific Review Committee Meeting, November 5-6, 2007 - BNL-79570-2007

Volume 86 - Global Analysis of Polarized Parton Distributions in the RHIC Era, October 8, 2007 - BNL-79457-2007

Volume 85 - Parity-Violating Spin Asymmetries at RHIC-BNL, April 26-27, 2007 - BNL-79146-2007

Volume 84 - Domain Wall Fermions at Ten Years, March 15-17, 2007 - BNL 77857-2007

Volume 83 - QCD in Extreme Conditions, July 31 - August 2, 2006 - BNL-76933-2006

Volume 82 - RHIC Physics in the Context of the Standard Model, June 18-23, 2006 - BNL-76863-2006

Volume 81 - Parton Orbital Angular Momentum (Joint RBRC/University of New Mexico Workshop) February 24-26, 2006 BNL-75937-2006

Volume 80 - Can We Discover the QCD Critical Point at RHIC?, March 9-10, 2006 - BNL-75692-2006

Volume 79 - Strangeness in Collisions, February 16-17, 2006 - BNL-79763-2008

Volume 78 - Heavy Flavor Productions and Hot/Dense Quark Matter, December 12-14, 2005 - BNL-76915-2006

Volume 77 - RBRC Scientific Review Committee Meeting, October 10-12, 2005 - BNL-52649-2005

Volume 76 - Odderon Searches at RHIC, September 27-29, 2005 - BNL-75092-2005

Volume 75 - Single Spin Asymmetries, June 1-3, 2005 - BNL-74717-2005

Volume 74 - RBRC QCDOC Computer Dedication and Symposium on RBRC QCDOC, May 26, 2005 - BNL-74813-2005

Volume 73 - Jet Correlations at RHIC, March 10-11, 2005 - BNL-73910-2005

Volume 72 - RHIC Spin Collaboration Meetings XXXI (January 14, 2005), XXXII (February 10, 2005), XXXIII (March 11, 2005) - BNL-73866-2005

Volume 71 - Classical and Quantum Aspects of the Color Glass Condensate - BNL-73793-2005

Volume 70 - Strongly Coupled Plasmas: Electromagnetic, Nuclear \& Atomic - BNL-73867-2005

Volume 69 - RBRC Scientific Review Committee - BNL-73546-2004

Volume 68 - Workshop on the Physics Programme of the RBRC and UKQCD QCDOC Machines - BNL-73604-2004

Volume 67 - High Performance Computing with BlueGene/L and QCDOC Architectures

Volume 66 - RHIC Spin Collaboration Meeting XXIX, October 8-9, 2004, Torino Italy - BNL-73534-2004

Volume 65 - RHIC Spin Collaboration Meetings XXVII (July 22, 2004), XXVIII (September 2, 2004), XXX (December 6, 2004) - BNL-73506-2004

Volume 64 - Theory Summer Program on RHIC Physics - BNL-73263-2004 
Volume 63 - RHIC Spin Collaboration Meetings XXIV (May 21, 2004), XXV (May 27, 2004), XXVI (June 1, 2004) - BNL72397-2004

Volume 62 - New Discoveries at RHIC, May 14-15, 2004 - BNL- 72391-2004

Volume 61 - RIKEN-TODAI Mini Workshop on "Topics in Hadron Physics at RHIC", March 23-24, 2004 - BNL-723362004

Volume 60 - Lattice QCD at Finite Temperature and Density - BNL-72083-2004

Volume 59 - RHIC Spin Collaboration Meeting XXI (January 22, 2004), XXII (February 27, 2004), XXIII (March 19, 2004)BNL-72382-2004

Volume 58 - RHIC Spin Collaboration Meeting XX - BNL-71900-2004

Volume 57 - High pt Physics at RHIC, December 2-6, 2003 - BNL-72069-2004

Volume 56 - RBRC Scientific Review Committee Meeting - BNL-71899-2003

Volume 55 - Collective Flow and QGP Properties - BNL-71898-2003

Volume 54

Volume 53

Volume 52

Volume 51

Volume 50

Volume 49

Volume 48

Volume 47

Volume 46

Volume 45

Volume 44

Volume 43

Volume 42

Volume 41

Volume 40

Volume 39

Volume 38

Volume 37

Volume 36

Volume 35

- RHIC Spin Collaboration Meetings XVII, XVIII, XIX - BNL-71751-2003

- Theory Studies for Polarized pp Scattering - BNL-71747-2003

- RIKEN School on QCD "Topics on the Proton" - BNL-71694-2003

- RHIC Spin Collaboration Meetings XV, XVI - BNL-71539-2003

- High Performance Computing with QCDOC and BlueGene - BNL-71147-2003

- RBRC Scientific Review Committee Meeting - BNL-52679

- RHIC Spin Collaboration Meeting XIV - BNL-71300-2003

- RHIC Spin Collaboration Meetings XII, XIII - BNL-71118-2003

- Large-Scale Computations in Nuclear Physics using the QCDOC - BNL-52678

- Summer Program: Current and Future Directions at RHIC - BNL-71035

- RHIC Spin Collaboration Meetings VIII, IX, X, XI - BNL-71117-2003

- RIKEN Winter School - Quark-Gluon Structure of the Nucleon and QCD - BNL-52672

- Baryon Dynamics at RHIC - BNL-52669

- Hadron Structure from Lattice QCD - BNL-52674

- Theory Studies for RHIC-Spin - BNL-52662

- RHIC Spin Collaboration Meeting VII - BNL-52659

- RBRC Scientific Review Committee Meeting - BNL-52649

- RHIC Spin Collaboration Meeting VI (Part 2) - BNL-52660

- RHIC Spin Collaboration Meeting VI - BNL-52642

- RIKEN Winter School - Quarks, Hadrons and Nuclei - QCD Hard Processes and the Nucleon Spin - BNL52643

Volume 34 - High Energy QCD: Beyond the Pomeron - BNL-52641

Volume 33 - Spin Physics at RHIC in Year-1 and Beyond - BNL-52635

Volume 32 - RHIC Spin Physics V - BNL-52628

Volume 31 - RHIC Spin Physics III \& IV Polarized Partons at High Q^2 Region - BNL-52617

Volume 30 - RBRC Scientific Review Committee Meeting - BNL-52603

Volume 29 - Future Transversity Measurements - BNL-52612

Volume 28 - Equilibrium \& Non-Equilibrium Aspects of Hot, Dense QCD - BNL-52613

Volume 27 - Predictions and Uncertainties for RHIC Spin Physics \& Event Generator for RHIC Spin Physics III - Towards Precision Spin Physics at RHIC - BNL-52596

Volume 26

Volume 25

Volume 24

- Circum-Pan-Pacific RIKEN Symposium on High Energy Spin Physics - BNL-52588

- RHIC Spin - BNL-52581

- Physics Society of Japan Biannual Meeting Symposium on QCD Physics at RIKEN BNL Research Center BNL-52578

Volume 23

Volume 22

Volume 21

Volume 20

Volume 19

Volume 18

Volume 17

Volume 16

Volume 15

Volume 14

Volume 13

- Coulomb and Pion-Asymmetry Polarimetry and Hadronic Spin Dependence at RHIC Energies - BNL-52589

- OSCAR II: Predictions for RHIC - BNL-52591

- RBRC Scientific Review Committee Meeting - BNL-52568

- Gauge-Invariant Variables in Gauge Theories - BNL-52590

- Numerical Algorithms at Non-Zero Chemical Potential - BNL-52573

- Event Generator for RHIC Spin Physics - BNL-52571

- Hard Parton Physics in High-Energy Nuclear Collisions - BNL-52574

- RIKEN Winter School - Structure of Hadrons - Introduction to QCD Hard Processes - BNL-52569

- QCD Phase Transitions - BNL-52561

- Quantum Fields In and Out of Equilibrium - BNL-52560

Volume 12

- Physics of the 1 Teraflop RIKEN-BNL-Columbia QCD Project First Anniversary Celebration - BNL-66299

- Quarkonium Production in Relativistic Nuclear Collisions - BNL-52559 
Volume 11 - Event Generator for RHIC Spin Physics - BNL-66116

Volume 10 - Physics of Polarimetry at RHIC - BNL-65926

Volume 9 - High Density Matter in AGS, SPS and RHIC Collisions - BNL-65762

Volume 8 - Fermion Frontiers in Vector Lattice Gauge Theories - BNL-65634

Volume 7 - RHIC Spin Physics - BNL-65615

Volume 6 - Quarks and Gluons in the Nucleon - BNL-65234

Volume 5 - Color Superconductivity, Instantons and Parity (Non?)-Conservation at High Baryon Density - BNL-65105

Volume 4 - Inauguration Ceremony and Non-Equilibrium Many Body Dynamics - BNL-64912

Volume 3 - Hadron Spin-Flip at RHIC Energies - BNL-64724

Volume 2 - Perturbative QCD as a Probe of Hadron Structure - BNL-64723

Volume 1 - Open Standards for Cascade Models for RHIC - BNL-64722 


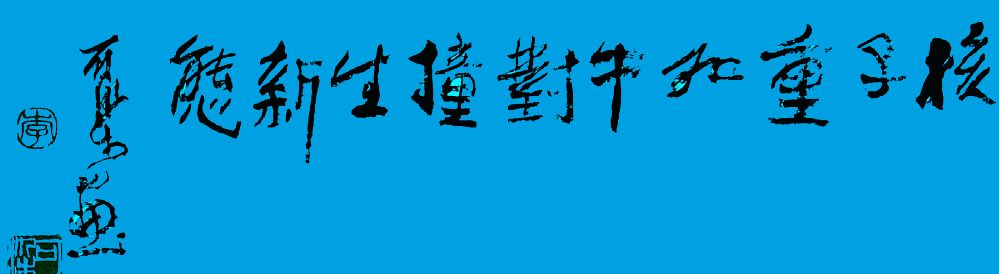

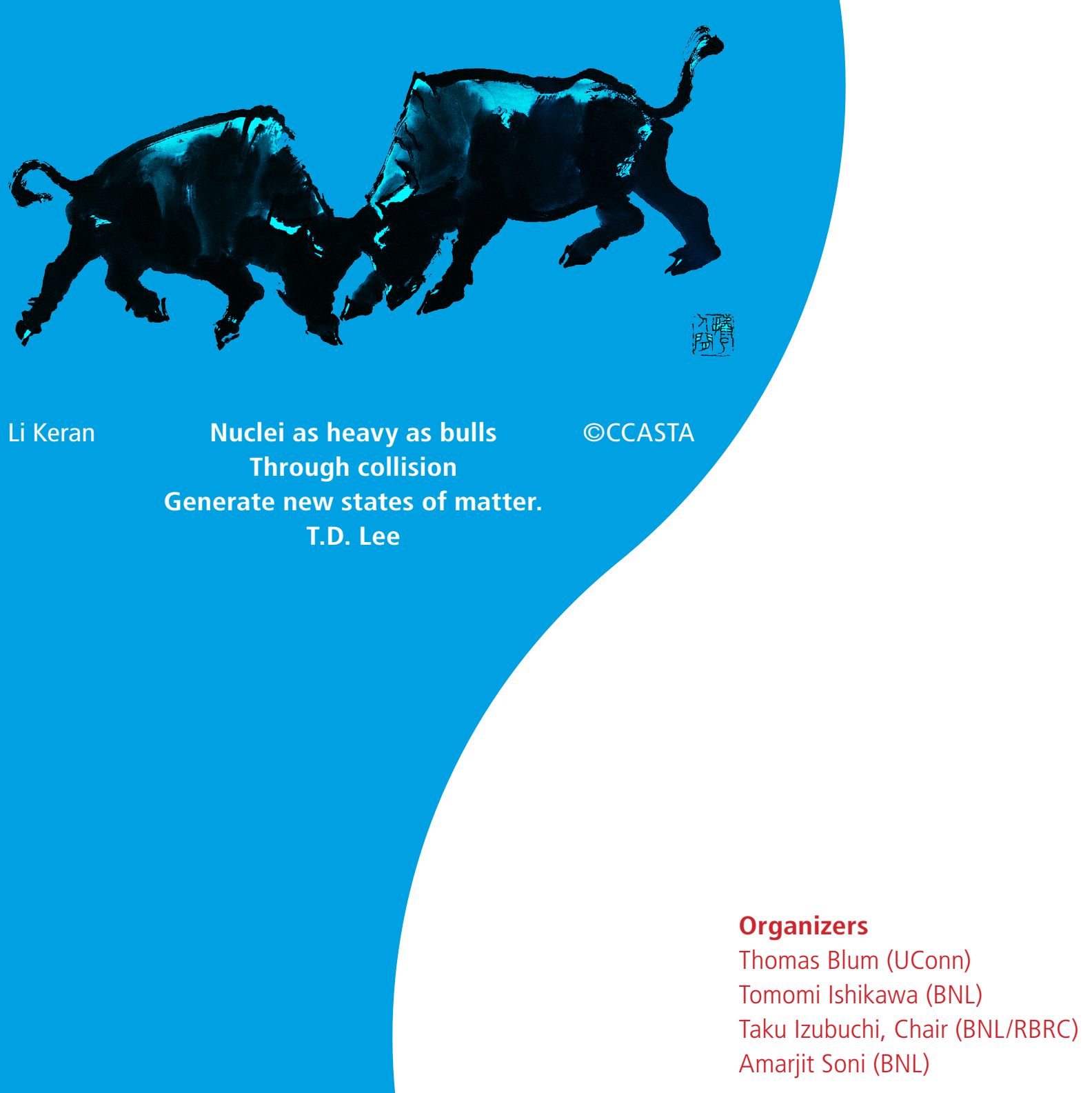

